PNNL-18723

WTP-RPT-192, Rev 0

\title{
Pretreatment Engineering Platform (PEP) Integrated Test B Run Report-Caustic and Oxidative Leaching in UFP-VSL-T02A
}
JGH Geeting
EC Golovich
DE Kurath
OP Bredt
CE Guzman-Leong
GJ Sevigny
CA Burns
GB Josephson
RL Aaberg

December 2009

Pacific Northwest

NATIONAL LABORATORY

Proudly Operated by Battelle Since 1965 
PNNL-18723

WTP-RPT-192, Rev 0

\section{Pretreatment Engineering Platform (PEP) Integrated Test B Run Report- Caustic Leaching and Oxidative Leaching in UFP-VSL-T02A}
JGH Geeting
EC Golovich
DE Kurath
OP Bredt
CE Guzman-Leong
GJ Sevigny
CA Burns
GB Josephson
RL Aaberg

December 2009

Test Specification: 24590-PTF-TSP-RT-07-001 Rev 2

Work Authorization: WA\# 2007-024

Test Plan: TP-RPP-WTP-506, Rev. 0.4; TP-WTP-PEP-044, Rev 0.2

Test Exceptions: 24590-PTF-TEF-RT-08-00002

24590-WTP-TEF-RT-09-00003

24590-PTF-TEF-RT-09-00001

24590-WTP-TEF-RT-09-00002 Rev 0

24590-PTF-TEF-RT-09-00001 Rev 1

R\&T focus area: Pretreatment

Test Scoping Statement(s): NA

Prepared for

the U.S. Department of Energy

under Contract DE-AC05-76RL01830

Pacific Northwest National Laboratory

Richland, Washington 99352 


\section{Completeness of Testing}

This report describes the results of work and testing specified by Test Specification 24590-PTF-TSP-RT-07-001 Rev 2 "Pretreatment Engineering Platform (PEP) Testing (Phase 1)" and Test Plans TP-RPP-WTP-506 Rev 0.4 "Pretreatment Engineering Platform (PEP) Testing (Phase 1) and TP-WTP-PEP-044 Rev 0.2" Test Plan for the PEP Parallel Laboratory Testing." The work and any associated testing followed the quality assurance requirements outlined in the Test Specification/Plan. The descriptions provided in this test report are an accurate account of both the conduct of the work and the data collected. Test Plan results are reported. Also reported are any unusual or anomalous occurrences that are different from expected results. The test results and this report have been reviewed and verified.

\section{Approved:}

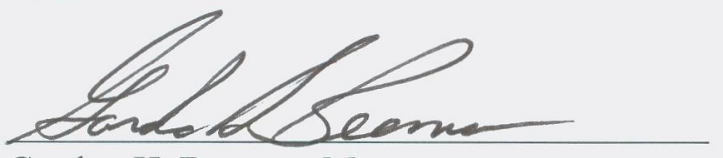

Gordon H. Beeman, Manager WTP R\&T Support Project

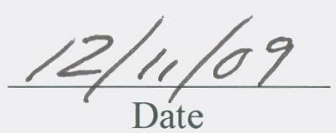




\section{Contents}

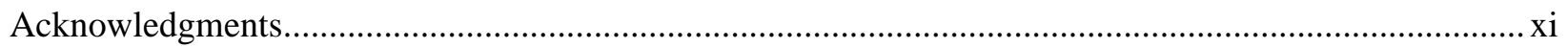

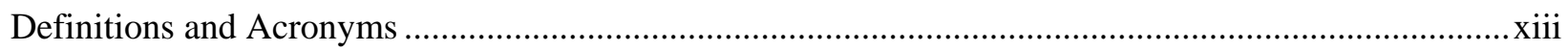

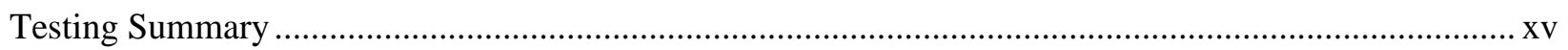

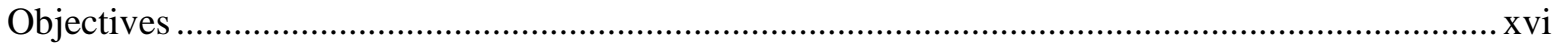

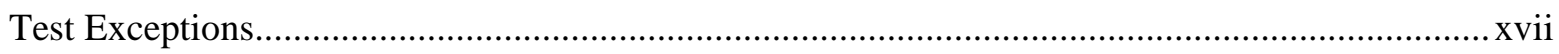

Results and Performance Against Success Criteria ....................................................................... Xx

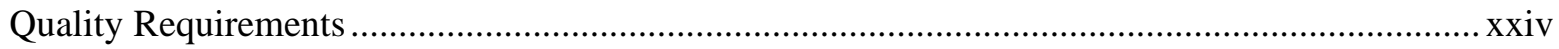

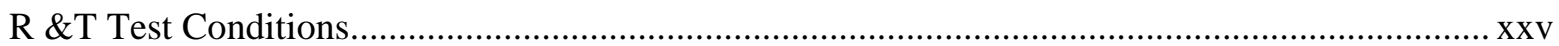

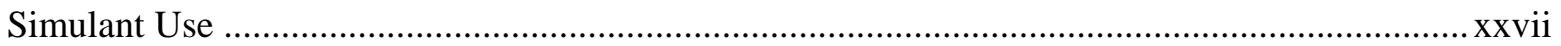

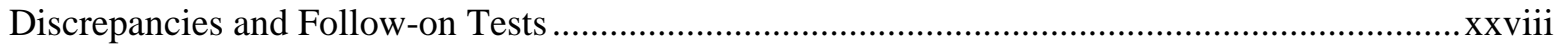

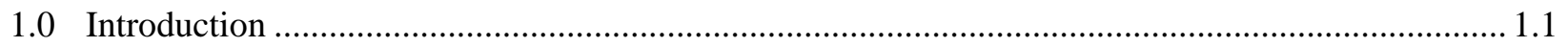

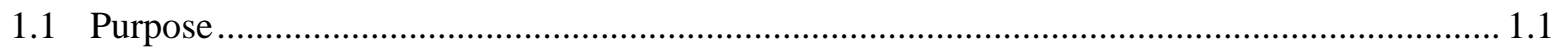

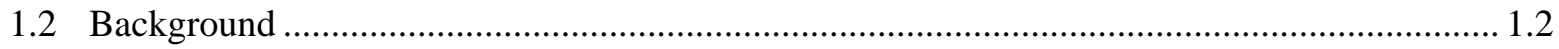

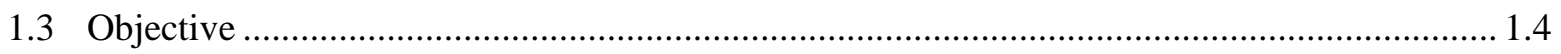

2.0 Quality Assurance Requirements ........................................................................................ 2.1

3.0 Experimental Methods and Apparatus.......................................................................................... 3.1

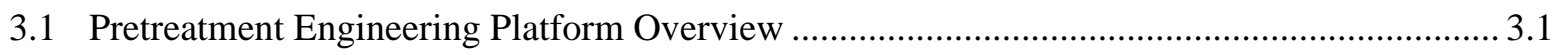

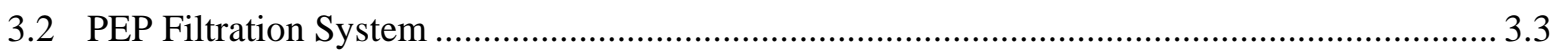

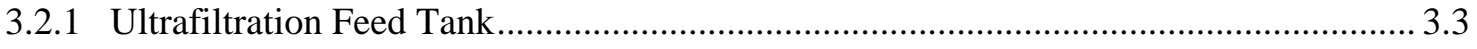

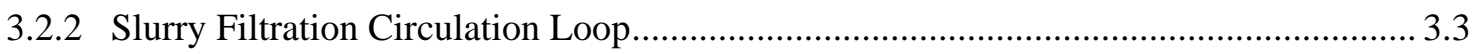

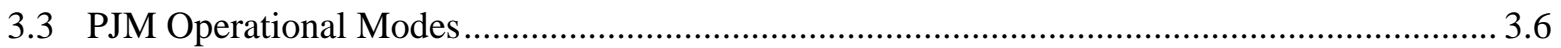

3.3.1 Standard Mode (Regular) Description ….................................................................... 3.7

3.3.2 Standard Mode-Short Cycle ................................................................................... 3.8

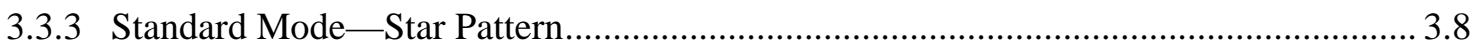

3.3.4 Simple PJM Mode Description .................................................................................... 3.9

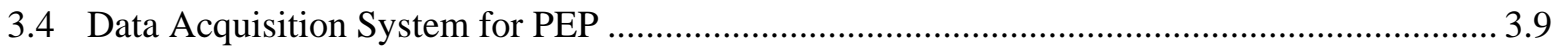

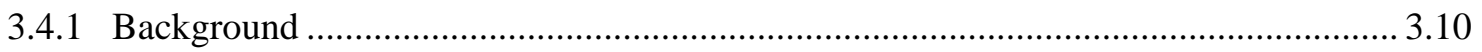

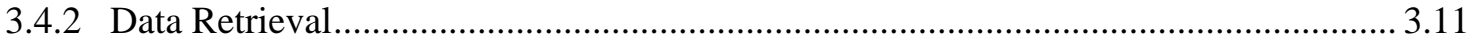

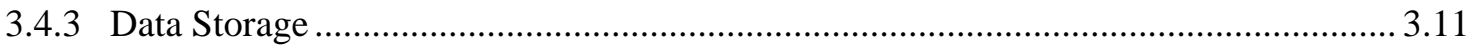

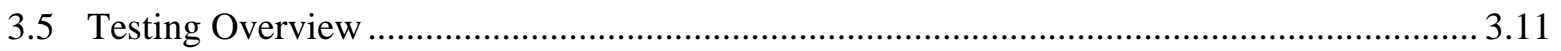

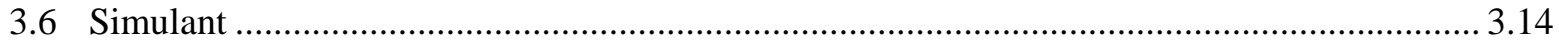

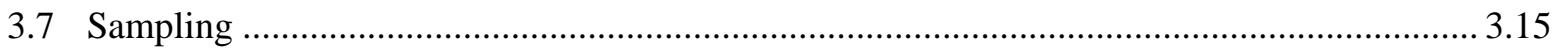

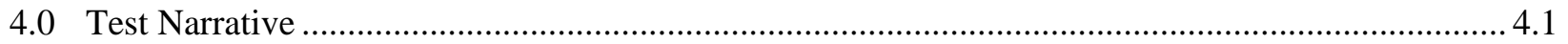

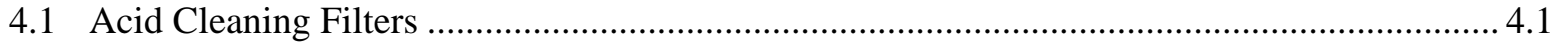

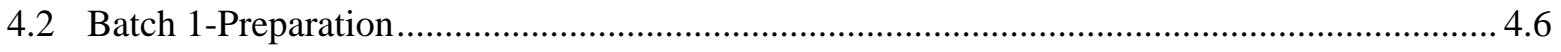

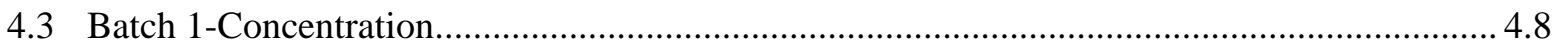




\section{Contents}

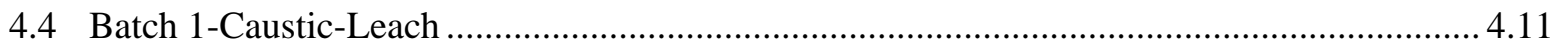

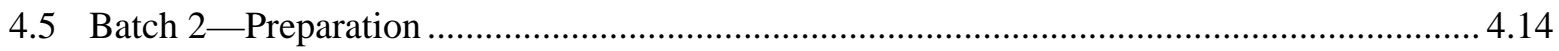

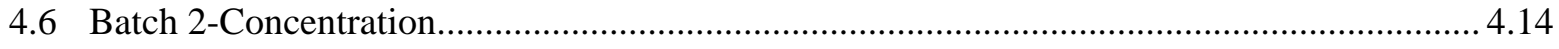

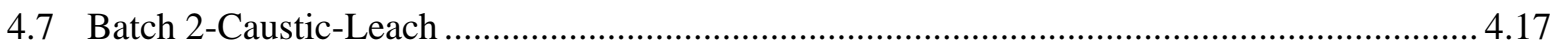

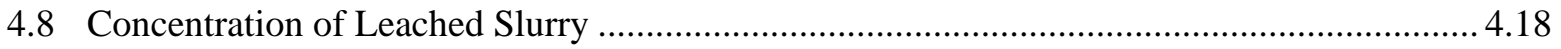

4.9 Filter-Loop Bypass Test with Cs Br Tracer ..........................................................................23

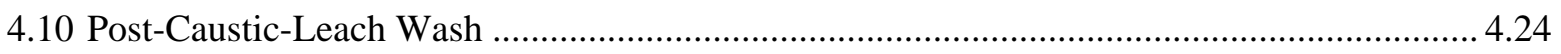

4.11 Chromium Slurry Addition, Concentration, and Wash ........................................................ 4.27

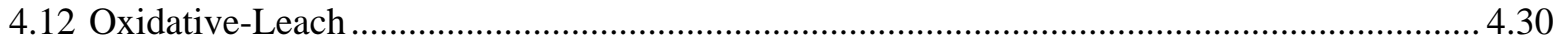

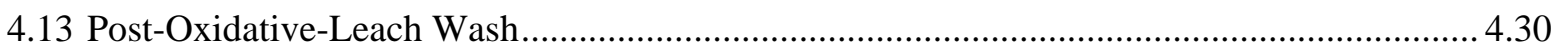

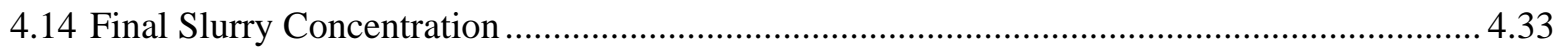

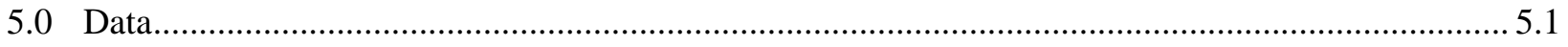

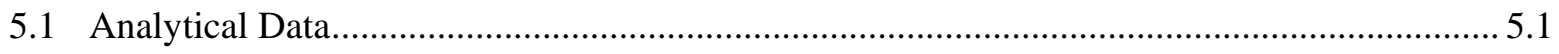

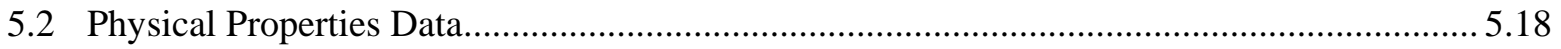

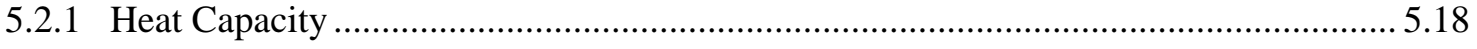

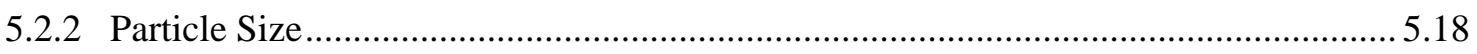

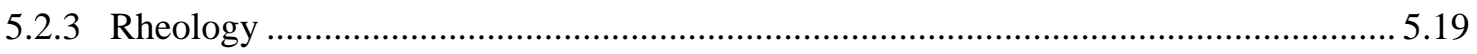

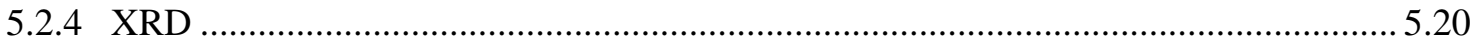

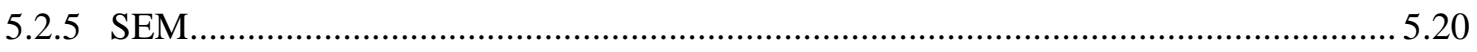

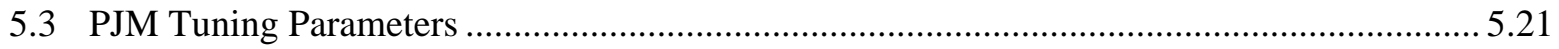

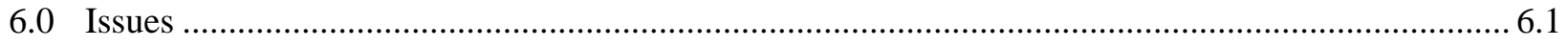

6.1 Maintaining Targeted Flow Rate in Filter-Loop ............................................................... 6.1

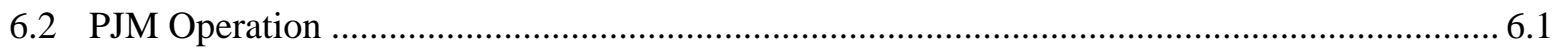

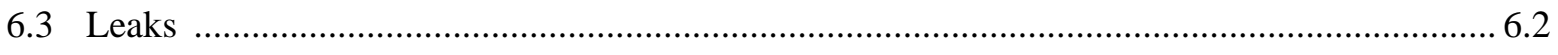

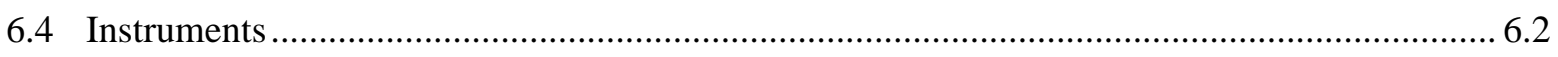

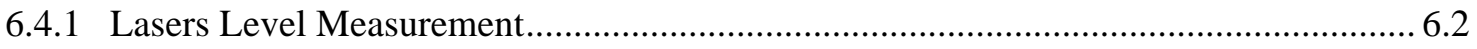

6.4.2 Bubblers Level/Density Measurement ........................................................................... 6.3

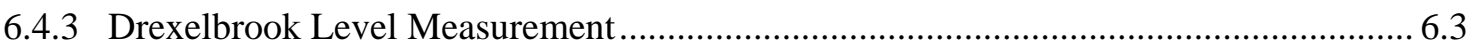

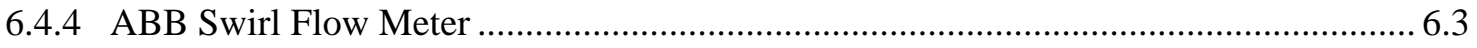

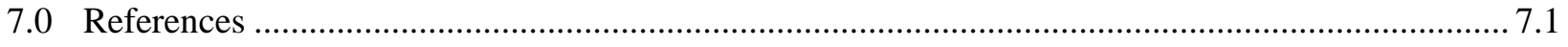

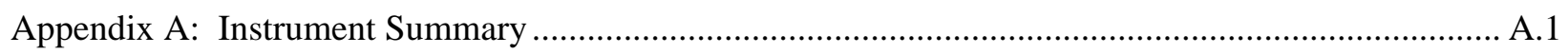

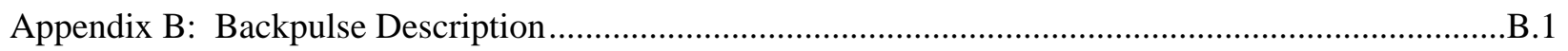

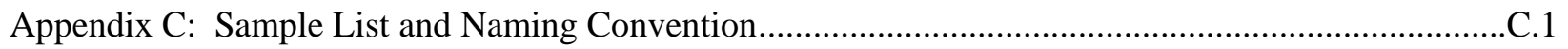

Appendix D: Coriolis Sampler Elevations ........................................................................................... D.1

Appendix E: Sample Processing and Analytical Methods........................................................................ 1

Appendix F: Evaluation of PJM Peak Average Velocity .........................................................................1 


\section{Contents}

Appendix G: Nitric Acid Cleaning Instructions for the PEP Filters .....................................................

Appendix H: Pump Operating Guidance ....................................................................................... H.1

Appendix I: T02A Systems Operations Guidance for Integrated Test B................................................1

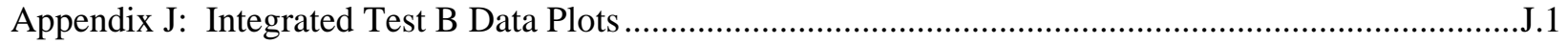

Appendix K: Integrated Test B Summary ........................................................................................... K.1

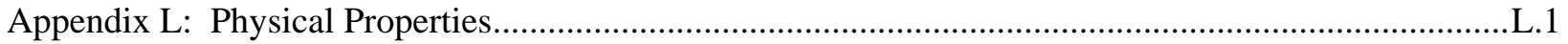

Appendix M: Integrated Test B Operational Process Sheet (Run Sheet) ...............................................1

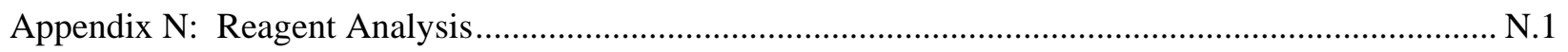




\section{Figures}

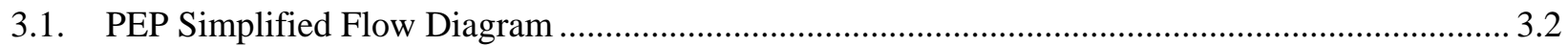

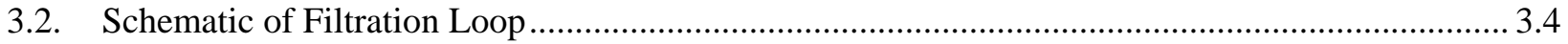

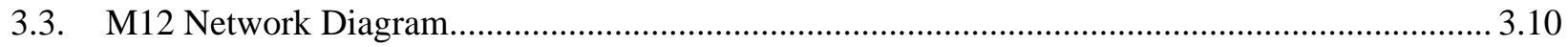

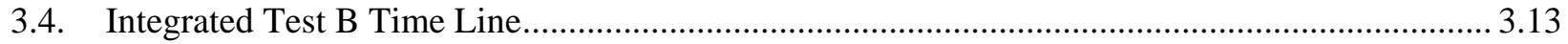

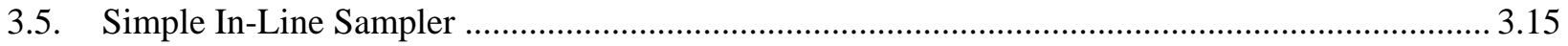

3.6. In-Tank Sampling Showing the Three Radial Positions at Three Heights and Sampling

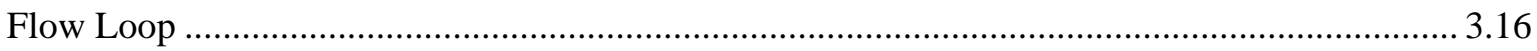

4.1. TMP for the First Filter Cleaning Event, 03/01/09, Preceding Integrated Test B ......................... 4.2

4.2. Axial Velocity Based on Flow Measurements at the Suction to Pump T42A and the Discharge to Pump T43A for the First Filter Cleaning Event, 3/01/09, Preceding Integrated Test B

4.3. Tank T02A Temperature (TTK-0619) for the First Filter Cleaning Event, 3/01/09, Preceding Integrated Test B

4.4. Permeate Production Rate for the First Filter Cleaning Event, 3/01/09, Preceding Integrated Test B

4.5. TMP for the Second Filter Cleaning Event, 3/07/09, Preceding Integrated Test B

4.6. Axial Velocity Based on Flow Measurements at the Suction to Pump T42A and the Discharge to Pump T43A for the Second Filter Cleaning Event, 3/07/09, Preceding Integrated Test B

4.7. Tank T02A Temperature (TTK-0619) for the Second Filter Cleaning Event, 3/07/09, Preceding Integrated Test B

4.8. Permeate Production Rate for the Second Filter Cleaning Event, 3/07/09, Preceding Integrated Test B

4.9. TMP for Pre-Caustic-Leach Concentration, Integrated Test B, Simulant Batch 1.

4.10. Axial Velocity Based on Flow Measurements at the Suction to Pump T42A and the Discharge to Pump T43A for Pre-Caustic-Leach Concentration, Integrated Test B, Simulant Batch 1

4.11. Tank T02A Temperature (TTK-0619) for Pre-Caustic-Leach Concentration, Integrated Test B, Simulant Batch 1

4.12. Permeate Production Rate for Pre-Caustic-Leach Concentration, Integrated Test B, Simulant Batch 1

4.13. Tank T02A Temperature During Batch 1 Caustic-Leach.....

4.14. TMP for Pre-Caustic-Leach Concentration, Integrated Test B, Simulant Batch 2.

4.15. Axial Velocity Based on Flow Measurements at the Suction to Pump T42A and the Discharge to Pump T43A for Pre-Caustic-Leach Concentration, Integrated Test B, Simulant Batch 2 


\section{Figures}

4.16. Tank T02A Temperature (TTK-0619) for Pre-Caustic-Leach Concentration, Integrated Test B, Simulant Batch 2

4.17. Permeate Production Rate for Pre-Caustic-Leach Concentration, Integrated Test B, Simulant Batch 2

4.18. Tank T02A Temperature During Batch 2 Caustic-Leach.............................................................18

4.19. TMP for Post-Caustic-Leach Concentration, Integrated Test B................................................ 4.19

4.20. Axial Velocity Based on Flow Measurements at the Suction to Pump T42A and the Discharge to Pump T43A for Post-Caustic-Leach Concentration, Integrated Test B

4.21. Tank T02A Temperature (TTK-0619) for Post-Caustic-Leach Concentration, Integrated Test B

4.22. Permeate Production Rate for Post-Caustic-Leach Concentration, Integrated Test B

4.23. Tank T02A Level and Filter-Loop Flow Rate During Concentration After Caustic-Leach Batch 2

4.24. Filter-Loop Flow Rate During Tracer Test.

4.25. TMP for Post-Caustic-Leach Washing, Integrated Test B

4.26. Axial Velocity Based on Flow Measurements at the Suction to Pump T42A and the Discharge to Pump T43A for Post-Caustic-Leach Washing, Integrated Test B

4.27. Tank T02A Temperature (TTK-0619) for Post-Caustic-Leach Washing, Integrated Test B........ 4.26

4.28. Permeate Production Rate for Post-Caustic-Leach Washing, Integrated Test B 4.26

4.29. TMP for Concentration and Washing Operations Following Chromium Slurry Addition in Integrated Test B

4.30. Axial Velocity Based on Flow Measurements at the Suction to Pump T42A and the Discharge to Pump T43A for Concentration and Washing Operations Following Chromium Slurry Addition in Integrated Test B.....

4.31. Tank T02A Temperature (TTK-0619) for Concentration and Washing Operations Following Chromium Slurry Addition in Integrated Test B

4.32. Permeate Production Rate for Concentration and Washing Operations Following Chromium Slurry Addition in Integrated Test B

4.33. Tank T02A Prototypic Temperature During Oxidative Leaching.............................................. 4.30

4.34. TMP for Post-Oxidative-Leach Washing, Integrated Test B .................................................... 4.31

4.35. Axial Velocity Based on Flow Measurements at the Suction to Pump T42A and the Discharge to Pump T43A for Post-Oxidative-Leach Washing, Integrated Test B.

4.36. Tank T02A Temperature (TTK-0619) for Post-Oxidative-Leach Washing, Integrated Test B .... 4.32

4.37. Permeate Production Rate for Post-Oxidative-Leach Washing, Integrated Test B.....

5.1. SEM Micrographs 5.21

5.2. Peak Average Velocity in Tank T01A at 11:09 on 3/12/09 5.23

5.3. Peak Average Velocity in Tank T02A at 13:58 on 3/12/09 5.23 


\section{Figures}

5.4. Peak Average Velocity in Tank T02A at $05: 15$ on 3/13/09 ...................................................... 5.24

5.5. Peak Average Velocity in Tank T01B at 14:26 on 3/14/09...................................................... 5.24

5.6. Peak Average Velocity in Tank T01A at 13:07 on 3/13/09 ......................................................... 5.25

5.7. Peak Average Velocity in Tank T02A at 14:08 on 3/14/09 .................................................... 5.25

5.8. Peak Average Velocity in Tank T02A at 21:29 on 3/15/09 ..................................................... 5.26

5.9. Peak Average Velocity in Tank T02A at 10:35 on 3/19/09 ..................................................... 5.26

\section{Tables}

S.1. Summary of Test Objectives and Results .....................................................................................

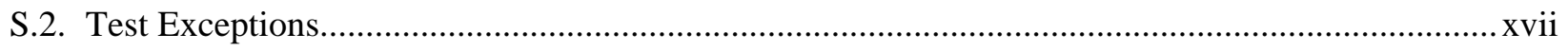

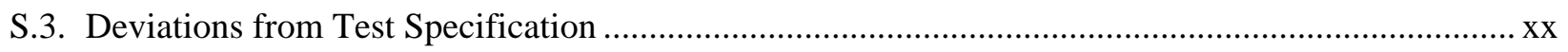

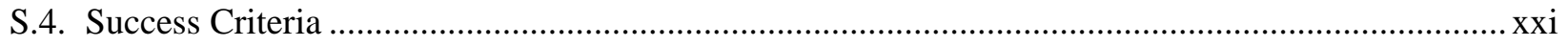

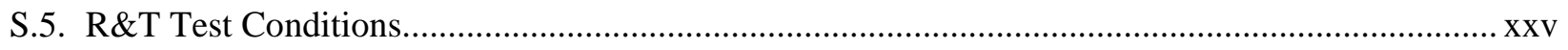

2.1. Description of NCRs Active During Integrated Test B........................................................... 2.2

3.1. Specifications of the Five PEP Cross-Flow Filtration Bundles....................................................... 3.5

3.2. Permeate Metering and Pulse-Pot Configurations for PEP ......................................................... 3.6

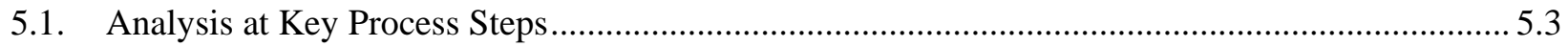

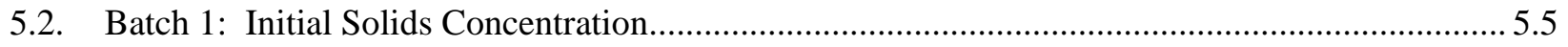

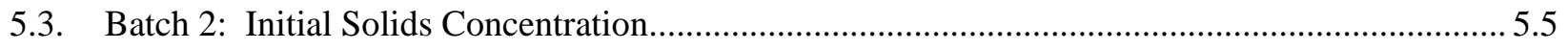

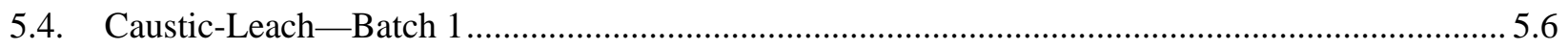

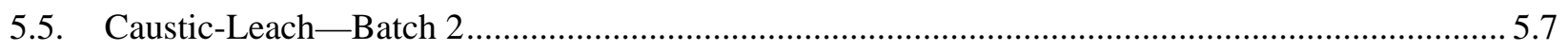

5.6. Post-Caustic-Leach Concentration of Solids ....................................................................... 5.8

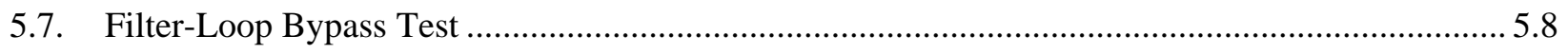

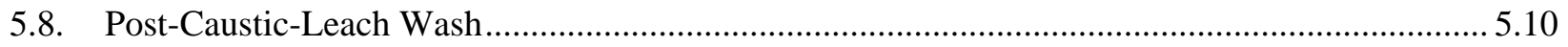

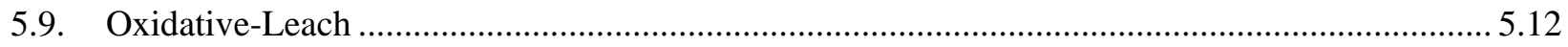

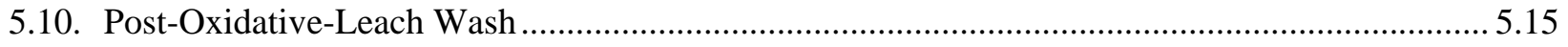

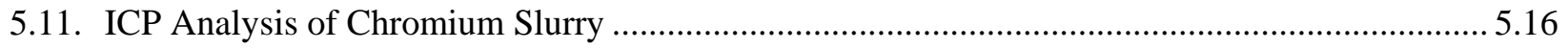

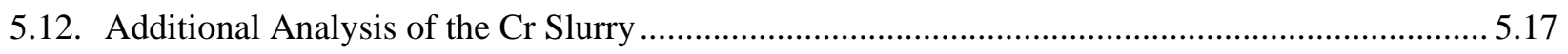

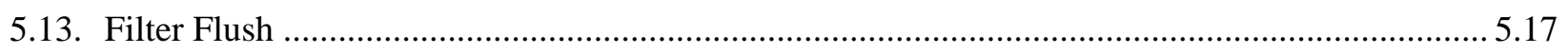

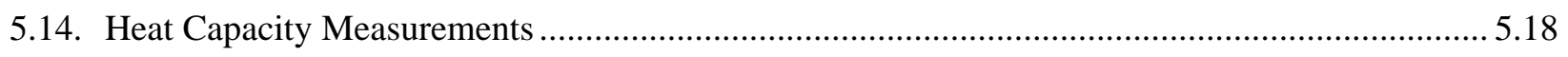

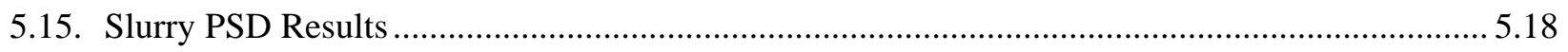

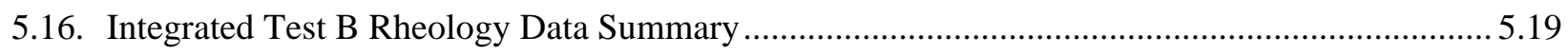

5.17. Integrated Test B Shear Strength Data …................................................................................ 5.20 
5.18. Measured Concentration and Estimated Crystallite Sizes of Identified Phases .......................... 5.20

5.19. PJM Parameters for Integrated Test B (TI-WTP-PEP-066) ......................................................... 5.22 



\section{Acknowledgments}

This report was supported by many dedicated staff involved in laboratory experiments, sampling, data acquisition, and technical reviews. Individuals on the operations and sampling crew are especially recognized for supporting the Pretreatment Engineering Platform Project by working unpredictable, long, and off-hour shifts for the 10 months of PEP operations. The tests and reports could not have gotten this far without their extraordinary effort. The authors gratefully acknowledge the help and support of the following staff:

$\begin{array}{lll}\text { JM Alzheimer } & \text { MS Fountain } & \text { JH Sachs } \\ \text { EJ Antonio } & \text { K Groves } & \text { R Shannon } \\ \text { EBK Baer } & \text { AD Guzman } & \text { SV Shoemaker } \\ \text { R Bang } & \text { SD Halstead } & \text { DS Sklarew } \\ \text { NN Bauman } & \text { GL Harvey } & \text { C Smith } \\ \text { JD Bohlke } & \text { RE Hohimer } & \text { DM Smith } \\ \text { JR Bontha } & \text { DE Hurley } & \text { SA Smith } \\ \text { CF Brown } & \text { S Lehrman } & \text { JJ Soelberg } \\ \text { WC Buchmiller } & \text { ML Kimura } & \text { B Stiver } \\ \text { KJ Cantrell } & \text { SN Kundu } & \text { Y Su } \\ \text { CC Chapman } & \text { JB Lang } & \text { JC Tucker } \\ \text { J Chun } & \text { JS Loveland } & \text { PLJ Valdez } \\ \text { DA Clark } & \text { JR Markillie } & \text { D Vonghalath } \\ \text { E Cordova } & \text { ES Mast } & \text { BE Wells } \\ \text { BP Crume } & \text { K Miller } & \text { A Woodstock } \\ \text { JM Cuta } & \text { DP Mendoza } & \text { S Wright } \\ \text { RL Daubert } & \text { KE Parker } & \text { ST Yokuda } \\ \text { C Dreyer } & \text { RP Pires } & \text { JK Young } \\ \text { EP Dresel } & \text { K Quigley } & \text { L Zhong } \\ \text { BM Dunn } & \text { BJ Riley } & \text { MR Zumhoff } \\ \text { A Edmondson } & \text { CP Rodriguez } & \end{array}$

The authors also thank Wayne Cosby for valuable editorial support, Dave MacPherson and Kirsten Meier for Quality Assurance support throughout the project, Chrissy Charron, Andrea Boehler, and Mona Champion for their administrative support through the months of PEP testing, and Wesley Lawrence, Dean Kurath, James Huckaby, Steve Barnes, John Truax, Donald Bachand, Robert Burk, and Gordon Beeman for their leadership and technical guidance during PEP operations. We also thank Brady Hanson and Michael Minette for the management and coordination of data analysis activities. 



\section{Definitions and Acronyms}

\begin{tabular}{|c|c|}
\hline AFA & antifoam agent \\
\hline ASO & Analytical Support Operations \\
\hline ASR & Analytical Services Request \\
\hline ASME & American Society of Mechanical Engineers \\
\hline BNI & Bechtel National Inc. \\
\hline BS & blank spike \\
\hline $\mathrm{CD}$ & Coriolis densitometer \\
\hline CFD & computational fluid dynamics \\
\hline CFR & Code of Federal Regulations \\
\hline CUF & Cell Unit Filter \\
\hline CXP & cesium ion exchange process system \\
\hline DAS & Data Acquisition System \\
\hline DIW & deionized water \\
\hline DOE & U.S. Department of Energy \\
\hline EFRT & External Flowsheet Review Team \\
\hline FEP & Feed Evaporator Process (vessel) \\
\hline FRP & Feed Receipt Process (vessel) \\
\hline GPC & gel permeation chromatography \\
\hline HDI & $\begin{array}{l}\text { "How Do I...?" System for managing the delivery of laboratory-level policies, } \\
\text { requirements, and procedures. }\end{array}$ \\
\hline HLP & HLW lag storage and feed blending process system \\
\hline HLW & high-level waste \\
\hline HMI & human-machine interface \\
\hline IC & ion chromatography \\
\hline ICP & inductively coupled plasma \\
\hline ICP-AES & inductively coupled plasma-atomic emission spectrometry \\
\hline ICP-MS & inductively coupled plasma-mass spectroscopy \\
\hline IW & inhibited water \\
\hline JTG & Joint Test Group \\
\hline LCS & laboratory control sample \\
\hline LTE & lead test engineer \\
\hline LRB & laboratory record book \\
\hline M\&TE & measuring and test equipment \\
\hline MS & matrix spike \\
\hline NCR & nonconformance report \\
\hline
\end{tabular}




\begin{tabular}{ll} 
PDMS & polydimethylsiloxane \\
PB & preparation blank \\
PDL-W & Process Development Laboratory-West \\
PEP & Pretreatment Engineering Platform \\
P\&ID & piping and instrumentation diagram \\
PJM & pulse-jet mixer \\
PLM & polarized light microscopy \\
PNNL & Pacific Northwest National Laboratory \\
PPG & polypropylene glycol \\
PSD & particle-size distribution \\
PTF & Pretreatment Facility \\
QA & quality assurance \\
QAM & Quality Assurance Manual \\
QAP & Quality Assurance Plan \\
QARD & Quality Assurance Requirements and Descriptions \\
QC & Quality Control \\
Re & Reynolds number \\
R\&T & Research and Technology \\
RPP & River Protection Project \\
RTD & resistance temperature detector \\
SEM & scanning electron microscopy \\
SwRI & Southwest Research Institute \\
TAT & turn around time \\
TDS & total dissolved solids \\
TIC & total inorganic carbon \\
TOC & total organic carbon \\
THF & tetrahydrofuran \\
TMP & transmembrane pressure \\
UDS & undissolved solids \\
UFP & ultrafiltration process \\
VFD & variable frequency drive \\
VSL & vessel \\
wt\% & weight percent \\
Hanford Tank Waste Treatment and Immobilization Plant & \\
XRD & Xiffraction \\
\hline
\end{tabular}




\section{Testing Summary}

Pacific Northwest National Laboratory (PNNL) has been tasked by Bechtel National Inc. (BNI) on the River Protection Project-Hanford Tank Waste Treatment and Immobilization Plant (RPP-WTP) project to perform research and development activities to resolve technical issues identified for the Pretreatment Facility (PTF). The Pretreatment Engineering Platform (PEP) was designed, constructed and operated as part of a plan to respond to issue M12, "Undemonstrated Leaching Processes" of the External Flowsheet Review Team (EFRT) issue response plan. ${ }^{\text {(a) }}$ The PEP is a ${ }^{1} / 4.5$-scale test platform designed to simulate the WTP pretreatment caustic leaching, oxidative leaching, ultrafiltration solids concentration, and slurry washing processes. ${ }^{(b)}$ The PEP replicates the WTP leaching processes using prototypic equipment and control strategies. The PEP also includes nonprototypic ancillary equipment to support the core processing.

Two process flowsheets are currently being evaluated for the ultrafiltration process (UFP) and leaching operations. The baseline flowsheet (Integrated Test A) has caustic leaching conducted in the UFP-1 ultrafiltration feed preparation vessels (i.e., vessels UFP-VSL-T01A and B in the PEP; vessels UFP-VSL-00001A and B [UFP-1] in the WTP PTF). The alternative scenario (Integrated Tests B and D) has caustic leaching performed in the UFP-2 ultrafiltration feed vessels (i.e., vessel UFP-VSL-T02A ${ }^{(\mathrm{c})}$ in the PEP and vessels UFP-VSL-00002A and B [UFP-2] in the WTP PTF).

In Integrated Test $\mathrm{B}$, a $19 \mathrm{M}$ sodium hydroxide solution $(\mathrm{NaOH}$, caustic) was added to the waste slurry in the UFP-VSL-T02A vessel after the solids were concentrated to $20 \%$ undissolved solids. The $\mathrm{NaOH}$ was added to leach solid aluminum compounds (e.g., gibbsite, boehmite). Caustic addition was followed by heating to $98^{\circ} \mathrm{C}$ using direct injection of steam to accelerate the leaching process. Following the caustic leach, the UFP-VSL-T02A vessel contents were cooled using the vessel cooling jacket. The slurry was then concentrated to $17-\mathrm{wt} \%$ and washed with inhibited water (IW) to remove $\mathrm{NaOH}$ and other soluble salts. Next, the slurry was oxidatively leached using sodium permanganate to solubilize chromium. The slurry was then washed to remove the dissolved chromium and concentrated.

This is one in a series of reports that summarizes Phase 1 results from testing in the PEP located in the Process Development Laboratory-West (PDL-W), located in Richland, Washington. Separate run reports have been prepared for each Phase 1 test (i.e., Shakedown/Functional Testing [Josephson et al. 2009, WTP-RPT-190], Integrated Test A [Guzman-Leong et al. 2009, WTP-RPT-191], and Integrated Test D [Sevigny et al. 2009, WTP-RPT-193]). In addition, reports have been written that specifically address the following:

1. Filtration Scale-up (Daniel et al. 2009, WTP-RPT-185)

(a) SM Barnes, and R Voke. 2006. "Issue Response Plan for Implementation of External Flowsheet Review Team (EFRT) Recommendations - M12: Undemonstrated Leaching Process." 24590-WTP-PL-ENG-06-0024 Rev. 0.

(b) The scale of $1 / 4.5$ was chosen because this scale enables the ultrafiltration loop to be configured to meet two important criteria: 1) using one filter bundle, the ratio of solids in the feed tank to filter surface area will be the same as in the plant, and 2) using five filter bundles, the type and extent of mixing in the feed vessel will be approximately prototypic during the solids washing processes.

(c) In this report, the UFP vessels are generally denoted as Tank T01A/B and Tank T02A. In some cases alternative designations are used to maintain continuity with previous documentation (e.g., Test Specification/Plan). 
2. Caustic Leaching Scale-up (Mahoney et al. 2009, WTP-RPT-186)

3. Solids Washing (Baldwin et al. 2009, WTP-RPT-187)

4. Oxidative Leaching Scale-up (Rapko et al. 2009, WTP-RPT-188).

This report focuses on Integrated Test B and summarizes test conduct and results; it includes only very limited data analysis. Integrated Test B was conducted under Test Plan TP-RPP-WTP-506 ${ }^{(a)}$ and in accordance with Test Instruction TI-WTP-PEP-066. Supporting data files and analytical reports have been supplied to WTP separately because of the large amount of data.

\section{Objectives}

Table S.1 summarizes the objectives for the entire PEP testing program along with a discussion of contributions made by the results of Integrated Test B to meet these objectives.

Table S.1. Summary of Test Objectives and Results

\begin{tabular}{|c|c|c|}
\hline Test Objective & $\begin{array}{l}\text { Objective } \\
\text { Met? }\end{array}$ & Discussion \\
\hline $\begin{array}{l}\text { Caustic leach process: Compare } \\
\text { engineering- and laboratory-scale } \\
\text { results to determine impact of } \\
\text { scale-up. }\end{array}$ & Yes & $\begin{array}{l}\text { Data from Integrated Test B were analyzed and compared with } \\
\text { laboratory-scale data with results presented in reports WTP-RPT-186 } \\
\text { and WTP-RPT-197. }\end{array}$ \\
\hline $\begin{array}{l}\text { Oxidative-leach process: } \\
\text { Compare engineering- and } \\
\text { laboratory-scale results to } \\
\text { determine impact of scale-up. }\end{array}$ & Yes & $\begin{array}{l}\text { Data from Integrated Test B were analyzed and compared with } \\
\text { laboratory-scale data with results presented in reports WTP-RPT-188 } \\
\text { and WTP-RPT-197. }\end{array}$ \\
\hline $\begin{array}{l}\text { Cross-flow Ultrafiltration: } \\
\text { Monitor cross-flow filter } \\
\text { performance at engineering- and } \\
\text { laboratory-scale to determine } \\
\text { scale-up. }\end{array}$ & Yes & $\begin{array}{l}\text { Data from Integrated Test B were analyzed and compared with } \\
\text { laboratory-scale data with results presented in reports WTP-RPT-185 } \\
\text { and WTP-RPT-197. }\end{array}$ \\
\hline $\begin{array}{l}\text { Slurry wash process: Determine } \\
\text { the post-caustic and oxidative } \\
\text { leaching slurry wash efficiencies. }\end{array}$ & Yes & $\begin{array}{l}\text { Data from Integrated Test B were analyzed and compared with } \\
\text { laboratory-scale data with results presented in reports WTP-RPT-187 } \\
\text { and WTP-RPT-197. }\end{array}$ \\
\hline $\begin{array}{l}\text { Process Integration: Evaluate the } \\
\text { chemical addition, filter operation } \\
\text { cycle performance, and } \\
\text { pressure-pot operations. } \\
\text { Also perform mass balances for } \\
\text { aluminum, chromium, } \\
\text { manganese, sodium, hydroxide, } \\
\text { oxalate, phosphate, sulfate, and } \\
\text { water and monitor permeates for } \\
\text { post-filtration precipitation. }\end{array}$ & Yes & $\begin{array}{l}\text { Supporting data from Integrated Test B are presented, and results to } \\
\text { meet this objective are discussed in WTP-RPT-197. }\end{array}$ \\
\hline $\begin{array}{l}\text { Monitor the performance of the } \\
\text { recirculation system pumps, }\end{array}$ & Yes & $\begin{array}{l}\text { The data required to meet this objective were provided on compact } \\
\text { discs transmitted in the following reference: Letter from GH Beeman }\end{array}$ \\
\hline
\end{tabular}

(a) GB Josephson, OP Bredt, JK Young, and DE Kurath. 2009. Test Plan for Pretreatment Engineering Platform (PEP) Testing (Phase I). TP-RPP-WTP-506, Rev. 0.4, Pacific Northwest National Laboratory, Richland, Washington. 


\begin{tabular}{||l|l|l||}
\hline \multicolumn{1}{|c|}{ Test Objective } & $\begin{array}{c}\text { Objective } \\
\text { Met? }\end{array}$ & \multicolumn{1}{|c|}{ Discussion } \\
\hline $\begin{array}{l}\text { filters, and heat exchanger to } \\
\text { support engineering fabrication } \\
\text { decisions for these components. }\end{array}$ & $\begin{array}{l}\text { to H Hazen, "Subcontract No. 24590-QL-HC9-WA49-00001, Project } \\
\text { No. 53569 (WA-024) Engineering Ties Data Transmittal: The } \\
\text { Electronic File Enclosed with this letter has been reviewed for } \\
\text { technical accuracy per the QA Program, } \\
\text { WTP/RPP-MOA-PNNL-00392, dated 4/10/09. }\end{array}$ \\
\hline
\end{tabular}

\section{Test Exceptions}

A summary description of the Test Exceptions applied to these tests is shown in Table S.2.

Table S.2. Test Exceptions

\begin{tabular}{|c|c|}
\hline Test Exceptions & Description of Test Exceptions \\
\hline $\begin{array}{l}\text { 1) 24590-PTF-TEF-RT-08- } \\
\text { 00002, incorporated into ICN-1 } \\
\text { to Test Plan TP-RPP-WTP-506. }\end{array}$ & $\begin{array}{l}\text { This Test Exception: } \\
\text { 1. Added a stage during the filter conditioning section of the } \\
\text { Shakedown/Functional Test where the simulant slurry is concentrated from } \\
\text { approximately } 5 \text {-wt\% solids to } 20 \text {-wt\% solids in one operation. This is in } \\
\text { addition to the previously specified low-solids filter and high-solids filter } \\
\text { testing. } \\
\text { 2. Documented the Joint Test Group (JTG) decision regarding the number of } \\
\text { replicate samples to be collected at various processing times. } \\
\text { 3. Revised the terminology specifying the Coriolis densitometer (CD) sample } \\
\text { locations changed to be consistent with PEP operating procedures. Renamed } \\
\text { the "center" array to "inner." } \\
\text { 4. The sampling specified in the low-solids filtration test over specifies the } \\
\text { sample collection timing required. The technical requirement is to get } 30 \\
\text { unique samples. The sampling schedule specified is not required to achieve } \\
\text { this test objective. }\end{array}$ \\
\hline $\begin{array}{l}\text { 2) } 24590-P T F-T E F-R T-09- \\
00001 \text { incorporated into ICN-2 } \\
\text { and ICN-3 to Test Plan } \\
\text { TP-RPP-WTP-506. }\end{array}$ & $\begin{array}{l}\text { 1. In several steps, the sampling location was changed from the filer loop in-line } \\
\text { location to a middle-low CD sample loop location in the UFP-T02A vessel. } \\
\text { This change impacted sampling in the functional and all Integrated tests } \\
\text { (ref CCN 187749). } \\
\text { 2. Added a step to the Shakedown/Functional test (step A.1.31) to add sodium } \\
\text { permanganate to UFP-VSL-T02A to assess possible foaming issue (ref CCN } \\
\text { 187749). } \\
\text { 3. Changed location of second sample for laboratory-scale Cells Unit Filter } \\
\text { (CUF) testing from the in-line filter-loop to the middle-low CD port in the } \\
\text { UFP-VSL-T02A (step A.1.10; Shakedown/Functional Test) (ref CCN } \\
\text { 187749). } \\
\text { 4. Collected samples for laboratory-scale laboratory leaching test before and } \\
\text { after caustic addition in UFP-VSL-T01A (A.1.20; Shakedown/Functional } \\
\text { Test) and UFP-VSL-T02A (step A.1.15; Shakedown/Functional Test) and in } \\
\text { the Integrated test steps (B1.2; Integrated Test A, B2.6; Integrated Tests B/D) } \\
\text { (ref CCN: 192734). } \\
\text { 5. Deleted reconfiguration of the filter-loop to bypass UFP-VSL-T02A and } \\
\text { circulate flush water with UFP-PMP-T42A and/or UFP-PMP-43A to allow a } \\
\text { representative in-line sample to be collected. This step (step A.1.17; } \\
\text { Shakedown/Functional Test) could not be done under the operating } \\
\text { restrictions in place on the operation of the filter-loop (ref CCN 192734). } \\
\text { 6liminated step A.1.25 (filter-loop by-pass test with tracer) from the }\end{array}$ \\
\hline
\end{tabular}


Table S.2. Test Exceptions

\begin{tabular}{|c|c|}
\hline Test Exceptions & Description of Test Exceptions \\
\hline & 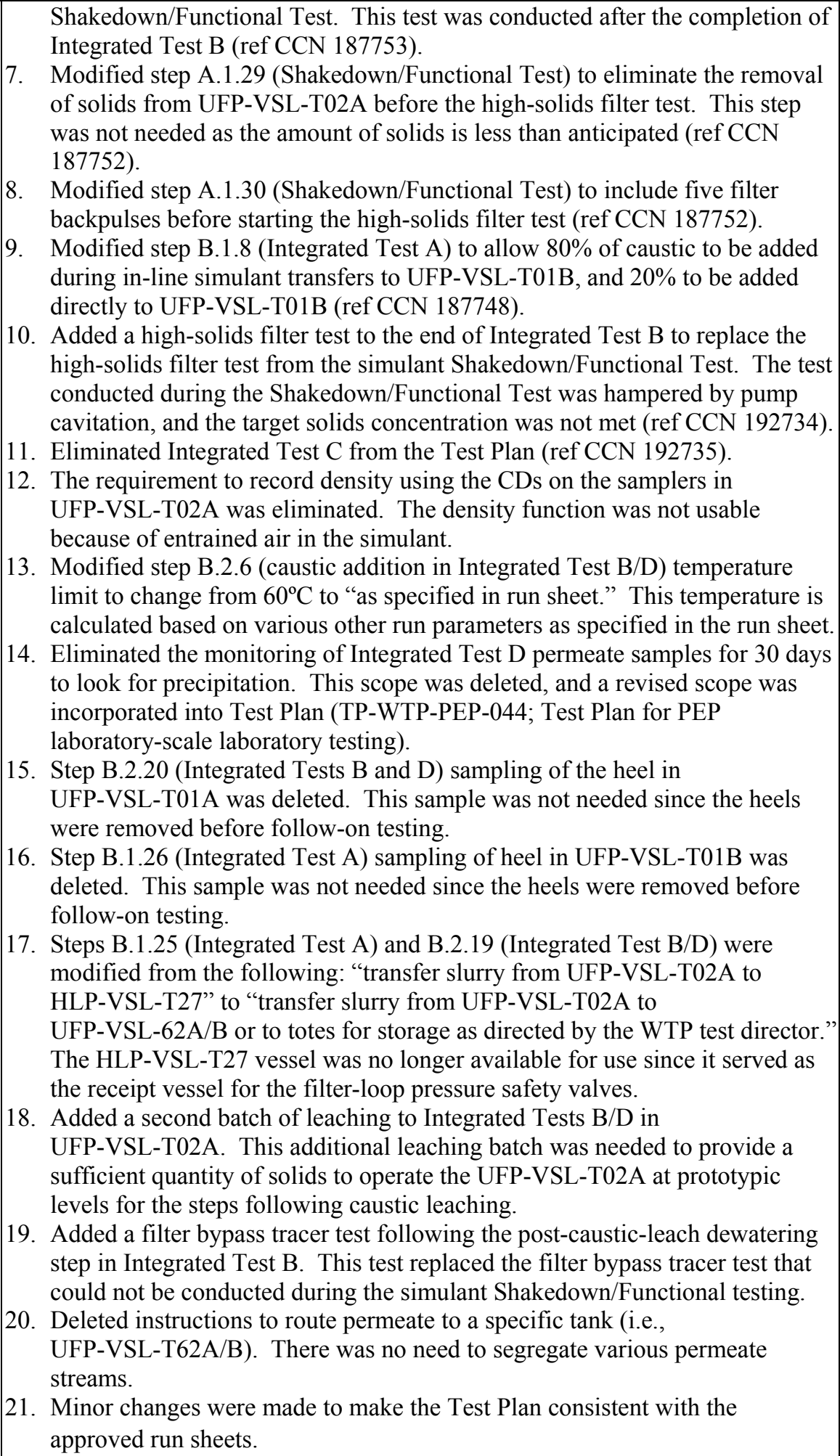 \\
\hline
\end{tabular}


Table S.2. Test Exceptions

\begin{tabular}{|c|c|}
\hline Test Exceptions & Description of Test Exceptions \\
\hline $\begin{array}{l}\text { 3) } 24590 \text {-WTP-TEF-RT-09- } \\
\text { 00003 incorporated into ICN-1 } \\
\text { to Test Plan TP-WTP-PEP-044. }\end{array}$ & $\begin{array}{l}\text { This Test Exception specified activities to be performed with permeate samples } \\
\text { obtained from Integrated Test D. The Integrated Test D permeate samples were } \\
\text { originally stored in a temperature-controlled environment and then moved to a } \\
\text { location with a reduced temperature where precipitation was likely to occur. The } \\
\text { Test Exception requested that the approximate size distribution of the solids be } \\
\text { measured in several ( } 3 \text { or 4) selected PEP samples from Integrated Test D using } \\
\text { polarized light microscopy (PLM). Size-calibrated photographs should be } \\
\text { provided along with the analysis. If possible, record the mineral identification of } \\
\text { the solids phase(s) along with the particle-size distribution (PSD). WTP } \\
\text { personnel will select the samples in consultation with the subcontractor, and this } \\
\text { will be based in part on observing which samples contain the most solids or } \\
\text { appear to contain different types of solids. Repeat the size-distribution analysis } \\
\text { approximately } 1 \text { week after the initial measurements to determine whether there } \\
\text { was a significant change in crystal size, habit, or composition. } \\
\text { Perform each size-distribution analysis by measuring the diameter (or length and } \\
\text { width for elongated crystals) of approximately } 100 \text { individual particles in each } \\
\text { sample. The size may be measured either on the microscope slide, using a } \\
\text { calibrated ocular scale, or on the size-calibrated photographs. The program } \\
\text { recognizes the limitations of the statistical significance of a size-distribution } \\
\text { measurement based on such a small population. This Test Exception did not } \\
\text { affect any of the existing Test Plan objectives. }\end{array}$ \\
\hline $\begin{array}{l}\text { 4) } 24590 \text {-WTP-TEF-RT-09- } \\
\text { 00002 Rev 0, incorporated into } \\
\text { ICN-4 to Test Plan } \\
\text { TP-RPP-WTP-506. }\end{array}$ & $\begin{array}{l}\text { This Test Exception: } \\
\text { 1. Requests a report summarizing the lessons learned during scale-up, } \\
\text { manufacture, and transport of the PEP simulant. } \\
\text { 2. Specifies the sampling and analysis scope to be performed to complete the } \\
\text { prototypic nitric acid PEP filter cleaning process. } \\
\text { 3. Deletes the Engineering Ties report scope. } \\
\text { 4. Specifies additional experimental and analytical work required to estimate the } \\
\text { amount of excess caustic in caustic-leachate samples and post-caustic-leach } \\
\text { wash solutions containing } \approx 3.5 \mathrm{M} \mathrm{Na} \text {. }\end{array}$ \\
\hline $\begin{array}{l}\text { 5) } 24590 \text {-WTP-TEF-RT-09- } \\
\text { 00001 Rev } 1 \text { incorporated into } \\
\text { ICN-2 to Test Plan } \\
\text { TP-WTP-PEP-044 }\end{array}$ & $\begin{array}{l}\text { This Test Exception specifies additional work to be conducted with caustic-leach } \\
\text { solutions and post-caustic-leach washing permeate samples obtained from PEP } \\
\text { Integrated Tests A, B and D. It contains the following tasks: } \\
\text { 1. Determine precipitate mineralogy, precipitate phase compositions, and } \\
\text { solution saturation composition. } \\
\text { 2. Determine rate of approach to saturation concentrations. } \\
\text { 3. Identify and characterize precipitates formed in post-caustic-leach filtrate. } \\
\text { 4. Determine the dilution required to redissolve the precipitate. } \\
\text { 5. Determine super-saturation in post-caustic-leach filtrates from Integrated Test } \\
\text { B in the PEP. } \\
\text { 6. Determine the effects of blending during the post-caustic-leach dewatering } \\
\text { and wash cycle. }\end{array}$ \\
\hline
\end{tabular}

As documented in the PEP Test Plan, the deviations from the Test Specification are provided in Table S.3. 
Table S.3. Deviations from Test Specification

\begin{tabular}{|c|c|}
\hline Test Specification Reference & Exception Taken \\
\hline $\begin{array}{l}\text { Section 6.4.4 "Analytical measurements will be } \\
\text { made in conformance to the Guidelines for } \\
\text { Performing Chemical Physical, and Rheological } \\
\text { Properties Measurements }{ }^{(\mathrm{a})} \text { as applicable." }\end{array}$ & $\begin{array}{l}\text { Three method exceptions are required under this Test Plan: } \\
\text { 1. Caustic-leach and oxidative-leach samples taken during this } \\
\text { testing must be separated more quickly than the standard } \\
\text { method using syringes. This testing will use a modified } \\
\text { method using a shorter centrifuge time and applying higher } \\
\text { g forces (e.g., } 4000 \mathrm{~g} \text { vs. } 1000 \mathrm{~g} \text { ). } \\
\text { Impact on results: If the standard method were used, the } \\
\text { longer time could very well lead to greater precipitation } \\
\text { and inaccurate results. Laboratory testing will be } \\
\text { conducted with simulants to confirm that this method of } \\
\text { sample handling is adequate. } \\
\text { 2. Densities of samples smaller than } 10 \text {-mL can only be } \\
\text { established within two significant figures of accuracy. } \\
\text { Density measurements for this Test Plan require greater } \\
\text { accuracy. Therefore, a more accurate method employing a } \\
\text { pycnometer will be used. } \\
\text { Impact on results: The change to a pycnometer will } \\
\text { generate more precise results than the standard method. } \\
\text { The main impact is expected to be on analysis time. The } \\
\text { pycnometer method will be slower. } \\
\text { The process for determining the wt } \% \text { undissolved solids } \\
\text { content of the slurries will in some cases be determined } \\
\text { with the use of a moisture analyzer. In addition, the } \\
\text { method of drying samples will be modified to allow the use } \\
\text { of glass fiber filters to aid in drying the samples. } \\
\text { Impact on results: Both modifications are intended to } \\
\text { decrease the time required to obtain results. }\end{array}$ \\
\hline
\end{tabular}

\section{Results and Performance Against Success Criteria}

The PEP system tests were designed to generate the data necessary to:

- Provide engineering-scale system performance data. This information is used to support the WTP computer process model projections of the waste-processing campaign.

- Confirm the operability and functionality of UFP system components.

The WTP Research and Technology (R\&T) success criteria for achieving these objectives are discussed in Table S.4. The success criteria for the entire PEP testing program are provided with discussion limited to the success criteria covered by the scope of this report. The success criteria not addressed in this report are shaded in gray.

(a) GL Smith and K Prindiville. 2002. Guidelines for Performing Chemical, Physical, and Rheological Properties Measurements. 24590-WTP-GPG-RTD-001, Rev 0, Bechtel National, Inc., Richland, Washington. 
Table S.4. Success Criteria

\begin{tabular}{|c|c|}
\hline Success Criteria & How Testing Did or Did Not Meet Success Criteria \\
\hline \multicolumn{2}{|l|}{ UFP System Process Performance } \\
\hline $\begin{array}{l}\text { Measure the aluminum leaching } \\
\text { performance of the PEP and } \\
\text { laboratory systems as a function of } \\
\text { time under WTP UFP-1 and UFP-2 } \\
\text { projected leaching conditions at } \\
\text { bounding high and low process } \\
\text { temperatures (nominally } 100^{\circ} \mathrm{C} \text { and } \\
80^{\circ} \mathrm{C} \text { ). }\end{array}$ & $\begin{array}{l}\text { Aluminum leaching at } 98^{\circ} \mathrm{C} \text { was measured as a function of time under WTP } \\
\text { UFP-2 projected leaching conditions during Integrated Test B. Results are } \\
\text { presented in this report and in report WTP-RPT-186 and WTP-RPT-197. }\end{array}$ \\
\hline $\begin{array}{l}\text { Compare aluminum leach } \\
\text { performance in UFP-1 where all of } \\
\text { the } \mathrm{NaOH} \text { is added in-line to the } \\
\text { case where a fraction of the total } \\
\mathrm{NaOH} \text { is added directly to the tank. }\end{array}$ & Results to meet this success criterion are discussed in report WTP-RPT-197. \\
\hline $\begin{array}{l}\text { Measure chromium leaching } \\
\text { performance in the PEP and } \\
\text { laboratory systems as a function of } \\
\text { time at the WTP projected } \\
\text { conditions in UFP-2 for both the } \\
\text { UFP-1 and UFP-2 aluminum } \\
\text { leaching flowsheets. }\end{array}$ & $\begin{array}{l}\text { Chromium leaching was measured as a function of time under WTP UFP-2 } \\
\text { projected leaching conditions during Integrated Test B. Results are presented } \\
\text { in report WTP-RPT-188 and WTP-RPT-197. }\end{array}$ \\
\hline $\begin{array}{l}\text { Evaluate the process control } \\
\text { strategy for specification of } \\
\text { required reagent additions, } \\
\text { including } \mathrm{NaOH}, \mathrm{NaMnO}_{4} \text {, and } \\
\text { wash solutions provided in the PEP } \\
\text { Phase } 1 \text { Testing Process } \\
\text { Description. }\end{array}$ & $\begin{array}{l}\text { The process control strategy for specifying the amount of reagent was to } \\
\text { analyze a sample of the simulant feed for aluminum and chromium content } \\
\text { and specify the amount of } \mathrm{NaOH} \text { and } \mathrm{NaMnO}_{4} \text { based on the result. The } \\
\text { amount of reagent added was correct within the analytical error of the } \\
\text { analysis method. A comparison of targeted and delivered reagent additions } \\
\text { is provided in report WTP-RPT-188 for Integrated Tests A and B. } \\
\text { Additional discussion and results for Integrated Test D are provided in report } \\
\text { WTP-RPT-197. }\end{array}$ \\
\hline $\begin{array}{l}\text { Measure the filter system } \\
\text { performance at the nominal flow } \\
\text { velocity and transmembrane } \\
\text { pressures (TMPs) for the solids } \\
\text { concentration and washing stages } \\
\text { for the UFP-1 and UFP-2 aluminum } \\
\text { leaching flowsheets. }\end{array}$ & $\begin{array}{l}\text { Filter performance was measured at a nominal axial flow velocity of } 15 \mathrm{ft} / \mathrm{sec} \\
\text { and a TMP of } 40 \text { psid during solids concentration and washing during } \\
\text { Integrated Test B. Actual permeate flux and filtration conditions are } \\
\text { provided in this report. A summary of filter system performance is provided } \\
\text { in WTP-RPT-197. }\end{array}$ \\
\hline $\begin{array}{l}\text { Evaluate the control strategy for } \\
\text { make-up additions from } \\
\text { UFP-VSL-00001A/B to } \\
\text { UFP-VSL-00002A/B during initial } \\
\text { dewatering process. }\end{array}$ & $\begin{array}{l}\text { The control strategy for make-up additions between UFP-VSL-T01A and } \\
\text { UFP-VSL-T02A during the initial dewatering process was demonstrated } \\
\text { during initial dewatering during Integrated Test B. The control strategy was } \\
\text { determined to be adequate, although maintaining a high operating level in } \\
\text { UFP-VSL-T02A until the end of processing should reduce the possibility of } \\
\text { entraining air into the recirculation pumps. Results are presented in report } \\
\text { WTP-RPT-197. }\end{array}$ \\
\hline
\end{tabular}


Table S.4. Success Criteria

\begin{tabular}{|c|c|}
\hline Success Criteria & How Testing Did or Did Not Meet Success Criteria \\
\hline $\begin{array}{l}\text { Measure the wash-water volumes } \\
\text { required to remove or reduce the } \\
\text { free hydroxide following the } \\
\text { aluminum leaching stage and } \\
\text { dissolved chromium after the } \\
\text { oxidative leaching process to the } \\
\text { specified concentrations. }\end{array}$ & $\begin{array}{l}\text { The volume of wash water required to reduce free hydroxide following the } \\
\text { aluminum leach was evaluated for Integrated Test B in report WTP-RPT- } 187 \\
\text { and WTP-RPT-197. In summary, } 436 \text { gallons of IW ( } 39 \text { IW batches) was } \\
\text { required to reduce the free hydroxide to } 0.25 \mathrm{M} \text {. Wash-water volumes for } \\
\text { the post-oxidative-leaching process are not provided since there is no } \\
\text { washing specification for Cr. }\end{array}$ \\
\hline $\begin{array}{l}\text { Perform mass balances for selected } \\
\text { constituents, including aluminum, } \\
\text { chromium, manganese, sodium, } \\
\text { hydroxide, oxalate, phosphate, } \\
\text { sulfate, and water, to evaluate } \\
\text { leaching and washing process } \\
\text { performance. }\end{array}$ & $\begin{array}{l}\text { Results necessary for performing mass balances for selected constituents for } \\
\text { Integrated Test B are presented in this report. Results to meet this success } \\
\text { criterion are discussed for Cr in the oxidative leaching process for Integrated } \\
\text { Tests A and B in report WTP-RPT-188 and are fully discussed for all } \\
\text { constituents in report WTP-RPT-197. }\end{array}$ \\
\hline $\begin{array}{l}\text { Measure solids distribution under } \\
\text { scaled mixing conditions before and } \\
\text { after caustic leaching evolutions. }\end{array}$ & $\begin{array}{l}\text { Solids distribution before and after caustic leaching is presented in report } \\
\text { WTP-RPT-190 and WTP-RPT-197. }\end{array}$ \\
\hline $\begin{array}{l}\text { Measure the rheology of the slurry } \\
\text { simulant and the shear strength of } \\
\text { the settled solids before and after } \\
\text { each leaching and washing unit } \\
\text { operation and following the final } \\
\text { concentration. }\end{array}$ & $\begin{array}{l}\text { The rheology of the slurry simulant was measured for Integrated Test B and } \\
\text { is provided in this report. }\end{array}$ \\
\hline $\begin{array}{l}\text { Estimate the quantity of excess } \\
\text { hydroxide added in the process that } \\
\text { may not be needed to keep } \\
\text { aluminate in solution following } \\
\text { filtration. }\end{array}$ & $\begin{array}{l}\text { Results to meet this success criterion are expected to be discussed in report } \\
\text { WTP-RPT-197. }\end{array}$ \\
\hline $\begin{array}{l}\text { Collect and retain permeate samples } \\
\text { for extended precipitation studies } \\
\text { (including permeate/simulated } \\
\text { supernatant blended cases) from } \\
\text { each concentration cycle. }\end{array}$ & $\begin{array}{l}\text { Permeate samples were collected during Integrated Test B for precipitation } \\
\text { studies. The results of the precipitation studies are discussed in } \\
\text { WTP-RPT-197, WTP-RPT-200, and WTP-RPT-205. }\end{array}$ \\
\hline \multicolumn{2}{|c|}{ UFP System Operability and Functionality } \\
\hline $\begin{array}{l}\text { Verify that the dual, in-series pump } \\
\text { configuration is controllable and } \\
\text { maintains the required slurry } \\
\text { velocity and pressures for ultrafilter } \\
\text { operation. }\end{array}$ & $\begin{array}{l}\text { The data required to meet this success criterion were provided on compact } \\
\text { disks transmitted in the following reference: Letter from GH Beeman to } \\
\text { H Hazen, "Subcontract No. 24590-QL-HC9-WA49-00001, Project No. } \\
53569 \text { (WA-024) Engineering Ties Data Transmittal: The Electronic File } \\
\text { Enclosed with this Letter Has Been Reviewed for Technical Accuracy Per } \\
\text { the quality assurance (QA) Program," WTP/RPP-MOA-PNNL-00392, dated } \\
\text { 4/10/09. }\end{array}$ \\
\hline
\end{tabular}


Table S.4. Success Criteria

\begin{tabular}{|c|c|}
\hline Success Criteria & How Testing Did or Did Not Meet Success Criteria \\
\hline $\begin{array}{l}\text { Measure the operating } \\
\text { characteristics for the cooling heat } \\
\text { exchanger for the UFP-VSL-00002 } \\
\text { filter recirculation loop } \\
\text { (temperature changes as a function } \\
\text { of flow to determine how to achieve } \\
\text { the desired performance in the PTF } \\
\text { analog). }\end{array}$ & $\begin{array}{l}\text { The data required to meet this success criterion were provided on compact } \\
\text { disks transmitted in the following reference: Letter from GH Beeman to } \\
\text { H Hazen, "Subcontract No. 24590-QL-HC9-WA49-00001, Project No. } \\
53569 \text { (WA-024) Engineering Ties Data Transmittal: The Electronic File } \\
\text { Enclosed with this Letter Has Been Reviewed for Technical Accuracy Per } \\
\text { the QA Program," WTP/RPP-MOA-PNNL-00392, dated 4/10/09. }\end{array}$ \\
\hline $\begin{array}{l}\text { Confirm whether the WTP process } \\
\text { control strategies for ultrafilter } \\
\text { system filling, operating, } \\
\text { backpulsing, draining, flushing, and } \\
\text { cleaning are adequate for stable } \\
\text { operation. Provide to WTP data to } \\
\text { determine whether backpulsing is a } \\
\text { required and effective means of } \\
\text { restoring the filter permeate rates to } \\
\text { confirm that production throughput } \\
\text { is maintained and whether operation } \\
\text { of the backpulse system induces } \\
\text { any process or equipment } \\
\text { operations issues. }\end{array}$ & Results to meet this success criterion are discussed in report WTP-RPT-197. \\
\hline $\begin{array}{l}\text { Use only the process information } \\
\text { and data available to the WTP PTF } \\
\text { operating staff during WTP } \\
\text { operations (e.g., caustic and } \\
\text { permanganate addition volumes, } \\
\text { permeate mass balances for solids } \\
\text { concentration, etc.) to operate the } \\
\text { PEP. }\end{array}$ & $\begin{array}{l}\text { This success criterion was met by developing a run sheet of all the operating } \\
\text { parameters (e.g., transfer volumes, reagent addition volumes, control levels, } \\
\text { etc.) based on prototypic characterization data before the start of Integrated } \\
\text { Test B. Changes to the run sheet made during the test itself were based only } \\
\text { on data that would be available to the plant, and were not, for example, based } \\
\text { on information from nonprototypic samples. This success criterion is fully } \\
\text { discussed in WTP-RPT-197. }\end{array}$ \\
\hline $\begin{array}{l}\text { Confirm whether the elevated } \\
\text { temperature PJM operating strategy } \\
\text { is adequate for stable PEP and WTP } \\
\text { operation. }\end{array}$ & Results to meet this success criterion are discussed in report WTP-RPT-197. \\
\hline $\begin{array}{l}\text { Measure the heat-up rate and } \\
\text { controllability of the PEP } \\
\text { UFP-VSL- } 00001 \text { and } \\
\text { UFP-VSL- } 00002 \text { vessels and the } \\
\text { cooling performance for UFP } \\
\text { vessels. }\end{array}$ & $\begin{array}{l}\text { The heat-up, thermal control at } 98^{\circ} \mathrm{C} \text {, and cool-down performance of } \\
\text { UFP-VSL-T02A was measured during Integrated Test B. Thermal profiles } \\
\text { are provided in this report. This information for the other tests is discussed } \\
\text { in the other run reports and summarized in WTP-RPT- } 197 \text {. }\end{array}$ \\
\hline $\begin{array}{l}\text { Measure the performance of the } \\
\text { in-line addition of process } \\
\text { chemicals into the simulated wastes } \\
\text { and determine the extent of } \\
\text { blending in the process vessels. }\end{array}$ & $\begin{array}{l}\text { The blending of process chemicals added in-line is presented in report } \\
\text { WTP-RPT-190 and WTP-RPT- } 197 .\end{array}$ \\
\hline
\end{tabular}


Table S.4. Success Criteria

\begin{tabular}{|c|c|}
\hline Success Criteria & How Testing Did or Did Not Meet Success Criteria \\
\hline $\begin{array}{l}\text { Monitor ultrafilter performance (to } \\
\text { include visual inspection of the } \\
\text { filter tubes, tube sheets, and heads } \\
\text { from an ultrafilter for any evidence } \\
\text { of flow mal-distribution and/or } \\
\text { solids buildup at least once during } \\
\text { Phase 1). }\end{array}$ & $\begin{array}{l}\text { Ultrafilter visual inspection results are presented in report WTP-RPT-193 } \\
\text { and WTP-RPT-197. }\end{array}$ \\
\hline $\begin{array}{l}\text { Measure, record, and control } \\
\text { ultrafiltration temperature, TMP, } \\
\text { and slurry flow during filter-loop } \\
\text { operations. }\end{array}$ & $\begin{array}{l}\text { Slurry flow rate, temperature, and TMP were recorded and controlled during } \\
\text { Integrated Test B. Results are provided in this report. }\end{array}$ \\
\hline $\begin{array}{l}\text { Record any solids accumulations } \\
\text { observed during any operating stage } \\
\text { or maintenance evolution. }\end{array}$ & Results to meet this success criterion are discussed in report WTP-RPT-197. \\
\hline $\begin{array}{l}\text { Monitor the permeate production } \\
\text { rate of each ultrafilter assembly in } \\
\text { operation. }\end{array}$ & $\begin{array}{l}\text { Permeate production rate of each ultrafilter was recorded during Integrated } \\
\text { Test B. Results are provided in this report. The permeate production rates } \\
\text { for each test are presented in the relevant run report. }\end{array}$ \\
\hline $\begin{array}{l}\text { Record operating time of each } \\
\text { ultrafilter assembly. }\end{array}$ & $\begin{array}{l}\text { The operating time of each ultrafilter assembly was recorded during } \\
\text { Integrated Test B. Results are provided in this report. The operating time of } \\
\text { each filter assembly for each test is discussed in the relevant run report and } \\
\text { summarized in WTP-RPT-197. }\end{array}$ \\
\hline $\begin{array}{l}\text { Record each ultrafilter assembly } \\
\text { cleaning event (backpulse, flush, } \\
\text { chemical cleaning, etc.). }\end{array}$ & $\begin{array}{l}\text { Each ultrafilter cleaning event was recorded in Integrated Test B. A } \\
\text { summary of the results is provided in this report, with more details recorded } \\
\text { in the Operations and Testing Laboratory Record Books. Additional filter } \\
\text { cleaning events are discussed in WTP-RPT-190, WTP-RPT-193, and } \\
\text { summarized in WTP-RPT-197. }\end{array}$ \\
\hline $\begin{array}{l}\text { Evaluate the pulse-pot operation } \\
\text { and backpulse operation strategies } \\
\text { contained in PEP Phase } 1 \text { Testing } \\
\text { Process Description. }\end{array}$ & $\begin{array}{l}\text { Backpulsing was conducted to increase the declining permeate rate through } \\
\text { the course of post-caustic-leach concentration and data are presented in this } \\
\text { report. Evaluation of backpulse operations is discussed in report } \\
\text { WTP-RPT-197. }\end{array}$ \\
\hline $\begin{array}{l}\text { Evaluate permeate and permeate } \\
\text { blends for precipitation of solids, } \\
\text { particularly aluminum and oxalate } \\
\text { solids. }\end{array}$ & $\begin{array}{l}\text { Permeate samples were collected during Integrated Test B for precipitation } \\
\text { studies. Results to meet this success criterion are discussed in reports } \\
\text { WTP-RPT-197, WTP-RPT-200, and WTP-RPT-205. }\end{array}$ \\
\hline
\end{tabular}

\section{Quality Requirements}

The PNNL Quality Assurance (QA) Program is based upon the requirements as defined in the U.S. Department of Energy (DOE) Order 414.1C, Quality Assurance and 10 CFR 830, Energy/Nuclear Safety Management, Subpart A -- Quality Assurance Requirements (a.k.a. the Quality Rule). PNNL has chosen to implement the following consensus standards in a graded approach:

- ASME NQA-1-2000, Quality Assurance Requirements for Nuclear Facility Applications, Part 1, Requirements for Quality Assurance Programs for Nuclear Facilities 
- ASME NQA-1-2000, Part II, Subpart 2.7, Quality Assurance Requirements for Computer Software for Nuclear Facility Applications

- ASME NQA-1-2000, Part IV, Subpart 4.2, Graded Approach Application of Quality Assurance Requirements for Research and Development.

The procedures necessary to implement the requirements are documented in PNNL's "How Do I...?"a

The RPP-WTP quality requirements are implemented by performing work in accordance with the River Protection Project-Waste Treatment Plant Support Program (RPP-WTP) Quality Assurance Plan (RPP-WTP-QA-001, QAP). Work was performed to the quality requirements of NQA-1-1989, Part I, Basic and Supplementary Requirements, NQA-2a-1990, Part 2.7, and DOE/RW-0333P, Rev 13, Quality Assurance Requirements and Descriptions (QARD), as applicable. These quality requirements are implemented through the River Protection Project-Waste Treatment Plant Support Program (RPP-WTP) Quality Assurance Manual (RPP-WTP-QA-003, QAM). The requirements of DOE/RW-0333P Rev 13, Quality Assurance Requirements and Descriptions (QARD), and 10 CFR 830 Subpart A were not required for this work.

The RPP-WTP addresses internal verification and validation activities by conducting an Independent Technical Review of the final data report in accordance with RPP-WTP's procedure QA-RPP-WTP-604. This review procedure is part of PNNL's RPP-WTP Quality Assurance Manual (RPP-WTP-QA-003). Following this procedure, a technical review would verify that the reported results are traceable, that inferences and conclusions are soundly based, and the reported work satisfies the objectives.

\section{R \& T Test Conditions}

The WTP R\&T test conditions as defined in the Test Specification are summarized in Table S.5. The R\&T test conditions for the entire PEP testing program are provided with discussion limited to the R\&T test conditions covered by the scope of this report. R\&T test conditions not addressed in this report are shaded in gray.

Table S.5. R\&T Test Conditions

\begin{tabular}{|c|c|}
\hline List R\&T Test Conditions & Were Test Conditions Followed? \\
\hline \multicolumn{2}{|l|}{ General Requirements } \\
\hline $\begin{array}{l}\text { Perform mass balances for selected constituents; } \\
\text { including aluminum, chromium, manganese, sodium, } \\
\text { hydroxide, oxalate, phosphate, sulfate, and water to } \\
\text { evaluate leaching and washing process performance. }\end{array}$ & $\begin{array}{l}\text { The necessary samples were taken to conduct a } \\
\text { mass balance for Integrated Test B. Mass balance } \\
\text { results are provided in WTP-RPT-197. }\end{array}$ \\
\hline $\begin{array}{l}\text { Evaluate ultrafilter performance (to include visual } \\
\text { inspection of the filter tubes, tube sheets, and heads } \\
\text { from an ultrafilter for any evidence of flow } \\
\text { mal-distribution and/or solids buildup or evidence of } \\
\text { potential failure). }\end{array}$ & $\begin{array}{l}\text { This R\&T test condition is discussed in reports } \\
\text { WTP-RPT-193 and WTP-RPT- } 197 .\end{array}$ \\
\hline $\begin{array}{l}\text { Assess the blending achieved during in-line additions } \\
\text { of leaching and washing solutions. }\end{array}$ & $\begin{array}{l}\text { In-line addition of wash water during Integrated } \\
\text { Tests A and B is discussed in WTP-RPT-187 and } \\
\text { WTP-RPT-197. }\end{array}$ \\
\hline
\end{tabular}

(a) HDI is a system for managing the delivery of laboratory-level policies, requirements, and procedures. 
Table S.5. R\&T Test Conditions

\begin{tabular}{|c|c|}
\hline List R\&T Test Conditions & Were Test Conditions Followed? \\
\hline $\begin{array}{l}\text { Record any solids accumulations observed during } \\
\text { any operating stage or maintenance evolution (e.g., } \\
\text { photography, PSD). }\end{array}$ & $\begin{array}{l}\text { This R\&T test condition is discussed in report } \\
\text { WTP-RPT-197. }\end{array}$ \\
\hline \multicolumn{2}{|l|}{ Leaching Operations } \\
\hline $\begin{array}{l}\text { Maintain caustic leaching temperature at the required } \\
\text { setpoint and record steam usage to remain in the } \\
\text { temperature range. }\end{array}$ & $\begin{array}{l}\text { The temperature during caustic leaching was } \\
\text { maintained at setpoint with steam usage measured } \\
\text { during Integrated Test B. Additional discussion of } \\
\text { this condition is provided in WTP-RPT-186 and } \\
\text { WTP-RPT-197. }\end{array}$ \\
\hline $\begin{array}{l}\text { Maintain oxidative leaching temperature at the } \\
\text { required setpoint. }\end{array}$ & $\begin{array}{l}\text { The temperature during oxidative leaching was } \\
\text { maintained at setpoint during Integrated Test B. } \\
\text { Additional discussion of this condition is provided } \\
\text { in WTP-RPT-188 and WTP-RPT-197. }\end{array}$ \\
\hline $\begin{array}{l}\text { Obtain periodic samples during the leaching } \\
\text { operations to monitor the amount of aluminum or } \\
\text { chromium that has dissolved and concentrations of } \\
\text { the reactants and products in the liquid fraction in the } \\
\text { vessel. }\end{array}$ & $\begin{array}{l}\text { This R\&T condition was met for the caustic } \\
\text { leaching tests discussed in this report. Additional } \\
\text { discussion of this R\&T condition is provided in } \\
\text { WTP-RPT-186, WTP-RPT-188, and } \\
\text { WTP-RPT-197. }\end{array}$ \\
\hline $\begin{array}{l}\text { Provide data to demonstrate the WTP process control } \\
\text { strategy for the caustic and permanganate addition. }\end{array}$ & $\begin{array}{l}\text { This R\&T test condition is discussed in report } \\
\text { WTP-RPT-197. }\end{array}$ \\
\hline $\begin{array}{l}\text { Measure the rheology of the slurry simulant and } \\
\text { shear strength of the settled solids before and } \\
\text { following each leaching unit operation. }\end{array}$ & $\begin{array}{l}\text { Slurry rheology was measured with samples taken } \\
\text { before and after each leaching unit operation. } \\
\text { Results are provided in this report. Rheology } \\
\text { results for the other tests are included in the } \\
\text { relevant run reports. }\end{array}$ \\
\hline \multicolumn{2}{|l|}{ Concentration Operations } \\
\hline $\begin{array}{l}\text { Monitor the permeate production rate of each } \\
\text { ultrafilter assembly in operation. }\end{array}$ & $\begin{array}{l}\text { Permeate production for each ultrafilter assembly } \\
\text { was monitored during testing and results are } \\
\text { provided in this report. Permeate production rates } \\
\text { for the other tests are included in the relevant run } \\
\text { reports. }\end{array}$ \\
\hline Record operating time of each ultrafilter assembly. & $\begin{array}{l}\text { Operating time of each ultrafilter was recorded } \\
\text { during Integrated Test B, with results discussed in } \\
\text { report WTP-RPT-197. }\end{array}$ \\
\hline $\begin{array}{l}\text { Record each ultrafilter assembly "cleaning" event } \\
\text { (backpulse, flush, chemical cleaning, etc.). }\end{array}$ & $\begin{array}{l}\text { Ultrafilter cleaning events were recorded with } \\
\text { results of the cleaning prior to Integrated Test B } \\
\text { provided in this report. A summary of the filter } \\
\text { cleaning events is provided in WTP-RPT-197, and } \\
\text { details of the final prototypic filter cleaning are } \\
\text { presented in WTP-RPT-193. Additional filter } \\
\text { cleaning events are also discussed in } \\
\text { WTP-RPT-190. }\end{array}$ \\
\hline $\begin{array}{l}\text { Confirm pulse-pot operation and backpulse operation } \\
\text { strategies. }\end{array}$ & $\begin{array}{l}\text { This R\&T test condition is discussed in report } \\
\text { WTP-RPT-197. }\end{array}$ \\
\hline
\end{tabular}


Table S.5. R\&T Test Conditions

\begin{tabular}{|c|c|}
\hline List R\&T Test Conditions & Were Test Conditions Followed? \\
\hline $\begin{array}{l}\text { Control ultrafiltration temperature, TMP, and slurry } \\
\text { flow as specified in test-specific run sheets. }\end{array}$ & $\begin{array}{l}\text { Ultrafilter temperature, TMP and slurry flow rate } \\
\text { were controlled. Deviations from values specified } \\
\text { in the Integrated Test B run sheet are noted in this } \\
\text { report. R\&T conditions for the other tests are } \\
\text { included in the relevant run reports. }\end{array}$ \\
\hline $\begin{array}{l}\text { Collect and retain permeate samples for extended } \\
\text { precipitation studies (including permeate/simulated } \\
\text { supernatant blended cases) from each concentration } \\
\text { cycle. }\end{array}$ & $\begin{array}{l}\text { Samples were collected and retained for extended } \\
\text { precipitation studies. The results of the } \\
\text { precipitation studies are discussed in } \\
\text { WTP-RPT-197, WTP-RPT-200, and } \\
\text { WTP-RPT-205. }\end{array}$ \\
\hline $\begin{array}{l}\text { Demonstrate WTP ultrafiltration system control } \\
\text { scheme in normal operating modes (e.g., fill and } \\
\text { startup, operation, backpulsing, flush and drain, } \\
\text { cleaning, and return to service). }\end{array}$ & $\begin{array}{l}\text { This R\&T test condition is discussed in report } \\
\text { WTP-RPT-197. }\end{array}$ \\
\hline \multicolumn{2}{|l|}{ Washing Operations } \\
\hline $\begin{array}{l}\text { Wash slurries using a washing protocol to be } \\
\text { specified in test-specific run sheets. }\end{array}$ & $\begin{array}{l}\text { Slurries were washed as specified in the Integrated } \\
\text { Test B run sheet. Washing results are discussed in } \\
\text { reports WTP-RPT-187 and WTP-RPT-197. }\end{array}$ \\
\hline $\begin{array}{l}\text { Sample permeate immediately before each wash } \\
\text { solution addition to monitor washing } \\
\text { performance/efficiency. }\end{array}$ & $\begin{array}{l}\text { Permeate was sampled and monitored during } \\
\text { washing with results provided in reports } \\
\text { WTP-RPT-187 and WTP-RPT-197. }\end{array}$ \\
\hline Measure rheology of the washed solids. & $\begin{array}{l}\text { The rheology of washed solids was measured with } \\
\text { results provided in this report. Rheology results for } \\
\text { the other tests are included in the relevant run } \\
\text { reports with selected results also discussed in } \\
\text { WTP-RPT-187 and WTP-RPT-197. }\end{array}$ \\
\hline
\end{tabular}

\section{Simulant Use}

PEP process testing was performed with a nonradioactive aqueous slurry of simulant waste chemicals and solids. The simulant composition and make-up recipe were provided by WTP as documented in Simulant Recommendation for Phase 1 Testing in the Pretreatment Engineering Platform. ${ }^{(a)}$ Aqueous chemical concentrations were within the ranges expected for waste feeds to the PTF. The hydroxide concentration was marginally one standard deviation lower than the average concentration expected in the feeds to the plant. The oxalate and phosphate components were at the lower end of the expected ranges, but the oxalate component was at the solubility limit, and the phosphate component was at or near the solubility limit. The solids components and blend were selected to obtain targeted solids mass loss (aluminum and chromium leaching and oxalate washing) and treatment time. The simulant was not selected to represent any particular Hanford tank waste type.

The simulant was blended from the components listed below. The basis for selecting the individual components and the comparison to actual waste behavior is provided where applicable in the indicated references.

(a) P Sundar. 2008. Simulant Recommendation for Phase 1 Testing in the Pretreatment Engineering Platform, 24590-PTF-RPT-RT-08-006 Rev 0, Bechtel National, Inc., Richland, WA. 
- Boehmite (for Al) (Russell et al. 2009a)

- Gibbsite (for Al) (Russell et al. 2009b)

- Chromium oxyhydroxide (CrOOH) slurry (Rapko et al. 2007)

- Sodium oxalate

- Filtration simulant (Russell et al. 2009c)

- Supernate.

A separate chromium solids slurry simulant was prepared and added to the PEP process after post-caustic-leach washing (a nonprototypic addition) during the Shakedown/Functional Tests and Integrated Tests A and B. This approach was taken because laboratory-scale tests had shown that the high-temperature caustic leaching step dissolved significant amounts of the $\mathrm{CrOOH}$ solids (Russell et al. 2009a). In Integrated Test D, the chromium solids component of the simulant was added during the simulant make-up process to demonstrate the PTF permanganate addition strategy. Simulant was procured from NOAH Technologies Corporation (San Antonio, TX). Samples of each simulant batch were characterized to make certain that chemical and physical property requirements were met. Batches of the simulant were procured as follows:

- A 15-gallon trial batch of the blended simulant for laboratory testing to demonstrate the efficacy of the simulant fabrication procedure.

- A 250-gallon scale-up batch of the blended simulant to demonstrate scale-up of the simulant fabrication procedure to an intermediate scale.

- Batches 0, 1, and 2, each nominally 3500 gallons, of blended simulant for the Shakedown/Functional Tests and Integrated Tests A and B. These batches did not contain the $\mathrm{CrOOH}$ component.

- The CrOOH solids slurry for the Shakedown/Functional Test and Integrated Tests A and B was obtained in two separate batches, containing nominally 18 and $36 \mathrm{~kg}$ of $\mathrm{Cr}$ as $\mathrm{CrOOH}$.

\section{Discrepancies and Follow-on Tests}

No discrepancies or follow-on tests were identified. 


\subsection{Introduction}

This is one in a series of reports that summarize Phase 1 testing results from the Pretreatment Engineering Platform (PEP) located in the Process Development Engineering Laboratory-West (PDL-W) in Richland, Washington. Separate run reports have been prepared for each Phase 1 test (i.e., Shakedown/Functional Testing [Josephson et al. 2009], Integrated Test A [Guzman-Leong et al. 2009], Integrated Test B [Geeting et al. 2009], and Integrated Test D [Sevigny et al. 2009]). In addition, reports have been written that specifically address the following:

1. Filtration (Daniel et al. 2009)

2. Caustic Leaching (Mahoney et al. 2009)

3. Washing (Baldwin et al. 2009)

4. Oxidative Leaching (Rapko et al. 2009).

This report focuses on Integrated Test B and summarizes test conduct and results; it includes only very limited data analysis. Data files have been supplied in an electronic format because of the large amount of data. The test reported here was conducted according to TI-WTP-PEP-066, which was written in accordance with Test Plan TP-RPP-WTP-506, Rev 0.4, which was written in response to Test Specification 24590-PTF-TSP-RT-07-001, Rev 2.

\subsection{Purpose}

The purpose of this testing program is to provide WTP with data to "...confirm the ultrafiltration process (UFP) system design and sludge treatment process flowsheet."(a) The system design and treatment process flowsheet were confirmed by evaluating the following process steps on an engineering-scale integrated platform (the PEP):

- Solids concentration

- Aluminum leaching

- Washing

- Chromium leaching.

Tests were used to collect data needed for design input and to improve prediction of full-scale plant performance:

- Demonstration of component operation and determination of operating characteristics

- Integrated simulations of leaching scenarios.

The PEP data will be used by WTP along with laboratory-scale data and appropriate application of scaling to improve the WTP plant models used to predict plant operating performance.

(a) S Barnes and R Voke. 2006. "Issue Response Plan for Implementation of External Flowsheet Review Team (EFRT) Recommendations - M12, Undemonstrated Leaching Processes.” 24590-WTP-PL-ENG-06-0024 Rev. 0. 


\subsection{Background}

In October 2005, an External Flowsheet Review Team (EFRT) was assembled to provide a critical review of the design of the Hanford Tank Waste Treatment and Immobilization Plant (WTP). The review $^{(a)}$ identified a number of issues and potential issues that if not addressed could impact operation of the plant. One of the issues that must be addressed in the Pretreatment Facility (PTF) is "Undemonstrated Leaching Processes." The EFRT reported that neither the caustic leaching nor the oxidative leaching process has been demonstrated at greater than bench scale. Without a scale-up study, the EFRT indicated that the ability to predict the effectiveness of these processes is limited.

The WTP project under Bechtel National Inc. (BNI) and the U.S. Department of Energy (DOE) developed the "Issue Response Plan for Implementation of External Flowsheet Review Team (EFRT) Recommendations-M12, Undemonstrated Leaching Processes" to resolve the "Undemonstrated Leaching Processes" issue. ${ }^{(b)}$ The plan addresses related topics that are not specifically in response to EFRT concerns. These include caustic addition and leaching concerns that were better understood after the EFRT report was issued, information to support revision of the contract design basis for the PTF, including system capacities, and earlier initiatives on enhancing plant throughput capacity. The solution for closing this issue includes conducting engineering-scale testing of all leaching (caustic and oxidative), washing, and filtration processes.

The flowsheet and equipment design demonstration will be performed in the following two phases:

- Phase 1 - Confirm the UFP system design and sludge treatment process flowsheet.

- Phase 2-Confirm the UFP system performance over a range of anticipated plant process conditions, including the principal types of WTP feeds.

The data provided by the Phase 1 engineering-scale testing will be used to confirm the performance of the selected process flowsheet design and equipment based on post-Phase 1 test modeling.

On 10/23/06 and 10/24/06, a WTP project ultrafiltration leaching process technical review meeting was held to review the Issue Response Plan for M12. ${ }^{\text {(b) }}$ Participants included members of DOE, EFRT, the WTP project, and Pacific Northwest National Laboratory (PNNL). At the meeting, PNNL made recommendations on the methodology and scale for the leaching processes.

Members of the EFRT also presented their assessment (Integrated Test Platform, M12 - Test Review) with specific recommendations. The general system requirements recommended by EFRT and PNNL staff members included the following:

- Principal processing equipment elements of the UFP system need to be included in the demonstration (including UFP-VSL-00001A/B, UFP-VSL-00002, the ultrafiltration loop recirculation pumps, and the ultrafilters with supporting equipment.).

(a) JP Henschel. March 17, 2006. Contract No. DE-AC27-01RV14136 - Report of External Flowsheet Review Team for the Hanford Tank Waste Treatment and Immobilization Plant: "Comprehensive Review of the Waste Treatment Plant Flowsheet and Throughput.” Letter to RJ Schepens. CCN: 132846, Bechtel National, Inc., Richland, Washington.

(b) SM Barnes, and R Voke. 2006. "Issue Response Plan for Implementation of External Flowsheet Review Team (EFRT) Recommendations - M12: Undemonstrated Leaching Process.” 24590-WTP-PL-ENG-06-0024 Rev. 0. 
- The demonstration system needs to be flexible to accommodate testing of the baseline processes and process options for a range of operating conditions and processing times.

- Geometric similarity (prototypic) is needed in the key pieces of processing equipment (including UFP-VSL-00001A/B and UFP-VSL-00002).

- Ultrafilter elements must be prototypic length and diameter to obtain expected filter performance data. The test equipment should be scaled down by using fewer filter elements in each assembly.

The following system parameters were developed and approved by the EFRT consultants:

- The engineering-scale test facility should be a factor of 4.5 smaller than the full-scale PTF.

- Each ultrafilter assembly should contain 12 full-scale elements.

- Five ultrafilter assemblies should be provided (based on M-13 recommendations).

Additional bases for the engineering-scale test system factor of 4.5 are provided in Scaling Relationships for the Pretreatment Engineering Platform (Kuhn et al. 2008). Some of the scaling factors are listed below.

- Selected processes within the PEP will be operated at both scale-time (4.5 times shorter than plant time) and plant time in separate test runs to address scaling issues.

- Prototypic tanks will be dimensionally scaled with a radius $1 / 4.5$ times their full-scale radius and operating height of $1 / 4.5$ that of full-scale. The tank volume will be $1 /(4.5)^{3}$ or $\sim^{1 / 90}$ of the plant.

- The filtration design is scaled by filter area. The total filtration area is $1 /(4.5)^{2}$ or $\sim 1 / 20$ that of the plant.

- Fluid velocities in pipes are kept approximately the same (using commercially available pipe diameters) to mimic solids settling that may occur in plant piping.

- Chemical reaction times are not scaled.

- The pulse-jet mixer (PJM) nozzle velocity in the PEP will be scaled to provide the same mixing power per volume of tank contents. The plant targets a nozzle discharge velocity of $8 \mathrm{~m} / \mathrm{s}$ in UFP-VSL-00001A/B and $12 \mathrm{~m} / \mathrm{s}$ in UFP-VSL-00002A/B. For non-Newtonian fluids, the nozzle velocity will be the same in the PEP and WTP. For Newtonian fluids, the velocity is scaled by $1 /(4.5)^{0.333}$. The total PJM cycle time will be scaled to maintain the fraction of the cycle in active drive mode. It is recognized that there is not a sharp distinction from Newtonian to non-Newtonian behavior. For the purpose of Phase 1 testing, we will scale the PJM velocities to maintain equal power per volume for the initial concentration and caustic leach. After the slurry is concentrated following the caustic leach, the PJMs will be adjusted to have the same PJM nozzle velocities as the plant (non-Newtonian scaling) and kept in that regime for the remainder of the process (post-caustic-leach wash, slurry wash, oxidative leaching, final slurry washing, and final concentration).

Section 6.4 of the Pretreatment Engineering Platform (PEP) Testing (Phase 1) Test Specification ${ }^{(a)}$ identifies the requirements for engineering-scale tests. The PEP data will be used by WTP along with laboratory-scale data and appropriate application of scaling to improve the WTP plant models used to predict plant operating performance.

(a) JL Huckaby and JR Markillie. 2008. Pretreatment Engineering Platform (PEP) Testing (Phase I). 24590-PTF-TSP-RT-07-001, Rev 2, Bechtel National, Inc., Richland, Washington. 


\subsection{Objective}

The objective of this report is to provide the Integrated Test B narrative, all sample analyses specified by the Test Plan, graphical PEP monitoring data, evaluations or summaries of problems encountered, and deviations from the test protocols. Analysis of Integrated Test B results will be provided by Kurath et al. (2009). Specific test objectives and summary results are provided in the summary section of this report. 


\subsection{Quality Assurance Requirements}

The PNNL QA program is based upon the requirements as defined in DOE Order 414.1C, Quality Assurance, and 10 CFR 830, Energy/Nuclear Safety Management, Subpart A-Quality Assurance Requirements (a.k.a. the Quality Rule). PNNL has chosen to implement the following consensus standards in a graded approach:

- ASME NQA-1-2000, Quality Assurance Requirements for Nuclear Facility Applications, Part 1, Requirements for Quality Assurance Programs for Nuclear Facilities.

- ASME NQA-1-2000, Part II, Subpart 2.7, Quality Assurance Requirements for Computer Software for Nuclear Facility Applications.

- ASME NQA-1-2000, Part IV, Subpart 4.2, Graded Approach Application of Quality Assurance Requirements for Research and Development.

The procedures necessary to implement the requirements are documented through PNNL's "How Do I...?" (HDI). ${ }^{\text {(a) }}$ PNNL implements the RPP-WTP quality requirements by performing work in accordance with the River Protection Project-Waste Treatment Plant Support Program (RPP-WTP) Quality Assurance Plan (RPP-WTP-QA-001, QAP). Work was performed to the quality requirements of NQA-1-1989, Part I, Basic and Supplementary Requirements, NQA-2a-1990, Part 2.7, and DOE/RW-0333P, Rev 13, Quality Assurance Requirements and Descriptions (QARD) as applicable. These quality requirements are implemented through the River Protection Project-Waste Treatment Plant Support Program (RPP-WTP) Quality Assurance Manual (RPP-WTP-QA-003, QAM). The requirements of DOE/RW-0333P, Rev 13, Quality Assurance Requirements and Descriptions (QARD) and 10 CFR 830 Subpart A were not required for this work.

RPP-WTP addresses internal verification and validation activities by conducting an Independent Technical Review of the final data report in accordance with RPP-WTP's procedure QA-RPP-WTP-604. This review procedure is part of PNNL's RPP-WTP Quality Assurance Manual (RPP-WTP-QA-003). Following this procedure, a technical review would verify that the reported results are traceable, that inferences and conclusions are soundly based, and the reported work satisfies the objectives.

Key analytes in the laboratory control sample (LCS) and PEP control sample were plotted over time to look for anomalies. The PEP control sample is a project-provided material generated from material very similar to the initial simulant feed. In general, the plots constructed to date associated with the inductively coupled plasma (ICP) and ion chromatography (IC) analysis of solutions shows recovery within limits of $80 \%$ to $120 \%$.

Table 2.1 shows a list of nonconformance reports (NCRs) active during Integrated Test B.

(a) HDI is a system for managing the delivery of laboratory-level policies, requirements, and procedures. 
Table 2.1. Description of NCRs Active During Integrated Test B

\begin{tabular}{|c|c|}
\hline $\begin{array}{l}\text { Nonconformance } \\
\text { Renort Number }\end{array}$ & \\
\hline \multirow[t]{4}{*}{ NCR 38767.1} & Measurement: Air flow rate to spargers in UFP-VSL-T02A. \\
\hline & $\begin{array}{l}\text { Issue: Micro-Motion identifies flow rates, below which the uncertainty is } \\
\text { greater than } 0.5 \% \text {. In the case of the PEP, air flow rates below } 0.090 \mathrm{~kg} / \mathrm{min} \\
\text { have uncertainties greater than } 0.5 \% \text {. For the lowest flow rate reported } \\
(0.012 \mathrm{~kg} / \mathrm{min} \text { on FT-1977), the estimated uncertainty is } \sim 4 \% \text {. }\end{array}$ \\
\hline & $\begin{array}{l}\text { Affected instruments follow: FT-1901, FT-1973, FT-1977, FT-1981, and } \\
\text { FT-1995. }\end{array}$ \\
\hline & $\begin{array}{l}\text { What it means: Recognize the greater uncertainty that accompanies data with air } \\
\text { flow rates }<0.090 \mathrm{~kg} / \mathrm{min} \text {. }\end{array}$ \\
\hline \multirow[t]{3}{*}{ NCR 43398.1} & Measurement: Temperature measurement in Tank UFP-VSL-T01B. \\
\hline & $\begin{array}{l}\text { Issue: TTK- } 0427 \text { failed at installation. All data from TTK- } 0427 \text { are invalid and } \\
\text { unusable. }\end{array}$ \\
\hline & $\begin{array}{l}\text { What it means: Do not use data from TTK- } 0427 \text {. (There are } 19 \text { other } \\
\text { temperature measurements available from this tank, with TTK-0425 the } \\
\text { prototypic measurement.) }\end{array}$ \\
\hline \multirow[t]{6}{*}{ NCR 42402.1 } & Measurement: Temperature measurement of the slurry in the filter-loop. \\
\hline & $\begin{array}{l}\text { Issue: The following resistance temperature detector (RTD) thermowells in the } \\
\text { filter-loop do not extend into process stream: }\end{array}$ \\
\hline & $\begin{array}{l}\text { TT-0513: UFP-HX-T02A (cooling) slurry outlet temperature. No data are to be } \\
\text { used for quality-affecting work. Data may be used for qualitative purposes only. }\end{array}$ \\
\hline & $\begin{array}{l}\text { TT-0515: UFP-HX-T03A (heating) slurry outlet temperature. No data are to be } \\
\text { used for quality-affecting work. Data may be used for qualitative purposes only. }\end{array}$ \\
\hline & $\begin{array}{l}\text { TT-0537: Filter } 5 \text { (UFP-FILT-T05A) outlet temperature (UFP-HX-T02A inlet). } \\
\text { No data are to be used for quality-affecting work. Data may be used for } \\
\text { qualitative purposes only. }\end{array}$ \\
\hline & $\begin{array}{l}\text { TT-0791: Filter } 1 \text { (UFP-FILT-T01A) inlet temperature. Data from this should } \\
\text { not be used at all. Tank temperature data will be used for quality-affecting filter } \\
\text { processing data. }\end{array}$ \\
\hline \multirow[t]{3}{*}{ NCR 41589.1} & $\begin{array}{l}\text { Measurement: On line density measurement in Tank T01B Coriolis sample } \\
\text { station. }\end{array}$ \\
\hline & Issue: DT-2101 failed. \\
\hline & What it means: All readings after $12 / 23 / 2008$ are not valid. \\
\hline
\end{tabular}




\subsection{Experimental Methods and Apparatus}

\subsection{Pretreatment Engineering Platform Overview}

The following section provides an overview of the PEP to orient the reader. This section does not provide details on PEP equipment or instrumentation. The interested reader is referred to the following documents for more details on the PEP:

- P\&IDs and mechanical data books.

- Pretreatment Engineering Platform (PEP) Phase I Testing Process Description, 24590-WTP-RPT-PET-07-002, Rev 1.

- Functional Requirements for Pretreatment Engineering Platform (PEP), 24590-PTF-3YD-UFP-00002, Rev 1.

The PEP test system is designed to perform engineering-scale demonstrations of most WTP pretreatment processes. These include (but are not limited to) vessel-to-vessel transfers, waste pumping, cross-flow filtration, filter cleaning, solids washing, chemical reagent addition and mixing, waste slurry heating and cooling, and waste chemical leaching. Figure 3.1 presents a simplified process diagram showing the vessels, pumps, heat exchangers, and filter systems associated with the PEP. Equipment that has been considered critical for evaluating the integrated system performance has been scaled to be prototypic. Specifically, the ultrafiltration feed preparation and feed vessels have been scaled to be geometrically similar to WTP with a $1 / 4.5$ scale; the working height and diameter are scaled to $1 / 4.5$. Pipe sizes are scaled to have approximately $1 / 4.5$ the diameter, but the fluid velocity is to be approximately the same as the full-scale plant.

Waste simulant feed can be provided from three primary sources: High Level Waste (HLW) Feed Receipt Vessel (HLP-VSL-T22), Feed Receipt Process (FRP) Vessels (FRP-VSL-T01), or Feed Evaporator Process (FEP) Vessel (FEP-VSL-T01). Feed is received into the Ultrafiltration Feed Preparation Vessels UFP-VSL-T01A/B (henceforth Tank T01A and Tank T01B). In Integrated Test B, feed from Tanks T01A/B was used to provide simulant feed to UFP-VSL-T02A (henceforth Tank T02A)

during simulant concentration. Simulant concentration, caustic leaching, oxidative leaching, and washing were conducted in Tank T02A and the associated filter-loop. The concentrated solids were transferred into totes while the liquid fraction (permeate) was stored in permeate collection vessels (UFP-VSL-T62A/B). Wash and leach solutions were sent to UFP-VSL-T62A/B. 


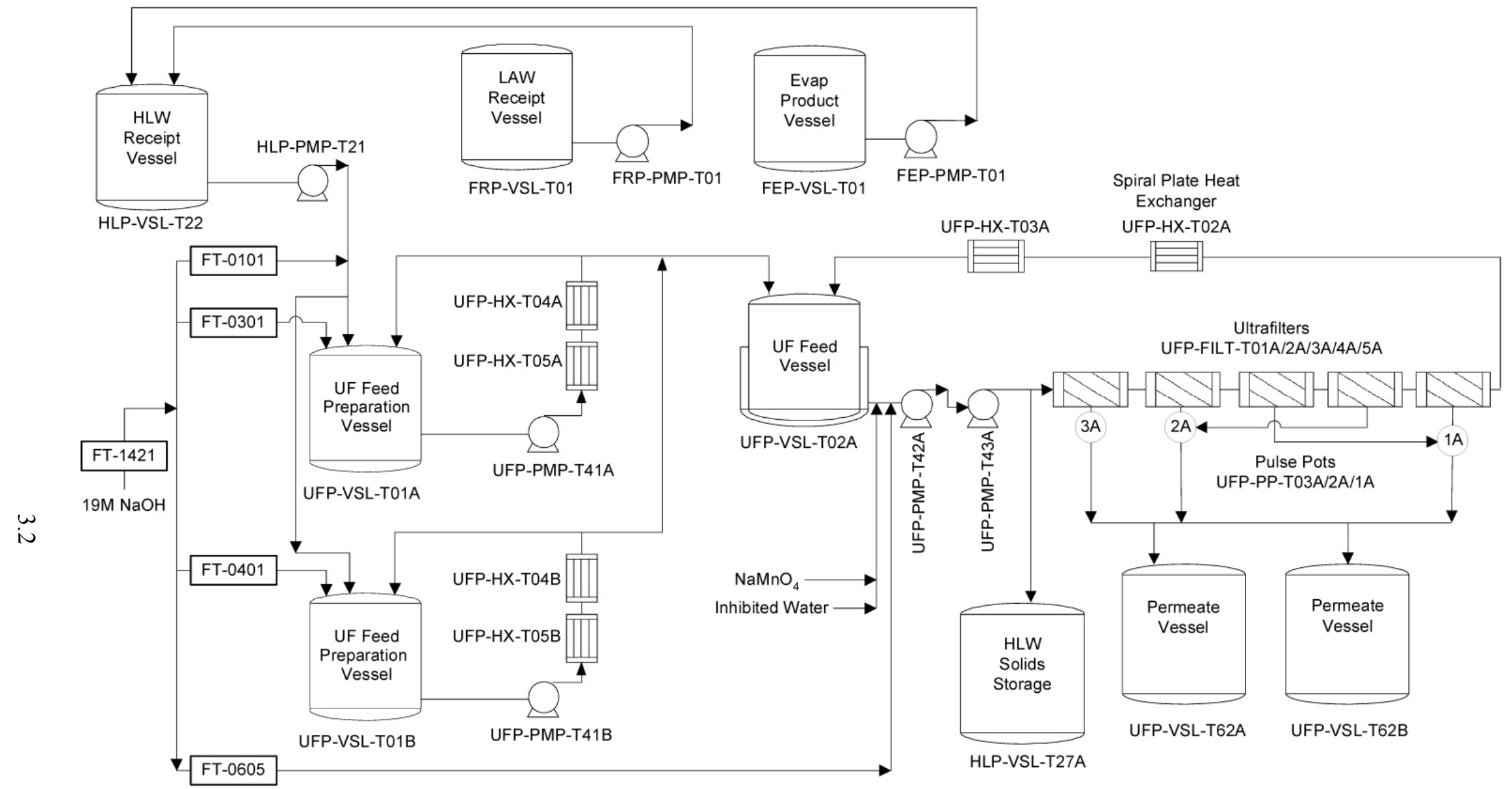

Figure 3.1. PEP Simplified Flow Diagram 


\subsection{PEP Filtration System}

The PEP filtration system is composed of an ultrafiltration feed tank, Tank T02A, a slurry circulation and filtration loop, a permeate metering and collection system, and a filter backpulse and cleaning system. A simplified schematic of the filtration system is shown in Figure 3.2. The PEP filtration system is configured to measure the feed flow rate, temperatures, and axial and transmembrane pressure (TMP) for each filter. In addition, the system is configurable such that filters 1 through 5 may be connected in series to the slurry circulation loop or bypassed such that flow is directed through filter bundle 1 or through filters 2 through 5. A summary of process instrumentation is provided in Appendix A. In the following paragraphs, key process equipment for slurry filtration operations are identified and discussed. Interested readers are referred to the documents listed in Section 3.1 if more information is needed.

\subsubsection{Ultrafiltration Feed Tank}

Tank T02A serves as a primary supply and mixing reservoir for slurry being circulated through the filtration loop. The contents of this tank are mixed using an array of six PJMs and the filter-loop return jet. Ancillary systems for Tank T02A include air spargers, which aid in mixing and also limit inflammable gas hold-up in the actual waste treatment system, bubblers to measure slurry density and level, laser level sensors, and an array of RTDs to measure the tank temperature profile. Specific locations of the RTD arrays for Tanks T02A and T01A are available in laboratory record book (LRB) 59944, pages 12-17. Tank T02A is equipped with a water jacket to cool and a steam ring to heat the contained slurry.

\subsubsection{Slurry Filtration Circulation Loop}

The filtration loop contains process equipment key to slurry dewatering and washing operations. Two centrifugal slurry pumps, UFP-PMP-T42A and UFP-PMP-T43A (hereafter referred to as Pump T42A and Pump T43A, respectively), are operated in series to provide the required slurry flow rate and pressure for the cross-flow filters. The suction to Pump T42A is fed from Tank T02A. In addition, the feed to Pump T42A is connected to process inhibited water (IW) ${ }^{(a)}$ supplies used for slurry washing and dilution operations. The discharge from Pump T42A feeds Pump T43A. Slurry discharge from Pump T43A can be fed through, or bypassed around, the cross-flow filters. Pumps T42A and T43A are identical pumps rated at $150 \mathrm{gpm}$ and $243 \mathrm{psi}$.

The cross-flow filter system is the core of slurry liquid-solid separations. It is composed of five filters connected in series. These filters are designated as UFP-FILT-T01A to -T05A (hereafter referred to as Filters 1 through 5). The filter-loop is equipped with slurry bypass valves to allow slurry flow through Filter 1 and/or Filters 2 through 5.

(a) Inhibited water is $0.01 \mathrm{M} \mathrm{NaOH}$. 


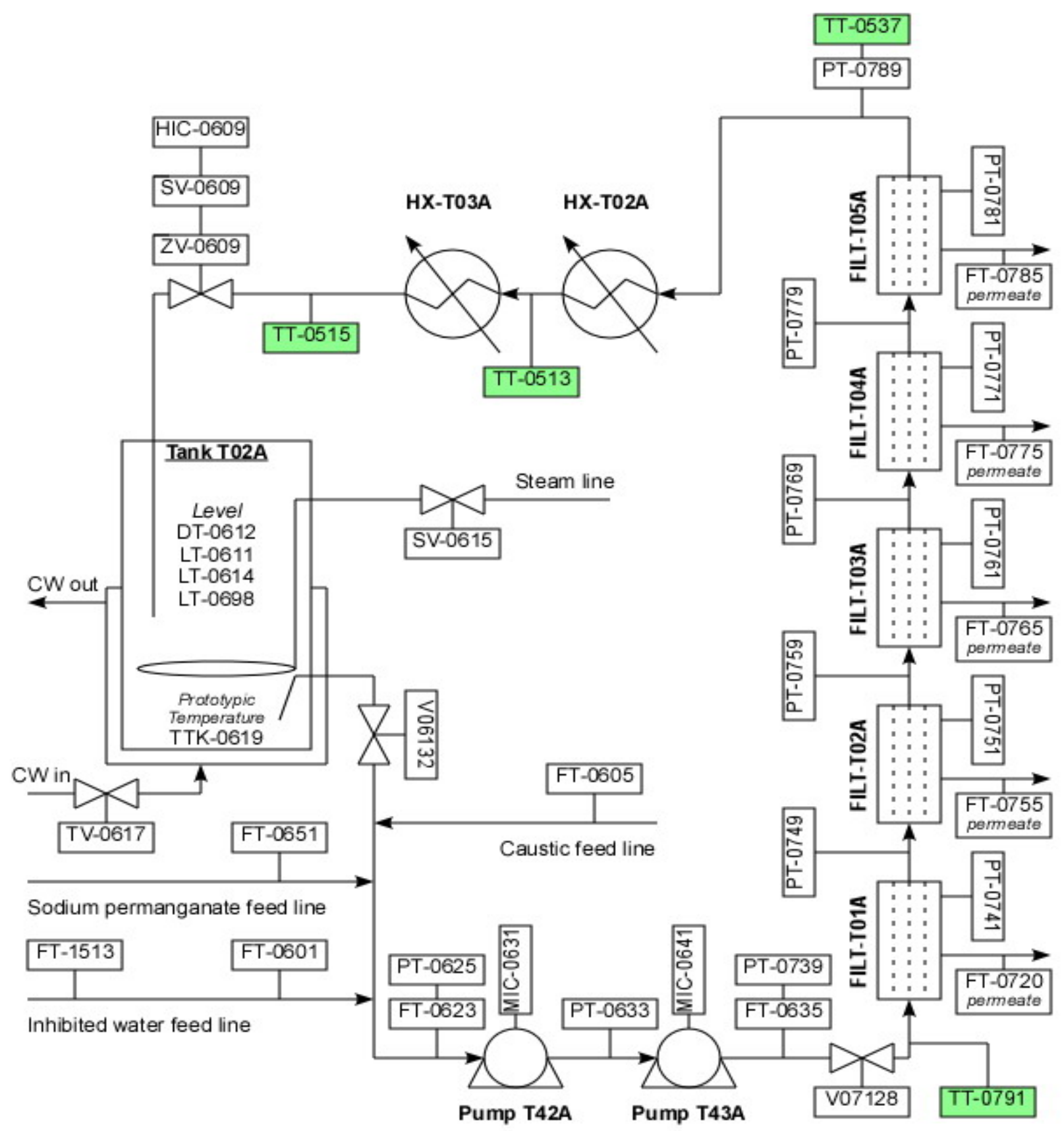

Figure 3.2. Schematic of Filtration Loop ${ }^{(a)}$

(a) $\mathrm{DT}=$ density transmitter, $\mathrm{HX}=$ heat exchanger, $\mathrm{LT}=$ level transmitter, $\mathrm{FT}=$ flow transmitter (includes the matching FE or Flow Element), PT = pressure transmitter (includes the matching PI or Pressure Instrument), $\mathrm{TT}=$ temperature transmitter (included the matching TE or Thermocouple Element), MIC $=$ Motor Indicating Controller, V=Valve, SV=Solenoid Valve, $\mathrm{ZV}=$ Positioning Valve, and $\mathrm{HIC}=$ Human Interface Control. Instruments with Nonconformance Reports issued during testing are shaded. Note that UFP-HX-T03A was bypassed during Integrated Test B. 
The filters purchased for the PEP were obtained from the Mott Corporation (Farmington, CT) using the same specifications for the filters being purchased for the PTF. The filters are constructed of porous sintered 316 stainless steel with an effective filtration rating of $0.1-\mu \mathrm{m}$. The PEP test system employs a combination of 8-ft-long and 10-ft-long filter elements (which were formed by welding either four or five 2-ft filter elements together). As such, the PEP elements have the same radial dimensions and filtration ratings as the 2-ft elements, but have a longer filtration length of either 96 inches or 120 inches. A summary of the geometries of the five filters is provided in Table 3.1.

Tubeside slurry flow rate and pressure are monitored by a series of flow meters and pressure transducers. As shown in Figure 3.2, slurry flow to Pump T42A is measured by magnetic flow meter FT-0623. Slurry discharge flow from Pump T43A is measured by a second magnetic flow meter (FT-0635). Circulation loop pressure is monitored by a series of pressure transducers located at the entrance to each slurry pump, filter bundle, and heat exchanger.

The temperature in both Tank T02A and the slurry filtration loop is controlled with two in-line heat exchangers. The first heat exchanger, UFP-HX-T02A, is a (prototypic) spiral plate heat exchanger that uses chilled water to cool the circulating slurry. The second heat exchanger, UFP-HX-T03A, is a (nonprototypic) steam exchanger used to heat the circulating slurry, if needed. Both heat exchangers are equipped with a bypass loop so that they can be isolated from slurry flow. RTDs installed in thermowells monitor the performance of the heat exchanger. ${ }^{\text {(a) }}$ For the current testing, UFP-HX-T03A was not used and was bypassed. The final process element in the slurry circulation loop is a pressure control valve (ZV-0609) that can be adjusted in combination with the slurry pumps to provide adequate backpressure for permeate production. The filter-loop in-line sampler is located immediately downstream of ZV-0609. After passing through ZV-0609 and past the in-line sampler, the circulating slurry is recycled back into Tank T02A.

Table 3.1. Specifications of the Five PEP Cross-Flow Filtration Bundles

\begin{tabular}{cccccc}
\hline Filter \# & Filter ID & $\begin{array}{c}\text { Number of } \\
\text { Elements in } \\
\text { Bundle }\end{array}$ & $\begin{array}{c}\text { Element } \\
\text { Inside } \\
\text { Diameter } \\
\text { (inches) }\end{array}$ & $\begin{array}{c}\text { Element } \\
\text { Length }(\mathrm{ft})\end{array}$ & $\begin{array}{c}\text { Burface Area } \\
\left(\mathrm{ft}^{2}\right)\end{array}$ \\
\hline 1 & UFP-FILT-T01A & 12 & 0.5 & 10 & 15.7 \\
2 & UFP-FILT-T02A & 12 & 0.5 & 10 & 15.7 \\
3 & UFP-FILT-T03A & 12 & 0.5 & 10 & 15.7 \\
4 & UFP-FILT-T04A & 12 & 0.5 & 8 & 12.6 \\
5 & UFP-FILT-T05A & 12 & 0.5 & 8 & 12.6 \\
\hline Total & -- & -- & -- & -- & 72.3 \\
\hline
\end{tabular}

(a) The RTD measuring the slurry outlet temperature (TT-0513) did not extend into the process stream, and data were to be used for qualitative purposes only. See discussion in Section 2 for a description of this nonconformance (NCR 42402.1). The impact for testing was that during Integrated Test B, the temperature control for the cooling heat exchanger in the filter-loop, UFP-HX-T02A, was with a hybrid control method: the cooling water control valve (TV-0513) was adjusted (in automatic) to control the outlet slurry temperature TT-0513 to a targeted setpoint. However, the temperature setpoint was changed manually by an operator to try to maintain the temperature setting in Tank T02A. 


\subsubsection{Permeate Metering and Collection Systems/Filtration Backpulse Systems}

The permeate metering and collection systems consist of Coriolis mass flow meters for monitoring permeate production rates, three pulse-pots connected to high-pressure air supplies for backpulsing the filters, and permeate collection tanks.

Permeate (shellside) mass production rates from Filters 1 through 5 are monitored by Coriolis flow meters. Permeate flow from each of the filters is directed to three pulse-pots (designated as UFP-PP-T01A to UFP-PP-T03A). Pulse-pot UFP-PP-T03A serves Filter 1, pulse-pot UFP-PP-T02A serves Filters 2 and 4, and pulse-pot UFP-PP-T01A serves Filters 3 and 5. The pulse-pots are filled with a sufficient volume of collected permeate to backpulse the filters. Overflow from the pulse-pots may be directed to 1) permeate or process slurry collection tanks (UFP-VSL-T62A and -T62B) during slurry dewatering operations, or 2) a return line to Tank T02A during continuous recycle filtration operations. Table 3.2 summarizes the permeate metering and pulse-pot systems.

Table 3.2. Permeate Metering and Pulse-Pot Configurations for PEP

\begin{tabular}{lll}
\hline Filter Bundle No. / ID & $\begin{array}{c}\text { Permeate } \\
\text { Coriolis Meter }\end{array}$ & $\begin{array}{c}\text { Associated Pulse } \\
\text { Pot }\end{array}$ \\
\hline 1 - UFP-FILT-T01A & FT-0720 & UFP-PP-T03A \\
2- UFP-FILT-T02A & FT-0755 & UFP-PP-T02A \\
3 - UFP-FILT-T03A & FT-0765 & UFP-PP-T01A \\
4- UFP-FILT-T04A & FT-0775 & UFP-PP-T02A \\
5 - UFP-FILT-T05A & FT-0785 & UFP-PP-T01A \\
\hline
\end{tabular}

During backpulsing, one of the pulse-pots is isolated, drained to a specific level, and charged with high-pressure air until the pulse-pot pressure exceeds the slurry pressure at the filter inlet by a given amount (typically 40 psid). After the target pulse-pot pressure is reached, a large, fast-acting valve isolating the pulse-pot from the filter is opened, and a fraction of the liquid in the pulse-pot (typically less than 1 gal) flows back to the filter bundle, forcing permeate from the shellside to the tubeside of the filter tubes. The backflow of permeate forces loosens any particles that are weakly entrained in the filter pores or that have caked on the filter surface. A description of the backpulsing process, as it was implemented in the PEP, is provided in Appendix B.

\subsection{PJM Operational Modes}

The leaching vessels UFP-VSL-T02A and UFP-VSL-T01A and B were equipped with PJMs and several different operating programs to allow plant prototypic mixing, low-level operation, and additional data collection.

PJM operating modes have the same basic cycle: Drive Phase, Vent Phase, and Vacuum Phase, followed by a Hold (or Vent) Phase. PJM mixing technology involves a pulse-tube coupled with a jet nozzle. One end of the tube is immersed in the tank while periodic pressure, vacuum, and venting are supplied to the opposite end. Changing the applied pressure creates four operating phases for the pulse-tube: 1) the drive phase, when pressure is applied to discharge the contents of the PJM tube through the nozzle, 2) the vent phase, when the pressure is vented to the atmosphere, 3 ) the refill phase, 
when vacuum is applied to refill the pulse-tube (at temperatures above $60^{\circ} \mathrm{C}$, the vacuum is disabled and the pulse-tubes refill by gravity.), and 4) the hold phase, when all valves to the PJMs are closed, causing the fluid level in each PJM to remain constant until the next drive phase. The PJM system uses these operating phases to produce a sequence of drive cycles that provide mixing in the vessel.

A timer set to the overall Cycle Time starts at the beginning of each Drive Phase. A new drive phase begins when either the cycle timer runs out or the cycle enters the final Hold/Vent Phase, whichever is later. This condition exists to make sure the Vacuum Phase has completed before moving on to the next cycle.

PJM operations at PEP consisted of one of several control modes: standard mode (regular), standard mode (star pattern-Tank T02A only), standard mode (short cycle), and simple mode. However, PJM controls at PEP were not prototypic. During Integrated Test B, the standard mode (regular) was used whenever possible, and the standard mode (star pattern) was used when the level was low, typically $<23$ inches. If standard mode was non-functional (e.g., during caustic leaching, the PJM Drexelbrook level probes ceased functioning), the PJMs were operated in simple mode. For all tanks, if the PJMs were operating in standard mode, the short cycle function would automatically activate when the tank level dropped to 12 inches and would remain active until the tank level rose above 22 for Tank T01 A and Tank T01B. The short cycle activation occurred automatically in Tank T02A when the level dropped to 11.5 inches and remained until the level returned to 16 inches.

\subsubsection{Standard Mode (Regular) Description}

Standard mode operations in PEP control each PJM individually based on that PJM level instrument. The PJMs have one overall cycle time, which keeps all PJMs in synch at the beginning of each cycle. Each cycle is controlled by five main variables: Cycle Time, Drive Time Set Point, Vent Time Set Point, L1 Level, and L2 Level. L1 and L2 Levels are user-definable upper and lower level set points used by the control software to indicate an overblow. Additionally, each PJM could be enabled or disabled individually.

The entered Cycle Time controls the peak-to-peak distance between cycles. However, if the value of the cycle time set point is shorter than the combined entered times for the PJM phases, the cycle time set point would be over-ridden, and the actual PJM cycle time would be the time it took the PJMs to complete the first three phases (Drive, Vent, and Vacuum). The fourth phase would be skipped.

The Drive Phase is controlled by the time entered as the Drive Time Set Point (corrected for temperature if enabled). Each PJM begins the Drive Phase at the same instant but can have different lengths of time specified for driving each PJM. If any PJM does not finish within 30 seconds of the PJM with the shortest drive time, that PJM is disabled, and the cycle continues to the next phase.

The Vent Phase is controlled by the Vent Time Set Point, which has no correction factors. Each PJM begins the Vent Phase at the same instant and can have different lengths of time specified for each PJM. If any PJM does not finish within 30 seconds of the PJM with the shortest vent time, that PJM will be disabled, and the cycle will continue to the next phase. Generally, each Vent Time Set Point was set to a small number such as 500 milliseconds to smooth out the transition between the Drive and Vacuum Phases. 
The Vacuum Phase is controlled by the L1 Level, which is set at the top of the desired PJM stroke. All PJMs begin the Vacuum Phase at the same instant, but each PJM L1 Level is set separately. If any PJM does not reach the L1 Level within 30 seconds of the first PJM to reach its L1 Level, that PJM is disabled, and the cycle continues to the next phase. During high temperature $\left(>60^{\circ} \mathrm{C}\right)$ operations, the vacuum was disabled, and each PJM tube was gravity filled up to its L1 Level. If the tank level was less than 44 inches (T01A/B) or 46 inches (T02A), then the PJM would switch operation to Standard ModeShort Cycle until either the temperature dropped below the $60^{\circ} \mathrm{C}$ threshold or the level rose above the 44/46-in. threshold.

The final phase in standard mode is a Hold Phase. All valves to the PJM are closed, causing the fluid level in each PJM to remain constant at the L1 Level.

The standard mode operation also contains a temperature linearization variable that reduces the drive time to prevent overblows due to the difference in slurry properties as the temperature increases. The variable is expressed as a slope, the percent of drive time decrease per degree Celsius increase from zero degrees Celsius. This function was largely untested. A modest $0.25 \%$ linearization factor was used in early testing and adopted for the remainder of testing simply because it seemed to work. Further optimization was not conducted.

\subsubsection{Standard Mode-Short Cycle}

A Short Cycle consists of a Drive Phase in which each enabled PJM drives for $20 \%$ of the Drive Time Set Point followed by a 10 -second Vent Phase. The short-cycle function would automatically activate when the tank level dropped to 12 inches and would remain active until the tank level rose above 22 inches for Tank T01A and Tank T01B. The short cycle activation occurred automatically in Tank T02A when the level dropped to 11.5 inches and remained until the level returned to 16 inches. Additionally, the short cycle would activate during high temperature operations $\left(>60^{\circ} \mathrm{C}\right)$ if the tank level fell below 44 inches (T01A/B) or 46 inches (T02A) to confirm that there was enough fluid to gravity fill above the L1 Level for each PJM due to the disabling of the vacuum at high temperatures.

\subsubsection{Standard Mode-Star Pattern}

Tank T02A additionally can operate in standard mode - star pattern. The star pattern option follows the same rules as the regular Standard PJM Mode. The difference is that only two PJMs are enabled at a given time. When the operator sets the star-pattern button on the human-machine interface (HMI), the center PJM and one other PJM will be enabled for five cycles. Then the outer PJM will be disabled, and a different outer PJM will be enabled for another five cycles. The pattern of enabling and disabling the five outer PJMs forms a five point star. The disabled PJMs are vented.

The star pattern was developed by PEP test engineers in an effort reduce the level fluctuations in Tank T02A and operate the PJMs in a manner that could be implemented in the PTF. During Integrated Test B, the star pattern was enabled when the level in Tank T02A was approximately 23 inches or when the filter-loop recirculation target could not be achieved. Enabling the star pattern increased the achievable filter-loop recirculation rate because the star pattern reduced the tank level fluctuations, as fewer PJMs were filling and discharging in this mode. 


\subsubsection{Simple PJM Mode Description}

As mentioned above, the simple mode operates substantially the same as the standard mode. Each cycle consists of Drive, Vent, Vacuum, and Hold/Vent phases. There are two main differences. The first difference is that all PJMs operate together as one unit. There are no individual levels used or individual enabling or disabling of PJMs. The second difference is that each phase is controlled by a time (instead of level). An operator sets the Cycle Time, Drive Time Set Point (uncorrected), Vent Time Set Point, and Vacuum Time Set Point (uncorrected). Additionally, the operator can choose whether the last phase holds the PJM level or vents (returning PJM level to the same as the tank level).

During Integrated Test B, the simple mode was used when the standard mode was not working. The standard mode did not work during leaching operations because the PJM level probe ceased to function at elevated temperatures in high-caustic solutions.

The Drive Time had additional correction factors for temperature (same as in standard mode) and tank level. The tank level correction (if enabled) reduced the drive time according to the user-entered slope ( $\%$ drive time/inch) for every inch below the top of the PJM head (46 inches by default). For example, if the Drive Time set point was 10,000 milliseconds, the Drive Slope was 1\%/inch, and if the tank level was at 36 inches, then the corrected drive time would be $(10,000$ milliseconds $) *\{1.00--[0.01 /$ inch $*(46-36$ inches $)]\}=9000$ milliseconds. The operator determines the tank level used in the calculation with either the tank bubbler, the laser, or the Drexelbrook probe.

The Vacuum time also contained a correction factor for tank level, but not for temperature. As the tank level decreased, the PJM fill level would decrease during the Vacuum Phase; therefore, a vacuum correction was implemented. The vacuum correction (if enabled) increased the vacuum time according to a user-entered vacuum slope (\% vacuum time/inch) for every inch the tank level was below 46 inches. For example, if the Vacuum Time set point was 10,000 milliseconds, the vacuum slope was 1\%/inch, and the tank level was at 36 inches, then the corrected drive time would be $(10,000$ milliseconds $) *\{1.00+[0.01 /$ inch $*(46-36$ inches $)]\}=11,000$ milliseconds.

The operator selectable Hold Phase was created to give the option to more closely resemble PJM Standard Mode operation. The Hold Phase prevented the PJM level from dropping after completing the Vacuum Phase. However, since the PJM phase set points were time based, and there was no level checking in the PJMs in Simple Mode, the PJM fill level could increase over successive cycles when using the Hold Phase - whether or not the drive or the vacuum time set points were decreased or increased, respectively. The successive increase in PJM level over time could result in fluid traveling all the way up into the hoses on top of the tank. Similarly, if the vacuum time was too low, the PJM fill level could decrease over successive cycles, eventually resulting in an overblow. If the Hold Phase was disabled, each PJM just vented to the tank level during the remainder of the PJM cycle.

\subsection{Data Acquisition System for PEP}

The data acquisition system (DAS) for PEP is composed of four data acquisition servers and one graphical user interface (GUI) PC. They are networked together, along with the rest of the PEP equipment as depicted in Figure 3.3. 


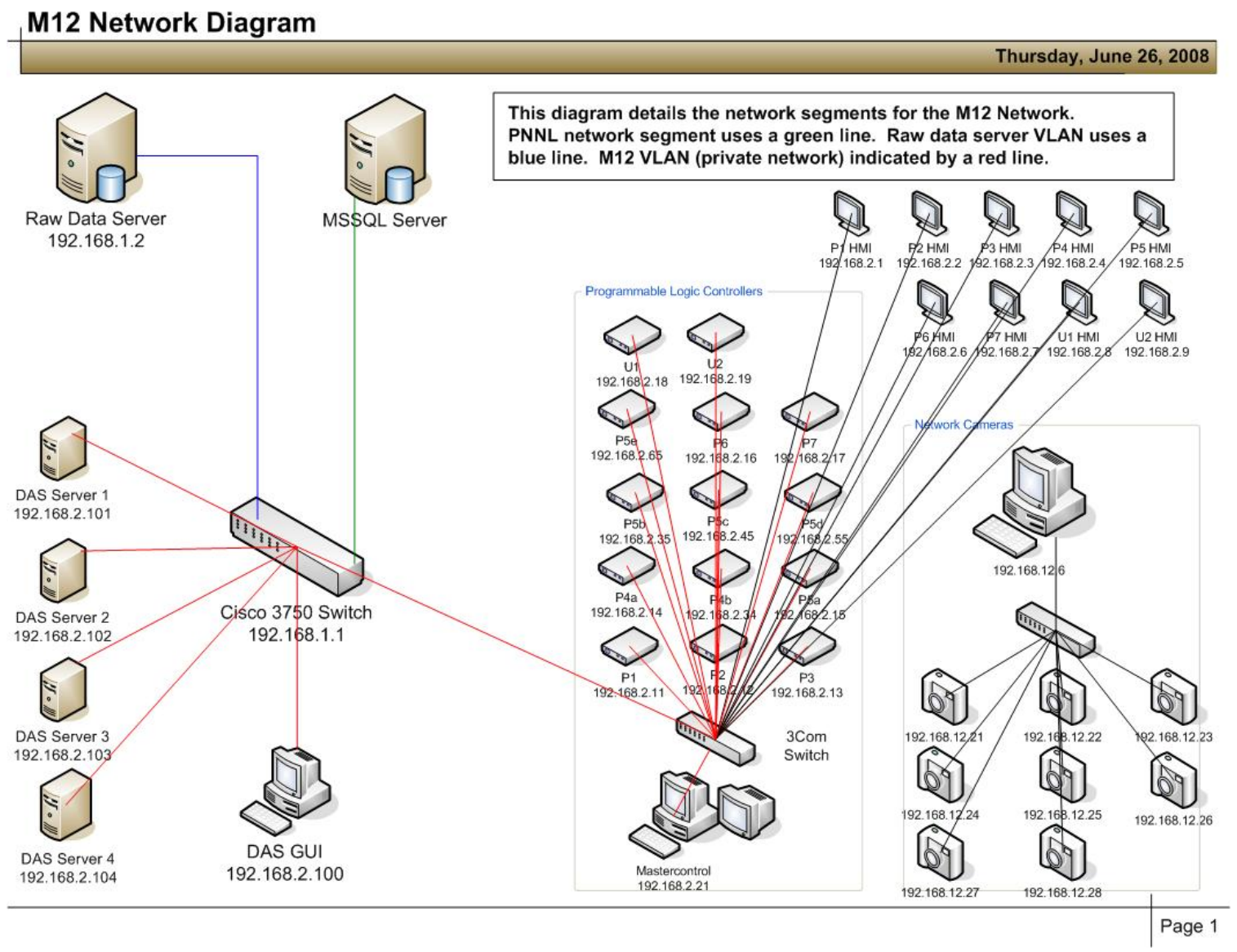

Figure 3.3. M12 Network Diagram

The GUI PC resides in the control room and is used to start and stop the data acquisition servers and set the recording rate. Each server that is located out in the high bay is independent of the others while collecting data. A global positioning system (GPS) receiver is used to provide a time stamp to each DAS server.

\subsubsection{Background}

The PEP data acquisition and control system was designed to be very robust and flexible in operation. While the same sensors are used for controlling the PEP and recording data, the sensor outputs are split into two loops to make certain that the DAS is completely separate from the control system. This requirement was put into the system to allow the PEP control system to be changed without the rigorous Verification and Validation process that is required of any quality-affecting software program. This allowed the control system to be changed many times while the PEP system was operating without affecting the quality of the data. The DAS and all related software did undergo PNNL software control procedures to make certain that the data meet quality standards.

From each signal splitter, one loop went to the control system, and the other went to the DAS. Four to twenty-ma outputs from all sensors were selected to confirm the reliability of the system and prevent long cable runs from introducing error in the signals. The data acquisition servers are manufactured by 
Microstar Laboratories, located in Bellevue, WA. Each server is capable of storing a large number of channels at a high sampling rate. For PEP, they have been set to a maximum sampling rate of $100 \mathrm{~Hz}$. Each server stores its information in a file local to that server. After data were collected, they were transferred to the raw data server and to the database server for analysis.

\subsubsection{Data Retrieval}

During operations of the PEP during shakedown and Phase 1 testing, over a terabyte of data was recorded by the four data acquisition servers. Those data are stored in binary files that are not human readable. Software was developed to provide analysts with an easy tool to retrieve the data they required by specifying the date/time, instruments, and sampling rate. This tool is composed of three main components: the data ingest server, the database itself, and the data retrieval tool. The data ingest server watches a specified directory on the database server, and when new files are put there, it uploads the data from that location into the database and transfers those data files into a directory where processed files are stored. The database itself uses Microsoft's SQL server as a basis for the large database that stores all data and sensor information for the PEP. Finally, the data retrieval tool is a web-based application that allows staff to recover the data they need by specifying the time/date, instruments, and sampling rate necessary. The software produces a text file that can easily be imported into Excel or any other analysis package. This method provides a data file that meets quality standards for the PEP project.

Another method for retrieving data was commonly referred to as the DAS widget. This tool is a simple application that allows users with a detailed knowledge of the system to retrieve data if they do not have access to the web-based tool, or if they need access to the data before the data have been imported into the database. This tool did not go through the software control procedure, and it does not provide data that meets quality standards for PEP. It will read a file from one server and produce a file with all the raw (ma) values from every instrument on that server. With that data, and the information in the measurement and test equipment (M\&TE) list, staff can retrieve the data from all sensors in engineering units. It is more time consuming to retrieve data in this manner, but it allows data to be read in near real-time. This method was commonly used to provide near real-time data for PJM tuning.

\subsubsection{Data Storage}

All data recorded during operation of the PEP were first copied to the raw data server in PDL-W and then copied to the database server in LSB. This procedure is detailed in the data management plan and provides redundant storage of all data to make certain that data are not lost.

\subsection{Testing Overview}

The following is a summary level overview of testing reported herein. Refer to the test narrative (Section 4), Test Instruction TI-WTP-PEP-066, and the LRBs listed below for additional details. The instruction for acid cleaning is provided in Appendix G, and observations during the cleaning are recorded in Laboratory Record Book BNW-60270. The operations log during testing is recorded in BNW-60270 and BNW-60271. The lead test engineer (LTE) observations were recorded in logbook BNW-60279. 
1. Acid clean UFP-FILT-T01A through -T05A with $2 \mathrm{M} \mathrm{HNO}_{3}$.

2. Rinse filters and filter-loop to achieve targeted $\mathrm{pH}$ of $\sim 3$.

3. Transfer simulant from HLP-VSL-T22 to Tank T01A.

4. Transfer simulant from Tank T01A to Tank T02A and concentrate solids to 20 -wt $\%$ using all five filters, UFP-FILT-T01A through -T05A.

5. Caustic-leach concentrated simulant at $98^{\circ} \mathrm{C}$ for 16 hours (Batch 1).

6. Transfer Batch 1 leached simulant to Tank T01B for temporary storage.

7. Transfer simulant from HLP-VSL-T22 to Tank T01A.

8. Transfer simulant from Tank T01A to Tank T02A and concentrate solids to $20-w t \%$ using one filter, UFP-FILT-T01A.

9. Caustic-leach concentrated simulant at $98^{\circ} \mathrm{C}$ for 16 hours (Batch 2).

10. Concentrate leached solids (Batch 1 and 2) using one filter, UFP-FILT-T01A.

11. Conduct filter-loop bypass (CsBr tracer) test.

12. Wash leached slurry and remove permeate using all five filters, UFP-FILT-T01A through -T05A.

13. Add chromium slurry simulant.

14. Re-concentrate slurry and wash using all five filters, UFP-FILT-T01A through -T05A.

15. Conduct oxidative-leach on slurry for 6 hours at $25^{\circ} \mathrm{C}$.

16. Wash oxidatively leached slurry using all five filters, UFP-FILT-T01A through -T05A.

17. Final concentration of slurry.

18. Slurry from Integrated Test B was then combined with slurry saved from Integrated Test A, and a "high-solids filter test" was conducted (reported in Integrated Test A run report, [Guzman-Leong et al. 2009]).

Figure 3.4 shows the overall time line for Integrated Test B testing. 


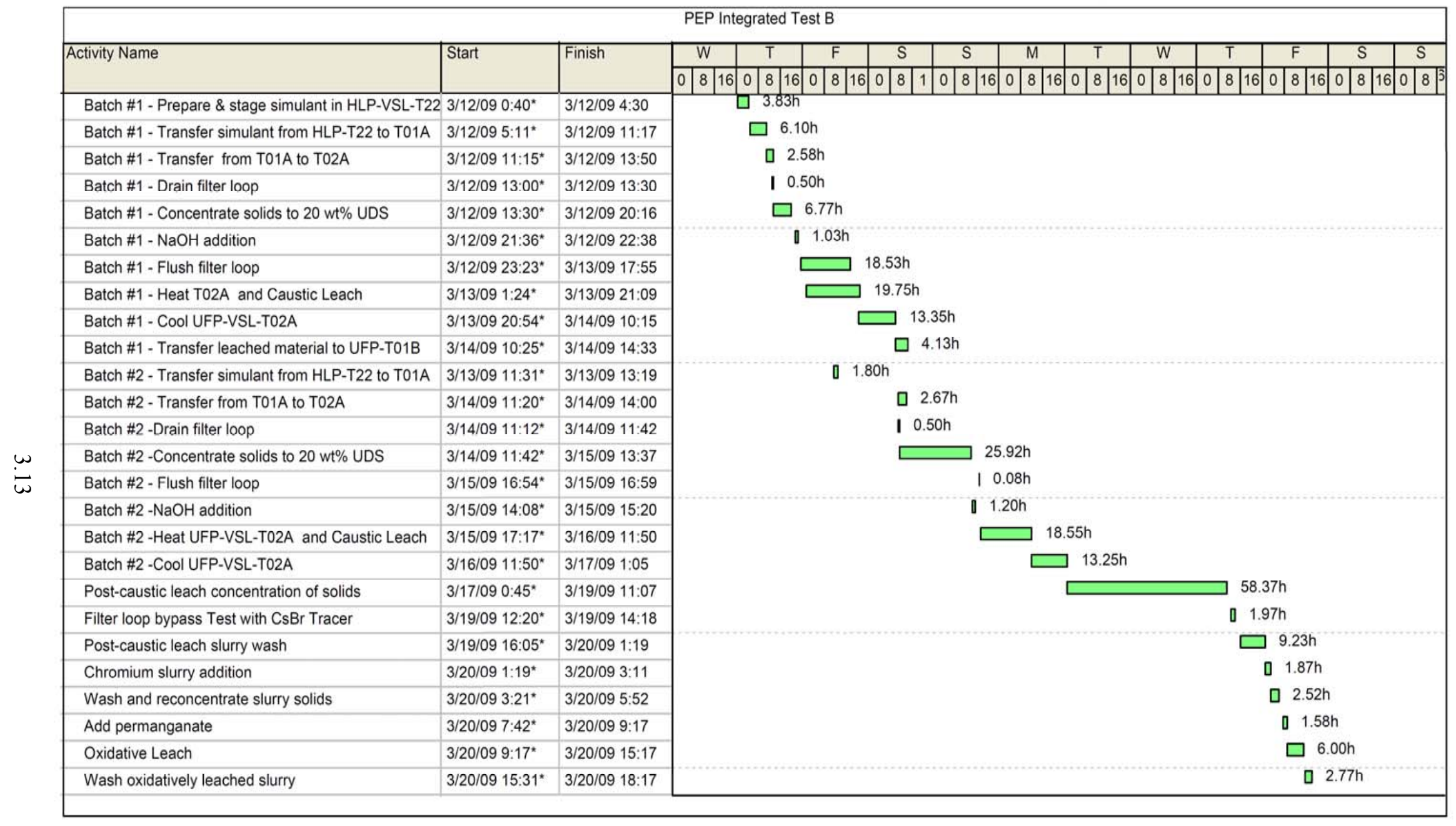

Figure 3.4. Integrated Test B Time Line 


\subsection{Simulant}

PEP process testing was performed with nonradioactive aqueous slurry of simulant waste solids and liquids. The simulant composition and make-up recipe were provided by WTP as documented in Simulant Recommendation for Phase 1 Testing in the Pretreatment Engineering Platform. ${ }^{\text {(a) }}$ Aqueous chemical concentrations were within the ranges expected for waste feeds to the PTF. The hydroxide concentration was marginally one standard deviation lower than the average concentration expected in the feeds to the plant. The oxalate and phosphate components were at the lower end of the expected ranges, but the oxalate component was at the solubility limit, and the phosphate component was at or near the solubility limit. The solids components and blend were selected to obtain targeted solids mass loss (aluminum and chromium leaching and oxalate washing) and treatment time. The simulant was not selected to represent any particular Hanford tank waste type.

The simulant was blended from the components listed below. The basis for selecting the individual components and the comparison to actual waste behavior is provided where applicable in the indicated references.

- Boehmite (for Al) (Russell et al. 2009a)

- Gibbsite (for Al) (Russell et al. 2009b)

- Chromium oxyhydroxide (CrOOH) slurry (Rapko et al. 2007)

- Sodium oxalate

- Filtration simulant (Russell et al. 2009c)

- Supernate.

A separate chromium solids slurry simulant was prepared and added to the PEP process after post-caustic-leach washing (a nonprototypic addition) during the Shakedown/Functional Tests and Integrated Tests A and B. This approach was taken because laboratory-scale tests had shown that the high-temperature caustic leaching step dissolved significant amounts of the $\mathrm{CrOOH}$ solids (Russell et al. 2009a). In Integrated Test D, the chromium solids component of the simulant was added during the simulant make-up process to demonstrate the PTF permanganate addition strategy. Simulant was procured from NOAH Technologies Corporation (San Antonio, TX). Samples of each simulant batch were characterized to make certain that chemical and physical property requirements were met. Batches of the simulant were procured as follows:

- A 15-gallon trial batch of the blended simulant for laboratory testing to demonstrate the efficacy of the simulant fabrication procedure.

- A 250-gallon scale-up batch of the blended simulant to demonstrate scale-up of the simulant fabrication procedure to an intermediate scale.

- Batches 0, 1, and 2, each nominally 3500 gallons, of blended simulant for the Shakedown/Functional Tests and Integrated Tests A and B. These batches did not contain the $\mathrm{CrOOH}$ component.

(a) P Sundar. 2008. Simulant Recommendation for Phase 1 Testing in the Pretreatment Engineering Platform, 24590-PTF-RPT-RT-08-006 Rev 0, Bechtel National, Inc., Richland, WA. 
- Batch 3, nominally 1200 gal, for Integrated Test D. This batch contained the $\mathrm{CrOOH}$ solids component.

- The CrOOH solids slurry for the Shakedown/Functional Test and Integrated Tests A and B was obtained in two separate batches containing nominally 18 and $36 \mathrm{~kg}$ of $\mathrm{Cr}$ as $\mathrm{CrOOH}$.

Table 5.2 provides the simulant composition at the start of testing. Table 5.11 and Table 5.12 provide the composition of the $\mathrm{Cr}$ bearing simulant added before the oxidative-leach.

\subsection{Sampling}

A list of samples taken and their purpose are provided in Appendix C. Actual sample times were recorded in the Test Instruction.

In general, slurry samples were collected using either an in-tank sampler or an in-line sampler. In-line samples were obtained from the slurry recirculation loop or transfer piping by drawing a side stream from the process flow as shown in Figure 3.5. To obtain a sample, the downstream valve was fully opened, and then the upstream valve was opened sufficiently to allow samples to be safely obtained. The sample line and valves were purged with at least three line volumes before a sample was collected. The vent and the pressure gauge shown are for a different sampling method, which would allow samples to be collected from the volume between the two main processing valves while isolated from the process flow. This method was not implemented in Phase 1 testing.

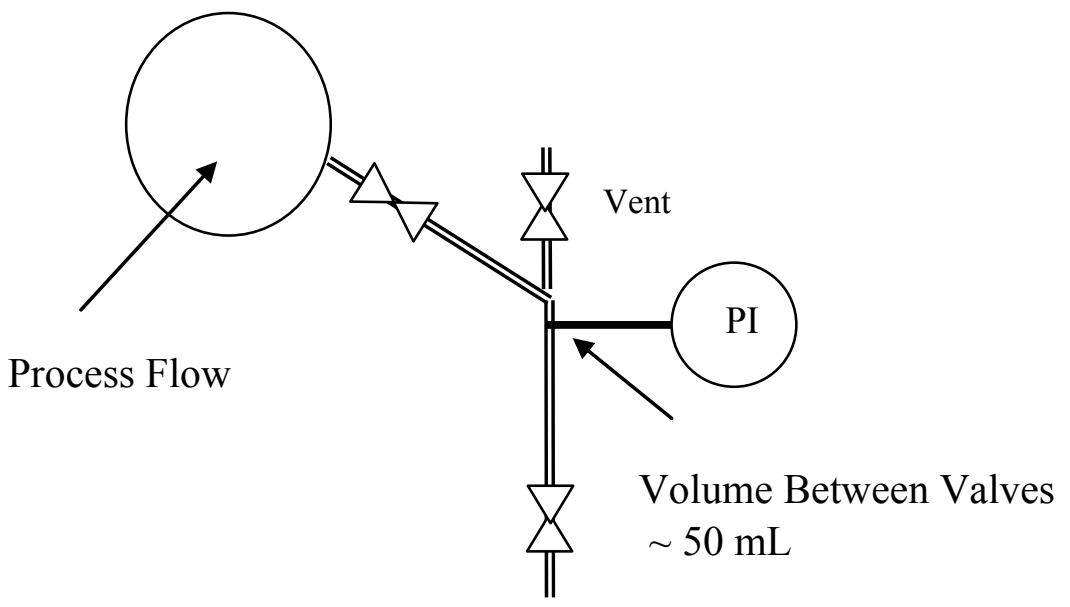

Figure 3.5. Simple In-Line Sampler

Figure 3.6 is a schematic of the in-tank sampling system for Tanks T01A/B and Tank T02A.

Samples were obtained with the sample loop in recirculation mode with slurry returned to the vessel. To obtain a sample, a valve was used to divert the entire flow to the sample bottle. The sampling valve and line were purged before each sample to minimize cross contamination with previous sampling events. Sample line inlet heights are provided in Appendix D.

Permeate (liquid) samples were taken from the permeate piping between the filter and the pulse-pot. The sample line and valves were purged with at least three line volumes before each sampling event. 
Sample handling and analytical methods are discussed in Appendix E.

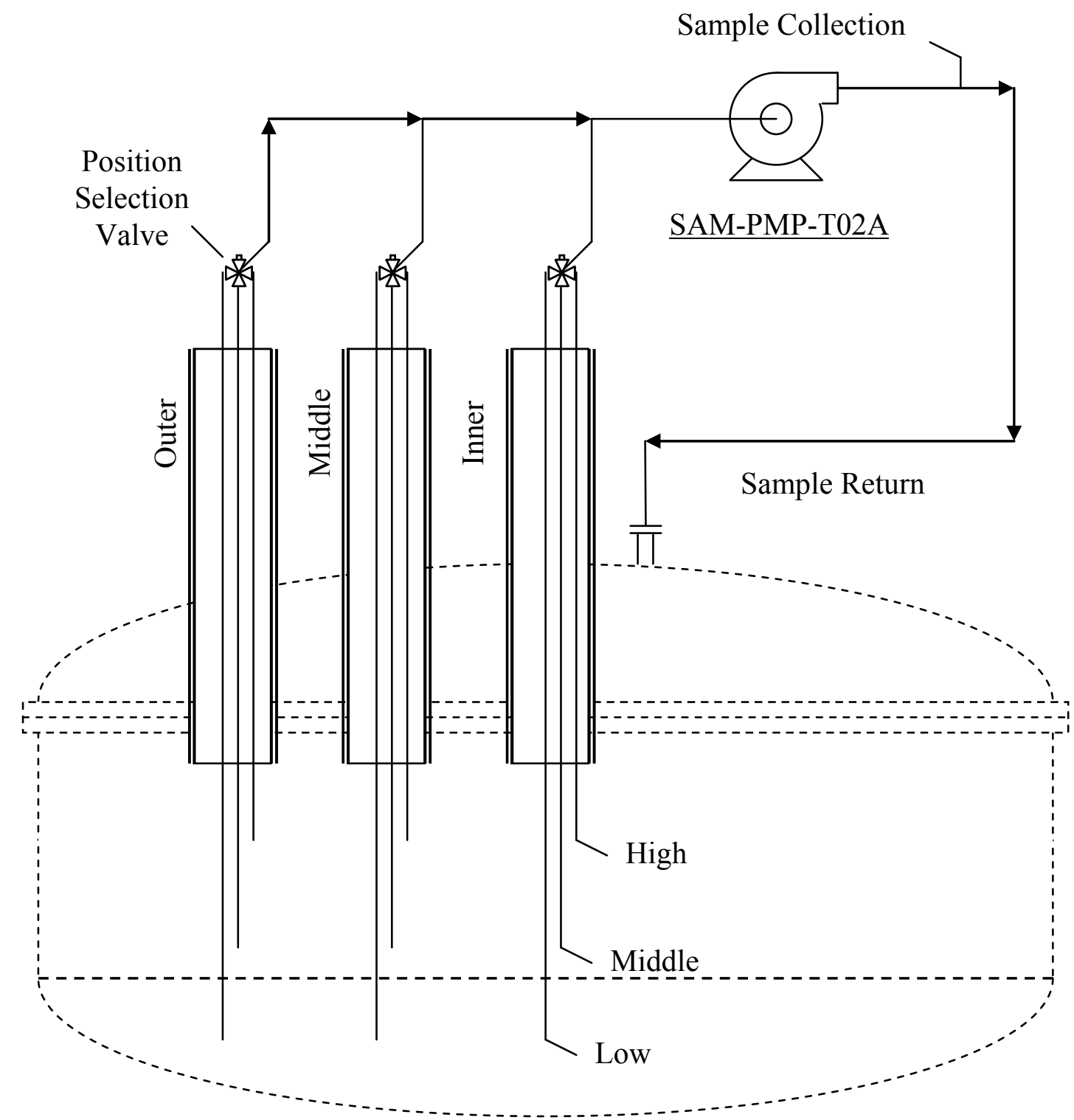

Figure 3.6. In-Tank Sampling Showing the Three Radial Positions at Three Heights and Sampling Flow Loop 


\subsection{Test Narrative}

This section describes the pre-test activities surrounding acid cleaning of the filters and the execution of Integrated Test B, which was run from 3/12/09 to 3/21/09.

Testing was conducted in accordance with Test Instruction TI-WTP-PEP-066. The Run Sheet section of the Test Instruction provided the LTE target or setpoint values for key process operations. A reproduction of the run sheet, with actual values from the LTE or other data source, is provided in Appendix M. Observations were written in this Test Instruction and in laboratory record books BNW-60279, BNW-60270, and BNW-60271.

Integrated Test B demonstrated caustic leaching in vessel Tank T02A using nonradioactive simulant. The reader is cautioned that data reported in this narrative section were generally taken from the Test Instruction and are not NQA-1-compliant, as data recorded in the Test Instruction were obtained from the HMI (rather than the DAS). NQA-1 data can be obtained from the electronic DAS files maintained by WTP, from the figures in this section, and the figures in Appendix J, which were plotted using DAS data and are NQA-1 compliant. A summary of the targeted test conditions provided to the LTE and the actual conditions during testing is provided in Appendix M.

The following subsections summarize the process steps executed for Integrated Test B. Data in plots shown in this section were averaged over 1 min intervals, except for the acid cleaning, which is provided as $1 \mathrm{~Hz}$ data. Sampling results are provided in Sections 5.1 and 5.2.

\subsection{Acid Cleaning Filters}

The filters were cleaned with $2 \mathrm{M}$ nitric acid before starting Integrated Test B. Refer to Appendix G for cleaning instructions for the first and second attempts.

The initial attempt at cleaning the filters started with Tank T02A and with the filter-loop drained. The next step pumped nitric acid into Tank T02A on 3/01/09. The $2 \mathrm{M}$ nitric acid was circulated and filtered for a short time ( $<20$ minutes) when a leak developed in the primary recirculation Pump T42A. However, for the 20 minutes of filtering, the operating parameters were $109 \mathrm{gpm}(15 \mathrm{ft} / \mathrm{s})$ while maintaining TMPs across the filters as high as 40 psid, but typically less than 20 psid. The TMP could not be maintained because the filter fluxes increased rapidly to 7 to $10 \mathrm{~kg} / \mathrm{min}$, and the high pressure drops in the permeate piping prevent a high TMP (i.e., the hydraulic resistance through the permeate line was greater than that through the filter, and a limiting permeate rate [and TMP] was reached.) A higher TMP can be achieved in filter 5 because it has a bigger flow control valve than the other filter assemblies, and consequently, there is a lower hydraulic resistance in the permeate line. Permeate rates just before cleaning were $\sim 2 \mathrm{~kg} / \mathrm{min}$. Temperatures were allowed to float at $\sim 40^{\circ} \mathrm{C}$ during the cleaning. See Figure 4.1 through Figure 4.4 for TMP, axial velocity, temperature, and permeate production during the first acid cleaning. 


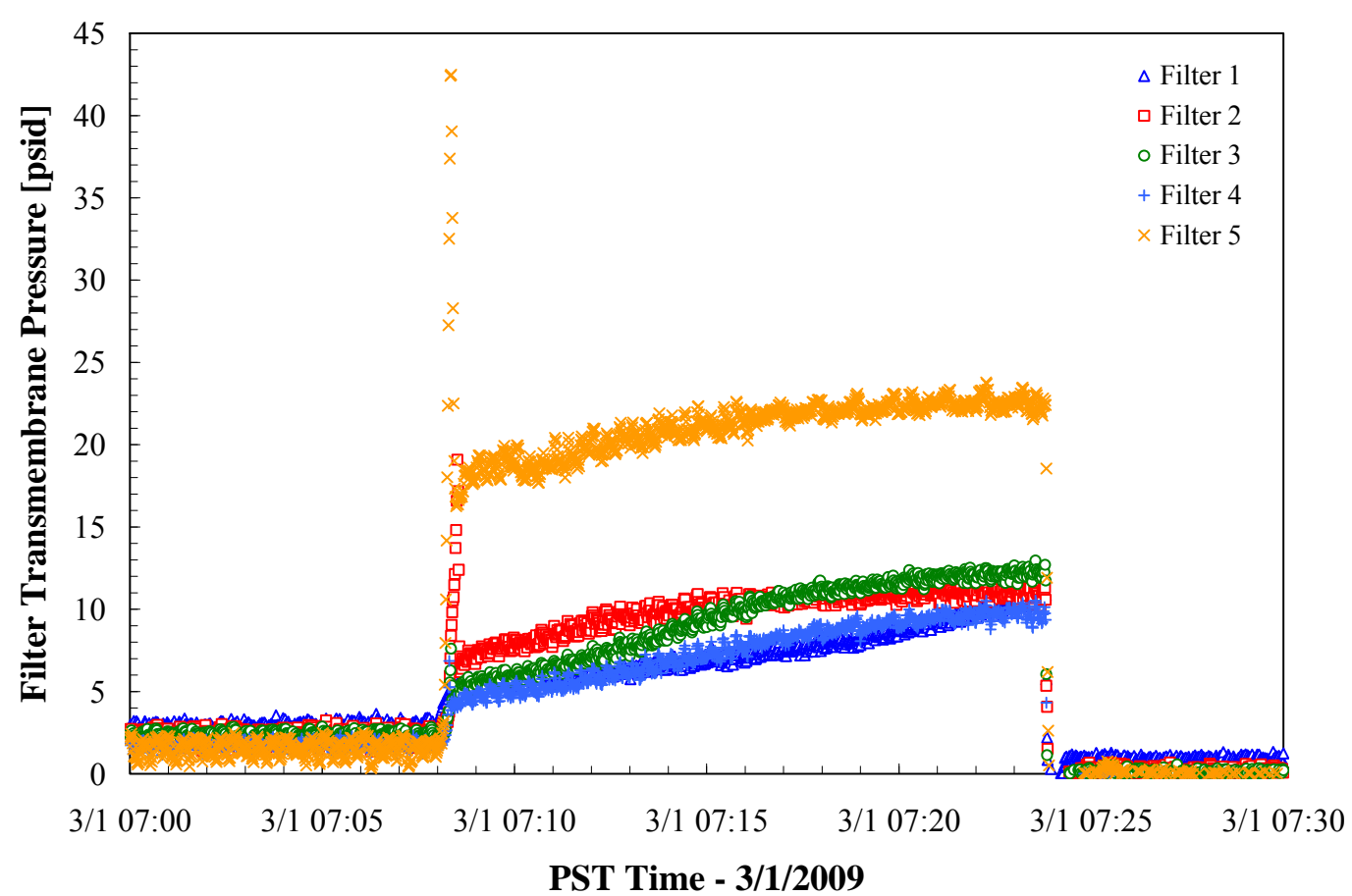

Figure 4.1. TMP for the First Filter Cleaning Event, 03/01/09, Preceding Integrated Test B

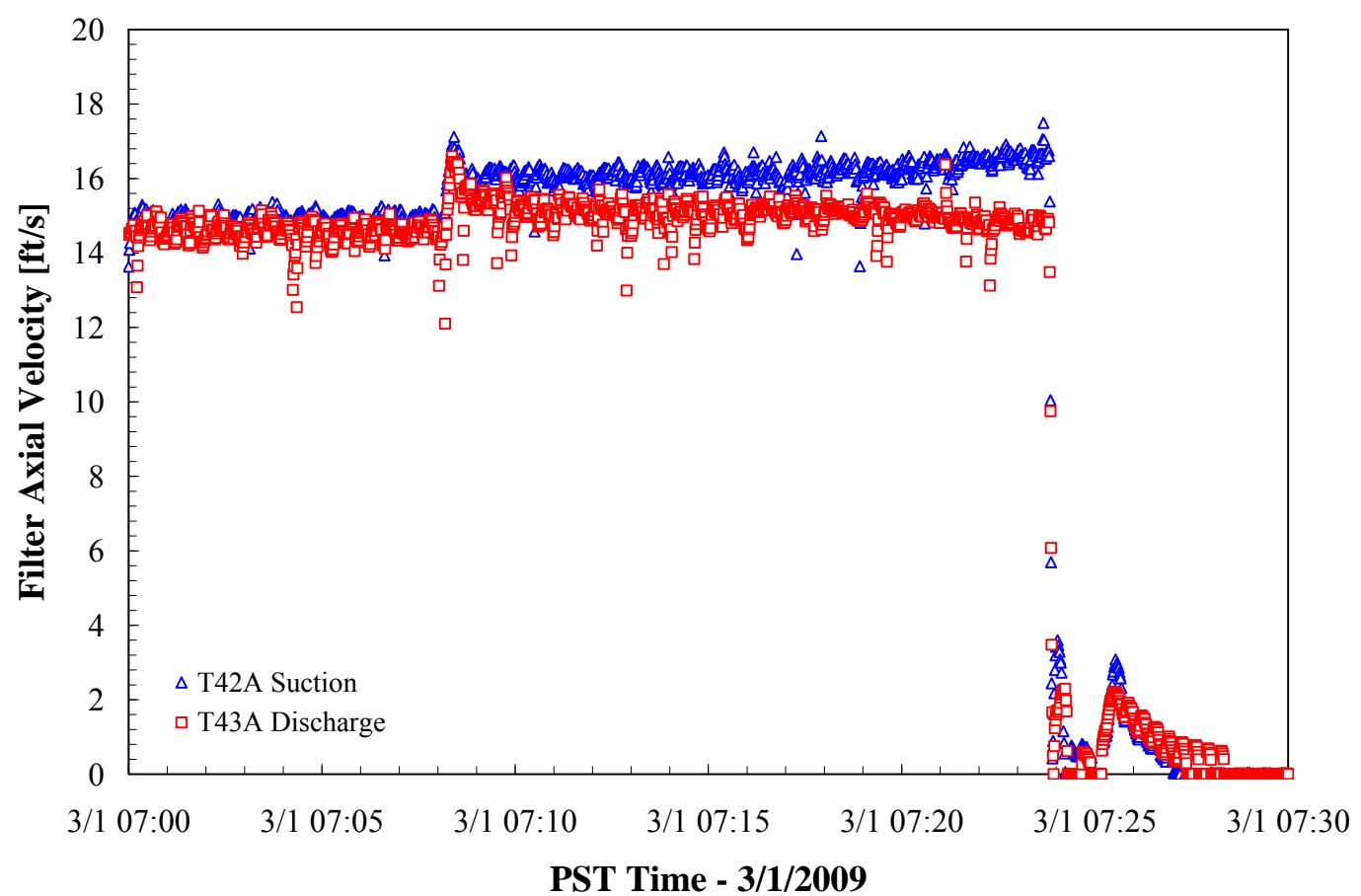

Figure 4.2. Axial Velocity Based on Flow Measurements at the Suction to Pump T42A and the Discharge to Pump T43A for the First Filter Cleaning Event, 3/01/09, Preceding Integrated Test B 


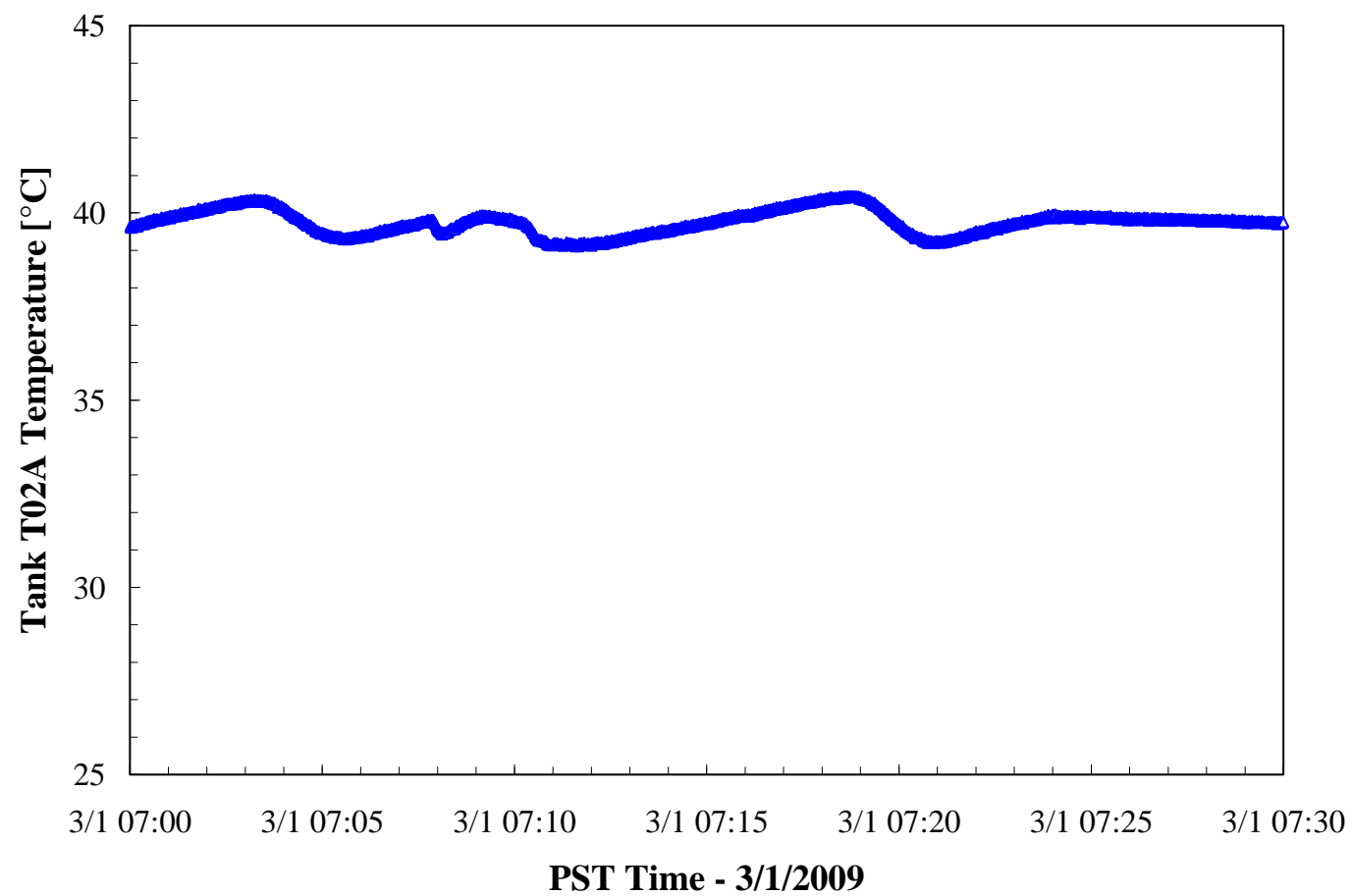

Figure 4.3. Tank T02A Temperature (TTK-0619) for the First Filter Cleaning Event, 3/01/09, Preceding Integrated Test B

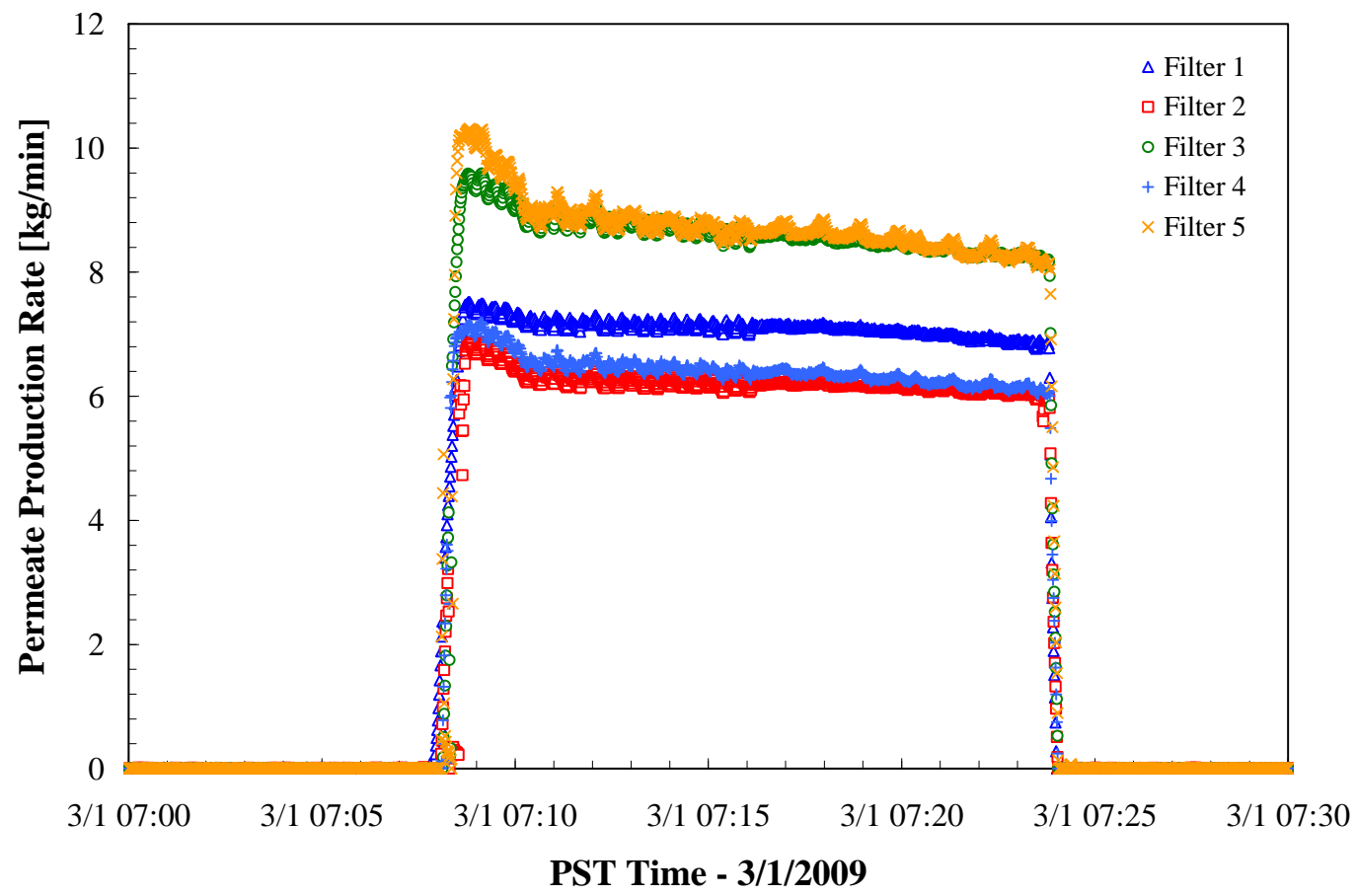

Figure 4.4. Permeate Production Rate for the First Filter Cleaning Event, 3/01/09, Preceding Integrated Test B 
The figures show a comparatively high permeate production rate at low TMP. However, the permeate rates started to decrease after the initial peak rates. The cleaning was terminated early because a leak developed in Pump T42A because some of the internal parts were not acid resistant. The correct pump materials were ordered, but the wrong materials were delivered. The pump parts that failed were nonprototypic. The system was subsequently drained so repairs could be made.

While pump replacement parts were being acquired, a second cleaning was performed on 3/07/09, using a portable 1-in. diaphragm pump to circulate the acid solution through the filter-loop with the Pumps T42A and T43A bypassed (and isolated). During the second attempt, nitric acid was backpulsed 10 times from the pulse-pots back through the filters, and a 1-in. diaphragm pump was used to recirculate nitric acid around the loop and through the filters. A summary of the instructions from an operating procedure are provided in Appendix G. The diaphragm pump could only achieve an axial velocity of $\sim 5 \mathrm{ft} / \mathrm{s}$ in the filter tubes. The filter cleaning instructions were modified to allow permeate through only one filter at a time, if necessary, so a reasonable pressure could be maintained in the loop. The flow rates and pressures pulsated during the cleaning because of the action of the diaphragm pump. The temperature was maintained below $21^{\circ} \mathrm{C}$. Figure 4.5 through Figure 4.8 show TMP, axial velocity, temperature, and permeate production during the second acid cleaning. After cleaning with nitric acid, the system was flushed with deionized water (DIW) and drained for the start of the Integrated Test B.

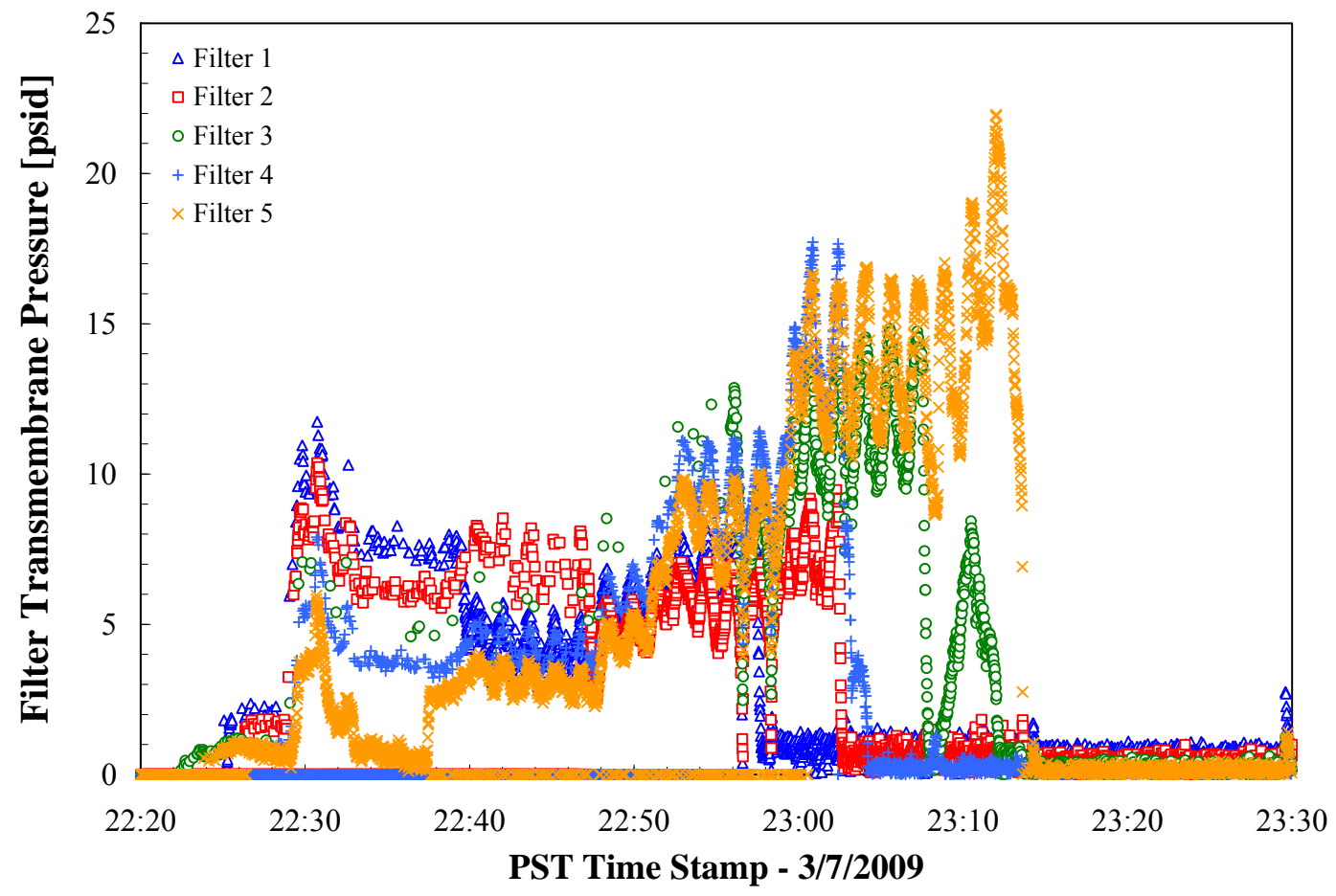

Figure 4.5. TMP for the Second Filter Cleaning Event, 3/07/09, Preceding Integrated Test B 


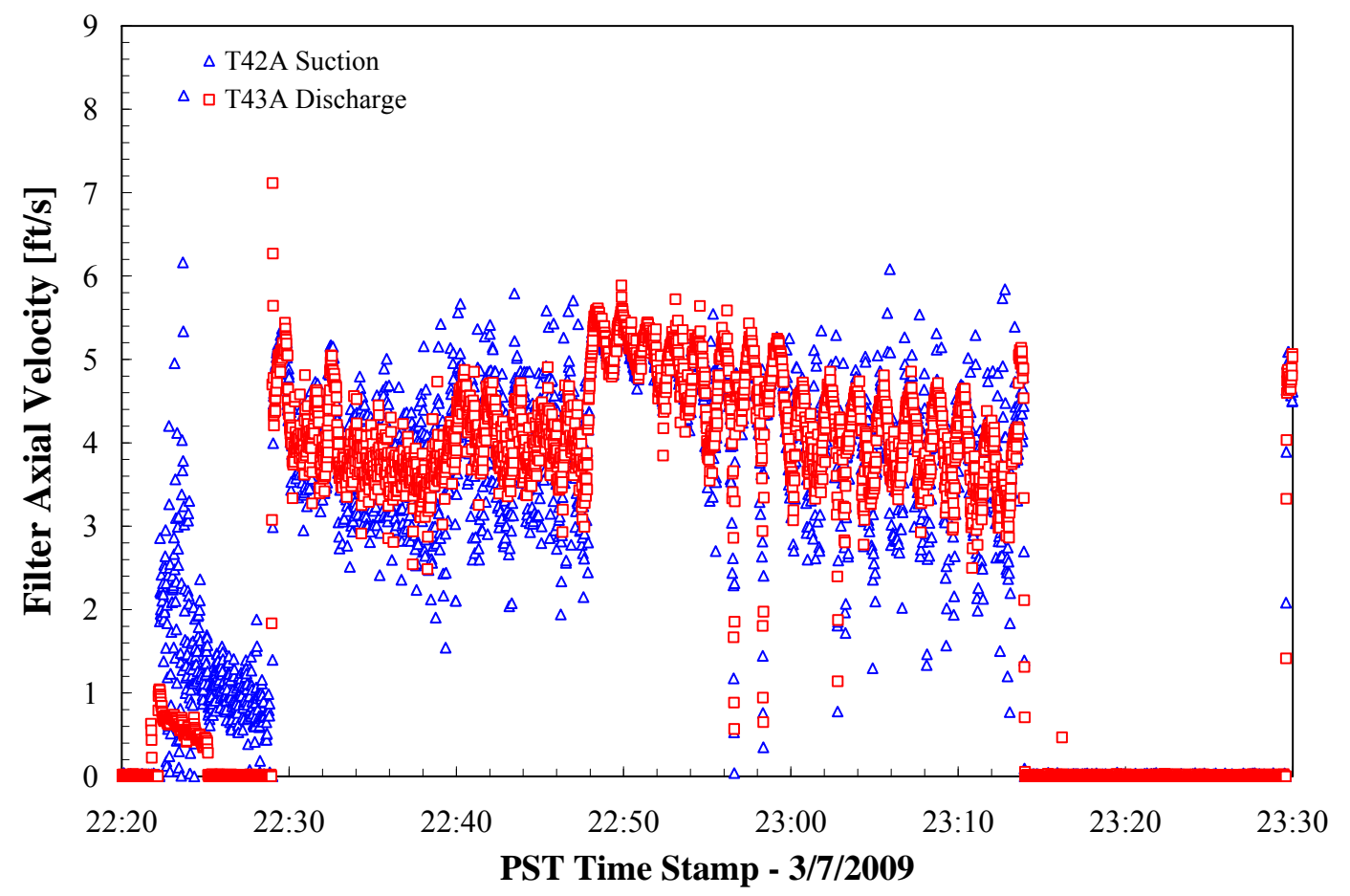

Figure 4.6. Axial Velocity Based on Flow Measurements at the Suction to Pump T42A and the Discharge to Pump T43A for the Second Filter Cleaning Event, 3/07/09, Preceding Integrated Test B

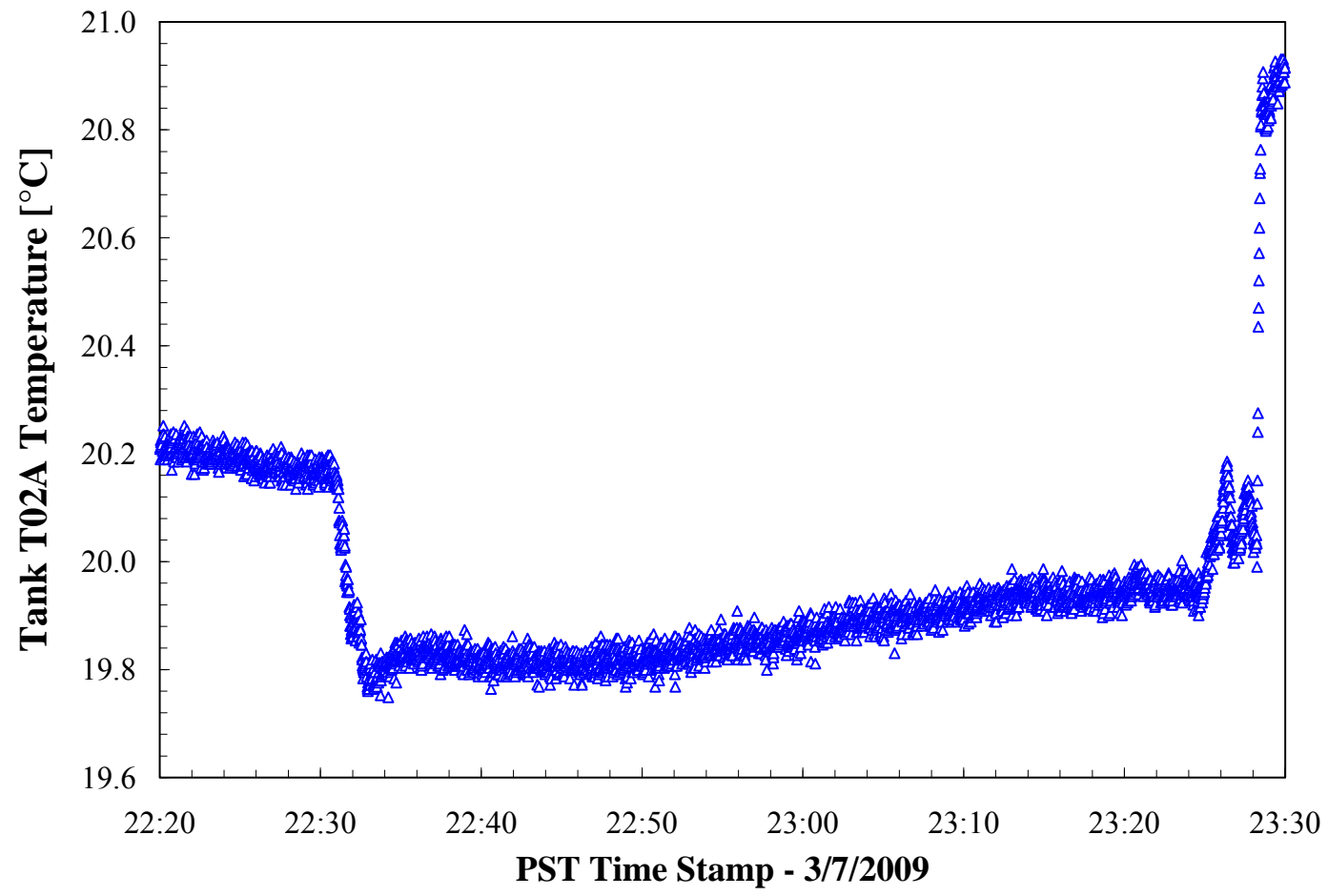

Figure 4.7. Tank T02A Temperature (TTK-0619) for the Second Filter Cleaning Event, 3/07/09, Preceding Integrated Test B 


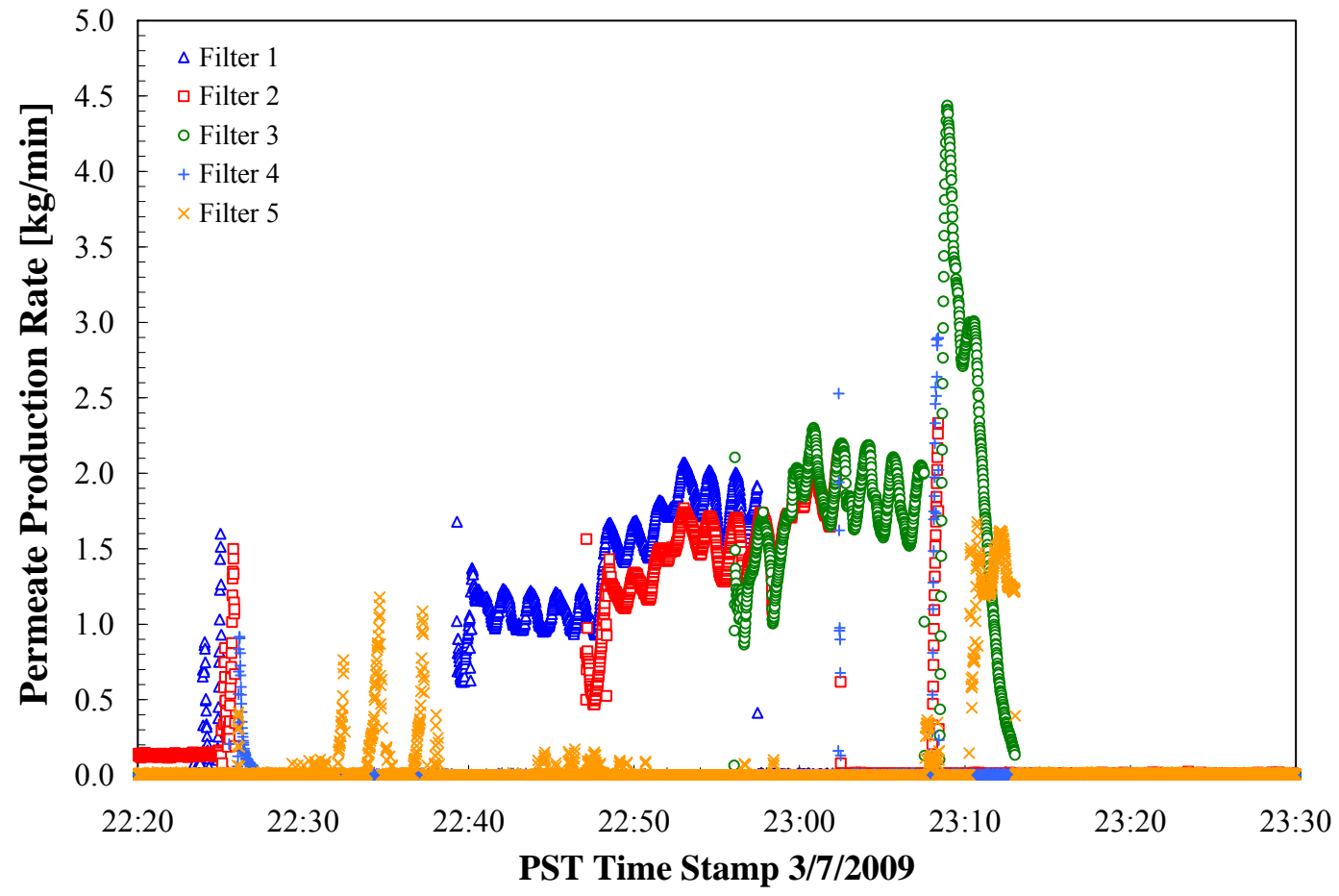

Figure 4.8. Permeate Production Rate for the Second Filter Cleaning Event, 3/07/09, Preceding Integrated Test B

After the main recirculation pumps were repaired, the pumps were tested with water (with the filters bypassed), and no leaks were observed. Dilute acid ( $\mathrm{pH} \sim 3$, as determined with $\mathrm{pH}$ paper) solution was maintained on the filters (shellside and tubeside) to prevent iron precipitation and filter fouling. This solution was drained from the filters immediately before filtering in Integrated Test B as indicated in Section 4.2.

\subsection{Batch 1-Preparation}

The pre-test checklist was completed by the night-shift crew during the early morning of 3/12/09. Start-up configuration included the following baseline conditions: process vessels (Tank T01A, Tank T01B, and Tank T02A, clean and empty; filter-loop (clean, filled with dilute nitric acid and bypass drained); sufficient simulant and reagents available; and sufficient storage space available in UFP waste tanks UFP-VSL-T62A and -62B. In addition, simulant transfer piping between HLP-VSL-T22 and Tank T01A was charged with simulant; simulant lines into Tank T02A were rinsed and drained, and the $19 \mathrm{M}$ $\mathrm{NaOH}$ manifold was charged with $\mathrm{NaOH}$.

Section 7.1 of the Test Instruction for Integrated Test B began with the preparation of HLP-VSL-T22 for transfer to process vessels. Constant mechanical agitation in HLP-VSL-T22 kept the slurry well mixed, and recirculation was started with the flow rate set for compliance with pump operating instructions. Simulant was sampled from HLP-VSL-T22 at three depths before it was transferred to process vessels for use in baseline chemical analyses. 
Simulant was transferred from HLP-VSL-T22 to Tank T01A (Test Instruction Section 7.2) on 3/12/09 at the end of night shift to provide feed for Batch 1 solids concentration and subsequent leaching in Tank T02A. The initial 593-gallon transfer was for Batch 1 solids concentration and subsequent leaching in Tank T02A. In-line sampling was performed, and 1000-mL of Dow Corning Q2-3183A Antifoam (hereafter-AFA) was added to Tank T01A during the transfer, with a targeted AFA concentration of $350 \mathrm{ppm}$. PJMs were started in Tank T01A when the fluid level reached 15 inches.

A stable level measurement ${ }^{(a)}$ was taken in Tank T01A after the transfer was completed. The tank level reading of 67 inches ${ }^{(b)}$ matched the expectation. However, problems were encountered with the PJMs in Tank T01A during the morning of 3/12/09. As noted in the LRB BNW-60279 at 09:55, one of the PJMs in Tank T01A (PJM-1605) appeared to plug intermittently. Examination of the pressure and level data indicate that the air supply was functioning properly, but the stroke length was very short. The normal working stroke length was about 25 inches. When the PJM appeared plugged, the stroke length was reduced to several inches. In addition, the level probes indicated that the pulse-tube was not completely refilling. The apparent plug was temporarily cleared at 10:04 by conducting an intentional overblow. This appeared to resolve the issue for approximately 1 hour. However, PJM-1605 appeared plugged again and was disabled when it could not be cleared by overblowing. PJM parameters (drive pressure and drive time) were collected and adjusted around 11:09 on 3/12/09 to achieve a peak average nozzle velocity of $4.7 \mathrm{~m} / \mathrm{s}$ and $82 \%$ stroke length in Tank T01A. (Note that Section 5.3 presents targeted versus actual PJM parameters, and Appendix F discusses the PJM tuning approach.)

The transfer of 220 gallons of simulant from Tank T01A to Tank T02A (Test Instruction Section 7.3) was started at about 12:00. This was the feed material for Batch 1 solids concentration and subsequent leaching in Tank T02A. PJMs were started in Tank T02A during the transfer when an adequate level was reached. A stable level measurement in Tank T02A, taken at 12:30 after the transfer was completed, was 53.5 inches (laser) compared with the expected tank level of 52 inches. PJM parameters (drive pressure and drive time) were then adjusted to achieve a peak average nozzle velocity of $7.1 \mathrm{~m} / \mathrm{s}$ and $83 \%$ stroke length in Tank T02A. (Section 5.3 presents targeted versus actual PJM parameters.)

Section 7.4 of the Test Instruction, placing Tank T01A in Auto Transfer Mode and Level Monitoring, was conducted concurrently with Section 7.5, Tank T02A Dewater Operations for Batch 1. The shellside and tubeside of the filter-loop was drained of dilute acid before starting filter pumps T42A and T43A. The loop was aligned to send slurry through all five filters and the cooling heat exchanger (UFP-HX-T02A). The heating heat exchanger (UFP-HX-T03A) was bypassed with the outlet slurry valve locked open.

At 14:30, Tank T01A was placed in auto transfer mode (Test Instruction step 7.4.1), and the Tank T02A slurry outlet valve (V06132) was opened. At 15:00, sparger flow rates were reset, and (ultrafilter) Pumps T42A and T43A were enabled and slowly powered up (Test Instruction step 7.5.4). Pumps 42A and 42B were operated throughout Integrated Test B in accordance with the Pump Operational Guidance,

(a) A stable level measurement required turning off equipment that would affect the tank level, including recirculation pumps, PJMs, steam ring air purge, air sparger, and direct steam injection. Level readings were taken 2 minutes after the appropriate equipment was turned off. Stable level measurements were generally taken before and after major evolutions impacting tank level, including filtration, transfers, and chemical additions, as directed in the test instruction.

(b) All levels indicated in the narrative are from the appropriate laser level instrument unless noted otherwise. 
provided in Appendix H. Variable frequency drive (VFD) settings for pumps 42A and 43A were 25\% and $85 \%$, respectively, and the filter-loop backpressure control valve (ZV-0609) was 50\% open when target conditions were achieved at 15:26. The target flow rate was $109 \mathrm{gpm}$ with a TMP of $40 \mathrm{psig}$.

Four auto-transfers were made before opening the permeate valves at 15:27. Tank T01A provided make-up feed to Tank T02A as filtration progressed, initiating an 11-gallon make-up batch whenever the level in Tank T02A dropped to 28 inches. The Test Instruction specified that forty-seven 11-gallon batches were to be transferred from Tank T01A to Tank T02A during the course of filtration.

Tank T02A CD sampling commenced at 15:30 and was performed every three make-up transfers until the targeted 47 transfers were completed at 19:07. Permeate and in-line samples were also collected as per the Test Instruction.

The stable level in Tank T01A (laser level) was about 25 inches at 16:00 before refill from HLP-VSL-T22. Refilling Tank T01A with 369 gallons of simulant (Test Instruction step 7.4.4) was completed at 17:05. AFA was added to Tank T01A after the simulant transfer. Stable level measurement after refill was about 63 inches (laser). Auto-transfers to Tank T02A were restarted at about 17:30 (Test Instruction step 7.4.7).

\subsection{Batch 1-Concentration}

Filtration of Batch 1, using all five filters, was started on 3/12/09 at 15:27 and concluded at 20:13 on the same date. The filters were idled (permeate valves closed) from approximately 15:50 until 17:30 while Tank T01A was being refilled with simulant from HLP-VSL-T22. After Tank T01A was refilled, filtration continued until 19:04. At this point, the filters were again idled to take a stable level measurement in Tank T02A to determine if the targeted level of 21.5 inches had been reached. It took two iterations of filtering and then stopping filtration (to the measured Tank T02A level) to achieve the targeted level in Tank T02A of 21.5 \pm 2 inches. Filtering was concluded at 20:13 when the stable level measurement (laser level) in Tank T02A was 22.2 inches.

Permeate flux was high in all filters because the filters had just been cleaned and were not conditioned before testing. The filtration of Batch 1 provided some filter conditioning for subsequent filtering, but substantially less than the filter conditioning was performed in Integrated Test A. Permeate flow decreased from about 4 to $6 \mathrm{~kg} / \mathrm{min} /$ filter to about 1.5 to $2.5 \mathrm{~kg} / \mathrm{min} /$ filter by the end of concentration. The total permeate removed was $2328 \mathrm{~kg}$, and the resulting UDS concentration was 20.3-wt\%. The drop in axial flow rate near the end of filtration was due to the low level in Tank T02A which resulted in air entrainment and loss of pump performance. Visual inspection of permeate samples taken during concentration (three samples/filter evenly spaced during concentration) showed no sign of particle break-through.

During Integrated Test B, the temperature control for the cooling heat exchanger in the filter-loop, UFP-HX-T02A, was a hybrid method of control. The cooling water control valve (TV-0513) was in automatic, controlling off of the outlet slurry temperature TT-0513. However, the temperature setpoint was manually changed to try to control the temperature in Tank T02A at $25^{\circ} \mathrm{C}$. This was done because it was discovered that this temperature sensor TT-0513 was not sufficiently exposed to slurry flow to reliably indicate slurry temperature. Data from this sensor were considered "for information only" (see 
Section 2 for a description of this nonconformance). This hybrid control scheme resulted in more temperature variation than under automatic control.

Figure 4.9 through Figure 4.12 illustrate TMP, axial velocity, temperature, and permeate production during the concentration of Batch 1 .

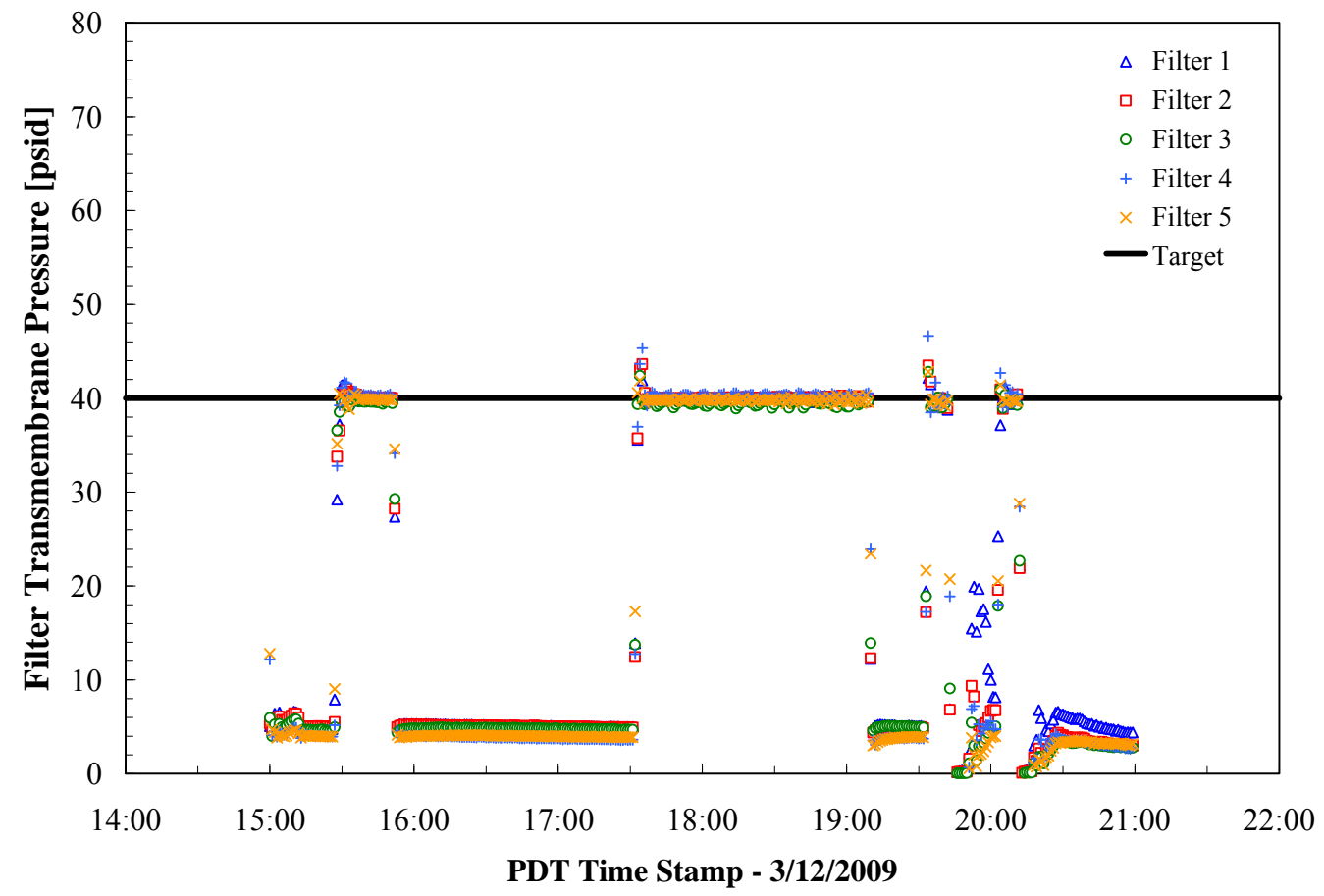

Figure 4.9. TMP for Pre-Caustic-Leach Concentration, Integrated Test B, Simulant Batch 1 


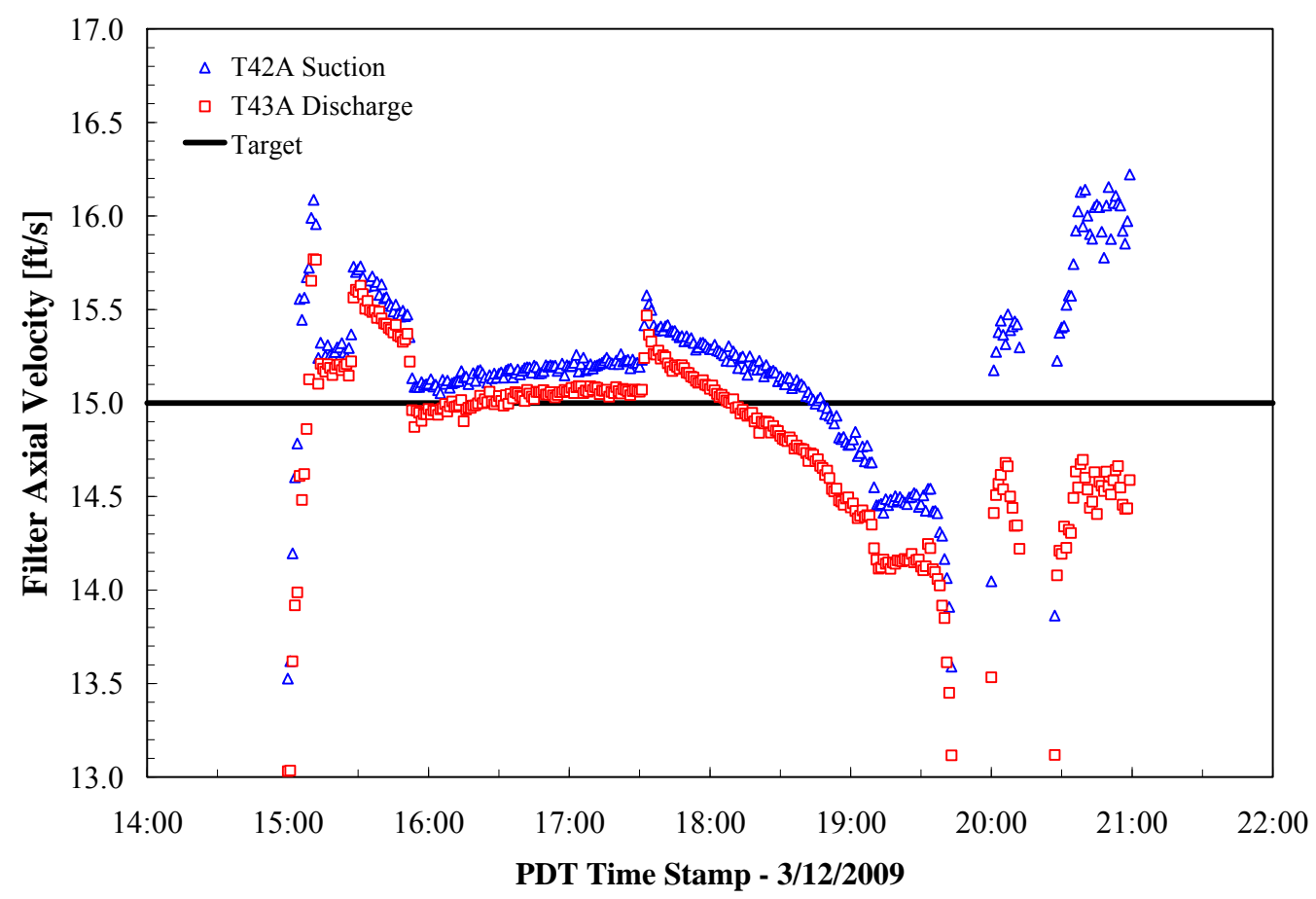

Figure 4.10. Axial Velocity Based on Flow Measurements at the Suction to Pump T42A and the Discharge to Pump T43A for Pre-Caustic-Leach Concentration, Integrated Test B, Simulant Batch 1

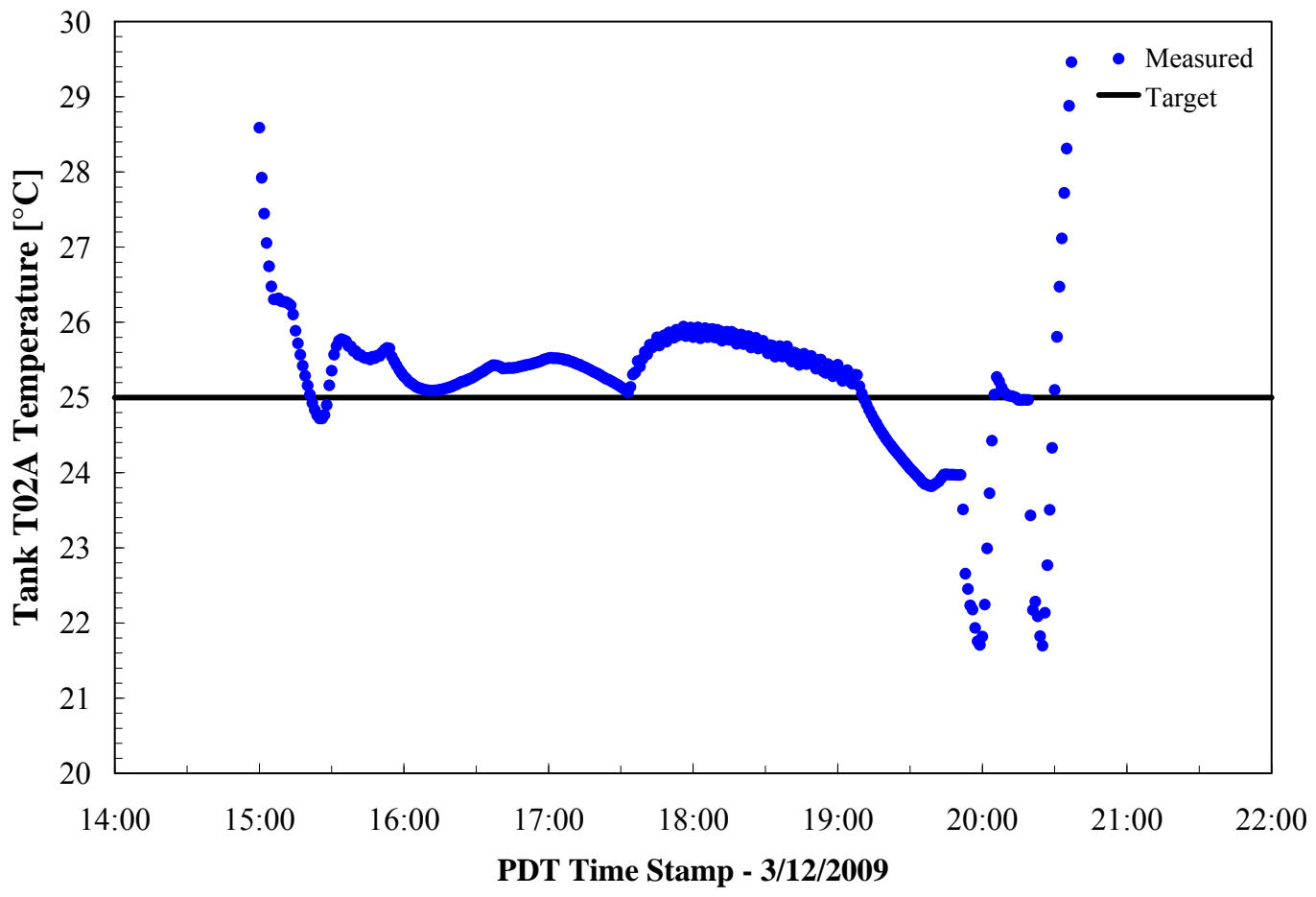

Figure 4.11. Tank T02A Temperature (TTK-0619) for Pre-Caustic-Leach Concentration, Integrated Test B, Simulant Batch 1 


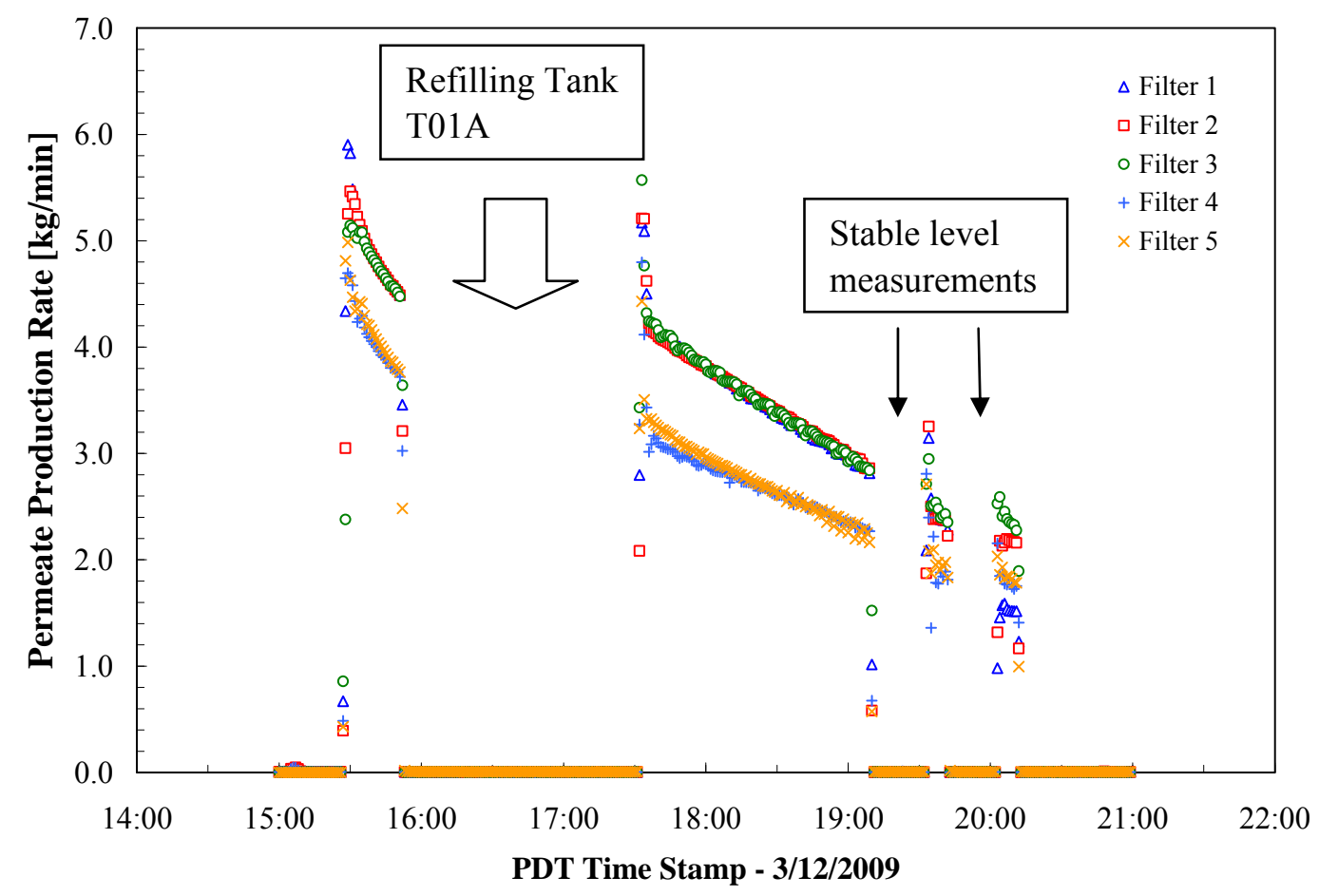

Figure 4.12. Permeate Production Rate for Pre-Caustic-Leach Concentration, Integrated Test B, Simulant Batch 1

\subsection{Batch 1-Caustic-Leach}

At the conclusion of Batch 1 concentration, the permeate valves were closed, the temperature set point of UFP-HX-T02A was increased to $71^{\circ} \mathrm{C}$, and AFA was added to Tank T02A in preparation for caustic-leach for Batch 1 (Test Instruction, Section 7.6). Recirculation of the filter-loop continued as $721 \mathrm{~kg}$ of $19 \mathrm{M} \mathrm{NaOH}$ was added to the suction side of Pump T42A, and the slurry was allowed to heat. (Note that a sample of the sodium hydroxide reagent was analyzed, and the reported free $\mathrm{OH}^{-}$ concentration was 19.0 M. See Appendix N.) Caustic addition started at 21:36 and concluded at 22:38 on 3/12/09 at a rate of $14 \mathrm{~kg} / \mathrm{min}$ under manual control. Samples were taken from the Tank T02A CD loop after a short period of mixing.

At the conclusion of caustic addition, the level in Tank T02A was about 7 inches higher than expected. A visual inspection of the tank was not conducted because of the lockout/tagout requirement to have the steam locked out before opening an access port. The bubbler does not provide accurate readings when Pumps T42A and/or T43A are operating. These pumps were turned off at 23:00 (3/12/09) in accordance with the Test Instruction. Comparison of the laser and bubbler, after the pumps were shut down, indicate that the bubbler reading was 3 inches lower than the laser (see Appendix J, p J.21 for plot), suggesting a 3-in. foam layer, as bubblers do not measure foam.

The initial heat-up temperature of $71^{\circ} \mathrm{C}$ was achieved at 23:00 without the use of UFP-HX-T03A. The ultrafilter pumps were turned off at 23:00, and the filter-loop was flushed to Tank T02A with $74 \mathrm{~kg}$ of IW at 23:23 before final heating with direct steam. Figure 4.13 shows the temperature as a function of time in Tank T02A during heating, leaching, and cooling of Batch 1. 


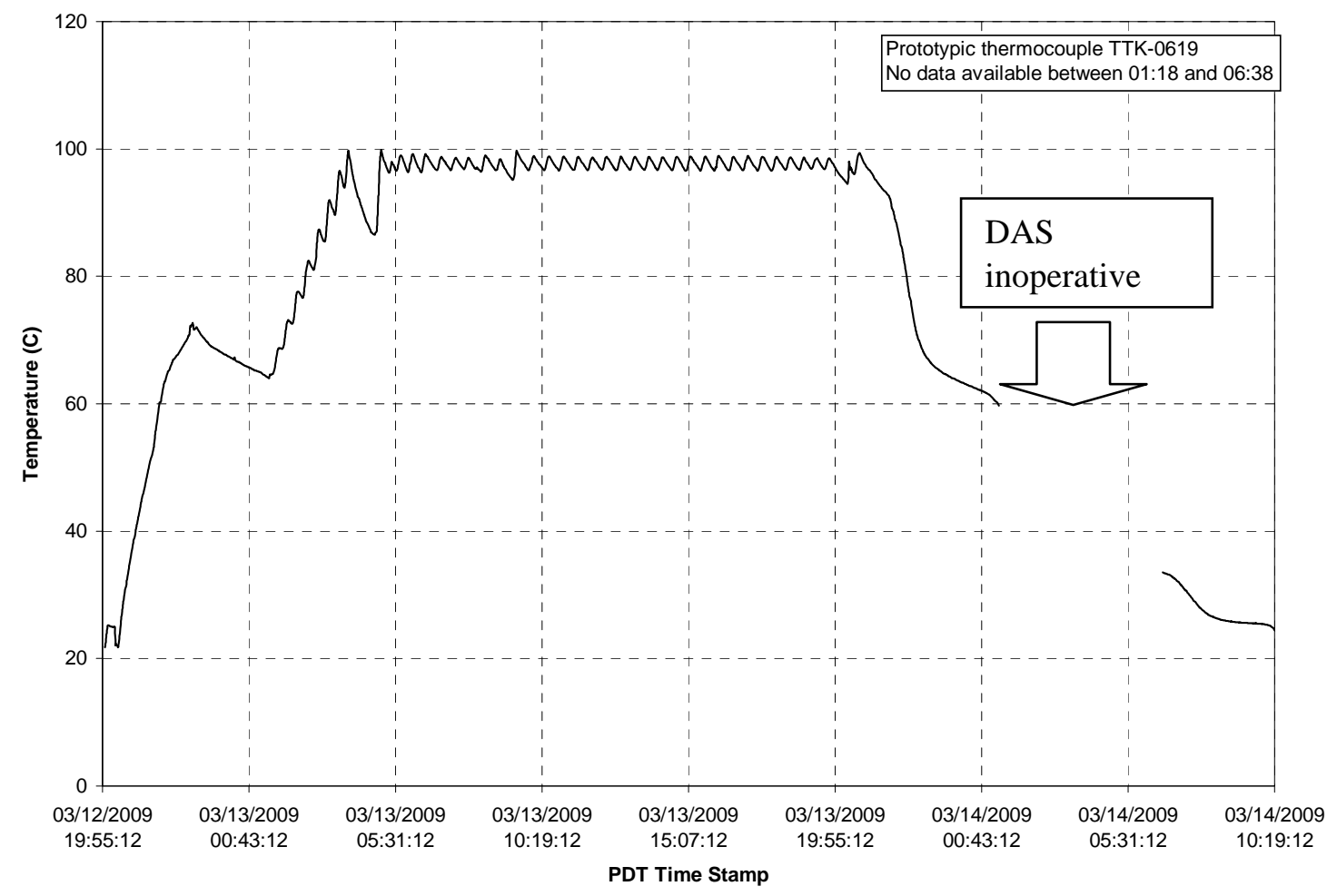

Figure 4.13. Tank T02A Temperature During Batch 1 Caustic-Leach

A slurry piping seal gasket on the suction side of Pump T42A was found to be leaking at 00:00 on 3/13/08. Testing was put on hold until the leak was evaluated by maintenance. Clearance to proceed with the test was granted from operations approximately an hour later, at 01:12. During this time, the temperature of Tank T02A had cooled from $71^{\circ} \mathrm{C}$ to $64^{\circ} \mathrm{C}$. It was also noted that the tank level (63 inches) was now 9 inches higher than the expected level (54 inches). Direct steam injection was initiated at 01:24 to heat the contents of the vessel to $98^{\circ} \mathrm{C}$ with a 2.6-hr heat-up time. During the heat-up of Batch 1, the PJMs started to overblow because the PJM level sensors were not responding correctly at elevated temperatures. PJMs were set to simple mode at 01:30 on 3/13/09.

Samples were taken during caustic leach, first at $88^{\circ} \mathrm{C}$ and then at $98^{\circ} \mathrm{C}$, and as scheduled throughout the leaching period. The leach samples were cooled immediately to prevent further leaching of aluminum. Samples were chilled in a water bath, centrifuged, and separated into solid and liquid fractions. Samples were sent to the Southwest Research Institute (SwRI) for analysis to determine the aluminum concentration in the liquid and solid fractions as a function of leach time.

When the temperature in the vessel reached $90^{\circ} \mathrm{C}$, the Test Instruction called for adjusting the lower air spargers from $0.4 \mathrm{~kg} / \mathrm{min}$ to $0.08 \mathrm{~kg} / \mathrm{min}$; however, they were mistakenly set to $0.8 \mathrm{~kg} / \mathrm{min}$. The extra air to the sparger added to the foaming/air entrainment issue and raised the apparent level in the vessel to approximately 74 inches at the end of the 2.6-hr heat-up time, which was 11 inches higher than expected. The specific gravity, measured by the bubbler (LT-0611/DT-0612), was reading 0.8. The specific gravity increased rapidly upon correctly setting the air sparger rate at 04:50 (see Appendix J, p. J.39). 
The high sparge rate also removed more heat from the vessel, based on a comparison of heat-up curves for Batch 1 and Batch 2. At 03:55, the steam injection to Tank T02A was shut off because of a high/high alarm. At 04:50, the incorrect sparger air setting was identified and corrected, which reduced the observed level from 76 to 65 inches, and leaching (at temperature) was allowed to proceed. Because the steam heating to Tank T02A had been off between about 04:00 and 05:00 on 3/13/09, the contents of Tank T02A had cooled from $98^{\circ} \mathrm{C}$ to $86^{\circ} \mathrm{C}$. When steam was turned on, a 15 -min ramp time was employed to increase the temperature to $98^{\circ} \mathrm{C}$. The measured specific gravity of the slurry in Tank T02A returned to 1.25 (from 0.5) upon correctly setting the air sparger setting (see Appendix J, p. J.39).

Stable levels measured (by laser) at $0,4,8$, and 12 hours after the start of caustic leach (Test Instruction step 7.6.22) were 64.7 inches at $04: 47 ; 67.0$ inches at $08: 11 ; 69.0$ inches at $12: 03 ; 70.0$ inches at 16:05, and 72.7 inches at 20:28.

Tuning of the PJMs in Tank T02A was completed at 06:15 on 3/13/09 (with PJMs in simple mode) to provide a $7.5 \mathrm{~m} / \mathrm{s}$ nozzle velocity and a $78 \%$ stroke.

At 18:38 on 3/13/09, PJM-1607 (from Tank T01A) was taken out of service for 10 minutes during the troubleshooting of PJM-1605. PJM-1607 was returned to service at 18:48. Troubleshooting of PJM-1605 was unsuccessful.

Automatic steam injection into Tank T02A switched off, and steam injection was switched to manual mode, without operator intervention, at 20:07 and 20:31. In both cases, the operators subsequently switched control back to automatic. The first time, the steam was off for 10 minutes, and the temperature in the vessel fell to $95^{\circ} \mathrm{C}$.

The leach time for Batch 1 was extended for 53 minutes (16 hours 53 minutes total leach time) to compensate for the time during which the temperature was less than $96^{\circ} \mathrm{C}$. Leaching was thus continued until about 21:00, and another sample was added to the Test Instruction.

Cooling of Batch 1 in Tank T02A (Test Instruction Section 7.7) was started at 20:54 on 3/13/09 when the steam supply was turned off. The stable level reading in Tank T02A was 73.1 inches (laser), compared with a target of $65.2 \pm 5$ inches. Approval for continuing was granted, and Batch 1 was subsequently cooled to $25^{\circ} \mathrm{C}$ during a time period of 13.2 hours to match a prototypic cooling curve.

The cooling rate was guided by comparing the temperature in Tank T02A every 15 minutes with the target temperature (in the Test Instruction) and adjusting the cooling water jacket control valve (TV-0617) manually. Cooling was completed at $10: 15$ on 3/14/09. There was a period of approximately 5 hours (01:17 through 06:37 on 3/14/09) in which no data are available during Batch 1 cooling, caused by a crash of the DAS. To minimize the impact of any subsequent DAS failure, the DAS was checked every half-hour during the remainder of testing, and a software alert was implemented.

At the cooling was completed, Batch 1 was transferred to Tank T01B for storage (Test Instruction Section 7.8) until the completion of Batch 2. The transfer was completed at 11:03 on 3/14/09. The PJMs in Tank T01B were checked and found to have a nozzle velocity of $5.1 \mathrm{~m} / \mathrm{s}(4.8 \mathrm{~m} / \mathrm{s}$ target $)$ with a $58 \%$ stroke ( $80 \%$ target). The test director directed the lead test engineer to leave PJMs "as is." (Section 5.3 discusses targeted versus actual PJM parameters.) 


\subsection{Batch 2-Preparation}

A second batch of concentrated and leached simulant was necessary because the filter-loop volume in the PEP is larger than prototypic of the WTP, thus requiring additional volume to conduct post-leach dewatering and washing at prototypic levels in Tank T02A. The preparation of Batch 2, described in Sections 7.9 through 7.12 of TI-066, is very similar to the preparation of Batch 1, described in Sections 7.2 through 7.5 .

Batch 2 was started at 11:33 on 3/13/09 with the transfer of 369 gallons of simulant from HLP-VSL-T22 to Tank T01A (Test Instruction Section 7.9). AFA was added to Tank T01A at 12:45. A stable level measurement taken in Tank T01A at 12:37 was 66 inches (laser), which matched well with the expected level of 67 inches. Informal PJM tuning (Tank T01A) was performed, and $10 \mathrm{~Hz}$ data were taken at about 13:00. These data were later analyzed, and the PJMs were found to have a nozzle velocity of $4.6 \mathrm{~m} / \mathrm{s}$ and a stroke of $64 \%$. A summary of target and actual PJM parameters can be found in Section 5.3 of this document.

The initial 220-gal transfer of simulant from Tank T01A to Tank T02A for Batch 2 (Test Instruction step 7.10.4) occurred between 12:19 and 12:59 on 3/14/09. PJMs in Tank T02A were started when the level reached 16 inches. The stable level in Tank T02A at 14:00, after simulant transfer, was 53.7 inches (laser); slightly higher than the expected level (52 inches). PJMs were tuned, and data were taken (Test Instruction step 7.10.5) between 14:05 and 14:33 on 3/14/09. Analysis of the data showed a nozzle velocity of $6.6 \mathrm{~m} / \mathrm{s}$ and a stroke of $73 \%$ (Section 5.3 , this document).

\subsection{Batch 2-Concentration}

Automatic transfers from Tank T01A (Test Instruction Section 7.11) and Tank T02A Dewatering (Test Instruction Section 7.12) were performed concurrently for Batch 2 as they were for Batch 1 (Test Instruction Sections 7.4 and 7.5). The filter-loop was drained at 11:42 on 3/14/09, and the slurry outlet valve (V06132) was opened at 14:00. The steam heat exchanger (UFP-HX-T03A) was bypassed (with the outlet slurry valve locked open), and the cooling heat exchanger (UFP-HX-T02A) was aligned. Pumps T42A and T43A were started at 14:35, and target conditions in the filter-loop (slurry flow rate $109 \mathrm{gpm}$, TMP $40 \mathrm{psi}$ ) were achieved at 14:42. The filter-loop was aligned to send slurry through all five filters, but with only the Filter 1 permeate valve open to concentrate Batch 2. Tank T01A provided make-up feed as the filtration progressed, initiating an 11-gallon make-up batch each time the level in Tank T02A fell to 28 inches.

Tank T01A was refilled with simulant from HLP-VSL-T22 to provide enough simulant feed. An additional 369 gallons of simulant was transferred between 20:37 and 21:35 on 3/14/09. The filtration was idled during most of this transfer to allow simpler inventory tracking. AFA was added to Tank T01A after the transfer was completed. The level in Tank T01A after the transfer was 66.6 inches (laser).

Forty-seven 11-gallon batches were transferred from Tank T01A to Tank T02A during filtration. After batch transfer 47, the level in Tank T02A was reduced to a target of 21.5 inches to complete the concentration. The filtration of Batch 2 was started on 3/14/09 at 16:37 and concluded at 13:32 on 3/15/09. Permeate flow in UFP-FILT-T01A decreased from about $4 \mathrm{~kg} / \mathrm{min}$ to $1.5 \mathrm{~kg} / \mathrm{min}$ during dewatering. The total permeate removed was $2611 \mathrm{~kg}$, and the resulting UDS concentration was 
21.8-wt $\%$. Visual inspection of permeate samples taken during concentration (three samples evenly spaced during concentration) showed no sign of particle break-through.

Figure 4.14 through Figure 4.17 show TMP, axial velocity, temperature, and permeate production during the concentration of Batch 2. The two obvious discontinuities in the permeate production rate, seen in Figure 4.17, are from temporarily suspending filtration while transferring simulant from HLP-VSL-T22 to Tank T01A. Changing the state of the recirculation pumps (on to off; or off to on) resulted in temperature fluctuations in Tank T02A. The drop in the axial flow rate near the end of filtration was due to the low level in Tank T02A.

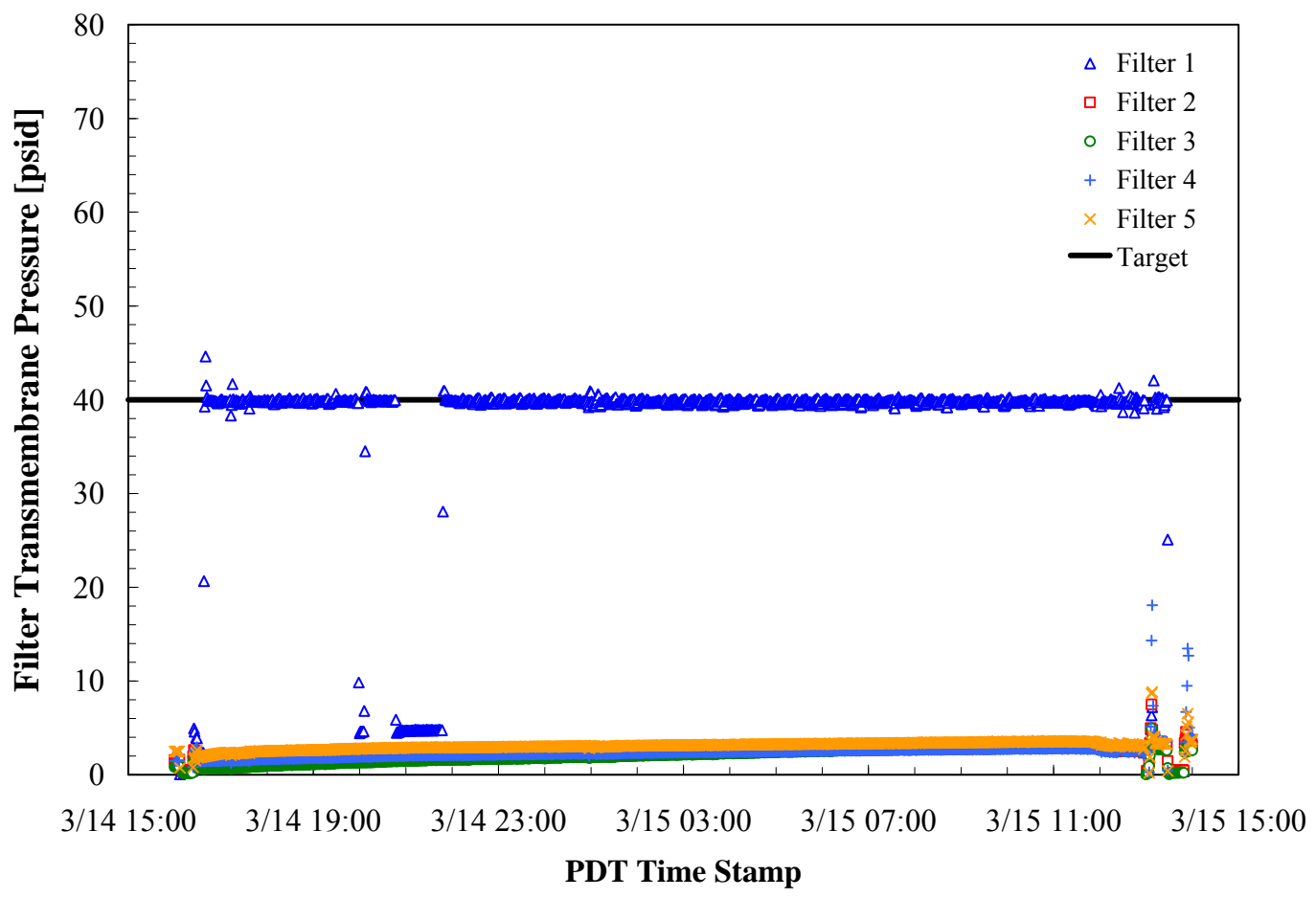

Figure 4.14. TMP for Pre-Caustic-Leach Concentration, Integrated Test B, Simulant Batch 2 


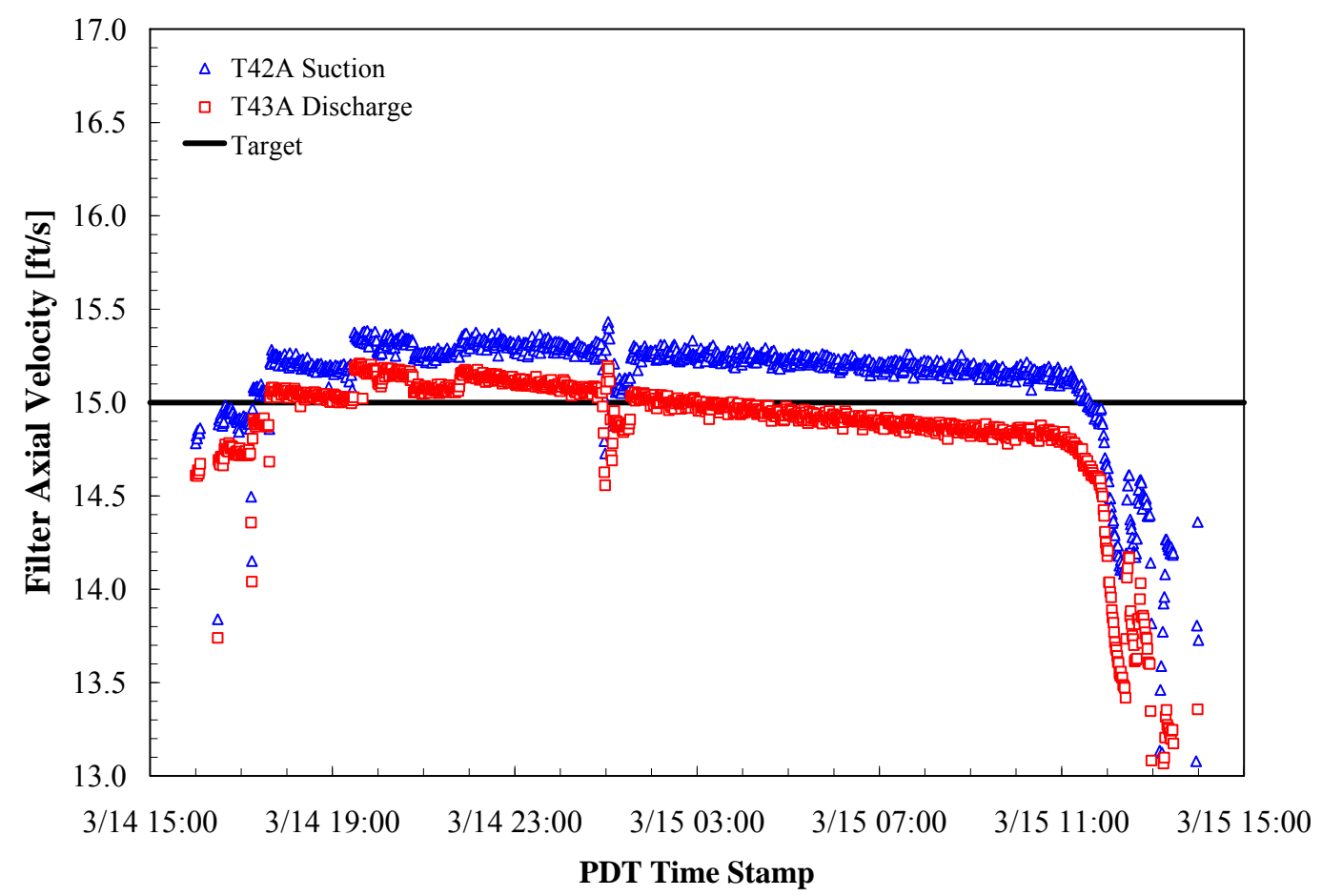

Figure 4.15. Axial Velocity Based on Flow Measurements at the Suction to Pump T42A and the Discharge to Pump T43A for Pre-Caustic-Leach Concentration, Integrated Test B, Simulant Batch 2

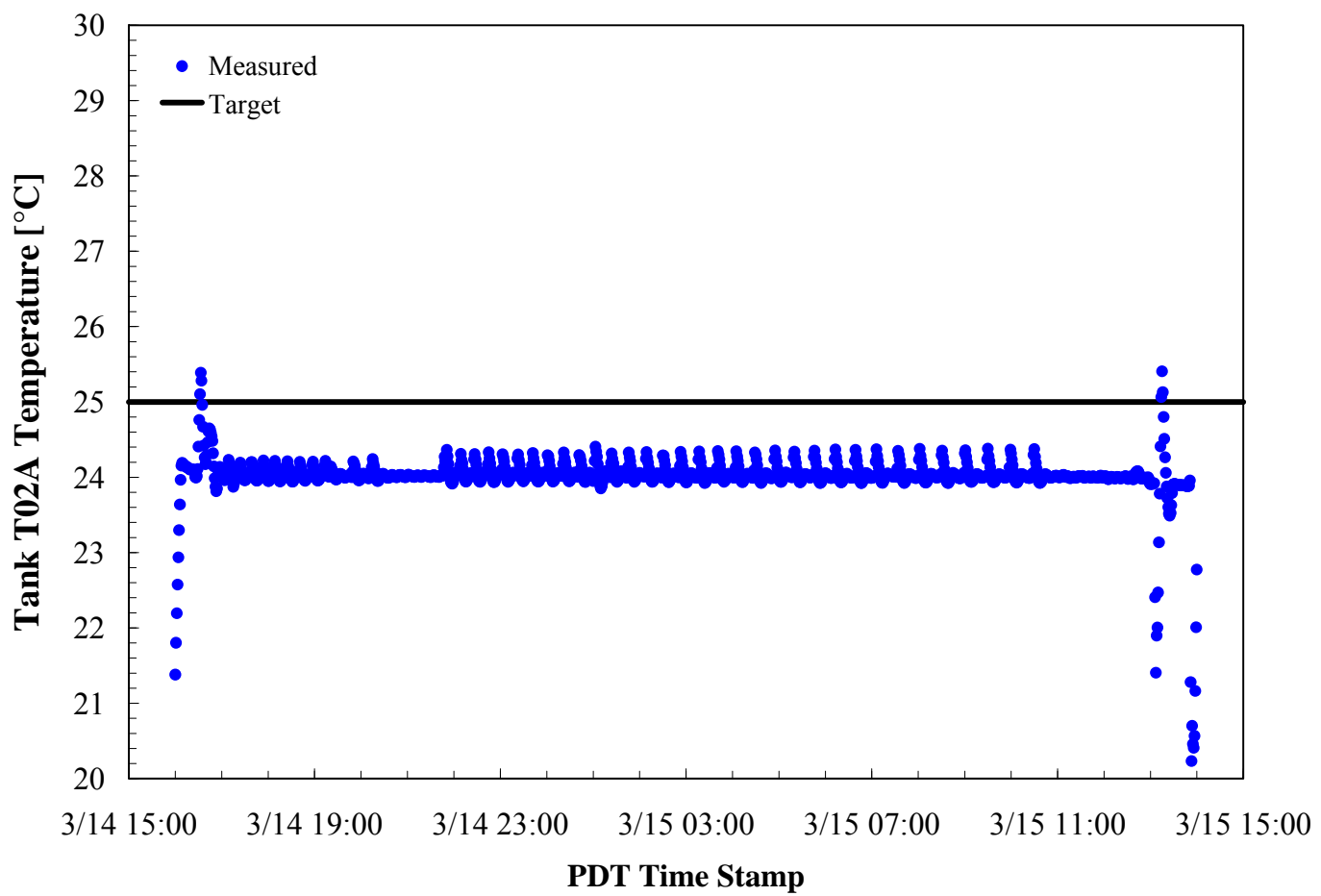

Figure 4.16. Tank T02A Temperature (TTK-0619) for Pre-Caustic-Leach Concentration, Integrated Test B, Simulant Batch 2 


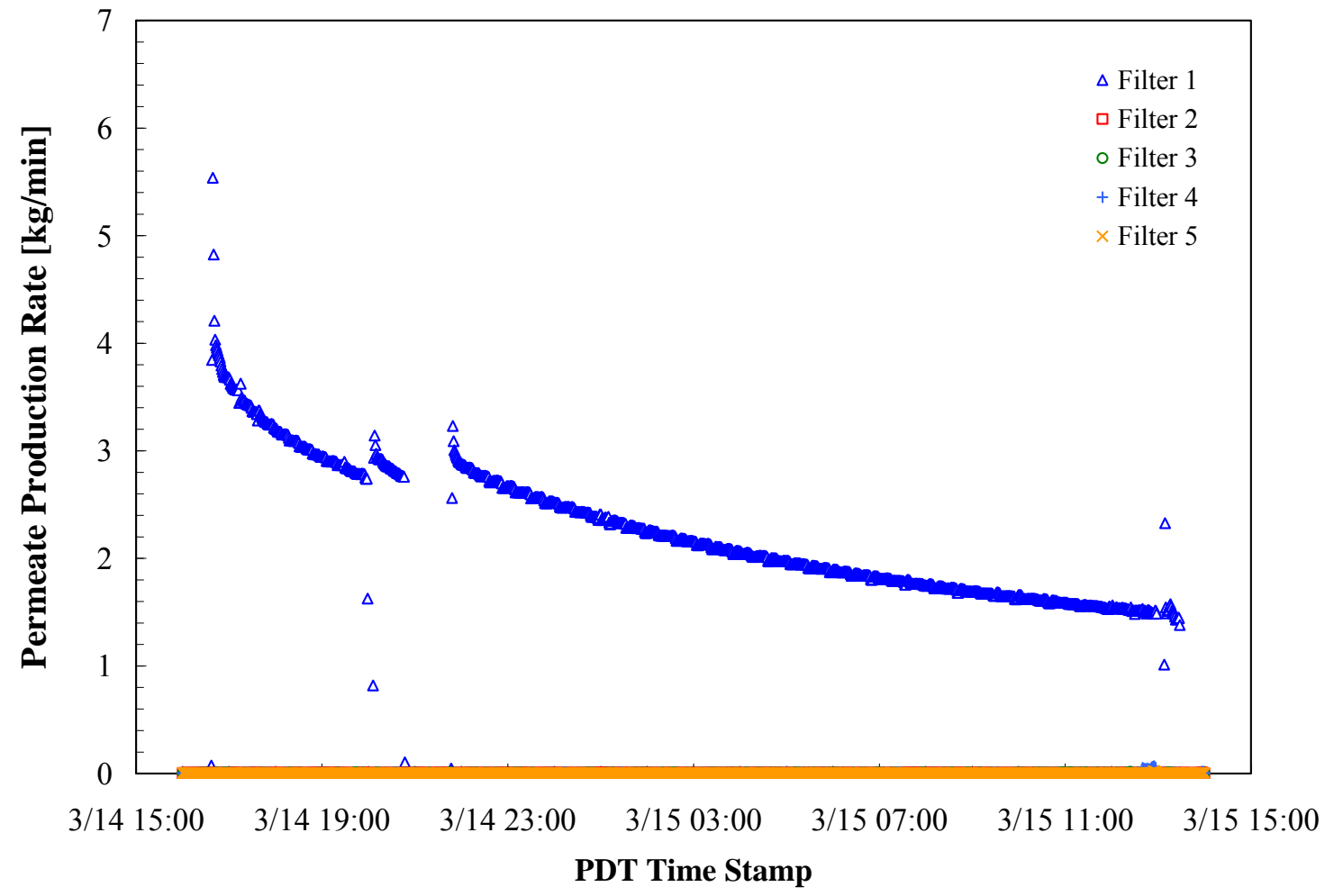

Figure 4.17. Permeate Production Rate for Pre-Caustic-Leach Concentration, Integrated Test B, Simulant Batch 2

\subsection{Batch 2-Caustic-Leach}

After Batch 2 was dewatered, Tank T02A and the filter-loop were prepared for caustic leaching (Test Instruction Section 7.13). The slurry was raised to an initial heat-up temperature both by adding caustic and by the energy added from the circulation pumps. AFA was added at 14:08 to maintain the concentration at $350 \mathrm{ppm}$ while compensating for additions of $\mathrm{NaOH}$ and steam condensate. The temperature set point of the cooling heat exchanger (UFP-HX-T02A) was increased to $80^{\circ} \mathrm{C}$, and the slurry was allowed to heat up during the caustic addition. Pumps T42A and T43A operated (permeate valves closed) while $720 \mathrm{~kg}$ of $19 \mathrm{M} \mathrm{NaOH}$ was added to Tank T02A via the suction side of Pump T42A $(10.2 \mathrm{~kg} / \mathrm{min})$, concluding at 15:20 on 3/15/09. After caustic addition was completed, the level in Tank T02A was 52.6 inches, only about 3 inches higher than expected (compared with +7 inches for Batch 1). At 16:59, some of the slurry in the filter-loop was flushed to Tank T02A (TI Step 7.13.11) using $74 \mathrm{~kg}$ of IW.

Direct steam injection was initiated at $17: 17$ on 3/15/09 to heat the contents of Tank T02A to $98^{\circ} \mathrm{C}$ with a 2.6-hr heat-up time. Samples were taken when the temperature reached $88^{\circ} \mathrm{C}$ at 19:15 (Test Instruction step 7.13.15). When the temperature reached $90^{\circ} \mathrm{C}$, the lower air spargers were adjusted from $0.4 \mathrm{~kg} / \mathrm{min}$ to $0.08 \mathrm{~kg} / \mathrm{min}$. The contents of Tank T02A reached $98^{\circ} \mathrm{C}$ at $19: 50$ on $3 / 15 / 09$ (Test Instruction step 7.13.17). At that time, the level in Tank T02A was 61.8 inches (laser) versus the 60.4 inches expected. Samples were taken hourly as leaching proceeded for the next 16 hours. Samples 
were cooled immediately to prevent further reaction, as were the Batch 1 leach samples. Figure 4.18 shows the temperature in Tank T02A as a function of time during the heating, leaching, and cooling.

During the heat-up of Batch 2, the PJMs in Tank T02A were not operating correctly under standard mode. PJMs were switched to simple mode at 19:08 with parameters matching those from Batch 1. PJMs in Tank T02A were tuned in simple mode while at the target leach temperature (Test Instruction step 7.13.19) at $19: 59$ on 3/15/09 to provide $7.5 \mathrm{~m} / \mathrm{s}$ nozzle velocity and $78 \%$ stroke. (Test Instruction Section 5.3 lists targeted versus actual PJM parameters.)

Slurry remaining in the filter-loop was flushed to UFP-VSL-T62B (Test Instruction step 7.13.21) with $693 \mathrm{~kg}$ of IW between 22:10 and 22:41 on 3/15/09.

The Batch 2 caustic-leach was completed at 11:50 on 3/16/09. Batch 2 was subsequently cooled to $25^{\circ} \mathrm{C}$ over 13.2 hours to match a prototypic cooling curve. The temperature in Tank T02A was monitored every 15 minutes; a valve on the cooling jacket (TV-0617) was adjusted manually to maintain the cooling rate (Test Instruction steps 7.14.6, 7.14.7). Cooling was completed at 01:05 on 3/17/09.

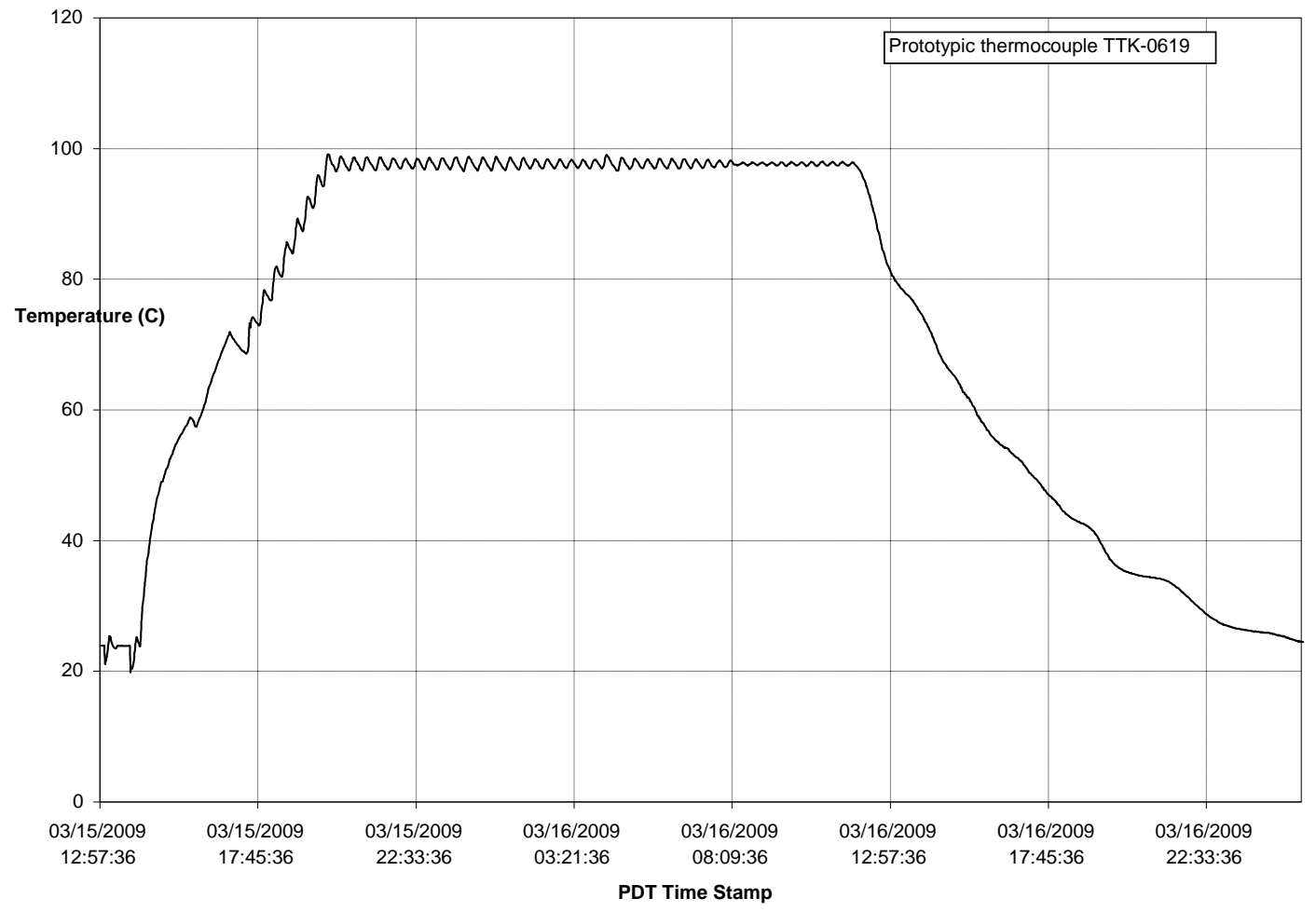

Figure 4.18. Tank T02A Temperature During Batch 2 Caustic-Leach

\subsection{Concentration of Leached Slurry}

At the completion of cooling Batch 2, concentration of the caustic-leached solids began (Test Instruction Section 7.15). 
The tubeside of the filter-loop was drained before starting the filter Pump T42A and Pump T43A. The loop was aligned to send slurry through all five filters and the cooling heat exchanger (UFP-HX-T02A), with permeate withdrawn from Filter 1 only. The heating heat exchanger (UFP-HX-T03A) was bypassed, with the slurry outlet valve locked open. The targeted slurry flow rate in the filter-loop was 109 gpm with a TMP of 40 psig. Pre-filtration samples (Test Instruction step 7.15.6) were taken at 01:55, 3/17/09. The permeate valve on Filter 1, aligned to UFP-VSL-T62B, was opened at 02:09 (Test Instruction step 7.15.7). Tank T01B provided make-up feed (from Batch 1 leached slurry). Filtration of the leached slurry started at 02:09 on 3/17/09. Batch transfers were initiated when the level in Tank T02A reached 47 inches, initiating an 11-gal make-up batch transferred at $6.9 \mathrm{gpm}$. Eighteen 11-gallon batches were transferred from Tank T01B to Tank T02A during the course of filtration, which brought the level in Tank T01B down to the low level alarm. Samples were taken every two make-up batches, as specified in the Test Instruction (Test Instruction steps 7.15.13-23). Visual inspection of permeate samples taken during concentration (three samples/filter evenly spaced during concentration) showed no sign of particle break-through. Figure 4.19 through Figure 4.22 show TMP, axial velocity, temperature, and permeate production during the concentration of leached slurry.

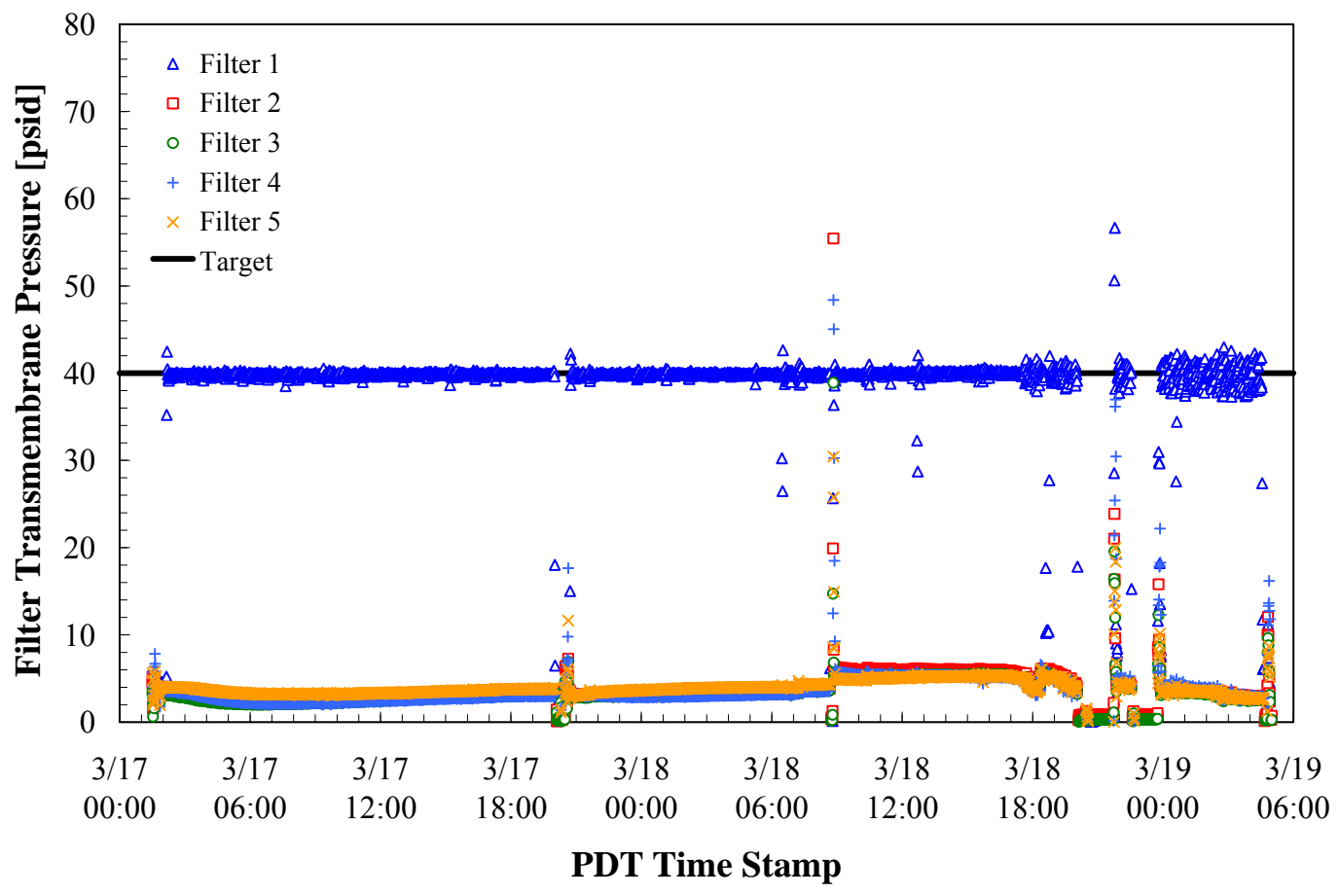

Figure 4.19. TMP for Post-Caustic-Leach Concentration, Integrated Test B 


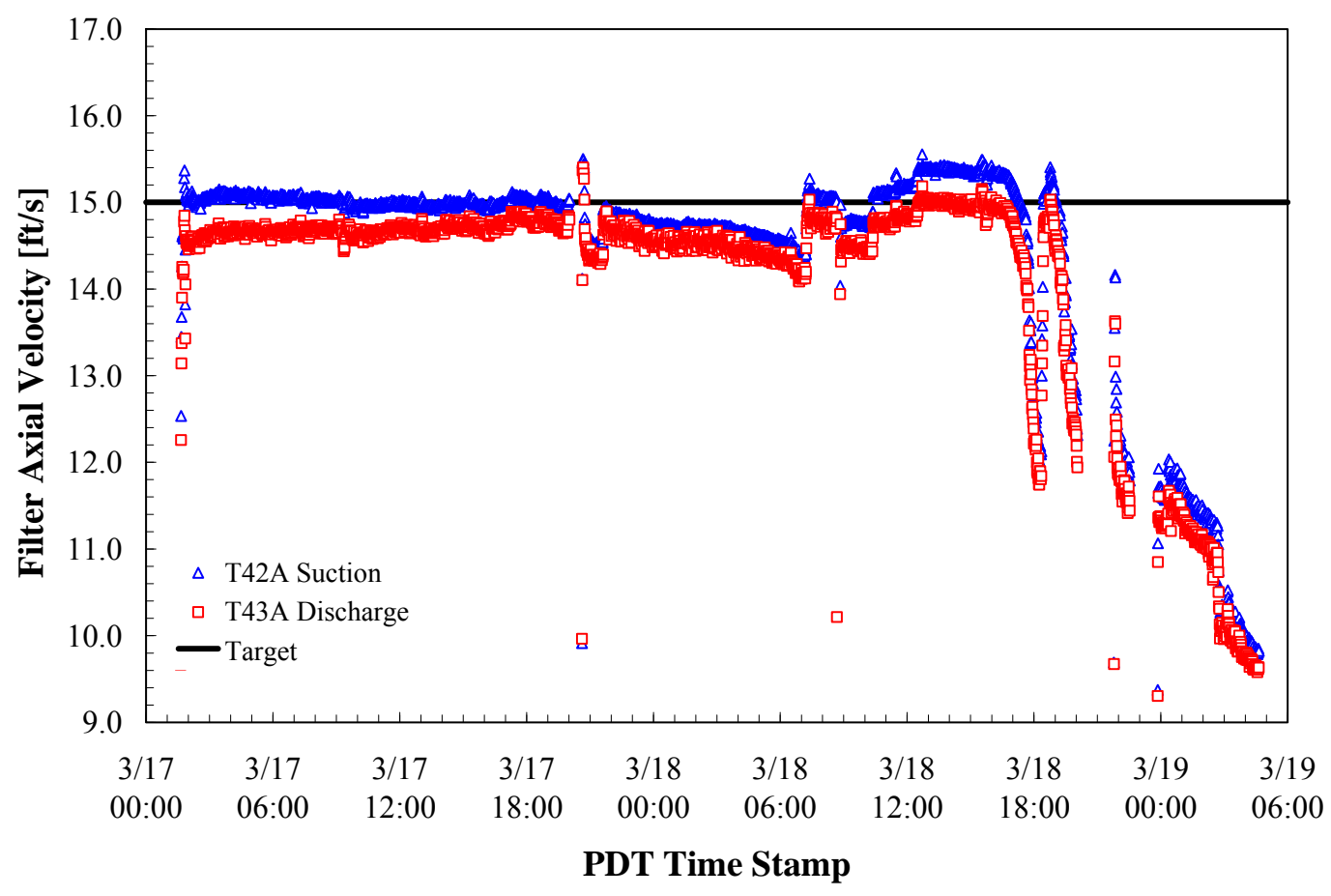

Figure 4.20. Axial Velocity Based on Flow Measurements at the Suction to Pump T42A and the Discharge to Pump T43A for Post-Caustic-Leach Concentration, Integrated Test B

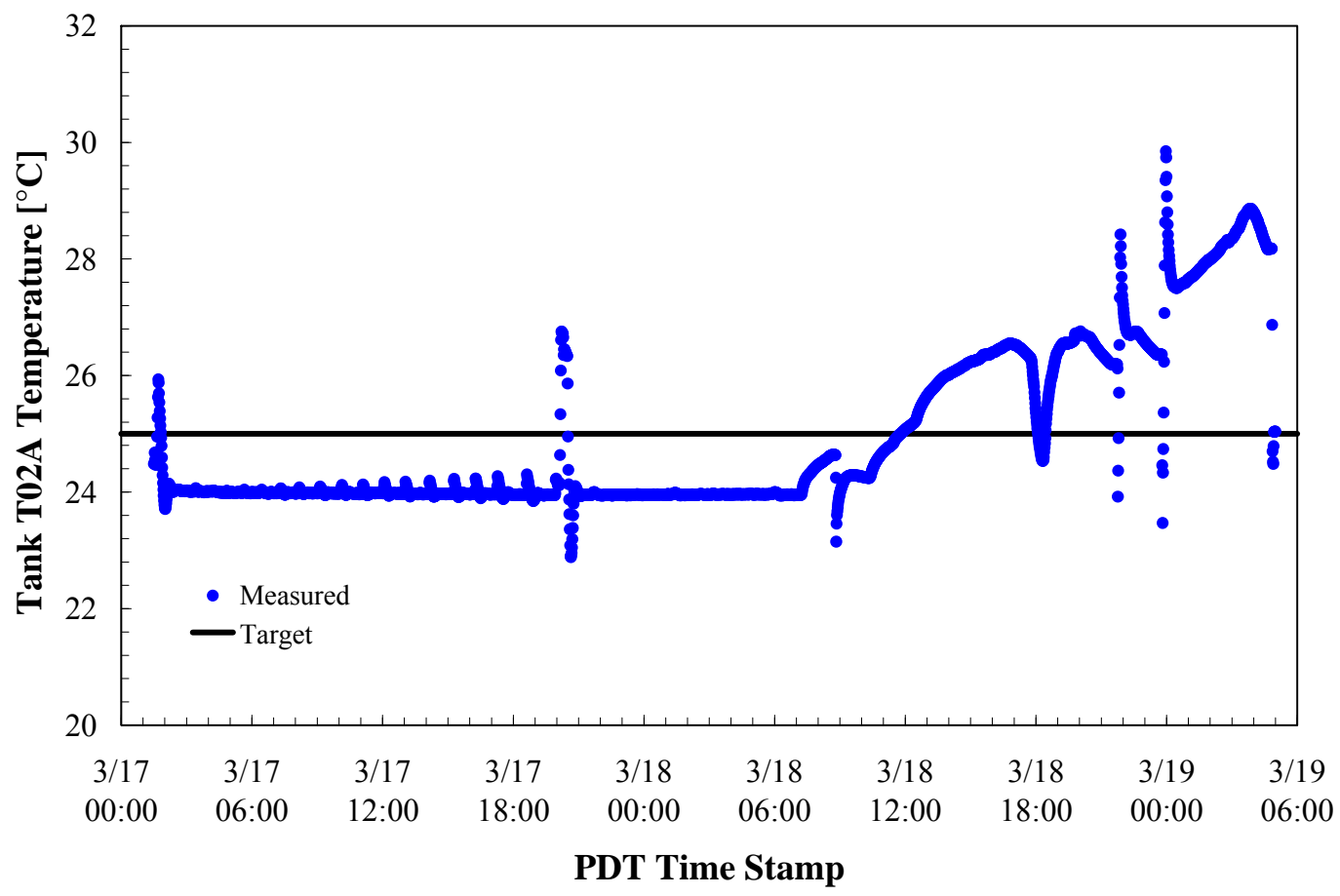

Figure 4.21. Tank T02A Temperature (TTK-0619) for Post-Caustic-Leach Concentration, Integrated Test B 


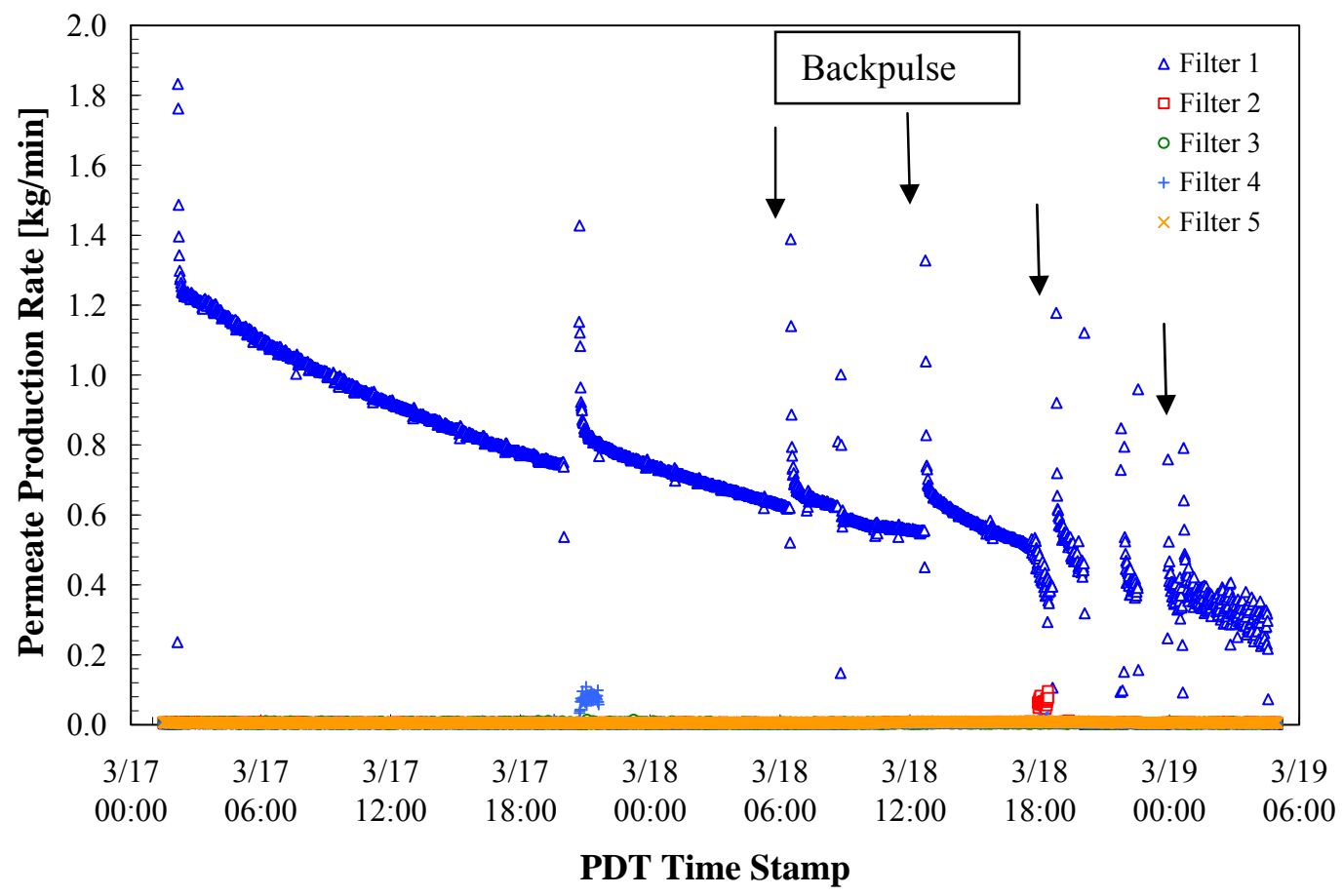

Figure 4.22. Permeate Production Rate for Post-Caustic-Leach Concentration, Integrated Test B

A diaphragm pump was used to pump the last 74 gallons to transfer all of the leached solids from Tank T01B to Tank T02A (via the drain in the bottom of Tank T01B). The regular transfer pump (UFP-PMP-T41B) was not used as the low/low level alarm disabled the pump at these low levels. The permeate valve was closed, and filtration was suspended between 20:01 and 20:44 on 3/17/09 during this transfer.

Instructions to backpulse Filter 1 were given on 3/17/09, with the following guidelines:

- Backpulse when the permeate rate drops below $0.6 \mathrm{~kg} / \mathrm{min}$

- Backpulse no more often than once every 6 hours

- Parameters: Overpressure $=40$ psi; Deadband $=5$ psi, Pulse-pot level $=10$ inches.

Backpulses were conducted according to the instructions above at the following times:

$$
\begin{gathered}
06: 30,3 / 18 / 09 \\
12: 44,3 / 18 / 09 \\
18: 36,3 / 18 / 09 \\
00: 37,3 / 19 / 09 .
\end{gathered}
$$

The effect of backpulsing on permeate production was transitory. Suspending filtration or turning the circulation pumps off also resulted in similar transient improvements of permeate production, as seen in Figure 4.22 . 
At 18:26 on 3/18/09, the level in Tank T02A was at about 20.5 inches at the low point of the PJM cycle, and the targeted flow rate in the filter-loop could not be maintained. PJMs were turned to star pattern mode (where only two of six PJMs are firing at the same time, resulting in less variation in tank level). The flow rate immediately improved to within the targeted range. This improvement was temporary while the level decreased as dewatering continued. The filter-loop flow rates again could not be maintained at the targeted $109 \pm 10 \mathrm{gpm}$, and the level bubbler and the stream ring air purge were turned off in accordance with the Degassing Protocol (Appendix I). This resulted in some temporary improvement to the flow rate. Figure 4.23 shows the slurry flow rate with the tank level.

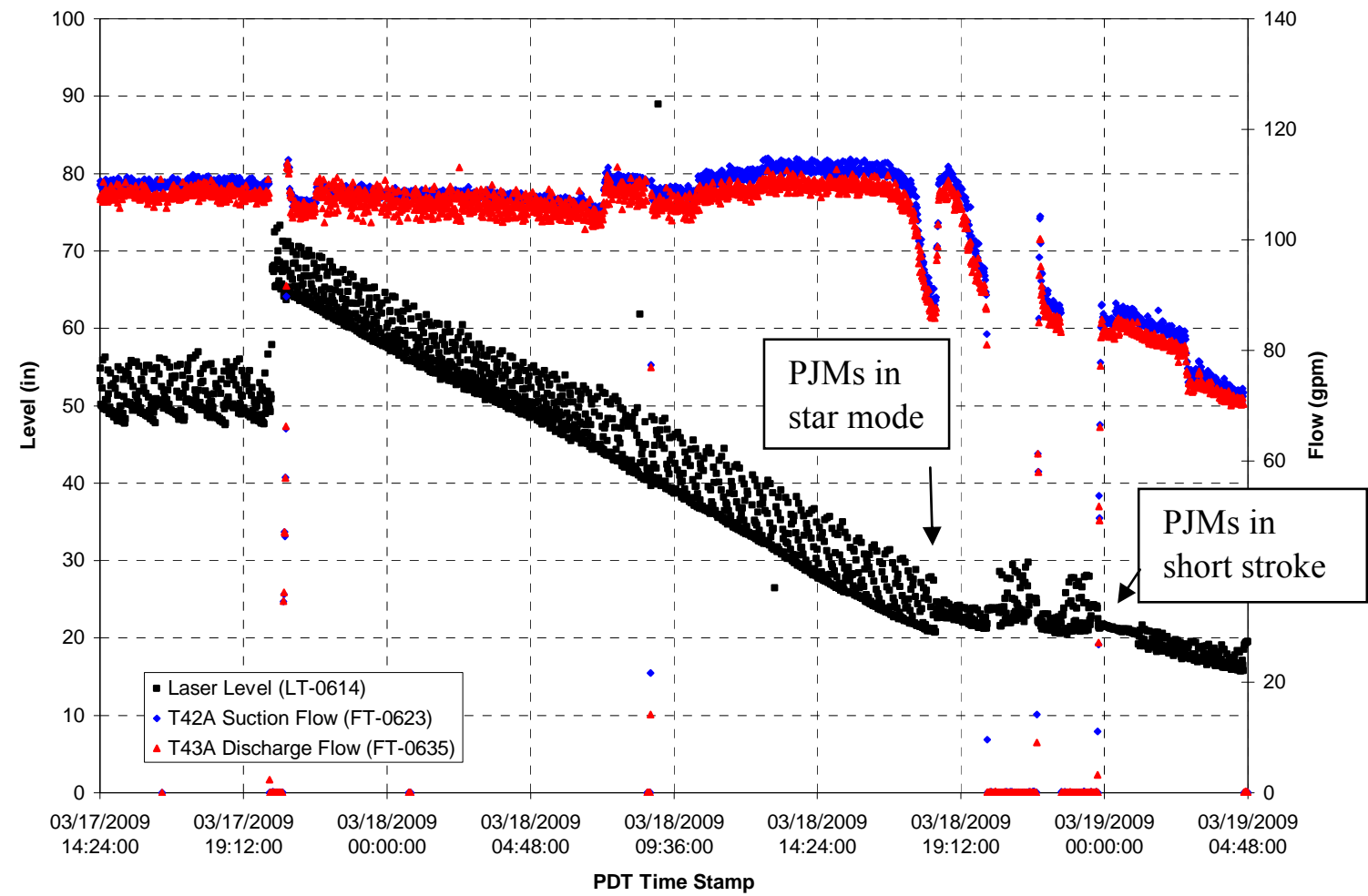

Figure 4.23. Tank T02A Level and Filter-loop Flow Rate During Concentration After Caustic-Leach Batch 2

Increasing the VFD on Pumps T42A and T43A did not result in sustained flow improvement. Significant air entrainment did not seem to be at issue as flow meters in the filer-loop (FI--0635 and FI-0623) readings were within about $3 \mathrm{gpm}$. When air entrainment was at issue, these flow meters diverged significantly, as was observed in Integrated Test A. A sample taken indicated 2.6 vol\% air held up in the slurry, which was not considered significant.

At 20:00, after 30 minutes, the "Initial Degassing Protocol" was executed. The filter-loop pumps were turned off, and a stable level measurement was taken in Tank T02A. The lower air spargers were set to $0.40 \mathrm{~kg} / \mathrm{min}$, and the PJMs were turned on. This configuration was held for 50 minutes, and then the PJMs and the air spargers were turned off. After 10 minutes, another stable level measurement was taken. 
The stable level in Tank T02A was measured before and after the slurry degassing protocol was executed, and the level increased from 23.5 inches to 24.8 inches, suggesting that the degassing protocol added gas to the system. The filter-loop pumps were turned back on, but the targeted flow rate could not be achieved. At 21:57, the lower air spargers were turned off in accordance with step 6 of the Tank T02A Systems Operation Guidance for Integrated Test B. At 22:30, the flow in the filter-loop was $\sim 85 \mathrm{gpm}$, and a second degassing protocol was executed in accordance with step 8 of the T02A Systems Operation Guidance for Integrated Test B. At the conclusion of the second degassing protocol, the flow rate in the loop could not be restored to the targeted value. An attempt was made to determine if the lower air spargers contributed to the flow-rate issues, and each of the spargers was turned on in succession with no adverse impact to the flow rate. It was concluded that the lower air spargers at idle flow were not impacting the flow rate.

At 00:37, the PJMs were turned back to "star" mode following the second degassing protocol; however, the PJMs were stuck in short stroke. This was fixed, and the PJMs were operating correctly in star pattern at 01:15,3/19/09. The filtration continued, at reduced flow rate, until the targeted concentration was achieved. The filtration was concluded at 04:34 on 3/19/09. The permeate flow decreased over the concentration period from $\sim 1.5 \mathrm{~kg} / \mathrm{min}$ to $\sim 0.2 \mathrm{~kg} / \mathrm{min}$. The total permeate removed was $2007 \mathrm{~kg}$, resulting in a UDS concentration of $17.3-\mathrm{wt} \%$.

The pumping issues resulted in an increased heat load to the cooling heat exchanger UFP-HX-T02A. At $0323,3 / 19 / 09$, the Tank T02A temperature was $28.7^{\circ} \mathrm{C}$, with the cooling water supply fully open. The cooling water set point was decreased from $42^{\circ} \mathrm{F}$ to $36^{\circ} \mathrm{F}$ in response. The Tank T02A temperature began to decrease after this change was made.

After the concentration, the PJMs in UFP-VSL-T02A were formally tuned in standard mode to $11.7 \mathrm{~m} / \mathrm{s}$ nozzle velocity and $70 \%$ drive stroke. This tuning was completed at 11:07 on 3/19/09. (See Section 5.3 for targeted versus actual PJM parameters.)

\subsection{Filter-Loop Bypass Test with Cs Br Tracer}

$\mathrm{A} \mathrm{CsBr}$ tracer test was conducted to evaluate whether the slurry returning to Tank T02A mixed well with tank contents. The stable level measurement in Tank T02A before testing started was 18.6 inches. Because of this low tank level, the pumps were operating at a reduced flow rate (64 to $73 \mathrm{gpm}$ ), and the PJMs were operating in star pattern. Figure 4.24 shows a plot of the filter-loop flow rate during the tracer test. In addition, to reduce air entrainment issues, the steam ring air purge and the air spargers were off. The tracer solution contained $108 \mathrm{~g}$ of $\mathrm{CsBr}$ and was added to the suction side of Pump T42A using a small piston pump. Tracer solution was added at 13:17 on 3/19/09. It took approximately 30 seconds to add the tracer. The test was started when the tracer addition to the loop was initiated. Samples from Tank T02A and the filter-loop were taken at 1, 2, 4, 8, 16, 30, 40, 50, and 60 minutes after the tracer was added. 


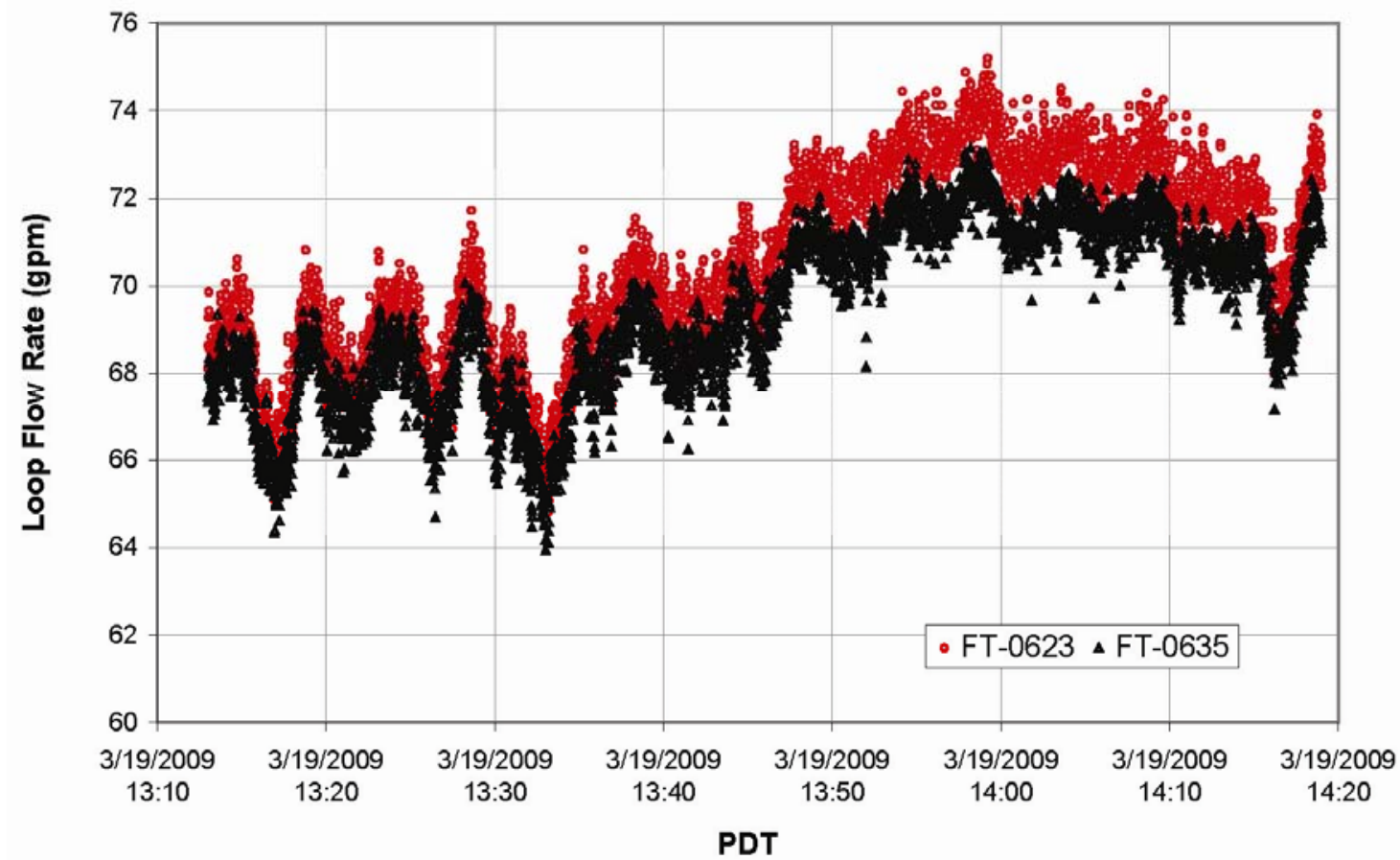

Figure 4.24. Filter-Loop Flow Rate During Tracer Test. FT-0623 precedes the recirculation pumps, and FT-0635 is after the recirculation pumps.

\subsection{Post-Caustic-Leach Wash}

The next step in the test was to water wash the caustic-leached slurry (Test Instruction step 7.17). Washing, which started at approximately $16: 25$ on 3/19/09 and concluded at $01: 10$ on 3/20/09, was conducted at scale-time using all five filters. IW was introduced into the suction of Pump T42A. As the slurry was recirculated through the loop, the liquid was extracted through the ultrafilters and collected in UFP-VSL-T62B. The wash water was delivered in fifty-two $41.6 \mathrm{~kg}$ batches for a total of $2204 \mathrm{~kg}$ of wash water. Wash-water batches were triggered when the level in Tank T02A decreased to 16.7 inches. AFA was added after every third wash batch addition to maintain the targeted concentration of greater than $350 \mathrm{ppm}$.

The PJMs in Tank T02A were operated in the star pattern during the wash. Operating at the low target level in Tank T02A (16.7 inches) led to air entrainment during the wash which prevented the filter-loop pumps from achieving $109 \mathrm{gpm}$. The wash was carried out with a filter-loop flow rate of approximately 70 to $95 \mathrm{gpm}$, with pump performance improving over the course of the wash. Likewise, the permeate flux increased over the course of the wash from approximately $0.5 \mathrm{~kg} / \mathrm{min} /$ filter to 1.3 to $1.7 \mathrm{~kg} / \mathrm{min} /$ filter. The total permeate produced was approximately $2472 \mathrm{~kg}$. The filtration rate improved during the post-caustic-leach wash in the PEP, as has been observed in the laboratory, and generally remained high through the remainder of the test.

See Figure 4.25 through Figure 4.28 for TMP, axial velocity, temperature, and permeate production during washing of the leached slurry. 


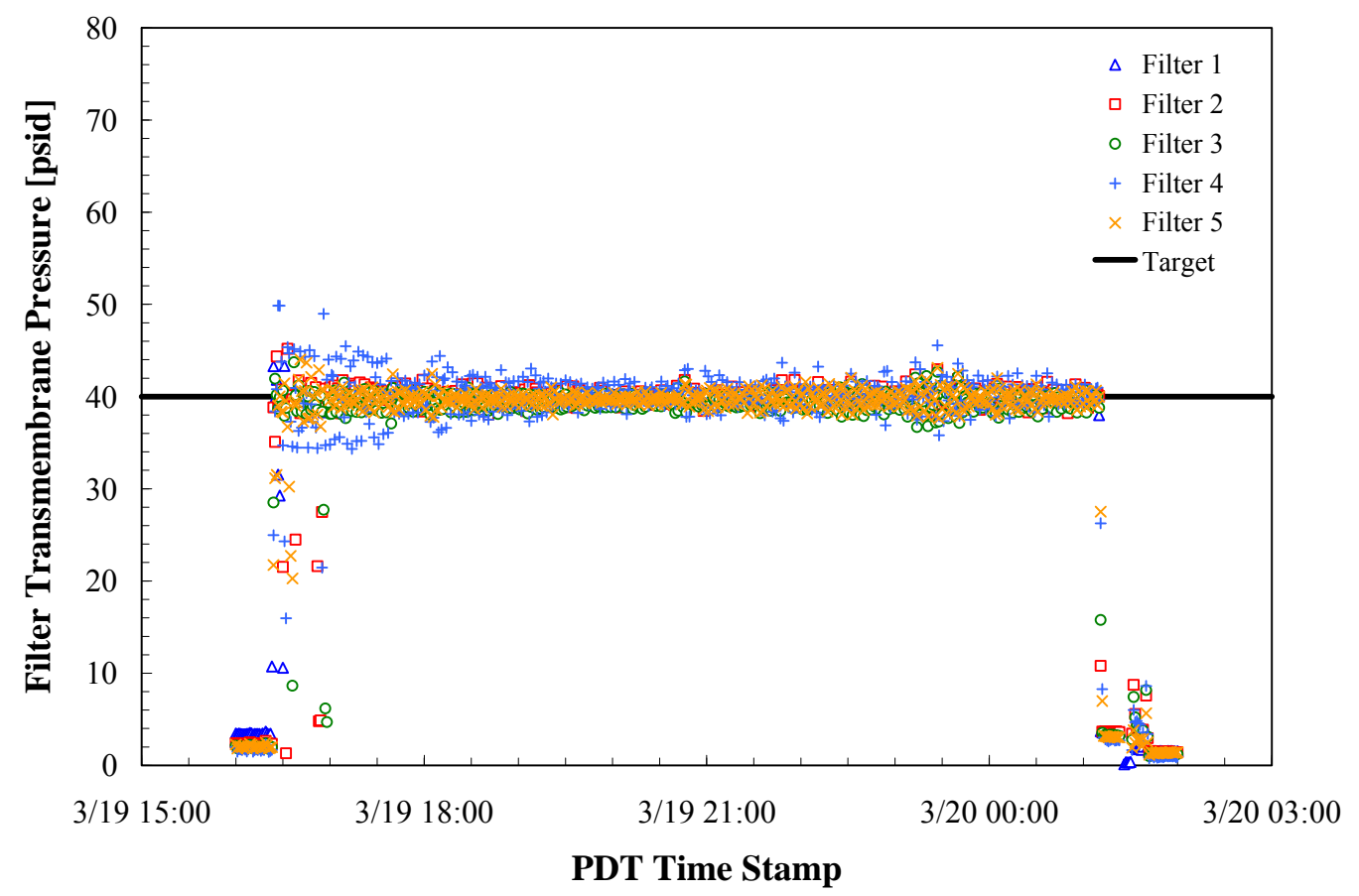

Figure 4.25. TMP for Post-Caustic-Leach Washing, Integrated Test B

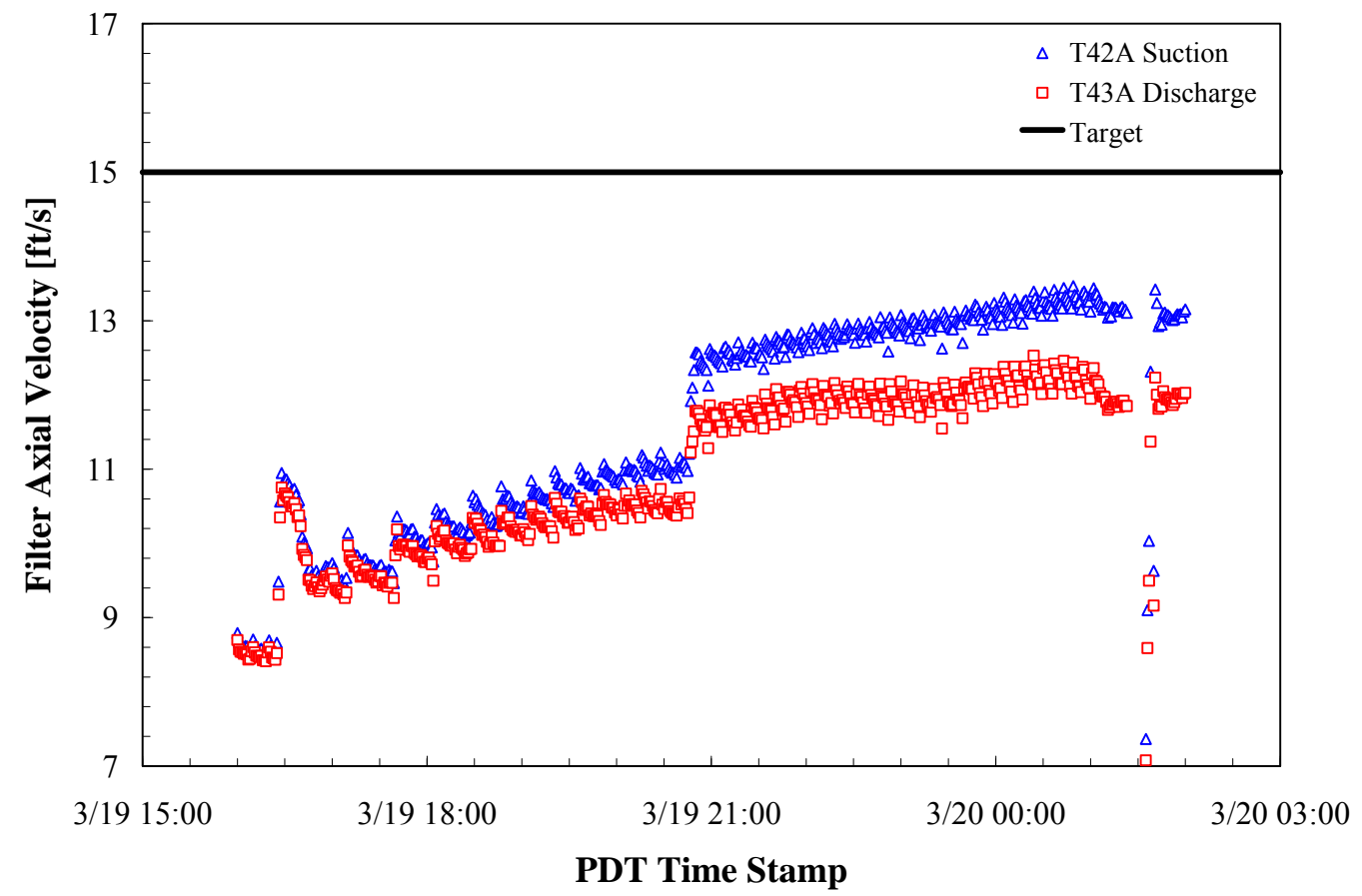

Figure 4.26. Axial Velocity Based on Flow Measurements at the Suction to Pump T42A and the Discharge to Pump T43 A for Post-Caustic-Leach Washing, Integrated Test B 


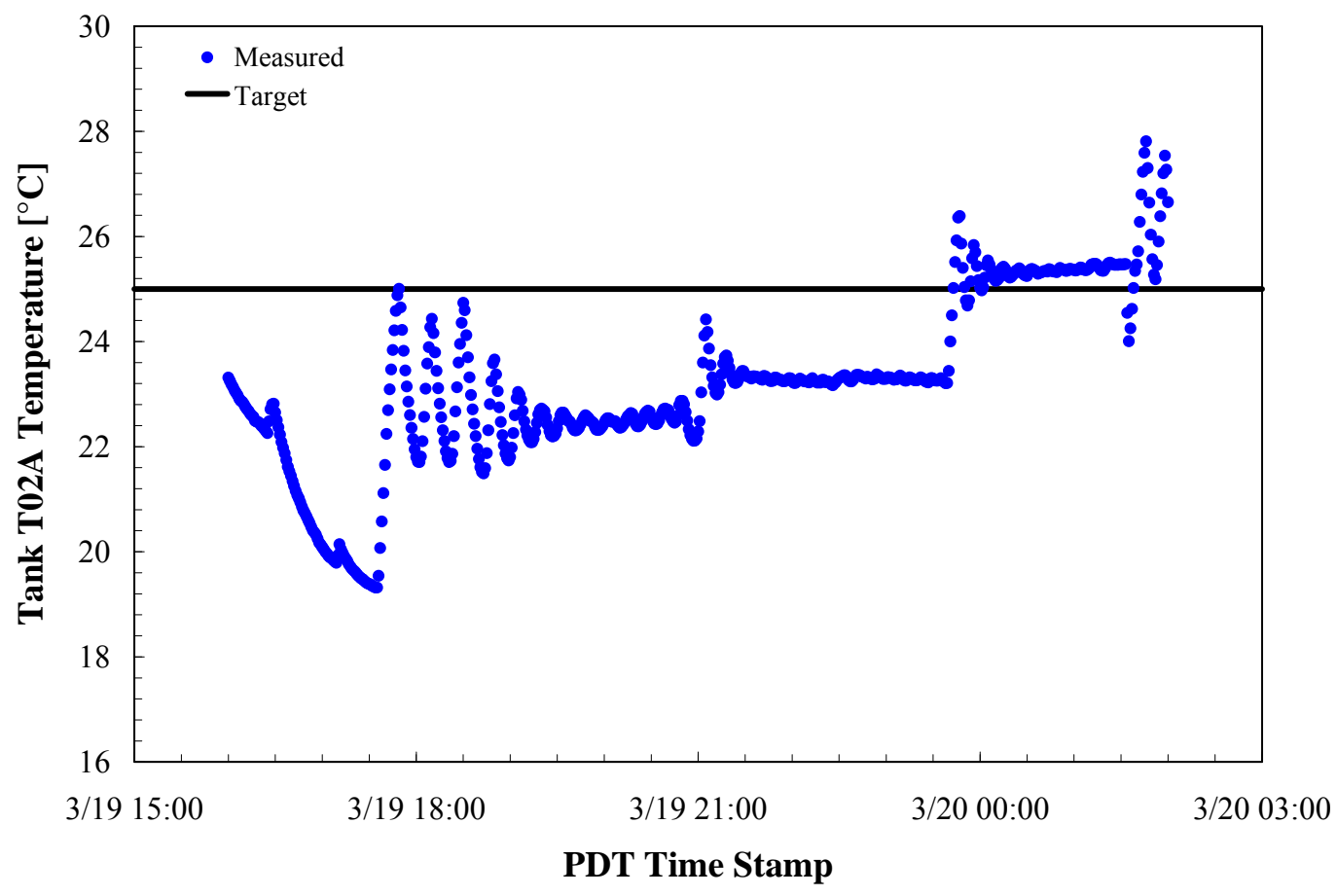

Figure 4.27. Tank T02A Temperature (TTK-0619) for Post-Caustic-Leach Washing, Integrated Test B

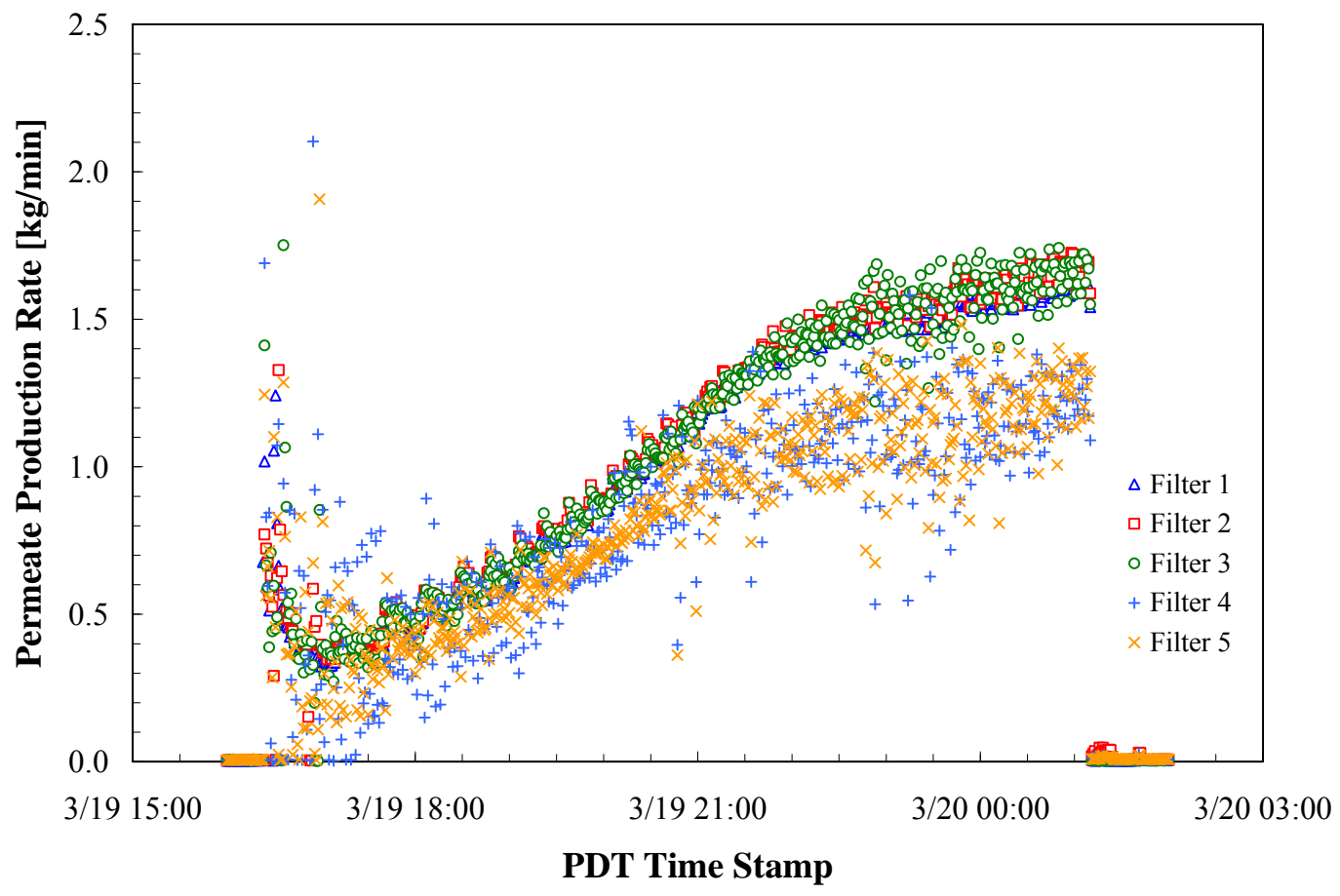

Figure 4.28. Permeate Production Rate for Post-Caustic-Leach Washing, Integrated Test B 
Tank T02A temperature variation is a result of changing the cooling water control valve setpoint (measured just downstream of the heat exchanger UFP-HX-T02A) to $21^{\circ} \mathrm{C}$ at $15: 13$, to $22^{\circ} \mathrm{C}$ at $21: 00$, and to $24^{\circ} \mathrm{C}$ at 23:39. These changes were made as operators were targeting a Tank T02A temperature of $25^{\circ} \mathrm{C}$. See Section 4.2 for write up on how tank temperature was controlled.

\subsection{Chromium Slurry Addition, Concentration, and Wash}

Chromium was added as a $\mathrm{CrOOH}$ slurry to the simulant after removing aluminum and after the washing and concentration steps. Adding the chromium-bearing slurry and subsequent wash is a nonprototypic aspect of this test, but it is necessary because chromium in the simulant was found to oxidize to the $\mathrm{Cr}^{6+}$ state during the caustic leach. Approximately $371 \mathrm{~kg}$ of chromium-bearing slurry was added to the suction side of Pump T42A, starting at 02:34 and concluding at 03:11 on 3/20/09. (Table 5.11 and Table 5.12 show the composition of the chromium bearing slurry). AFA was added to maintain a concentration at $350 \mathrm{ppm}$. After the Cr slurry addition, permeate was removed to reduce the level of the slurry in Tank T02A to 21.4 inches, followed by twenty-one additional $41.6 \mathrm{~kg}$ wash batches. AFA was added after every third wash batch addition to maintain the targeted concentration. The filter-loop flow rate was approximately $90 \mathrm{gpm}$ (versus $109 \mathrm{gpm}$ target condition) during the concentration and wash. Comparing the filter-loop magnetic flow meters before (FT-0623) and after (FT-0635) the recirculation pumps (Pumps T42A and T43A) shows an increasing difference between the measured flow rate as the concentration and wash continued. This suggests that at the low tank level (21.3 inches at the conclusion of the wash) at which these operations were conducted resulted in air entrainment into the slurry. The simulant was concentrated and washed from 03:21 through 05:52 on 3/20/09.

Figure 4.29 through Figure 4.32 show TMP, axial velocity, temperature, and permeate production during concentration and washing before oxidative leaching. 


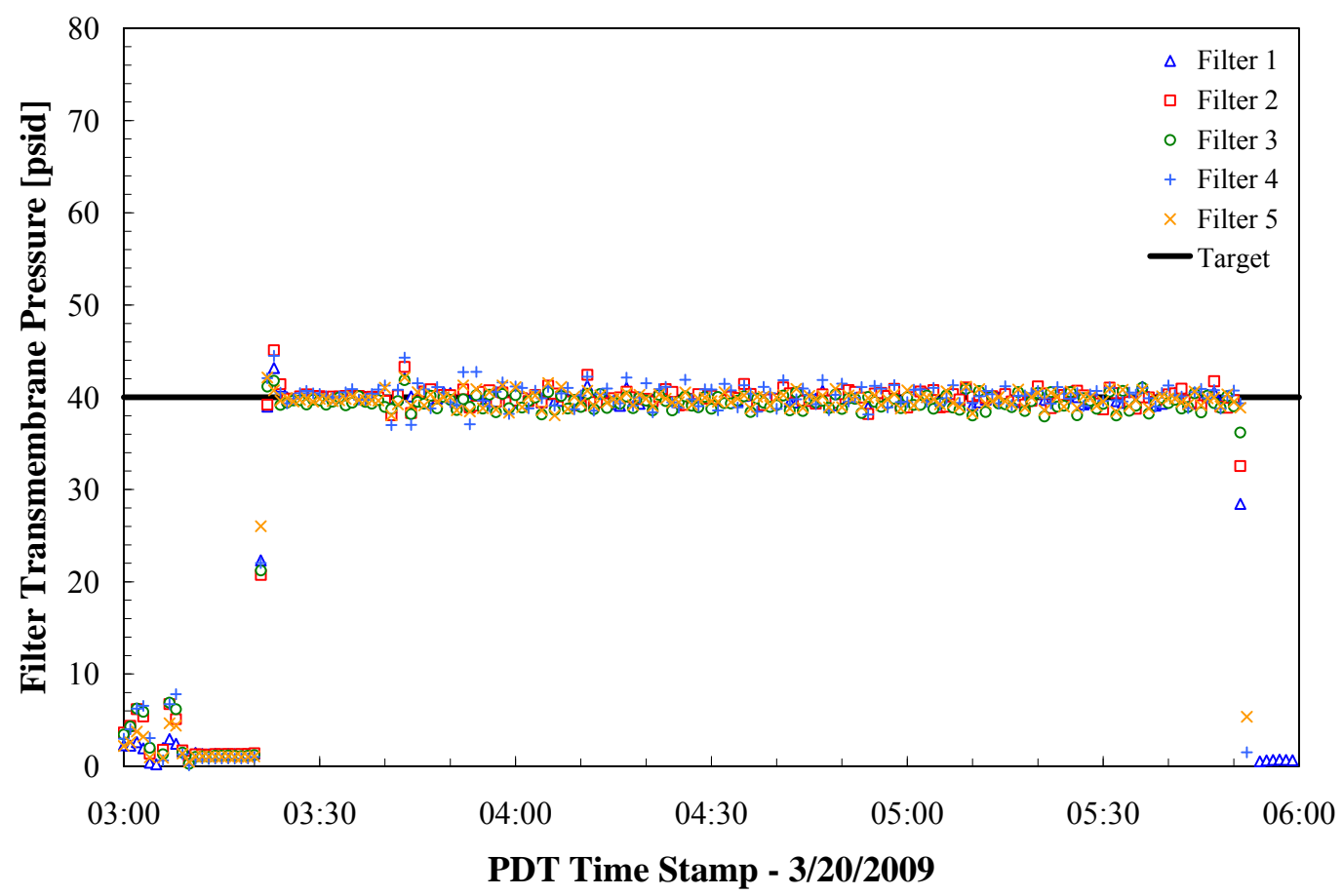

Figure 4.29. TMP for Concentration and Washing Operations Following Chromium Slurry Addition in Integrated Test B

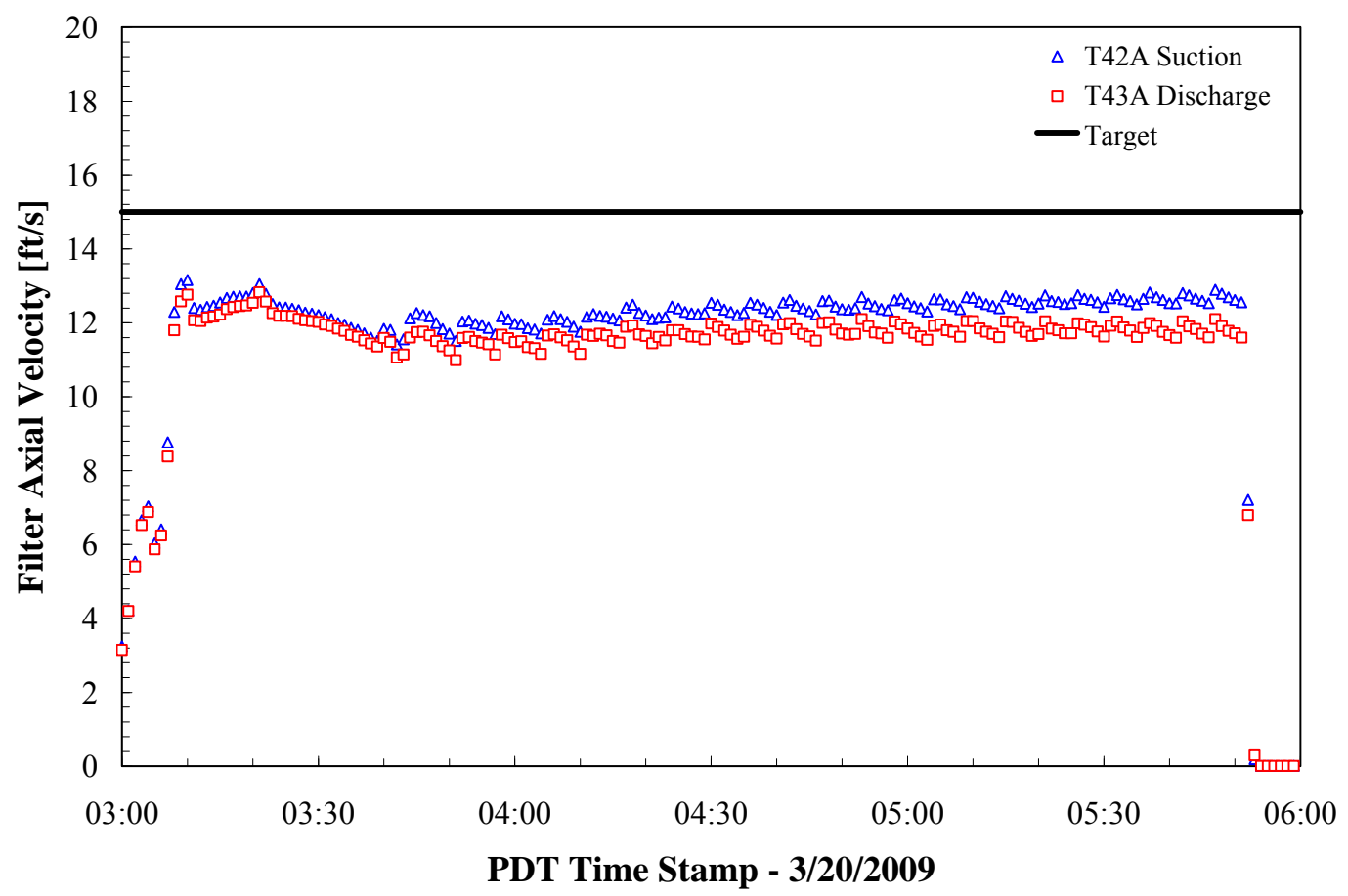

Figure 4.30. Axial Velocity Based on Flow Measurements at the Suction to Pump T42A and the Discharge to Pump T43A for Concentration and Washing Operations Following Chromium Slurry Addition in Integrated Test B 


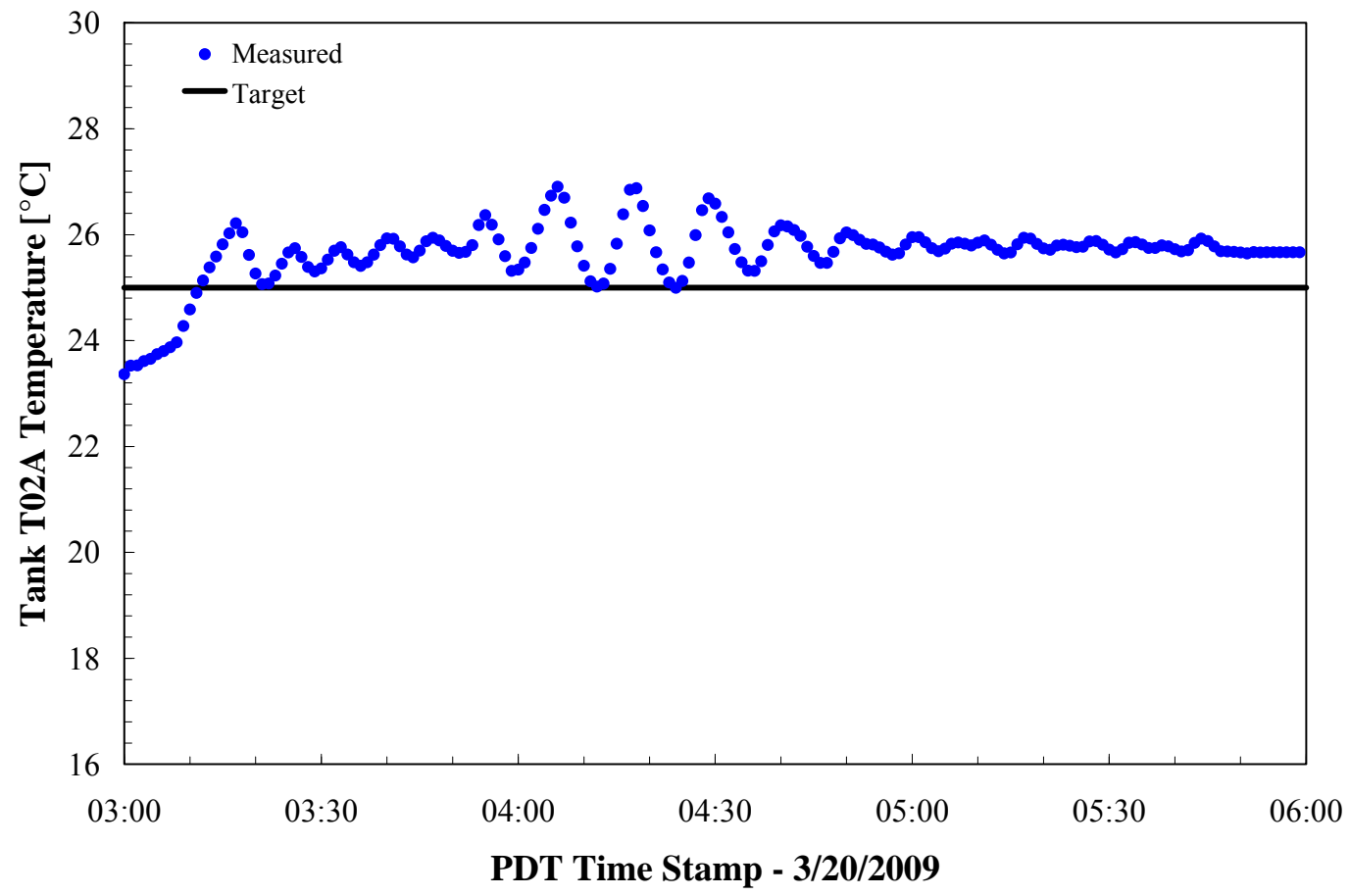

Figure 4.31. Tank T02A Temperature (TTK-0619) for Concentration and Washing Operations Following Chromium Slurry Addition in Integrated Test B

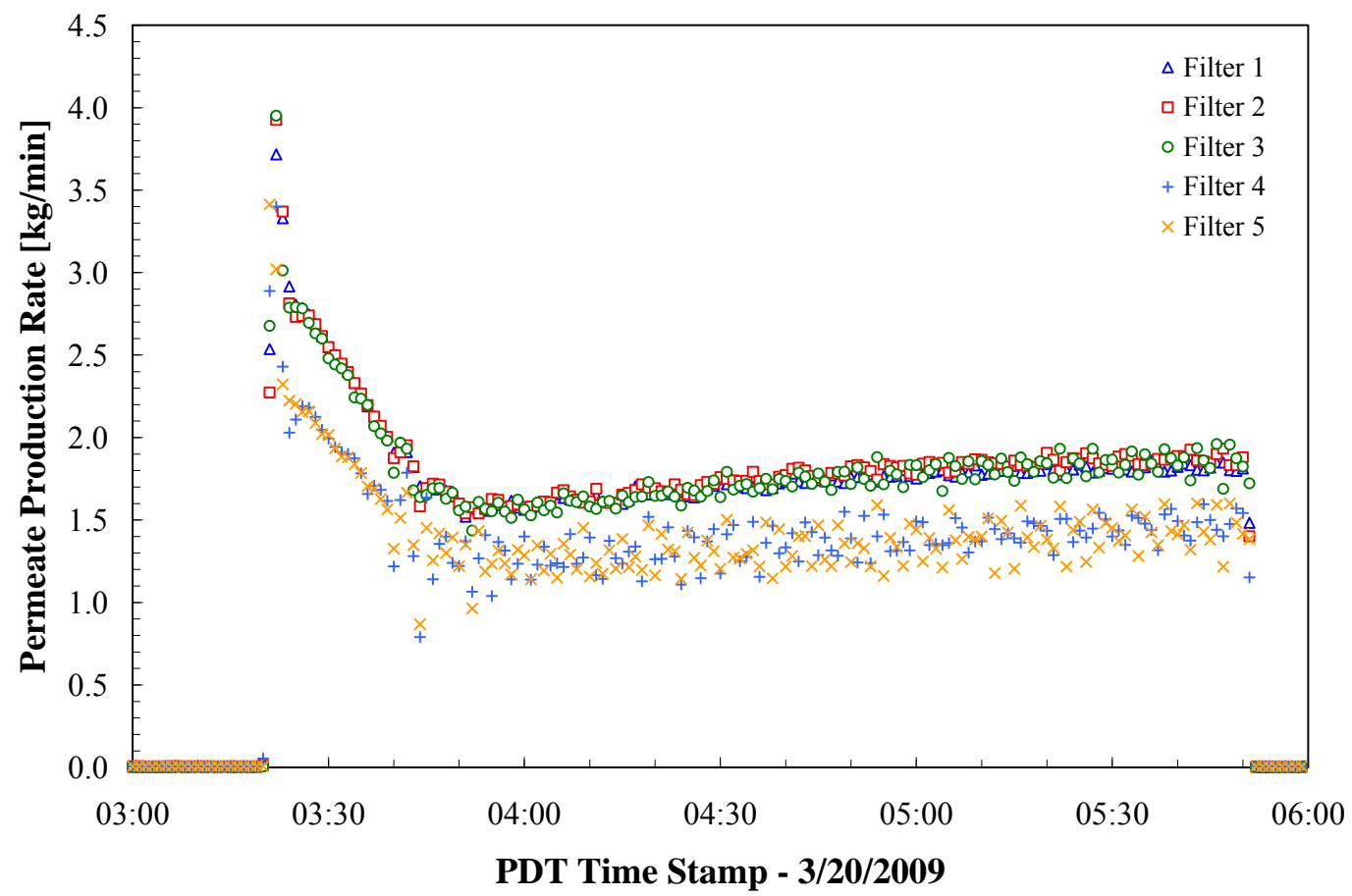

Figure 4.32. Permeate Production Rate for Concentration and Washing Operations Following Chromium Slurry Addition in Integrated Test B 


\subsection{Oxidative-Leach}

After the concentration and wash were completed, $1 \mathrm{M}$ sodium permanganate (20.9 gallons) was added (as the oxidizer) to the suction side of Pump T42A. (Note that a sample of the sodium permanganate reagent was analyzed, and the reported $\mathrm{MnO}_{4}{ }^{-}$concentration was $1.06 \mathrm{M}$. See Appendix N.) The oxidative-leach started at 09:17 on 3/20/09. Slurry samples were taken 5 minutes after permanganate addition and hourly for the 6-hr oxidative leaching test, which concluded at 15:17. During the oxidative-leach, Tank T02A was mixed from the operation of the PJMs in star pattern and the recirculation of the filter-loop ( $88 \mathrm{gpm}$ ). The targeted filter-loop flow rate of $109 \mathrm{gpm}$ was not attainable, presumably due to the low operating level in Tank T02A. Comparison of the filter-loop magnetic flow meters before (FT-0623) and after (FT-0635) the recirculation pumps (Pumps T42A and T43A) indicate a difference of $6 \mathrm{gpm}$, presumably due to the compression of air entrained in the slurry (see Appendix J, p. J.292). The level bubbler, air spargers, and steam ring air purge were off. There were no operational issues with foaming during the oxidative-leach.

As seen in Figure 4.33, the temperature in T02A shows an initial temperature increase of several degrees $\mathrm{C}$, presumably because of the heat of mixing of $1 \mathrm{M}$ permanganate with the slurry and/or, more likely, the heat of reaction of permanganate with the $\mathrm{Cr}$ in the slurry. As the temperature controller on UFP-HX-T02A reacts, the temperature oscillates for the first hour and then essentially remains constant over the remainder of the 6 -hr reaction time.

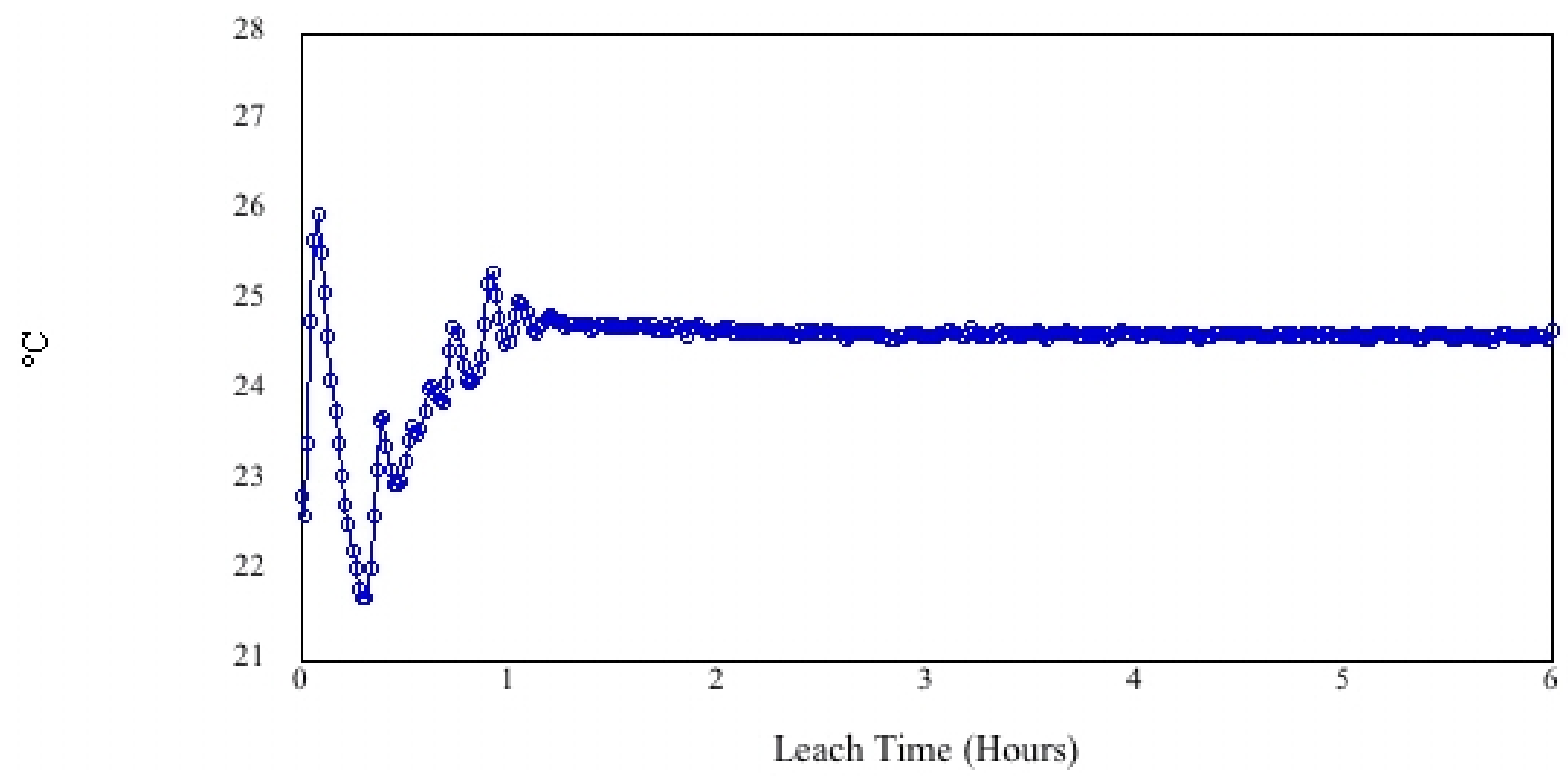

Figure 4.33. Tank T02A Prototypic Temperature During Oxidative Leaching

\subsection{Post-Oxidative-Leach Wash}

The final process steps of Integrated Test B were a final water wash and a concentration of the slurry to $20-\mathrm{wt} \%$ (Test Instruction Section 7.19). Washing was conducted at scale-time using all five filters. IW was introduced into the suction of Pump T42A. As the slurry was circulated through the filter-loop, 
liquid was extracted through the ultrafilters and collected in UFP-VSL-T62B. The wash water was delivered in forty-seven $41.6 \mathrm{~kg}$ batches. Wash water batches were triggered when the level in Tank T02A reached 21.4 inches. AFA was added after every third wash batch addition to maintain the targeted concentration of greater than $350 \mathrm{ppm}$. The wash started at 15:53 and concluded at 18:17 on $3 / 20 / 09$. Permeate flow decreased during the wash because of decreased flow rates as the level in Tank T02A decreased. Permeate flow rates started at $\sim 2$ to $4 \mathrm{~kg} / \mathrm{min} /$ filter and ended at $\sim 1$ to $2 \mathrm{~kg} / \mathrm{min} /$ filter. Changing the state of the recirculation pumps (on to off; or off to on) resulted in temperature fluctuations in Tank T02A.

See Figure 4.34 through Figure 4.37 for TMP, axial velocity, temperature, and permeate production during concentration and washing before oxidative leaching.

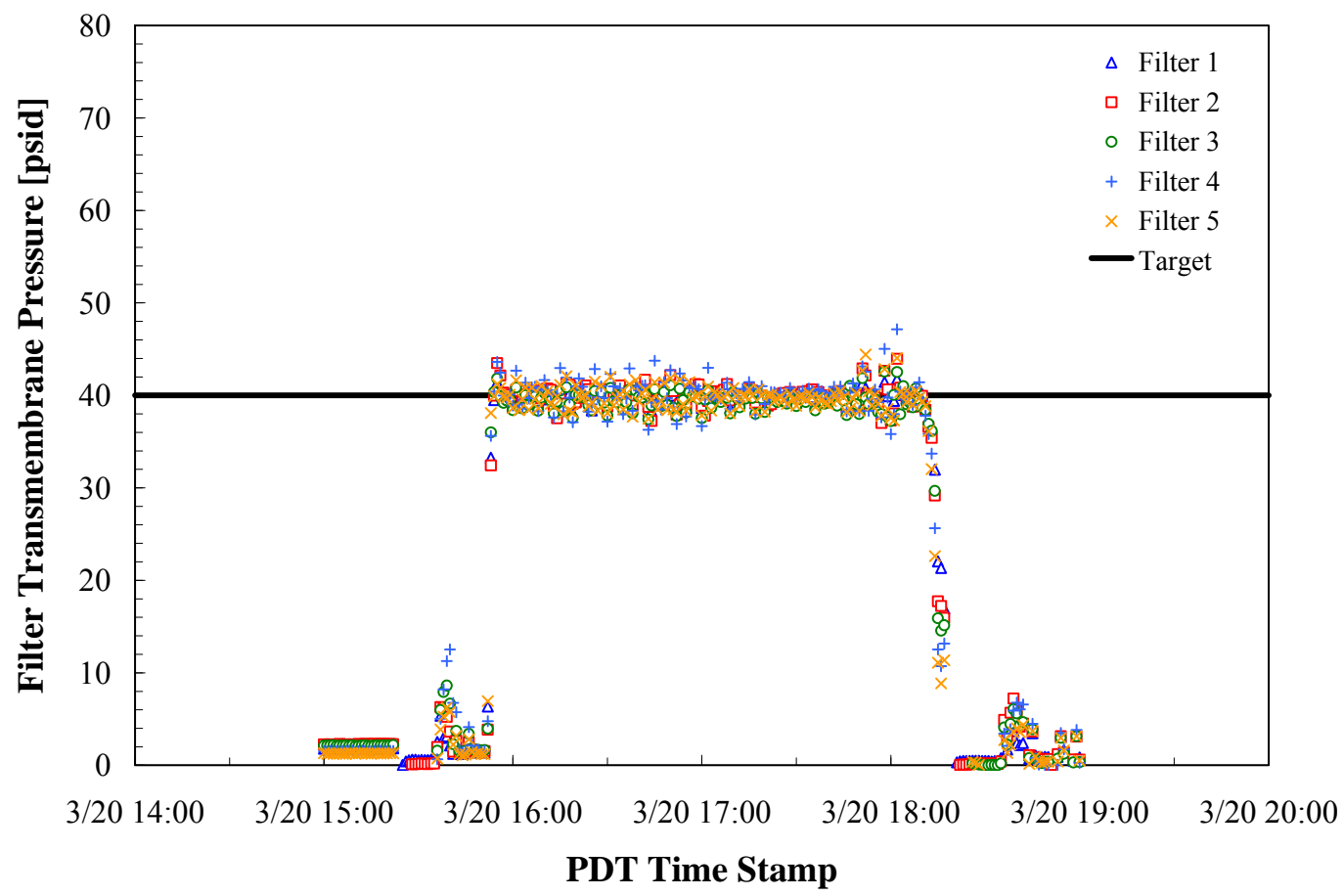

Figure 4.34. TMP for Post-Oxidative-Leach Washing, Integrated Test B 


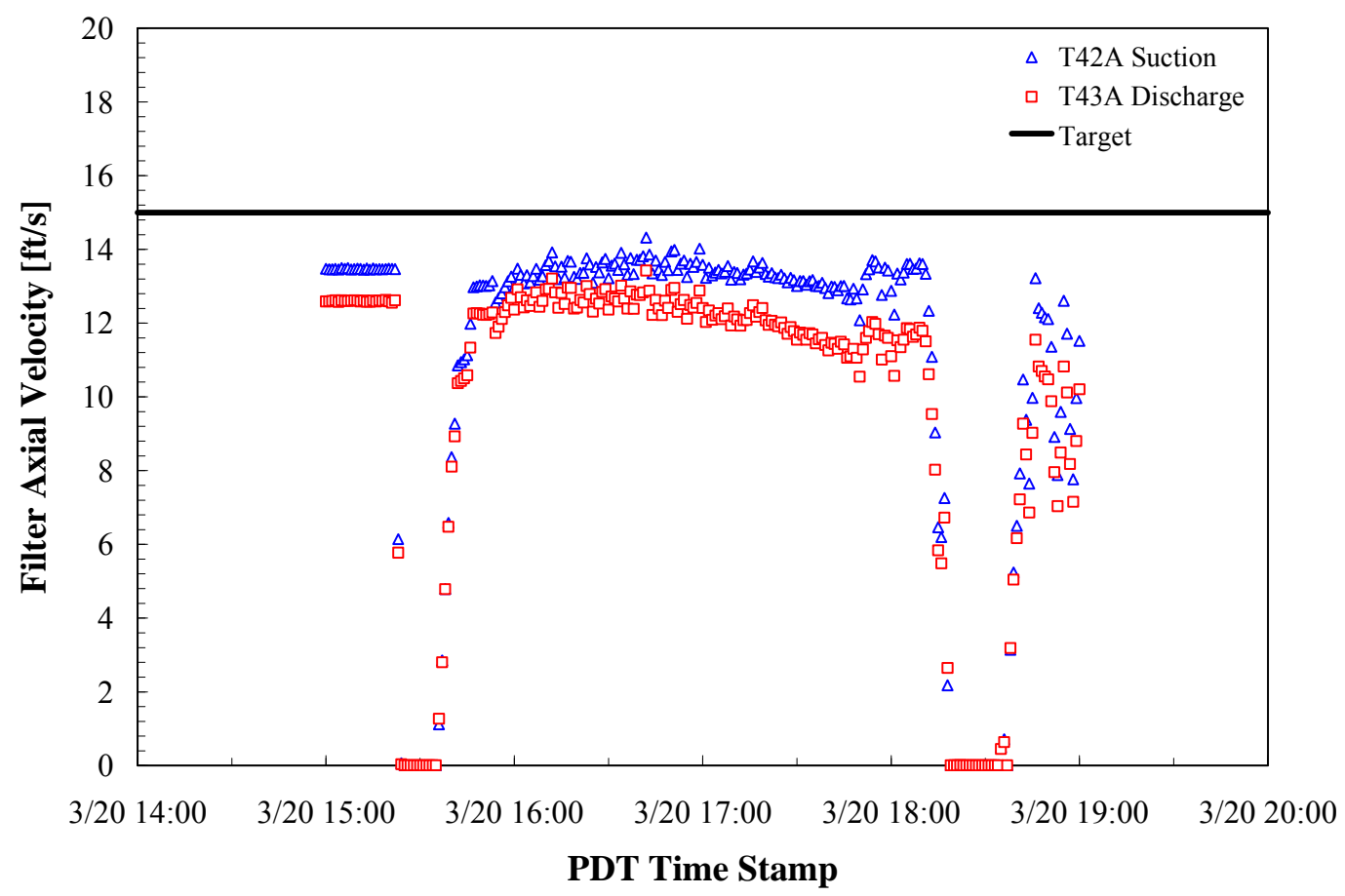

Figure 4.35. Axial Velocity Based on Flow Measurements at the Suction to Pump T42A and the Discharge to Pump T43A for Post-Oxidative-Leach Washing, Integrated Test B

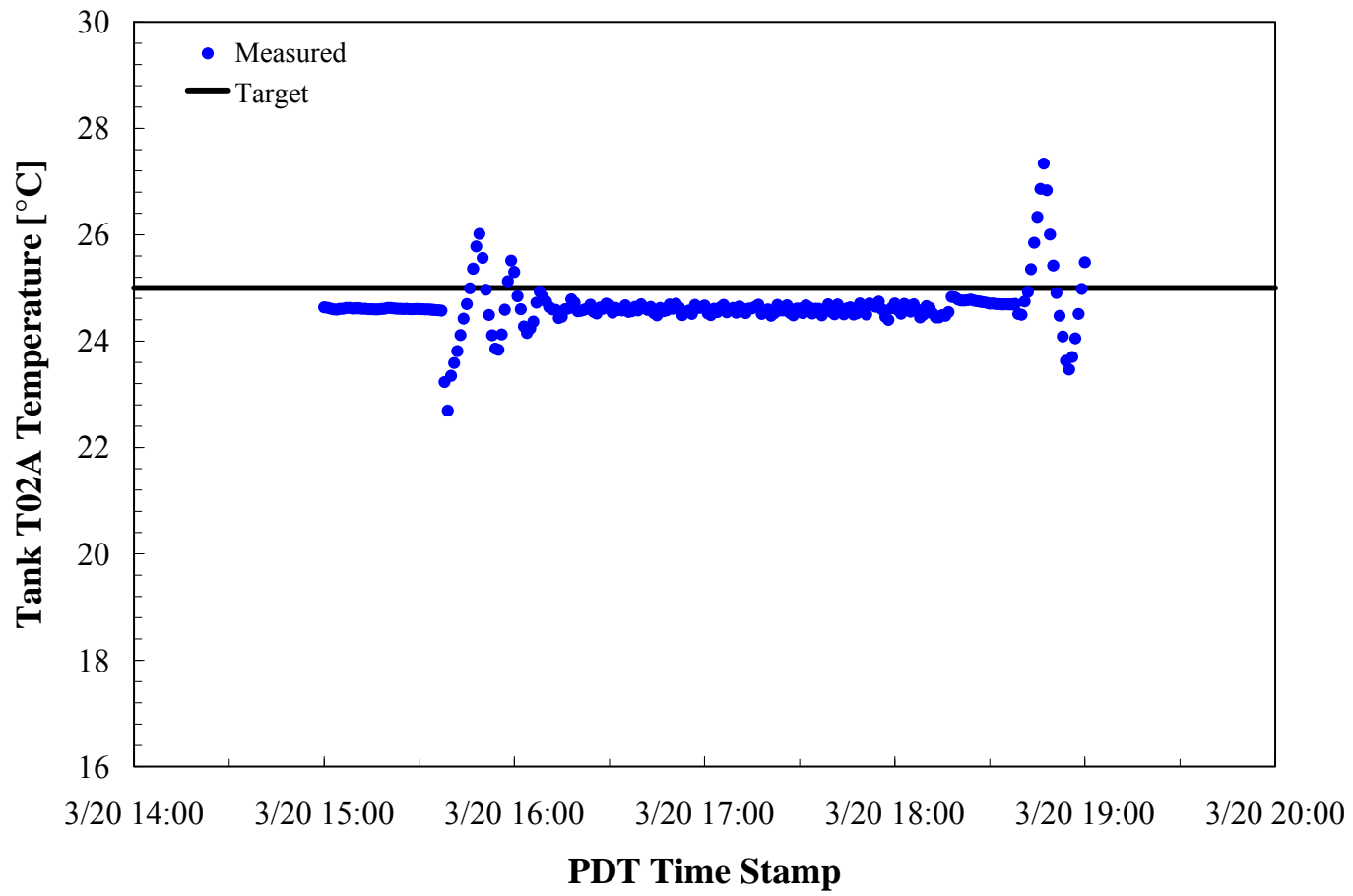

Figure 4.36. Tank T02A Temperature (TTK-0619) for Post-Oxidative-Leach Washing, Integrated Test B 


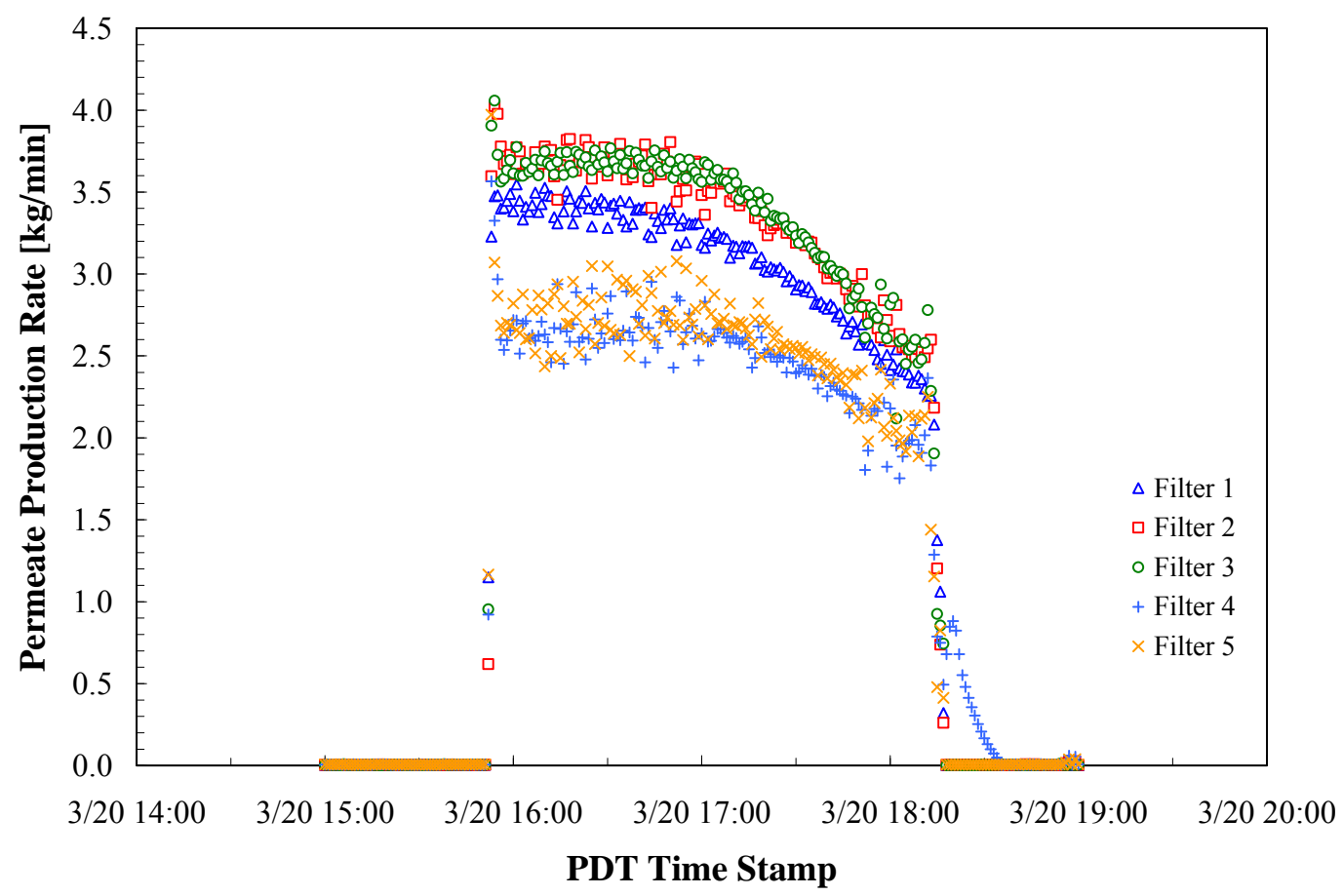

Figure 4.37. Permeate Production Rate for Post-Oxidative-Leach Washing, Integrated Test B

\subsection{Final Slurry Concentration}

At 18:18 on 3/20/09, Pumps T42A and T43A were turned off as part of the stable level measurement taken in Tank T02A (22.9 inches). At the conclusion of the measurement, the pumps were turned back on, but operators were not able to achieve a steady filter-loop flow rate above $57 \mathrm{gpm}(7.8 \mathrm{ft} / \mathrm{s}$ in the filter tubes) due to the impact of air entrainment. The minimum target was $67 \pm 10 \mathrm{gpm}$. The degassing protocol (Appendix I) was initiated to no effect. At 20:41, the effort to increase the flow rate was halted, and the final concentration of the slurry was not conducted as part of Integrated Test B. Instead, the slurry was used to conduct a high-solids filter test.

The high-solids filter test was conducted as part of TI-WTP-PEP-065 with results reported in the Integrated Test A run report (Guzman-Leong et al. 2009). Briefly, this test combined leached solids from Integrated Test A with those from Integrated Test B, and the combined slurry was further concentrated. The goal was to measure the permeate flux as a function of $\mathrm{wt} \%$ solids.

At the conclusion of the concentration, the filter-loop and Tank T02A were drained and flushed. 



\subsection{Data}

Tables summarizing the results of sample measurements taken during testing are provided in Section 5.1 (analytical results) and Section 5.2 (physical-property results). The analytical data are analyzed in Sections 9, 10, 11, and 12 of the Pretreatment Engineering Platform Phase 1 Final Test Report (Kurath et al. 2009). PJM peak average velocity and stroke length, tuned iteratively to target values during testing, are provided in Section 5.3. Selected data obtained from the DAS during Integrated Test B have been plotted in 12-hr intervals, and this is provided in Appendix J.

\subsection{Analytical Data}

This section provides analytical summary tables for key processes. Reporting limits, uncertainties, and special analytical notes are in the final analytical reports submitted by each analytical laboratory. These reports are identified for each sample in Appendix C, which provides a list of all samples collected, the disposition of each sample, and references to final electronic analytical reports.

In many cases, such as during caustic and oxidative leaching, the slurry samples taken from PEP were analyzed after phase separation was performed. The wet centrifuged solids and the supernatant liquid from the samples were analyzed separately. In other cases, data are presented in a more raw form: the species concentration in the supernatant, the concentration in the wet centrifuged solids, and the masses of tare sample vial, vial plus total slurry, and vial plus wet centrifuged solids or the calculated value of the $\mathrm{wt} \%$ centrifuged solids. The latter three masses can be used to calculate the mass fraction of wet centrifuged solids in the slurry, which in turn can be used in calculating the total species concentration in the original samples slurry. (See Mahoney et al. 2009, Section A.5 of Appendix A for more information on calculating the species concentration in the slurry.)

Table 5.1, Analysis at Key Process Steps, provides the concentration of key metal analytes, anions, total inorganic carbon (TIC), wt \% UDS, density, etc. at key process steps. Two separate slurry samples were collected for the wt $\%$ UDS and slurry density. The supernatant density is a higher accuracy density than can be provided by the UDS analysis. The concentrations of the metals and TIC in the slurry were calculated from samples phase-separated at PDL-W. The liquid analysis results are supernatant analytical results obtained from samples phase-separated in PDL-W. The $\mathrm{wt} \%$ of wet centrifuged solids in the slurry can be used in calculating the total species concentration in the original samples slurry. See Section A.5 in Appendix A of Mahoney et al. (2009) for more information on calculating the species concentration in the slurry. An aliquot of the wet centrifuged solids was dried at $105^{\circ} \mathrm{C}$ to determine the percent solids that are reported with the reported analytes in the wet centrifuged solids.

Table 5.2 and Table 5.3 provide the wt $\%$ UDS for slurry samples collected during the concentration of the first and second batch of slurry (respectively) before caustic leaching in Tank T02A.

Table 5.4 and Table 5.5 provide analytical results for samples collected during the Batch 1 and Batch 2 caustic leach in Tank T02A, respectively. The slurry density results were performed on the slurry sample submitted to the analytical laboratory. The wt $\%$ UDS, aluminum, iron, and strontium slurry concentrations were calculated from samples phase-separated at PDL-W. The liquid analysis results are 
analytical results obtained from the phase-separated supernatants. Raman results, shown in brackets [ ], are provided as part of the liquid analyses and are presented in molar concentration (mole/liter). 
Table 5.1. Analysis at Key Process Steps

\begin{tabular}{|c|c|c|c|c|c|c|c|c|}
\hline \multirow[b]{2}{*}{ Analyte } & \multicolumn{2}{|c|}{ Simulant Feed composition $^{(\mathrm{f})}$} & \multicolumn{2}{|c|}{$\begin{array}{c}\text { Post Concentration of } \\
\text { Solid Batch \#1 } \\
\end{array}$} & \multicolumn{2}{|c|}{$\begin{array}{c}\text { Post Concentration of } \\
\text { Solid Batch \#2 } \\
\end{array}$} & \multicolumn{2}{|c|}{$\begin{array}{l}\text { Initial Slurry Composition for } \\
\text { Post-Caustic-Leach Wash }\end{array}$} \\
\hline & $\begin{array}{c}\text { Slurry } \\
\mu \mathrm{g} / \mathrm{g}\end{array}$ & $\begin{array}{c}\text { Liquid Phase } \\
\mu \mathrm{g} / \mathrm{g}\end{array}$ & $\begin{array}{c}\text { wet centrifuged } \\
\text { solids } \\
\mu \mathrm{g} / \mathrm{g} \\
\end{array}$ & $\begin{array}{c}\text { Liquid Phase } \\
\mu \mathrm{g} / \mathrm{g}\end{array}$ & $\begin{array}{c}\text { wet centrifuged } \\
\text { solids } \\
\mu \mathrm{g} / \mathrm{g} \\
\end{array}$ & $\begin{array}{c}\text { Liquid Phase } \\
\mu \mathrm{g} / \mathrm{g}\end{array}$ & $\begin{array}{c}\text { wet centrifuged } \\
\text { solids } \\
\mu \mathrm{g} / \mathrm{g} \\
\end{array}$ & $\begin{array}{c}\text { Liquid Phase } \\
\mu \mathrm{g} / \mathrm{g}\end{array}$ \\
\hline $\mathrm{Al}$ & $18794 \pm 318^{(\mathrm{a})}$ & $3163 \pm 55$ & $130000 \pm 2534$ & $3317 \pm 116$ & $146000 \pm 5691$ & $3687 \pm 128$ & $97700 \pm 6600$ & $17600 \pm 1060$ \\
\hline $\mathrm{Ca}$ & $154.7 \pm 3.0$ & $\mathrm{n} / \mathrm{d}$ & $1447 \pm 32$ & $\mathrm{n} / \mathrm{d}$ & $1530 \pm 60$ & $\mathrm{n} / \mathrm{d}$ & $2280 \pm 154$ & $<1.24 \pm 0.62$ \\
\hline $\mathrm{Ce}$ & $51.6 \pm 1.0$ & $0.013 \pm 0.001$ & $528 \pm 10$ & $0.0205 \pm 0.0016$ & $561 \pm 22$ & $0.0317 \pm 0.0018$ & $822 \pm 56$ & $0.011 \pm 0.003$ \\
\hline $\mathrm{Cr}$ & $8.0 \pm 0.9$ & $1.42 \pm 0.04$ & $161 \pm 7$ & $1.8 \pm 0.1$ & $71 \pm 4$ & $1.9 \pm 0.1$ & $175 \pm 13$ & $6.43 \pm 0.41$ \\
\hline $\mathrm{Fe}$ & $4945 \pm 98$ & $\mathrm{n} / \mathrm{d}$ & $46267 \pm 1808$ & $\mathrm{n} / \mathrm{d}$ & $49200 \pm 1921$ & $5.8 \pm 1.4$ & $71600 \pm 4830$ & $10.70 \pm 2.55$ \\
\hline $\mathrm{K}$ & $999 \pm 17$ & $1063 \pm 19$ & $567 \pm 29$ & $986 \pm 36$ & $493 \pm 28$ & $1001 \pm 36$ & $272 \pm 39$ & $497 \pm 35$ \\
\hline $\mathrm{La}$ & $39.5 \pm 0.8$ & $0.012 \pm 0.001$ & $405 \pm 16$ & $0.018 \pm 0.002$ & $428 \pm 17$ & $0.0285 \pm 0.0017$ & $623 \pm 42$ & $0.0104 \pm 0.0026$ \\
\hline $\mathrm{Mg}$ & $94.9 \pm 1.8$ & $\mathrm{n} / \mathrm{d}$ & $955 \pm 42$ & $\mathrm{n} / \mathrm{d}$ & $997 \pm 39$ & $\mathrm{n} / \mathrm{d}$ & $1390 \pm 94$ & $<2.47 \pm 1.24$ \\
\hline $\mathrm{Mn}$ & $1058 \pm 21$ & $\mathrm{n} / \mathrm{d}$ & $9153 \pm 357$ & $0.164 \pm 0.036$ & $10167 \pm 396$ & $\mathrm{n} / \mathrm{d}$ & $15500 \pm 1050$ & $0.14 \pm 0.06$ \\
\hline $\mathrm{Na}$ & $90174 \pm 1400$ & $93233 \pm 1615$ & $66033 \pm 2589$ & $88433 \pm 3064$ & $66067 \pm 2596$ & $90733 \pm 3145$ & $115000 \pm 7780$ & $134000 \pm 8040$ \\
\hline $\mathrm{Nd}$ & $107.1 \pm 2.1$ & $0.031 \pm 0.001$ & $1097 \pm 43$ & $0.047 \pm 0.002$ & $1160 \pm 45$ & $0.076 \pm 0.003$ & $1700 \pm 115$ & $0.029 \pm 0.003$ \\
\hline $\mathrm{Ni}$ & $145.3 \pm 2.8$ & $\mathrm{n} / \mathrm{d}$ & $1567 \pm 61$ & $0.309 \pm 0.071$ & $1677 \pm 65$ & $0.47 \pm 0.07$ & $2370 \pm 160$ & $<0.247 \pm 0.124$ \\
\hline$P$ & $1581 \pm 25$ & $1643 \pm 29$ & $1123 \pm 44$ & $1677 \pm 58$ & $1050 \pm 41$ & $1677 \pm 58$ & $1680 \pm 113$ & $740 \pm 45$ \\
\hline $\mathrm{Si}^{(\mathrm{h})}$ & $106.5 \pm 8.0$ & $\mathrm{n} / \mathrm{d}$ & $685 \pm 38$ & $27.2 \pm 1.1$ & $959 \pm 47$ & $43.3 \pm 1.6$ & $771 \pm 72$ & $140 \pm 8$ \\
\hline $\mathrm{Sr}$ & $44.2 \pm 0.9$ & $0.120 \pm .017$ & $401 \pm 16$ & $\mathrm{n} / \mathrm{d}$ & $433 \pm 17$ & $\mathrm{n} / \mathrm{d}$ & $645 \pm 44$ & $<0.124 \pm 0.062$ \\
\hline $\mathrm{Zr}$ & $140.9 \pm 2.7$ & $0.726 \pm 0.021$ & $1467 \pm 57$ & $0.756 \pm 0.075$ & $1587 \pm 62$ & $1.075 \pm 0.080$ & $2190 \pm 148$ & $6.75 \pm 0.42$ \\
\hline $\mathrm{Cl}$ & - & $1013 \pm 18$ & - & $968 \pm 34$ & - & $961 \pm 34$ & - & $408 \pm 26$ \\
\hline $\mathrm{NO}_{2}$ & - & $17867 \pm 348$ & - & $17633 \pm 713$ & - & $17533 \pm 701$ & - & $8160 \pm 798$ \\
\hline $\mathrm{NO}_{3}$ & - & $75967 \pm 1333$ & - & $74267 \pm 2620$ & - & $73867 \pm 2602$ & - & $32000 \pm 2100$ \\
\hline $\mathrm{PO}_{4}$ & - & $4893 \pm 85$ & - & $4873 \pm 170$ & - & $4853 \pm 169$ & - & $2070 \pm 128$ \\
\hline $\mathrm{SO}_{4}$ & - & $13967 \pm 247$ & - & $13767 \pm 490$ & - & $13667 \pm 485$ & - & $6130 \pm 415$ \\
\hline $\mathrm{C}_{2} \mathrm{O}_{4}$ & - & $642 \pm 11$ & - & $684 \pm 24$ & - & $664 \pm 24$ & - & $69 \pm 10$ \\
\hline \% Solids & - & - & 62.10 & - & 65.99 & - & 63.12 & - \\
\hline Free $\mathrm{OH}(\mu \mathrm{g} / \mathrm{mL})$ & - & $12623 \pm 93^{(\mathrm{c})}$ & - & $1.73 E+04$ & - & $2.04 E+04$ & - & $9.44 \mathrm{E}+04$ \\
\hline $\mathrm{TIC}^{(b)}$ & $2023 \pm 90$ & $5577 \pm 107$ & - & - & - & - & - & - \\
\hline PDMS (mg/g) & $\mathrm{n} / \mathrm{d}^{(\mathrm{d}, \mathrm{e})}$ & $\mathrm{n} / \mathrm{d}^{(\mathrm{d})}$ & - & - & - & - & $\mathrm{n} / \mathrm{d}^{(\mathrm{d})}$ & $\mathrm{n} / \mathrm{d}^{(\mathrm{d})}$ \\
\hline PPG (mg/g) & $\mathrm{n} / \mathrm{d}^{(\mathrm{d}, \mathrm{e})}$ & $\mathrm{n} / \mathrm{d}^{(\mathrm{d})}$ & - & - & - & - & $\mathrm{n} / \mathrm{d}^{(\mathrm{d})}$ & $\mathrm{n} / \mathrm{d}^{(\mathrm{d})}$ \\
\hline Density $(\mathrm{g} / \mathrm{mL})$ & $1.276 \pm 0.006$ & $1.233 \pm 0.005$ & - & $1.234 \pm 0.011$ & - & $1.235 \pm 0.011$ & - & $1.328 \pm 0.021$ \\
\hline $\mathrm{Wt} \% \mathrm{H}_{2} \mathrm{O}$ & - & $73.4 \pm .3$ & - & - & - & - & $52.4 \pm 0.8$ & - \\
\hline Slurry wt $\%$ UDS & $5.20 \pm 0.02$ & - & $20.3 \pm 0.2$ & - & $21.8 \pm 0.2$ & - & $17.3 \pm 0.3$ & - \\
\hline Slurry Density $(\mathrm{g} / \mathrm{mL})$ & $1.276 \pm 0.006$ & - & $1.379 \pm 0.012$ & - & $1.408 \pm 0.013$ & - & $1.481 \pm 0.023$ & - \\
\hline $\begin{array}{c}\mathrm{Wt} \% \text { Centrifuged Solids } \\
(\%)\end{array}$ & 14.2 & - & 43.0 & - & 41.9 & - & 43.2 & - \\
\hline $\begin{array}{l}\text { (a) Fraction of solid-phase } \mathrm{A} \\
\text { (b) TIC = total inorganic cart } \\
\text { (c) Free OH is expressed in } \\
\text { (d) Single sample non-detect } \\
\text { (e) Analysis performed on w } \\
\text { (f) Uncertainty values report } \\
\text { throughout the rest of the tabl }\end{array}$ & $\begin{array}{l}1 \text { that is in gibbsite }=\mathrm{c} \\
\text { bon } \\
\mathrm{ng} / \mathrm{kg} \\
\text { with analysis results } \\
\text { et centrifuged solids } \\
\text { ed for the Simulant } \mathrm{F} \\
\text { le }\end{array}$ & Composition are 10 & $\begin{array}{l}\text { mit } \\
\text { stead of the } 2 \sigma \text { value }\end{array}$ & \begin{tabular}{l|l} 
& (g) Liqu \\
& (h) Si re \\
& n/d Trip \\
& - Anal \\
& SX Faile \\
orted & Bold nur
\end{tabular} & $\begin{array}{l}\text { phase data contains } \\
\text { lts from general ICP } \\
\text { ate samples with ana } \\
\text { is not required by Te } \\
\text { - the organic phase } \\
\text { ers are calculated val }\end{array}$ & $\begin{array}{l}\text { results from the dece } \\
\text { lysis rather than from } \\
\text { s results below metho } \\
\text { lan } \\
\text { ed to separate from th }\end{array}$ & $\begin{array}{l}\text { supernate. No rinss } \\
\text { ples taken to specifi } \\
\text { orting limit. } \\
\text { leous phase and no r }\end{array}$ & $\begin{array}{l}\text { sults are included. } \\
\text { track AFA. } \\
\text { s were obtained }\end{array}$ \\
\hline
\end{tabular}


Table 5.1 (contd)

\begin{tabular}{|c|c|c|c|c|c|c|c|c|c|}
\hline \multirow[b]{2}{*}{ Analyte } & \multicolumn{2}{|c|}{$\begin{array}{l}\text { Post-Caustic-Leach, Concentration, } \\
\text { and Wash Sample }\end{array}$} & \multicolumn{3}{|c|}{$\begin{array}{l}\text { Starting Slurry Composition Before } \\
\text { Permanganate Addition }\end{array}$} & \multicolumn{2}{|c|}{$\begin{array}{l}\text { Initial Slurry Composition for } \\
\text { Post-Oxidative-Leach Wash }\end{array}$} & \multicolumn{2}{|c|}{$\begin{array}{l}\text { Post-Oxidative-Leach, Concentration, and } \\
\text { Wash Sample }\end{array}$} \\
\hline & $\begin{array}{c}\text { wet centrifuged } \\
\text { solids } \\
\mu \mathrm{g} / \mathrm{g}\end{array}$ & $\begin{array}{c}\text { Liquid Phase } \\
\mu \mathrm{g} / \mathrm{g}\end{array}$ & $\begin{array}{c}\text { rinsed wet } \\
\text { centrifuged solids } \\
\mu \mathrm{g} / \mathrm{g}\end{array}$ & Liquic & $\begin{array}{l}\text { Phase }^{(g)} \\
\mathrm{g} / \mathrm{g}\end{array}$ & $\begin{array}{c}\text { rinsed wet } \\
\text { centrifuged solids } \\
\mu \mathrm{g} / \mathrm{g}\end{array}$ & $\begin{array}{c}\text { Liquid Phase }^{(\mathrm{g})} \\
\mu \mathrm{g} / \mathrm{g}\end{array}$ & $\begin{array}{c}\text { wet centrifuged } \\
\text { solids } \\
\mu \mathrm{g} / \mathrm{g}\end{array}$ & $\begin{array}{c}\text { Liquid Phase } \\
\mu \mathrm{g} / \mathrm{g}\end{array}$ \\
\hline $\mathrm{Al}$ & $130000 \pm 8780$ & $395 \pm 24$ & $107333 \pm 4185$ & & $6 \pm 5$ & $120000 \pm 5728$ & $165 \pm 6$ & - & $20.3 \pm 1.7$ \\
\hline $\mathrm{Ca}$ & $3460 \pm 234$ & $<1.24 \pm 0.62$ & $3207 \pm 125$ & & $\mathrm{l} / \mathrm{d}$ & $3460 \pm 165$ & $\mathrm{n} / \mathrm{d}$ & - & $<1.23 \pm 0.62$ \\
\hline $\mathrm{Ce}$ & $1090 \pm 74$ & $0.00878 \pm 0.00254$ & $1067 \pm 42$ & 0.0122 & \pm 0.0015 & $1155 \pm 55$ & $0.0115 \pm 0.0015$ & - & $<0.00491 \pm 0.00247$ \\
\hline $\mathrm{Cr}$ & $337 \pm 23$ & $<0.248 \pm 0.125$ & $18533 \pm 722$ & 11. & \pm 0.4 & $1700 \pm 81$ & $5550 \pm 193$ & - & $251 \pm 15$ \\
\hline $\mathrm{Fe}$ & $107000 \pm 7220$ & $<2.48 \pm 1.24$ & $93767 \pm 3656$ & & $\mathrm{a} / \mathrm{d}$ & $100500 \pm 4798$ & $4.24 \pm 0.74$ & - & $<2.45 \pm 1.23$ \\
\hline $\mathrm{K}$ & $89.5 \pm 13.2$ & $10.5 \pm 3.8$ & $57 \pm 7$ & & $\mathrm{a} / \mathrm{d}$ & $144 \pm 11$ & $33.8 \pm 2.5$ & - & $<7.36 \pm 6.38$ \\
\hline $\mathrm{La}$ & $819 \pm 55$ & $0.00691 \pm 0.00251$ & $806 \pm 31$ & 0.0108 & \pm 0.0015 & $874 \pm 42$ & $0.0115 \pm 0.0015$ & - & $<0.00491 \pm 0.00246$ \\
\hline $\mathrm{Mg}$ & $2090 \pm 141$ & $<2.48 \pm 1.24$ & $1917 \pm 75$ & & $\mathrm{l} / \mathrm{d}$ & $1945 \pm 93$ & $\mathrm{n} / \mathrm{d}$ & - & $<2.45 \pm 1.23$ \\
\hline $\mathrm{Mn}$ & $23200 \pm 1570$ & $0.157 \pm 0.063$ & $20667 \pm 805$ & 0.176 & $\pm \mathbf{0 . 0 3 0}$ & $41450 \pm 1976$ & $0.433 \pm 0.039$ & - & $<0.123 \pm 0.062$ \\
\hline $\mathrm{Na}$ & $12300 \pm 881$ & $7910 \pm 499$ & $11133 \pm 521$ & 841 & \pm 292 & $12850 \pm 647$ & $10300 \pm 368$ & - & $952 \pm 59$ \\
\hline $\mathrm{Nd}$ & $2260 \pm 153$ & $0.0191 \pm 0.0027$ & $2230 \pm 87$ & 0.0294 & \pm 0.0018 & $2415 \pm 115$ & $0.0321 \pm 0.0018$ & - & $0.0112 \pm 0.0026$ \\
\hline $\mathrm{Ni}$ & $3640 \pm 246$ & $<0.124 \pm 0.062$ & $3330 \pm 130$ & & $\mathrm{a} / \mathrm{d}$ & $3585 \pm 171$ & $0.559 \pm 0.053$ & - & $<0.123 \pm 0.062$ \\
\hline $\mathrm{P}$ & $759 \pm 51$ & $29.6 \pm 1.9$ & $544 \pm 27$ & 23. & \pm 0.8 & $551 \pm 26$ & $24.8 \pm 0.9$ & - & $5.67 \pm 0.70$ \\
\hline $\mathrm{Si}^{(\mathrm{h})}$ & $1830 \pm 128$ & $18.8 \pm 1.2$ & $1673 \pm 69$ & 23.3 & \pm 0.86 & $1770 \pm 89$ & $20.90 \pm 0.78$ & - & $8.44 \pm 0.71$ \\
\hline $\mathrm{Sr}$ & $968 \pm 66$ & $<0.124 \pm 0.062$ & $803 \pm 31$ & & $\mathrm{l} / \mathrm{d}$ & $855 \pm 41$ & $\mathrm{n} / \mathrm{d}$ & - & $<0.123 \pm 0.062$ \\
\hline $\mathrm{Zr}$ & $2960 \pm 200$ & $<0.124 \pm 0.062$ & $2740 \pm 107$ & & $\mathrm{a} / \mathrm{d}$ & $2950 \pm 141$ & $\mathrm{n} / \mathrm{d}$ & - & $<0.123 \pm 0.062$ \\
\hline $\mathrm{Cl}$ & - & $<19.6 \pm 9.8$ & - & 149. & $7 \pm 7.5$ & - & $152 \pm 8$ & - & $20.9 \pm 9.9$ \\
\hline $\mathrm{NO}_{2}$ & - & $188 \pm 34$ & - & & \pm 18 & - & $112 \pm 19$ & - & $<64.5 \pm 32.4$ \\
\hline $\mathrm{NO}_{3}$ & - & $689 \pm 60$ & - & 375 & \pm 132 & - & $3863 \pm 136$ & - & $244 \pm 46$ \\
\hline $\mathrm{PO}_{4}$ & - & $100 \pm 31$ & - & & \pm 17 & - & $96 \pm 81$ & - & $<60.0 \pm 30.1$ \\
\hline $\mathrm{SO}_{4}$ & - & $126 \pm 12$ & - & & \pm 6 & - & $153 \pm 8$ & - & $<19.6 \pm 9.9$ \\
\hline $\mathrm{C}_{2} \mathrm{O}_{4}$ & - & $9890 \pm 625$ & - & 151 & $7 \pm 53$ & - & $1573 \pm 55$ & - & $97.8 \pm 11.4$ \\
\hline$\%$ Solids & 55.66 & - & 52.11 & & - & 57.16 & - & - & - \\
\hline Free $\mathrm{OH}(\mu \mathrm{g} / \mathrm{mL})$ & - & $1.99 \mathrm{E}+03$ & - & 4.8 & $E+03$ & - & $2.61 E+03$ & - & $4.18 \mathrm{E}+02$ \\
\hline $\mathrm{TIC}^{(b)}$ & - & - & - & & - & - & - & - & - \\
\hline PDMS (mg/g) & 0.0044 & $\mathrm{n} / \mathrm{d}^{(\mathrm{d})}$ & 0.0077 & & $\mathrm{~d}^{(\mathrm{d})}$ & $3.8 \mathrm{E}-03$ & $5.0 \mathrm{E}-04$ & - & - \\
\hline PPG (mg/g) & 1.1 & SX Failed & 1 & & Failed & 1.1 & SX Failed & - & - \\
\hline Density $(\mathrm{g} / \mathrm{mL})$ & - & $1.009 \pm 0.019$ & - & 1.01 & \pm .009 & - & $1.026 \pm 0.009$ & - & $1.000 \pm 0.016$ \\
\hline $\mathrm{Wt} \% \mathrm{H}_{2} \mathrm{O}$ & - & - & - & & - & - & - & - & - \\
\hline Slurry wt $\%$ UDS & $20.5 \pm 0.3$ & - & $17.0 \pm 0.2$ & & - & $18.3 \pm 0.2$ & - & $20.2 \pm 0.3$ & - \\
\hline Slurry Density $(\mathrm{g} / \mathrm{mL})$ & $1.123 \pm 0.017$ & - & $1.071 \pm 0.010$ & & - & $1.144 \pm 0.010$ & - & $1.126 \pm 0.018$ & - \\
\hline $\begin{array}{c}\mathrm{Wt} \% \text { Centrifuged Solids } \\
(\%)\end{array}$ & 38.7 & - & 39.8 & & - & 32.9 & - & - & - \\
\hline $\begin{array}{l}\text { (a) Fraction of solid-phase } \mathrm{A} \\
\text { (b) TIC = total inorganic cart } \\
\text { (c) Free OH is expressed in } \\
\text { (d) Single sample non-detect } \\
\text { (e) Analysis performed on w } \\
\text { (f) Uncertainty values report } \\
\text { throughout the rest of the tabl }\end{array}$ & $\begin{array}{l}\text { that is in gibbsite }=0 \text {. } \\
\text { on } \\
\mathrm{g} / \mathrm{kg} \\
\text { with analysis results } \\
\text { t centrifuged solids } \\
\mathrm{d} \text { for the Simulant } \mathrm{Fe} \\
\end{array}$ & $\begin{array}{l}\text { low method reporting } 1 \\
\text { d Composition are } 1 \sigma, \text { i }\end{array}$ & tead of the $2 \sigma$ value & oorted & $\begin{array}{l}\text { (g) Liqu } \\
\text { (h) Si re } \\
\text { n/d Trip } \\
\text { - Anal } \\
\text { SX Faile } \\
\text { Bold nur }\end{array}$ & $\begin{array}{l}\text { phase data contains on } \\
\text { llts from general ICP a } \\
\text { cate samples with analy } \\
\text { is not required by Test } \\
\text { - the organic phase fa } \\
\text { bers are calculated valu }\end{array}$ & $\begin{array}{l}\text { results from the deca } \\
\text { ysis rather than from } \\
\text { results below metho } \\
\text { an } \\
\text { d to separate from th }\end{array}$ & $\begin{array}{l}\text { ted supernate. No rinsat } \\
\text { aples taken to specific } \\
\text { reporting limit. } \\
\text { aqueous phase and no re }\end{array}$ & $\begin{array}{l}\text { results are included. } \\
\text { ly track AFA. } \\
\text { llts were obtained }\end{array}$ \\
\hline
\end{tabular}


Table 5.2. Batch 1: Initial Solids Concentration

\begin{tabular}{cc}
\hline Sample location: & \\
Tank T02A & Wt\% UDS (slurry) \\
\hline Prior to Concentration & 5.98 \\
After Batch 7 & 6.56 \\
After Batch 10 & 7.66 \\
After Batch 13 & 8.21 \\
After Batch 16 & 8.74 \\
After Batch 19 & 9.31 \\
After Batch 22 & 10.0 \\
After Batch 25 & 10.8 \\
After Batch 28 & 11.5 \\
After Batch 31 & 12.5 \\
After Batch 34 & 12.9 \\
After Batch 37 & 13.7 \\
After Batch 40 & 14.4 \\
After Batch 43 & 15.0 \\
After Batch 46 & 15.8 \\
After Concentration & (a) \\
After Caustic Addition & $\mathbf{2 0 . 3}$ \\
and Filter Flush & $\mathbf{6 . 9 3}$ \\
\hline Bod
\end{tabular}

Bold numbers are calculated values.

(a) Analytical results duplicated in Analysis at Key Process Steps table in column Post Concentration of Solid Batch \#1.

Table 5.3. Batch 2: Initial Solids Concentration

\begin{tabular}{|c|c|}
\hline $\begin{array}{c}\text { Sample location: } \\
\text { Tank T02A }\end{array}$ & $\mathrm{Wt} \%$ UDS (slurry) \\
\hline Prior to Concentration & 4.92 \\
\hline After Batch 3 & 6.03 \\
\hline After Batch 6 & 6.72 \\
\hline After Batch 9 & 7.46 \\
\hline After Batch 12 & 8.21 \\
\hline After Batch 15 & 8.88 \\
\hline After Batch 18 & 9.64 \\
\hline After Batch 21 & 10.4 \\
\hline After Batch 24 & 11.2 \\
\hline After Batch 27 & 11.8 \\
\hline After Batch 30 & 12.6 \\
\hline After Batch 33 & 13.2 \\
\hline After Batch 36 & 14.0 \\
\hline After Batch 39 & 14.8 \\
\hline After Batch 42 & 15.5 \\
\hline After Batch 45 & 16.2 \\
\hline After Batch 47 & 16.6 \\
\hline After Concentration ${ }^{(a)}$ & 21.8 \\
\hline $\begin{array}{c}\text { After Caustic Addition and } \\
\text { Filter Flush }\end{array}$ & 8.37 \\
\hline
\end{tabular}

Bold numbers are calculated values.

(a) Analytical results duplicated in Analysis at Key Process Steps table in column Post Concentration of Solid Batch \#2. 
Table 5.4. Caustic-Leach-Batch 1

\begin{tabular}{|c|c|c|c|c|c|c|c|c|c|c|c|c|c|}
\hline \multirow[b]{2}{*}{$\begin{array}{l}\text { Sample location: } \\
\text { Tank T02A, } \\
\text { Condition: }\end{array}$} & \multicolumn{5}{|c|}{ Slurry Analyses } & \multicolumn{8}{|c|}{ Liquid Analyses } \\
\hline & $\begin{array}{l}\text { UDS } \\
\mathrm{Wt} \% \\
\end{array}$ & $\begin{array}{c}\text { Slurry } \\
\text { Density } \\
\mathrm{g} / \mathrm{mL} \\
\end{array}$ & $\begin{array}{r}\mathrm{Al} \\
\mu \mathrm{g} / \mathrm{g} \\
\end{array}$ & $\begin{array}{r}\mathrm{Fe} \\
\mu \mathrm{g} / \mathrm{g} \\
\end{array}$ & $\begin{array}{r}\mathrm{Sr} \\
\mu \mathrm{g} / \mathrm{g} \\
\end{array}$ & $\begin{array}{l}\text { Liquid } \\
\text { Density } \\
\mathrm{g} / \mathrm{mL} \\
\end{array}$ & $\begin{array}{c}\mathrm{Al} \\
{\left[\mathrm{Al}(\mathrm{OH})_{4}^{-}\right.} \\
\text {by } \mathrm{Raman}] \\
\mu \mathrm{g} / \mathrm{g} \\
{[\mathrm{M}]}\end{array}$ & $\begin{array}{c}\text { Free OH- } \\
\text { [Raman] } \\
\mathrm{M} \\
{[\mathrm{M}]} \\
\end{array}$ & $\begin{array}{c}\mathrm{C}_{2} \mathrm{O}_{4} \\
{[\mathrm{Raman}]} \\
\mu \mathrm{g} / \mathrm{g} \\
{[\mathrm{M}]} \\
\end{array}$ & $\begin{array}{r}\mathrm{Cl}- \\
\mu \mathrm{g} / \mathrm{g} \\
\end{array}$ & $\begin{array}{c}\mathrm{NO}_{3} \\
\text { [Raman] } \\
\mu \mathrm{g} / \mathrm{g} \\
{[\mathrm{M}]} \\
\end{array}$ & $\begin{array}{c}\mathrm{NO}_{2} \\
{[\mathrm{Raman}]} \\
\mu \mathrm{g} / \mathrm{g} \\
{[\mathrm{M}]} \\
\end{array}$ & $\begin{array}{c}\mathrm{SO}_{4} \\
\text { [Raman] } \\
\mu \mathrm{g} / \mathrm{g} \\
{[\mathrm{M}]} \\
\end{array}$ \\
\hline $\begin{array}{l}\text { After } \mathrm{NaOH} \\
\text { addition }\end{array}$ & 6.93 & 1.445 & 29696 & 9690 & 87.1 & 1.382 & $\begin{array}{c}13733 \\
{[0.596]}\end{array}$ & $\begin{array}{c}7.69 \\
{[7.87]}\end{array}$ & $\begin{array}{l}29.7 \\
{[\mathrm{n} / \mathrm{d}]}\end{array}$ & 443 & $\begin{array}{c}37100 \\
{[0.918]}\end{array}$ & $\begin{array}{c}9290 \\
{[0.312]}\end{array}$ & $\begin{array}{c}2737 \\
{[0.038]}\end{array}$ \\
\hline $88^{\circ} \mathrm{C}$ & 6.44 & 1.419 & - & - & - & 1.366 & 13100 & 7.00 & 35.1 & 423 & 35200 & 8890 & 4160 \\
\hline $98^{\circ} \mathrm{C}-$ hour 0 & 5.60 & 1.408 & 28867 & 8745 & 78.2 & 1.347 & $\begin{array}{c}12600 \\
{[0.565]}\end{array}$ & $\begin{array}{c}6.56 \\
{[6.820]}\end{array}$ & $\begin{array}{l}37.2 \\
{[\mathrm{n} / \mathrm{d}]}\end{array}$ & 403 & $\begin{array}{c}33533 \\
{[0.829]}\end{array}$ & $\begin{array}{c}8443 \\
{[0.268]}\end{array}$ & $\begin{array}{c}6393 \\
{[0.093]}\end{array}$ \\
\hline $98^{\circ} \mathrm{C}-$ hour 1 & 5.69 & 1.411 & - & - & - & 1.358 & 12800 & 6.76 & 45.4 & 407 & 34000 & 8600 & 6580 \\
\hline $98^{\circ} \mathrm{C}-$ hour 2 & 5.52 & 1.397 & - & - & - & 1.349 & 13200 & 6.51 & 41.0 & 398 & 33200 & 8400 & 6480 \\
\hline $98^{\circ} \mathrm{C}-$ hour 4 & 5.31 & 1.390 & 26770 & 8737 & 78.3 & 1.351 & 13900 & 6.34 & 43.7 & 396 & 32800 & 8310 & 6450 \\
\hline $98^{\circ} \mathrm{C}-$ hour 8 & 5.12 & 1.383 & 26005 & 8532 & 76.2 & 1.350 & 14700 & 6.05 & 51.9 & 387 & 31800 & 8020 & 6160 \\
\hline $98^{\circ} \mathrm{C}-$ hour 10 & 5.07 & 1.377 & - & - & - & 1.343 & 15500 & 5.78 & 52.2 & 373 & 31200 & 7890 & 6070 \\
\hline $\begin{array}{l}98^{\circ} \mathrm{C}- \\
\text { hour } 12\end{array}$ & 4.84 & 1.372 & 25558 & 8232 & 74.5 & 1.337 & $\begin{array}{c}15867 \\
{[0.673]}\end{array}$ & $\begin{array}{c}5.79 \\
{[5.904]}\end{array}$ & $\begin{array}{l}53.3 \\
{[\mathrm{n} / \mathrm{d}]}\end{array}$ & 371 & $\begin{array}{c}31133 \\
{[0.732]}\end{array}$ & $\begin{array}{c}7827 \\
{[0.236]}\end{array}$ & $\begin{array}{c}5940 \\
{[0.084]}\end{array}$ \\
\hline $98^{\circ} \mathrm{C}$ - hour 14 & 4.13 & 1.371 & - & - & - & 1.324 & 16000 & 5.52 & 63.8 & 360 & 30100 & 7690 & 5770 \\
\hline $98^{\circ} \mathrm{C}-$ hour 16 & 4.50 & 1.359 & 24758 & 7897 & 71.4 & 1.318 & $\begin{array}{c}16500 \\
{[0.707]}\end{array}$ & $\begin{array}{c}5.4 \\
{[5.607]}\end{array}$ & $\begin{array}{l}64.5 \\
{[\mathrm{n} / \mathrm{d}]}\end{array}$ & 367 & $\begin{array}{c}30000 \\
{[0.711]}\end{array}$ & $\begin{array}{c}7623 \\
{[0.231]}\end{array}$ & $\begin{array}{c}5823 \\
{[0.082]}\end{array}$ \\
\hline $\begin{array}{c}\text { After cool down } \\
\text { to } 25^{\circ}\end{array}$ & 4.15 & 1.366 & 25523 & 8265 & 74.3 & 1.337 & 17200 & 5.51 & 59.3 & 378 & 31300 & 7860 & 6150 \\
\hline
\end{tabular}

[Raman] results are provided as bracketed values [].

M Molarity

$\mathrm{Ca}, \mathrm{Mg}$, and $\mathrm{Nd}$ are not provided in this table because they were not used as tracers in the caustic leach data analysis.

Bold numbers are calculated values.

- Analysis not required by Test Plan.

$\mathrm{n} / \mathrm{d}$ Triplicate samples with analysis results below method reporting limit. 
Table 5.5. Caustic-Leach-Batch 2

\begin{tabular}{|c|c|c|c|c|c|c|c|c|c|c|c|c|c|}
\hline \multirow[b]{2}{*}{$\begin{array}{c}\text { Sample location: } \\
\text { Tank T02A, } \\
\text { Condition: }\end{array}$} & \multicolumn{5}{|c|}{ Slurry Analyses } & \multicolumn{8}{|c|}{ Liquid Analyses } \\
\hline & $\begin{array}{l}\text { UDS } \\
\mathrm{Wt} \%\end{array}$ & $\begin{array}{l}\text { Slurry } \\
\text { Density } \\
\text { g/mL }\end{array}$ & $\begin{array}{c}\mathrm{Al} \\
\mu \mathrm{g} / \mathrm{g}\end{array}$ & $\begin{array}{r}\mathrm{Fe} \\
\mu \mathrm{g} / \mathrm{g} \\
\end{array}$ & $\begin{array}{r}\mathrm{Sr} \\
\mu \mathrm{g} / \mathrm{g} \\
\end{array}$ & \begin{tabular}{|l} 
\\
Liquid \\
Density \\
$\mathrm{g} / \mathrm{mL}$
\end{tabular} & $\begin{array}{l}\mathrm{Al} \\
{\left[\mathrm{Al}(\mathrm{OH})_{4}^{-} \text {by }\right.} \\
\text { Raman }] \\
\mu \mathrm{g} / \mathrm{g} \\
{[\mathrm{M}]}\end{array}$ & $\begin{array}{c}\text { Free OH- } \\
\text { [Raman] } \\
\mathrm{M} \\
{[\mathrm{M}]}\end{array}$ & $\begin{array}{c}\mathrm{C}_{2} \mathrm{O}_{4} \\
{[\mathrm{Raman}]} \\
\mu \mathrm{g} / \mathrm{g} \\
{[\mathrm{M}]}\end{array}$ & $\begin{array}{l}\mathrm{Cl}- \\
\mu \mathrm{g} / \mathrm{g} \\
\end{array}$ & $\begin{array}{c}\mathrm{NO}_{3} \\
{[\mathrm{Raman}]} \\
\mu \mathrm{g} / \mathrm{g} \\
{[\mathrm{M}]}\end{array}$ & $\begin{array}{c}\mathrm{NO}_{2} \\
\text { [Raman] } \\
\mu \mathrm{g} / \mathrm{g} \\
{[\mathrm{M}]}\end{array}$ & $\begin{array}{c}\mathrm{SO}_{4} \\
\text { [Raman] } \\
\mu \mathrm{g} / \mathrm{g} \\
{[\mathrm{M}]} \\
\end{array}$ \\
\hline $\begin{array}{c}\text { After } \mathrm{NaOH} \\
\text { addition }\end{array}$ & 8.37 & 1.441 & 35727 & 10662 & 98.6 & 1.377 & $\begin{array}{c}16400 \\
{[0.677]}\end{array}$ & $\begin{array}{c}7.15 \\
{[7.456]}\end{array}$ & $\begin{array}{l}25.0 \\
{[\mathrm{n} / \mathrm{d}]}\end{array}$ & 476 & $\begin{array}{c}38667 \\
{[0.969]}\end{array}$ & $\begin{array}{c}9620 \\
{[0.323]}\end{array}$ & $\begin{array}{c}3230 \\
{[0.047]}\end{array}$ \\
\hline $88^{\circ} \mathrm{C}$ & 7.37 & 1.420 & 33809 & 10456 & 93.0 & 1.367 & 16700 & 6.30 & 39.6 & 443 & 36100 & 8990 & 4870 \\
\hline $98^{\circ} \mathrm{C}-$ hour 0 & 6.68 & 1.408 & 32398 & 10078 & 89.8 & 1.355 & $\begin{array}{c}16500 \\
{[0.635]}\end{array}$ & $\begin{array}{c}6.35 \\
{[6.457]}\end{array}$ & $\begin{array}{l}36.2 \\
{[\mathrm{n} / \mathrm{d}]}\end{array}$ & 428 & $\begin{array}{c}34600 \\
{[0.851]}\end{array}$ & $\begin{array}{c}8713 \\
{[0.268]}\end{array}$ & $\begin{array}{c}6950 \\
{[0.099]}\end{array}$ \\
\hline $98^{\circ} \mathrm{C}-$ hour 1 & 6.77 & 1.399 & 32436 & 10307 & 92.4 & 1.354 & 16600 & 6.33 & 34.7 & 416 & 34000 & 8540 & 6700 \\
\hline $98^{\circ} \mathrm{C}-$ hour 2 & 6.41 & 1.398 & 32105 & 10038 & 89.6 & 1.346 & 17000 & 6.19 & 34.0 & 416 & 33800 & 8460 & 6730 \\
\hline $98^{\circ} \mathrm{C}$ - hour 4 & 6.34 & 1.395 & 32374 & 9458 & 86.8 & 1.345 & 17800 & 5.97 & 32.9 & 405 & 33300 & 8400 & 6590 \\
\hline $98^{\circ} \mathrm{C}-$ hour 8 & 5.93 & 1.390 & 32536 & 9261 & 85.7 & 1.339 & 19000 & 5.71 & 40.7 & 407 & 32700 & 8190 & 6440 \\
\hline $98^{\circ} \mathrm{C}-$ hour 10 & 5.74 & 1.386 & 31008 & 9416 & 86.0 & 1.339 & 19400 & 5.62 & 42.5 & 398 & 32200 & 8170 & 6560 \\
\hline $98^{\circ} \mathrm{C}-$ hour 12 & 5.75 & 1.360 & 31009 & 9245 & 84.2 & 1.338 & 19067 & 5.46 & 49.0 & 388 & 31333 & 7967 & 6197 \\
\hline $98^{\circ} \mathrm{C}-$ hour 14 & 5.65 & 1.358 & 31099 & 9499 & 84.7 & 1.338 & 20200 & 5.36 & 42.3 & 383 & 30600 & 7780 & 6110 \\
\hline $98^{\circ} \mathrm{C}$ - hour 16 & 5.64 & 1.372 & 30924 & 8867 & 80.7 & 1.330 & $\begin{array}{c}20433 \\
{[0.758]}\end{array}$ & $\begin{array}{c}5.25 \\
{[5.448]}\end{array}$ & $\begin{array}{l}50.0 \\
{[\mathrm{n} / \mathrm{d}]}\end{array}$ & 379 & $\begin{array}{c}30700 \\
{[0.732]}\end{array}$ & $\begin{array}{c}7776 \\
{[0.237]}\end{array}$ & $\begin{array}{c}6167 \\
{[0.087]}\end{array}$ \\
\hline $\begin{array}{l}\text { After cool down } \\
\text { to } 25^{\circ}\end{array}$ & 5.32 & 1.388 & 32296 & 8995 & 83.0 & 1.340 & 21400 & 5.41 & 35.2 & 395 & 31500 & 7990 & 6300 \\
\hline \multicolumn{14}{|c|}{$\begin{array}{l}\text { [Raman] results are provided as bracketed values []. } \\
\mathrm{M} \mathrm{Molarity.} \\
\mathrm{Ca}, \mathrm{Mg} \text {, and Nd are not provided in this table because they were not used as tracers in the caustic leach data analysis. } \\
\text { Bold numbers are calculated values. } \\
\text { - Analysis not required by Test Plan. }\end{array}$} \\
\hline
\end{tabular}


Table 5.6 provides analytical results for samples collected during solids concentration of the caustic-leached slurry in Tank T02A. This concentration combined the leached solids from Batch 1 and 2 as described in Section 4. The wt $\%$ UDS and densities were obtained following analytical methods described in Appendix E. The Si data presented are from wet centrifuged solids. The $\mathrm{wt} \%$ of wet centrifuged solids in the slurry can be used in calculating the total species concentration in the original samples slurry. See Section A.5 in Appendix A of Mahoney et al. (2009) for more information on calculating the species concentration in the slurry. An aliquot of the wet centrifuged solids was dried at $105^{\circ} \mathrm{C}$ to determine the percent solids that are reported with the reported analytes in the wet centrifuged solids.

Table 5.6. Post-Caustic-Leach Concentration of Solids

\begin{tabular}{|c|c|c|c|c|}
\hline \multirow[b]{2}{*}{$\begin{array}{c}\text { Sample location: } \\
\text { Tank T02A }\end{array}$} & \multicolumn{3}{|c|}{ Slurry } & \multirow{2}{*}{$\begin{array}{l}\text { Liquid } \\
\text { Density } \\
\mathrm{g} / \mathrm{mL}\end{array}$} \\
\hline & $\begin{array}{l}\text { UDS } \\
\mathrm{Wt} \%\end{array}$ & $\begin{array}{l}\text { Density } \\
\mathrm{g} / \mathrm{mL}\end{array}$ & $\begin{array}{c}\mathrm{Si} \\
\mu \mathrm{g} / \mathrm{g}\end{array}$ & \\
\hline $\begin{array}{c}\text { Prior to } \\
\text { Concentration }\end{array}$ & 5.09 & 1.359 & - & 1.267 \\
\hline After Batch 2 & 5.73 & 1.359 & - & 1.264 \\
\hline After Batch 4 & 6.09 & 1.349 & - & 1.267 \\
\hline After Batch 6 & 6.12 & 1.361 & - & 1.269 \\
\hline After Batch 8 & 7.06 & 1.380 & 一 & 1.278 \\
\hline After Batch 10 & 7.03 & 1.406 & — & 1.303 \\
\hline After Batch 12 & 7.84 & 1.392 & - & 1.272 \\
\hline After Batch 14 & 7.69 & 1.417 & - & 1.299 \\
\hline After Batch 16 & 7.85 & 1.424 & - & 1.283 \\
\hline After Batch 18 & 7.65 & 1.408 & - & 1.291 \\
\hline After Batch 23 & 10.31 & 1.413 & - & 1.279 \\
\hline After Concentration & 17.3 & 1.481 & $815^{(\mathrm{a})}$ & 1.328 \\
\hline \multicolumn{5}{|c|}{$\begin{array}{l}\text { (a) Wet centrifuged solids. Slurry sample was phase separated at SwRI with the following } \\
\text { information reported: percent total solids in the wet centrifuged solids was } 64.09 \% \text {; liquic } \\
\text { fraction of slurry sample had } 144-\mu \mathrm{g} / \mathrm{g} \text { Si. } \\
\text { - Analysis not required by Test Plan. }\end{array}$} \\
\hline
\end{tabular}

Table 5.7 provides the Cs concentration measured during the filter-loop bypass test (see Section 4 for description). Samples were taken from Tank T02A (outer lower port on the CD sampler) and the filter-loop in-line sample station. The test was meant to evaluate if the slurry was mixing well within Tank T02A at low levels and high-solids loading in the slurry.

Table 5.7. Filter-Loop Bypass Test

\begin{tabular}{ccc}
\hline & \multicolumn{2}{c}{ Liquid Analyses } \\
\cline { 2 - 3 } Condition & $\mu \mathrm{gg} / \mathrm{g}$ & $\mu \mathrm{g} / \mathrm{g}$ \\
\hline Before tracer addition & 0.00580 & - \\
1 min after tracer addition & 126 & 62.2 \\
2 min after tracer addition & 145 & 94.6 \\
4 min after tracer addition & 108 & 107 \\
8 min after tracer addition & 104 & 101 \\
16 min after tracer addition & 105 & 103 \\
30 min after tracer addition & 101 & 103 \\
40 min after tracer addition & 98.4 & 101 \\
50 min after tracer addition & 98.5 & 99.1 \\
60 min after tracer addition & 96.9 & 96.0 \\
\hline - Analysis not required by Test Plan. \\
\hline
\end{tabular}


Table 5.8 provides analytical results for samples collected during the post-caustic-leach washing in Tank T02A. Two separate slurry samples were collected for the wt $\%$ UDS and slurry density. The supernatant density is a higher accuracy density than can be provided by the UDS analysis. The silicon slurry concentrations were obtained by direct analysis of the slurry. The liquid analysis results are supernatant analytical results obtained from samples phase-separated in PDL-W. Raman results, shown in brackets [ ], are provided as part of the liquid analyses and presented in molar concentration (mole/liter). The $\mathrm{wt} \%$ of wet centrifuged solids in the slurry can be used in calculating the total species concentration in the original samples slurry. See Section A.5 in Appendix A of Mahoney et al. (2009) for more information on calculating the species concentration in the slurry. An aliquot of the wet centrifuged solids was dried at $105^{\circ} \mathrm{C}$ to determine the percent solids that are reported with the reported analytes in the wet centrifuged solids.

Table 5.9 provides analytical results for samples collected during the oxidative leaching in Tank T02A. The slurry density results and silicon slurry concentrations were performed on intact slurry submitted to the analytical laboratory. The wt $\%$ UDS were calculated from samples phase-separated at PDL-W. The liquid analysis results are supernatant analytical results obtained from samples phase-separated at PDL-W. The solids analyses results are analytical results of rinsed solids obtained from sample phase separation and rinsing at PDL-W. The wet centrifuged solids were immediately rinsed after phase separation to remove the remaining supernatant from the wet solids in an attempt to interrupt further reaction. The rinsate results are analytical results of composited rinse solutions used during the rinsing of phase-separated solids. Raman results, shown in brackets, [ ], are provided as part of the liquid analyses and presented in molar concentration (mole/liter). 
Table 5.8. Post-Caustic-Leach Wash

\begin{tabular}{|c|c|c|c|c|c|c|c|c|c|c|c|c|c|c|}
\hline \multirow{4}{*}{$\begin{array}{c}\text { Sample location: } \\
\text { Tank T02A } \\
\end{array}$} & \multicolumn{2}{|c|}{ Slurry Analyses } & \multicolumn{12}{|c|}{ Supernatant Analyses } \\
\hline & UDS & $\underset{\text { (a) }}{\text { Density }}$ & $\operatorname{TDS}^{(\mathrm{c})}$ & $\begin{array}{l}\text { Liquid } \\
\text { Density } \\
\text { (a) }\end{array}$ & $\begin{array}{c}\mathrm{Al} \\
{[\mathrm{Al}(\mathrm{OH}) 4-} \\
\text { by Raman] }\end{array}$ & $\begin{array}{l}\text { Free OH- } \\
\text { [Raman] }\end{array}$ & $\mathrm{Na}$ & $\begin{array}{c}\mathrm{C}_{2} \mathrm{O}_{4} \\
{[\mathrm{Raman}]}\end{array}$ & $\begin{array}{c}\mathrm{NO}_{3} \\
{[\mathrm{Raman}]}\end{array}$ & $\begin{array}{c}\mathrm{NO}_{2} \\
{[\mathrm{Raman}]}\end{array}$ & $\begin{array}{c}\mathrm{SO}_{4} \\
{[\mathrm{Raman}]}\end{array}$ & $\begin{array}{c}\mathrm{PO}_{4} \\
{[\mathrm{Raman}]}\end{array}$ & PDMS & PPG \\
\hline & & & & & $\mu \mathrm{g} / \mathrm{g}$ & M & & $\mu \mathrm{g} / \mathrm{g}$ & $\mu \mathrm{g} / \mathrm{g}$ & $\mu \mathrm{g} / \mathrm{g}$ & $\mu \mathrm{g} / \mathrm{g}$ & $\mu \mathrm{g} / \mathrm{g}$ & & \\
\hline & $\mathrm{Wt} \%$ & $\mathrm{~g} / \mathrm{mL}$ & Wt $\%$ & $\mathrm{~g} / \mathrm{mL}$ & {$[\mathrm{M}]$} & {$[\mathrm{M}]$} & $\mu \mathrm{g} / \mathrm{g}$ & {$[\mathrm{M}]$} & {$[\mathrm{M}]$} & {$[\mathrm{M}]$} & {$[\mathrm{M}]$} & {$[\mathrm{M}]$} & $\mathrm{mg} / \mathrm{g}$ & $\mathrm{mg} / \mathrm{g}$ \\
\hline After Wash 3 & - & - & 28.4 & 1.256 & $\begin{array}{c}14600 \\
{[0.616]}\end{array}$ & $\begin{array}{c}4.27 \\
{[4.519]}\end{array}$ & 117000 & $\begin{array}{c}149 \\
{[<0.01]}\end{array}$ & $\begin{array}{c}26900 \\
{[0.604]}\end{array}$ & $\begin{array}{c}7260 \\
{[0.205]}\end{array}$ & $\begin{array}{c}5190 \\
{[0.070]}\end{array}$ & $\begin{array}{c}999 \\
{[0.017]^{(\mathrm{d})}}\end{array}$ & - & - \\
\hline After Wash 6 & 17.8 & 1.339 & 25.0 & 1.224 & 11800 & 3.41 & 92900 & 335 & 21900 & 5970 & 4210 & 1570 & - & - \\
\hline After Wash 9 & - & - & 19.6 & 1.173 & 9610 & 2.81 & 77900 & 628 & 18000 & 4800 & 3490 & 2330 & - & - \\
\hline After Wash 12 & 19.8 & 1.300 & 16.7 & 1.148 & 7690 & 2.19 & 61100 & 1060 & 15900 & 3850 & 2850 & 1900 & - & - \\
\hline After Wash 15 & - & - & 13.2 & 1.108 & 6390 & 1.69 & 51600 & 1660 & 12700 & 2980 & 2250 & 1520 & - & - \\
\hline After Wash 18 & 20.8 & 1.220 & 11.1 & 1.095 & 5010 & 1.28 & 40700 & 2510 & 10100 & 2340 & 1790 & 1260 & - & - \\
\hline After Wash 21 & - & - & 9.02 & 1.067 & 4140 & 1.06 & 34400 & 3430 & 8180 & 1890 & 1460 & 1030 & - & - \\
\hline After Wash 24 & 20.7 & 1.199 & 7.57 & 1.063 & 3170 & 0.79 & 27200 & 4630 & 6290 & 1470 & 1150 & 807 & - & - \\
\hline After Wash 27 & - & - & 6.42 & 1.031 & 2550 & 0.64 & 23100 & 6610 & 4920 & 1150 & 910 & 648 & - & - \\
\hline After Wash 30 & 20.4 & 1.176 & 5.62 & 1.045 & $\begin{array}{c}1980 \\
{[0.071]}\end{array}$ & $\begin{array}{c}0.5 \\
{[0.536]^{(\mathrm{d})}}\end{array}$ & 19000 & $\begin{array}{c}7680 \\
{[0.076]}\end{array}$ & $\begin{array}{c}3730 \\
{[0.074]}\end{array}$ & $\begin{array}{c}889 \\
{[0.040]^{(\mathrm{d})}}\end{array}$ & $\begin{array}{c}695 \\
{[0.008]^{(\mathrm{d})}}\end{array}$ & $\begin{array}{c}496 \\
{[<0.01]}\end{array}$ & - & - \\
\hline After Wash 33 & - & - & 4.97 & 1.022 & 1700 & 0.41 & 17900 & 8940 & 3010 & 722 & 572 & 394 & - & - \\
\hline After Wash 36 & 19.9 & 1.129 & 4.56 & 1.04 & 1270 & 0.32 & 15100 & 10900 & 2230 & 544 & 422 & 315 & - & - \\
\hline After Wash 39 & - & - & 4.08 & 1.017 & 1050 & 0.25 & 14500 & 12300 & 1790 & 450 & 338 & 254 & - & - \\
\hline After Wash 42 & 18.9 & 1.139 & 4.01 & 1.034 & 798 & 0.20 & 13000 & 15500 & 1390 & 354 & 265 & 203 & - & - \\
\hline After Wash 45 & - & - & 3.85 & 1.014 & 671 & 0.15 & 13300 & 16100 & 1120 & 291 & 206 & 173 & - & - \\
\hline After Wash 48 & 18.5 & 1.121 & 3.13 & 1.022 & 525 & 0.14 & 10600 & 13200 & 879 & 237 & 165 & 130 & - & - \\
\hline After Wash 51 & - & - & 2.48 & 1.007 & 430 & 0.12 & 8570 & 10700 & 728 & 197 & 135 & 107 & - & - \\
\hline After Final Wash ${ }^{(\mathrm{e})}$ & 20.5 & 1.123 & 2.36 & 1.009 & 395 & 0.11 & 7910 & 9890 & 689 & 188 & 126 & 100 & $\mathrm{n} / \mathrm{d}^{(\mathrm{b})}$ & Failed \\
\hline $\begin{array}{l}\text { (a) If high-accuracy d } \\
\text { (b) Single sample nor } \\
\text { (c) TDS results deter } \\
\text { (d) Detected result is } \\
\text { (e) Analytical results } \\
\text { - Analysis not requi } \\
\text { [Raman] results are pr } \\
\text { M-Molarity. } \\
\text { Failed - the organic }\end{array}$ & $\begin{array}{l}\text { in } \\
\text { t Alan. } \\
\text { bracke }\end{array}$ & $\begin{array}{l}\text { then they } \\
\text { below } \\
\text { when ave } \\
\text { it }<\text { EQL } \\
\text { y Proces } \\
\text { ]. }\end{array}$ & Steps tab & ble column & $\begin{array}{l}\text { ion limit). } \\
\text { heading Initi }\end{array}$ & ial Slurry Co & omposi & for Pos & austic-L & h Wash. & UDS & & & \\
\hline
\end{tabular}


Table 5.8 (contd)

\begin{tabular}{|c|c|c|c|c|c|c|c|}
\hline \multirow[b]{2}{*}{ Sample location: Tank T02A } & \multicolumn{7}{|c|}{ Wet Centrifuged Solids $^{(\mathrm{f})}$} \\
\hline & $\begin{array}{c}\text { PDMS } \\
\mathrm{mg} / \mathrm{g}\end{array}$ & $\begin{array}{l}\mathrm{PPG} \\
\mathrm{mg} / \mathrm{g}\end{array}$ & $\begin{array}{c}\mathrm{Si} \\
\mu \mathrm{g} / \mathrm{g} \\
\end{array}$ & $\begin{array}{c}\mathrm{Al} \\
\mu \mathrm{g} / \mathrm{g}\end{array}$ & $\begin{array}{c}\mathrm{Na} \\
\mu \mathrm{g} / \mathrm{g} \\
\end{array}$ & $\begin{array}{c}\mathrm{P} \\
\mu \mathrm{g} / \mathrm{g}\end{array}$ & $\begin{array}{c}\mathrm{S} \\
\mu \mathrm{g} / \mathrm{g}\end{array}$ \\
\hline After Final Wash & $4.4 \mathrm{E}-03$ & 1.10 & $1660^{(g)}$ & 130000 & 12300 & 759 & 42.7 \\
\hline
\end{tabular}

(g) Original intact slurry sample submitted to analytical lab. Phase separation performed at analytical laboratory indicated $54.2 \%$ solids in the submitted sample. The measured $\mathrm{Si}$ in the liquid fraction of the submitted sample was $75.1-\mu \mathrm{g} / \mathrm{g}$.

Bold numbers are calculated values. 
Table 5.9. Oxidative-Leach

\begin{tabular}{|c|c|c|c|c|c|c|c|c|c|c|c|c|c|c|c|c|c|}
\hline \multirow[b]{2}{*}{$\begin{array}{l}\text { Sample location: } \\
\text { Tank T02A, } \\
\text { Condition: }\end{array}$} & \multicolumn{2}{|c|}{ Slurry Analyses } & \multicolumn{15}{|c|}{ Liquid Analyses } \\
\hline & $\begin{array}{l}\text { UDS } \\
\mathrm{Wt} \%\end{array}$ & $\begin{array}{c}\text { Slurry } \\
\text { Density } \\
\mathrm{g} / \mathrm{mL}\end{array}$ & $\begin{array}{l}\text { Liquid } \\
\text { Density } \\
\mathrm{g} / \mathrm{mL}\end{array}$ & $\begin{array}{c}\mathrm{Al} \\
{\left[\mathrm{Al}(\mathrm{OH})_{4}^{-}\right.} \\
\text {by Raman }] \\
\mu \mathrm{g} / \mathrm{g} \\
{[\mathrm{M}]}\end{array}$ & $\begin{array}{c}\mathrm{Al} \\
\text { (rinsate) } \\
{\left[\mathrm{Al}(\mathrm{OH})_{4}{ }^{-}\right.} \\
\text {by Raman] } \\
\mu \mathrm{g} / \mathrm{g} \\
{[\mathrm{M}]}\end{array}$ & $\begin{array}{c}\text { Free OH- } \\
\text { [Raman] } \\
\mathrm{M} \\
{[\mathrm{M}]}\end{array}$ & $\begin{array}{c}\mathrm{Cr} \\
{\left[\mathrm{CrO}_{4}^{-2} \text { by }\right.} \\
\left.\mathrm{Raman}^{2}\right] \\
\mu \mathrm{g} / \mathrm{g} \\
{[\mathrm{M}]}\end{array}$ & $\begin{array}{c}\mathrm{Cr} \text { (rinsate) } \\
{\left[\mathrm{CrO}_{4}^{-2} \text { by }\right.} \\
\left.\mathrm{Raman}^{2}\right] \\
\mu \mathrm{g} / \mathrm{g} \\
{[\mathrm{M}]}\end{array}$ & $\begin{array}{l}\mathrm{Mn} \\
\mu \mathrm{g} / \mathrm{g}\end{array}$ & $\begin{array}{c}\mathrm{Mn} \\
\text { (rinsate) } \\
\mu \mathrm{g} / \mathrm{g}\end{array}$ & $\begin{array}{l}\text { PDMS } \\
\mathrm{mg} / \mathrm{g}\end{array}$ & $\begin{array}{l}\text { PPG } \\
\mathrm{mg} / \mathrm{g}\end{array}$ & $\begin{array}{c}\mathrm{C}_{2} \mathrm{O}_{4} \\
{[\mathrm{Raman}]} \\
\mu \mathrm{g} / \mathrm{g} \\
{[\mathrm{M}]}\end{array}$ & $\begin{array}{l}\mathrm{Cl}- \\
\mu \mathrm{g} / \mathrm{g}\end{array}$ & $\begin{array}{c}\mathrm{NO}_{3} \\
{[\mathrm{Raman}]} \\
\mu \mathrm{g} / \mathrm{g} \\
{[\mathrm{M}]}\end{array}$ & $\begin{array}{c}\mathrm{NO}_{2} \\
\text { [Raman] } \\
\mu \mathrm{g} / \mathrm{g} \\
{[\mathrm{M}]}\end{array}$ & $\begin{array}{c}\mathrm{SO}_{4} \\
{[\mathrm{Raman}]} \\
\mu \mathrm{g} / \mathrm{g} \\
{[\mathrm{M}]}\end{array}$ \\
\hline Before $\mathrm{MnO}_{4}$ Addition ${ }^{(\mathrm{e})}$ & 16.9 & 1.071 & 1.015 & $\begin{array}{c}146 \\
{[\mathrm{n} / \mathrm{d}]}\end{array}$ & $\begin{array}{l}\mathbf{4 6 . 8} \\
{[\mathrm{n} / \mathrm{d}]}\end{array}$ & $\begin{array}{c}0.28 \\
{[0.300]^{(d)}}\end{array}$ & $\begin{array}{l}11.7 \\
{[\mathrm{n} / \mathrm{d}]}\end{array}$ & $\begin{array}{l}6.23 \\
{[\mathrm{n} / \mathrm{d}]}\end{array}$ & 0.176 & 0.407 & $\mathrm{n} / \mathrm{d}^{(\mathrm{c})}$ & Failed & $\begin{array}{c}1517 \\
{[0.014]^{(\mathrm{d})}} \\
{[<0.01]} \\
{[<0.01]}\end{array}$ & 150 & $\begin{array}{c}3757 \\
{[0.070]}\end{array}$ & $\begin{array}{c}93.2 \\
{[0.032]^{(\mathrm{d})}} \\
{[0.026]^{(\mathrm{d})}} \\
{[<0.03]}\end{array}$ & $\begin{array}{c}\mathbf{5 4 . 9} \\
{[<0.001]} \\
{[<0.001]} \\
{[0.001]^{(\mathrm{d})}}\end{array}$ \\
\hline After $\mathrm{MnO}_{4}$ Addition- $\mathrm{Hr} 0$ & 18.2 & 1.135 & 1.027 & $\begin{array}{c}53.2 \\
{[<0.01]}\end{array}$ & - & $\begin{array}{c}0.07 \\
{[0.067]^{(\mathrm{d})}}\end{array}$ & $\begin{array}{c}7030 \\
{[0.132]}\end{array}$ & - & 0.375 & - & - & - & $\begin{array}{c}1340 \\
{[<0.01]}\end{array}$ & 136 & $\begin{array}{c}3270 \\
{[0.064]}\end{array}$ & $\begin{array}{c}91.7 \\
{[<0.03]}\end{array}$ & $\begin{array}{c}155 \\
{[0.002]^{(\mathrm{d})}}\end{array}$ \\
\hline After $\mathrm{MnO}_{4}$ Addition- $\mathrm{Hr} 1$ & 18.3 & 1.136 & 1.030 & 111 & - & 0.09 & 6620 & - & 0.328 & - & - & - & 1460 & 143 & 3520 & 103.0 & 168.0 \\
\hline After $\mathrm{MnO}_{4}$ Addition- $\mathrm{Hr} 2$ & 18.3 & 1.142 & 1.031 & 130 & - & 0.11 & 6330 & - & 0.391 & - & - & - & 1460 & 144 & 3580 & 107.0 & 156.0 \\
\hline After $\mathrm{MnO}_{4}$ Addition- $\mathrm{Hr} 3$ & 18.3 & 1.141 & 1.029 & 144 & - & 0.13 & 6140 & - & 0.582 & - & - & - & 1510 & 147 & 3660 & 109.0 & 155.0 \\
\hline After $\mathrm{MnO}_{4}$ Addition- $\mathrm{Hr} 4$ & 18.3 & 1.115 & 1.028 & 149 & - & 0.15 & 5670 & - & 0.402 & - & - & - & 1560 & 153 & 3820 & 110.0 & 158.0 \\
\hline After $\mathrm{MnO}_{4}$ Addition- $\mathrm{Hr} 5$ & 18.3 & 1.141 & 1.028 & 162 & - & 0.14 & 5740 & - & 0.361 & - & - & - & 1530 & 147 & 3790 & 112.0 & 148.0 \\
\hline $\begin{array}{c}\text { After } \mathrm{MnO}_{4} \text { Addition-Hr } 6 \\
\text { (Final after } \\
\text { Oxidative-Leach) }{ }^{(\mathrm{f})}\end{array}$ & 18.3 & 1.144 & 1.027 & $\begin{array}{c}166 \\
{[\mathrm{n} / \mathrm{d}]}\end{array}$ & $\begin{array}{l}\mathbf{6 0 . 0} \\
{[\mathrm{n} / \mathrm{d}]}\end{array}$ & $\begin{array}{c}0.16 \\
{[0.141]^{(\mathrm{d})}}\end{array}$ & $\begin{array}{c}5550 \\
{[0.102]}\end{array}$ & $\begin{array}{c}1495 \\
{[0.026]}\end{array}$ & 0.433 & 0.240 & 5.E-04 & Failed & $\begin{array}{c}1573 \\
{[<0.01]} \\
{[<0.01]} \\
{[0.015]^{\text {(d) }}}\end{array}$ & 152 & $\begin{array}{c}3863 \\
{[0.071]}\end{array}$ & $\begin{array}{c}112 \\
{[0.026]^{(\mathrm{d})}} \\
{[<0.03]} \\
{[<0.03]}\end{array}$ & $\begin{array}{c}153 \\
{[0.002]^{(\mathrm{d})}}\end{array}$ \\
\hline
\end{tabular}

ur (a) Original intact slurry submitted to analytical lab so phase separation was performed at analytical laboratory.

$\bar{N}$ (b) Slurry sample was phase-separated at PDL-W.

(c) Single sample non-detect with analysis results below method reporting limit.

(d) Detected result is qualitative. Result $>$ IDL but $<$ EQL (estimated quantitation limit).

(e) Analytical results duplicated in Analysis at Key Process Steps table column heading Starting Slurry Composition Before Permanganate Addition, including wt\% solids data.

(f) Analytical results duplicated in Analysis at Key Process Steps table column heading Initial Slurry Composition for Post-Oxidative-Leach Wash, including wt\% solids data.

Failed - the organic phase failed to separate from the aqueous phase and no results were obtained.

[Raman] results are provided as bracketed values [].

Bold numbers are calculated values.

- Analysis not required by Test Plan. 
Table 5.9 (contd)

\begin{tabular}{|c|c|c|c|c|c|c|c|}
\hline \multirow{2}{*}{$\begin{array}{l}\text { Sample location: } \\
\text { Tank T02A, } \\
\text { Condition: }\end{array}$} & \multicolumn{7}{|c|}{ Rinsed Wet Centrifuged Solids Analyses } \\
\hline & $\begin{array}{c}\mathrm{Al} \\
\mu \mathrm{g} / \mathrm{g}\end{array}$ & $\begin{array}{c}\mathrm{Cr} \\
\mu \mathrm{g} / \mathrm{g}\end{array}$ & $\begin{array}{c}\mathrm{Mn} \\
\mu \mathrm{g} / \mathrm{g}\end{array}$ & $\begin{array}{c}\mathrm{Sr} \\
\mu \mathrm{g} / \mathrm{g}\end{array}$ & $\begin{array}{c}\mathrm{Si} \\
\mu \mathrm{g} / \mathrm{g}\end{array}$ & $\begin{array}{c}\mathrm{PDMS} \\
\mathrm{mg} / \mathrm{g}\end{array}$ & $\begin{array}{l}\mathrm{PPG} \\
\mathrm{mg} / \mathrm{g}\end{array}$ \\
\hline Before $\mathrm{MnO}_{4}$ Addition ${ }^{(\mathrm{e})}$ & 107333 & 18533 & 20667 & 803 & $1520^{(\mathrm{a})}$ & $7.7 \mathrm{E}-03$ & 1.0 \\
\hline After $\mathrm{MnO}_{4}$ Addition- $\mathrm{Hr} 1$ & - & - & - & - & - & - & - \\
\hline After $\mathrm{MnO}_{4}$ Addition- $\mathrm{Hr} 2$ & - & - & - & - & - & - & - \\
\hline After $\mathrm{MnO}_{4}$ Addition- $\mathrm{Hr} 3$ & - & - & - & - & - & - & - \\
\hline $\begin{array}{c}\text { After } \mathrm{MnO}_{4} \text { Addition-Hr } 6 \\
\text { (Final after } \\
\text { Oxidative-Leach) })^{(\mathrm{f})}\end{array}$ & 120000 & 1700 & 41450 & 855 & $1410^{(\mathrm{b})}$ & $3.8 \mathrm{E}-03$ & 1.1 \\
\hline
\end{tabular}

(a) Original intact slurry sample submitted to analytical lab. Phase separation performed at analytical laboratory indicated $48.56 \%$ solids in the submitted sample.

The measured $\mathrm{Si}$ in the liquid fraction of the submitted sample was $67.8-\mu \mathrm{g} / \mathrm{g}$.

(b) Slurry sample was phase-separated at PDL-W, and then submitted to the analytical lab. Measurement indicated $48.81 \%$ solids in the phase-separated solids sample. The measured $\mathrm{Si}$ in the phase-separated liquid sample was $35.4-\mu \mathrm{g} / \mathrm{g}$.

(e) Analytical results duplicated in Analysis at Key Process Steps table column heading Starting Slurry Composition Before Permanganate Addition, including wt $\%$ solids data.

(f) Analytical results duplicated in Analysis at Key Process Steps table column heading Initial Slurry Composition for Post-Oxidative-Leach Wash, including wt\% solids data.

Bold numbers are calculated values. 
Table 5.10, Post-Oxidative-Leach Wash, provides analytical results for samples collected during the post-oxidative-leach washing in Tank T02A. Two separate slurry samples were collected for the wt $\%$ UDS and slurry density. The supernatant density is a higher accuracy density than can be provided by the UDS analysis. The liquid analysis results are supernatant analytical results obtained from samples phase-separated in PDL-W. Raman results, shown in brackets, [ ], are provided as part of the liquid analyses and presented in molar concentration (mole/liter).

Table 5.11, ICP Analysis of Chromium Slurry, provides the ICP analysis of the chromium-bearing slurry, which was added immediately before the oxidative-leach. Table 5.12 provides the IC, TIC/TOC, $\mathrm{OH}-$, of the liquid fraction and physical analyses of the chromium slurry. 
Table 5.10. Post-Oxidative-Leach Wash

\begin{tabular}{|c|c|c|c|c|c|c|c|c|c|c|c|c|c|c|}
\hline \multirow[b]{2}{*}{$\begin{array}{c}\text { Sample location: } \\
\text { Tank T02A, } \\
\text { Condition: }\end{array}$} & \multicolumn{2}{|c|}{ Slurry Analyses } & \multirow[b]{2}{*}{$\begin{array}{c} \\
\operatorname{TDS}^{(c)} \\
\mathrm{wt}^{(\mathrm{c}} \% \\
\end{array}$} & \multicolumn{11}{|c|}{ Liquid Analyses } \\
\hline & $\begin{array}{l}\text { UDS } \\
\mathrm{wt}^{0} \%\end{array}$ & $\begin{array}{c}\text { Slurry } \\
\text { Density }^{(a)} \\
\text { g/mL } \\
\end{array}$ & & $\begin{array}{c}\text { Liquid } \\
\underset{\text { (a) }}{\text { Density }} \\
\mathrm{g} / \mathrm{mL} \\
\end{array}$ & $\begin{array}{c}\mathrm{Al} \\
{\left[\mathrm{Al}(\mathrm{OH})_{4}^{-}\right.} \\
\text {by } \\
\text { Raman] } \\
\mu \mathrm{g} / \mathrm{g} \\
{[\mathrm{M}]}\end{array}$ & $\begin{array}{c}\mathrm{Na} \\
\mu \mathrm{g} / \mathrm{g}\end{array}$ & $\begin{array}{c}\mathrm{Cr} \\
{\left[\mathrm{CrO}_{4}^{-2} \text { by }\right.} \\
\left.\mathrm{Raman}^{-}\right] \\
\mu \mathrm{g} / \mathrm{g} \\
{[\mathrm{M}]}\end{array}$ & $\begin{array}{c}\mathrm{Sr} \\
\mu \mathrm{g} / \mathrm{g}\end{array}$ & $\begin{array}{c}\text { Free } \\
\text { OH- } \\
\text { [Raman] } \\
\mathrm{M} \\
{[\mathrm{M}]}\end{array}$ & $\begin{array}{c}\mathrm{NO}_{3} \\
{[\mathrm{Raman}]} \\
\mu \mathrm{g} / \mathrm{g} \\
{[\mathrm{M}]} \\
\end{array}$ & $\begin{array}{c}\mathrm{NO}_{2} \\
{[\mathrm{Raman}]} \\
\mu \mathrm{g} / \mathrm{g} \\
{[\mathrm{M}]} \\
\end{array}$ & $\begin{array}{c}\mathrm{SO}_{4} \\
{[\mathrm{Raman}]} \\
\mu \mathrm{g} / \mathrm{g} \\
{[\mathrm{M}]} \\
\end{array}$ & $\begin{array}{c}\mathrm{C}_{2} \mathrm{O}_{4} \\
{[\mathrm{Raman}]} \\
\mu \mathrm{g} / \mathrm{g} \\
{[\mathrm{M}]} \\
\end{array}$ & $\begin{array}{c}\mathrm{Cl}- \\
\mu \mathrm{g} / \mathrm{g}\end{array}$ \\
\hline After Wash 3 & 18.1 & 1.196 & 2.97 & 1.022 & $\begin{array}{c}148 \\
{[<0.01]}\end{array}$ & 9060 & $\begin{array}{c}4460 \\
{[0.084]}\end{array}$ & $<0.124$ & $\begin{array}{c}0.02 \\
{[0.129]^{(b)}}\end{array}$ & $\begin{array}{c}3200 \\
{[0.062]}\end{array}$ & $\begin{array}{c}109 \\
{[<0.03]}\end{array}$ & $\begin{array}{c}127 \\
{[0.002]^{(b)}}\end{array}$ & $\begin{array}{c}1340 \\
{[<0.01]}\end{array}$ & 131 \\
\hline After Wash 6 & - & - & 2.28 & 0.995 & 122 & 7400 & 3580 & $<0.124$ & 0.10 & 2550 & 93.0 & 103 & 1080 & 104 \\
\hline After Wash 9 & 17.9 & 1.176 & 1.94 & 1.001 & 98.8 & 5970 & 2840 & $<0.124$ & 0.10 & 2040 & 87.1 & 84.6 & 872 & 84.2 \\
\hline After Wash 12 & - & - & 1.52 & 0.992 & 90.1 & 5270 & 2470 & $<0.124$ & 0.09 & 1700 & 76.1 & 69.8 & 737 & 71.7 \\
\hline After Wash 15 & 18.2 & 1.172 & 1.34 & 1.006 & 69.1 & 4110 & 1890 & $<0.125$ & 0.07 & 1310 & 70.5 & 54.6 & 573 & 54.1 \\
\hline After Wash 18 & - & - & 1.08 & 0.981 & 63.2 & 3600 & 1610 & $<0.125$ & 0.05 & 1120 & 69.0 & 45.5 & 487 & 48.0 \\
\hline After Wash 21 & - & 1.122 & 0.92 & 0.980 & $\begin{array}{c}51.4 \\
{[<0.01]}\end{array}$ & 2980 & $\begin{array}{c}1310 \\
{[0.023]}\end{array}$ & $<0.124$ & $\begin{array}{c}0.05 \\
{[<0.04]}\end{array}$ & $\begin{array}{c}882 \\
{[0.026]}\end{array}$ & $\begin{array}{c}<61.5 \\
{[<0.03]}\end{array}$ & $\begin{array}{c}41.9 \\
{[<0.001]}\end{array}$ & $\begin{array}{c}394 \\
{[<0.01]}\end{array}$ & 39.6 \\
\hline After Wash 24 & - & - & 0.72 & 0.983 & 42.5 & 2480 & 1010 & $<0.125$ & 0.02 & 721 & $<65.5$ & 36.9 & 301 & 36.3 \\
\hline After Wash 27 & 18.8 & 1.162 & 0.66 & 1.001 & 36.2 & 2030 & 811 & $<0.123$ & 0.02 & 593 & $<64.9$ & 23.3 & 248 & 26.4 \\
\hline After Wash 30 & - & - & 0.52 & 0.978 & 32.8 & 1800 & 677 & $<0.125$ & 0.04 & 487 & $<63.3$ & 21.0 & 210 & 22.5 \\
\hline After Wash 33 & 18.7 & 1.135 & 0.42 & 1.003 & 27.5 & 1490 & 533 & $<0.123$ & 0.03 & 406 & $<65.2$ & $<19.8$ & 173 & 20.2 \\
\hline After Wash 36 & - & - & $<0.5$ & 0.984 & 26.1 & 1360 & 444 & $<0.125$ & 0.03 & 347 & $<63.6$ & $<19.3$ & 136 & $<19.3$ \\
\hline After Wash 40 & 18.9 & 1.122 & 0.24 & 1.003 & 21.7 & 1090 & 330 & $<0.124$ & 0.03 & 272 & $<59.9$ & $<18.2$ & 106 & $<18.2$ \\
\hline After Wash 44 & - & - & $<0.5$ & 0.982 & 18.6 & 974 & 251 & $<0.125$ & 0.03 & 233 & $<63.1$ & $<19.2$ & 85.8 & $<19.2$ \\
\hline After Wash 47 & 20.2 & 1.126 & 0.20 & 1.000 & 20.3 & 952 & 251 & $<0.123$ & 0.02 & 244 & $<64.5$ & $<19.6$ & 97.8 & $<20.9$ \\
\hline
\end{tabular}

(a) If high accuracy density results are available, then they are provided. If high accuracy density results are not available, then density results from the UDS are provided.

(b) Detected result is qualitative. Result $>$ IDL but $<$ EQL (estimated quantitation limit).

(c) TDS results determined during UDS analysis when available. Otherwise, TDS results determined from supernate samples phase-separated at PDL-W.

- Analysis not required by Test Plan.

[Raman] results are provided as bracketed Molar concentrations [ ].

M Molarity. 
Table 5.11. ICP Analysis of Chromium Slurry

\begin{tabular}{|c|c|c|c|}
\hline \multirow[t]{3}{*}{$\begin{array}{l}\text { Batch Name } \\
\text { Sample ID }\end{array}$} & \multicolumn{3}{|c|}{$\begin{array}{l}\text { CrOOH Final Batch } \\
\text { Cr-080917-Final-SL }\end{array}$} \\
\hline & \multicolumn{3}{|c|}{ Total Slurry Fraction } \\
\hline & $\mu \mathrm{g} / \mathrm{mL}$ & $\mathrm{Mol} / \mathrm{L}$ & $\%$ \\
\hline $\mathrm{Al}$ & 0.77 & 0.00003 & 0.0001 \\
\hline B & 3.3 & 0.0003 & 0.0003 \\
\hline $\mathrm{Ba}$ & 13.4 & 0.0001 & 0.0011 \\
\hline $\mathrm{Bi}$ & $<1.5$ & $<0.0000$ & $<0.000$ \\
\hline $\mathrm{Ca}$ & 19.1 & 0.0005 & 0.0016 \\
\hline $\mathrm{Cd}$ & 0.99 & 0.0000 & 0.0001 \\
\hline $\mathrm{Ce}$ & $<0.5$ & $<0.0000$ & $<0.000$ \\
\hline $\mathrm{Cr}$ & 14,100 & 0.2712 & 1.1899 \\
\hline $\mathrm{Fe}$ & 7.0 & 0.0001 & 0.0006 \\
\hline $\mathrm{K}$ & 419 & 0.0107 & 0.0354 \\
\hline $\mathrm{La}$ & 0.94 & 0.0000 & 0.0001 \\
\hline $\mathrm{Mg}$ & 0.72 & 0.0000 & 0.0001 \\
\hline $\mathrm{Mn}$ & 0.23 & 0.0000 & 0.0000 \\
\hline $\mathrm{Na}$ & $<99,100$ & $<4.311$ & $<8.363$ \\
\hline $\mathrm{Nd}$ & $<1.0$ & $<0.0000$ & $<0.000$ \\
\hline $\mathrm{Ni}$ & 1.21 & 0.0000 & 0.0001 \\
\hline $\mathrm{P}$ & $<2.5$ & $<0.0001$ & $<0.000$ \\
\hline $\mathrm{Pb}$ & $<1.6$ & $<0.0000$ & $<0.000$ \\
\hline $\mathrm{S}$ & 30 & 0.0009 & 0.0025 \\
\hline $\mathrm{Si}$ & 15 & 0.0005 & 0.0013 \\
\hline $\mathrm{Sr}$ & 30.6 & 0.0003 & 0.0026 \\
\hline Th & 26.8 & 0.0001 & 0.0023 \\
\hline $\mathrm{Zn}$ & 9.43 & 0.0001 & 0.0008 \\
\hline $\mathrm{Zr}$ & 0.073 & 0.0000 & 0.0000 \\
\hline
\end{tabular}


Table 5.12. Additional Analysis of the Cr Slurry

\begin{tabular}{|c|c|c|c|}
\hline $\begin{array}{l}\text { Batch Name } \\
\text { Sample ID }\end{array}$ & \multicolumn{3}{|c|}{$\begin{array}{l}\text { CrOOH Final Batch } \\
\text { Cr-080917-Final-SL }\end{array}$} \\
\hline & \multicolumn{3}{|c|}{ Liquid Fraction Only } \\
\hline Parameter & $\mu \mathrm{g} / \mathrm{mL}$ & $\mathrm{Mol} / \mathrm{L}$ & $\%$ \\
\hline $\mathrm{F}$ & 4.6 & 0.0002 & 0.0004 \\
\hline $\mathrm{Cl}$ & 2,185 & 0.0616 & 0.1844 \\
\hline $\mathrm{NO}_{2}$ & $<3$ & $<0.0001$ & $<0.0003$ \\
\hline $\mathrm{SO}_{4}$ & 60.1 & 0.0006 & 0.0051 \\
\hline $\mathrm{C}_{2} \mathrm{O}_{4}$ & $<44$ & $<0.0005$ & $<0.0037$ \\
\hline $\mathrm{NO}_{3}$ & 55,300 & 0.8918 & 4.6667 \\
\hline $\mathrm{PO}_{4}$ & 24 & 0.0003 & 0.0020 \\
\hline TIC & 185 & 0.0154 & 0.0156 \\
\hline TOC & $<94$ & $<0.0039$ & $<0.0079$ \\
\hline $\mathrm{OH}$ & 53,718 & 3.1585 & 4.5332 \\
\hline UDS & 2.47 & \multicolumn{2}{|c|}{ wt $\%$ UDS $(\%)$} \\
\hline DS & 17.26 & \multicolumn{2}{|c|}{$\mathrm{wt} \%$ DS (\%) } \\
\hline PSD & 12.37 & \multicolumn{2}{|c|}{$50 \%$ Percentile } \\
\hline Slurry Liquid & 1.164 & \multicolumn{2}{|c|}{ Liquid Density $(\mathrm{g} / \mathrm{mL})$} \\
\hline Slurry & 1.185 & \multicolumn{2}{|c|}{ Slurry Density $(\mathrm{g} / \mathrm{mL})$} \\
\hline
\end{tabular}

Table 5.13 provides analytical results for samples collected before caustic-leach after the filter-loop was flushed back into Tank T02A and for samples collected during the end of the testing (Test Instruction step 7.20.5) when the filter-loop was being flushed. The wt $\%$ UDS and densities were obtained following analytical methods described in Section 3 for slurry not phase-separated. The liquid analysis results are supernatant analytical results obtained from samples phase-separated in PDL-W.

Table 5.13. Filter Flush

\begin{tabular}{|c|c|c|c|c|c|c|c|c|c|c|c|c|c|}
\hline \multirow[b]{3}{*}{ Sample Location } & \multicolumn{2}{|c|}{ Slurry Analyses } & \multicolumn{11}{|c|}{ Liquid Analyses } \\
\hline & UDS & Density & TDS & $\mathrm{Al}$ & $\mathrm{Na}$ & $\mathrm{Cr}$ & $\mathrm{Sr}$ & $\begin{array}{l}\text { Free } \\
\mathrm{OH}^{-}\end{array}$ & $\mathrm{NO}_{3}$ & $\mathrm{NO}_{2}$ & $\mathrm{SO}_{4}$ & $\mathrm{C}_{2} \mathrm{O}_{4}$ & $\mathrm{Cl}-$ \\
\hline & $\mathrm{wt} \%$ & $\mathrm{~g} / \mathrm{mL}$ & $\mathrm{wt} \%$ & $\mu \mathrm{g} / \mathrm{g}$ & $\mu \mathrm{g} / \mathrm{g}$ & $\mu \mathrm{g} / \mathrm{g}$ & $\mu \mathrm{g} / \mathrm{g}$ & $\mathrm{M}$ & $\mu \mathrm{g} / \mathrm{g}$ & $\mu \mathrm{g} / \mathrm{g}$ & $\mu \mathrm{g} / \mathrm{g}$ & $\mu \mathrm{g} / \mathrm{g}$ & $\mu \mathrm{g} / \mathrm{g}$ \\
\hline $\begin{array}{l}\text { Batch 1: } 5 \text { min after } \\
\text { circulating flush water } \\
\text { in the loop with } \\
\text { Tank T02A bypassed: } \\
\text { Flushed loop contents }\end{array}$ & 3.15 & 1.175 & 17.7 & 6000 & 70900 & 1.79 & $<0.119$ & 2.50 & 17500 & 4690 & 3470 & 966 & 206 \\
\hline $\begin{array}{l}\text { Batch 2: } 5 \text { min after } \\
\text { circulating flush water } \\
\text { in the loop with Tank } \\
\text { T02A bypassed: } \\
\text { Flushed loop contents }\end{array}$ & 3.90 & 1.202 & 20.8 & 7390 & 77000 & 1.45 & $<0.121$ & 2.78 & 18800 & 4940 & 3730 & 754 & 222 \\
\hline $\begin{array}{l}5 \text { min after circulating } \\
\text { flush water in the loop } \\
\text { with Tank T02A } \\
\text { bypassed: Flushed loop } \\
\text { contents }\end{array}$ & 11.8 & 1.474 & 33.6 & 329 & 156000 & 205 & $<0.124$ & 9.40 & 445 & 103 & 63.9 & 37.6 & $<16.7$ \\
\hline $\begin{array}{l}\text { Prior to transfer of } \\
\text { slurry from Tank T02A } \\
\text { to HLP-VSL-T62A/B: } \\
\text { From Tank T02A } \\
\text { middle-low port }\end{array}$ & 13.8 & 1.480 & 31.2 & 305 & 135000 & 233 & $<0.125$ & 7.52 & 453 & 108 & 62.2 & 98.2 & $<18.6$ \\
\hline M Molarity. & & & & & & & & & & & & & \\
\hline
\end{tabular}




\subsection{Physical Properties Data}

This section summarizes the heat capacity, PSD, rheology, XRD, and SEM results. The interested reader is referred to Appendix L for additional information.

\subsubsection{Heat Capacity}

Table 5.14, Heat Capacity Measurements, provides a summary of the heat capacity data obtained at process steps of interest at both 40 and $80^{\circ} \mathrm{C}$. Heat capacity data over the temperature range 35 to $90^{\circ} \mathrm{C}$ for the samples listed can be found in Appendix L.

Table 5.14. Heat Capacity Measurements

\begin{tabular}{ccc}
\hline & Heat Capacity & Heat Capacity \\
\cline { 2 - 3 } Sample location: Tank T02A & $\mathrm{J} / \mathrm{g}{ }^{\circ} \mathrm{C} @ 400^{\circ} \mathrm{C}$ & $\mathrm{J} / \mathrm{g}{ }^{\circ} \mathrm{C} @ 80^{\circ} \mathrm{C}$ \\
\hline Initial slurry material & $3.33 \pm 0.02$ & $3.35 \pm 0.02$ \\
After Concentration - Batch 1 & $2.97 \pm 0.00$ & $2.96 \pm 0.05$ \\
After caustic addition - Batch 1 & $3.22 \pm 0.01$ & $3.29 \pm 0.01$ \\
88C - Batch 1 & $3.25 \pm 0.02$ & $3.29 \pm 0.02$ \\
98C - Start of Caustic-Leach - Batch 1 & $3.23 \pm 0.09$ & $3.23 \pm 0.08$ \\
\hline
\end{tabular}

\subsubsection{Particle Size}

Table 5.15, Slurry PSD Results, provides the PSD data at key process steps, before and after sonication. The average particle size, i.e., the average equivalent diameter, is defined as the diameter where 50 mass- $\%$ (of the particles) have a larger equivalent diameter, and the other 50 mass- $\%$ have a smaller equivalent diameter. Hence the average particle size is denoted as equivalent $\mathrm{d}(0.500)$. The definition of $\mathrm{d}(0.100)$ is the equivalent diameter where 10 mass- $\%$ (of the particles) has a smaller diameter (and hence the remaining $90 \%$ is coarser). The definition of $\mathrm{d}(0.900)$ can be derived similarly.

Table 5.15. Slurry PSD Results

\begin{tabular}{|c|c|c|c|c|c|c|}
\hline \multirow[b]{2}{*}{ Process Step Description } & \multicolumn{3}{|c|}{$\begin{array}{l}\text { Pre-Sonication } \\
\text { (microns) }\end{array}$} & \multicolumn{3}{|c|}{$\begin{array}{l}\text { Sonicated } \\
\text { (microns) }\end{array}$} \\
\hline & $\mathrm{d}(0.100)$ & $\mathrm{d}(0.500)$ & $\mathrm{d}(0.900)$ & $\mathrm{d}(0.100)$ & $\mathrm{d}(0.500)$ & $\mathrm{d}(0.900)$ \\
\hline Initial simulant characterization from UFP-VSL-FRP $(02 / 19 / 09)$ & 1.758 & 7.589 & 25.064 & 1.720 & 7.061 & 19.974 \\
\hline \multirow{2}{*}{ Initial simulant characterization at test start from HLP-VSL-T22 $2^{(a)}$} & 1.721 & 8.196 & 30.935 & 1.674 & 7.249 & 21.499 \\
\hline & 1.762 & 8.458 & 32.574 & 1.668 & 7.366 & 22.553 \\
\hline \multirow{2}{*}{ Batch \#1 - pre-leach concentration in Tank T02A - final slurry ${ }^{(a)}$} & 1.048 & 5.111 & 16.73 & 1.203 & 5.504 & 17.016 \\
\hline & 0.968 & 5.033 & 16.726 & 1.168 & 5.41 & 18.13 \\
\hline \multirow{2}{*}{ Batch \#2 - pre-leach concentration in Tank T02A - final slurry } & 0.959 & 4.182 & 14.073 & 1.129 & 4.626 & 14.143 \\
\hline & 0.959 & 4.182 & 14.073 & 1.129 & 4.626 & 14.143 \\
\hline Post-leach concentration - initial unwashed slurry & 0.793 & 1.928 & 3.892 & 0.754 & 1.736 & 3.438 \\
\hline \multirow{2}{*}{ Post-leach concentration - final unwashed slurry ${ }^{(a)}$} & 0.708 & 1.737 & 6.975 & 0.625 & 1.795 & 8.302 \\
\hline & 0.660 & 1.577 & 4.102 & 0.582 & 1.343 & 4.118 \\
\hline
\end{tabular}

(a) Sample taken was sub-sampled and analyzed more than once. 


\subsubsection{Rheology}

Table 5.16, Integrated Test B Rheology Data, provides a summary of rheology measurements performed at key points throughout Integrated Test B. For simplicity, only Bingham yield stress and consistency fits for the down ramps have been reported here. A more extensive analysis of the rheology measurements taken is provided in Appendix L with flow curve analysis using both the Bingham and Casson models for both the up and down ramps. Table 5.17, Integrated Test B Shear Strength Data, provides additional shear strength measurements.

Table 5.16. Integrated Test B Rheology Data Summary

\begin{tabular}{|c|c|c|c|c|}
\hline Process Test Step & $\begin{array}{l}\text { Shear } \\
\text { Strength } \\
{[\mathrm{Pa}]^{(\mathrm{b})}}\end{array}$ & $\begin{array}{l}\text { Bingham } \\
\text { Yield } \\
\frac{\mathrm{Pa}]^{(\mathrm{a})}}{}\end{array}$ & $\begin{array}{c}\text { Bingham } \\
\text { Consistency } \\
{[\mathrm{mPa} \cdot \mathrm{s}]^{(\mathrm{a})}}\end{array}$ & $\begin{array}{l}\text { Supernate } \\
\text { Viscosity } \\
{[\mathrm{mPa} \cdot \mathrm{s}]^{(\mathrm{a})}}\end{array}$ \\
\hline Feed UFP-VSL-FRP & 10.36 & - & - & - \\
\hline Feed UFP-VSL-FRP & - & $0^{(c)}$ & 4.079 & - \\
\hline Feed UFP-VSL-FRP & - & - & - & 2.746 \\
\hline Feed UFP-VSL-T22 & - & $0^{(\mathrm{c})}$ & 4.089 & - \\
\hline $\begin{array}{l}\text { Batch \#1 - Pre-leach concentration in Tank T02A - } \\
\text { initial slurry }\end{array}$ & - & $0^{(\mathrm{c})}$ & 4.718 & - \\
\hline $\begin{array}{l}\text { Batch \#1 - Pre-leach concentration in Tank T02A - final } \\
\text { slurry }\end{array}$ & - & 2.553 & 10.56 & - \\
\hline $\begin{array}{l}\text { Batch \#2 - Pre-leach concentration in Tank T02A - } \\
\text { initial slurry }\end{array}$ & - & - & - & 5.182 \\
\hline $\begin{array}{l}\text { Batch \#2 - Pre-leach concentration in Tank T02A - final } \\
\text { slurry }\end{array}$ & - & 2.31 & 11.02 & - \\
\hline $\begin{array}{l}\text { Post-caustic-leach concentration in Tank T02A - initial } \\
\text { slurry }\end{array}$ & - & - & - & 8.628 \\
\hline $\begin{array}{l}\text { Post-caustic-leach concentration in Tank T02A - initial } \\
\text { slurry }\end{array}$ & - & 0.199 & 14.01 & - \\
\hline $\begin{array}{l}\text { Post-caustic-leach concentration in Tank T02A - final } \\
\text { slurry }\end{array}$ & - & 7.594 & 30.35 & - \\
\hline $\begin{array}{l}\text { Post-caustic-leach concentration in Tank T02A - final } \\
\text { slurry }\end{array}$ & - & - & - & 8.853 \\
\hline Tracer test - 1 minute after tracer injection & - & 7.587 & 30.46 & - \\
\hline Post-caustic-leach wash - intermediate slurry & - & 7.802 & 7.925 & - \\
\hline Post-caustic-leach wash - intermediate slurry & - & - & - & 1.172 \\
\hline Post-caustic-leach wash - final slurry & - & 11.6 & 7.632 & - \\
\hline Post-caustic-leach wash - final slurry & - & - & - & 1.047 \\
\hline Oxidative-leach - before permanganate addition & 23.24 & - & - & - \\
\hline Oxidative-leach - before permanganate addition & - & 11.887 & 7.684 & - \\
\hline Oxidative-leach - final slurry & - & - & - & 1.054 \\
\hline Post-oxidative-leach wash - intermediate slurry & - & 4.081 & 5.991 & - \\
\hline Post-oxidative-leach wash - intermediate slurry & - & - & - & 0.9916 \\
\hline Post-oxidative-leach wash - final slurry & - & 2.991 & 6.296 & - \\
\hline Post-oxidative-leach wash - final slurry & - & - & - & 0.9507 \\
\hline \multicolumn{5}{|c|}{$\begin{array}{l}\text { (a) All results derived from flow curve analysis correspond to the down-ran } \\
\text { (b) Shear strength measurements correspond to a } 72 \mathrm{hr} \text { gel time } \\
\text { (c) Newtonian slurry treated as a Bingham Plastic with a zero yield stress } \\
\text { - Analysis not required by Test Plan }\end{array}$} \\
\hline
\end{tabular}


Table 5.17. Integrated Test B Shear Strength Data

\begin{tabular}{ccc}
\hline \multirow{2}{*}{$\begin{array}{c}\text { Settling } \\
\text { Time } \\
(\mathrm{hr})\end{array}$} & $\begin{array}{c}\text { Feed } \\
\text { UFP-VSL-FRP }\end{array}$ & $\begin{array}{c}\text { Maximum Shear } \\
\text { Oxidative-leach - before } \\
\text { permanganate addition }\end{array}$ \\
\hline 1 & Stress (Pa) & $\begin{array}{c}\text { Maximum Shear Stress } \\
(\mathrm{Pa})\end{array}$ \\
\hline 2 & 0.986 & 11.450 \\
4 & 2.237 & 10.660 \\
8 & 2.886 & 11.920 \\
16 & 3.880 & 12.340 \\
32 & 4.932 & 12.520 \\
72 & 10.350 & 13.740 \\
\hline
\end{tabular}

\subsubsection{XRD}

Table 5.18 summarizes the results of the XRD analysis of the initial simulant characterization samples taken before the commencement of Integrated Test B. The balance of the unaccounted material in the sample is assumed to be amorphous. Boehmite was the major phase detected. More information, including the XRD pattern of the simulant used for Integrated Test B, is provided in Appendix L.

Table 5.18. Measured Concentration and Estimated Crystallite Sizes of Identified Phases

\begin{tabular}{l|cc|cc}
\hline & \multicolumn{2}{|c|}{ Boehmite } & \multicolumn{2}{c}{ Gibbsite } \\
\cline { 2 - 5 } Sample ID & $\begin{array}{c}\text { Amount } \\
(\mathrm{wt} \%)\end{array}$ & $\begin{array}{c}\text { Size } \\
(\AA)\end{array}$ & $\begin{array}{c}\text { Amount } \\
(\mathrm{wt} \%)\end{array}$ & $\begin{array}{c}\text { Size } \\
(\AA)\end{array}$ \\
\hline B_FRPGM_001_XX_2713_XSP_4_B & 51.6 & 414 & 32.1 & 160 \\
\hline
\end{tabular}

\subsubsection{SEM}

Figure 5.1 is an SEM micrograph collage of the initial slurry characterization sample taken before testing. Appendix L contains the SEM micrograph along with information on the sample preparation, analysis, and further results. 


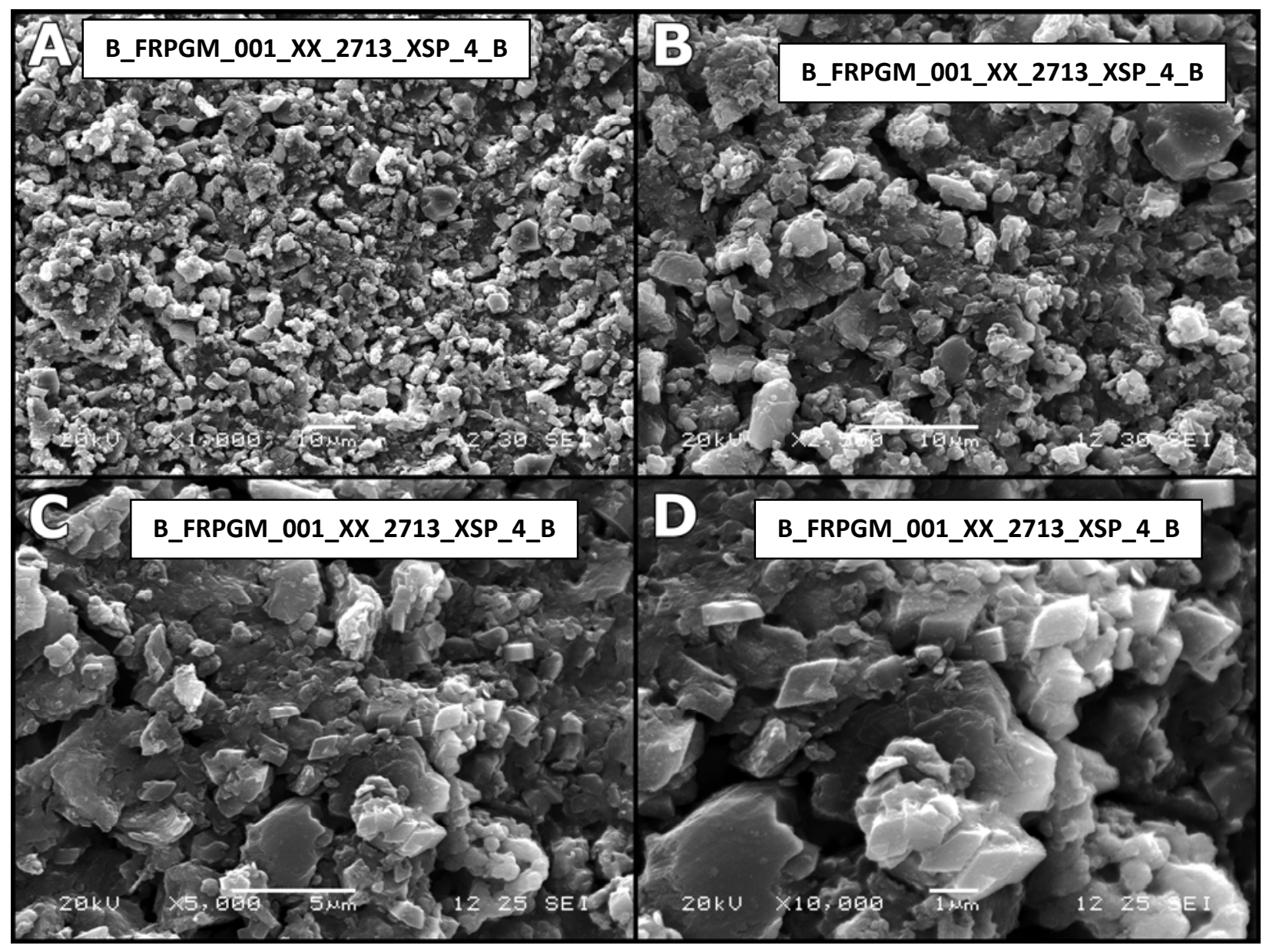

Figure 5.1. SEM Micrographs (Magnification—A: 1000×, B: $2500 \times$, C: $5000 \times$, and D: 10,000×)

\subsection{PJM Tuning Parameters}

In the PJM operation of the RPP-WTP PEP testing, air pressure was used to drive liquid inside the PJM tubes to mobilize and mix tank contents. The data obtained in the test runs included liquid surface levels in steady periodic vertical motion inside the PJM tubes and the PJM tank, and the steady periodic driving PJM tube pressures. The measured liquid levels in the PJM tubes and the PJM tank as well as the driving pressure in the PJM tubes were used to determine the PJM jet velocity (peak average velocity). The measured liquid levels in the PJM tubes and the PJM tank were used to determine the average PJM stroke length. In the RPP-WTP PEP testing, the drive pressures and cycle times were iteratively adjusted to meet the target drive velocities and stroke lengths specified by the Test Instructions. Table 5.19 shows the PJM parameters for Integrated Test B, and Figure 5.2 through Figure 5.9 provide plots of the data used to calculate the peak average velocity. Appendix F provides details on how the PJM peak average velocities were determined. 
Table 5.19. PJM Parameters for Integrated Test B (TI-WTP-PEP-066)

\begin{tabular}{|c|c|c|c|c|c|c|c|c|c|c|}
\hline $\begin{array}{c}\text { Data Set } \\
\text { Date:Time } \\
(\mathrm{mm} / \mathrm{dd} / \mathrm{yr}) \\
(\mathrm{hr}: \mathrm{min})\end{array}$ & $\begin{array}{l}\text { TI step \# } \\
\text { (TI p\#) }\end{array}$ & Vessel & $\begin{array}{l}\text { PJM } \\
\text { operation } \\
\text { mode }\end{array}$ & $\begin{array}{c}\text { Process step } \\
\text { description }\end{array}$ & $\begin{array}{c}\text { Target PJM } \\
\text { nozzle } \\
\text { velocity } \\
(\mathrm{m} / \mathrm{s})\end{array}$ & $\begin{array}{c}\text { Actual PJM } \\
\text { nozzle } \\
\text { velocity } \\
(\mathrm{m} / \mathrm{s})\end{array}$ & $\begin{array}{l}\text { Target stroke } \\
\text { length } \\
\text { (in.) } \\
(\%)\end{array}$ & $\begin{array}{c}\text { Actual } \\
\text { stroke } \\
\text { length } \\
\text { (in.) } \\
(\%)\end{array}$ & $\begin{array}{c}\text { Target } \\
\text { PJM } \\
\text { cycle } \\
\text { time }(\mathrm{sec}) \\
\end{array}$ & $\begin{array}{l}\text { Actual } \\
\text { PJM cycle } \\
\text { time }(\mathrm{sec})\end{array}$ \\
\hline $\begin{array}{c}3 / 12 / 09 \\
11: 02-11: 17\end{array}$ & $\begin{array}{l}7.2 .6 \\
(p 36)\end{array}$ & Tank T01A & Standard & $\begin{array}{l}\text { After transfer from } \\
\text { HLP-VSL-T22 for } \\
\text { batch } 1\end{array}$ & $4.8 \pm 0.3$ & 4.7 & $\begin{array}{c}28.1 \pm 1.7 \\
80 \pm 5 \%\end{array}$ & $\begin{array}{l}28.6 " \\
82 \%\end{array}$ & $35 \pm 1$ & 35.2 \\
\hline $\begin{array}{c}3 / 12 / 09 \\
13: 50-14: 05\end{array}$ & 7.3.5.1 (p 40) & Tank T02A & Standard & $\begin{array}{l}\text { After transfer from } \\
\text { Tank T01A for batch } 1\end{array}$ & $7.3 \pm 0.4$ & 7.1 & $\begin{array}{c}30.3 \pm 1.8 \% \\
80 \pm 5 \%\end{array}$ & $\begin{array}{l}31.5 \% \\
83 \%\end{array}$ & $33 \pm 1$ & 33.2 \\
\hline $\begin{array}{l}\text { 3/13/09 } \\
\sim 06: 20\end{array}$ & $7.6 .20(\mathrm{p} 60)$ & Tank T02A & Simple & $\begin{array}{c}\text { Caustic leach @99 C } \\
\text { for batch } 1\end{array}$ & $7.3 \pm 0.4$ & 7.5 & $\begin{array}{c}30.3 \pm 1.8 \% \\
80 \pm 5 \%\end{array}$ & $\begin{array}{l}29.7 \% \\
78 \%\end{array}$ & $33 \pm 1$ & 33.2 \\
\hline $\begin{array}{c}3 / 14 / 09 \\
14: 05-14: 33\end{array}$ & $\begin{array}{l}7.8 .4 .1 \\
(\mathrm{p} 71)\end{array}$ & Tank T01B & Standard & $\begin{array}{l}\text { After transfer of leach } \\
\text { slurry from Tank T02A } \\
\text { for batch } 1\end{array}$ & $4.8 \pm 0.3$ & 5.1 & $\begin{array}{c}28.1 \pm 1.7 \\
80 \pm 5 \%\end{array}$ & $\begin{array}{l}20.4 \% \\
58 \%\end{array}$ & $35 \pm 1$ & 35.1 \\
\hline $\begin{array}{c}3 / 13 / 09 \\
13: 01-13: 19\end{array}$ & $\begin{array}{l}7.9 .7 \\
(p 75)\end{array}$ & Tank T01A & Standard & $\begin{array}{c}\text { After transfer from } \\
\text { HLP-VSL-T22 for } \\
\text { batch } 2\end{array}$ & $4.8 \pm 0.3$ & 4.6 & $\begin{array}{c}28.1 \pm 1.7 \\
80 \pm 5 \%\end{array}$ & $\begin{array}{l}22.4 ” \\
64 \%\end{array}$ & $35 \pm 1$ & 35.2 \\
\hline $\begin{array}{c}3 / 14 / 09 \\
14: 05-14: 33\end{array}$ & $\begin{array}{l}7.10 .5 .1 \\
(\mathrm{p} 78)\end{array}$ & Tank T02A & Simple & $\begin{array}{c}\text { After transfer from } \\
\text { Tank T01A for batch } 2\end{array}$ & $7.3 \pm 0.4$ & 6.6 & $\begin{array}{c}30.3 \pm 1.8 \\
80 \pm 5 \%\end{array}$ & $\begin{array}{l}27.5 \% \\
73 \%\end{array}$ & $33 \pm 1$ & 33.2 \\
\hline $\begin{array}{c}3 / 15 / 09 \\
20: 05-22: 04\end{array}$ & $\begin{array}{l}7.13 .19 .1 \\
(\mathrm{p} 96)\end{array}$ & Tank T02A & Simple & $\begin{array}{c}\text { Caustic leach @99 C } \\
\text { for batch } 2\end{array}$ & $7.3 \pm 0.4$ & 7.5 & $\begin{array}{c}30.3 \pm 1.8 \% \\
80 \pm 5 \%\end{array}$ & $\begin{array}{l}29.6 \% \\
78 \%\end{array}$ & $33 \pm 1$ & 33.2 \\
\hline $\begin{array}{c}3 / 19 / 09 \\
08: 25-11: 07\end{array}$ & $\begin{array}{l}7.15 .29 .1 \\
(p 116)\end{array}$ & Tank T02A & Standard & $\begin{array}{l}\text { After concentration to } \\
20-w t \% \text { UDS }\end{array}$ & $12 \pm 0.4$ & 11.7 & $\begin{array}{c}30.3 \pm 1.8 \\
80 \pm 5 \%\end{array}$ & $\begin{array}{c}26.7^{\prime} \\
70 \%\end{array}$ & $20 \pm 1$ & 20.2 \\
\hline
\end{tabular}


T01A at 11:09 on 3/ 12/ 09: Peak Average $=4.7(\mathrm{~m} / \mathrm{s})$

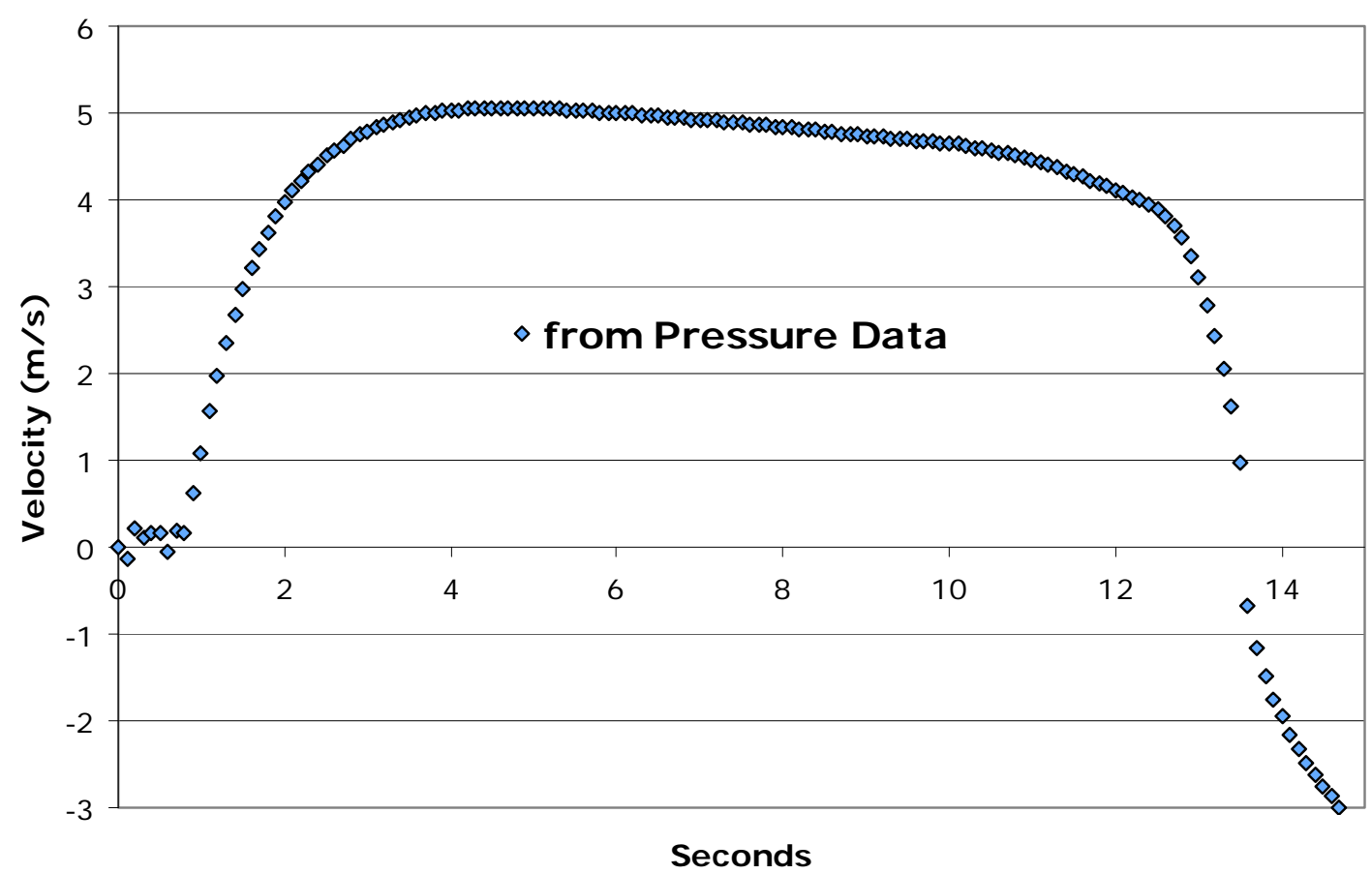

Figure 5.2. Peak Average Velocity in Tank T01A at 11:09 on 3/12/09

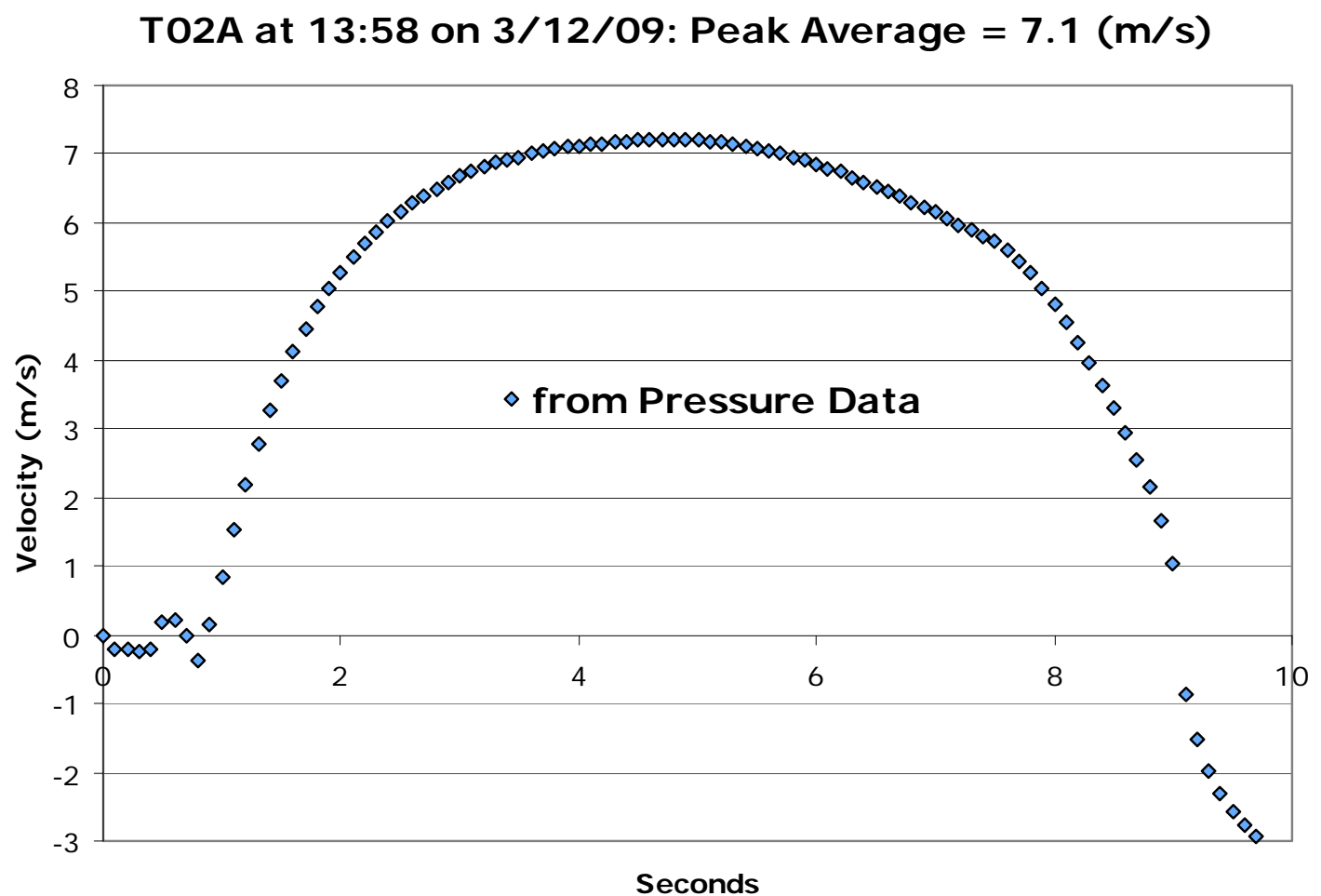

Figure 5.3. Peak Average Velocity in Tank T02A at 13:58 on 3/12/09 
T02A at 5:15AM on 3/ 13/ 09:Peak Average $=7.5(\mathrm{~m} / \mathrm{s})$

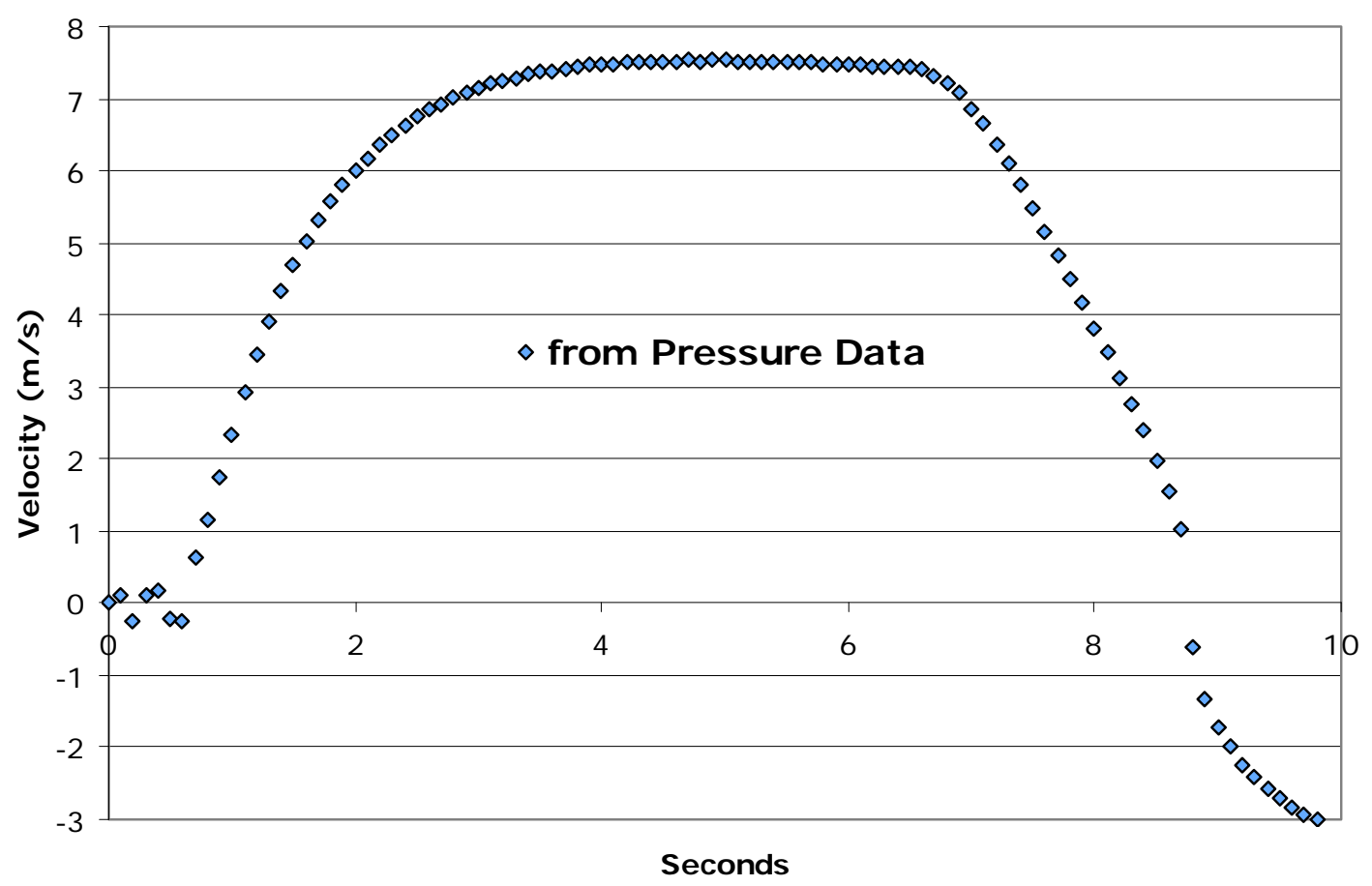

Figure 5.4. Peak Average Velocity in Tank T02A at $05: 15$ on 3/13/09

T01B at 14:26 on 3/ 14/ 09: Peak Average $=5.1(\mathrm{~m} / \mathrm{s})$

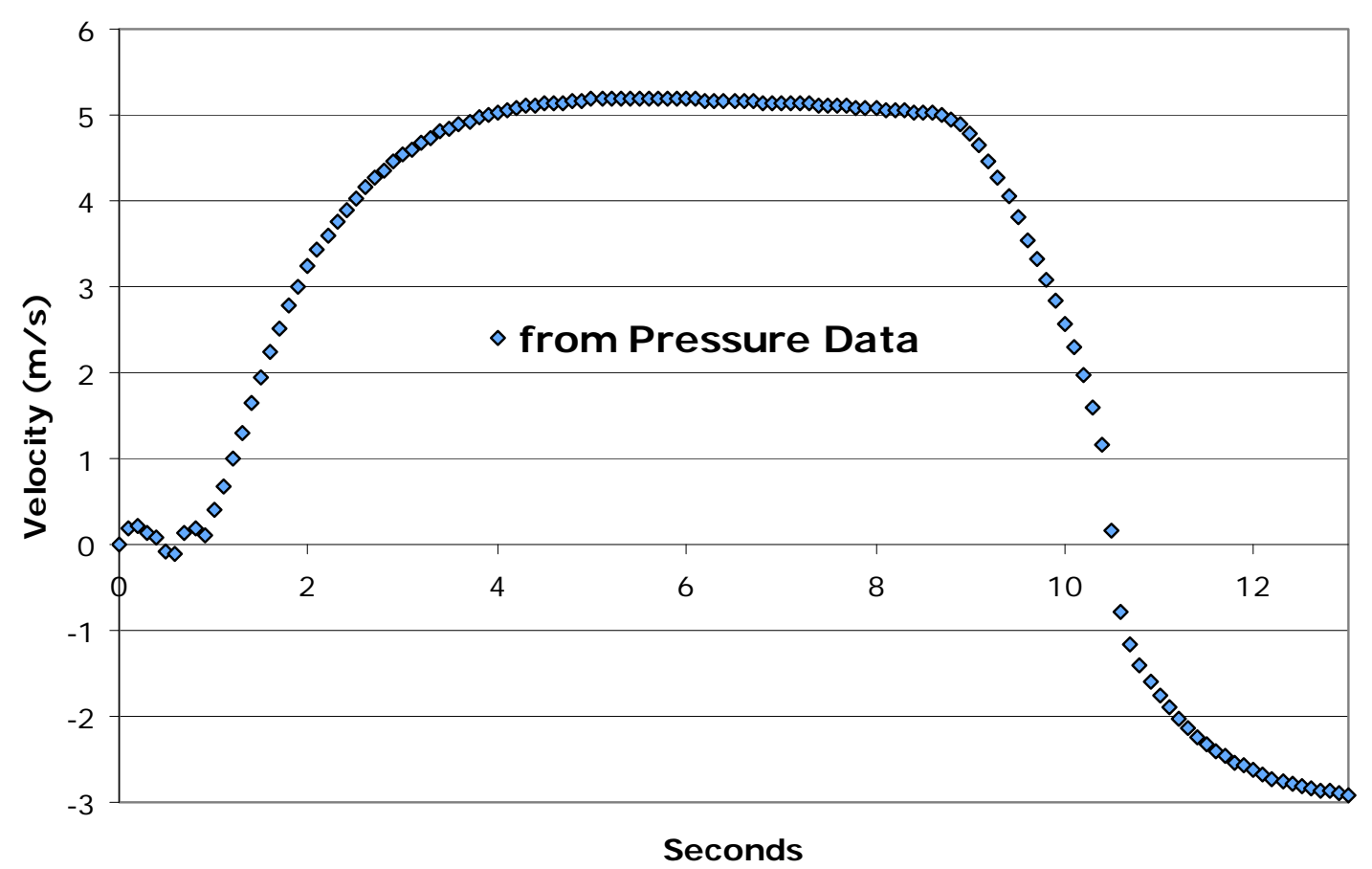

Figure 5.5. Peak Average Velocity in Tank T01B at $14: 26$ on $3 / 14 / 09$ 
T01A at 13:07 on 3/ 13/ 09: Peak Average $=4.6(\mathrm{~m} / \mathrm{s})$

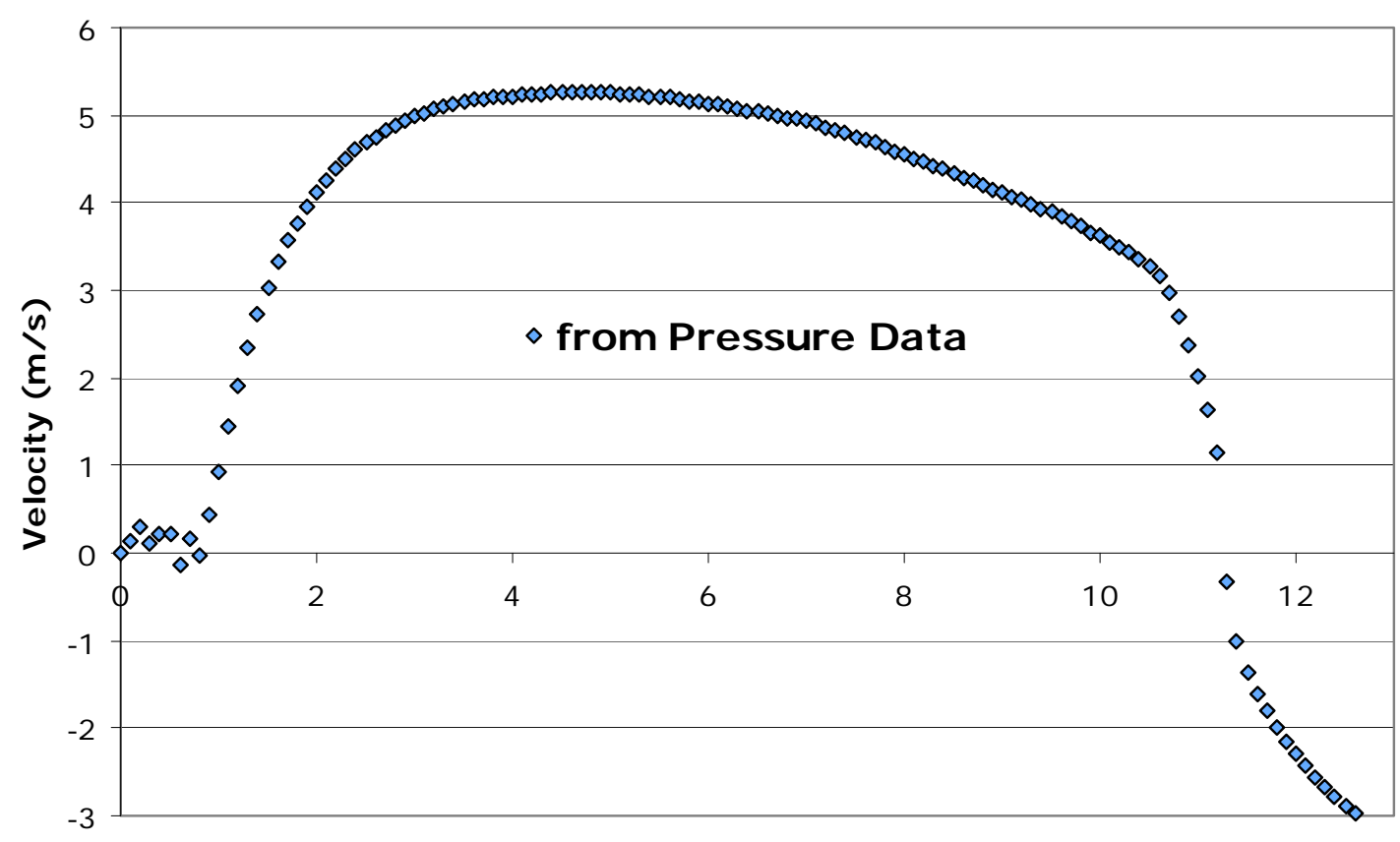

Seconds

Figure 5.6. Peak Average Velocity in Tank T01A at 13:07 on 3/13/09

T02A at 14:08 on 3/ 14/ 09: Peak Average $=6.6(\mathrm{~m} / \mathrm{s})$

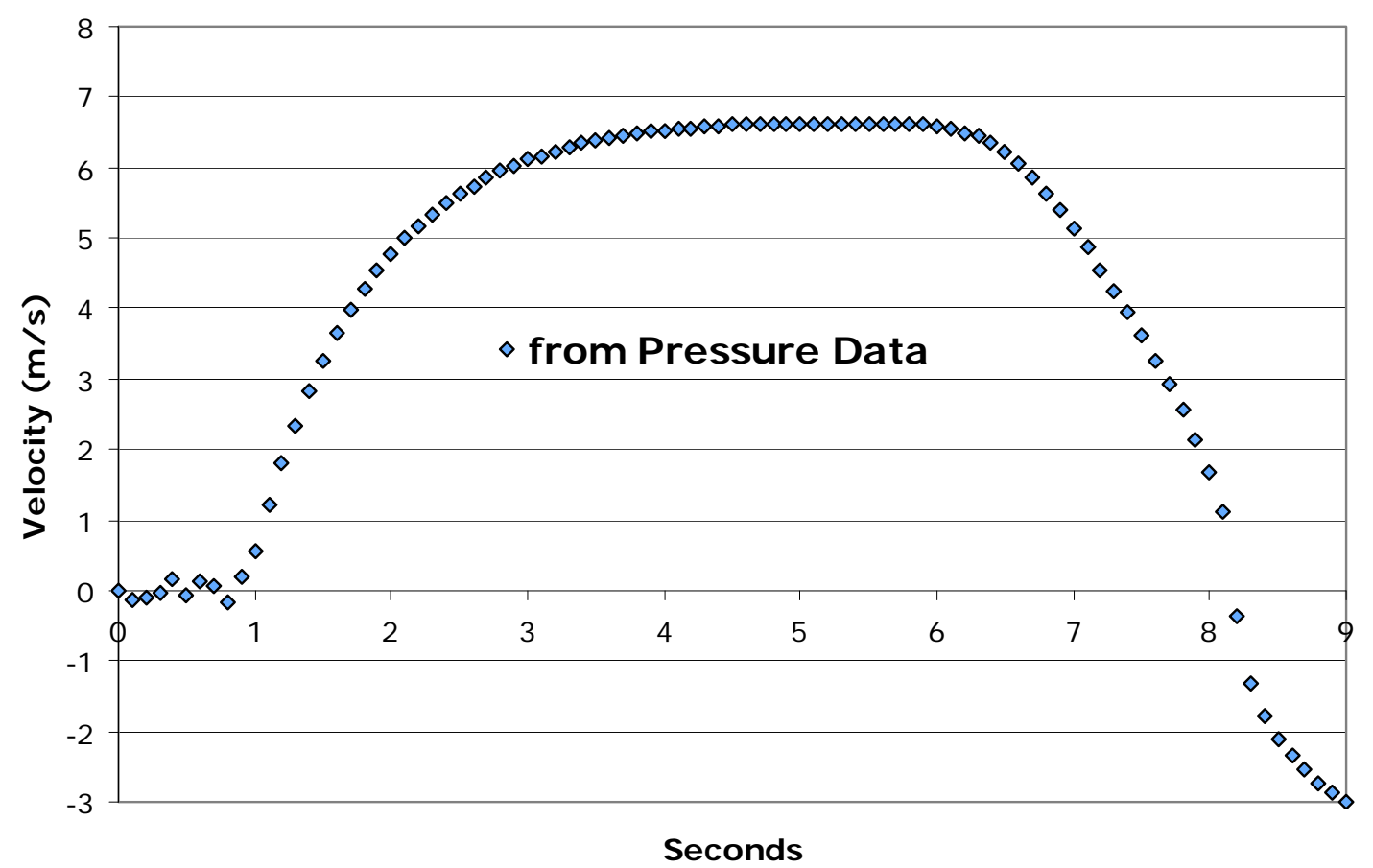

Figure 5.7. Peak Average Velocity in Tank T02A at 14:08 on 3/14/09 
T02A at 21:29 on 3/ 15/ 09: Peak Average $=7.5(\mathrm{~m} / \mathrm{s})$

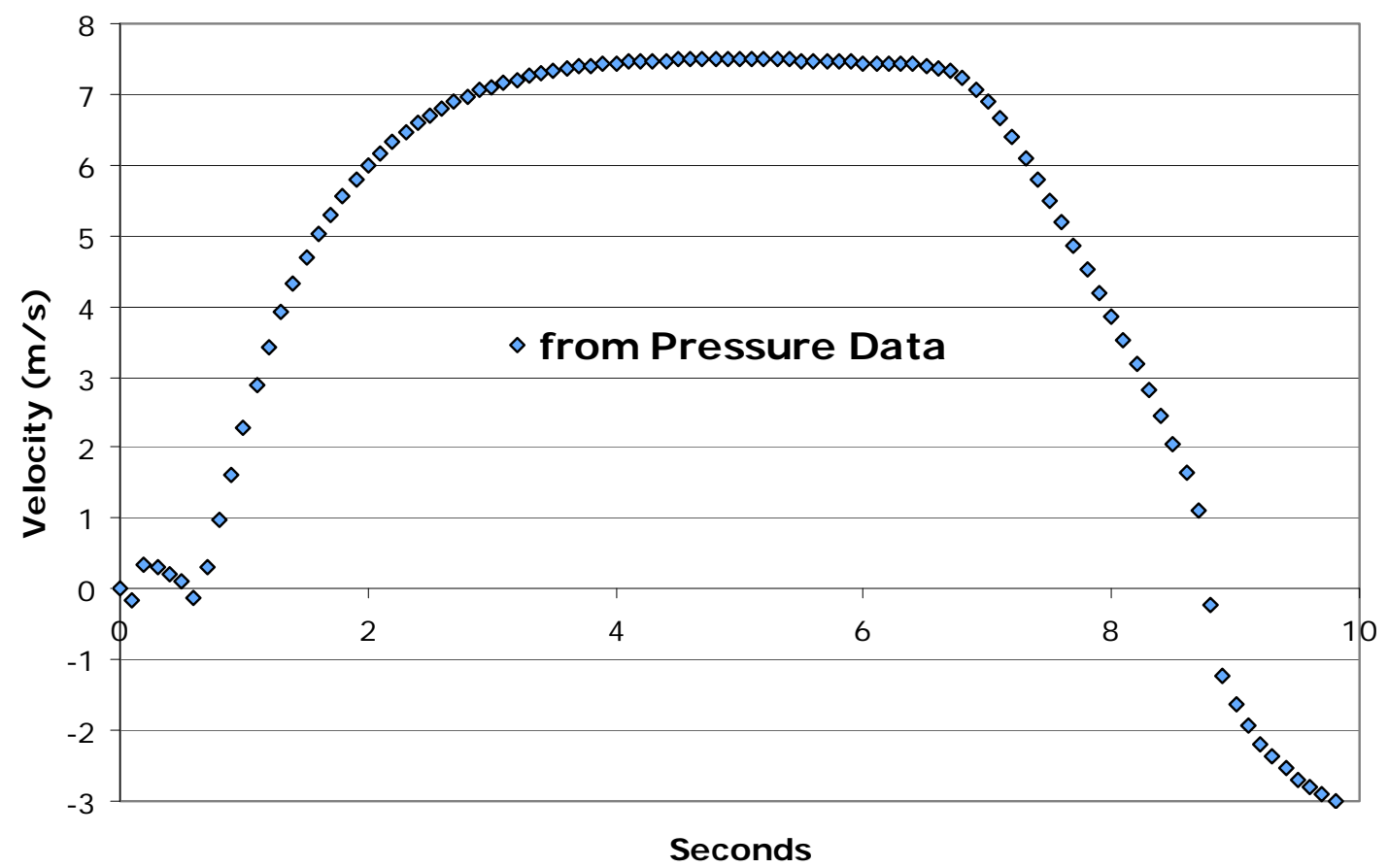

Figure 5.8. Peak Average Velocity in Tank T02A at 21:29 on 3/15/09

T02A at 10:35AM on 3/ 19/09:Peak Average $=11.7(\mathrm{~m} / \mathrm{s})$

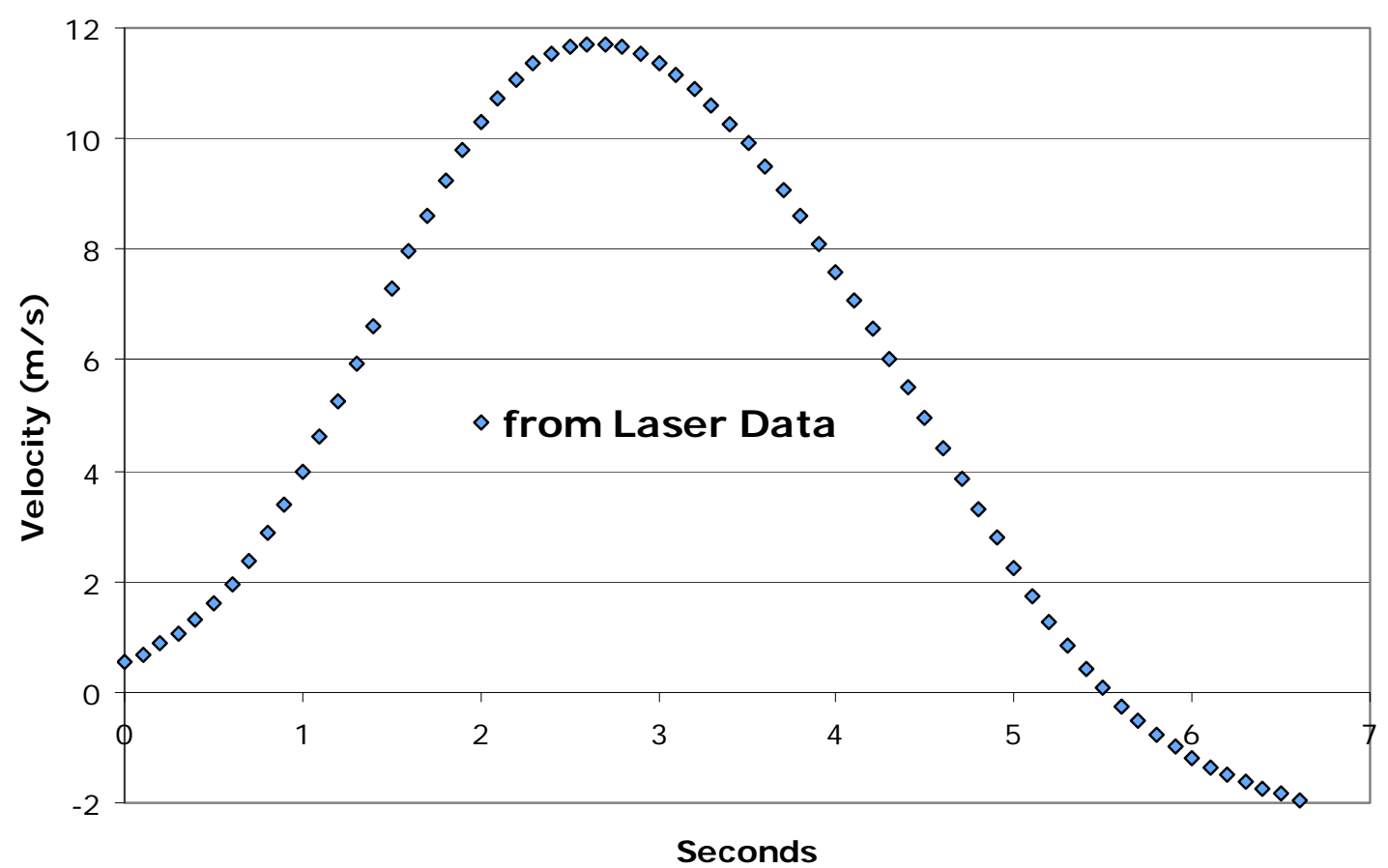

Figure 5.9. Peak Average Velocity in Tank T02A at 10:35 on 3/19/09 


\subsection{Issues}

\subsection{Maintaining Targeted Flow Rate in Filter-Loop}

It was not possible to meet the targeted flow rate of $109 \mathrm{gpm}$ when the level in the tank was below $\sim 22$ inches. This is the approximate level of the filter-loop return nozzle. The result is air entrainment into the slurry, which causes apparent cavitation and decreased performance of the pumps. (Actual cavitation is the formation of gas bubbles due to low pressure at the pump impeller, resulting in decreased pump performance.) The entrained gas would mostly release after the slurry returned to Tank T02A. A sample taken, when pumping performance was at issue, indicated $2.5 \mathrm{vol} \%$ air held up in the slurry. This was measured by comparing the volume before and after centrifugation. (A similar sample taken during Integrated Test A was found to have $\sim 7.5 \mathrm{vol} \%$ air held up in the slurry, and the degassing protocol that was applied improved pump performance.) However, the degassing protocol applied in Integrated Test B did not result in increased pumping rates, and it was concluded that air held up in the slurry was not at issue; rather, transient air entrainment due to the returning slurry impinging on the exposed slurry surface in Tank T02A was at issue.

Air entrainment is believed to cause an offset in flow rate readings for the magnetic flow meter upstream (FT-0623) and downstream (FT-0635) of the filter-loop pumps. The upstream flow meter (under vacuum) read higher flow rates than the downstream flow meter, which was under pressure. In Integrated Test B, the offset between the flow meters was much less severe than for Integrated Test A, with the conclusion being that air entrainment was less of an issue forIntegrated Test B than it was for Integrated Test A. It is believed that the two magnetic flow meters in the filter-loop provided volumetrically accurate readings, and the offset between the two (FT-0623 and FT-0635) was typically due to air entrainment. However, this issue was not studied.

\subsection{PJM Operation}

Standard Mode-The Drexelbrook level instruments in the PJMs (which determined how long pressure and vacuum to the PJMs would be applied) did not work at elevated temperatures in caustic solution. As a result, while heating during the caustic-leach step, the PJMs would have to be switched from standard mode to simple mode. (See Section 3.5 for PJM operational details.)

Simple Mode - In simple mode, pressure and vacuum are applied for predetermined time intervals. These time intervals are modified by tank level and temperature. These corrections generally worked, but operators would have to closely monitor the PJMs because these correction factors did not work perfectly. The PJMs would overblow (that is, pressurized air would be on too long, causing the air to come out the bottom of the PJM) and overdraw (that is vacuum being applied too long and drawing slurry up into the vacuum delivery piping). Both issues caused operational problems.

In Tank T02A, air from PJM overblows was drawn into the pump suction causing severe pump vibration. Because the pump flow rate in UFP-VSL-T01A/B was much lower, PJM overblows in these tanks did not present an immediate operational issue. 
Overdraws did not cause immediate problems, but could eventually be observed if there were any leaks in the air delivery piping. Also, it is expected that over long durations, this would cause plugging of the air supply line.

PJM-1605 (in Tank T01A) was inoperative during most of Integrated Test B and appeared to be plugged. An intentional overblow resolved the issue for approximately 1 hour. However, PJM-1605 appeared plugged again and was disabled when it could not be cleared by additional overblows. The source of the apparent plug could not be verified since this would have required disrupting the test. However, we suspect that the plug occurred in the air supply line above the PJM. The Drexelbrook level probes were inserted through the air line at the top of the PJM to access the inside of the pulse-tubes. A plastic spacer was added to the level probe to stabilize it. This spacer provided significant constriction in the air supply line a few inches above the top of the PJM tube. It is likely that some of the simulant caked around the level probe spacer and eventually plugged the air supply line. Extensive post-test inspections did not reveal any plug in the air line or the PJM nozzle. A simulant plug would likely have been rinsed out by the flushing operations that were conducted before the inspection. The inspection also verified that the air supply was properly functioning, including the valves and the regulator. The level probe was also verified to work properly. This problem is not expected to occur in plant PJMs because they do not contain the Drexelbrook level probes and spacer. In addition, the plant has a wash-down system for the air line that the PEP did not have.

\subsection{Leaks}

The PEP was subject to periodic leaks. Leaks reported during Integrated Test B follow:

1. Leak in Pump T42A suction gasket

2. Leak in site glass of seal pot for Pump T43A

3. Leak in acid line gasket $(08: 00,3 / 14 / 09)$.

Item 1 delayed testing for 1 hour and 12 minutes. During this time, the temperature of Tank T02A had cooled from $71^{\circ} \mathrm{C}$ to $64^{\circ} \mathrm{C}$. For items 2 and 3, crafts were able to repair these leaks in a timely manner with no impact on the test or results.

\subsection{Instruments}

\subsubsection{Lasers Level Measurement}

The issues with the laser reported in Integrated Test $\mathrm{A}^{(\mathrm{a})}$ were largely corrected for Integrated Test B. Two software filters were used to remove spurious readings. The first was an out-of-range filter that ignored any high or low readings that were out of range. The second filter ignored readings when the rate of change was greater than 3 inches in 0.5 seconds.

(a) Laser performance was unreliable throughout Integrated Test A efforts, but especially during the leaching and post-caustic-leach concentration. One of the symptoms of unstable laser level performance was high and low level spikes, which interrupted steam injection, PJM, and pump operation. When concentrating post-caustic-leach slurry, the UFP-VSL-T02A low-level spikes erroneously triggered batches from the feed vessel and/or shut down PJMs and filter-loop pumps. 
Two configurational changes, initiated in Integrated Test A, were also used in Integrated Test B. The first was to open a tank vent, which allowed air to sweep through the tank head, effectively reducing the aerosol concentration. The second was to remove the laser vent plugs, which allowed air to flow directly past the lens.

It is believed that the laser level instruments read level within their calibration tolerance ( 0.25 inches) and were considered the most reliable level measurement during testing. Mixing of the slurry, via the PJMs or from pump recirculation, resulted in the slurry surface rising and falling and caused variability in laser readings. Also, it is believed that the lasers read the air/foam interface rather than the air/liquid interface. Consequently, the laser readings were biased high when foam was present. The effect of these phenomena on instrument accuracy was not studied as part of testing.

\subsubsection{Bubblers Level/Density Measurement}

Bubblers were susceptible to plugging on a regular basis. The plugs prevented accurate determination of the slurry level and density in the vessels. Blowing high pressure air through the bubbler piping, which was sometimes followed by water (steam condensate and IW were used for bubbler cleaning), left bubblers operational - sometimes. When bubbler cleaning was successful, the duration of functionality was unpredictable.

Another issue with the bubbler is that their readings in Tank T02A were affected when the filter-loop pumps (UFP-PMP-T42A and/or UFP-PMP-T43A) were running. The flow from these pumps would cause pressure changes at the bubbler dip legs. Consequently, the bubbler level and density readings were wrong when the filter-loop pumps were on. The bubblers in Tanks T01A/B were not influenced by the recirculation pumps in these tanks because of lower flow or better placement within the tank.

The lesson learned on the bubblers is that they need to be equipped with cleaning capability, such as steam and/or water, and the placement of the dip legs should be chosen (or engineered) so as not to be influenced by moving liquid within the tank.

\subsubsection{Drexelbrook Level Measurement}

The Drexelbrook level indicators in the PJMs did not function properly at elevated temperatures and elevated caustic solutions. During the temperature ramp from ambient to $98^{\circ} \mathrm{C}$ in both batches, the PJMs quit functioning in standard mode (which relied on the Drexelbrook to hit vacuum and drive level set points) and had to be switched to simple mode (which did not rely on the Drexelbrook level).

\subsubsection{ABB Swirl Flow Meter}

Swirl flow meters require a minimum Reynolds number to provide an accurate flow rate. The flow meters are programmed to output zero flow rate below the minimum Reynolds number. At issue in the PEP was that the ABB swirl flow meters were susceptible to ambient vibration (from pumps, people walking in close proximity to the flow meter, etc.) causing some of the PEP swirl flow meters to read flow when there was none in the pipe. The flow meters were used for totalizing inputs and could have some error. Steps were taken to reduce the vibration by isolating the swirl flow meters. Such steps 
reduced, but did not eliminate, errant readings. Swirl flow meter DAS data should be compared against the Test Instruction to validate whether flow indicated is actual or due to vibration. 


\subsection{References}

10 CFR 830, Energy/Nuclear Safety Management, Subpart A-Quality Assurance Requirements

Baldwin DL, PP Schonewill, JJ Toth, JL Huckaby, PW Eslinger, BD Hanson, DE Kurath, and MJ Minette. 2009. EFRT M12 Issue Resolution: Solids Washing. WTP-RPT-187, PNNL-18499, Pacific Northwest National Laboratory, Richland, Washington.

Barnes HA and NQ Dzuy. 2001. "Rotating vane rheometry - a review.” J. Non-Newtonian Fluid Mech., 98, 1-14.

Daniel RC, JM Billing, JR Bontha, CF Brown, PW Eslinger, BD Hanson, JL Huckaby, NK Karri, ML Kimura, DE Kurath, and MJ Minette. 2009. EFRT M12 Issue Resolution: Comparison of Filter Performance at PEP and CUF Scale. WTP-RPT-185, PNNL-18498, Pacific Northwest National Laboratory, Richland, Washington.

Guzman-Leong CE, OP Bredt, CA Burns, RC Daniel, YF Su, JGH Geeting, EC Golovich, GB Josephson, DE Kurath, GJ Sevigny, DM Smith, PLJ Valdez, ST Yokuda, and JK Young. 2009. PEP Run Report for Integrated Test A; Caustic Leaching in UFP-VSL-T01A, Oxidative Leaching in UFP-VSL-T02A. WTP-RPT-191, PNNL-18755, Pacific Northwest National Laboratory, Richland, Washington.

Josephson GB, JGH Geeting, OP Bredt, CA Burns, EC Golovich, CE Guzman-Leong, DE Kurath, and GH Sevigny. 2009. PEP Run Report for Simulant Shakedown/Functional Testing. WTP-RPT-190, PNNL-18823, Pacific Northwest National Laboratory, Richland, Washington.

Kuhn WL, ST Arm, JL Huckaby, DE Kurath, SD Rassat. 2008. Technical Basis for Scaling Relationships for the Pretreatment Engineering Platform. WTP-RPT-160, PNNL-16948, Pacific Northwest National Laboratory, Richland, Washington.

Kurath DE, BD Hanson, MJ Minette, DL Baldwin, BM Rapko, LA Mahoney, PP Schonewill, RC Daniel, PW Eslinger, JL Huckaby, JM Billing, PS Sundar, GJ Josephson, JJ Toth, ST Yokuda, EBK Baer, SM Barnes, EC Golovich, SD Rassat, CF Brown, JGH Geeting, GJ Sevigny, JR Bontha, RL Aaberg, PM Aker, CE Guzman-Leong, ML Kimura, SK Sundaram, RP Pires, BE Wells, and OP Bredt. 2009. Pretreatment Engineering Platform Phase I Final Test Report. WTP-RPT-197, PNNL-18894, Pacific Northwest National Laboratory, Richland, Washington.

Mahoney, LA, SD Rassat, PW Eslinger, RL Aaberg, PM Aker, EC Golovich, BD Hanson, TS Hausmann, JL Huckaby, DE Kurath, MJ Minette, SK Sundaram, ST Yokuda. 2009. EFRT M-12 Issue Resolution: Caustic-Leach Rate Constants from PEP and Laboratory-Scale Tests Process. WTP-RPT-186, PNNL-18502, Pacific Northwest National Laboratory, Richland, Washington.

Rapko BM, GJ Lumetta, JR Deschane, and RA Peterson. 2007. Process Development for Permanganate Addition During Oxidative Leaching of Hanford Tank Sludge Simulants. WTP-RPT-164, PNNL-16794, Pacific Northwest National Laboratory, Richland, Washington. 
Rapko BM, CF Brown, PW Eslinger, MS Fountain, TS Hausmann, JL Huckaby, BD Hanson, DE Kurath, and MJ Minette. 2009. EFRT M12 Issue Resolution: Comparison of PEP and Bench-Scale Oxidative Leaching Results. WTP-RPT-188, PNNL-18500, Pacific Northwest National Laboratory, Richland, Washington.

Russell RL, RA Peterson, HD Smith, DE Rinehart, PM Aker, and EC Buck. 2009a. Development and Characterization of Boehmite Component Simulant. WTP-RPT-184, PNNL-18176, Pacific Northwest National Laboratory, Richland, Washington.

Russell RL, Peterson RA, DE Rinehart, HD Smith. 2009b. Development and Characterization of Gibbsite Component Simulant. WTP-RPT-176, PNNL-18013, Pacific Northwest National Laboratory, Richland Washington.

Russell RL, JM Billing, RA Peterson, DE Rinehart, and HD Smith. 2009c. Development and Demonstration of Ultrafiltration Simulants. WTP-RPT-183, PNNL-18090, Pacific Northwest National Laboratory, Richland, Washington.

Sevigny GJ, OP Bredt, CA Burns, DE Kurath, JGH Geeting, EC Golovich, CE Guzman-Leong, and GB Josephson. 2009. PEP Integrated Test D Run Report-Caustic and Oxidative Leaching in UFP-VSL-T02A. WTP-RPT-193, PNNL-18741, Pacific Northwest National Laboratory, Richland, Washington. 
Appendix A

Instrument Summary 



\section{Appendix A: Instrument Summary}

This table provides a partial list of instruments used in the PEP. The interested reader is referred to the P\&IDs and the M\&TE list for a complete list of instruments.

\begin{tabular}{|c|c|c|c|c|c|c|}
\hline \multirow[b]{2}{*}{ Component } & \multicolumn{3}{|l|}{ Instrument } & \multicolumn{3}{|c|}{ Tolerance } \\
\hline & ID & Min & Max & Units & $( \pm)$ & Notes \\
\hline \multicolumn{7}{|l|}{ Vessel T02A } \\
\hline T02A level (bubbler) & LT-0611 & 0.00 & 200.00 & in $\mathrm{H}_{2} \mathrm{O}$ & 0.15 & \\
\hline T02A density (bubbler) & DT-0612 & 0.00 & 200.00 & in $\mathrm{H}_{2} \mathrm{O}$ & 0.15 & \\
\hline T02A level (laser) & LT-0614 & -3.95 & 96.00 & in & 0.25 & \\
\hline T02A prototypic temperature & TTK-0619 & 10.00 & 110.00 & $\mathrm{C}$ & 2 & \\
\hline \multicolumn{7}{|l|}{ T02A Temperature Array } \\
\hline $\mathrm{x}=\mathrm{A}, \mathrm{B}, \mathrm{D}, \mathrm{E}, \mathrm{F}, \mathrm{G}, \mathrm{H}, \mathrm{J}, \mathrm{K}, \mathrm{L}$ & TTx-0619 & 10.00 & 110.00 & $\mathrm{C}$ & 2 & \\
\hline $\mathrm{x}=\mathrm{A}, \mathrm{B}, \mathrm{D}, \mathrm{E}, \mathrm{F}, \mathrm{G}, \mathrm{H}, \mathrm{J}, \mathrm{K}, \mathrm{L}$ & TTx-0621 & 10.00 & 110.00 & $\mathrm{C}$ & 2 & \\
\hline$x=1-7$ & N39-x & 10.00 & 110.00 & $\mathrm{C}$ & 2 & \\
\hline$x=1-7$ & $\mathrm{~N} 41-\mathrm{x}$ & 10.00 & 110.00 & $\mathrm{C}$ & 2 & \\
\hline$x=1-7$ & N42-X & 10.00 & 110.00 & $\mathrm{C}$ & 2 & \\
\hline$x=1-7$ & N52-X & 10.00 & 110.00 & $\mathrm{C}$ & 2 & \\
\hline \multicolumn{7}{|l|}{ HX-T02A } \\
\hline HX-T02A cooling water flow & FT-0541 & 0.00 & 70.00 & gpm & 0.88 & \\
\hline $\begin{array}{l}\text { HX-T02A cooling water inlet } \\
\text { temperature }\end{array}$ & TT-0539 & 0.00 & 110.00 & $\mathrm{C}$ & 1.8 & \\
\hline HX-T02A cooling water outlet & TT-0540 & 0.00 & 110.00 & $\mathrm{C}$ & 1.8 & \\
\hline $\begin{array}{l}\text { HX-T02A process fluid inlet } \\
\text { temperature }\end{array}$ & TT-0537 & 0.00 & 110.00 & $\mathrm{C}$ & 1.8 & $\begin{array}{l}\text { For Information Only } \\
\text { re: NCR } 42402.1\end{array}$ \\
\hline $\begin{array}{l}\text { HX-T02A process fluid outlet } \\
\text { temperature }\end{array}$ & TT-0513 & 0.00 & 110.00 & $\mathrm{C}$ & 1.8 & $\begin{array}{l}\text { For Information Only } \\
\text { re: NCR 42402.1 }\end{array}$ \\
\hline \multicolumn{7}{|l|}{ Pumps T42A/T43A } \\
\hline $\begin{array}{l}\text { T42A pump discharge pressure } \\
\text { (T43A suction) }\end{array}$ & PT-0633 & 0.00 & 348.00 & psig & 0.26 & \\
\hline T42A pump inlet flow & FT-0623 & 0.00 & 220.00 & gpm & 1.6 & \\
\hline T42A pump speed & ST-0627 & 0.00 & 1785.00 & rpm & 4 & \\
\hline T42A pump suction pressure & PT-0625 & -260.00 & 260.00 & in $\mathrm{H} 2 \mathrm{O}$ & 0.4 & \\
\hline T42A pump motor HP & JT-0629 & 0.00 & 100.00 & HP & 1.5 & For Information Only \\
\hline T43A outlet temperature & TT-0791 & 0.00 & 110.00 & $\mathrm{C}$ & 1.8 & $\begin{array}{l}\text { Should not be used as } \\
\text { an indicator of } \\
\text { temperature re: NCR } \\
42402.1\end{array}$ \\
\hline T43A pump discharge pressure & PT-0739 & 0.00 & 348.00 & psig & 0.26 & \\
\hline T43A pump outlet flow & FT-0635 & 0.00 & 220.00 & gpm & 1.6 & \\
\hline T43A pump speed & ST-0639 & 0.00 & 1785.00 & $\mathrm{rpm}$ & 4 & \\
\hline T43A pump motor HP & JT-0637 & 0.00 & 100.00 & HP & 1.5 & For Information Only \\
\hline \multicolumn{7}{|l|}{$\begin{array}{c}\text { Pumps Air Entrainment Trouble } \\
\text { Shoot }\end{array}$} \\
\hline $\begin{array}{l}\text { Air purge flow to steam sparger } \\
\text { FT-1995 }\end{array}$ & FT-1995 & 0.00 & 0.73 & $\mathrm{~kg} / \mathrm{min}$ & 0.02 & $\begin{array}{l}\text { Accuracy revised at } \\
\text { flow rates below } 0.090 \\
\mathrm{~kg} / \mathrm{min} \text { re: NCR } \\
38767.1(0.00045 \\
\mathrm{kg} / \mathrm{min} / \text { flow rate in } \\
\mathrm{kg} / \mathrm{min} \times 100 \%)\end{array}$ \\
\hline
\end{tabular}




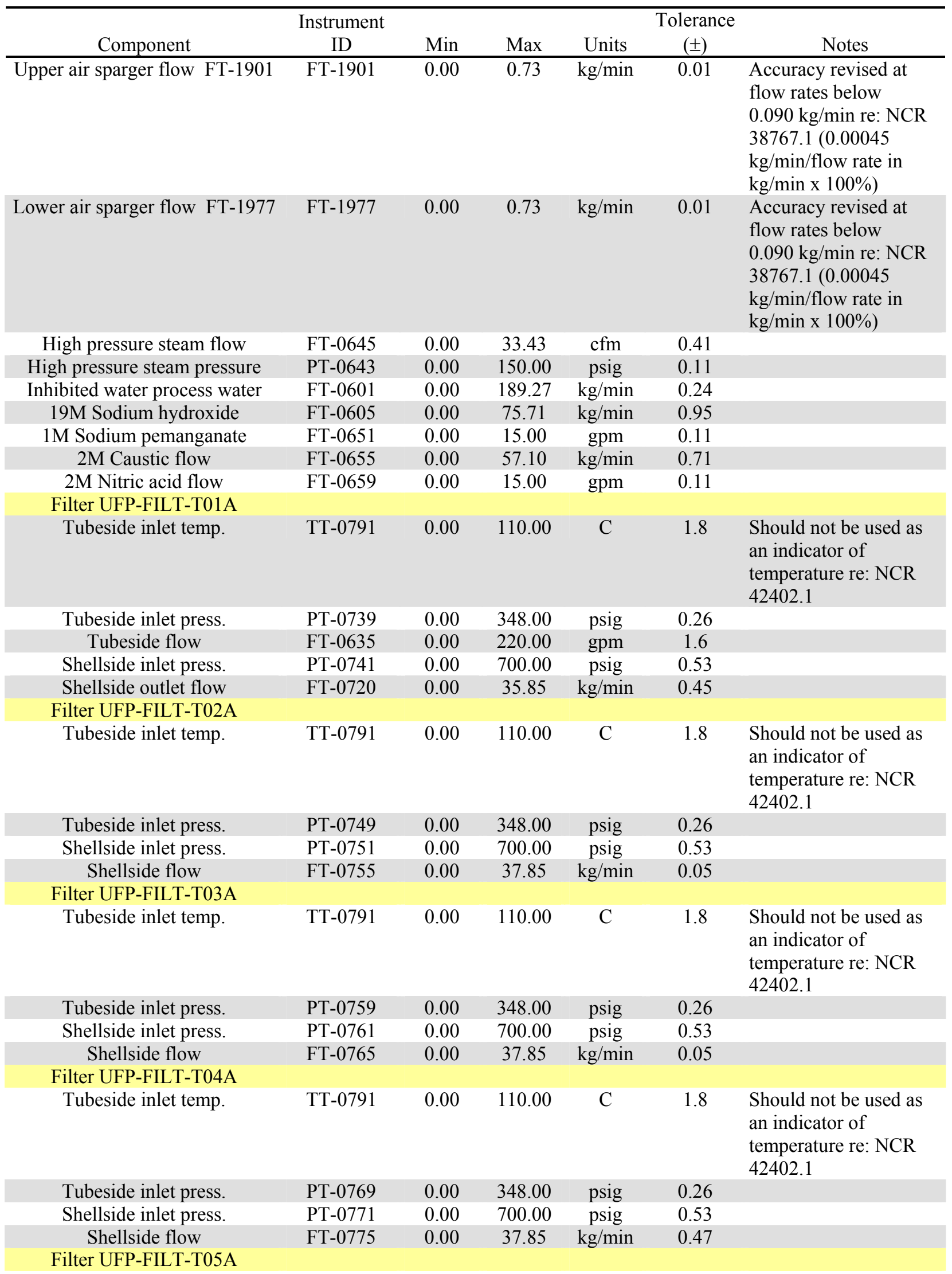




\begin{tabular}{|c|c|c|c|c|c|c|}
\hline \multirow[b]{2}{*}{ Component } & \multicolumn{3}{|l|}{ Instrument } & \multicolumn{3}{|c|}{ Tolerance } \\
\hline & ID & Min & $\operatorname{Max}$ & Units & $( \pm)$ & Notes \\
\hline Tubeside inlet temp. & TT-0791 & 0.00 & 110.00 & $\mathrm{C}$ & 1.8 & $\begin{array}{l}\text { Should not be used as } \\
\text { an indicator of } \\
\text { temperature re: NCR } \\
42402.1\end{array}$ \\
\hline Tubeside inlet press. & PT-0779 & 0.00 & 348.00 & psig & 0.26 & \\
\hline Tubeside outlet press. & PT-0789 & 0.00 & 348.00 & psig & 0.26 & \\
\hline Shellside inlet press. & PT-0781 & 0.00 & 700.00 & psig & 0.53 & \\
\hline Shellside flow & FT-0785 & 0.00 & 37.85 & $\mathrm{~kg} / \mathrm{min}$ & 0.24 & \\
\hline \multicolumn{7}{|l|}{ Pulse pot UFP-PP-T03A } \\
\hline Level & LT-0733 & 0.00 & 44.00 & inches & 0.1 & For Information Only \\
\hline Permeate flow from FILT T01A & FT-0720 & 0.00 & 35.85 & $\mathrm{~kg} / \mathrm{min}$ & 0.45 & \\
\hline Inlet pressure & PT-0731 & 0.00 & 700.00 & psig & 0.53 & \\
\hline Outlet pressure & PT-0741 & 0.00 & 700.00 & psig & 0.53 & \\
\hline \multicolumn{7}{|l|}{ Pulse pot UFP-PP-T02A } \\
\hline Level & LT-0725 & 0.00 & 44.00 & inches & 0.1 & For Information Only \\
\hline Permeate flow from FILT T02A & FT-0755 & 0.00 & 37.85 & $\mathrm{~kg} / \mathrm{min}$ & 0.05 & \\
\hline Permeate flow from FILT T04A & FT-0775 & 0.00 & 37.85 & $\mathrm{~kg} / \mathrm{min}$ & 0.47 & \\
\hline Inlet pressure & PT-0723 & 0.00 & 700.00 & psig & 0.53 & \\
\hline Outlet pressure to FILT T02A & PT-0751 & 0.00 & 700.00 & psig & 0.53 & \\
\hline Outlet pressure to FILT T04A & PT-0771 & 0.00 & 700.00 & psig & 0.53 & \\
\hline \multicolumn{7}{|l|}{ Pulse pot UFP-PP-T01A } \\
\hline Level & LT-0717 & 0.00 & 44.00 & inches & 0.1 & For Information Only \\
\hline Permeate flow from FILT T03A & FT-0765 & 0.00 & 37.85 & $\mathrm{~kg} / \mathrm{min}$ & 0.05 & \\
\hline Permeate flow from FILT T05A & FT-0785 & 0.00 & 37.85 & $\mathrm{~kg} / \mathrm{min}$ & 0.24 & \\
\hline Inlet pressure & PT-0715 & 0.00 & 700.00 & psig & 0.53 & \\
\hline Outlet pressure to FILT T03A & PT-0761 & 0.00 & 700.00 & psig & 0.53 & \\
\hline Outlet pressure to FILT T05A & PT-0781 & 0.00 & 700.00 & psig & 0.53 & \\
\hline
\end{tabular}



Appendix B

\section{Backpulse Description}





\section{Appendix B: Backpulse Description}

Backpulse Description:

The backpulsing function of the filter-loop can be operated only when actively filtering T02A contents. There are three variables that can be set by the operator: Level Drain Set Point, Backpulse Pressure Set Point, and Pressure Deadband for Completion.

The Level Drain Set Point is the height of fluid in the pulse-pot used for the backpulse.

The Backpulse Pressure Set Point is the amount above the filter inlet pressure that the pulse-pot should be charged to (i.e., if the inlet pressure is 100 psig and the Backpulse Pressure Set Point is set to $40 \mathrm{psig}$, the control system will charge the pulse-pot to $140 \mathrm{psig}$ ).

The Pressure Deadband for Completion is the amount above the filter inlet pressure that will cause the backpulse to be marked as finished (i.e., if the inlet pressure is 100 psig and the Deadband is set to $10 \mathrm{psig}$, the backpulse will complete when the pulse-pot pressure indicator gets down to $110 \mathrm{psig}$ ).

During filtering, the operator presses the "Start Backpulse" button in the HMI, which initiates the backpulse cycle. The first step is to close all valves entering and leaving the pulse-pot. Next, the high pressure air line is opened, and the pulse-pot is pressurized to $50 \mathrm{psig}$. The high pressure air valve is closed, and the drain valve to T62A/B is opened. The pulse-pot fluid level falls until reaching the Level Drain Set Point when the drain valve is closed. The high pressure air valve is opened again and pressurizes the pulse-pot to the sum of the filter inlet pressure plus the Backpulse Pressure Set Point $(100 \mathrm{psig}+40 \mathrm{psig}=140 \mathrm{psig}$ in the above example). The air valve is closed and the backpulse cycle pauses for 15 seconds. The fast acting valve then opens, and the pressure in the pulse-pot pushes fluid back through the filter until the pressure in the pulse-pot is equal to the filter inlet pressure plus the Deadband (100 psig $+10 \mathrm{psig}=110 \mathrm{psig}$ in the above example). The final step is to return to filtering conditions. The fast acting valve closes and the filter outlet valve and pulse-pot outlet valve to T62A/B (not the drain valve) are opened. 

Appendix C

Sample List and Naming Convention 



\section{Appendix C: Sample List and Naming Convention}

\subsection{Sample Naming Convention}

Every sample was given a unique sample name. The sample names were a composite of either six or seven separate descriptors. The descriptors are defined in Table C.1, and the acronyms for each descriptor are defined in Table C.2. The spacings between descriptors were separated by either an underscore symbol or a space. Sample naming examples are provided after Table C.1.

Table C.1. Sample Naming Nomenclature Definitions

\begin{tabular}{|c|c|c|}
\hline Descriptor & Identification & Explanation \\
\hline Descriptor 1 & Test Name & $\begin{array}{l}\text { Refers to the Shakedown Test, Functional Test, Integrated } \\
\text { Test A, Integrated Test B, or Integrated Test D. }\end{array}$ \\
\hline Descriptor 2 & Location & $\begin{array}{l}\text { Identifies the location that the sample is being collected from } \\
\text { based on Table } 3 \text {. For example, a sample collected from a } \\
\text { tank will include the acronym of the tank followed by the } \\
\text { location within the tank. }\end{array}$ \\
\hline Descriptor 3 & Test Process Step & $\begin{array}{l}\text { Refers to the process step as identified in the sample collection } \\
\text { and analysis table in the governing Test Instruction. The test } \\
\text { process step includes an identifier for processes that are } \\
\text { repeated during testing. }\end{array}$ \\
\hline Descriptor 4 & Sequential Number & $\begin{array}{l}\text { Unique number identifier that increments by } 1 \text { each time a } \\
\text { sample was collected. All sub-samples and separated samples } \\
\text { generated from the original sample had the same unique } \\
\text { number identifier. }\end{array}$ \\
\hline Descriptor 5 & Sample Routing & $\begin{array}{l}\text { Refers to the sample destination, type of analysis, storage, or } \\
\text { archive. }\end{array}$ \\
\hline Descriptor 6 & Store code & Final destination code. \\
\hline Descriptor 7 & $\begin{array}{l}\text { Separated-sample } \\
\text { identifier }\end{array}$ & $\begin{array}{l}\text { Refers to the type of sub-sample; e.g., decantate, solids, rinse } \\
\text { solution, etc. For a separated-sample, a descriptor of the } \\
\text { matrix was added after the sample routing identifier. }\end{array}$ \\
\hline
\end{tabular}


Table C.2. Sample Naming Acronyms

\begin{tabular}{|c|c|}
\hline Acronym & Definition \\
\hline \multicolumn{2}{|c|}{ Test Name (1 character) Descriptor 1} \\
\hline $\mathrm{S}$ & Shakedown testing \\
\hline $\mathrm{F}$ & Functional testing \\
\hline A & First integrated test to be performed \\
\hline B & Second integrated test to be performed \\
\hline $\mathrm{C}$ & Third integrated test to be performed \\
\hline $\mathrm{D}$ & Fourth integrated test to be performed \\
\hline \multirow{2}{*}{\multicolumn{2}{|c|}{$\begin{array}{l}\text { Location (maximum of } 5 \text { characters) Descriptor } 2 \\
\text { Vessel Name ( } 3 \text { characters) }\end{array}$}} \\
\hline & \\
\hline $\mathrm{T} 22$ & HLP-VSL-T22 \\
\hline FRP & FRP-VSL-T01 \\
\hline FEP & FEP-VSL-T01 \\
\hline $01 \mathrm{~A}$ & UFP-VSL-T01A \\
\hline 01B & UFP-VSL-T01B \\
\hline $02 \mathrm{~A}$ & UFP-VSL-T02A \\
\hline $\mathrm{T} 27$ & HLP-VSL-T27A \\
\hline $62 \mathrm{~A}$ & UFP-VSL-T62A \\
\hline $62 \mathrm{~B}$ & UFP-VSL-T62B \\
\hline \multicolumn{2}{|c|}{ Vessel Sampling Location (2 characters) } \\
\hline $\mathrm{IH}$ & Inner High \\
\hline IM & Inner Middle \\
\hline IL & Inner Low \\
\hline $\mathrm{MH}$ & Middle High \\
\hline MM & Middle Middle \\
\hline ML & Middle Low \\
\hline $\mathrm{OH}$ & Outer High \\
\hline $\mathrm{OM}$ & Outer Middle \\
\hline OL & Outer Low \\
\hline RL & Recirculation line \\
\hline GT & Grab sample at top of a tank \\
\hline GM & Grab sample at middle of a tank \\
\hline GB & Grab sample at bottom of a tank \\
\hline \multicolumn{2}{|c|}{ In-Line/Transfer Sampling Locations (5 characters) } \\
\hline $\mathrm{T} 221 \mathrm{~A}$ & In-line transfer from HT22 to UT01A \\
\hline 01A2A & In-line transfer from UT01A to UT02A \\
\hline 01B2A & In-line transfer from UT01B to UT02A \\
\hline $\mathrm{T} 221 \mathrm{~B}$ & In-line transfer from HT22 to UT01B \\
\hline 000FL & Filter-loop in-line sample \\
\hline 00PF1 & Permeate filter number 1 \\
\hline 00PF2 & Permeate filter number 2 \\
\hline 00PF3 & Permeate filter number 3 \\
\hline
\end{tabular}


Table C.2. Sample Naming Acronyms

\begin{tabular}{|c|c|}
\hline Acronym & Definition \\
\hline 00PF4 & Permeate filter number 4 \\
\hline 00PF5 & Permeate filter number 5 \\
\hline $00 \mathrm{C} 01$ & Downstream of CS-PMP-T01 \\
\hline $00 \mathrm{C} 02$ & Downstream of CS-PMP-T02 \\
\hline $00 \mathrm{C} 03$ & Downstream of CS-PMP-T03 \\
\hline \multicolumn{2}{|c|}{ Test Process Step $\left(3\right.$ characters ${ }^{(a)}$ plus 2 repetitive process characters $\left.{ }^{(b)}\right)$ Descriptor 3} \\
\hline (a) 001 through 032 (initial 3 characters) & As described in the guiding document such as \\
\hline $\begin{array}{l}\text { (b) A two-character field to identify any } \\
\text { repetitive process. }\end{array}$ & $\begin{array}{l}\text { Appendix A or B in TP-RPP-WTP-506 Rev. } 0 \text { or } \\
\text { the governing Test Instruction. }\end{array}$ \\
\hline \multicolumn{2}{|l|}{ Repetitive Process (2 characters) } \\
\hline $\mathrm{XX}$ & No repeating process \\
\hline bT & $10^{\circ} \mathrm{C}$ below set-point temperature \\
\hline 00 & $\begin{array}{l}\text { Time when set-point temperature reached (in } \\
\text { hours) }\end{array}$ \\
\hline 01 through 16 & $\begin{array}{l}\text { Time after set-point temperature reached (in } \\
\text { hours) or cycle number, depending on the process } \\
\text { step. }\end{array}$ \\
\hline
\end{tabular}

Sequence Number (4 characters) Descriptor 4 0001 through 9999

Sequential number incremented by 1 for replicate samples

Sample Routing (3 characters) Descriptor 5

\begin{tabular}{ll}
\hline ARC & Archive \\
UDS & Undissolved solids \\
AFA & XRD, SEM, and/or PSD \\
SOX & Antifoaming agent analysis \\
HTC & Solid oxalate analysis \\
DEN & Heat capacity \\
ICP & Density \\
& Inductively coupled plasma optical emission \\
RAM & spectrometry (ICP) analysis \\
RHE & Raman analysis \\
TDS & Rheology (shear strength/shear stress) \\
CUF & Total dissolved solids \\
TFI & Cross-flow ultrafilter (CUF)/parallel testing \\
& Total inorganic carbon analysis (TIC)/free \\
& hydroxide analysis (FOH)/ion chromatography \\
ALK & (IC) analysis \\
ADT & Total alkalinity \\
OST & Acidity titration \\
\hline
\end{tabular}


Table C.2. Sample Naming Acronyms

\begin{tabular}{ll}
\hline Acronym & Definition \\
\hline OTR & Other \\
SUP & Supernate, used when no Raman required \\
Separated-samples (1 character) Descriptor 6 & \\
\hdashline 0 & RTL-520 Store \\
1 & RTL-520 Archive \\
2 & SWRI \\
3 & RPL \\
4 & APEL \\
5 & Dow Corning \\
6 & Other \\
Separated-samples (1 character) Descriptor 7 & \\
\hline D & \\
B & Decanted supernatant (decantate) \\
R & Bulk solids not rinsed \\
S & Rinsate composite \\
\hline F & Rinsed solids \\
\hline
\end{tabular}

The sample names are constructed in the following manner:

\section{Descriptor1_Descriptor2_Descriptor3a_Descriptor3b_Descriptor4_Descriptor5_Descriptor6_ Descriptor7}

The following description is an example of sample naming for samples collected from HLP-VSL-T22 during Shakedown/Functional testing.

- The sample name for the first Functional Test grab slurry sample collected from the top of HLP-VSL-T22 during process step 1 that was to be archived would have been:

F_T22GT_001_XX_0001_ARC_1.

- For the same sample location and type described above, analyzed for PSD on a bulk solid that was not rinsed and ICP on the decantate, the original sample vial would have been labeled: F_T22GM_001_XX_0009_XSP_4_B for the solid.

- Since the solid phase always stayed in the original sample vial, and the liquid was decanted off into a new vial, then the new vial would have been labeled:

F_T22GM_001_XX_0009_DEN_0_D for the decantate.

- The decantate from the above sample container could have been sub-sampled for ICP and Raman; the ICP container would have been labeled as follows:

F_T22GM_001_XX_0009_ICP_2_D.

- The Raman container would have been labeled as follows: F_T22GM_001_XX_0009_RAM_3_D. 


\subsection{Sample List}

This appendix contains a listing of all the samples collected during Integrated Test B. The sample listing in the table below includes:

- Sample name

- Test Instruction number

- Test Instruction step number

- Test Instruction step description

- Sample disposition.

The sample disposition was analysis, storage, or archive. If analysis was performed, it is identified. Archive samples are samples that were identified in the Pretreatment Engineering Platform (PEP) Testing (Phase I) Test Plan (TP-RPP-WTP-506) as samples to be collected and archived. Samples submitted for storage were excess sample material. In addition to the sample disposition, a reference is provided to an electronic folder and electronic analytical report containing sample results.

Analysis acronyms are used in the table, so brief acronym descriptions are provided.

- Density slurry - analysis performed on slurry

- Density supernate - analysis performed on supernate

- Free hydroxide $(\mathrm{OH})$

- Inductively coupled plasma supernate (ICP supernate) — analysis performed on the supernate

- Inductively coupled plasma wet solids (ICP wet solids) — analysis performed on wet solids

- Ion chromatography (IC)

- Particle size distribution (PSD)

- Rheology is a general term used to describe either shear strength or shear stress vs. shear rate analysis - the actual analytical report specifies the specific analysis

- $\quad$ Scanning electron microscope (SEM)

- Total dissolved solids (TDS)

- $\quad$ THF Wet Solids, THF Supernate, Toluene Wet Solids, and Toluene Supernate are the solvent extractions performed on either the supernate or wet solid before being analyzed by Gel permeation chromatography (GPC)

- Total inorganic carbon and total organic carbon (TIC/TOC)

- Weight Percent Undissolved Solids (UDS)

- $\mathrm{X}$-ray diffraction (XRD). 

Integrated Test B Sample List

\begin{tabular}{|c|c|c|c|c|c|c|}
\hline TI\# & TI Step & Step Description & Sample ID & Analysis & File Folder & Final Electronic Report \# \\
\hline TI-WTP-PEP-066 & N/A & Prepare and stage simulant & B FRPGM 001 XX 2700 UDS 2 & UDS & SWRI-090204 & UDS R1 Shake and Test A and Test B Step001 Date 08182009 \\
\hline TI-WTP-PEP-066 & N/A & Prepare and stage simulant & B FRPGM 001 XX 2701 UDS 2 & UDS & SWRI-090204 & UDS R1 Shake and Test A and Test B Step001 Date 08182009 \\
\hline TI-WTP-PEP-066 & N/A & Prepare and stage simulant & B FRPGM 001 XX 2702 UDS 2 & UDS & SWRI-090204 & UDS R1 Shake and Test A and Test B Step001 Date 08182009 \\
\hline TI-WTP-PEP-066 & N/A & Prepare and stage simulant & B FRPGM 001 XX 2706 HTC 3 & Heat Capacity & Heat Capacity & TDP-WTP-368 \\
\hline TI-WTP-PEP-066 & N/A & Prepare and stage simulant & B FRPGM 001 XX 2707 RHE 4 & Rheology & APEL Rheology & TDP WTP 359 Int Test A RHE Data on PEP Slurry \\
\hline TI-WTP-PEP-066 & N/A & Prepare and stage simulant & B FRPGM 001 XX 2708 RHE 4 & Rheology & APEL Rheology & TDP WTP 359 Int Test A RHE Data on PEP Slurry \\
\hline TI-WTP-PEP-066 & N/A & Prepare and stage simulant & B FRPGM 001 XX 2709 RHE 4 & Rheology & APEL Rheology & TDP WTP 359 Int Test A RHE Data on PEP Slurry \\
\hline TI-WTP-PEP-066 & $\mathrm{N} / \mathrm{A}$ & Prepare and stage simulant & B FRPGM 001 XX 2710 ICP 2 B & ICP Wet Solids & SWRI-090204 & 090204 and 090205 ICP and ICPMS \\
\hline TI-WTP-PEP-066 & N/A & Prepare and stage simulant & B FRPGM 001 XX 2710 ICP 2 D & ICP Supernate & SWRI-090204 & 090204 and 090205 ICP and ICPMS \\
\hline TI-WTP-PEP-066 & N/A & Prepare and stage simulant & B FRPGM 001 XX 2710 DEN 0 D & Storage & & \\
\hline TI-WTP-PEP-066 & N/A & Prepare and stage simulant & B FRPGM 001 XX 2710 TFI 2 D & IC & SWRI-090204 & 090204-8 IC TIC and TOC \\
\hline TI-WTP-PEP-066 & N/A & Prepare and stage simulant & B FRPGM 001 XX 2710 TFI 2 D & TIC TOC & SWRI-090204 & 090204-8 IC TIC and TOC \\
\hline TI-WTP-PEP-066 & N/A & Prepare and stage simulant & B FRPGM 001 XX 2710 RAM 3 D & Raman & ASO_ASR8343 & ASR 8343 RAM rev 1 \\
\hline TI-WTP-PEP-066 & $\mathrm{N} / \mathrm{A}$ & Prepare and stage simulant & B FRPGM 001 XX 2710 RAM 3 D & $\mathrm{OH}$ & ASO_ASR8343 & ASR 8343_1 OH \\
\hline TI-WTP-PEP-066 & N/A & Prepare and stage simulant & B FRPGM 001 XX 2711 ICP 2 B & ICP Wet Solids & SWRI-090204 & 090204 and 090205 ICP and ICPMS \\
\hline TI-WTP-PEP-066 & N/A & Prepare and stage simulant & B FRPGM 001 XX 2711 ICP 2 D & ICP Supernate & SWRI-090204 & 090204 and 090205 ICP and ICPMS \\
\hline TI-WTP-PEP-066 & N/A & Prepare and stage simulant & B FRPGM 001 XX 2711 DEN 0 D & Storage & & \\
\hline TI-WTP-PEP-066 & $\mathrm{N} / \mathrm{A}$ & Prepare and stage simulant & B FRPGM 001 XX 2711 TFI 2 D & IC & SWRI-090204 & 090204-8 IC TIC and TOC \\
\hline TI-WTP-PEP-066 & N/A & Prepare and stage simulant & B FRPGM 001 XX 2711 TFI 2 D & TIC TOC & SWRI-090204 & 090204-8 IC TIC and TOC \\
\hline TI-WTP-PEP-066 & N/A & Prepare and stage simulant & B FRPGM 001 XX 2711 RAM 3 D & Raman & ASO_ASR8343 & ASR 8343 RAM rev 1 \\
\hline TI-WTP-PEP-066 & N/A & Prepare and stage simulant & B FRPGM 001 XX 2711 RAM 3 D & $\mathrm{OH}$ & ASO_ASR8343 & ASR 8343_1 OH \\
\hline TI-WTP-PEP-066 & N/A & Prepare and stage simulant & B FRPGM 001 XX 2712 ICP 2 B & ICP Wet Solids & SWRI-090204 & 090204 and 090205 ICP and ICPMS \\
\hline TI-WTP-PEP-066 & N/A & Prepare and stage simulant & B FRPGM 001 XX 2712 ICP 2 D & ICP Supernate & SWRI-090204 & 090204 and 090205 ICP and ICPMS \\
\hline TI-WTP-PEP-066 & $\mathrm{N} / \mathrm{A}$ & Prepare and stage simulant & B FRPGM 001 XX 2712 DEN 0 D & Storage & & \\
\hline TI-WTP-PEP-066 & N/A & Prepare and stage simulant & B FRPGM 001 XX 2712 TFI 2 D & IC & SWRI-090204 & 090204-8 IC TIC and TOC \\
\hline TI-WTP-PEP-066 & N/A & Prepare and stage simulant & B FRPGM 001 XX 2712 TFI 2 D & TIC TOC & SWRI-090204 & 090204-8 IC TIC and TOC \\
\hline TI-WTP-PEP-066 & N/A & Prepare and stage simulant & B FRPGM 001 XX 2712 RAM 3 D & Raman & ASO_ASR8343 & ASR 8343 RAM rev 1 \\
\hline TI-WTP-PEP-066 & $\mathrm{N} / \mathrm{A}$ & Prepare and stage simulant & B FRPGM 001 XX 2712 RAM 3 D & $\mathrm{OH}$ & ASO_ASR8343 & ASR 8343_1 OH \\
\hline TI-WTP-PEP-066 & N/A & Prepare and stage simulant & B FRPGM 001 XX 2713 XSP 0 D & Storage & & \\
\hline TI-WTP-PEP-066 & N/A & Prepare and stage simulant & B FRPGM 001 XX 2713 XSP 4 B & XRD; SEM; PSD & $\begin{array}{l}\text { APEL SEM - } \\
\text { XRD APEL PSD }\end{array}$ & $\begin{array}{l}\text { TDP WTP } 357 \text { Int Test A XRD Data on PEP Slurry; TDP WTP } 356 \text { Int Test } \\
\text { A SEM Data Analysis; TDP WTP } 358 \text { Int Test A PSD Data Analysis }\end{array}$ \\
\hline TI-WTP-PEP-066 & N/A & Prepare and stage simulant & B FRPGM 001 XX 2714 SOX 2 & TIC TOC & SWRI-090204 & 090204-8 IC TIC and TOC \\
\hline TI-WTP-PEP-066 & $\mathrm{N} / \mathrm{A}$ & Prepare and stage simulant & B FRPGM 001 XX 2715 AFA 2 & ICP Supernate & SWRI-090204 & 090204-16 and 090206-4 and 090210-14 and 090210-15 ICP and ICPMS \\
\hline TI-WTP-PEP-066 & N/A & Prepare and stage simulant & B FRPGM 001 XX 2715 AFA 2 & ICP Wet Solids & SWRI-090204 & $090204-16$ and $090206-4$ and $090210-14$ and $090210-15$ ICP and ICPMS \\
\hline
\end{tabular}


Integrated Test B Sample List

\begin{tabular}{|c|c|c|c|c|c|c|}
\hline TI \# & TI Step & Step Description & Sample ID & Analysis & File Folder & Final Electronic Report \# \\
\hline TI-WTP-PEP-066 & N/A & Prepare and stage simulant & B FRPGM 001 XX 2716 AFA 5 B & Toluene Wet Solids & DOW & PNNL 48531 Report.pdf \\
\hline TI-WTP-PEP-066 & N/A & Prepare and stage simulant & B FRPGM 001 XX 2716 AFA 5 D & Toluene Supernate & DOW & PNNL 48531 Report.pdf \\
\hline TI-WTP-PEP-066 & N/A & Prepare and stage simulant & B FRPGM 001 XX 2717 AFA 5 B & THF Wet Solids & DOW & PNNL 48531 Report.pdf \\
\hline TI-WTP-PEP-066 & N/A & Prepare and stage simulant & B FRPGM 001 XX 2717 AFA 5 D & THF Supernate & DOW & PNNL 48531 Report.pdf \\
\hline TI-WTP-PEP-066 & N/A & Prepare and stage simulant & B FRPGM 001 XX 2718 RHE 4 & Rheology & APEL Rheology & TDP WTP 359 Int Test A RHE Data on PEP Slurry \\
\hline TI-WTP-PEP-066 & $\mathrm{N} / \mathrm{A}$ & Prepare and stage simulant & B FRPGM 001 XX 2719 UDS 3 & Storage & & \\
\hline TI-WTP-PEP-066 & N/A & Prepare and stage simulant & B FRPGM 001 XX 2720 UDS & Storage & & \\
\hline TI-WTP-PEP-066 & N/A & Prepare and stage simulant & B FRPGM 001 XX 2721 UDS 3 & Storage & & \\
\hline TI-WTP-PEP-066 & 7.1.4 & Prior to transfer of simulant from HLP-T22 to UFP-VSL-T01A & B T22GT 002 XX 2900 ARC 1 & Archive & & \\
\hline TI-WTP-PEP-066 & 7.1.4 & Prior to transfer of simulant from HLP-T22 to UFP-VSL-T01A & B T22GM 002 XX 2901 ARC 1 & Archive & & \\
\hline TI-WTP-PEP-066 & 7.1.4 & Prior to transfer of simulant from HLP-T22 to UFP-VSL-T01A & B T22GB 002 XX 2902 ARC 1 & Archive & & \\
\hline TI-WTP-PEP-066 & 7.1.4 & Prior to transfer of simulant from HLP-T22 to UFP-VSL-T01A & B T22GM 002 XX 2903 UDS 2 & UDS & SWRI-090317 & 090317-16UDS R1 \\
\hline TI-WTP-PEP-066 & 7.1.4 & Prior to transfer of simulant from HLP-T22 to UFP-VSL-T01A & B T22GM 002 XX 2904 RHE 4 & Rheology & APEL Rheology & TDP WTP 363 Int Test B RHE Data on PEP slurry \\
\hline TI-WTP-PEP-066 & 7.1.4 & Prior to transfer of simulant from HLP-T22 to UFP-VSL-T01A & B T22GM 002 XX 2905 XSP 4 & PSD & APEL PSD & TDP WTP 362 Int Test B PSD Data Analysis \\
\hline TI-WTP-PEP-066 & 7.2.1 & Transfer simulant from HLP-T22 to UFP-VSL-TO1A & B T221A 002 XX 2906 ARC 1 & Archive & & \\
\hline TI-WTP-PEP-066 & 7.2 .1 & Transfer simulant from HLP-T22 to UFP-VSL-TO1A & B T221A 002 XX 2907 ARC 1 & Archive & & \\
\hline TI-WTP-PEP-066 & 7.2 .1 & Transfer simulant from HLP-T22 to UFP-VSL-TO1A & B T221A 002 XX 2908 ARC 1 & Archive & & \\
\hline TI-WTP-PEP-066 & 7.2.1 & Transfer simulant from HLP-T22 to UFP-VSL-TO1A & B T221A 002 XX 2909 ARC 1 & Archive & & \\
\hline TI-WTP-PEP-066 & 7.3 .4 & Transfer simulant from UFP-VSL-T01A to UFP-VSL-T02A & B 01A2A 003 XX 2910 ARC 1 & Archive & & \\
\hline TI-WTP-PEP-066 & 7.3.4 & Transfer simulant from UFP-VSL-T01A to UFP-VSL-T02A & B 01A2A 003 XX 2911 ARC 1 & Archive & & \\
\hline TI-WTP-PEP-066 & 7.3.4 & Transfer simulant from UFP-VSL-T01A to UFP-VSL-T02A & B 01A2A 003 XX 2912 ARC 1 & Archive & & \\
\hline TI-WTP-PEP-066 & 7.3.4 & Transfer simulant from UFP-VSL-T01A to UFP-VSL-T02A & B 01A2A 003 XX 2913 ARC 1 & Archive & & \\
\hline TI-WTP-PEP-066 & 7.5.7 & Concentrate solids in UFP-VSL-T02A to 20-wt\% UDS & B 01A2A 005 XX 2914 ARC 1 & Archive & & \\
\hline TI-WTP-PEP-066 & 7.5.7 & Concentrate solids in UFP-VSL-T02A to 20 -wt $\%$ UDS & B 01A2A 005 XX 2915 ARC 1 & Archive & & \\
\hline TI-WTP-PEP-066 & 7.5.7 & Concentrate solids in UFP-VSL-T02A to 20 -wt $\%$ UDS & B 01A2A 005 XX 2916 ARC 1 & Archive & & \\
\hline TI-WTP-PEP-066 & 7.5.7 & Concentrate solids in UFP-VSL-T02A to 20 -wt $\%$ UDS & B 01A2A 005 XX 2917 ARC 1 & Archive & & \\
\hline TI-WTP-PEP-066 & 7.5.7 & Concentrate solids in UFP-VSL-T02A to 20-wt\% UDS & B 01A2A 005 XX 2918 ARC 1 & Archive & & \\
\hline TI-WTP-PEP-066 & 7.5 .7 & Concentrate solids in UFP-VSL-T02A to 20 -wt $\%$ UDS & B 00PF1 005 XX 2919 ARC 1 & Archive & & \\
\hline TI-WTP-PEP-066 & 7.5.7 & Concentrate solids in UFP-VSL-T02A to 20 -wt $\%$ UDS & B 00PF2 005 XX 2920 ARC 1 & Archive & & \\
\hline TI-WTP-PEP-066 & 7.5 .7 & Concentrate solids in UFP-VSL-T02A to 20 -wt $\%$ UDS & B 00PF3 005 XX 2921 ARC 1 & Archive & & \\
\hline TI-WTP-PEP-066 & 7.5.7 & Concentrate solids in UFP-VSL-T02A to 20 -wt $\%$ UDS & B 00PF4 005 XX 4440 ARC 1 & Archive & & \\
\hline TI-WTP-PEP-066 & 7.5 .7 & Concentrate solids in UFP-VSL-T02A to 20 -wt $\%$ UDS & B 00PF5 005 XX 4441 ARC 1 & Archive & & \\
\hline TI-WTP-PEP-066 & 7.5 .7 & Concentrate solids in UFP-VSL-T02A to 20-wt\% UDS & B 00PF1 005 XX 4442 ARC 1 & Archive & & \\
\hline
\end{tabular}


Integrated Test B Sample List

\begin{tabular}{|c|c|c|c|c|c|c|}
\hline TI \# & TI Step & Step Description & Sample ID & Analysis & File Folder & Final Electronic Report \# \\
\hline TI-WTP-PEP-066 & 7.5.7 & Concentrate solids in UFP-VSL-T02A to $20-\mathrm{wt} \%$ UDS & B 00PF2 005 XX 4443 ARC 1 & Archive & & \\
\hline TI-WTP-PEP-066 & 7.5.7 & Concentrate solids in UFP-VSL-T02A to 20 -wt $\%$ UDS & B 00PF3 005 XX 4444 ARC 1 & Archive & & \\
\hline TI-WTP-PEP-066 & 7.5.7 & Concentrate solids in UFP-VSL-T02A to 20 -wt $\%$ UDS & B 00PF4 005 XX 4445 ARC 1 & Archive & & \\
\hline TI-WTP-PEP-066 & 7.5.7 & Concentrate solids in UFP-VSL-T02A to 20 -wt $\%$ UDS & B 00PF5 005 XX 4446 ARC 1 & Archive & & \\
\hline TI-WTP-PEP-066 & 7.5.7 & Concentrate solids in UFP-VSL-T02A to 20 -wt $\%$ UDS & B 00PF1 005 XX 4447 ARC 1 & Archive & & \\
\hline TI-WTP-PEP-066 & 7.5.7 & Concentrate solids in UFP-VSL-T02A to 20 -wt $\%$ UDS & B 00PF2 005 XX 4448 ARC 1 & Archive & & \\
\hline TI-WTP-PEP-066 & 7.5 .7 & Concentrate solids in UFP-VSL-T02A to $20-\mathrm{wt} \%$ UDS & B 00PF3 005 XX 4449 ARC 1 & Archive & & \\
\hline TI-WTP-PEP-066 & 7.5.7 & Concentrate solids in UFP-VSL-T02A to 20 -wt $\%$ UDS & B 00PF4 005 XX 4450 ARC 1 & Archive & & \\
\hline TI-WTP-PEP-066 & 7.5.7 & Concentrate solids in UFP-VSL-T02A to 20 -wt\% UDS & B 00PF5 005 XX 4451 ARC 1 & Archive & & \\
\hline TI-WTP-PEP-066 & 7.5.7 & Concentrate solids in UFP-VSL-T02A to 20 -wt $\%$ UDS & B 02AML 005 XX 2922 UDS 2 & UDS & SWRI-090317 & 090317-16UDS R1 \\
\hline TI-WTP-PEP-066 & 7.5.7 & Concentrate solids in UFP-VSL-T02A to 20 -wt $\%$ UDS & B 02AML 005 XX 2923 RHE 4 & Rheology & APEL Rheology & TDP WTP 363 Int Test B RHE Data on PEP slurry \\
\hline TI-WTP-PEP-066 & 7.5.7 & Concentrate solids in UFP-VSL-T02A to $20-\mathrm{wt} \%$ UDS & В 02AML 005 XX 2924 UDS 2 & UDS & SWRI-090317 & 090317-16UDS R1 \\
\hline TI-WTP-PEP-066 & 7.5.7 & Concentrate solids in UFP-VSL-T02A to $20-\mathrm{wt} \%$ UDS & B 02AML 005 XX 2925 UDS 2 & UDS & SWRI-090317 & 090317-16UDS R1 \\
\hline TI-WTP-PEP-066 & 7.5.7 & Concentrate solids in UFP-VSL-T02A to 20 -wt $\%$ UDS & В 02AML 005 XX 2926 UDS 2 & UDS & SWRI-090317 & 090317-16UDS R1 \\
\hline TI-WTP-PEP-066 & 7.5.7 & Concentrate solids in UFP-VSL-T02A to 20 -wt $\%$ UDS & B 02AML 005 XX 2927 UDS 2 & UDS & SWRI-090317 & 090317-16UDS R1 \\
\hline TI-WTP-PEP-066 & 7.5.7 & Concentrate solids in UFP-VSL-T02A to 20 -wt $\%$ UDS & B 02AML 005 XX 2928 UDS 2 & UDS & SWRI-090317 & 090317-16UDS R1 \\
\hline TI-WTP-PEP-066 & 7.5.7 & Concentrate solids in UFP-VSL-T02A to $20-\mathrm{wt} \%$ UDS & B 02AML 005 XX 2929 UDS 2 & UDS & SWRI-090317 & 090317-16UDS R1 \\
\hline TI-WTP-PEP-066 & 7.5.7 & Concentrate solids in UFP-VSL-T02A to 20 -wt $\%$ UDS & B 02AML 005 XX 2930 UDS 2 & UDS & SWRI-090317 & 090317-16UDS R1 \\
\hline TI-WTP-PEP-066 & 7.5.7 & Concentrate solids in UFP-VSL-T02A to 20-wt\% UDS & B 02AML 005 XX 2931 UDS 2 & UDS & SWRI-090317 & 090317-16UDS R1 \\
\hline TI-WTP-PEP-066 & 7.5.7 & Concentrate solids in UFP-VSL-T02A to 20 -wt $\%$ UDS & B 02AML 005 XX 2932 UDS 2 & UDS & SWRI-090317 & 090317-16UDS R1 \\
\hline TI-WTP-PEP-066 & 7.5.7 & Concentrate solids in UFP-VSL-T02A to 20 -wt $\%$ UDS & B 02 AML 005 XX 2933 UDS 2 & UDS & SWRI-090317 & 090317-16UDS R1 \\
\hline TI-WTP-PEP-066 & 7.5.7 & Concentrate solids in UFP-VSL-T02A to 20 -wt $\%$ UDS & B 02AML 005 XX 2934 UDS 2 & UDS & SWRI-090317 & 090317-16UDS R1 \\
\hline TI-WTP-PEP-066 & 7.5.7 & Concentrate solids in UFP-VSL-T02A to 20 -wt $\%$ UDS & B 02AML 005 XX 2935 UDS 2 & UDS & SWRI-090317 & 090317-16UDS R1 \\
\hline TI-WTP-PEP-066 & 7.5 .7 & Concentrate solids in UFP-VSL-T02A to $20-\mathrm{wt} \%$ UDS & B 02AML 005 XX 2936 UDS 2 & UDS & SWRI-090317 & 090317-16UDS R1 \\
\hline TI-WTP-PEP-066 & 7.5.7 & Concentrate solids in UFP-VSL-T02A to 20 -wt $\%$ UDS & B 02AML 005 XX 2937 UDS 2 & UDS & SWRI-090317 & 090317-16UDS R1 \\
\hline TI-WTP-PEP-066 & 7.5 .9 & Concentrate solids in UFP-VSL-T02A to 20 -wt $\%$ UDS & B 02AML 005 XX 2943 UDS 2 & UDS & SWRI-090317 & 090317-16UDS R1 \\
\hline TI-WTP-PEP-066 & 7.5 .9 & Concentrate solids in UFP-VSL-T02A to 20 -wt\% UDS & B 02AML 005 XX 2944 UDS 2 & UDS & SWRI-090317 & 090317-16UDS R1 \\
\hline TI-WTP-PEP-066 & 7.5.9 & Concentrate solids in UFP-VSL-T02A to 20 -wt $\%$ UDS & B 02AML 005 XX 2945 UDS 2 & UDS & SWRI-090317 & 090317-16UDS R1 \\
\hline TI-WTP-PEP-066 & 7.5.9 & Concentrate solids in UFP-VSL-T02A to 20 -wt $\%$ UDS & B 02AML 005 XX 2946 DEN 2 & Density Slurry & SWRI-090317 & 090317-17, 090317-33, 090317-38 DEN slurry and liquid \\
\hline TI-WTP-PEP-066 & 7.5 .9 & Concentrate solids in UFP-VSL-T02A to 20 -wt $\%$ UDS & B 02AML 005 XX 2946 DEN 2 & Density Supernate & SWRI-090317 & 090317-17, 090317-33, 090317-38 DEN slurry and liquid \\
\hline TI-WTP-PEP-066 & 7.5.9 & Concentrate solids in UFP-VSL-T02A to 20 -wt $\%$ UDS & B 02AML 005 XX 2947 DEN 2 & Density Slurry & SWRI-090317 & 090317-17, 090317-33, 090317-38 DEN slurry and liquid \\
\hline TI-WTP-PEP-066 & 7.5 .9 & Concentrate solids in UFP-VSL-T02A to 20-wt\% UDS & B 02AML 005 XX 2947 DEN 2 & Density Supernate & SWRI-090317 & 090317-17, 090317-33, 090317-38 DEN slurry and liquid \\
\hline TI-WTP-PEP-066 & 7.5.9 & Concentrate solids in UFP-VSL-T02A to 20 -wt $\%$ UDS & B 02AML 005 XX 2948 DEN 2 & Density Slurry & SWRI-090317 & 090317-17, 090317-33, 090317-38 DEN slurry and liquid \\
\hline
\end{tabular}


Integrated Test B Sample List

\begin{tabular}{|c|c|c|c|c|c|c|}
\hline TI \# & TI Step & Step Description & Sample ID & Analysis & File Folder & Final Electronic Report \# \\
\hline TI-WTP-PEP-066 & 7.5 .9 & Concentrate solids in UFP-VSL-T02A to 20 -wt $\%$ UDS & B 02AML 005 XX 2948 DEN 2 & Density Supernate & SWRI-090317 & 090317-17, 090317-33, 090317-38 DEN slurry and liquid \\
\hline TI-WTP-PEP-066 & 7.5 .9 & Concentrate solids in UFP-VSL-T02A to 20 -wt $\%$ UDS & B 02AML 005 XX 2949 HTC 3 & Heat Capacity & Heat Capacity & TDP-WTP-372 \\
\hline TI-WTP-PEP-066 & 7.5 .9 & After solids concentration completed to $20-\mathrm{wt} \%$ UDS & B 02AML 005 XX 2950 RHE 4 & Rheology & APEL Rheology & TDP WTP 363 Int Test B RHE Data on PEP slurry \\
\hline TI-WTP-PEP-066 & 7.5 .9 & After solids concentration completed to $20-\mathrm{w} t \%$ UDS & B 02AML 005 XX 2951 ICP 2 B & ICP Wet Solids & SWRI-090317 & $\begin{array}{l}090317-23 \text { and } 090317-43 \text { and } 090318-10 \text { and } 090320-10 \text { and } 090320-13 \\
\text { ICP ICPMS }\end{array}$ \\
\hline TI-WTP-PEP-066 & 7.5 .9 & After solids concentration completed to $20-\mathrm{wt} \%$ UDS & B 02AML 005 XX 2951 ICP 2 D & ICP Supernate & SWRI-090317 & $\begin{array}{l}090317-22 \text { and } 090317-42 \text { and } 090318-6 \text { and } 090318-10 \text { and } 090320-10 \\
\text { and 090320-12 ICP and ICPMS }\end{array}$ \\
\hline TI-WTP-PEP-066 & 7.5.9 & After solids concentration completed to 20 -wt $\%$ UDS & B 02AML 005 XX 2951 TFI 2 D & IC & SWRI-090317 & 090317-26 090317-41 IC \\
\hline TI-WTP-PEP-066 & 7.5 .9 & After solids concentration completed to $20-\mathrm{w} t \%$ UDS & B 02AML 005 XX 2951 TFI 3 D & $\mathrm{OH}$ & ASO_ASR8383 & ASR $8383 \mathrm{OH}$ rev 1 \\
\hline TI-WTP-PEP-066 & 7.5 .9 & After solids concentration completed to $20-\mathrm{w} t \%$ UDS & B 02AML 005 XX 2951 SUP 0 D & Storage & & \\
\hline TI-WTP-PEP-066 & 7.5 .9 & After solids concentration completed to $20-\mathrm{w} t \%$ UDS & B 02AML 005 XX 2952 ICP 2 B & ICP Wet Solids & SWRI-090317 & $\begin{array}{l}090317-23 \text { and } 090317-43 \text { and } 090318-10 \text { and } 090320-10 \text { and } 090320-13 \\
\text { ICP ICPMS }\end{array}$ \\
\hline TI-WTP-PEP-066 & 7.5 .9 & After solids concentration completed to $20-\mathrm{w} t \%$ UDS & B 02AML 005 XX 2952 ICP 2 D & ICP Supernate & SWRI-090317 & $\begin{array}{l}090317-22 \text { and } 090317-42 \text { and } 090318-6 \text { and } 090318-10 \text { and } 090320-10 \\
\text { and } 090320-12 \text { ICP and ICPMS }\end{array}$ \\
\hline TI-WTP-PEP-066 & 7.5 .9 & After solids concentration completed to $20-\mathrm{w} t \%$ UDS & B 02AML 005 XX 2952 TFI 2 D & IC & SWRI-090317 & 090317-26 090317-41 IC \\
\hline TI-WTP-PEP-066 & 7.5 .9 & After solids concentration completed to $20-\mathrm{w} t \%$ UDS & B 02AML 005 XX 2952 TFI 3 D & $\mathrm{OH}$ & ASO_ASR8383 & ASR $8383 \mathrm{OH}$ rev 1 \\
\hline TI-WTP-PEP-066 & 7.5 .9 & After solids concentration completed to 20 -wt $\%$ UDS & B 02AML 005 XX 2952 SUP 0 D & Storage & & \\
\hline TI-WTP-PEP-066 & 7.5 .9 & After solids concentration completed to $20-\mathrm{w} t \%$ UDS & B 02AML 005 XX 2953 ICP 2 B & ICP Wet Solids & SWRI-090317 & $\begin{array}{l}090317-23 \text { and } 090317-43 \text { and } 090318-10 \text { and } 090320-10 \text { and } 090320-13 \\
\text { ICP ICPMS }\end{array}$ \\
\hline TI-WTP-PEP-066 & 7.5 .9 & After solids concentration completed to 20 -wt $\%$ UDS & B 02AML 005 XX 2953 ICP 2 D & ICP Supernate & SWRI-090317 & $\begin{array}{l}\text { 090317-22 and 090317-42 and 090318-6 and 090318-10 and 090320-10 } \\
\text { and 090320-12 ICP and ICPMS }\end{array}$ \\
\hline TI-WTP-PEP-066 & 7.5 .9 & After solids concentration completed to $20-\mathrm{wt} \%$ UDS & B 02AML 005 XX 2953 TFI 2 D & IC & SWRI-090317 & 090317-26 090317-41 IC \\
\hline TI-WTP-PEP-066 & 7.5.9 & After solids concentration completed to 20 -wt $\%$ UDS & B 02AML 005 XX 2953 TFI 3 D & $\mathrm{OH}$ & ASO_ASR8383 & ASR $8383 \mathrm{OH}$ rev 1 \\
\hline TI-WTP-PEP-066 & 7.5 .9 & After solids concentration completed to $20-\mathrm{w} t \%$ UDS & B 02AML 005 XX 2953 SUP 0 D & Storage & & \\
\hline TI-WTP-PEP-066 & 7.5 .9 & After solids concentration completed to 20 -wt $\%$ UDS & B 02AML 005 XX 2954 XSP 4 & PSD & APEL PSD & TDP WTP 362 Int Test B PSD Data Analysis \\
\hline TI-WTP-PEP-066 & 7.5 .9 & After solids concentration completed to $20-\mathrm{w} t \%$ UDS & B 02AML 005 XX 2955 CUF 4 & Parallel Lab Test & & \\
\hline TI-WTP-PEP-066 & 7.5 .9 & At end of concentration of solids & B 00PF1 005 XX 2956 ARC 4 & Precipitation Study & & \\
\hline TI-WTP-PEP-066 & 7.5 .9 & At end of concentration of solids & B 00PF2 005 XX 4452 ARC 4 & Archive & & \\
\hline TI-WTP-PEP-066 & 7.5 .9 & At end of concentration of solids & B 00PF3 005 XX 4453 ARC 4 & Archive & & \\
\hline TI-WTP-PEP-066 & 7.5 .9 & At end of concentration of solids & B 00PF4 005 XX 4454 ARC 4 & Archive & & \\
\hline TI-WTP-PEP-066 & 7.5 .9 & At end of concentration of solids & B 00PF5 005 XX 4455 ARC 4 & Archive & & \\
\hline TI-WTP-PEP-066 & 7.6.11 & Flush filter-loop & B 000FL 007 XX 2957 UDS 2 & UDS & SWRI-090317 & 090317-39UDS R1 \\
\hline TI-WTP-PEP-066 & 7.6.11 & Flush filter-loop & B 000FL 007 XX 2958 ICP 0 B & Storage & & \\
\hline TI-WTP-PEP-066 & 7.6.11 & Flush filter-loop & B 000FL 007 XX 2958 ICP 2 D & ICP Supernate & SWRI-090317 & $\begin{array}{l}090317-22 \text { and } 090317-42 \text { and } 090318-6 \text { and } 090318-10 \text { and } 090320-10 \\
\text { and 090320-12 ICP and ICPMS }\end{array}$ \\
\hline
\end{tabular}


Integrated Test B Sample List

\begin{tabular}{|c|c|c|c|c|c|c|}
\hline TI \# & TI Step & Step Description & Sample ID & Analysis & File Folder & Final Electronic Report \# \\
\hline TI-WTP-PEP-066 & 7.6.11 & Flush filter-loop & B 000FL 007 XX 2958 TFI 2 D & IC & SWRI-090317 & 090317-26 090317-41 IC \\
\hline TI-WTP-PEP-066 & 7.6.11 & Flush filter-loop & B 000FL 007 XX 2958 TFI 3 D & $\mathrm{OH}$ & ASO_ASR 8383 & ASR $8383 \mathrm{OH}$ rev 1 \\
\hline TI-WTP-PEP-066 & 7.6.11 & Flush filter-loop & B 000FL 007 XX 2958 SUP 0 D & Storage & & \\
\hline TI-WTP-PEP-066 & 7.6.11 & $\begin{array}{l}\text { At least } 10 \text { min after UFP-VSL-T02A has been isolated - after } \\
\text { caustic added before leach begins }\end{array}$ & B 02AML 007 XX 2959 UDS 2 B & UDS & SWRI-090317 & 090317-34UDS R1 \\
\hline TI-WTP-PEP-066 & 7.6.11 & $\begin{array}{l}\text { At least } 10 \text { min after UFP-VSL-T02A has been isolated - after } \\
\text { caustic added before leach begins }\end{array}$ & B 02AML 007 XX 2959 UDS 2 D & UDS & SWRI-090317 & 090317-34UDS R1 \\
\hline TI-WTP-PEP-066 & 7.6.11 & $\begin{array}{l}\text { At least } 10 \text { min after UFP-VSL-T02A has been isolated - after } \\
\text { caustic added before leach begins }\end{array}$ & B 02AML 007 XX 2960 UDS 2 B & UDS & SWRI-090317 & 090317-34UDS R1 \\
\hline TI-WTP-PEP-066 & 7.6.11 & $\begin{array}{l}\text { At least } 10 \text { min after UFP-VSL-T02A has been isolated - after } \\
\text { caustic added before leach begins }\end{array}$ & B 02AML 007 XX 2960 UDS 2 D & UDS & SWRI-090317 & 090317-34UDS R1 \\
\hline TI-WTP-PEP-066 & 7.6.11 & $\begin{array}{l}\text { At least } 10 \text { min after UFP-VSL-T02A has been isolated - after } \\
\text { caustic added before leach begins }\end{array}$ & B 02AML 007 XX 2961 UDS 2 B & UDS & SWRI-090317 & 090317-34UDS R1 \\
\hline TI-WTP-PEP-066 & 7.6.11 & $\begin{array}{l}\text { At least } 10 \text { min after UFP-VSL-T02A has been isolated - after } \\
\text { caustic added before leach begins }\end{array}$ & B 02AML 007 XX 2961 UDS 2 D & UDS & SWRI-090317 & 090317-34UDS R1 \\
\hline TI-WTP-PEP-066 & 7.6.11 & $\begin{array}{l}\text { At least } 10 \text { min after UFP-VSL-T02A has been isolated - after } \\
\text { caustic added before leach begins }\end{array}$ & B 02AML 007 XX 2962 DEN 2 & Density Slurry & SWRI-090317 & 090317-17, 090317-33, 090317-38 DEN slurry and liquid \\
\hline TI-WTP-PEP-066 & 7.6.11 & $\begin{array}{l}\text { At least } 10 \text { min after UFP-VSL-T02A has been isolated - after } \\
\text { caustic added before leach begins }\end{array}$ & B 02AML 007 XX 2963 DEN 2 & Density Slurry & SWRI-090317 & 090317-17, 090317-33, 090317-38 DEN slurry and liquid \\
\hline TI-WTP-PEP-066 & 7.6.11 & $\begin{array}{l}\text { At least } 10 \text { min after UFP-VSL-T02A has been isolated - after } \\
\text { caustic added before leach begins }\end{array}$ & B 02AML 007 XX 2964 DEN 2 & Density Slurry & SWRI-090317 & $090317-17,090317-33,090317-38$ DEN slurry and liquid \\
\hline TI-WTP-PEP-066 & 7.6.11 & $\begin{array}{l}\text { At least } 10 \text { min after UFP-VSL-T02A has been isolated - after } \\
\text { caustic added before leach begins }\end{array}$ & B 02AML 007 XX 2965 HTC 3 & Heat Capacity & Heat Capacity & TDP-WTP-372 \\
\hline TI-WTP-PEP-066 & 7.6.11 & $\begin{array}{l}\text { At least } 10 \text { min after UFP-VSL-T02A has been isolated - after } \\
\text { caustic added before leach begins }\end{array}$ & B 02AML 007 XX 2966 ICP 2 B & ICP Wet Solids & SWRI-090317 & 090317-32 ICP and ICPMS \\
\hline TI-WTP-PEP-066 & 7.6.11 & $\begin{array}{l}\text { At least } 10 \text { min after UFP-VSL-T02A has been isolated - after } \\
\text { caustic added before leach begins }\end{array}$ & B 02AML 007 XX 2966 ICP 2 D & ICP Supernate & SWRI-090317 & 090317-30 and 090317-31 ICP and ICPMS \\
\hline TI-WTP-PEP-066 & 7.6.11 & $\begin{array}{l}\text { At least } 10 \text { min after UFP-VSL-T02A has been isolated - after } \\
\text { caustic added before leach begins }\end{array}$ & B 02AML 007 XX 2966 TFI 2 D & IC & SWRI-090317 & 090317-36IC \\
\hline TI-WTP-PEP-066 & 7.6.11 & $\begin{array}{l}\text { At least } 10 \text { min after UFP-VSL-T02A has been isolated - after } \\
\text { caustic added before leach begins }\end{array}$ & B 02AML 007 XX 2966 TFI 3 D & $\mathrm{OH}$ & ASO_ASR8383 & ASR 8383 OH rev 1 \\
\hline TI-WTP-PEP-066 & 7.6.11 & $\begin{array}{l}\text { At least } 10 \text { min after UFP-VSL-T02A has been isolated - after } \\
\text { caustic added before leach begins }\end{array}$ & B 02AML 007 XX 2966 RAM 3 D & Raman & ASO_ASR8382 & ASR 8382 RAM \\
\hline TI-WTP-PEP-066 & 7.6.11 & $\begin{array}{l}\text { At least } 10 \text { min after UFP-VSL-T02A has been isolated - after } \\
\text { caustic added before leach begins }\end{array}$ & B 02AML 007 XX 2966 DEN 0 D & Storage & & \\
\hline TI-WTP-PEP-066 & 7.6.11 & $\begin{array}{l}\text { At least } 10 \text { min after UFP-VSL-T02A has been isolated - after } \\
\text { caustic added before leach begins }\end{array}$ & B 02AML 007 XX 2967 ICP 2 B & ICP Wet Solids & SWRI-090317 & 090317-32 ICP and ICPMS \\
\hline
\end{tabular}


Integrated Test B Sample List

\begin{tabular}{|c|c|c|c|c|c|c|}
\hline TI \# & TI Step & Step Description & Sample ID & Analysis & File Folder & Final Electronic Report \# \\
\hline TI-WTP-PEP-066 & 7.6.11 & $\begin{array}{l}\text { At least } 10 \text { min after UFP-VSL-T02A has been isolated - after } \\
\text { caustic added before leach begins }\end{array}$ & B 02AML 007 XX 2967 ICP 2 D & ICP Supernate & SWRI-090317 & 090317-30 and 090317-31 ICP and ICPMS \\
\hline TI-WTP-PEP-066 & 7.6.11 & $\begin{array}{l}\text { At least } 10 \text { min after UFP-VSL-T02A has been isolated - after } \\
\text { caustic added before leach begins }\end{array}$ & B 02AML 007 XX 2967 TFI 2 D & IC & SWRI-090317 & 090317-36IC \\
\hline TI-WTP-PEP-066 & 7.6.11 & $\begin{array}{l}\text { At least } 10 \text { min after UFP-VSL-T02A has been isolated - after } \\
\text { caustic added before leach begins }\end{array}$ & B 02AML 007 XX 2967 TFI 3 D & $\mathrm{OH}$ & ASO_ASR8383 & ASR $8383 \mathrm{OH}$ rev 1 \\
\hline TI-WTP-PEP-066 & 7.6.11 & $\begin{array}{l}\text { At least } 10 \text { min after UFP-VSL-T02A has been isolated - after } \\
\text { caustic added before leach begins }\end{array}$ & B 02AML 007 XX 2967 RAM 3 D & Raman & ASO_ASR8382 & ASR 8382 RAM \\
\hline TI-WTP-PEP-066 & 7.6.11 & $\begin{array}{l}\text { At least } 10 \text { min after UFP-VSL-T02A has been isolated - after } \\
\text { caustic added before leach begins }\end{array}$ & B 02AML 007 XX 2967 DEN 0 D & Storage & & \\
\hline TI-WTP-PEP-066 & 7.6.11 & $\begin{array}{l}\text { At least } 10 \text { min after UFP-VSL-T02A has been isolated - after } \\
\text { caustic added before leach begins }\end{array}$ & B 02AML 007 XX 2968 ICP 2 B & ICP Wet Solids & SWRI-090317 & 090317-32 ICP and ICPMS \\
\hline TI-WTP-PEP-066 & 7.6.11 & $\begin{array}{l}\text { At least } 10 \text { min after UFP-VSL-T02A has been isolated - after } \\
\text { caustic added before leach begins }\end{array}$ & B 02AML 007 XX 2968 ICP 2 D & ICP Supernate & SWRI-090317 & 090317-30 and 090317-31 ICP and ICPMS \\
\hline TI-WTP-PEP-066 & 7.6.11 & $\begin{array}{l}\text { At least } 10 \text { min after UFP-VSL-T02A has been isolated - after } \\
\text { caustic added before leach begins }\end{array}$ & B 02AML 007 XX 2968 TFI 2 D & IC & SWRI-090317 & 090317-36IC \\
\hline TI-WTP-PEP-066 & 7.6.11 & $\begin{array}{l}\text { At least } 10 \text { min after UFP-VSL-T02A has been isolated - after } \\
\text { caustic added before leach begins }\end{array}$ & B 02AML 007 XX 2968 TFI 3 D & $\mathrm{OH}$ & ASO_ASR8383 & ASR $8383 \mathrm{OH}$ rev 1 \\
\hline TI-WTP-PEP-066 & 7.6.11 & $\begin{array}{l}\text { At least } 10 \text { min after UFP-VSL-T02A has been isolated - after } \\
\text { caustic added before leach begins }\end{array}$ & B 02AML 007 XX 2968 RAM 3 D & Raman & ASO_ASR8382 & ASR 8382 RAM \\
\hline TI-WTP-PEP-066 & 7.6.11 & $\begin{array}{l}\text { At least } 10 \text { min after UFP-VSL-T02A has been isolated - after } \\
\text { caustic added before leach begins }\end{array}$ & B 02AML 007 XX 2968 DEN 0 D & Storage & & \\
\hline TI-WTP-PEP-066 & 7.6.11 & $\begin{array}{l}\text { At least } 10 \text { min after UFP-VSL-T02A has been isolated - after } \\
\text { caustic added before leach begins }\end{array}$ & B 02AML 007 XX 2969 CUF 4 & Parallel Lab Test & & \\
\hline TI-WTP-PEP-066 & 7.6.16 & (a) $88^{\circ} \mathrm{C}\left(\operatorname{target} \mathrm{T}-10^{\circ} \mathrm{C}\right)$ & B 02AML 008 BT 2970 UDS 2 B & UDS & SWRI-090317 & 090317-34UDS R1 \\
\hline TI-WTP-PEP-066 & 7.6.16 & @ $88^{\circ} \mathrm{C}\left(\operatorname{target} \mathrm{T}-10^{\circ} \mathrm{C}\right)$ & B 02AML 008 BT 2970 UDS 2 D & UDS & SWRI-090317 & 090317-34UDS R1 \\
\hline TI-WTP-PEP-066 & 7.6.16 & (a) $88^{\circ} \mathrm{C}\left(\operatorname{target} \mathrm{T}-10^{\circ} \mathrm{C}\right)$ & B 02AML 008 BT 2971 DEN 2 & Density Slurry & SWRI-090317 & $090317-17,090317-33,090317-38$ DEN slurry and liquid \\
\hline TI-WTP-PEP-066 & 7.6.16 & (a) $88^{\circ} \mathrm{C}\left(\operatorname{target} \mathrm{T}-10^{\circ} \mathrm{C}\right)$ & В 02AML 008 BT 2972 HTC 3 & Heat Capacity & Heat Capacity & TDP-WTP-372 \\
\hline TI-WTP-PEP-066 & 7.6.16 & @ $88^{\circ} \mathrm{C}\left(\operatorname{target} \mathrm{T}-10^{\circ} \mathrm{C}\right)$ & B 02AML 008 BT 2973 ICP 0 B & Storage & & \\
\hline TI-WTP-PEP-066 & 7.6.16 & @ $88^{\circ} \mathrm{C}\left(\operatorname{target} \mathrm{T}-10^{\circ} \mathrm{C}\right)$ & B 02AML 008 BT 2973 SUP 0 D & Storage & & \\
\hline TI-WTP-PEP-066 & 7.6.16 & @ $88^{\circ} \mathrm{C}\left(\operatorname{target} \mathrm{T}-10^{\circ} \mathrm{C}\right)$ & B 02AML 008 BT 2973 ICP 2 D & ICP Supernate & SWRI-090317 & 090317-30 and 090317-31 ICP and ICPMS \\
\hline TI-WTP-PEP-066 & 7.6.16 & @ $88^{\circ} \mathrm{C}$ (target $\mathrm{T}-10^{\circ} \mathrm{C}$ ) & B 02AIL 008 BT 2974 ARC 1 B & Archive & & \\
\hline TI-WTP-PEP-066 & 7.6.16 & @ $88^{\circ} \mathrm{C}\left(\operatorname{target} \mathrm{T}-10^{\circ} \mathrm{C}\right)$ & B 02AIL 008 BT 2974 ARC 1 D & Archive & & \\
\hline TI-WTP-PEP-066 & 7.6.16 & @ $88^{\circ} \mathrm{C}\left(\operatorname{target} \mathrm{T}-10^{\circ} \mathrm{C}\right)$ & B 02AIH 008 BT 2975 ARC 1 B & Archive & & \\
\hline TI-WTP-PEP-066 & 7.6.16 & @ $88^{\circ} \mathrm{C}\left(\operatorname{target} \mathrm{T}-10^{\circ} \mathrm{C}\right)$ & B 02AIH 008 BT 2975 ARC 1 D & Archive & & \\
\hline TI-WTP-PEP-066 & 7.6.16 & @ $88^{\circ} \mathrm{C}\left(\operatorname{target} \mathrm{T}-10^{\circ} \mathrm{C}\right)$ & B 02AIM 008 BT 2976 ARC 1 B & Archive & & \\
\hline
\end{tabular}


Integrated Test B Sample List

\begin{tabular}{|c|c|c|c|c|c|c|}
\hline TI \# & TI Step & Step Description & Sample ID & Analysis & File Folder & Final Electronic Report \# \\
\hline TI-WTP-PEP-066 & 7.6.16 & (a) $88^{\circ} \mathrm{C}$ (target $\left.\mathrm{T}-10^{\circ} \mathrm{C}\right)$ & B 02AIM 008 BT 2976 ARC 1 D & Archive & & \\
\hline TI-WTP-PEP-066 & 7.6.16 & (a) $88^{\circ} \mathrm{C}$ (target $\mathrm{T}-10^{\circ} \mathrm{C}$ ) & B 02AMM 008 BT 2977 ARC 1 B & Archive & & \\
\hline TI-WTP-PEP-066 & 7.6 .16 & (a) $88^{\circ} \mathrm{C}\left(\right.$ target $\left.\mathrm{T}-10^{\circ} \mathrm{C}\right)$ & B 02AMM 008 BT 2977 ARC 1 D & Archive & & \\
\hline TI-WTP-PEP-066 & 7.6.16 & (a) $88^{\circ} \mathrm{C}$ (target $\left.\mathrm{T}-10^{\circ} \mathrm{C}\right)$ & В 02AMH 008 BT 2978 ARC 1 B & Archive & & \\
\hline TI-WTP-PEP-066 & 7.6.16 & (a) $88^{\circ} \mathrm{C}\left(\right.$ target $\left.\mathrm{T}-10^{\circ} \mathrm{C}\right)$ & B 02AMH 008 BT 2978 ARC 1 D & Archive & & \\
\hline TI-WTP-PEP-066 & 7.6.16 & (a) $88^{\circ} \mathrm{C}$ (target $\mathrm{T}-10^{\circ} \mathrm{C}$ ) & B 02AOL 008 BT 2979 ARC 1 B & Archive & & \\
\hline TI-WTP-PEP-066 & 7.6.16 & (a) $88^{\circ} \mathrm{C}$ (target $\left.\mathrm{T}-10^{\circ} \mathrm{C}\right)$ & B 02AOL 008 BT 2979 ARC 1 D & Archive & & \\
\hline TI-WTP-PEP-066 & 7.6.16 & (a) $88^{\circ} \mathrm{C}$ (target $\mathrm{T}-10^{\circ} \mathrm{C}$ ) & B 02AOM 008 BT 2980 ARC 1 B & Archive & & \\
\hline TI-WTP-PEP-066 & 7.6.16 & (a) $88^{\circ} \mathrm{C}$ (target $\left.\mathrm{T}-10^{\circ} \mathrm{C}\right)$ & B 02AOM 008 BT 2980 ARC 1 D & Archive & & \\
\hline TI-WTP-PEP-066 & 7.6.16 & (a) $88^{\circ} \mathrm{C}$ (target $\left.\mathrm{T}-10^{\circ} \mathrm{C}\right)$ & B $02 \mathrm{AOH} 008 \mathrm{BT} 2981$ ARC 1 B & Archive & & \\
\hline TI-WTP-PEP-066 & 7.6.16 & (a) $88^{\circ} \mathrm{C}$ (target $\left.\mathrm{T}-10^{\circ} \mathrm{C}\right)$ & B 02AOH 008 BT 2981 ARC 1 D & Archive & & \\
\hline TI-WTP-PEP-066 & 7.6.22 & (a) $98^{\circ} \mathrm{C}$ (target $\left.\mathrm{T}, 0 \mathrm{hr}\right)$ & B 02AML 008002982 UDS 2 B & UDS & SWRI-090317 & 090317-34UDS R1 \\
\hline TI-WTP-PEP-066 & 7.6.22 & (a) $98^{\circ} \mathrm{C}$ (target T, 0 hr) & B 02AML 008002982 UDS 2 D & UDS & SWRI-090317 & 090317-34UDS R1 \\
\hline TI-WTP-PEP-066 & 7.6.22 & (a) $98^{\circ} \mathrm{C}$ (target T, 0 hr) & B 02AML 008002983 UDS 2 B & UDS & SWRI-090317 & 090317-34UDS R1 \\
\hline TI-WTP-PEP-066 & 7.6.22 & (a) $98^{\circ} \mathrm{C}$ (target T, 0 hr) & B 02AML 008002983 UDS 2 D & UDS & SWRI-090317 & 090317-34UDS R1 \\
\hline TI-WTP-PEP-066 & 7.6.22 & (a) $98^{\circ} \mathrm{C}$ (target $\mathrm{T}, 0 \mathrm{hr}$ ) & B 02AML 008002984 UDS 2 B & UDS & SWRI-090317 & 090317-34UDS R1 \\
\hline TI-WTP-PEP-066 & 7.6.22 & @ $98^{\circ} \mathrm{C}$ (target $\mathrm{T}, 0 \mathrm{hr}$ ) & В 02AML 008002984 UDS 2 D & UDS & SWRI-090317 & 090317-34UDS R1 \\
\hline TI-WTP-PEP-066 & 7.6.22 & (a) $98^{\circ} \mathrm{C}$ (target T, 0 hr) & B 02AML 008002985 DEN 2 & Density Slurry & SWRI-090317 & 090317-17, 090317-33, 090317-38 DEN slurry and liquid \\
\hline TI-WTP-PEP-066 & 7.6.22 & (a) $98^{\circ} \mathrm{C}$ (target T, 0 hr) & B 02AML 008002986 DEN 2 & Density Slurry & SWRI-090317 & 090317-17, 090317-33, 090317-38 DEN slurry and liquid \\
\hline TI-WTP-PEP-066 & 7.6.22 & (a) $98^{\circ} \mathrm{C}$ (target T, 0 hr) & B 02AML 008002987 DEN 2 & Density Slurry & SWRI-090317 & 090317-17, 090317-33, 090317-38 DEN slurry and liquid \\
\hline TI-WTP-PEP-066 & 7.6.22 & (a) $98^{\circ} \mathrm{C}$ (target T, 0 hr) & B 02AML 008002988 HTC 3 & Heat Capacity & Heat Capacity & TDP-WTP-372 \\
\hline TI-WTP-PEP-066 & 7.6.22 & (a) $98^{\circ} \mathrm{C}$ (target T, 0 hr) & B 02AML 008002989 DEN 0 B & ICP Wet Solids & $090625-24$ & 090625-24 ICP and ICPMS \\
\hline TI-WTP-PEP-066 & 7.6.22 & (a) $98^{\circ} \mathrm{C}$ (target T, 0 hr) & B 02AML 008002989 DEN 0 D & Storage & & \\
\hline TI-WTP-PEP-066 & 7.6.22 & @ $98^{\circ} \mathrm{C}$ (target T, 0 hr) & B 02AML 008002989 ICP 2 D & ICP Supernate & SWRI-090317 & 090317-30 and 090317-31 ICP and ICPMS \\
\hline TI-WTP-PEP-066 & 7.6.22 & (a) $98^{\circ} \mathrm{C}$ (target $\mathrm{T}, 0 \mathrm{hr}$ ) & B 02AML 008002989 TFI 2 D & IC & SWRI-090317 & $090317-36 \mathrm{IC}$ \\
\hline TI-WTP-PEP-066 & 7.6.22 & (a) $98^{\circ} \mathrm{C}$ (target T, 0 hr) & B 02AML 008002989 RAM 3 D & Raman & ASO_ASR8382 & ASR 8382 RAM \\
\hline TI-WTP-PEP-066 & 7.6.22 & (a) $98^{\circ} \mathrm{C}$ (target T, $0 \mathrm{hr}$ ) & B 02AML 008002990 DEN 0 B & ICP Wet Solids & $090625-24$ & 090625-24 ICP and ICPMS \\
\hline TI-WTP-PEP-066 & 7.6.22 & @ $98^{\circ} \mathrm{C}$ (target $\mathrm{T}, 0 \mathrm{hr}$ ) & B 02AML 008002990 DEN 0 D & Storage & & \\
\hline TI-WTP-PEP-066 & 7.6.22 & @ $98^{\circ} \mathrm{C}$ (target T, 0 hr) & B 02AML 008002990 ICP 2 D & ICP Supernate & SWRI-090317 & 090317-30 and 090317-31 ICP and ICPMS \\
\hline TI-WTP-PEP-066 & 7.6.22 & @ $98^{\circ} \mathrm{C}$ (target T, 0 hr) & B 02AML 008002990 TFI 2 D & IC & SWRI-090317 & $090317-36 \mathrm{IC}$ \\
\hline TI-WTP-PEP-066 & 7.6.22 & (a) $98^{\circ} \mathrm{C}$ (target T, 0 hr) & B 02AML 008002990 RAM 3 D & Raman & ASO_ASR8382 & ASR 8382 RAM \\
\hline TI-WTP-PEP-066 & 7.6.22 & (a) $98^{\circ} \mathrm{C}$ (target $\mathrm{T}, 0 \mathrm{hr}$ ) & B 02AML 008002991 DEN 0 B & ICP Wet Solids & 090625-24 & 090625-24 ICP and ICPMS \\
\hline TI-WTP-PEP-066 & 7.6 .22 & @ $98^{\circ} \mathrm{C}$ (target $\left.\mathrm{T}, 0 \mathrm{hr}\right)$ & B 02AML 008002991 DEN 0 D & Storage & & \\
\hline
\end{tabular}


Integrated Test B Sample List

\begin{tabular}{|c|c|c|c|c|c|c|}
\hline TI \# & TI Step & Step Description & Sample ID & Analysis & File Folder & Final Electronic Report \# \\
\hline TI-WTP-PEP-066 & 7.6 .22 & (a) $98^{\circ} \mathrm{C}$ (target $\left.\mathrm{T}, 0 \mathrm{hr}\right)$ & B 02AML 008002991 ICP 2 D & ICP Supernate & SWRI-090317 & 090317-30 and 090317-31 ICP and ICPMS \\
\hline TI-WTP-PEP-066 & 7.6.22 & (a) $98^{\circ} \mathrm{C}$ (target T, 0 hr) & B 02AML 008002991 TFI 2 D & IC & SWRI-090317 & 090317-36IC \\
\hline TI-WTP-PEP-066 & 7.6.22 & @ $98^{\circ} \mathrm{C}$ (target T, 0 hr) & B 02AML 008002991 RAM 3 D & Raman & ASO_ASR8382 & ASR 8382 RAM \\
\hline TI-WTP-PEP-066 & 7.6.22 & (a) $98^{\circ} \mathrm{C}$ (target $\mathrm{T}, 0 \mathrm{hr}$ ) & B 02AIL 008002992 ARC 1 B & Archive & & \\
\hline TI-WTP-PEP-066 & 7.6.22 & (a) $98^{\circ} \mathrm{C}$ (target $\mathrm{T}, 0 \mathrm{hr}$ ) & B 02AIL 008002992 ARC 1 D & Archive & & \\
\hline TI-WTP-PEP-066 & 7.6.22 & (a) $98^{\circ} \mathrm{C}$ (target $\left.\mathrm{T}, 0 \mathrm{hr}\right)$ & B 02AIH 008002993 ARC 1 B & Archive & & \\
\hline TI-WTP-PEP-066 & 7.6.22 & (a) $98^{\circ} \mathrm{C}$ (target $\left.\mathrm{T}, 0 \mathrm{hr}\right)$ & B 02AIH 008002993 ARC 1 D & Archive & & \\
\hline TI-WTP-PEP-066 & 7.6.22 & (a) $98^{\circ} \mathrm{C}$ (target $\mathrm{T}, 0 \mathrm{hr}$ ) & B 02AIM 008002994 ARC 1 B & Archive & & \\
\hline TI-WTP-PEP-066 & 7.6.22 & (a) $98^{\circ} \mathrm{C}$ (target $\mathrm{T}, 0 \mathrm{hr}$ ) & B 02AIM 008002994 ARC 1 D & Archive & & \\
\hline TI-WTP-PEP-066 & 7.6.22 & (a) $98^{\circ} \mathrm{C}$ (target T, 0 hr) & B 02AMM 008002995 ARC 1 B & Archive & & \\
\hline TI-WTP-PEP-066 & 7.6.22 & (a) $98^{\circ} \mathrm{C}$ (target $\mathrm{T}, 0 \mathrm{hr}$ ) & B 02AMM 008002995 ARC 1 D & Archive & & \\
\hline TI-WTP-PEP-066 & 7.6.22 & (a) $98^{\circ} \mathrm{C}$ (target $\left.\mathrm{T}, 0 \mathrm{hr}\right)$ & В 02AMH 008002996 ARC 1 B & Archive & & \\
\hline TI-WTP-PEP-066 & 7.6.22 & (a) $98^{\circ} \mathrm{C}$ (target $\left.\mathrm{T}, 0 \mathrm{hr}\right)$ & B 02AMH 008002996 ARC 1 D & Archive & & \\
\hline TI-WTP-PEP-066 & 7.6.22 & (a) $98^{\circ} \mathrm{C}$ (target T, 0 hr) & B 02AOL 008002997 ARC 1 B & Archive & & \\
\hline TI-WTP-PEP-066 & 7.6.22 & (a) $98^{\circ} \mathrm{C}$ (target $\mathrm{T}, 0 \mathrm{hr}$ ) & B 02AOL 008002997 ARC 1 D & Archive & & \\
\hline TI-WTP-PEP-066 & 7.6 .22 & (a) $98^{\circ} \mathrm{C}$ (target $\left.\mathrm{T}, 0 \mathrm{hr}\right)$ & B 02AOM 008002998 ARC 1 B & Archive & & \\
\hline TI-WTP-PEP-066 & 7.6.22 & (a) $98^{\circ} \mathrm{C}$ (target $\mathrm{T}, 0 \mathrm{hr}$ ) & B 02AOM 008002998 ARC 1 D & Archive & & \\
\hline TI-WTP-PEP-066 & 7.6.22 & (a) $98^{\circ} \mathrm{C}$ (target $\left.\mathrm{T}, 0 \mathrm{hr}\right)$ & B 02AOH 008002999 ARC 1 B & Archive & & \\
\hline TI-WTP-PEP-066 & 7.6.22 & (a) $98^{\circ} \mathrm{C}$ (target $\left.\mathrm{T}, 0 \mathrm{hr}\right)$ & B $02 A O H ~ 008002999$ ARC 1 D & Archive & & \\
\hline TI-WTP-PEP-066 & 7.6.22 & Target T, $1 \mathrm{hr}$ & В 02AML 008013000 UDS 2 D & UDS & SWRI-090317 & 090317-34UDS R1 \\
\hline TI-WTP-PEP-066 & 7.6.22 & Target T, $1 \mathrm{hr}$ & B 02AML 008013000 UDS 2 B & UDS & SWRI-090317 & 090317-34UDS R1 \\
\hline TI-WTP-PEP-066 & 7.6.22 & Target T, $1 \mathrm{hr}$ & B 02AML 008013001 DEN 2 & Density Slurry & SWRI-090317 & 090317-17, 090317-33, 090317-38 DEN slurry and liquid \\
\hline TI-WTP-PEP-066 & 7.6.22 & Target T, $1 \mathrm{hr}$ & B 02AML 008013002 ICP 0 B & Storage & & \\
\hline TI-WTP-PEP-066 & 7.6.22 & Target T, $1 \mathrm{hr}$ & B 02AML 008013002 SUP 0 D & Storage & & \\
\hline TI-WTP-PEP-066 & 7.6.22 & Target T, $1 \mathrm{hr}$ & B 02AML 008013002 ICP 2 D & ICP Supernate & SWRI-090317 & 090317-30 and 090317-31 ICP and ICPMS \\
\hline TI-WTP-PEP-066 & 7.6.22 & Target T, $1 \mathrm{hr}$ & B 02AML 008013002 TFI 2 D & IC & SWRI-090317 & 090317-36IC \\
\hline TI-WTP-PEP-066 & 7.6.22 & Target T, $1 \mathrm{hr}$ & B 02AML 008013002 TFI 3 D & $\mathrm{OH}$ & ASO_ASR8383 & ASR $8383 \mathrm{OH}$ rev 1 \\
\hline TI-WTP-PEP-066 & 7.6.22 & Target T, $2 \mathrm{hr}$ & B 02AML 008023003 UDS 2 D & UDS & SWRI-090317 & 090317-34UDS R1 \\
\hline TI-WTP-PEP-066 & 7.6.22 & Target T, $2 \mathrm{hr}$ & B 02AML 008023003 UDS 2 B & UDS & SWRI-090317 & 090317-34UDS R1 \\
\hline TI-WTP-PEP-066 & 7.6.22 & Target T, $2 \mathrm{hr}$ & B 02AML 008023004 DEN 2 & Density Slurry & SWRI-090317 & 090317-17, 090317-33, 090317-38 DEN slurry and liquid \\
\hline TI-WTP-PEP-066 & 7.6.22 & Target T, $2 \mathrm{hr}$ & B 02AML 008023005 ICP 0 B & Storage & & \\
\hline TI-WTP-PEP-066 & 7.6.22 & Target T, $2 \mathrm{hr}$ & B 02AML 008023005 SUP 0 D & Storage & & \\
\hline TI-WTP-PEP-066 & 7.6.22 & Target T, 2 hr & B $02 A M L 008023005$ ICP 2 D & ICP Supernate & SWRI-090317 & 090317-30 and 090317-31 ICP and ICPMS \\
\hline
\end{tabular}


Integrated Test B Sample List

\begin{tabular}{|c|c|c|c|c|c|c|}
\hline TI \# & TI Step & Step Description & Sample ID & Analysis & File Folder & Final Electronic Report \# \\
\hline TI-WTP-PEP-066 & 7.6 .22 & Target T, $2 \mathrm{hr}$ & B 02AML 008023005 TFI 2 D & IC & SWRI-090317 & $090317-36 \mathrm{IC}$ \\
\hline TI-WTP-PEP-066 & 7.6.22 & Target T, $2 \mathrm{hr}$ & B 02AML 008023005 TFI 3 D & $\mathrm{OH}$ & ASO_ASR8383 & ASR $8383 \mathrm{OH}$ rev 1 \\
\hline TI-WTP-PEP-066 & 7.6.22 & Target T, $3 \mathrm{hr}$ & B 02AML 008033006 ARC 1 B & Storage & & \\
\hline TI-WTP-PEP-066 & 7.6.22 & Target T, $3 \mathrm{hr}$ & B 02AML 008033006 ARC 1 D & Storage & & \\
\hline TI-WTP-PEP-066 & 7.6.22 & Target T, $4 \mathrm{hr}$ & B 02AML 008043007 UDS 2 B & UDS & SWRI-090317 & 090317-34UDS R1 \\
\hline TI-WTP-PEP-066 & 7.6.22 & Target T, $4 \mathrm{hr}$ & B 02AML 008043007 UDS 2 D & UDS & SWRI-090317 & 090317-34UDS R1 \\
\hline TI-WTP-PEP-066 & 7.6.22 & Target T, $4 \mathrm{hr}$ & B 02AML 008043008 DEN 2 & Density Slurry & SWRI-090317 & 090317-17, 090317-33, 090317-38 DEN slurry and liquid \\
\hline TI-WTP-PEP-066 & 7.6.22 & Target T, $4 \mathrm{hr}$ & B $02 A M L 008043009$ ICP 2 B & ICP Wet Solids & SWRI-090317 & 090317-32 ICP and ICPMS \\
\hline TI-WTP-PEP-066 & 7.6.22 & Target T, $4 \mathrm{hr}$ & B 02AML 008043009 ICP 2 D & ICP Supernate & SWRI-090317 & 090317-30 and 090317-31 ICP and ICPMS \\
\hline TI-WTP-PEP-066 & 7.6.22 & Target T, $4 \mathrm{hr}$ & B 02AML 008043009 SUP 0 D & Storage & & \\
\hline TI-WTP-PEP-066 & 7.6.22 & Target T, $4 \mathrm{hr}$ & B 02AML 008043009 TFI 2 D & IC & SWRI-090317 & 090317-36IC \\
\hline TI-WTP-PEP-066 & 7.6.22 & Target T, $4 \mathrm{hr}$ & B 02AML 008043009 TFI 3 D & $\mathrm{OH}$ & ASO_ASR8383 & ASR $8383 \mathrm{OH}$ rev 1 \\
\hline TI-WTP-PEP-066 & 7.6.22 & Target T, $4 \mathrm{hr}$ & B 02AIL 008043010 ARC 1 B & Archive & & \\
\hline TI-WTP-PEP-066 & 7.6.22 & Target T, $4 \mathrm{hr}$ & B 02AIL 008043010 ARC 1 D & Archive & & \\
\hline TI-WTP-PEP-066 & 7.6.22 & Target T, $4 \mathrm{hr}$ & B 02AIH 008043011 ARC 1 B & Archive & & \\
\hline TI-WTP-PEP-066 & 7.6.22 & Target T, $4 \mathrm{hr}$ & B 02AIH 008043011 ARC 1 D & Archive & & \\
\hline TI-WTP-PEP-066 & 7.6.22 & Target T, $4 \mathrm{hr}$ & B 02AIM 008043012 ARC 1 B & Archive & & \\
\hline TI-WTP-PEP-066 & 7.6.22 & Target T, $4 \mathrm{hr}$ & B 02AML 008043012 ARC 1 D & Archive & & \\
\hline TI-WTP-PEP-066 & 7.6.22 & Target T, $4 \mathrm{hr}$ & B 02AMM 008043013 ARC 1 B & Archive & & \\
\hline TI-WTP-PEP-066 & 7.6.22 & Target T, $4 \mathrm{hr}$ & B 02AMM 008043013 ARC 1 D & Archive & & \\
\hline TI-WTP-PEP-066 & 7.6.22 & Target T, $4 \mathrm{hr}$ & B $02 \mathrm{AMH} 008043014$ ARC 1 B & Archive & & \\
\hline TI-WTP-PEP-066 & 7.6.22 & Target T, $4 \mathrm{hr}$ & B 02AMH 008043014 ARC 1 D & Archive & & \\
\hline TI-WTP-PEP-066 & 7.6.22 & Target T, $4 \mathrm{hr}$ & B 02AOL 008043015 ARC 1 B & Archive & & \\
\hline TI-WTP-PEP-066 & 7.6.22 & Target T, $4 \mathrm{hr}$ & B 02AOL 008043015 ARC 1 D & Archive & & \\
\hline TI-WTP-PEP-066 & 7.6.22 & Target T, $4 \mathrm{hr}$ & B 02AOM 008043016 ARC 1 B & Archive & & \\
\hline TI-WTP-PEP-066 & 7.6.22 & Target T, $4 \mathrm{hr}$ & B 02AOM 008043016 ARC 1 D & Archive & & \\
\hline TI-WTP-PEP-066 & 7.6.22 & Target T, $4 \mathrm{hr}$ & B $02 \mathrm{AOH} 008043017$ ARC 1 B & Archive & & \\
\hline TI-WTP-PEP-066 & 7.6.22 & Target T, $4 \mathrm{hr}$ & B 02AOH 008043017 ARC 1 D & Archive & & \\
\hline TI-WTP-PEP-066 & 7.6.22 & Target T, $5 \mathrm{hr}$ & B 02AML 008053018 ARC 1 B & Archive & & \\
\hline TI-WTP-PEP-066 & 7.6.22 & Target T, $5 \mathrm{hr}$ & B 02AML 008053018 ARC 1 D & Archive & & \\
\hline TI-WTP-PEP-066 & 7.6.22 & Target T, $6 \mathrm{hr}$ & B 02AML 008063019 ARC 1 B & Archive & & \\
\hline TI-WTP-PEP-066 & 7.6.22 & Target T, $6 \mathrm{hr}$ & B 02AML 008063019 ARC 1 D & Archive & & \\
\hline TI-WTP-PEP-066 & 7.6.22 & Target T, $7 \mathrm{hr}$ & B 02AML 008073020 ARC 1 B & Archive & & \\
\hline
\end{tabular}


Integrated Test B Sample List

\begin{tabular}{|c|c|c|c|c|c|c|}
\hline TI \# & TI Step & Step Description & Sample ID & Analysis & File Folder & Final Electronic Report \# \\
\hline TI-WTP-PEP-066 & 7.6.22 & Target T, $7 \mathrm{hr}$ & B 02AML 008073020 ARC 1 D & Archive & & \\
\hline TI-WTP-PEP-066 & 7.6.22 & Target T, $8 \mathrm{hr}$ & B 02AML 008083021 UDS 2 B & UDS & SWRI-090317 & 090317-34UDS R1 \\
\hline TI-WTP-PEP-066 & 7.6.22 & Target T, $8 \mathrm{hr}$ & B 02AML 008083021 UDS 2 D & UDS & SWRI-090317 & 090317-34UDS R1 \\
\hline TI-WTP-PEP-066 & 7.6.22 & Target T, $8 \mathrm{hr}$ & B 02AML 008083022 DEN 2 & Density Slurry & SWRI-090317 & 090317-17, 090317-33, 090317-38 DEN slurry and liquid \\
\hline TI-WTP-PEP-066 & 7.6.22 & Target T, $8 \mathrm{hr}$ & B 02AML 008083023 ICP 2 B & ICP Wet Solids & SWRI-090317 & 090317-32 ICP and ICPMS \\
\hline TI-WTP-PEP-066 & 7.6.22 & Target T, $8 \mathrm{hr}$ & B 02AML 008083023 ICP 2 D & ICP Supernate & SWRI-090317 & 090317-30 and 090317-31 ICP and ICPMS \\
\hline TI-WTP-PEP-066 & 7.6.23 & Target T, $8 \mathrm{hr}$ & B 02AML 008083023 SUP 0 D & Storage & & \\
\hline TI-WTP-PEP-066 & 7.6.22 & Target T, $8 \mathrm{hr}$ & B 02AML 008083023 TFI 2 D & IC & SWRI-090317 & 090317-36IC \\
\hline TI-WTP-PEP-066 & 7.6.22 & Target T, $8 \mathrm{hr}$ & B 02AML 008083023 TFI 3 D & $\mathrm{OH}$ & ASO_ASR8383 & ASR $8383 \mathrm{OH}$ rev 1 \\
\hline TI-WTP-PEP-066 & 7.6.22 & Target T, $8 \mathrm{hr}$ & B 02AIL 008083024 ARC 1 B & Archive & & \\
\hline TI-WTP-PEP-066 & 7.6.22 & Target T, $8 \mathrm{hr}$ & B 02AIL 008083024 ARC 1 D & Archive & & \\
\hline TI-WTP-PEP-066 & 7.6.22 & Target T, $8 \mathrm{hr}$ & B $02 \mathrm{AIH} 008083025$ ARC $1 \mathrm{~B}$ & Archive & & \\
\hline TI-WTP-PEP-066 & 7.6.22 & Target T, $8 \mathrm{hr}$ & B 02AIH 008083025 ARC 1 D & Archive & & \\
\hline TI-WTP-PEP-066 & 7.6.22 & Target T, $8 \mathrm{hr}$ & B 02AIM 008083026 ARC 1 B & Archive & & \\
\hline TI-WTP-PEP-066 & 7.6.22 & Target T, $8 \mathrm{hr}$ & B 02AIM 008083026 ARC 1 D & Archive & & \\
\hline TI-WTP-PEP-066 & 7.6.22 & Target T, $8 \mathrm{hr}$ & В 02AMM 008083027 ARC 1 B & Archive & & \\
\hline TI-WTP-PEP-066 & 7.6.22 & Target T, $8 \mathrm{hr}$ & B 02AMM 008083027 ARC 1 D & Archive & & \\
\hline TI-WTP-PEP-066 & 7.6.22 & Target T, $8 \mathrm{hr}$ & В $02 \mathrm{AMH} 008083028$ ARC 1 B & Archive & & \\
\hline TI-WTP-PEP-066 & 7.6.22 & Target T, $8 \mathrm{hr}$ & B 02AMH 008083028 ARC 1 D & Archive & & \\
\hline TI-WTP-PEP-066 & 7.6.22 & Target T, $8 \mathrm{hr}$ & B 02AOL 008083029 ARC 1 B & Archive & & \\
\hline TI-WTP-PEP-066 & 7.6.22 & Target T, $8 \mathrm{hr}$ & B 02AOL 008083029 ARC 1 D & Archive & & \\
\hline TI-WTP-PEP-066 & 7.6.22 & Target T, $8 \mathrm{hr}$ & B $02 \mathrm{AOM} 008083030$ ARC $1 \mathrm{~B}$ & Archive & & \\
\hline TI-WTP-PEP-066 & 7.6 .22 & Target T, $8 \mathrm{hr}$ & B 02AOM 008083030 ARC 1 D & Archive & & \\
\hline TI-WTP-PEP-066 & 7.6.22 & Target T, $8 \mathrm{hr}$ & B $02 \mathrm{AOH} 008083031$ ARC $1 \mathrm{~B}$ & Archive & & \\
\hline TI-WTP-PEP-066 & 7.6.22 & Target T, $8 \mathrm{hr}$ & B $02 \mathrm{AOH} 008083031$ ARC 1 D & Archive & & \\
\hline TI-WTP-PEP-066 & 7.6.22 & Target T, $9 \mathrm{hr}$ & B 02AML 008093032 ARC 1 B & Archive & & \\
\hline TI-WTP-PEP-066 & 7.6.22 & Target T, $9 \mathrm{hr}$ & B 02AML 008093032 ARC 1 D & Archive & & \\
\hline TI-WTP-PEP-066 & 7.6.22 & Target $\mathrm{T}, 10 \mathrm{hr}$ & B 02AML 008103033 UDS 2 B & UDS & SWRI-090317 & 090317-34UDS R1 \\
\hline TI-WTP-PEP-066 & 7.6.22 & Target $\mathrm{T}, 10 \mathrm{hr}$ & B 02AML 008103033 UDS 2 D & UDS & SWRI-090317 & 090317-34UDS R1 \\
\hline TI-WTP-PEP-066 & 7.6.22 & Target $\mathrm{T}, 10 \mathrm{hr}$ & B 02AML 008103034 DEN 2 & Density Slurry & SWRI-090317 & 090317-17, 090317-33, 090317-38 DEN slurry and liquid \\
\hline TI-WTP-PEP-066 & 7.6.22 & Target $\mathrm{T}, 10 \mathrm{hr}$ & B 02AML 008103035 ICP 0 B & Storage & & \\
\hline TI-WTP-PEP-066 & 7.6.22 & Target $\mathrm{T}, 10 \mathrm{hr}$ & B 02AML 008103035 SUP 0 D & Storage & & \\
\hline TI-WTP-PEP-066 & 7.6.22 & Target $\mathrm{T}, 10 \mathrm{hr}$ & B 02AML 008103035 ICP 2 D & ICP Supernate & SWRI-090317 & 090317-30 and 090317-31 ICP and ICPMS \\
\hline
\end{tabular}


Integrated Test B Sample List

\begin{tabular}{|c|c|c|c|c|c|c|}
\hline TI \# & TI Step & Step Description & Sample ID & Analysis & File Folder & Final Electronic Report \# \\
\hline TI-WTP-PEP-066 & 7.6.22 & Target $\mathrm{T}, 10 \mathrm{hr}$ & B 02AML 008103035 TFI 2 D & IC & SWRI-090317 & 090317-36IC \\
\hline TI-WTP-PEP-066 & 7.6.22 & Target $\mathrm{T}, 10 \mathrm{hr}$ & B 02AML 008103035 TFI 3 D & $\mathrm{OH}$ & ASO_ASR8383 & ASR $8383 \mathrm{OH}$ rev 1 \\
\hline TI-WTP-PEP-066 & 7.6.22 & Target $\mathrm{T}, 11 \mathrm{hr}$ & B 02AML 008113036 ARC 1 B & Storage & & \\
\hline TI-WTP-PEP-066 & 7.6.22 & Target T, $11 \mathrm{hr}$ & B 02AML 008113036 ARC 1 D & Storage & & \\
\hline TI-WTP-PEP-066 & 7.6.22 & Target $\mathrm{T}, 12 \mathrm{hr}$ & B 02AML 008123037 UDS 2 B & UDS & SWRI-090317 & 090317-34UDS R1 \\
\hline TI-WTP-PEP-066 & 7.6.22 & Target $\mathrm{T}, 12 \mathrm{hr}$ & B 02AML 008123037 UDS 2 D & UDS & SWRI-090317 & 090317-34UDS R1 \\
\hline TI-WTP-PEP-066 & 7.6.22 & Target T, $12 \mathrm{hr}$ & B 02AML 008123038 UDS 2 B & UDS & SWRI-090317 & 090317-34UDS R1 \\
\hline TI-WTP-PEP-066 & 7.6.22 & Target $\mathrm{T}, 12 \mathrm{hr}$ & B 02AML 008123038 UDS 2 D & UDS & SWRI-090317 & 090317-34UDS R1 \\
\hline TI-WTP-PEP-066 & 7.6.22 & Target T, $12 \mathrm{hr}$ & B 02AML 008123039 UDS 2 B & UDS & SWRI-090317 & 090317-34UDS R1 \\
\hline TI-WTP-PEP-066 & 7.6.22 & Target $\mathrm{T}, 12 \mathrm{hr}$ & B 02AML 008123039 UDS 2 D & UDS & SWRI-090317 & 090317-34UDS R1 \\
\hline TI-WTP-PEP-066 & 7.6.22 & Target T, $12 \mathrm{hr}$ & B 02AML 008123040 DEN 2 & Density Slurry & SWRI-090317 & 090317-17, 090317-33, 090317-38 DEN slurry and liquid \\
\hline TI-WTP-PEP-066 & 7.6.22 & Target $\mathrm{T}, 12 \mathrm{hr}$ & B 02AML 008123041 DEN 2 & Density Slurry & SWRI-090317 & $090317-17,090317-33,090317-38 \mathrm{DEN}$ slurry and liquid \\
\hline TI-WTP-PEP-066 & 7.6.22 & Target $\mathrm{T}, 12 \mathrm{hr}$ & B 02AML 008123042 DEN 2 & Density Slurry & SWRI-090317 & 090317-17, 090317-33, 090317-38 DEN slurry and liquid \\
\hline TI-WTP-PEP-066 & 7.6.22 & Target $\mathrm{T}, 12 \mathrm{hr}$ & B 02AML 008123043 ICP 2 B & ICP Wet Solids & SWRI-090317 & 090317-32 ICP and ICPMS \\
\hline TI-WTP-PEP-066 & 7.6.22 & Target $\mathrm{T}, 12 \mathrm{hr}$ & B 02AML 008123043 ICP 2 D & ICP Supernate & SWRI-090317 & 090317-30 and 090317-31 ICP and ICPMS \\
\hline TI-WTP-PEP-066 & 7.6.22 & Target $\mathrm{T}, 12 \mathrm{hr}$ & B 02AML 008123043 DEN 0 D & Storage & & \\
\hline TI-WTP-PEP-066 & 7.6.22 & Target T, $12 \mathrm{hr}$ & B 02AML 008123043 TFI 2 D & IC & SWRI-090317 & 090317-36IC \\
\hline TI-WTP-PEP-066 & 7.6.22 & Target $\mathrm{T}, 12 \mathrm{hr}$ & B 02AML 008123043 TFI 3 D & $\mathrm{OH}$ & ASO_ASR8383 & ASR $8383 \mathrm{OH}$ rev 1 \\
\hline TI-WTP-PEP-066 & 7.6.22 & Target T, $12 \mathrm{hr}$ & B 02AML 008123043 RAM 3 D & Raman & ASO_ASR8382 & ASR 8382 RAM \\
\hline TI-WTP-PEP-066 & 7.6.22 & Target $\mathrm{T}, 12 \mathrm{hr}$ & B 02AML 008123044 ICP 2 B & ICP Wet Solids & SWRI-090317 & 090317-32 ICP and ICPMS \\
\hline TI-WTP-PEP-066 & 7.6.22 & Target $\mathrm{T}, 12 \mathrm{hr}$ & B 02AML 008123044 ICP 2 D & ICP Supernate & SWRI-090317 & 090317-30 and 090317-31 ICP and ICPMS \\
\hline TI-WTP-PEP-066 & 7.6.22 & Target $\mathrm{T}, 12 \mathrm{hr}$ & B 02AML 008123044 DEN 0 D & Storage & & \\
\hline TI-WTP-PEP-066 & 7.6 .22 & Target T, $12 \mathrm{hr}$ & B 02AML 008123044 TFI 2 D & $\mathrm{IC}$ & SWRI-090317 & 090317-36IC \\
\hline TI-WTP-PEP-066 & 7.6.22 & Target $\mathrm{T}, 12 \mathrm{hr}$ & B 02AML 008123044 TFI 3 D & $\mathrm{OH}$ & ASO_ASR8383 & ASR $8383 \mathrm{OH}$ rev 1 \\
\hline TI-WTP-PEP-066 & 7.6 .22 & Target T, $12 \mathrm{hr}$ & B 02AML 008123044 RAM 3 D & Raman & ASO_ASR8382 & ASR 8382 RAM \\
\hline TI-WTP-PEP-066 & 7.6.22 & Target $\mathrm{T}, 12 \mathrm{hr}$ & B 02 AML 008123045 ICP 2 B & ICP Wet Solids & SWRI-090317 & 090317-32 ICP and ICPMS \\
\hline TI-WTP-PEP-066 & 7.6.22 & Target T, $12 \mathrm{hr}$ & B 02AML 008123045 ICP 2 D & ICP Supernate & SWRI-090317 & $090317-30$ and $090317-31$ ICP and ICPMS \\
\hline TI-WTP-PEP-066 & 7.6.22 & Target $\mathrm{T}, 12 \mathrm{hr}$ & B 02AML 008123045 DEN 0 D & Storage & & \\
\hline TI-WTP-PEP-066 & 7.6.22 & Target T, $12 \mathrm{hr}$ & B 02AML 008123045 TFI 2 D & IC & SWRI-090317 & 090317-36IC \\
\hline TI-WTP-PEP-066 & 7.6.22 & Target $\mathrm{T}, 12 \mathrm{hr}$ & B 02AML 008123045 TFI 3 D & $\mathrm{OH}$ & ASO_ASR8383 & ASR $8383 \mathrm{OH}$ rev 1 \\
\hline TI-WTP-PEP-066 & 7.6 .22 & Target T, $12 \mathrm{hr}$ & B 02AML 008123045 RAM 3 D & Raman & ASO_ASR8382 & ASR 8382 RAM \\
\hline TI-WTP-PEP-066 & 7.6.22 & Target $\mathrm{T}, 12 \mathrm{hr}$ & B 02AIL 008123046 ARC 1 B & Archive & & \\
\hline TI-WTP-PEP-066 & 7.6.22 & Target T, $12 \mathrm{hr}$ & B 02AIL 008123046 ARC 1 D & Archive & & \\
\hline
\end{tabular}


Integrated Test B Sample List

\begin{tabular}{|c|c|c|c|c|c|c|}
\hline TI \# & TI Step & Step Description & Sample ID & Analysis & File Folder & Final Electronic Report \# \\
\hline TI-WTP-PEP-066 & 7.6 .22 & Target T, $12 \mathrm{hr}$ & B 02AIH 008123047 ARC 1 B & Archive & & \\
\hline TI-WTP-PEP-066 & 7.6.22 & Target T, $12 \mathrm{hr}$ & B 02AIH 008123047 ARC 1 D & Archive & & \\
\hline TI-WTP-PEP-066 & 7.6.22 & Target T, $12 \mathrm{hr}$ & B 02AIM 008123048 ARC 1 B & Archive & & \\
\hline TI-WTP-PEP-066 & 7.6.22 & Target T, $12 \mathrm{hr}$ & B 02AIM 008123048 ARC 1 D & Archive & & \\
\hline TI-WTP-PEP-066 & 7.6.22 & Target T, $12 \mathrm{hr}$ & B 02AMM 008123049 ARC 1 B & Archive & & \\
\hline TI-WTP-PEP-066 & 7.6.22 & Target T, $12 \mathrm{hr}$ & B 02AMM 008123049 ARC 1 D & Archive & & \\
\hline TI-WTP-PEP-066 & 7.6.22 & Target T, $12 \mathrm{hr}$ & B 02AMH 008123050 ARC 1 B & Archive & & \\
\hline TI-WTP-PEP-066 & 7.6.22 & Target T, $12 \mathrm{hr}$ & В 02AMH 008123050 ARC 1 D & Archive & & \\
\hline TI-WTP-PEP-066 & 7.6.22 & Target T, $12 \mathrm{hr}$ & B 02AOL 008123051 ARC 1 B & Archive & & \\
\hline TI-WTP-PEP-066 & 7.6.22 & Target T, $12 \mathrm{hr}$ & B 02AOL 008123051 ARC 1 D & Archive & & \\
\hline TI-WTP-PEP-066 & 7.6.22 & Target T, $12 \mathrm{hr}$ & B 02AOM 008123052 ARC 1 B & Archive & & \\
\hline TI-WTP-PEP-066 & 7.6.22 & Target T, $12 \mathrm{hr}$ & B 02AOM 008123052 ARC 1 D & Archive & & \\
\hline TI-WTP-PEP-066 & 7.6.22 & Target T, $12 \mathrm{hr}$ & B $02 \mathrm{AOH} 008123053$ ARC $1 \mathrm{~B}$ & Archive & & \\
\hline TI-WTP-PEP-066 & 7.6.22 & Target T, $12 \mathrm{hr}$ & B 02AOH 008123053 ARC 1 D & Archive & & \\
\hline TI-WTP-PEP-066 & 7.6.22 & Target T, $13 \mathrm{hr}$ & B 02AML 008133054 ARC 1 B & Archive & & \\
\hline TI-WTP-PEP-066 & 7.6.22 & Target T, $13 \mathrm{hr}$ & B 02AML 008133054 ARC 1 D & Archive & & \\
\hline TI-WTP-PEP-066 & 7.6.22 & Target T, $14 \mathrm{hr}$ & B 02AML 008143055 UDS 2 B & UDS & SWRI-090317 & 090317-34UDS R1 \\
\hline TI-WTP-PEP-066 & 7.6.22 & Target T, $14 \mathrm{hr}$ & B 02AML 008143055 UDS 2 D & UDS & SWRI-090317 & 090317-34UDS R1 \\
\hline TI-WTP-PEP-066 & 7.6.22 & Target T, $14 \mathrm{hr}$ & B 02AML 008143056 DEN 2 & Density Slurry & SWRI-090317 & 090317-17, 090317-33, 090317-38 DEN slurry and liquid \\
\hline TI-WTP-PEP-066 & 7.6.22 & Target T, $14 \mathrm{hr}$ & B 02AML 008143057 ICP 0 B & Storage & & \\
\hline TI-WTP-PEP-066 & 7.6.22 & Target T, $14 \mathrm{hr}$ & B 02AML 008143057 SUP 0 D & Storage & & \\
\hline TI-WTP-PEP-066 & 7.6.22 & Target T, $14 \mathrm{hr}$ & B 02AML 008143057 ICP 2 D & ICP Supernate & SWRI-090317 & 090317-30 and 090317-31 ICP and ICPMS \\
\hline TI-WTP-PEP-066 & 7.6.22 & Target T, $14 \mathrm{hr}$ & B 02AML 008143057 TFI 2 D & IC & SWRI-090317 & $090317-36 \mathrm{IC}$ \\
\hline TI-WTP-PEP-066 & 7.6.22 & Target T, $14 \mathrm{hr}$ & B 02AML 008143057 TFI 3 D & $\mathrm{OH}$ & ASO_ASR8383 & ASR $8383 \mathrm{OH}$ rev 1 \\
\hline TI-WTP-PEP-066 & 7.6.22 & Target T, $15 \mathrm{hr}$ & B 02AML 008153058 ARC 1 B & Archive & & \\
\hline TI-WTP-PEP-066 & 7.6.22 & Target T, $15 \mathrm{hr}$ & B 02AML 008153058 ARC 1 D & Archive & & \\
\hline TI-WTP-PEP-066 & 7.6.22 & Target T, $16 \mathrm{hr}$ & B 02AML 008 BL 4458 ARC 1 B & Archive & & \\
\hline TI-WTP-PEP-066 & 7.6.22 & Target T, $16 \mathrm{hr}$ & B 02AML 008 BL 4458 ARC 1 D & Archive & & \\
\hline TI-WTP-PEP-066 & 7.6.22 & Target T, $16 \mathrm{hr}$ & B 02AML 008163059 UDS 2 B & UDS & SWRI-090317 & 090317-34UDS R1 \\
\hline TI-WTP-PEP-066 & 7.6.22 & Target T, $16 \mathrm{hr}$ & B 02AML 008163059 UDS 2 D & UDS & SWRI-090317 & 090317-34UDS R1 \\
\hline TI-WTP-PEP-066 & 7.6.22 & Target T, $16 \mathrm{hr}$ & B 02AML 008163060 UDS 2 B & UDS & SWRI-090317 & 090317-34UDS R1 \\
\hline TI-WTP-PEP-066 & 7.6.22 & Target T, $16 \mathrm{hr}$ & B 02AML 008163060 UDS 2 D & UDS & SWRI-090317 & 090317-34UDS R1 \\
\hline TI-WTP-PEP-066 & 7.6.22 & Target T, $16 \mathrm{hr}$ & B 02AML 008163061 UDS 2 B & UDS & SWRI-090317 & 090317-34UDS R1 \\
\hline
\end{tabular}


Integrated Test B Sample List

\begin{tabular}{|c|c|c|c|c|c|c|}
\hline TI \# & TI Step & Step Description & Sample ID & Analysis & File Folder & Final Electronic Report \# \\
\hline TI-WTP-PEP-066 & 7.6.22 & Target $\mathrm{T}, 16 \mathrm{hr}$ & B 02AML 008163061 UDS 2 D & UDS & SWRI-090317 & 090317-34UDS R1 \\
\hline TI-WTP-PEP-066 & 7.6.22 & Target $\mathrm{T}, 16 \mathrm{hr}$ & B 02AML 008163062 DEN 2 & Density Slurry & SWRI-090317 & 090317-17, 090317-33, 090317-38 DEN slurry and liquid \\
\hline TI-WTP-PEP-066 & 7.6.22 & Target $\mathrm{T}, 16 \mathrm{hr}$ & B 02AML 008163063 DEN 2 & Density Slurry & SWRI-090317 & 090317-17, 090317-33, 090317-38 DEN slurry and liquid \\
\hline TI-WTP-PEP-066 & 7.6.22 & Target $\mathrm{T}, 16 \mathrm{hr}$ & B 02AML 008163064 DEN 2 & Density Slurry & SWRI-090317 & 090317-17, 090317-33, 090317-38 DEN slurry and liquid \\
\hline TI-WTP-PEP-066 & 7.6.22 & Target T, $16 \mathrm{hr}$ & B 02AML 008163065 ICP 2 B & ICP Wet Solids & SWRI-090317 & 090317-32 ICP and ICPMS \\
\hline TI-WTP-PEP-066 & 7.6.22 & Target T, $16 \mathrm{hr}$ & В 02AML 008163065 ICP 2 D & ICP Supernate & SWRI-090317 & 090317-30 and 090317-31 ICP and ICPMS \\
\hline TI-WTP-PEP-066 & 7.6.22 & Target $\mathrm{T}, 16 \mathrm{hr}$ & B 02AML 008163065 DEN 0 D & Storage & & \\
\hline TI-WTP-PEP-066 & 7.6.22 & Target $\mathrm{T}, 16 \mathrm{hr}$ & B 02AML 008163065 TFI 2 D & IC & SWRI-090317 & 090317-36IC \\
\hline TI-WTP-PEP-066 & 7.6.22 & Target T, $16 \mathrm{hr}$ & B 02AML 008163065 TFI 3 D & $\mathrm{OH}$ & ASO_ASR8383 & ASR $8383 \mathrm{OH}$ rev 1 \\
\hline TI-WTP-PEP-066 & 7.6.22 & Target $\mathrm{T}, 16 \mathrm{hr}$ & B 02AML 008163065 RAM 3 D & Raman & ASO_ASR8382 & ASR 8382 RAM \\
\hline TI-WTP-PEP-066 & 7.6.22 & Target T, $16 \mathrm{hr}$ & B 02AML 008163066 ICP 2 B & ICP Wet Solids & SWRI-090317 & 090317-32 ICP and ICPMS \\
\hline TI-WTP-PEP-066 & 7.6.22 & Target T, $16 \mathrm{hr}$ & B 02AML 008163066 ICP 2 D & ICP Supernate & SWRI-090317 & 090317-30 and 090317-31 ICP and ICPMS \\
\hline TI-WTP-PEP-066 & 7.6.22 & Target T, $16 \mathrm{hr}$ & B 02AML 008163066 DEN 0 D & Storage & & \\
\hline TI-WTP-PEP-066 & 7.6.22 & Target T, $16 \mathrm{hr}$ & B 02AML 008163066 TFI 2 D & IC & SWRI-090317 & 090317-36IC \\
\hline TI-WTP-PEP-066 & 7.6.22 & Target $\mathrm{T}, 16 \mathrm{hr}$ & B 02AML 008163066 TFI 3 D & $\mathrm{OH}$ & ASO_ASR8383 & ASR $8383 \mathrm{OH}$ rev 1 \\
\hline TI-WTP-PEP-066 & 7.6.22 & Target $\mathrm{T}, 16 \mathrm{hr}$ & B 02AML 008163066 RAM 3 D & Raman & ASO_ASR8382 & ASR 8382 RAM \\
\hline TI-WTP-PEP-066 & 7.6.22 & Target T, $16 \mathrm{hr}$ & B 02AML 008163067 ICP 2 B & ICP Wet Solids & SWRI-090317 & 090317-32 ICP and ICPMS \\
\hline TI-WTP-PEP-066 & 7.6.22 & Target $\mathrm{T}, 16 \mathrm{hr}$ & B 02AML 008163067 ICP 2 D & ICP Supernate & SWRI-090317 & $090317-30$ and $090317-31$ ICP and ICPMS \\
\hline TI-WTP-PEP-066 & 7.6.22 & Target T, $16 \mathrm{hr}$ & B 02AML 008163067 DEN 0 D & Storage & & \\
\hline TI-WTP-PEP-066 & 7.6.22 & Target T, $16 \mathrm{hr}$ & B 02AML 008163067 TFI 2 D & IC & SWRI-090317 & 090317-36IC \\
\hline TI-WTP-PEP-066 & 7.6.22 & Target T, $16 \mathrm{hr}$ & B 02AML 008163067 TFI 3 D & $\mathrm{OH}$ & ASO_ASR8383 & ASR $8383 \mathrm{OH}$ rev 1 \\
\hline TI-WTP-PEP-066 & 7.6.22 & Target T, $16 \mathrm{hr}$ & B 02AML 008163067 RAM 3 D & Raman & ASO_ASR8382 & ASR 8382 RAM \\
\hline TI-WTP-PEP-066 & 7.6 .22 & Target T, $16 \mathrm{hr}$ & B 02AIL 008163068 ARC 1 B & Archive & & \\
\hline TI-WTP-PEP-066 & 7.6.22 & Target T, $16 \mathrm{hr}$ & B 02AIL 008163068 ARC 1 D & Archive & & \\
\hline TI-WTP-PEP-066 & 7.6.22 & Target T, $16 \mathrm{hr}$ & B 02AIH 008163069 ARC 1 B & Archive & & \\
\hline TI-WTP-PEP-066 & 7.6.22 & Target T, $16 \mathrm{hr}$ & B 02AIH 008163069 ARC 1 D & Archive & & \\
\hline TI-WTP-PEP-066 & 7.6.22 & Target T, $16 \mathrm{hr}$ & B 02AIM 008163070 ARC 1 B & Archive & & \\
\hline TI-WTP-PEP-066 & 7.6.22 & Target $\mathrm{T}, 16 \mathrm{hr}$ & B 02AIM 008163070 ARC 1 D & Archive & & \\
\hline TI-WTP-PEP-066 & 7.6 .22 & Target T, $16 \mathrm{hr}$ & B 02AMM 008163071 ARC 1 B & Archive & & \\
\hline TI-WTP-PEP-066 & 7.6.22 & Target $\mathrm{T}, 16 \mathrm{hr}$ & B 02AMM 008163071 ARC 1 D & Archive & & \\
\hline TI-WTP-PEP-066 & 7.6.22 & Target T, $16 \mathrm{hr}$ & B 02AMH 008163072 ARC 1 B & Archive & & \\
\hline TI-WTP-PEP-066 & 7.6.22 & Target T, $16 \mathrm{hr}$ & В 02AMH 008163072 ARC 1 D & Archive & & \\
\hline TI-WTP-PEP-066 & 7.6.22 & Target T, $16 \mathrm{hr}$ & B 02AOL 008163073 ARC 1 B & Archive & & \\
\hline
\end{tabular}


Integrated Test B Sample List

\begin{tabular}{|c|c|c|c|c|c|c|}
\hline TI \# & TI Step & Step Description & Sample ID & Analysis & File Folder & Final Electronic Report \# \\
\hline TI-WTP-PEP-066 & 7.6 .22 & Target T, $16 \mathrm{hr}$ & B 02AOL 008163073 ARC 1 D & Archive & & \\
\hline TI-WTP-PEP-066 & 7.6.22 & Target T, $16 \mathrm{hr}$ & B 02AOM 008163074 ARC 1 B & Archive & & \\
\hline TI-WTP-PEP-066 & 7.6.22 & Target T, $16 \mathrm{hr}$ & B 02AOM 008163074 ARC 1 D & Archive & & \\
\hline TI-WTP-PEP-066 & 7.6.22 & Target T, $16 \mathrm{hr}$ & B 02AOH 008163075 ARC 1 B & Archive & & \\
\hline TI-WTP-PEP-066 & 7.6.22 & Target $\mathrm{T}, 16 \mathrm{hr}$ & B 02AOH 008163075 ARC 1 D & Archive & & \\
\hline TI-WTP-PEP-066 & 7.7.6 & Cool-down of UFP-VSL-T02A using cooling jacket & B 02AML 009 XX 3084 UDS 2 & UDS & SWRI-090317 & 090317-34UDS R1 \\
\hline TI-WTP-PEP-066 & 7.7.6 & Cool-down of UFP-VSL-T02A using cooling jacket & B 02AML 009 XX 3085 DEN 2 & Density Supernate & SWRI-090317 & 090317-17, 090317-33, 090317-38 DEN slurry and liquid \\
\hline TI-WTP-PEP-066 & 7.7.6 & Cool-down of UFP-VSL-T02A using cooling jacket & B 02AML 009 XX 3085 DEN 2 & Density Slurry & SWRI-090317 & 090317-17, 090317-33, 090317-38 DEN slurry and liquid \\
\hline TI-WTP-PEP-066 & 7.7.6 & Cool-down of UFP-VSL-T02A using cooling jacket & B 02AML 009 XX 3086 ICP 2 B & ICP Wet Solids & SWRI-090317 & 090317-32 ICP and ICPMS \\
\hline TI-WTP-PEP-066 & 7.7.6 & Cool-down of UFP-VSL-T02A using cooling jacket & B 02AML 009 XX 3086 ICP 2 D & ICP Supernate & SWRI-090317 & 090317-30 and 090317-31 ICP and ICPMS \\
\hline TI-WTP-PEP-066 & 7.7.6 & Cool-down of UFP-VSL-T02A using cooling jacket & B 02AML 009 XX 3086 SUP 0 D & Storage & & \\
\hline TI-WTP-PEP-066 & 7.7.6 & Cool-down of UFP-VSL-T02A using cooling jacket & B 02AML 009 XX 3086 TFI 2 D & IC & SWRI-090317 & 090317-37IC \\
\hline TI-WTP-PEP-066 & 7.7.6 & Cool-down of UFP-VSL-TO2A using cooling jacket. & B 02AML 009 XX 3086 TFI 3 D & $\mathrm{OH}$ & ASO_ASR8383 & ASR $8383 \mathrm{OH}$ rev 1 \\
\hline TI-WTP-PEP-066 & 7.7.6 & Cool-down of UFP-VSL-T02A using cooling jacket & B 02AIL 009 XX 3076 ARC 1 & Archive & & \\
\hline TI-WTP-PEP-066 & 7.7.6 & Cool-down of UFP-VSL-T02A using cooling jacket & B 02AIM 009 XX 3077 ARC 1 & Archive & & \\
\hline TI-WTP-PEP-066 & 7.7.6 & Cool-down of UFP-VSL-T02A using cooling jacket & B 02AOL 009 XX 3078 ARC 1 & Archive & & \\
\hline TI-WTP-PEP-066 & 7.7.6 & Cool-down of UFP-VSL-T02A using cooling jacket & B 02AMM 009 XX 3079 ARC 1 & Archive & & \\
\hline TI-WTP-PEP-066 & 7.7.6 & Cool-down of UFP-VSL-T02A using cooling jacket & B 02AOM 009 XX 3080 ARC 1 & Archive & & \\
\hline TI-WTP-PEP-066 & 7.7.6 & Cool-down of UFP-VSL-T02A using cooling jacket & B 02AIH 009 XX 3081 ARC 1 & Archive & & \\
\hline TI-WTP-PEP-066 & 7.7.6 & Cool-down of UFP-VSL-T02A using cooling jacket & B 02AMH 009 XX 3082 ARC 1 & Archive & & \\
\hline TI-WTP-PEP-066 & 7.7.6 & Cool-down of UFP-VSL-T02A using cooling jacket & B 02AOH 009 XX 3083 ARC 1 & Archive & & \\
\hline TI-WTP-PEP-066 & 7.9.1 & HLP-T22 TO TO1A transfer & B T221A 011 XX 3087 ARC 1 & Archive & & \\
\hline TI-WTP-PEP-066 & 7.9 .1 & HLP-T22 TO TO1A transfer & B T221A 011 XX 3088 ARC 1 & Archive & & \\
\hline TI-WTP-PEP-066 & 7.9.1 & HLP-T22 TO TO1A transfer & B T221A 011 XX 3089 ARC 1 & Archive & & \\
\hline TI-WTP-PEP-066 & 7.9 .1 & HLP-T22 TO TO1A transfer & B T221A 011 XX 3090 ARC 1 & Archive & & \\
\hline TI-WTP-PEP-066 & 7.10 .4 & TO1A to TO2A transfer & B 01A2A 012 XX 3091 ARC 1 & Archive & & \\
\hline TI-WTP-PEP-066 & 7.10 .4 & TO1A to TO2A transfer & B 01A2A 012 XX 3092 ARC 1 & Archive & & \\
\hline TI-WTP-PEP-066 & 7.10 .4 & TO1A to TO2A transfer & B 01A2A 012 XX 3093 ARC 1 & Archive & & \\
\hline TI-WTP-PEP-066 & 7.10 .4 & TO1A to TO2A transfer & B 01A2A 012 XX 3094 ARC 1 & Archive & & \\
\hline TI-WTP-PEP-066 & 7.12 .7 & Concentrate solids in UFP-VSL-T02A to $20-\mathrm{wt} \%$ UDS & B 01A2A 014 XX 3095 ARC 1 & Archive & & \\
\hline TI-WTP-PEP-066 & 7.12 .7 & Concentrate solids in UFP-VSL-T02A to 20 -wt $\%$ UDS & B 01A2A 014 XX 3096 ARC 1 & Archive & & \\
\hline TI-WTP-PEP-066 & 7.12 .7 & Concentrate solids in UFP-VSL-T02A to 20 -wt $\%$ UDS & B 01A2A 014 XX 3097 ARC 1 & Archive & & \\
\hline TI-WTP-PEP-066 & 7.12 .7 & Concentrate solids in UFP-VSL-T02A to 20 -wt $\%$ UDS & B 01A2A 014 XX 3098 ARC 1 & Archive & & \\
\hline
\end{tabular}


Integrated Test B Sample List

\begin{tabular}{|c|c|c|c|c|c|c|}
\hline TI \# & TI Step & Step Description & Sample ID & Analysis & File Folder & Final Electronic Report \# \\
\hline TI-WTP-PEP-066 & 7.12 .7 & Concentrate solids in UFP-VSL-T02A to 20 -wt $\%$ UDS & B 01A2A 014 XX 3099 ARC 1 & Archive & & \\
\hline TI-WTP-PEP-066 & 7.12.7 & Concentrate solids in UFP-VSL-T02A to 20 -wt\% UDS & B 00PF1 014 XX 4000 ARC 1 & Archive & & \\
\hline TI-WTP-PEP-066 & 7.12.7 & Concentrate solids in UFP-VSL-T02A to 20 -wt $\%$ UDS & B 00PF1 014 XX 4001 ARC 1 & Archive & & \\
\hline TI-WTP-PEP-066 & 7.12 .7 & Concentrate solids in UFP-VSL-T02A to 20 -wt $\%$ UDS & B 00PF1 014 XX 4002 ARC 1 & Archive & & \\
\hline TI-WTP-PEP-066 & 7.12.7 & Concentrate solids in UFP-VSL-T02A to 20 -wt $\%$ UDS & B 02AML 014 XX 4003 UDS 2 & UDS & SWRI-090317 & 090317-39UDS R1 \\
\hline TI-WTP-PEP-066 & 7.12.7 & Concentrate solids in UFP-VSL-T02A to 20-wt\% UDS & B 02AML 014 XX 4004 RHE 4 & Rheology & APEL Rheology & TDP WTP 363 Int Test B RHE Data on PEP slurry \\
\hline TI-WTP-PEP-066 & 7.12.7 & Concentrate solids in UFP-VSL-T02A to 20 -wt $\%$ UDS & B 02AML 014 XX 4005 UDS 2 & UDS & SWRI-090317 & 090317-39UDS R1 \\
\hline TI-WTP-PEP-066 & 7.12 .7 & Concentrate solids in UFP-VSL-T02A to 20 -wt $\%$ UDS & B 02AML 014 XX 4006 UDS 2 & UDS & SWRI-090317 & 090317-39UDS R1 \\
\hline TI-WTP-PEP-066 & 7.12 .7 & Concentrate solids in UFP-VSL-T02A to 20 -wt $\%$ UDS & B 02AML 014 XX 4007 UDS 2 & UDS & SWRI-090317 & 090317-39UDS R1 \\
\hline TI-WTP-PEP-066 & 7.12 .7 & Concentrate solids in UFP-VSL-T02A to 20 -wt $\%$ UDS & B 02AML 014 XX 4008 UDS 2 & UDS & SWRI-090317 & 090317-39UDS R1 \\
\hline TI-WTP-PEP-066 & 7.12.7 & Concentrate solids in UFP-VSL-T02A to 20 -wt $\%$ UDS & B 02AML 014 XX 4009 UDS 2 & UDS & SWRI-090317 & 090317-39UDS R1 \\
\hline TI-WTP-PEP-066 & 7.12 .7 & Concentrate solids in UFP-VSL-T02A to 20 -wt $\%$ UDS & B 02AML 014 XX 4010 UDS 2 & UDS & SWRI-090317 & 090317-39UDS R1 \\
\hline TI-WTP-PEP-066 & 7.12 .7 & Concentrate solids in UFP-VSL-T02A to 20 -wt $\%$ UDS & B 02AML 014 XX 4011 UDS 2 & UDS & SWRI-090317 & 090317-39UDS R1 \\
\hline TI-WTP-PEP-066 & 7.12 .7 & Concentrate solids in UFP-VSL-T02A to 20 -wt $\%$ UDS & B 02AML 014 XX 4012 UDS 2 & UDS & SWRI-090317 & 090317-39UDS R1 \\
\hline TI-WTP-PEP-066 & 7.12.7 & Concentrate solids in UFP-VSL-T02A to 20 -wt $\%$ UDS & B 02AML 014 XX 4013 UDS 2 & UDS & SWRI-090317 & 090317-39UDS R1 \\
\hline TI-WTP-PEP-066 & 7.12.7 & Concentrate solids in UFP-VSL-T02A to 20 -wt $\%$ UDS & B 02AML 014 XX 4014 UDS 2 & UDS & SWRI-090317 & 090317-39UDS R1 \\
\hline TI-WTP-PEP-066 & 7.12 .7 & Concentrate solids in UFP-VSL-T02A to 20 -wt $\%$ UDS & B 02AML 014 XX 4015 UDS 2 & UDS & SWRI-090317 & 090317-39UDS R1 \\
\hline TI-WTP-PEP-066 & 7.12 .7 & Concentrate solids in UFP-VSL-T02A to 20 -wt $\%$ UDS & B 02AML 014 XX 4016 UDS 2 & UDS & SWRI-090317 & 090317-39UDS R1 \\
\hline TI-WTP-PEP-066 & 7.12 .7 & Concentrate solids in UFP-VSL-T02A to 20 -wt $\%$ UDS & B 02AML 014 XX 4017 UDS 2 & UDS & SWRI-090317 & 090317-39UDS R1 \\
\hline TI-WTP-PEP-066 & 7.12 .7 & Concentrate solids in UFP-VSL-T02A to 20 -wt $\%$ UDS & B 02AML 014 XX 4018 UDS 2 & UDS & SWRI-090317 & 090317-39UDS R1 \\
\hline TI-WTP-PEP-066 & 7.12 .7 & Concentrate solids in UFP-VSL-T02A to 20 -wt $\%$ UDS & B 02AML 014 XX 4019 UDS 2 & UDS & SWRI-090317 & 090317-39UDS R1 \\
\hline TI-WTP-PEP-066 & 7.12 .7 & Concentrate solids in UFP-VSL-T02A to 20 -wt $\%$ UDS & B 02AML 014 XX 4020 UDS 2 & UDS & SWRI-090317 & 090317-39UDS R1 \\
\hline TI-WTP-PEP-066 & 7.12 .7 & Concentrate solids in UFP-VSL-T02A to 20 -wt $\%$ UDS & B 02AML 014 XX 4024 UDS 2 & UDS & SWRI-090317 & 090317-39UDS R1 \\
\hline TI-WTP-PEP-066 & 7.12.7 & Concentrate solids in UFP-VSL-T02A to 20 -wt\% UDS & B 02 AML 014 XX 4025 UDS 2 & UDS & SWRI-090317 & 090317-40UDS R1 \\
\hline TI-WTP-PEP-066 & 7.12 .7 & Concentrate solids in UFP-VSL-T02A to 20 -wt $\%$ UDS & B 02AML 014 XX 4026 UDS 2 & UDS & SWRI-090317 & 090317-40UDS R1 \\
\hline TI-WTP-PEP-066 & 7.12.7 & Concentrate solids in UFP-VSL-T02A to 20 -wt $\%$ UDS & B 02AML 014 XX 4027 DEN 2 & Density Slurry & SWRI-090317 & 090317-17 and 090317-33 and 090317-38 DEN slurry and liquid \\
\hline TI-WTP-PEP-066 & 7.12.7 & Concentrate solids in UFP-VSL-T02A to 20 -wt $\%$ UDS & B 02AML 014 XX 4027 DEN 2 & Density Supernate & SWRI-090317 & 090317-17 and 090317-33 and 090317-38 DEN slurry and liquid \\
\hline TI-WTP-PEP-066 & 7.12 .7 & Concentrate solids in UFP-VSL-T02A to 20 -wt $\%$ UDS & B 02AML 014 XX 4028 DEN 2 & Density Slurry & SWRI-090317 & 090317-17 and 090317-33 and 090317-38 DEN slurry and liquid \\
\hline TI-WTP-PEP-066 & 7.12 .7 & Concentrate solids in UFP-VSL-T02A to 20 -wt $\%$ UDS & B 02AML 014 XX 4028 DEN 2 & Density Supernate & SWRI-090317 & 090317-17 and 090317-33 and 090317-38 DEN slurry and liquid \\
\hline TI-WTP-PEP-066 & 7.12 .7 & Concentrate solids in UFP-VSL-T02A to 20 -wt $\%$ UDS & B 02AML 014 XX 4029 DEN 2 & Density Slurry & SWRI-090317 & 090317-17 and 090317-33 and 090317-38 DEN slurry and liquid \\
\hline TI-WTP-PEP-066 & 7.12 .7 & Concentrate solids in UFP-VSL-T02A to 20-wt\% UDS & B 02AML 014 XX 4029 DEN 2 & Density Supernate & SWRI-090317 & $090317-17$ and $090317-33$ and $090317-38$ DEN slurry and liquid \\
\hline TI-WTP-PEP-066 & 7.12 .7 & Concentrate solids in UFP-VSL-T02A to 20 -wt $\%$ UDS & B 02AML 014 XX 4030 HTC 3 & Storage & & \\
\hline TI-WTP-PEP-066 & 7.12 .7 & Concentrate solids in UFP-VSL-T02A to 20 -wt $\%$ UDS & B 02AML 014 XX 4031 RHE 4 & Rheology & APEL Rheology & TDP WTP 363 Int Test B RHE Data on PEP slurry \\
\hline
\end{tabular}


Integrated Test B Sample List

\begin{tabular}{|c|c|c|c|c|c|c|}
\hline TI\# & TI Step & Step Description & Sample ID & Analysis & File Folder & Final Electronic Report \# \\
\hline TI-WTP-PEP-066 & 7.12 .7 & Concentrate solids in UFP-VSL-T02A to 20 -wt $\%$ UDS & B 02AML 014 XX 4032 ICP 2 B & ICP Wet Solids & SWRI-090317 & $\begin{array}{l}090317-23 \text { and } 090317-43 \text { and } 090318-10 \text { and } 090320-10 \text { and } 090320-13 \\
\text { ICP ICPMS }\end{array}$ \\
\hline TI-WTP-PEP-066 & 7.12.7 & Concentrate solids in UFP-VSL-T02A to 20 -wt $\%$ UDS & B 02AML 014 XX 4032 ICP 2 D & ICP Supernate & SWRI-090317 & $\begin{array}{l}090317-22 \text { and } 090317-42 \text { and } 090318-6 \text { and } 090318-10 \text { and } 090320-10 \\
\text { and } 090320-12 \text { ICP and ICPMS }\end{array}$ \\
\hline TI-WTP-PEP-066 & 7.12 .7 & Concentrate solids in UFP-VSL-T02A to 20 -wt $\%$ UDS & B 02AML 014 XX 4032 SUP 0 D & Storage & & \\
\hline TI-WTP-PEP-066 & 7.12.7 & Concentrate solids in UFP-VSL-T02A to $20-\mathrm{wt} \%$ UDS & B 02AML 014 XX 4032 TFI 2 D & IC & SWRI-090317 & 090317-26 090317-41 IC \\
\hline TI-WTP-PEP-066 & 7.12 .7 & Concentrate solids in UFP-VSL-T02A to 20 -wt $\%$ UDS & B 02AML 014 XX 4032 TFI 3 D & $\mathrm{OH}$ & ASO_ASR8383 & ASR $8383 \mathrm{OH}$ rev 1 \\
\hline TI-WTP-PEP-066 & 7.12 .7 & Concentrate solids in UFP-VSL-T02A to 20 -wt $\%$ UDS & B 02AML 014 XX 4033 ICP 2 B & ICP Wet Solids & SWRI-090317 & $\begin{array}{l}\text { 090317-23 and 090317-43 and 090318-10 and 090320-10 and 090320-13 } \\
\text { ICP ICPMS }\end{array}$ \\
\hline TI-WTP-PEP-066 & 7.12 .7 & Concentrate solids in UFP-VSL-T02A to $20-\mathrm{wt} \%$ UDS & B 02AML 014 XX 4033 ICP 2 D & ICP Supernate & SWRI-090317 & $\begin{array}{l}\text { 090317-22 and 090317-42 and 090318-6 and 090318-10 and 090320-10 } \\
\text { and 090320-12 ICP and ICPMS }\end{array}$ \\
\hline TI-WTP-PEP-066 & 7.12 .7 & Concentrate solids in UFP-VSL-T02A to $20-\mathrm{wt} \%$ UDS & B 02AML 014 XX 4033 SUP 0 D & Storage & & \\
\hline TI-WTP-PEP-066 & 7.12.7 & Concentrate solids in UFP-VSL-T02A to 20 -wt $\%$ UDS & B 02AML 014 XX 4033 TFI 2 D & IC & SWRI-090317 & 090317-26 090317-41 IC \\
\hline TI-WTP-PEP-066 & 7.12 .7 & Concentrate solids in UFP-VSL-T02A to 20 -wt $\%$ UDS & B 02AML 014 XX 4033 TFI 3 D & $\mathrm{OH}$ & ASO_ASR8383 & ASR $8383 \mathrm{OH}$ rev 1 \\
\hline TI-WTP-PEP-066 & 7.12 .7 & Concentrate solids in UFP-VSL-T02A to 20 -wt $\%$ UDS & B 02AML 014 XX 4034 ICP 2 B & ICP Wet Solids & SWRI-090317 & $\begin{array}{l}090317-23 \text { and } 090317-43 \text { and } 090318-10 \text { and } 090320-10 \text { and } 090320-13 \\
\text { ICP ICPMS }\end{array}$ \\
\hline TI-WTP-PEP-066 & 7.12 .7 & Concentrate solids in UFP-VSL-T02A to 20 -wt $\%$ UDS & B 02AML 014 XX 4034 ICP 2 D & ICP Supernate & SWRI-090317 & $\begin{array}{l}090317-22 \text { and } 090317-42 \text { and } 090318-6 \text { and } 090318-10 \text { and } 090320-10 \\
\text { and 090320-12 ICP and ICPMS }\end{array}$ \\
\hline TI-WTP-PEP-066 & 7.12 .7 & Concentrate solids in UFP-VSL-T02A to 20 -wt $\%$ UDS & B 02AML 014 XX 4034 SUP 0 D & Storage & & \\
\hline TI-WTP-PEP-066 & 7.12 .7 & Concentrate solids in UFP-VSL-T02A to 20 -wt $\%$ UDS & B 02AML 014 XX 4034 TFI 2 D & IC & SWRI-090317 & 090317-26 090317-41 IC \\
\hline TI-WTP-PEP-066 & 7.12 .7 & Concentrate solids in UFP-VSL-T02A to 20 -wt $\%$ UDS & B 02AML 014 XX 4034 TFI 3 D & $\mathrm{OH}$ & ASO_ASR8383 & ASR $8383 \mathrm{OH}$ rev 1 \\
\hline TI-WTP-PEP-066 & 7.12 .7 & Concentrate solids in UFP-VSL-T02A to 20 -wt $\%$ UDS & B 02AML 014 XX 4035 XSP 4 & PSD & APEL PSD & TDP WTP 362 Int Test B PSD Data Analysis \\
\hline TI-WTP-PEP-066 & 7.12 .7 & Concentrate solids in UFP-VSL-T02A to 20 -wt $\%$ UDS & B 02AML 014 XX 4036 CUF 4 & Parallel Lab Test & & \\
\hline TI-WTP-PEP-066 & 7.12 .7 & Concentrate solids in UFP-VSL-T02A to 20 -wt $\%$ UDS & B 00PF1 014 XX 4037 ARC 4 & Precipitation Study & & \\
\hline TI-WTP-PEP-066 & 7.12 .7 & Concentrate solids in UFP-VSL-T02A to 20-wt\% UDS & B 00PF1 014 XX 4038 ARC 4 & Precipitation Study & & \\
\hline TI-WTP-PEP-066 & 7.13 .21 & Flush filter-loop & B 000FL 016 XX 4039 UDS 2 & UDS & SWRI-090317 & 090317-39UDS R1 \\
\hline TI-WTP-PEP-066 & 7.13 .21 & Flush filter-loop & B 000FL 016 XX 4040 ICP 0 B & Storage & & \\
\hline TI-WTP-PEP-066 & 7.13 .21 & Flush filter-loop & B 000FL 016 XX 4040 SUP 0 D & Storage & & \\
\hline TI-WTP-PEP-066 & 7.13 .21 & Flush filter-loop & B 000FL 016 XX 4040 ICP 2 D & ICP Supernate & SWRI-090317 & $\begin{array}{c}090317-22 \text { and } 090317-42 \text { and } 090318-6 \text { and } 090318-10 \text { and } 090320-10 \\
\text { and } 090320-12 \text { ICP and ICPMS }\end{array}$ \\
\hline TI-WTP-PEP-066 & 7.13.21 & Flush filter-loop & B 000FL 016 XX 4040 TFI 2 D & IC & SWRI-090317 & 090317-26 090317-41 IC \\
\hline TI-WTP-PEP-066 & 7.13 .21 & $\begin{array}{l}\text { Flush filter loop } \\
\end{array}$ & B 000FL 016 XX 4040 TFI 3 D & $\mathrm{OH}$ & ASO_ASR8383 & ASR $8383 \mathrm{OH}$ rev 1 \\
\hline TI-WTP-PEP-066 & 7.13.4 & $\begin{array}{l}\text { At least } 10 \text { min after UFP-VSL-T02A has been isolated - after } \\
\text { caustic added before leach begins }\end{array}$ & B 02AML 016 XX 4041 UDS 2 B & Storage & & \\
\hline
\end{tabular}


Integrated Test B Sample List

\begin{tabular}{|c|c|c|c|c|c|c|}
\hline TI \# & TI Step & Step Description & Sample ID & Analysis & File Folder & Final Electronic Report \# \\
\hline TI-WTP-PEP-066 & 7.13 .4 & $\begin{array}{l}\text { At least } 10 \text { min after UFP-VSL-T02A has been isolated - after } \\
\text { caustic added before leach begins }\end{array}$ & В 02AML 016 XX 4042 UDS 2 B & Storage & & \\
\hline TI-WTP-PEP-066 & 7.13 .4 & $\begin{array}{l}\text { At least } 10 \text { min after UFP-VSL-T02A has been isolated - after } \\
\text { caustic added before leach begins }\end{array}$ & B 02AML 016 XX 4043 UDS 2 B & Storage & & \\
\hline TI-WTP-PEP-066 & 7.13.4 & $\begin{array}{l}\text { At least } 10 \text { min after UFP-VSL-T02A has been isolated - after } \\
\text { caustic added before leach begins }\end{array}$ & B 02AML 016 XX 4044 DEN 2 & Storage & & \\
\hline TI-WTP-PEP-066 & 7.13.4 & $\begin{array}{l}\text { At least } 10 \text { min after UFP-VSL-T02A has been isolated - after } \\
\text { caustic added before leach begins }\end{array}$ & B 02AML 016 XX 4045 DEN 2 & Storage & & \\
\hline TI-WTP-PEP-066 & 7.13.4 & $\begin{array}{l}\text { At least } 10 \text { min after UFP-VSL-T02A has been isolated - after } \\
\text { caustic added before leach begins }\end{array}$ & B 02AML 016 XX 4046 DEN 2 & Storage & & \\
\hline TI-WTP-PEP-066 & 7.13.4 & $\begin{array}{l}\text { At least } 10 \text { min after UFP-VSL-T02A has been isolated - after } \\
\text { caustic added before leach begins }\end{array}$ & B 02AML 016 XX 4047 HTC 3 & Storage & & \\
\hline TI-WTP-PEP-066 & 7.13.4 & $\begin{array}{l}\text { At least } 10 \text { min after UFP-VSL-T02A has been isolated - after } \\
\text { caustic added before leach begins }\end{array}$ & В 02AML 016 XX 4048 ICP 2 B & Storage & & \\
\hline TI-WTP-PEP-066 & 7.13.4 & $\begin{array}{l}\text { At least } 10 \text { min after UFP-VSL-T02A has been isolated - after } \\
\text { caustic added before leach begins }\end{array}$ & В 02AML 016 XX 4049 ICP 2 B & Storage & & \\
\hline TI-WTP-PEP-066 & 7.13.4 & $\begin{array}{l}\text { At least } 10 \text { min after UFP-VSL-T02A has been isolated - after } \\
\text { caustic added before leach begins }\end{array}$ & В $02 \mathrm{AML} 016$ XX 4050 ICP 2 B & Storage & & \\
\hline TI-WTP-PEP-066 & 7.13.4 & $\begin{array}{l}\text { At least } 10 \text { min after UFP-VSL-T02A has been isolated - after } \\
\text { caustic added before leach begins }\end{array}$ & В 02AML 016 XX 4459 UDS 2 В & UDS & SWRI-090318 & 090318-2 UDS \\
\hline TI-WTP-PEP-066 & 7.13.4 & $\begin{array}{l}\text { At least } 10 \text { min after UFP-VSL-T02A has been isolated - after } \\
\text { caustic added before leach begins }\end{array}$ & В 02AML 016 XX 4459 UDS 2 D & UDS & SWRI-090318 & 090318-2 UDS \\
\hline TI-WTP-PEP-066 & 7.13.4 & $\begin{array}{l}\text { At least } 10 \text { min after UFP-VSL-T02A has been isolated - after } \\
\text { caustic added before leach begins }\end{array}$ & В 02AML 016 XX 4460 UDS 2 B & UDS & SWRI-090318 & 090318-2 UDS \\
\hline TI-WTP-PEP-066 & 7.13.4 & $\begin{array}{l}\text { At least } 10 \text { min after UFP-VSL-T02A has been isolated - after } \\
\text { caustic added before leach begins }\end{array}$ & B 02AML 016 XX 4460 UDS 2 D & UDS & SWRI-090318 & 090318-2 UDS \\
\hline TI-WTP-PEP-066 & 7.13.4 & $\begin{array}{l}\text { At least } 10 \text { min after UFP-VSL-T02A has been isolated - after } \\
\text { caustic added before leach begins }\end{array}$ & В $02 \mathrm{AML} 016 \mathrm{XX} 4461$ UDS 2 B & UDS & SWRI-090318 & 090318-2 UDS \\
\hline TI-WTP-PEP-066 & 7.13.4 & $\begin{array}{l}\text { At least } 10 \text { min after UFP-VSL-T02A has been isolated - after } \\
\text { caustic added before leach begins }\end{array}$ & B 02AML 016 XX 4461 UDS 2 D & UDS & SWRI-090318 & 090318-2 UDS \\
\hline TI-WTP-PEP-066 & 7.13 .4 & $\begin{array}{l}\text { At least } 10 \text { min after UFP-VSL-T02A has been isolated - after } \\
\text { caustic added before leach begins }\end{array}$ & B 02AML 016 XX 4462 DEN 2 & Density Slurry & SWRI-090318 & 090318-1 090319-3 090320-9 DEN slurry and liquid \\
\hline TI-WTP-PEP-066 & 7.13.4 & $\begin{array}{l}\text { At least } 10 \text { min after UFP-VSL-T02A has been isolated - after } \\
\text { caustic added before leach begins }\end{array}$ & B 02AML 016 XX 4463 DEN 2 & Density Slurry & SWRI-090318 & 090318-1 090319-3 090320-9 DEN slurry and liquid \\
\hline TI-WTP-PEP-066 & 7.13 .4 & $\begin{array}{l}\text { At least } 10 \text { min after UFP-VSL-T02A has been isolated - after } \\
\text { caustic added before leach begins }\end{array}$ & B 02AML 016 XX 4464 DEN 2 & Density Slurry & SWRI-090318 & 090318-1 090319-3 090320-9 DEN slurry and liquid \\
\hline TI-WTP-PEP-066 & 7.13.4 & $\begin{array}{l}\text { At least } 10 \text { min after UFP-VSL-T02A has been isolated - after } \\
\text { caustic added before leach begins }\end{array}$ & В $02 \mathrm{AML} 016$ XX 4465 HTC 3 & Storage & & \\
\hline
\end{tabular}


Integrated Test B Sample List

\begin{tabular}{|c|c|c|c|c|c|c|}
\hline TI \# & TI Step & Step Description & Sample ID & Analysis & File Folder & Final Electronic Report \# \\
\hline TI-WTP-PEP-066 & 7.13 .4 & $\begin{array}{l}\text { At least } 10 \text { min after UFP-VSL-T02A has been isolated - after } \\
\text { caustic added before leach begins }\end{array}$ & B 02AML 016 XX 4466 ICP 2 B & ICP Wet Solids & SWRI-090318 & 090318-4 and 090319-6 ICP ICPMS \\
\hline TI-WTP-PEP-066 & 7.13 .4 & $\begin{array}{l}\text { At least } 10 \text { min after UFP-VSL-T02A has been isolated - after } \\
\text { caustic added before leach begins }\end{array}$ & B 02AML 016 XX 4466 DEN 0 D & Storage & & \\
\hline TI-WTP-PEP-066 & 7.13 .4 & $\begin{array}{l}\text { At least } 10 \text { min after UFP-VSL-T02A has been isolated - after } \\
\text { caustic added before leach begins }\end{array}$ & B 02AML 016 XX 4466 ICP 2 D & ICP Supernate & SWRI-090318 & $\begin{array}{c}090317-22 \text { and } 090317-42 \text { and } 090318-6 \text { and } 090318-10 \text { and } 090320-10 \\
\text { and } 090320-12 \text { ICP and ICPMS }\end{array}$ \\
\hline TI-WTP-PEP-066 & 7.13 .4 & $\begin{array}{l}\text { At least } 10 \text { min after UFP-VSL-T02A has been isolated - after } \\
\text { caustic added before leach begins }\end{array}$ & B 02AML 016 XX 4466 TFI 2 D & IC & SWRI-090318 & 090318-5IC \\
\hline TI-WTP-PEP-066 & 7.13 .4 & $\begin{array}{l}\text { At least } 10 \text { min after UFP-VSL-T02A has been isolated - after } \\
\text { caustic added before leach begins }\end{array}$ & B 02AML 016 XX 4466 TFI 3 D & $\mathrm{OH}$ & ASO_ASR8386 & ASR $8386 \mathrm{OH}$ \\
\hline TI-WTP-PEP-066 & 7.13 .4 & $\begin{array}{l}\text { At least } 10 \text { min after UFP-VSL-T02A has been isolated - after } \\
\text { caustic added before leach begins }\end{array}$ & B 02AML 016 XX 4466 RAM 3 D & Raman & ASO_ASR8385 & ASR 8385 RAM \\
\hline TI-WTP-PEP-066 & 7.13 .4 & $\begin{array}{l}\text { At least } 10 \text { min after UFP-VSL-T02A has been isolated - after } \\
\text { caustic added before leach begins }\end{array}$ & B 02AML 016 XX 4467 ICP 2 B & ICP Wet Solids & SWRI-090318 & 090318-4 and 090319-6 ICP ICPMS \\
\hline TI-WTP-PEP-066 & 7.13 .4 & $\begin{array}{l}\text { At least } 10 \text { min after UFP-VSL-T02A has been isolated - after } \\
\text { caustic added before leach begins }\end{array}$ & B 02AML 016 XX 4467 DEN 0 D & Storage & & \\
\hline TI-WTP-PEP-066 & 7.13 .4 & $\begin{array}{l}\text { At least } 10 \text { min after UFP-VSL-T02A has been isolated - after } \\
\text { caustic added before leach begins }\end{array}$ & B 02AML 016 XX 4467 ICP 2 D & ICP Supernate & SWRI-090318 & $\begin{array}{c}090317-22 \text { and } 090317-42 \text { and } 090318-6 \text { and } 090318-10 \text { and } 090320-10 \\
\text { and } 090320-12 \text { ICP and ICPMS }\end{array}$ \\
\hline TI-WTP-PEP-066 & 7.13 .4 & $\begin{array}{l}\text { At least } 10 \text { min after UFP-VSL-T02A has been isolated - after } \\
\text { caustic added before leach begins }\end{array}$ & B 02AML 016 XX 4467 TFI 2 D & IC & SWRI-090318 & 090318-5IC \\
\hline TI-WTP-PEP-066 & 7.13 .4 & $\begin{array}{l}\text { At least } 10 \text { min after UFP-VSL-T02A has been isolated - after } \\
\text { caustic added before leach begins }\end{array}$ & B 02AML 016 XX 4467 TFI 3 D & $\mathrm{OH}$ & ASO_ASR8386 & ASR $8386 \mathrm{OH}$ \\
\hline TI-WTP-PEP-066 & 7.13 .4 & $\begin{array}{l}\text { At least } 10 \text { min after UFP-VSL-T02A has been isolated - after } \\
\text { caustic added before leach begins }\end{array}$ & B 02AML 016 XX 4467 RAM 3 D & Raman & ASO_ASR8385 & ASR 8385 RAM \\
\hline TI-WTP-PEP-066 & 7.13 .4 & $\begin{array}{l}\text { At least } 10 \text { min after UFP-VSL-T02A has been isolated - after } \\
\text { caustic added before leach begins }\end{array}$ & B 02AML 016 XX 4468 ICP 2 B & ICP Wet Solids & SWRI-090318 & 090318-4 and 090319-6 ICP ICPMS \\
\hline TI-WTP-PEP-066 & 7.13 .4 & $\begin{array}{l}\text { At least } 10 \text { min after UFP-VSL-T02A has been isolated - after } \\
\text { caustic added before leach begins }\end{array}$ & B 02AML 016 XX 4468 DEN 0 D & Storage & & \\
\hline TI-WTP-PEP-066 & 7.13.4 & $\begin{array}{l}\text { At least } 10 \text { min after UFP-VSL-T02A has been isolated - after } \\
\text { caustic added before leach begins }\end{array}$ & B 02AML 016 XX 4468 ICP 2 D & ICP Supernate & SWRI-090318 & $\begin{array}{c}090317-22 \text { and } 090317-42 \text { and } 090318-6 \text { and } 090318-10 \text { and } 090320-10 \\
\text { and } 090320-12 \text { ICP and ICPMS }\end{array}$ \\
\hline TI-WTP-PEP-066 & 7.13 .4 & $\begin{array}{l}\text { At least } 10 \text { min after UFP-VSL-T02A has been isolated - after } \\
\text { caustic added before leach begins }\end{array}$ & B 02AML 016 XX 4468 TFI 2 D & IC & SWRI-090318 & 090318-5IC \\
\hline TI-WTP-PEP-066 & 7.13 .4 & $\begin{array}{l}\text { At least } 10 \text { min after UFP-VSL-T02A has been isolated - after } \\
\text { caustic added before leach begins }\end{array}$ & B 02AML 016 XX 4468 TFI 3 D & $\mathrm{OH}$ & ASO_ASR8386 & ASR $8386 \mathrm{OH}$ \\
\hline TI-WTP-PEP-066 & 7.13 .4 & $\begin{array}{l}\text { At least } 10 \text { min after UFP-VSL-T02A has been isolated - after } \\
\text { caustic added before leach begins }\end{array}$ & B 02AML 016 XX 4468 RAM 3 D & Raman & ASO_ASR8385 & ASR 8385 RAM \\
\hline TI-WTP-PEP-066 & 7.13 .4 & $\begin{array}{l}\text { At least } 10 \text { min after UFP-VSL-TO2A is isolated from filter loop } \\
\text { (batch 2) }\end{array}$ & B 02AML 016 XX 4469 CUF 4 & Parallel Lab Test & & \\
\hline TI-WTP-PEP-066 & 7.13 .15 & (a) $88^{\circ} \mathrm{C}$ (target $\left.\mathrm{T}-10^{\circ} \mathrm{C}\right)$ & B 02AML 017 BT 4052 UDS 2 B & UDS & SWRI-090318 & 090318-2 UDS \\
\hline
\end{tabular}


Integrated Test B Sample List

\begin{tabular}{|c|c|c|c|c|c|c|}
\hline TI \# & TI Step & Step Description & Sample ID & Analysis & File Folder & Final Electronic Report \# \\
\hline TI-WTP-PEP-066 & 7.13 .15 & (a) $88^{\circ} \mathrm{C}\left(\operatorname{target} \mathrm{T}-10^{\circ} \mathrm{C}\right)$ & B 02AML 017 BT 4052 UDS 2 D & UDS & SWRI-090318 & 090318-2 UDS \\
\hline TI-WTP-PEP-066 & 7.13 .15 & (a) $88^{\circ} \mathrm{C}$ (target $\left.\mathrm{T}-10^{\circ} \mathrm{C}\right)$ & B 02AML 017 BT 4053 DEN 2 & Density Slurry & SWRI-090318 & 090318-1 090319-3 090320-9 DEN slurry and liquid \\
\hline TI-WTP-PEP-066 & 7.13 .15 & (a) $88^{\circ} \mathrm{C}$ (target $\left.\mathrm{T}-10^{\circ} \mathrm{C}\right)$ & B 02AML 017 BT 4054 HTC 3 & Storage & & \\
\hline TI-WTP-PEP-066 & 7.13 .15 & (a) $88^{\circ} \mathrm{C}$ (target $\left.\mathrm{T}-10^{\circ} \mathrm{C}\right)$ & B 02AML 017 BT 4055 ICP 0 B & ICP Wet Solids & SWRI-090402 & 090402-28 ICP and ICPMS \\
\hline TI-WTP-PEP-066 & 7.13 .15 & (a) $88^{\circ} \mathrm{C}$ (target $\left.\mathrm{T}-10^{\circ} \mathrm{C}\right)$ & B 02AML 017 BT 4055 SUP 0 D & Storage & & \\
\hline TI-WTP-PEP-066 & 7.13 .15 & (a) $88^{\circ} \mathrm{C}$ (target $\left.\mathrm{T}-10^{\circ} \mathrm{C}\right)$ & B 02AML 017 BT 4055 ICP 2 D & ICP Supernate & SWRI-090318 & 090318-3 and 090319-5 ICP and ICPMS \\
\hline TI-WTP-PEP-066 & 7.13 .15 & (a) $88^{\circ} \mathrm{C}$ (target $\left.\mathrm{T}-10^{\circ} \mathrm{C}\right)$ & B 02AML 017 BT 4055 TFI 2 D & IC & SWRI-090318 & $090318-5 \mathrm{IC}$ \\
\hline TI-WTP-PEP-066 & 7.13 .15 & (a) $88^{\circ} \mathrm{C}$ (target $\mathrm{T}-10^{\circ} \mathrm{C}$ ) & B 02AML 017 BT 4055 TFI 3 D & $\mathrm{OH}$ & ASO_ASR8386 & ASR $8386 \mathrm{OH}$ \\
\hline TI-WTP-PEP-066 & 7.13 .15 & (a) $88^{\circ} \mathrm{C}\left(\right.$ target $\left.\mathrm{T}-10^{\circ} \mathrm{C}\right)$ & B 02AIL 017 BT 4056 ARC 1 B & Archive & & \\
\hline TI-WTP-PEP-066 & 7.13 .15 & (a) $88^{\circ} \mathrm{C}$ (target $\mathrm{T}-10^{\circ} \mathrm{C}$ ) & B 02AIL 017 BT 4056 ARC 1 D & Archive & & \\
\hline TI-WTP-PEP-066 & 7.13 .15 & (a) $88^{\circ} \mathrm{C}$ (target $\left.\mathrm{T}-10^{\circ} \mathrm{C}\right)$ & B 02AIM 017 BT 4057 ARC 1 B & Archive & & \\
\hline TI-WTP-PEP-066 & 7.13 .15 & (a) $88^{\circ} \mathrm{C}$ (target $\left.\mathrm{T}-10^{\circ} \mathrm{C}\right)$ & B 02AIM 017 BT 4057 ARC 1 D & Archive & & \\
\hline TI-WTP-PEP-066 & 7.13 .15 & (a) $88^{\circ} \mathrm{C}\left(\operatorname{target} \mathrm{T}-10^{\circ} \mathrm{C}\right)$ & B 02AOL 017 BT 4058 ARC 1 B & Archive & & \\
\hline TI-WTP-PEP-066 & 7.13 .15 & (a) $88^{\circ} \mathrm{C}$ (target $\left.\mathrm{T}-10^{\circ} \mathrm{C}\right)$ & B 02AOL 017 BT 4058 ARC 1 D & Archive & & \\
\hline TI-WTP-PEP-066 & 7.13 .15 & (a) $88^{\circ} \mathrm{C}$ (target $\left.\mathrm{T}-10^{\circ} \mathrm{C}\right)$ & B 02AMM 017 BT 4059 ARC 1 B & Archive & & \\
\hline TI-WTP-PEP-066 & 7.13 .15 & (a) $88^{\circ} \mathrm{C}$ (target $\left.\mathrm{T}-10^{\circ} \mathrm{C}\right)$ & B 02AMM 017 BT 4059 ARC 1 D & Archive & & \\
\hline TI-WTP-PEP-066 & 7.13 .15 & (a) $88^{\circ} \mathrm{C}\left(\right.$ target $\left.\mathrm{T}-10^{\circ} \mathrm{C}\right)$ & B 02AOM 017 BT 4060 ARC 1 B & Archive & & \\
\hline TI-WTP-PEP-066 & 7.13 .15 & (a) $88^{\circ} \mathrm{C}$ (target $\left.\mathrm{T}-10^{\circ} \mathrm{C}\right)$ & B 02AOM 017 BT 4060 ARC 1 D & Archive & & \\
\hline TI-WTP-PEP-066 & 7.13 .15 & (a) $88^{\circ} \mathrm{C}$ (target $\left.\mathrm{T}-10^{\circ} \mathrm{C}\right)$ & B 02AMH 017 BT 4062 ARC 1 B & Archive & & \\
\hline TI-WTP-PEP-066 & 7.13 .15 & (a) $88^{\circ} \mathrm{C}$ (target $\mathrm{T}-10^{\circ} \mathrm{C}$ ) & B 02AMH 017 BT 4062 ARC 1 D & Archive & & \\
\hline TI-WTP-PEP-066 & 7.13 .15 & (a) $88^{\circ} \mathrm{C}$ (target $\mathrm{T}-10^{\circ} \mathrm{C}$ ) & B 02AOH 017 BT 4063 ARC 1 B & Archive & & \\
\hline TI-WTP-PEP-066 & 7.13 .15 & (a) $88^{\circ} \mathrm{C}$ (target $\mathrm{T}-10^{\circ} \mathrm{C}$ ) & B 02AOH 017 BT 4063 ARC 1 D & Archive & & \\
\hline TI-WTP-PEP-066 & 7.13 .22 & (a) $98^{\circ} \mathrm{C}$ (target T, 0 hr) & B 02AML 017004064 UDS 2 B & UDS & SWRI-090318 & 090318-2 UDS \\
\hline TI-WTP-PEP-066 & 7.13 .22 & (a) $98^{\circ} \mathrm{C}$ (target T, $0 \mathrm{hr}$ ) & B 02AML 017004064 UDS 2 D & UDS & SWRI-090318 & 090318-2 UDS \\
\hline TI-WTP-PEP-066 & 7.13 .22 & @ $98^{\circ} \mathrm{C}$ (target T, 0 hr) & B 02AML 017004065 UDS 2 B & UDS & SWRI-090318 & 090318-2 UDS \\
\hline TI-WTP-PEP-066 & 7.13 .22 & (a) $98^{\circ} \mathrm{C}$ (target T, 0 hr) & B 02AML 017004065 UDS 2 D & UDS & SWRI-090318 & 090318-2 UDS \\
\hline TI-WTP-PEP-066 & 7.13 .22 & @ $98^{\circ} \mathrm{C}$ (target T, 0 hr) & B 02AML 017004066 UDS 2 B & UDS & SWRI-090318 & 090318-2 UDS \\
\hline TI-WTP-PEP-066 & 7.13 .22 & (a) $98^{\circ} \mathrm{C}$ (target T, 0 hr) & B 02AML 017004066 UDS 2 D & UDS & SWRI-090318 & 090318-2 UDS \\
\hline TI-WTP-PEP-066 & 7.13 .22 & (a) $98^{\circ} \mathrm{C}$ (target T, 0 hr) & B 02AML 017004067 DEN 2 & Density Slurry & SWRI-090318 & 090318-1 090319-3 090320-9 DEN slurry and liquid \\
\hline TI-WTP-PEP-066 & 7.13 .22 & (@) $98^{\circ} \mathrm{C}$ (target T, 0 hr) & B 02AML 017004068 DEN 2 & Density Slurry & SWRI-090318 & 090318-1 090319-3 090320-9 DEN slurry and liquid \\
\hline TI-WTP-PEP-066 & 7.13 .22 & @ $98^{\circ} \mathrm{C}$ (target T, 0 hr) & B 02AML 017004069 DEN 2 & Density Slurry & SWRI-090318 & 090318-1 090319-3 090320-9 DEN slurry and liquid \\
\hline TI-WTP-PEP-066 & 7.13 .22 & (a) $98^{\circ} \mathrm{C}$ (target T, 0 hr) & B 02AML 017004070 HTC 3 & Storage & & \\
\hline TI-WTP-PEP-066 & 7.13 .22 & @ $98^{\circ} \mathrm{C}$ (target T, 0 hr) & B 02AML 017004071 ICP 0 B & ICP Wet Solids & SWRI-090402 & 090402-28 ICP and ICPMS \\
\hline
\end{tabular}


Integrated Test B Sample List

\begin{tabular}{|c|c|c|c|c|c|c|}
\hline TI \# & TI Step & Step Description & Sample ID & Analysis & File Folder & Final Electronic Report \# \\
\hline TI-WTP-PEP-066 & 7.13 .22 & @ $98^{\circ} \mathrm{C}$ (target T, 0 hr) & B 02AML 017004071 ICP 2 D & ICP Supernate & SWRI-090318 & 090318-3 and 090319-5 ICP and ICPMS \\
\hline TI-WTP-PEP-066 & 7.13.22 & (a) $98^{\circ} \mathrm{C}$ (target T, 0 hr) & B 02AML 017004071 DEN 0 D & Storage & & \\
\hline TI-WTP-PEP-066 & 7.13 .22 & @ $98^{\circ} \mathrm{C}$ (target T, 0 hr) & B 02AML 017004071 TFI 2 D & IC & SWRI-090318 & 090318-5IC \\
\hline TI-WTP-PEP-066 & 7.13.22 & (a) $98^{\circ} \mathrm{C}$ (target T, 0 hr) & B 02AML 017004071 TFI 3 D & $\mathrm{OH}$ & ASO_ASR8386 & ASR $8386 \mathrm{OH}$ \\
\hline TI-WTP-PEP-066 & 7.13.22 & (a) $98^{\circ} \mathrm{C}$ (target $\mathrm{T}, 0 \mathrm{hr}$ ) & B 02AML 017004071 RAM 3 D & Raman & ASO_ASR8385 & ASR 8385 RAM \\
\hline TI-WTP-PEP-066 & 7.13.22 & (a) $98^{\circ} \mathrm{C}$ (target $\mathrm{T}, 0 \mathrm{hr}$ ) & B 02AML 017004072 ICP 0 B & ICP Wet Solids & SWRI-090402 & 090402-28 ICP and ICPMS \\
\hline TI-WTP-PEP-066 & 7.13 .22 & (a) $98^{\circ} \mathrm{C}$ (target $\left.\mathrm{T}, 0 \mathrm{hr}\right)$ & B 02AML 017004072 ICP 2 D & ICP Supernate & SWRI-090318 & 090318-3 and 090319-5 ICP and ICPMS \\
\hline TI-WTP-PEP-066 & 7.13 .22 & (a) $98^{\circ} \mathrm{C}$ (target $\mathrm{T}, 0 \mathrm{hr}$ ) & B 02AML 017004072 DEN 0 D & Storage & & \\
\hline TI-WTP-PEP-066 & 7.13 .22 & (@) $98^{\circ} \mathrm{C}$ (target $\mathrm{T}, 0 \mathrm{hr}$ ) & B 02AML 017004072 TFI 2 D & IC & SWRI-090318 & 090318-5IC \\
\hline TI-WTP-PEP-066 & 7.13.22 & (a) $98^{\circ} \mathrm{C}$ (target T, 0 hr) & B 02AML 017004072 TFI 3 D & $\mathrm{OH}$ & ASO_ASR8386 & ASR $8386 \mathrm{OH}$ \\
\hline TI-WTP-PEP-066 & 7.13.22 & (a) $98^{\circ} \mathrm{C}$ (target $\mathrm{T}, 0 \mathrm{hr}$ ) & B 02AML 017004072 RAM 3 D & Raman & ASO_ASR8385 & ASR 8385 RAM \\
\hline TI-WTP-PEP-066 & 7.13.22 & @ $98^{\circ} \mathrm{C}$ (target $\mathrm{T}, 0 \mathrm{hr}$ ) & B 02AML 017004073 ICP 0 B & ICP Wet Solids & SWRI-090402 & 090402-28 ICP and ICPMS \\
\hline TI-WTP-PEP-066 & 7.13 .22 & (a) $98^{\circ} \mathrm{C}$ (target $\left.\mathrm{T}, 0 \mathrm{hr}\right)$ & B 02AML 017004073 ICP 2 D & ICP Supernate & SWRI-090318 & 090318-3 and 090319-5 ICP and ICPMS \\
\hline TI-WTP-PEP-066 & 7.13 .22 & (a) $98^{\circ} \mathrm{C}$ (target T, 0 hr) & B 02AML 017004073 DEN 0 D & Storage & & \\
\hline TI-WTP-PEP-066 & 7.13 .22 & (a) $98^{\circ} \mathrm{C}$ (target $\mathrm{T}, 0 \mathrm{hr}$ ) & B 02AML 017004073 TFI 2 D & IC & SWRI-090318 & 090318-5IC \\
\hline TI-WTP-PEP-066 & 7.13 .22 & @ $98^{\circ} \mathrm{C}$ (target $\mathrm{T}, 0 \mathrm{hr}$ ) & B 02AML 017004073 TFI 3 D & $\mathrm{OH}$ & ASO_ASR8386 & ASR 8386 OH \\
\hline TI-WTP-PEP-066 & 7.13 .22 & @ $98^{\circ} \mathrm{C}$ (target T, 0 hr) & B 02AML 017004073 RAM 3 D & Raman & ASO_ASR8385 & ASR 8385 RAM \\
\hline TI-WTP-PEP-066 & 7.13.22 & (a) $98^{\circ} \mathrm{C}$ (target $\left.\mathrm{T}, 0 \mathrm{hr}\right)$ & B 02AIL 017004074 ARC 1 B & ICP Wet Solids & SWRI-090827 & 090827-7 and 090827-8 ICP and ICPMS \\
\hline TI-WTP-PEP-066 & 7.13 .22 & (a) $98^{\circ} \mathrm{C}$ (target $\mathrm{T}, 0 \mathrm{hr}$ ) & B 02AIL 017004074 ARC 1 D & IC & SWRI 090827 & $090827-5$ and $0908027-6 \mathrm{IC}$ and TDS ${ }^{(1)}$ \\
\hline TI-WTP-PEP-066 & 7.13 .22 & (a) $98^{\circ} \mathrm{C}$ (target $\mathrm{T}, 0 \mathrm{hr}$ ) & B 02AIL 017004074 ARC 1 D & TDS & SWRI 090827 & 090827-5 and 0908027-6 IC and TDS ${ }^{(1)}$ \\
\hline TI-WTP-PEP-066 & 7.13 .22 & @ $98^{\circ} \mathrm{C}$ (target T, 0 hr) & B 02AIM 017004075 ARC 1 B & ICP Wet Solids & SWRI-090827 & $090827-7$ and $090827-8$ ICP and ICPMS \\
\hline TI-WTP-PEP-066 & 7.13.22 & @ $98^{\circ} \mathrm{C}$ (target T, 0 hr) & B 02AIM 017004075 ARC 1 D & IC & SWRI 090827 & 090827-5 and 0908027-6 IC and TDS ${ }^{(1)}$ \\
\hline TI-WTP-PEP-066 & 7.13 .22 & (a) $98^{\circ} \mathrm{C}$ (target $\mathrm{T}, 0 \mathrm{hr}$ ) & B 02AIM 017004075 ARC 1 D & TDS & SWRI 090827 & 090827-5 and 0908027-6 IC and TDS ${ }^{(1)}$ \\
\hline TI-WTP-PEP-066 & 7.13 .22 & (a) $98^{\circ} \mathrm{C}$ (target $\mathrm{T}, 0 \mathrm{hr}$ ) & B 02AOL 017004076 ARC 1 B & ICP Wet Solids & SWRI-090827 & $090827-7$ and $090827-8$ ICP and ICPMS \\
\hline TI-WTP-PEP-066 & 7.13 .22 & (a) $98^{\circ} \mathrm{C}$ (target $\left.\mathrm{T}, 0 \mathrm{hr}\right)$ & B 02AOL 017004076 ARC 1 D & IC & SWRI 090827 & $090827-5$ and $0908027-6$ IC and TDS ${ }^{(1)}$ \\
\hline TI-WTP-PEP-066 & 7.13.22 & @ $98^{\circ} \mathrm{C}$ (target T, $0 \mathrm{hr}$ ) & B 02AOL 017004076 ARC 1 D & TDS & SWRI 090827 & 090827-5 and 0908027-6 IC and TDS ${ }^{(1)}$ \\
\hline TI-WTP-PEP-066 & 7.13.22 & @ $98^{\circ} \mathrm{C}$ (target T, 0 hr) & В 02 AMM 017004077 ARC 1 B & ICP Wet Solids & SWRI-090827 & $090827-7$ and $090827-8$ ICP and ICPMS \\
\hline TI-WTP-PEP-066 & 7.13 .22 & (a) $98^{\circ} \mathrm{C}$ (target $\left.\mathrm{T}, 0 \mathrm{hr}\right)$ & B 02AMM 017004077 ARC 1 D & IC & SWRI 090827 & $090827-5$ and $0908027-6$ IC and TDS ${ }^{(1)}$ \\
\hline TI-WTP-PEP-066 & 7.13 .22 & (a) $98^{\circ} \mathrm{C}$ (target T, 0 hr) & B 02AMM 017004077 ARC 1 D & TDS & SWRI 090827 & $090827-5$ and $0908027-6$ IC and TDS ${ }^{(1)}$ \\
\hline TI-WTP-PEP-066 & 7.13 .22 & (a) $98^{\circ} \mathrm{C}$ (target $\mathrm{T}, 0 \mathrm{hr}$ ) & В $02 \mathrm{AOM} 017004078$ ARC 1 B & ICP Wet Solids & SWRI-090827 & 090827-7 and 090827-8 ICP and ICPMS \\
\hline TI-WTP-PEP-066 & 7.13 .22 & (a) $98^{\circ} \mathrm{C}$ (target $\mathrm{T}, 0 \mathrm{hr}$ ) & B 02AOM 017004078 ARC 1 D & IC & SWRI 090827 & $090827-5$ and $0908027-6$ IC and TDS ${ }^{(1)}$ \\
\hline TI-WTP-PEP-066 & 7.13.22 & @ $98^{\circ} \mathrm{C}$ (target T, 0 hr) & B 02AOM 017004078 ARC 1 D & TDS & SWRI 090827 & 090827-5 and 0908027-6 IC and TDS ${ }^{(1)}$ \\
\hline TI-WTP-PEP-066 & 7.13 .22 & (a) $98^{\circ} \mathrm{C}$ (target $\mathrm{T}, 0 \mathrm{hr}$ ) & B 02 AMH 017004080 ARC 1 B & ICP Wet Solids & SWRI-090827 & $090827-7$ and $090827-8$ ICP and ICPMS \\
\hline
\end{tabular}


Integrated Test B Sample List

\begin{tabular}{|c|c|c|c|c|c|c|}
\hline TI \# & TI Step & Step Description & Sample ID & Analysis & File Folder & Final Electronic Report \# \\
\hline TI-WTP-PEP-066 & 7.13.22 & (a) $98^{\circ} \mathrm{C}$ (target T, 0 hr) & B 02AMH 017004080 ARC 1 D & IC & SWRI 090827 & $090827-5$ and $0908027-6$ IC and TDS ${ }^{(1)}$ \\
\hline TI-WTP-PEP-066 & 7.13 .22 & (a) $98^{\circ} \mathrm{C}$ (target $\mathrm{T}, 0 \mathrm{hr}$ ) & В 02AMH 017004080 ARC 1 D & TDS & SWRI 090827 & 090827-5 and 0908027-6 IC and TDS ${ }^{(1)}$ \\
\hline TI-WTP-PEP-066 & 7.13.22 & (a) $98^{\circ} \mathrm{C}$ (target T, 0 hr) & B $02 \mathrm{AOH} 017004081$ ARC $1 \mathrm{~B}$ & ICP Wet Solids & SWRI-090827 & 090827-7 and 090827-8 ICP and ICPMS \\
\hline TI-WTP-PEP-066 & 7.13.22 & (a) $98^{\circ} \mathrm{C}$ (target $\mathrm{T}, 0 \mathrm{hr}$ ) & B 02AOH 017004081 ARC 1 D & IC & SWRI 090827 & $090827-5$ and $0908027-6$ IC and TDS ${ }^{(1)}$ \\
\hline TI-WTP-PEP-066 & 7.13 .22 & (a) $98^{\circ} \mathrm{C}$ (target $\left.\mathrm{T}, 0 \mathrm{hr}\right)$ & B $02 \mathrm{AOH} 017004081$ ARC $1 \mathrm{D}$ & TDS & SWRI 090827 & 090827-5 and 0908027-6 IC and TDS ${ }^{(1)}$ \\
\hline TI-WTP-PEP-066 & 7.13.22 & Target $\mathrm{T}, 1 \mathrm{hr}$ & B 02AML 017014082 UDS 2 B & UDS & SWRI-090318 & 090318-2 UDS \\
\hline TI-WTP-PEP-066 & 7.13 .22 & Target $\mathrm{T}, 1 \mathrm{hr}$ & B 02AML 017014082 UDS 2 D & UDS & SWRI-090318 & 090318-2 UDS \\
\hline TI-WTP-PEP-066 & 7.13.22 & Target T, $1 \mathrm{hr}$ & B 02AML 017014083 DEN 2 & Density Slurry & SWRI-090318 & 090318-1 090319-3 090320-9 DEN slurry and liquid \\
\hline TI-WTP-PEP-066 & 7.13.22 & Target T, $1 \mathrm{hr}$ & B 02AML 017014084 ICP 0 B & ICP Wet Solids & SWRI-090402 & 090402-28 ICP and ICPMS \\
\hline TI-WTP-PEP-066 & 7.13.22 & Target T, $1 \mathrm{hr}$ & B 02AML 017014084 ICP 2 D & ICP Supernate & SWRI-090318 & 090318-3 and 090319-5 ICP and ICPMS \\
\hline TI-WTP-PEP-066 & 7.13.22 & Target T, $1 \mathrm{hr}$ & B 02AML 017014084 SUP 0 D & Storage & & \\
\hline TI-WTP-PEP-066 & 7.13.22 & Target T, $1 \mathrm{hr}$ & B 02AML 017014084 TFI 2 D & IC & SWRI-090318 & 090318-5IC \\
\hline TI-WTP-PEP-066 & 7.13.22 & Target T, $1 \mathrm{hr}$ & B 02AML 017014084 TFI 3 D & $\mathrm{OH}$ & ASO_ASR8386 & ASR $8386 \mathrm{OH}$ \\
\hline TI-WTP-PEP-066 & 7.13 .22 & Target T, $2 \mathrm{hr}$ & B 02 AML 017024085 UDS 2 B & UDS & SWRI-090318 & 090318-2 UDS \\
\hline TI-WTP-PEP-066 & 7.13 .22 & Target T, $2 \mathrm{hr}$ & B 02AML 017024085 UDS 2 D & UDS & SWRI-090318 & 090318-2 UDS \\
\hline TI-WTP-PEP-066 & 7.13.22 & Target T, $2 \mathrm{hr}$ & B 02AML 017024086 DEN 2 & Density Slurry & SWRI-090318 & 090318-1 090319-3 090320-9 DEN slurry and liquid \\
\hline TI-WTP-PEP-066 & 7.13 .22 & Target T, $2 \mathrm{hr}$ & B 02AML 017024087 ICP 0 B & ICP Wet Solids & SWRI-090402 & 090402-28 ICP and ICPMS \\
\hline TI-WTP-PEP-066 & 7.13.22 & Target T, $2 \mathrm{hr}$ & B 02AML 017024087 ICP 2 D & ICP Supernate & SWRI-090318 & 090318-3 and 090319-5 ICP and ICPMS \\
\hline TI-WTP-PEP-066 & 7.13.22 & Target T, $2 \mathrm{hr}$ & B 02AML 017024087 SUP 0 D & Storage & & \\
\hline TI-WTP-PEP-066 & 7.13.22 & Target T, $2 \mathrm{hr}$ & B 02AML 017024087 TFI 2 D & IC & SWRI-090318 & 090318-5IC \\
\hline TI-WTP-PEP-066 & 7.13.22 & Target T, $2 \mathrm{hr}$ & B 02AML 017024087 TFI 3 D & $\mathrm{OH}$ & ASO_ASR8386 & ASR $8386 \mathrm{OH}$ \\
\hline TI-WTP-PEP-066 & 7.13.22 & Target T, $3 \mathrm{hr}$ & B 02AML 017034088 ARC 1 B & Archive & & \\
\hline TI-WTP-PEP-066 & 7.13 .22 & Target T, $3 \mathrm{hr}$ & B 02AML 017034088 ARC 1 D & Archive & & \\
\hline TI-WTP-PEP-066 & 7.13.22 & Target T, $4 \mathrm{hr}$ & B 02AML 017044089 UDS 2 B & UDS & SWRI-090318 & 090318-2 UDS \\
\hline TI-WTP-PEP-066 & 7.13.22 & Target T, $4 \mathrm{hr}$ & B 02 AML 017044089 UDS 2 D & UDS & SWRI-090318 & 090318-2 UDS \\
\hline TI-WTP-PEP-066 & 7.13 .22 & Target T, $4 \mathrm{hr}$ & B 02AML 017044090 DEN 2 & Density Slurry & SWRI-090318 & 090318-1 090319-3 090320-9 DEN slurry and liquid \\
\hline TI-WTP-PEP-066 & 7.13 .22 & Target T, $4 \mathrm{hr}$ & B 02AML 017044091 ICP 2 B & ICP Wet Solids & SWRI-090318 & 090318-4 and 090319-6 ICP ICPMS \\
\hline TI-WTP-PEP-066 & 7.13 .22 & Target T, $4 \mathrm{hr}$ & B 02AML 017044091 ICP 2 D & ICP Supernate & SWRI-090318 & 090318-3 and 090319-5 ICP and ICPMS \\
\hline TI-WTP-PEP-066 & 7.13 .22 & Target T, $4 \mathrm{hr}$ & B 02AML 017044091 SUP 0 D & Storage & & \\
\hline TI-WTP-PEP-066 & 7.13.22 & Target T, $4 \mathrm{hr}$ & B 02AML 017044091 TFI 2 D & IC & SWRI-090318 & 090318-5IC \\
\hline TI-WTP-PEP-066 & 7.13.22 & Target T, $4 \mathrm{hr}$ & B 02AML 017044091 TFI 3 D & $\mathrm{OH}$ & ASO_ASR8386 & ASR $8386 \mathrm{OH}$ \\
\hline TI-WTP-PEP-066 & 7.13 .22 & Target T, $4 \mathrm{hr}$ & B 02AIL 017044092 ARC 1 B & Archive & & \\
\hline TI-WTP-PEP-066 & 7.13 .22 & Target T, $4 \mathrm{hr}$ & B 02AIL 017044092 ARC 1 D & Archive & & \\
\hline
\end{tabular}


Integrated Test B Sample List

\begin{tabular}{|c|c|c|c|c|c|c|}
\hline TI \# & TI Step & Step Description & Sample ID & Analysis & File Folder & Final Electronic Report \# \\
\hline TI-WTP-PEP-066 & 7.13 .22 & Target T, $4 \mathrm{hr}$ & B 02AIM 017044093 ARC 1 B & Archive & & \\
\hline TI-WTP-PEP-066 & 7.13 .22 & Target T, $4 \mathrm{hr}$ & B 02AIM 017044093 ARC 1 D & Archive & & \\
\hline TI-WTP-PEP-066 & 7.13 .22 & Target T, $4 \mathrm{hr}$ & B 02AOL 017044094 ARC 1 B & Archive & & \\
\hline TI-WTP-PEP-066 & 7.13 .22 & Target T, $4 \mathrm{hr}$ & B 02AOL 017044094 ARC 1 D & Archive & & \\
\hline TI-WTP-PEP-066 & 7.13 .22 & Target T, $4 \mathrm{hr}$ & B 02AMM 017044095 ARC 1 B & Archive & & \\
\hline TI-WTP-PEP-066 & 7.13 .22 & Target T, $4 \mathrm{hr}$ & B 02AMM 017044095 ARC 1 D & Archive & & \\
\hline TI-WTP-PEP-066 & 7.13 .22 & Target T, $4 \mathrm{hr}$ & B $02 \mathrm{AOM} 017044096$ ARC $1 \mathrm{~B}$ & Archive & & \\
\hline TI-WTP-PEP-066 & 7.13 .22 & Target T, $4 \mathrm{hr}$ & B 02AOM 017044096 ARC 1 D & Archive & & \\
\hline TI-WTP-PEP-066 & 7.13 .22 & Target T, $4 \mathrm{hr}$ & B 02 AMH 017044098 ARC 1 B & Archive & & \\
\hline TI-WTP-PEP-066 & 7.13 .22 & Target T, $4 \mathrm{hr}$ & B $02 \mathrm{AMH} 017044098$ ARC 1 D & Archive & & \\
\hline TI-WTP-PEP-066 & 7.13 .22 & Target T, $4 \mathrm{hr}$ & B 02AOH 017044099 ARC 1 B & Archive & & \\
\hline TI-WTP-PEP-066 & 7.13 .22 & Target T, $4 \mathrm{hr}$ & B 02AOH 017044099 ARC 1 D & Archive & & \\
\hline TI-WTP-PEP-066 & 7.13 .22 & Target $\mathrm{T}, 5 \mathrm{hr}$ & B 02AML 017054100 ARC 1 B & Archive & & \\
\hline TI-WTP-PEP-066 & 7.13 .22 & Target T, $5 \mathrm{hr}$ & B 02AML 017054100 ARC 1 D & Archive & & \\
\hline TI-WTP-PEP-066 & 7.13 .22 & Target T, 6 hr & B 02AML 017064101 ARC 1 B & Archive & & \\
\hline TI-WTP-PEP-066 & 7.13 .22 & Target T, $6 \mathrm{hr}$ & B 02AML 017064101 ARC 1 D & Archive & & \\
\hline TI-WTP-PEP-066 & 7.13 .22 & Target T, $7 \mathrm{hr}$ & B 02AML 017074102 ARC 1 B & Archive & & \\
\hline TI-WTP-PEP-066 & 7.13 .22 & Target T, $7 \mathrm{hr}$ & B 02AML 017074102 ARC 1 D & Archive & & \\
\hline TI-WTP-PEP-066 & 7.13 .22 & Target T, $8 \mathrm{hr}$ & B 02 AML 017084103 UDS 2 B & UDS & SWRI-090318 & 090318-2 UDS \\
\hline TI-WTP-PEP-066 & 7.13 .22 & Target T, 8 hr & B 02AML 017084103 UDS 2 D & UDS & SWRI-090318 & 090318-2 UDS \\
\hline TI-WTP-PEP-066 & 7.13 .22 & Target $\mathrm{T}, 8 \mathrm{hr}$ & B 02AML 017084104 DEN 2 & Density Slurry & SWRI-090318 & 090318-1 090319-3 090320-9 DEN slurry and liquid \\
\hline TI-WTP-PEP-066 & 7.13.22 & Target $\mathrm{T}, 8 \mathrm{hr}$ & B 02AML 017084105 ICP 2 B & ICP Wet Solids & SWRI-090318 & 090318-4 and 090319-6 ICP ICPMS \\
\hline TI-WTP-PEP-066 & 7.13 .22 & Target T, $8 \mathrm{hr}$ & B 02AML 017084105 ICP 2 D & ICP Supernate & SWRI-090318 & 090318-3 and 090319-5 ICP and ICPMS \\
\hline TI-WTP-PEP-066 & 7.13 .22 & Target T, $8 \mathrm{hr}$ & B 02AML 017084105 SUP 0 D & Storage & & \\
\hline TI-WTP-PEP-066 & 7.13 .22 & Target T, $8 \mathrm{hr}$ & B 02AML 017084105 TFI 2 D & IC & SWRI-090318 & 090318-5IC \\
\hline TI-WTP-PEP-066 & 7.13.22 & Target T, $8 \mathrm{hr}$ & B 02AML 017084105 TFI 3 D & $\mathrm{OH}$ & ASO_ASR8386 & ASR $8386 \mathrm{OH}$ \\
\hline TI-WTP-PEP-066 & 7.13 .22 & Target T, $8 \mathrm{hr}$ & B 02AIL 017084106 ARC 1 B & Archive & & \\
\hline TI-WTP-PEP-066 & 7.13 .22 & Target T, $8 \mathrm{hr}$ & B 02AIL 017084106 ARC 1 D & Archive & & \\
\hline TI-WTP-PEP-066 & 7.13 .22 & Target T, $8 \mathrm{hr}$ & B 02AIM 017084107 ARC 1 B & Archive & & \\
\hline TI-WTP-PEP-066 & 7.13 .22 & Target T, $8 \mathrm{hr}$ & B 02 AIM 017084107 ARC 1 D & Archive & & \\
\hline TI-WTP-PEP-066 & 7.13 .22 & Target T, $8 \mathrm{hr}$ & B $02 \mathrm{AOL} 017084108$ ARC $1 \mathrm{~B}$ & Archive & & \\
\hline TI-WTP-PEP-066 & 7.13 .22 & Target $\mathrm{T}, 8 \mathrm{hr}$ & B 02AOL 017084108 ARC 1 D & Archive & & \\
\hline TI-WTP-PEP-066 & 7.13 .22 & Target T, $8 \mathrm{hr}$ & B 02 AMM 017084109 ARC 1 B & Archive & & \\
\hline
\end{tabular}


Integrated Test B Sample List

\begin{tabular}{|c|c|c|c|c|c|c|}
\hline TI \# & TI Step & Step Description & Sample ID & Analysis & File Folder & Final Electronic Report \# \\
\hline TI-WTP-PEP-066 & 7.13 .22 & Target T, $8 \mathrm{hr}$ & B 02AMM 017084109 ARC 1 D & Archive & & \\
\hline TI-WTP-PEP-066 & 7.13.22 & Target T, $8 \mathrm{hr}$ & B 02AOM 017084110 ARC 1 B & Archive & & \\
\hline TI-WTP-PEP-066 & 7.13 .22 & Target T, $8 \mathrm{hr}$ & B 02AOM 017084110 ARC 1 D & Archive & & \\
\hline TI-WTP-PEP-066 & 7.13 .22 & Target T, $8 \mathrm{hr}$ & B 02AMH 017084112 ARC 1 B & Archive & & \\
\hline TI-WTP-PEP-066 & 7.13 .22 & Target T, $8 \mathrm{hr}$ & B 02AMH 017084112 ARC 1 D & Archive & & \\
\hline TI-WTP-PEP-066 & 7.13.22 & Target $\mathrm{T}, 8 \mathrm{hr}$ & B $02 \mathrm{AOH} 017084113$ ARC $1 \mathrm{~B}$ & Archive & & \\
\hline TI-WTP-PEP-066 & 7.13 .22 & Target T, $8 \mathrm{hr}$ & B 02AOH 017084113 ARC 1 D & Archive & & \\
\hline TI-WTP-PEP-066 & 7.13.22 & Target T, $9 \mathrm{hr}$ & B 02AML 017094114 ARC 1 B & Archive & & \\
\hline TI-WTP-PEP-066 & 7.13 .22 & Target T, $9 \mathrm{hr}$ & B 02AML 017094114 ARC 1 D & Archive & & \\
\hline TI-WTP-PEP-066 & 7.13 .22 & Target T, $10 \mathrm{hr}$ & B 02AML 017104115 UDS 2 B & UDS & SWRI-090318 & 090318-2 UDS \\
\hline TI-WTP-PEP-066 & 7.13 .22 & Target $\mathrm{T}, 10 \mathrm{hr}$ & B 02AML 017104115 UDS 2 D & UDS & SWRI-090318 & 090318-2 UDS \\
\hline TI-WTP-PEP-066 & 7.13 .22 & Target T, $10 \mathrm{hr}$ & B 02AML 017104116 DEN 2 & Density Slurry & SWRI-090318 & 090318-1 and 090319-3 and 090320-9 DEN slurry and liquid \\
\hline TI-WTP-PEP-066 & 7.13 .22 & Target $\mathrm{T}, 10 \mathrm{hr}$ & B 02AML 017104117 ICP 0 B & ICP Wet Solids & SWRI-090402 & 090402-28 ICP and ICPMS \\
\hline TI-WTP-PEP-066 & 7.13 .22 & Target T, $10 \mathrm{hr}$ & B 02AML 017104117 ICP 2 D & ICP Supernate & SWRI-090318 & 090318-3 and 090319-5 ICP and ICPMS \\
\hline TI-WTP-PEP-066 & 7.13.22 & Target T, $10 \mathrm{hr}$ & B 02AML 017104117 SUP 0 D & Storage & & \\
\hline TI-WTP-PEP-066 & 7.13.22 & Target $\mathrm{T}, 10 \mathrm{hr}$ & B 02AML 017104117 TFI 2 D & IC & SWRI-090318 & 090318-5IC \\
\hline TI-WTP-PEP-066 & 7.13.22 & Target T, $10 \mathrm{hr}$ & B 02AML 017104117 TFI 3 D & $\mathrm{OH}$ & ASO_ASR8386 & ASR $8386 \mathrm{OH}$ \\
\hline TI-WTP-PEP-066 & 7.13.22 & Target T, $11 \mathrm{hr}$ & B 02AML 017114118 ARC 1 B & Archive & & \\
\hline TI-WTP-PEP-066 & 7.13 .22 & Target T, $11 \mathrm{hr}$ & B 02AML 017114118 ARC 1 D & Archive & & \\
\hline TI-WTP-PEP-066 & 7.13.22 & Target T, $12 \mathrm{hr}$ & B 02AML 017124121 UDS 2 B & UDS & SWRI-090319 & 090319-4UDS R1 \\
\hline TI-WTP-PEP-066 & 7.13 .22 & Target T, $12 \mathrm{hr}$ & B 02AML 017124121 UDS 2 D & UDS & SWRI-090319 & 090319-4UDS R1 \\
\hline TI-WTP-PEP-066 & 7.13.22 & Target T, $12 \mathrm{hr}$ & B 02AML 017124120 UDS 2 B & UDS & SWRI-090319 & 090319-4UDS R1 \\
\hline TI-WTP-PEP-066 & 7.13 .22 & Target $\mathrm{T}, 12 \mathrm{hr}$ & B 02AML 017124120 UDS 2 D & UDS & SWRI-090319 & 090319-4UDS R1 \\
\hline TI-WTP-PEP-066 & 7.13.22 & Target T, $12 \mathrm{hr}$ & B 02AML 017124119 UDS 2 B & UDS & SWRI-090319 & 090319-4UDS R1 \\
\hline TI-WTP-PEP-066 & 7.13 .22 & Target T, $12 \mathrm{hr}$ & B 02AML 017124119 UDS 2 D & UDS & SWRI-090319 & 090319-4UDS R1 \\
\hline TI-WTP-PEP-066 & 7.13.22 & Target T, $12 \mathrm{hr}$ & B 02AML 017124124 DEN 2 & Density Slurry & SWRI-090319 & 090318-1 090319-3 090320-9 DEN slurry and liquid \\
\hline TI-WTP-PEP-066 & 7.13 .22 & Target T, $12 \mathrm{hr}$ & B 02AML 017124122 DEN 2 & Density Slurry & SWRI-090319 & 090318-1 090319-3 090320-9 DEN slurry and liquid \\
\hline TI-WTP-PEP-066 & 7.13.22 & Target T, $12 \mathrm{hr}$ & B 02AML 017124123 DEN 2 & Density Slurry & SWRI-090319 & 090318-1 090319-3 090320-9 DEN slurry and liquid \\
\hline TI-WTP-PEP-066 & 7.13 .22 & Target T, $12 \mathrm{hr}$ & B 02AML 017124125 ICP 2 B & ICP Wet Solids & SWRI-090319 & 090318-4 and 090319-6 ICP ICPMS \\
\hline TI-WTP-PEP-066 & 7.13.22 & Target T, $12 \mathrm{hr}$ & B $02 A M L ~ 017124125$ ICP 2 D & ICP Supernate & SWRI-090319 & 090318-3 and 090319-5 ICP and ICPMS \\
\hline TI-WTP-PEP-066 & 7.13 .22 & Target T, $12 \mathrm{hr}$ & B 02AML 017124125 SUP 0 D & Storage & & \\
\hline TI-WTP-PEP-066 & 7.13.22 & Target T, $12 \mathrm{hr}$ & B 02AML 017124125 TFI 2 D & IC & SWRI-090318 & $090318-9$ and $090319-9$ and $090320-11$ and $090324-20$ IC and TIC TOC \\
\hline TI-WTP-PEP-066 & 7.13.22 & Target $\mathrm{T}, 12 \mathrm{hr}$ & B 02AML 017124125 TFI 3 D & $\mathrm{OH}$ & ASO_ASR 8388 & ASR $8388 \mathrm{OH}$ \\
\hline
\end{tabular}


Integrated Test B Sample List

\begin{tabular}{|c|c|c|c|c|c|c|}
\hline TI \# & TI Step & Step Description & Sample ID & Analysis & File Folder & Final Electronic Report \# \\
\hline TI-WTP-PEP-066 & 7.13 .22 & Target T, $12 \mathrm{hr}$ & B 02AML 017124126 ICP 2 B & ICP Wet Solids & SWRI-090319 & 090318-4 and 090319-6 ICP ICPMS \\
\hline TI-WTP-PEP-066 & 7.13 .22 & Target $\mathrm{T}, 12 \mathrm{hr}$ & B 02AML 017124126 ICP 2 D & ICP Supernate & SWRI-090319 & 090318-3 and 090319-5 ICP and ICPMS \\
\hline TI-WTP-PEP-066 & 7.13 .22 & Target $\mathrm{T}, 12 \mathrm{hr}$ & B 02AML 017124126 SUP 0 D & Storage & & \\
\hline TI-WTP-PEP-066 & 7.13 .22 & Target $\mathrm{T}, 12 \mathrm{hr}$ & B 02AML 017124126 TFI 2 D & $\mathrm{IC}$ & SWRI-090318 & 090318-9 and 090319-9 and 090320-11 and 090324-20 IC and TIC TOC \\
\hline TI-WTP-PEP-066 & 7.13 .22 & Target $\mathrm{T}, 12 \mathrm{hr}$ & B 02AML 017124126 TFI 3 D & $\mathrm{OH}$ & ASO_ASR8388 & ASR $8388 \mathrm{OH}$ \\
\hline TI-WTP-PEP-066 & 7.13 .22 & Target $\mathrm{T}, 12 \mathrm{hr}$ & B 02AML 017124390 ICP 2 B & ICP Wet Solids & SWRI-090319 & 090318-4 and 090319-6 ICP ICPMS \\
\hline TI-WTP-PEP-066 & 7.13 .22 & Target $\mathrm{T}, 12 \mathrm{hr}$ & B 02AML 017124390 ICP 2 D & ICP Supernate & SWRI-090319 & 090318-3 and 090319-5 ICP and ICPMS \\
\hline TI-WTP-PEP-066 & 7.13 .22 & Target $\mathrm{T}, 12 \mathrm{hr}$ & B 02AML 017124390 SUP 0 D & Storage & & \\
\hline TI-WTP-PEP-066 & 7.13 .22 & Target $\mathrm{T}, 12 \mathrm{hr}$ & B 02AML 017124390 TFI 2 D & IC & SWRI-090318 & 090318-9 and 090319-9 and 090320-11 and 090324-20 IC and TIC TOC \\
\hline TI-WTP-PEP-066 & 7.13 .22 & Target $\mathrm{T}, 12 \mathrm{hr}$ & B 02AML 017124390 TFI 3 D & $\mathrm{OH}$ & ASO_ASR8388 & ASR $8388 \mathrm{OH}$ \\
\hline TI-WTP-PEP-066 & 7.13 .22 & Target T, $12 \mathrm{hr}$ & B 02AIL 017124391 ARC 1 B & Archive & & \\
\hline TI-WTP-PEP-066 & 7.13 .22 & Target $\mathrm{T}, 12 \mathrm{hr}$ & B 02AIL 017124391 ARC 1 D & Archive & & \\
\hline TI-WTP-PEP-066 & 7.13 .22 & Target $\mathrm{T}, 12 \mathrm{hr}$ & B 02AIM 017124392 ARC 1 B & Archive & & \\
\hline TI-WTP-PEP-066 & 7.13 .22 & Target T, $12 \mathrm{hr}$ & B 02AIM 017124392 ARC 1 D & Archive & & \\
\hline TI-WTP-PEP-066 & 7.13.22 & Target $\mathrm{T}, 12 \mathrm{hr}$ & B 02AOL 017124393 ARC 1 B & Archive & & \\
\hline TI-WTP-PEP-066 & 7.13 .22 & Target $\mathrm{T}, 12 \mathrm{hr}$ & B 02AOL 017124393 ARC 1 D & Archive & & \\
\hline TI-WTP-PEP-066 & 7.13 .22 & Target $\mathrm{T}, 12 \mathrm{hr}$ & B 02AMM 017124394 ARC 1 B & Archive & & \\
\hline TI-WTP-PEP-066 & 7.13 .22 & Target $\mathrm{T}, 12 \mathrm{hr}$ & B 02AMM 017124394 ARC 1 D & Archive & & \\
\hline TI-WTP-PEP-066 & 7.13.22 & Target $\mathrm{T}, 12 \mathrm{hr}$ & B 02AOM 017124395 ARC 1 B & Archive & & \\
\hline TI-WTP-PEP-066 & 7.13 .22 & Target T, $12 \mathrm{hr}$ & В 02 AOM 017124395 ARC 1 D & Archive & & \\
\hline TI-WTP-PEP-066 & 7.13 .22 & Target $\mathrm{T}, 13 \mathrm{hr}$ & B 02AML 017134399 ARC 1 B & Archive & & \\
\hline TI-WTP-PEP-066 & 7.13 .22 & Target T, $13 \mathrm{hr}$ & B 02AML 017134399 ARC 1 D & Archive & & \\
\hline TI-WTP-PEP-066 & 7.13 .22 & Target $\mathrm{T}, 14 \mathrm{hr}$ & B 02AML 017144400 UDS 2 B & UDS & SWRI-090319 & 090319-4UDS R1 \\
\hline TI-WTP-PEP-066 & 7.13 .22 & Target T, $14 \mathrm{hr}$ & B 02AML 017144400 UDS 2 D & UDS & SWRI-090319 & 090319-4UDS R1 \\
\hline TI-WTP-PEP-066 & 7.13 .22 & Target $\mathrm{T}, 14 \mathrm{hr}$ & B 02AML 017144401 DEN 2 & Density Slurry & SWRI-090319 & 090318-1 and 090319-3 and 090320-9 DEN slurry and liquid \\
\hline TI-WTP-PEP-066 & 7.13.22 & Target T, $14 \mathrm{hr}$ & B 02AML 017144402 ICP 0 B & ICP Wet Solids & SWRI-090402 & 090402-28 ICP and ICPMS \\
\hline TI-WTP-PEP-066 & 7.13 .22 & Target T, $14 \mathrm{hr}$ & B 02AML 017144402 ICP 2 D & ICP Supernate & SWRI-090319 & 090318-3 and 090319-5 ICP and ICPMS \\
\hline TI-WTP-PEP-066 & 7.13.22 & Target $\mathrm{T}, 14 \mathrm{hr}$ & B 02AML 017144402 SUP 0 D & Storage & & \\
\hline TI-WTP-PEP-066 & 7.13.22 & Target T, $14 \mathrm{hr}$ & B 02AML 017144402 TFI 2 D & IC & SWRI-090318 & 090318-9 and 090319-9 and 090320-11 and 090324-20 IC and TIC TOC \\
\hline TI-WTP-PEP-066 & 7.13 .22 & Target T, $14 \mathrm{hr}$ & B 02AML 017144402 TFI 3 D & $\mathrm{OH}$ & ASO_ASR 8388 & ASR $8388 \mathrm{OH}$ \\
\hline TI-WTP-PEP-066 & 7.13 .22 & Target $\mathrm{T}, 15 \mathrm{hr}$ & B 02AML 017154403 ARC 1 B & Archive & & \\
\hline TI-WTP-PEP-066 & 7.13 .22 & Target T, $15 \mathrm{hr}$ & B 02AML 017154403 ARC 1 D & Archive & & \\
\hline TI-WTP-PEP-066 & 7.13 .22 & Target $\mathrm{T}, 16 \mathrm{hr}$ & B 02AML 017164404 UDS 2 B & UDS & SWRI-090319 & 090319-4UDS R1 \\
\hline
\end{tabular}


Integrated Test B Sample List

\begin{tabular}{|c|c|c|c|c|c|c|}
\hline TI \# & TI Step & Step Description & Sample ID & Analysis & File Folder & Final Electronic Report \# \\
\hline TI-WTP-PEP-066 & 7.13 .22 & Target $\mathrm{T}, 16 \mathrm{hr}$ & B 02AML 017164404 UDS 2 D & UDS & SWRI-090319 & 090319-4UDS R1 \\
\hline TI-WTP-PEP-066 & 7.13 .22 & Target T, $16 \mathrm{hr}$ & B 02AML 017164405 UDS 2 B & UDS & SWRI-090319 & 090319-4UDS R1 \\
\hline TI-WTP-PEP-066 & 7.13 .22 & Target $\mathrm{T}, 16 \mathrm{hr}$ & B 02AML 017164405 UDS 2 D & UDS & SWRI-090319 & 090319-4UDS R1 \\
\hline TI-WTP-PEP-066 & 7.13 .22 & Target T, $16 \mathrm{hr}$ & В 02AML 017164406 UDS 2 B & UDS & SWRI-090319 & 090319-4UDS R1 \\
\hline TI-WTP-PEP-066 & 7.13 .22 & Target T, $16 \mathrm{hr}$ & B 02AML 017164406 UDS 2 D & UDS & SWRI-090319 & 090319-4UDS R1 \\
\hline TI-WTP-PEP-066 & 7.13.22 & Target T, $16 \mathrm{hr}$ & B 02AML 017164407 DEN 2 & Density Slurry & SWRI-090319 & 090318-1 and 090319-3 and 090320-9 DEN slurry and liquid \\
\hline TI-WTP-PEP-066 & 7.13 .22 & Target T, $16 \mathrm{hr}$ & B 02AML 017164408 DEN 2 & Density Slurry & SWRI-090319 & 090318-1 and 090319-3 and 090320-9 DEN slurry and liquid \\
\hline TI-WTP-PEP-066 & 7.13 .22 & Target T, $16 \mathrm{hr}$ & B 02AML 017164409 DEN 2 & Density Slurry & SWRI-090319 & 090318-1 and 090319-3 and 090320-9 DEN slurry and liquid \\
\hline TI-WTP-PEP-066 & 7.13 .22 & Target T, $16 \mathrm{hr}$ & B 02AML 017164410 ICP 2 B & ICP Wet Solids & SWRI-090319 & 090318-4 and 090319-6 ICP ICPMS \\
\hline TI-WTP-PEP-066 & 7.13 .22 & Target T, $16 \mathrm{hr}$ & B 02AML 017164410 ICP 2 D & ICP Supernate & SWRI-090319 & 090318-3 and 090319-5 ICP and ICPMS \\
\hline TI-WTP-PEP-066 & 7.13 .22 & Target T, $16 \mathrm{hr}$ & B 02AML 017164410 DEN 0 D & Storage & & \\
\hline TI-WTP-PEP-066 & 7.13 .22 & Target T, $16 \mathrm{hr}$ & B 02AML 017164410 TFI 2 D & IC & SWRI-090318 & 090318-9 and 090319-9 and 090320-11 and 090324-20 IC and TIC TOC \\
\hline TI-WTP-PEP-066 & 7.13 .22 & Target T, $16 \mathrm{hr}$ & B 02AML 017164410 TFI 3 D & $\mathrm{OH}$ & ASO_ASR8388 & ASR $8388 \mathrm{OH}$ \\
\hline TI-WTP-PEP-066 & 7.13 .22 & Target T, $16 \mathrm{hr}$ & B 02AML 017164410 RAM 3 D & Raman & ASO_ASR8387 & ASR 8387 RAM \\
\hline TI-WTP-PEP-066 & 7.13 .22 & Target T, $16 \mathrm{hr}$ & B 02AML 017164411 ICP 2 B & ICP Wet Solids & SWRI-090319 & 090318-4 and 090319-6 ICP ICPMS \\
\hline TI-WTP-PEP-066 & 7.13 .22 & Target T, $16 \mathrm{hr}$ & B 02AML 017164411 ICP 2 D & ICP Supernate & SWRI-090319 & 090318-3 and 090319-5 ICP and ICPMS \\
\hline TI-WTP-PEP-066 & 7.13 .22 & Target T, $16 \mathrm{hr}$ & B 02AML 017164411 DEN 0 D & Storage & & \\
\hline TI-WTP-PEP-066 & 7.13 .22 & Target T, $16 \mathrm{hr}$ & B 02AML 017164411 TFI 2 D & IC & SWRI-090318 & 090318-9 and 090319-9 and 090320-11 and 090324-20 IC and TIC TOC \\
\hline TI-WTP-PEP-066 & 7.13 .22 & Target T, $16 \mathrm{hr}$ & B 02AML 017164411 TFI 3 D & $\mathrm{OH}$ & ASO_ASR8388 & ASR $8388 \mathrm{OH}$ \\
\hline TI-WTP-PEP-066 & 7.13 .22 & Target T, $16 \mathrm{hr}$ & B 02AML 017164411 RAM 3 D & Raman & ASO_ASR8387 & ASR 8387 RAM \\
\hline TI-WTP-PEP-066 & 7.13.22 & Target T, $16 \mathrm{hr}$ & B 02AML 017164412 ICP 2 B & ICP Wet Solids & SWRI-090319 & 090318-4 and 090319-6 ICP ICPMS \\
\hline TI-WTP-PEP-066 & 7.13 .22 & Target T, $16 \mathrm{hr}$ & B 02AML 017164412 ICP 2 D & ICP Supernate & SWRI-090319 & 090318-3 and 090319-5 ICP and ICPMS \\
\hline TI-WTP-PEP-066 & 7.13 .22 & Target T, $16 \mathrm{hr}$ & B 02AML 017164412 DEN 0 D & Storage & & \\
\hline TI-WTP-PEP-066 & 7.13 .22 & Target T, $16 \mathrm{hr}$ & B 02AML 017164412 TFI 2 D & IC & SWRI-090318 & 090318-9 and 090319-9 and 090320-11 and 090324-20 IC and TIC TOC \\
\hline TI-WTP-PEP-066 & 7.13 .22 & Target T, $16 \mathrm{hr}$ & B 02AML 017164412 TFI 3 D & $\mathrm{OH}$ & ASO_ASR8388 & ASR 8388 OH \\
\hline TI-WTP-PEP-066 & 7.13.22 & Target T, $16 \mathrm{hr}$ & B 02AML 017164412 RAM 3 D & Raman & ASO_ASR 8387 & ASR 8387 RAM \\
\hline TI-WTP-PEP-066 & 7.13 .22 & Target T, $16 \mathrm{hr}$ & B 02AIL 017164413 ARC 1 B & Archive & & \\
\hline TI-WTP-PEP-066 & 7.13.22 & Target T, $16 \mathrm{hr}$ & B 02AIL 017164413 ARC 1 D & Archive & & \\
\hline TI-WTP-PEP-066 & 7.13 .22 & Target T, $16 \mathrm{hr}$ & B 02AIM 017164414 ARC 1 B & Archive & & \\
\hline TI-WTP-PEP-066 & 7.13 .22 & Target T, $16 \mathrm{hr}$ & B 02AIM 017164414 ARC 1 D & Archive & & \\
\hline TI-WTP-PEP-066 & 7.13 .22 & Target T, $16 \mathrm{hr}$ & B 02AOL 017164415 ARC 1 B & Archive & & \\
\hline TI-WTP-PEP-066 & 7.13 .22 & Target T, $16 \mathrm{hr}$ & B 02AOL 017164415 ARC 1 D & Archive & & \\
\hline TI-WTP-PEP-066 & 7.13 .22 & Target T, $16 \mathrm{hr}$ & B 02AMM 017164416 ARC 1 B & Archive & & \\
\hline
\end{tabular}


Integrated Test B Sample List

\begin{tabular}{|c|c|c|c|c|c|c|}
\hline TI\# & TI Step & Step Description & Sample ID & Analysis & File Folder & Final Electronic Report \# \\
\hline TI-WTP-PEP-066 & 7.13 .22 & Target $\mathrm{T}, 16 \mathrm{hr}$ & B 02AMM 017164416 ARC 1 D & Archive & & \\
\hline TI-WTP-PEP-066 & 7.13 .22 & Target T, $16 \mathrm{hr}$ & B 02AOM 017164417 ARC 1 B & Archive & & \\
\hline TI-WTP-PEP-066 & 7.13 .22 & Target $\mathrm{T}, 16 \mathrm{hr}$ & B 02AOM 017164417 ARC 1 D & Archive & & \\
\hline TI-WTP-PEP-066 & 7.13 .22 & Target $\mathrm{T}, 16 \mathrm{hr}$ & B 02AIH 017164418 ARC 1 B & Archive & & \\
\hline TI-WTP-PEP-066 & 7.13 .22 & Target T, $16 \mathrm{hr}$ & B 02AIH 017164418 ARC 1 D & Archive & & \\
\hline TI-WTP-PEP-066 & 7.13.22 & Target T, $16 \mathrm{hr}$ & B 02AMH 017164419 ARC 1 B & Archive & & \\
\hline TI-WTP-PEP-066 & 7.13 .22 & Target T, $16 \mathrm{hr}$ & B 02AMH 017164419 ARC 1 D & Archive & & \\
\hline TI-WTP-PEP-066 & 7.13 .22 & Target T, $16 \mathrm{hr}$ & B 02AOH 017164420 ARC 1 B & Archive & & \\
\hline TI-WTP-PEP-066 & 7.13 .22 & Target $\mathrm{T}, 16 \mathrm{hr}$ & B 02AOH 017164420 ARC 1 D & Archive & & \\
\hline TI-WTP-PEP-066 & 7.14 .8 & Cool-down of UFP-T02A using cooling jacket & B 02AIL 018 XX 4421 ARC 1 & Archive & & \\
\hline TI-WTP-PEP-066 & 7.14.8 & Cool-down of UFP-T02A using cooling jacket & B 02AIM 018 XX 4422 ARC 1 & Archive & & \\
\hline TI-WTP-PEP-066 & 7.14 .8 & Cool-down of UFP-T02A using cooling jacket & B 02AOL 018 XX 4423 ARC 1 & Archive & & \\
\hline TI-WTP-PEP-066 & 7.14 .8 & Cool-down of UFP-T02A using cooling jacket & B 02AMM 018 XX 4424 ARC 1 & Archive & & \\
\hline TI-WTP-PEP-066 & 7.14 .8 & Cool-down of UFP-T02A using cooling jacket & B $02 \mathrm{AOM} 018 \mathrm{XX} 4425$ ARC 1 & Archive & & \\
\hline TI-WTP-PEP-066 & 7.14 .8 & Cool-down of UFP-T02A using cooling jacket & B 02AIH 018 XX 4426 ARC 1 & Archive & & \\
\hline TI-WTP-PEP-066 & 7.14 .8 & Cool-down of UFP-T02A using cooling jacket & B 02AMH 018 XX 4427 ARC 1 & Archive & & \\
\hline TI-WTP-PEP-066 & 7.14 .8 & Cool-down of UFP-T02A using cooling jacket & B $02 \mathrm{AOH} 018 \mathrm{XX} 4428 \mathrm{ARC} 1$ & Archive & & \\
\hline TI-WTP-PEP-066 & 7.14 .8 & Cool-down of UFP-T02A using cooling jacket & B 02AML 018 XX 4429 UDS 2 & UDS & SWRI-090318 & 090318-2 UDS \\
\hline TI-WTP-PEP-066 & 7.14 .8 & Cool-down of UFP-T02A using cooling jacket & B 02AML 018 XX 4430 DEN 2 & Density Supernate & SWRI-090318 & 090318-1 090319-3 090320-9 DEN slurry and liquid \\
\hline TI-WTP-PEP-066 & 7.14.8 & Cool-down of UFP-T02A using cooling jacket & B 02AML 018 XX 4430 DEN 2 & Density Slurry & SWRI-090318 & 090318-1 090319-3 090320-9 DEN slurry and liquid \\
\hline TI-WTP-PEP-066 & 7.14 .8 & Cool-down of UFP-T02A using cooling jacket & B 02AML 018 XX 4431 ICP 2 B & ICP Wet Solids & SWRI-090318 & 090318-4 and 090319-6 ICP ICPMS \\
\hline TI-WTP-PEP-066 & 7.14 .8 & Cool-down of UFP-T02A using cooling jacket & B 02AML 018 XX 4431 ICP 2 D & ICP Supernate & SWRI-090318 & 090318-3 and 090319-5 ICP and ICPMS \\
\hline TI-WTP-PEP-066 & 7.14 .8 & Cool-down of UFP-T02A using cooling jacket & B 02 AML 018 XX 4431 SUP 0 D & Storage & & \\
\hline TI-WTP-PEP-066 & 7.14 .8 & Cool-down of UFP-T02A using cooling jacket & B 02AML 018 XX 4431 TFI 2 D & IC & SWRI-090318 & 090318-5IC \\
\hline TI-WTP-PEP-066 & 7.14 .8 & Cool-down of UFP-T02A using cooling jacket & B 02AML 018 XX 4431 TFI 3 D & $\mathrm{OH}$ & ASO_ASR8386 & ASR $8386 \mathrm{OH}$ \\
\hline TI-WTP-PEP-066 & 7.15 .6 & Post-caustic-leach concentration of solids to 17 -wt $\%$ UDS & B 02AML 019 XX 4127 UDS 2 & UDS & SWRI-090318 & 090318-7UDS slurry R1 \\
\hline TI-WTP-PEP-066 & 7.15.6 & Post-caustic-leach concentration of solids to 17 -wt $\%$ UDS & B 02AML 019 XX 4128 RHE 4 & Rheology & APEL Rheology & TDP WTP 363 Int Test B RHE Data on PEP slurry \\
\hline TI-WTP-PEP-066 & 7.15.6 & Post-caustic-leach concentration of solids to 17 -wt $\%$ UDS & B 02AML 019 XX 4129 RHE 4 & Rheology & APEL Rheology & TDP WTP 363 Int Test B RHE Data on PEP slurry \\
\hline TI-WTP-PEP-066 & 7.15.6 & Post-caustic-leach concentration of solids to 17 -wt $\%$ UDS & B 02AML 019 XX 4130 XSP 4 & PSD & APEL PSD & TDP WTP 362 Int Test B PSD Data Analysis \\
\hline TI-WTP-PEP-066 & 7.15.6 & Post-caustic-leach concentration of solids to 17 -wt $\%$ UDS & B 02AML 019 XX 4131 XSP 4 & PSD & APEL PSD & TDP WTP 362 Int Test B PSD Data Analysis \\
\hline TI-WTP-PEP-066 & 7.15 .13 & Post-caustic-leach concentration of solids to 17 -wt $\%$ UDS & B 02AML 019 XX 4132 UDS 2 & UDS & SWRI-090318 & 090318-7UDS slurry R1 \\
\hline TI-WTP-PEP-066 & 7.15 .13 & Post-caustic-leach concentration of solids to 17 -wt $\%$ UDS & B 02AML 019 XX 4133 UDS 2 & UDS & SWRI-090318 & 090318-7UDS slurry R1 \\
\hline TI-WTP-PEP-066 & 7.15 .13 & Post-caustic-leach concentration of solids to $17-\mathrm{wt} \%$ UDS & B 02AML 019 XX 4134 UDS 2 & UDS & SWRI-090319 & 090319-10UDS R1 \\
\hline
\end{tabular}


Integrated Test B Sample List

\begin{tabular}{|c|c|c|c|c|c|c|}
\hline TI\# & TI Step & Step Description & Sample ID & Analysis & File Folder & Final Electronic Report \# \\
\hline TI-WTP-PEP-066 & 7.15 .13 & Post-caustic-leach concentration of solids to $17-\mathrm{wt} \%$ UDS & B 02AML 019 XX 4135 UDS 2 & UDS & SWRI-090319 & 090319-10UDS R1 \\
\hline TI-WTP-PEP-066 & 7.15 .13 & Post-caustic-leach concentration of solids to $17-\mathrm{wt} \%$ UDS & B 02AML 019 XX 4136 UDS 2 & UDS & SWRI-090319 & 090319-10UDS R1 \\
\hline TI-WTP-PEP-066 & 7.15 .13 & Post-caustic-leach concentration of solids to $17-\mathrm{wt} \%$ UDS & B 02 AML 019 XX 4137 UDS 2 & UDS & SWRI-090319 & 090319-10UDS R1 \\
\hline TI-WTP-PEP-066 & 7.15 .13 & Post-caustic-leach concentration of solids to $17-\mathrm{wt} \%$ UDS & B 02AML 019 XX 4138 UDS 2 & UDS & SWRI-090319 & 090319-10UDS R1 \\
\hline TI-WTP-PEP-066 & 7.15 .13 & Post-caustic-leach concentration of solids to 17 -wt $\%$ UDS & B 02AML 019 XX 4139 UDS 2 & UDS & SWRI-090319 & 090319-10UDS R1 \\
\hline TI-WTP-PEP-066 & 7.15 .13 & Post-caustic-leach concentration of solids to 17 -wt $\%$ UDS & B 02AML 019 XX 4456 UDS 2 & UDS & SWRI-090319 & 090319-10UDS R1 \\
\hline TI-WTP-PEP-066 & 7.15 .13 & Post-caustic-leach concentration of solids to $17-\mathrm{wt} \%$ UDS & B 02AML 019 XX 4457 UDS 2 & UDS & SWRI-090319 & 090319-10UDS R1 \\
\hline TI-WTP-PEP-066 & 7.15 .13 & Post-caustic-leach concentration of solids to $17-\mathrm{wt} \%$ UDS & B 02AML 019 XX 4470 RHE 4 & Rheology & APEL Rheology & TDP WTP 363 Int Test B RHE Data on PEP slurry \\
\hline TI-WTP-PEP-066 & 7.15 .13 & Post-caustic-leach concentration of solids to $17-\mathrm{wt} \%$ UDS & B 00PF1 019 XX 4140 ARC 1 & Archive & & \\
\hline TI-WTP-PEP-066 & 7.15 .13 & Post-caustic-leach concentration of solids to $17-\mathrm{wt} \%$ UDS & B 00PF1 019 XX 4141 ARC 1 & Archive & & \\
\hline TI-WTP-PEP-066 & 7.15 .13 & Post-caustic-leach concentration of solids to $17-\mathrm{wt} \%$ UDS & B 00PF1 019 XX 4142 ARC 1 & Archive & & \\
\hline TI-WTP-PEP-066 & 7.15 .26 & $\begin{array}{l}\text { Post-caustic-leach concentration of solids to } 17 \text {-wt } \% \text { UDS- after } \\
\text { solid concentration complete }\end{array}$ & B 02AML 019 XX 4143 UDS 2 & UDS & SWRI-090320 & 090320-8UDS R1 \\
\hline TI-WTP-PEP-066 & 7.15 .26 & $\begin{array}{l}\text { Post-caustic-leach concentration of solids to } 17 \text {-wt } \% \text { UDS- after } \\
\text { solid concentration complete }\end{array}$ & B 02AML 019 XX 4144 DEN 2 & Density Slurry & SWRI-090320 & 090318-1 090319-3 090320-9 DEN slurry and liquid \\
\hline TI-WTP-PEP-066 & 7.15 .26 & $\begin{array}{l}\text { Post-caustic-leach concentration of solids to } 17 \text {-wt } \% \text { UDS- after } \\
\text { solid concentration complete }\end{array}$ & B 02AML 019 XX 4144 DEN 2 & Density Supernate & SWRI-090320 & 090318-1 090319-3 090320-9 DEN slurry and liquid \\
\hline TI-WTP-PEP-066 & 7.15 .26 & $\begin{array}{l}\text { Post-caustic-leach concentration of solids to } 17 \text {-wt } \% \text { UDS- after } \\
\text { solid concentration complete }\end{array}$ & B 02AML 019 XX 4145 RHE 4 & Rheology & APEL Rheology & TDP WTP 363 Int Test B RHE Data on PEP slurry \\
\hline TI-WTP-PEP-066 & 7.15 .26 & $\begin{array}{l}\text { Post-caustic-leach concentration of solids to } 17 \text {-wt } \% \text { UDS- after } \\
\text { solid concentration complete }\end{array}$ & B 02AML 019 XX 4146 RHE 4 & Rheology & APEL Rheology & TDP WTP 363 Int Test B RHE Data on PEP slurry \\
\hline TI-WTP-PEP-066 & 7.15 .26 & $\begin{array}{l}\text { Post-caustic-leach concentration of solids to } 17 \text {-wt } \% \text { UDS- after } \\
\text { solid concentration complete }\end{array}$ & B 02AML 019 XX 4147 ICP 2 B & ICP Wet Solids & SWRI-090320 & $\begin{array}{l}090317-23 \text { and } 090317-43 \text { and } 090318-10 \text { and } 090320-10 \text { and } 090320-13 \\
\text { ICP ICPMS }\end{array}$ \\
\hline TI-WTP-PEP-066 & 7.15 .26 & $\begin{array}{l}\text { Post-caustic-leach concentration of solids to } 17 \text {-wt } \% \text { UDS- after } \\
\text { solid concentration complete }\end{array}$ & B 02AML 019 XX 4147 ICP 2 D & ICP Supernate & SWRI-090320 & $\begin{array}{c}090317-22 \text { and } 090317-42 \text { and } 090318-6 \text { and } 090318-10 \text { and } 090320-10 \\
\text { and } 090320-12 \text { ICP and ICPMS }\end{array}$ \\
\hline TI-WTP-PEP-066 & 7.15 .26 & $\begin{array}{l}\text { Post-caustic-leach concentration of solids to } 17 \text {-wt } \% \text { UDS- after } \\
\text { solid concentration complete }\end{array}$ & B 02AML 019 XX 4147 TFI 2 D & IC & $\begin{array}{l}\text { SWRI-090320- } \\
\quad 14\end{array}$ & 090320-14IC \\
\hline TI-WTP-PEP-066 & 7.15 .26 & $\begin{array}{l}\text { Post-caustic-leach concentration of solids to } 17 \text {-wt\% UDS- after } \\
\text { solid concentration complete }\end{array}$ & B 02AML 019 XX 4147 TFI 3 D & $\mathrm{OH}$ & ASR8391 & ASR 8391 OH Rev. 3 \\
\hline TI-WTP-PEP-066 & 7.15 .26 & $\begin{array}{l}\text { Post-caustic-leach concentration of solids to } 17 \text {-wt } \% \text { UDS- after } \\
\text { solid concentration complete }\end{array}$ & B 02AML 019 XX 4148 XSP 4 & PSD & APEL PSD & TDP WTP 362 Int Test B PSD Data Analysis \\
\hline TI-WTP-PEP-066 & 7.15 .26 & $\begin{array}{l}\text { Post-caustic-leach concentration of solids to } 17 \text {-wt } \% \text { UDS- after } \\
\text { solid concentration complete }\end{array}$ & B 02AML 019 XX 4149 SOX 2 & TIC/TOC & SWRI-090320 & $090318-9$ and $090319-9$ and $090320-11$ and $090324-20$ IC and TIC TOC \\
\hline TI-WTP-PEP-066 & 7.15 .26 & $\begin{array}{l}\text { Post-caustic-leach concentration of solids to } 17 \text {-wt } \% \text { UDS- after } \\
\text { solid concentration complete }\end{array}$ & B 02AML 019 XX 4150 AFA 2 & ICP Supernate & SWRI-090320 & $\begin{array}{l}090317-22 \text { and } 090317-42 \text { and } 090318-6 \text { and } 090318-10 \text { and } 090320-10 \\
\text { and 090320-12 ICP and ICPMS }\end{array}$ \\
\hline TI-WTP-PEP-066 & 7.15 .26 & $\begin{array}{l}\text { Post-caustic-leach concentration of solids to } 17 \text {-wt } \% \text { UDS- after } \\
\text { solid concentration complete }\end{array}$ & B 02AML 019 XX 4150 AFA 2 & ICP wet solids & SWRI-090320 & $\begin{array}{l}\text { 090317-23 and 090317-43 and 090318-10 and 090320-10 and 090320-13 } \\
\text { ICP ICPMS }\end{array}$ \\
\hline
\end{tabular}


Integrated Test B Sample List

\begin{tabular}{|c|c|c|c|c|c|c|}
\hline TI \# & TI Step & Step Description & Sample ID & Analysis & File Folder & Final Electronic Report \# \\
\hline TI-WTP-PEP-066 & 7.15 .26 & $\begin{array}{l}\text { Post-caustic-leach concentration of solids to } 17 \text {-wt } \% \text { UDS- after } \\
\text { solid concentration complete }\end{array}$ & B 02AML 019 XX 4151 AFA 5 B & THF Wet Solids & DOW & PNNL 49499 Report.pdf \\
\hline TI-WTP-PEP-066 & 7.15 .26 & $\begin{array}{l}\text { Post-caustic-leach concentration of solids to } 17 \text {-wt } \% \text { UDS- after } \\
\text { solid concentration complete }\end{array}$ & В 02AML 019 XX 4151 AFA 5 D & THF Supernate & DOW & PNNL 49499 Report.pdf \\
\hline TI-WTP-PEP-066 & 7.15 .26 & $\begin{array}{l}\text { Post-caustic-leach concentration of solids to } 17 \text {-wt } \% \text { UDS- after } \\
\text { solid concentration complete }\end{array}$ & B $02 \mathrm{AML} 019 \mathrm{XX} 4152$ AFA 5 B & Toluene Wet Solids & DOW & PNNL 49499 Report.pdf \\
\hline TI-WTP-PEP-066 & 7.15 .26 & $\begin{array}{l}\text { Post-caustic-leach concentration of solids to } 17 \text {-wt } \% \text { UDS- after } \\
\text { solid concentration complete }\end{array}$ & B 02AML 019 XX 4152 AFA 5 D & Toluene Supernate & DOW & PNNL 49499 Report.pdf \\
\hline TI-WTP-PEP-066 & 7.15 .26 & $\begin{array}{l}\text { Post-caustic-leach concentration of solids to } 17 \text {-wt } \% \text { UDS- after } \\
\text { solid concentration complete }\end{array}$ & B 00PF1 019 XX 4153 ARC 4 & Precipitation Study & & \\
\hline TI-WTP-PEP-066 & 7.15 .26 & $\begin{array}{l}\text { Post-caustic-leach concentration of solids to } 17 \text {-wt } \% \text { UDS- after } \\
\text { solid concentration complete }\end{array}$ & B 00PF1 019 XX 4154 ARC 4 & Precipitation Study & & \\
\hline TI-WTP-PEP-066 & 7.16 .4 & Evaluation bypass of filter-loop jet within UFP-VSL-T02A & B 0CSBR 020 XX 4155 ICP 2 & ICP Solution & SWRI-090320 & $090320-15$ and $090324-33$ and $090324-34$ and $090324-35$ ICP and ICPMS \\
\hline TI-WTP-PEP-066 & 7.16 .5 & Evaluation bypass of filter-loop jet within UFP-VSL-T02A & B 000FL 020 BT 4432 ICP 0 B & Storage & & \\
\hline TI-WTP-PEP-066 & 7.16 .5 & Evaluation bypass of filter-loop jet within UFP-VSL-T02A & B 000FL 020 BT 4432 ICP 2 D & ICP Supernate & SWRI-090324 & 090320-15 and 090324-33 and 090324-34 and 090324-35 ICP and ICPMS \\
\hline TI-WTP-PEP-066 & 7.16 .5 & Evaluation bypass of filter-loop jet within UFP-VSL-T02A & B 000FL 020014156 ICP 0 B & Storage & & \\
\hline TI-WTP-PEP-066 & 7.16 .5 & Evaluation bypass of filter-loop jet within UFP-VSL-T02A & B 000FL 020014156 ICP 2 D & ICP Supernate & SWRI-090324 & $090320-15$ and $090324-33$ and $090324-34$ and $090324-35$ ICP and ICPMS \\
\hline TI-WTP-PEP-066 & 7.16 .5 & Evaluation bypass of filter-loop jet within UFP-VSL-T02A & B 02AOL 020014165 RHE 4 & Rheology & TDP-WTP-363 & TDP WTP 363 Int Test B RHE Data on PEP slurry \\
\hline TI-WTP-PEP-066 & 7.16 .5 & Evaluation bypass of filter-loop jet within UFP-VSL-T02A & B 000FL 020024157 ICP 0 B & Storage & & \\
\hline TI-WTP-PEP-066 & 7.16 .5 & Evaluation bypass of filter-loop jet within UFP-VSL-T02A & B 000FL 020024157 ICP 2 D & ICP Supernate & SWRI-090324 & 090320-15 and 090324-33 and 090324-34 and 090324-35 ICP and ICPMS \\
\hline TI-WTP-PEP-066 & 7.16 .5 & Evaluation bypass of filter-loop jet within UFP-VSL-T02A & B 000FL 020044158 ICP 0 B & Storage & & \\
\hline TI-WTP-PEP-066 & 7.16 .5 & Evaluation bypass of filter-loop jet within UFP-VSL-T02A & B 000FL 020044158 ICP 2 D & ICP Supernate & SWRI-090324 & $090320-15$ and $090324-33$ and $090324-34$ and $090324-35$ ICP and ICPMS \\
\hline TI-WTP-PEP-066 & 7.16 .5 & Evaluation bypass of filter-loop jet within UFP-VSL-T02A & B 000FL 020084159 ICP 0 B & Storage & & \\
\hline TI-WTP-PEP-066 & 7.16 .5 & Evaluation bypass of filter-loop jet within UFP-VSL-T02A & B 000FL 020084159 ICP 2 D & ICP Supernate & SWRI-090324 & $090320-15$ and $090324-33$ and $090324-34$ and $090324-35$ ICP and ICPMS \\
\hline TI-WTP-PEP-066 & 7.16 .5 & Evaluation bypass of filter-loop jet within UFP-VSL-T02A & B 000FL 020164160 ICP 0 B & Storage & & \\
\hline TI-WTP-PEP-066 & 7.16 .5 & Evaluation bypass of filter-loop jet within UFP-VSL-T02A & B 000FL 020164160 ICP 2 D & ICP Supernate & SWRI-090324 & 090320-15 and 090324-33 and 090324-34 and 090324-35 ICP and ICPMS \\
\hline TI-WTP-PEP-066 & 7.16 .5 & Evaluation bypass of filter-loop jet within UFP-VSL-T02A & B 000FL 020304161 ICP 0 B & Storage & & \\
\hline TI-WTP-PEP-066 & 7.16 .5 & Evaluation bypass of filter-loop jet within UFP-VSL-T02A & B 000FL 020304161 ICP 2 D & ICP Supernate & SWRI-090324 & 090320-15 and 090324-33 and 090324-34 and 090324-35 ICP and ICPMS \\
\hline TI-WTP-PEP-066 & 7.16 .5 & Evaluation bypass of filter-loop jet within UFP-VSL-T02A & B 000FL 020404162 ICP 0 B & Storage & & \\
\hline TI-WTP-PEP-066 & 7.16 .5 & Evaluation bypass of filter-loop jet within UFP-VSL-T02A & B 000FL 020404162 ICP 2 D & ICP Supernate & SWRI-090324 & $090320-15$ and $090324-33$ and $090324-34$ and $090324-35$ ICP and ICPMS \\
\hline TI-WTP-PEP-066 & 7.16 .5 & Evaluation bypass of filter-loop jet within UFP-VSL-T02A & B 000FL 020504163 ICP 0 B & Storage & & \\
\hline TI-WTP-PEP-066 & 7.16 .5 & Evaluation bypass of filter-loop jet within UFP-VSL-T02A & B 000FL 020504163 ICP 2 D & ICP Supernate & SWRI-090324 & 090320-15 and 090324-33 and 090324-34 and 090324-35 ICP and ICPMS \\
\hline TI-WTP-PEP-066 & 7.16 .5 & Evaluation bypass of filter-loop jet within UFP-VSL-T02A & B 000FL 020604164 ICP 0 B & Storage & & \\
\hline TI-WTP-PEP-066 & 7.16 .5 & Evaluation bypass of filter-loop jet within UFP-VSL-T02A & B 000FL 020604164 ICP 2 D & ICP Supernate & SWRI-090324 & $090320-15$ and $090324-33$ and $090324-34$ and $090324-35$ ICP and ICPMS \\
\hline
\end{tabular}


Integrated Test B Sample List

\begin{tabular}{|c|c|c|c|c|c|c|}
\hline TI \# & TI Step & Step Description & Sample ID & Analysis & File Folder & Final Electronic Report \# \\
\hline TI-WTP-PEP-066 & 7.16 .5 & Evaluation bypass of filter-loop jet within UFP-VSL-T02A & B 02AOL 020014166 ICP 0 B & Storage & & \\
\hline TI-WTP-PEP-066 & 7.16 .5 & Evaluation bypass of filter-loop jet within UFP-VSL-T02A & B 02AOL 020014166 ICP 2 D & ICP Supernate & SWRI-090324 & 090320-15 and 090324-33 and 090324-34 and 090324-35 ICP and ICPMS \\
\hline TI-WTP-PEP-066 & 7.16 .5 & Evaluation bypass of filter-loop jet within UFP-VSL-T02A & B $02 \mathrm{AOL} 020024167$ ICP $0 \mathrm{~B}$ & Storage & & \\
\hline TI-WTP-PEP-066 & 7.16 .5 & Evaluation bypass of filter-loop jet within UFP-VSL-T02A & B 02AOL 020024167 ICP 2 D & ICP Supernate & SWRI-090324 & 090320-15 and 090324-33 and 090324-34 and 090324-35 ICP and ICPMS \\
\hline TI-WTP-PEP-066 & 7.16 .5 & Evaluation bypass of filter-loop jet within UFP-VSL-T02A & B 02AOL 020044168 ICP $0 \mathrm{~B}$ & Storage & & \\
\hline TI-WTP-PEP-066 & 7.16 .5 & Evaluation bypass of filter-loop jet within UFP-VSL-T02A & B 02AOL 020044168 ICP 2 D & ICP Supernate & SWRI-090324 & 090320-15 and 090324-33 and 090324-34 and 090324-35 ICP and ICPMS \\
\hline TI-WTP-PEP-066 & 7.16 .5 & Evaluation bypass of filter-loop jet within UFP-VSL-T02A & B $02 \mathrm{AOL} 020084169$ ICP 0 B & Storage & & \\
\hline TI-WTP-PEP-066 & 7.16 .5 & Evaluation bypass of filter-loop jet within UFP-VSL-T02A & B 02AOL 020084169 ICP 2 D & ICP Supernate & SWRI-090324 & 090320-15 and 090324-33 and 090324-34 and 090324-35 ICP and ICPMS \\
\hline TI-WTP-PEP-066 & 7.16 .5 & Evaluation bypass of filter-loop jet within UFP-VSL-T02A & B $02 \mathrm{AOL} 020164170$ ICP $0 \mathrm{~B}$ & Storage & & \\
\hline TI-WTP-PEP-066 & 7.16 .5 & Evaluation bypass of filter-loop jet within UFP-VSL-T02A & B 02AOL 020164170 ICP 2 D & ICP Supernate & SWRI-090324 & $090320-15$ and $090324-33$ and $090324-34$ and $090324-35$ ICP and ICPMS \\
\hline TI-WTP-PEP-066 & 7.16 .5 & Evaluation bypass of filter-loop jet within UFP-VSL-T02A & B 02AOL 020304171 ICP 0 B & Storage & & \\
\hline TI-WTP-PEP-066 & 7.16 .5 & Evaluation bypass of filter-loop jet within UFP-VSL-T02A & B 02AOL 020304171 ICP 2 D & ICP Supernate & SWRI-090324 & $090320-15$ and $090324-33$ and $090324-34$ and $090324-35$ ICP and ICPMS \\
\hline TI-WTP-PEP-066 & 7.16 .5 & Evaluation bypass of filter-loop jet within UFP-VSL-T02A & B 02AOL 020404172 ICP 0 B & Storage & & \\
\hline TI-WTP-PEP-066 & 7.16 .5 & Evaluation bypass of filter-loop jet within UFP-VSL-T02A & B 02AOL 020404172 ICP 2 D & ICP Supernate & SWRI-090324 & $090320-15$ and $090324-33$ and $090324-34$ and $090324-35$ ICP and ICPMS \\
\hline TI-WTP-PEP-066 & 7.16 .5 & Evaluation bypass of filter-loop jet within UFP-VSL-T02A & B 02AOL 020504173 ICP 0 B & Storage & & \\
\hline TI-WTP-PEP-066 & 7.16 .5 & Evaluation bypass of filter-loop jet within UFP-VSL-T02A & B 02AOL 020504173 ICP 2 D & ICP Supernate & SWRI-090324 & 090320-15 and 090324-33 and 090324-34 and 090324-35 ICP and ICPMS \\
\hline TI-WTP-PEP-066 & 7.16 .5 & Evaluation bypass of filter-loop jet within UFP-VSL-T02A & B 02AOL 020604174 ICP 0 B & Storage & & \\
\hline TI-WTP-PEP-066 & 7.16 .5 & Evaluation bypass of filter-loop jet within UFP-VSL-T02A & B $02 \mathrm{AOL} 020604174$ ICP 2 D & ICP Supernate & SWRI-090324 & 090320-15 and 090324-33 and 090324-34 and 090324-35 ICP and ICPMS \\
\hline TI-WTP-PEP-066 & 7.17.4 & Post-caustic-leach slurry wash & B 02AOL 020014175 DEN 0 B & Storage & & \\
\hline TI-WTP-PEP-066 & 7.17.4 & Post-caustic-leach slurry wash & B 02AOL 020014175 DEN 0 D & Storage & & \\
\hline TI-WTP-PEP-066 & 7.17.4 & Post-caustic-leach slurry wash & B $02 \mathrm{AOL} 020014175$ ICP $2 \mathrm{D}$ & TDS & SWRI-090324 & 090324-6 090324-26 090324-27 ICP ICPMS TDS and density \\
\hline TI-WTP-PEP-066 & 7.17.4 & Post-caustic-leach slurry wash & B $02 \mathrm{AOL} 020014175$ ICP $2 \mathrm{D}$ & ICP Supernate & SWRI-090324 & 090324-6 090324-26 090324-27 ICP ICPMS TDS and density \\
\hline TI-WTP-PEP-066 & 7.17.4 & Post-caustic-leach slurry wash & B 02AOL 020014175 TFI 2 D & $\mathrm{IC}$ & SWRI-090324 & 090324-22IC \\
\hline TI-WTP-PEP-066 & 7.17 .4 & Post-caustic-leach slurry wash & B 02AOL 020014175 TFI 3 D & $\mathrm{OH}$ & ASO_ASR8391 & ASR 8391 OH Rev. 3 \\
\hline TI-WTP-PEP-066 & 7.17.4 & Post-caustic-leach slurry wash & B 02AOL 020014175 RAM 3 D & Raman & ASO_ASR8392 & ASR 8392 RAM \\
\hline TI-WTP-PEP-066 & 7.17.4 & Post-caustic-leach slurry wash & B 02AOL 020024176 UDS 2 & UDS & SWRI-090324 & 090324-7UDS R1 \\
\hline TI-WTP-PEP-066 & 7.17.4 & Post-caustic-leach slurry wash & B 02AOL 020024177 DEN 2 & Density Slurry & SWRI-090324 & 090324-9 and 090324-10 and 090326-10 DEN slurry and liquid \\
\hline TI-WTP-PEP-066 & 7.17.4 & Post-caustic-leach slurry wash & B 02AOL 020024177 DEN 2 & Density Supernate & SWRI-090324 & 090324-9 090324-10 090326-10 DEN slurry and liquid \\
\hline TI-WTP-PEP-066 & 7.17.4 & Post-caustic-leach slurry wash & B $02 \mathrm{AOL} 020024178$ ICP 0 B & Storage & & \\
\hline TI-WTP-PEP-066 & 7.17 .4 & Post-caustic-leach slurry wash & B 02AML 020024178 SUP 0 D & Storage & & \\
\hline TI-WTP-PEP-066 & 7.17.4 & Post-caustic-leach slurry wash & B 02AOL 020024178 ICP 2 D & ICP Supernate & SWRI-090324 & 090324-6 090324-26 090324-27 ICP ICPMS TDS and density \\
\hline TI-WTP-PEP-066 & 7.17.4 & Post-caustic-leach slurry wash & B 02AOL 020024178 TFI 2 D & IC & SWRI-090324 & 090324-23IC \\
\hline TI-WTP-PEP-066 & 7.17.4 & Post-caustic-leach slurry wash & B 02AOL 020024178 TFI 3 D & $\mathrm{OH}$ & ASO_ASR8391 & ASR 8391 OH Rev. 3 \\
\hline
\end{tabular}


Integrated Test B Sample List

\begin{tabular}{|c|c|c|c|c|c|c|}
\hline TI \# & TI Step & Step Description & Sample ID & Analysis & File Folder & Final Electronic Report \# \\
\hline TI-WTP-PEP-066 & 7.17.4 & Post-caustic-leach slurry wash & B 02AOL 020034179 ICP 0 B & Storage & & \\
\hline TI-WTP-PEP-066 & 7.17.4 & Post-caustic-leach slurry wash & B 02AML 020034179 SUP 0 D & Storage & & \\
\hline TI-WTP-PEP-066 & 7.17.4 & Post-caustic-leach slurry wash & B 02AOL 020034179 ICP 2 D & TDS & SWRI-090324 & 090324-6 090324-26 090324-27 ICP ICPMS TDS and density \\
\hline TI-WTP-PEP-066 & 7.17.4 & Post-caustic-leach slurry wash & B 02AOL 020034179 ICP 2 D & ICP Supernate & SWRI-090324 & 090324-6 090324-26 090324-27 ICP ICPMS TDS and density \\
\hline TI-WTP-PEP-066 & 7.17.4 & Post-caustic-leach slurry wash & B 02AOL 020034179 TFI 2 D & $\mathrm{IC}$ & SWRI-090324 & 090324-23IC \\
\hline TI-WTP-PEP-066 & 7.17.4 & Post-caustic-leach slurry wash & B 02AOL 020034179 TFI 3 D & $\mathrm{OH}$ & ASO_ASR8391 & ASR 8391 OH Rev. 3 \\
\hline TI-WTP-PEP-066 & 7.17.4 & Post-caustic-leach slurry wash & B 02AOL 020044180 UDS 2 & UDS & SWRI-090324 & 090324-7UDS R1 \\
\hline TI-WTP-PEP-066 & 7.17.4 & Post-caustic-leach slurry wash & B 02AOL 020044181 DEN 2 & Density Slurry & SWRI-090324 & 090324-9 090324-10 090326-10 DEN slurry and liquid \\
\hline TI-WTP-PEP-066 & 7.17.4 & Post-caustic-leach slurry wash & B 02AOL 020044181 DEN 2 & Density Supernate & SWRI-090324 & 090324-9 090324-10 090326-10 DEN slurry and liquid \\
\hline TI-WTP-PEP-066 & 7.17.4 & Post-caustic-leach slurry wash & B 02AOL 020044182 ICP 0 B & Storage & & \\
\hline TI-WTP-PEP-066 & 7.17.4 & Post-caustic-leach slurry wash & B 02AML 020044182 SUP 0 D & Storage & & \\
\hline TI-WTP-PEP-066 & 7.17.4 & Post-caustic-leach slurry wash & B 02AOL 020044182 ICP 2 D & ICP Supernate & SWRI-090324 & 090324-6 090324-26 090324-27 ICP ICPMS TDS and density \\
\hline TI-WTP-PEP-066 & 7.17.4 & Post-caustic-leach slurry wash & B 02AOL 020044182 TFI 2 D & IC & SWRI-090324 & 090324-22IC \\
\hline TI-WTP-PEP-066 & 7.17.4 & Post-caustic-leach slurry wash & B 02AOL 020044182 TFI 3 D & $\mathrm{OH}$ & ASO_ASR8391 & ASR 8391 OH Rev. 3 \\
\hline TI-WTP-PEP-066 & 7.17.4 & Post-caustic-leach slurry wash & B $02 \mathrm{AOL} 020054183$ ICP $0 \mathrm{~B}$ & Storage & & \\
\hline TI-WTP-PEP-066 & 7.17.4 & Post-caustic-leach slurry wash & B 02AML 020054183 SUP 0 D & Storage & & \\
\hline TI-WTP-PEP-066 & 7.17.4 & Post-caustic-leach slurry wash & B 02AOL 020054183 ICP 2 D & TDS & SWRI-090324 & 090324-6 090324-26 090324-27 ICP ICPMS TDS and density \\
\hline TI-WTP-PEP-066 & 7.17.4 & Post-caustic-leach slurry wash & B 02AOL 020054183 ICP 2 D & ICP Supernate & SWRI-090324 & 090324-6 090324-26 090324-27 ICP ICPMS TDS and density \\
\hline TI-WTP-PEP-066 & 7.17.4 & Post-caustic-leach slurry wash & B 02AOL 020054183 TFI 2 D & IC & SWRI-090324 & 090324-22IC \\
\hline TI-WTP-PEP-066 & 7.17.4 & Post-caustic-leach slurry wash & B 02AOL 020054183 TFI 3 D & $\mathrm{OH}$ & ASO_ASR8391 & ASR 8391 OH Rev. 3 \\
\hline TI-WTP-PEP-066 & 7.17.4 & Post-caustic-leach slurry wash & B 02AOL 020064184 UDS 2 & UDS & SWRI-090324 & 090324-7UDS R1 \\
\hline TI-WTP-PEP-066 & 7.17.4 & Post-caustic-leach slurry wash & B 02AOL 020064185 DEN 2 & Density Slurry & SWRI-090324 & 090324-9 090324-10 090326-10 DEN slurry and liquid \\
\hline TI-WTP-PEP-066 & 7.17.4 & Post-caustic-leach slurry wash & B 02AOL 020064185 DEN 2 & Density Supernate & SWRI-090324 & 090324-9 090324-10 090326-10 DEN slurry and liquid \\
\hline TI-WTP-PEP-066 & 7.17 .4 & Post-caustic-leach slurry wash & B $02 \mathrm{AOL} 020064186$ ICP $0 \mathrm{~B}$ & Storage & & \\
\hline TI-WTP-PEP-066 & 7.17.4 & Post-caustic-leach slurry wash & B 02AML 020064186 SUP 0 D & Storage & & \\
\hline TI-WTP-PEP-066 & 7.17 .4 & Post-caustic-leach slurry wash & B $02 \mathrm{AOL} 020064186$ ICP 2 D & ICP Supernate & SWRI-090324 & 090324-6 090324-26 090324-27 ICP ICPMS TDS and density \\
\hline TI-WTP-PEP-066 & 7.17.4 & Post-caustic-leach slurry wash & B 02AOL 020064186 TFI 2 D & IC & SWRI-090324 & 090324-22IC \\
\hline TI-WTP-PEP-066 & 7.17 .4 & Post-caustic-leach slurry wash & B 02AOL 020064186 TFI 3 D & $\mathrm{OH}$ & ASO_ASR8391 & ASR 8391 OH Rev. 3 \\
\hline TI-WTP-PEP-066 & 7.17.4 & Post-caustic-leach slurry wash & B $02 \mathrm{AOL} 020074187$ ICP $0 \mathrm{~B}$ & Storage & & \\
\hline TI-WTP-PEP-066 & 7.17.4 & Post-caustic-leach slurry wash & B 02AML 020074187 SUP 0 D & Storage & & \\
\hline TI-WTP-PEP-066 & 7.17.4 & Post-caustic-leach slurry wash & B 02AOL 020074187 ICP 2 D & TDS & SWRI-090324 & 090324-6 090324-26 090324-27 ICP ICPMS TDS and density \\
\hline TI-WTP-PEP-066 & 7.17.4 & Post-caustic-leach slurry wash & B $02 \mathrm{AOL} 020074187$ ICP 2 D & ICP Supernate & SWRI-090324 & 090324-6 090324-26 090324-27 ICP ICPMS TDS and density \\
\hline TI-WTP-PEP-066 & 7.17.4 & Post-caustic-leach slurry wash & B 02AOL 020074187 TFI 2 D & IC & SWRI-090324 & 090324-22IC \\
\hline
\end{tabular}


Integrated Test B Sample List

\begin{tabular}{|c|c|c|c|c|c|c|}
\hline TI\# & TI Step & Step Description & Sample ID & Analysis & File Folder & Final Electronic Report \# \\
\hline TI-WTP-PEP-066 & 7.17 .4 & Post-caustic-leach slurry wash & B 02AOL 020074187 TFI 3 D & $\mathrm{OH}$ & ASO_ASR8391 & ASR 8391 OH Rev. 3 \\
\hline TI-WTP-PEP-066 & 7.17.4 & Post-caustic-leach slurry wash & B 02AOL 020084188 UDS 2 & UDS & SWRI-090324 & 090324-7UDS R1 \\
\hline TI-WTP-PEP-066 & 7.17.4 & Post-caustic-leach slurry wash & B 02AOL 020084189 DEN 2 & Density Slurry & SWRI-090324 & 090324-9 090324-10 090326-10 DEN slurry and liquid \\
\hline TI-WTP-PEP-066 & 7.17.4 & Post-caustic-leach slurry wash & B 02AOL 020084189 DEN 2 & Density Supernate & SWRI-090324 & 090324-9 090324-10 090326-10 DEN slurry and liquid \\
\hline TI-WTP-PEP-066 & 7.17.4 & Post-caustic-leach slurry wash & B $02 \mathrm{AOL} 020084190$ ICP $0 \mathrm{~B}$ & Storage & & \\
\hline TI-WTP-PEP-066 & 7.17.4 & Post-caustic-leach slurry wash & B 02AML 020084190 SUP 0 D & Storage & & \\
\hline TI-WTP-PEP-066 & 7.17.4 & Post-caustic-leach slurry wash & B 02AOL 020084190 ICP 2 D & ICP Supernate & SWRI-090324 & 090324-6 090324-26 090324-27 ICP ICPMS TDS and density \\
\hline TI-WTP-PEP-066 & 7.17.4 & Post-caustic-leach slurry wash & B 02AOL 020084190 TFI 2 D & IC & SWRI-090324 & 090324-22IC \\
\hline TI-WTP-PEP-066 & 7.17.4 & Post-caustic-leach slurry wash & B $02 \mathrm{AOL} 020084190$ TFI $3 \mathrm{D}$ & $\mathrm{OH}$ & ASO_ASR8391 & ASR 8391 OH Rev. 3 \\
\hline TI-WTP-PEP-066 & 7.17.4 & Post-caustic-leach slurry wash & B 02AOL 020094191 ICP 0 B & Storage & & \\
\hline TI-WTP-PEP-066 & 7.17.4 & Post-caustic-leach slurry wash & B 02AML 020094191 SUP 0 D & Storage & & \\
\hline TI-WTP-PEP-066 & 7.17.4 & Post-caustic-leach slurry wash & B 02AOL 020094191 ICP 2 D & TDS & SWRI-090324 & 090324-6 090324-26 090324-27 ICP ICPMS TDS and density \\
\hline TI-WTP-PEP-066 & 7.17.4 & Post-caustic-leach slurry wash & B 02AOL 020094191 ICP 2 D & ICP Supernate & SWRI-090324 & 090324-6 090324-26 090324-27 ICP ICPMS TDS and density \\
\hline TI-WTP-PEP-066 & 7.17.4 & Post-caustic-leach slurry wash & B $02 \mathrm{AOL} 020094191$ TFI $2 \mathrm{D}$ & IC & SWRI-090324 & 090324-22IC \\
\hline TI-WTP-PEP-066 & 7.17.4 & Post-caustic-leach slurry wash & B 02AOL 020094191 TFI 3 D & $\mathrm{OH}$ & ASO_ASR8391 & ASR 8391 OH Rev. 3 \\
\hline TI-WTP-PEP-066 & 7.17.4 & Post-caustic-leach slurry wash & B 02AOL 020104192 UDS 2 & UDS & SWRI-090324 & 090324-7UDS R1 \\
\hline TI-WTP-PEP-066 & 7.17.4 & Post-caustic-leach slurry wash & B 02AOL 020104193 DEN 2 & Density Slurry & SWRI-090324 & 090324-9 090324-10 090326-10 DEN slurry and liquid \\
\hline TI-WTP-PEP-066 & 7.17.4 & Post-caustic-leach slurry wash & B 02AOL 020104193 DEN 2 & Density Supernate & SWRI-090324 & 090324-9 090324-10 090326-10 DEN slurry and liquid \\
\hline TI-WTP-PEP-066 & 7.17.4 & Post-caustic-leach slurry wash & B 02AOL 020104194 RHE 4 & Rheology & APEL Rheology & TDP WTP 363 Int Test B RHE Data on PEP slurry \\
\hline TI-WTP-PEP-066 & 7.17.4 & Post-caustic-leach slurry wash & B 02AOL 020104195 RHE 4 & Rheology & APEL Rheology & TDP WTP 363 Int Test B RHE Data on PEP slurry \\
\hline TI-WTP-PEP-066 & 7.17.4 & Post-caustic-leach slurry wash & B 02AOL 020104196 DEN 0 B & Storage & & \\
\hline TI-WTP-PEP-066 & 7.17.4 & Post-caustic-leach slurry wash & B 02AOL 020104196 DEN 0 D & Storage & & \\
\hline TI-WTP-PEP-066 & 7.17.4 & Post-caustic-leach slurry wash & B 02AOL 020104196 ICP 2 D & ICP Supernate & SWRI-090324 & 090324-6 090324-26 090324-27 ICP ICPMS TDS and density \\
\hline TI-WTP-PEP-066 & 7.17.4 & Post-caustic-leach slurry wash & B $02 \mathrm{AOL} 020104196$ TFI $2 \mathrm{D}$ & IC & SWRI-090324 & 090324-22IC \\
\hline TI-WTP-PEP-066 & 7.17.4 & Post-caustic-leach slurry wash & B 02AOL 020104196 TFI 3 D & $\mathrm{OH}$ & ASO_ASR8391 & ASR 8391 OH Rev. 3 \\
\hline TI-WTP-PEP-066 & 7.17.4 & Post-caustic-leach slurry wash & B 02AOL 020104196 RAM 3 D & Raman & ASO_ASR8392 & ASR 8392 RAM \\
\hline TI-WTP-PEP-066 & 7.17.4 & Post-caustic-leach slurry wash & B 02AOL 020114197 ICP 0 B & Storage & & \\
\hline TI-WTP-PEP-066 & 7.17.4 & Post-caustic-leach slurry wash & B 02AML 020114197 SUP 0 D & Storage & & \\
\hline TI-WTP-PEP-066 & 7.17.4 & Post-caustic-leach slurry wash & B 02AOL 020114197 ICP 2 D & TDS & SWRI-090324 & 090324-6 090324-26 090324-27 ICP ICPMS TDS and density \\
\hline TI-WTP-PEP-066 & 7.17.4 & Post-caustic-leach slurry wash & B 02AOL 020114197 ICP 2 D & ICP Supernate & SWRI-090324 & 090324-6 090324-26 090324-27 ICP ICPMS TDS and density \\
\hline TI-WTP-PEP-066 & 7.17.4 & Post-caustic-leach slurry wash & B 02AOL 020114197 TFI 2 D & IC & SWRI-090324 & 090324-22IC \\
\hline TI-WTP-PEP-066 & 7.17 .4 & Post-caustic-leach slurry wash & B 02AOL 020114197 TFI 3 D & $\mathrm{OH}$ & ASO_ASR8391 & ASR 8391 OH Rev. 3 \\
\hline TI-WTP-PEP-066 & 7.17.4 & Post-caustic-leach slurry wash & B 02AOL 020124198 UDS 2 & UDS & SWRI-090324 & 090324-7UDS R1 \\
\hline
\end{tabular}


Integrated Test B Sample List

\begin{tabular}{|c|c|c|c|c|c|c|}
\hline TI\# & TI Step & Step Description & Sample ID & Analysis & File Folder & Final Electronic Report \# \\
\hline TI-WTP-PEP-066 & 7.17.4 & Post-caustic-leach slurry wash & B 02AOL 020124199 DEN 2 & Density Slurry & SWRI-090324 & 090324-9 090324-10 090326-10 DEN slurry and liquid \\
\hline TI-WTP-PEP-066 & 7.17.4 & Post-caustic-leach slurry wash & B 02AOL 020124199 DEN 2 & Density Supernate & SWRI-090324 & 090324-9 090324-10 090326-10 DEN slurry and liquid \\
\hline TI-WTP-PEP-066 & 7.17.4 & Post-caustic-leach slurry wash & B 02AOL 020124200 ICP 0 B & Storage & & \\
\hline TI-WTP-PEP-066 & 7.17.4 & Post-caustic-leach slurry wash & B 02AML 020124200 SUP 0 D & Storage & & \\
\hline TI-WTP-PEP-066 & 7.17.4 & Post-caustic-leach slurry wash & B 02AOL 020124200 ICP 2 D & ICP Supernate & SWRI-090324 & 090324-6 090324-26 090324-27 ICP ICPMS TDS and density \\
\hline TI-WTP-PEP-066 & 7.17.4 & Post-caustic-leach slurry wash & B 02AOL 020124200 TFI 2 D & IC & SWRI-090324 & 090324-22IC \\
\hline TI-WTP-PEP-066 & 7.17.4 & Post-caustic-leach slurry wash & B 02AOL 020124200 TFI 3 D & $\mathrm{OH}$ & ASO_ASR8391 & ASR 8391 OH Rev. 3 \\
\hline TI-WTP-PEP-066 & 7.17.4 & Post-caustic-leach slurry wash & B 02AOL 020134201 ICP 0 B & Storage & & \\
\hline TI-WTP-PEP-066 & 7.17.4 & Post-caustic-leach slurry wash & B 02AML 020134201 SUP 0 D & Storage & & \\
\hline TI-WTP-PEP-066 & 7.17.4 & Post-caustic-leach slurry wash & B 02AOL 020134201 ICP 2 D & TDS & SWRI-090324 & 090324-6 090324-26 090324-27 ICP ICPMS TDS and density \\
\hline TI-WTP-PEP-066 & 7.17.4 & Post-caustic-leach slurry wash & B 02AOL 020134201 ICP 2 D & ICP Supernate & SWRI-090324 & 090324-6 090324-26 090324-27 ICP ICPMS TDS and density \\
\hline TI-WTP-PEP-066 & 7.17.4 & Post-caustic-leach slurry wash & B 02AOL 020134201 TFI 2 D & IC & SWRI-090324 & 090324-22IC \\
\hline TI-WTP-PEP-066 & 7.17.4 & Post-caustic-leach slurry wash & B 02AOL 020134201 TFI 3 D & $\mathrm{OH}$ & ASO_ASR8391 & ASR 8391 OH Rev. 3 \\
\hline TI-WTP-PEP-066 & 7.17.4 & Post-caustic-leach slurry wash & B 02AOL 020144202 UDS 2 & UDS & SWRI-090324 & 090324-7UDS R1 \\
\hline TI-WTP-PEP-066 & 7.17.4 & Post-caustic-leach slurry wash & B 02AOL 020144203 DEN 2 & Density Slurry & SWRI-090324 & 090324-9 090324-10 090326-10 DEN slurry and liquid \\
\hline TI-WTP-PEP-066 & 7.17.4 & Post-caustic-leach slurry wash & B 02AOL 020144203 DEN 2 & Density Supernate & SWRI-090324 & 090324-9 090324-10 090326-10 DEN slurry and liquid \\
\hline TI-WTP-PEP-066 & 7.17.4 & Post-caustic-leach slurry wash & B 02AOL 020144204 ICP 0 B & Storage & & \\
\hline TI-WTP-PEP-066 & 7.17 .4 & Post-caustic-leach slurry wash & B 02AML 020144204 SUP 0 D & Storage & & \\
\hline TI-WTP-PEP-066 & 7.17.4 & Post-caustic-leach slurry wash & B 02AOL 020144204 ICP 2 D & ICP Supernate & SWRI-090324 & 090324-6 090324-26 090324-27 ICP ICPMS TDS and density \\
\hline TI-WTP-PEP-066 & 7.17.4 & Post-caustic-leach slurry wash & B 02AOL 020144204 TFI 2 D & IC & SWRI-090324 & 090324-22IC \\
\hline TI-WTP-PEP-066 & 7.17.4 & Post-caustic-leach slurry wash & B 02AOL 020144204 TFI 3 D & $\mathrm{OH}$ & ASO_ASR8391 & ASR 8391 OH Rev. 3 \\
\hline TI-WTP-PEP-066 & 7.17.4 & Post-caustic-leach slurry wash & B 02AOL 020154205 ICP 0 B & Storage & & \\
\hline TI-WTP-PEP-066 & 7.17.4 & Post-caustic-leach slurry wash & B 02AML 020154205 SUP 0 D & Storage & & \\
\hline TI-WTP-PEP-066 & 7.17 .4 & Post-caustic-leach slurry wash & B 02AOL 020154205 ICP 2 D & TDS & SWRI-090324 & 090324-6 090324-26 090324-27 ICP ICPMS TDS and density \\
\hline TI-WTP-PEP-066 & 7.17.4 & Post-caustic-leach slurry wash & B 02AOL 020154205 ICP 2 D & ICP Supernate & SWRI-090324 & 090324-6 090324-26 090324-27 ICP ICPMS TDS and density \\
\hline TI-WTP-PEP-066 & 7.17 .4 & Post-caustic-leach slurry wash & B 02AOL 020154205 TFI 2 D & IC & SWRI-090324 & 090324-22IC \\
\hline TI-WTP-PEP-066 & 7.17.4 & Post-caustic-leach slurry wash & B 02AOL 020154205 TFI 3 D & $\mathrm{OH}$ & ASO_ASR8391 & ASR 8391 OH Rev. 3 \\
\hline TI-WTP-PEP-066 & 7.17.4 & Post-caustic-leach slurry wash & B 02AOL 020164206 UDS 2 & UDS & SWRI-090324 & 090324-7UDS R1 \\
\hline TI-WTP-PEP-066 & 7.17.4 & Post-caustic-leach slurry wash & B 02AOL 020164207 DEN 2 & Density Slurry & SWRI-090324 & 090324-9 090324-10 090326-10 DEN slurry and liquid \\
\hline TI-WTP-PEP-066 & 7.17.4 & Post-caustic-leach slurry wash & B 02AOL 020164207 DEN 2 & Density Supernate & SWRI-090324 & 090324-9 090324-10 090326-10 DEN slurry and liquid \\
\hline TI-WTP-PEP-066 & 7.17.4 & Post-caustic-leach slurry wash & B 02AOL 020164208 ICP 0 B & Storage & & \\
\hline TI-WTP-PEP-066 & 7.17.4 & Post-caustic-leach slurry wash & B 02AML 020164208 SUP 0 D & Storage & & \\
\hline TI-WTP-PEP-066 & 7.17.4 & Post-caustic-leach slurry wash & B 02AOL 020164208 ICP 2 D & ICP Supernate & SWRI-090324 & 090324-6 090324-26 090324-27 ICP ICPMS TDS and density \\
\hline
\end{tabular}


Integrated Test B Sample List

\begin{tabular}{|c|c|c|c|c|c|c|}
\hline TI\# & TI Step & Step Description & Sample ID & Analysis & File Folder & Final Electronic Report \# \\
\hline TI-WTP-PEP-066 & 7.17 .4 & Post-caustic-leach slurry wash & B 02AOL 020164208 TFI 2 D & IC & SWRI-090324 & 090324-23IC \\
\hline TI-WTP-PEP-066 & 7.17.4 & Post-caustic-leach slurry wash & B 02AOL 020164208 TFI 3 D & $\mathrm{OH}$ & ASO_ASR8391 & ASR 8391 OH Rev. 3 \\
\hline TI-WTP-PEP-066 & 7.17.4 & Post-caustic-leach slurry wash & B 02AOL 020174209 ICP $0 \mathrm{~B}$ & Storage & & \\
\hline TI-WTP-PEP-066 & 7.17.4 & Post-caustic-leach slurry wash & B 02AML 020174209 SUP 0 D & Storage & & \\
\hline TI-WTP-PEP-066 & 7.17.4 & Post-caustic-leach slurry wash & B 02AOL 020174209 ICP 2 D & TDS & SWRI-090324 & 090324-6 090324-26 090324-27 ICP ICPMS TDS and density \\
\hline TI-WTP-PEP-066 & 7.17.4 & Post-caustic-leach slurry wash & B 02AOL 020174209 ICP 2 D & ICP Supernate & SWRI-090324 & 090324-6 090324-26 090324-27 ICP ICPMS TDS and density \\
\hline TI-WTP-PEP-066 & 7.17.4 & Post-caustic-leach slurry wash & B 02AOL 020174209 TFI 2 D & IC & SWRI-090324 & 090324-23IC \\
\hline TI-WTP-PEP-066 & 7.17.4 & Post-caustic-leach slurry wash & B 02AOL 020174209 TFI 3 D & $\mathrm{OH}$ & ASO_ASR8391 & ASR 8391 OH Rev. 3 \\
\hline TI-WTP-PEP-066 & 7.17.5 & Post-caustic-leach slurry wash & B 02AOL 020 XX 4214 UDS 2 & UDS & SWRI-090324 & 090324-7UDS R1 \\
\hline TI-WTP-PEP-066 & 7.17.5 & Post-caustic-leach slurry wash & B 02AOL $020 \mathrm{XX} 4215$ DEN 2 & Density Slurry & SWRI-090324 & 090324-9 090324-10 090326-10 DEN slurry and liquid \\
\hline TI-WTP-PEP-066 & 7.17.5 & Post-caustic-leach slurry wash & B 02AOL 020 XX 4215 DEN 2 & Density Supernate & SWRI-090324 & 090324-9 090324-10 090326-10 DEN slurry and liquid \\
\hline TI-WTP-PEP-066 & 7.17.5 & Post-caustic-leach slurry wash & B 02AOL 020 XX 4216 RHE 4 & Rheology & APEL Rheology & TDP WTP 363 Int Test B RHE Data on PEP slurry \\
\hline TI-WTP-PEP-066 & 7.17 .5 & Post-caustic-leach slurry wash & B 02AOL 020 XX 4217 RHE 4 & Rheology & APEL Rheology & TDP WTP 363 Int Test B RHE Data on PEP slurry \\
\hline TI-WTP-PEP-066 & 7.17.5 & Post-caustic-leach slurry wash & B 02AOL $020 \mathrm{XX} 4218$ ICP $2 \mathrm{~B}$ & ICP Wet Solids & SWRI-090324 & 090324-28 ICP and ICPMS \\
\hline TI-WTP-PEP-066 & 7.17 .5 & Post-caustic-leach slurry wash & B 02AOL 020 XX 4218 SUP 0 D & Storage & & \\
\hline TI-WTP-PEP-066 & 7.17.5 & Post-caustic-leach slurry wash & B 02AOL 020 XX 4218 ICP 2 D & ICP Supernate & SWRI-090324 & 090324-6 090324-26 090324-27 ICP ICPMS TDS and density \\
\hline TI-WTP-PEP-066 & 7.17.5 & Post-caustic-leach slurry wash & B 02AOL $020 \mathrm{XX} 4218$ TFI $2 \mathrm{D}$ & $\mathrm{IC}$ & SWRI-090324 & 090324-23IC \\
\hline TI-WTP-PEP-066 & 7.17.5 & Post-caustic-leach slurry wash & B $02 \mathrm{AOL} 020 \mathrm{XX} 4218$ TFI $3 \mathrm{D}$ & $\mathrm{OH}$ & ASO_ASR8391 & ASR 8391 OH Rev. 3 \\
\hline TI-WTP-PEP-066 & 7.17.5 & Post-caustic-leach slurry wash & B 02AOL 020 XX 4219 SOX 2 & TIC/TOC & SWRI-090324 & 090318-9 and 090319-9 and 090320-11 and 090324-20 IC and TIC TOC \\
\hline TI-WTP-PEP-066 & 7.17 .5 & Post-caustic-leach slurry wash & B $02 \mathrm{AOL} 020 \mathrm{XX} 4220$ AFA 2 & ICP Supernate & SWRI-090324 & 090324-21 ICP and ICPMS \\
\hline TI-WTP-PEP-066 & 7.17 .5 & Post-caustic-leach slurry wash & B 02AOL 020 XX 4220 AFA 2 & ICP Wet Solids & SWRI-090324 & 090324-21 ICP and ICPMS \\
\hline TI-WTP-PEP-066 & 7.17 .5 & Post-caustic-leach slurry wash & B 02AOL 020 XX 4221 AFA 5 B & THF Wet Solids & DOW & PNNL 49554 Report.pdf \\
\hline TI-WTP-PEP-066 & 7.17 .5 & Post-caustic-leach slurry wash & B 02AOL 020 XX 4221 AFA 5 D & THF Supernate & DOW & PNNL 49554 Report.pdf \\
\hline TI-WTP-PEP-066 & 7.17 .5 & Post-caustic-leach slurry wash & B $02 \mathrm{AOL} 020 \mathrm{XX} 4222 \mathrm{AFA} 5 \mathrm{~B}$ & Toluene Wet Solids & DOW & PNNL 49554 Report.pdf \\
\hline TI-WTP-PEP-066 & 7.17.5 & Post-caustic-leach slurry wash & B 02AOL 020 XX 4222 AFA 5 D & Toluene Supernate & DOW & PNNL 49554 Report.pdf \\
\hline TI-WTP-PEP-066 & 7.17.5 & Post-caustic-leach slurry wash & B 00PF1 020 XX 4223 ARC 4 & Precipitation Study & & \\
\hline TI-WTP-PEP-066 & 7.17.5 & Post-caustic-leach slurry wash & B 00PF1 $020 \mathrm{XX} 4224$ ARC 4 & Precipitation Study & & \\
\hline TI-WTP-PEP-066 & 7.17.5 & Post-caustic-leach slurry wash & B 00PF1 $020 \mathrm{XX} 4225$ ARC 4 & Precipitation Study & & \\
\hline TI-WTP-PEP-066 & 7.17 .5 & Post-caustic-leach slurry wash & B 00PF1 020 XX 4226 ARC 4 & Precipitation Study & & \\
\hline TI-WTP-PEP-066 & 7.17.5 & Post-caustic-leach slurry wash & B 00PF1 020 XX 4227 ARC 4 & Precipitation Study & & \\
\hline TI-WTP-PEP-066 & 7.17 .5 & Post-caustic-leach slurry wash & B 00PF1 020 XX 4228 ARC 4 & Precipitation Study & & \\
\hline TI-WTP-PEP-066 & 7.17.5 & Post-caustic-leach slurry wash & B 00PF1 020 XX 4229 ARC 4 & Precipitation Study & & \\
\hline TI-WTP-PEP-066 & 7.17 .5 & Post-caustic-leach slurry wash & B 00PF1 020 XX 4230 ARC 4 & Precipitation Study & & \\
\hline
\end{tabular}


Integrated Test B Sample List

\begin{tabular}{|c|c|c|c|c|c|c|}
\hline TI \# & TI Step & Step Description & Sample ID & Analysis & File Folder & Final Electronic Report \# \\
\hline TI-WTP-PEP-066 & 7.17 .5 & Post-caustic-leach slurry wash & B 00PF1 020 XX 4231 ARC 4 & Precipitation Study & & \\
\hline TI-WTP-PEP-066 & 7.17 .5 & Post-caustic-leach slurry wash & B 00PF1 020 XX 4232 ARC 4 & Precipitation Study & & \\
\hline TI-WTP-PEP-066 & 7.17 .5 & Post-caustic-leach slurry wash & B 00PF1 020 XX 4233 ARC 4 & Precipitation Study & & \\
\hline TI-WTP-PEP-066 & 7.17 .5 & Post-caustic-leach slurry wash & B 00PF1 020 XX 4234 ARC 4 & Precipitation Study & & \\
\hline TI-WTP-PEP-066 & 7.17 .5 & Post-caustic-leach slurry wash & B 00PF1 020 XX 4235 ARC 4 & Precipitation Study & & \\
\hline TI-WTP-PEP-066 & 7.17 .5 & Post-caustic-leach slurry wash & B 00PF1 020 XX 4236 ARC 4 & Precipitation Study & & \\
\hline TI-WTP-PEP-066 & 7.17 .5 & Post-caustic-leach slurry wash & B 00PF1 020 XX 4237 ARC 4 & Precipitation Study & & \\
\hline TI-WTP-PEP-066 & 7.17.5 & Post-caustic-leach slurry wash & B 00PF1 020 XX 4238 ARC 4 & Precipitation Study & & \\
\hline TI-WTP-PEP-066 & 7.17.5 & Post-caustic-leach slurry wash & B 00PF1 020 XX 4239 ARC 4 & Precipitation Study & & \\
\hline TI-WTP-PEP-066 & 7.17 .5 & Post-caustic-leach slurry wash & B 00PF1 020 XX 4240 ARC 4 & Precipitation Study & & \\
\hline TI-WTP-PEP-066 & 7.17.5 & Post-caustic-leach slurry wash & B 00PF1 020 XX 4241 ARC 4 & Precipitation Study & & \\
\hline TI-WTP-PEP-066 & 7.17.5 & Post-caustic-leach slurry wash & B 00PF1 020 XX 4242 ARC 4 & Precipitation Study & & \\
\hline TI-WTP-PEP-066 & 7.17.5 & Post-caustic-leach slurry wash & B 00PF1 020 XX 4243 ARC 4 & Precipitation Study & & \\
\hline TI-WTP-PEP-066 & 7.17 .5 & Post-caustic-leach slurry wash & B 00PF1 020 XX 4244 ARC 4 & Precipitation Study & & \\
\hline TI-WTP-PEP-066 & 7.17 .5 & Post-caustic-leach slurry wash & B 00PF1 020 XX 4245 ARC 4 & Precipitation Study & & \\
\hline TI-WTP-PEP-066 & 7.17 .5 & Post-caustic-leach slurry wash & B 00PF1 020 XX 4246 ARC 4 & Precipitation Study & & \\
\hline TI-WTP-PEP-066 & 7.17 .5 & Post-caustic-leach slurry wash & B 00PF1 020 XX 4247 ARC 4 & Precipitation Study & & \\
\hline TI-WTP-PEP-066 & 7.17.5 & Post-caustic-leach slurry wash & B 00PF1 020 XX 4248 ARC 4 & Precipitation Study & & \\
\hline TI-WTP-PEP-066 & 7.17 .5 & Post-caustic-leach slurry wash & B 00PF1 020 XX 4249 ARC 4 & Precipitation Study & & \\
\hline TI-WTP-PEP-066 & 7.17.5 & Post-caustic-leach slurry wash & B 00PF1 020 XX 4250 ARC 4 & Precipitation Study & & \\
\hline TI-WTP-PEP-066 & 7.17 .5 & Post-caustic-leach slurry wash & B 00PF1 020 XX 4251 ARC 4 & Precipitation Study & & \\
\hline TI-WTP-PEP-066 & 7.17 .5 & Post-caustic-leach slurry wash & B 00PF1 020 XX 4252 ARC 4 & Precipitation Study & & \\
\hline TI-WTP-PEP-066 & 7.17.5 & Post-caustic-leach slurry wash & B 00PF1 020 XX 4253 ARC 4 & Precipitation Study & & \\
\hline TI-WTP-PEP-066 & 7.17 .5 & Post-caustic-leach slurry wash & B 00PF1 020 XX 4254 ARC 4 & Precipitation Study & & \\
\hline TI-WTP-PEP-066 & 7.17.5 & Post-caustic-leach slurry wash & B 00PF1 020 XX 4255 ARC 4 & Precipitation Study & & \\
\hline TI-WTP-PEP-066 & 7.17.5 & Post-caustic-leach slurry wash & B 00PF1 020 XX 4256 ARC 4 & Precipitation Study & & \\
\hline TI-WTP-PEP-066 & 7.17.5 & Post-caustic-leach slurry wash & B 00PF1 020 XX 4257 ARC 4 & Precipitation Study & & \\
\hline TI-WTP-PEP-066 & 7.17 .5 & Post-caustic-leach slurry wash & B 00PF1 020 XX 4258 ARC 4 & Precipitation Study & & \\
\hline TI-WTP-PEP-066 & 7.17 .5 & Post-caustic-leach slurry wash & B 00PF1 020 XX 4259 ARC 4 & Precipitation Study & & \\
\hline TI-WTP-PEP-066 & 7.17.5 & Post-caustic-leach slurry wash & B 00PF1 020 XX 4260 ARC 4 & Precipitation Study & & \\
\hline TI-WTP-PEP-066 & 7.17.5 & Post-caustic-leach slurry wash & B 00PF1 020 XX 4261 ARC 4 & Precipitation Study & & \\
\hline TI-WTP-PEP-066 & 7.17.5 & Post-caustic-leach slurry wash & B 00PF1 020 XX 4262 ARC 4 & Precipitation Study & & \\
\hline TI-WTP-PEP-066 & 7.17.5 & Post-caustic-leach slurry wash & B 00PF1 020 XX 4263 ARC 4 & Precipitation Study & & \\
\hline
\end{tabular}


Integrated Test B Sample List

\begin{tabular}{|c|c|c|c|c|c|c|}
\hline TI \# & TI Step & Step Description & Sample ID & Analysis & File Folder & Final Electronic Report \# \\
\hline TI-WTP-PEP-066 & 7.17 .5 & Post-caustic-leach slurry wash & B 00PF1 020 XX 4264 ARC 4 & Precipitation Study & & \\
\hline TI-WTP-PEP-066 & 7.17 .5 & Post-caustic-leach slurry wash & B 00PF1 020 XX 4265 ARC 4 & Precipitation Study & & \\
\hline TI-WTP-PEP-066 & 7.17 .5 & Post-caustic-leach slurry wash & B 00PF1 020 XX 4266 ARC 4 & Precipitation Study & & \\
\hline TI-WTP-PEP-066 & 7.17.5 & Post-caustic-leach slurry wash & B 00PF1 020 XX 4267 ARC 4 & Precipitation Study & & \\
\hline TI-WTP-PEP-066 & 7.17 .5 & Post-caustic-leach slurry wash & B 00PF1 020 XX 4268 ARC 4 & Precipitation Study & & \\
\hline TI-WTP-PEP-066 & 7.17 .5 & Post-caustic-leach slurry wash & B 00PF1 020 XX 4269 ARC 4 & Precipitation Study & & \\
\hline TI-WTP-PEP-066 & 7.17 .5 & Post-caustic-leach slurry wash & B 00PF1 020 XX 4270 ARC 4 & Precipitation Study & & \\
\hline TI-WTP-PEP-066 & 7.17.5 & Post-caustic-leach slurry wash & B 00PF1 020 XX 4271 ARC 4 & Precipitation Study & & \\
\hline TI-WTP-PEP-066 & 7.17 .5 & Post-caustic-leach slurry wash & B 00PF1 020 XX 4272 ARC 4 & Precipitation Study & & \\
\hline TI-WTP-PEP-066 & 7.17.5 & Post-caustic-leach slurry wash & B 00PF1 020 XX 4273 ARC 4 & Precipitation Study & & \\
\hline TI-WTP-PEP-066 & 7.17 .5 & Post-caustic-leach slurry wash & B 00PF1 020 XX 4274 ARC 4 & Precipitation Study & & \\
\hline TI-WTP-PEP-066 & 7.18 .11 & Add permanganate - before permanganate addition & B 02AOL 023 XX 4282 UDS 2 & UDS & SWRI-090324 & 090324-7UDS R1 \\
\hline TI-WTP-PEP-066 & 7.18 .11 & Add permanganate - before permanganate addition & B 02AOL 023 XX 4283 UDS 2 & UDS & SWRI-090324 & 090324-7UDS R1 \\
\hline TI-WTP-PEP-066 & 7.18.11 & Add permanganate - before permanganate addition & В $02 \mathrm{AOL} 023 \mathrm{XX} 4284$ UDS 2 & UDS & SWRI-090324 & 090324-7UDS R1 \\
\hline TI-WTP-PEP-066 & 7.18 .11 & Add permanganate - before permanganate addition & B 02AOL 023 XX 4285 DEN 2 & Density Slurry & SWRI-090324 & 090324-9 090324-10 090326-10 DEN slurry and liquid \\
\hline TI-WTP-PEP-066 & 7.18 .11 & Add permanganate - before permanganate addition & B 02AOL 023 XX 4285 DEN 2 & Density Supernate & SWRI-090324 & 090324-9 090324-10 090326-10 DEN slurry and liquid \\
\hline TI-WTP-PEP-066 & 7.18.11 & Add permanganate - before permanganate addition & B $02 \mathrm{AOL} 023 \mathrm{XX} 4286$ DEN 2 & Density Slurry & SWRI-090324 & 090324-9 090324-10 090326-10 DEN slurry and liquid \\
\hline TI-WTP-PEP-066 & 7.18.11 & Add permanganate - before permanganate addition & B 02AOL $023 \mathrm{XX} 4286$ DEN 2 & Density Supernate & SWRI-090324 & 090324-9 090324-10 090326-10 DEN slurry and liquid \\
\hline TI-WTP-PEP-066 & 7.18 .11 & Add permanganate - before permanganate addition & B $02 \mathrm{AOL} 023 \mathrm{XX} 4287 \mathrm{DEN} 2$ & Density Slurry & SWRI-090324 & 090324-9 090324-10 090326-10 DEN slurry and liquid \\
\hline TI-WTP-PEP-066 & 7.18 .11 & Add permanganate - before permanganate addition & B 02AOL 023 XX 4287 DEN 2 & Density Supernate & SWRI-090324 & 090324-9 090324-10 090326-10 DEN slurry and liquid \\
\hline TI-WTP-PEP-066 & 7.18 .11 & Add permanganate - before permanganate addition & B 02AOL 023 XX 4288 RHE 4 & Rheology & APEL Rheology & TDP WTP 363 Int Test B RHE Data on PEP slurry \\
\hline TI-WTP-PEP-066 & 7.18 .11 & Add permanganate - before permanganate addition & B 02AOL 023 XX 4289 RHE 4 & Rheology & APEL Rheology & TDP WTP 363 Int Test B RHE Data on PEP slurry \\
\hline TI-WTP-PEP-066 & 7.18.11 & Add permanganate - before permanganate addition & B 02AOL 023 XX 4290 RHE 4 & Rheology & APEL Rheology & TDP WTP 363 Int Test B RHE Data on PEP slurry \\
\hline TI-WTP-PEP-066 & 7.18.11 & Add permanganate - before permanganate addition & B $02 \mathrm{AOL} 023 \mathrm{XX} 4291$ ICP $2 \mathrm{~S}$ & ICP Rinsed Solids & SWRI-090324 & 090324-28 ICP and ICPMS \\
\hline TI-WTP-PEP-066 & 7.18 .11 & Add permanganate - before permanganate addition & B 02AOL 023 XX 4291 DEN 0 D & Storage & & \\
\hline TI-WTP-PEP-066 & 7.18 .11 & Add permanganate - before permanganate addition & B 02AOL 023 XX 4291 DEN 0 R & Storage & & \\
\hline TI-WTP-PEP-066 & 7.18 .11 & Add permanganate - before permanganate addition & B 02AOL 023 XX 4291 ICP 2 D & ICP Supernate & SWRI-090324 & 090324-6 090324-26 090324-27 ICP ICPMS TDS and density \\
\hline TI-WTP-PEP-066 & 7.18 .11 & Add permanganate - before permanganate addition & B 02AOL 023 XX 4291 ICP 2 R & ICP Rinsate & SWRI-090324 & 090324-6 090324-26 090324-27 ICP ICPMS TDS and density \\
\hline TI-WTP-PEP-066 & 7.18 .11 & Add permanganate - before permanganate addition & B 02AOL 023 XX 4291 TFI 2 D & IC & SWRI-090324 & 090324-22IC \\
\hline TI-WTP-PEP-066 & 7.18.11 & Add permanganate - before permanganate addition & B 02AOL 023 XX 4291 TFI 3 D & $\mathrm{OH}$ & ASO_ASR8391 & ASR 8391 OH Rev. 3 \\
\hline TI-WTP-PEP-066 & 7.18 .11 & Add permanganate - before permanganate addition & B 02AOL 023 XX 4291 RAM 3 D & Raman & ASO_ASR8392 & ASR 8392 RAM \\
\hline TI-WTP-PEP-066 & 7.18 .11 & Add permanganate - before permanganate addition & B 02AOL 023 XX 4291 RAM 3 R & Raman & ASO_ASR8392 & ASR 8392 RAM \\
\hline TI-WTP-PEP-066 & 7.18 .11 & Add permanganate - before permanganate addition & B 02AOL 023 XX 4292 ICP $2 \mathrm{~S}$ & ICP Rinsed Solids & SWRI-090324 & 090324-28 ICP and ICPMS \\
\hline
\end{tabular}


Integrated Test B Sample List

\begin{tabular}{|c|c|c|c|c|c|c|}
\hline TI \# & TI Step & Step Description & Sample ID & Analysis & File Folder & Final Electronic Report \# \\
\hline TI-WTP-PEP-066 & 7.18 .11 & Add permanganate - before permanganate addition & B 02AOL 023 XX 4292 DEN 0 D & Storage & & \\
\hline TI-WTP-PEP-066 & 7.18 .11 & Add permanganate - before permanganate addition & B 02AOL 023 XX 4292 DEN 0 R & Storage & & \\
\hline TI-WTP-PEP-066 & 7.18 .11 & Add permanganate - before permanganate addition & B 02AOL 023 XX 4292 ICP 2 D & ICP Supernate & SWRI-090324 & 090324-6 090324-26 090324-27 ICP ICPMS TDS and density \\
\hline TI-WTP-PEP-066 & 7.18 .11 & Add permanganate - before permanganate addition & B 02AOL 023 XX 4292 ICP 2 R & ICP Rinsate & SWRI-090324 & 090324-6 090324-26 090324-27 ICP ICPMS TDS and density \\
\hline TI-WTP-PEP-066 & 7.18 .11 & Add permanganate - before permanganate addition & B 02AOL 023 XX 4292 TFI 2 D & $\mathrm{IC}$ & SWRI-090324 & 090324-22IC \\
\hline TI-WTP-PEP-066 & 7.18 .11 & Add permanganate - before permanganate addition & B 02AOL 023 XX 4292 TFI 3 D & $\mathrm{OH}$ & ASO_ASR 8391 & ASR 8391 OH Rev. 3 \\
\hline TI-WTP-PEP-066 & 7.18 .11 & Add permanganate - before permanganate addition & B 02AOL 023 XX 4292 RAM 3 D & Raman & ASO_ASR 8392 & ASR 8392 RAM \\
\hline TI-WTP-PEP-066 & 7.18 .11 & Add permanganate - before permanganate addition & B $02 \mathrm{AOL} 023$ XX 4292 RAM $3 \mathrm{R}$ & Raman & ASO_ASR 8392 & ASR 8392 RAM \\
\hline TI-WTP-PEP-066 & 7.18 .11 & Add permanganate - before permanganate addition & B 02AOL 023 XX 4293 ICP 2 S & ICP Rinsed Solids & SWRI-090324 & 090324-28 ICP and ICPMS \\
\hline TI-WTP-PEP-066 & 7.18 .11 & Add permanganate - before permanganate addition & B 02AOL 023 XX 4293 DEN 0 D & Storage & & \\
\hline TI-WTP-PEP-066 & 7.18 .11 & Add permanganate - before permanganate addition & B 02AOL 023 XX 4293 DEN 0 R & Storage & & \\
\hline TI-WTP-PEP-066 & 7.18 .11 & Add permanganate - before permanganate addition & B 02AOL 023 XX 4293 ICP 2 D & ICP Supernate & SWRI-090324 & 090324-6 090324-26 090324-27 ICP ICPMS TDS and density \\
\hline TI-WTP-PEP-066 & 7.18 .11 & Add permanganate - before permanganate addition & B $02 \mathrm{AOL} 023 \mathrm{XX} 4293$ ICP $2 \mathrm{R}$ & ICP Rinsate & SWRI-090324 & 090324-6 090324-26 090324-27 ICP ICPMS TDS and density \\
\hline TI-WTP-PEP-066 & 7.18 .11 & Add permanganate - before permanganate addition & B $02 A O L ~ 023$ XX 4293 TFI 2 D & IC & SWRI-090324 & 090324-22IC \\
\hline TI-WTP-PEP-066 & 7.18 .11 & Add permanganate - before permanganate addition & B 02AOL 023 XX 4293 TFI 3 D & $\mathrm{OH}$ & ASO_ASR8391 & ASR 8391 OH Rev. 3 \\
\hline TI-WTP-PEP-066 & 7.18 .11 & Add permanganate - before permanganate addition & B 02AOL 023 XX 4293 RAM 3 D & Raman & ASO_ASR8392 & ASR 8392 RAM \\
\hline TI-WTP-PEP-066 & 7.18 .11 & Add permanganate - before permanganate addition & B 02AOL 023 XX 4293 RAM 3 R & Raman & ASO_ASR8392 & ASR 8392 RAM \\
\hline TI-WTP-PEP-066 & 7.18 .11 & Add permanganate - before permanganate addition & B 02AOL 023 XX 4294 AFA 2 & ICP Supernate & SWRI-090324 & 090324-21 ICP and ICPMS \\
\hline TI-WTP-PEP-066 & 7.18 .11 & Add permanganate - before permanganate addition & B 02AOL 023 XX 4294 AFA 2 & ICP Wet Solids & SWRI-090324 & 090324-21 ICP and ICPMS \\
\hline TI-WTP-PEP-066 & 7.18 .11 & Add permanganate - before permanganate addition & B 02AOL 023 XX 4295 AFA 5 B & Toluene Wet Solids & DOW & PNNL 49554 Report.pdf \\
\hline TI-WTP-PEP-066 & 7.18 .11 & Add permanganate - before permanganate addition & B 02AOL 023 XX 4295 AFA 5 D & Toluene Supernate & DOW & PNNL 49554 Report.pdf \\
\hline TI-WTP-PEP-066 & 7.18 .11 & Add permanganate - before permanganate addition & B 02AOL 023 XX 4296 AFA 5 B & THF Wet Solids & DOW & PNNL 49554 Report.pdf \\
\hline TI-WTP-PEP-066 & 7.18 .11 & Add permanganate - before permanganate addition & B 02AOL 023 XX 4296 AFA 5 D & THF Supernate & DOW & PNNL 49554 Report.pdf \\
\hline TI-WTP-PEP-066 & 7.18 .11 & Add permanganate - before permanganate addition & B 02AOL 023 XX 4297 CUF 4 & Parallel Lab Test & & \\
\hline TI-WTP-PEP-066 & 7.18 .14 & Add permanganate - 5 min after permanganate addition & B 02AOL 023 XX 4471 OTR $0 \mathrm{~F}$ & Storage & & \\
\hline TI-WTP-PEP-066 & 7.18 .14 & Add permanganate - 5 min after permanganate addition & В 02AOL 023 XX 4298 UDS 2 B & UDS & SWRI-090324 & 090324-8UDS R1 \\
\hline TI-WTP-PEP-066 & 7.18 .14 & Add permanganate - 5 min after permanganate addition & B 02AOL 023 XX 4298 UDS 2 D & UDS & SWRI-090324 & 090324-8UDS R1 \\
\hline TI-WTP-PEP-066 & 7.18 .14 & Add permanganate - 5 min after permanganate addition & B 02AOL 023 XX 4299 DEN 2 & Density Slurry & SWRI-090324 & 090324-9 090324-10 090326-10 DEN slurry and liquid \\
\hline TI-WTP-PEP-066 & 7.18 .14 & Add permanganate - 5 min after permanganate addition & B 02AOL 023 XX 4299 DEN 2 & Density Supernate & SWRI-090324 & 090324-9 090324-10 090326-10 DEN slurry and liquid \\
\hline TI-WTP-PEP-066 & 7.18 .14 & Add permanganate - 5 min after permanganate addition & B 02AOL 023 XX 4300 DEN 0 B & Storage & & \\
\hline TI-WTP-PEP-066 & 7.18 .14 & Add permanganate - 5 min after permanganate addition & B 02AOL 023 XX 4300 DEN 0 D & Storage & & \\
\hline TI-WTP-PEP-066 & 7.18 .14 & Add permanganate - 5 min after permanganate addition & B $02 \mathrm{AOL} 023 \mathrm{XX} 4300$ ICP $2 \mathrm{D}$ & ICP Supernate & SWRI-090324 & 090324-6 090324-26 090324-27 ICP ICPMS TDS and density \\
\hline TI-WTP-PEP-066 & 7.18 .14 & Add permanganate - 5 min after permanganate addition & B $02 \mathrm{AOL} 023 \mathrm{XX} 4300$ TFI $2 \mathrm{D}$ & IC & SWRI-090324 & 090324-22IC \\
\hline
\end{tabular}


Integrated Test B Sample List

\begin{tabular}{|c|c|c|c|c|c|c|}
\hline TI \# & TI Step & Step Description & Sample ID & Analysis & File Folder & Final Electronic Report \# \\
\hline TI-WTP-PEP-066 & 7.18 .14 & Add permanganate - $5 \mathrm{~min}$ after permanganate addition & B 02AOL 023 XX 4300 TFI 3 D & $\mathrm{OH}$ & ASO_ASR8391 & ASR 8391 OH Rev. 3 \\
\hline TI-WTP-PEP-066 & 7.18 .14 & Add permanganate - $5 \mathrm{~min}$ after permanganate addition & B 02AOL 023 XX 4300 RAM 3 D & Raman & ASO_ASR8392 & ASR 8392 RAM \\
\hline TI-WTP-PEP-066 & 7.18 .14 & Add permanganate - 5 min after permanganate addition & B 02AOL 023 XX 4301 OTR 6 P & Storage & & \\
\hline TI-WTP-PEP-066 & 7.18 .14 & Add permanganate - $5 \mathrm{~min}$ after permanganate addition & B 02AOL 023 XX 4301 OTR $0 \mathrm{~F}$ & Storage & & \\
\hline TI-WTP-PEP-066 & 7.18 .14 & Oxidative-leach & B 02AOL 024014302 UDS 2 B & UDS & SWRI-090324 & 090324-8UDS R1 \\
\hline TI-WTP-PEP-066 & 7.18 .14 & Oxidative-leach & B 02AOL 024014302 UDS 2 D & UDS & SWRI-090324 & 090324-8UDS R1 \\
\hline TI-WTP-PEP-066 & 7.18 .14 & Oxidative-leach & B 02AOL 024014303 DEN 2 & Density Slurry & SWRI-090324 & 090324-9 090324-10 090326-10 DEN slurry and liquid \\
\hline TI-WTP-PEP-066 & 7.18 .14 & Oxidative-leach & B 02AOL 024014304 ICP 0 B & Storage & & \\
\hline TI-WTP-PEP-066 & 7.18 .14 & Oxidative-leach & B 02AOL 024014304 SUP 0 D & Storage & & \\
\hline TI-WTP-PEP-066 & 7.18 .14 & Oxidative-leach & B 02AOL 024014304 ICP 2 D & ICP Supernate & SWRI-090324 & 090324-6 090324-26 090324-27 ICP ICPMS TDS and density \\
\hline TI-WTP-PEP-066 & 7.18 .14 & Oxidative-leach & B 02AOL 024014304 TFI 2 D & $\mathrm{IC}$ & SWRI-090324 & 090324-22IC \\
\hline TI-WTP-PEP-066 & 7.18 .14 & Oxidative-leach & B 02AOL 024014304 TFI 3 D & $\mathrm{OH}$ & ASO_ASR8391 & ASR 8391 OH Rev. 3 \\
\hline TI-WTP-PEP-066 & 7.18 .14 & Oxidative-leach & B 02AOL 024014305 OTR 6 P & Storage & & \\
\hline TI-WTP-PEP-066 & 7.18 .14 & Oxidative-leach & B 02AOL 024014305 OTR $0 \mathrm{~F}$ & Storage & & \\
\hline TI-WTP-PEP-066 & 7.18 .14 & Oxidative-leach & B 02AOL 024024306 UDS 2 B & UDS & SWRI-090324 & 090324-8UDS R1 \\
\hline TI-WTP-PEP-066 & 7.18 .14 & Oxidative-leach & B 02AOL 024024306 UDS 2 D & UDS & SWRI-090324 & 090324-8UDS R1 \\
\hline TI-WTP-PEP-066 & 7.18 .14 & Oxidative-leach & B 02AOL 024024307 DEN 2 & Density Slurry & SWRI-090324 & 090324-9 090324-10 090326-10 DEN slurry and liquid \\
\hline TI-WTP-PEP-066 & 7.18 .14 & Oxidative-leach & B 02AOL 024024308 ICP 0 B & Storage & & \\
\hline TI-WTP-PEP-066 & 7.18 .14 & Oxidative-leach & B 02AOL 024024308 SUP 0 D & Storage & & \\
\hline TI-WTP-PEP-066 & 7.18 .14 & Oxidative-leach & B 02AOL 024024308 ICP 2 D & ICP Supernate & SWRI-090324 & 090324-6 090324-26 090324-27 ICP ICPMS TDS and density \\
\hline TI-WTP-PEP-066 & 7.18 .14 & Oxidative-leach & B 02AOL 024024308 TFI 2 D & IC & SWRI-090324 & 090324-23IC \\
\hline TI-WTP-PEP-066 & 7.18 .14 & Oxidative-leach & B 02AOL 024024308 TFI 3 D & $\mathrm{OH}$ & ASO_ASR8391 & ASR 8391 OH Rev. 3 \\
\hline TI-WTP-PEP-066 & 7.18 .14 & Oxidative-leach & B 02AOL 024024309 OTR 6 P & Storage & & \\
\hline TI-WTP-PEP-066 & 7.18 .14 & Oxidative-leach & B 02AOL 024024309 OTR $0 \mathrm{~F}$ & Storage & & \\
\hline TI-WTP-PEP-066 & 7.18 .14 & Oxidative-leach & B 02AOL 024034310 UDS 2 B & UDS & SWRI-090324 & 090324-8UDS R1 \\
\hline TI-WTP-PEP-066 & 7.18 .14 & Oxidative-leach & B 02AOL 024034310 UDS 2 D & UDS & SWRI-090324 & 090324-8UDS R1 \\
\hline TI-WTP-PEP-066 & 7.18 .14 & Oxidative-leach & B 02AOL 024034311 DEN 2 & Density Slurry & SWRI-090324 & 090324-9 090324-10 090326-10 DEN slurry and liquid \\
\hline TI-WTP-PEP-066 & 7.18 .14 & Oxidative-leach & B 02AOL 024034312 ICP 0 B & Storage & & \\
\hline TI-WTP-PEP-066 & 7.18 .14 & Oxidative-leach & B 02AOL 024034312 SUP 0 D & Storage & & \\
\hline TI-WTP-PEP-066 & 7.18 .14 & Oxidative-leach & B 02AOL 024034312 ICP 2 D & ICP Supernate & SWRI-090324 & 090324-6 090324-26 090324-27 ICP ICPMS TDS and density \\
\hline TI-WTP-PEP-066 & 7.18 .14 & Oxidative-leach & B 02 AOL 024034312 TFI 2 D & $\mathrm{IC}$ & SWRI-090324 & 090324-23IC \\
\hline TI-WTP-PEP-066 & 7.18 .14 & Oxidative-leach & B 02AOL 024034312 TFI 3 D & $\mathrm{OH}$ & ASO_ASR8391 & ASR 8391 OH Rev. 3 \\
\hline TI-WTP-PEP-066 & 7.18 .14 & Oxidative-leach & B 02AOL 024034313 OTR 6 P & Storage & & \\
\hline
\end{tabular}


Integrated Test B Sample List

\begin{tabular}{|c|c|c|c|c|c|c|}
\hline TI \# & TI Step & Step Description & Sample ID & Analysis & File Folder & Final Electronic Report \# \\
\hline TI-WTP-PEP-066 & 7.18 .14 & Oxidative-leach & B 02AOL 024034313 OTR $0 \mathrm{~F}$ & Storage & & \\
\hline TI-WTP-PEP-066 & 7.18 .14 & Oxidative-leach & B 02AOL 024044314 UDS 2 B & UDS & SWRI-090324 & 090324-8UDS R1 \\
\hline TI-WTP-PEP-066 & 7.18 .14 & Oxidative-leach & B 02AOL 024044314 UDS 2 D & UDS & SWRI-090324 & 090324-8UDS R1 \\
\hline TI-WTP-PEP-066 & 7.18 .14 & Oxidative-leach & B 02AOL 024044315 DEN 2 & Density Slurry & SWRI-090324 & 090324-9 090324-10 090326-10 DEN slurry and liquid \\
\hline TI-WTP-PEP-066 & 7.18 .14 & Oxidative-leach & B 02AOL 024044316 ICP 0 B & Storage & & \\
\hline TI-WTP-PEP-066 & 7.18 .14 & Oxidative-leach & B 02AOL 024044316 SUP 0 D & Storage & & \\
\hline TI-WTP-PEP-066 & 7.18 .14 & Oxidative-leach & B $02 \mathrm{AOL} 024044316$ ICP 2 D & ICP Supernate & SWRI-090324 & 090324-6 090324-26 090324-27 ICP ICPMS TDS and density \\
\hline TI-WTP-PEP-066 & 7.18 .14 & Oxidative-leach & B 02AOL 024044316 TFI 2 D & IC & SWRI-090324 & 090324-22IC \\
\hline TI-WTP-PEP-066 & 7.18 .14 & Oxidative-leach & B 02AOL 024044316 TFI 3 D & $\mathrm{OH}$ & ASO_ASR8391 & ASR 8391 OH Rev. 3 \\
\hline TI-WTP-PEP-066 & 7.18 .14 & Oxidative-leach & B 02AOL 024044317 OTR 6 P & Storage & & \\
\hline TI-WTP-PEP-066 & 7.18 .14 & Oxidative-leach & B 02AOL 024044317 OTR $0 \mathrm{~F}$ & Storage & & \\
\hline TI-WTP-PEP-066 & 7.18 .14 & Oxidative-leach & B 02 AOL 024054318 UDS 2 B & UDS & SWRI-090324 & 090324-8UDS R1 \\
\hline TI-WTP-PEP-066 & 7.18 .14 & Oxidative-leach & B 02AOL 024054318 UDS 2 D & UDS & SWRI-090324 & 090324-8UDS R1 \\
\hline TI-WTP-PEP-066 & 7.18 .14 & Oxidative-leach & B 02AOL 024054319 DEN 2 & Density Slurry & SWRI-090324 & 090324-9 090324-10 090326-10 DEN slurry and liquid \\
\hline TI-WTP-PEP-066 & 7.18 .14 & Oxidative-leach & B 02AOL 024054320 ICP 0 B & Storage & & \\
\hline TI-WTP-PEP-066 & 7.18 .14 & Oxidative-leach & B 02AOL 024054320 SUP 0 D & Storage & & \\
\hline TI-WTP-PEP-066 & 7.18 .14 & Oxidative-leach & B 02AOL 024054320 ICP 2 D & ICP Supernate & SWRI-090324 & 090324-6 090324-26 090324-27 ICP ICPMS TDS and density \\
\hline TI-WTP-PEP-066 & 7.18 .14 & Oxidative-leach & B $02 \mathrm{AOL} 024054320$ TFI $2 \mathrm{D}$ & IC & SWRI-090324 & $090324-23 I C$ \\
\hline TI-WTP-PEP-066 & 7.18 .14 & Oxidative-leach & B $02 \mathrm{AOL} 024054320$ TFI $3 \mathrm{D}$ & $\mathrm{OH}$ & ASO_ASR8391 & ASR 8391 OH Rev. 3 \\
\hline TI-WTP-PEP-066 & 7.18 .14 & Oxidative-leach & B $02 \mathrm{AOL} 024054321$ OTR 6 P & Storage & & \\
\hline TI-WTP-PEP-066 & 7.18 .14 & Oxidative-leach & B 02AOL 024054321 OTR $0 \mathrm{~F}$ & Storage & & \\
\hline TI-WTP-PEP-066 & 7.18 .14 & Oxidative-leach & B 02AOL 024064322 UDS 2 B & UDS & SWRI-090324 & 090324-8UDS R1 \\
\hline TI-WTP-PEP-066 & 7.18 .14 & Oxidative-leach & B 02 AOL 024064322 UDS 2 D & UDS & SWRI-090324 & 090324-8UDS R1 \\
\hline TI-WTP-PEP-066 & 7.18 .14 & Oxidative-leach & B 02AOL 024064323 UDS 2 B & UDS & SWRI-090324 & 090324-8UDS R1 \\
\hline TI-WTP-PEP-066 & 7.18 .14 & Oxidative-leach & B 02AOL 024064323 UDS 2 D & UDS & SWRI-090324 & 090324-8UDS R1 \\
\hline TI-WTP-PEP-066 & 7.18 .14 & Oxidative-leach & B 02AOL 024064324 UDS 2 B & UDS & SWRI-090324 & 090324-8UDS R1 \\
\hline TI-WTP-PEP-066 & 7.18 .14 & Oxidative-leach & B 02AOL 024064324 UDS 2 D & UDS & SWRI-090324 & 090324-8UDS R1 \\
\hline TI-WTP-PEP-066 & 7.18 .14 & Oxidative-leach & B 02AOL 024064325 DEN 2 & Density Slurry & SWRI-090324 & 090324-9 090324-10 090326-10 DEN slurry and liquid \\
\hline TI-WTP-PEP-066 & 7.18 .14 & Oxidative-leach & B 02AOL 024064326 DEN 2 & Density Slurry & SWRI-090324 & 090324-9 090324-10 090326-10 DEN slurry and liquid \\
\hline TI-WTP-PEP-066 & 7.18 .14 & Oxidative-leach & B 02AOL 024064327 DEN 2 & Density Slurry & SWRI-090324 & 090324-9 090324-10 090326-10 DEN slurry and liquid \\
\hline TI-WTP-PEP-066 & 7.18 .14 & Oxidative-leach & B 02AOL 024064328 RHE 4 & Rheology & APEL Rheology & TDP WTP 363 Int Test B RHE Data on PEP slurry \\
\hline TI-WTP-PEP-066 & 7.18 .14 & Oxidative-leach & B 02AOL 024064329 ICP $2 \mathrm{~S}$ & ICP Rinsed Solids & SWRI-090324 & 090324-28 ICP and ICPMS \\
\hline TI-WTP-PEP-066 & 7.18 .14 & Oxidative-leach & B 02AOL 024064329 DEN 0 D & Storage & & \\
\hline
\end{tabular}


Integrated Test B Sample List

\begin{tabular}{|c|c|c|c|c|c|c|}
\hline TI \# & TI Step & Step Description & Sample ID & Analysis & File Folder & Final Electronic Report \# \\
\hline TI-WTP-PEP-066 & 7.18 .14 & Oxidative-leach & B 02AOL 024064329 DEN 0 R & Storage & & \\
\hline TI-WTP-PEP-066 & 7.18 .14 & Oxidative-leach & B 02AOL 024064329 ICP 2 D & ICP Supernate & SWRI-090324 & 090324-6 090324-26 090324-27 ICP ICPMS TDS and density \\
\hline TI-WTP-PEP-066 & 7.18 .14 & Oxidative-leach & B 02AOL 024064329 ICP 2 R & ICP Rinsate & SWRI-090324 & 090324-6 090324-26 090324-27 ICP ICPMS TDS and density \\
\hline TI-WTP-PEP-066 & 7.18 .14 & Oxidative-leach & B 02AOL 024064329 TFI 2 D & IC & SWRI-090324 & 090324-24 IC \\
\hline TI-WTP-PEP-066 & 7.18 .14 & Oxidative-leach & B 02AOL 024064329 TFI 3 D & $\mathrm{OH}$ & ASO_ASR8391 & ASR 8391 OH Rev. 3 \\
\hline TI-WTP-PEP-066 & 7.18 .14 & Oxidative-leach & B 02AOL 024064329 RAM 3 D & Raman & ASO_ASR8392 & ASR 8392 RAM \\
\hline TI-WTP-PEP-066 & 7.18 .14 & Oxidative-leach & B 02AOL 024064329 RAM 3 R & Raman & ASO_ASR8392 & ASR 8392 RAM \\
\hline TI-WTP-PEP-066 & 7.18 .14 & Oxidative-leach & B 02AOL 024064330 OTR 6 P & Storage & & \\
\hline TI-WTP-PEP-066 & 7.18 .14 & Oxidative-leach & B 02AOL 024064330 OTR $0 \mathrm{~F}$ & Storage & & \\
\hline TI-WTP-PEP-066 & 7.18 .14 & Oxidative-leach & B 02 AOL 024064331 ICP $2 \mathrm{~S}$ & Sample broke & & \\
\hline TI-WTP-PEP-066 & 7.18 .14 & Oxidative-leach & B 02AOL 024064331 DEN 0 D & Storage & & \\
\hline TI-WTP-PEP-066 & 7.18 .14 & Oxidative-leach & B 02AOL 024064331 DEN 0 R & Storage & & \\
\hline TI-WTP-PEP-066 & 7.18 .14 & Oxidative-leach & B 02AOL 024064331 ICP 2 D & ICP Supernate & SWRI-090324 & 090324-6 090324-26 090324-27 ICP ICPMS TDS and density \\
\hline TI-WTP-PEP-066 & 7.18 .14 & Oxidative-leach & B 02AOL 024064331 TFI 2 D & IC & SWRI-090324 & 090324-24 IC \\
\hline TI-WTP-PEP-066 & 7.18 .14 & Oxidative-leach & B $02 \mathrm{AOL} 024064331$ TFI $3 \mathrm{D}$ & $\mathrm{OH}$ & ASO_ASR8391 & ASR 8391 OH Rev. 3 \\
\hline TI-WTP-PEP-066 & 7.18 .14 & Oxidative-leach & B 02AOL 024064331 RAM 3 D & Raman & ASO_ASR8392 & ASR 8392 RAM \\
\hline TI-WTP-PEP-066 & 7.18 .14 & Oxidative-leach & B 02AOL 024064332 OTR 6 P & Storage & & \\
\hline TI-WTP-PEP-066 & 7.18 .14 & Oxidative-leach & B $02 A O L ~ 024064332$ OTR $0 \mathrm{~F}$ & Storage & & \\
\hline TI-WTP-PEP-066 & 7.18 .14 & Oxidative-leach & B $02 \mathrm{AOL} 024064333$ ICP $2 \mathrm{~S}$ & ICP Rinsed Solids & SWRI-090324 & 090324-28 ICP and ICPMS \\
\hline TI-WTP-PEP-066 & 7.18 .14 & Oxidative-leach & B 02AOL 024064333 DEN 0 D & Storage & & \\
\hline TI-WTP-PEP-066 & 7.18 .14 & Oxidative-leach & B 02AOL 024064333 DEN 0 R & Storage & & \\
\hline TI-WTP-PEP-066 & 7.18 .14 & Oxidative-leach & B 02AOL 024064333 ICP 2 D & ICP Supernate & SWRI-090324 & 090324-6 090324-26 090324-27 ICP ICPMS TDS and density \\
\hline TI-WTP-PEP-066 & 7.18 .14 & Oxidative-leach & B 02 AOL 024064333 ICP 2 R & ICP Rinsate & SWRI-090324 & 090324-6 090324-26 090324-27 ICP ICPMS TDS and density \\
\hline TI-WTP-PEP-066 & 7.18 .14 & Oxidative-leach & B 02AOL 024064333 TFI 2 D & IC & SWRI-090324 & 090324-24 IC \\
\hline TI-WTP-PEP-066 & 7.18 .14 & Oxidative-leach & B 02AOL 024064333 TFI 3 D & $\mathrm{OH}$ & ASO_ASR8391 & ASR 8391 OH Rev. 3 \\
\hline TI-WTP-PEP-066 & 7.18 .14 & Oxidative-leach & B 02AOL 024064333 RAM 3 D & Raman & ASO_ASR8392 & ASR 8392 RAM \\
\hline TI-WTP-PEP-066 & 7.18 .14 & Oxidative-leach & B 02AOL 024064333 RAM 3 R & Raman & ASO_ASR8392 & ASR 8392 RAM \\
\hline TI-WTP-PEP-066 & 7.18 .14 & Oxidative-leach & B 02AOL 024064335 AFA 2 B & ICP Wet Solids & SWRI-090324 & 090324-21 ICP and ICPMS \\
\hline TI-WTP-PEP-066 & 7.18 .14 & Oxidative-leach & B 02AOL 024064335 AFA 2 D & ICP Supernate & SWRI-090324 & 090324-21 ICP and ICPMS \\
\hline TI-WTP-PEP-066 & 7.18 .14 & Oxidative-leach & B $02 \mathrm{AOL} 024064336$ AFA 5 B & THF Wet Solids & DOW & PNNL 49554 Report.pdf \\
\hline TI-WTP-PEP-066 & 7.18 .14 & Oxidative-leach & B 02AOL 024064336 AFA 5 D & THF Supernate & DOW & PNNL 49554 Report.pdf \\
\hline TI-WTP-PEP-066 & 7.18 .14 & Oxidative-leach & В 02AOL 024064337 AFA 5 B & Toluene Wet Solids & DOW & PNNL 49554 Report.pdf \\
\hline TI-WTP-PEP-066 & 7.18 .14 & Oxidative-leach & B 02AOL 024064337 AFA 5 D & Toluene Supernate & DOW & PNNL 49554 Report.pdf \\
\hline
\end{tabular}


Integrated Test B Sample List

\begin{tabular}{|c|c|c|c|c|c|c|}
\hline TI \# & TI Step & Step Description & Sample ID & Analysis & File Folder & Final Electronic Report \# \\
\hline TI-WTP-PEP-066 & 7.19 .6 & Post-oxidative-leach wash & B 02AOL 025014338 UDS 2 & UDS & SWRI-090324 & 090324-7UDS R1 \\
\hline TI-WTP-PEP-066 & 7.19 .6 & Post-oxidative-leach wash & B 02AOL 025014339 DEN 0 B & Storage & & \\
\hline TI-WTP-PEP-066 & 7.19 .6 & Post-oxidative-leach wash & B 02AOL 025014339 DEN 0 D & Storage & & \\
\hline TI-WTP-PEP-066 & 7.19 .6 & Post-oxidative-leach slurry wash & B 02AOL 025014339 ICP 2 D & Density Supernate & SWRI-090324 & 090324-6 090324-26 090324-27 ICP ICPMS TDS and density \\
\hline TI-WTP-PEP-066 & 7.19 .6 & Post-oxidative-leach slurry wash & B 02AOL 025014339 ICP 2 D & ICP Supernate & SWRI-090324 & 090324-6 090324-26 090324-27 ICP ICPMS TDS and density \\
\hline TI-WTP-PEP-066 & 7.19 .6 & Post-oxidative-leach slurry wash & B 02AOL 025014339 TFI 2 D & IC & SWRI-090324 & 090324-23IC \\
\hline TI-WTP-PEP-066 & 7.19 .6 & Post-oxidative-leach slurry wash & B 02AOL 025014339 TFI 3 D & $\mathrm{OH}$ & ASO_ASR8391 & ASR 8391 OH Rev. 3 \\
\hline TI-WTP-PEP-066 & 7.19.6 & Post-oxidative-leach slurry wash & B 02AOL 025014339 RAM 3 D & Raman & ASO_ASR8392 & ASR 8392 RAM \\
\hline TI-WTP-PEP-066 & 7.19 .6 & Post-oxidative-leach slurry wash & B $02 \mathrm{AOL} 025024340$ ICP $0 \mathrm{~B}$ & Storage & & \\
\hline TI-WTP-PEP-066 & 7.19 .6 & Post-oxidative-leach slurry wash & B 02AOL 025024340 SUP 0 D & Storage & & \\
\hline TI-WTP-PEP-066 & 7.19 .6 & Post-oxidative-leach slurry wash & B 02AOL 025024340 ICP 2 D & TDS & SWRI-090324 & 090324-6 090324-26 090324-27 ICP ICPMS TDS and density \\
\hline TI-WTP-PEP-066 & 7.19 .6 & Post-oxidative-leach slurry wash & B 02AOL 025024340 ICP 2 D & ICP Supernate & SWRI-090324 & 090324-6 090324-26 090324-27 ICP ICPMS TDS and density \\
\hline TI-WTP-PEP-066 & 7.19 .6 & Post-oxidative-leach slurry wash & B $02 \mathrm{AOL} 025024340$ TFI $2 \mathrm{D}$ & IC & SWRI-090324 & 090324-23IC \\
\hline TI-WTP-PEP-066 & 7.19 .6 & Post-oxidative-leach slurry wash & B 02AOL 025024340 TFI 3 D & $\mathrm{OH}$ & ASO_ASR8391 & ASR 8391 OH Rev. 3 \\
\hline TI-WTP-PEP-066 & 7.19 .6 & Post-oxidative-leach slurry wash & B $02 A O L 025034341$ UDS 2 & UDS & SWRI-090324 & 090324-7UDS R1 \\
\hline TI-WTP-PEP-066 & 7.19 .6 & Post-oxidative-leach slurry wash & B $02 \mathrm{AOL} 025034342$ ICP $0 \mathrm{~B}$ & Storage & & \\
\hline TI-WTP-PEP-066 & 7.19 .6 & Post-oxidative-leach slurry wash & B 02AOL 025034342 SUP 0 D & Storage & & \\
\hline TI-WTP-PEP-066 & 7.19 .6 & Post-oxidative-leach slurry wash & B 02AOL 025034342 ICP 2 D & Density Supernate & SWRI-090324 & 090324-6 090324-26 090324-27 ICP ICPMS TDS and density \\
\hline TI-WTP-PEP-066 & 7.19 .6 & Post-oxidative-leach slurry wash & B $02 \mathrm{AOL} 025034342$ ICP 2 D & ICP Supernate & SWRI-090324 & 090324-6 090324-26 090324-27 ICP ICPMS TDS and density \\
\hline TI-WTP-PEP-066 & 7.19 .6 & Post-oxidative-leach slurry wash & B 02 AOL 025034342 TFI 2 D & $\mathrm{IC}$ & SWRI-090324 & 090324-23IC \\
\hline TI-WTP-PEP-066 & 7.19.6 & Post-oxidative-leach slurry wash & B 02 AOL 025034342 TFI 3 D & $\mathrm{OH}$ & ASO_ASR8391 & ASR 8391 OH Rev. 3 \\
\hline TI-WTP-PEP-066 & 7.19 .6 & Post-oxidative-leach slurry wash & B $02 \mathrm{AOL} 025044343$ ICP $0 \mathrm{~B}$ & Storage & & \\
\hline TI-WTP-PEP-066 & 7.19 .6 & Post-oxidative-leach slurry wash & B 02AOL 025044343 SUP 0 D & Storage & & \\
\hline TI-WTP-PEP-066 & 7.19 .6 & Post-oxidative-leach slurry wash & B $02 A O L ~ 025044343$ ICP 2 D & TDS & SWRI-090324 & 090324-6 090324-26 090324-27 ICP ICPMS TDS and density \\
\hline TI-WTP-PEP-066 & 7.19.6 & Post-oxidative-leach slurry wash & B $02 \mathrm{AOL} 025044343$ ICP $2 \mathrm{D}$ & ICP Supernate & SWRI-090324 & 090324-6 090324-26 090324-27 ICP ICPMS TDS and density \\
\hline TI-WTP-PEP-066 & 7.19 .6 & Post-oxidative-leach slurry wash & B $02 \mathrm{AOL} 025044343$ TFI 2 D & IC & SWRI-090324 & 090324-23IC \\
\hline TI-WTP-PEP-066 & 7.19 .6 & Post-oxidative-leach slurry wash & B 02AOL 025044343 TFI 3 D & $\mathrm{OH}$ & ASO_ASR8391 & ASR 8391 OH Rev. 3 \\
\hline TI-WTP-PEP-066 & 7.19 .6 & Post-oxidative-leach slurry wash & B 02AOL 025054344 UDS 2 & UDS & SWRI-090324 & 090324-7UDS R1 \\
\hline TI-WTP-PEP-066 & 7.19 .6 & Post-oxidative-leach slurry wash & B $02 \mathrm{AOL} 025054345$ ICP $0 \mathrm{~B}$ & Storage & & \\
\hline TI-WTP-PEP-066 & 7.19.6 & Post-oxidative-leach slurry wash & B 02AOL 025054345 SUP 0 D & Storage & & \\
\hline TI-WTP-PEP-066 & 7.19 .6 & Post-oxidative-leach slurry wash & B $02 \mathrm{AOL} 025054345$ ICP 2 D & Density Supernate & SWRI-090324 & 090324-6 090324-26 090324-27 ICP ICPMS TDS and density \\
\hline TI-WTP-PEP-066 & 7.19 .6 & Post-oxidative-leach slurry wash & B $02 \mathrm{AOL} 025054345$ ICP 2 D & ICP Supernate & SWRI-090324 & 090324-6 090324-26 090324-27 ICP ICPMS TDS and density \\
\hline TI-WTP-PEP-066 & 7.19.6 & Post-oxidative-leach slurry wash & B $02 \mathrm{AOL} 025054345$ TFI $2 \mathrm{D}$ & IC & SWRI-090324 & 090324-23IC \\
\hline
\end{tabular}


Integrated Test B Sample List

\begin{tabular}{|c|c|c|c|c|c|c|}
\hline TI \# & TI Step & Step Description & Sample ID & Analysis & File Folder & Final Electronic Report \# \\
\hline TI-WTP-PEP-066 & 7.19 .6 & Post-oxidative-leach slurry wash & B 02 AOL 025054345 TFI 3 D & $\mathrm{OH}$ & ASO_ASR8391 & ASR 8391 OH Rev. 3 \\
\hline TI-WTP-PEP-066 & 7.19 .6 & Post-oxidative-leach slurry wash & B $02 \mathrm{AOL} 025064346$ ICP 0 B & Storage & & \\
\hline TI-WTP-PEP-066 & 7.19 .6 & Post-oxidative-leach slurry wash & B 02AOL 025064346 SUP 0 D & Storage & & \\
\hline TI-WTP-PEP-066 & 7.19 .6 & Post-oxidative-leach slurry wash & B 02 AOL 025064346 ICP 2 D & TDS & SWRI-090324 & 090324-6 090324-26 090324-27 ICP ICPMS TDS and density \\
\hline TI-WTP-PEP-066 & 7.19 .6 & Post-oxidative-leach slurry wash & B 02AOL 025064346 ICP 2 D & ICP Supernate & SWRI-090324 & 090324-6 090324-26 090324-27 ICP ICPMS TDS and density \\
\hline TI-WTP-PEP-066 & 7.19 .6 & Post-oxidative-leach slurry wash & B $02 \mathrm{AOL} 025064346$ TFI 2 D & IC & SWRI-090324 & 090324-23IC \\
\hline TI-WTP-PEP-066 & 7.19 .6 & Post-oxidative-leach slurry wash & B $02 \mathrm{AOL} 025064346$ TFI $3 \mathrm{D}$ & $\mathrm{OH}$ & ASO_ASR8391 & ASR 8391 OH Rev. 3 \\
\hline TI-WTP-PEP-066 & 7.19.6 & Post-oxidative-leach slurry wash & B 02AOL 025074347 DEN 2 & Density Slurry & SWRI-090324 & 090324-9 090324-10 090326-10 DEN slurry and liquid \\
\hline TI-WTP-PEP-066 & 7.19.6 & Post-oxidative-leach slurry wash & B 02AOL 025074348 RHE 4 & Rheology & APEL Rheology & TDP WTP 363 Int Test B RHE Data on PEP slurry \\
\hline TI-WTP-PEP-066 & 7.19 .6 & Post-oxidative-leach slurry wash & B 02AOL 025074349 RHE 4 & Rheology & APEL Rheology & TDP WTP 363 Int Test B RHE Data on PEP slurry \\
\hline TI-WTP-PEP-066 & 7.19 .6 & Post-oxidative-leach slurry wash & B 02AOL 025074350 DEN 0 B & Storage & & \\
\hline TI-WTP-PEP-066 & 7.19 .6 & Post-oxidative-leach slurry wash & B 02AOL 025074350 DEN 0 D & Storage & & \\
\hline TI-WTP-PEP-066 & 7.19 .6 & Post-oxidative-leach slurry wash & B 02AOL 025074350 ICP 2 D & TDS & SWRI-090324 & 090324-6 090324-26 090324-27 ICP ICPMS TDS and density \\
\hline TI-WTP-PEP-066 & 7.19 .6 & Post-oxidative-leach slurry wash & B 02AOL 025074350 ICP 2 D & ICP Supernate & SWRI-090324 & 090324-6 090324-26 090324-27 ICP ICPMS TDS and density \\
\hline TI-WTP-PEP-066 & 7.19 .6 & Post-oxidative-leach slurry wash & B $02 \mathrm{AOL} 025074350$ TFI $2 \mathrm{D}$ & IC & SWRI-090324 & 090324-23IC \\
\hline TI-WTP-PEP-066 & 7.19 .6 & Post-oxidative-leach slurry wash & B $02 \mathrm{AOL} 025074350$ TFI $3 \mathrm{D}$ & $\mathrm{OH}$ & ASO_ASR8391 & ASR 8391 OH Rev. 3 \\
\hline TI-WTP-PEP-066 & 7.19 .6 & Post-oxidative-leach slurry wash & B 02AOL 025074350 RAM 3 D & Raman & ASO_ASR8392 & ASR 8392 RAM \\
\hline TI-WTP-PEP-066 & 7.19 .6 & Post-oxidative-leach slurry wash & B $02 \mathrm{AOL} 025084351$ ICP 0 B & Storage & & \\
\hline TI-WTP-PEP-066 & 7.19.6 & Post-oxidative-leach slurry wash & B $02 A O L ~ 025084351$ SUP 0 D & Storage & & \\
\hline TI-WTP-PEP-066 & 7.19.6 & Post-oxidative-leach slurry wash & B 02AOL 025084351 ICP 2 D & TDS & SWRI-090324 & 090324-6 090324-26 090324-27 ICP ICPMS TDS and density \\
\hline TI-WTP-PEP-066 & 7.19 .6 & Post-oxidative-leach slurry wash & B 02AOL 025084351 ICP 2 D & ICP Supernate & SWRI-090324 & 090324-6 090324-26 090324-27 ICP ICPMS TDS and density \\
\hline TI-WTP-PEP-066 & 7.19 .6 & Post-oxidative-leach slurry wash & B $02 \mathrm{AOL} 025084351$ TFI $2 \mathrm{D}$ & IC & SWRI-090324 & 090324-23IC \\
\hline TI-WTP-PEP-066 & 7.19 .6 & Post-oxidative-leach slurry wash & B $02 \mathrm{AOL} 025084351$ TFI $3 \mathrm{D}$ & $\mathrm{OH}$ & ASO_ASR8391 & ASR 8391 OH Rev. 3 \\
\hline TI-WTP-PEP-066 & 7.19 .6 & Post-oxidative-leach slurry wash & B 02AOL 025094352 UDS 2 & UDS & SWRI-090324 & 090324-7UDS R1 \\
\hline TI-WTP-PEP-066 & 7.19 .6 & Post-oxidative-leach slurry wash & B $02 \mathrm{AOL} 025094353$ ICP $0 \mathrm{~B}$ & Storage & & \\
\hline TI-WTP-PEP-066 & 7.19 .6 & Post-oxidative-leach slurry wash & B 02AOL 025094353 SUP 0 D & Storage & & \\
\hline TI-WTP-PEP-066 & 7.19 .6 & Post-oxidative-leach slurry wash & B 02AOL 025094353 ICP 2 D & Density Supernate & SWRI-090324 & 090324-6 090324-26 090324-27 ICP ICPMS TDS and density \\
\hline TI-WTP-PEP-066 & 7.19 .6 & Post-oxidative-leach slurry wash & B $02 \mathrm{AOL} 025094353$ ICP 2 D & ICP Supernate & SWRI-090324 & 090324-6 090324-26 090324-27 ICP ICPMS TDS and density \\
\hline TI-WTP-PEP-066 & 7.19.6 & Post-oxidative-leach slurry wash & B 02AOL 025094353 TFI 2 D & IC & SWRI-090324 & 090324-23IC \\
\hline TI-WTP-PEP-066 & 7.19 .6 & Post-oxidative-leach slurry wash & B $02 A O L 025094353$ TFI 3 D & $\mathrm{OH}$ & ASO_ASR8391 & ASR 8391 OH Rev. 3 \\
\hline TI-WTP-PEP-066 & 7.19 .6 & Post-oxidative-leach slurry wash & B 02AOL 025104354 ICP 0 B & Storage & & \\
\hline TI-WTP-PEP-066 & 7.19 .6 & Post-oxidative-leach slurry wash & B 02AOL 025104354 SUP 0 D & Storage & & \\
\hline TI-WTP-PEP-066 & 7.19 .6 & Post-oxidative-leach slurry wash & B 02AOL 025104354 ICP 2 D & TDS & SWRI-090324 & 090324-6 090324-26 090324-27 ICP ICPMS TDS and density \\
\hline
\end{tabular}


Integrated Test B Sample List

\begin{tabular}{|c|c|c|c|c|c|c|}
\hline TI\# & TI Step & Step Description & Sample ID & Analysis & File Folder & Final Electronic Report \# \\
\hline TI-WTP-PEP-066 & 7.19 .6 & Post-oxidative-leach slurry wash & B 02AOL 025104354 ICP 2 D & ICP Supernate & SWRI-090324 & 090324-6 090324-26 090324-27 ICP ICPMS TDS and density \\
\hline TI-WTP-PEP-066 & 7.19 .6 & Post-oxidative-leach slurry wash & B 02AOL 025104354 TFI 2 D & IC & SWRI-090324 & 090324-23IC \\
\hline TI-WTP-PEP-066 & 7.19 .6 & Post-oxidative-leach slurry wash & B 02 AOL 025104354 TFI 3 D & $\mathrm{OH}$ & ASO_ASR8391 & ASR 8391 OH Rev. 3 \\
\hline TI-WTP-PEP-066 & 7.19 .6 & Post-oxidative-leach slurry wash & B 02AOL 025114355 UDS 2 & UDS & SWRI-090324 & 090324-7UDS R1 \\
\hline TI-WTP-PEP-066 & 7.19 .6 & Post-oxidative-leach slurry wash & B $02 \mathrm{AOL} 025114356$ ICP $0 \mathrm{~B}$ & Storage & & \\
\hline TI-WTP-PEP-066 & 7.19 .6 & Post-oxidative-leach slurry wash & B 02AOL 025114356 SUP 0 D & Storage & & \\
\hline TI-WTP-PEP-066 & 7.19 .6 & Post-oxidative-leach slurry wash & B 02AOL 025114356 ICP 2 D & Density Supernate & SWRI-090324 & 090324-6 090324-26 090324-27 ICP ICPMS TDS and density \\
\hline TI-WTP-PEP-066 & 7.19 .6 & Post-oxidative-leach slurry wash & B 02AOL 025114356 ICP 2 D & ICP Supernate & SWRI-090324 & 090324-6 090324-26 090324-27 ICP ICPMS TDS and density \\
\hline TI-WTP-PEP-066 & 7.19 .6 & Post-oxidative-leach slurry wash & B 02AOL 025114356 TFI 2 D & IC & SWRI-090324 & 090324-23IC \\
\hline TI-WTP-PEP-066 & 7.19 .6 & Post-oxidative-leach slurry wash & B 02AOL 025114356 TFI 3 D & $\mathrm{OH}$ & ASO_ASR8391 & ASR 8391 OH Rev. 3 \\
\hline TI-WTP-PEP-066 & 7.19 .6 & Post-oxidative-leach slurry wash & B $02 \mathrm{AOL} 025124357$ ICP $0 \mathrm{~B}$ & Storage & & \\
\hline TI-WTP-PEP-066 & 7.19 .6 & Post-oxidative-leach slurry wash & B 02AOL 025124357 SUP 0 D & Storage & & \\
\hline TI-WTP-PEP-066 & 7.19 .6 & Post-oxidative-leach slurry wash & B 02AOL 025124357 ICP 2 D & TDS & SWRI-090324 & 090324-6 090324-26 090324-27 ICP ICPMS TDS and density \\
\hline TI-WTP-PEP-066 & 7.19 .6 & Post-oxidative-leach slurry wash & B 02AOL 025124357 ICP 2 D & ICP Supernate & SWRI-090324 & 090324-6 090324-26 090324-27 ICP ICPMS TDS and density \\
\hline TI-WTP-PEP-066 & 7.19 .6 & Post-oxidative-leach slurry wash & B $02 \mathrm{AOL} 025124357$ TFI 2 D & IC & SWRI-090324 & 090324-24 IC \\
\hline TI-WTP-PEP-066 & 7.19 .6 & Post-oxidative-leach slurry wash & B $02 \mathrm{AOL} 025124357$ TFI $3 \mathrm{D}$ & $\mathrm{OH}$ & ASO_ASR8391 & ASR 8391 OH Rev. 3 \\
\hline TI-WTP-PEP-066 & 7.19 .6 & Post-oxidative-leach slurry wash & B 02AOL 025134358 UDS 2 & UDS & SWRI-090324 & 090324-7UDS R1 \\
\hline TI-WTP-PEP-066 & 7.19.6 & Post-oxidative-leach slurry wash & B 02AOL 025134359 ICP 0 B & Storage & & \\
\hline TI-WTP-PEP-066 & 7.19 .6 & Post-oxidative-leach slurry wash & B 02AOL 025134359 SUP 0 D & Storage & & \\
\hline TI-WTP-PEP-066 & 7.19 .6 & Post-oxidative-leach slurry wash & B 02AOL 025134359 ICP 2 D & Density Supernate & SWRI-090324 & 090324-6 090324-26 090324-27 ICP ICPMS TDS and density \\
\hline TI-WTP-PEP-066 & 7.19.6 & Post-oxidative-leach slurry wash & B 02AOL 025134359 ICP 2 D & ICP Supernate & SWRI-090324 & 090324-6 090324-26 090324-27 ICP ICPMS TDS and density \\
\hline TI-WTP-PEP-066 & 7.19 .6 & Post-oxidative-leach slurry wash & B 02AOL 025134359 TFI 2 D & IC & SWRI-090324 & 090324-24 IC \\
\hline TI-WTP-PEP-066 & 7.19 .6 & Post-oxidative-leach slurry wash & B $02 \mathrm{AOL} 025134359$ TFI $3 \mathrm{D}$ & $\mathrm{OH}$ & ASO_ASR8391 & ASR 8391 OH Rev. 3 \\
\hline TI-WTP-PEP-066 & 7.19 .6 & Post-oxidative-leach slurry wash & B $02 \mathrm{AOL} 025144360$ ICP $0 \mathrm{~B}$ & Storage & & \\
\hline TI-WTP-PEP-066 & 7.19.6 & Post-oxidative-leach slurry wash & B 02AOL 025144360 SUP 0 D & Storage & & \\
\hline TI-WTP-PEP-066 & 7.19 .6 & Post-oxidative-leach slurry wash & B $02 \mathrm{AOL} 025144360 \mathrm{ICP} 2 \mathrm{D}$ & TDS & SWRI-090324 & 090324-6 090324-26 090324-27 ICP ICPMS TDS and density \\
\hline TI-WTP-PEP-066 & 7.19 .6 & Post-oxidative-leach slurry wash & B $02 \mathrm{AOL} 025144360 \mathrm{ICP} 2 \mathrm{D}$ & ICP Supernate & SWRI-090324 & 090324-6 090324-26 090324-27 ICP ICPMS TDS and density \\
\hline TI-WTP-PEP-066 & 7.19 .6 & Post-oxidative-leach slurry wash & B 02AOL 025144360 TFI 2 D & IC & SWRI-090324 & 090324-24 IC \\
\hline TI-WTP-PEP-066 & 7.19 .6 & Post-oxidative-leach wash & B 02AOL 025144360 TFI 3 D & $\mathrm{OH}$ & ASO_ASR8391 & ASR 8391 OH Rev. 3 \\
\hline TI-WTP-PEP-066 & 7.19.7 & Post-oxidative-leach wash & B 02AOL 025 XX 4363 UDS 2 & UDS & SWRI-090324 & 090324-7UDS R1 \\
\hline TI-WTP-PEP-066 & 7.19.7 & Post-oxidative-leach wash & B 02AOL 025 XX 4364 DEN 2 & Density Slurry & SWRI-090324 & 090324-9 090324-10 090326-10 DEN slurry and liquid \\
\hline TI-WTP-PEP-066 & 7.19 .7 & Post-oxidative-leach wash & B 02AOL 025 XX 4364 DEN 2 & Density Supernate & SWRI-090324 & 090324-9 090324-10 090326-10 DEN slurry and liquid \\
\hline TI-WTP-PEP-066 & 7.19.7 & Post oxidative-leach wash & B 02AOL 025 XX 4365 RHE 4 & Rheology & APEL Rheology & TDP WTP 363 Int Test B RHE Data on PEP slurry \\
\hline
\end{tabular}


Integrated Test B Sample List

\begin{tabular}{|c|c|c|c|c|c|c|}
\hline TI \# & TI Step & Step Description & Sample ID & Analysis & File Folder & Final Electronic Report \# \\
\hline TI-WTP-PEP-066 & 7.19.7 & Post oxidative-leach wash & B 02AOL 025 XX 4366 RHE 4 & Rheology & APEL Rheology & TDP WTP 363 Int Test B RHE Data on PEP slurry \\
\hline TI-WTP-PEP-066 & 7.19 .7 & Post oxidative-leach wash & B $02 \mathrm{AOL} 025 \mathrm{XX} 4367$ ICP $0 \mathrm{~B}$ & Storage & & \\
\hline TI-WTP-PEP-066 & 7.19 .7 & Post oxidative-leach wash & B 02AOL 025 XX 4367SUP 0 D & Storage & & \\
\hline TI-WTP-PEP-066 & 7.19.7 & Post oxidative-leach wash & B $02 \mathrm{AOL} 025 \mathrm{XX} 4367$ ICP $2 \mathrm{D}$ & ICP Supernate & SWRI-090324 & 090324-6 090324-26 090324-27 ICP ICPMS TDS and density \\
\hline TI-WTP-PEP-066 & 7.19.7 & Post oxidative-leach wash & В 02AOL 025 XX 4367 TFI 2 D & IC & SWRI-090324 & 090324-24 IC \\
\hline TI-WTP-PEP-066 & 7.19.7 & Post oxidative-leach wash & B 02AOL 025 XX 4367 TFI 3 D & $\mathrm{OH}$ & ASO_ASR8391 & ASR 8391 OH Rev. 3 \\
\hline TI-WTP-PEP-066 & 7.20 .5 & $\begin{array}{l}\text { Collect filter loop in line sample } 5 \text { min after circulating the loop } \\
\text { with flush water and with UFP-VSL-T02 bypassed }\end{array}$ & B 000FL 027 XX 4379 UDS 2 & UDS & SWRI-090324 & 090324-29UDS R1 \\
\hline TI-WTP-PEP-066 & 7.20 .5 & $\begin{array}{l}\text { Collect filter loop in line sample } 5 \text { min after circulating the loop } \\
\text { with flush water and with UFP-VSL-T02 bypassed }\end{array}$ & B 000FL 027 XX 4380 ICP 0 B & Storage & & \\
\hline TI-WTP-PEP-066 & 7.20 .5 & Prior to transfer of slurry from UFP-T02A to UFP-VSL-T62A/B & B 000FL 027 XX 4380 SUP 0 D & Storage & & \\
\hline TI-WTP-PEP-066 & 7.20 .5 & Prior to transfer of slurry from UFP-T02A to UFP-VSL-T62A/B & B 000FL 027 XX 4380 ICP 2 D & ICP Supernate & SWRI-090324 & 090324-31 and 090326-13 ICP and ICPMS \\
\hline TI-WTP-PEP-066 & 7.20 .5 & Prior to transfer of slurry from UFP-T02A to UFP-VSL-T62A/B & B 000FL 027 XX 4380 TFI 2 D & IC & SWRI-090324 & 090324-32 IC \\
\hline TI-WTP-PEP-066 & 7.20 .5 & Prior to transfer of slurry from UFP-T02A to UFP-VSL-T62A/B & B 000FL 027 XX 4380 TFI 3 D & $\mathrm{OH}$ & ASO_ASR8391 & ASR 8391 OH Rev. 3 \\
\hline TI-WTP-PEP-066 & 7.20 .5 & Prior to transfer of slurry from UFP-T02A to UFP-VSL-T62A/B & B 000FL 027 XX 4472 UDS 2 & UDS & $\begin{array}{l}\text { SWRI-090331- } \\
\quad 10\end{array}$ & 090331-10UDS R1 \\
\hline TI-WTP-PEP-066 & 7.20 .5 & Prior to transfer of slurry from UFP-T02A to UFP-VSL-T62A/B & B 000FL 027 XX 4473 ICP 0 B & Storage & & \\
\hline TI-WTP-PEP-066 & 7.20 .5 & Prior to transfer of slurry from UFP-T02A to UFP-VSL-T62A/B & B 000FL 027 XX 4473 SUP 0 D & Storage & & \\
\hline TI-WTP-PEP-066 & 7.20 .5 & Flush filter loop forward after all permeate samples are taken & B 000FL 027 XX 4473 ICP 2 D & ICP Supernate & SWRI-090331 & 090331-12 and 090331-22 and 090331-23 ICP and ICPMS \\
\hline TI-WTP-PEP-066 & 7.20 .5 & Flush filter loop forward after all permeate samples are taken & B 000FL 027 XX 4473 TFI 2 D & IC & SWRI-090331 & 090331-14 IC \\
\hline TI-WTP-PEP-066 & 7.20 .5 & Flush filter loop forward after all permeate samples are taken & B 000FL 027 XX 4473 TFI 3 D & $\mathrm{OH}$ & ASO_ASR8396 & ASR $8396 \mathrm{OH}$ \\
\hline TI-WTP-PEP-066 & 7.20 .5 & Prior to transfer of slurry from UFP-T02A to UFP-VSL-T62A/B & B 02AML 028 XX 4381 UDS 2 & UDS & SWRI-090324 & 090324-29UDS R1 \\
\hline TI-WTP-PEP-066 & 7.20 .5 & Prior to transfer of slurry from UFP-T02A to UFP-VSL-T62A/B & В 02AML 028 XX 4382 ICP 0 B & Storage & & \\
\hline TI-WTP-PEP-066 & 7.20 .5 & Prior to transfer of slurry from UFP-T02A to UFP-VSL-T62A/B & B 02 AML 028 XX 4382 SUP 0 D & Storage & & \\
\hline TI-WTP-PEP-066 & 7.20 .5 & Prior to transfer of slurry from UFP-T02A to UFP-VSL-T62A/B & B 02AML 028 XX 4382 ICP 2 D & ICP Supernate & SWRI-090324 & 090324-31 and 090326-13 ICP and ICPMS \\
\hline TI-WTP-PEP-066 & 7.20 .5 & Prior to transfer of slurry from UFP-T02A to UFP-VSL-T62A/B & B 02AML 028 XX 4382 TFI 2 D & IC & SWRI-090324 & 090324-32 IC \\
\hline TI-WTP-PEP-066 & 7.20 .5 & Prior to transfer of slurry from UFP-T02A to UFP-VSL-T62A/B & B 02AML 028 XX 4382 TFI 3 D & $\mathrm{OH}$ & ASO_ASR8391 & ASR 8391 OH Rev. 3 \\
\hline
\end{tabular}

(1)Pending final report receipt

TI Step \# have been adjusted to allow samples collected in a single sampling event to listed together

"Storage" indicates samples were sent to storage/archive in RTL.

Density slurry - analysis performed on slurry

Density supernate - analysis performed on supernate

Free hydroxide $(\mathrm{OH})$

Inductively coupled plasma supernate (ICP Supernate) ICP performed on the supernate

Inductively coupled plasma wet solids (ICP wet solids) ICP performed on wet solids 
Integrated Test B Sample List

TI \# TI Ste

Step Description Sample ID

Analysis

File Folder

Final Electronic Report \#

Ion chromatography (IC)

Particle size distribution (PSD)

Rheology is a general term used to describe either Shear strength or Shear stress vs. shear rate analysis - actual analytical report specify specific analysis

Scanning electron microscope (SEM)

(

THF Wet Solids, THF Supernate, Toluene Wet Solids, Toluene Supernate are the solvent extraction performed on either the supernate or wet solid prior to analysis by Gel Permeation chromatography (GPC)

Total inorganic carbon and total organic carbon (TIC/TOC)

Weight Percent Undissolved Solids (UDS)

X-ray diffraction (XRD) 
Appendix D

\section{Coriolis Sampler Elevations}





\section{Appendix D: Coriolis Sampler Elevations (Kurath 2008)}

$\begin{array}{llll}\text { Date: } & \mathbf{1 2 / 3 1 / 2 0 0 8} & \text { Project No.: } & <53032> \\ \text { To: } & \text { Dean Kurath } & \text { Internal } & \text { File/LB } \\ \text { From: } & \begin{array}{l}\text { Matt Fountain } \\ \text { Gary Josephson }\end{array} & \text { Distribution: } & \\ & & & \end{array}$

Subject: Coriolis Sampler Elevations

'As installed' elevations of the Coriolis samplers were estimated by bubbling air through each sampler leg and measuring the bubbler pressure on the tank's lower bubbler leg calibrated pressure transmitter.

Method: After recording the pressure of the lowest bubbler leg the bubbler tubing was disconnected from the air supply and the supply attached to the Coriolis sampler stand via the low point drain and connecting Teflon tubing with Swagelock fittings. Air was bubbled through the tubing to each sampler leg and the pressure recorded. In UFP-1A and UFP-1B the PJMs were operating and the "lowest" pressure was recorded, when the PJMs were filled. Levels in UFP-1A, UFP-1B, and UFP-2A were measured on LT-0323, LT-0423, and LT-0611, respectively.

The measured pressures were compared with the measured differences in tubing lengths between the bottom-middle and middle-upper to make the final estimate sample elevations. Measured tubing differences were considered absolute and measured elevations were adjusted as much as an inch so that the offsets matched measured lengths.

Below are listed the "expected" sampler elevations based upon initial design and the results of this study:

\begin{tabular}{|c|c|c|c|c|c|c|}
\hline \multirow{2}{*}{$\begin{array}{l}\text { Vessel } \\
\text { UFP-1A }\end{array}$} & \multicolumn{3}{|c|}{ Initial design elevation } & \multicolumn{3}{|c|}{ "as installed" sample tube elevation } \\
\hline & Low & Middle & High & Low & Middle & High \\
\hline Inner & 2 & 33 & 64 & 11 & 33 & 64 \\
\hline Middle & 11 & 33 & 55 & 9 & 31 & 53 \\
\hline Outer & 11 & 33 & 55 & 9 & 31 & 53 \\
\hline \multicolumn{7}{|l|}{ UFP-1B } \\
\hline Inner & 2 & 33 & 64 & 10 & 32 & 63 \\
\hline Middle & 11 & 33 & 55 & 6 & 28 & 51 \\
\hline Outer & 11 & 33 & 55 & 6 & 51 & 29 \\
\hline \multicolumn{7}{|l|}{ UFP-2A } \\
\hline Inner & 50 & 57 & 64 & 51 & 58 & 65 \\
\hline Middle & 2" off bottom & 33 & 55 & 6 & 34 & 56 \\
\hline Outer & 11 & 33 & 55 & 11 & 33 & 56 \\
\hline
\end{tabular}


Conclusions: The 'as installed' sampler levels agree quite well with the design with the following exceptions:

- The UFP-1B outer/middle and outer/high sampling tubing has been switched. (An NCR is being written and the tubing needs to be corrected and the correction date recorded. Samples taken until then are incorrect.)

- The inner low sampler tubes were cut shorter than originally anticipated. Instead of sampling very close (2") from the bottom of the vessel the sample is 10"-11" off the bottom.

- The middle and outer samplers in UFP-1B were installed about 4"-5" lower than initially intended.

The design of the UFP-2A middle/low position was designed to be 2 inches off the vessel bottom at the middle radial location. With a 2:1 elliptical head the elevation rise for the middle sampling location is estimated to be $\sim 3.5$ " so the measured 6 " elevation from the center tank bottom is actually very close. 
Appendix E

Sample Processing and Analytical Methods 



\section{Appendix E: Sample Processing and Analytical Methods}

\section{E.1 Sample Processing}

The samples were collected in pre-labeled sample containers that were prepared and staged within PDL-W based upon the Sample Collection and Analysis table in the governing Test Instruction. The required analysis determined the sample volume and sample collection container. Sample handling flow diagrams are given in Figure E.1 through Figure E. 3 for samples collected and processed in PDL-W. Figure E.2 is a continuation of Figure E.1 and follows the handling of the wet centrifuged solids. Figure E. 3 indicates the sample flow for samples requiring UDS measurement. The letters "D", "B", "R," and "S," which are shown in the figures, were included in the sample names for phase-separated processed samples. The letter "D" was included in the decanted supernatant sample names. The letter "B" was included in the wet centrifuged solids sample names. The letter "R" was included in the sample name of the combined rinse solution. The letter " $S$ " was included in the sample names of the wet rinsed centrifuged solids. These figures represent two basic sample processing methods. One approach was used during non-leaching test steps, and another was used during leaching.

\section{E.1.1 Non-Leaching Samples}

Non-leaching samples were not always processed immediately after collection. Because sample collection could occur at anytime during testing and testing was being performed 24 hours per day, non-leached samples collected after 0600 hour were typically processed and prepared for shipment by 0600 hour the morning following sample collection. Non-leach samples used for the analyses listed below were shipped as collected in the container size specified and with the exception of the archive samples, tare and full sample weights were not required.

- weight percent undissolved solids (UDS) (50-mL)

- density $(50-\mathrm{mL})$

- heat capacity $(50-\mathrm{mL})$

- $\quad$ shear strength $(2 \times 1-\mathrm{L})$

- shear stress vs. shear rate on original intact slurry $(100-\mathrm{mL})$

- shear stress vs. shear rate on supernatant (100-mL)

- total organic carbon on the slurry $(50-\mathrm{mL})$

- inductively coupled plasma - Silicon $(50-\mathrm{mL})$

- $\operatorname{archive}(50-\mathrm{mL})$.

During a sampling event if an intact slurry sample was collected for density analysis and another intact slurry sample was collected for weight percent UDS, then density measurements were performed on the supernatant in addition to the intact slurry submitted for density analysis. These density measurements were higher accuracy than density measurements obtained following the weight percent 
UDS method described in Bechtel procedure, Guidelines for Performing Chemical, Physical, and Rheological Properties Measurements (24590-WTP-GPG-RTD-001 Rev. 0). ${ }^{\text {(a) }}$

(a) GL Smith and K Prindiville. 2002. Guidelines for Performing Chemical, Physical, and Rheological Properties Measurements. 24590-WTP-GPG-RTD-001 Rev. 0, Bechtel National, Inc., Richland, Washington. 


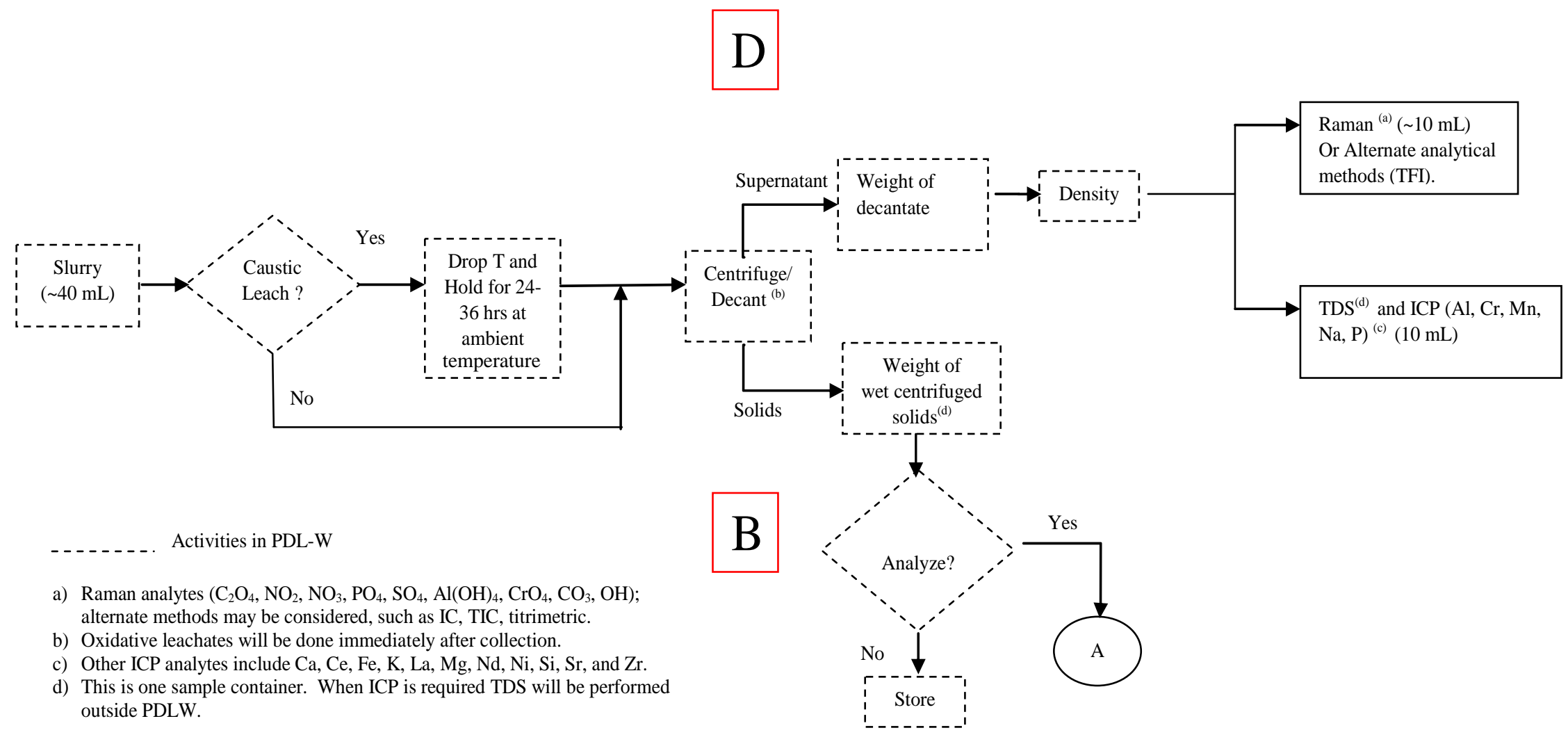

Figure E.1. Sample Flow Diagram 


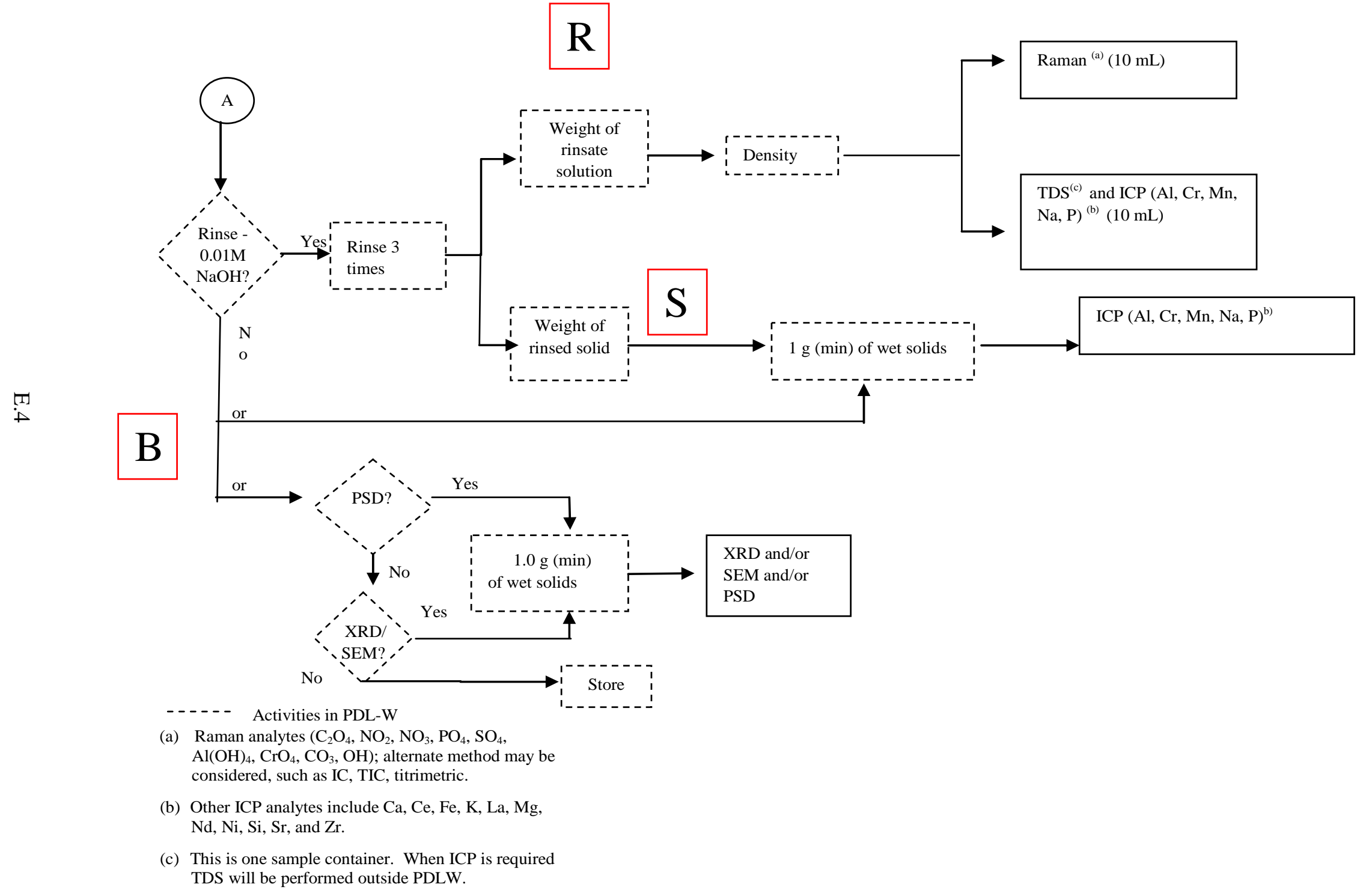

Figure E.2. Sample Flow Diagram (continued) 


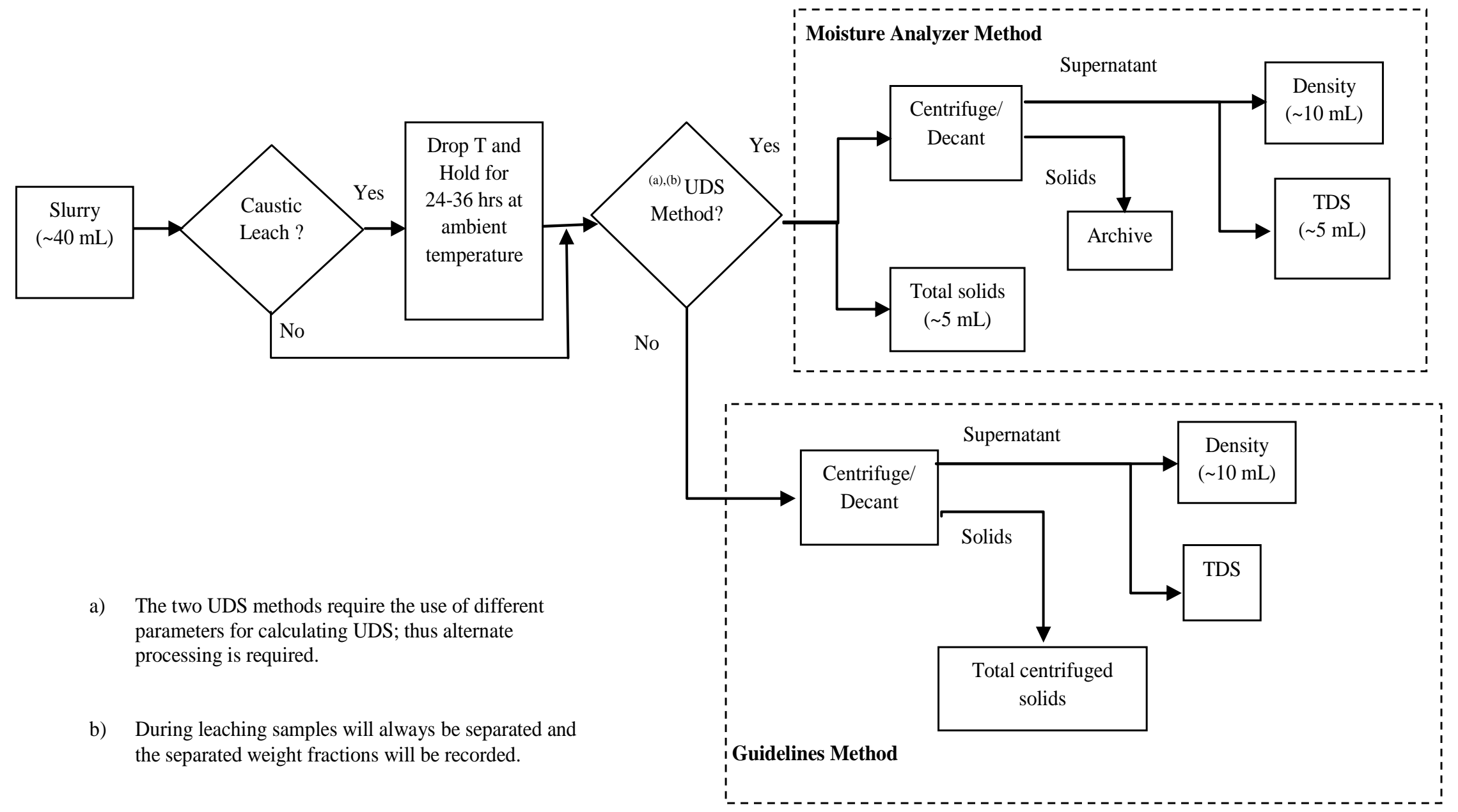

Figure E.3. Sample Flow Diagram for UDS Measurement 
A single 50-mL sample was centrifuged and the supernatant decanted to allow multiple laboratories to perform several analyses simultaneously on the supernatant. To perform this phase separation, the original sample was centrifuged at $\sim 4500 \mathrm{G}$ with a swinging bucket rotor in PDLW. The centrifuging time was initially set for 10 minutes. This centrifuging time was sufficient to cause phase separation during shakedown, Integrated Test A, and oxidative leaching. However, during some of the process steps, phase separation was not achieved after 10 minutes, so the samples were centrifuged for an hour. The actual centrifuging time is recorded on the sample bench sheets. After centrifuging, the supernatant was decanted and submitted for some or all the analyses listed below:

- ICP

- total dissolved solids

- density

- IC

- $\mathrm{TOC}$

- Raman

- free hydroxide.

Not all these analyses were necessarily performed on every decanted supernatant. The required analysis was identified in the Sample Collection and Analysis table in the governing Test Instruction. Density was determined on the decanted supernatant at PDL-W for all samples being analyzed by Raman spectroscopy before the samples were transported for analysis. In addition, at times, density was determined by Southwest Research Institute (SwRI) before ICP analysis. The wet centrifuged solids from this same container were either submitted for ICP analysis, if it was required, or stored in the original sample collection container as excess. A tare weight (before filling) and gross weight (after filling) of the sample container were documented on sample bench sheets.

For Dow Corning to quantify the AFA compounds by gel permeation chromatography (GPC) in the solid and supernatant fractions of the slurry, two 50-mL slurry samples were collected and centrifuged at $4500 \mathrm{G}$ for 10 minutes, and the supernatant was decanted into two additional properly labeled $50-\mathrm{mL}$ containers before shipping. Weighing empty and filled containers was not required.

For SwRI to quantify silicon by ICP in the AFA, a 50-mL slurry sample was collected and shipped as collected during non-leaching process steps. Once at SwRI, the slurry was centrifuged at $2200 \mathrm{G}$ for an hour, the supernatant was separated from the wet centrifuged solids and then each phase was analyzed separately. During leaching, the phase separation occurred in PDL-W following the standard practice of centrifuging at $\sim 4500 \mathrm{G}$ for 10 minutes.

A single 50-mL container was filled for particle-size distribution (PSD), XRD, and SEM. Initially, these samples were centrifuged. The supernatant was decanted and stored while the wet solids were submitted for analysis. Intact slurry samples were submitted after shakedown and Integrated Test A and initial Integrated Test B simulant characterization. If a phase-separated sample was submitted, then descriptor 7 was included in the sample name. If descriptor 7 was not in the name, then an intact sample was submitted for analysis. Weighing empty and filled containers was not required. 
For parallel CUF and bench-scale testing, varying amounts of either permeate or slurry samples were collected. A tare weight (before filling) and gross weight (after filling) of the sample container was recorded on the sample container. These samples were delivered to APEL by the next business day after sample collection.

\section{E.1.2 Leaching Samples}

The core critical analyses for leached samples were weight percent UDS, slurry density, inductively coupled plasma, ion chromatography, Raman and free hydroxide analyses. Two 50-mL samples were collected for the UDS and density analyses. Another 50-mL sample was collected for the remaining core analyses.

- During caustic leaching, the original samples were immediately placed in a thermostatically controlled water bath at $20^{\circ} \pm 2^{\circ} \mathrm{C}$ to decrease the sample temperature to $25 \pm 5^{\circ} \mathrm{C}$ after collection. Once the desired temperature was reached, which typically took less than 10 minutes for $50-\mathrm{mL}$ containers, the samples were allowed to sit for 24 hours in PDL-W. The density sample was shipped as collected. The two other samples to be used for analysis were centrifuged following the sample process described for non-leached samples.

- During oxidative leaching, sample processing began immediately after sample collection. The oxidative leach density sample was shipped as collected while the other two samples were phase-separated immediately after collection by centrifuging and decanting the supernatant as described above for non-leached samples. To allow for consistency in comparing analytical results, the samples for ICP analysis collected immediately before adding $\mathrm{NaMnO}_{4}$ were phase-separated immediately after collection. The oxidative-leached centrifuged solids from samples collected immediately before adding $\mathrm{NaMnO}_{4}$ and at the end of oxidative leaching for analysis were weighed and rinsed three times with $0.01 \mathrm{M} \mathrm{NaOH}$ solution. The actual quantity of rinse is recorded on sample bench sheets. The wet solids were vortexed three times to suspend and rinse the solids after each addition of $\mathrm{NaOH}$ solution. The rinsate was collected and weighed. The density of the rinsate was measured at PDL-W, and then it was split into sub-samples for ICP and Raman analysis.

During leaching, additional 50-mL samples were collected for archiving. Caustic leached archived samples were also cooled and phase-separated as described above before storage. The oxidative leached archived samples were also immediately phase-separated after collection as described above. The centrifuged oxidative leached solids that went to storage were not rinsed.

\section{E.2 Analytical Methods}

This section describes the analytical methods used to determine the chemical composition of the PEP simulant samples collected during testing. Analytical results are provided in Section 5.

\section{E.2.1 Preparation for ICP or ICP-MS}

For liquid samples, aliquots were diluted with hydrochloric acid and then analyzed. Dilutions were made with a representative aliquot of approximately 2 grams combined with $5-\mathrm{mL}$ of $50 \%$ hydrochloric acid, and then diluted to a final volume of $50-\mathrm{mL}$ with DI water. 
For solid samples, two preparative techniques were used. The first preparative technique was lithium metaborate/tetraborate fusion, referred to as prep method "80/20 Fusion." Aluminum, barium, cerium, chromium, iron, lanthanum, manganese, neodymium, silicon, and strontium were reported from the fusion. The second preparative method used concentrated nitric, perchloric, hydrofluoric, and hydrochloric acids in an open vessel and was referred to as the "Teflon" prep method. If residue remained from the "Teflon" method, then the residue was separated, dried, and fused using the lithium metaborate/tetraborate fusion technique. Both the "Teflon" and residue preparations were analyzed. The remaining metals were reported from either the "Teflon" digestion only or the combination of the two. If an analyte was detected in both the Teflon digestate and the residue fusions, then the reported result was the sum of the results obtained from the two preparations. These results were identified as "combined" on Sample Analysis Data Sheets while analytes reported from only the fusion were identified as "80/20 Fusion," and analytes reported solely from the acid digestions were identified as "Teflon" on the Sample Analysis Data Sheets. The preparative QC samples included a sample duplicate, preparation blank (PB), solid laboratory control samples (LCS) consisting of obsidian rock and basalt rock, and an aqueous laboratory control sample (LCS) and a matrix spike (MS), for "Teflon". The results are reported on an "as received" or wet weight basis. The percent solids were determined at $105^{\circ} \mathrm{C}$ and reported on the Sample Analysis Data Sheets for all solids.

\section{E.2.2 Metals Analysis by ICP-AES}

All metals were determined by ICP-AES using SwRI procedure TAP01-0406-130 with the exception of cerium, cesium, lanthanum, and neodymium. The preparative QC samples (duplicate, PB, LCS, MS) were processed along with analytical workstation QC (initial and continuing calibration verifications, initial and continuing blanks, interference check samples and post-digestion spikes).

\section{E.2.3 Metals Analysis by ICP-MS}

Cerium, cesium, lanthanum, and neodymium were determined by ICP-MS in accordance with SwRI procedure TAP01-0406-046. The preparative QC samples (duplicate, PB, LCS, MS) were processed along with analytical workstation QC (initial and continuing calibration verifications, initial and continuing blanks, interference check samples and post-digestion spikes).

\section{E.2.4 Anions (IC)}

Decanted supernatant and rinsants were analyzed by IC for chloride, nitrate, nitrite phosphate, sulfate, and oxalate at SwRI in accordance with procedure SWRI TAP 01-0406-042. Approximately $0.25 \mathrm{~g}$ of the sample was diluted to $50-\mathrm{mL}$ using DIW. Since the dilution was performed by weight, the sample results are reported on a weight basis. The standard reporting by the laboratory is nitrate as N, nitrite as N, and phosphate as P. Shortly after PEP testing began, PNNL requested that results be reported as nitrate, nitrite, and phosphate and not as nitrogen or phosphorus. The IC analytical report narrative identifies conversion factors used to report as anions. QC samples generated at the analytical workstation included a sample replicate determination, preparation blank, LCS, and matrix spike (MS). 


\section{E.2.5 Total Inorganic Carbon/Total Organic Carbon (TIC/TOC)}

The samples were analyzed for total carbon (TC) using a Dohrman DC-80 Carbon Analyzer in accordance with procedure SwRI TAP 01-0406-001. The liquids were directly injected, and the slurry was analyzed using the soil/sludge sampler. Another aliquot of the sample was acidified with sulfuric acid and sparged to remove inorganic carbon and then analyzed for TOC. The TIC is calculated from the difference in the TC and TOC results. All samples were analyzed in duplicate and average results reported when the relative percent differences (RPDs) were less than 20\%. If the RPD was greater than $20 \%$, then the sample was analyzed twice more, and the average of the quadruplicate analysis was reported. The liquids were corrected for density, and all sample results were reported on a weight basis. QC samples were generated at the analytical workstation and included a sample replicate determination, preparation blank, LCS, and MS.

\section{E.2.6 Hydroxide}

The free-hydroxide concentration was determined by potentiometric titration with standardized $\mathrm{HCl}$ according to procedure RPG-CMC-228, "Determination of Hydroxyl (OH-) and Alkalinity of Aqueous Solutions, Leachates, and Supernates and Operation of Brinkman 636 Auto-Titrator." The free hydroxide was defined as the first inflection point on the titration curve. QC samples were generated at the analytical workstation and included a sample replicate determination, preparation blank, and blank spike (BS).

\section{E.2.7 Raman}

Raman was used to quantify aluminate, carbonate, chromate, hydroxide, nitrate, nitrite, oxalate, phosphate, and sulfate following procedure RPG-CMC-240. If precipitate formed in the solution samples submitted for Raman before the analysis, then the samples were centrifuged, and aliquots of the liquid were pipetted and analyzed. Two sets of Raman results were reported for shakedown, Integrated Test A, and some of the Integrated Test B samples. The first set of results was generated using calibrations that were periodically adjusted to optimize performance of QC check samples. The second set of results was recalculated based on the original calibration parameters. The generation of these two sets of results and the discovery of the calibration adjustments are documented as RPP-WTP CAR, number 42708.1. Only Raman results from the recalculation are provided. As a result of using the original calibration parameters, an occasional QC sample falls outside of established performance limits. QC samples were generated at the analytical workstation and included a sample replicate determination, preparation blank, $\mathrm{BS}$, and MS.

\section{E.2.8 Preparation for Gel Permeation Chromatography (AFA components)}

Duplicate samples were collected to support this analysis. Both samples were centrifuged and phase-separated at PDL-W before shipping. Once at the analytical laboratory, one of the two liquid fractions was mixed with toluene while the other was mixed with tetrahydrofuran (THF). Both were shaken for an hour and allowed to settle overnight. The upper organic layer was removed and allowed to concentrate. The toluene extract was dried at room temperature to completeness and brought to $3.0-\mathrm{mL}$ 
toluene. The THF extracts were concentrated to approximately 2-mL at room temperature and then placed in an $80^{\circ} \mathrm{C}$ oven to dry to completeness and brought up to 3.0-mL THF. One of the two solids fractions was extracted with $10-\mathrm{mL}$ of toluene while the other was extracted with $10-\mathrm{mL}$ of THF. In both cases, the solvent was added directly into the receipt vessel, and the initial sample and solvent were shaken for 2 hours. All solutions were filtered through $0.45-\mu \mathrm{m}$ PTFE syringe filters. Calibration verification was performed prior to sample analysis and after every 15 samples.

\section{E.2.9 Gel Permeation Chromatography (AFA components)}

The toluene extract allowed polydimethylsiloxane (PDMS) to be analyzed by GPC while the THF extract allowed polypropylene glycol (PPG) to be analyzed by GPC. The toluene extract was analyzed by a Waters 717 autosampler and a Waters 2410 differential refractometer. Two columns, a PLgel 5- $\mu \mathrm{m}$ guard column and a PLgel 5- $\mu \mathrm{m}$ Mixed-C column, were used for separation, and an HPLC grade toluene at a flow of $1.0-\mathrm{mL} /$ minute was used as the eluent. Both the columns and detector were heated to $45^{\circ} \mathrm{C}$. The THF extract was analyzed by a Waters 2695 Separation Module equipped with a vacuum degasser and a Waters 2410 differential refractometer. Two columns, a PLgel 5- $\mu \mathrm{m}$ guard column and a PLgel $5-\mu \mathrm{m}$ Mixed-C column, were used for separation, and a certified grade THF at a flow of $1.0-\mathrm{mL} / \mathrm{minute}$ was used as the eluent. Both the columns and detector were heated to $35^{\circ} \mathrm{C}$. The quantity of PDMS and PPG was used to determine the amount of AFA, Q2-3183A Antifoam, in the samples. Since the AFA is a proprietary composition belonging to Dow Corning, they performed the analytical work. Calibration verification was performed prior to sample analysis and after every fifteen samples.

\section{E.3 Physical Properties}

This section describes the methods used to determine the physical properties of the PEP simulant samples, the crystal form and habit of the solids, density, wt $\%$ UDS, rheology, particle-size attributes, and heat capacity. A more detailed outline of the methods used in this section is given, where applicable, in the appropriate test data package supplied with the sample results for each characterization.

\section{E.3.1 Percent UDS and Density}

Weight percent UDS, weight percent dissolved solids, bulk slurry density, and supernatant density were determined following Bechtel procedure 24590-WTP-GPG-RTD-001, Rev 0. UDS was measured at PNNL only when results were needed the same day the sample was collected. A halogen moisture analyzer (HG63, Mettler Toledo) was used to determine the wt $\%$ UDS under procedure TPR-RPP-WTP-648. If results for multiple samples were required, then a modified version of the oven method outlined in Guidelines for Performing Chemical, Physical, and Rheological Properties Measurements (24590-WTP-GPG-RTD-001 Rev. 0) was used. ${ }^{\text {(a) }}$ Some steps were not performed because the results generated from these steps were not needed, or steps were slightly modified to reduce analysis time. These modifications are not believed to impact the final results.

(a) GL Smith and K Prindiville. 2002. Guidelines for Performing Chemical, Physical, and Rheological Properties Measurements. 24590-WTP-GPG-RTD-001 Rev. 0, Bechtel National, Inc., Richland, Washington. 
Slurry sample UDS analysis was performed with the following modifications:

- Steps 4, 6, and 7 were omitted because the settling data were not required.

- Steps 8 and 9. Centrifuge the cones at $\sim 1000 \mathrm{G}$ for 1 hour. Record the volume of the total sample and the volume of centrifuged solids on the physical properties data sheet. After this, the WTP procedures required that the supernatant be decanted into a pre-weighed graduated cylinder to obtain the supernatant mass and volume. Rather than use a graduated cylinder, the volume of supernatant was calculated as the difference between the volume of the total sample and the volume of centrifuged solids.

- Step 11 requires decanting the centrifuged supernatant liquid to a pre-weighed graduated cylinder. Because of the high concentration of $\mathrm{NaOH}$ in some of the samples, some of the liquid was left in the cylinder. Rather than decant, the centrifuged supernatant liquid was pipetted to a pre-weighed glass Petri dish or vial, and the mass of the supernatant liquid was recorded.

- Step 12 was omitted because air-drying was not necessary.

Separated slurry samples UDS analysis was performed with the following modifications:

- Step 8 requires centrifuging at $\sim 1000 \mathrm{G}$ for 1 hour. Samples were centrifuged at $\sim 4500 \mathrm{G}$ for either 10 minutes or 1 hour. The centrifuge time is documented on the sample handling benchsheets in TDP-WTP-349.

- Wet solids (designated as "B") and decanted supernatants (designated as "D") were sent to SwRI along with empty vial and separated sample weights. These weights are also documented on the sample handing benchsheets in TDP-WTP-349.

- The wet solids were transferred to a tared container using DIW to completely remove the solids. The solids and DIW rinses were then oven dried to constant weight.

- The total weight of the supernatant was calculated from the sample-handling benchsheets. Since it was not feasible for the entire volume of supernatant to be oven dried, approximately 5-mL was transferred to a Petri dish for drying.

- Density was determined on the supernatant liquid using a 5-mL volumetric flask.

\section{E.3.2 Density}

Density measurements were performed at PDL-W on samples that were analyzed by Raman. Density was measured in accordance with TPR-WTP-PEP-054, Determination of Density using Pycnometer or Graduated Cylinder. All other density values were analyzed externally by SWRI.

\section{E.3.3 Rheology Measurements}

Rheological testing was conducted in accordance with RPL-COLLIOD-02, Measurement of Physical and Rheological Properties of Solutions, Slurries and Sludges. For the current study, two regions of tank waste flow behavior were considered: 1) incipient motion in settled tank waste solids (shear strength); and 2) non-elastic flow of tank waste slurries and supernates (flow curve). 


\section{E.3.3.1 Rheology Instrumentation}

Rheological characterization was accomplished using an Anton Parr Rheometer (MCR 301) for shear-rate measurements, and shear strength was performed on a Rotovisco RV20 Measuring System M equipped with an M5 measuring head and RC20 controller sold by HAAKE Mess-Technik GmbH u Co. (now the Thermo Electron Corporation). The M5 measuring head is a "Searle" type viscometer capable of producing rotational speeds up to $500 \mathrm{rpm}$ and measuring torques up to $0.049 \mathrm{~N} \cdot \mathrm{m}$. The minimum rotational speed and torque resolution achievable by this measuring head are $0.05 \mathrm{RPM}$ and $0.49 \mathrm{mN} \cdot \mathrm{m}$, respectively.

Calibration and analysis were performed according to TPR-WTP-PEP-049.

Specific measurement tools, such as cup-and-rotor assemblies and shear vanes, are attached to measure selected rheological properties. Shear-strength measurements employ an $8 \mathrm{~mm} \times 16 \mathrm{~mm}(\mathrm{R} \times \mathrm{H})$ shear vane tool. Flow-curve measurements employed an MV1 stainless steel measuring cup and rotor. The dimensions of the MV1 and vane measuring systems are listed in Table E.1.

Table E.1. Vane and Cup and Rotor Measuring System Dimensions

\begin{tabular}{lcccc}
\hline Measuring System & $\begin{array}{c}\text { Vane/Rotor Radius } \\
(\mathrm{mm})\end{array}$ & $\begin{array}{c}\text { Vane/Rotor Height } \\
(\mathrm{mm})\end{array}$ & $\begin{array}{c}\text { Container Radius } \\
(\mathrm{mm})\end{array}$ & $\begin{array}{c}\text { Gap Width } \\
(\mathrm{mm})\end{array}$ \\
\hline Vane Tool & 8 & 16 & $>16^{(\text {a) }}$ & $>8$ \\
MV1 & 20.04 & 60 & 21 & 0.96 \\
\hline
\end{tabular}

The temperature is controlled with a combination of the standard measuring system temperature jacket and a temperature-controlled recirculator. The jacket temperature is monitored using a Type-K thermocouple calibrated over $0^{\circ}$ to $100^{\circ} \mathrm{C}$ and connected to a calibrated multichannel temperature display. The temperature control is employed only for flow-curve measurements. Shear-strength measurements are carried out at ambient cell temperature.

The rheometer was controlled, and data were acquired with a remote computer connection using the RheoWin Pro Job Manager Software, Version 2.96.

\section{E.3.3.2 Rheology Materials and Methods}

No sample treatment was performed before analysis with the exception of the mechanical agitation required to mix and sub-sample selected sample containers.

\section{E.3.3.3 Shear-Strength Testing}

Before testing, the simulant slurries that were provided for shear-strength testing were mixed thoroughly and subsequently allowed to settle for at least 48 to $72 \mathrm{~h}$. When possible, the shear strength was measured by immersing the $16-\times 16-\mathrm{mm}$ vane tool to a depth of $15 \mathrm{~mm}$ into the settled solids. The vane was slowly rotated $(0.03 \mathrm{1} / \mathrm{sec})$ for $120 \mathrm{~s}$. For the entire duration of rotation, the time, rotational rate, and vane torque were continuously monitored and recorded. At the end of the measurement, shear 
stress versus time data were parsed, and the maximum measured shear stress (i.e., the material's shear strength) was determined.

\section{E.3.3.4 Flow-Curve Testing}

Each flow curve was measured over an 11-minute period and split into three intervals. Over the first 5 minutes, the shear rate was smoothly increased from zero to $1000 \mathrm{~s}^{-1}$. For the next minute, the shear rate was held constant at $1000 \mathrm{~s}^{-1}$. For the final 5 minutes, the shear rate was smoothly reduced back to zero. During this time, the resisting torque and rotational rate were continuously monitored and recorded.

Results are provided in the test data packages listed below, with summary results provided in Appendix L.

- Integrated Tests A and B TDP-WTP-359

- Integrated Test B TDP-WTP-363

\section{E.3.4 Particle-Size Distribution}

Particle sizes were characterized according to procedure RPL-COLLOID-01, Rev. 1, Particle Size Analysis Using Malvern MS2000. This procedure uses a Mastersizer 2000 (Malvern Instruments, Inc., Southborough, MA 01772 USA) with a Hydro S wet dispersion accessory. Malvern lists the Mastersizer particle-size measurement range as nominally 0.02 to $2000-\mu \mathrm{m}$. The actual PSD measurement range is dependent on the accessory used as well as the properties of the solids being analyzed. The Malvern 2000 uses laser diffraction technology to define PSD.

The Hydro S wet-dispersion accessory consisted of a 150-mL dispersion unit coupled with a sample flow cell with a continuous variable and independent pump and stirrer and ultrasound. Flow, stirring rate, and sonication can be controlled and altered during measurement. PSD measurements can be made before, during, and after sonication, allowing the influence of each on the sample PSD to be determined. The primary measurement functions of the Malvern analyzer were controlled through Mastersizer 2000 software (Malvern Instruments, Ltd. Copyright 1998-2002). The properties applied to the test samples are summarized in Table E.2.

The PSD measurements were conducted in either DIW or in a $0.01 \mathrm{M} \mathrm{NaOH}$ dispersion solution matrix, depending on the sample being analyzed. The sample dispersion was added drop-wise to the dispersion unit (while the pump and stirrer were active) until an obscuration in the range of 10 to $20 \%$ was reached. 
Table E.2. Properties Applied to Group 8 Test Materials

\begin{tabular}{cc}
\hline Property & \\
\hline $\begin{array}{c}\text { Material selected for optical } \\
\text { properties }\end{array}$ & Ferric Oxide Hydroxide \\
Refractive Index (RI) & 2.94 \\
Absorption & 0 \\
Analysis mode & General purpose \\
Sensitivity & normal \\
Suspending Phase & Water/0.01 M NaOH \\
\hline
\end{tabular}

The size distributions of particles were measured under varying flow conditions before and after sonication. For each condition, multiple measurements of PSD were taken. The analyzer software then generated an average of these measurements. Both the individual measurement and average were saved to the analyzer data file.

\section{E.3.5 X-Ray Diffraction}

The sample mounts for XRD examination were prepared by first cleaning the solids. This procedure included centrifuging the solids into a pellet and decanting the solute. Fresh washing solution was added to the pellet, and it was resuspended. The pellets in solution were vortexed to reconstitute them into the solution, and the centrifuging procedure was repeated three times. Following the final centrifuging and decant, the remaining pellet was left to dry in a $105^{\circ} \mathrm{C}$ oven overnight. The pellet was then pulverized to a powder with a tungsten carbide milling chamber for 1 minute in the Angstrom shaker mill, mixed with an internal standard (rutile, $\mathrm{TiO}_{2}$, or alumina, $\mathrm{Al}_{2} \mathrm{O}_{3}$ ), milled for another 2 minutes to make sure that the two powders were a homogenous mixture, and then mounted into an off-axis, zero background, quartz sample holder. The XRD examination was conducted according to procedure APEL-PAD-V, Operation-of Scintag Pad-VX-Ray Diffractometer. The XRD instrument used for these samples was the PNNL Scintag PAD V XRD (property number WD33356), located in Laboratory 102 in the APEL building. The data range for the sample was $5^{\circ}$ to $80^{\circ} 2 \theta$, with a step size of $0.02^{\circ} 2 \theta$ and count time of 2.0 seconds per step. Copper $\mathrm{K} \alpha \mathrm{X}$-rays were used. The X-ray tube operating conditions were $45 \mathrm{kV}$ and $40 \mathrm{~mA}$. Phase identification was done by use of the JADE search match routines (Version 6.0, Materials Data Inc.) with comparison to the International Centre for Diffraction Data (ICDD) database PDF-2, release 1999, which includes the Inorganic Crystal Structure Database (ICSD) maintained by Fachinformationszentrum (FIZ), Karlsruhe, Germany. The chemistry provided for Group 7, in order of decreasing concentration, was $\mathrm{Fe}, \mathrm{Na}, \mathrm{U}, \mathrm{P}, \mathrm{Ca}, \mathrm{Al}, \mathrm{Si}, \mathrm{Bi}, \mathrm{Sr}$, and $\mathrm{Mg}$. Phase identifications were first done without chemistry restrictions. Searches were restricted to the PDF and ICSD inorganic sections.

The pattern was also examined using RIQAS (release 4.0.0.26, 6/10/2002, Materials Data Inc.) Rietveld analysis software. The phases identified above were input into the analysis along with a polynomial background and an amorphous hump at $\sim 35^{\circ} 2 \theta$.

Details of the XRD analysis and results are provided in test data package, TDP-WTP-357, with summary results provided in Appendix L. 


\section{E.3.6 Scanning Electron Microscopy}

Within the same sample processing as above for the XRD sample preparation, the final step just before the supernatant was decanted, the specimen was vortexed, and a small volume of slurry was drawn up using a pipette and placed on an aluminum stub. The slurry was placed in an oven at $105^{\circ} \mathrm{C}$ to dry overnight. The sample was then coated with gold-palladium using a Polaron Range plasma sputter coater and analyzed with a JEOL SEM (property number WD30596) according to APEL-102-SEM, Scanning Electron Microscope Examination. Selected sample areas were evaluated by X-ray energy dispersive spectroscopy (EDS) for qualitative elemental composition.

Results are provided in the test data package, TDP-WTP-356, provided in Appendix L.

\section{E.3.7 Heat Capacity}

Approximately eight samples at key process steps were collected and analyzed for heat capacity during Integrated Tests A and B. Three nominal 30-mg subsamples were taken from each of these samples as they were vigorously stirred using a pipette with an enlarged tip to confirm that the slurry samples were representative. Each slurry sample was analyzed in triplicate, recognizing that obtaining a $30-\mathrm{mg}$ representative sample is challenging.

A Perkin-Elmer DSC7 differential scanning calorimeter (DSC) was used to determine the heat capacity of each subsample at temperatures between $20^{\circ} \mathrm{C}$ and $95^{\circ} \mathrm{C}$. The DSC7 was temperature- and enthalpy-calibrated using a gallium standard (NIST SRM 2234) (m.p. $29.8^{\circ} \mathrm{C}$ ) and/or an indium standard (NIST SRM 2232) (m.p. $156.6^{\circ} \mathrm{C}$ ).

The method used for heat-capacity measurement was as provided in the DSC7 operating manual. In this method, the heat capacity of the empty sample pan is measured to provide the "baseline"; the sample pan was a gold "volatile" sample pan designed to contain volatile samples such as the water in these PEP slurries. The heat capacity of a reference or standard material such as sapphire or high-purity water is then determined; the purity of these water standards was determined by measuring their electrical conductivity. Finally, the heat capacity of the sample is determined. The measured heat capacity of the sample is adjusted to remove the contribution of the empty pan. We further adjusted the reported heat capacity by applying a measured response factor for the high-purity water.

Optimally, the heat-capacity measurement approach would include analyzing a standard or reference material in the same container in which the sample was analyzed; however, because the analysis of these slurry samples required a hermetic seal, which cannot be broken and resealed, an equivalent gold volatile sample pan was used for the water. In addition, we characterized a sapphire standard to demonstrate instrument performance; the heat capacity of sapphire $\left(0.8 \mathrm{~J} / \mathrm{g}{ }^{\circ} \mathrm{C}\right)$ is significantly lower than that of the PEP slurries $(\sim 3.5 \mathrm{~J} / \mathrm{g})$ and does not provide an adequate calibration adjustment.

Results are provided in the heat capacity test data packages listed below and given in Appendix L.

- Integrated Tests A and B TDP-WTP-368

- Integrated Test B TDP-WTP-372 

Appendix F

Evaluation of PJM Peak Average Velocity 



\section{Appendix F: Evaluation of PJM Peak Average Velocity}

\section{F.1 Introduction}

A number of Microsoft Excel macros were developed in the Visual Basic language to evaluate the peak average velocity and average PJM stroke length. The definitions of the peak average velocity and stroke length and the background theory of evaluating these quantities are given in Section F.2. In Section F.3, the Excel macros developed to evaluate the peak average velocity and stroke length, and their implementation procedures are described. Finally, the loss coefficients used for the nozzle velocity evaluation from the PJM tube pressures are presented in Section F.4.

\section{F.2 Background Theory}

This section defines the peak average velocity and stroke length, and describes the methods used to evaluate these quantities.

\section{F.2.1 Definitions of Peak Average Velocity and Stroke Length}

In the PJM operation, steady periodic variation in PJM tube pressure drives flow through the PJM nozzle, which results in cyclic vertical motion of the liquid level in the PJM tube and the PJM tank. Typical nozzle velocity and PJM tube level profiles as a function of time for a complete operating cycle are shown in Figure F.1 and Figure F.2, respectively.

From Figure F.1, the peak average time $t_{\text {peak }}$ and the discharge time $t_{\text {disch arge }}$ are defined as:

$$
\begin{gathered}
t_{\text {peak }}=t_{\text {end }}-t_{\max } \\
t_{\text {disch arge }}=t_{\text {end }}-t_{o}
\end{gathered}
$$

where $t_{o}$ is the start of pressurization (start of drive cycle), $t_{\text {end }}$ is the end of pressurization (nozzle velocity begins to decrease rapidly), and $t_{\max }$ is the time at maximum discharge.

The peak average velocity $\bar{U}_{\text {peak }}$ for the discharge process is defined as:

$$
\bar{U}_{\text {peak }}=\frac{1}{t_{\text {peak }}} \int_{t_{\max }}^{t_{\text {end }}} U(t) d t
$$

where $U(t)$ is the nozzle velocity. 


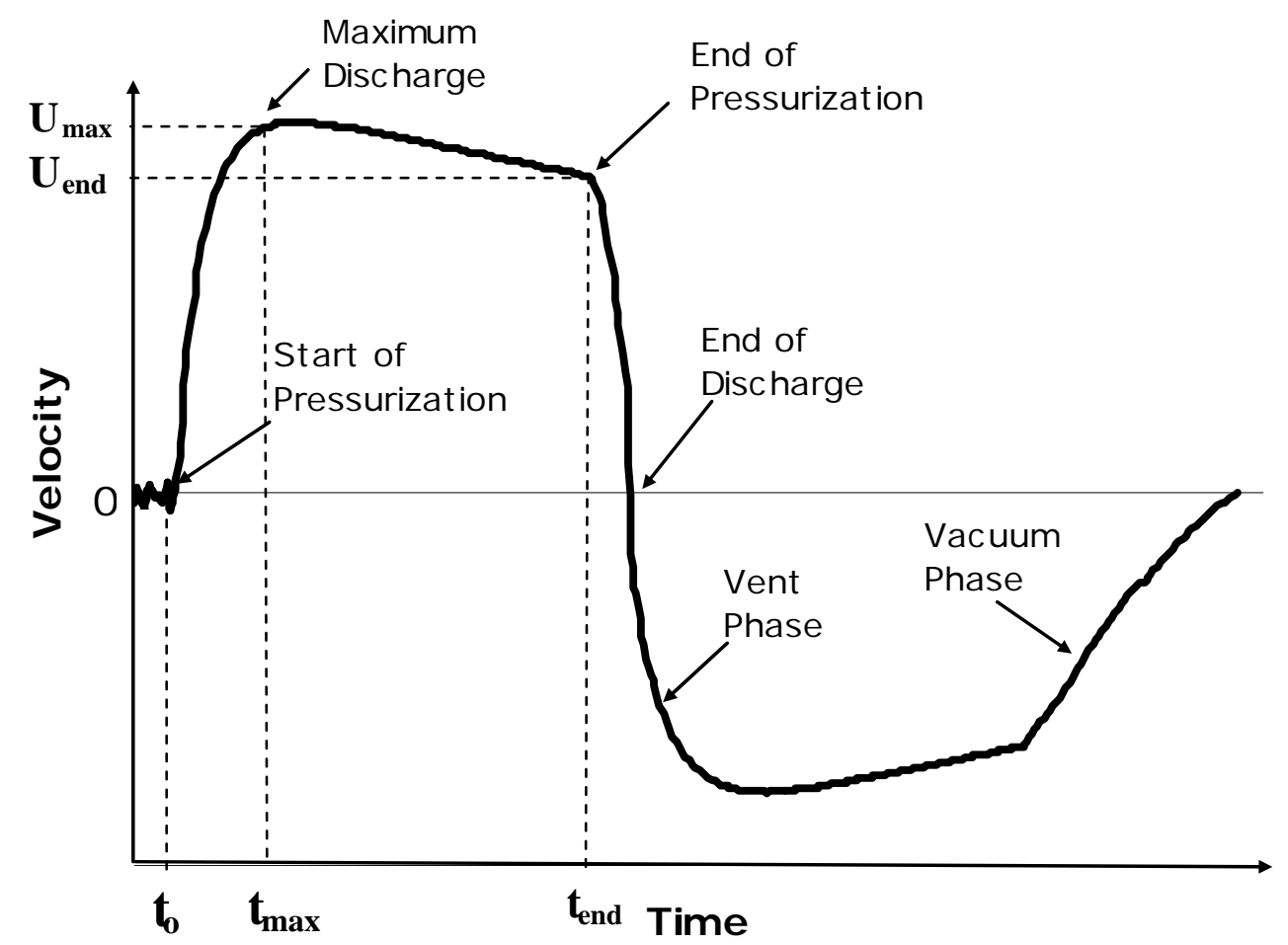

Figure F.1. Typical Nozzle Velocity Time Distribution

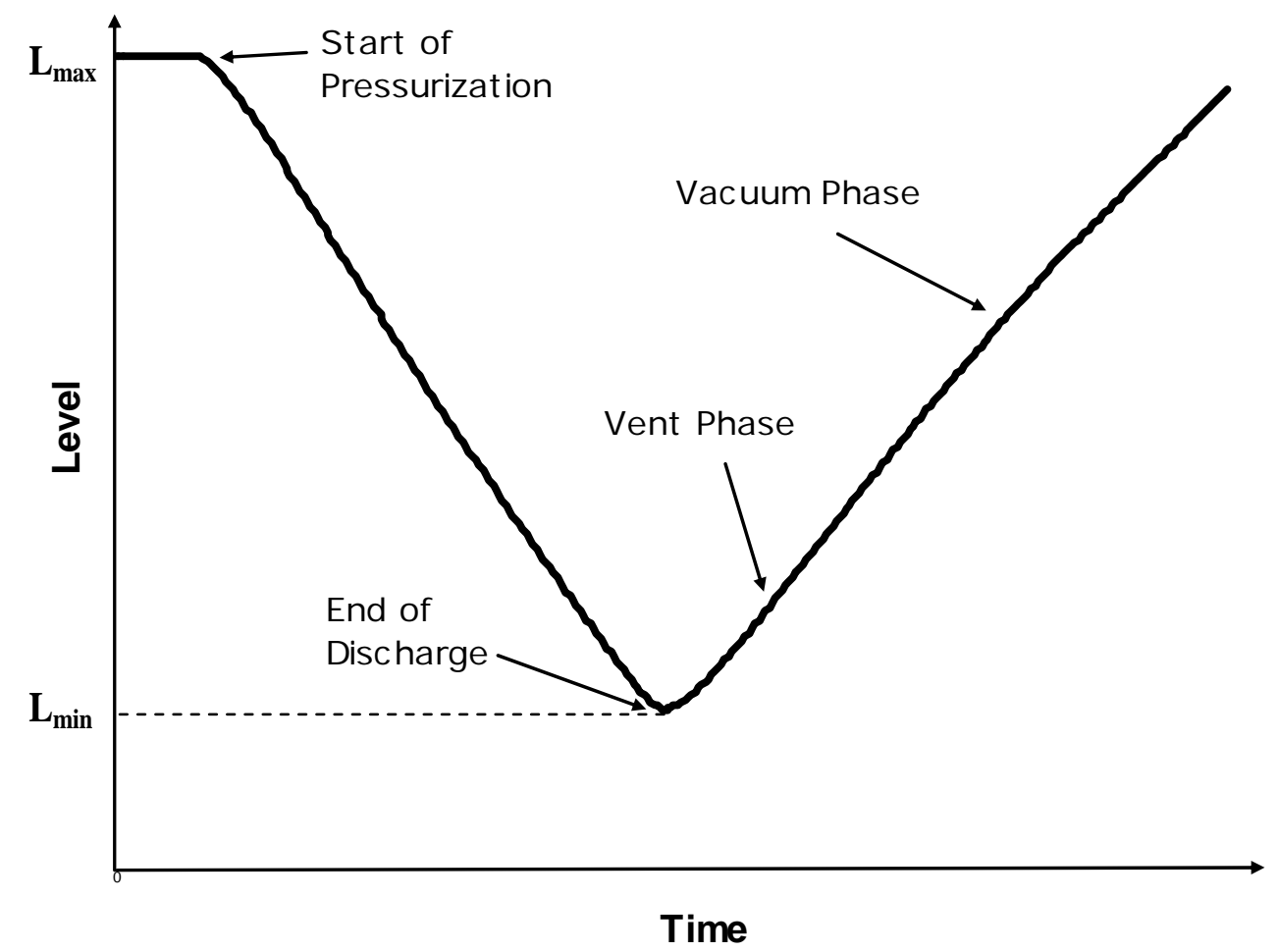

Figure F.2. Typical PJM Tube Level Time Distribution 
The nozzle velocity $U(t)$ is evaluated from the measured PJM tube liquid level and the PJM tank level, and the PJM tube pressure. Calculation of the nozzle velocity $U(t)$ is described in section F. 3 in detail. The Trapezoidal rule is used for the numerical integration of Equation (F.3).

From Figure F.2, the PJM tube stroke length $L_{S T R}$ is defined as:

$$
L_{S T R}=L_{\max }-L_{\min }
$$

where $L_{\max }$ is the PJM tube level at start of pressurization, and $L_{\min }$ is the PJM tube level at the end of discharge.

Along with Equation (F.4), the PJM tube stroke length fraction $L_{S T R P}$ is defined as:

$$
L_{S T R P}=\frac{L_{S T R} \cdot A_{\text {tube }}}{V}
$$

where $A_{\text {tube }}$ is the PJM tube cross-sectional area, and $V$ is the PJM tube volume. Namely, the PJM tube stroke length fraction $L_{\text {STRP }}$ is defined as the ratio of the volume of the PJM liquid displacement to the entire PJM tube volume.

\section{F.3 Methods}

The methods used to evaluate the nozzle velocity $U(t)$ from the level and pressure data are described in this section.

\section{F.3.1 Nozzle Velocity Evaluation from PJM Tube and Tank Liquid Level Data}

Significantly large fluctuations were contained in the level data measured for the PJM tubes and the PJM tanks, due to the hydrodynamics of the mixing operation. To smooth out these fluctuations and capture the effective level motion in an operating cycle, the level measurements were averaged. The measured level data was averaged over multiple sequential sampling cycles (typically 10 cycles) to obtain the average cyclic level distribution for each tube, or for the PJM tank. In addition, the averages for each PJM tube were combined to obtain the average cyclic level distribution for all of the PJM tubes. Prior to the data averaging, the PJM tank level data were filtered to remove the outliers.

For the case in which averaging was insufficient to remove fluctuations, the averaged liquid level distribution was further smoothed by applying the $6^{\text {th }}$ power binomial coefficients $(1,6,15,20,15,6$, 1)/64 for smoothing of the interior data points, the $3^{\text {rd }}$ power binomial coefficients $(1,4,6,4,1) / 16$ for smoothing at the $3^{\text {rd }}$ exterior data point, the $2^{\text {nd }}$ power binomial coefficients $(1,2,1) / 4$ for smoothing at the $2^{\text {nd }}$ exterior data point.

The averaged liquid surface levels with or without smoothing were used to evaluate the nozzle velocity $U$ as: 


$$
U=\left(\frac{A}{A_{n}}\right) \frac{d L}{d t}
$$

where

$$
\begin{aligned}
L & =\text { averaged liquid level } \\
A / A_{n} & =\text { area ratio } \\
A & =\text { area of the PJM tube or the PJM tank liquid surface } \\
A_{n} & =\text { area of the PJM nozzle. }
\end{aligned}
$$

The finite central difference method is used to evaluate $\frac{d L}{d t}$.

\section{F.3.2 Nozzle Velocity Evaluation from Tube Pressure Data}

The average of the PJM tube pressure data was taken prior to the nozzle velocity evaluation, using the same approach as described above for averaging of the level measurements. The averaged cyclic distribution in pressure was used to evaluate the nozzle velocity by applying the Bernoulli equation with the pressure loss effect taken into account.

The Bernoulli's equation taking account of the pressure loss coefficient is given as:

$$
P_{t}+\rho g\left(L_{i n i}-x-H\right)-P_{H S P}=\frac{1}{2} \rho\left[\left(1+k_{f}\right)\left(\frac{R}{R_{n}}\right)^{4}-1\right] \dot{x}^{2}
$$

where $\quad \dot{x}=$ liquid velocity in the PJM tube

$x=$ length of the displaced liquid inside the PJM tube

$P_{t}=$ averaged PJM tube pressure

$P_{H S P}=$ headspace pressure of the PJM tank

$L_{i n i}=$ initial liquid level height inside the PJM tube

$H=$ liquid level height inside the tank

$\rho=$ liquid density

$g=$ gravitational acceleration $9.81\left(\mathrm{~m} / \mathrm{s}^{2}\right)$

$k_{f}=$ empirically determined loss coefficient

$R=$ PJM tube radius

$R_{n}=$ nozzle radius.

The coefficient $\left(\frac{R}{R_{n}}\right)^{2}$ in Equation (F.7) is exactly equivalent to the area ratio defined in

Equation (F.6) for the ratio of the pulse tube area to the pulse tube nozzle area. The parameters $x, P_{t}$, $P_{H S P}, L_{i n i}$, and $H$ used for Equation (F.7) are illustrated in the schematic diagram of Figure F.3. 
Along with Equation (F.7), the nozzle velocity $U$, the length of the displaced liquid $x$, the liquid level height of the tank $H$ is evaluated as:

$$
\begin{gathered}
U=\left(\frac{R}{R_{n}}\right)^{2} \dot{x} \\
x=x_{\text {old }}+\Delta t \dot{x} \\
H=H_{\text {ini }}+\left(\frac{A_{P L}}{A_{T L}}\right) x
\end{gathered}
$$

where $\Delta t=$ sampling time

$x_{\text {old }}=$ length of the displaced liquid at the previous pressure measurement time

$H_{\text {ini }}=$ initial tank liquid level height

$A_{P L}=$ total liquid surface area of all of the PJM tubes

$A_{T L}=$ tank liquid surface area.

In order to solve Equation (F.7) numerically, the initial liquid level height inside the PJM tube $L_{i n i}$ is needed as a starting point. This can be determined by imposing the zero initial velocity condition for the given initial tank liquid level height $H_{i n i}$ as:

$$
L_{i n i}=\left(\frac{P_{H S P}-P_{t i}}{\rho g}\right)+H_{i n i}
$$

where $P_{t i}$ is the averaged PJM tube pressure at the pressurization start point of the PJM pressure cycle process (see Figure F.1). 


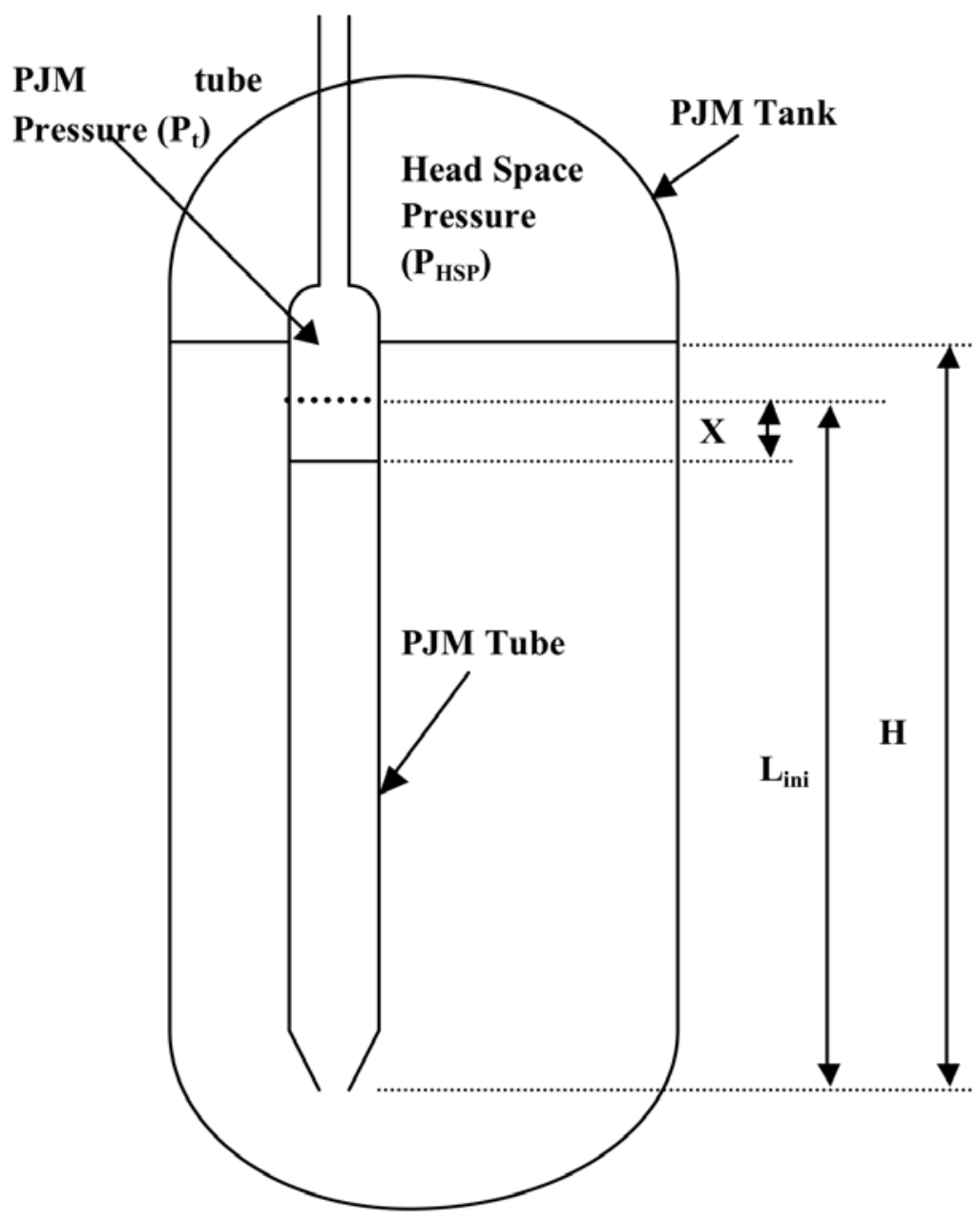

Figure F.3. Schematic of PJM Tank and Tube

The loss coefficients were obtained by iterating to a 'best fit' of the nozzle velocity distribution evaluated by applying Equation (F.7) through Equation (F.11), compared to the distribution determined from the tank level data measured by a laser anemometer.

\section{F.4 Loss Coefficient Evaluations}

In order to evaluate the nozzle velocity time distribution from the PJM pressure data with Equation (F.7) through Equation (F.11), the empirically determined loss coefficients were needed. The loss coefficients were obtained by iterating to a 'best fit' of the nozzle velocity distribution evaluated from the pressure data, compared to the distribution determined from the tank level data measured by a laser anemometer.

This section presents the loss coefficients evaluated in the RPP-WTP PEP testing in Table F.1. The plots used for the evaluations of the loss coefficients are given in Figure F.4 through Figure F.7. 
Table F.1. Loss Coefficients Evaluated

\begin{tabular}{cccc}
\hline Tank & $\begin{array}{c}\text { Loss Coefficient for } \\
\text { Forward Flow }\end{array}$ & $\begin{array}{c}\text { Loss Coefficient for } \\
\text { Backward Flow }\end{array}$ & $\begin{array}{c}\text { Target Velocity } \\
(\mathrm{m} / \mathrm{s})\end{array}$ \\
\hline UFP-VSL-T01A & 0.25 & 1.2 & 4.8 \\
UFP-VSL-T01B & 0.38 & 1.3 & 4.8 \\
UFP-VSL-T02A & 0.34 & 0.1 & 7.3 \\
UFP-VSL-T02A & 0.1 & 0.1 & 12 \\
\hline
\end{tabular}

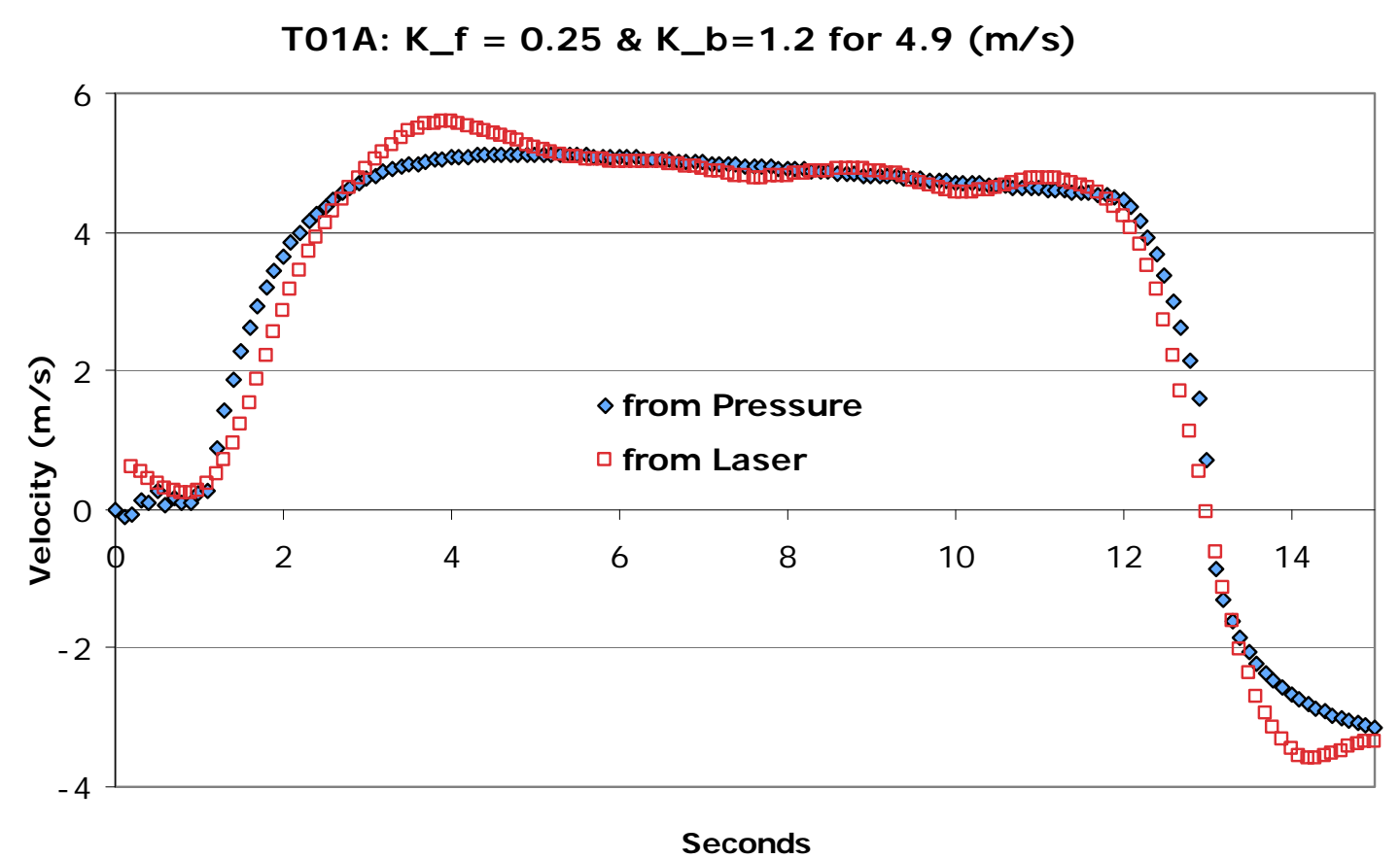

Figure F.4. Nozzle Velocity Distributions Used to Evaluate the Loss Coefficients for UFP-VSL-T01A Tank at the Peak Average Velocity of $4.9(\mathrm{~m} / \mathrm{s}) ; \mathrm{k} \_\mathrm{f}=0.25$ is the loss coefficient of the forward flow, and $\mathrm{k} \_\mathrm{b}=1.2$ is the loss coefficient of the backward flow. 


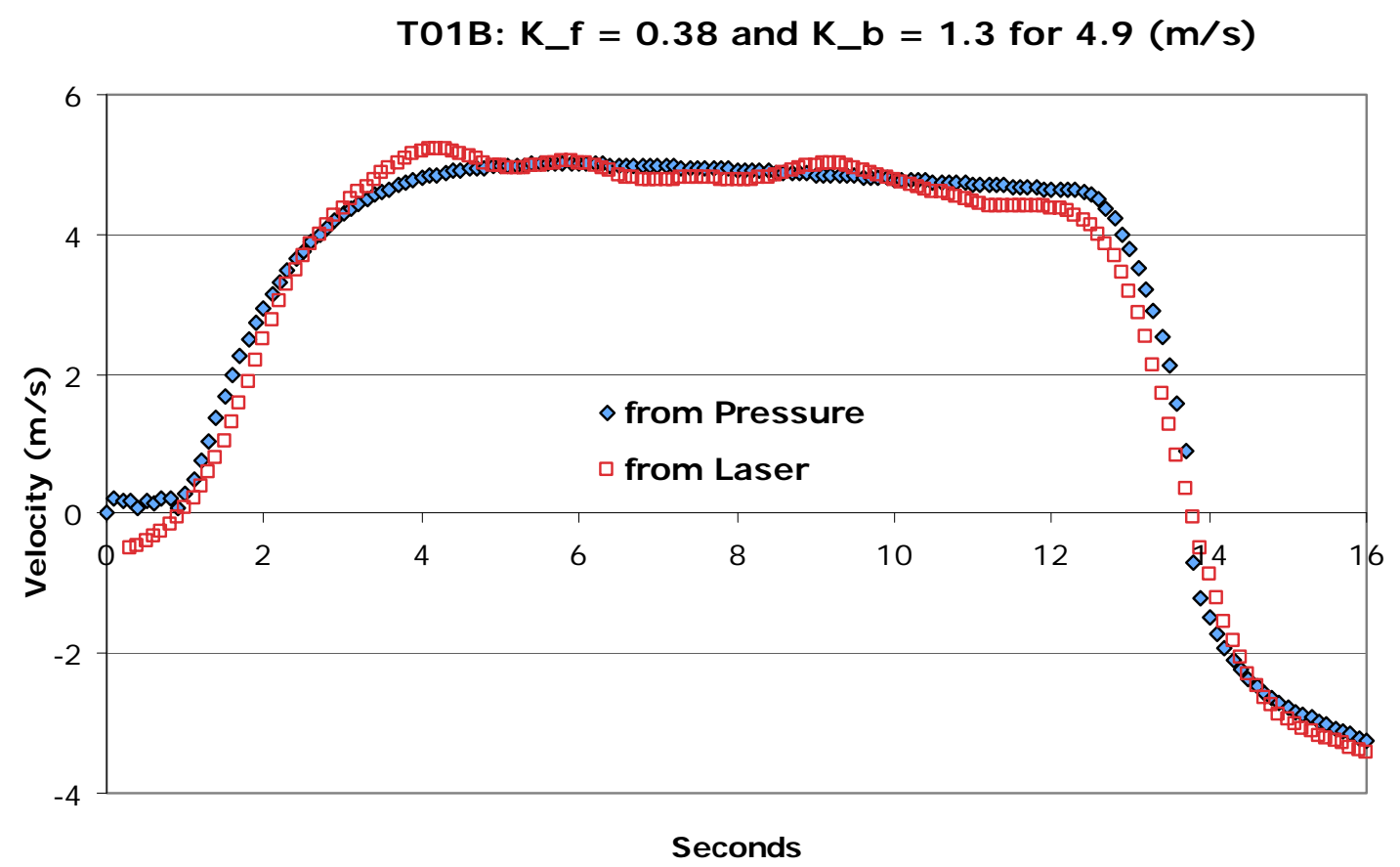

Figure F.5. Nozzle Velocity Distributions Used to Evaluate the Loss Coefficients for UFP-VSL-T01B Tank at the Peak Average Velocity of $4.9(\mathrm{~m} / \mathrm{s}) ; \mathrm{k}_{-} \mathrm{f}=0.38$ is the loss coefficient of the forward flow, and $\mathrm{k} \_\mathrm{b}=1.3$ is the loss coefficient of the backward flow. 


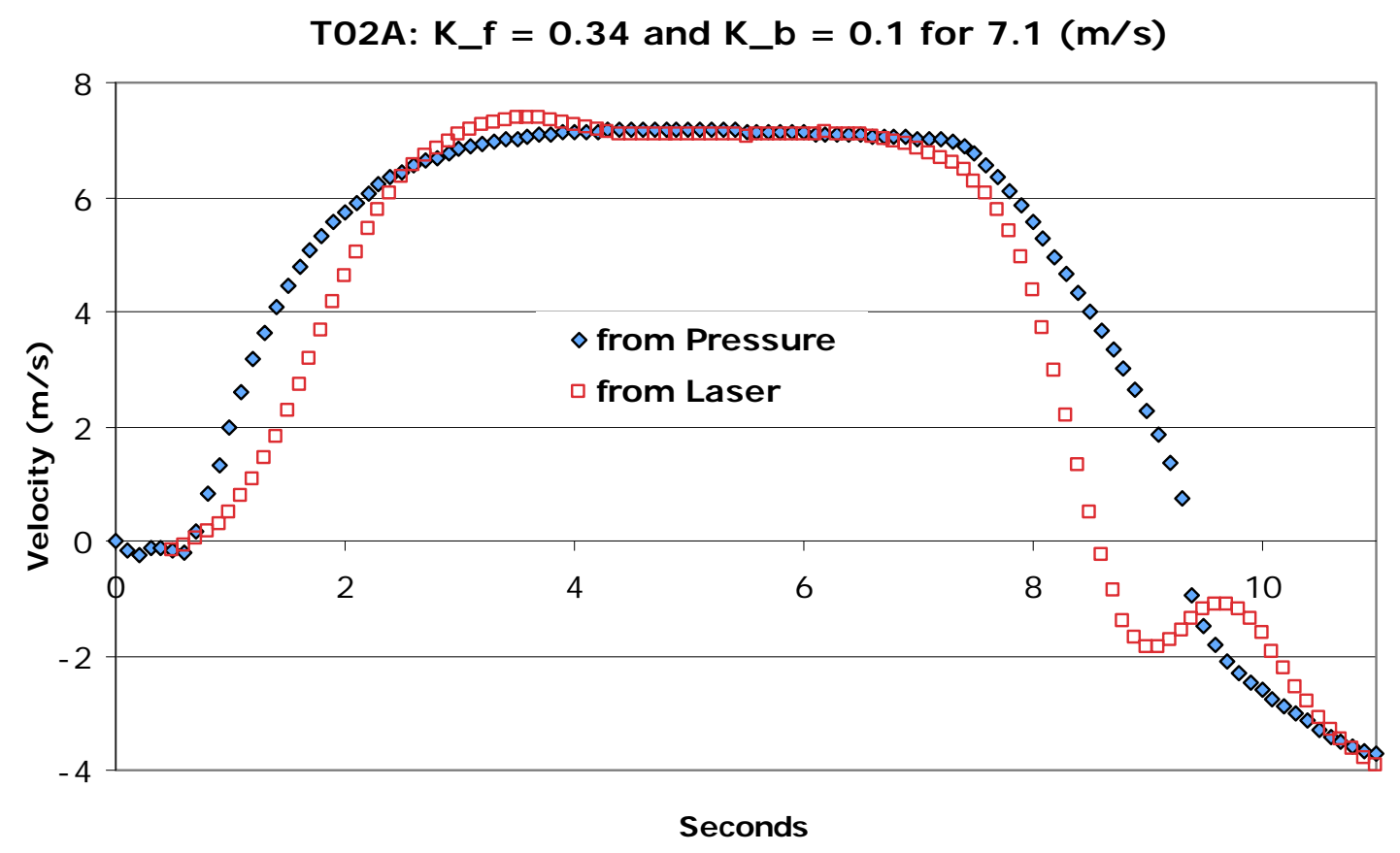

Figure F.6. Nozzle Velocity Distributions Used to Evaluate the Loss Coefficients for UFP-VSL-T02A Tank at the Peak Average Velocity of $7.1(\mathrm{~m} / \mathrm{s}) ; \mathrm{k}_{-} \mathrm{f}=0.34$ is the loss coefficient of the forward flow, and $\mathrm{k} \_\mathrm{b}=0.1$ is the loss coefficient of the backward flow.

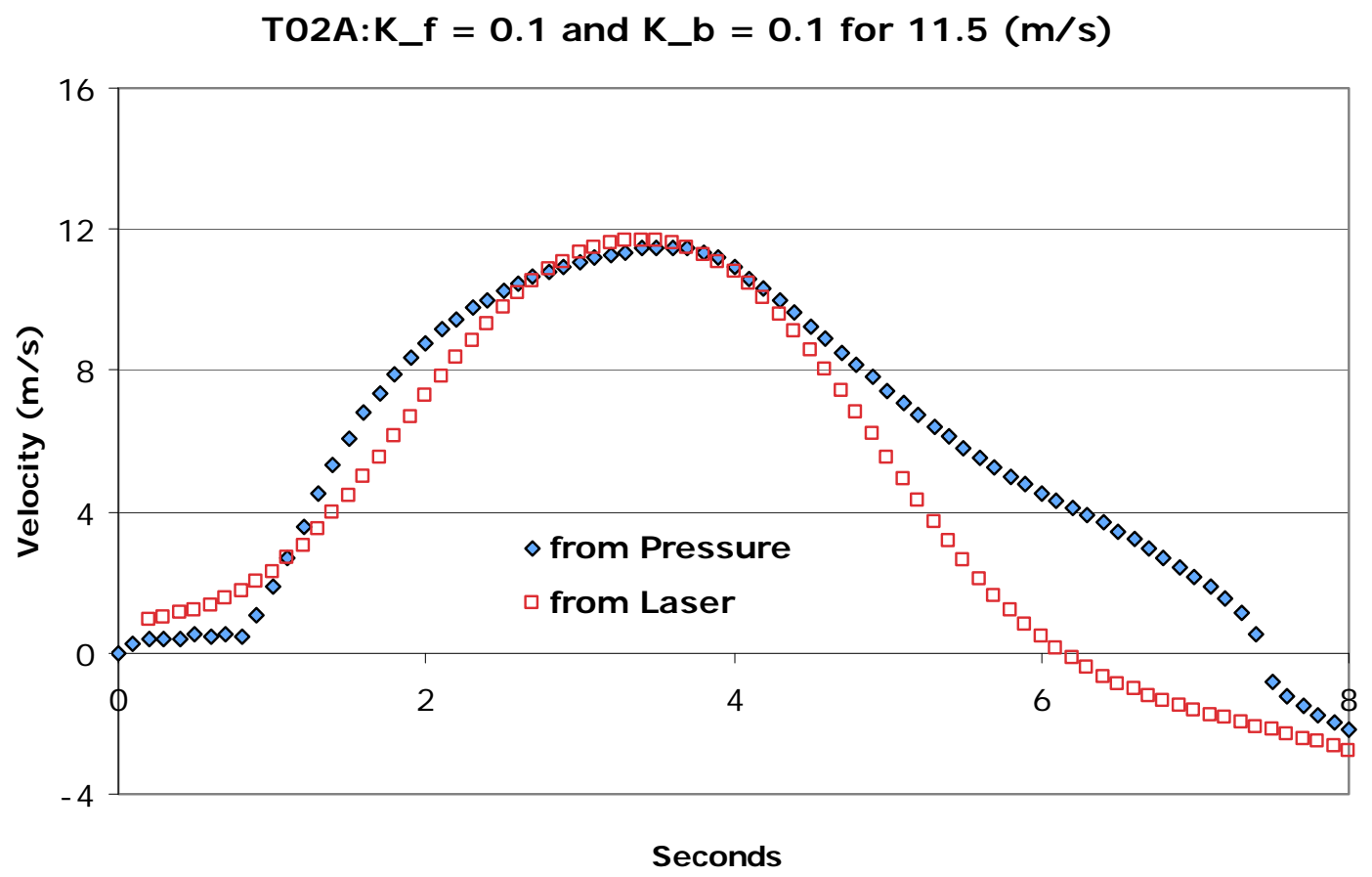

Figure F.7. Nozzle Velocity Distributions Used to Evaluate the Loss Coefficients for UFP-VSL-T02A Tank at the Peak Average Velocity of $11.5(\mathrm{~m} / \mathrm{s}) ; \mathrm{k}_{-} \mathrm{f}=0.1$ is the loss coefficient of the forward flow, and $\mathrm{k} \_\mathrm{b}=0.1$ is the loss coefficient of the backward flow. 

Appendix G

Nitric Acid Cleaning Instructions for the PEP Filters 



\section{Appendix G: Nitric Acid Cleaning Instructions for the PEP Filters}

The following instructions up to step 7 were used for the first cleaning attempt prior to initiating Integrated Test B. During step 7, a leak in pump 42A occurred, and the cleaning was terminated. No backpulses were recorded.

\section{Instructions for Nitric Acid Cleaning of PEP Filters (First Attempt)}

1. Ensure DAS system is operating.

2. Drain the UFP-VSL-T02A and the recirculation loop (both sides of the filters, pulse-pots, heat exchangers, piping and all dead-legs) to UFP-VSL-T62A per procedure OP-411 and OP-413.

3. Ensure NAR-VSL-T01 contains $>200$ gallons of $2 \mathrm{M}$ nitric acid solution.

4. Transfer at least 200 gallons of 2M nitric acid to UFP-VSL-T02A per procedure OP-305.

5. Start the PJMs in UFP-VSL-T02A using normal mode.

6. Align recirculation loop for flow through all five filters and the chiller recirculation system with permeate to UFP-VSL-T02A per procedure OP-408. Start recirculation loop with a flow of $109 \mathrm{gpm}$. Set chiller controller to $45^{\circ} \mathrm{C}$.

7. Start filtration according to procedure OP-409 for all filters with recirculation back to UFP-VSL-T02A. When the UFP-VSL-T02A has reached $40^{\circ} \mathrm{C}$ (due to the pump recirculation) initiate filter backpulsing and reduce the chiller set point to $40^{\circ} \mathrm{C}$. Backpulse each filter 10 times. Record the permeate rate prior to the backpulse, the pulse-pot starting level, the times when the fast acting valve opens and closes, pulse-pot ending levels, permeate rates immediately following the backpulse, and the time to refill the pulse-pot in the logbook. Contact the Test Director prior to initiating step 10 to determine if additional backpulsing is required. TMP controller shall be set at 40 psi.

8. Sample UFP-VSL-T02A from recirculation loop sampler using procedure OP-401. Check sample $\mathrm{pH}$ and then add $20-\mathrm{mL}$ of $2 \mathrm{M} \mathrm{NaOH}$ to $\sim 4-\mathrm{mL}$ of sample to check for precipitation while completing steps 10 through 13.

9. Stop PJMs and pumps.

10. Drain UFP-VSL-T02A and the filter-loop to UFP-VSL-T62A or T62B as directed by Shift Supervisor. The draining should include the filters (both sides), pulse-pots, heat exchangers, piping and all dead-legs of the nitric acid solution.

11. Add at least 200 gallons of deionized water (DIW) or inhibited water to UFP-VSL-T02A such that the tank contents are in a range $\mathrm{pH}$ range of $2-3.5$ to flush tank and filter-loop. If DIW is used, add manually from tote to the inlet of the T42A pump.

12. Start PJMs and run for 30 minutes. 
13. Start recirculation and filtration according to procedure OP-408 and OP-409 for all filters. Operate filters with recirculation back to UFP-VSL-T02A for at least 30 minutes and backpulse the filter at least 5 times. TMP set at 40 psig.

14. Sample UFP-VSL-T02A from recirculation loop sampler using procedure OP-401. Record sample $\mathrm{pH}$ in the logbook.

15. Stop filtration and recirculation pumps.

16. Isolate the filter-loop from UFP-VSL-T02A.

17. Drain UFP-VSL-T02A.

18. Do not drain the filter-loop. This will be coordinated with the execution of TI-66.

The following is a summary of the instructions were used for the second cleaning attempt prior to initiating Integrated Test B. Low pumping rates causes the operation to delete the backpulses with the DIW.

\section{Initial Nitric Acid Cleaning with Diaphragm Pump}

1. Install a diaphragm pump to circulate the nitric acid currently in T02A through the filter assembly, by passing the recirculation pumps. Connect the pump inlet to the T02A outlet and the pump outlet to one of the ports ahead of the filters.

2. Fill the pulse pots directly from the NAR vessel with nitric acid for the back pulse procedure.

3. When pulse-pots are ready, backpulse each filter.

4. Add nitric acid to T02A and start recirculation through the loop.

5. Perform ten backpulses using the normal procedure.

6. Start filtering.

7. Drain UFP-VSL-T02A and the filter-loop to UFP-VSL-T62A or T62B as directed by Shift Supervisor. The draining should include the filters (both sides), pulse-pots, heat exchangers, piping and all dead-legs of the nitric acid solution.

8. Add DIW to UFP-VSL-T02A such that the tank contents are in a $\mathrm{pH}$ range of 2 - 3 to flush tank and filter-loop.

9. Start PJMs and run for 30 minutes (30 minutes operation was not performed).

10. Sample UFP-VSL-T02A from recirculation loop sampler. Record sample $\mathrm{pH}$ in the logbook. Adjust $\mathrm{pH}$ as needed maintain the $\mathrm{pH}$ range of 2 - 3 .

11. Stop filtration and diaphragm pump.

12. Isolate the filter-loop from UFP-VSL-T02A. Drain UFP-T02A and the remainder of the sample loop. 
Appendix $\mathrm{H}$

\section{Pump Operating Guidance}





\section{Appendix H: Pump Operating Guidance}

1. Initial Pump Startup.

a. Ensure that seal pot pressures are within spec.

b. START pump T42A in MANUAL at $25 \%$.

c. If not already positioned, POSITION flow control valve 0609 to $50 \%$ OPEN.

d. Allow system parameters to stabilize for one minute or when flow rates and pressures appear stable, whichever is longer.

e. START pump T43A in MANUAL at $25 \%$.

f. Increase pump T43A speed in $10 \%$ increments, waiting one minute before each increase, until the target flow rate is achieved.

g. MONITOR system pressures, ensure that PT-0625 is not lower than -200 inches water, PT-0633 is positive, and filter pressures are within spec.

h. If pump T43A speed is at $95 \%$ and desired flow rate has NOT been achieved, INCREASE pump T42A speed in 5\% increments and DECREASE pump T43A to obtain desired flow rate.

i. If desired flow rate is not achieved, OPEN flow control valve $06095 \%$, do not exceed $60 \%$ OPEN.

j. MONITOR system pressures, ensure that PT-0625 is not lower than -200 inches water, PT-0633 is positive, and filter pressures are within spec.

k. If desired flow rate is not achieved, repeat previous three steps.

2. MONITOR and MAINTAIN system flow rate during testing.

a. Monitor system flow rates and pressures for oscillations or erratic behavior. If unstable or erratic system performance is observed restore stable operation by performing the following in order of preference from most preferred to least preferred:

i. Reduce sources of air entrainment.

ii. Reduce flow rate by closing flow control valve and reducing pump speed in alternating 5\% increments until lowest acceptable flow rate is reached.

iii. Increase level in tank T02A to highest acceptable level.

3. Pump SHUT DOWN.
a. Turn pump T43A OFF.
b. Adjust pump T42A to $25 \%$ speed.
c. CLOSE flow control valve 0609.
d. Turn pump T42A OFF. 



\section{Appendix I}

\section{T02A Systems Operations Guidance for Integrated Test B}





\section{Appendix I: T02A Systems Operations Guidance for Integrated Test B}

\section{I.1 T02A Systems Operations Guidance for Integrated Test B}

In the event that filter-loop flow rates cannot be maintained at the targeted $109 \pm 10$ gpm using the pump-control guidance and there is evidence that there is significant entrained air in the slurry, the following actions are to be taken.

\section{I.1.1 T02A Systems Guidance}

1. Turn level bubbler off.

2. Turn steam ring air purge off.

3. Allow 30 minutes to determine if the T02A recirculation system recovers the desired flow rate. If flow is recovered, proceed with testing.

4. If flow does not recover, implement the initial degassing protocol listed below.

5. If flow recovers, proceed with testing.

6. If flow does not recover, turn off lower air spargers.

7. Allow 30 minutes to see if the system recovers flow. If flow is recovered, proceed with testing.

8. If flow does not recover, implement initial degassing protocol.

9. If flow recovers, proceed with testing.

10. If flow does not recover contact the on-call Test Director.

11. Perform the extended degassing protocol listed below as needed to fully degas the system.

12. Reinitiate testing.

13. If flow rates cannot be maintained at the targeted $109 \pm 10$ gpm perform the initial degassing protocol.

14. If flow does not recover, reduce the filter recirculation flow to $88 \pm 10 \mathrm{gpm}$ filter-loop flow rate (gives $\sim 12 \mathrm{ft} / \mathrm{s}$ axial velocity in the filter tubes).

15. If $88 \mathrm{gpm}$ cannot be maintained perform the extended degassing protocol as needed to fully degas T02A.

16. Reinitiate testing with the filter recirculation loop flow rate reduced to $67 \pm 10$ gpm filter-loop flow rate (gives $\sim 9 \mathrm{ft} / \mathrm{s}$ axial velocity in the filter tubes and power/volume scaled mixing from the return nozzle jet).

17. If none of these actions are successful, begin raising the T02A level by diluting with IW. Raise the tank level in 2 inch increments, allowing at least 30 minutes between IW additions. 
Initial degassing protocol

1. Turn off filter-loop pumps.

2. Take a stable level measurement.

3. Adjust lower air sparge flow rate to normal full flow $(0.40 \mathrm{~kg} / \mathrm{min}, \sim 2.4 \mathrm{scfm} / \mathrm{tube})$ and turn on PJMs.

4. Allow this configuration to run for $50 \mathrm{~min}$.

5. Turn off spargers and PJMs.

6. Wait for $10 \mathrm{~min}$.

7. Take a stable level measurement.

8. Turn pumps on and attempt to achieve target filter-loop flow rate.

9. Restore T02A mixing systems per the stage of the T02A systems operating guidance above where the initial degassing process was referenced.

Extended degassing protocol

1. Turn off filter-loop pumps and take a stable level measurement.

2. Adjust lower air sparge flow rate to normal full flow $(0.40 \mathrm{~kg} / \mathrm{min}, \sim 2.4 \mathrm{scfm} / \mathrm{tube})$ and turn on PJMs.

3. Allow this configuration to run for 4.5 hours.

4. Turn off spargers and PJMs.

5. Wait for 20 minutes.

6. Operate the filter-loop pumps for 10 minutes.

7. Take a stable level measurement.

8. Repeat steps 2 through 7 as needed to degas T02A as determined by prior degassed level readings or by less than $5 \%$ gas volume holdup measured by centrifuging a slurry sample.

9. Turn pumps on and attempt to achieve target filter-loop flow rate.

10. Restore T02A mixing systems per the T02A systems operating guidance above. 
Appendix $\mathbf{J}$

\section{Integrated Test B Data Plots}



Note: In Appendix J, it is recognized that the correct units are psid although axial pressure drop and transmembrane pressure (TMP) are displayed as psig.

\section{Appendix J: Integrated Test B Data Plots 03/12/09 00:00 to 03/12/09 12:00}

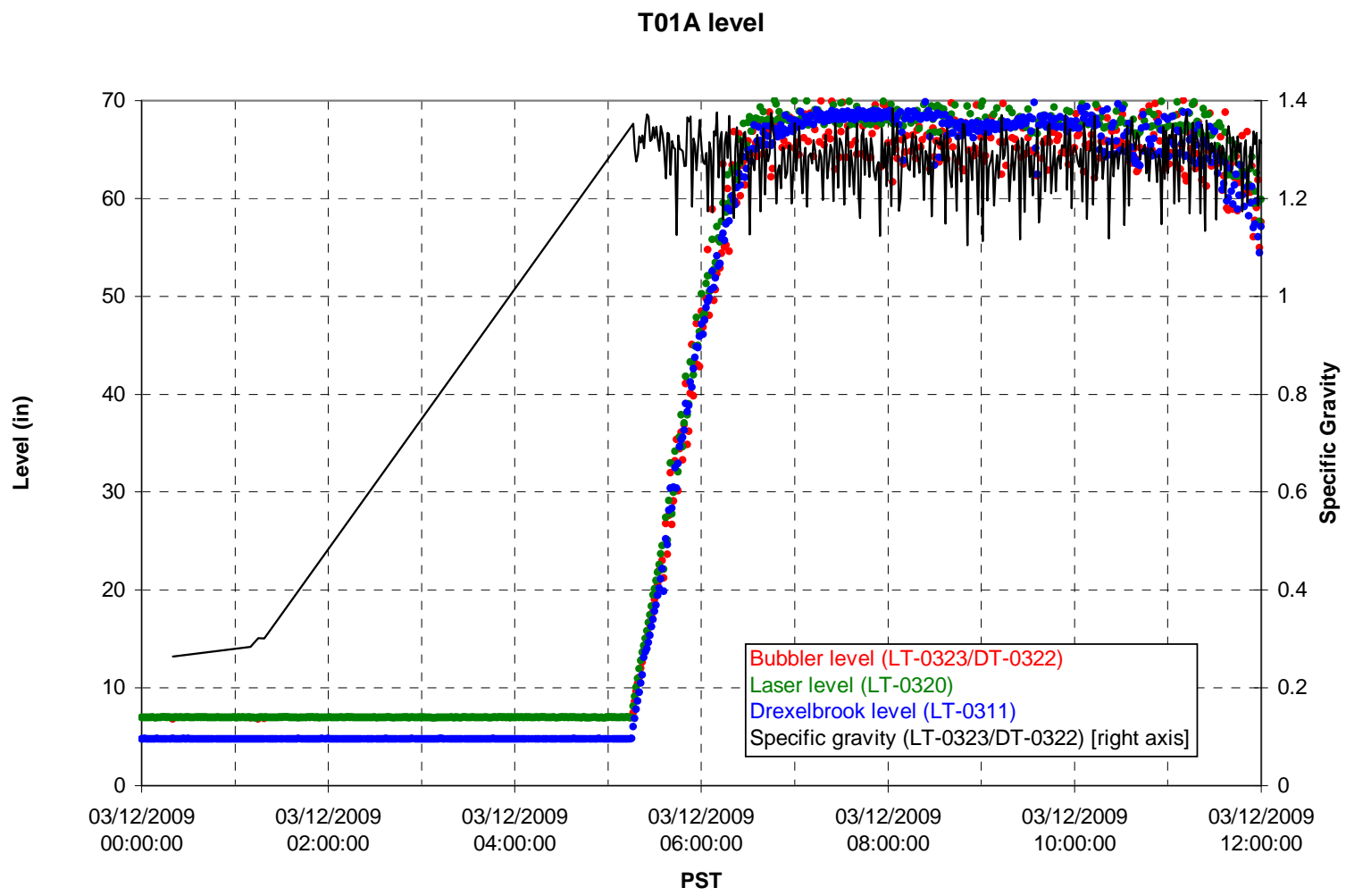


T01A temperatures

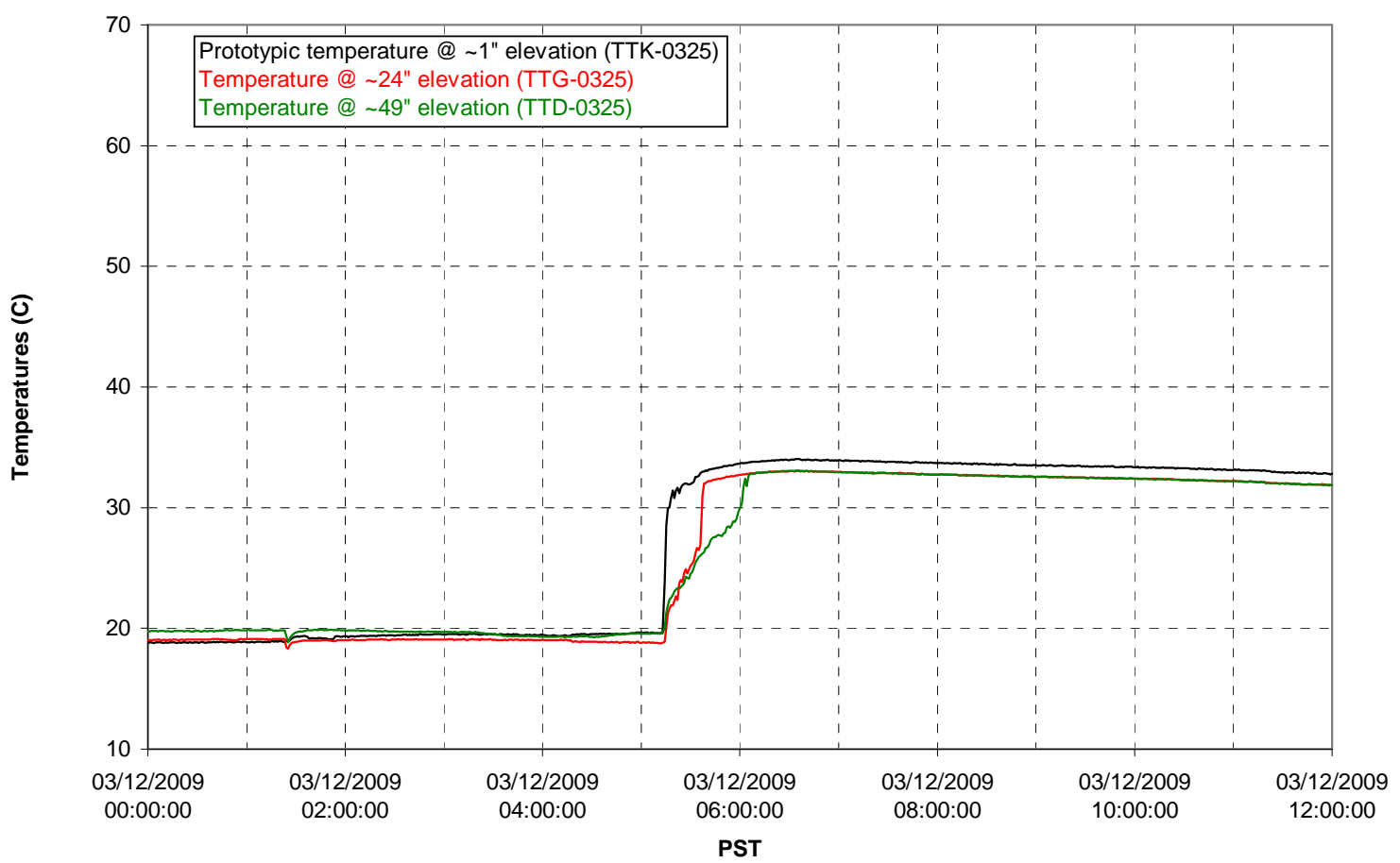

T01B level

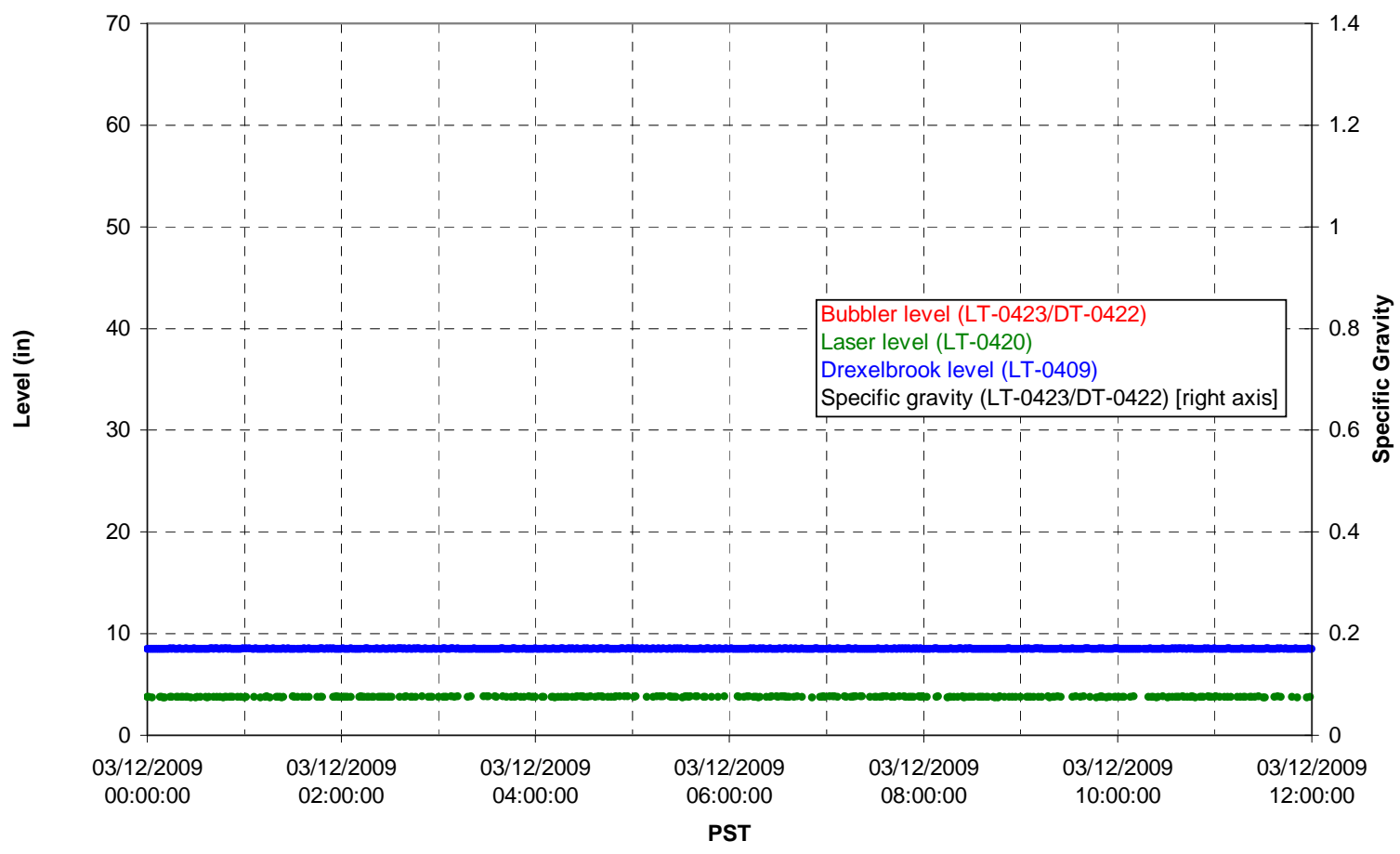


T01B temperatures

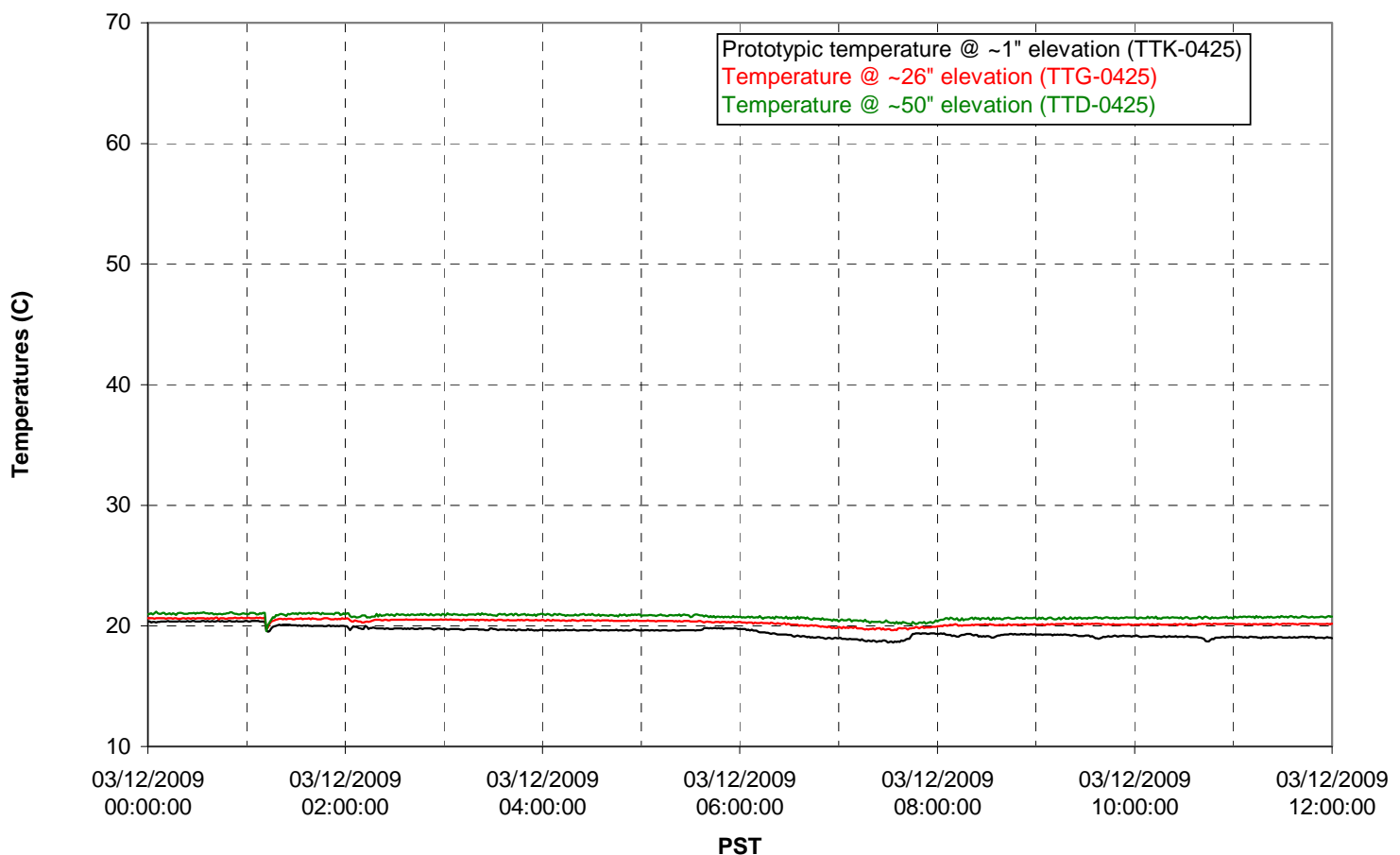

T02A level

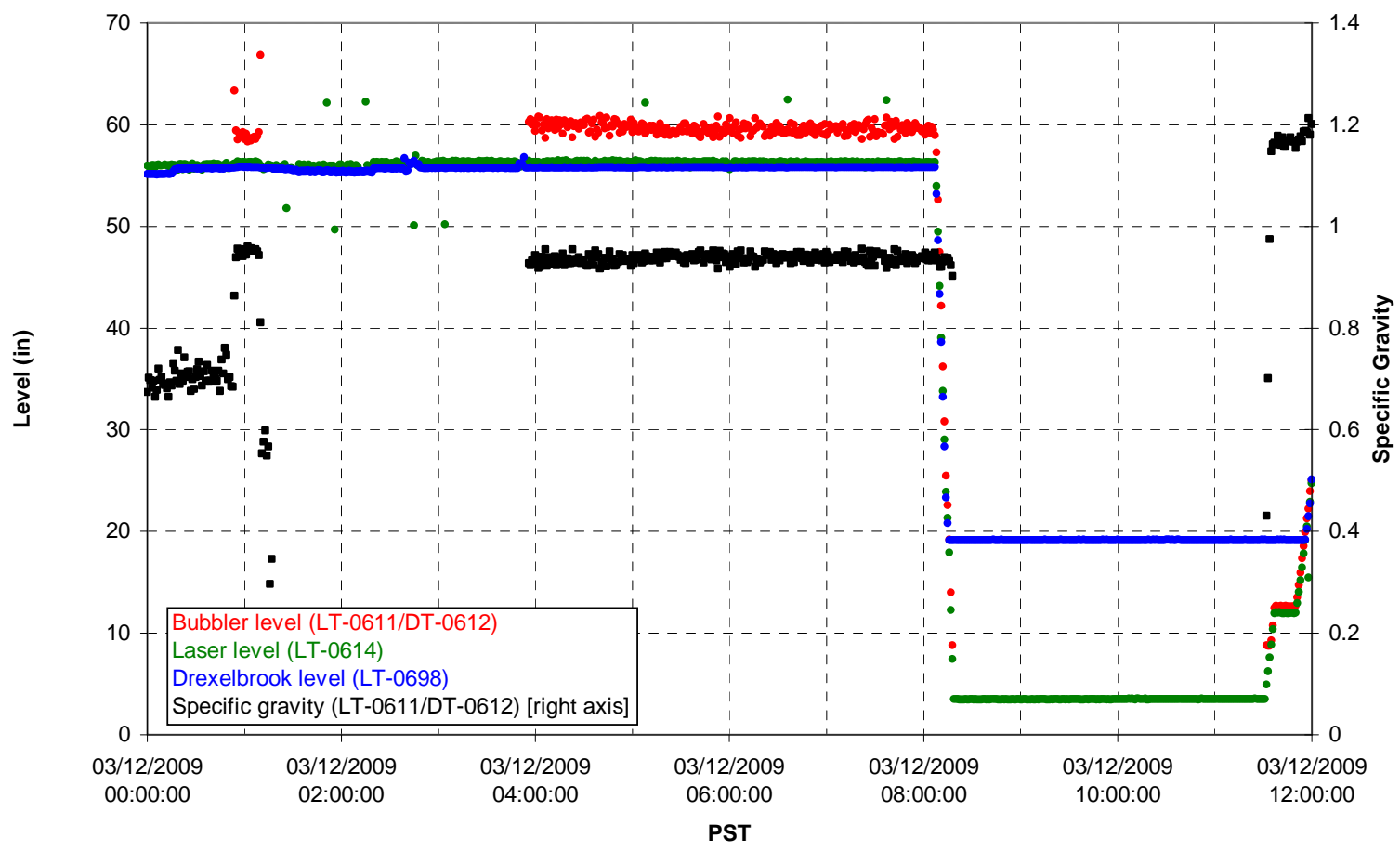


T02A temperatures

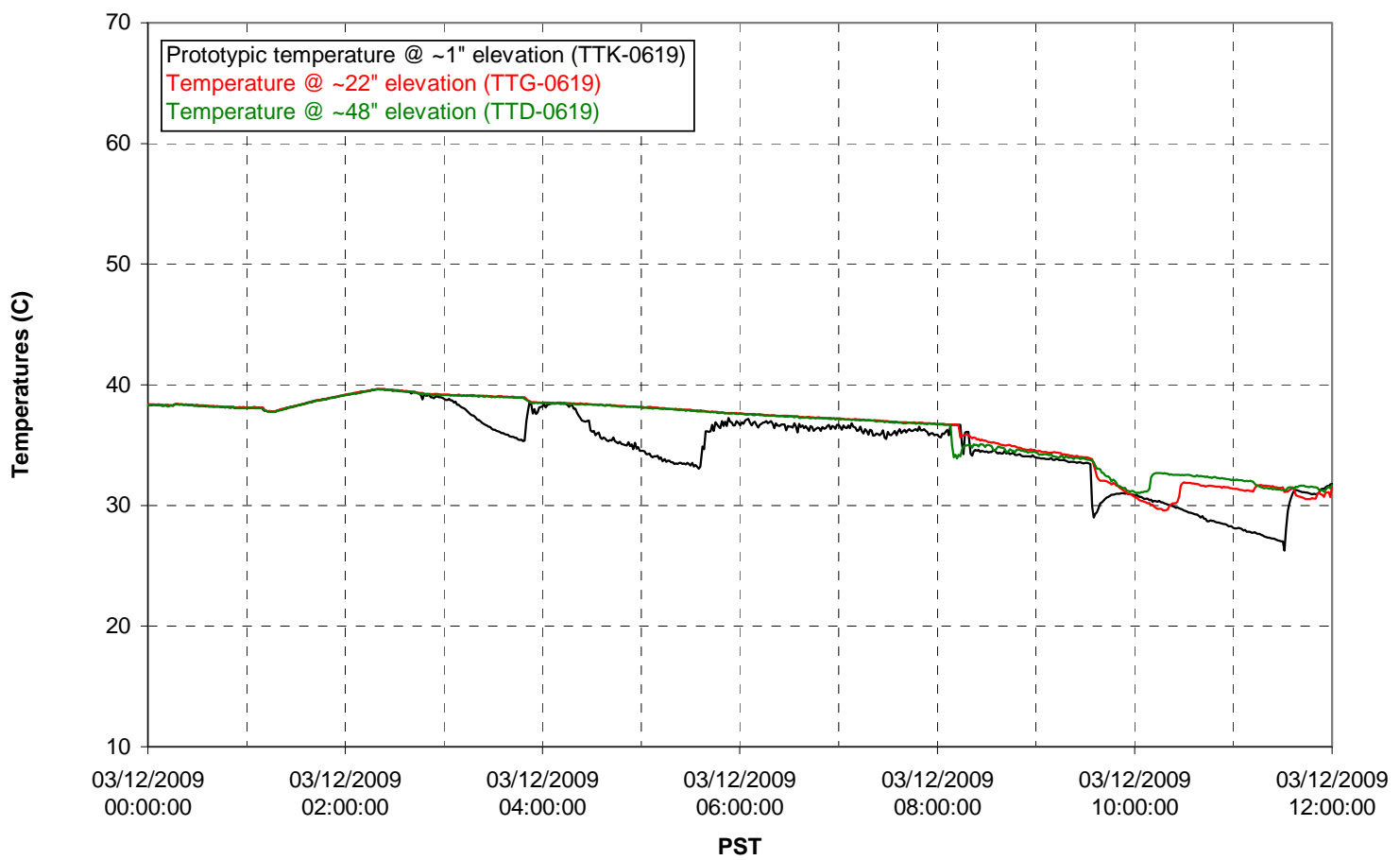

T02A and filter loop temperatures

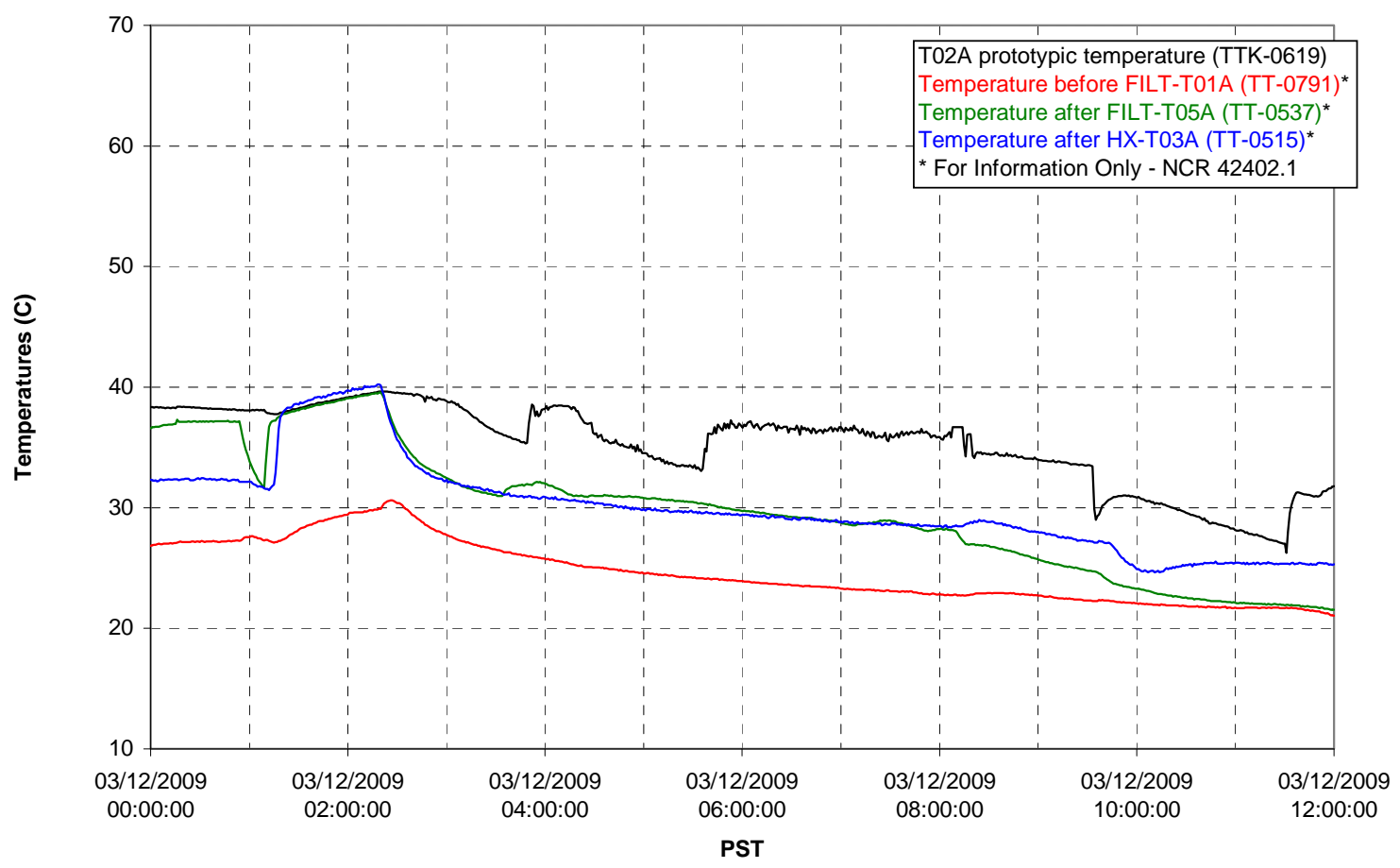


Pump Pressures and Flow

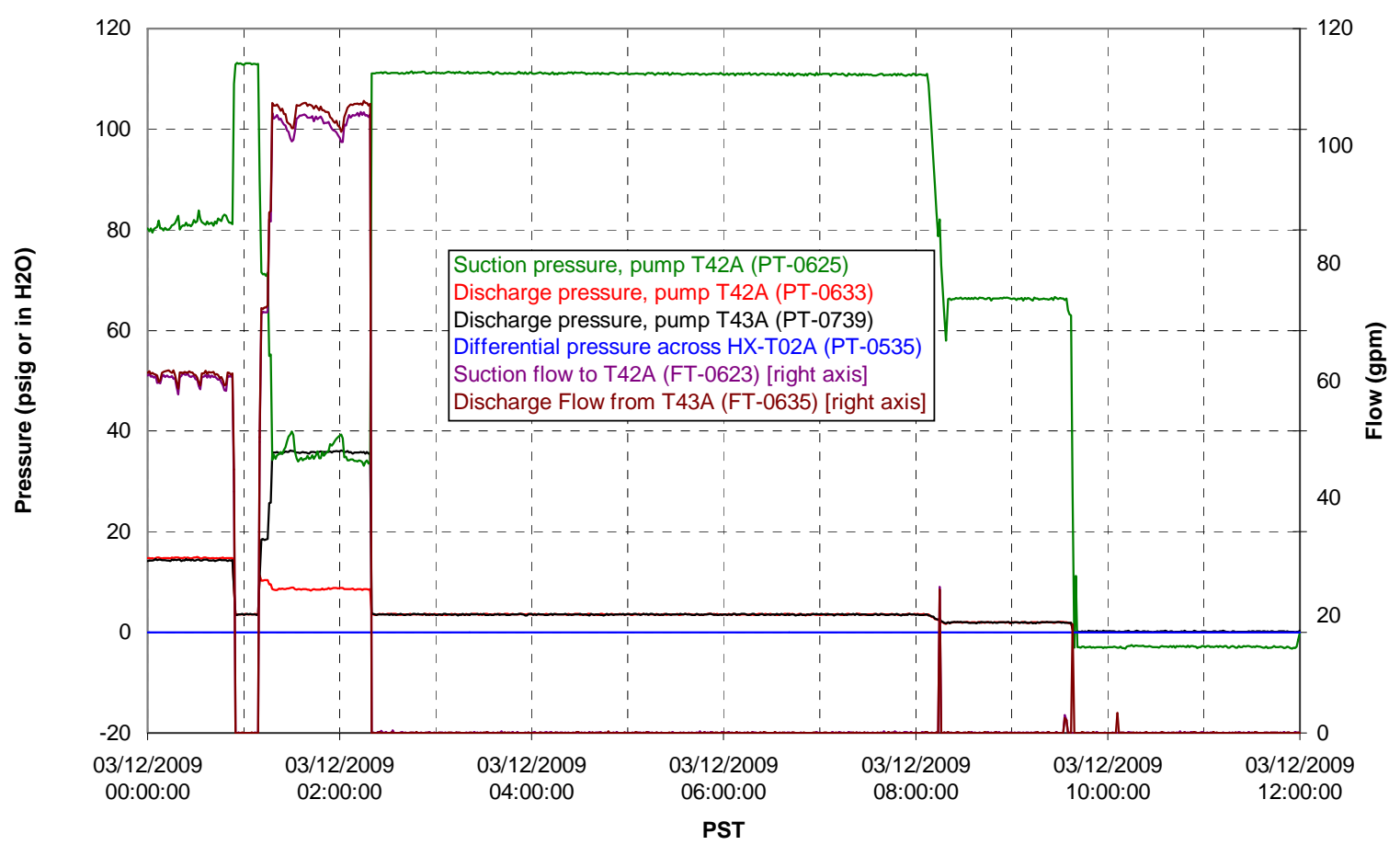

Axial pressure drop

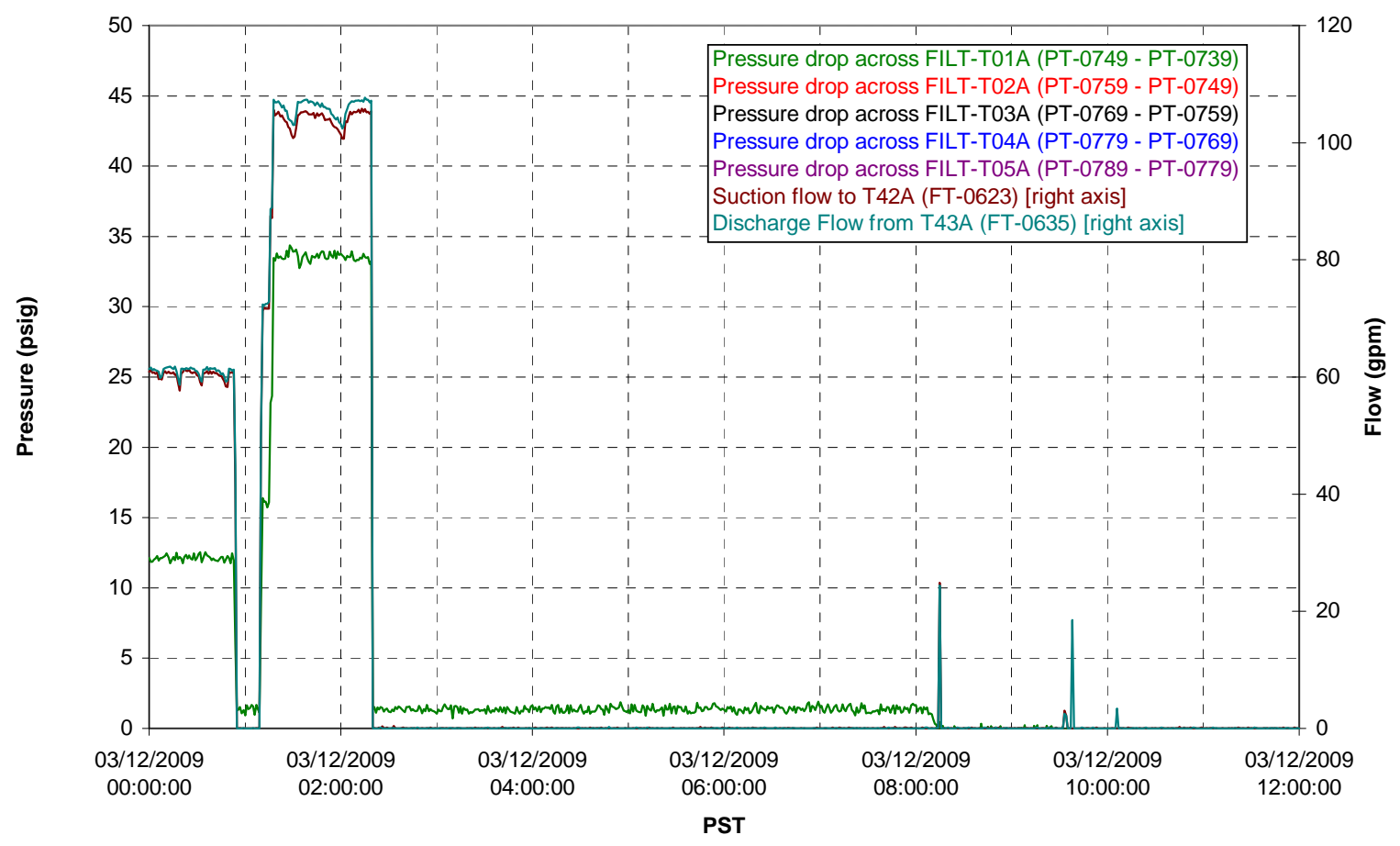


Permeate flow rates

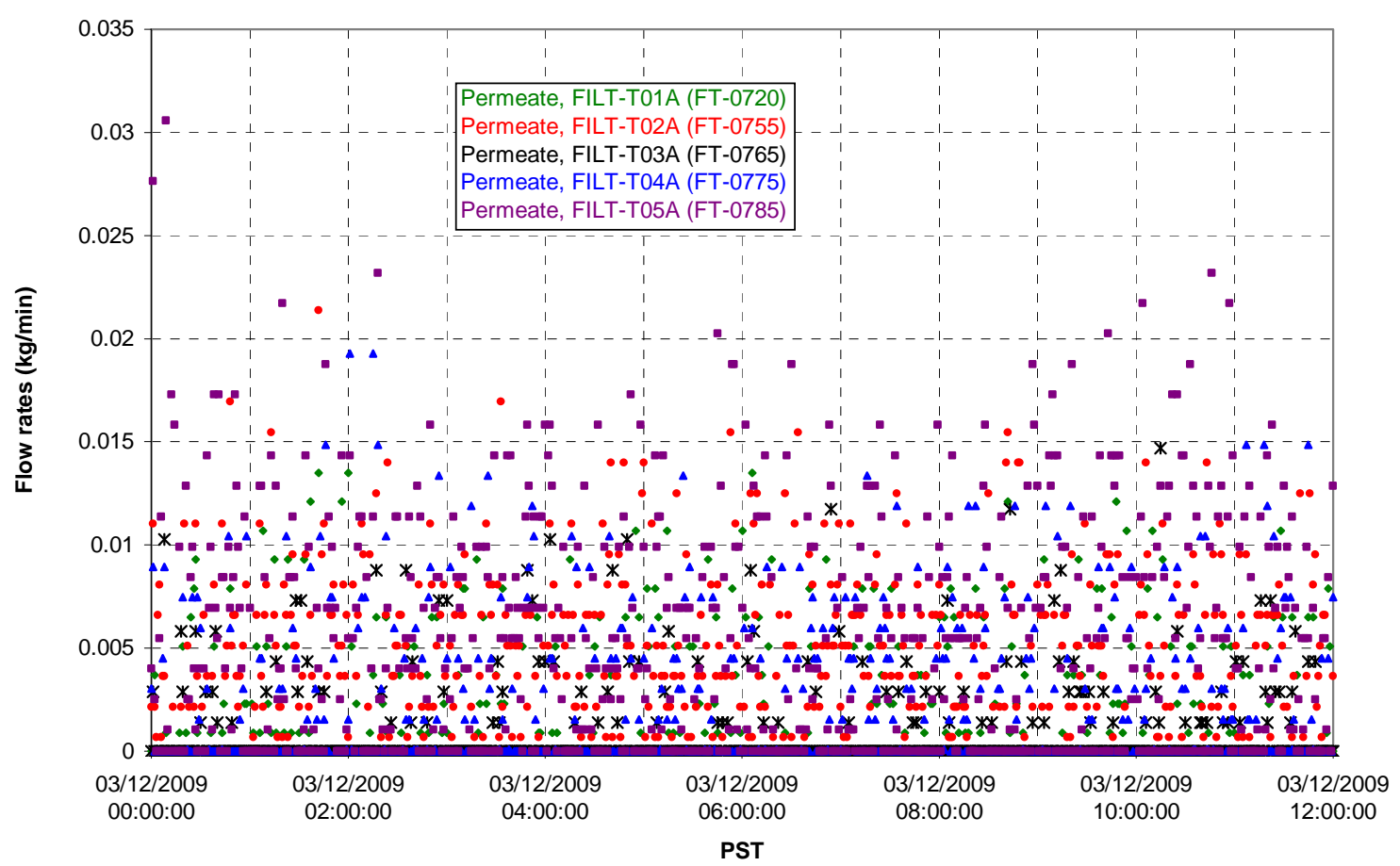

T02A Inner Temperature Tree

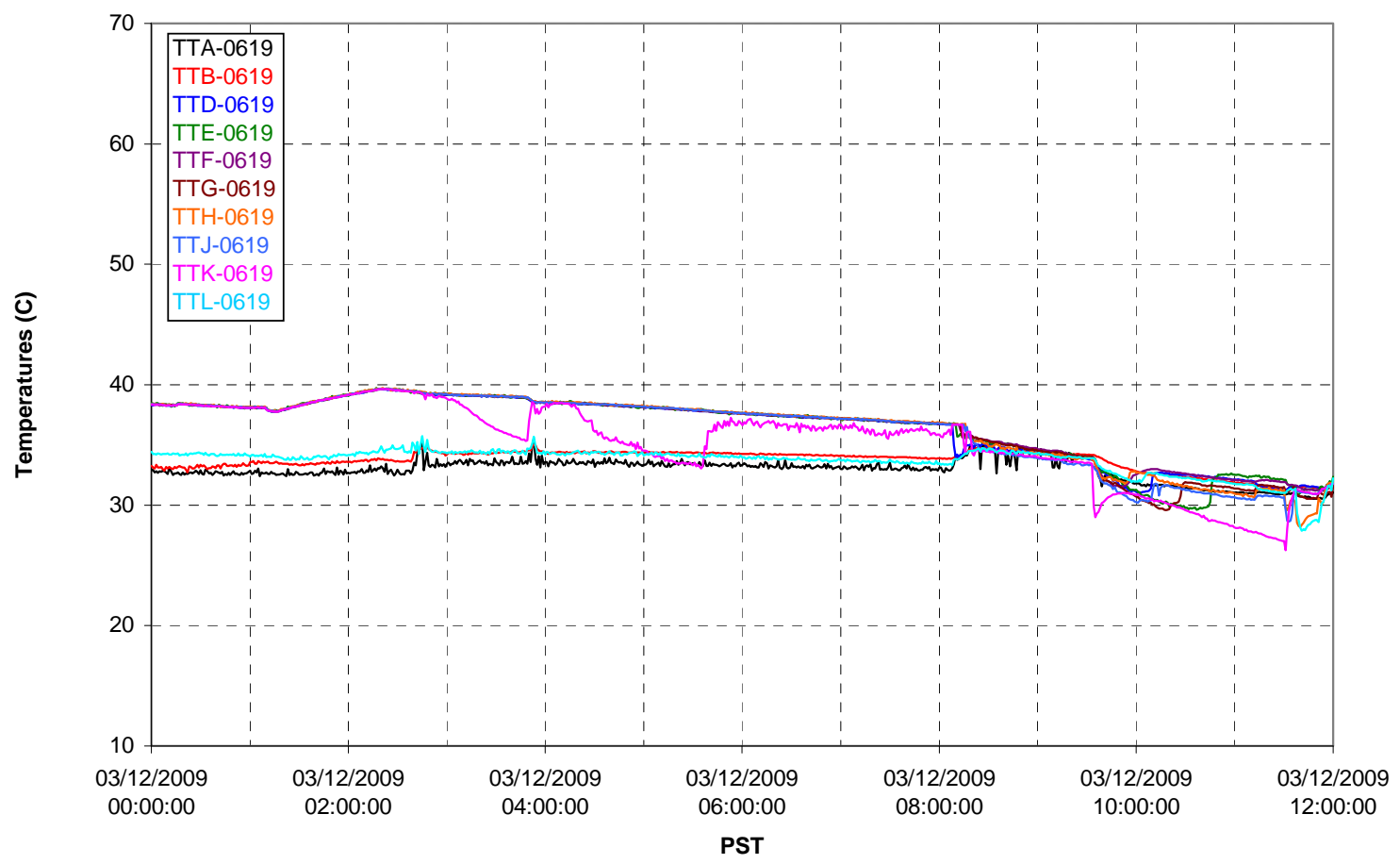


T02A Outer Temperature Tree

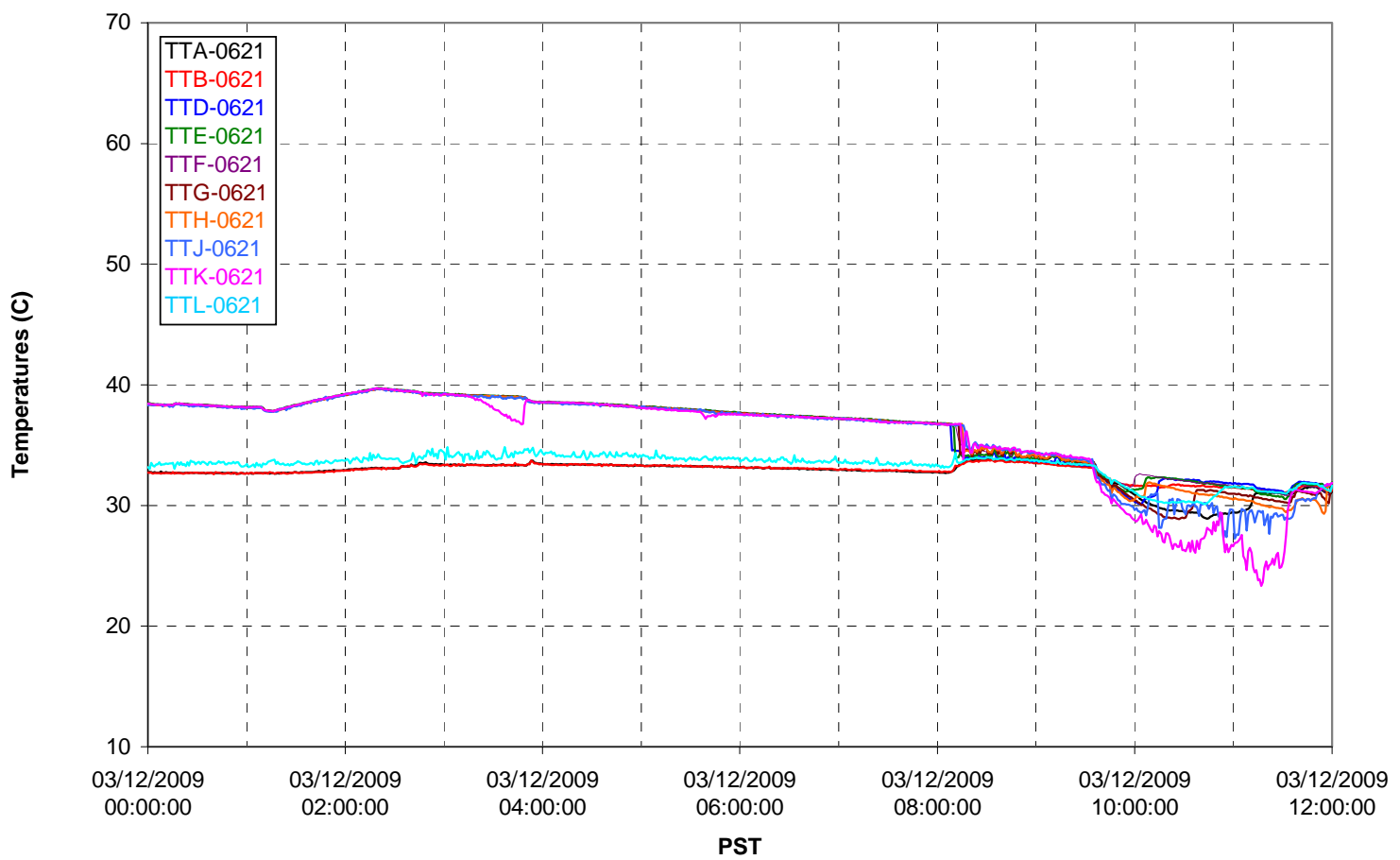

T02A temperatures

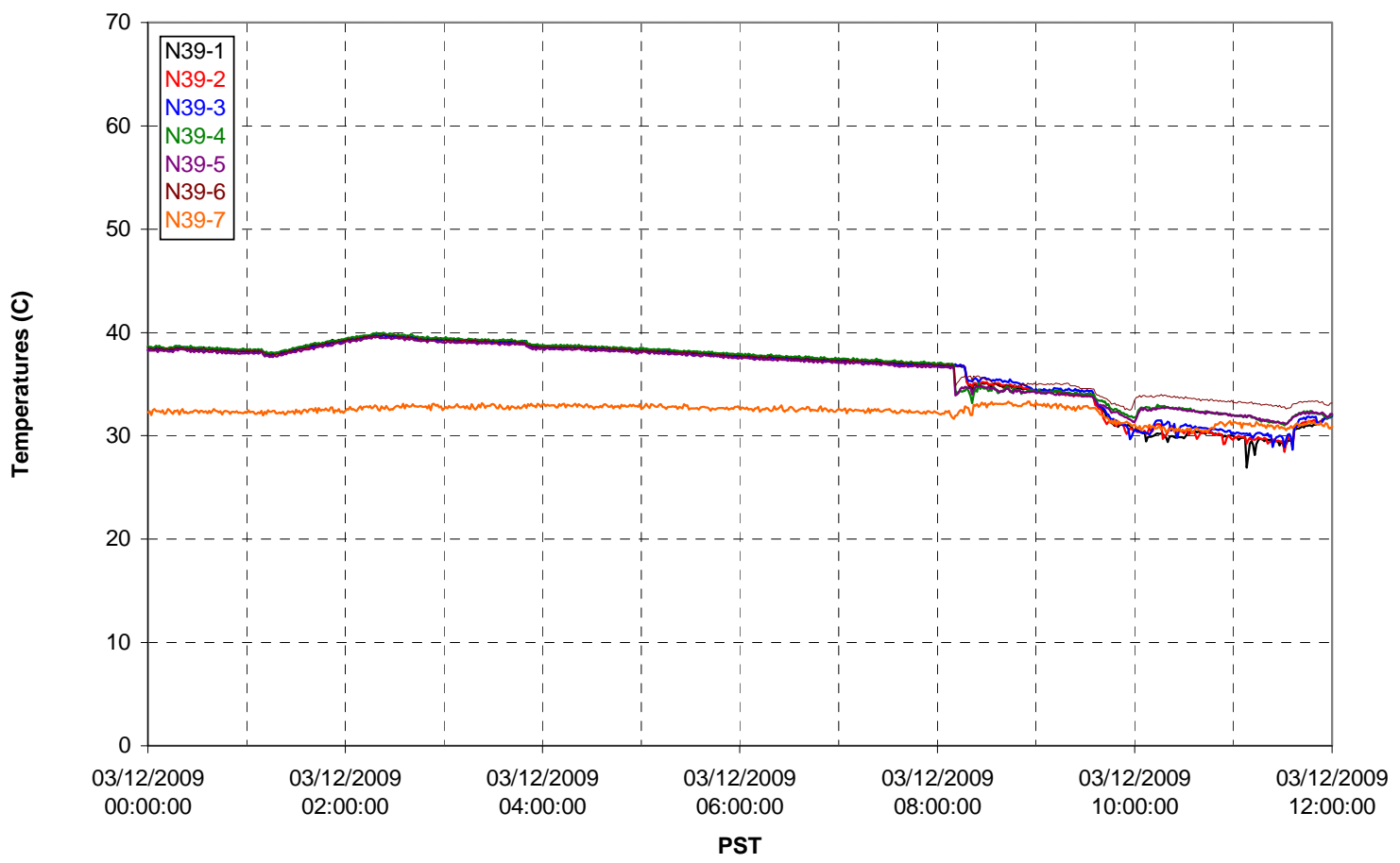


T02A temperatures

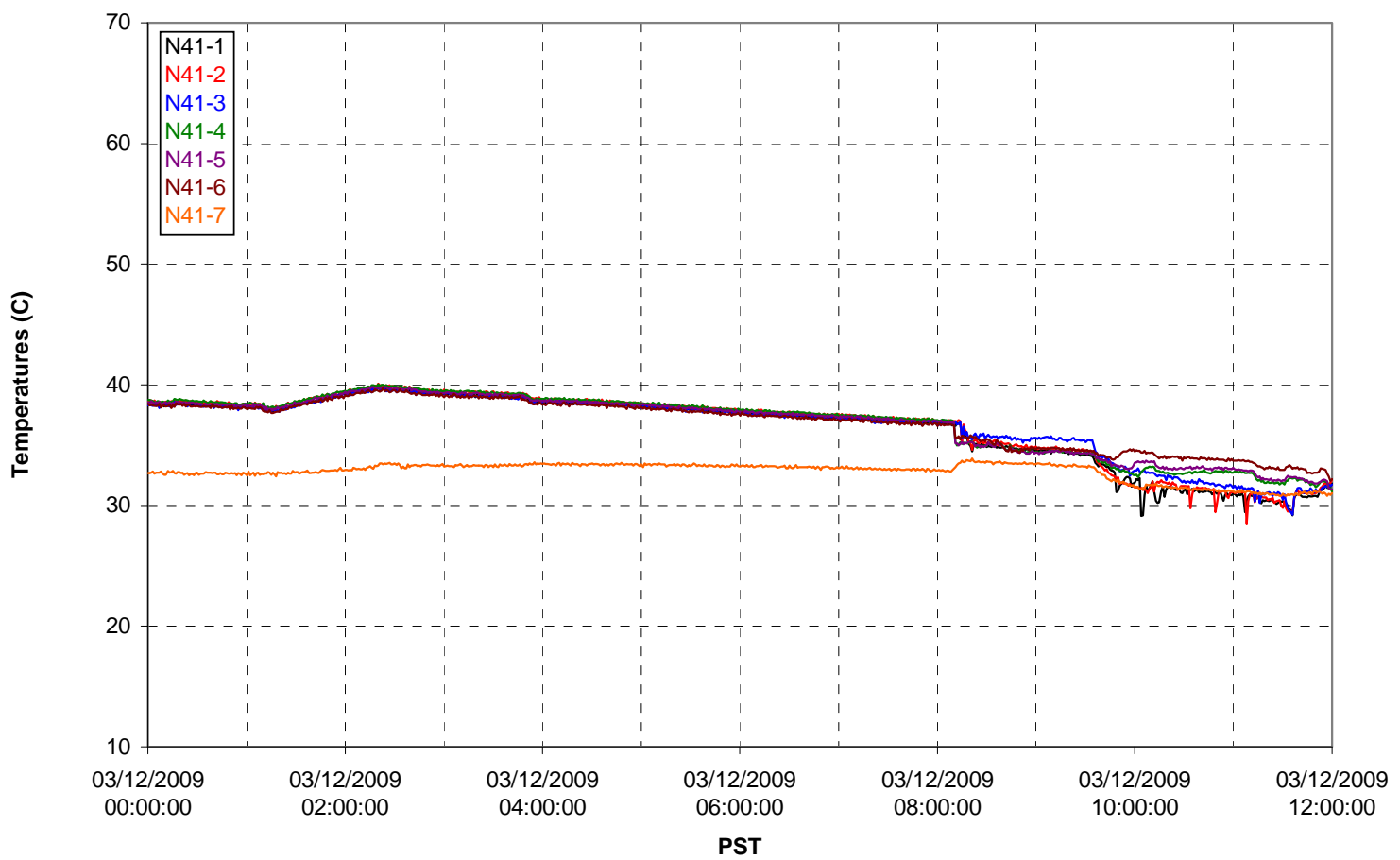

T02A temperatures

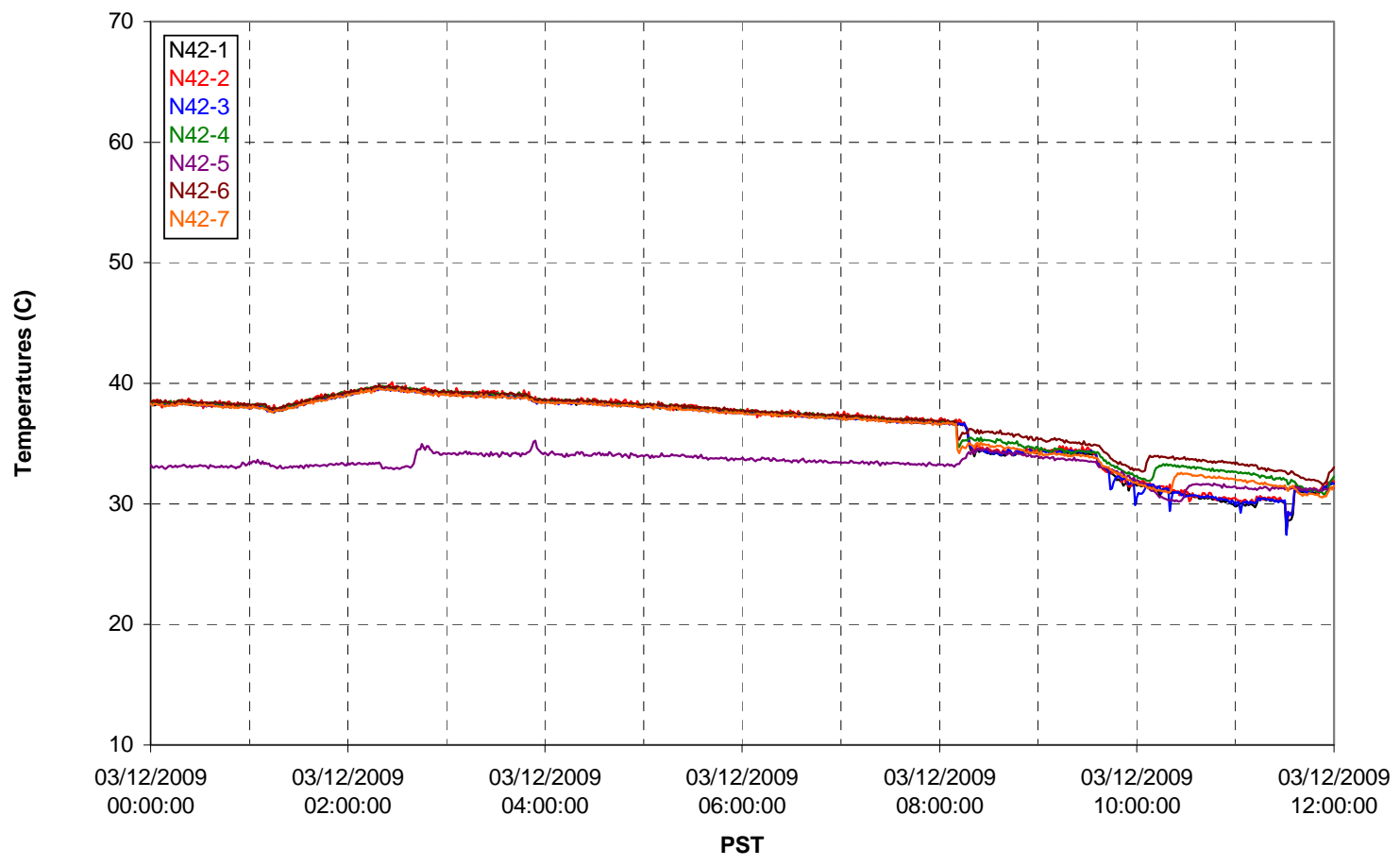


T02A temperatures

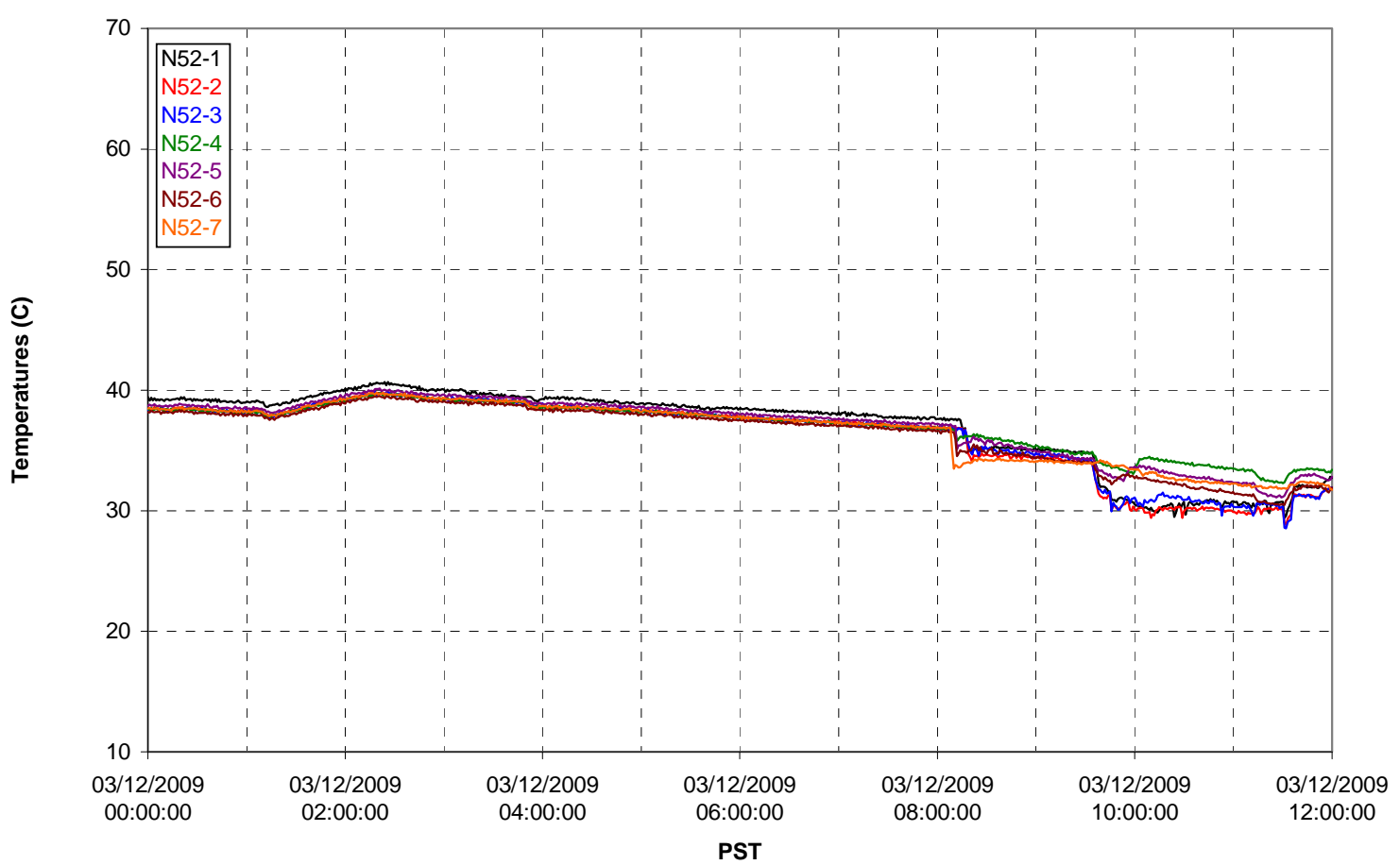

T02A Heating and Cooling

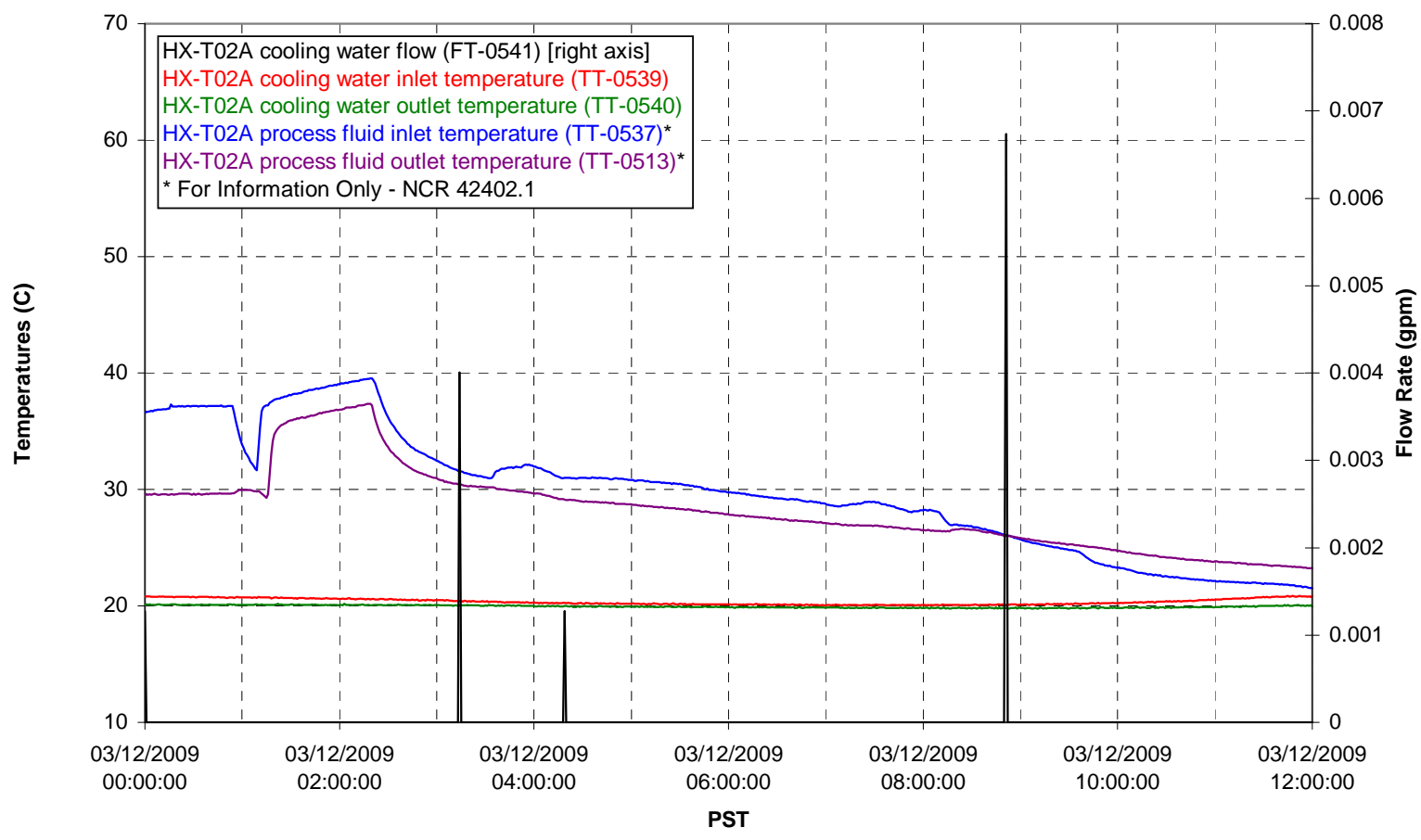


Pump Operation

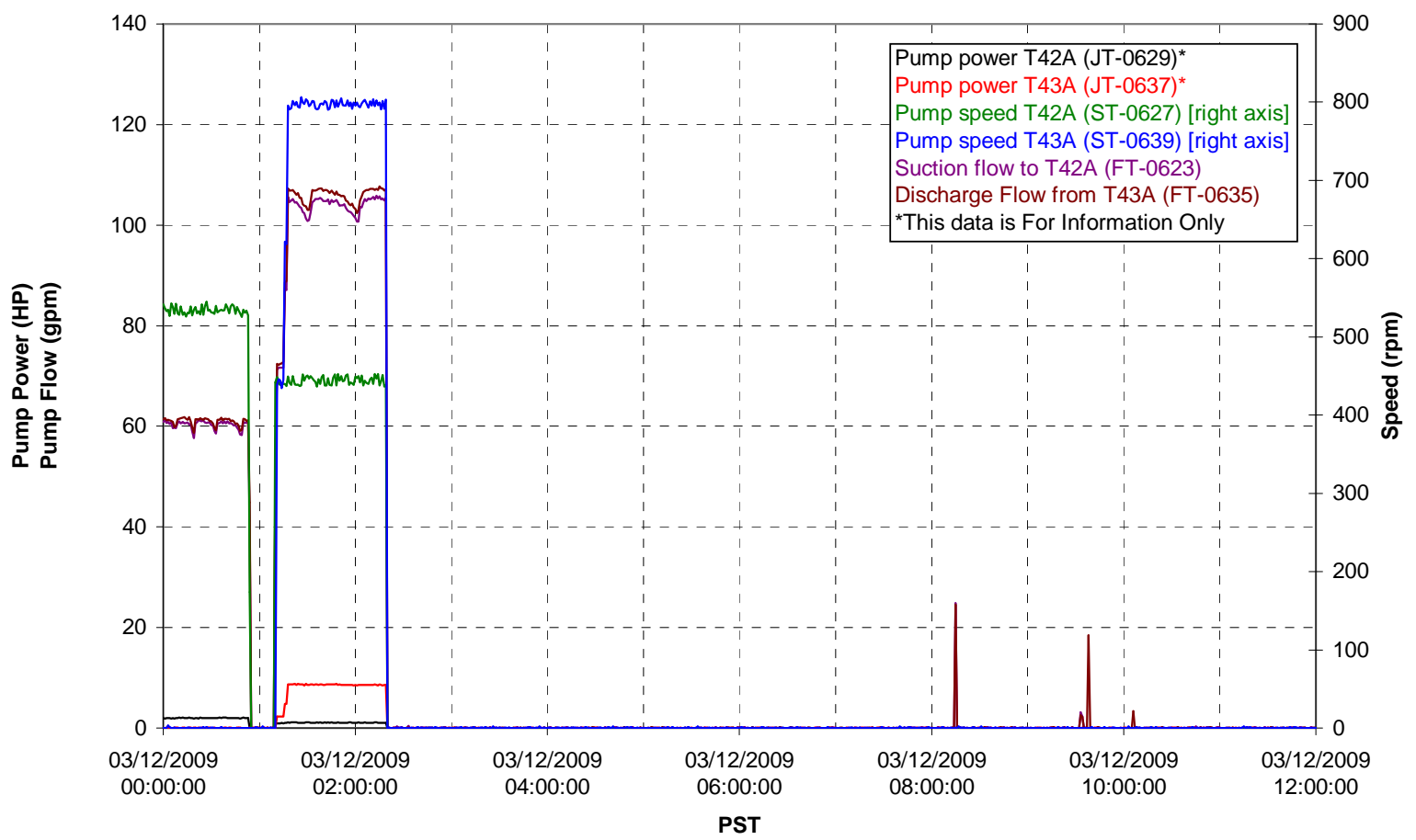

Pulsepot UFP-PP-T01A

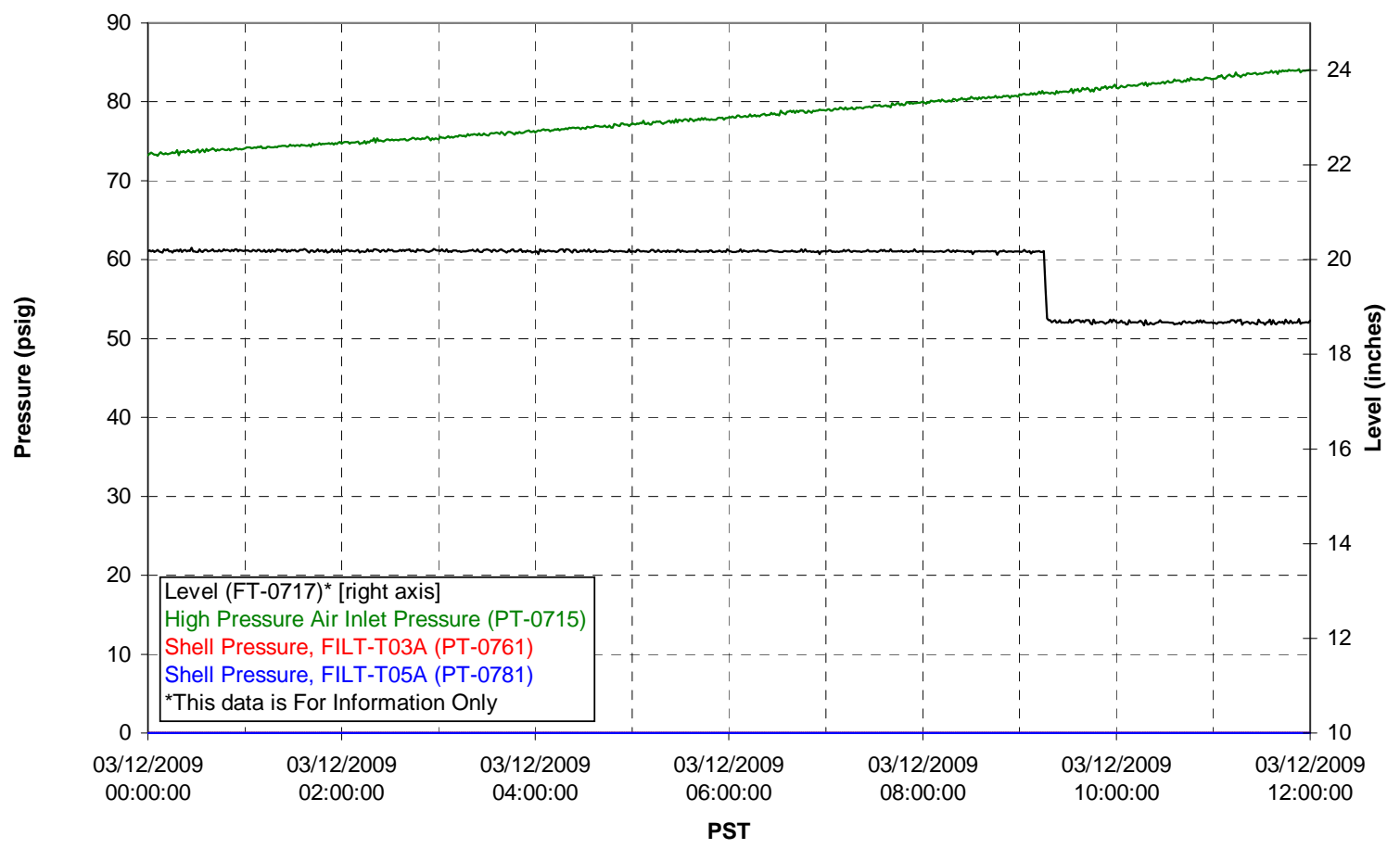


Pulsepot UFP-PP-T02A

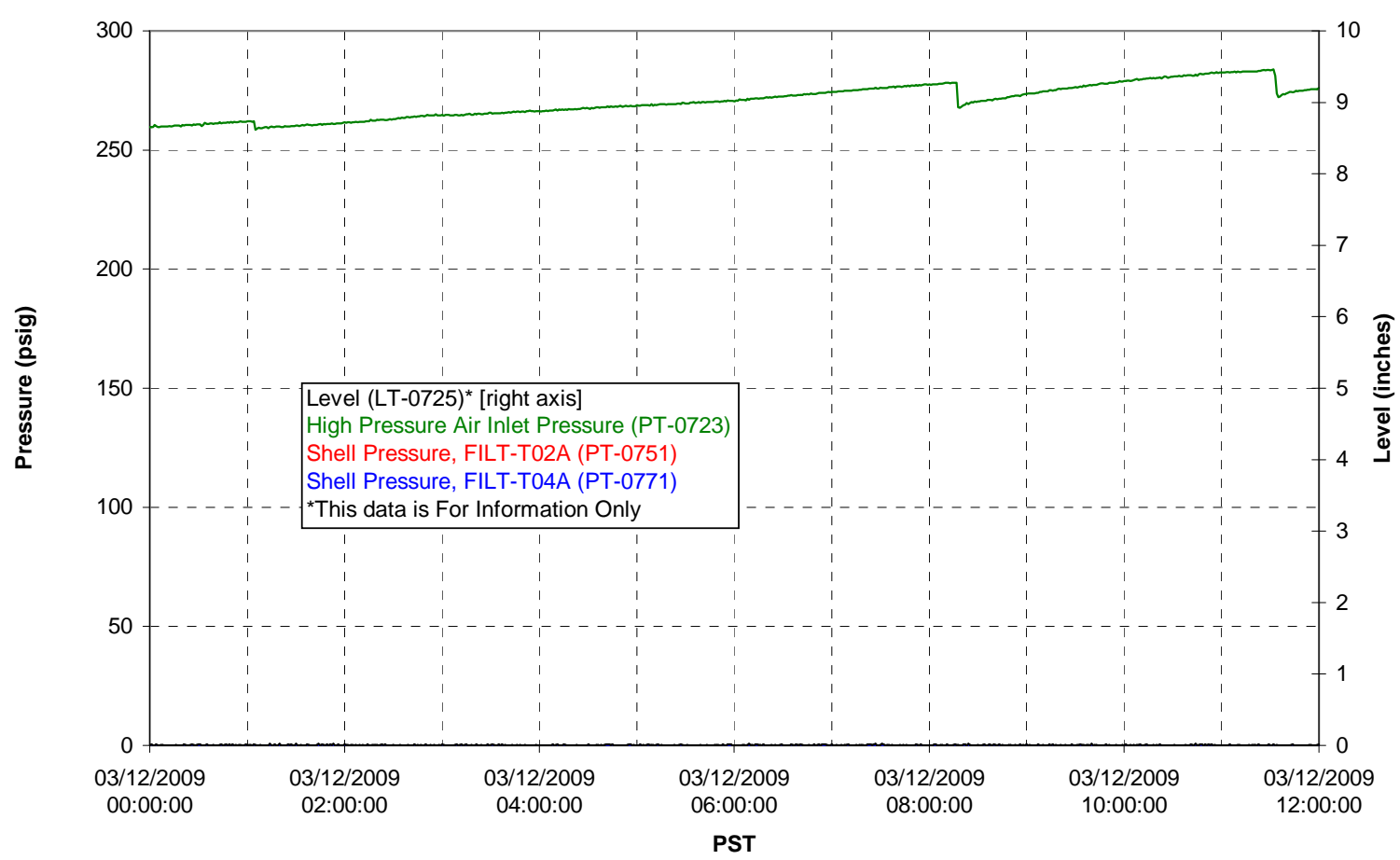

Pulsepot UFP-PP-T03A

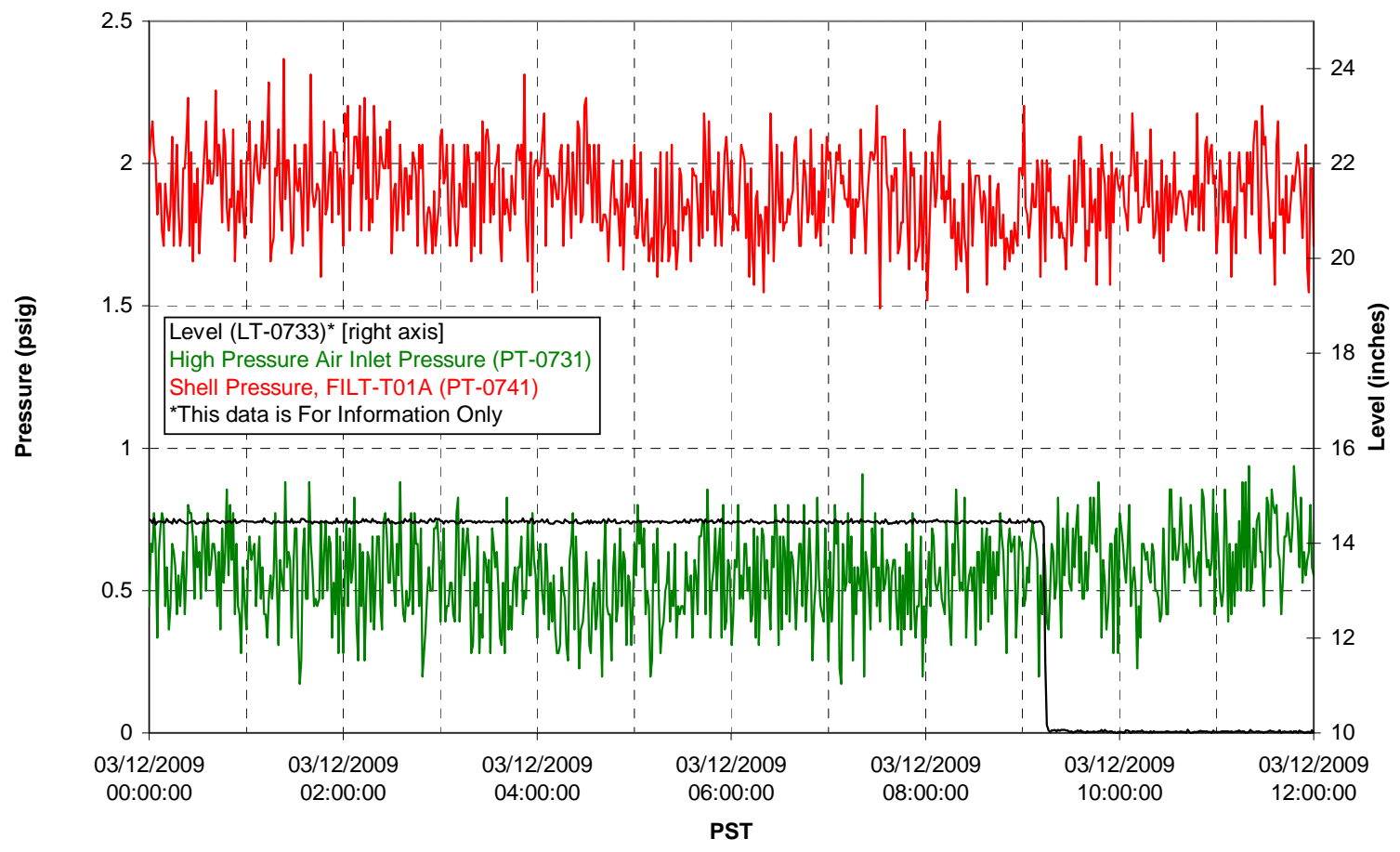


Pulsepot Levels

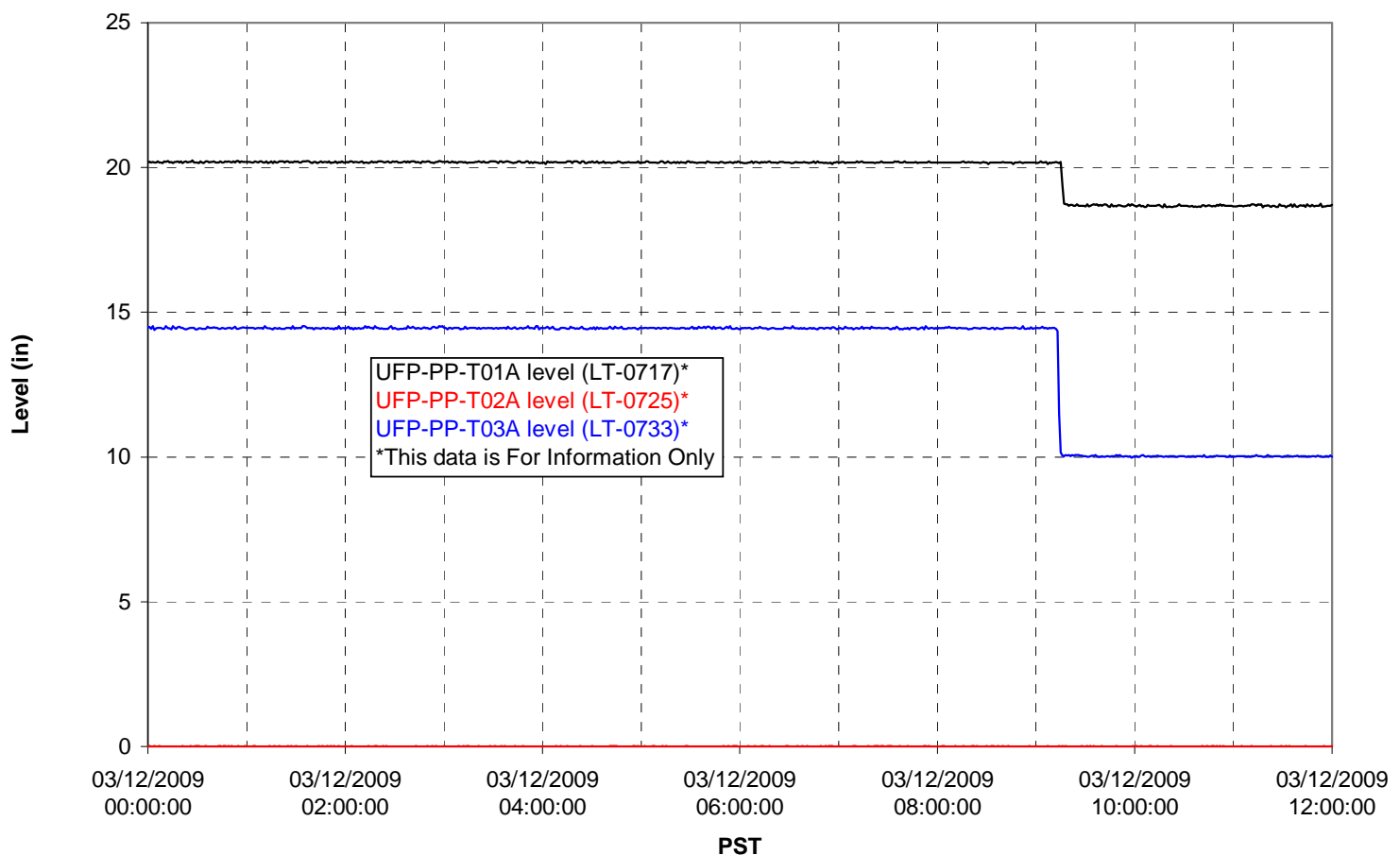

Filter UFP-FILT-T01A

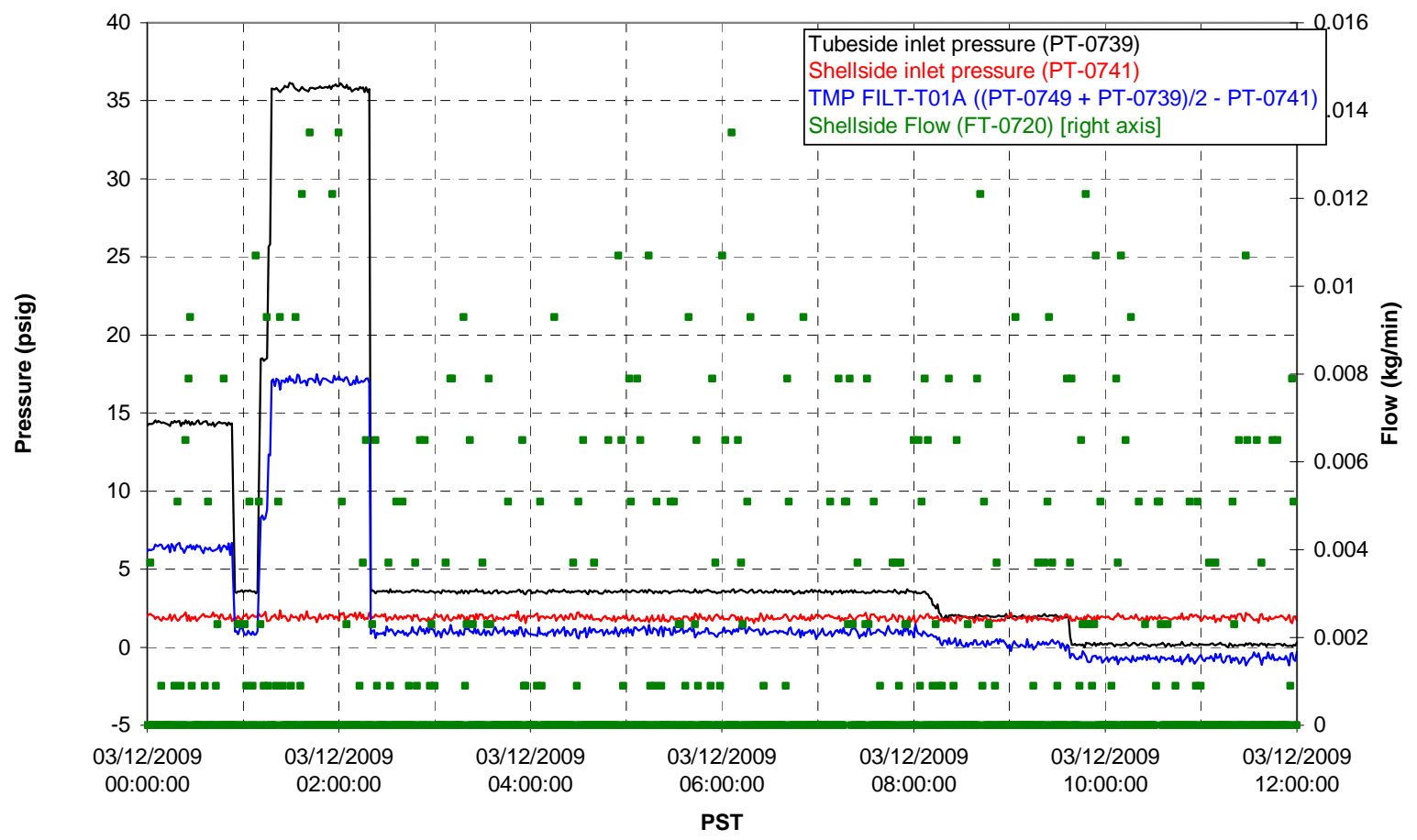


Filter UFP-FILT-T02A

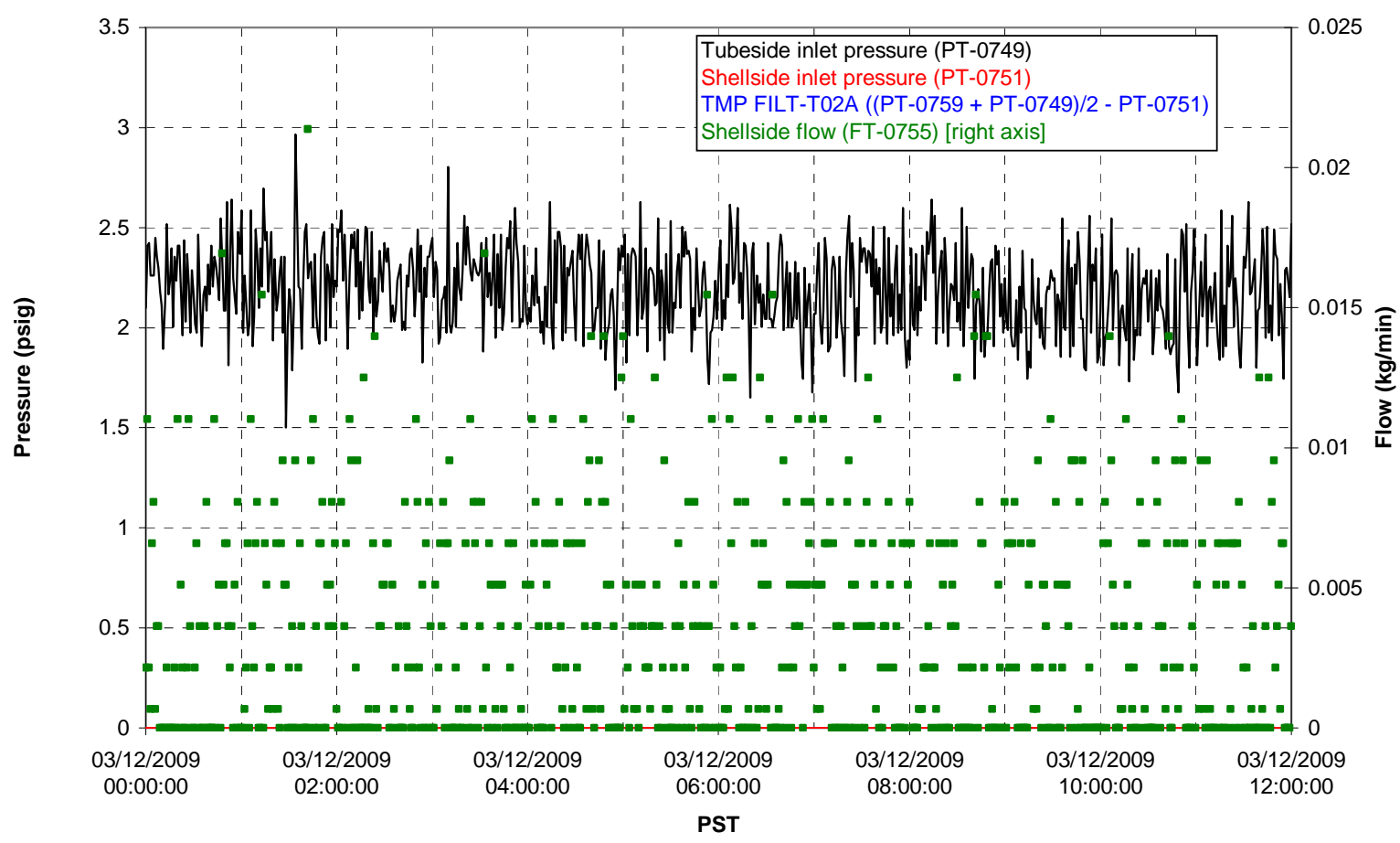

Filter UFP-FILT-T03A

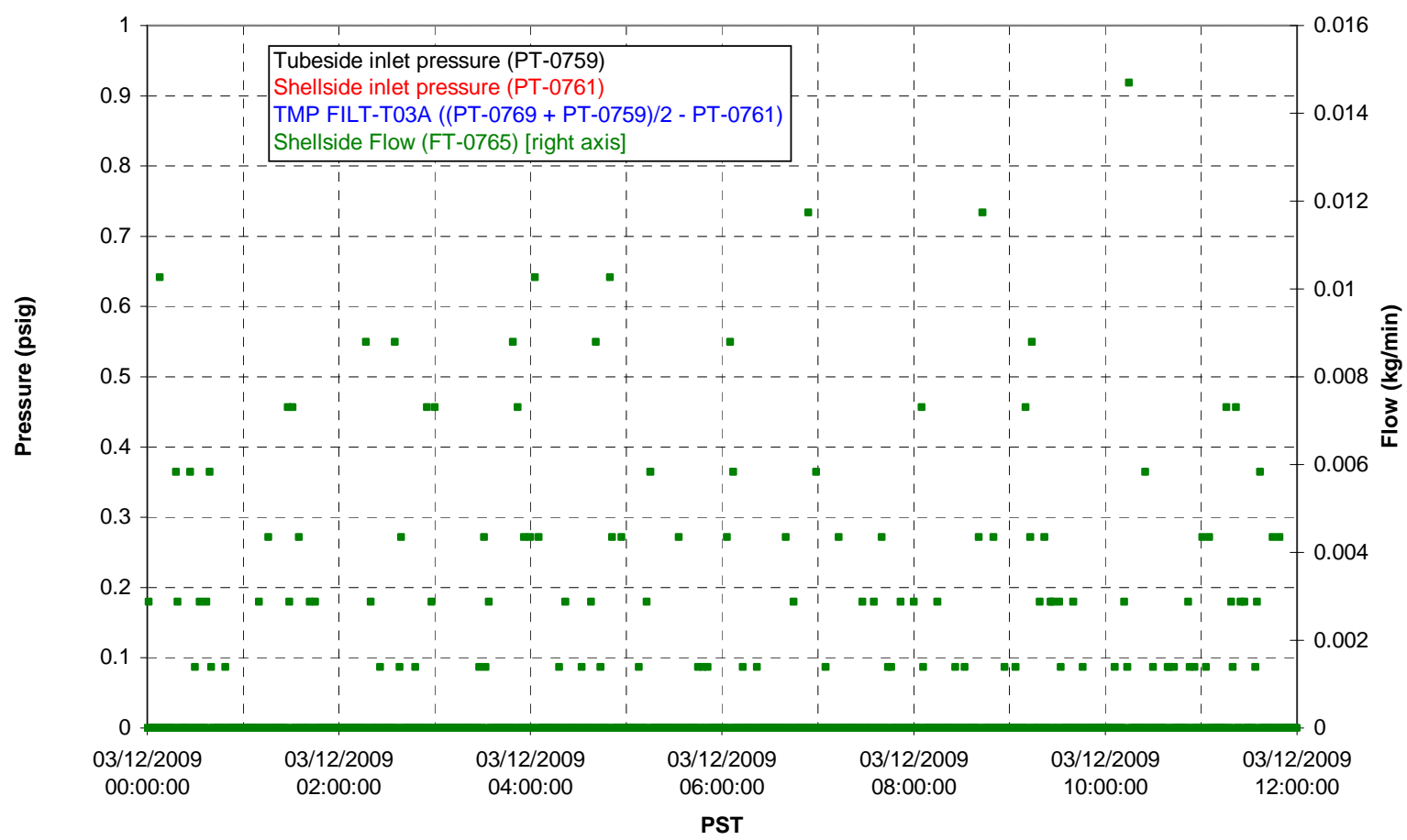


Filter UFP-FILT-T04A

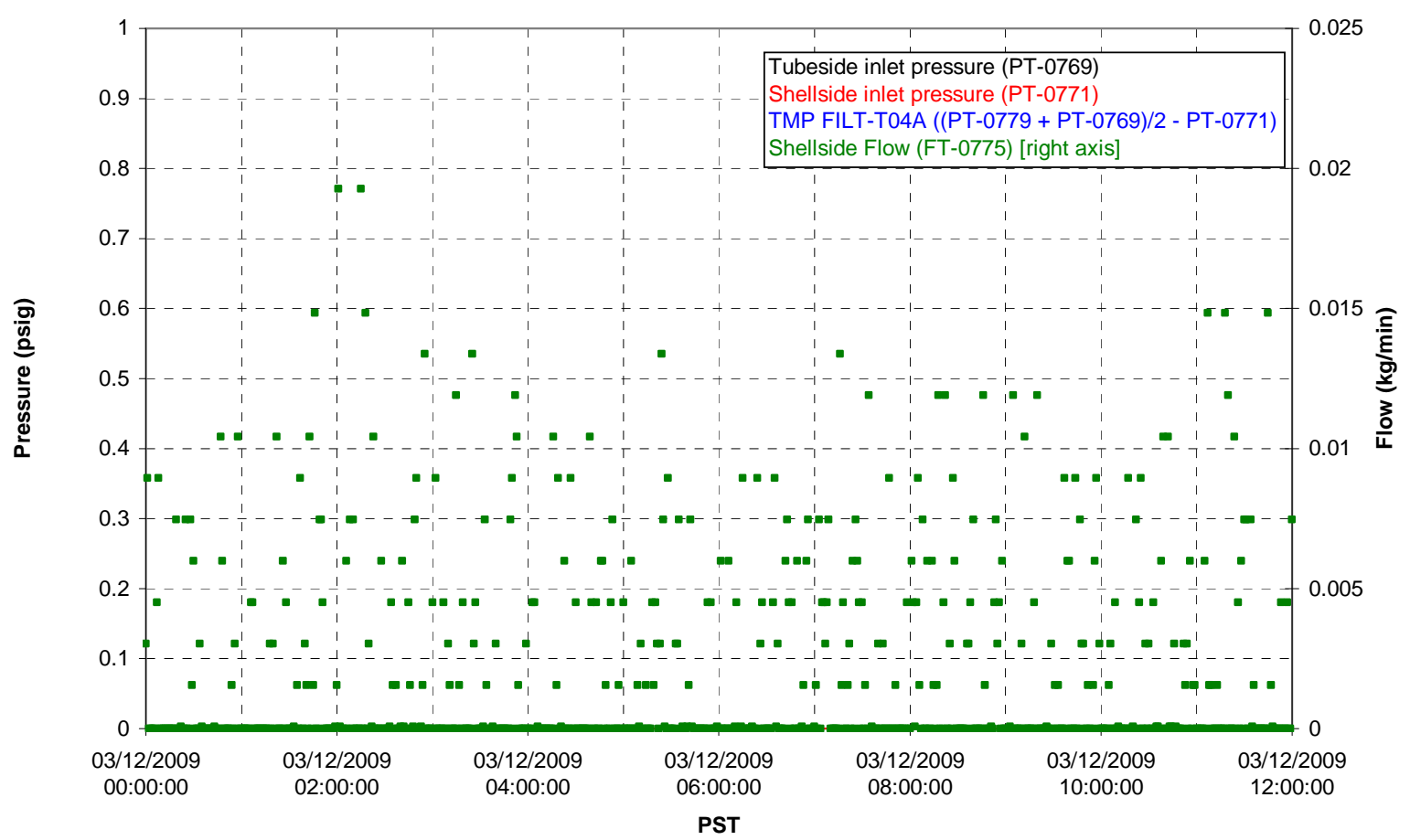

Filter UFP-FILT-T05A

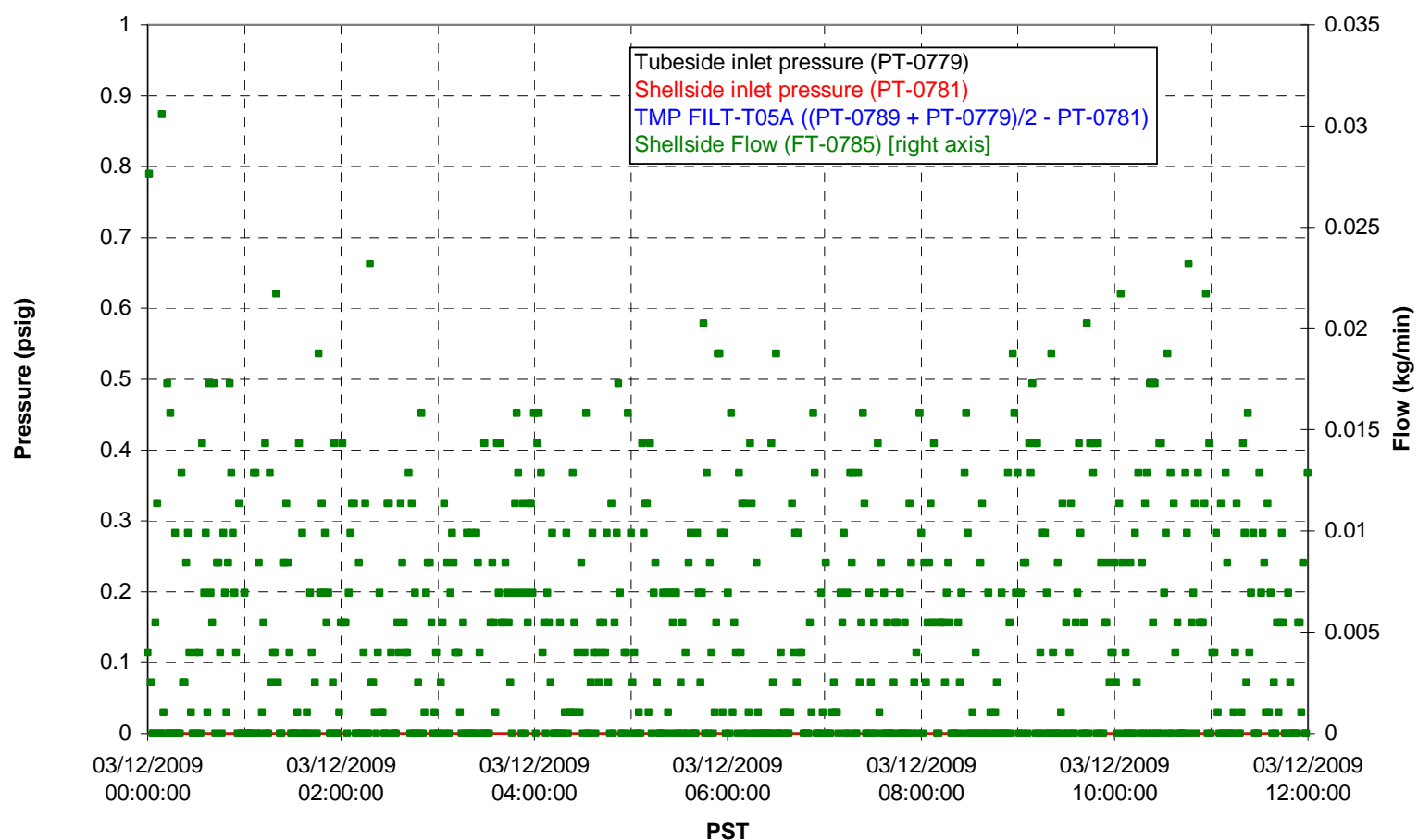



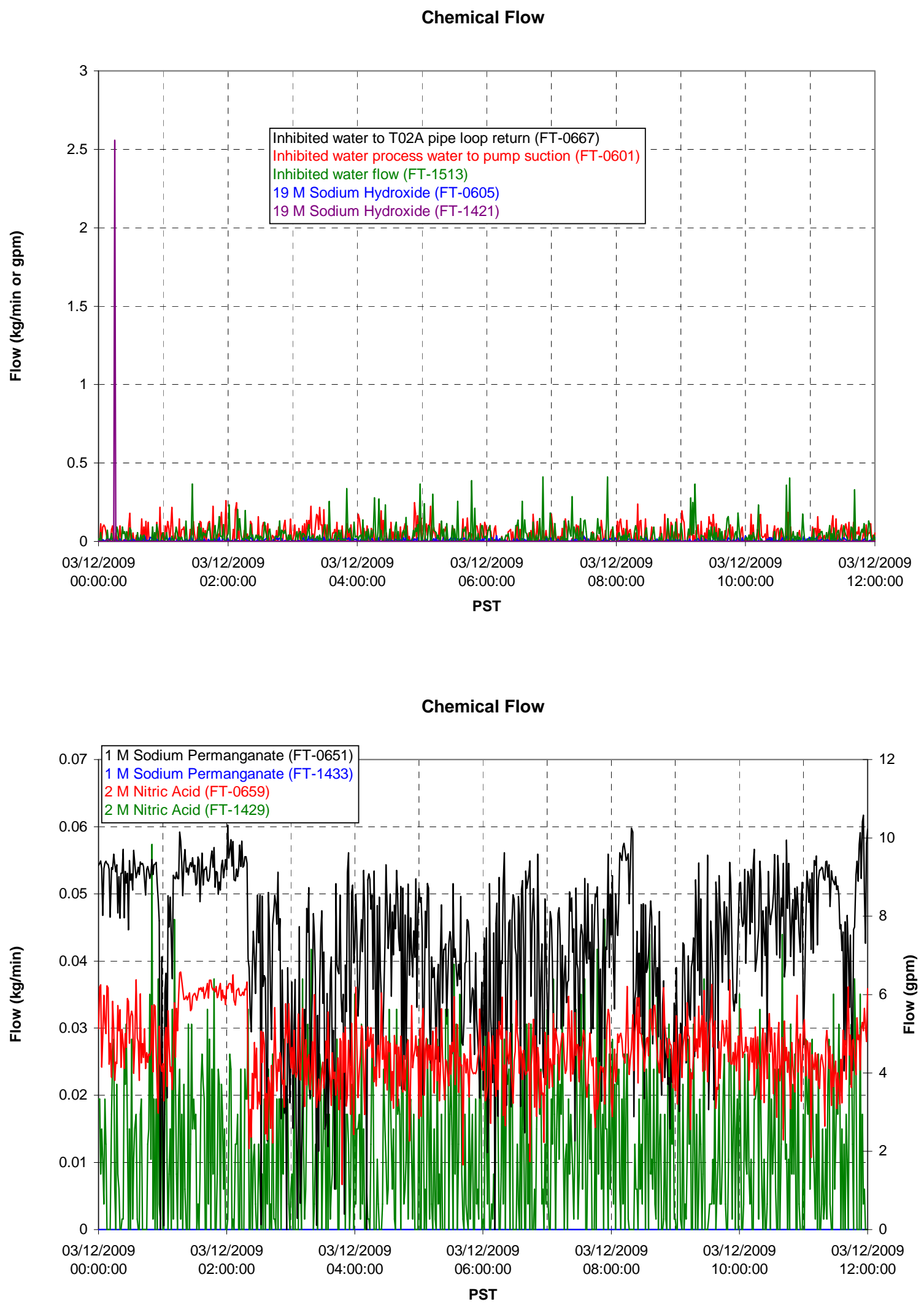
Air Flows

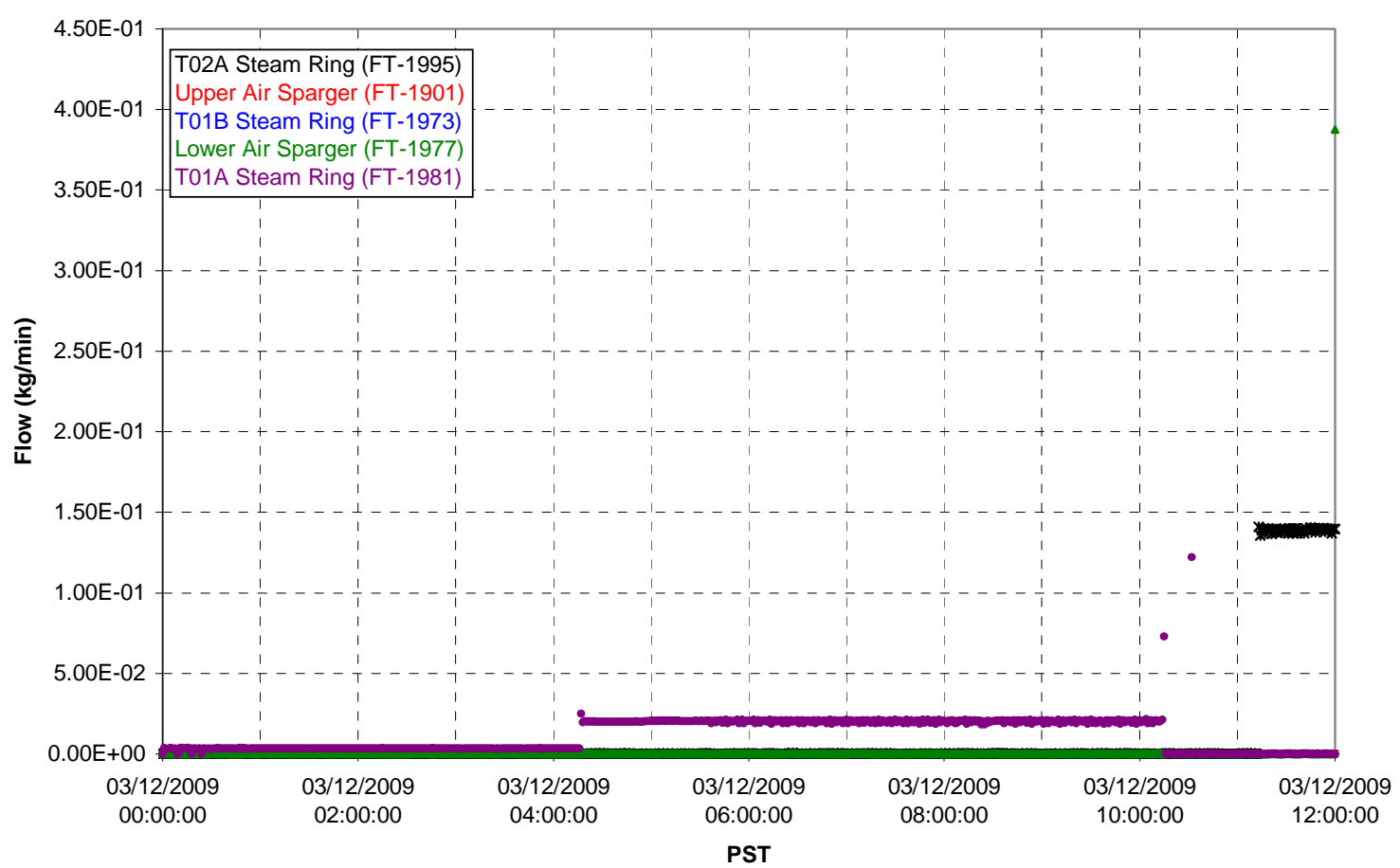

T02A Steam

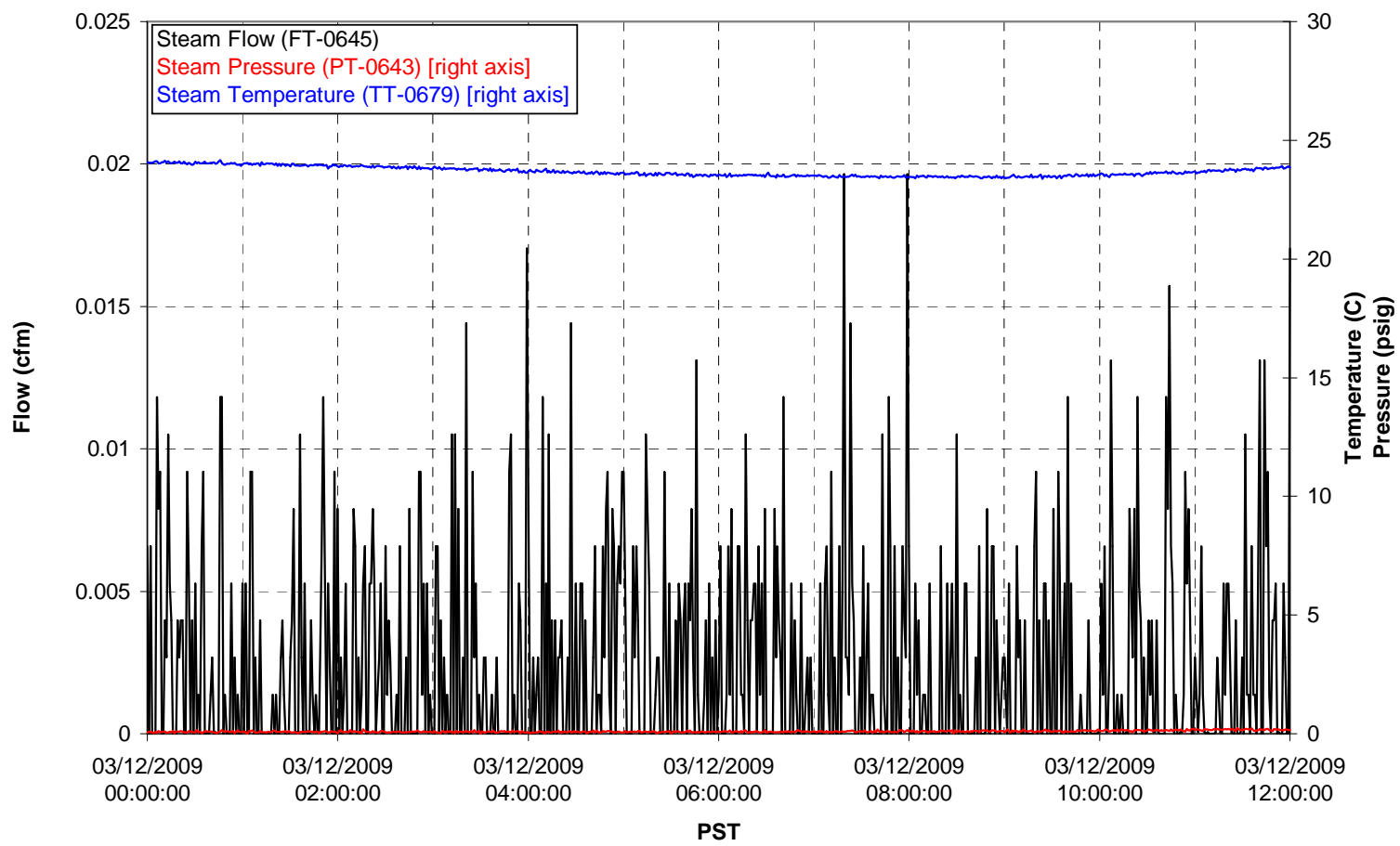


T01A Steam

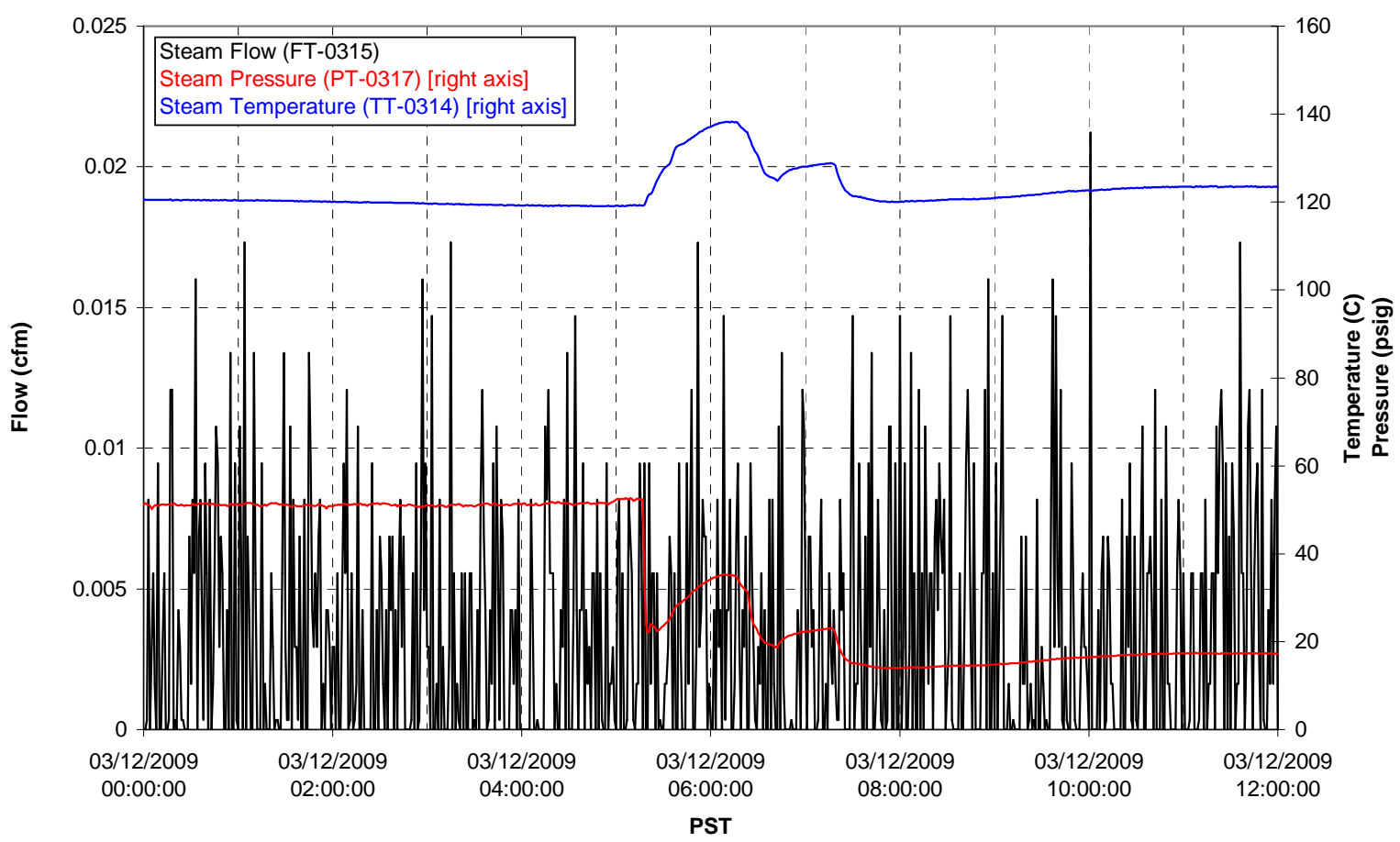

T01B Steam

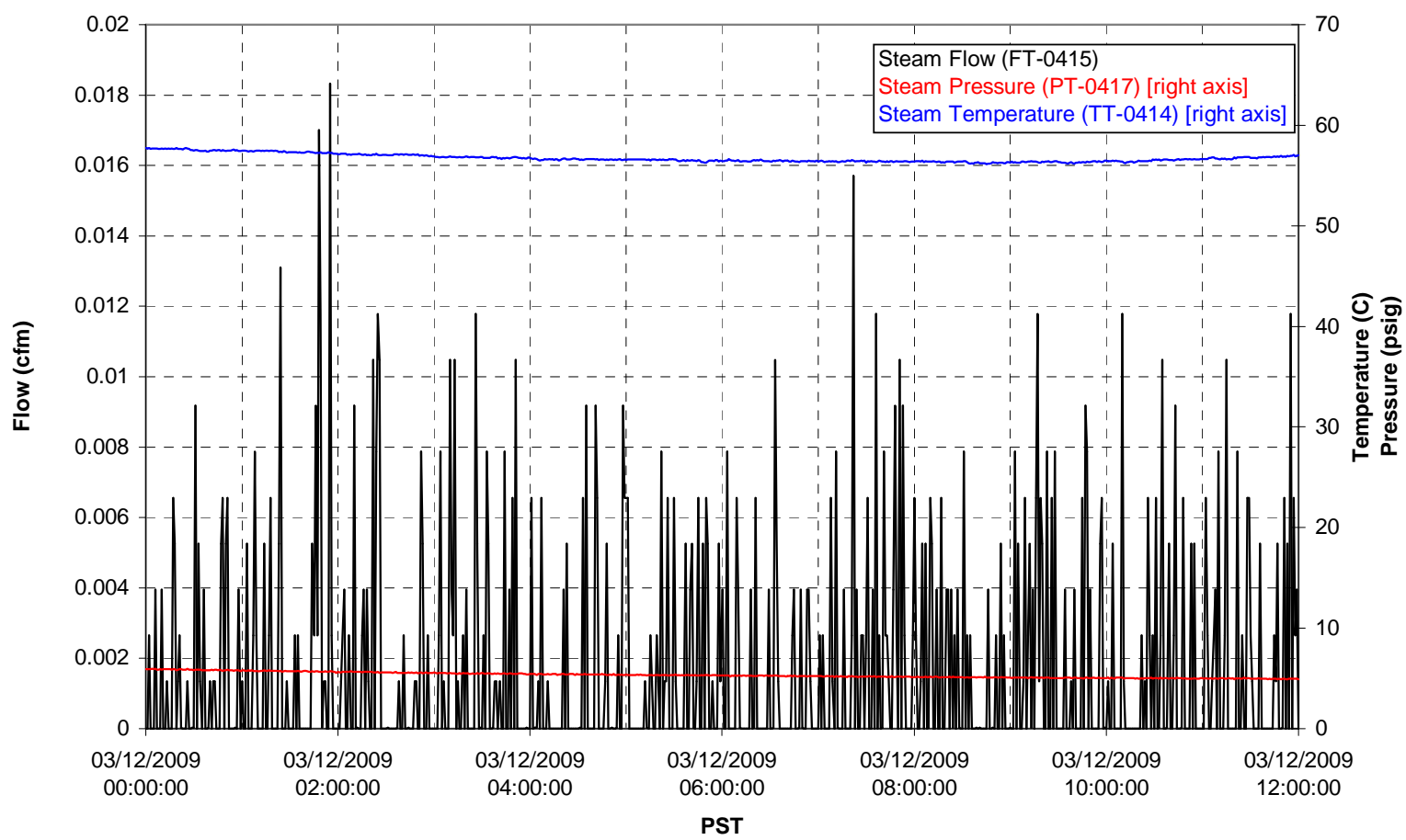


Integrated Test B Data Plots 03/12/09 12:00 - 03/13/09 00:00 
T01A level

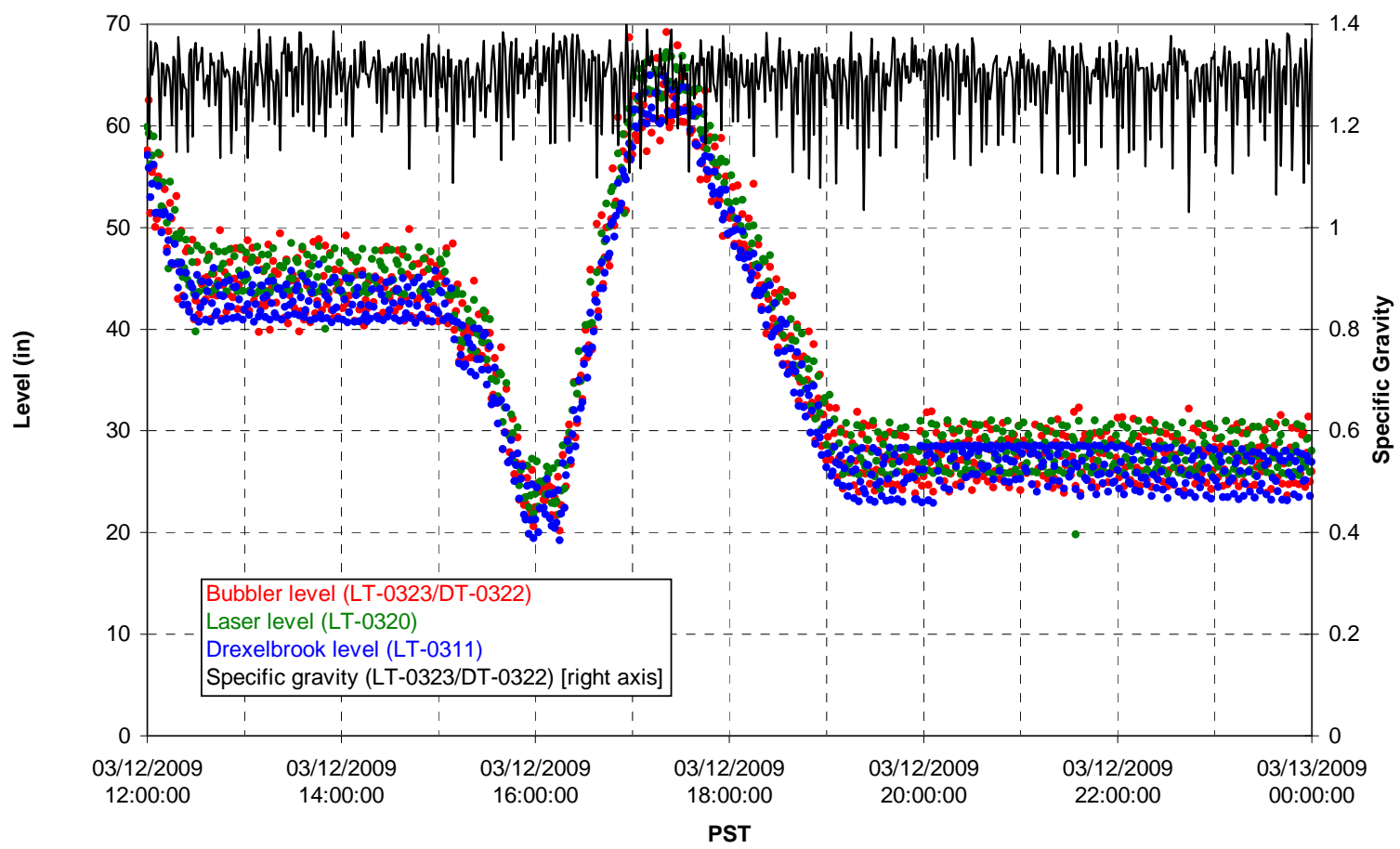

T01A temperatures

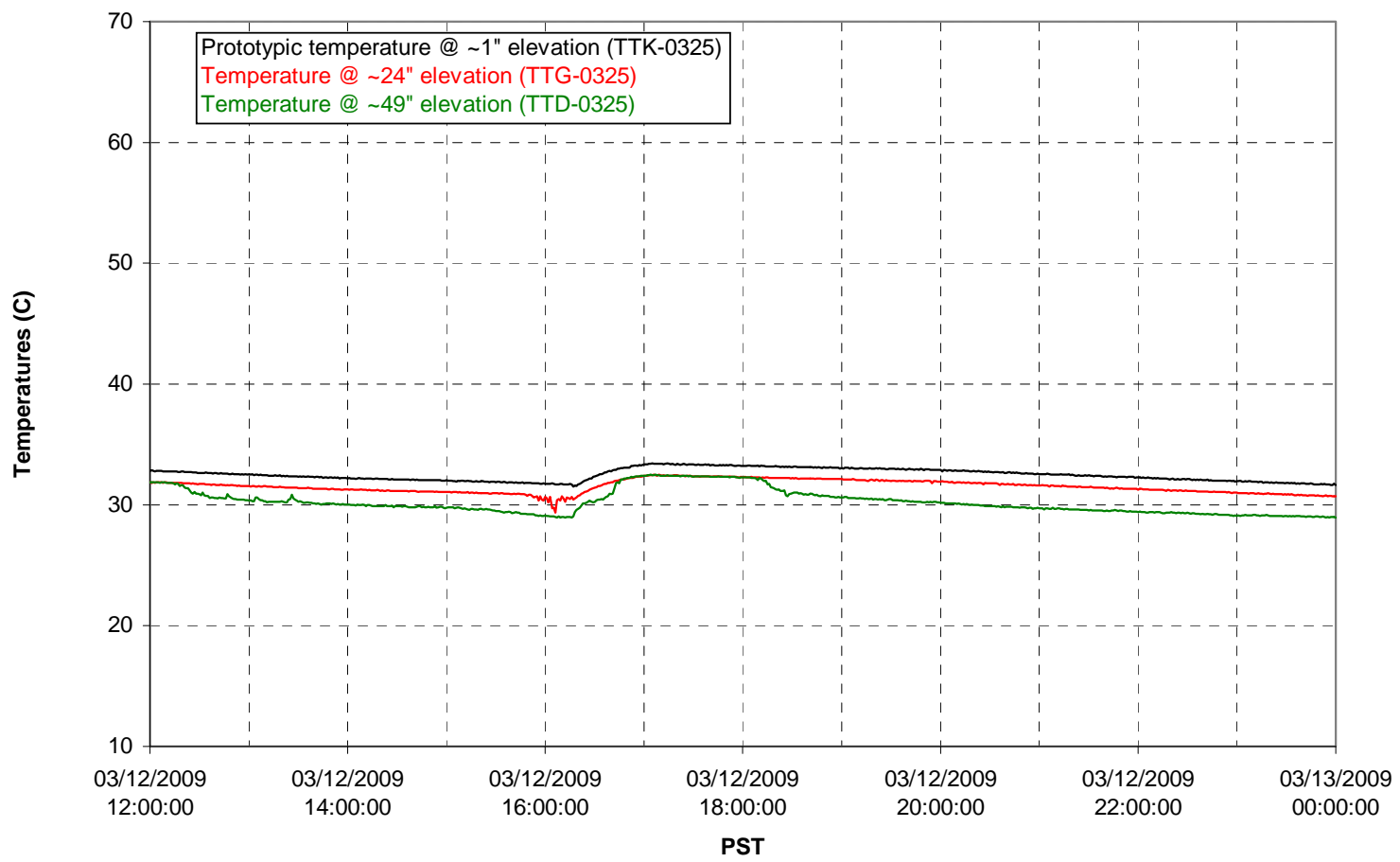


T01B level

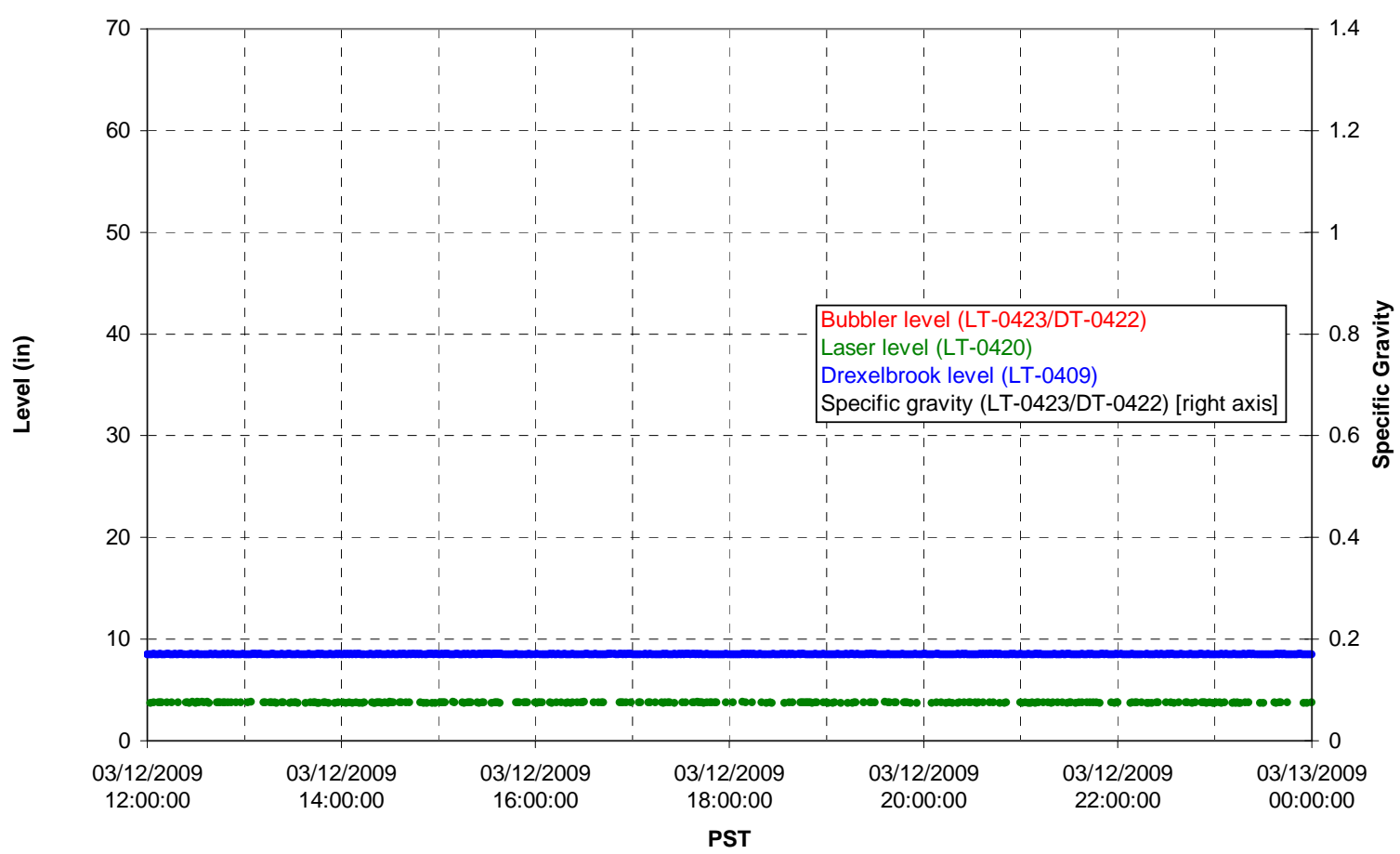

T01B temperatures

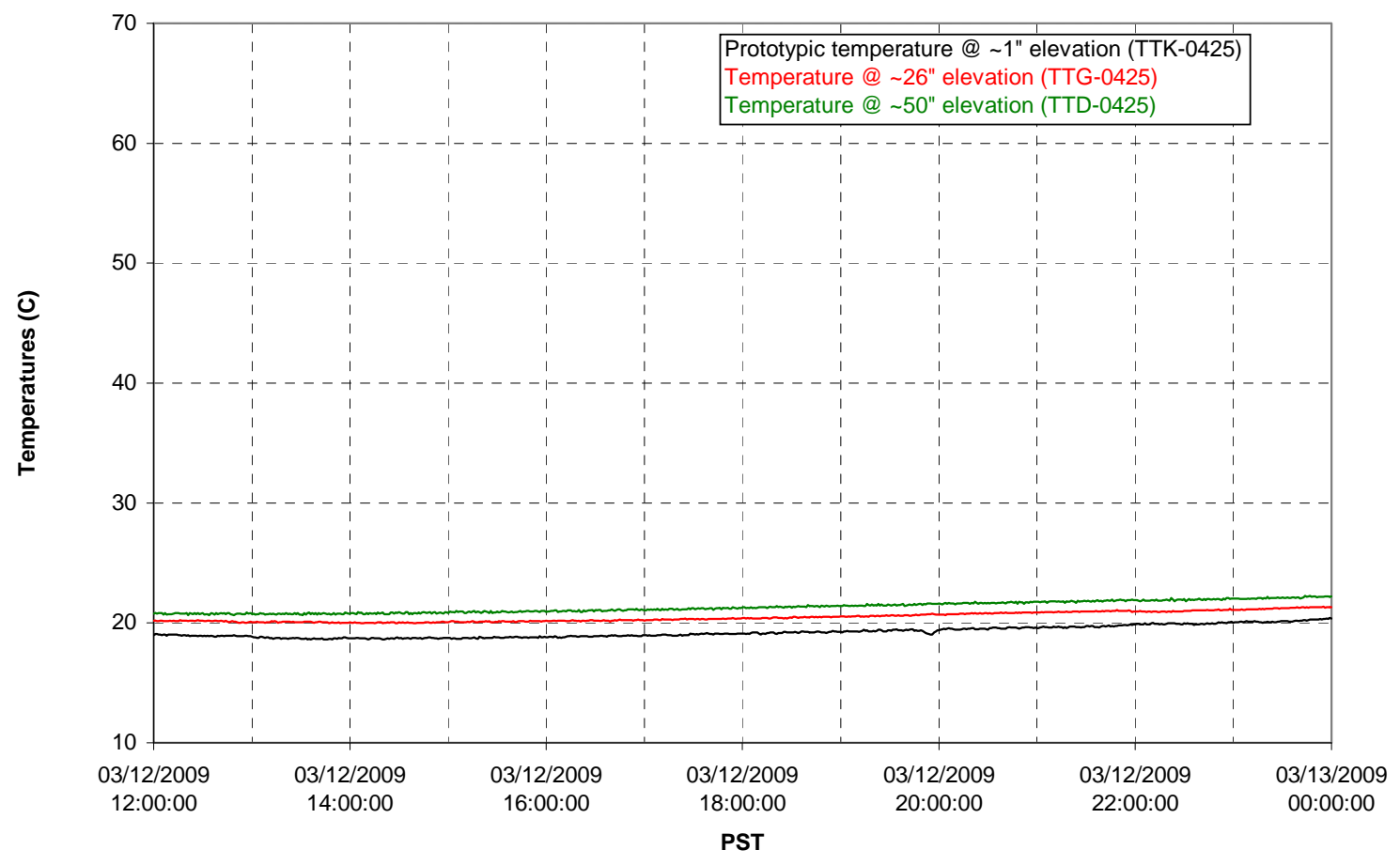


T02A level

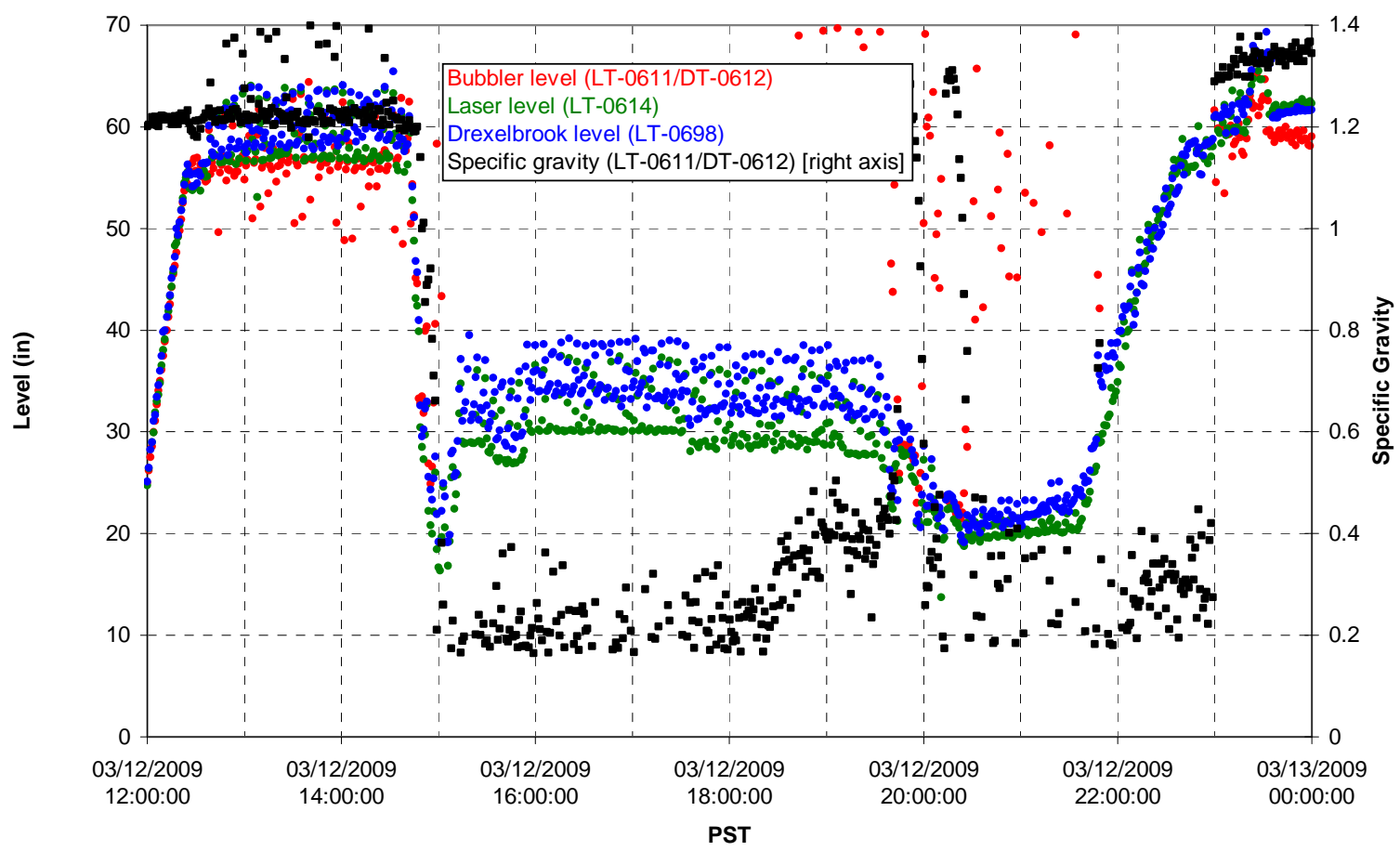

T02A temperatures

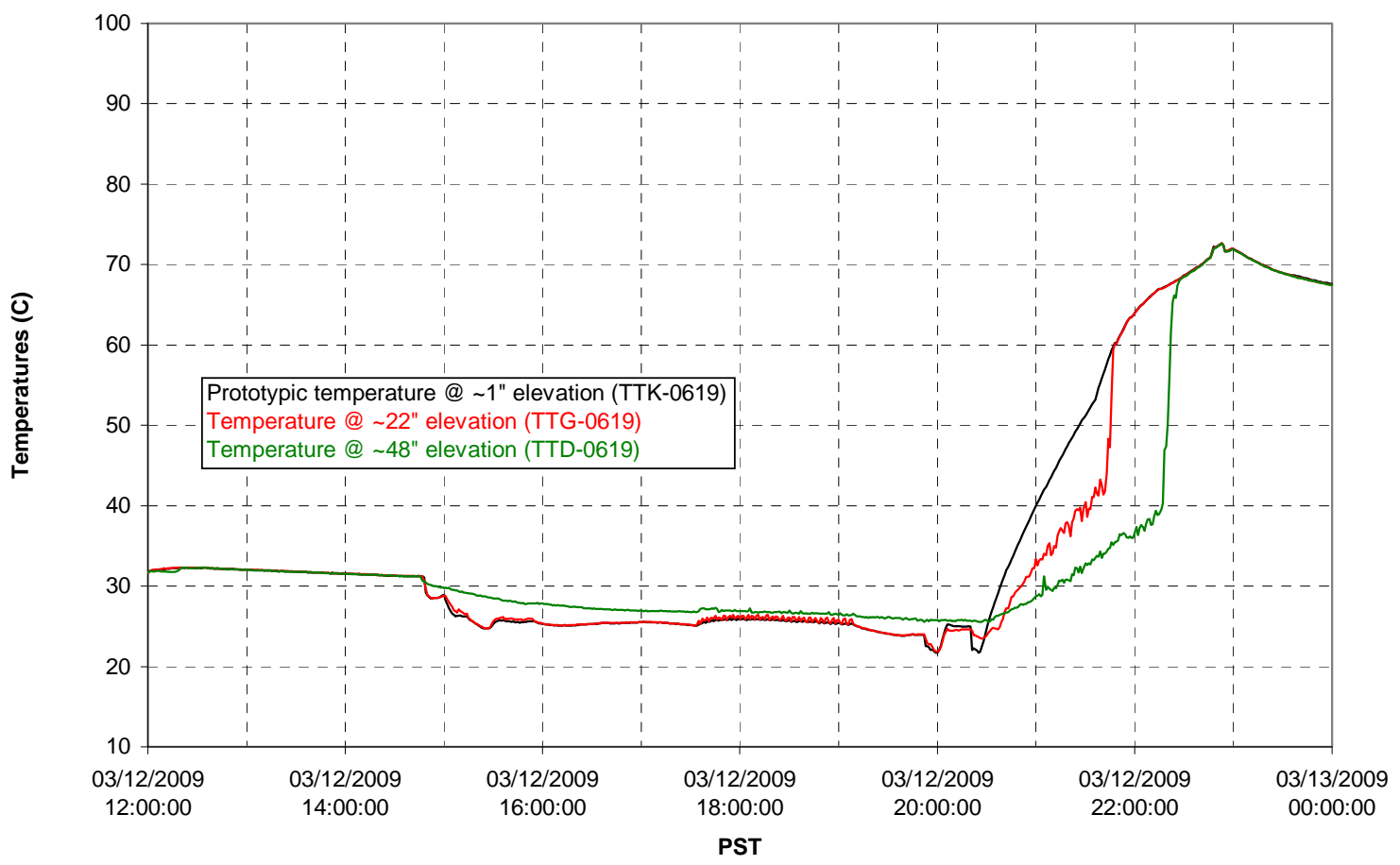


T02A and filter loop temperatures

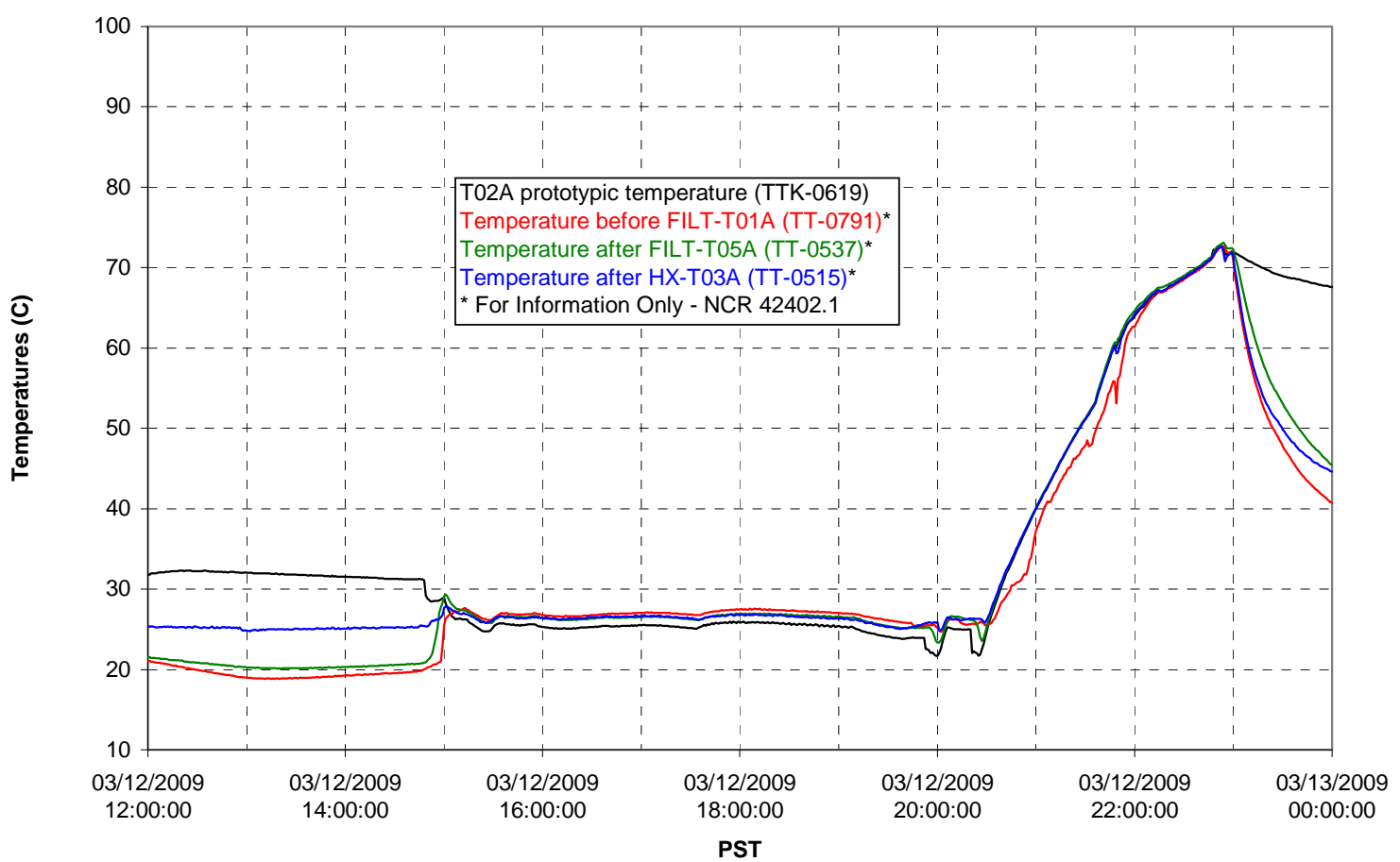

Pump Pressures and Flow

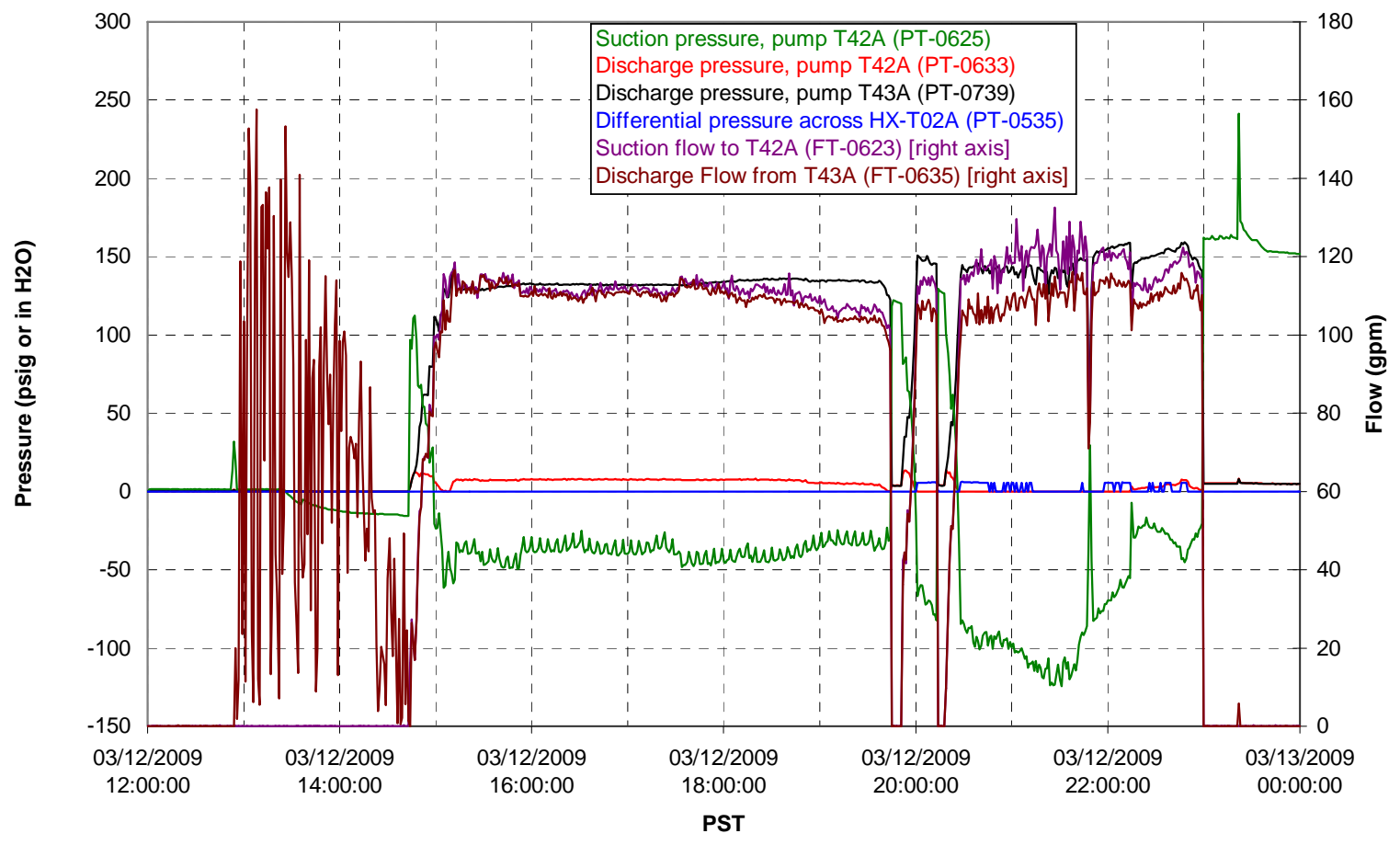


Axial pressure drop

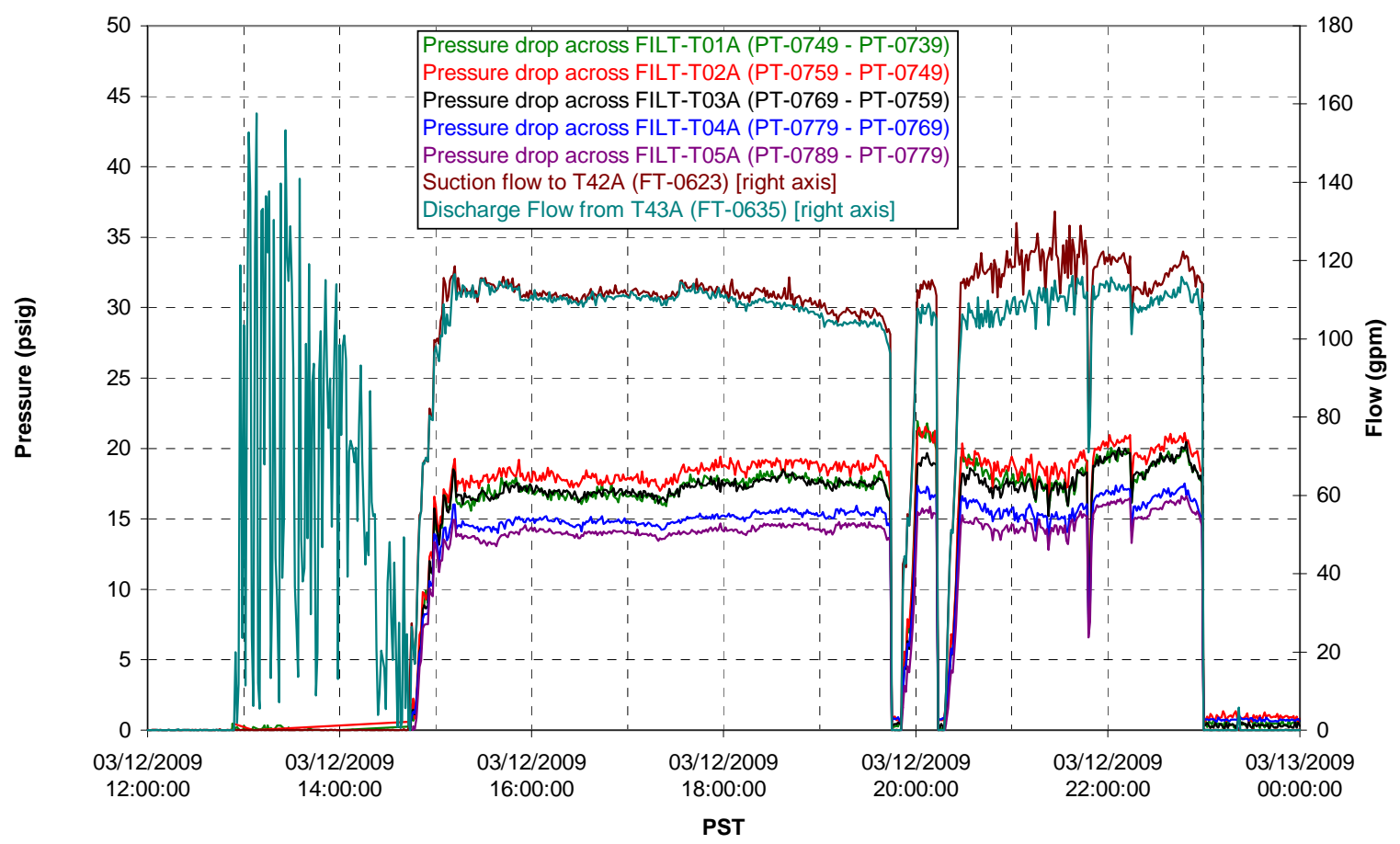

Permeate flow rates

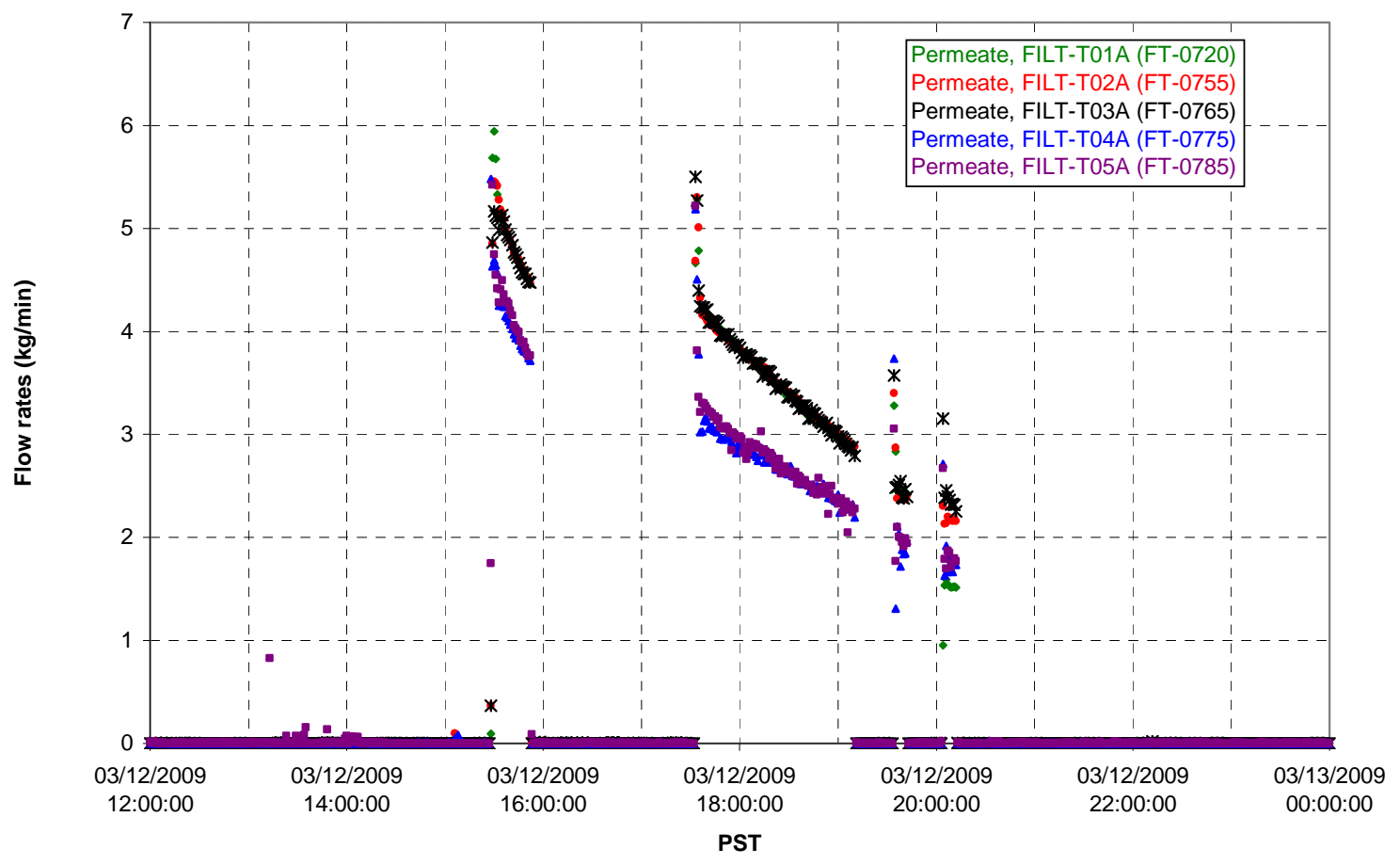


T02A Inner Temperature Tree

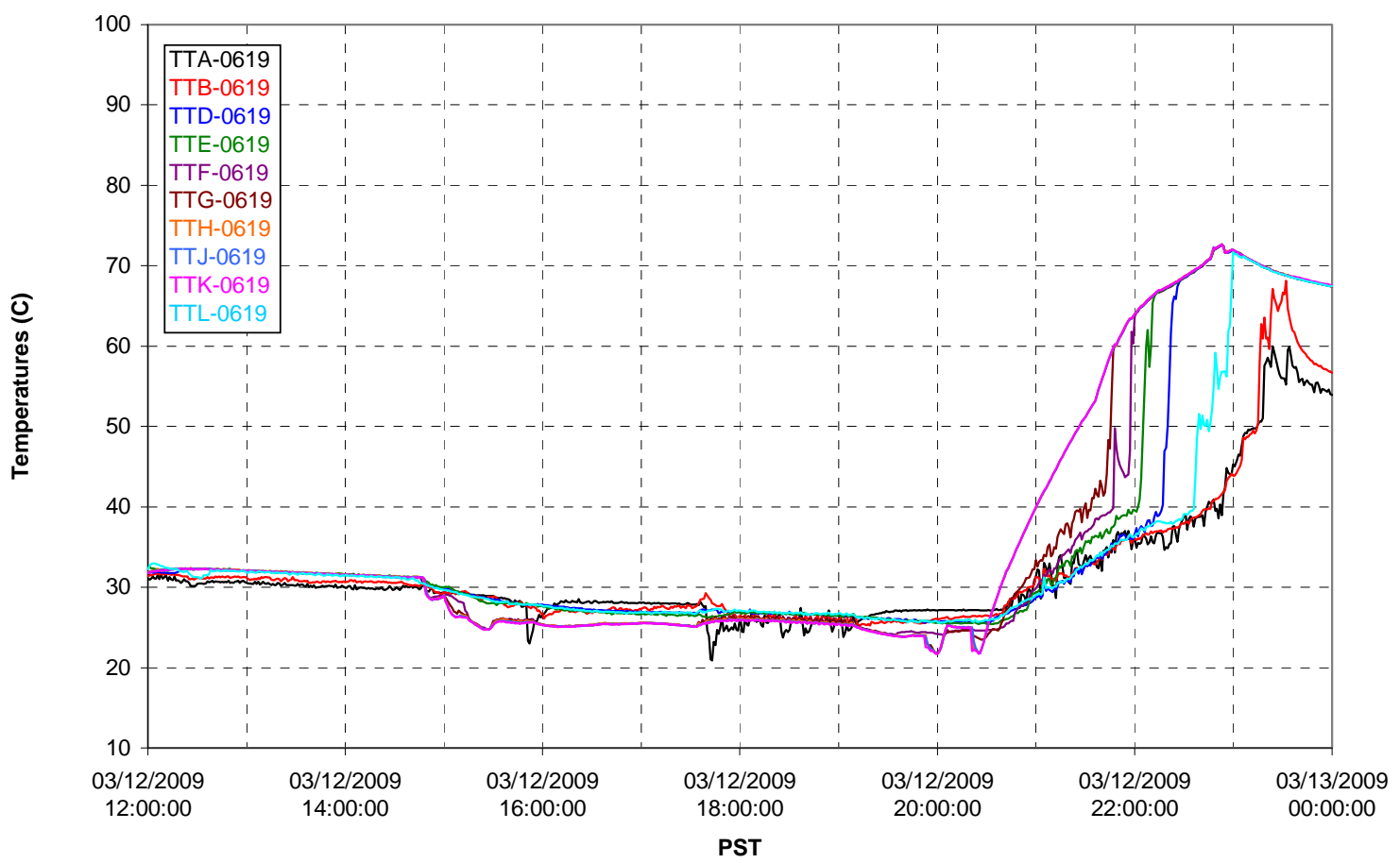

T02A Outer Temperature Tree

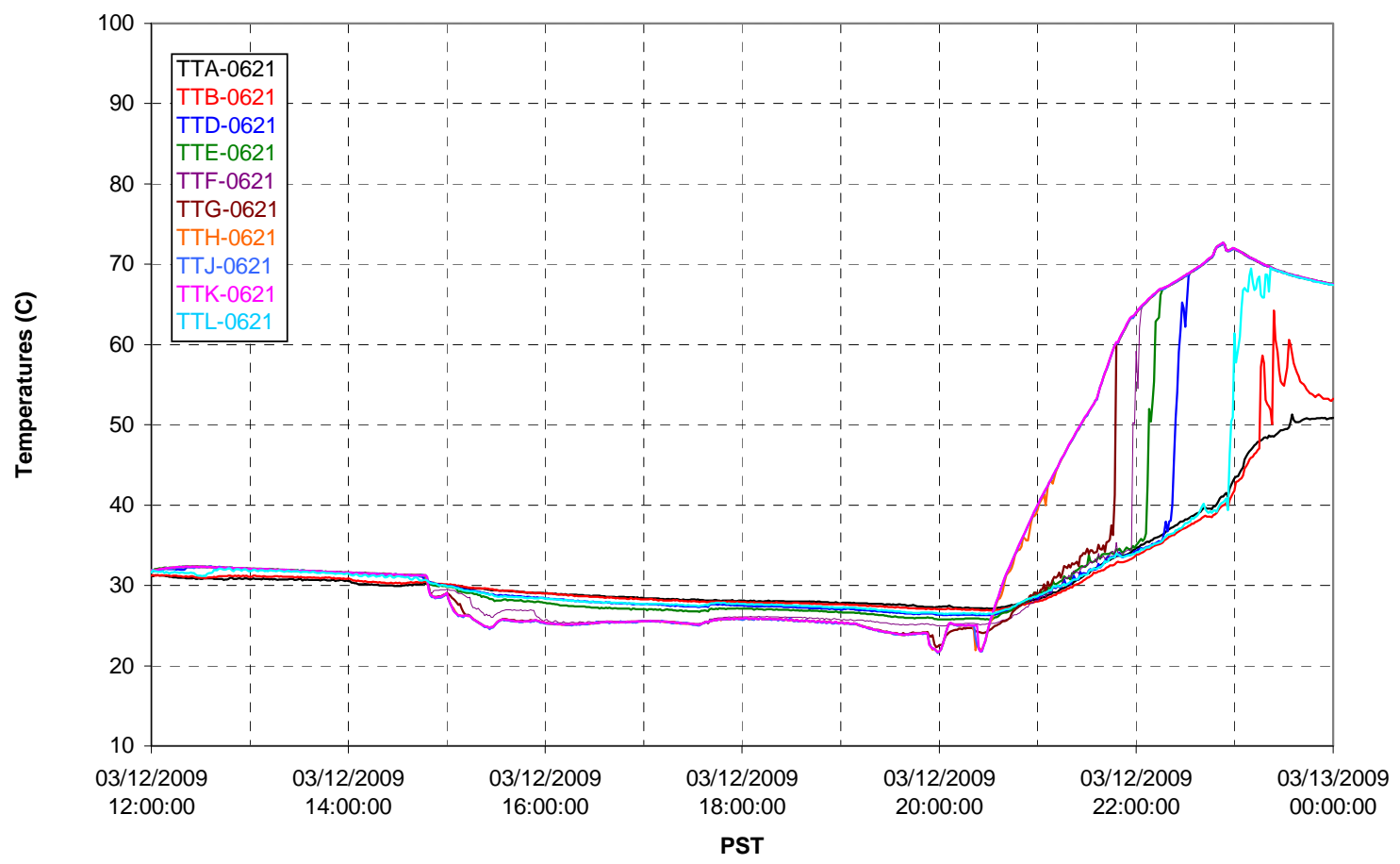


T02A temperatures

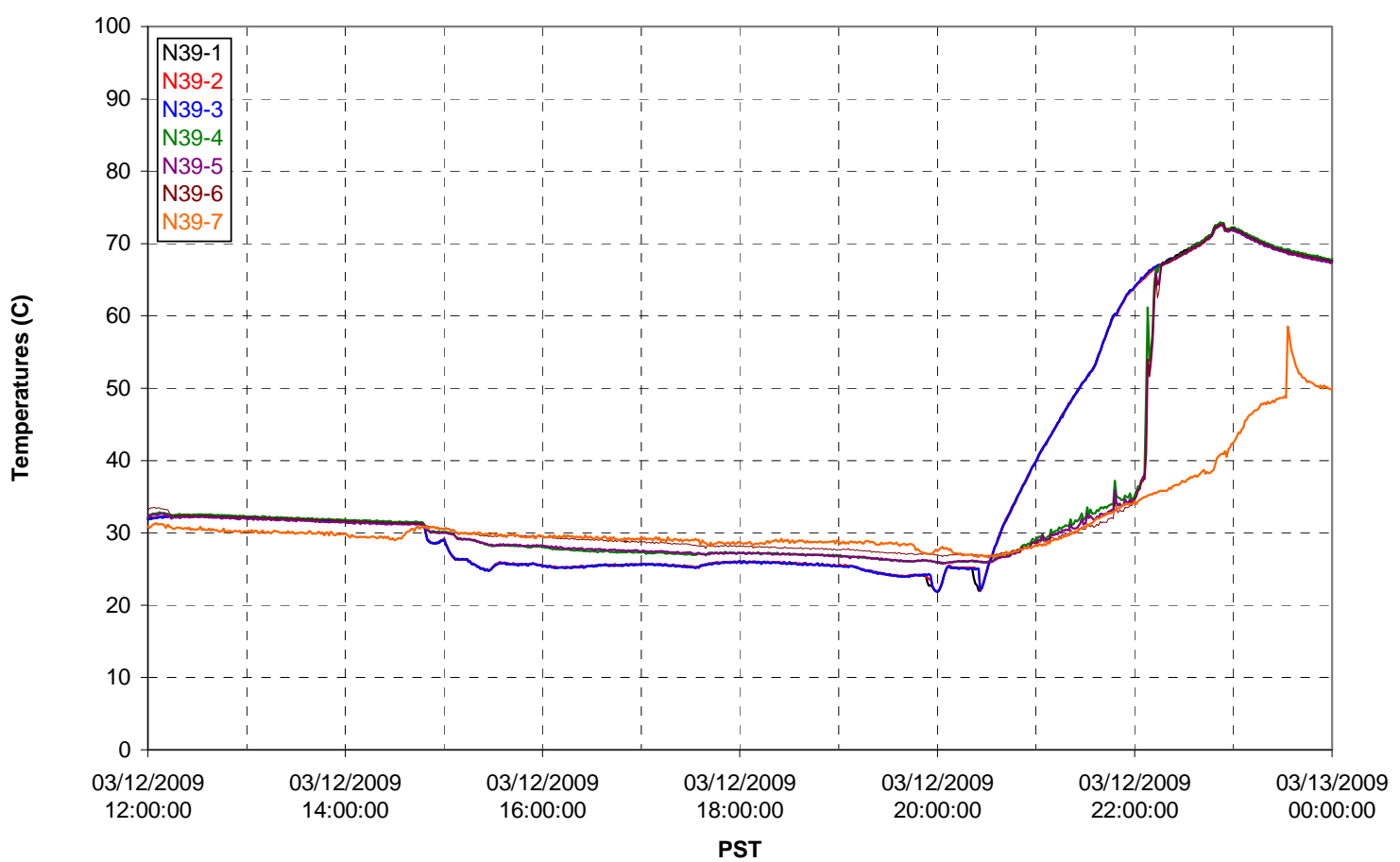

T02A temperatures

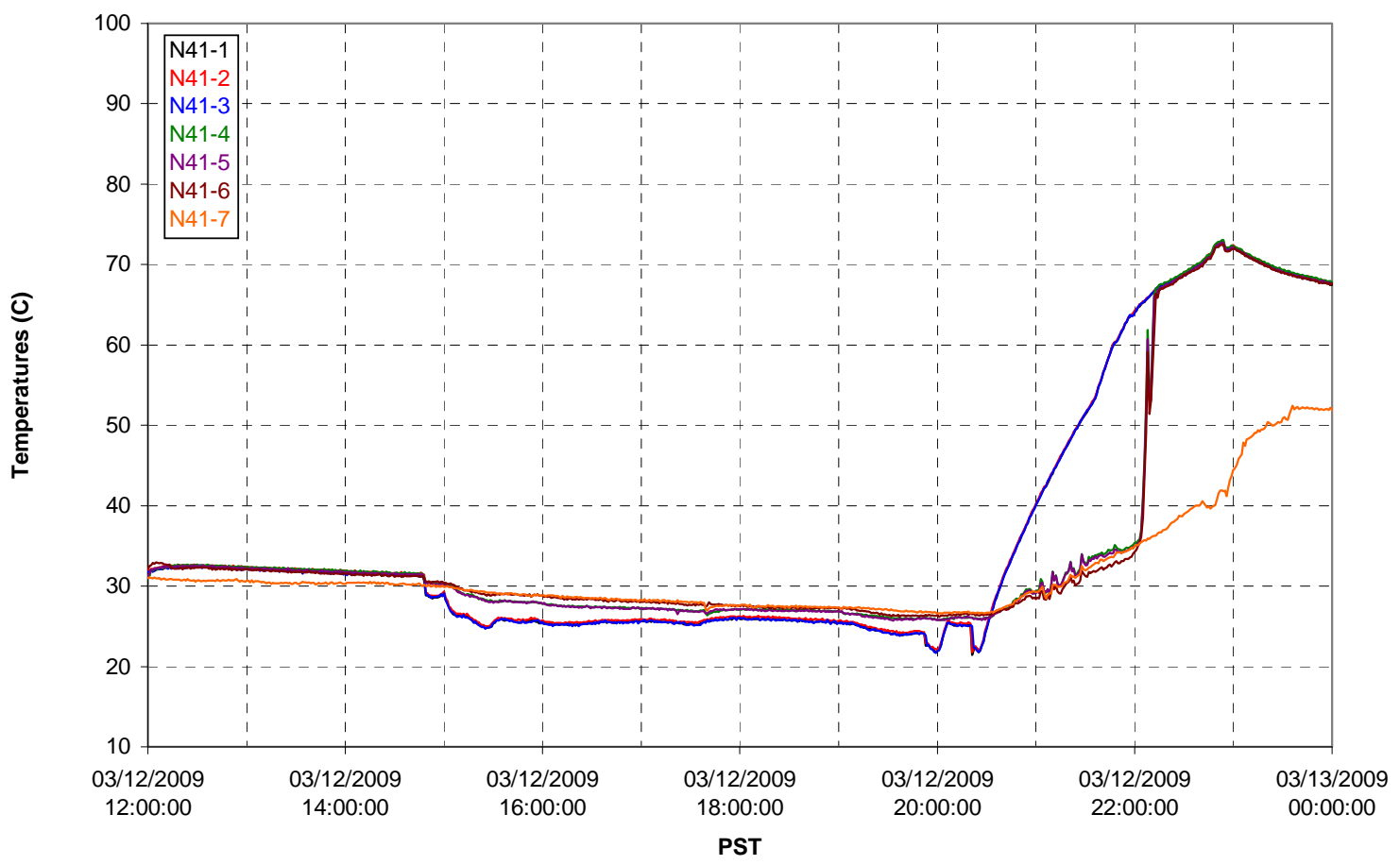


T02A temperatures

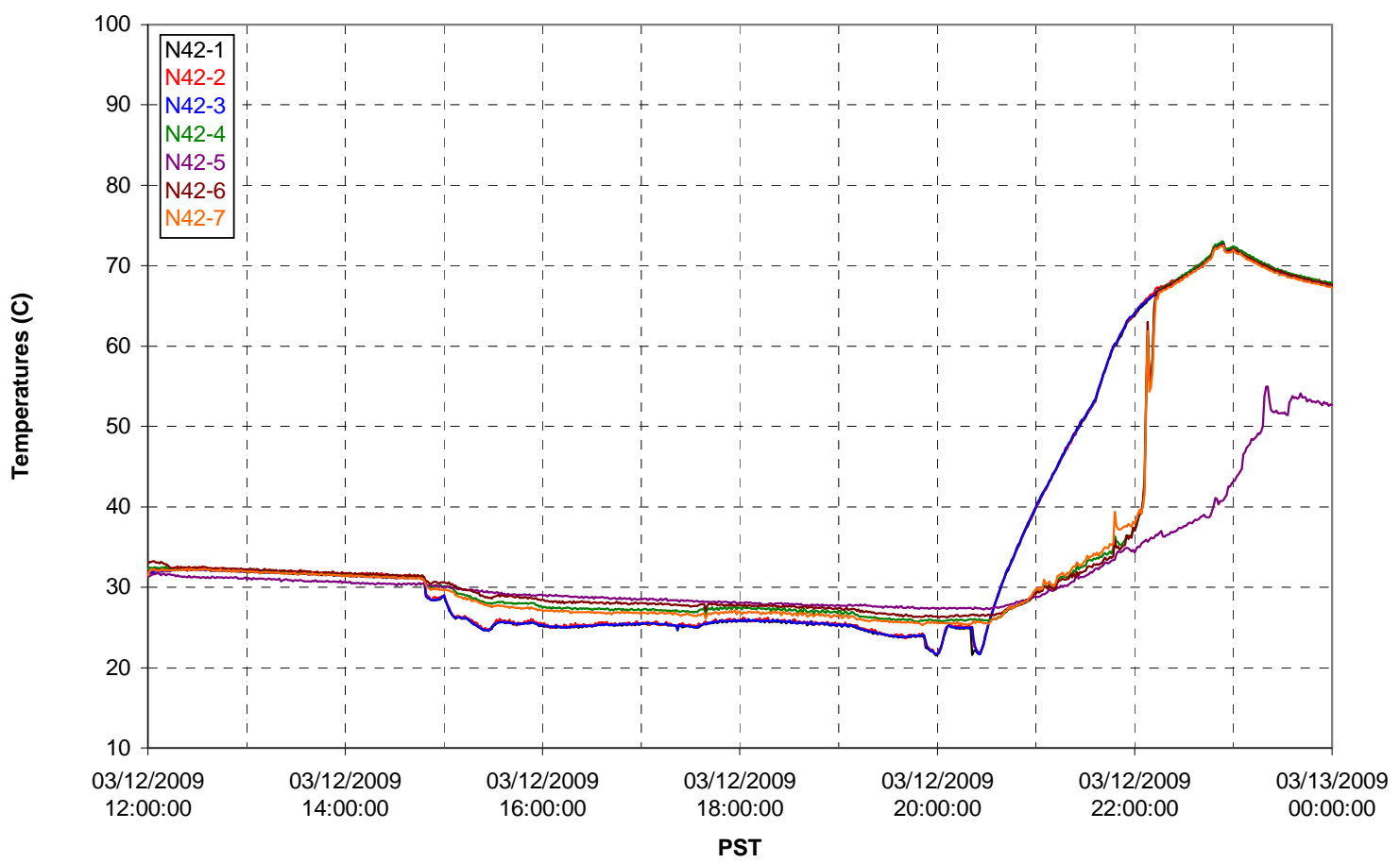

T02A temperatures

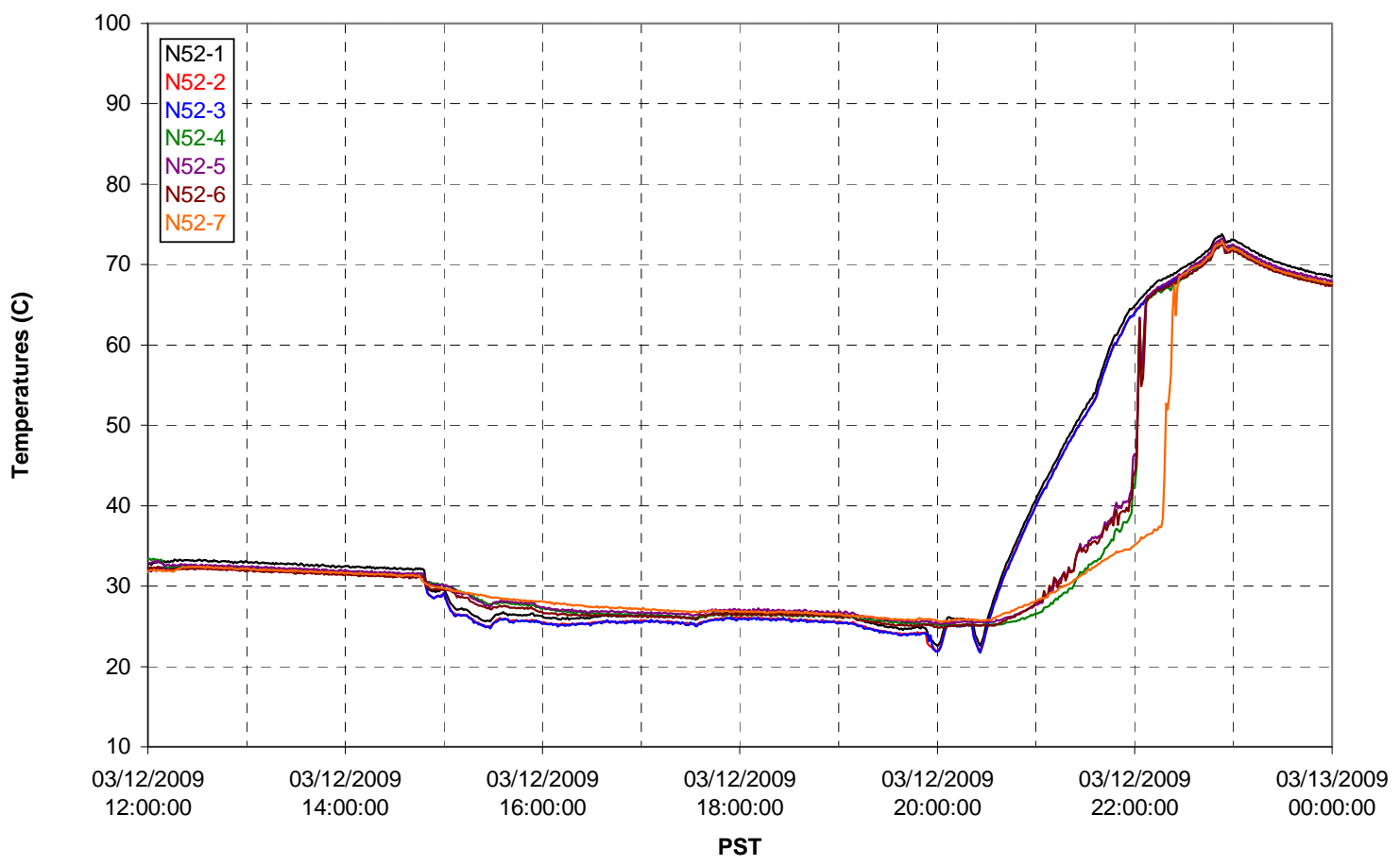


T02A Heating and Cooling
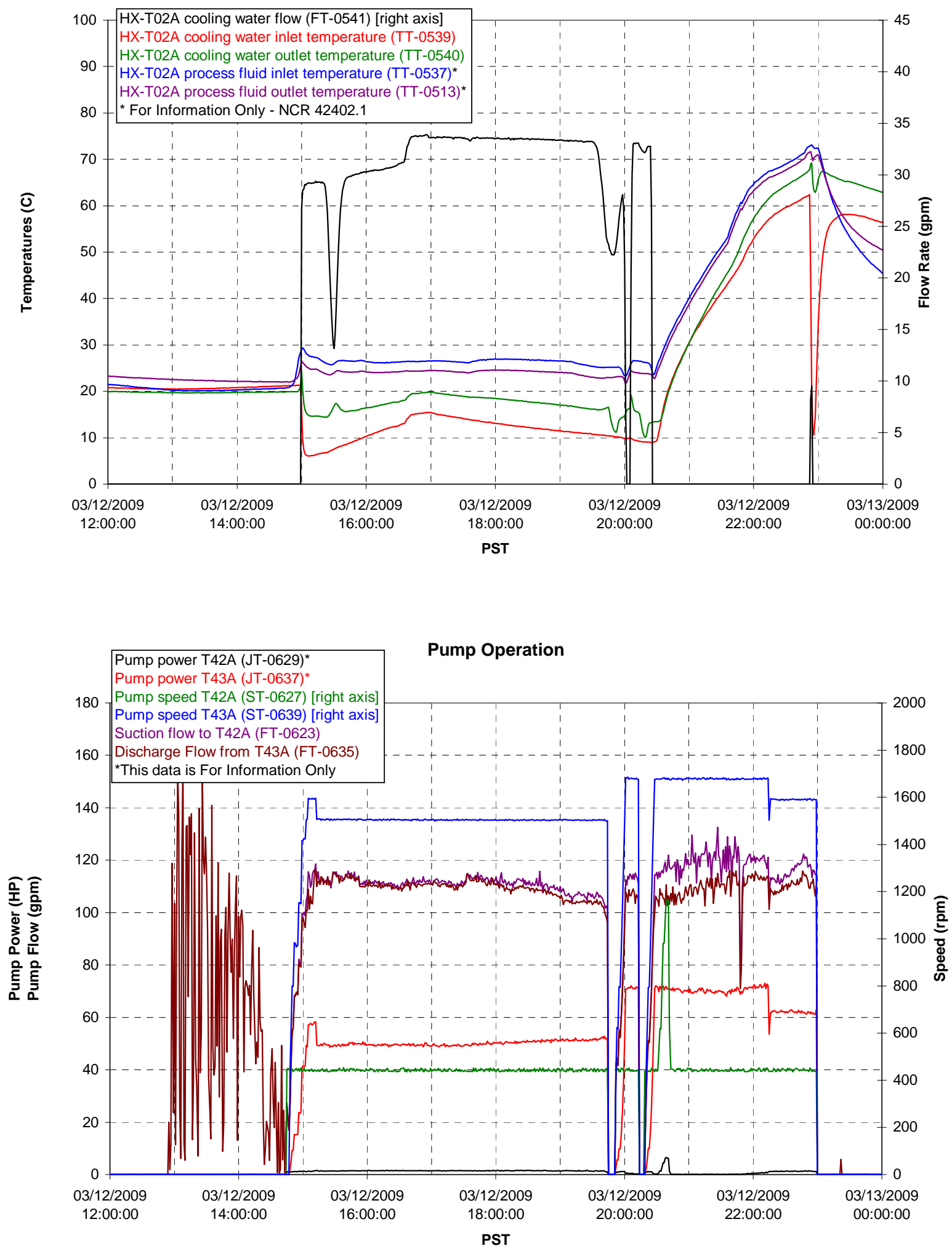
Pulsepot UFP-PP-T01A

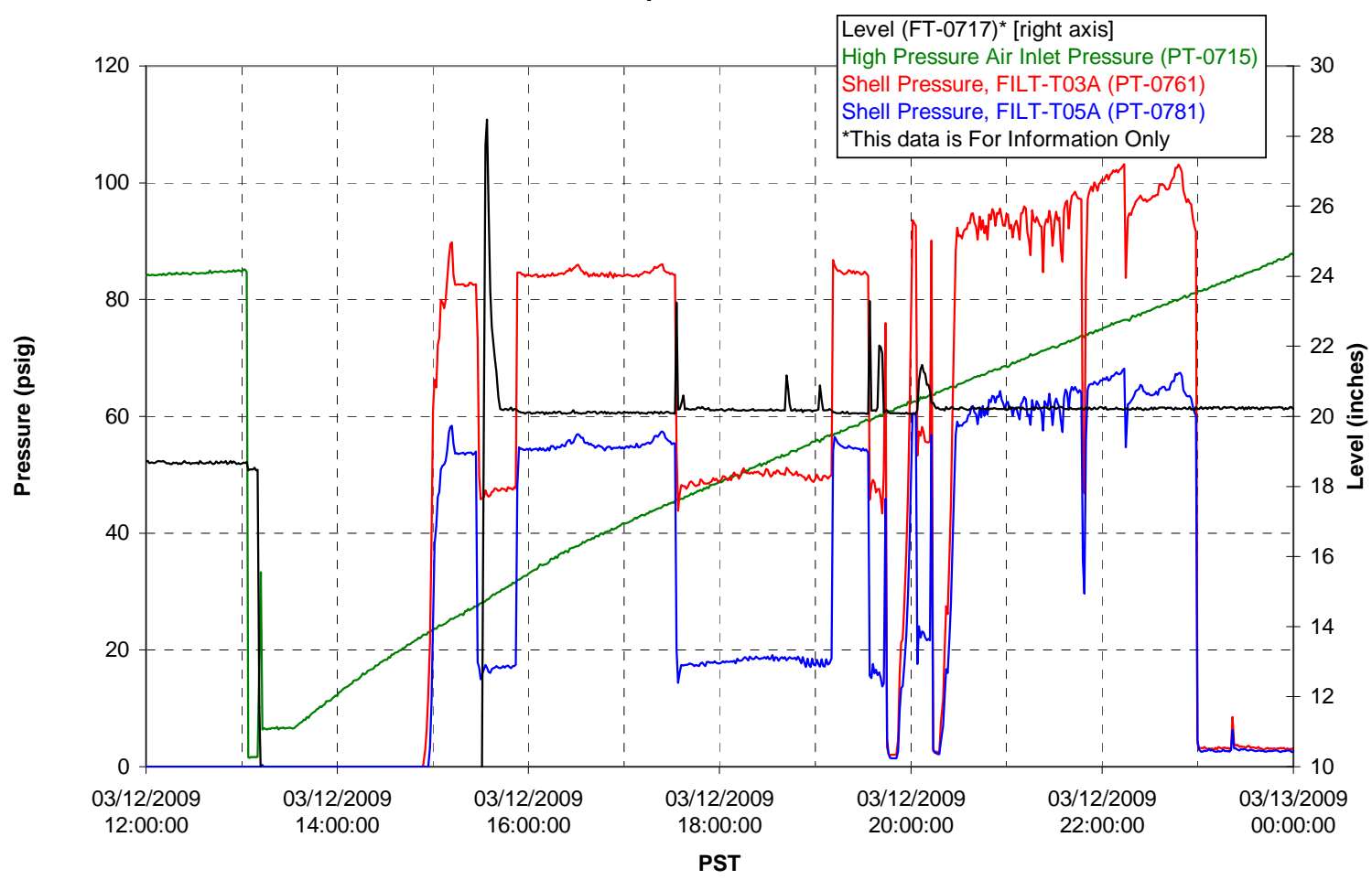

Pulsepot UFP-PP-T02A

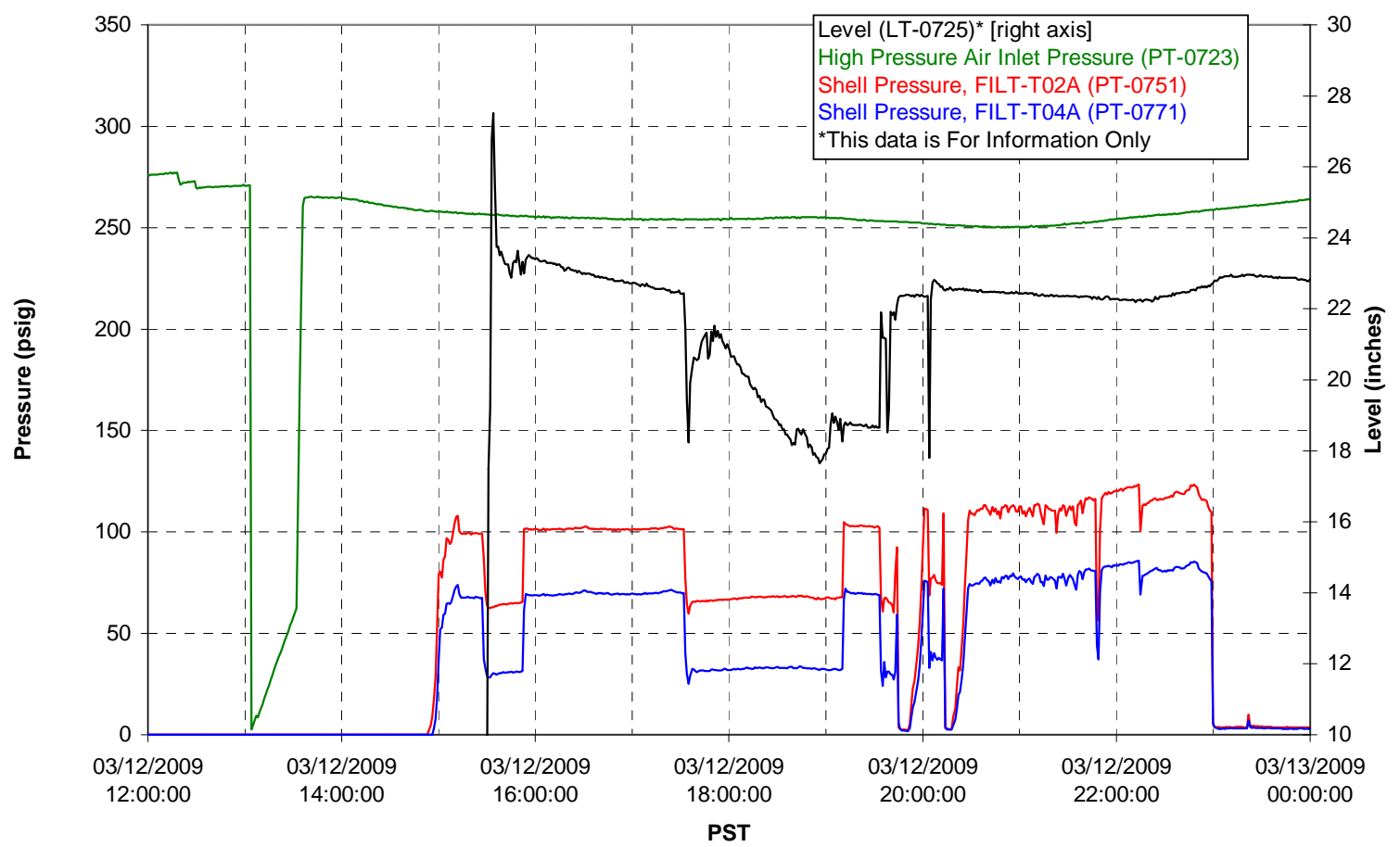


Pulsepot UFP-PP-T03A

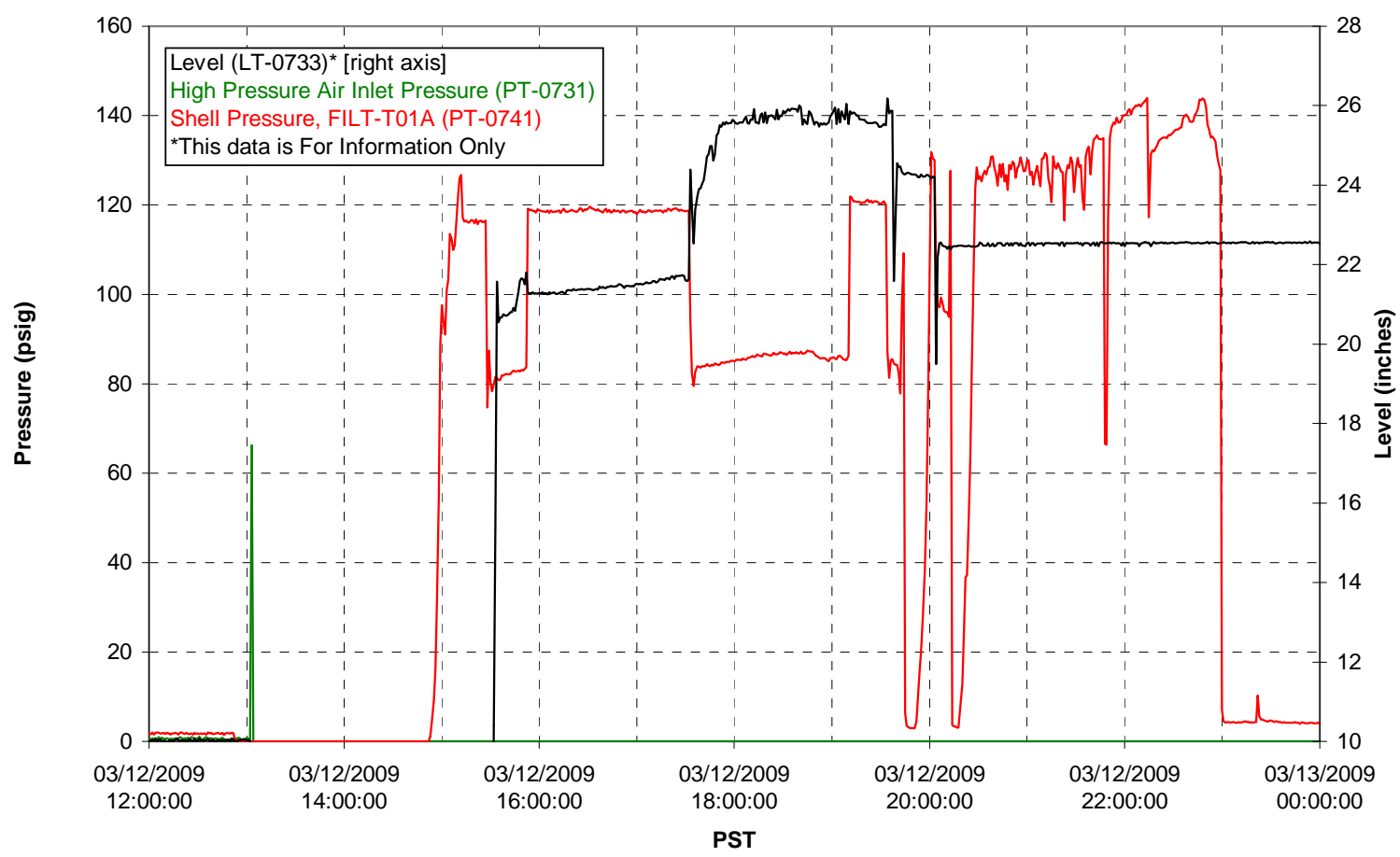

Pulsepot Levels

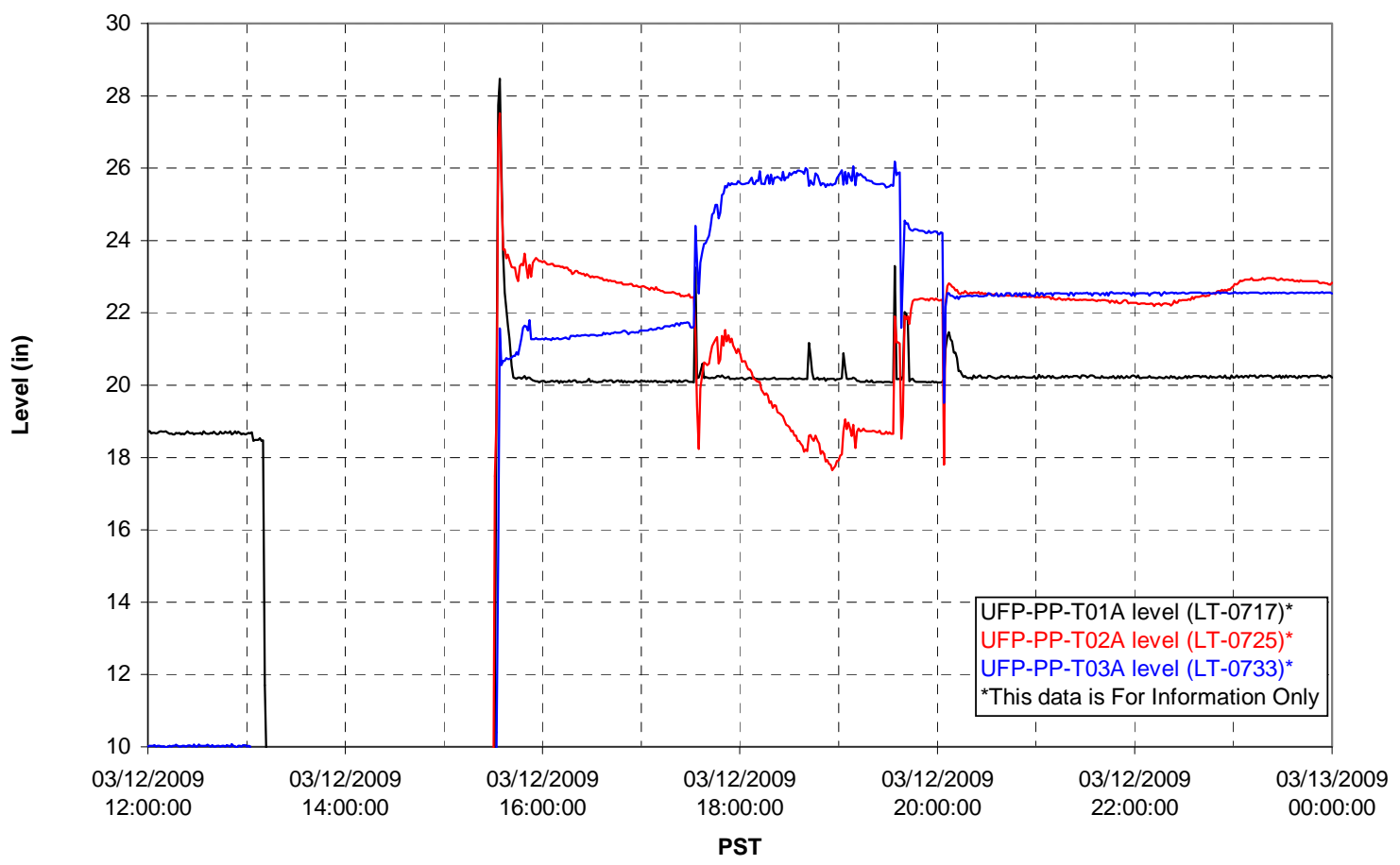


Filter UFP-FILT-T01A

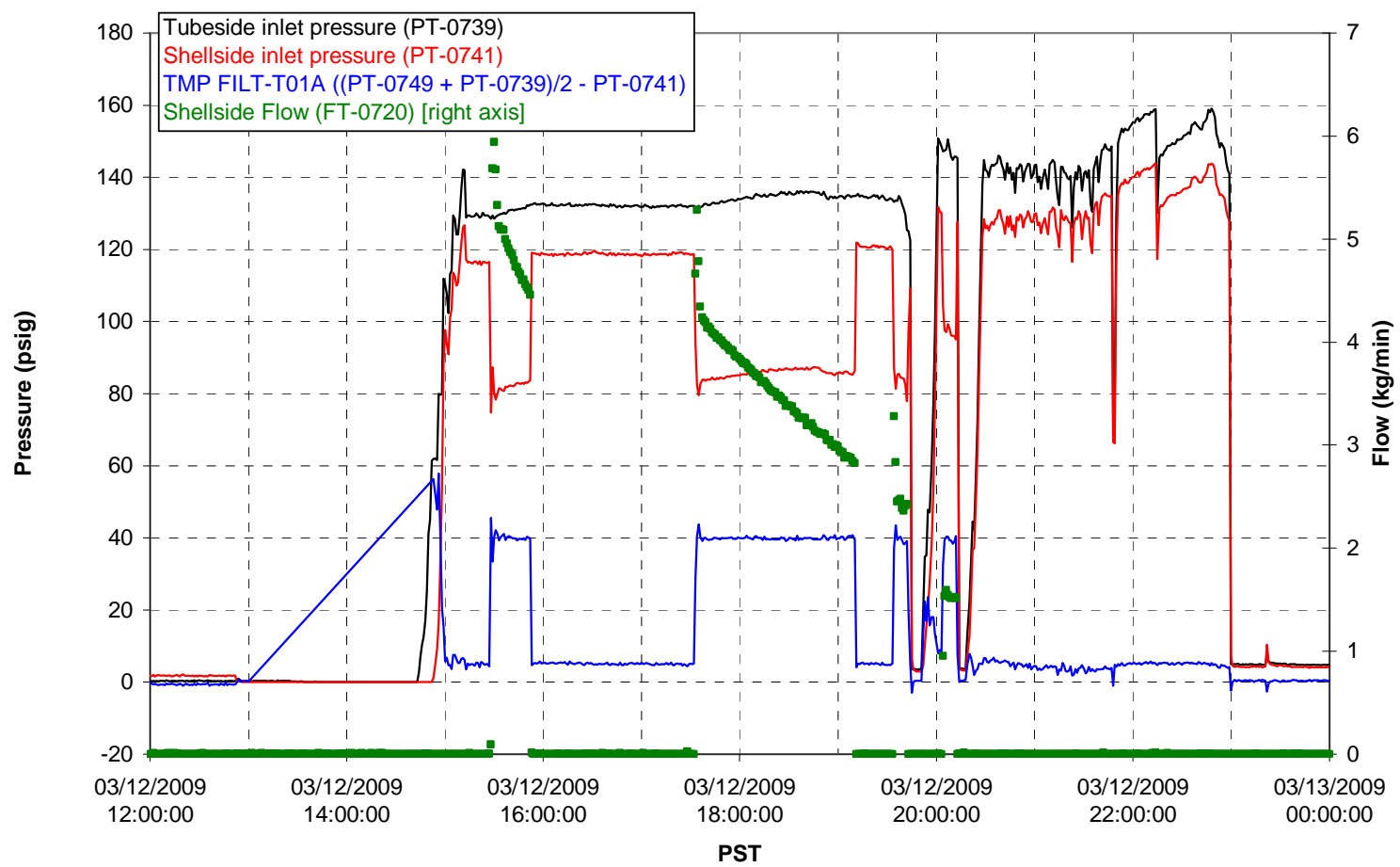

Filter UFP-FILT-T02A

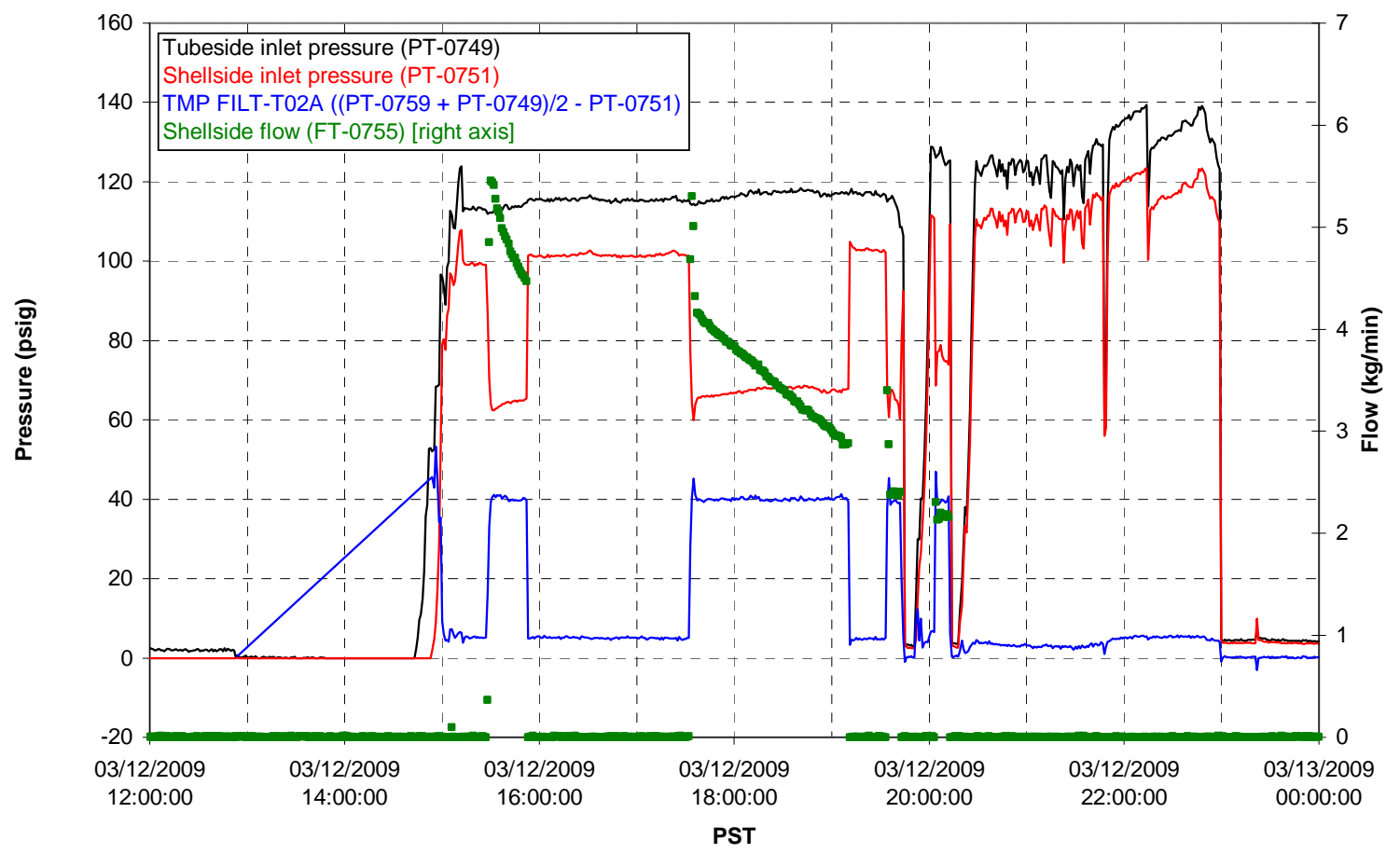


Filter UFP-FILT-T03A

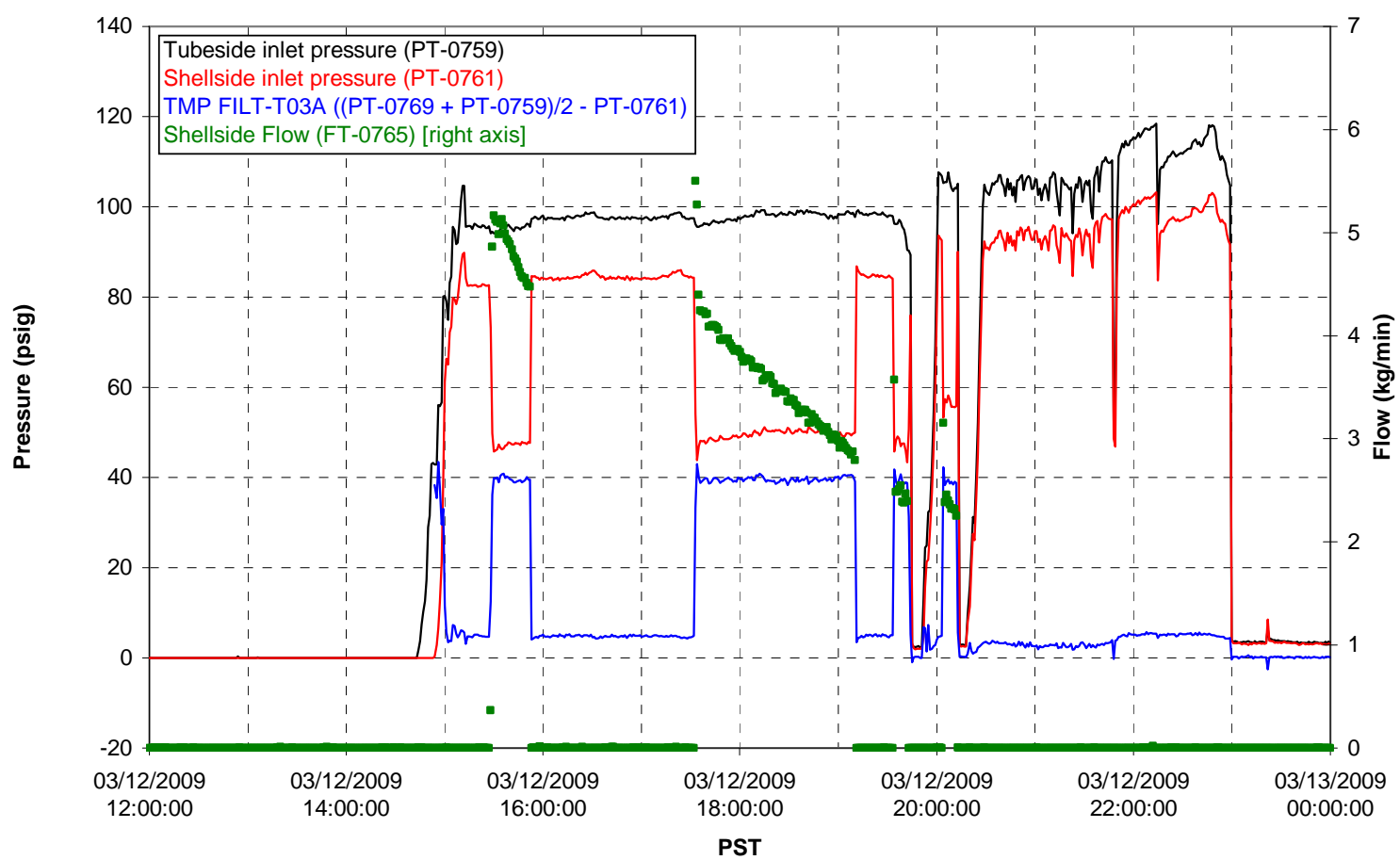

Filter UFP-FILT-T04A

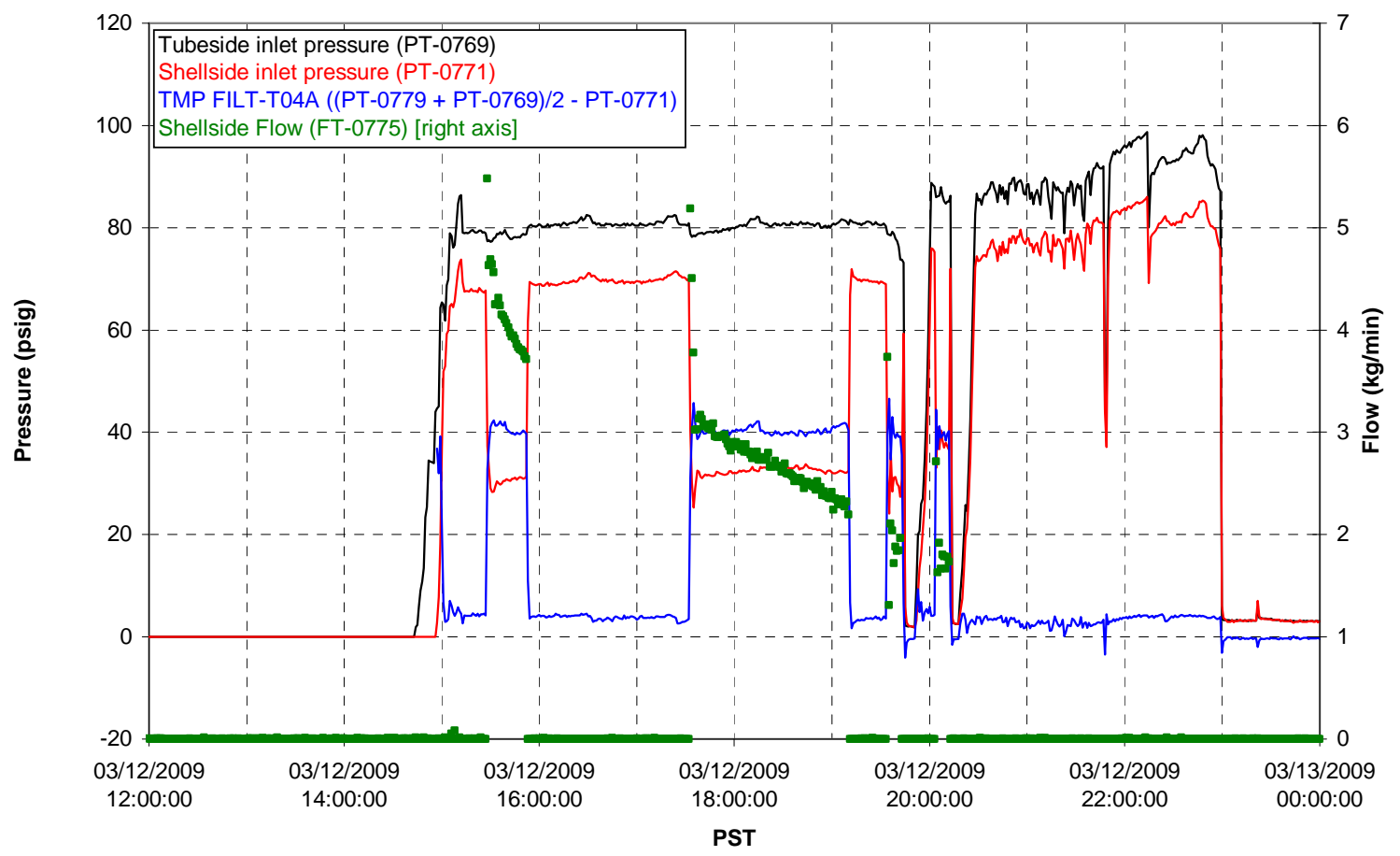


Filter UFP-FILT-T05A

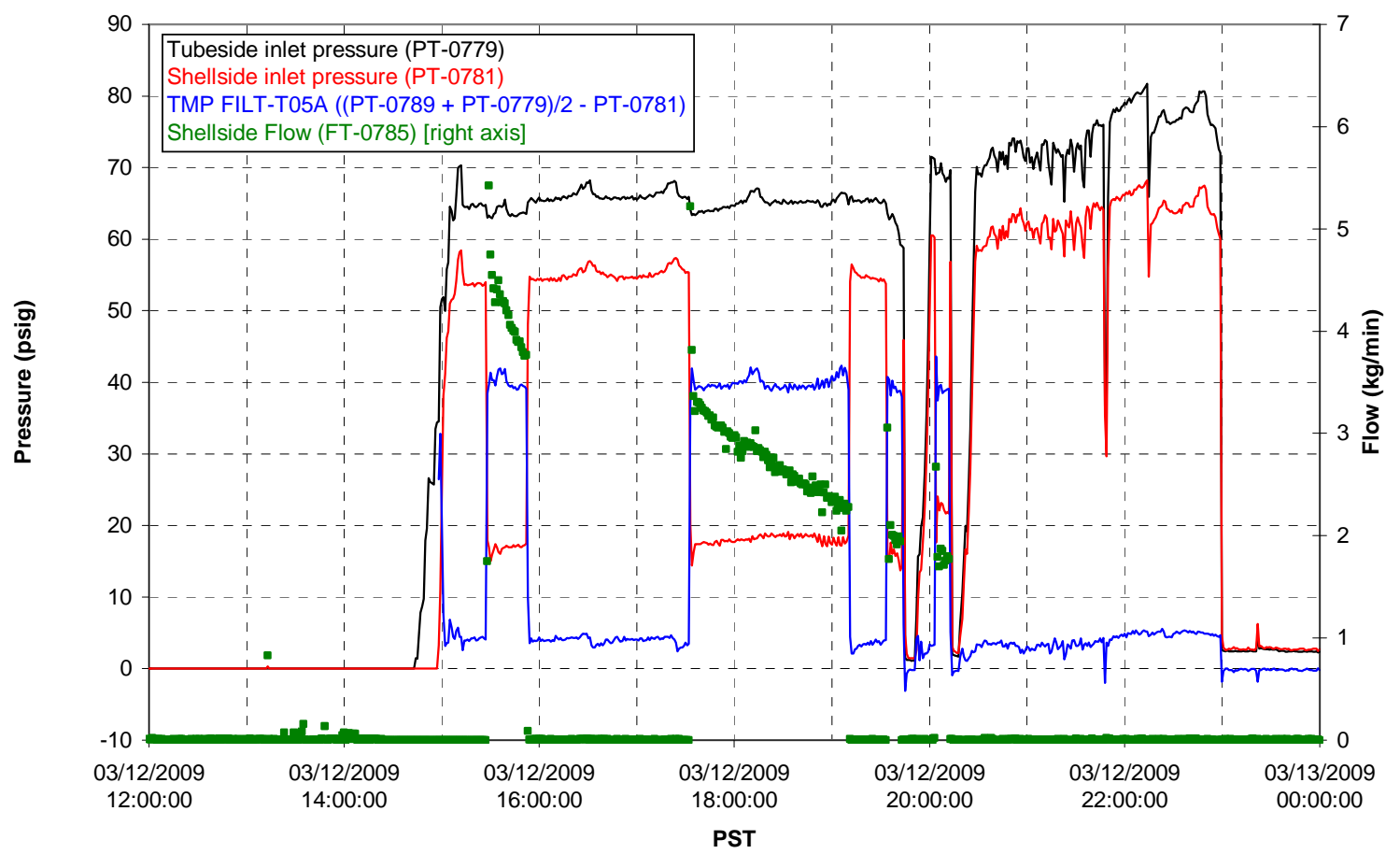

Chemical Flow

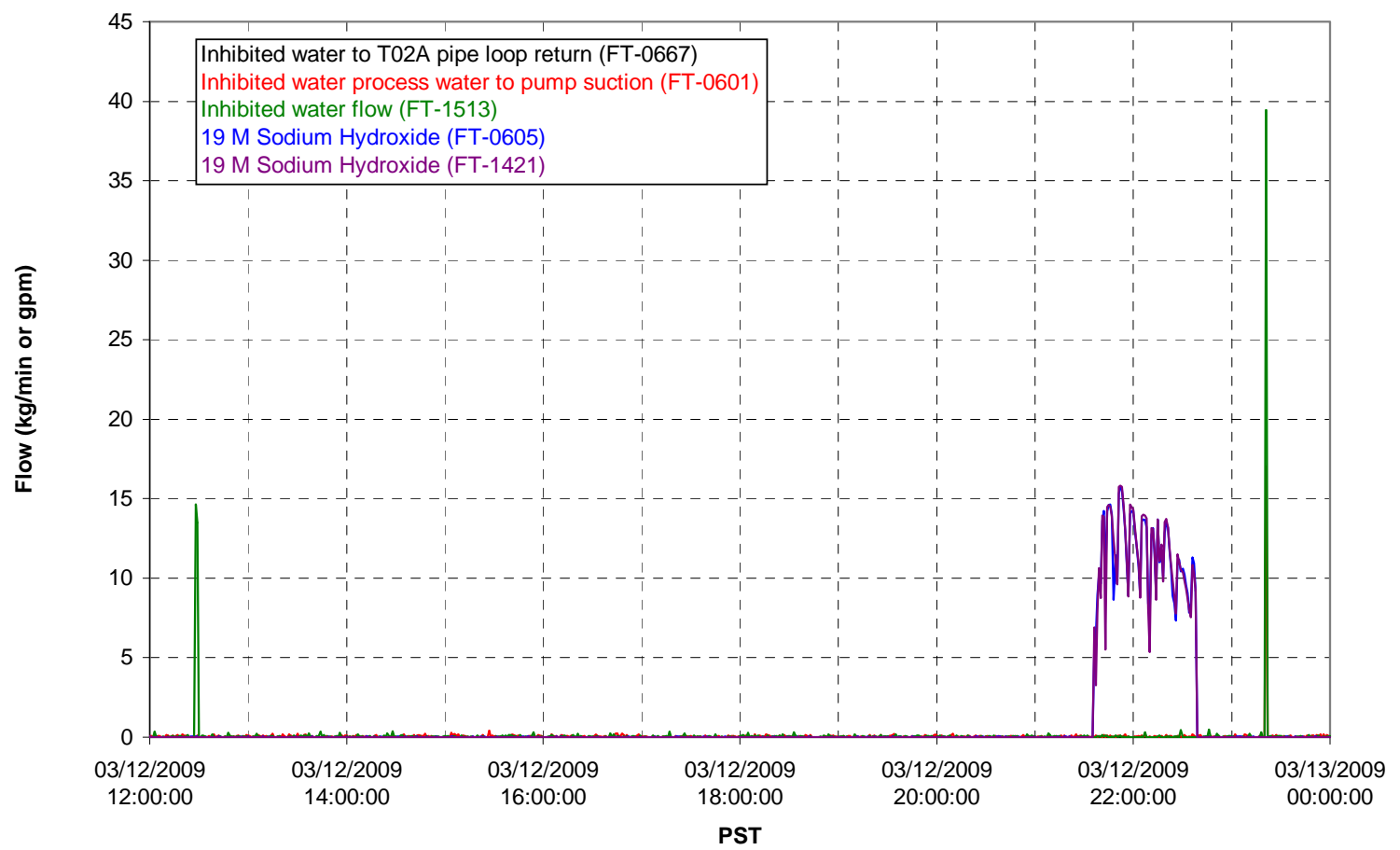




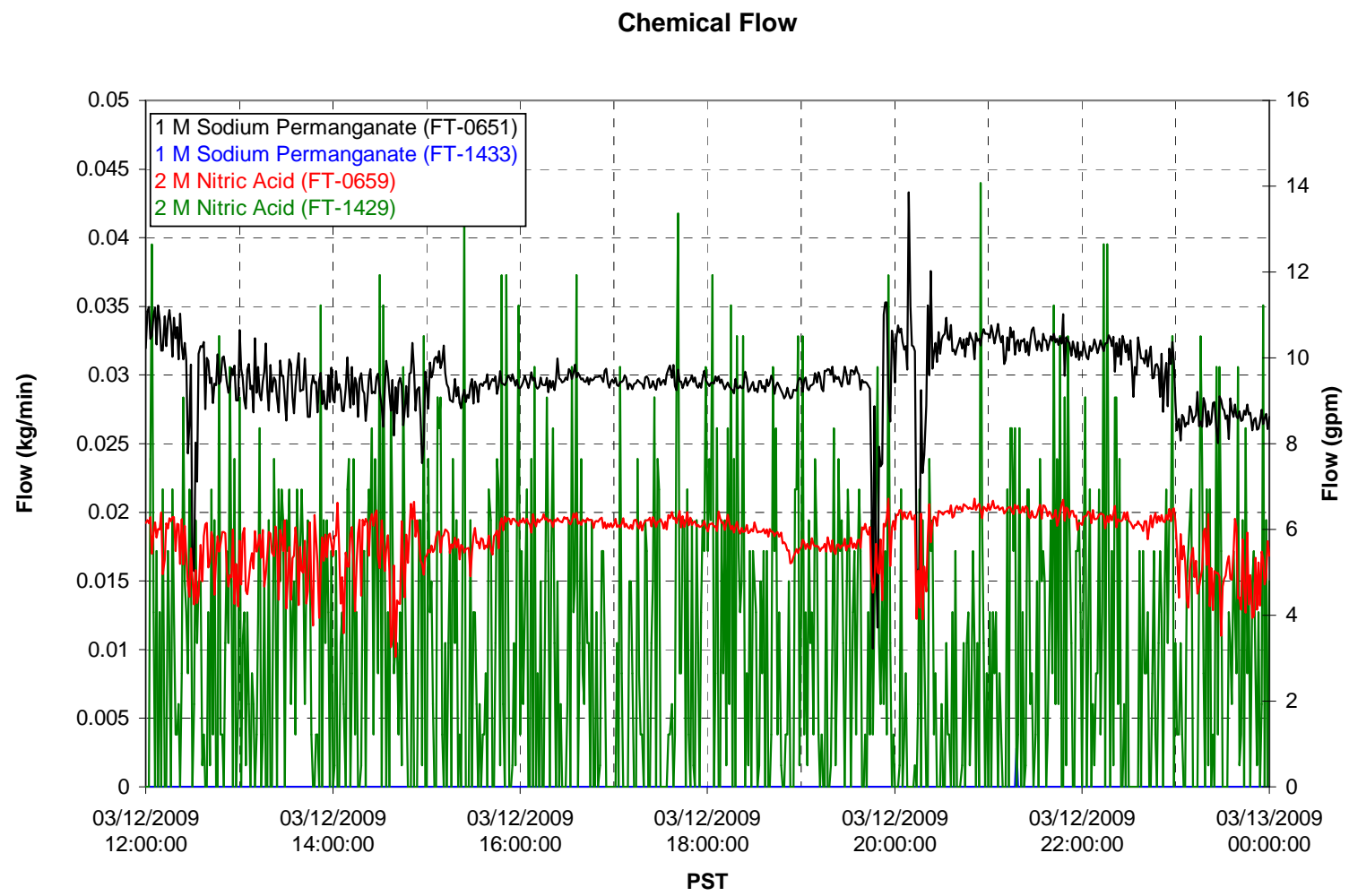

Air Flows

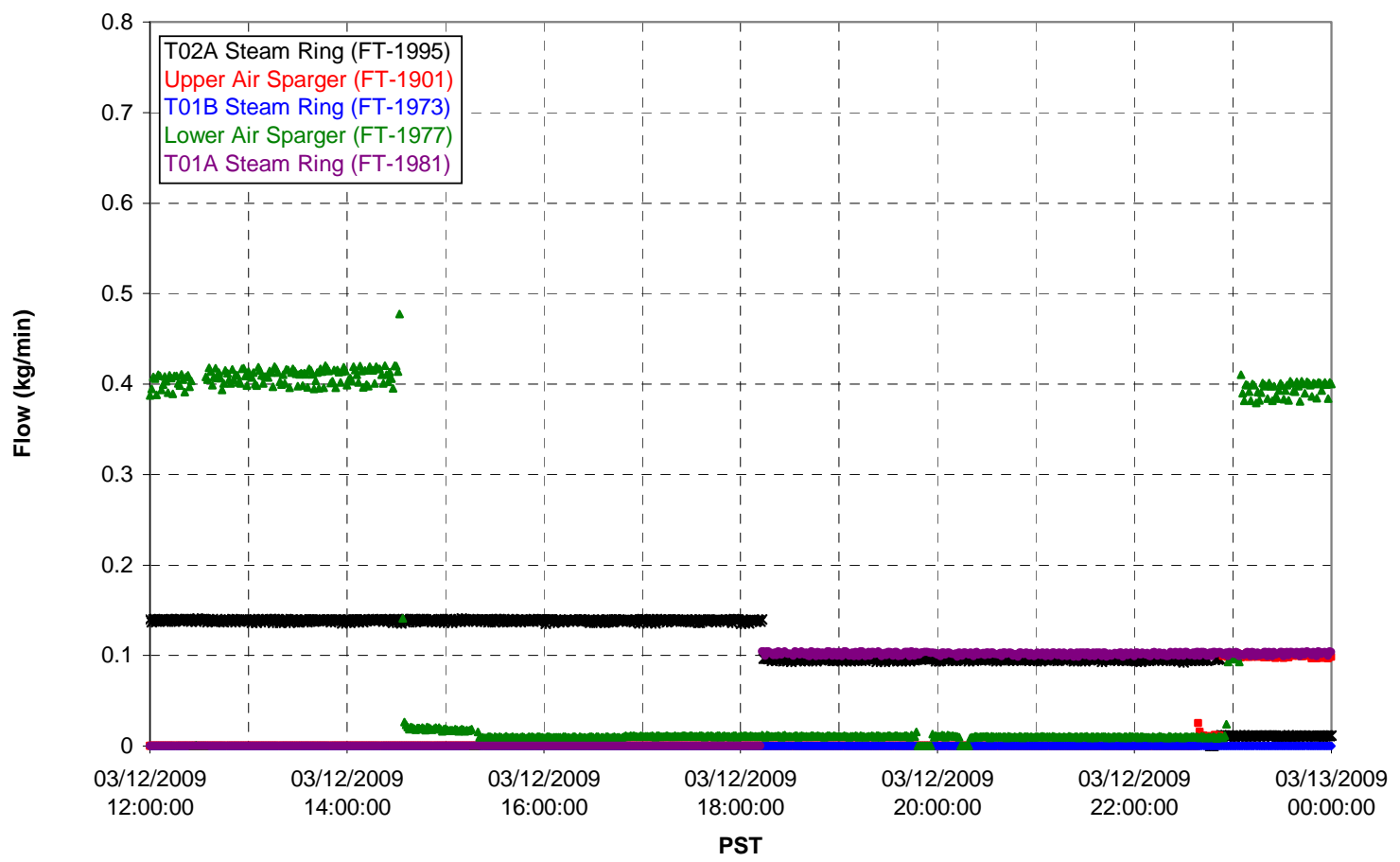


T02A Steam

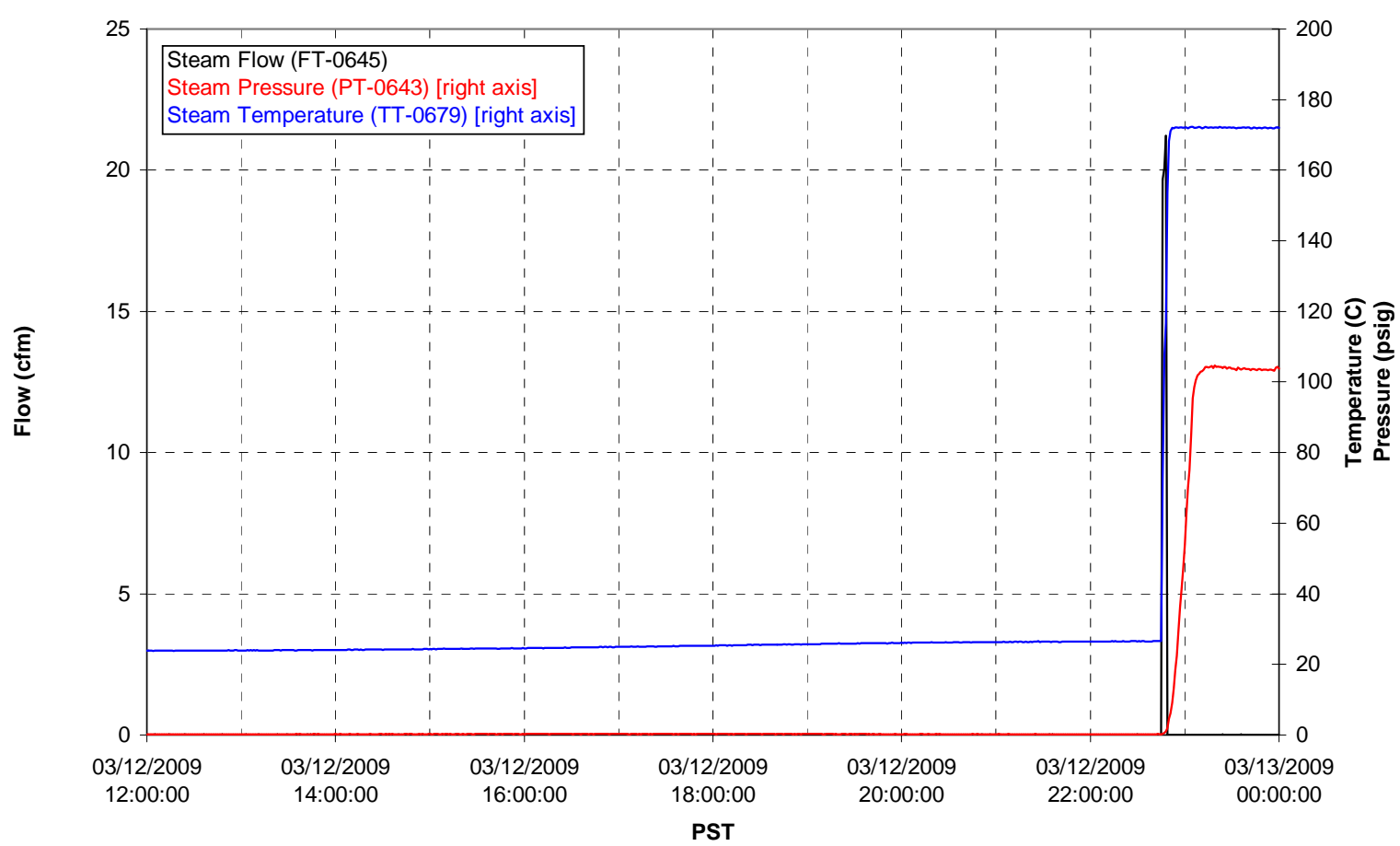

T01A Steam

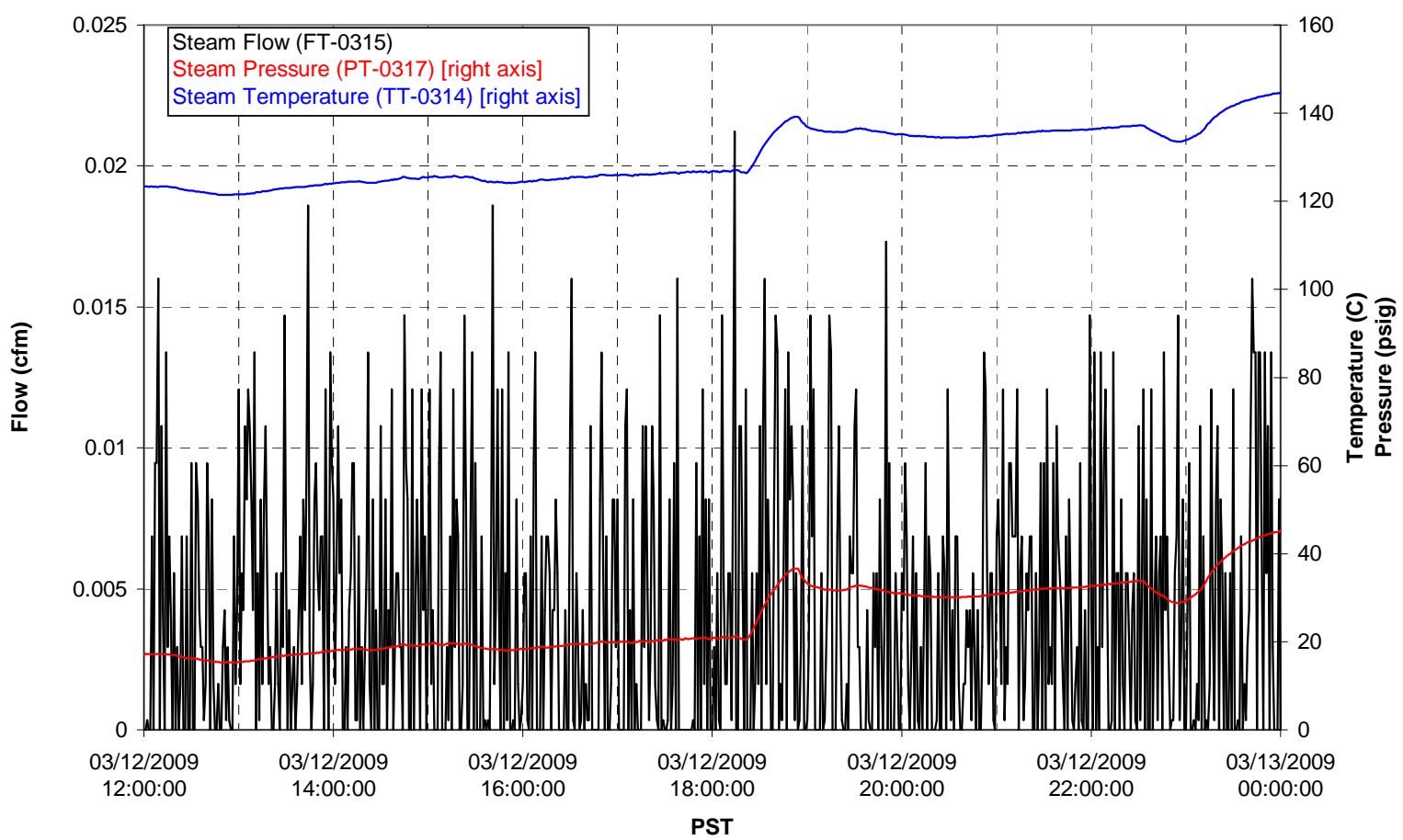


T01B Steam

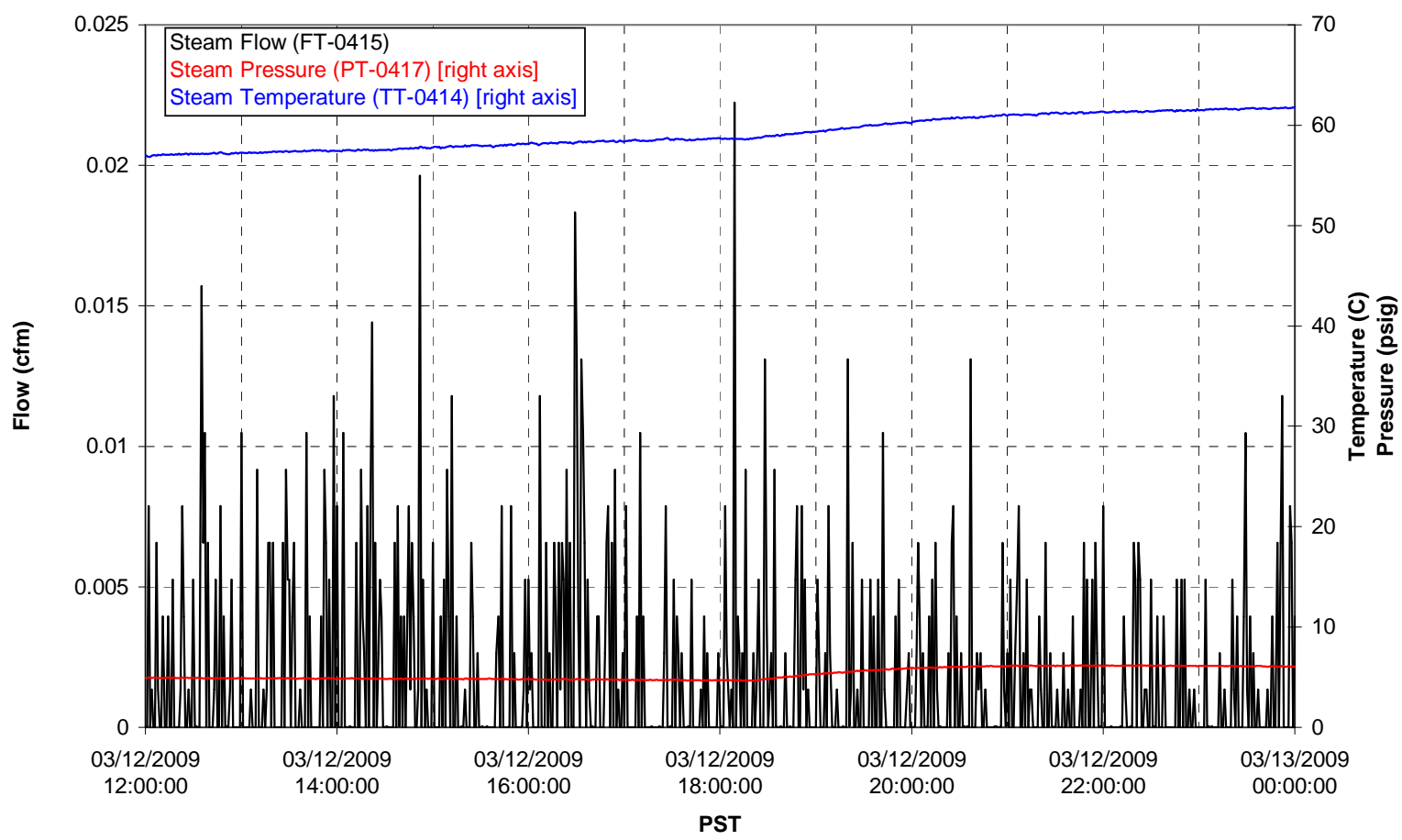




\section{Integrated Test B Data Plots 03/13/09 00:00 - 03/13/09 12:00}


T01A level

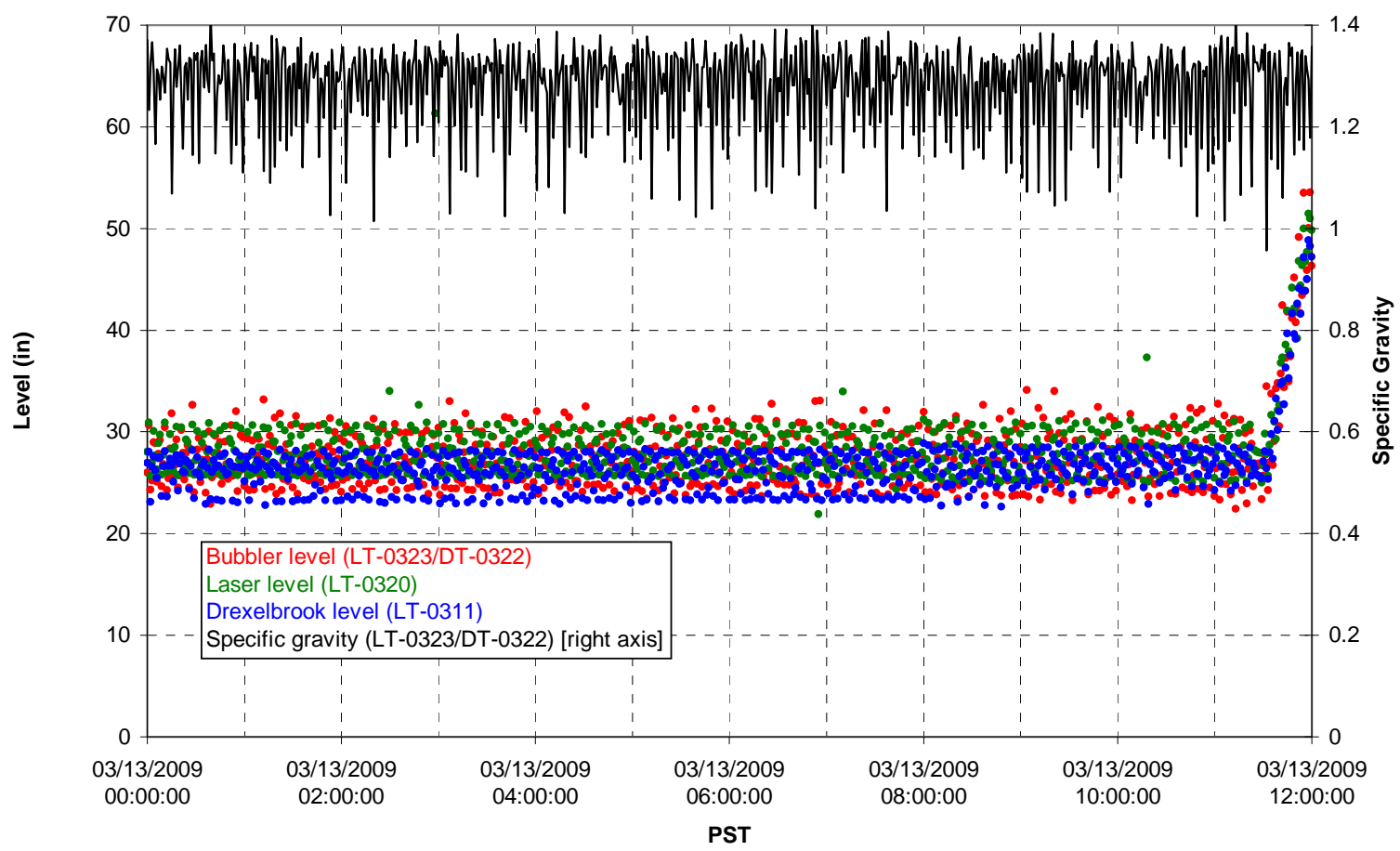

T01A temperatures

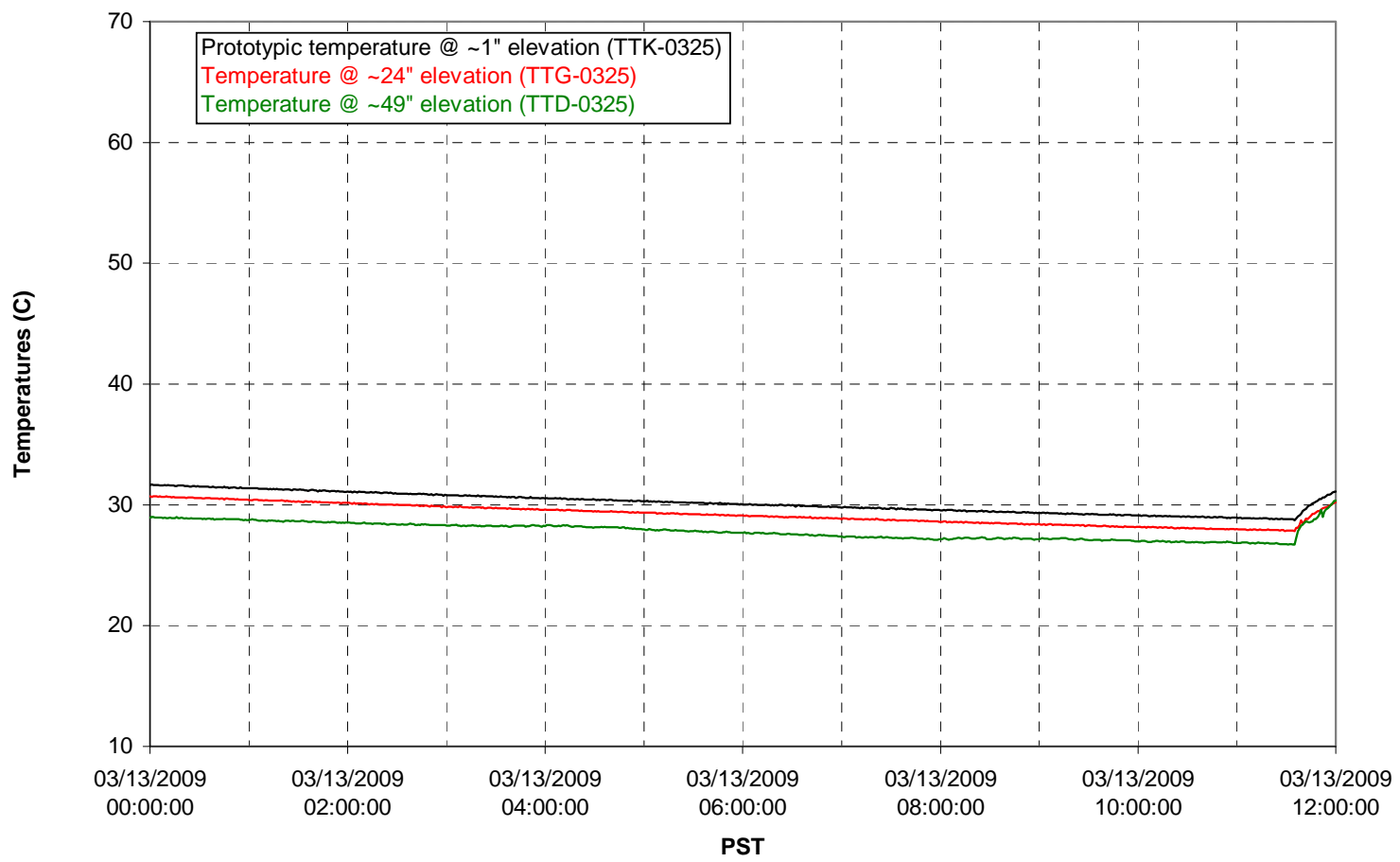


T01B level

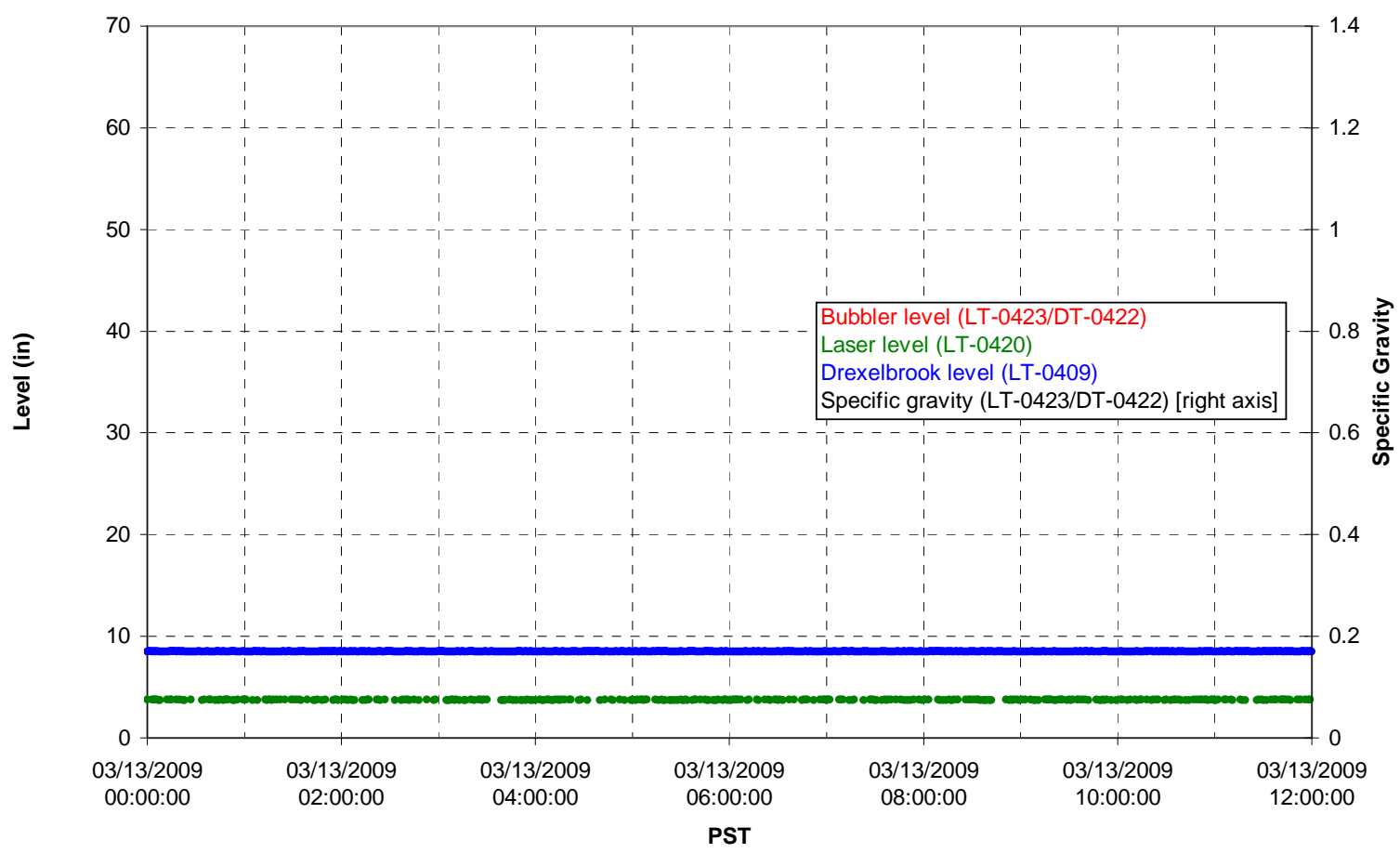

T01B temperatures

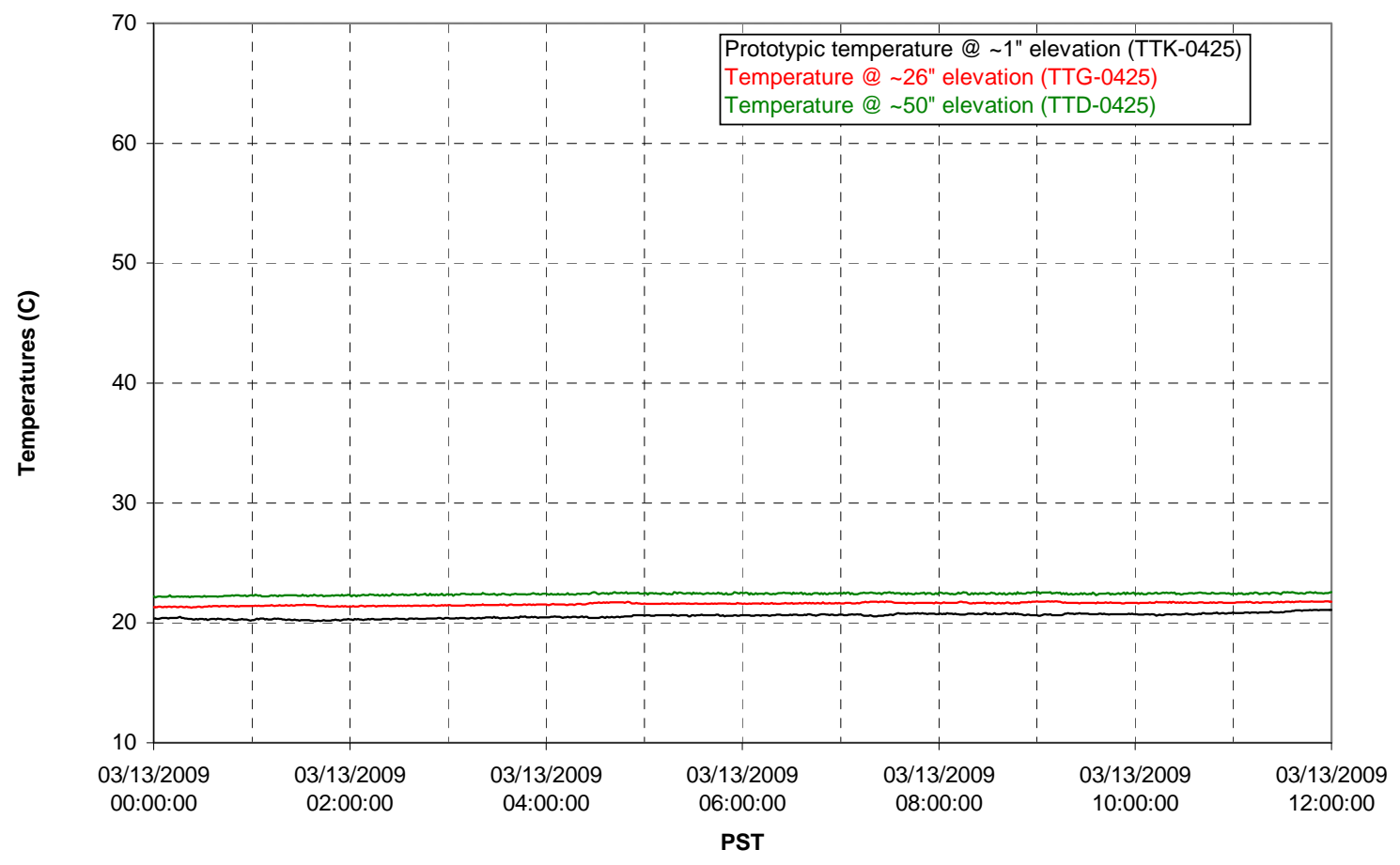


T02A level

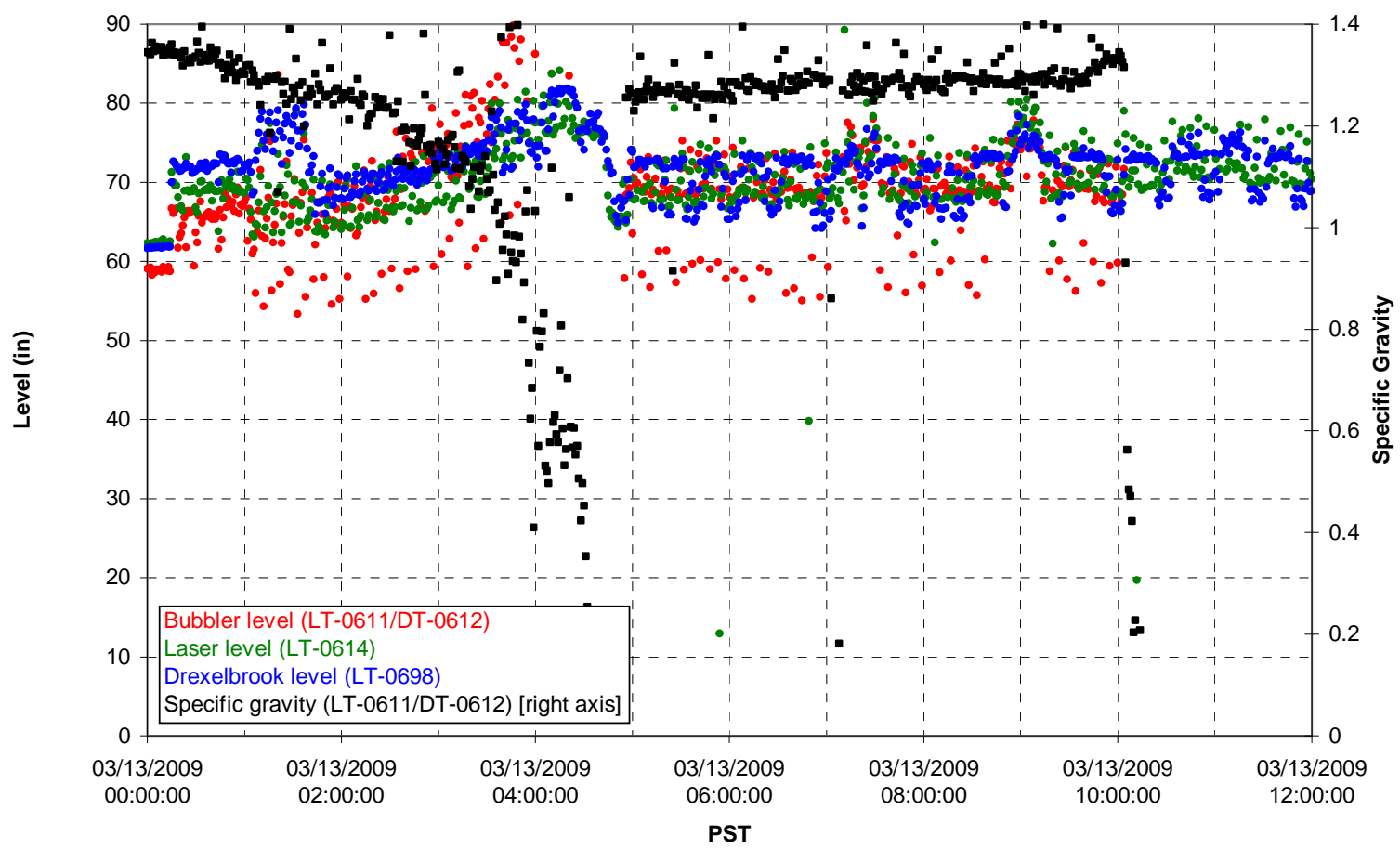

T02A temperatures

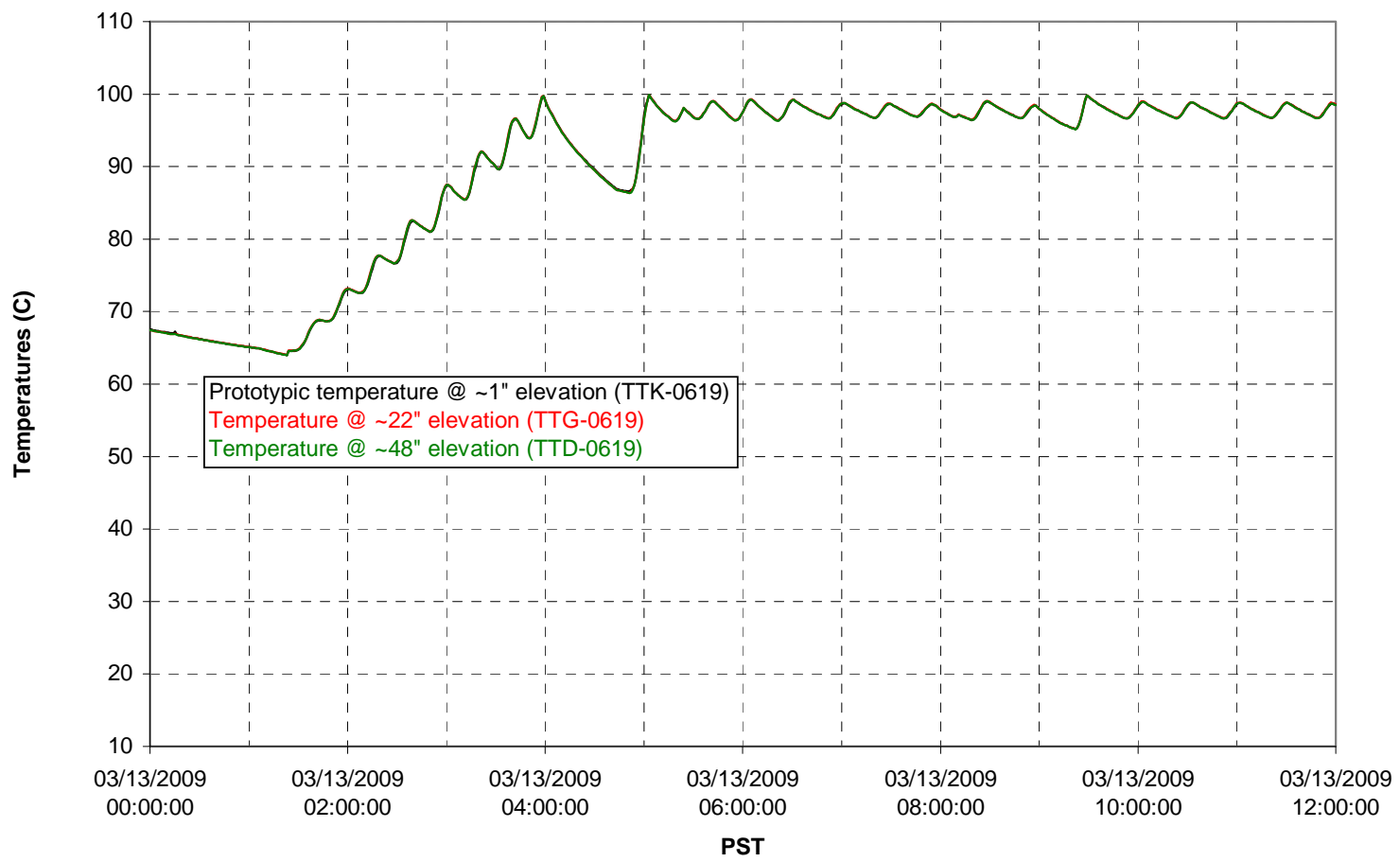


T02A and filter loop temperatures

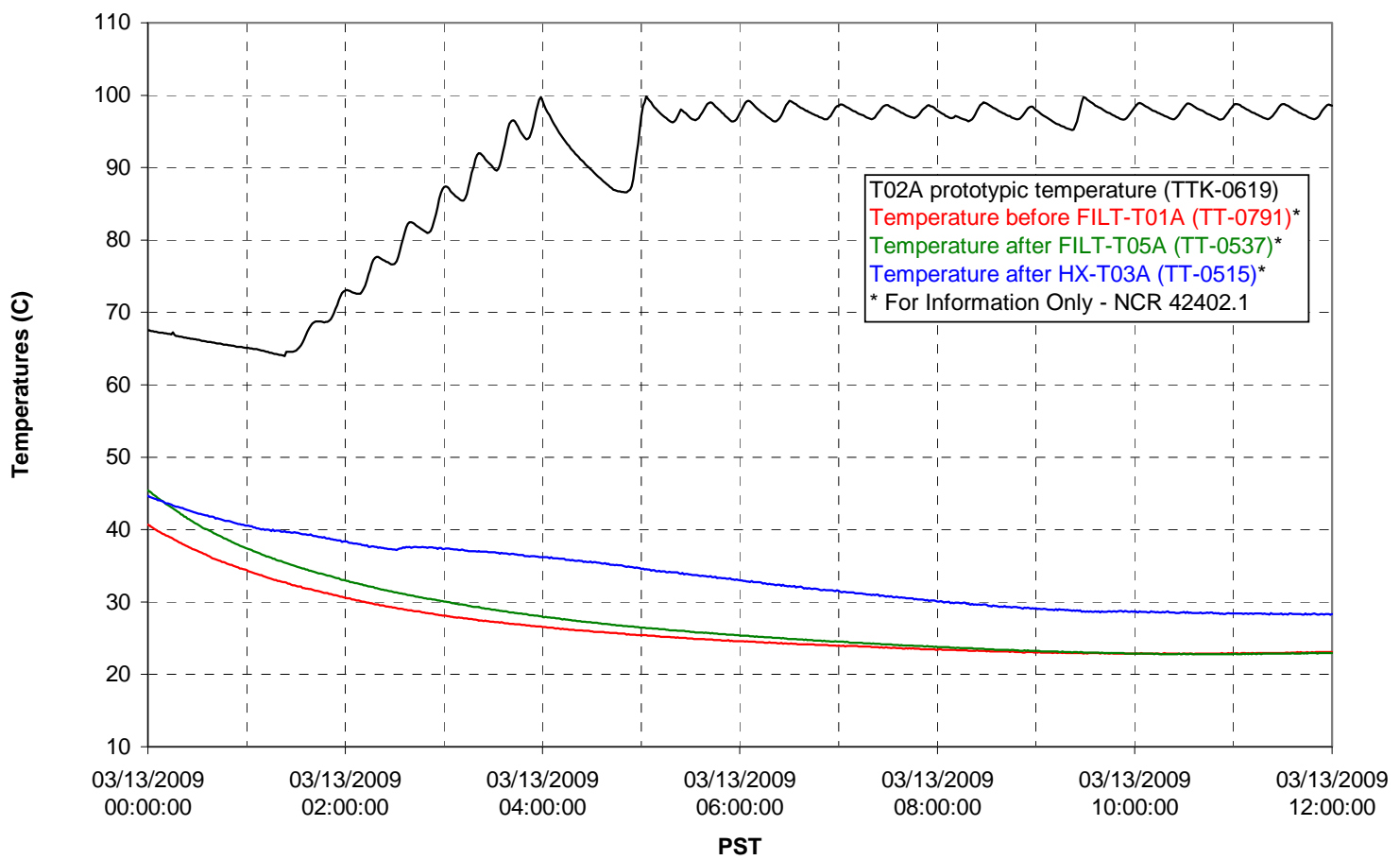

Pump Pressures and Flow

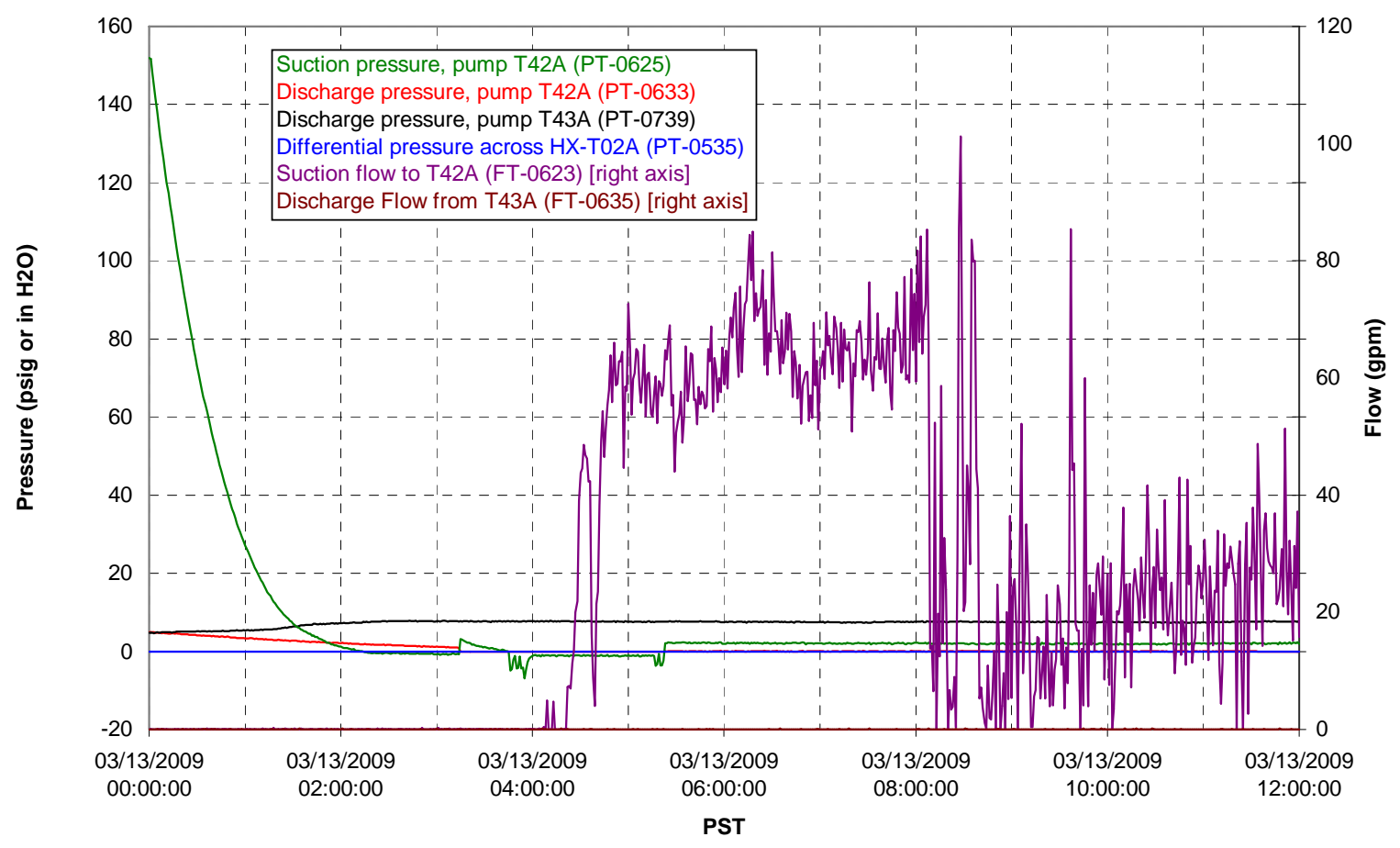


Axial pressure drop

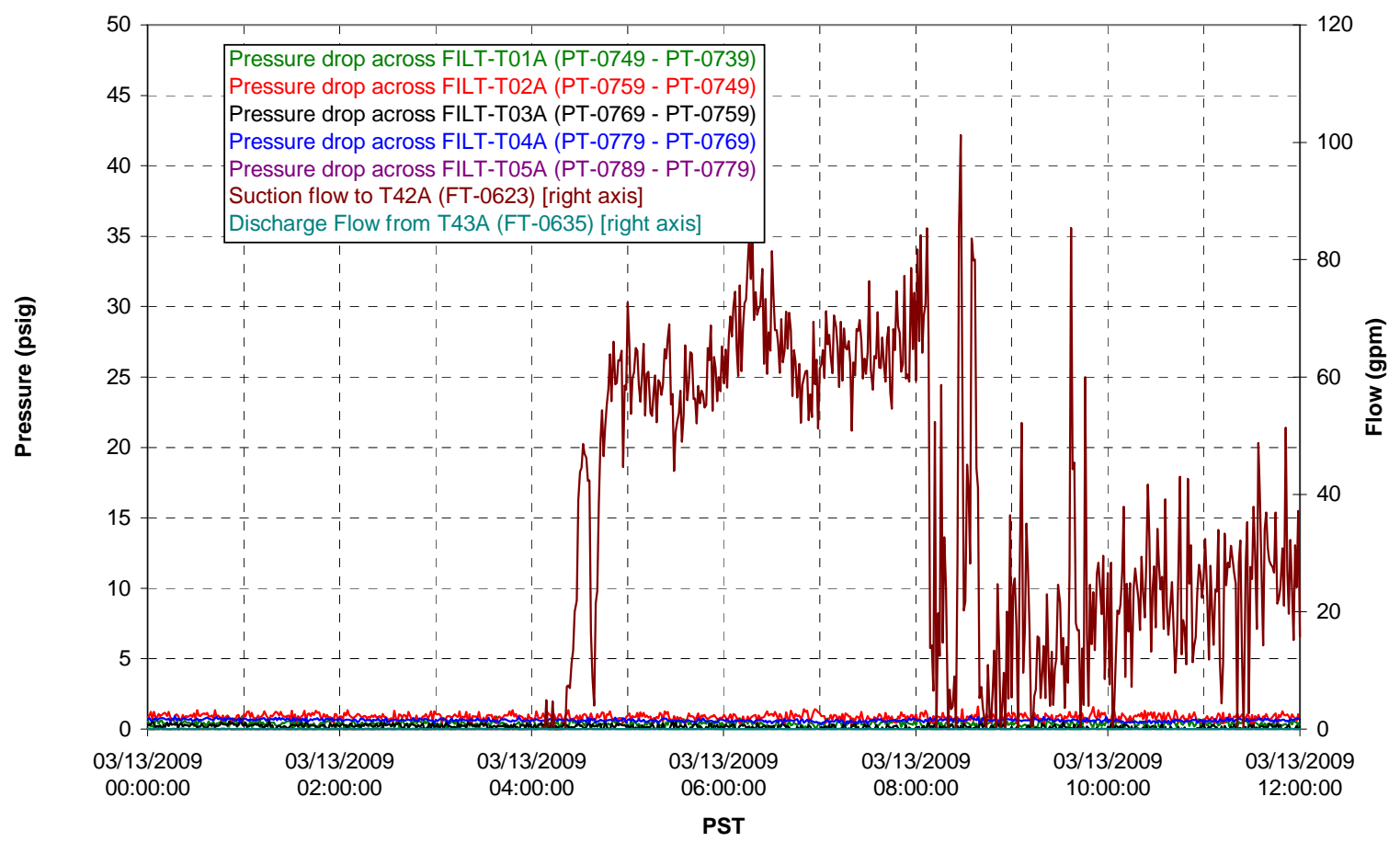

Permeate flow rates

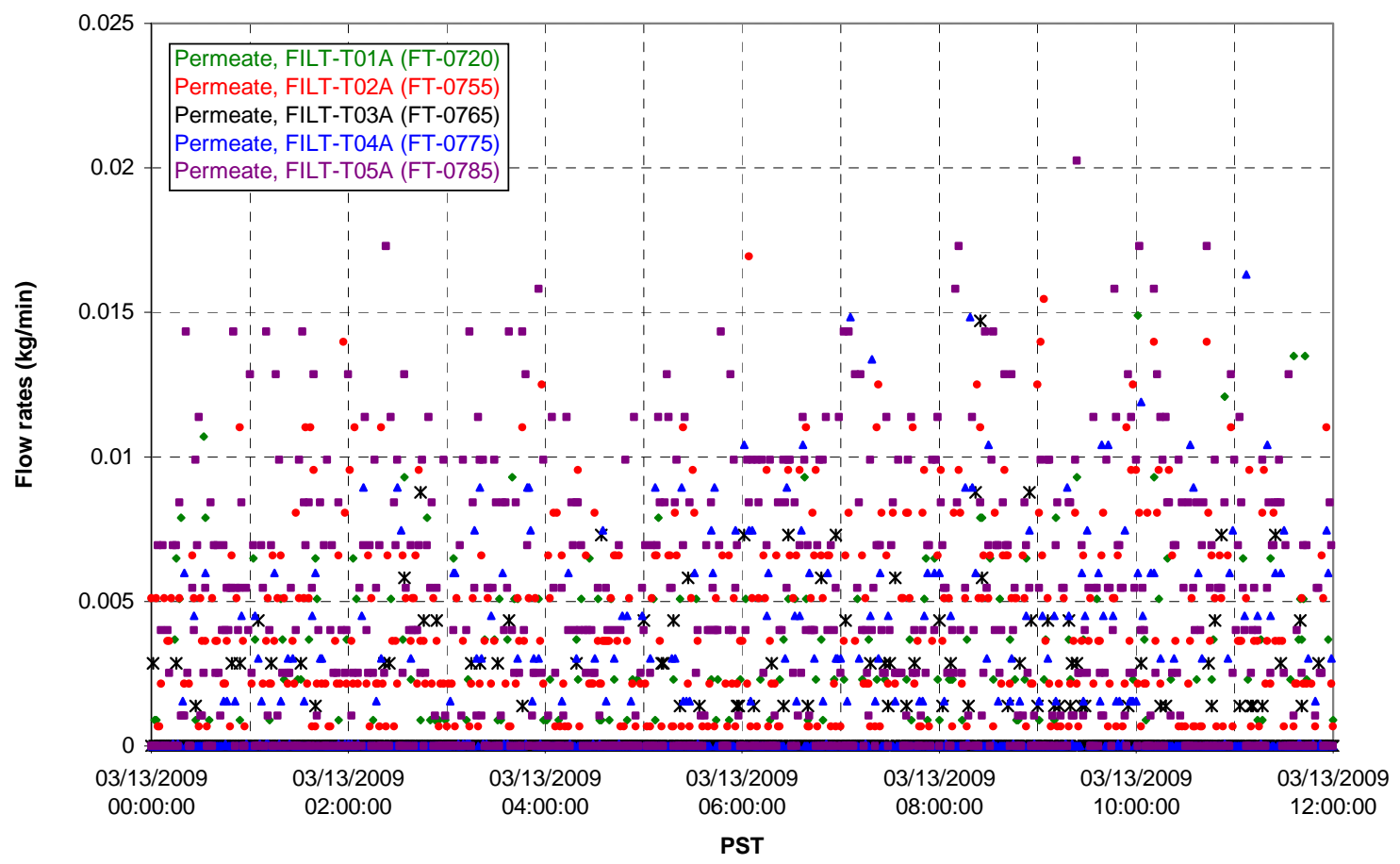


T02A Inner Temperature Tree

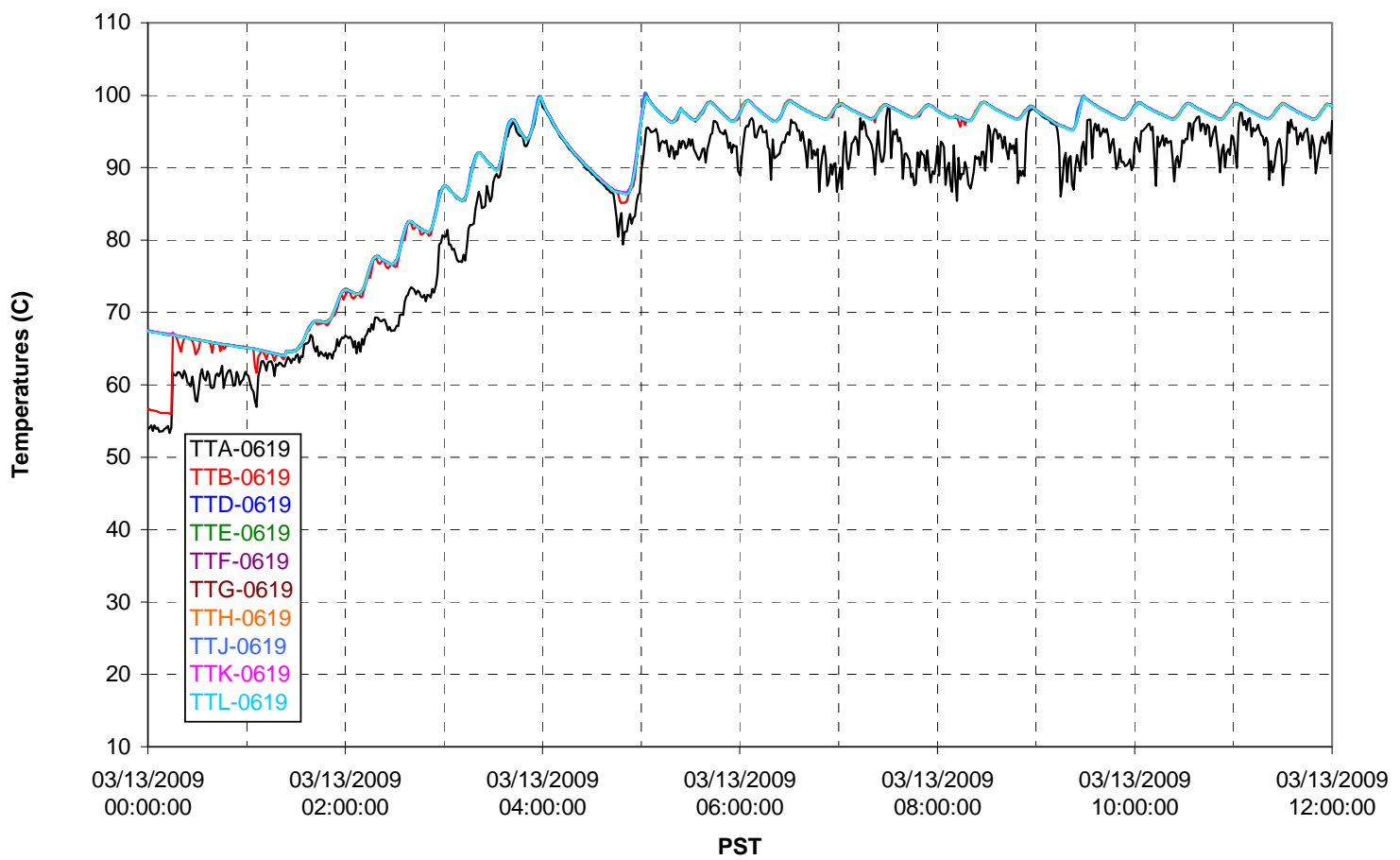

T02A Outer Temperature Tree

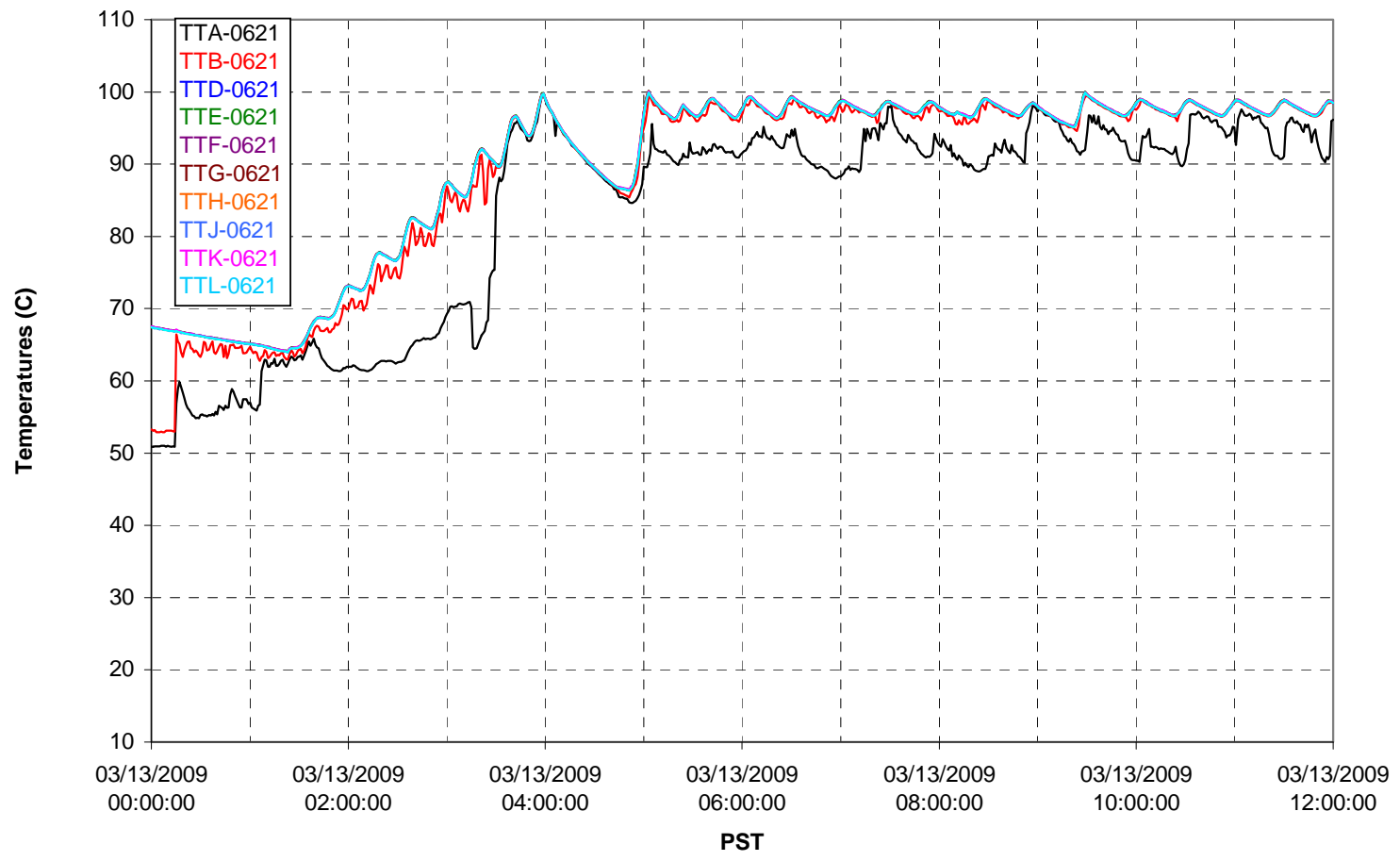


T02A temperatures

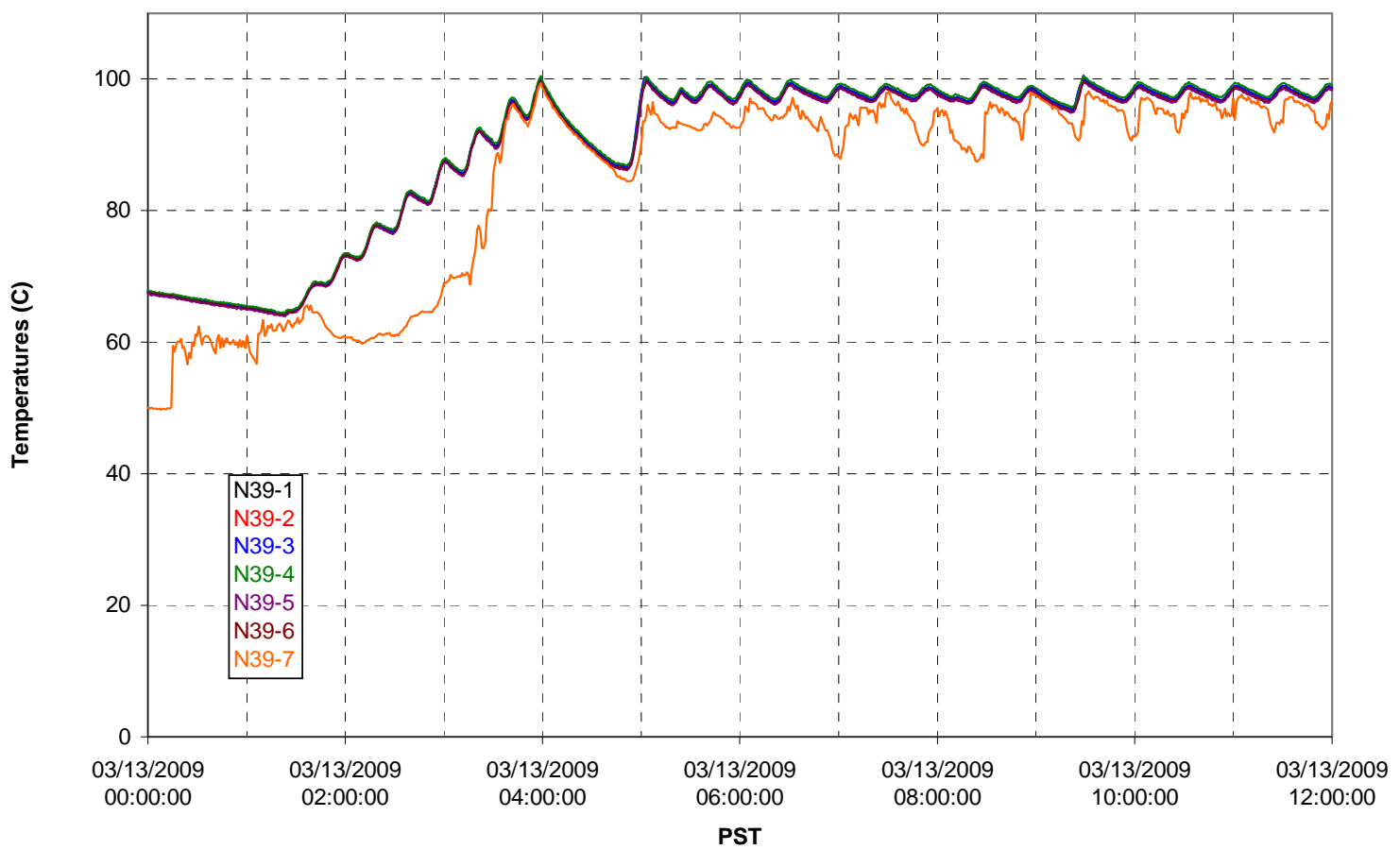

T02A temperatures

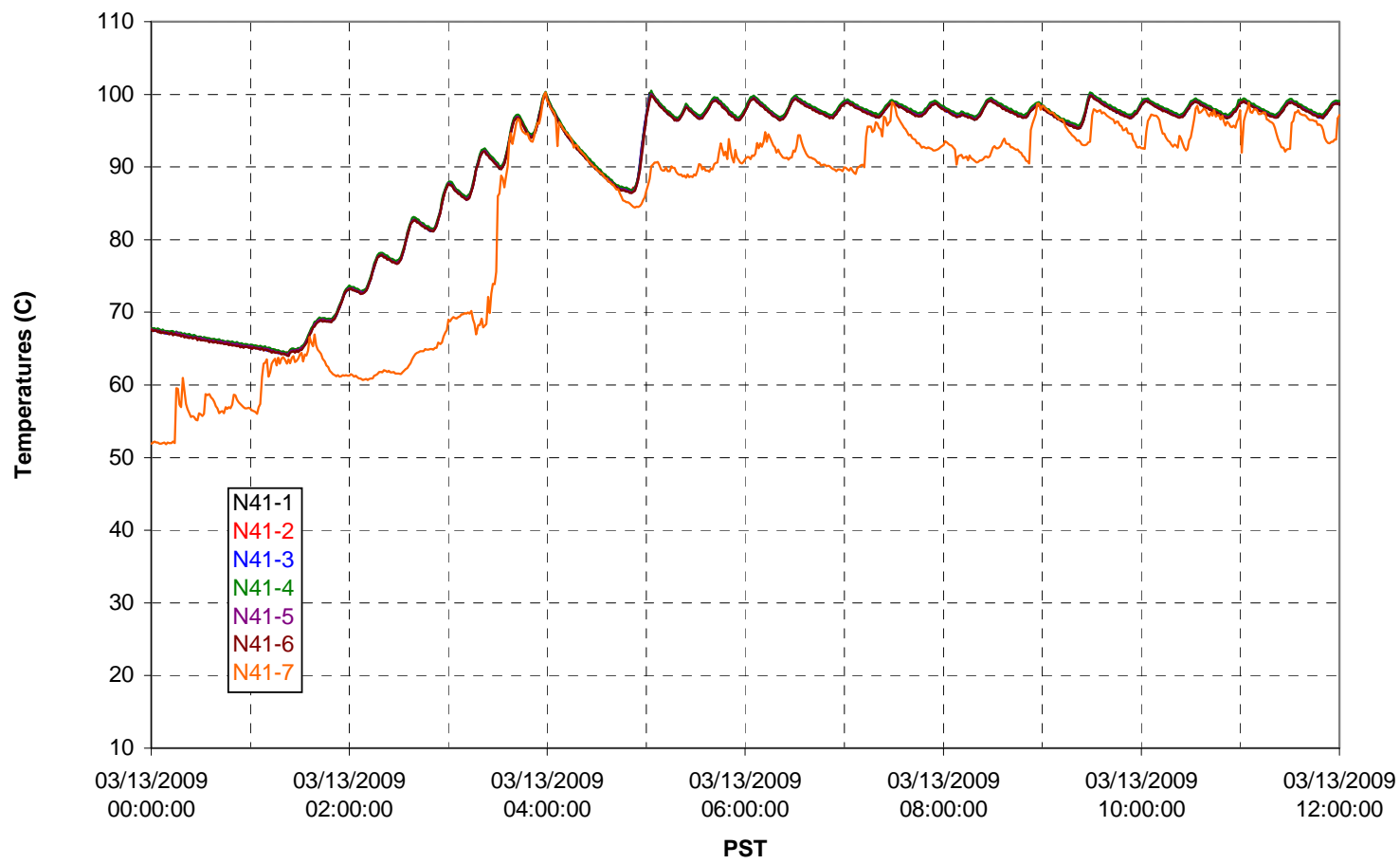


T02A temperatures

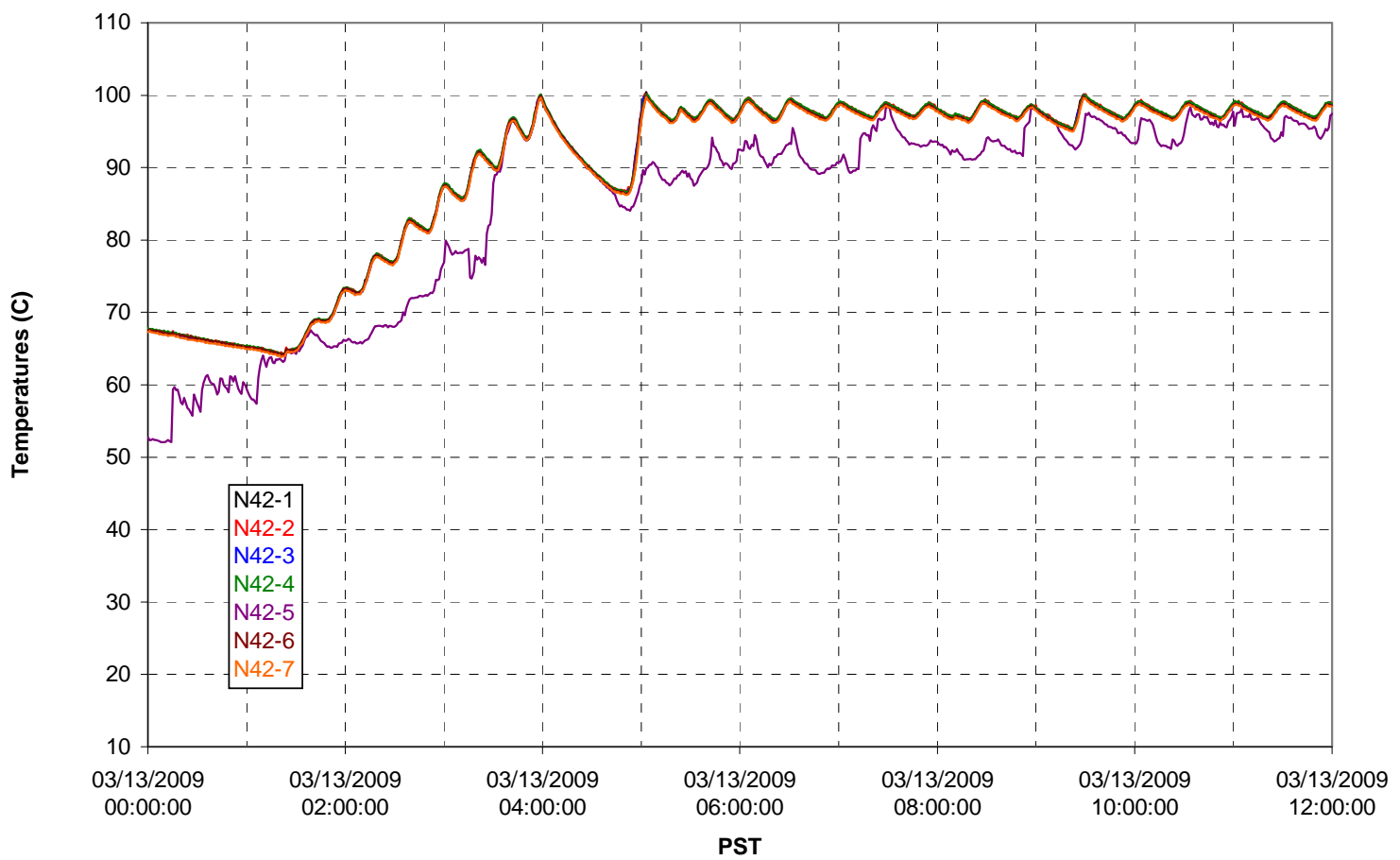

T02A temperatures

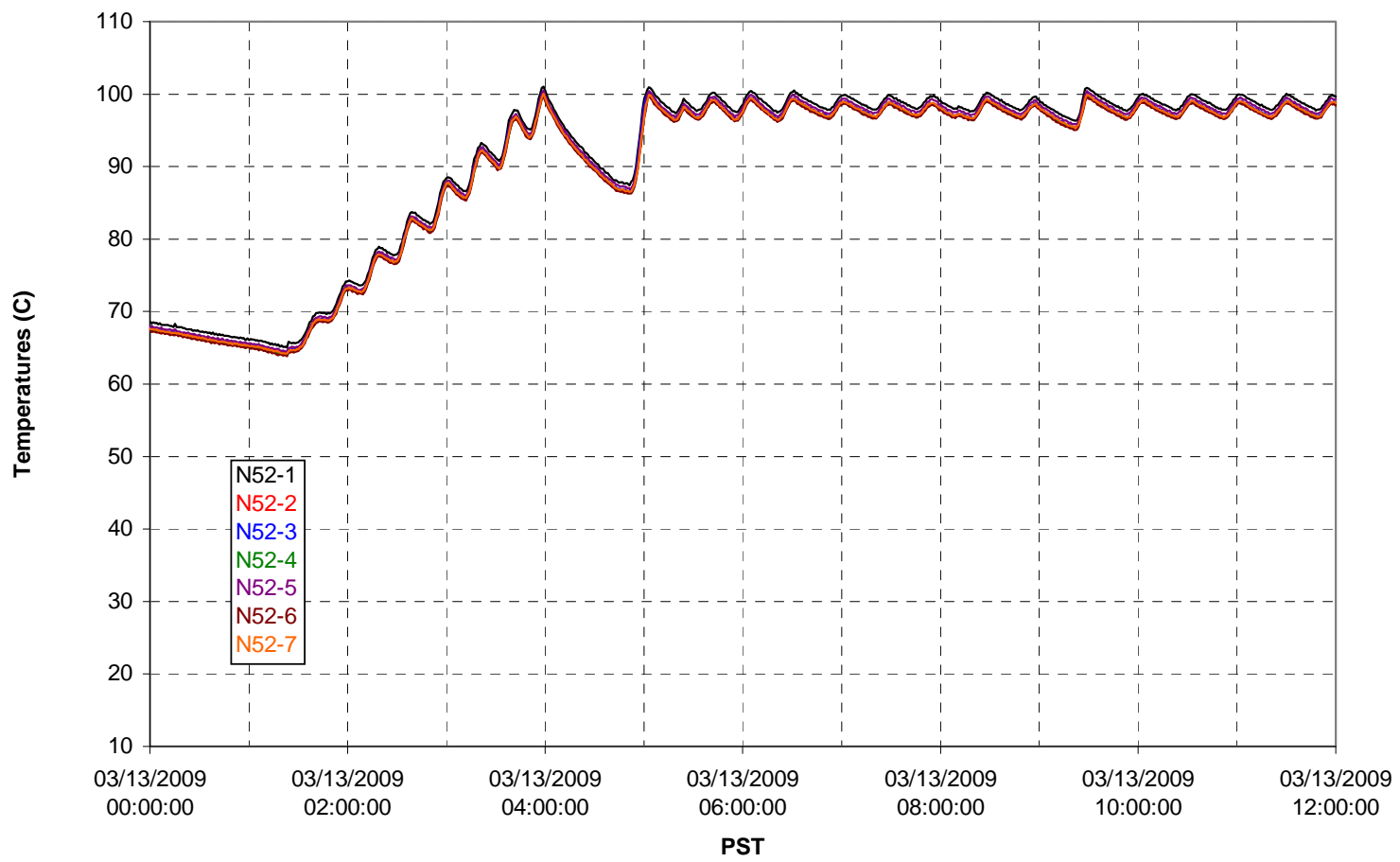


T02A Heating and Cooling

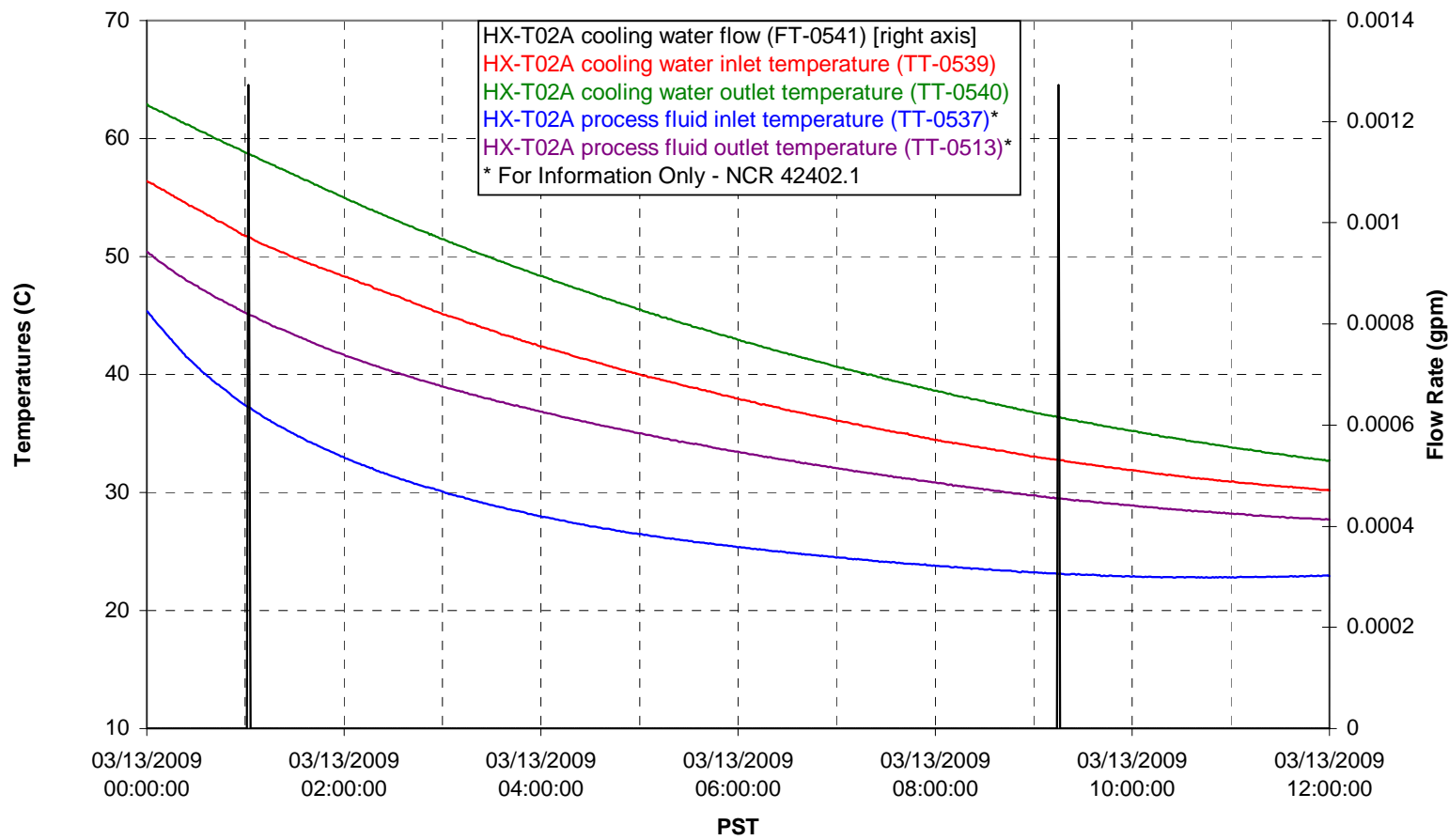

Pump Operation

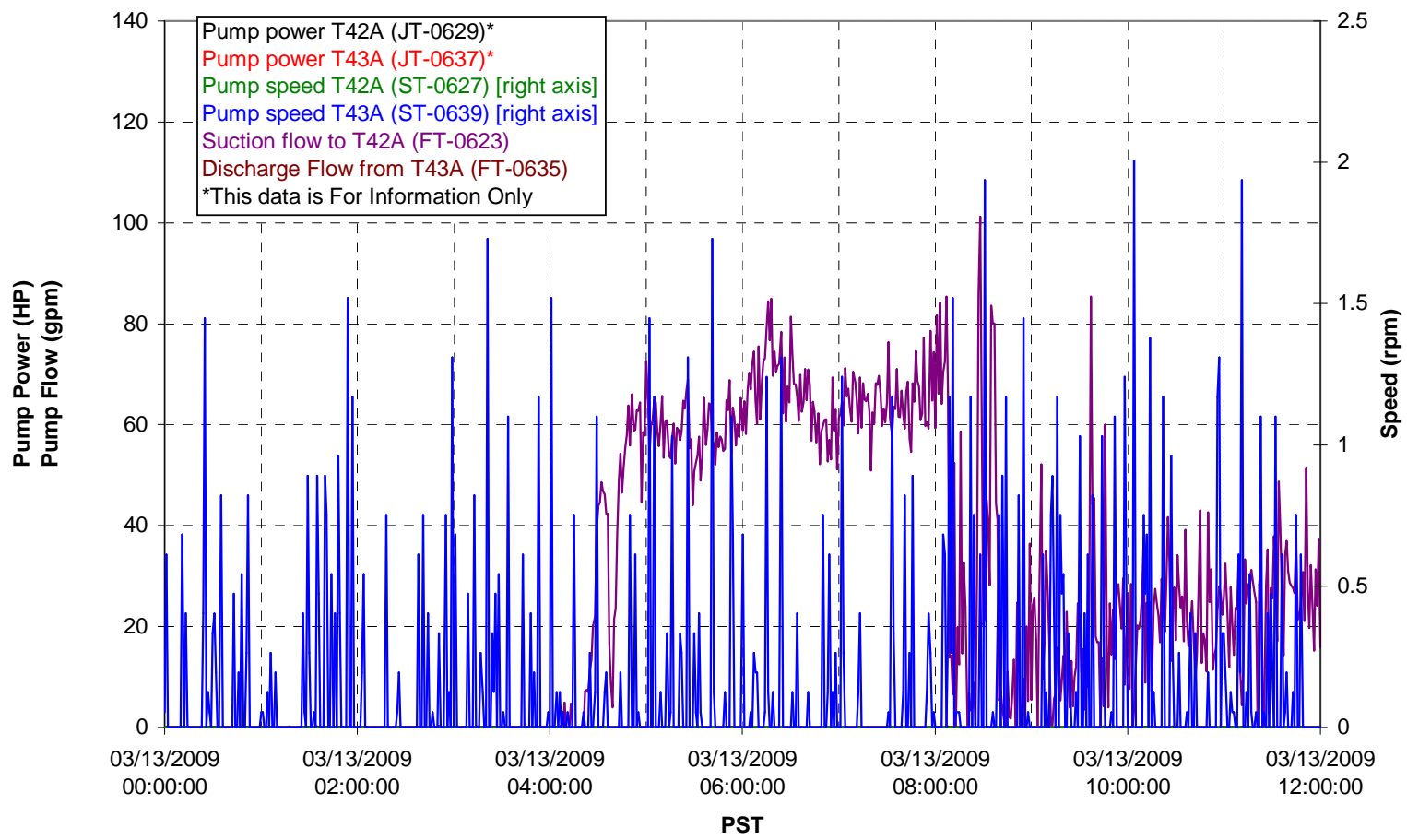


Pulsepot UFP-PP-T01A

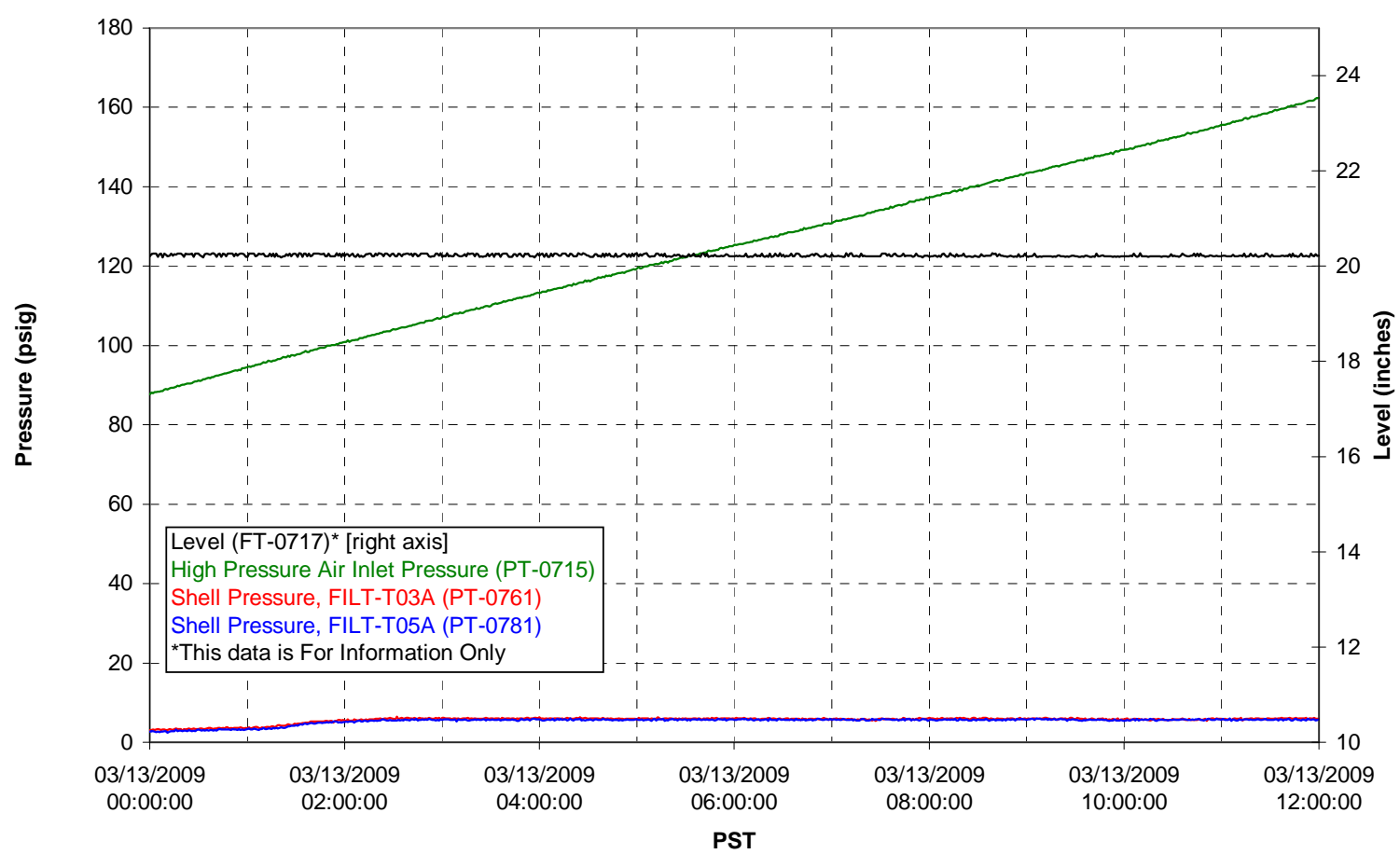

Pulsepot UFP-PP-T02A

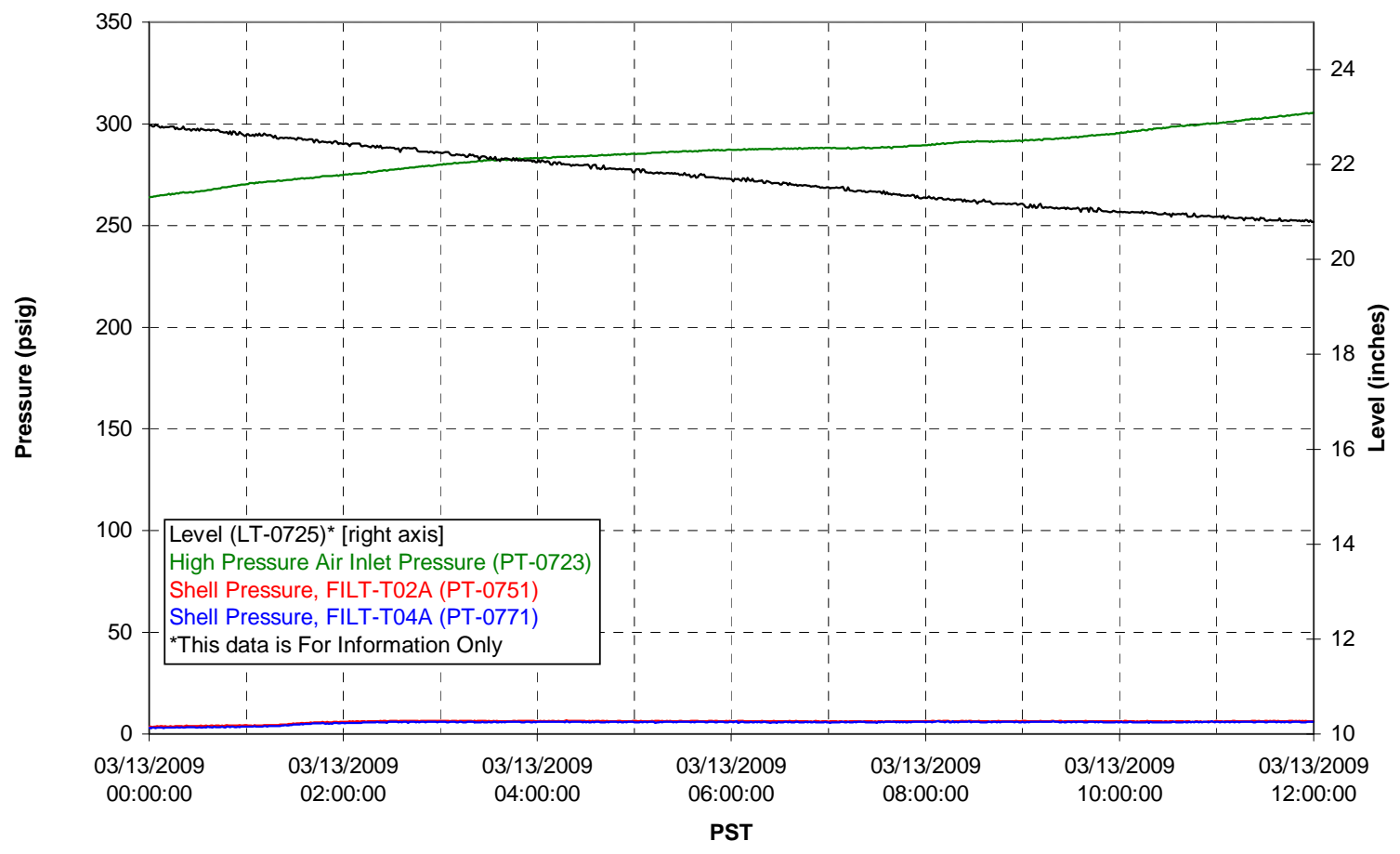


Pulsepot UFP-PP-T03A

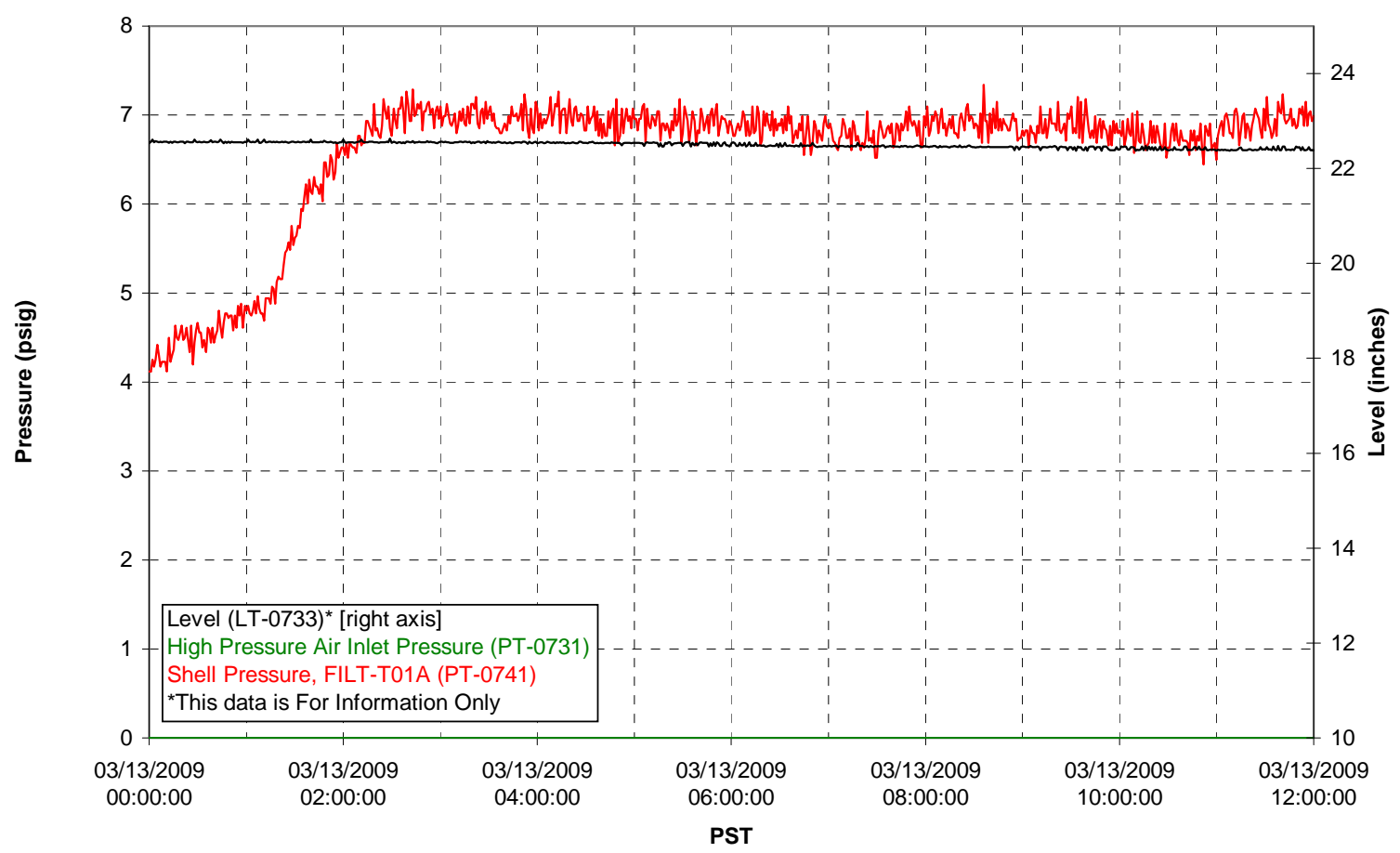

Pulsepot Levels

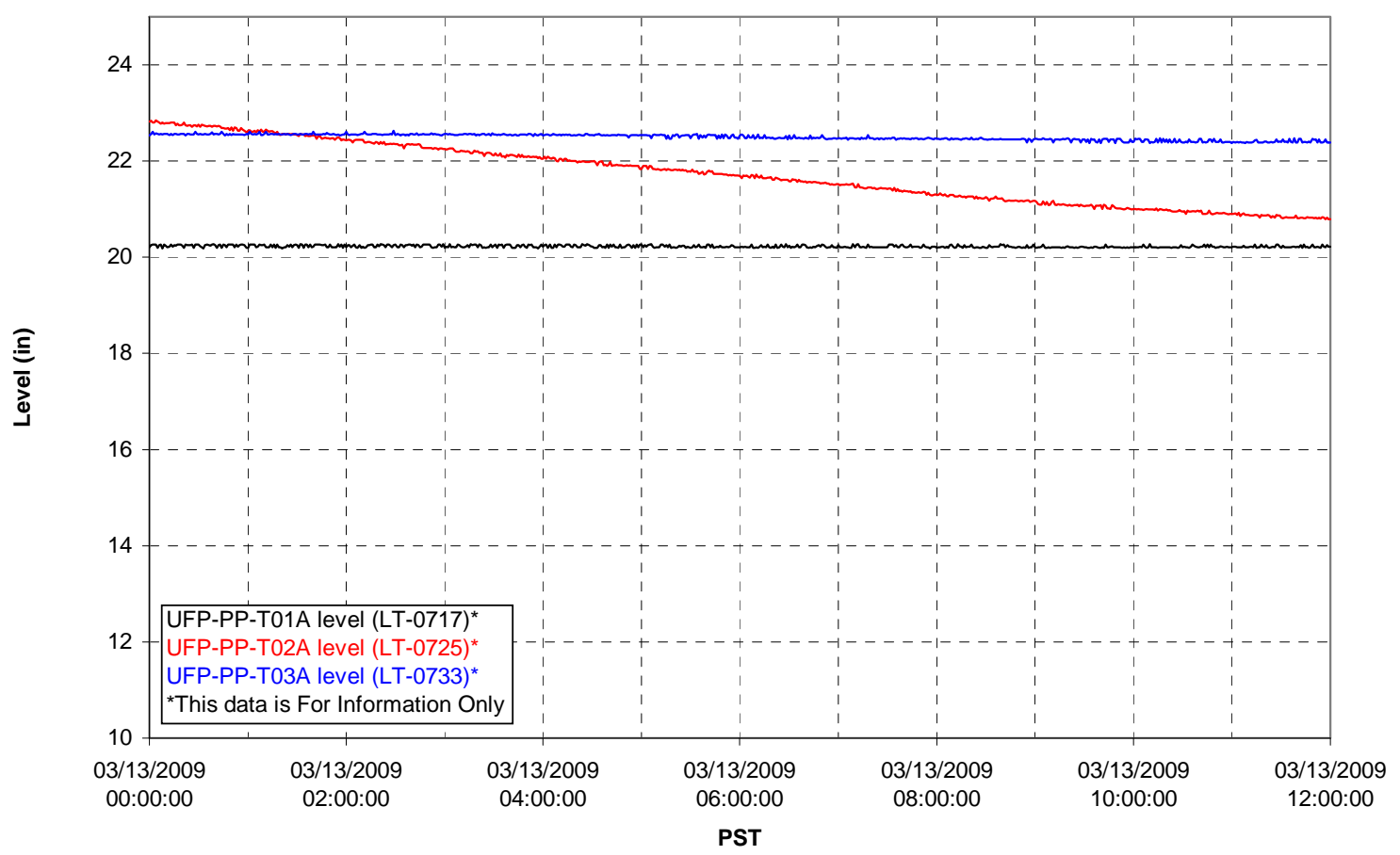


Filter UFP-FILT-T01A

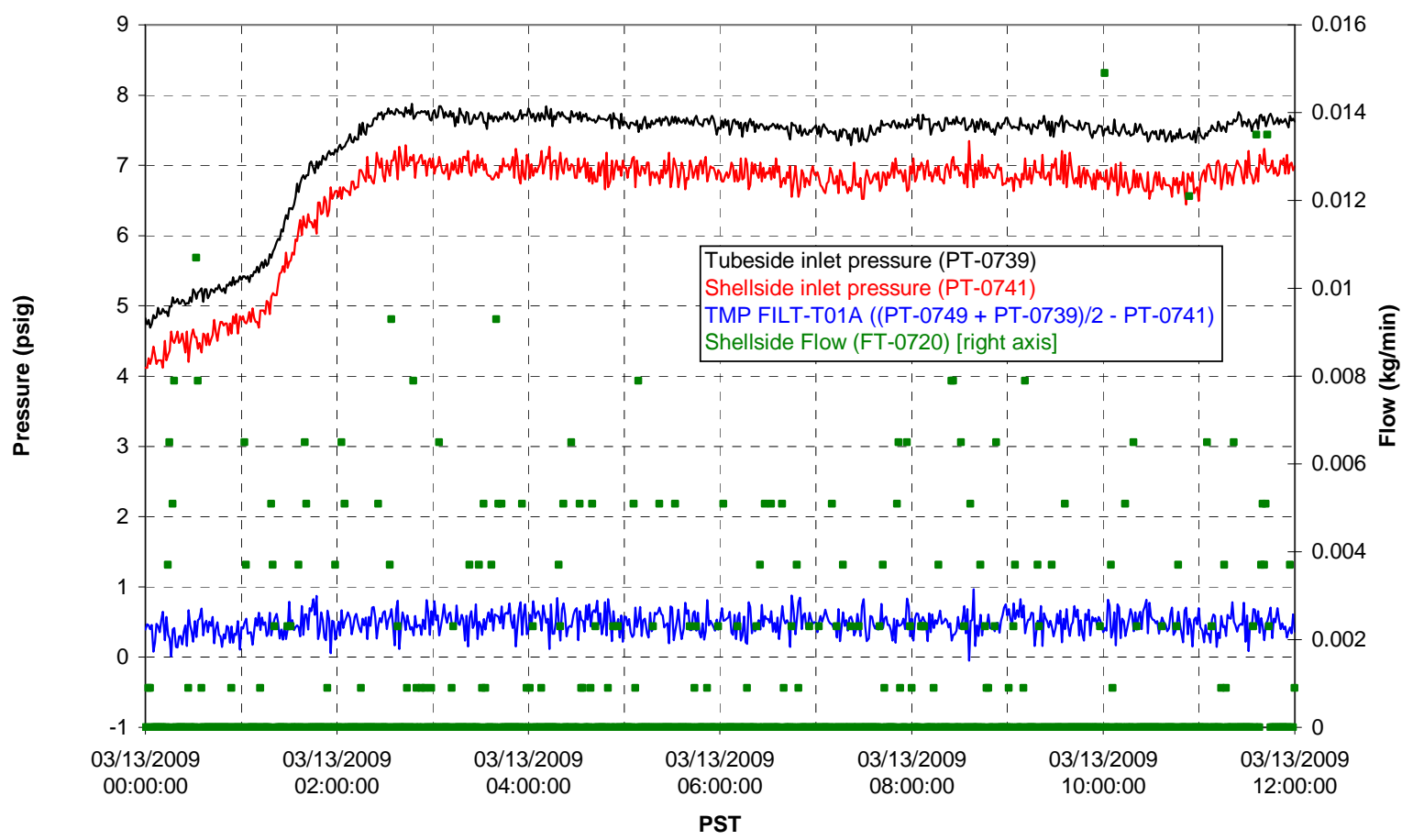

Filter UFP-FILT-T02A

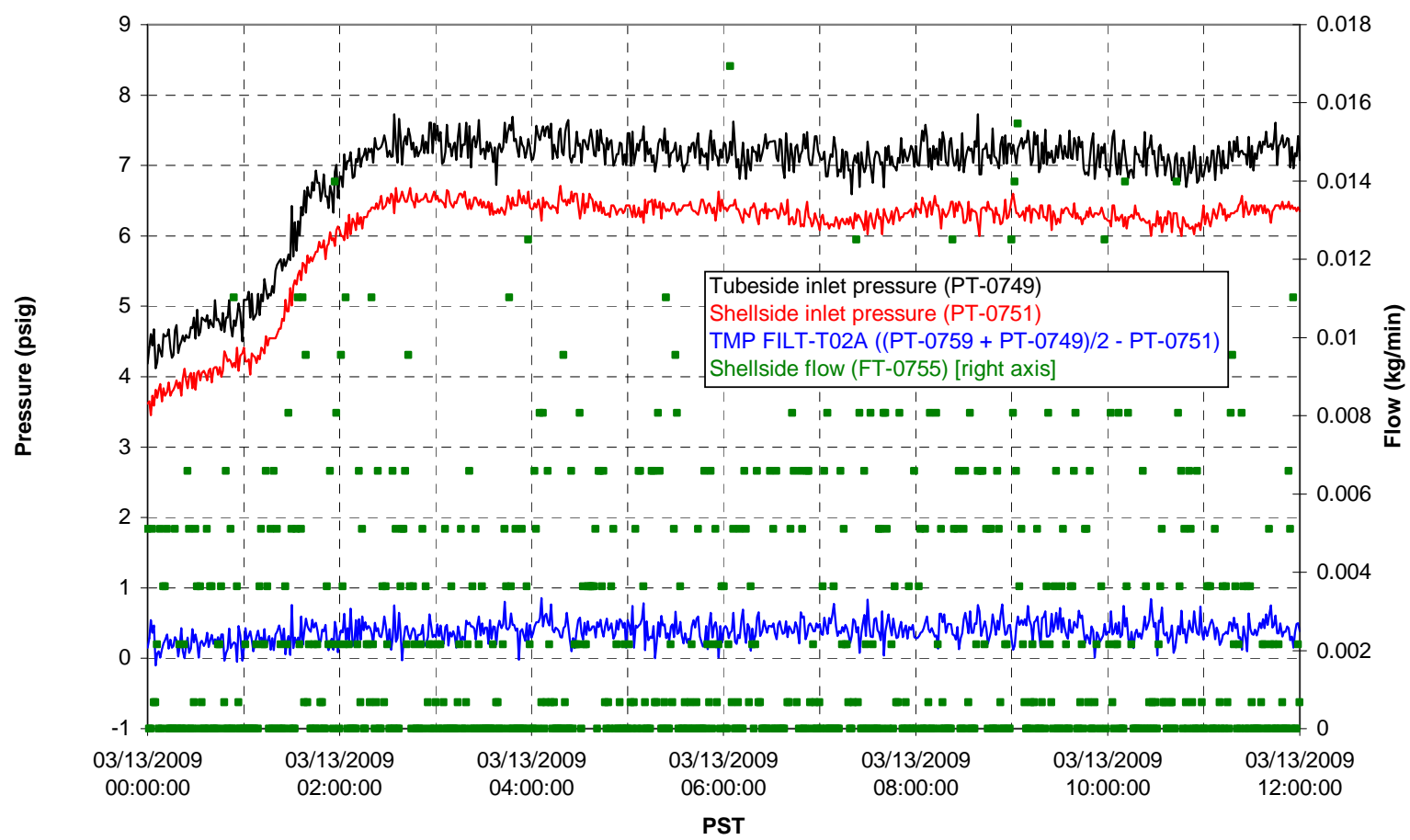


Filter UFP-FILT-T03A

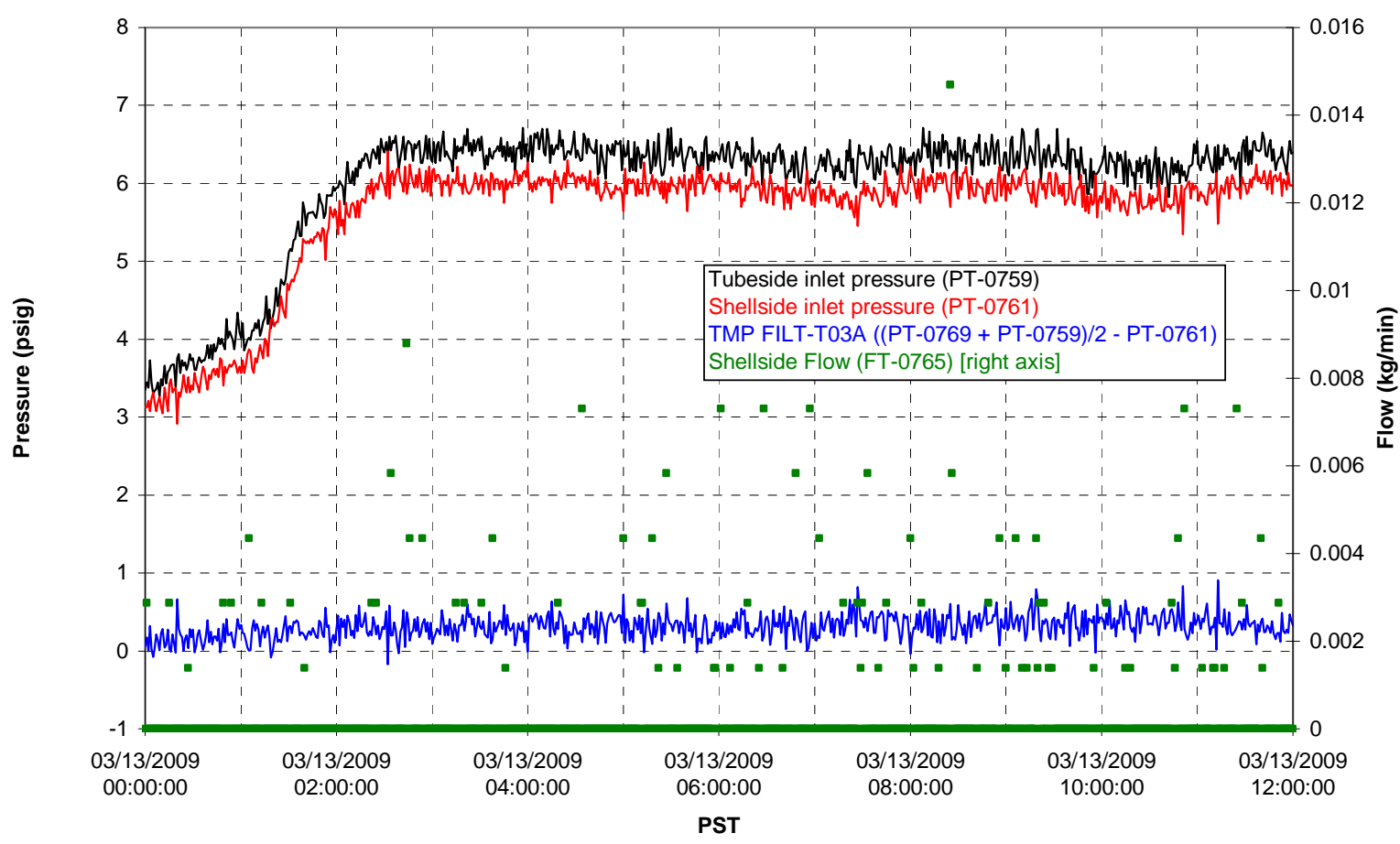

Filter UFP-FILT-T04A

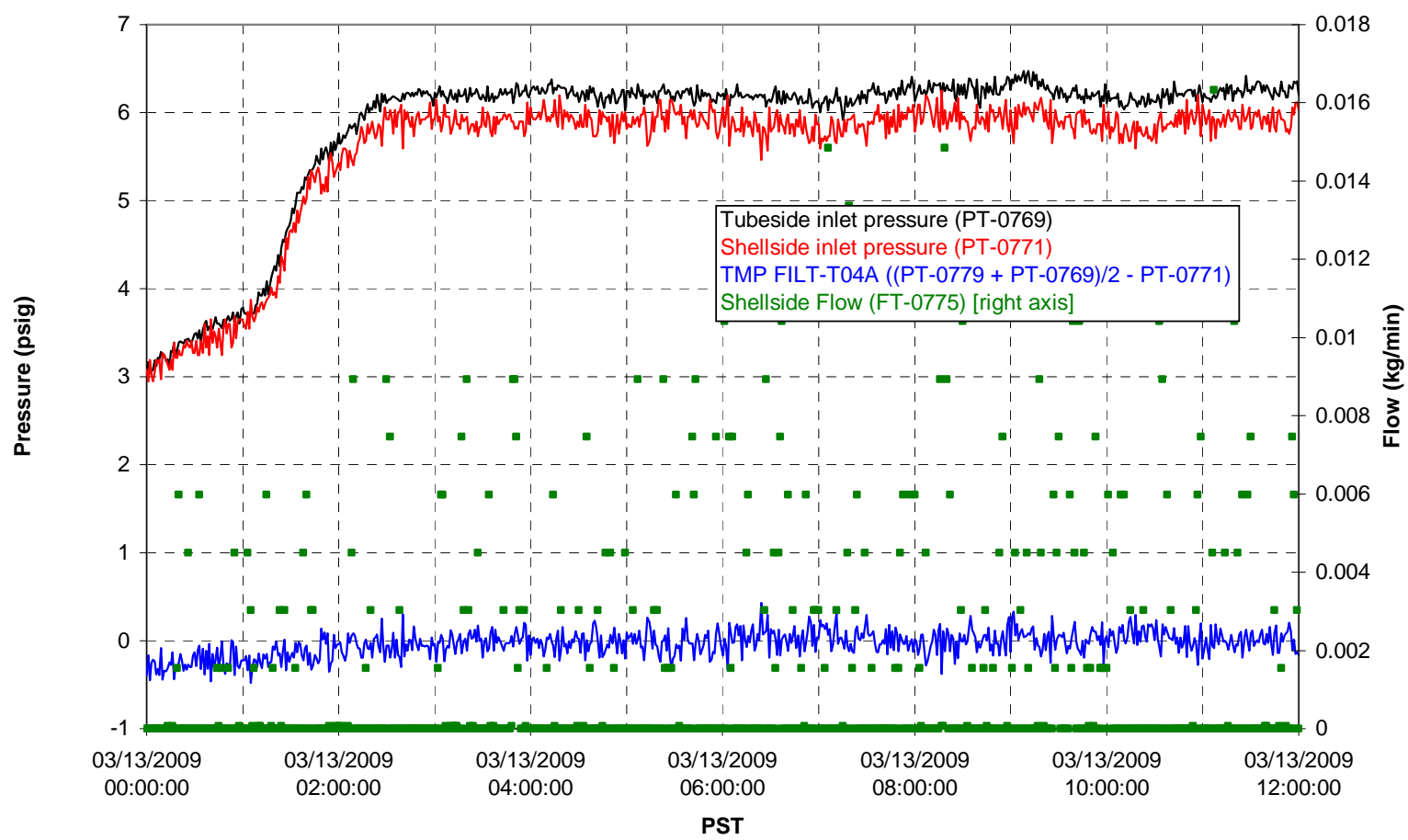


Filter UFP-FILT-T05A
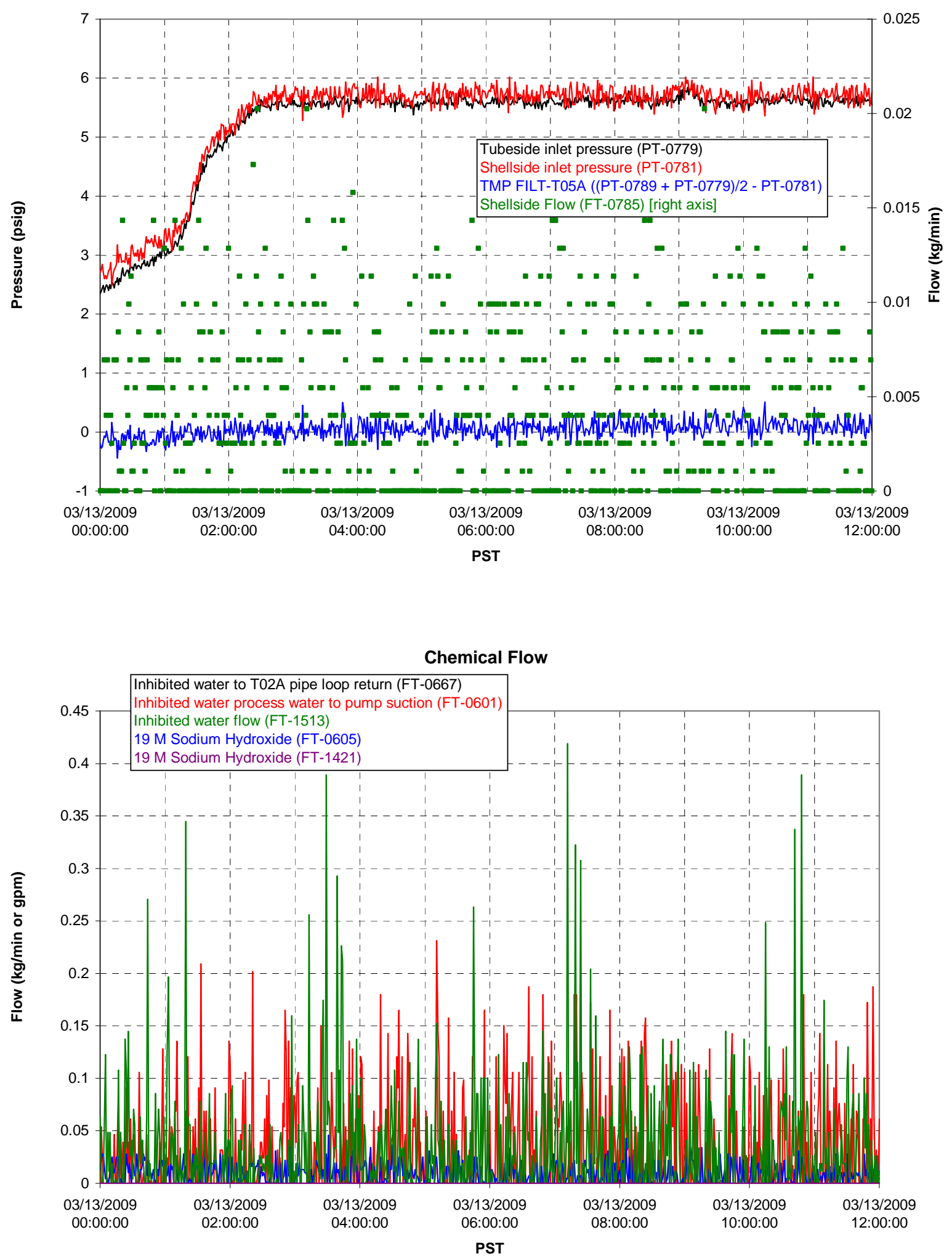


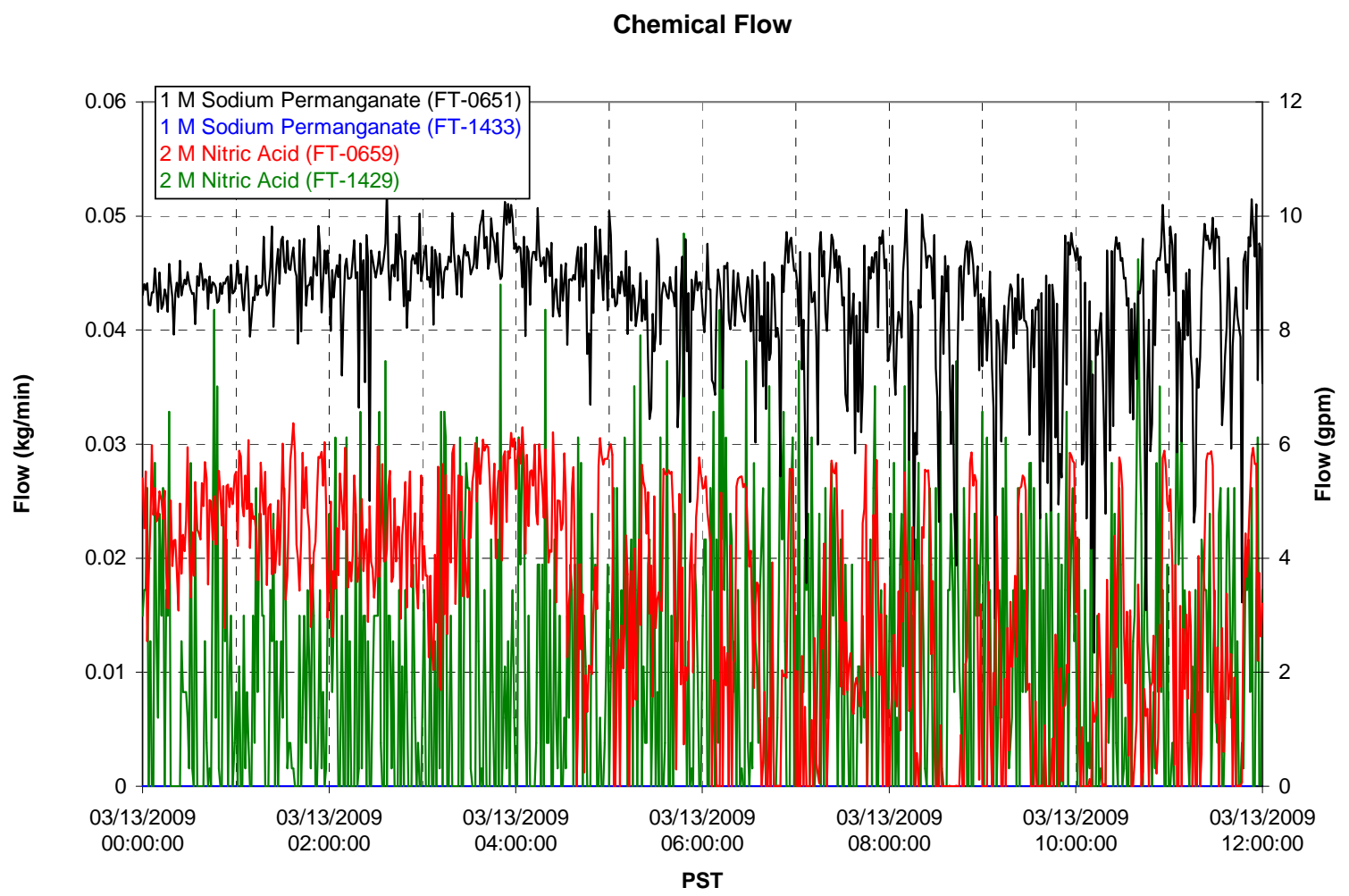

Air Flows

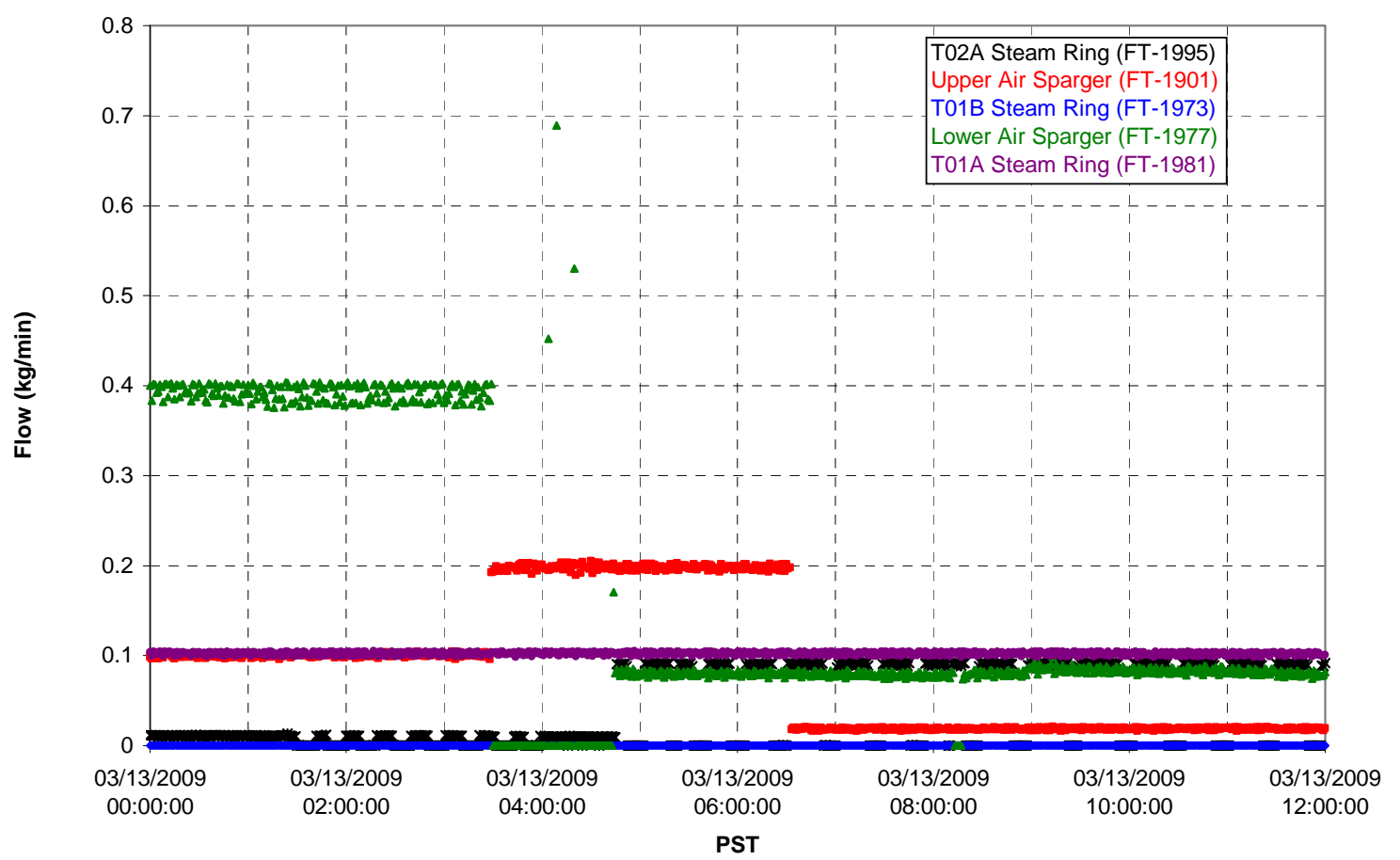


T02A Steam

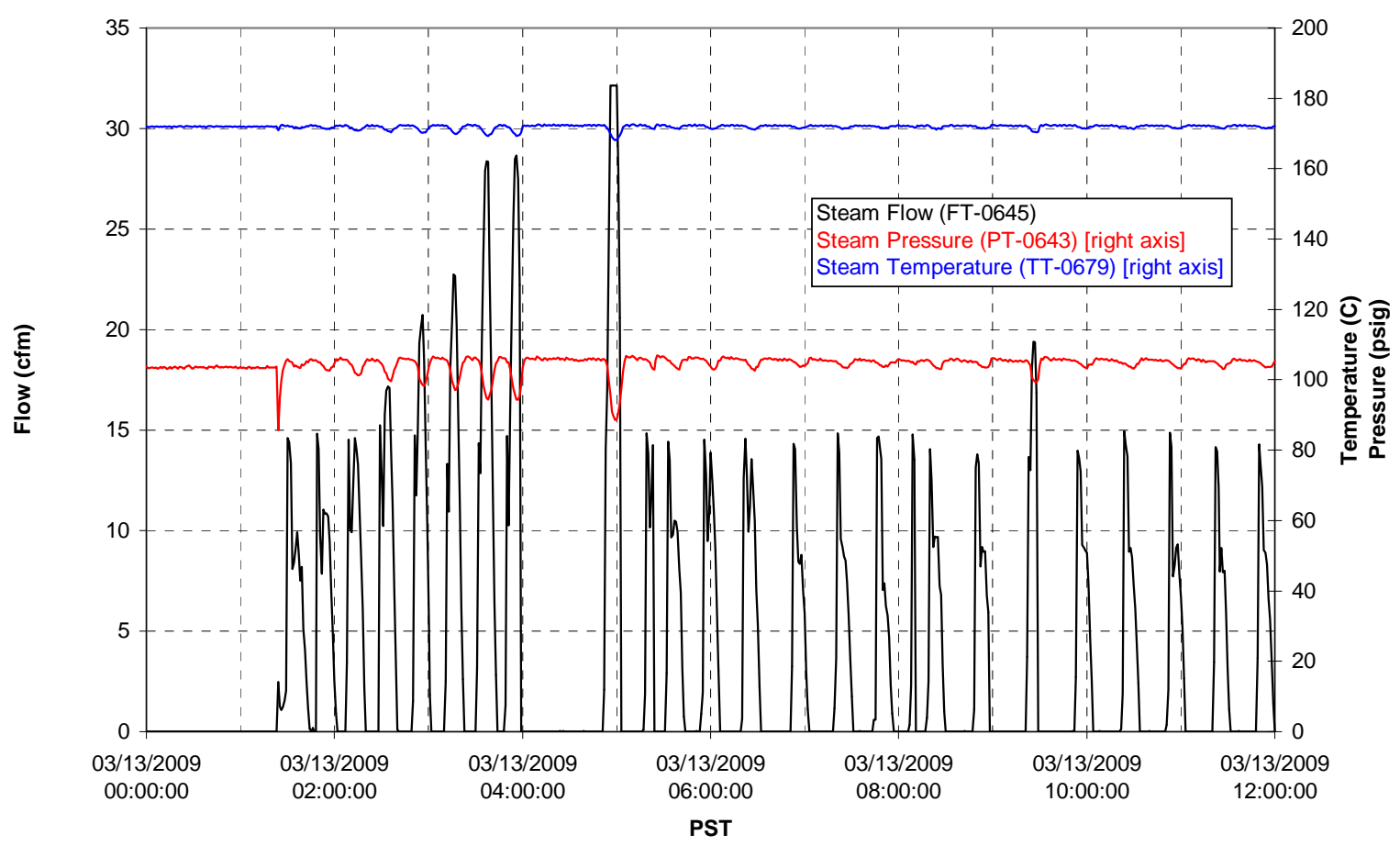

T01A Steam

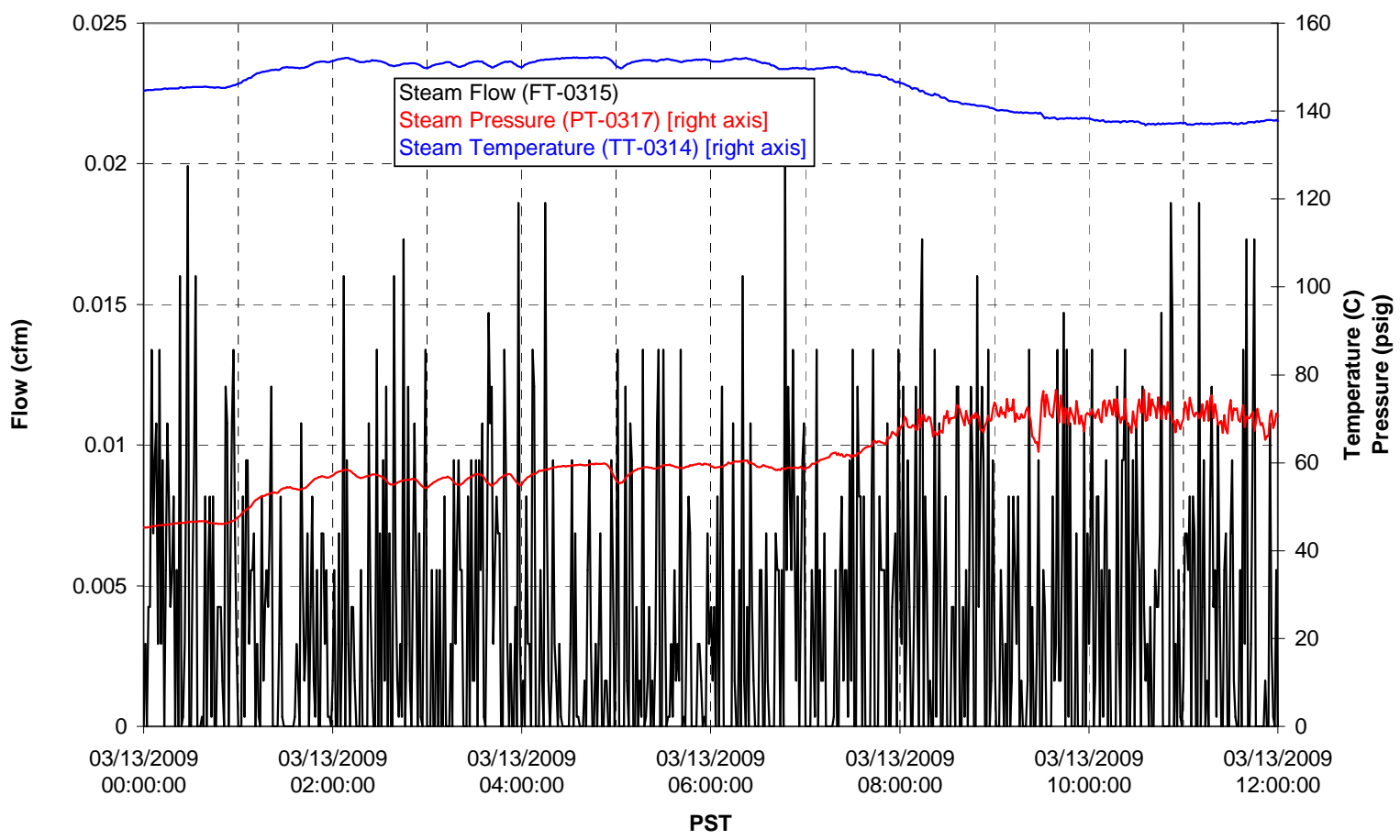


T01B Steam

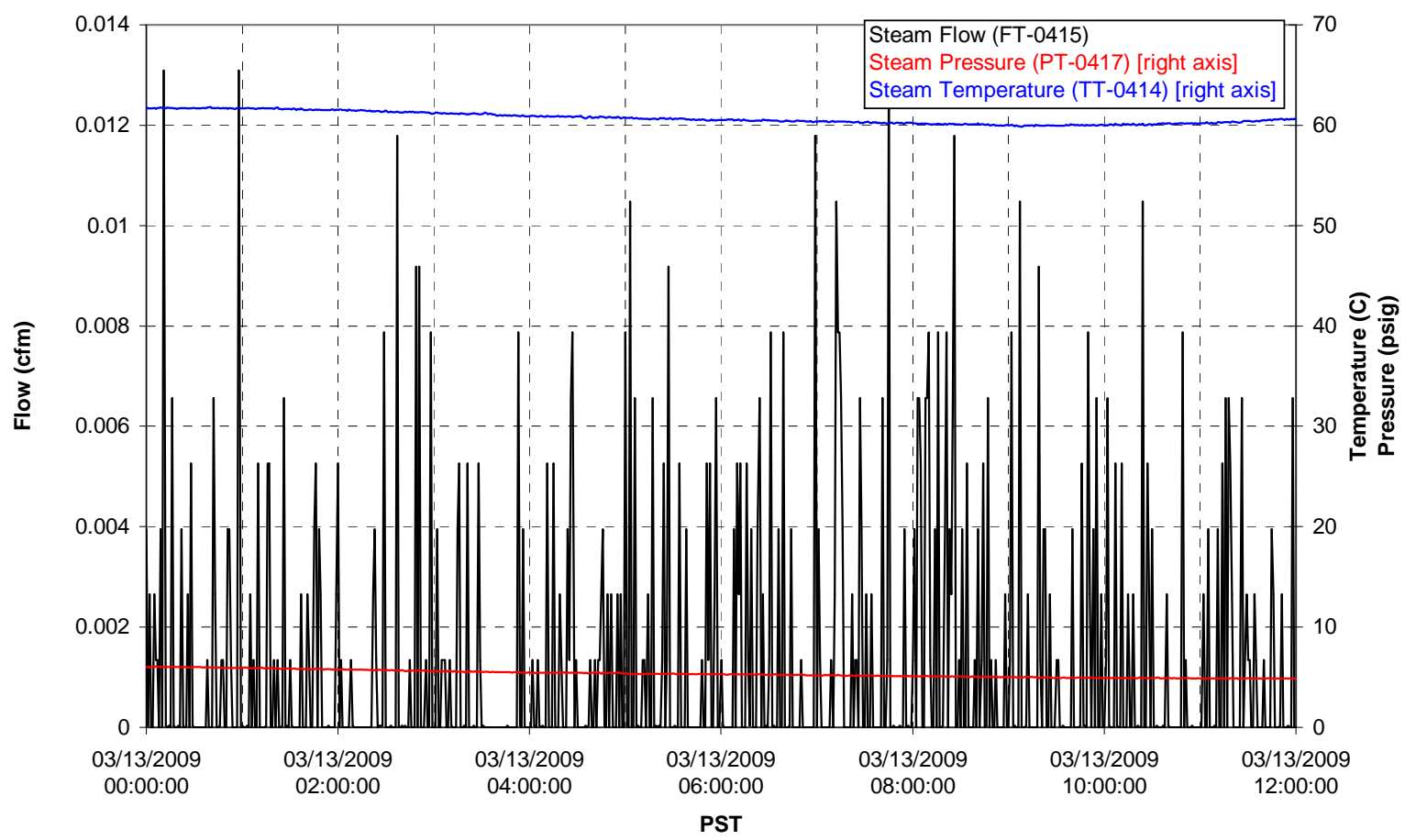


Integrated Test B Data Plots 03/13/09 12:00 - 03/14/09 00:00 
T01A level

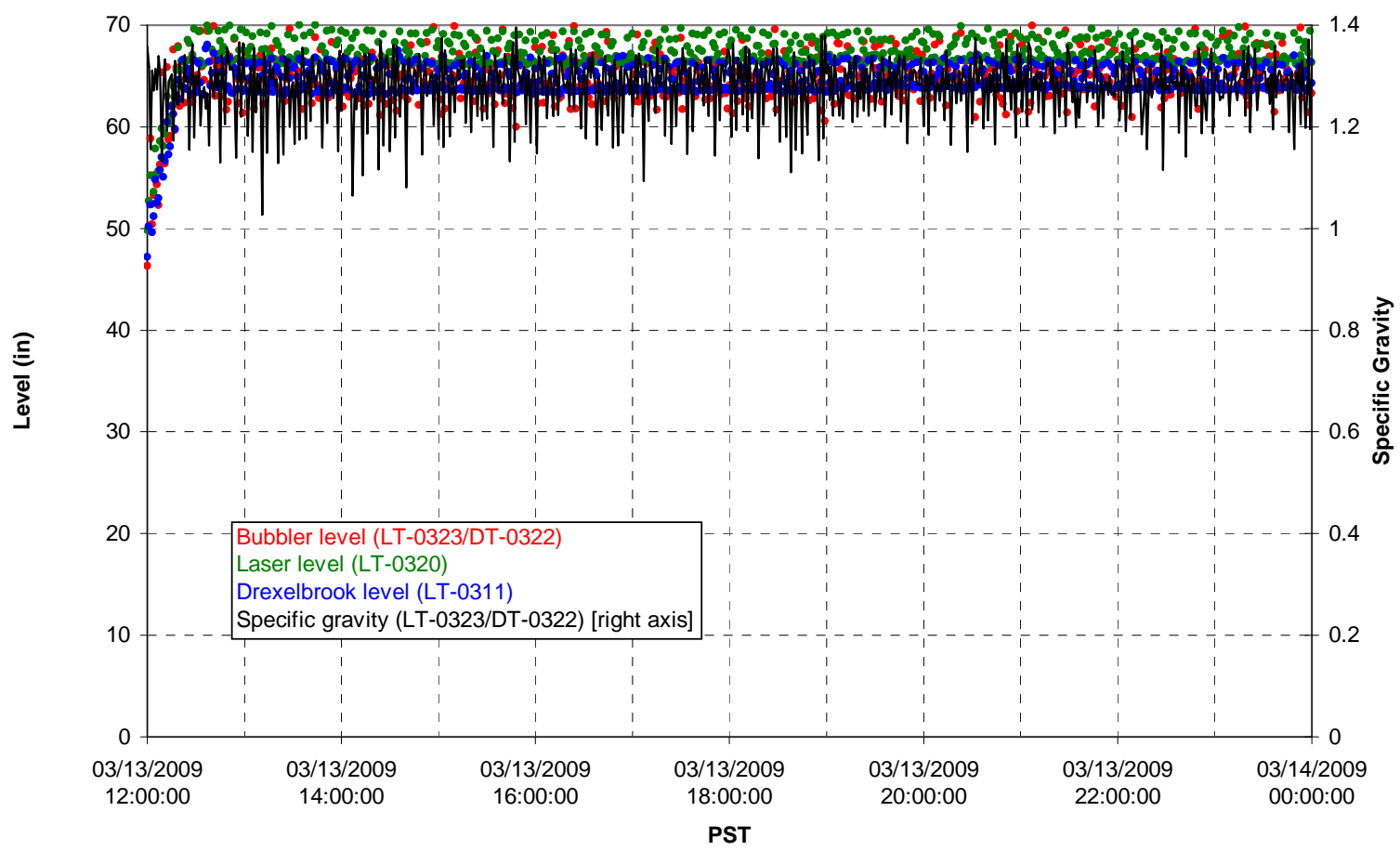

T01A temperatures

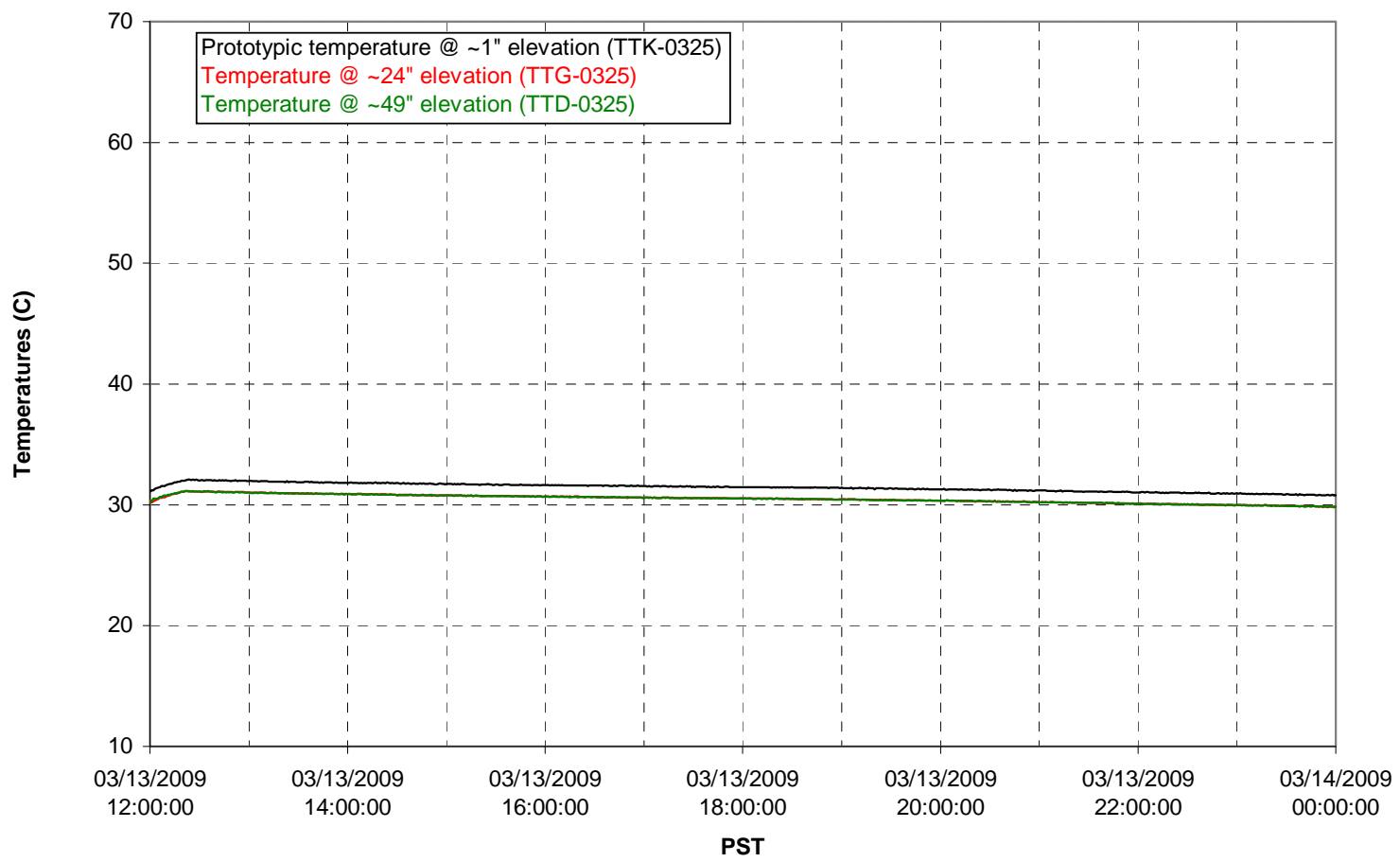


T01B level

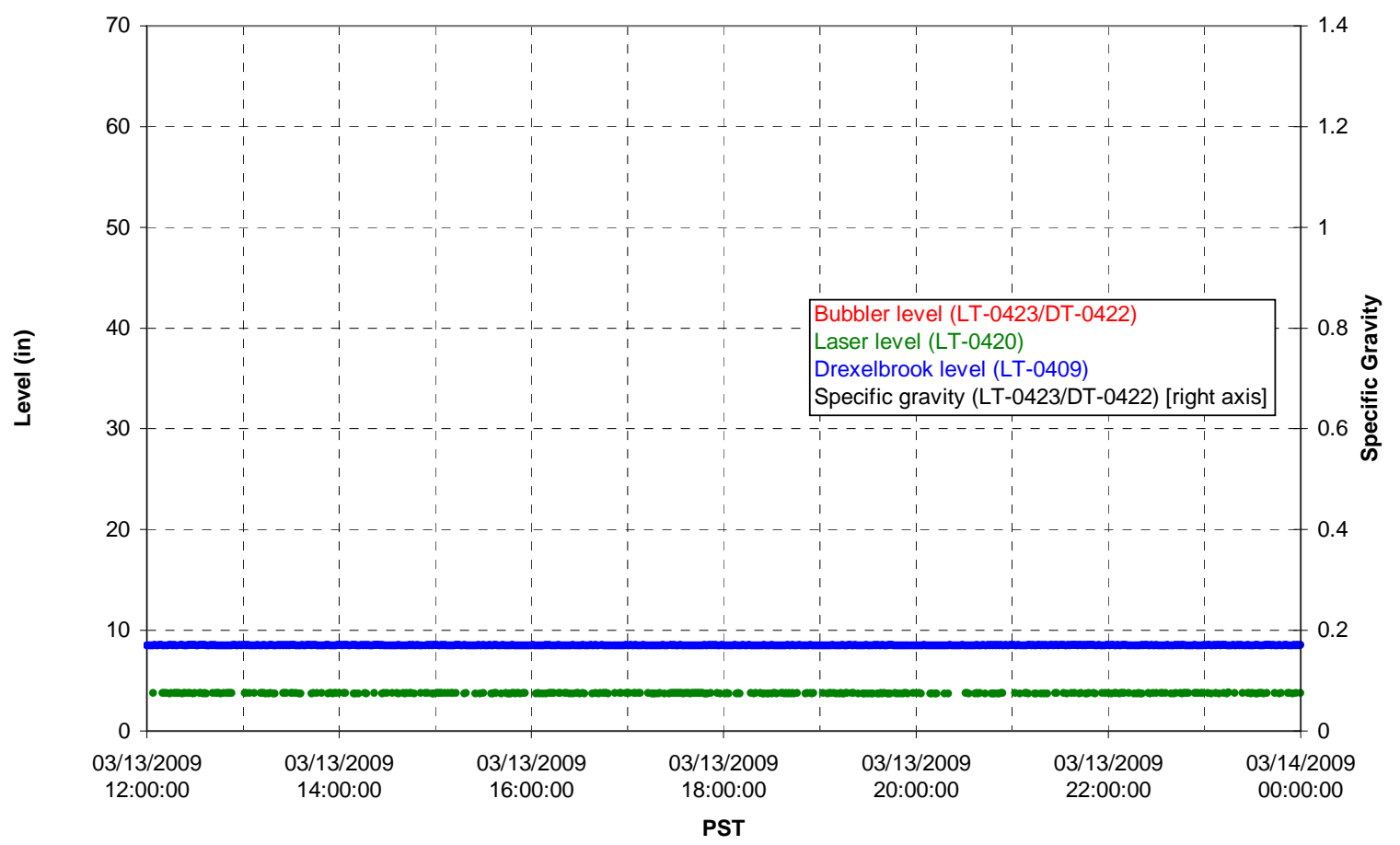

T01B temperatures

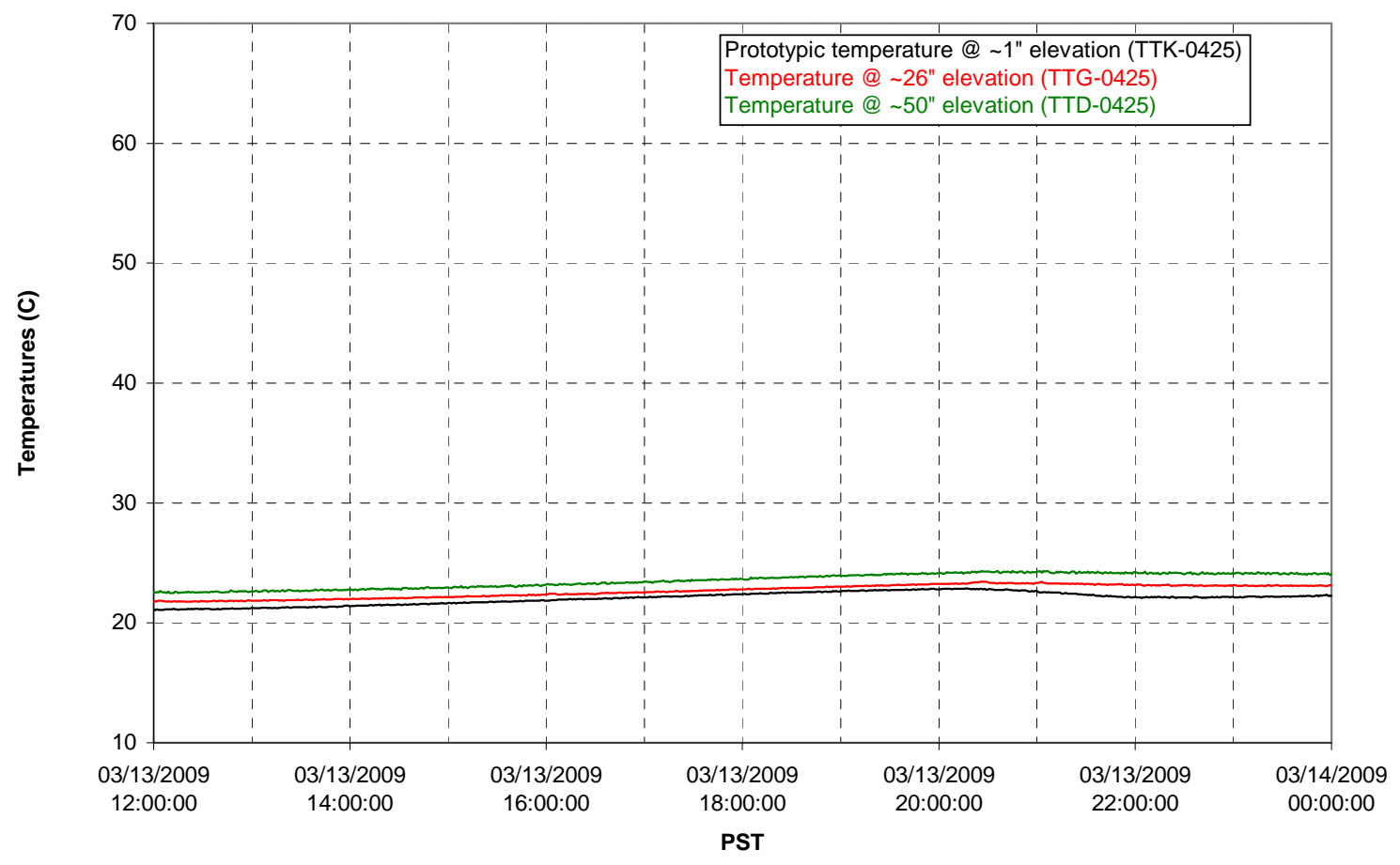


T02A level

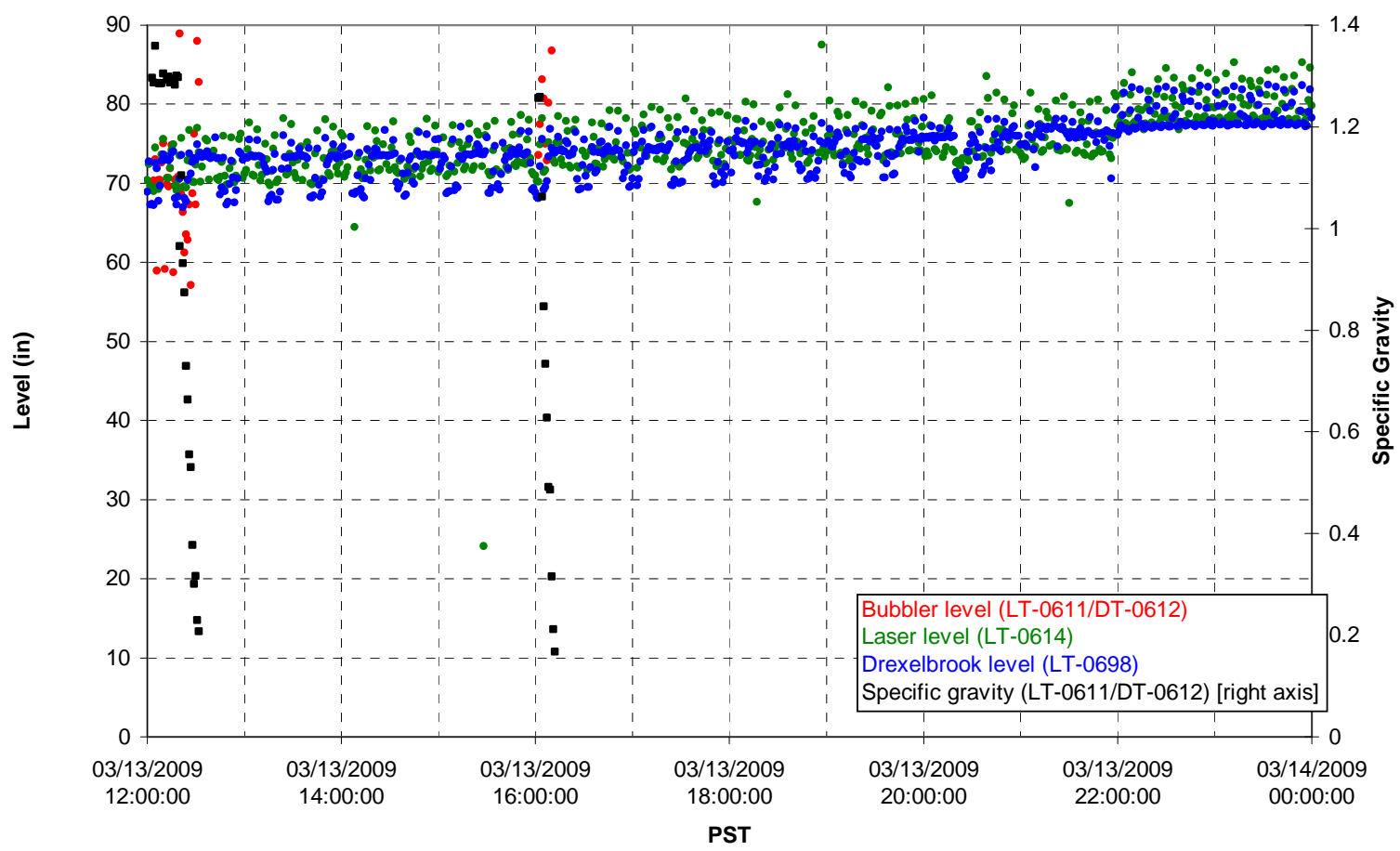

T02A temperatures

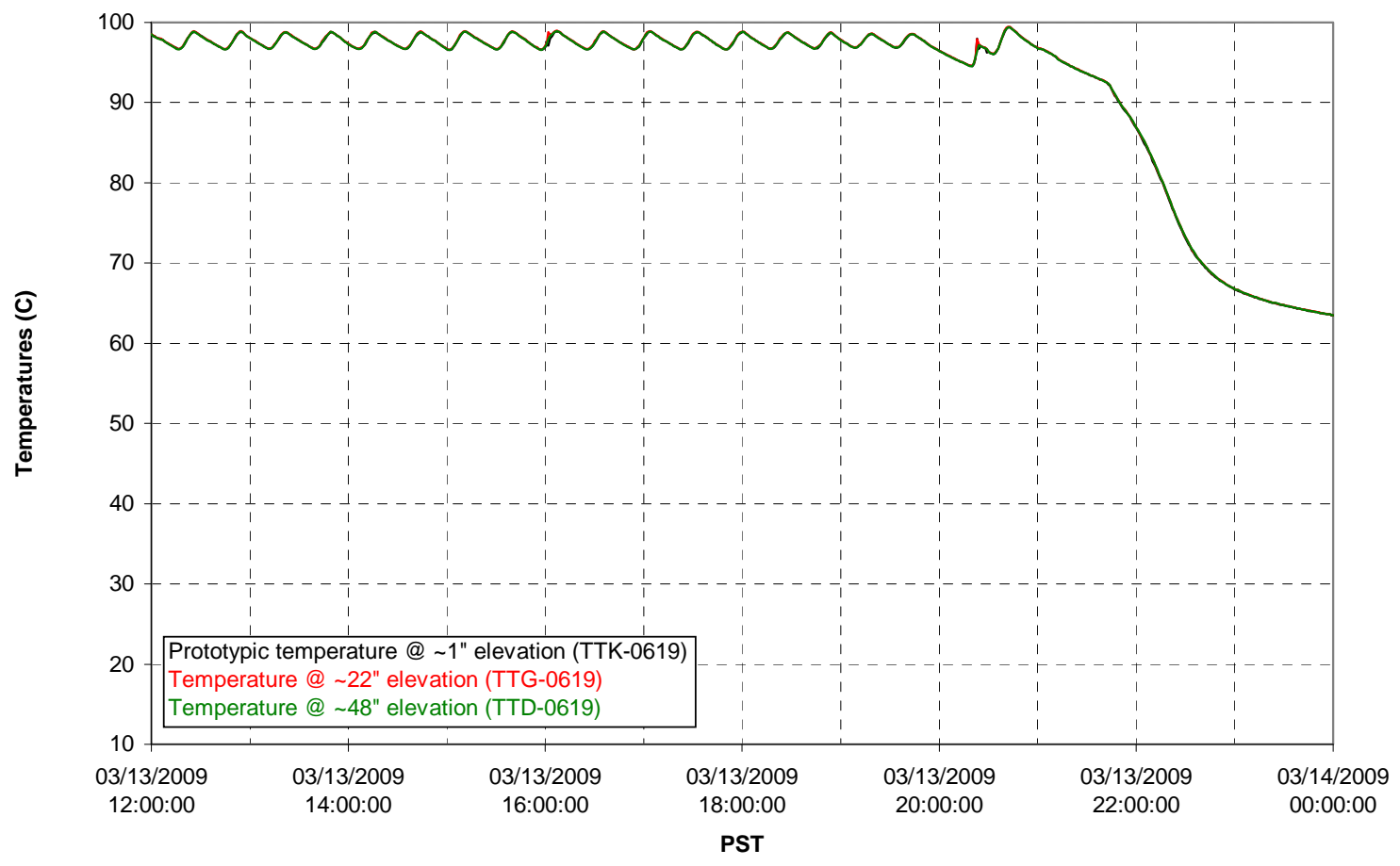


T02A and filter loop temperatures

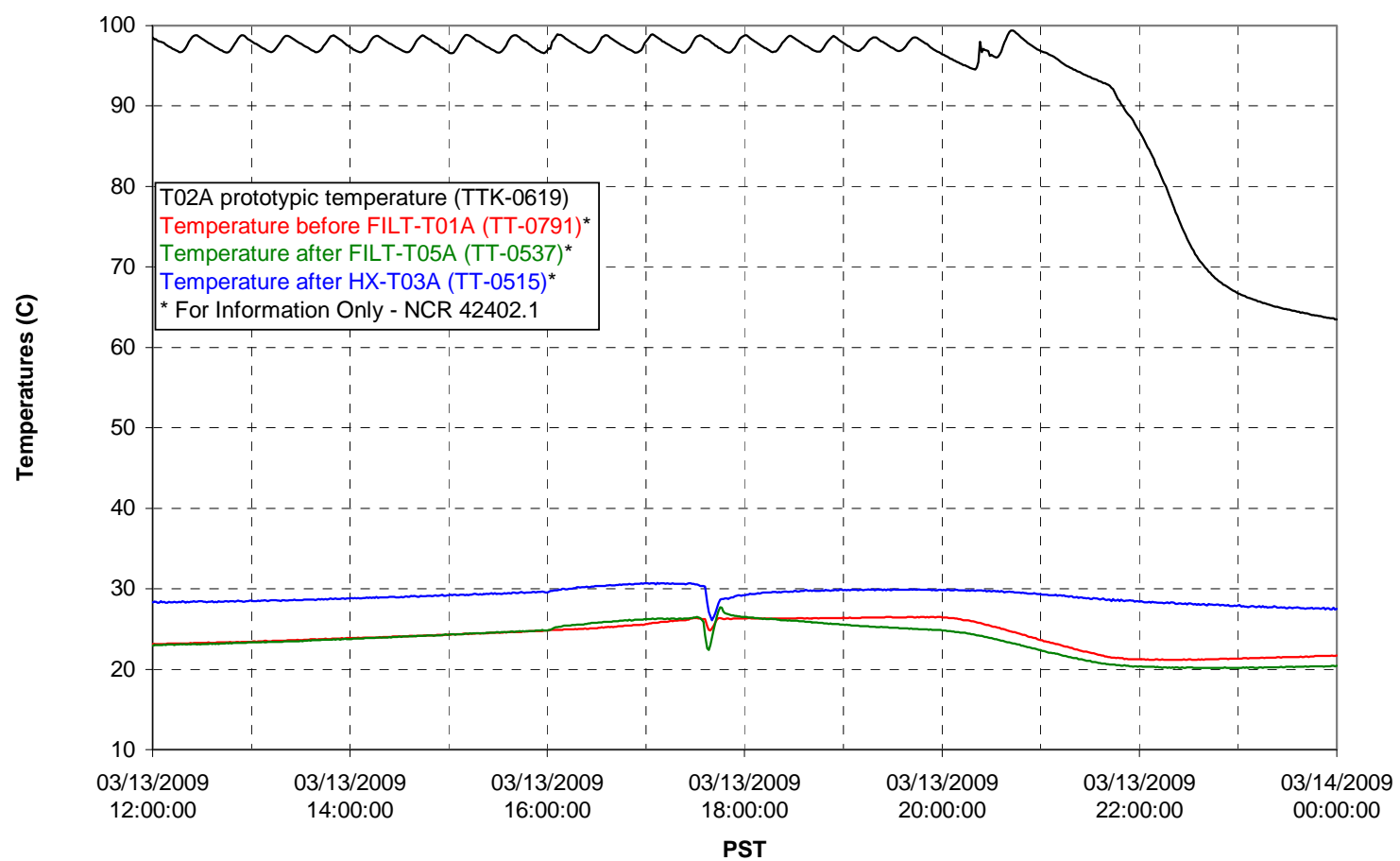

Pump Pressures and Flow

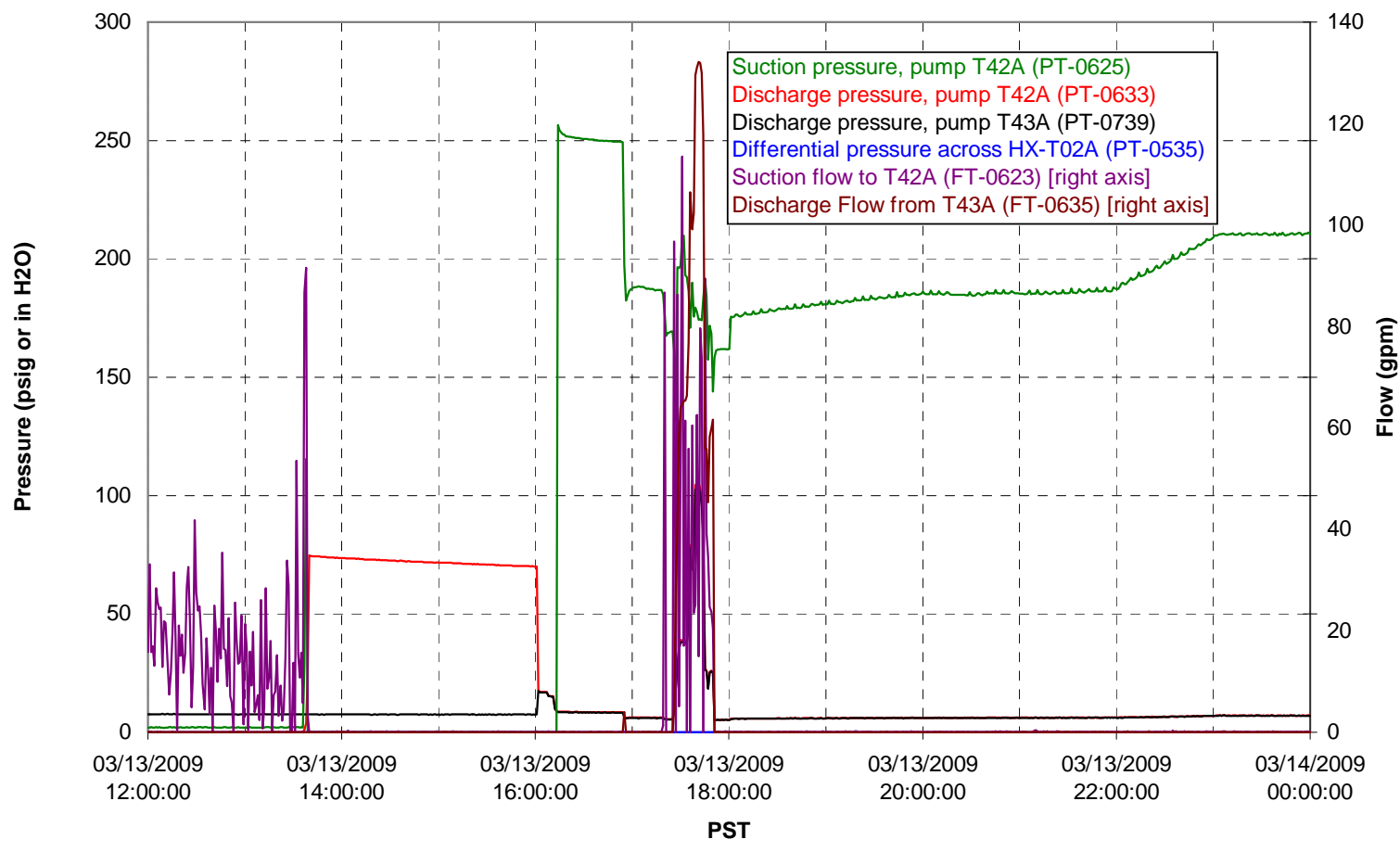


Axial pressure drop

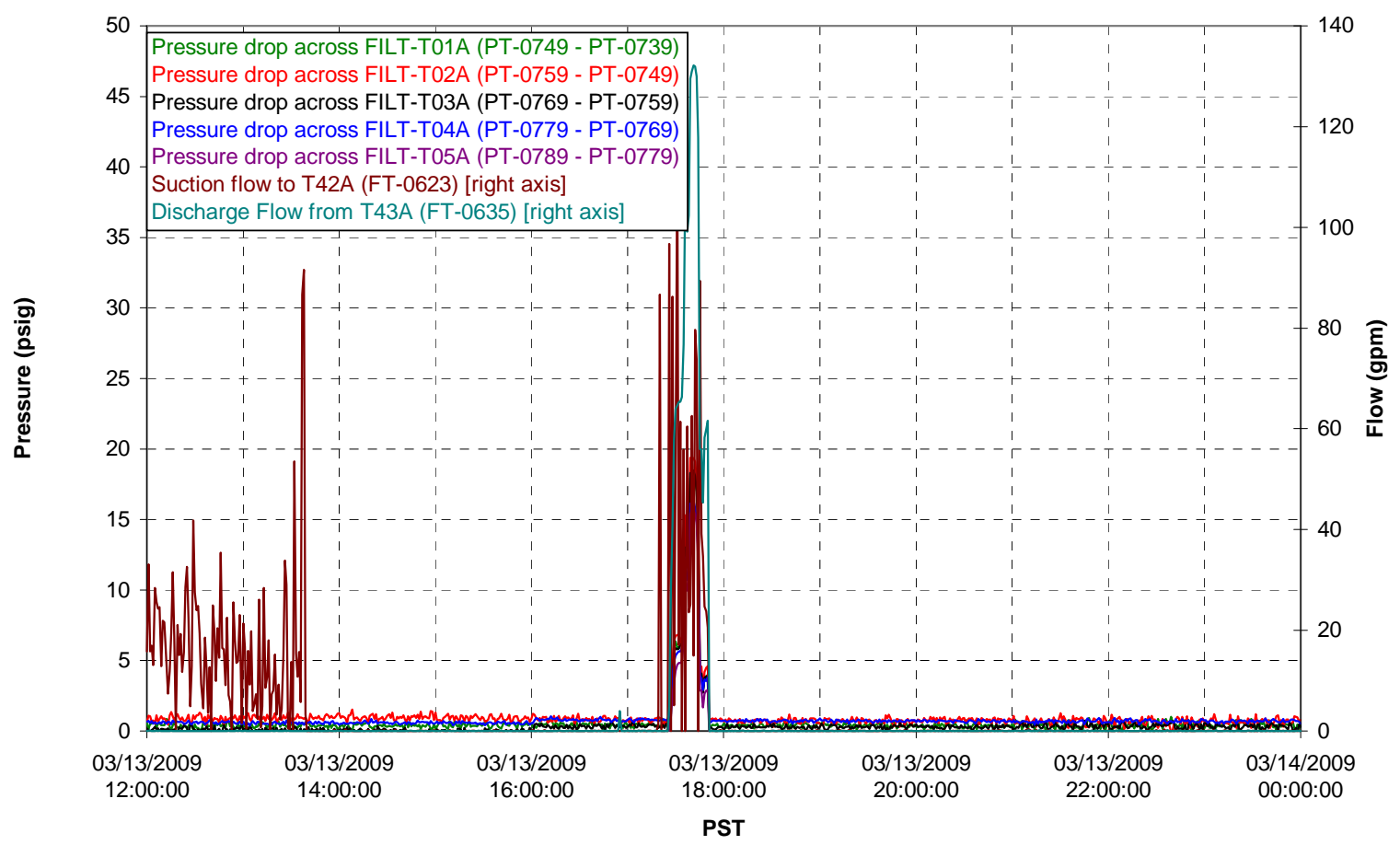

Permeate flow rates

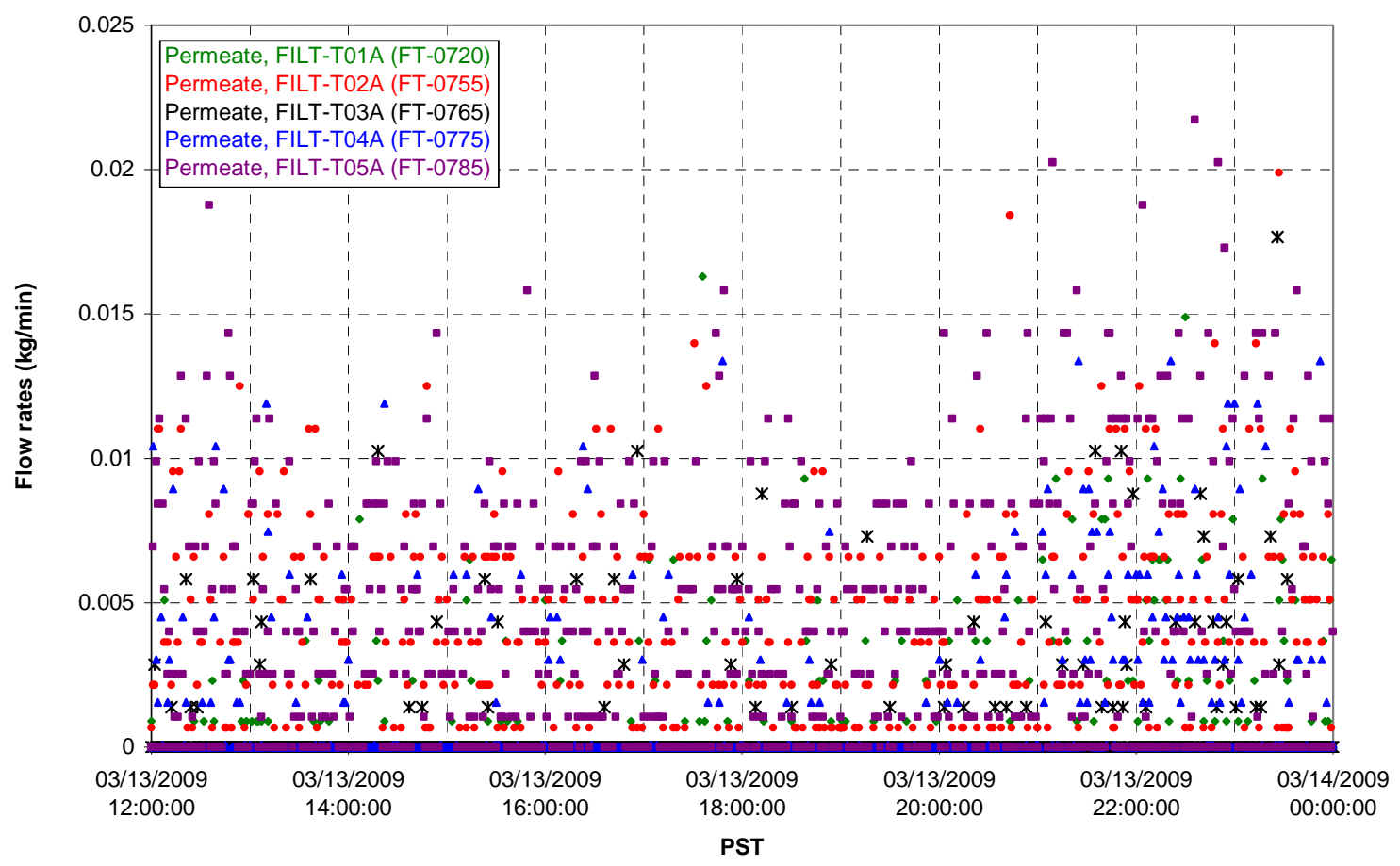


T02A Inner Temperature Tree

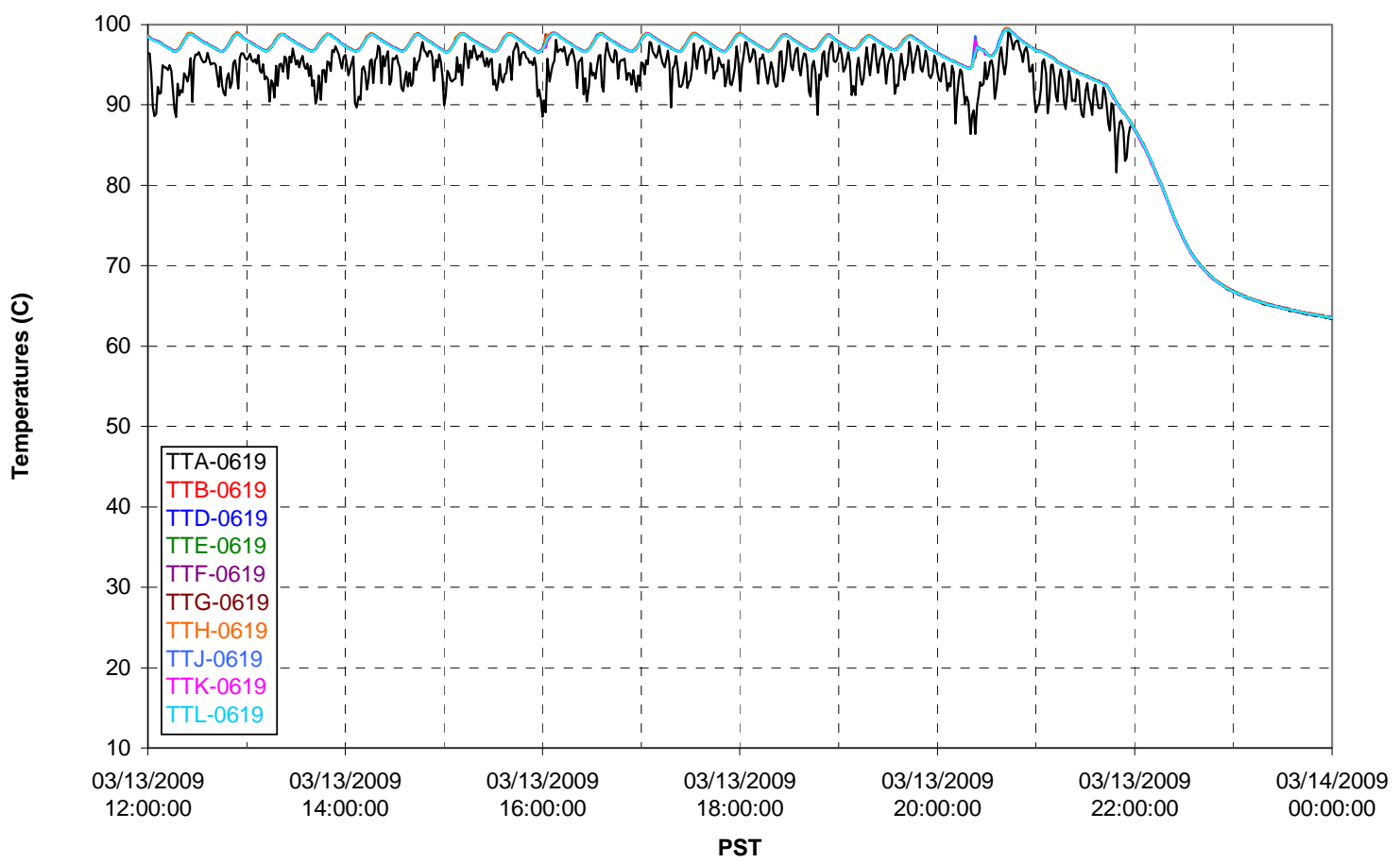

T02A Outer Temperature Tree

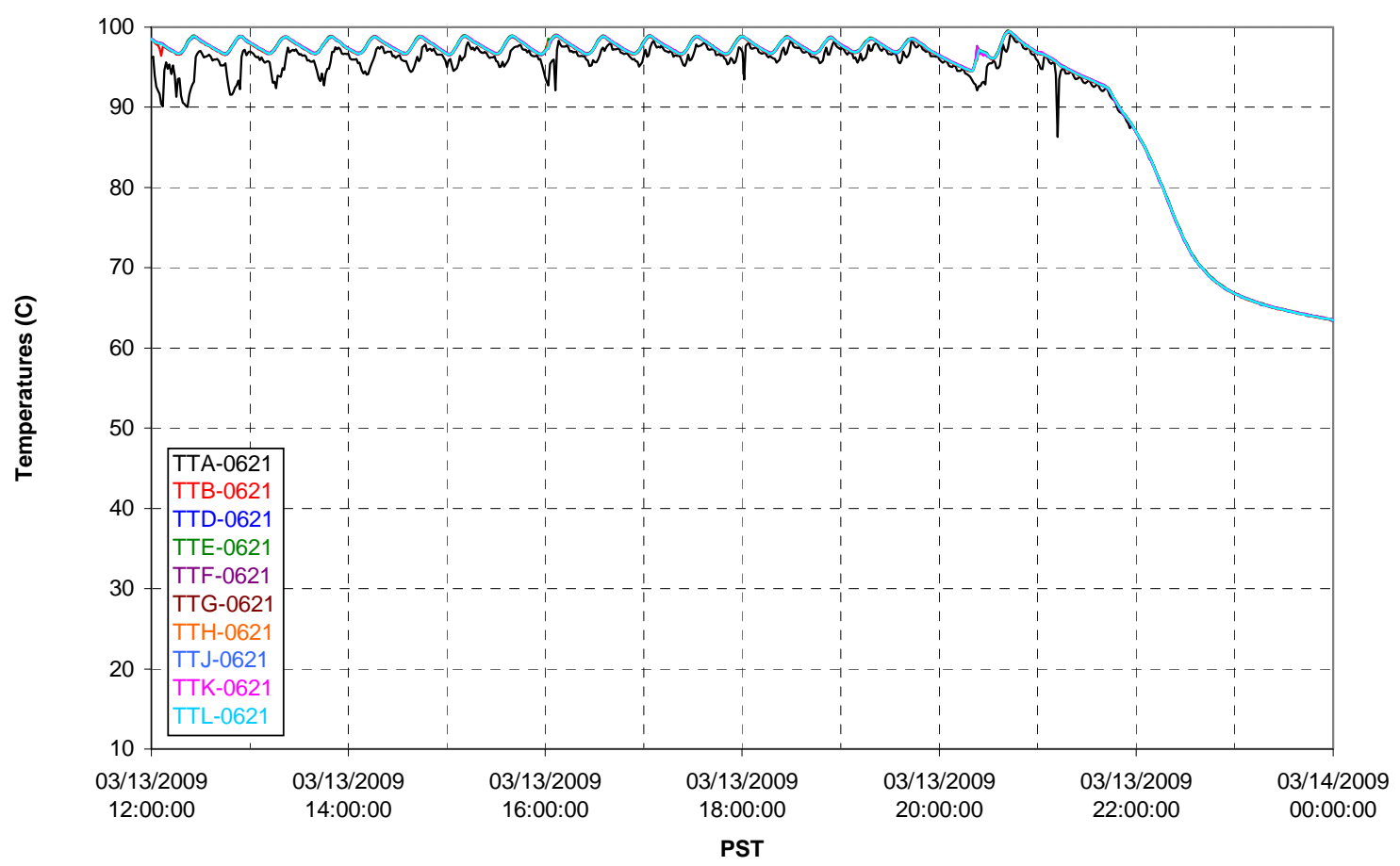


T02A temperatures

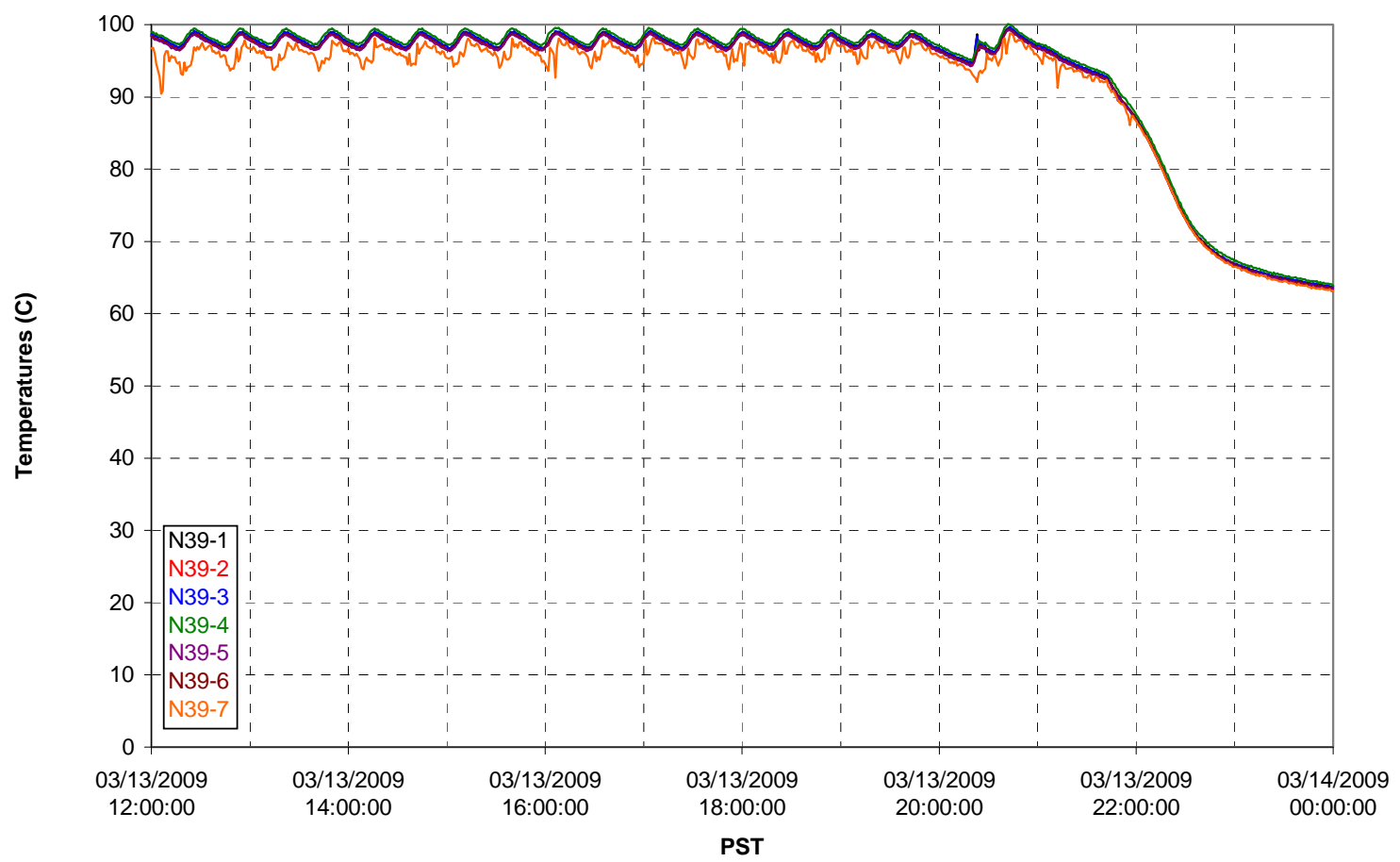

T02A temperatures

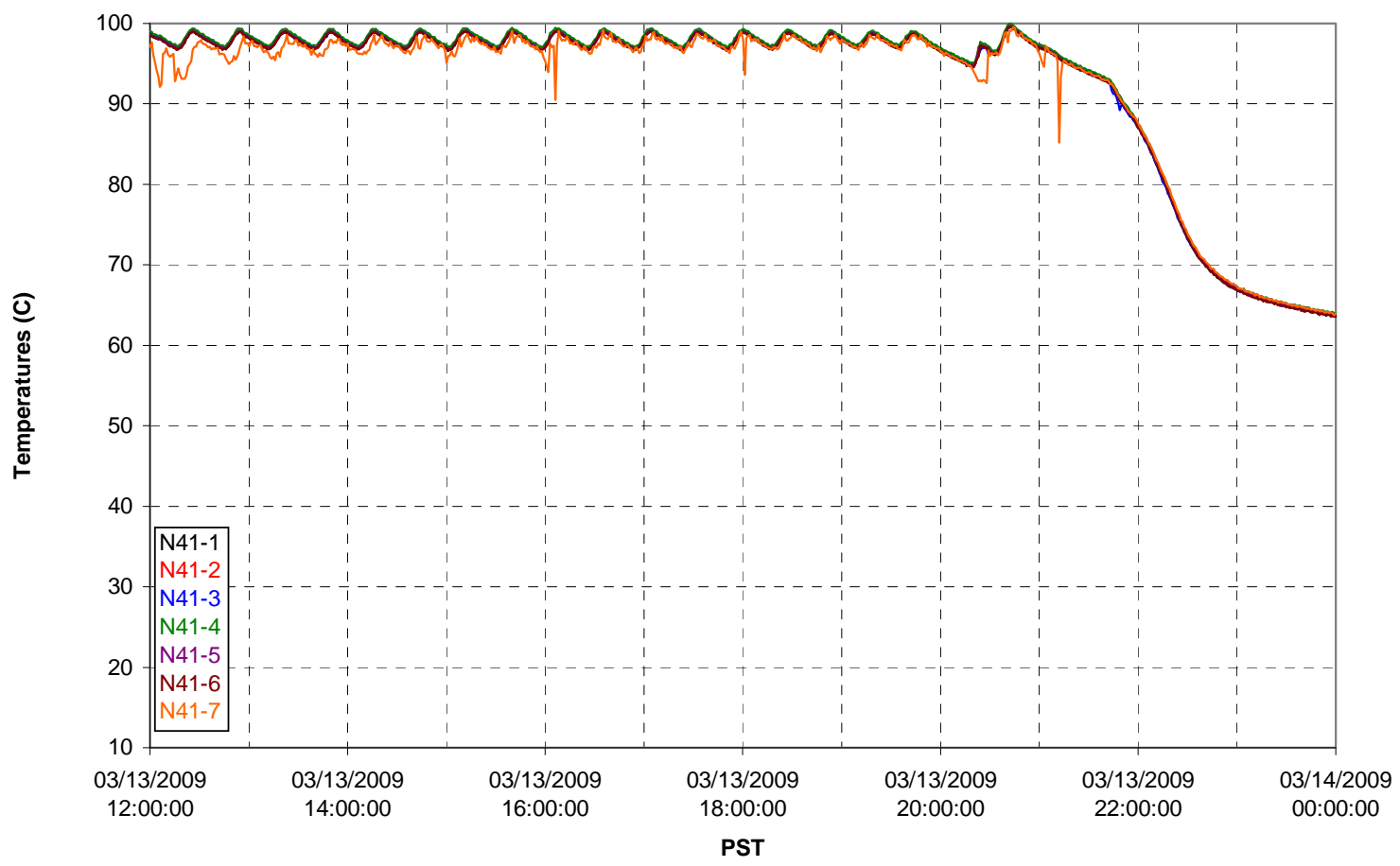


T02A temperatures

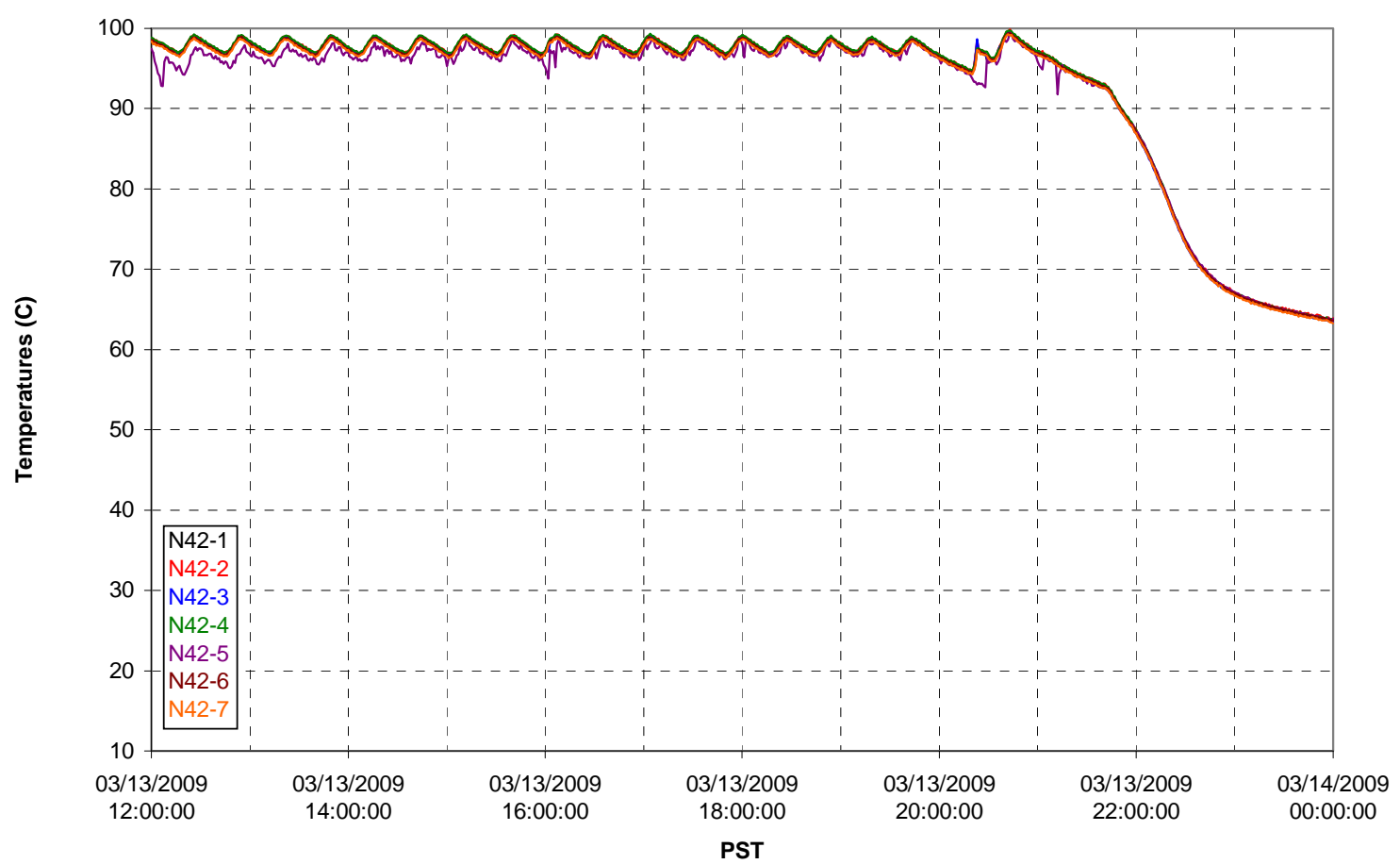

T02A temperatures

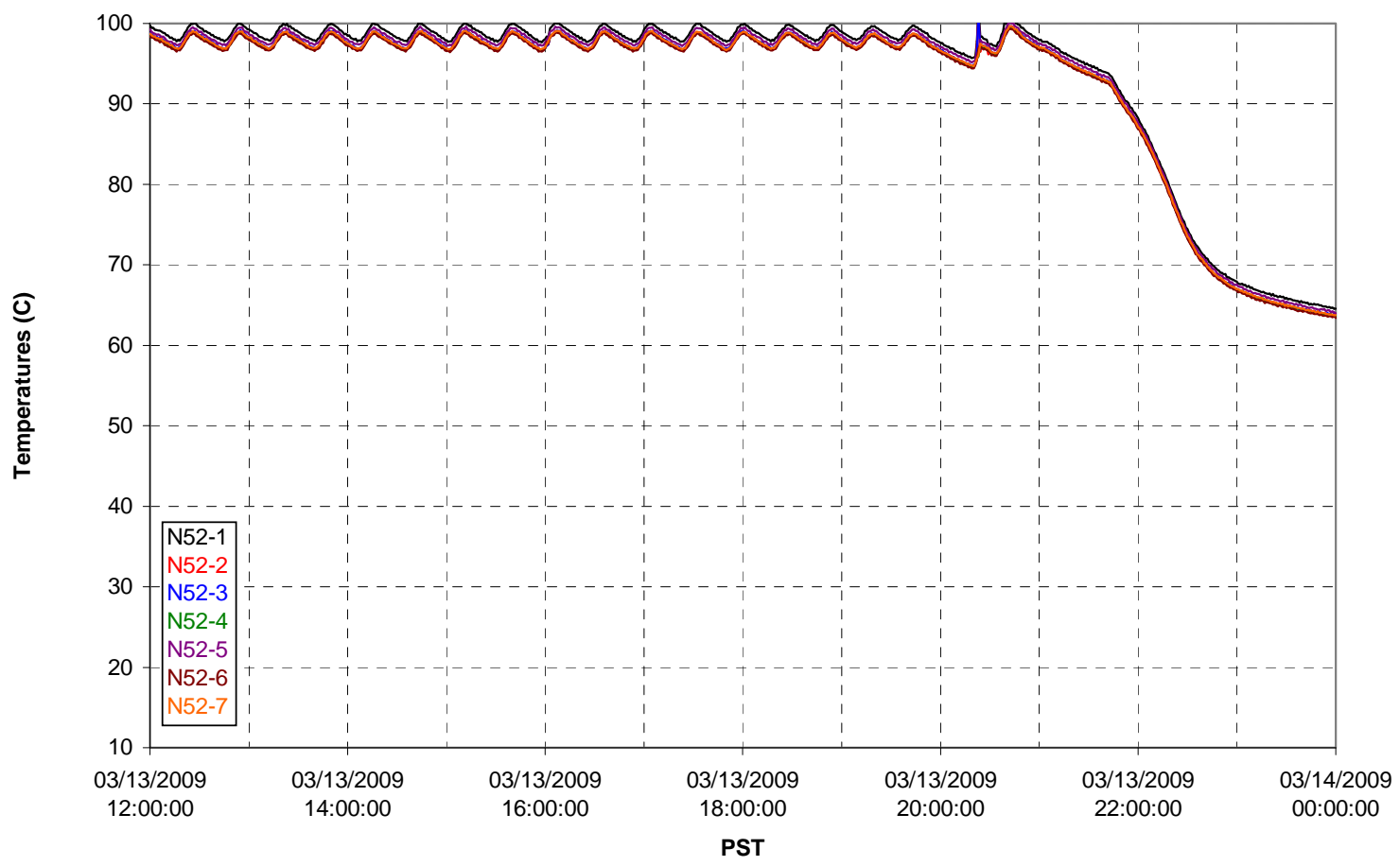


T02A Heating and Cooling

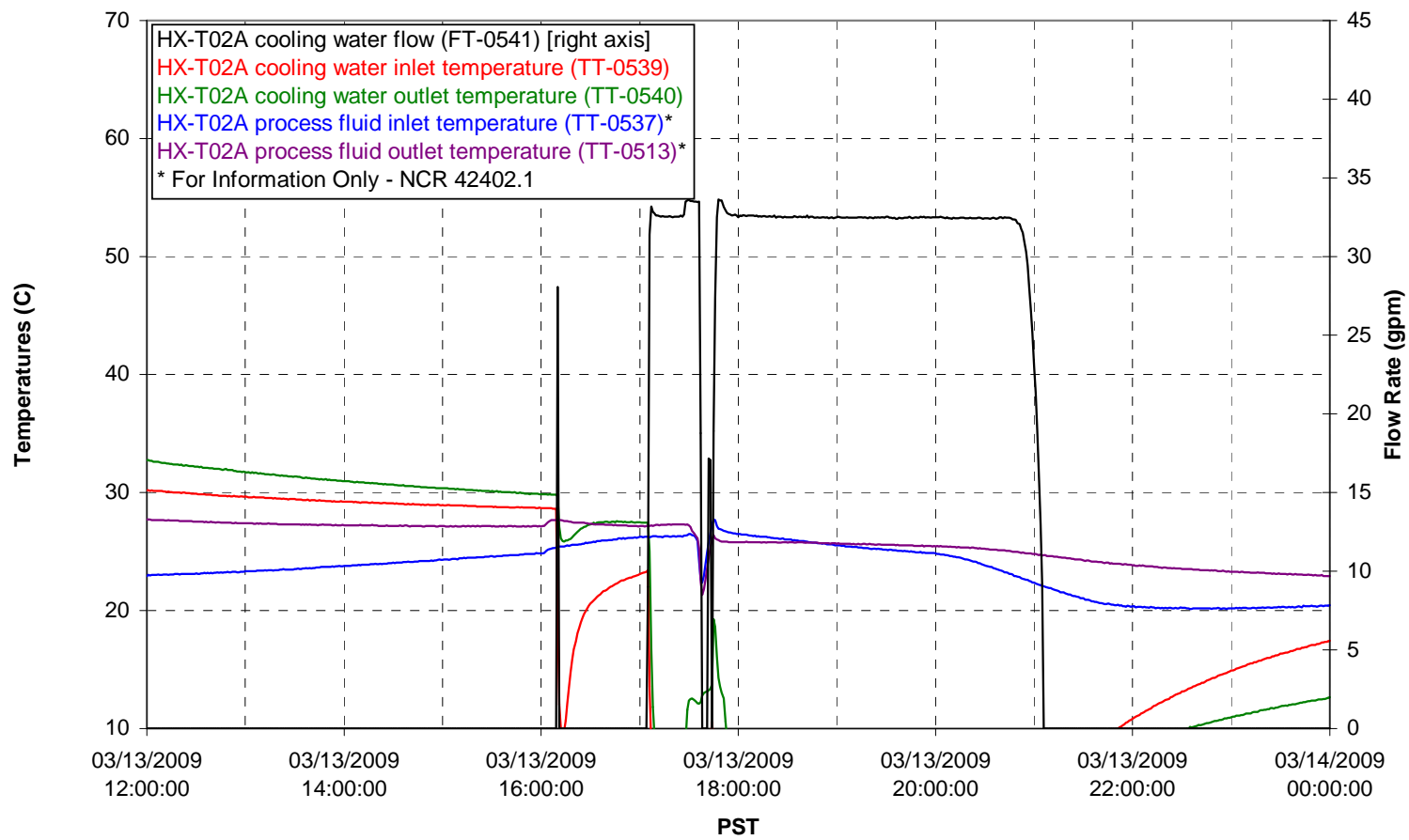

Pump Operation

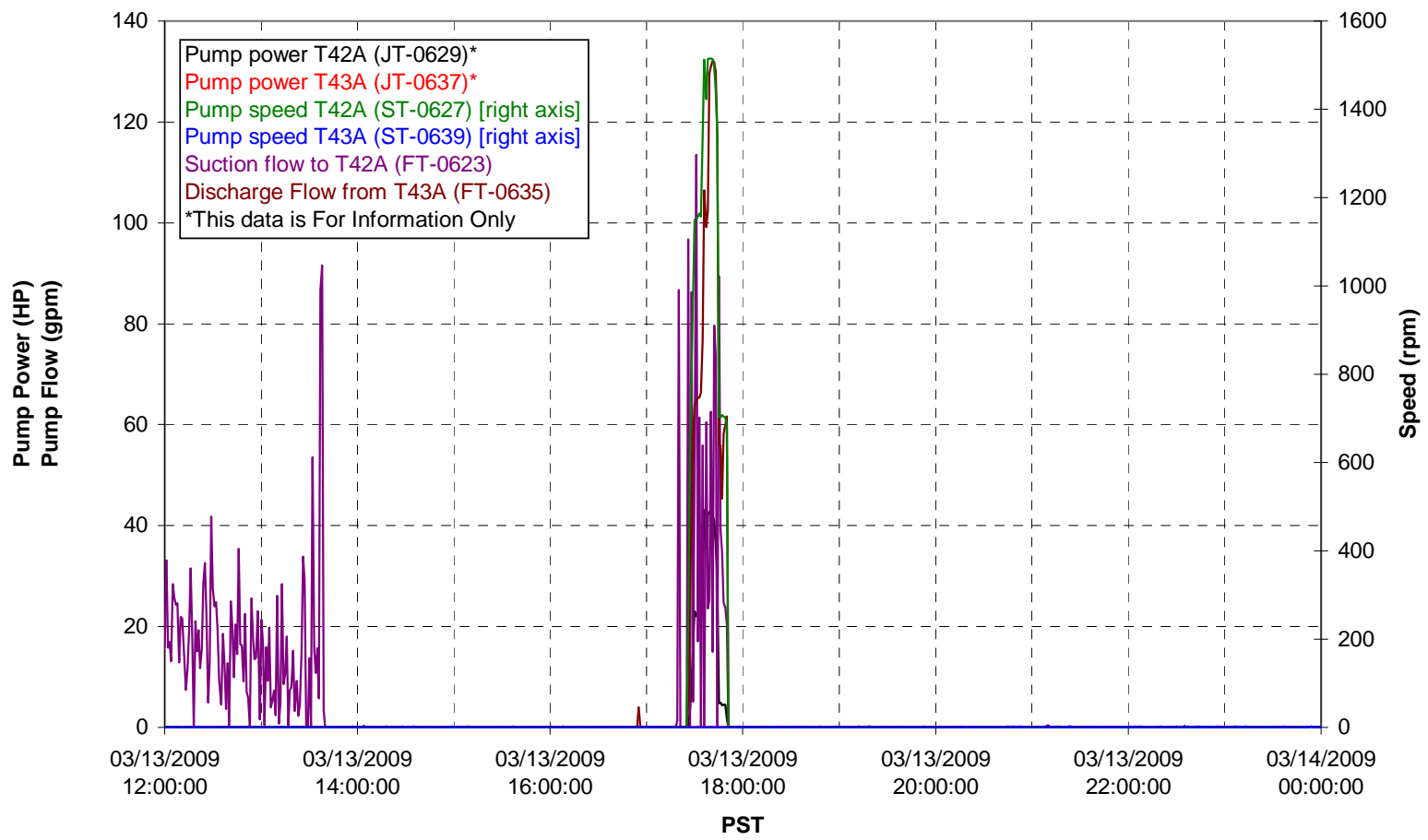


Pulsepot UFP-PP-T01A

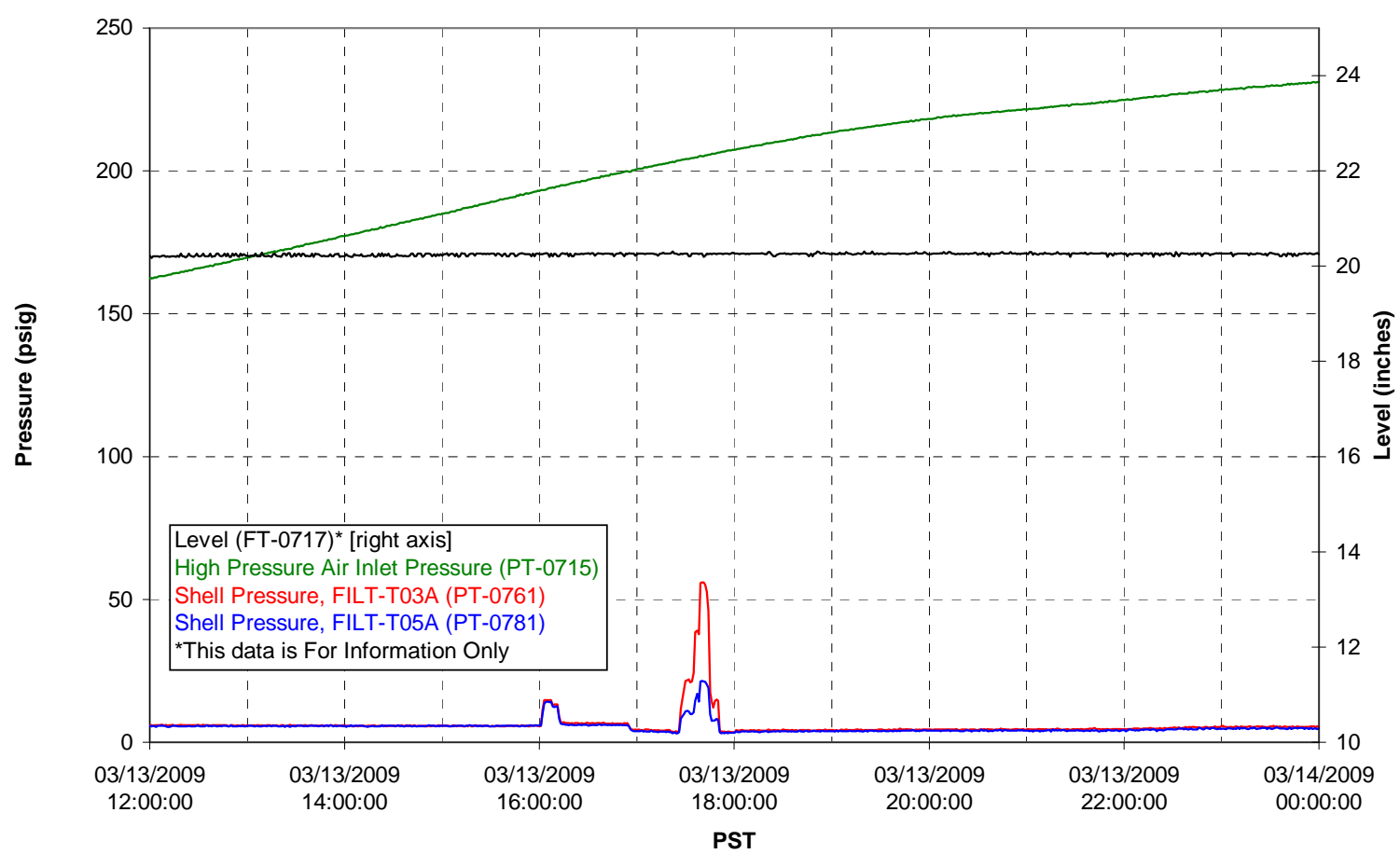

Pulsepot UFP-PP-T02A

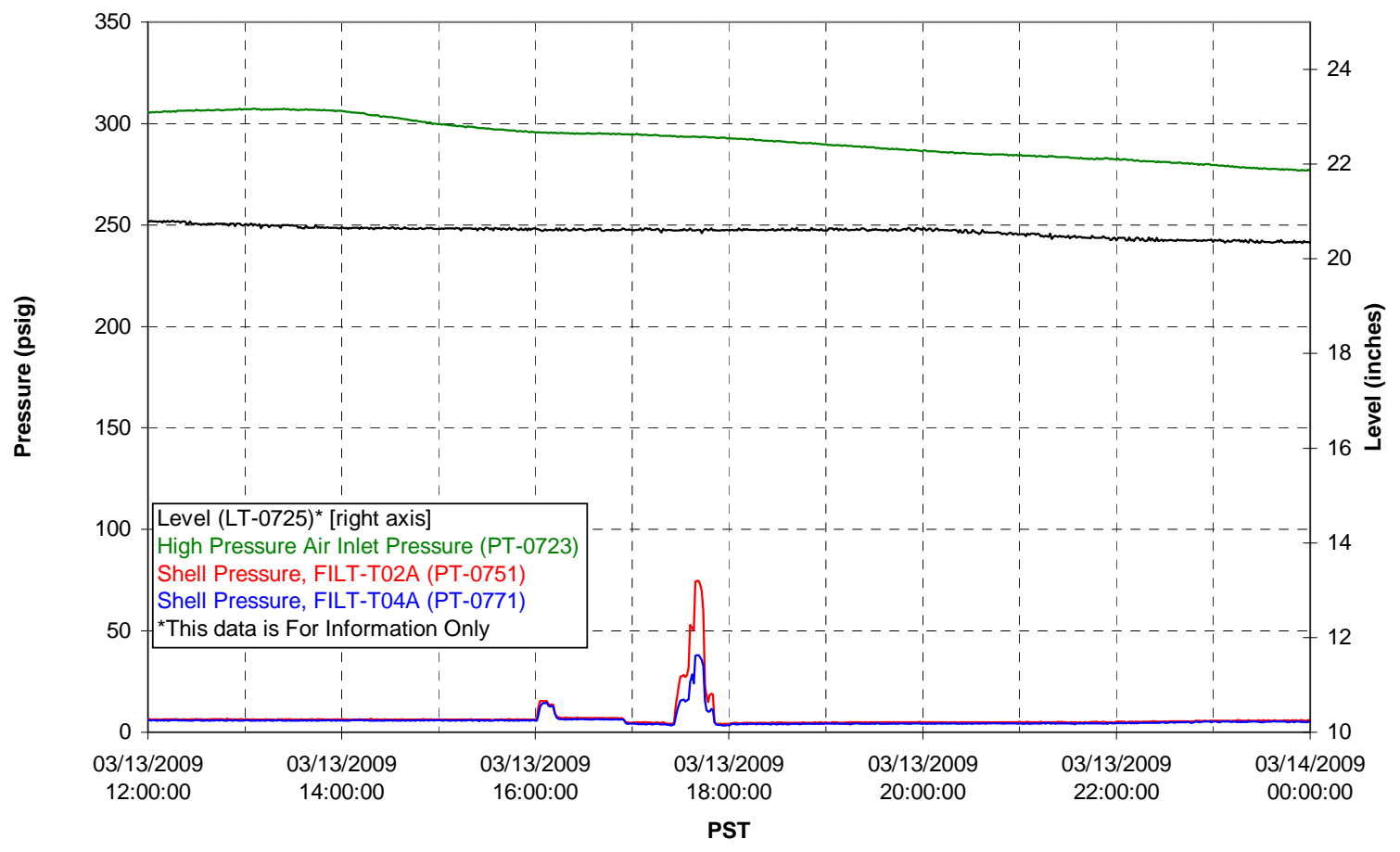


Pulsepot UFP-PP-T03A

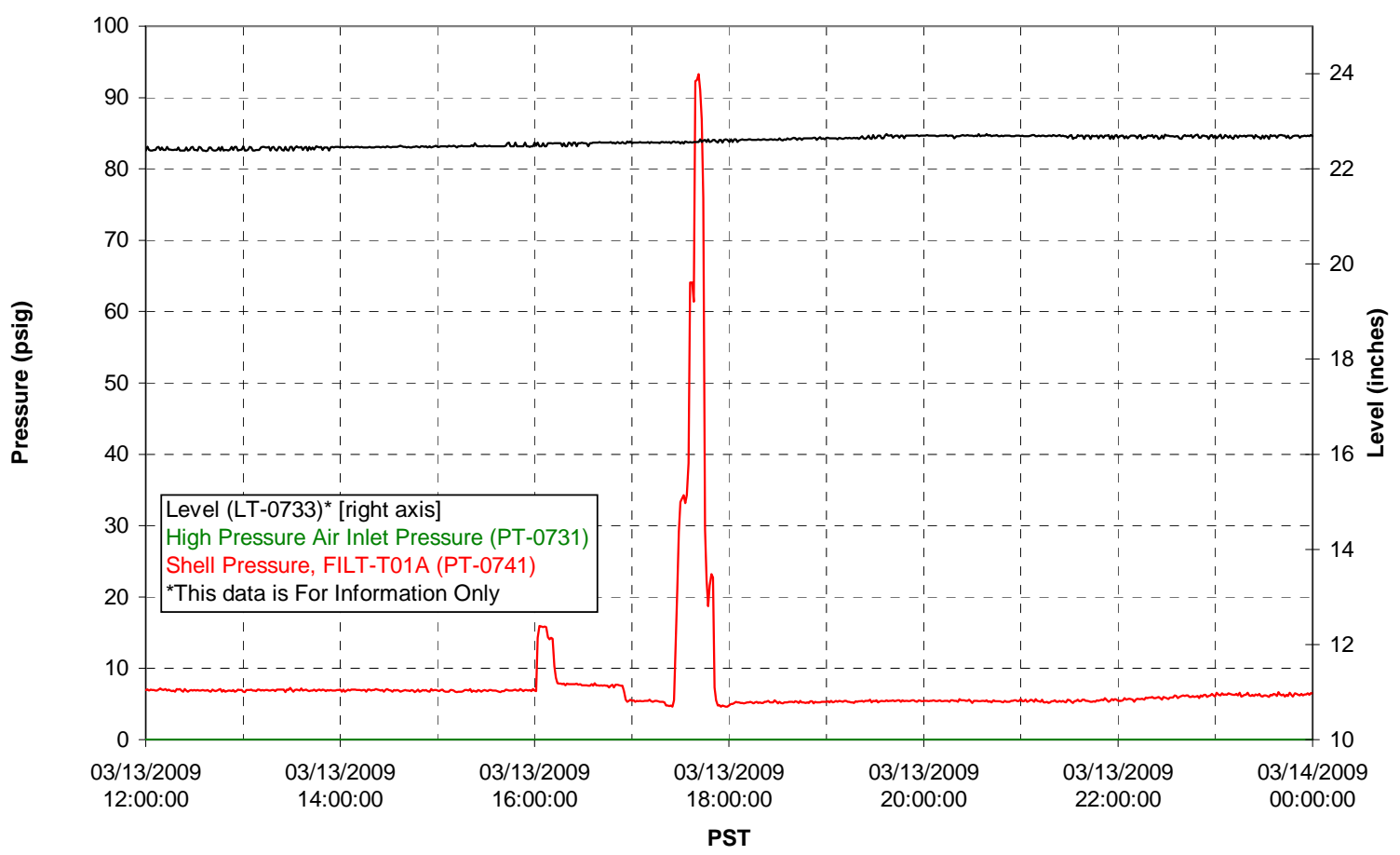

Pulsepot Levels

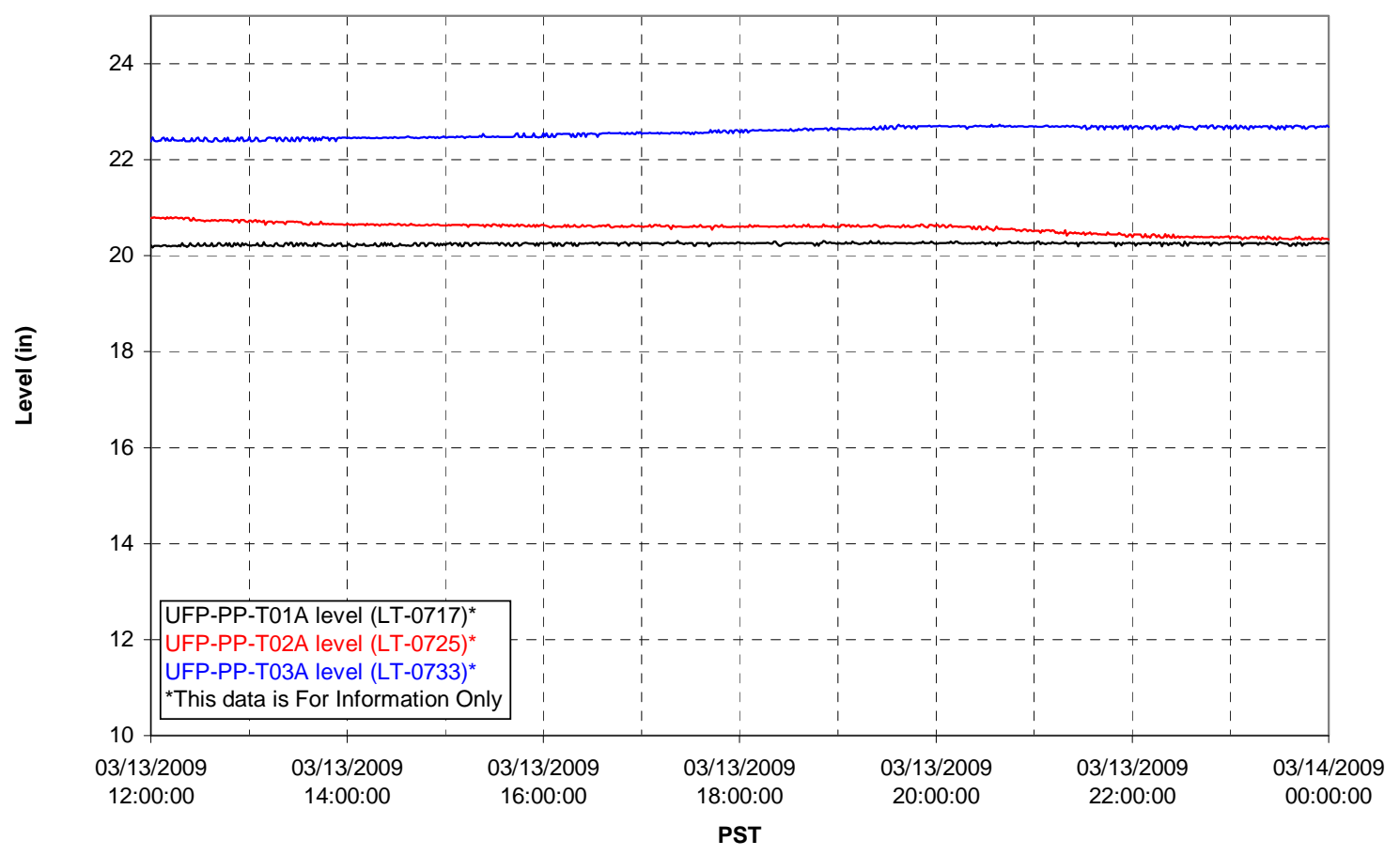


Filter UFP-FILT-T01A

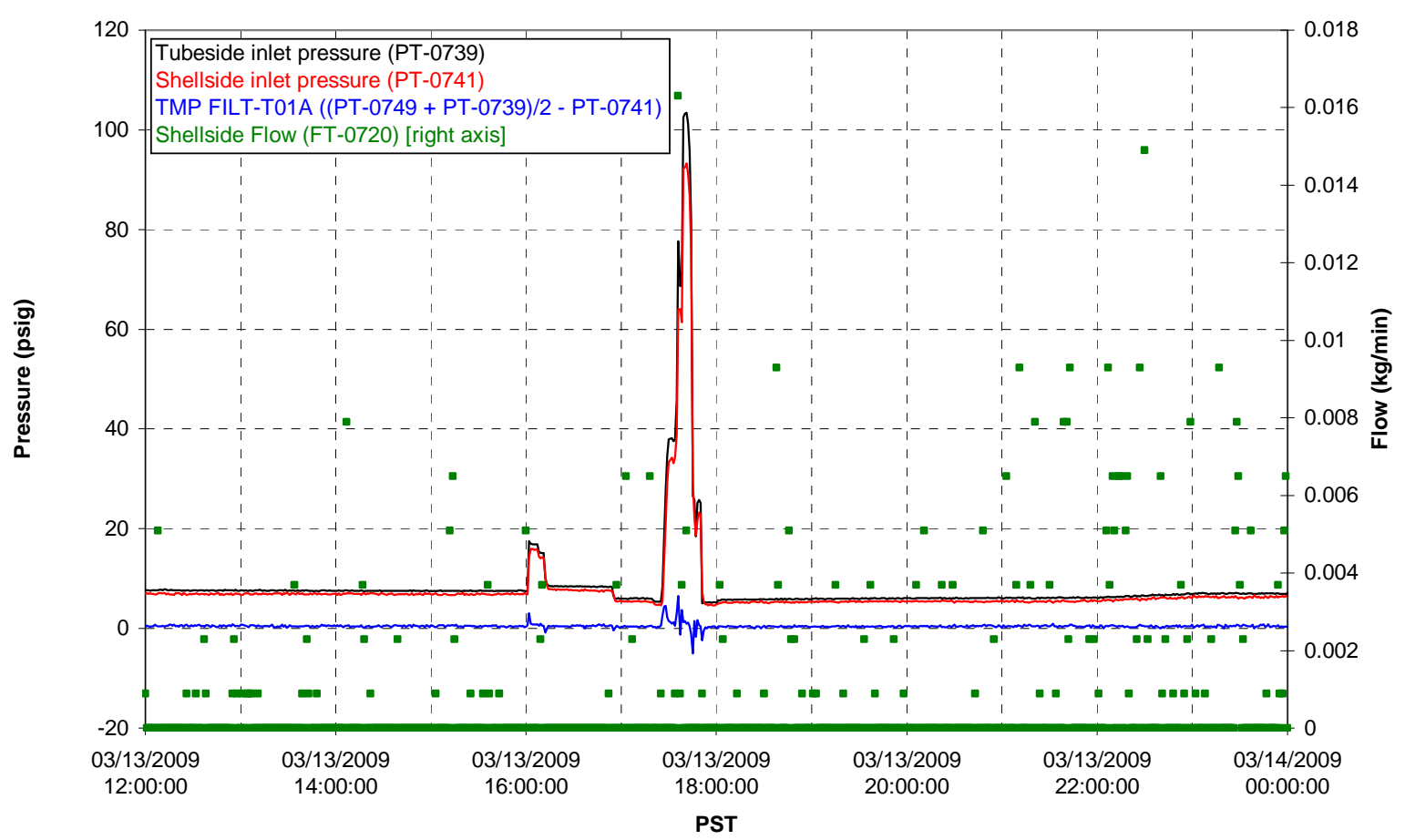

Filter UFP-FILT-T02A

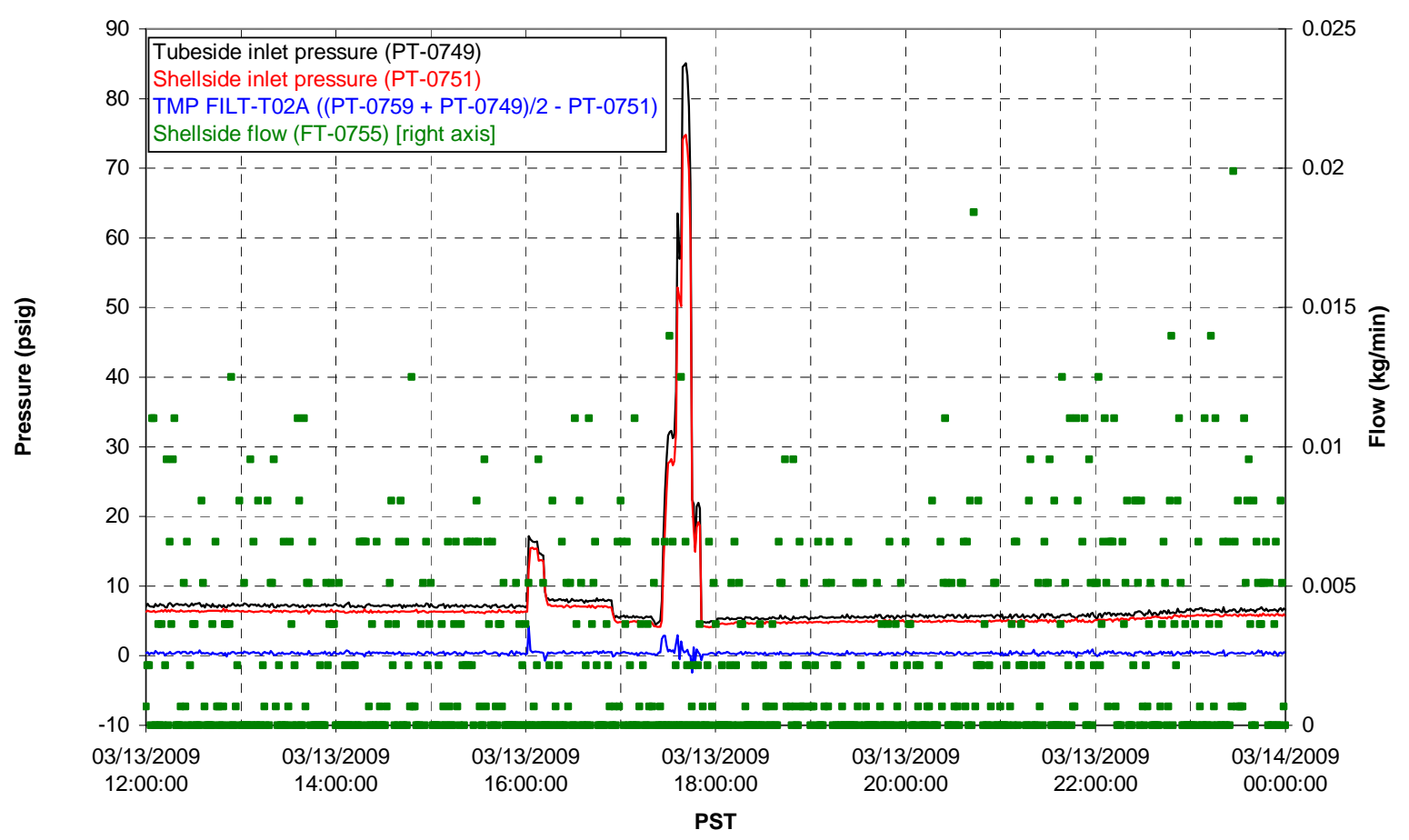


Filter UFP-FILT-T03A

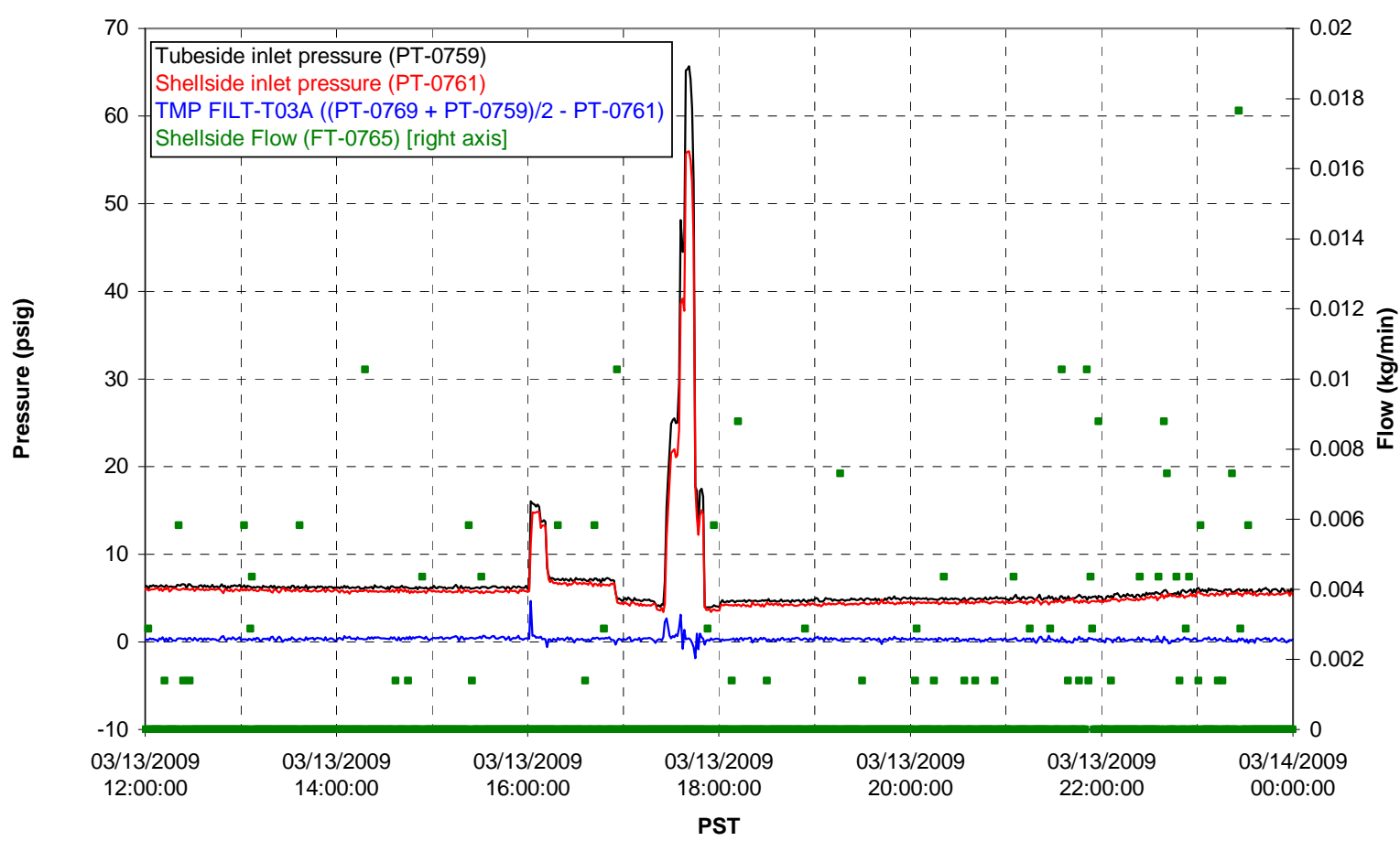

Filter UFP-FILT-T04A

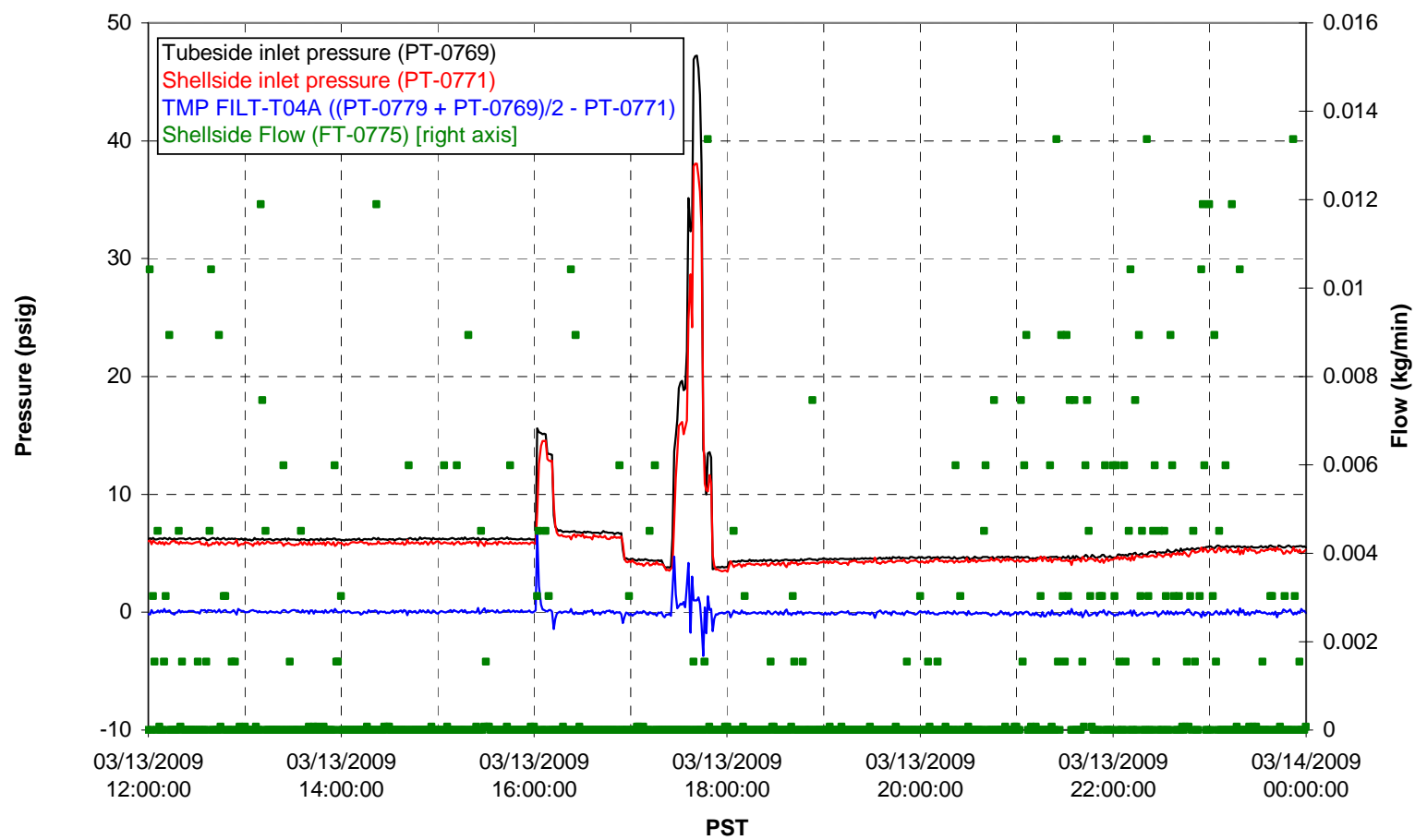


Filter UFP-FILT-T05A

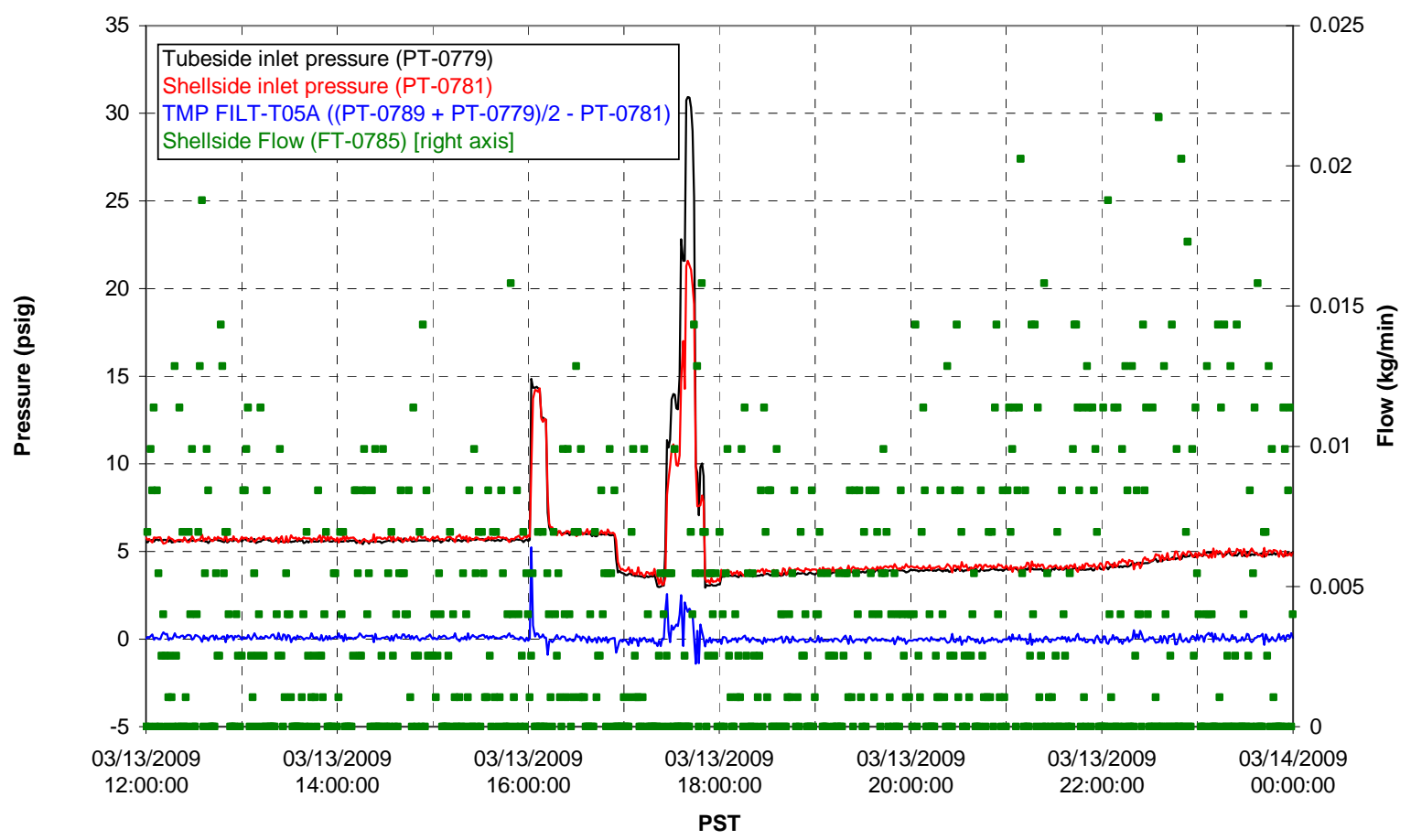

Chemical Flow

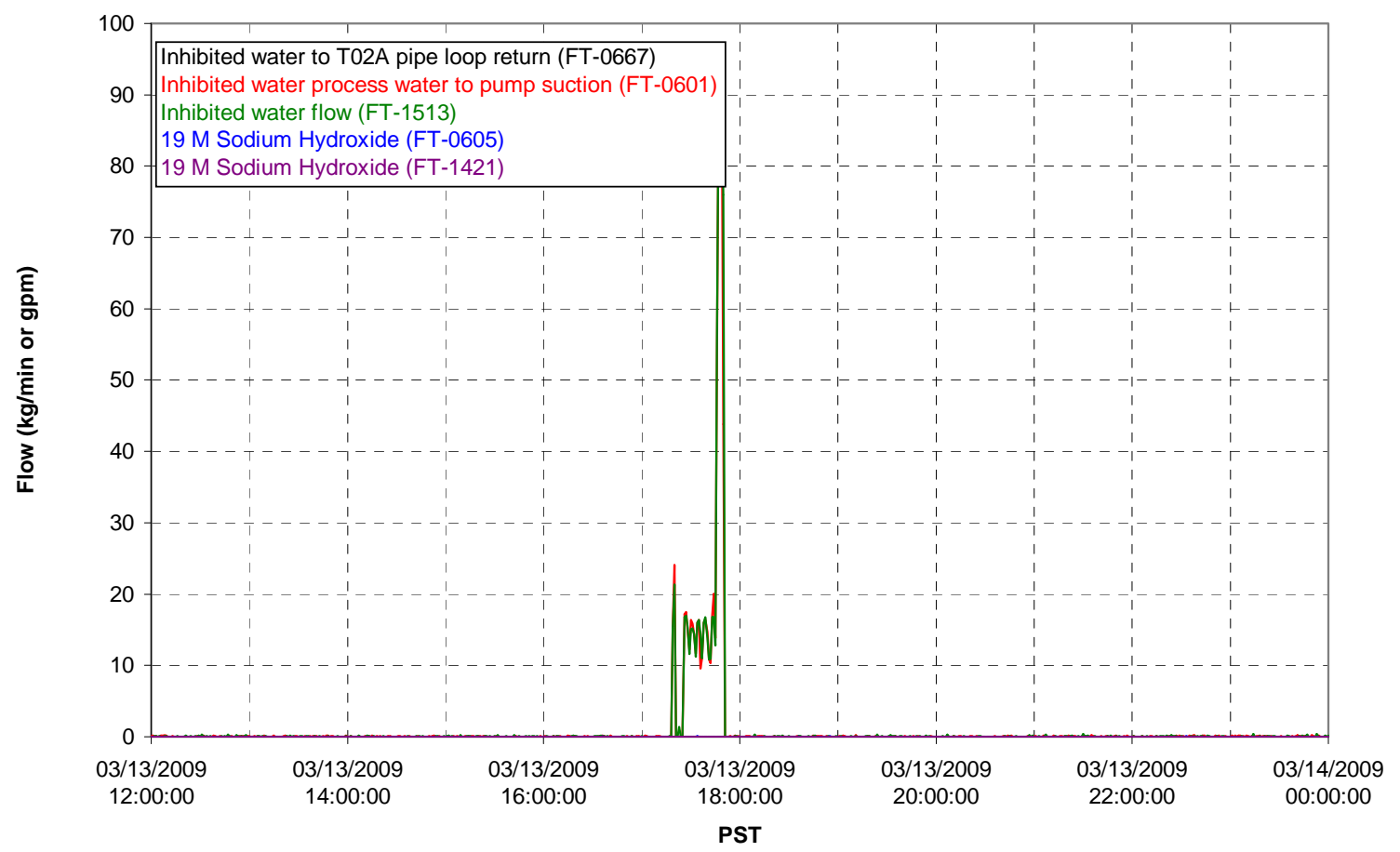




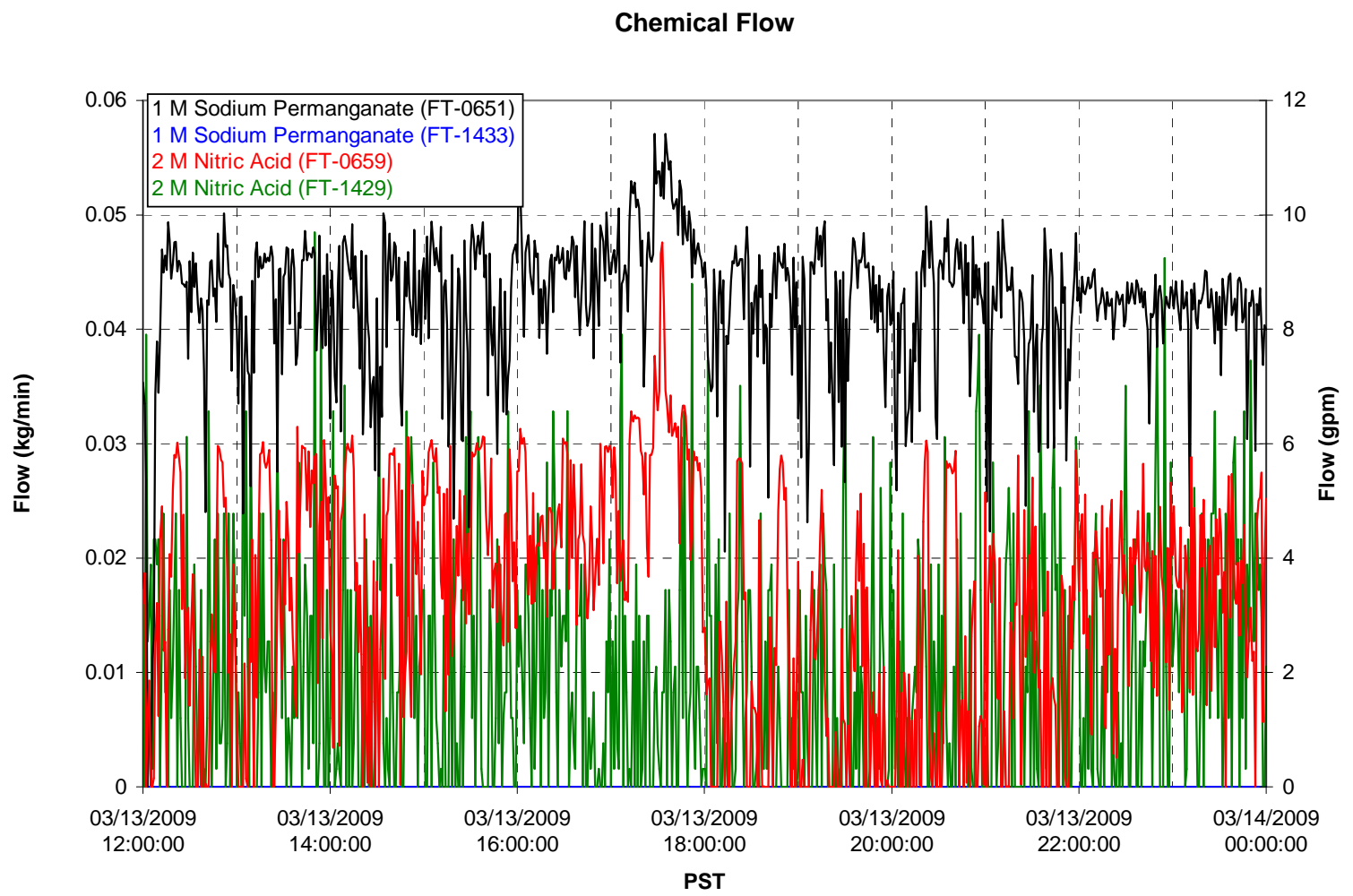

Air Flows

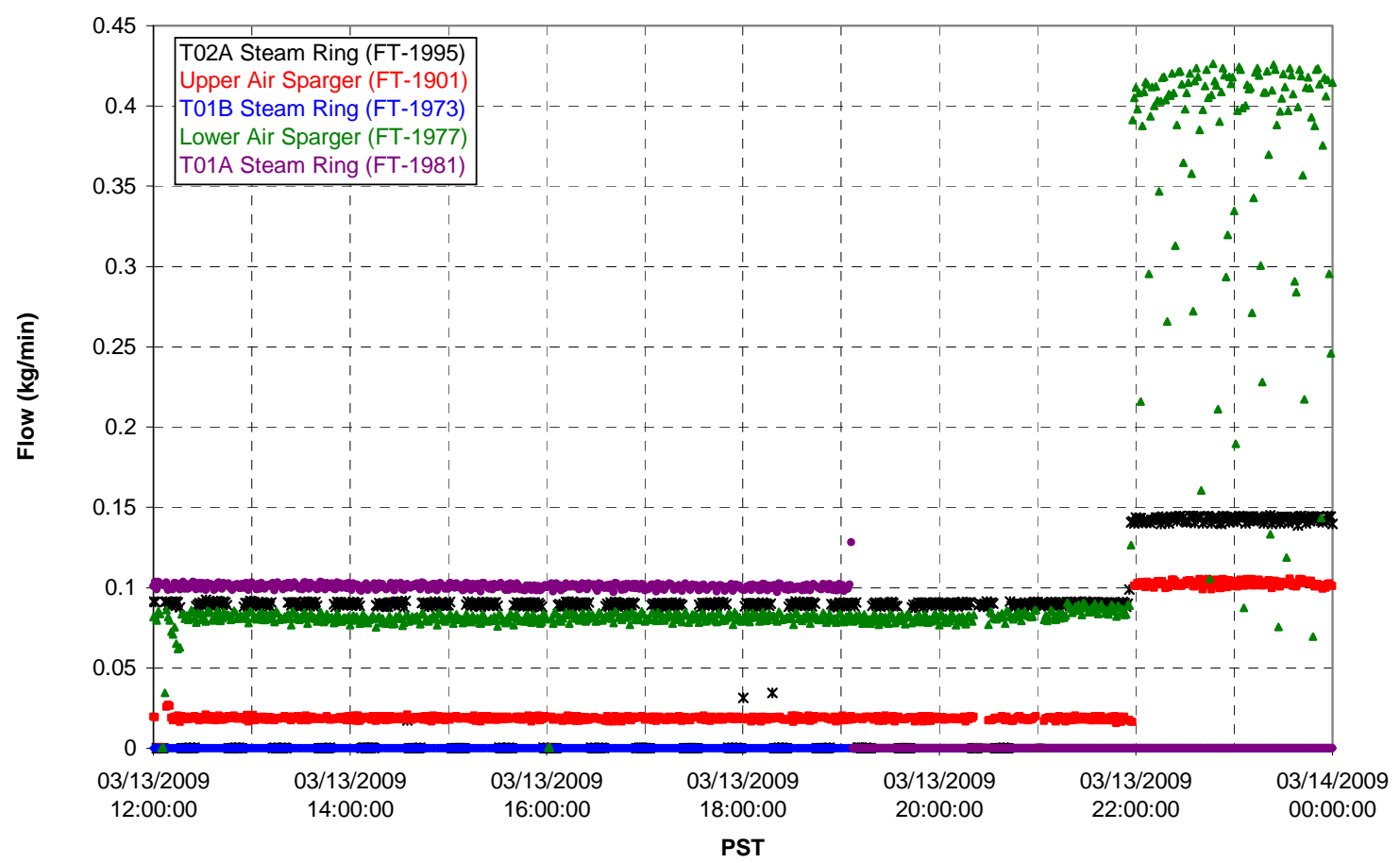


T02A Steam

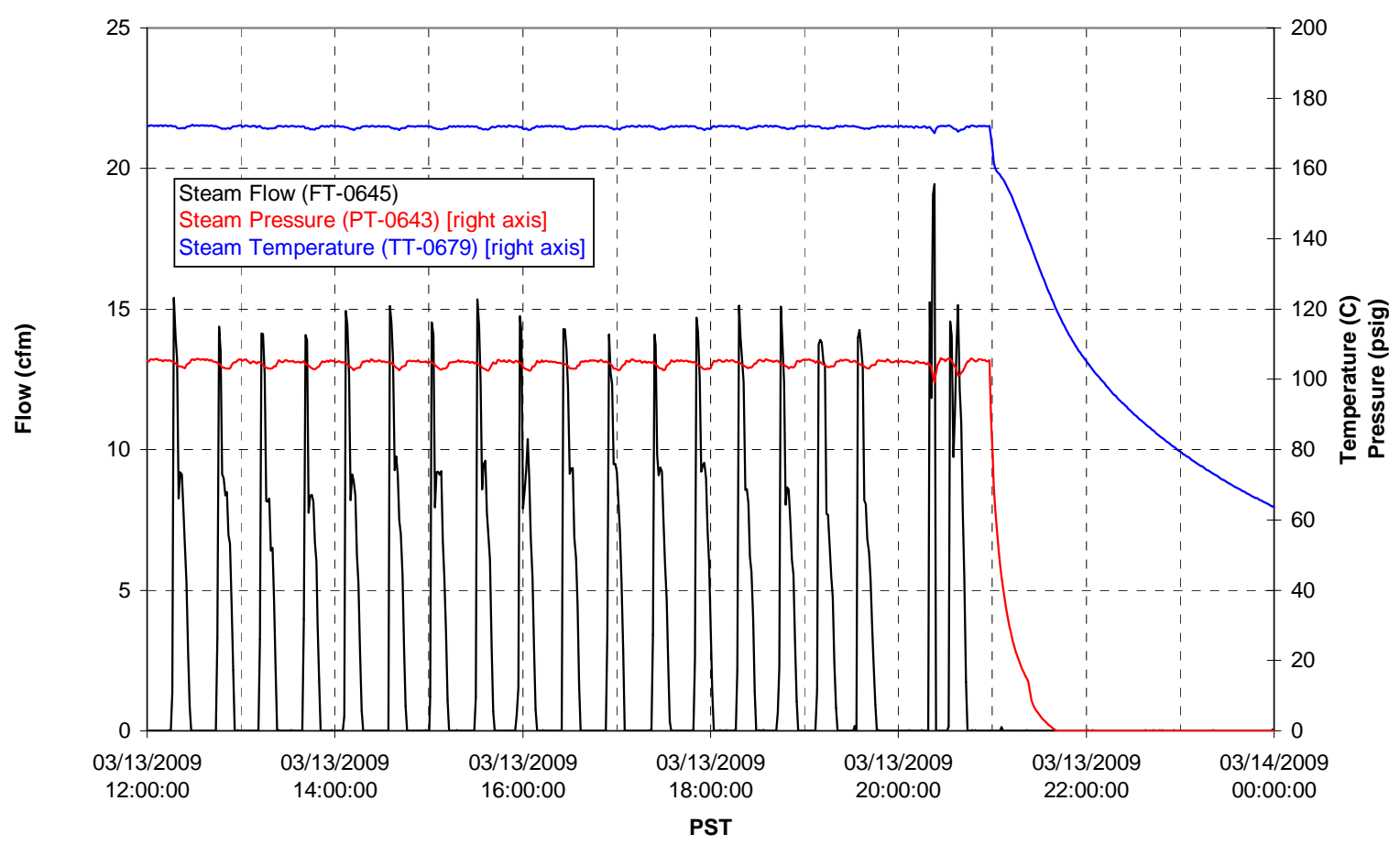

T01A Steam

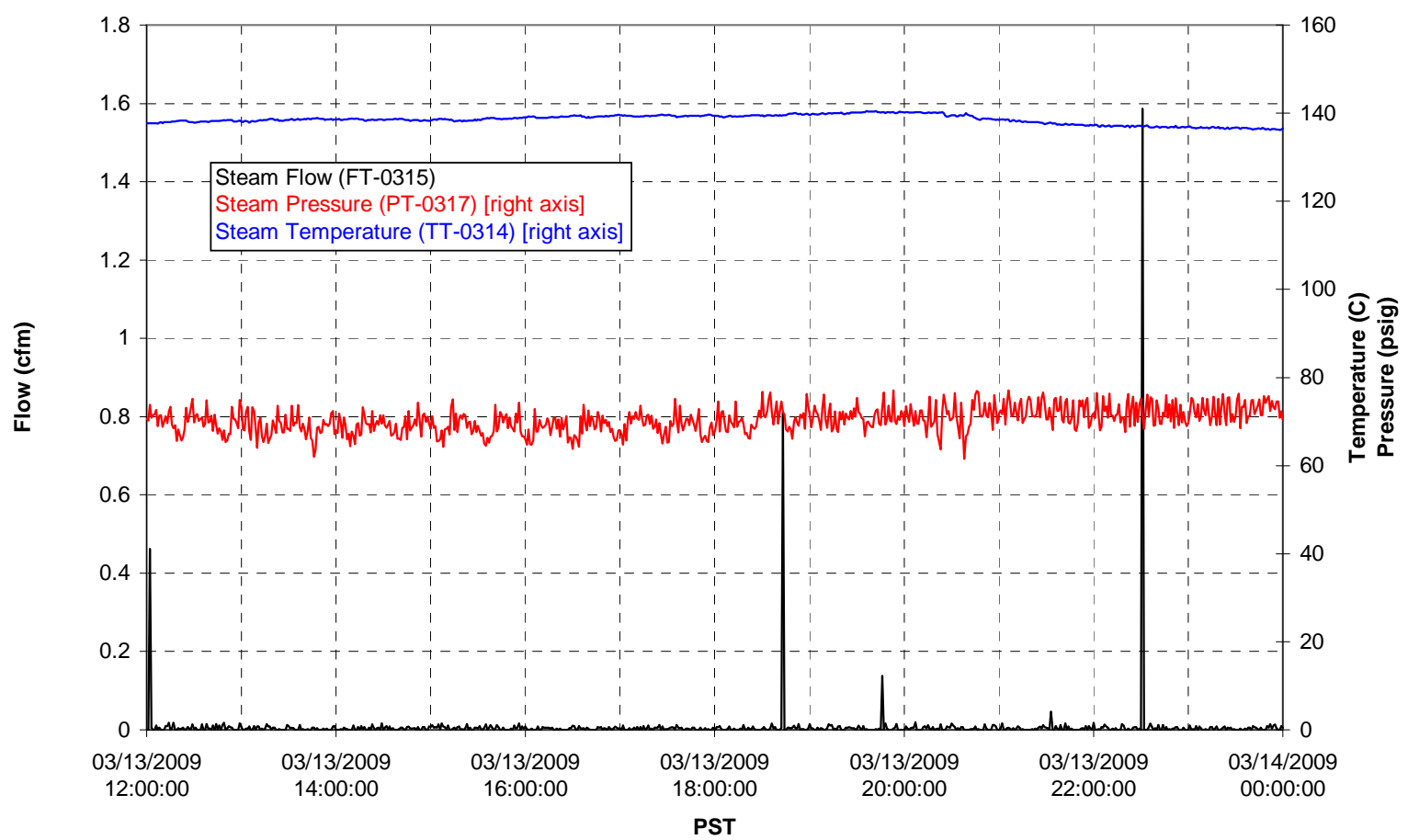


T01B Steam

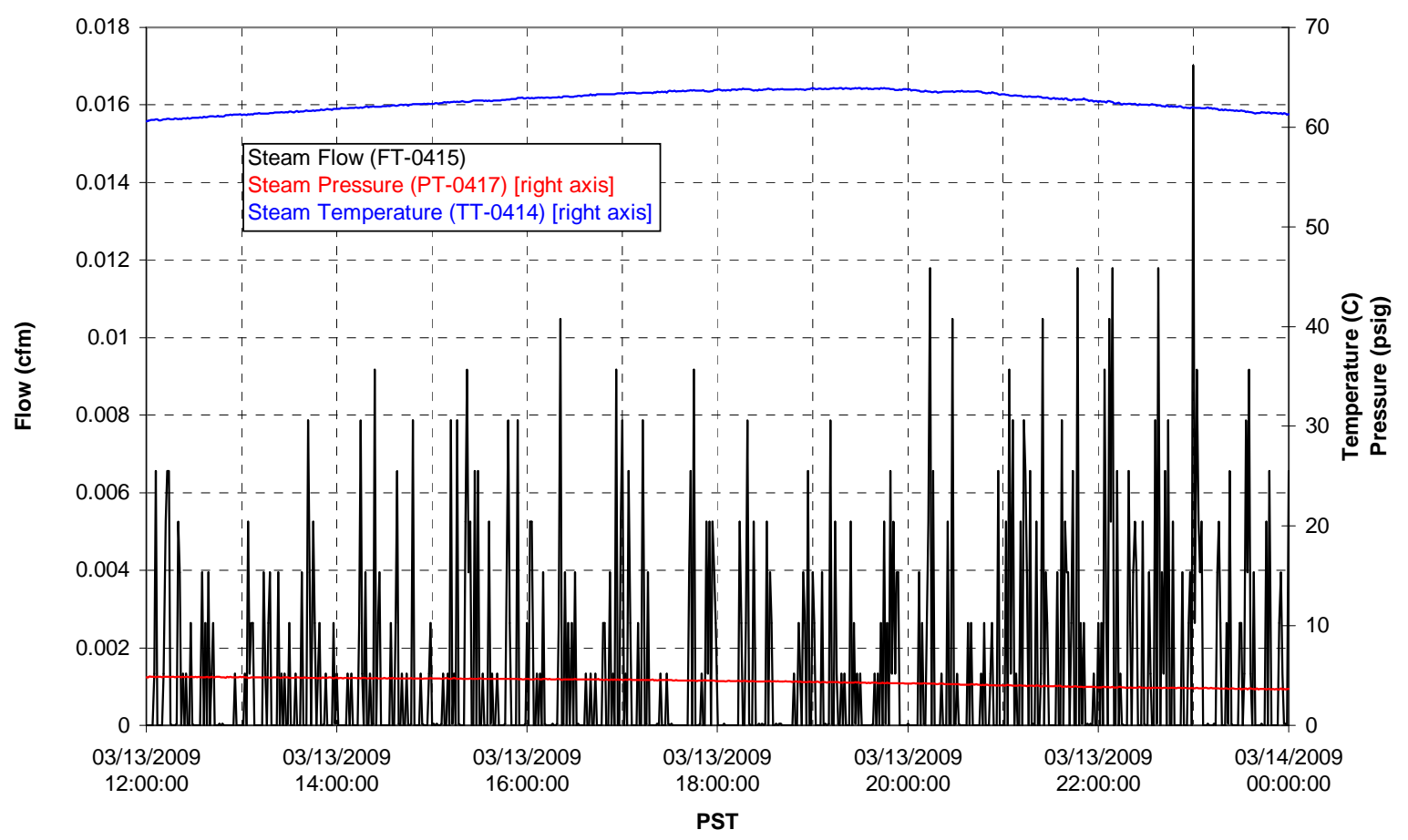




\section{Integrated Test B Data Plots \\ 03/14/09 00:00 - 03/14/09 12:00}

During this period of testing, the DAS crashed and no data was saved for some sensors between 01:18 and 06:38. 
T01A level

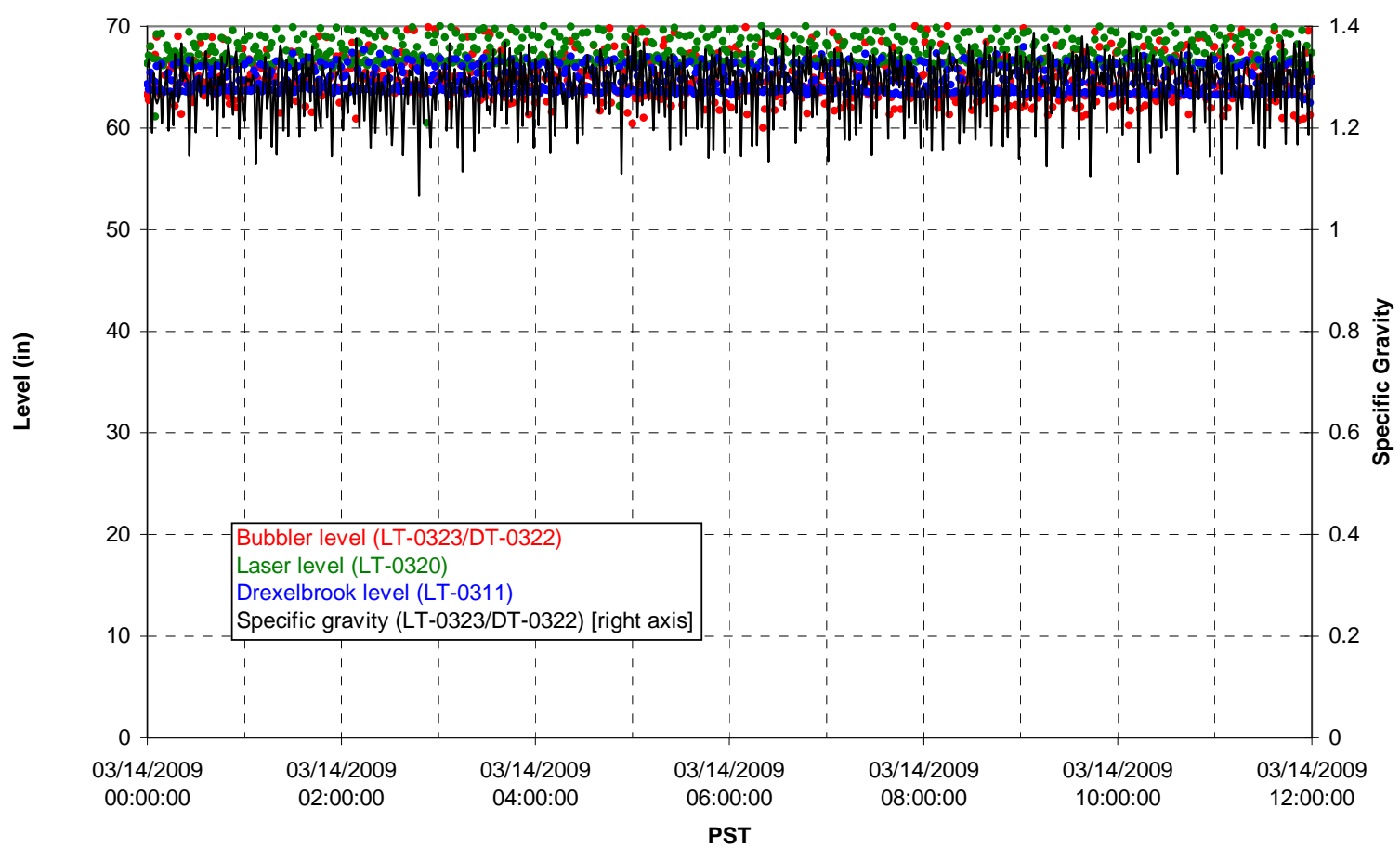

T01A temperatures

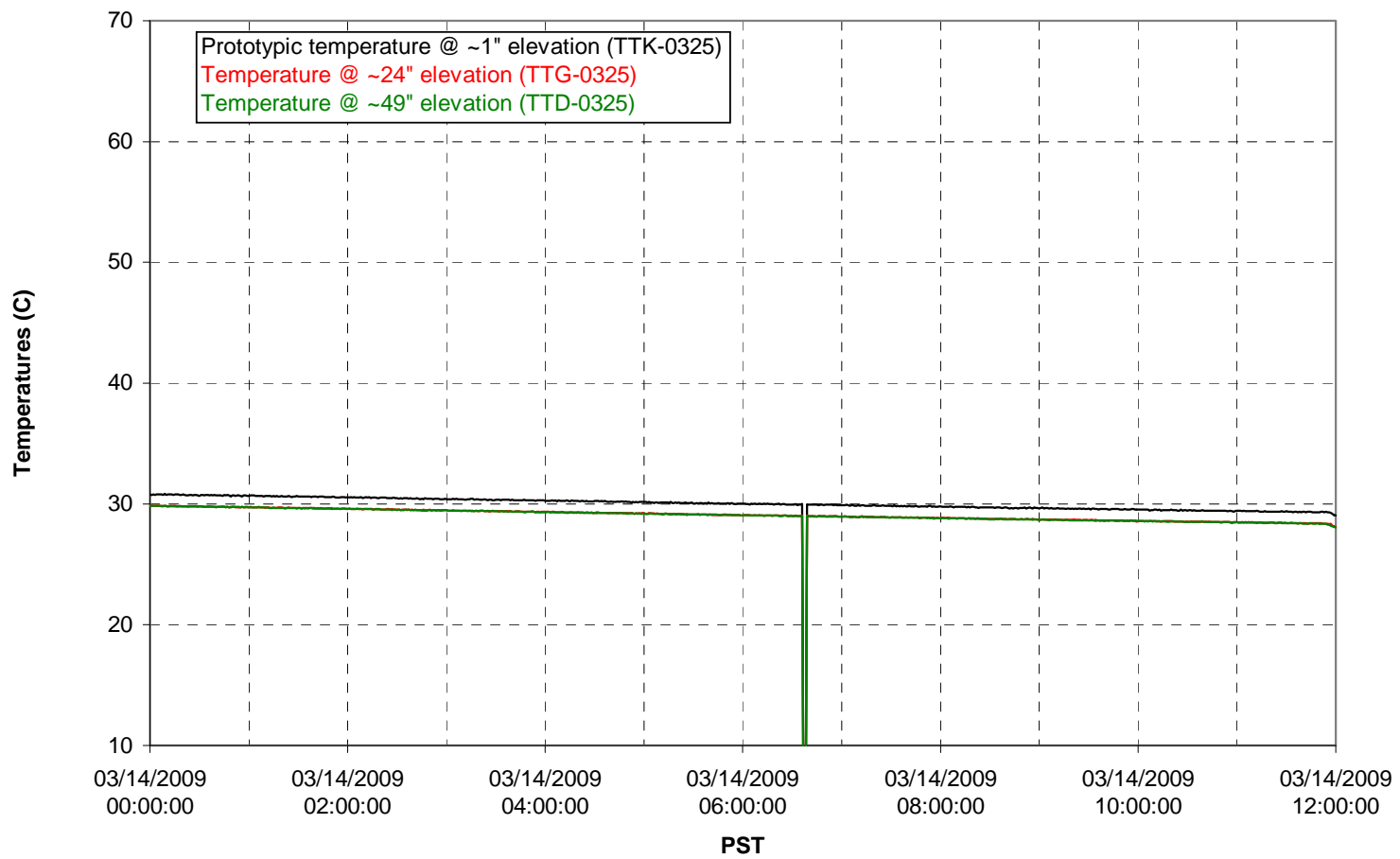


T01B level

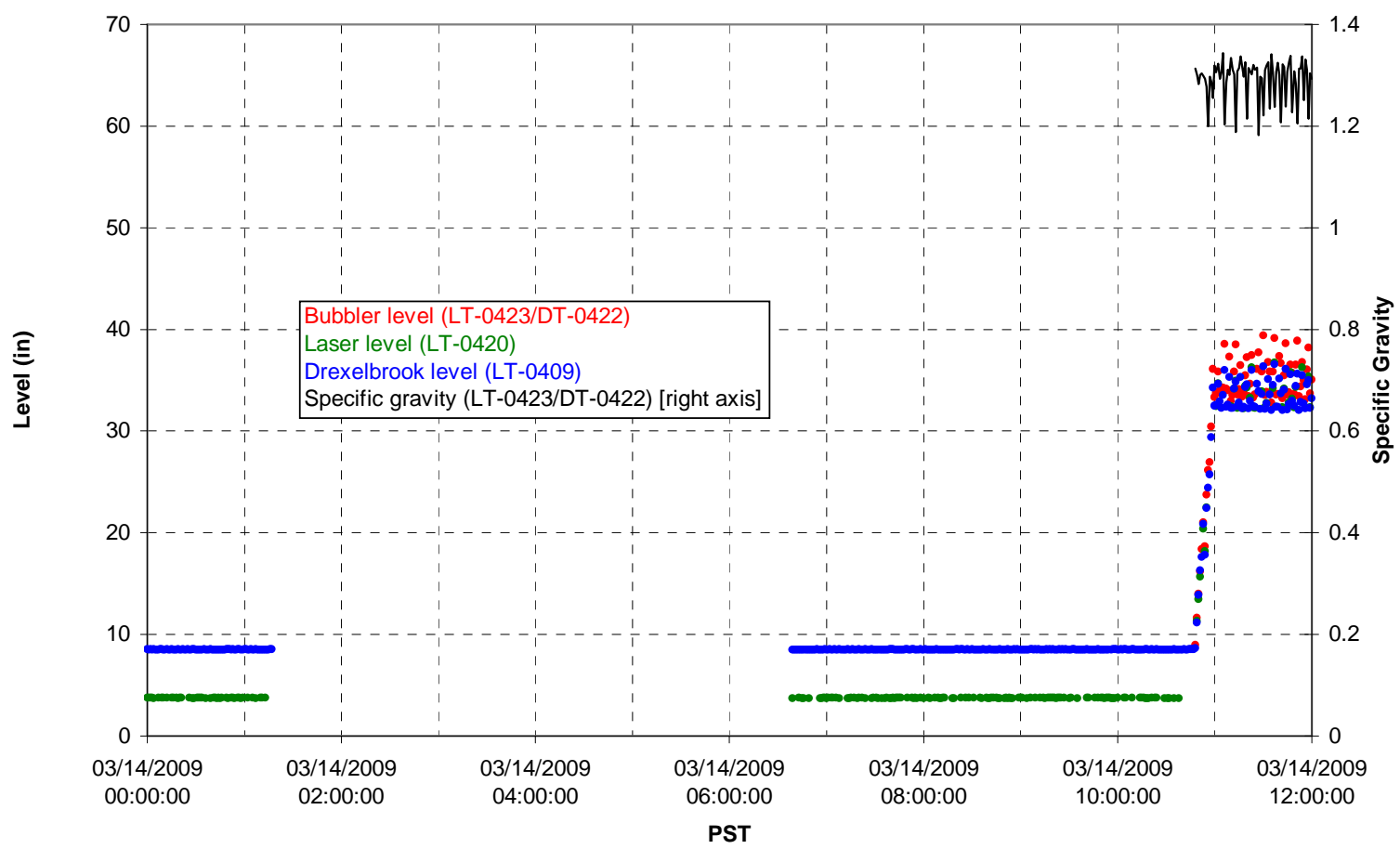

T01B temperatures

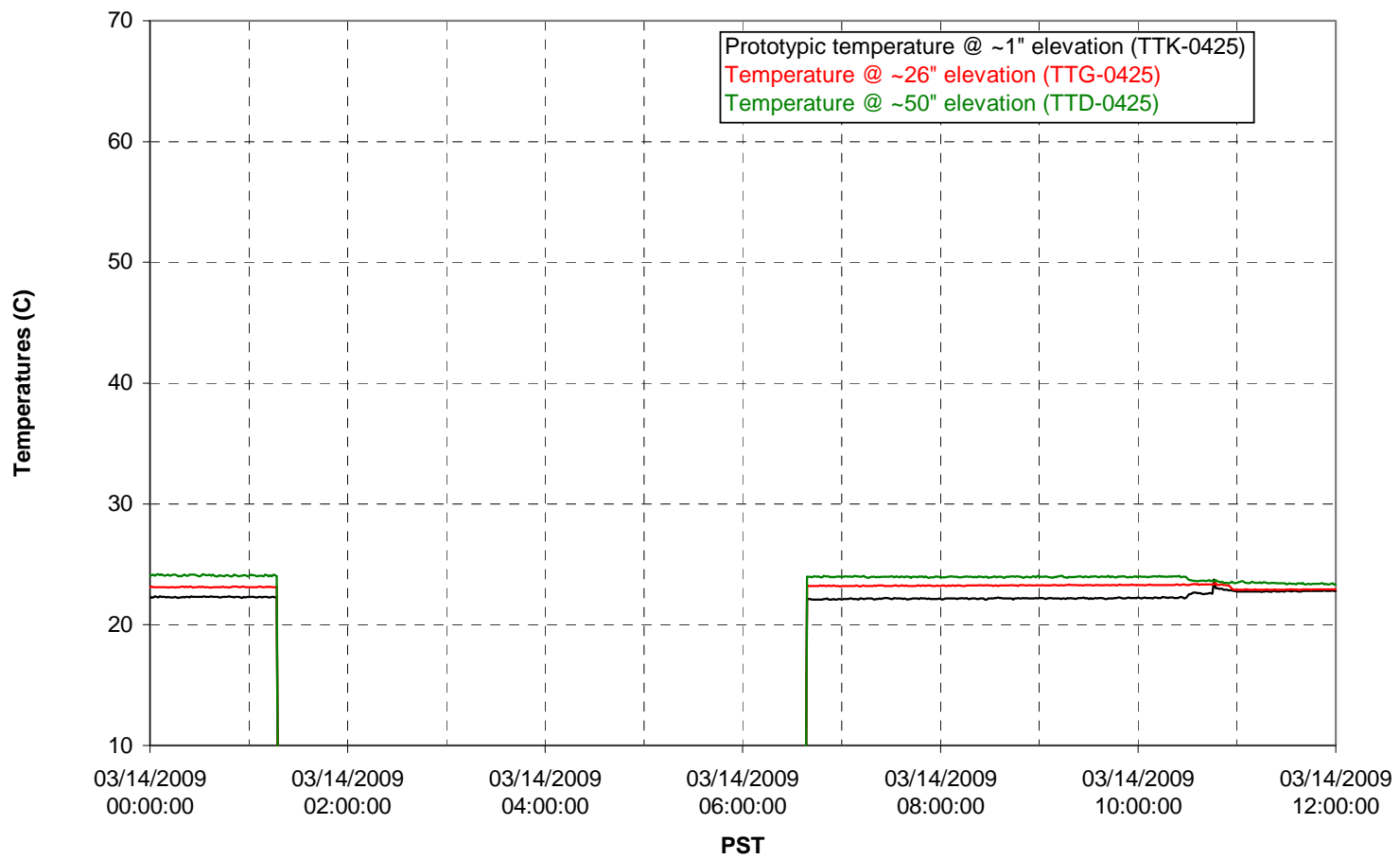


T02A level

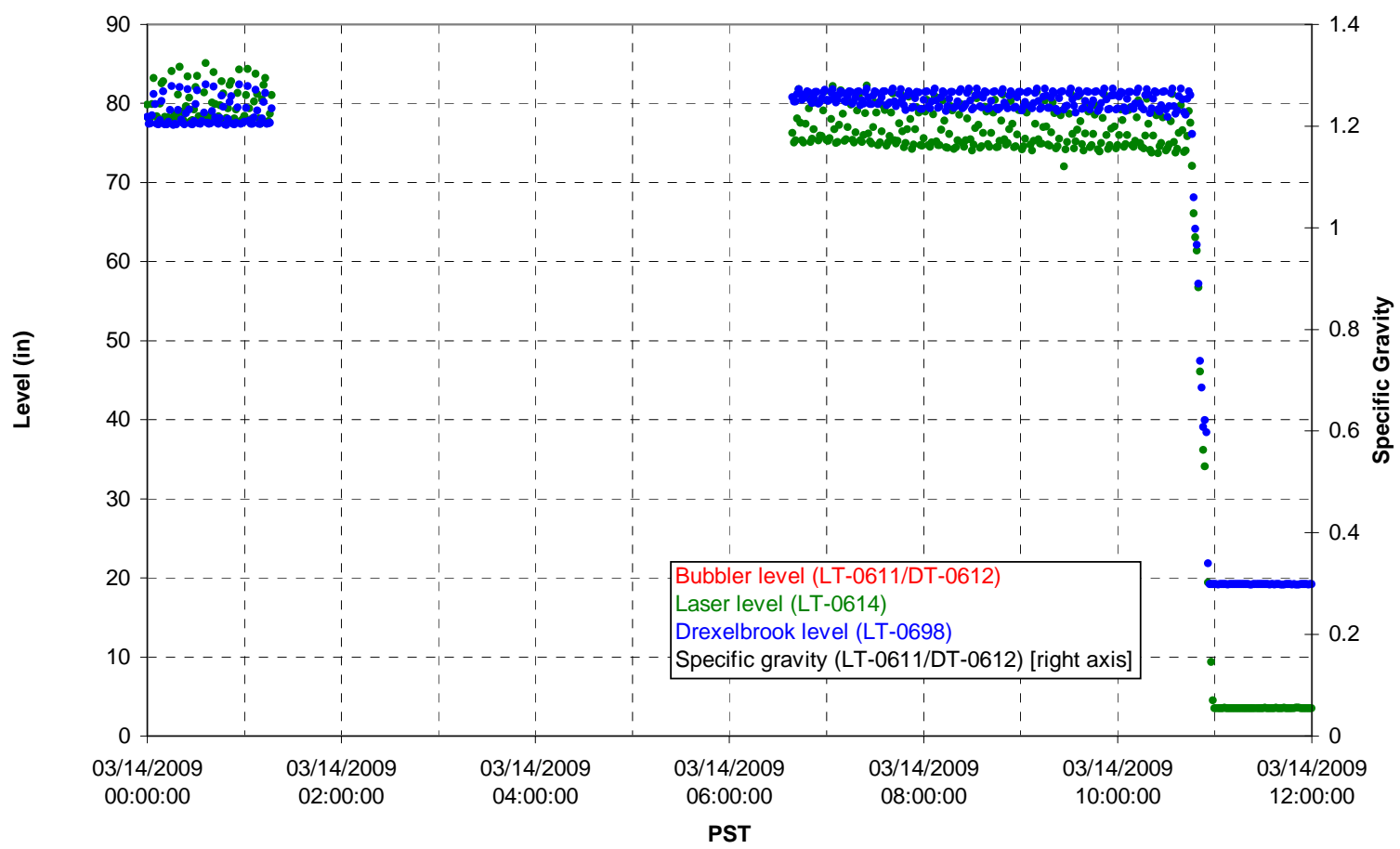

T02A temperatures

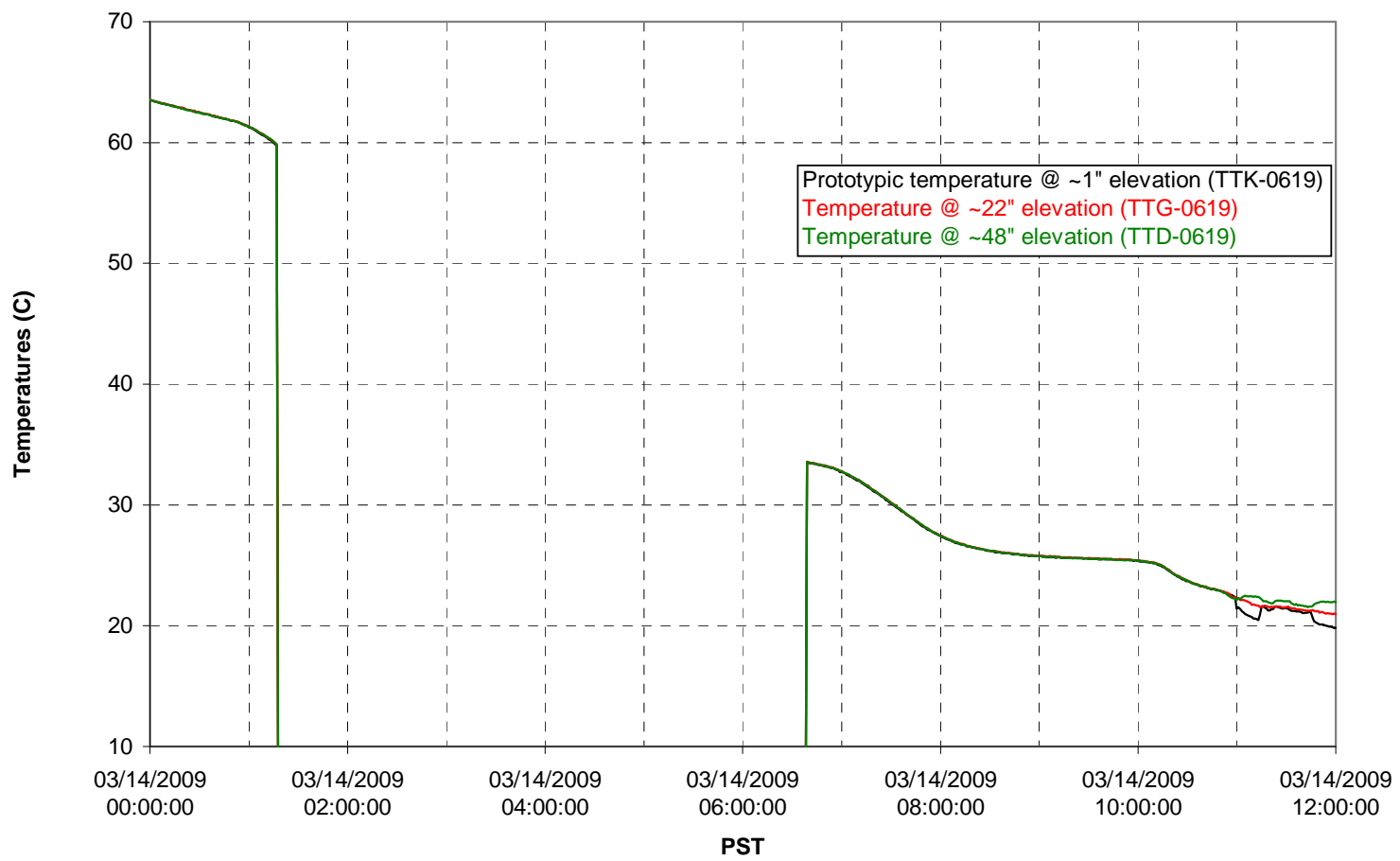


T02A and filter loop temperatures

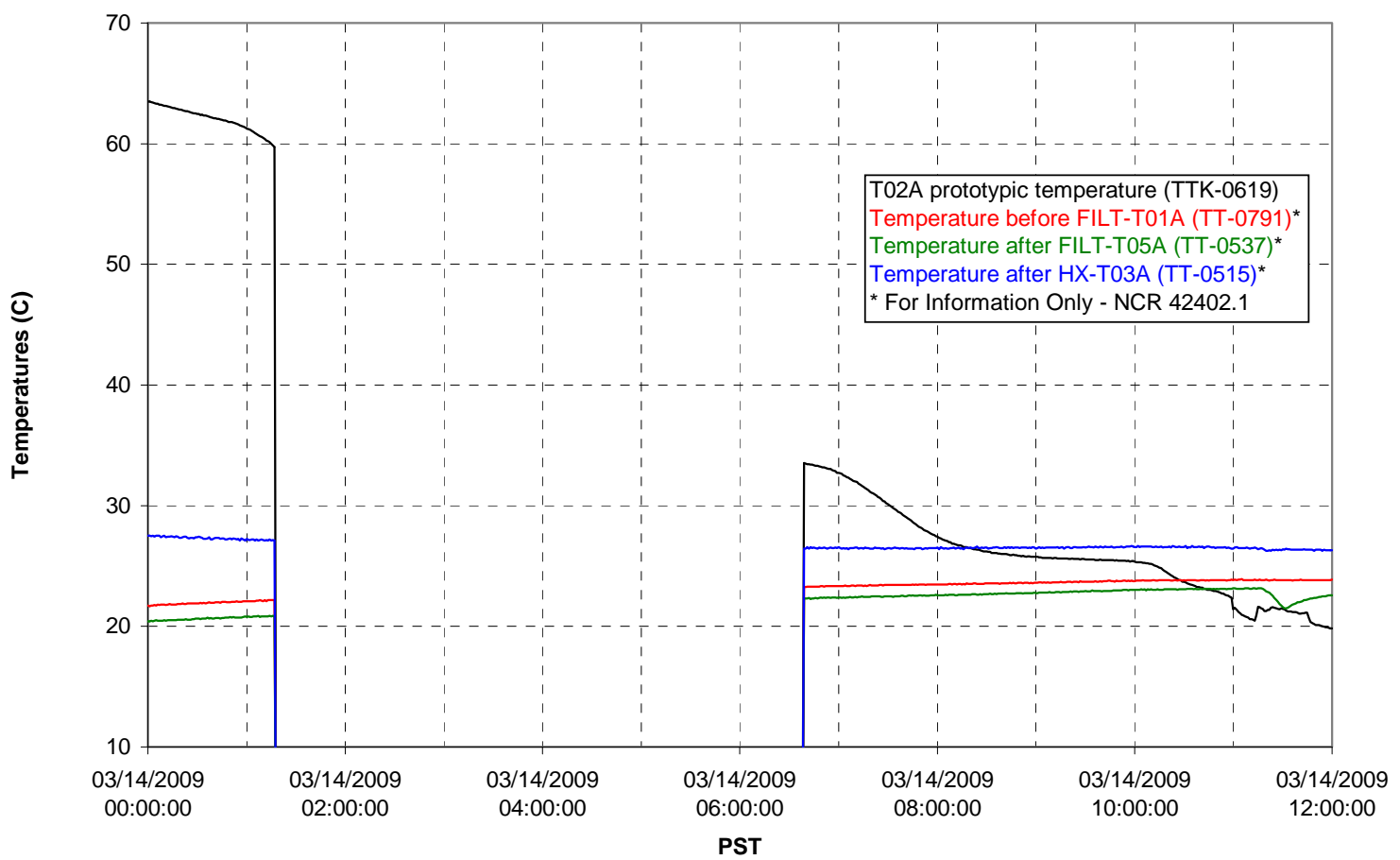

Pump Pressures and Flow

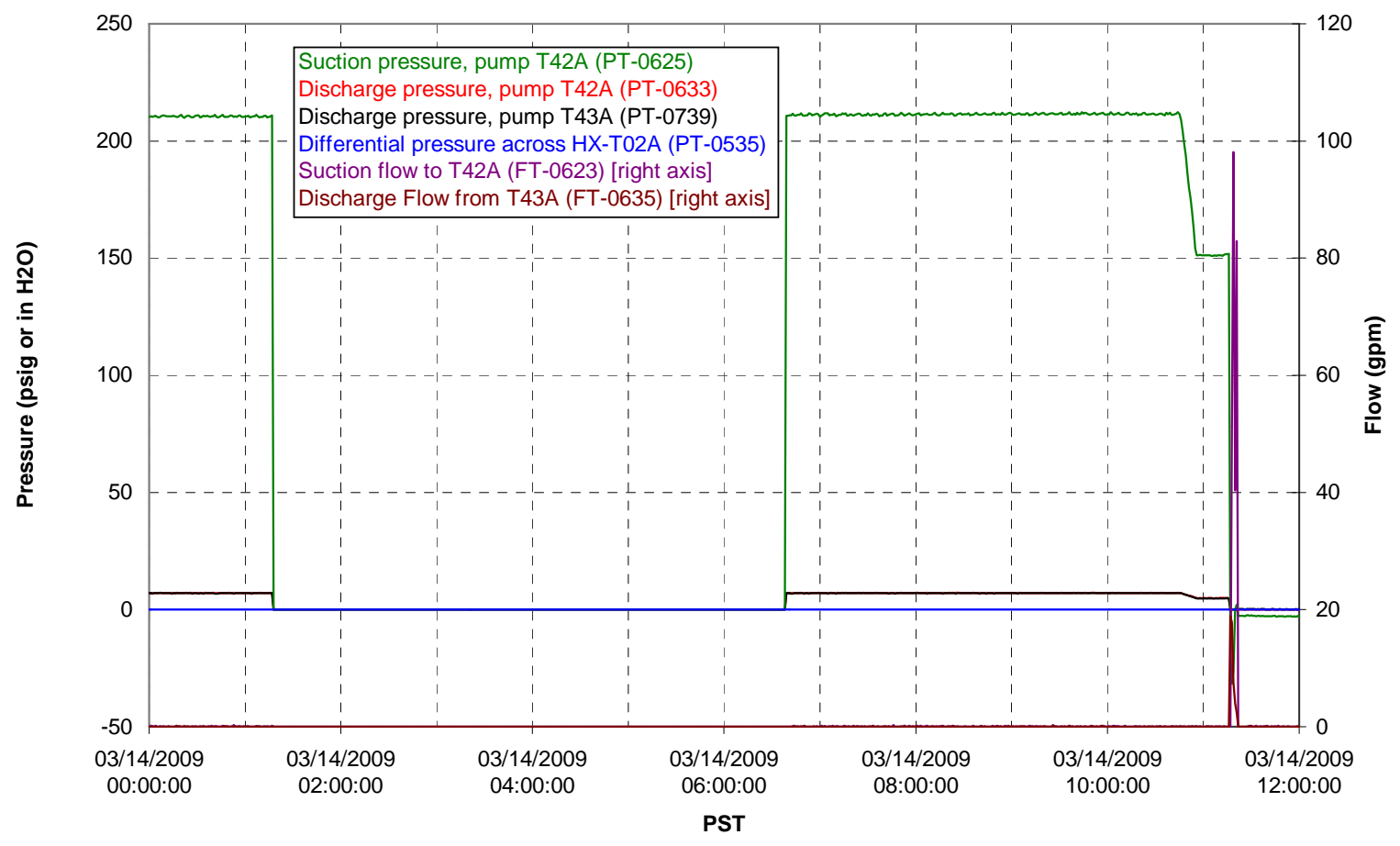




\section{Axial pressure drop}

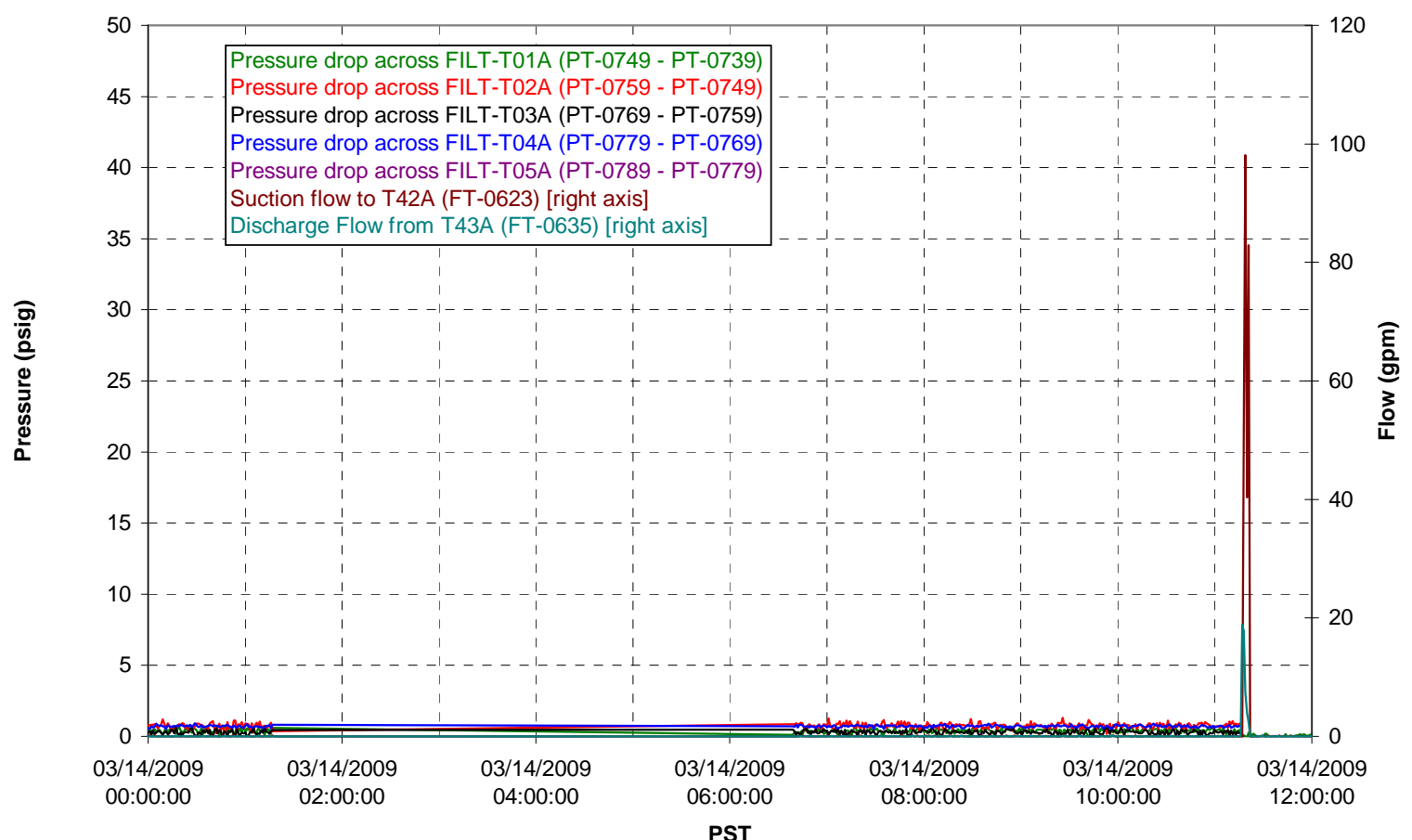

Permeate flow rates

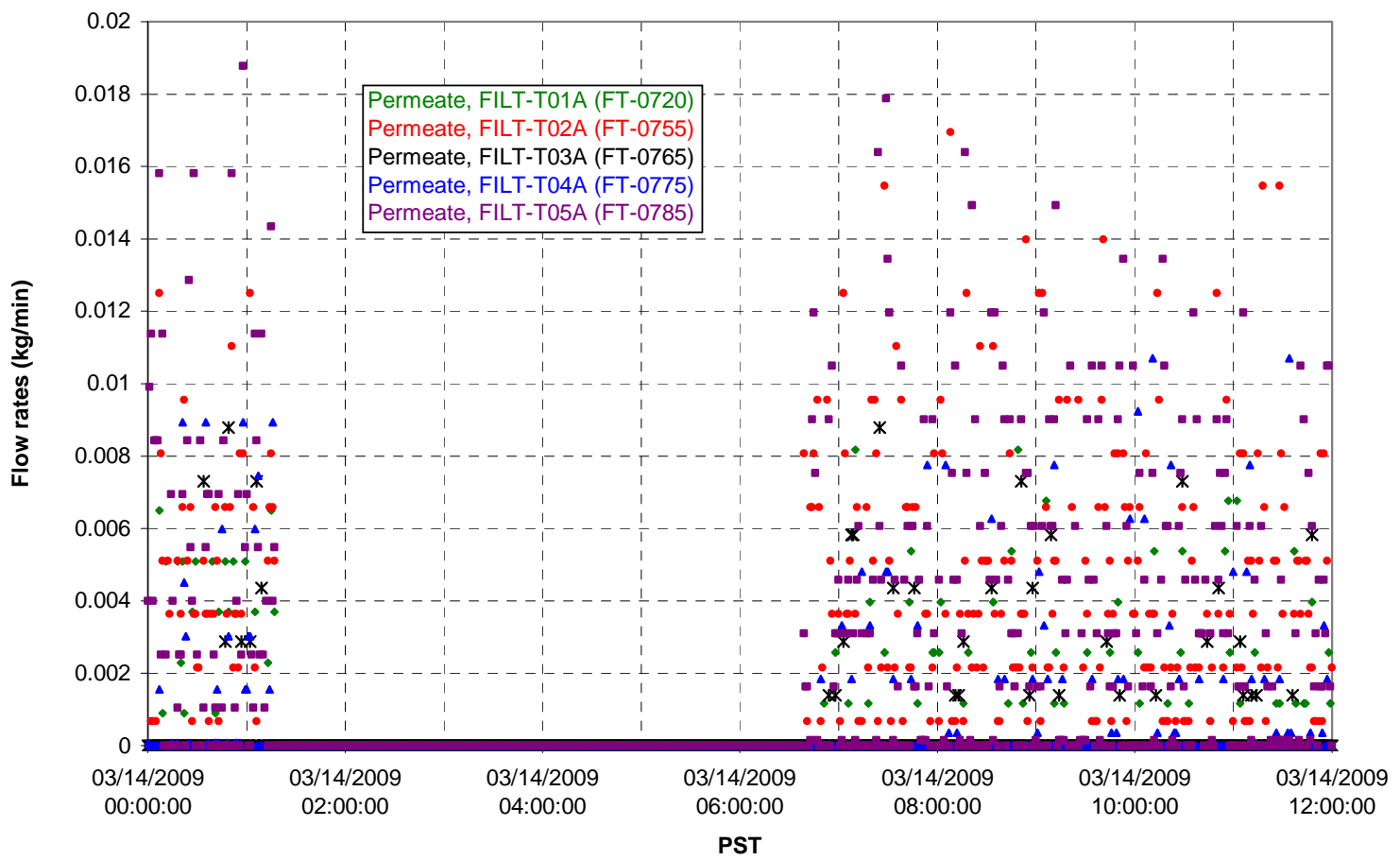


T02A Inner Temperature Tree

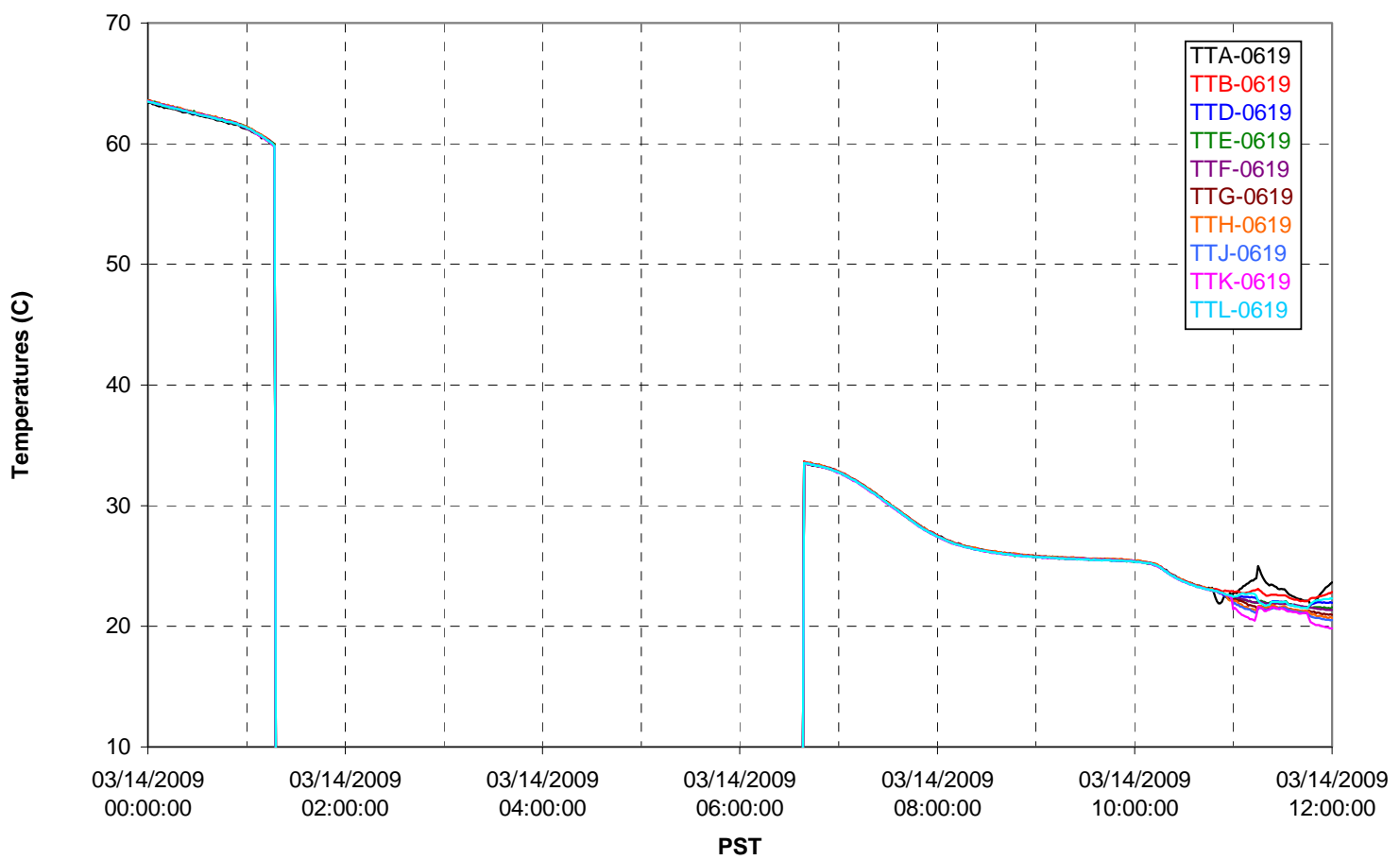

T02A Outer Temperature Tree

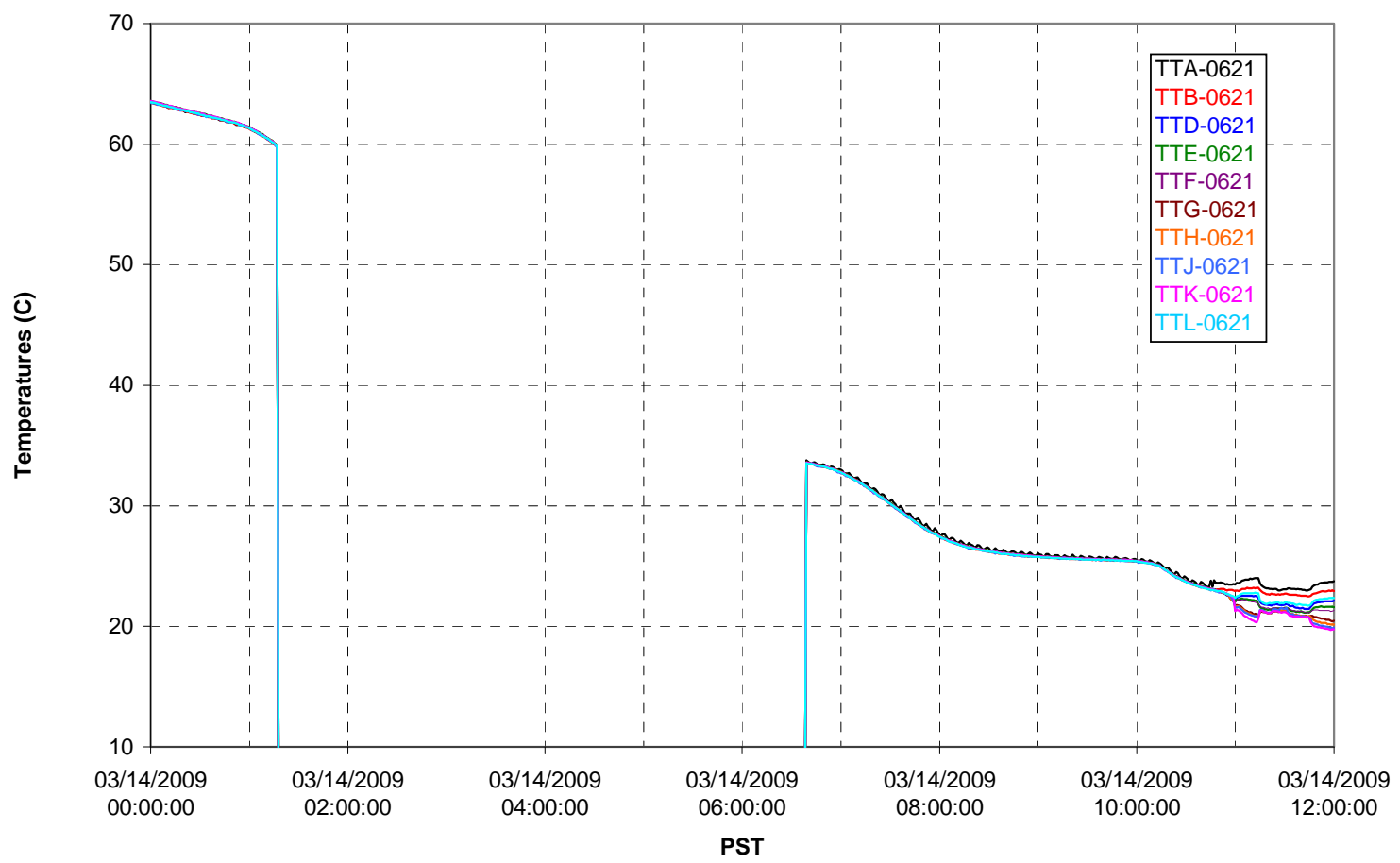


T02A temperatures

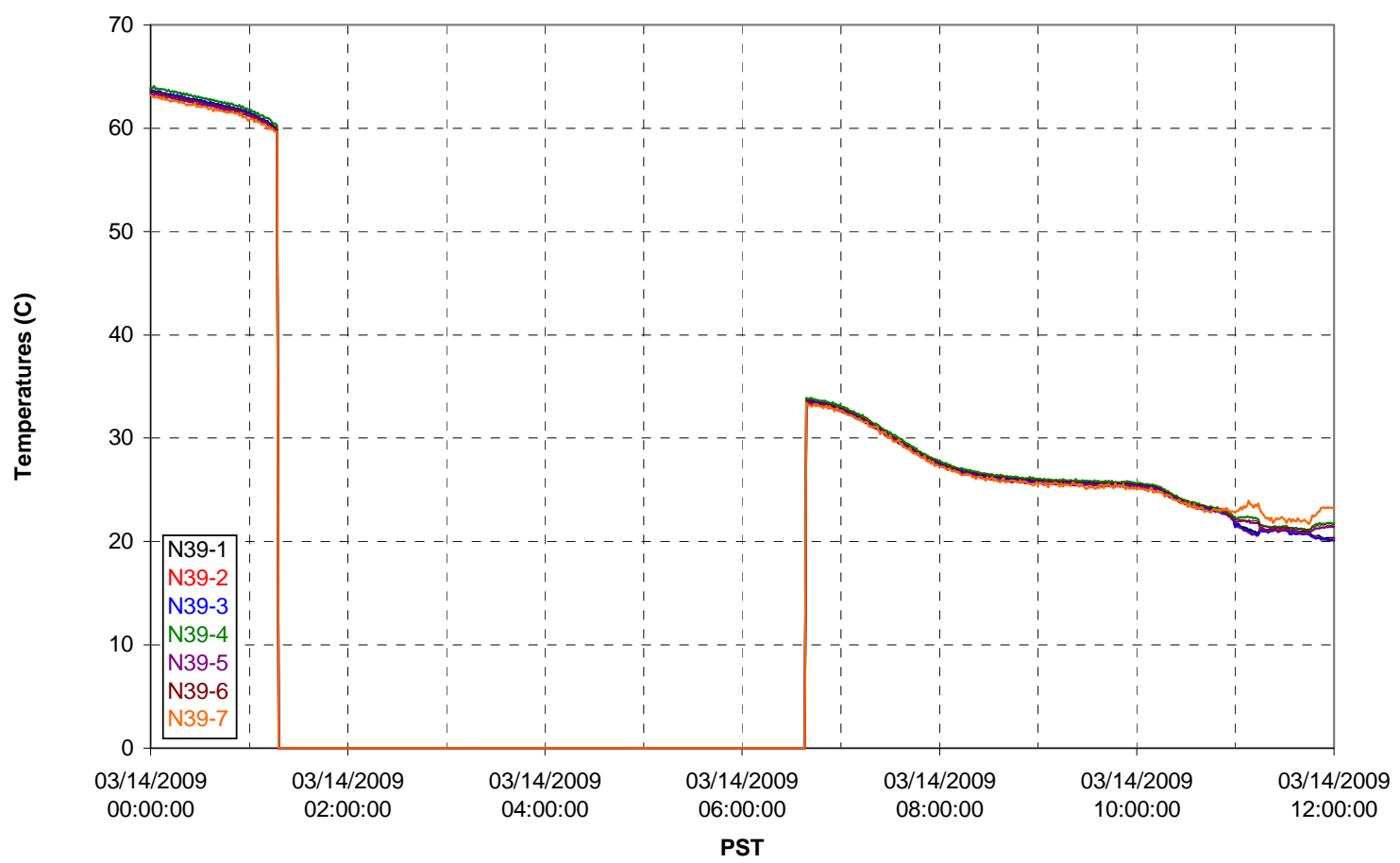

T02A temperatures

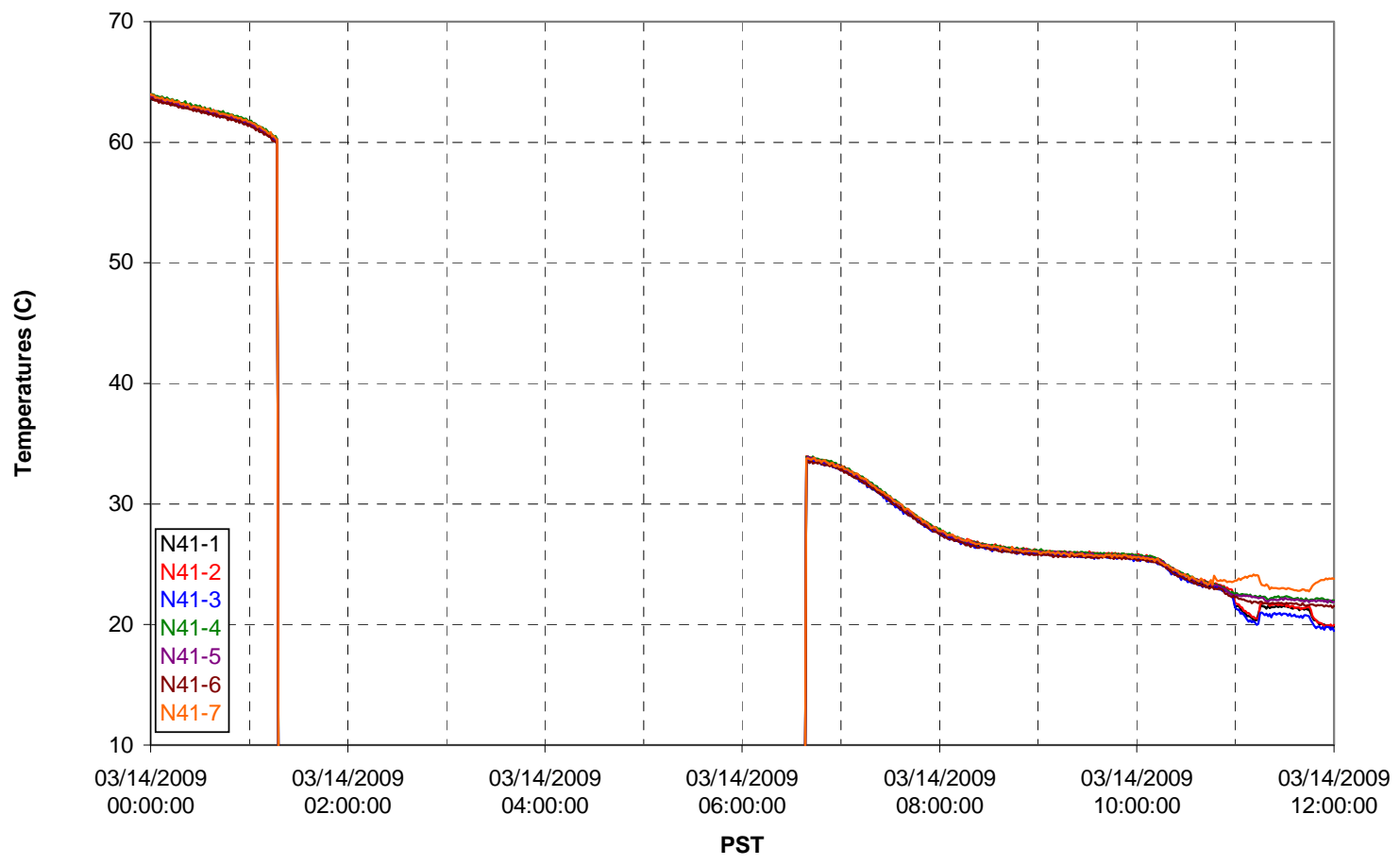


T02A temperatures

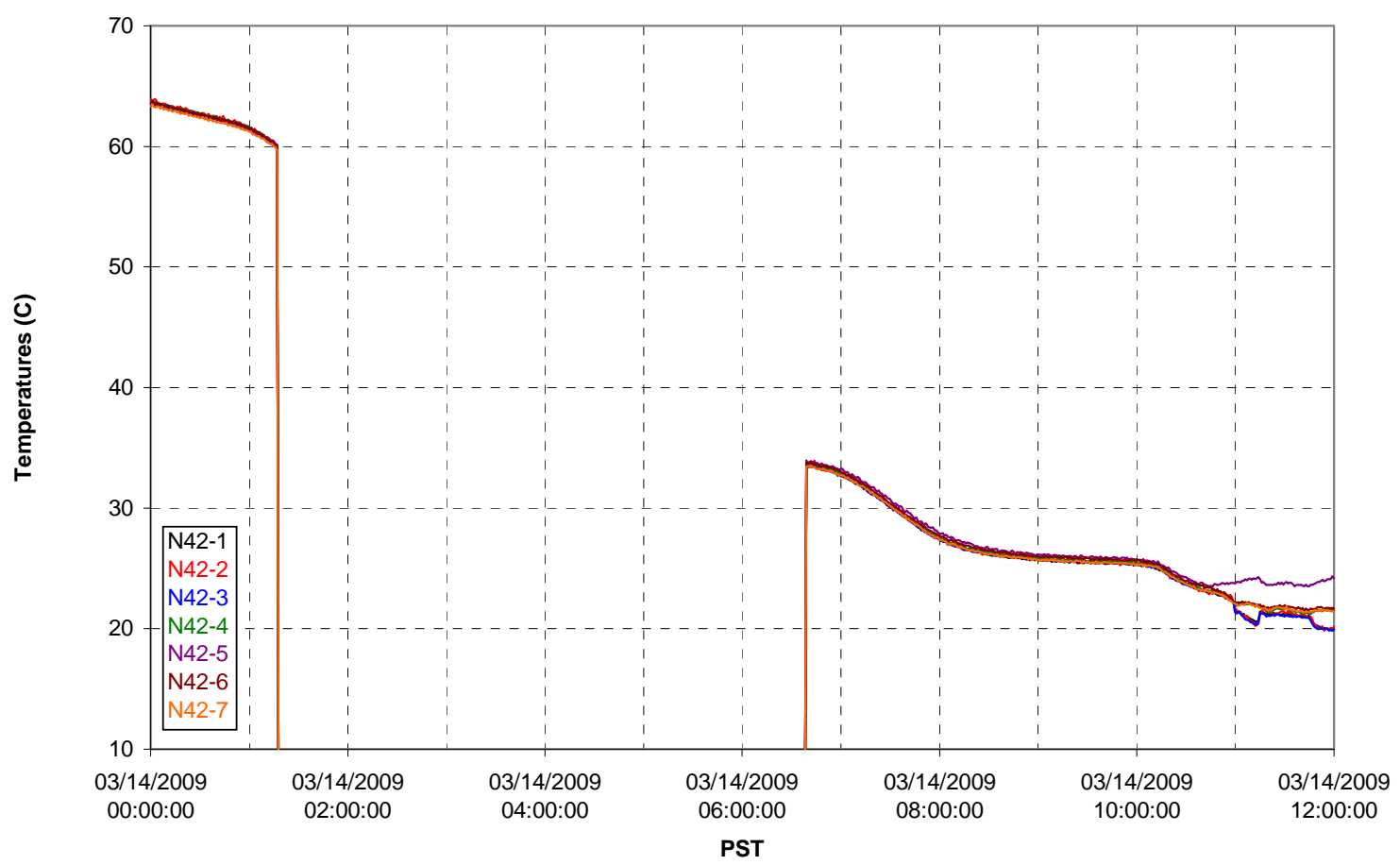

T02A temperatures

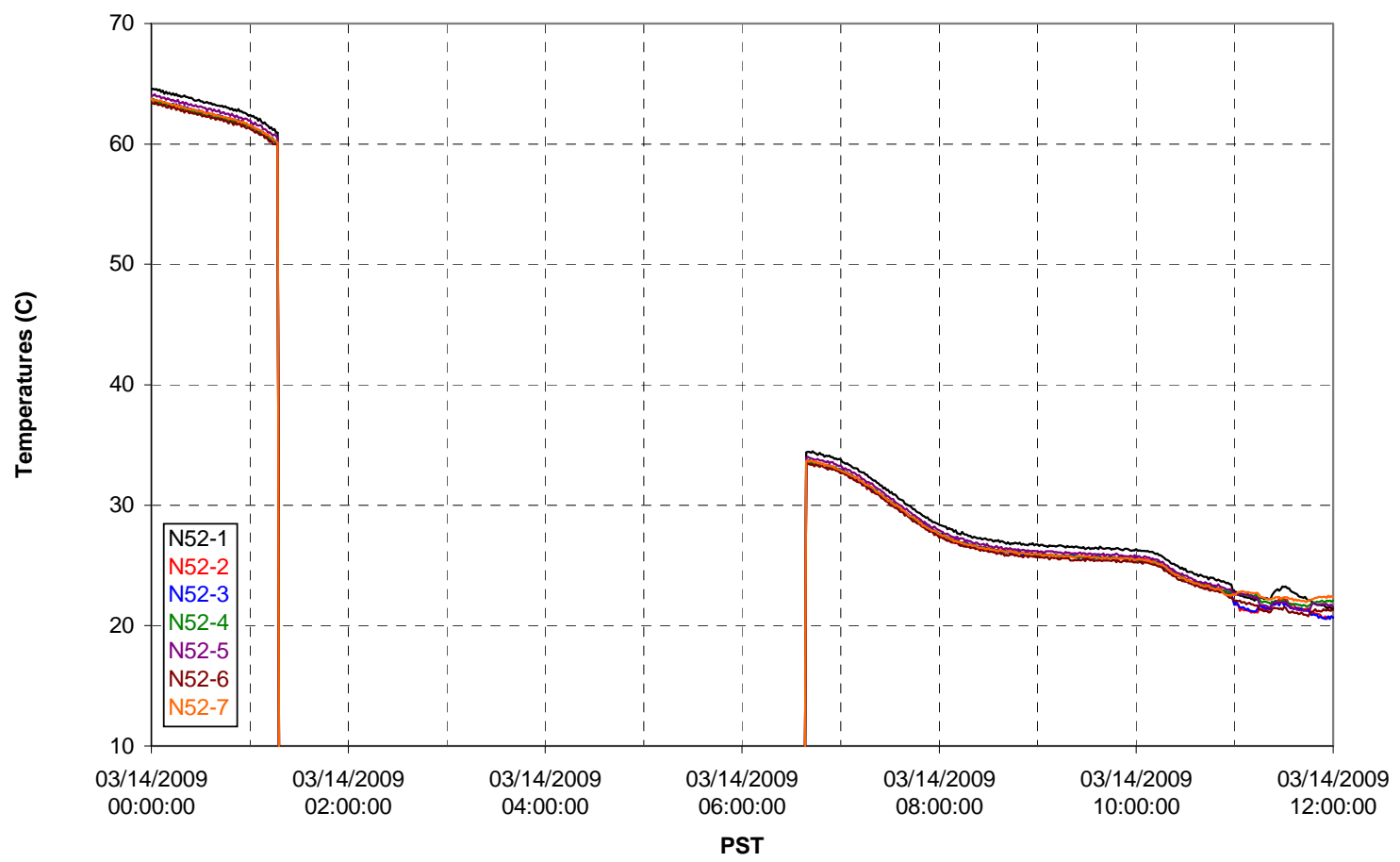


T02A Heating and Cooling

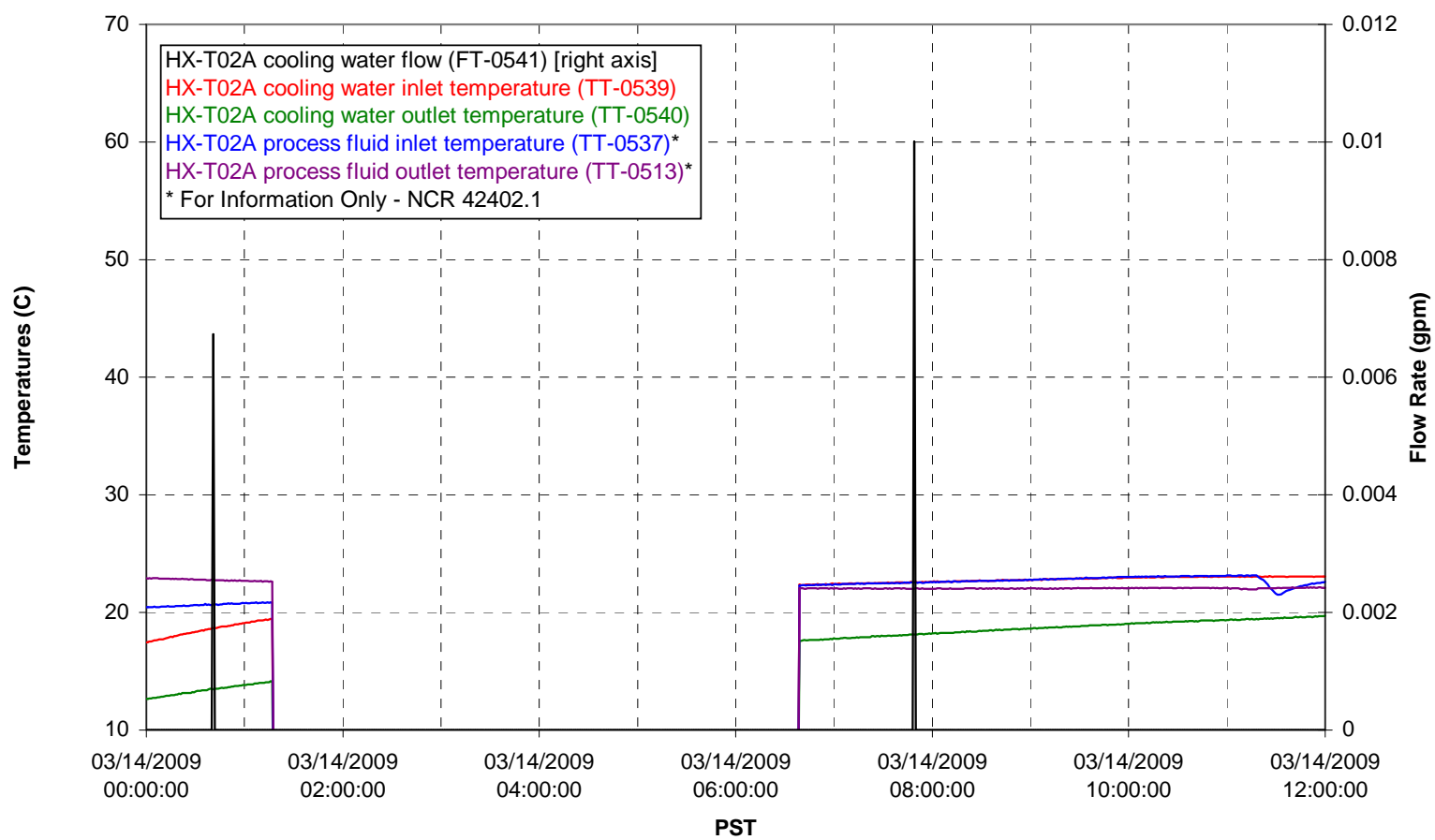

Pump Operation

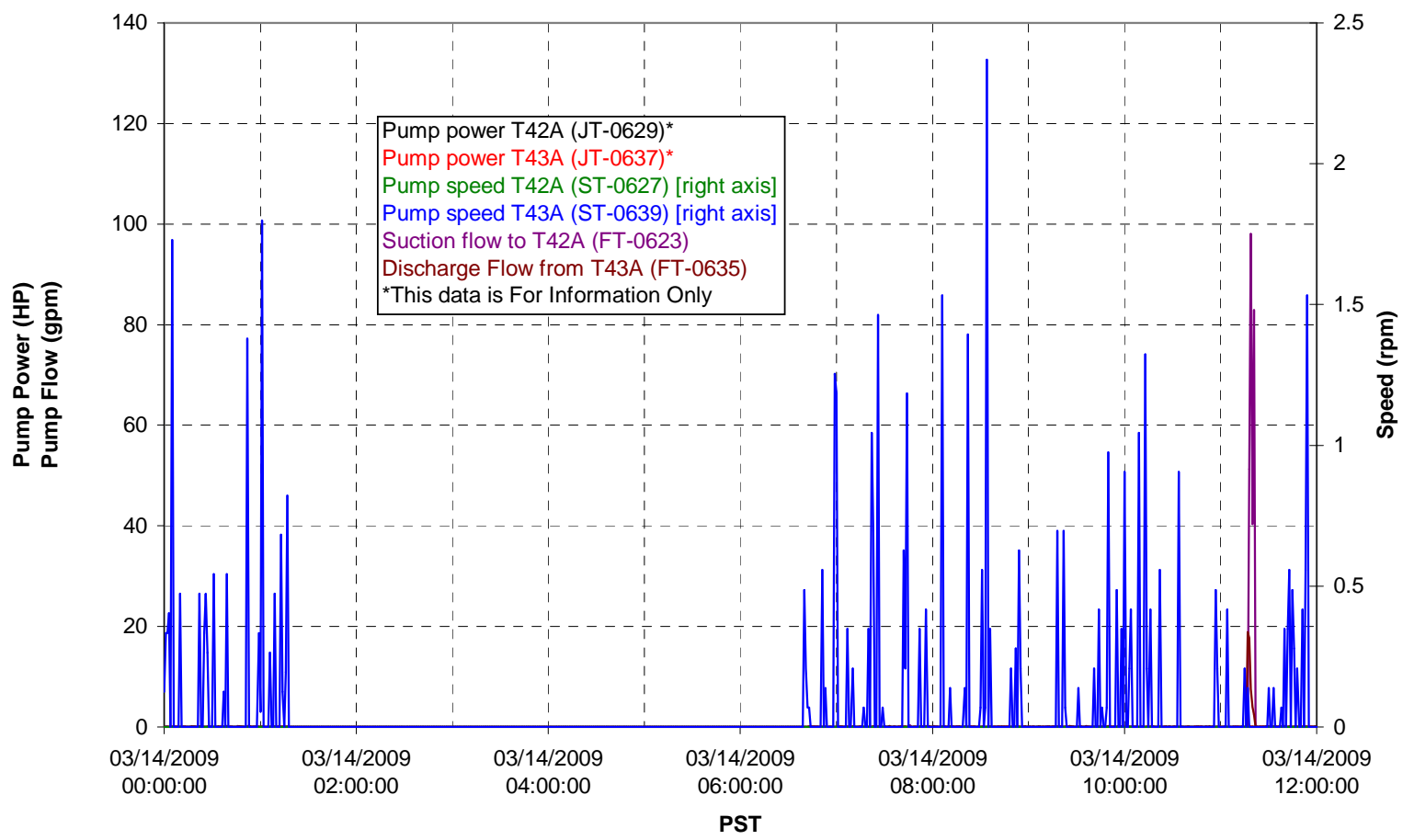


Pulsepot UFP-PP-T01A

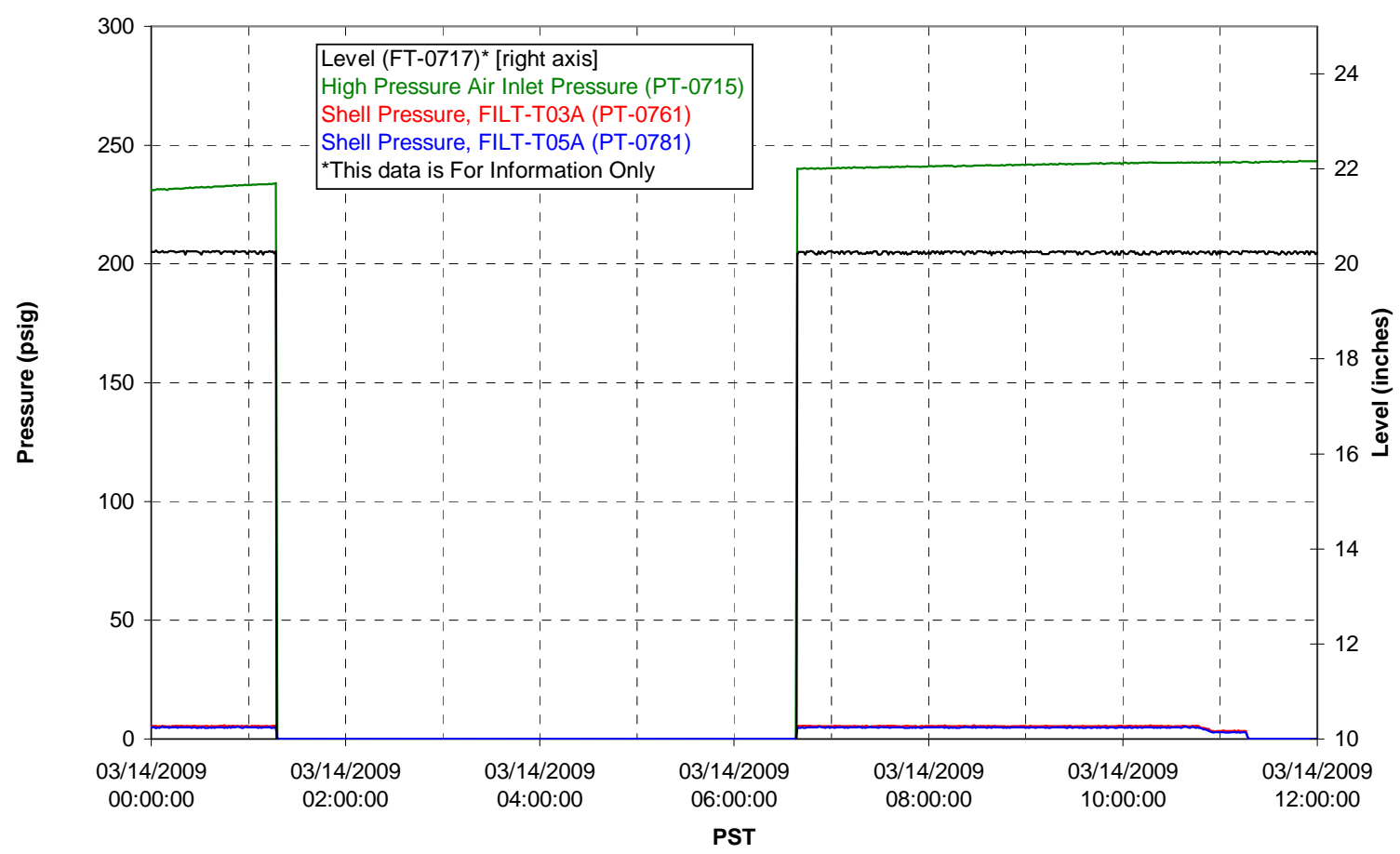

Pulsepot UFP-PP-T02A

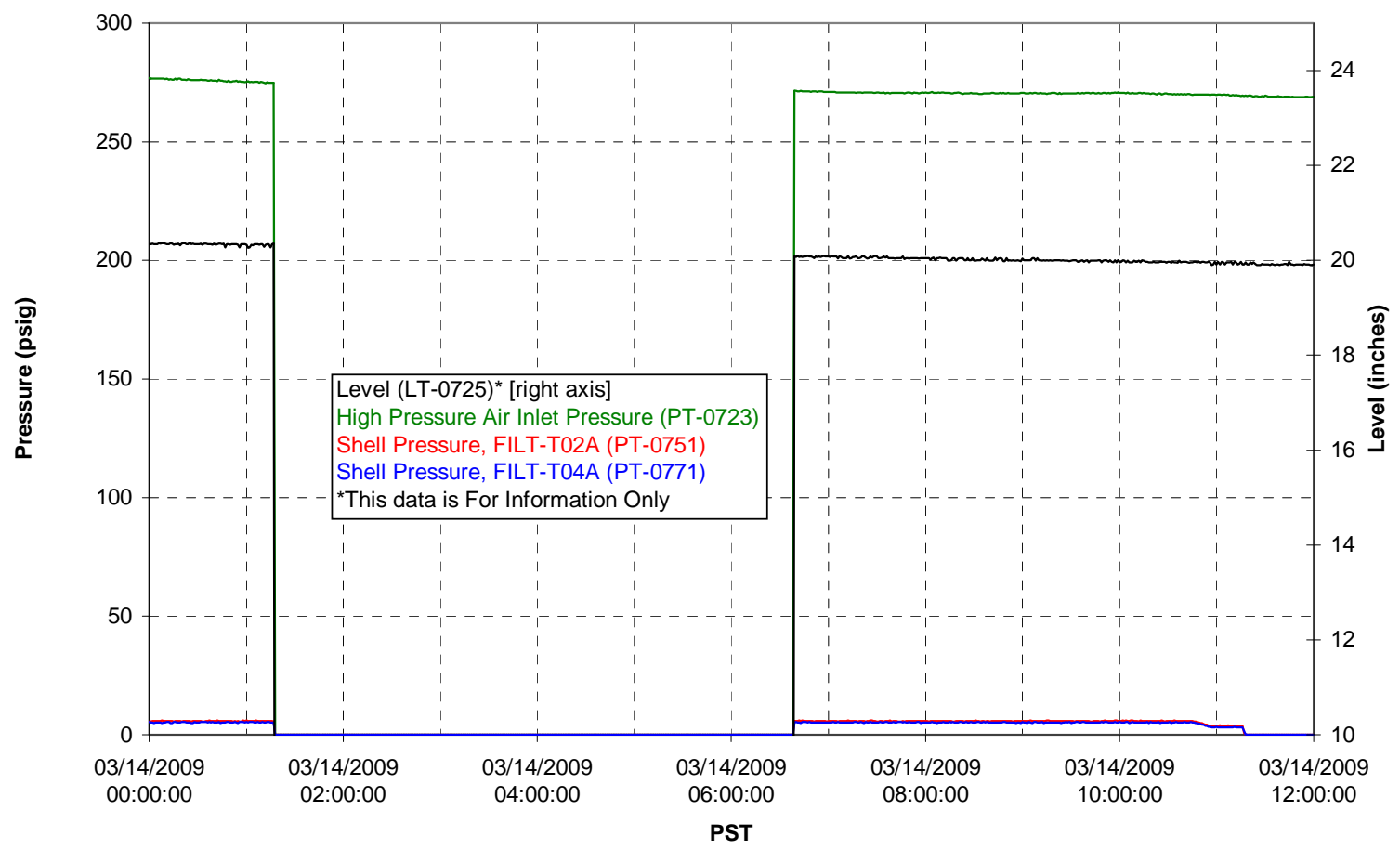


Pulsepot UFP-PP-T03A

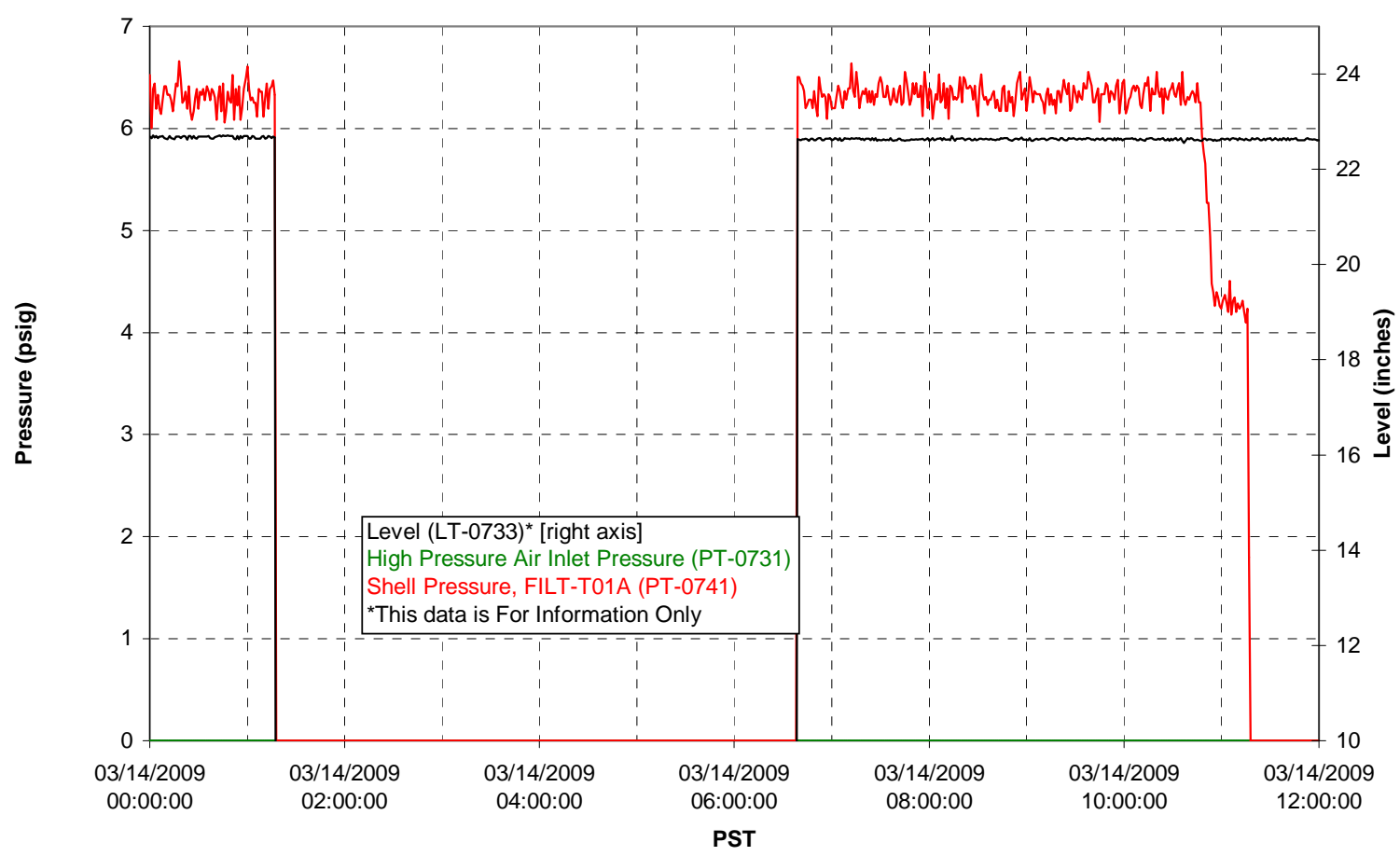

Pulsepot Levels

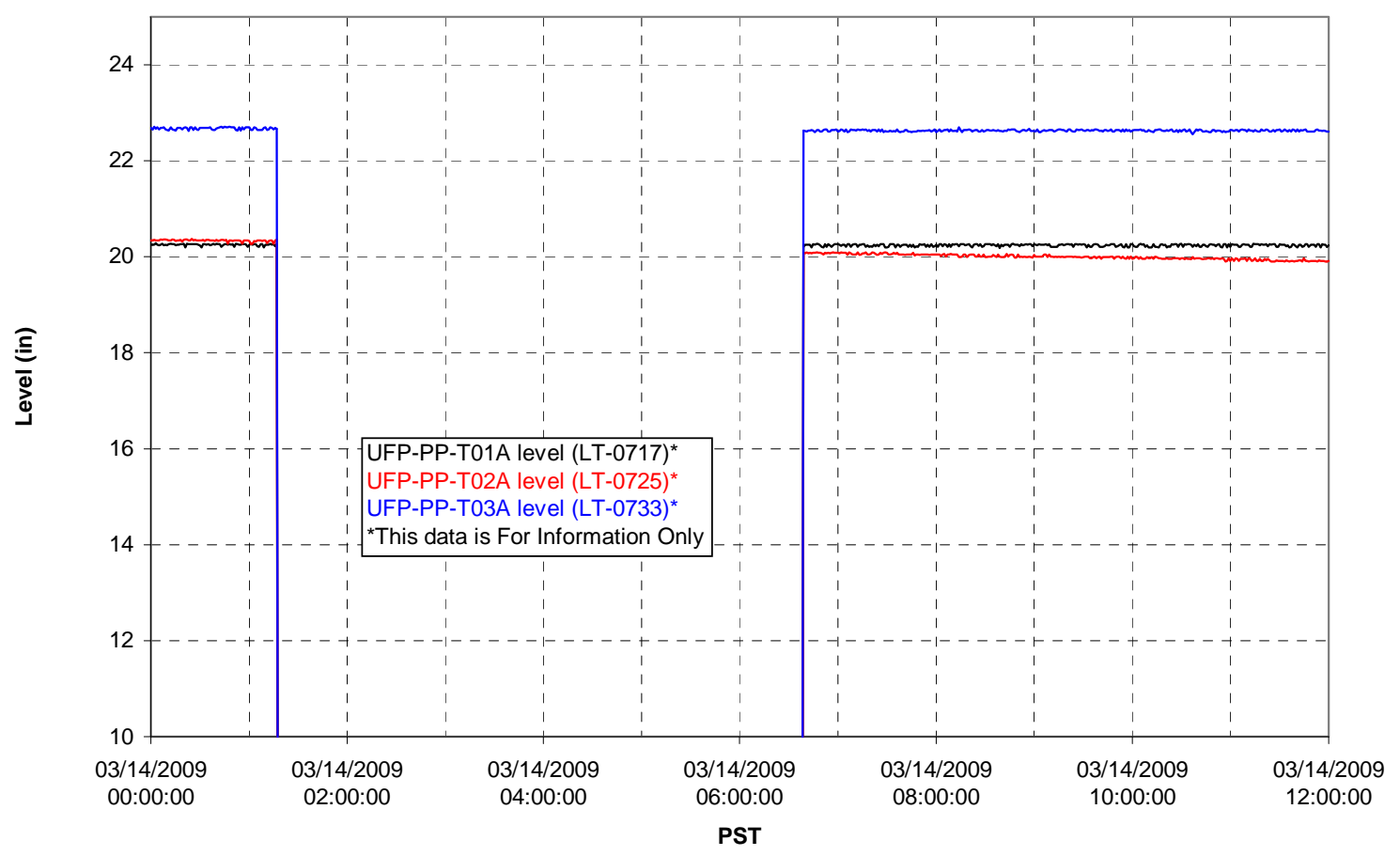


Filter UFP-FILT-T01A

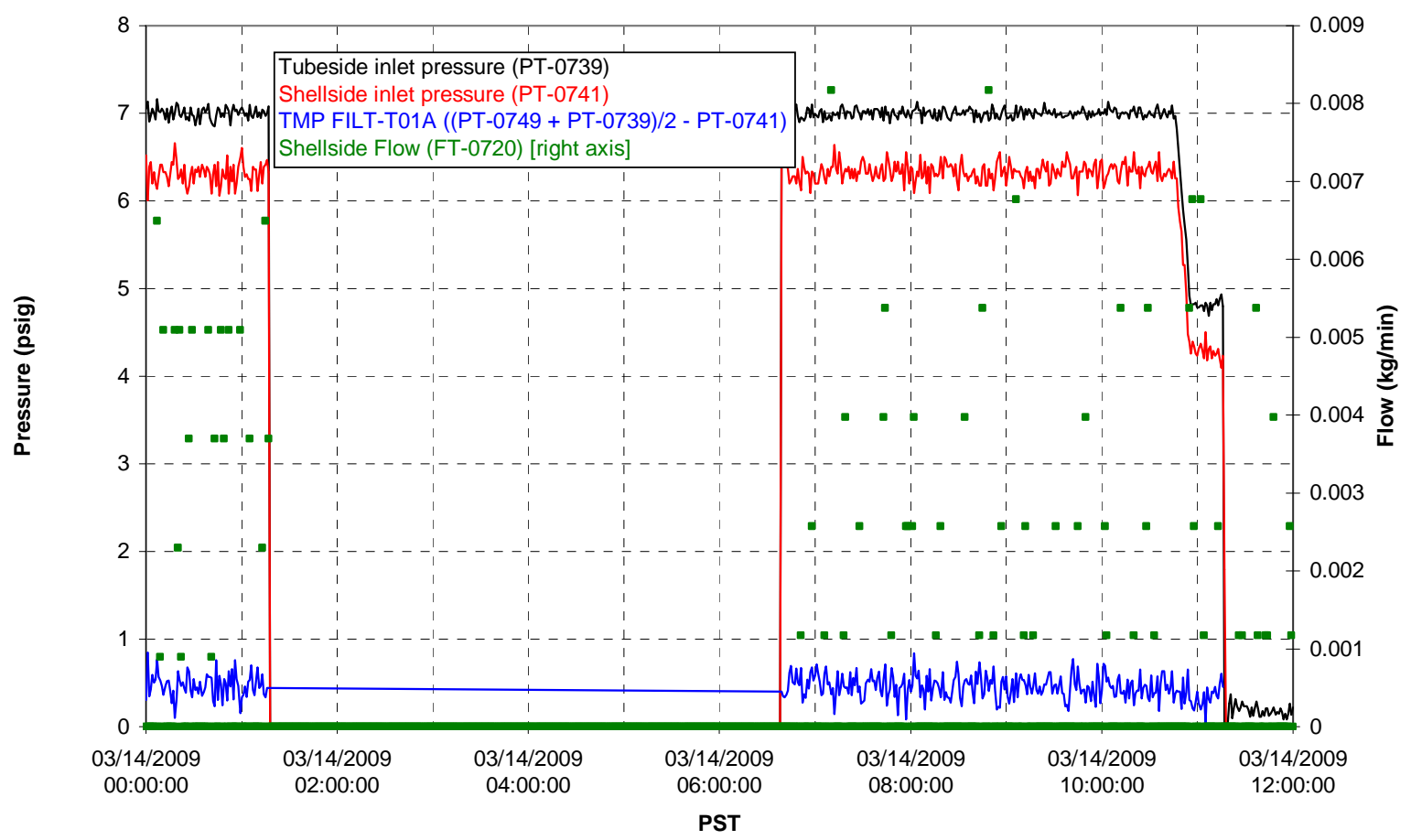

Filter UFP-FILT-T02A

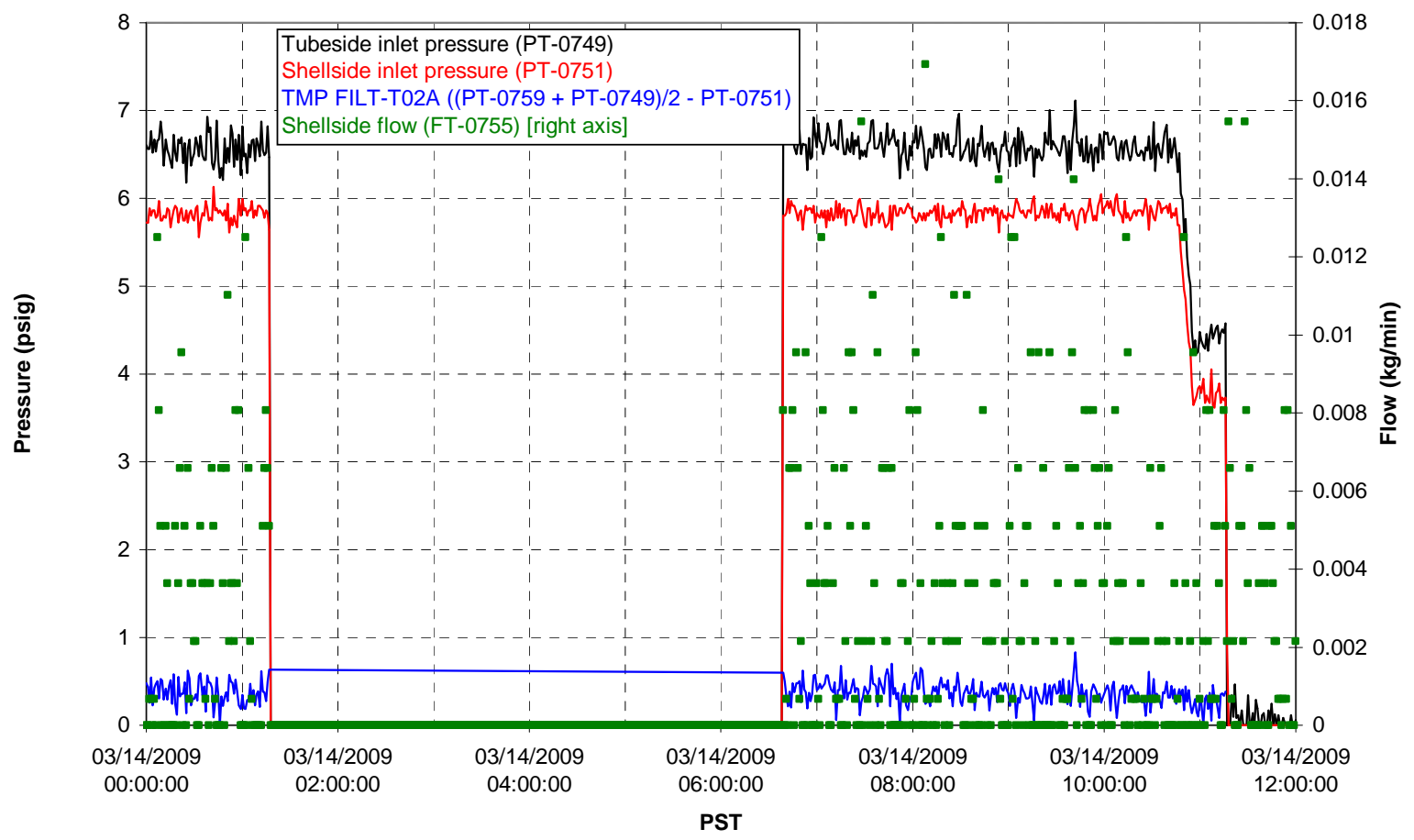


Filter UFP-FILT-T03A

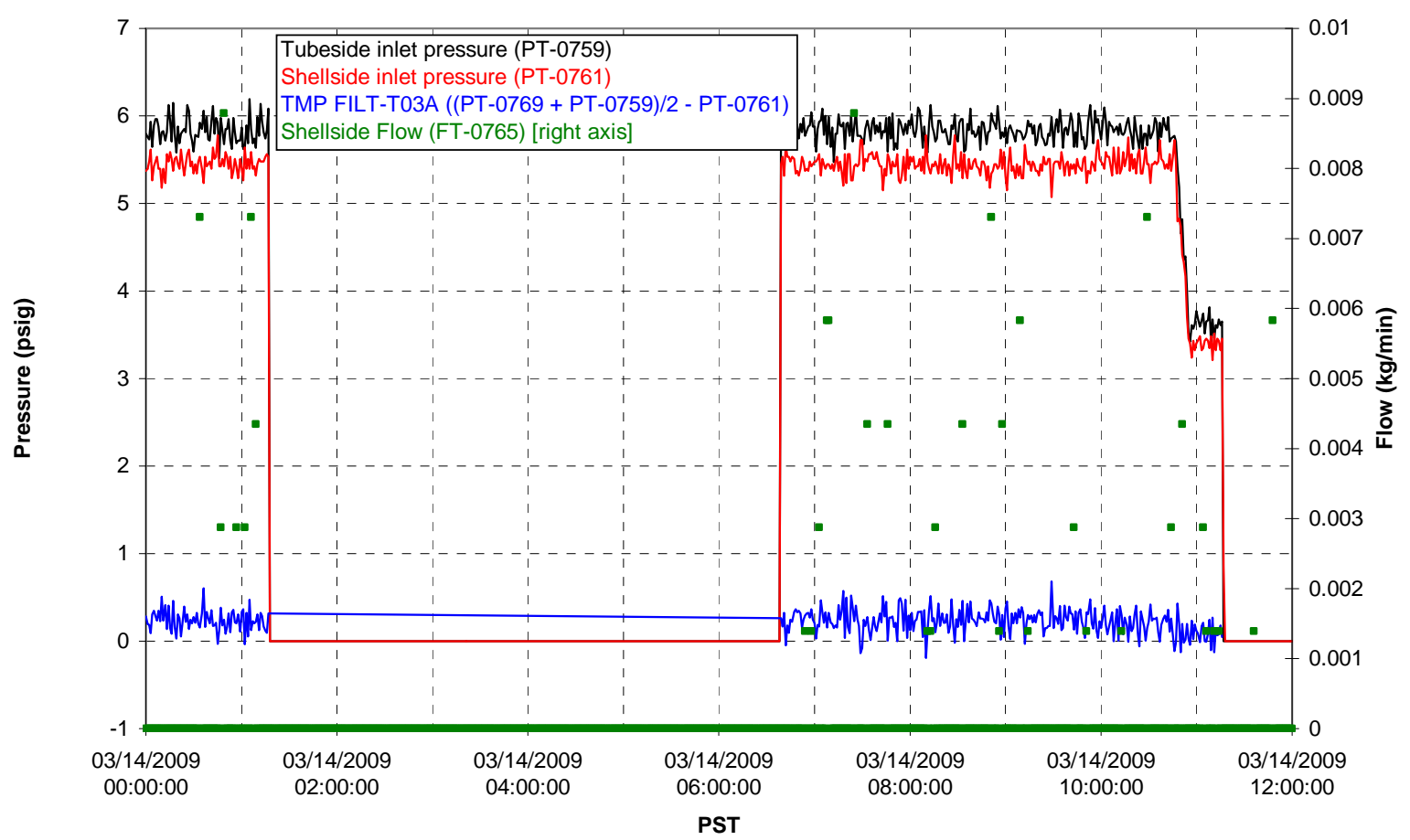

Filter UFP-FILT-T04A

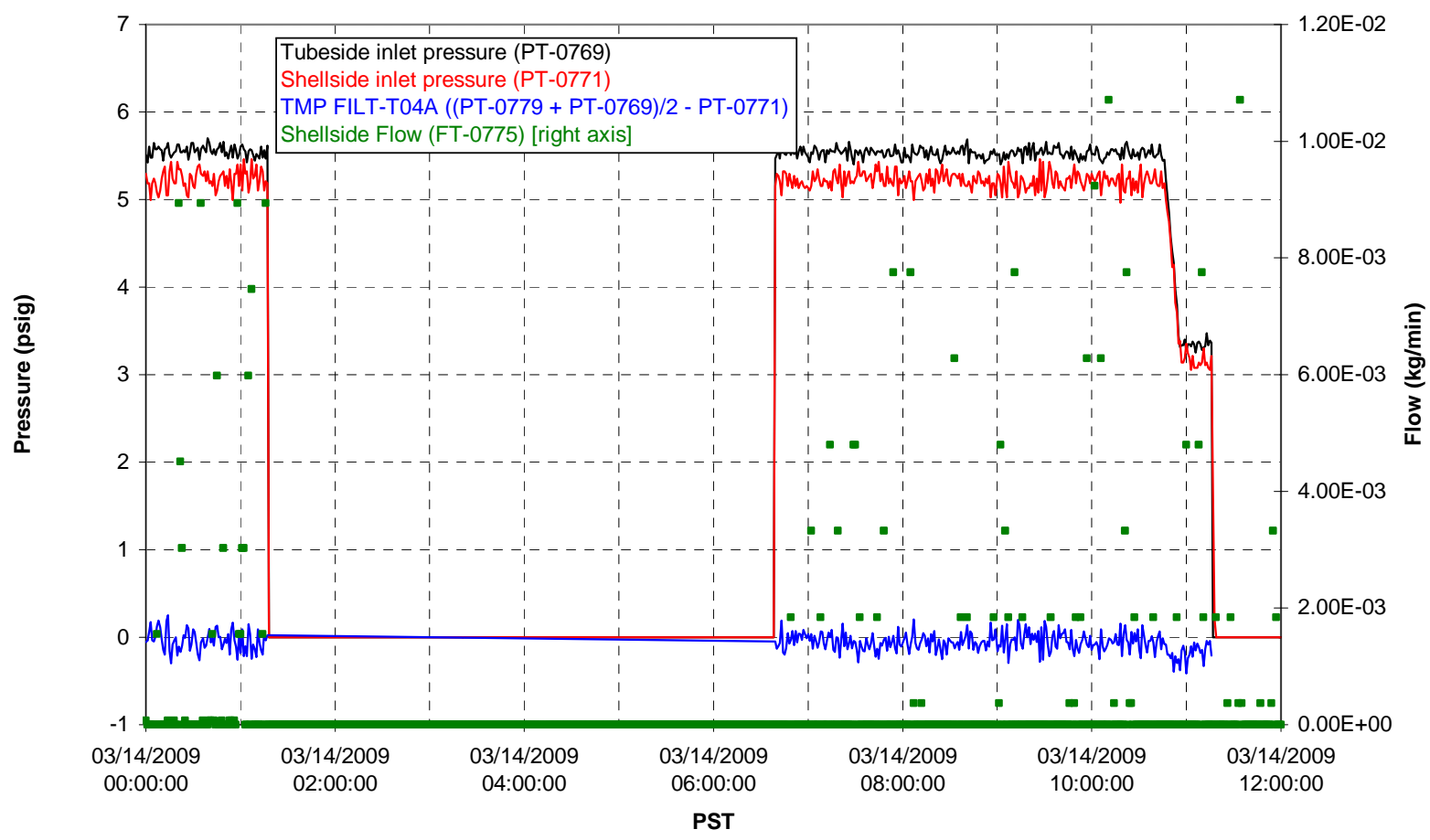


Filter UFP-FILT-T05A

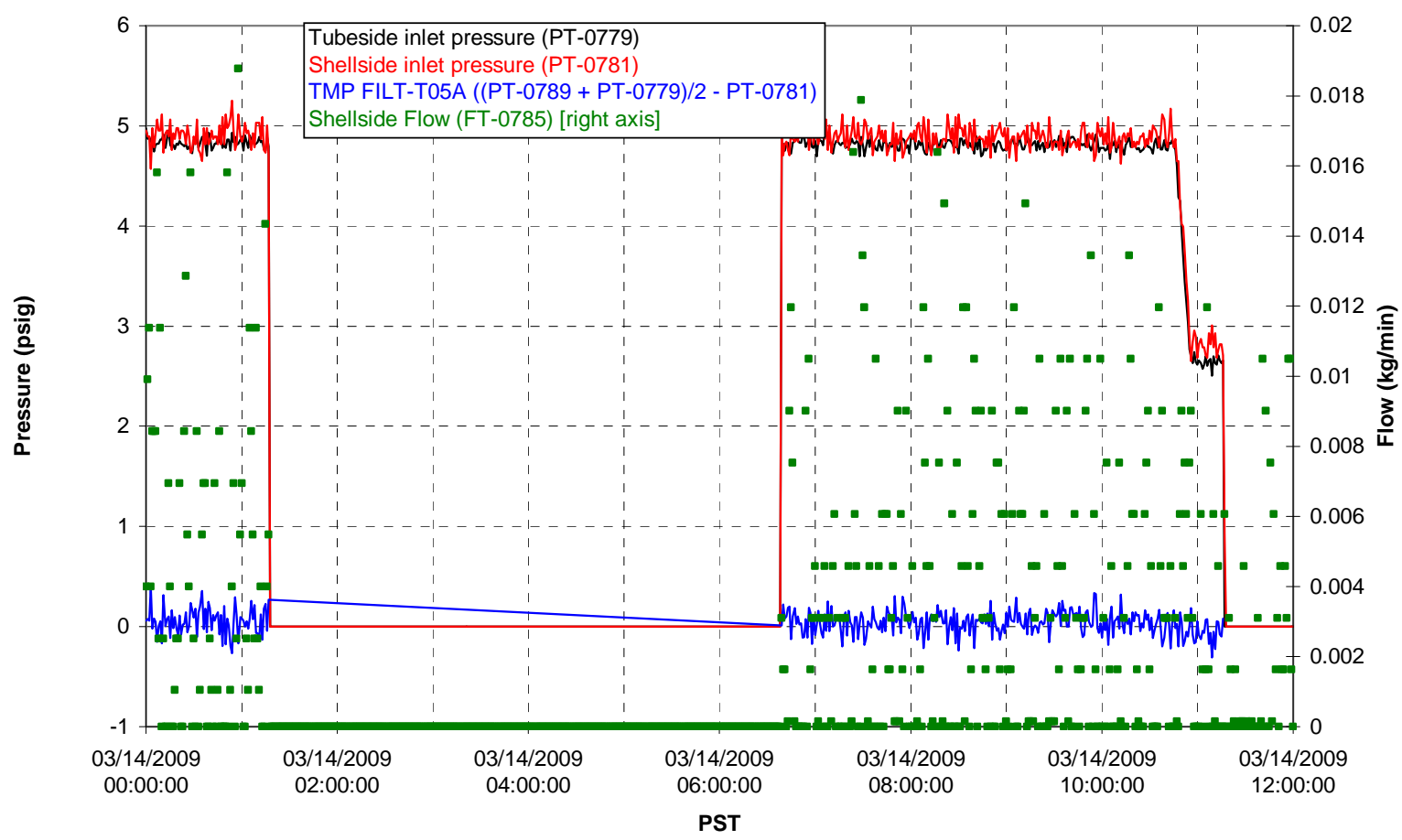

Chemical Flow

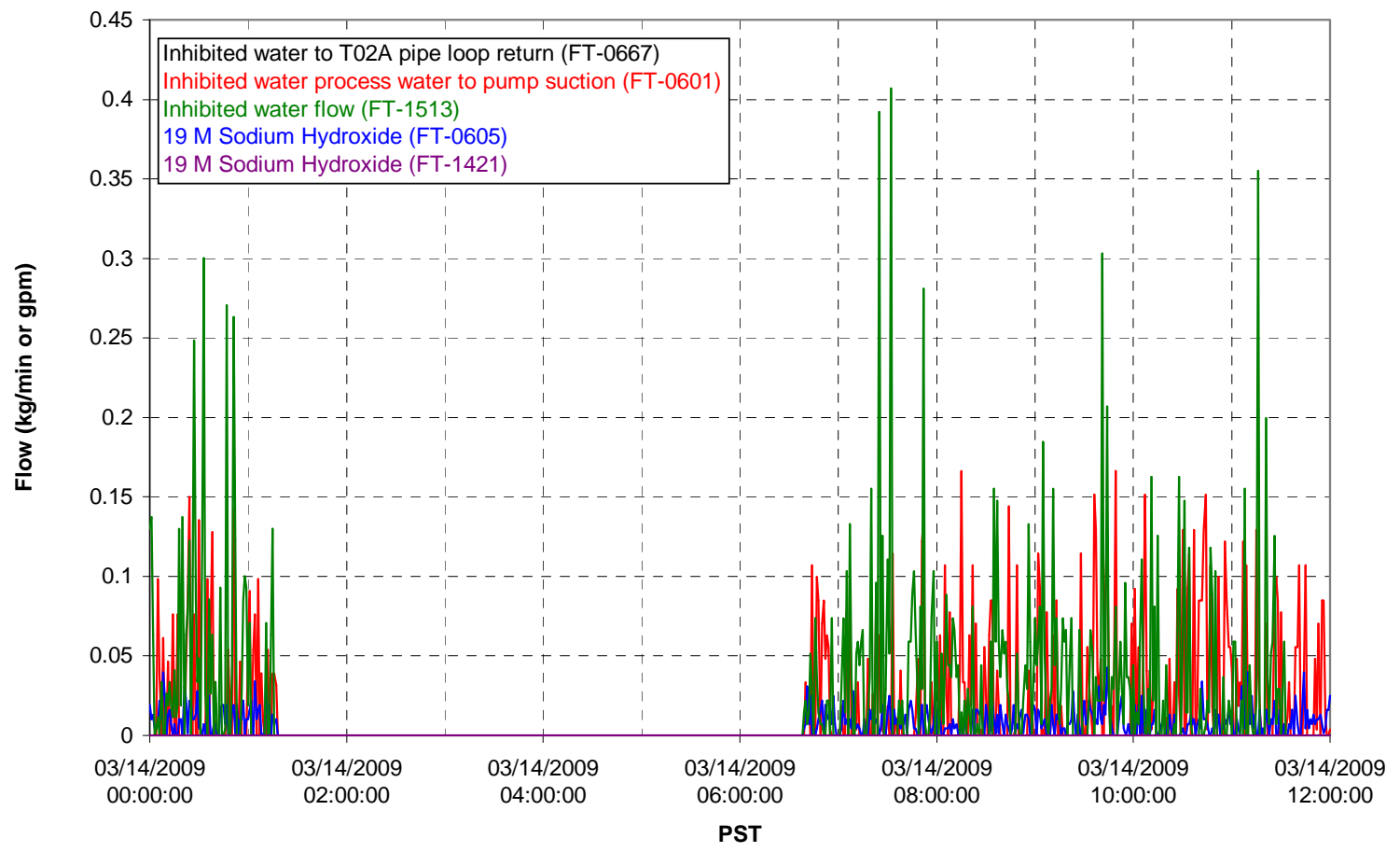




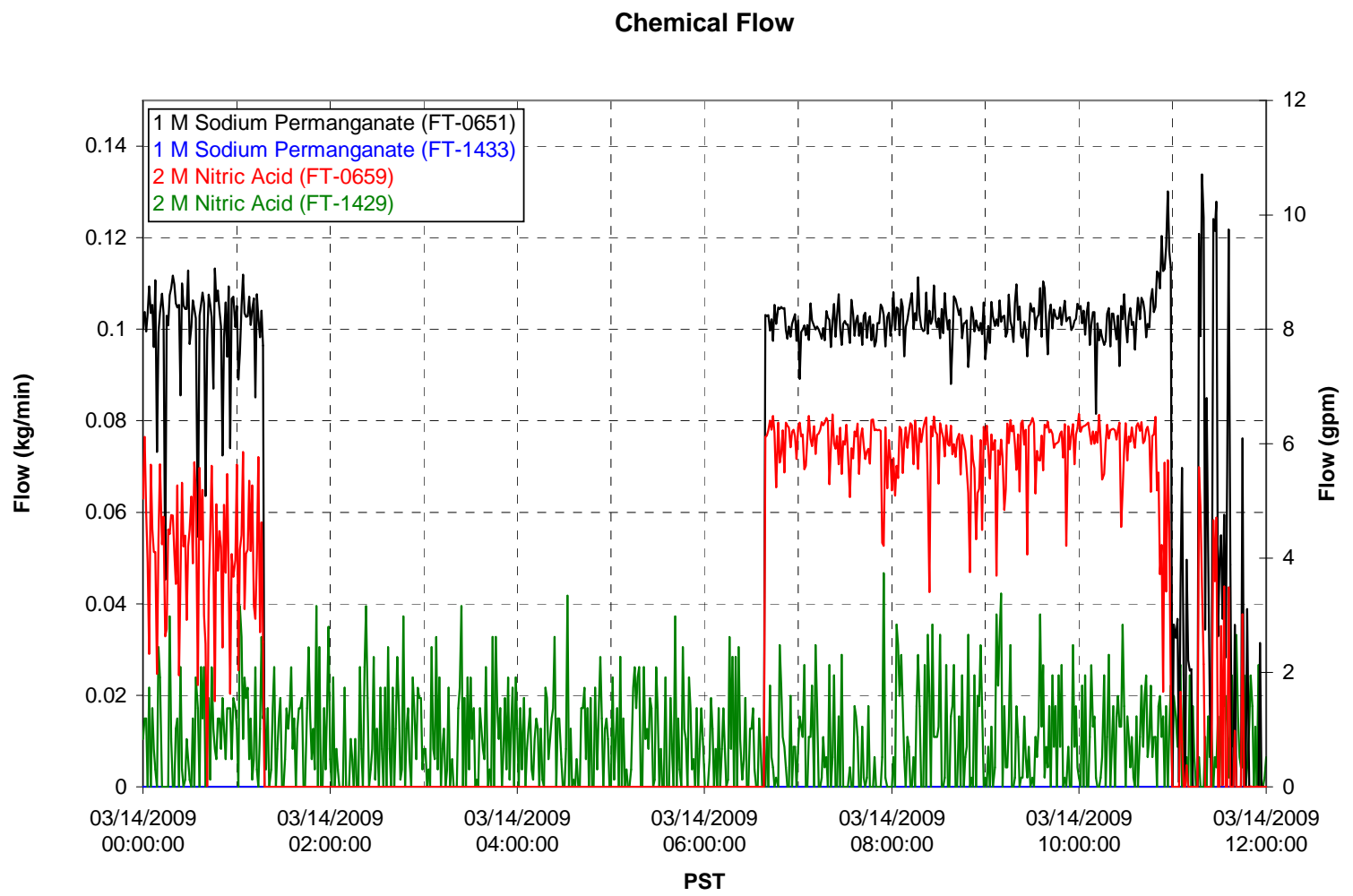

Air Flows

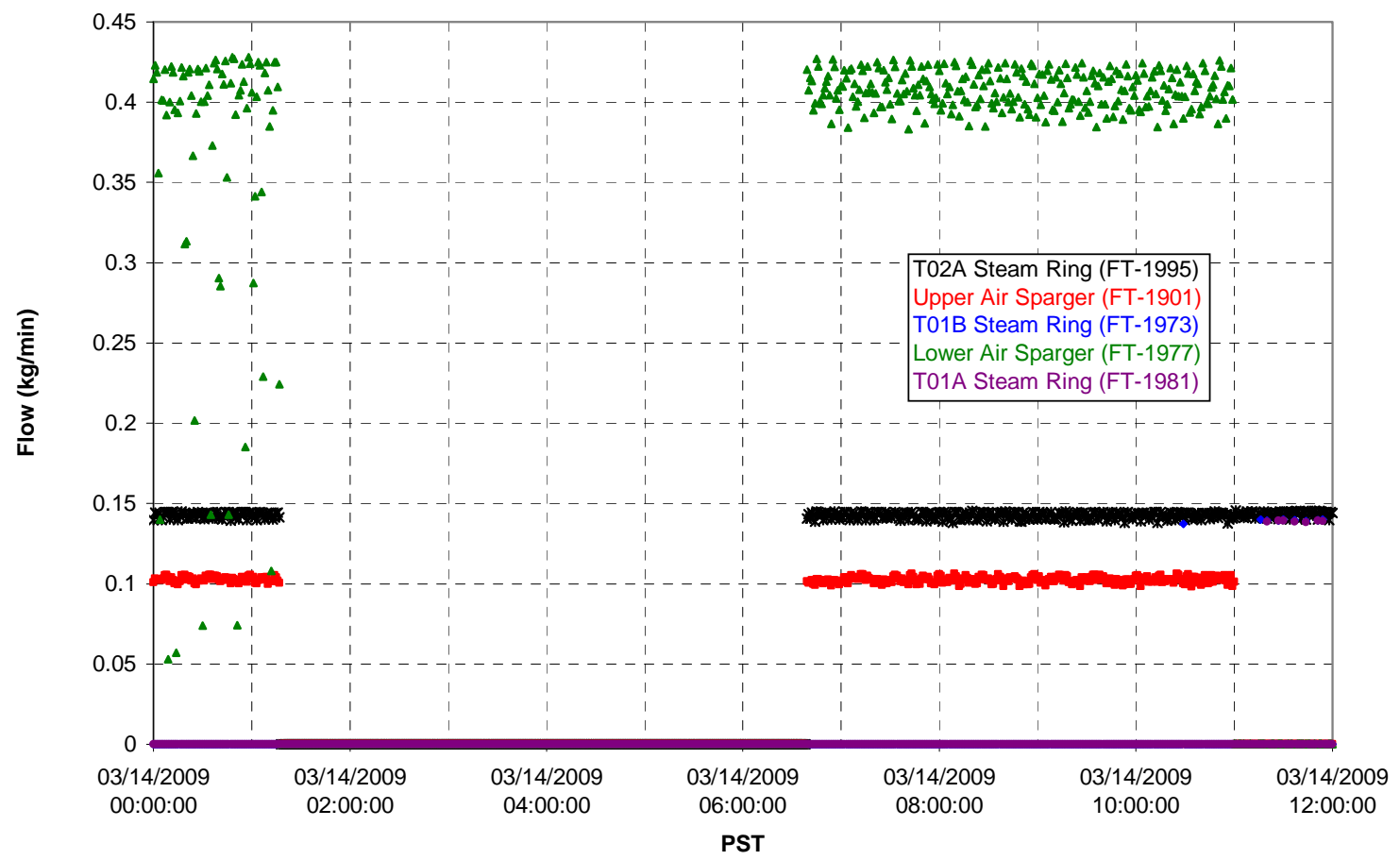


T02A Steam

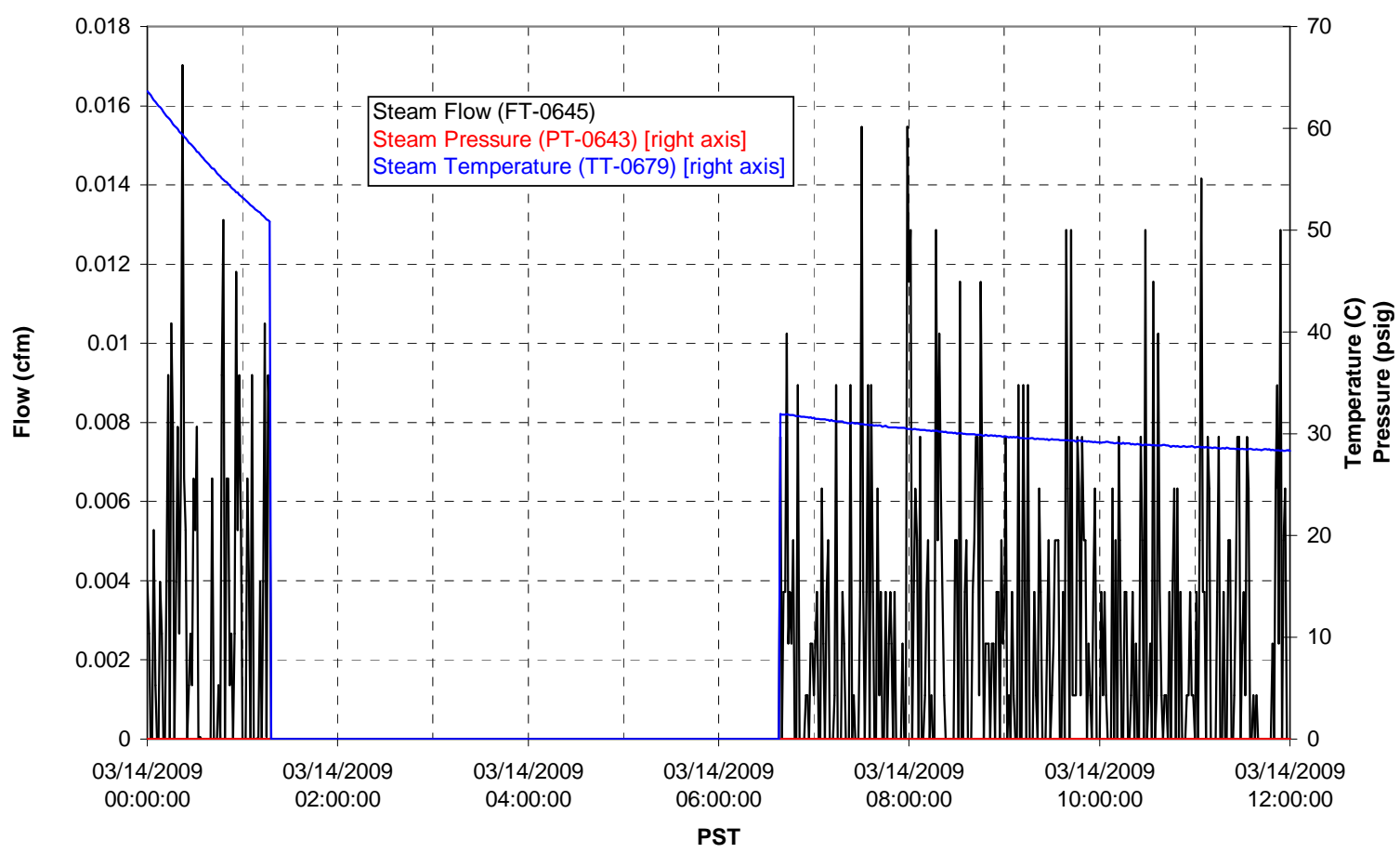

T01A Steam

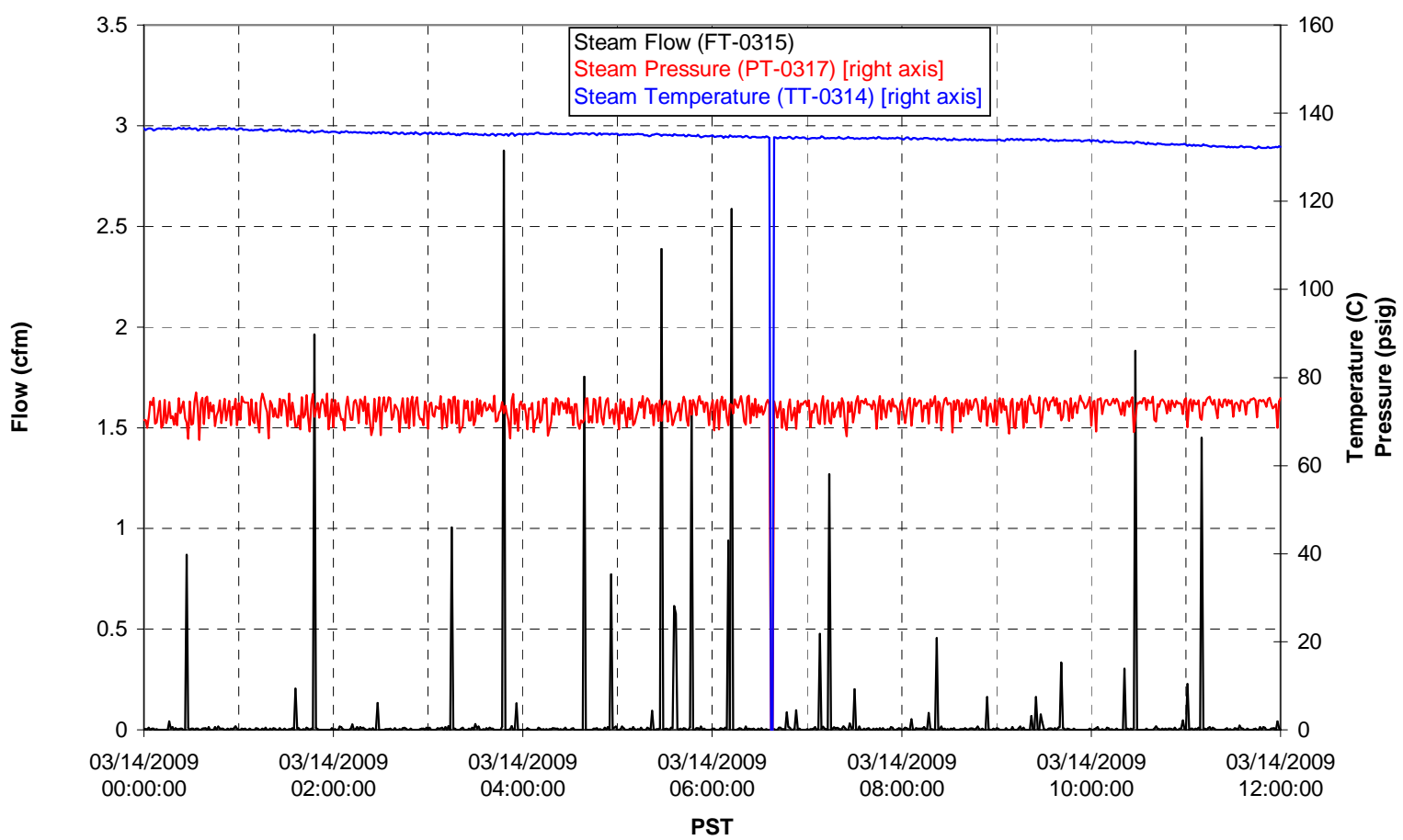


T01B Steam

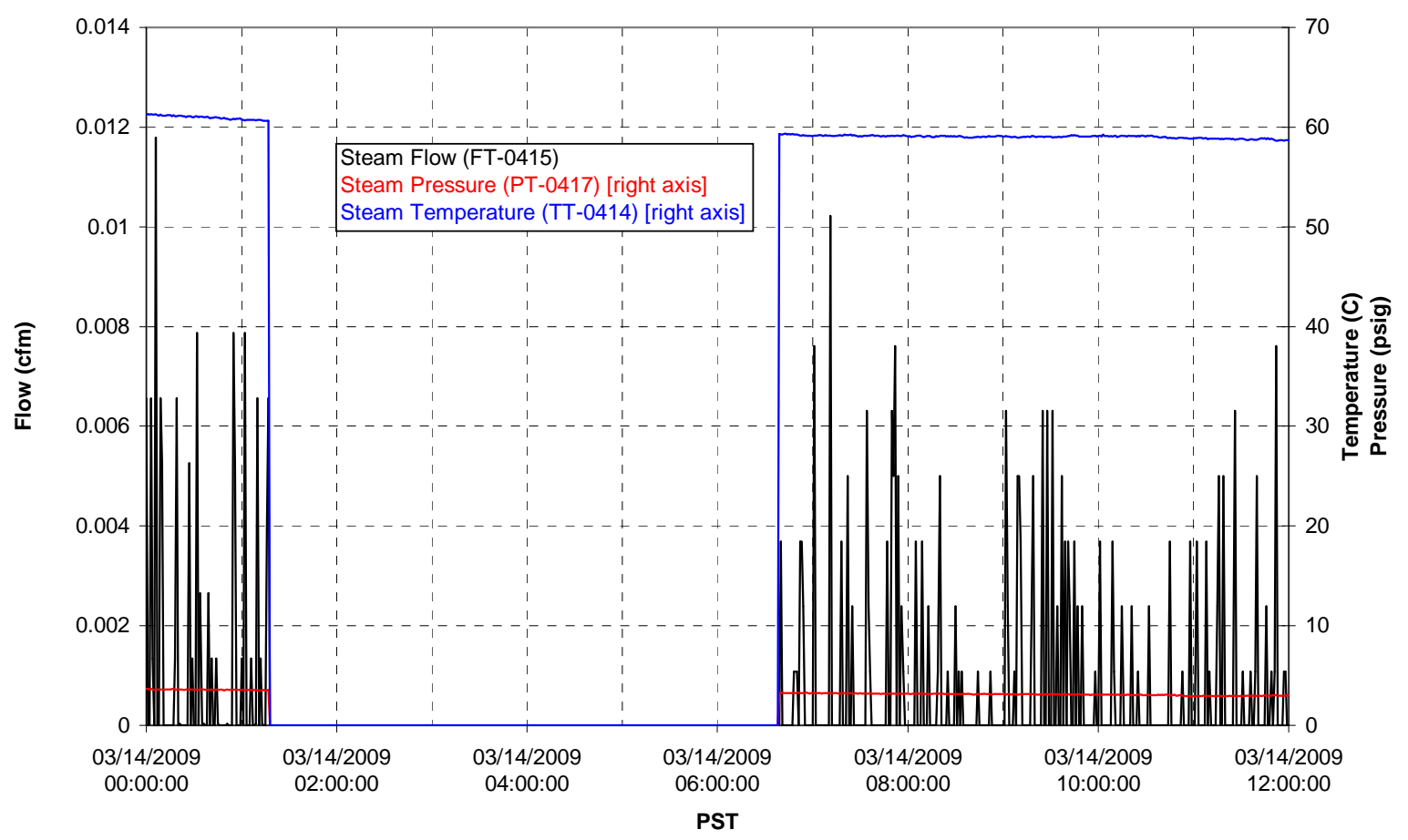


Integrated Test B Data Plots 03/14/09 12:00 - 03/15/09 00:00 
T01A level

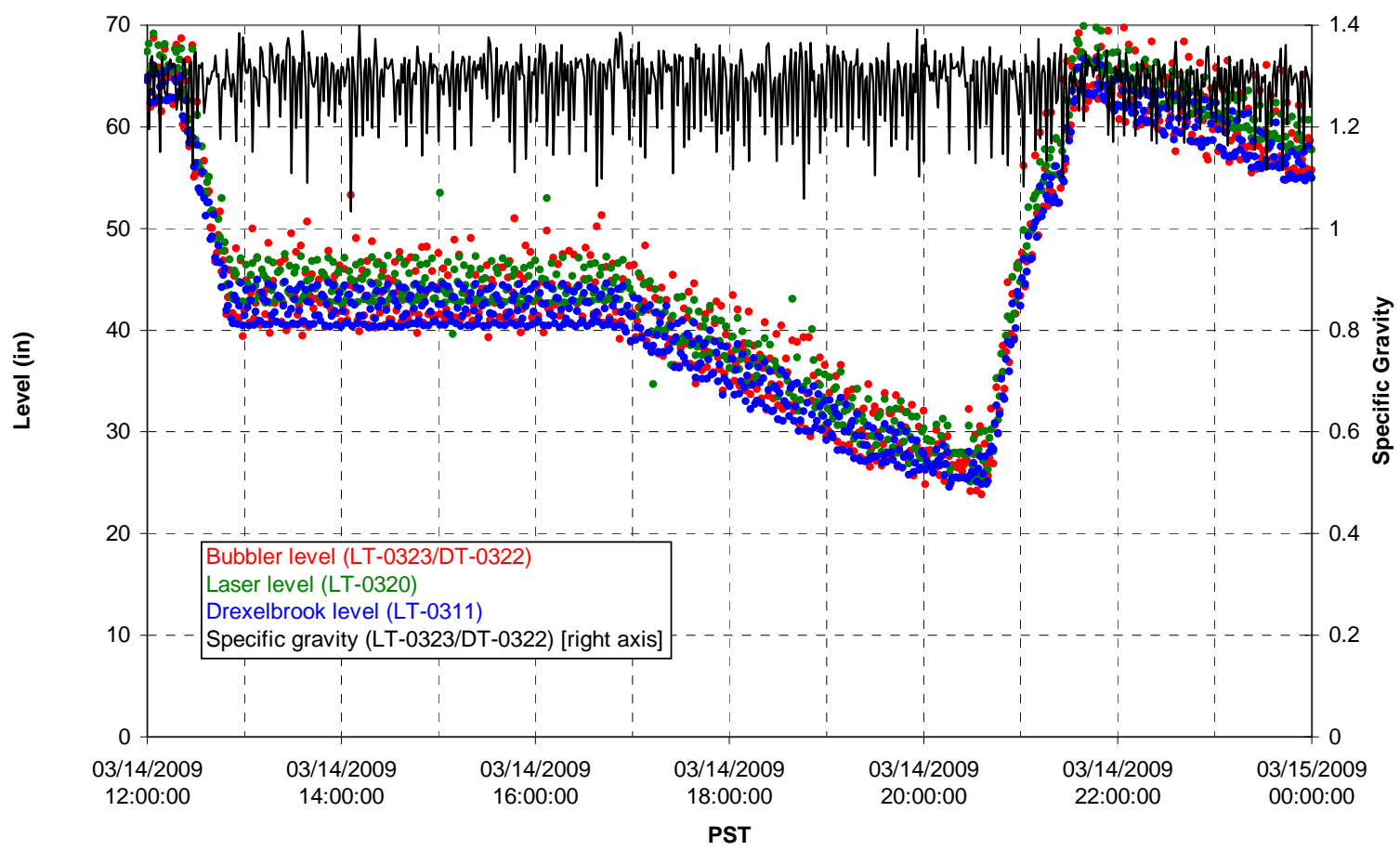

T01A temperatures

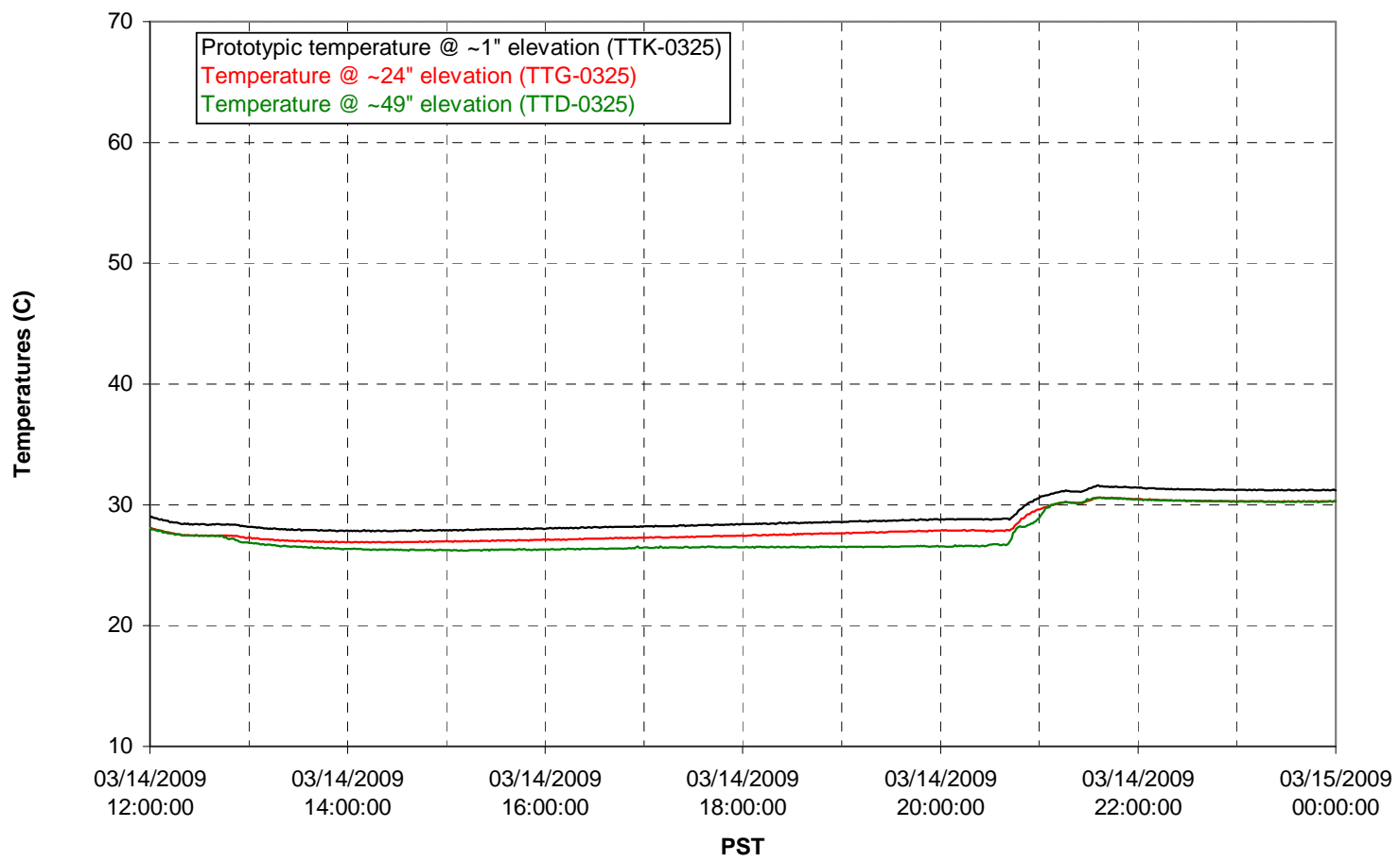


T01B level

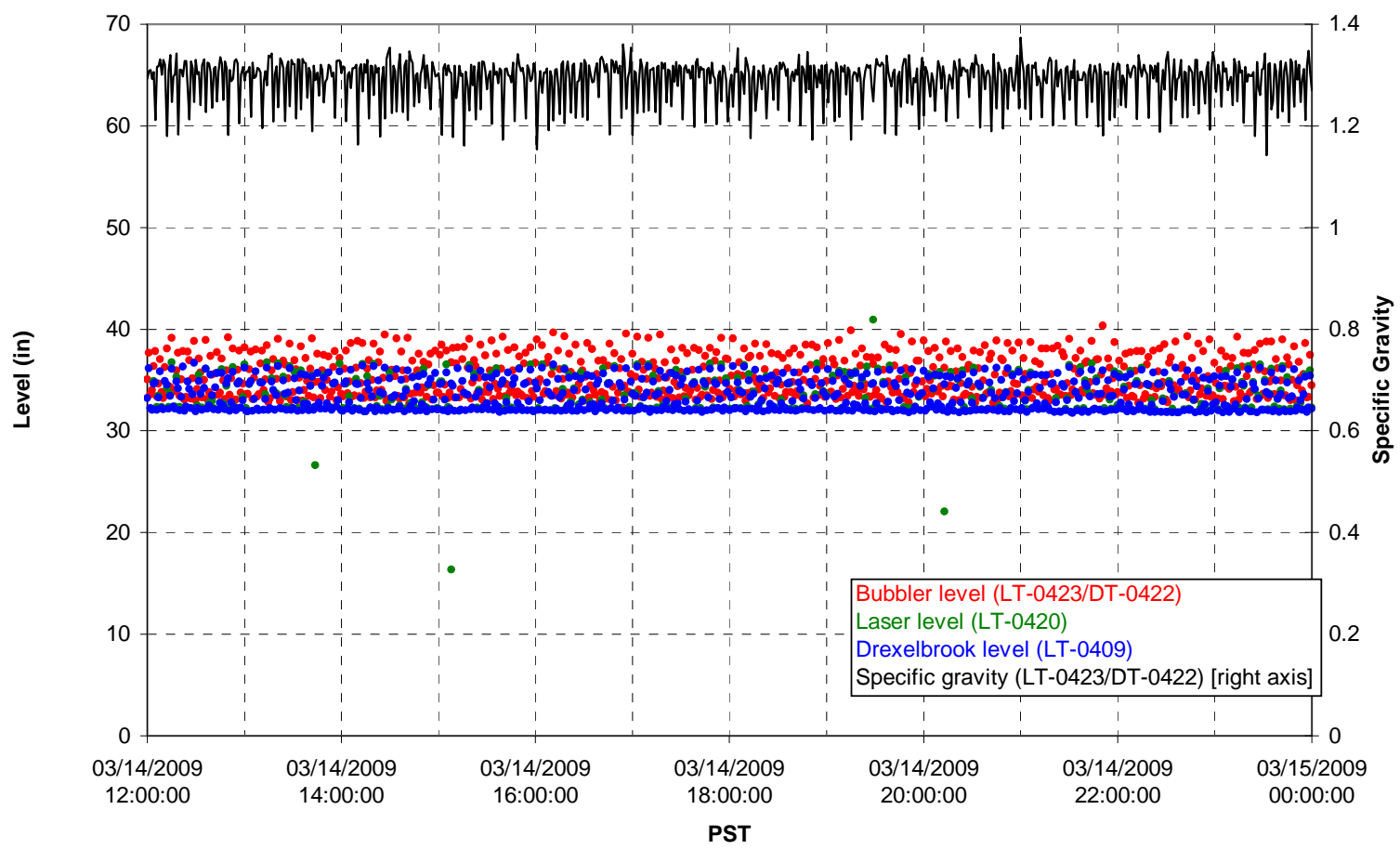

T01B temperatures

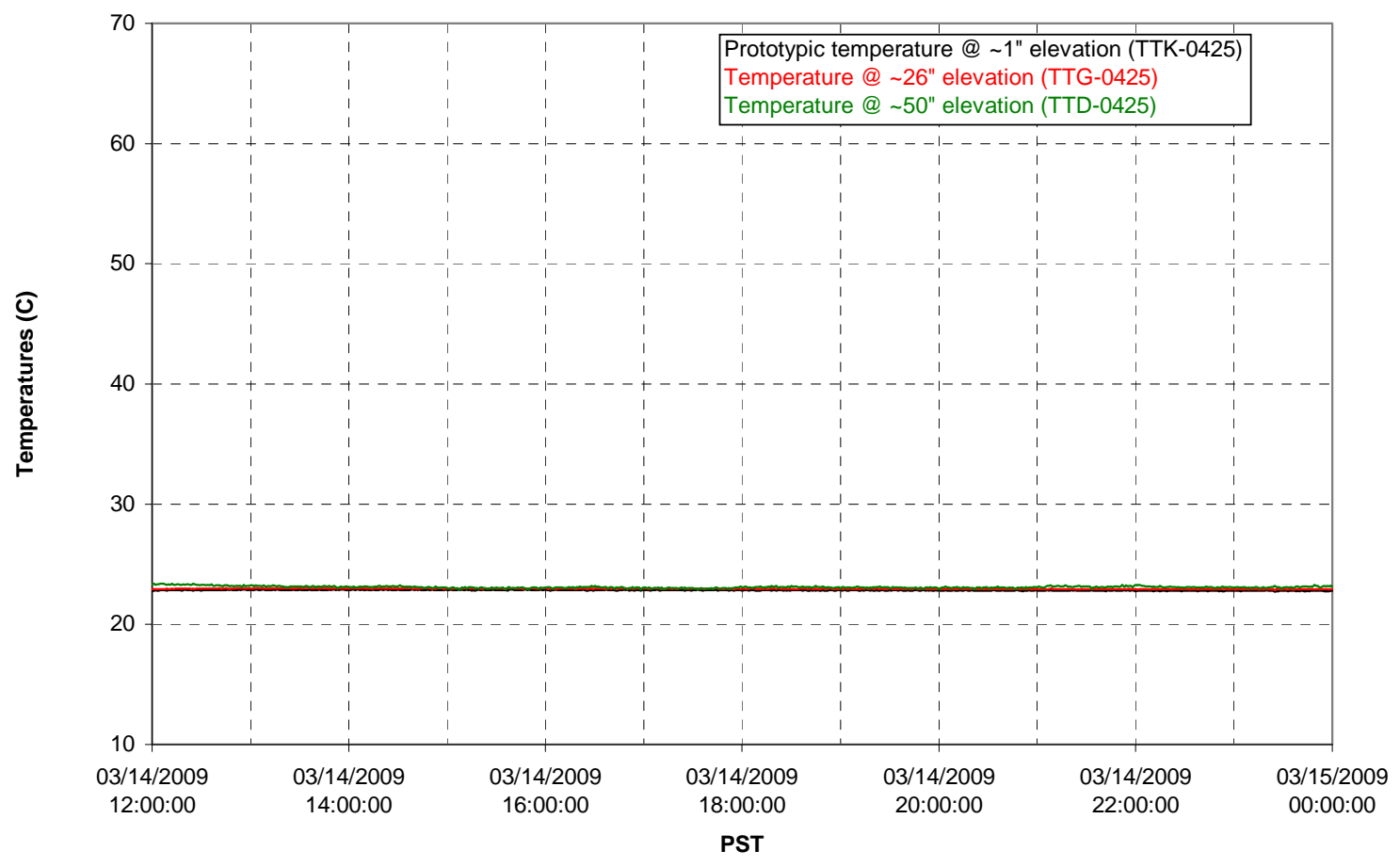


T02A level

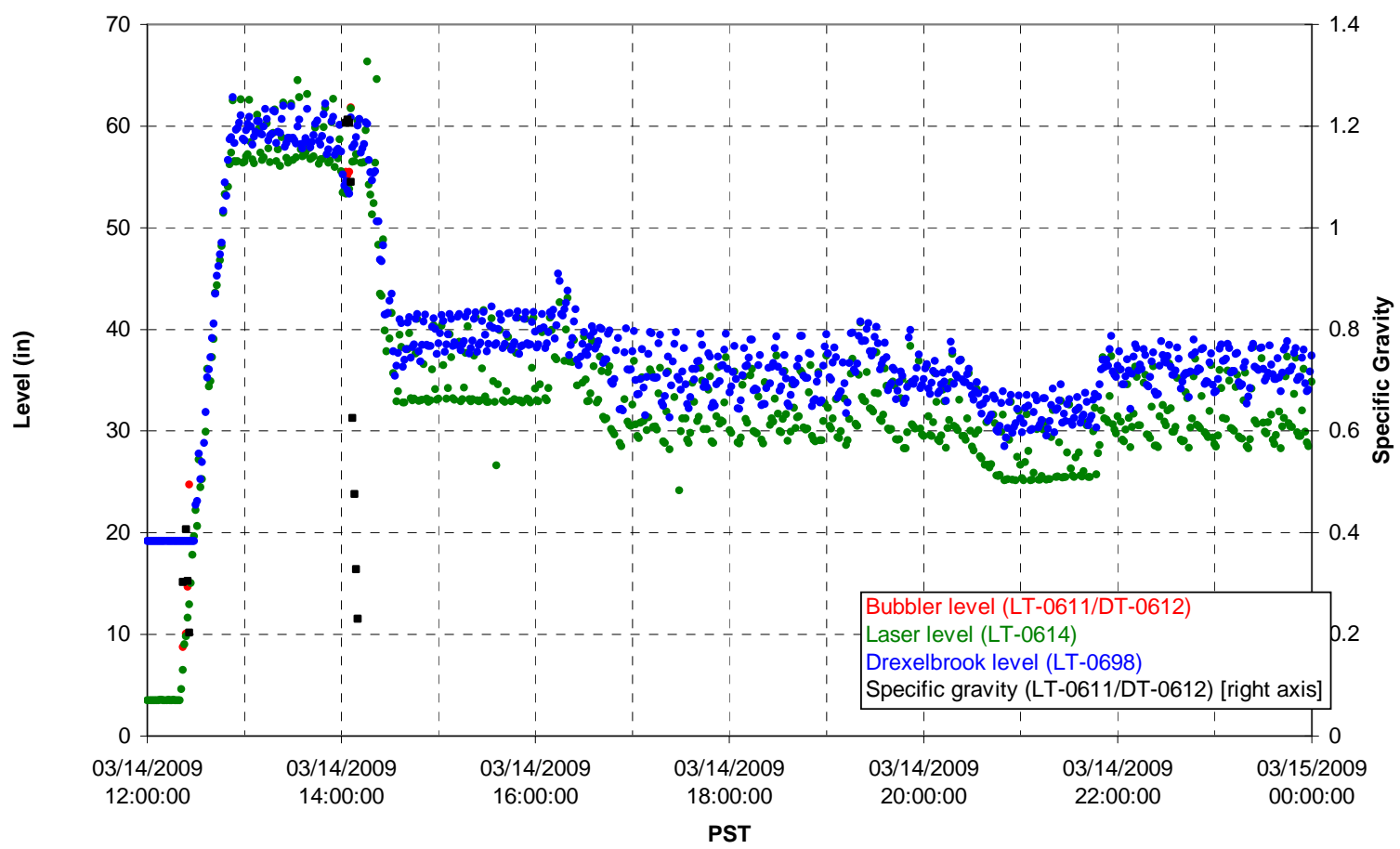

T02A temperatures

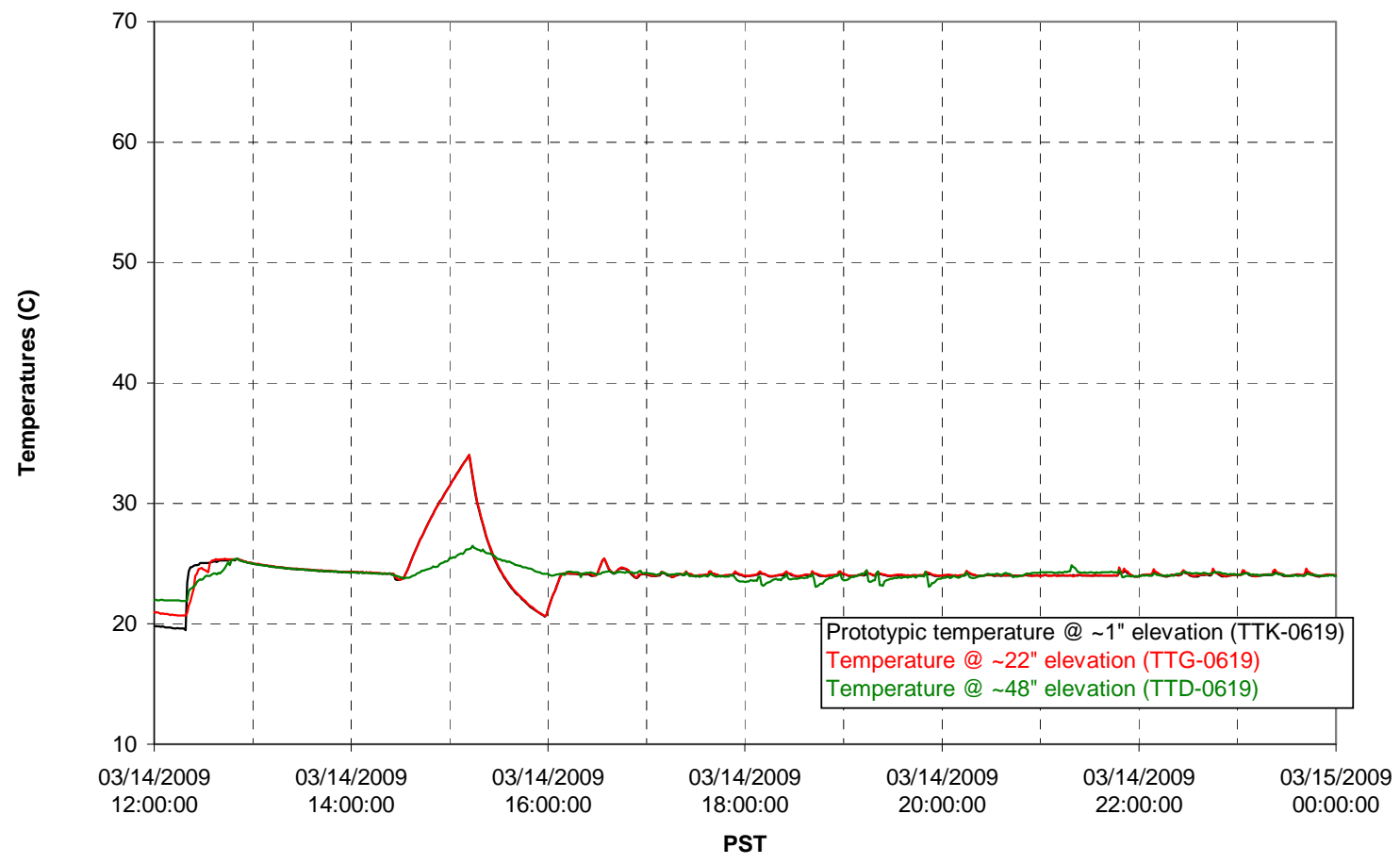


T02A and filter loop temperatures

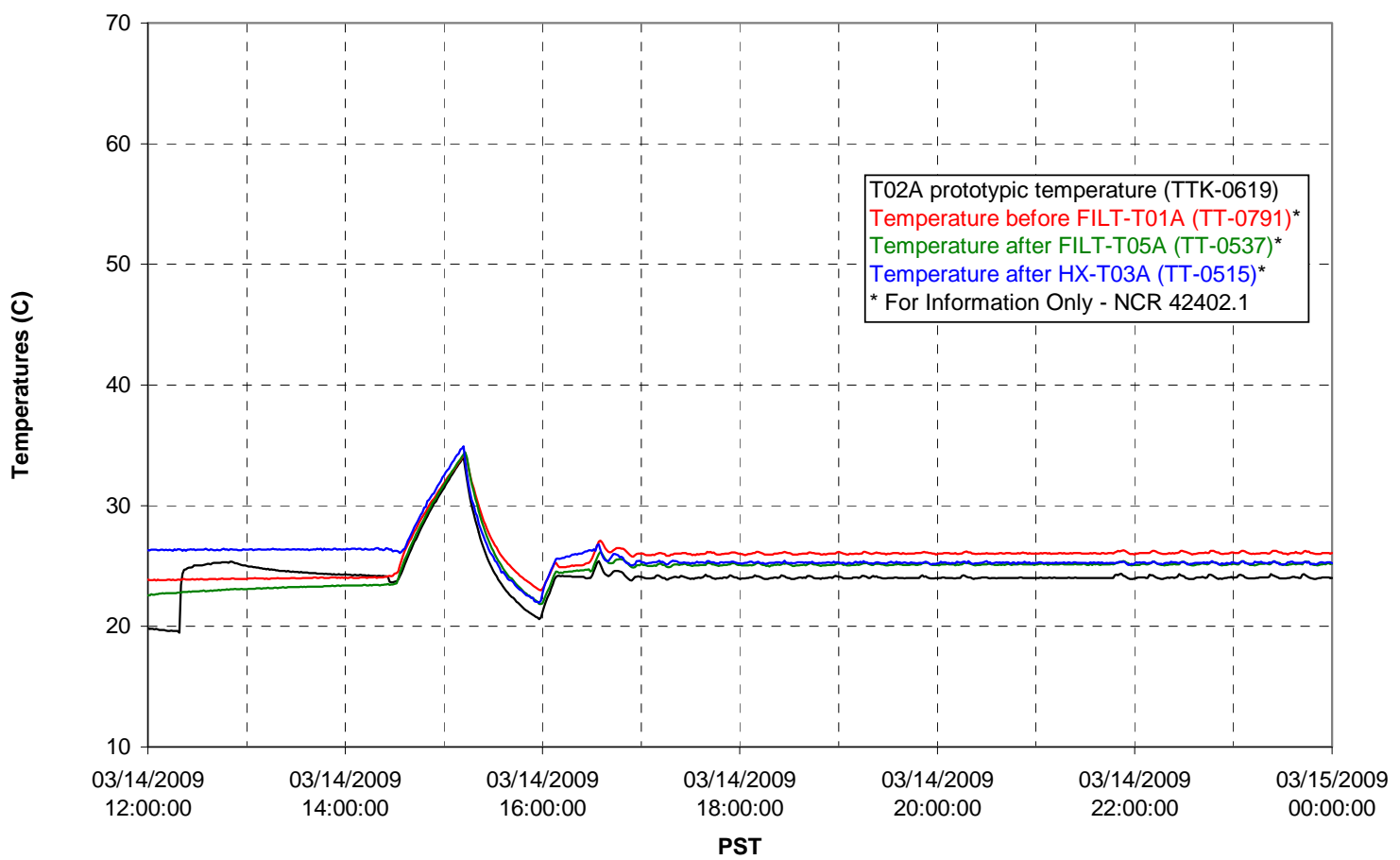

Pump Pressures and Flow

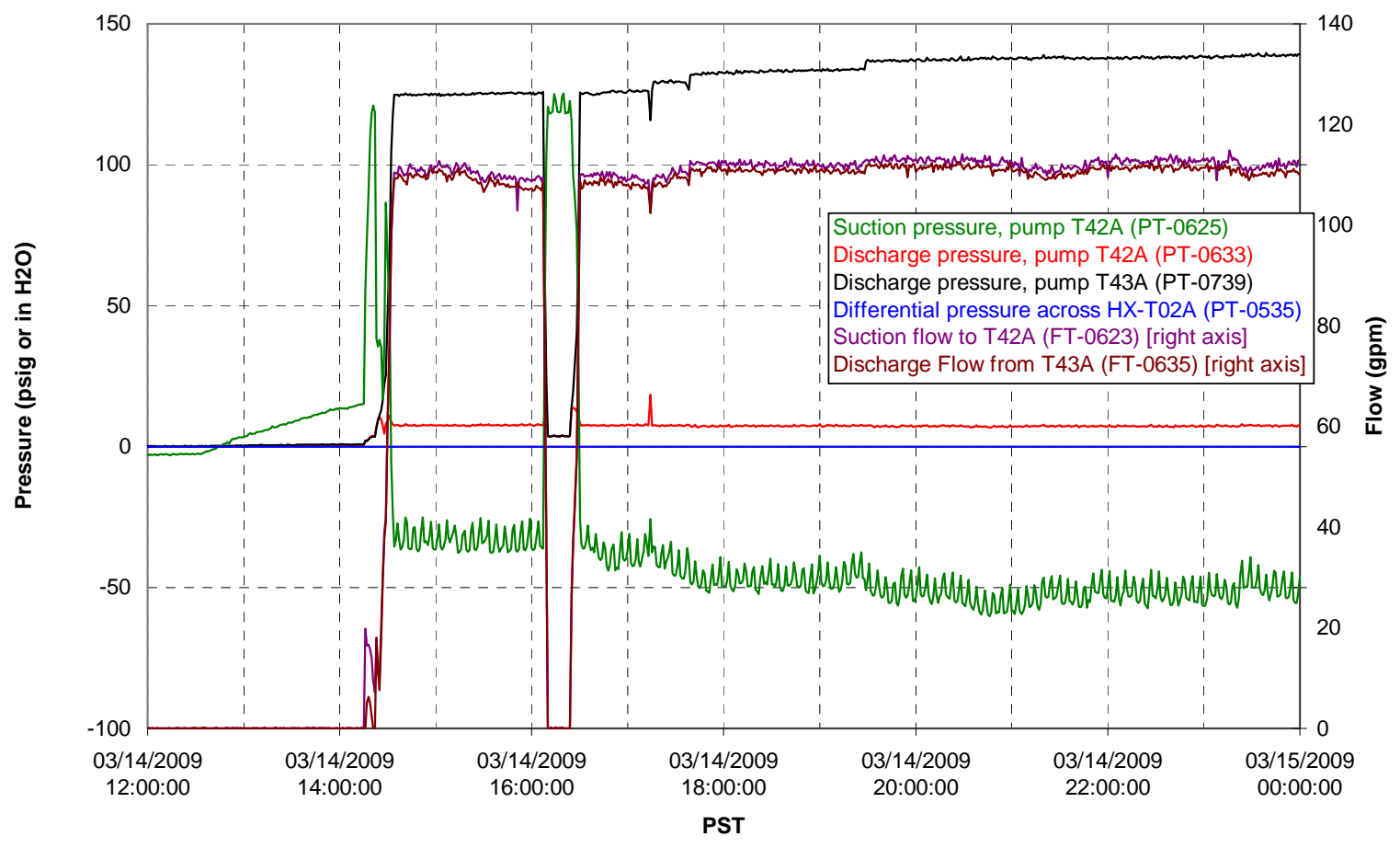




\section{Axial pressure drop}

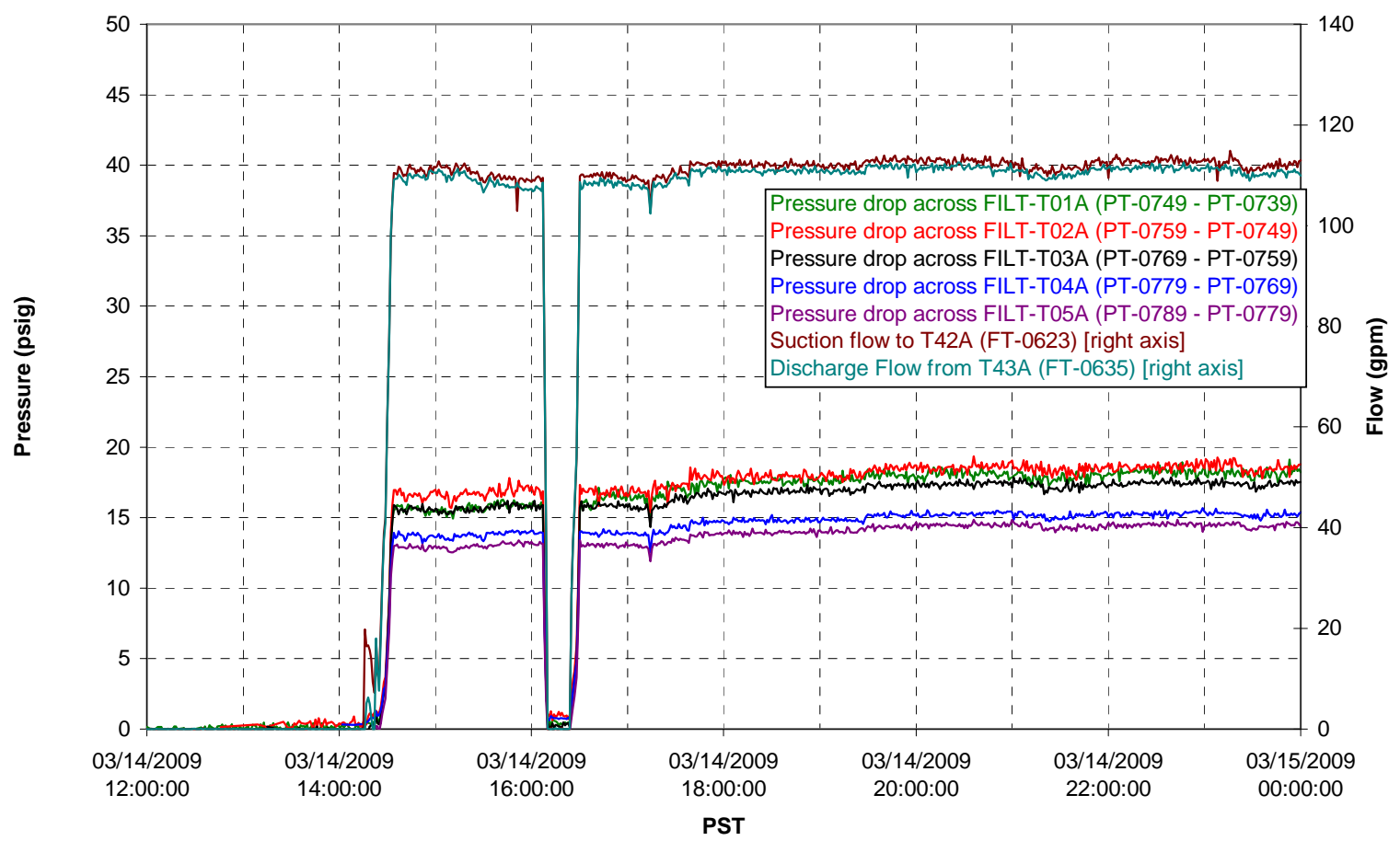

Permeate flow rates

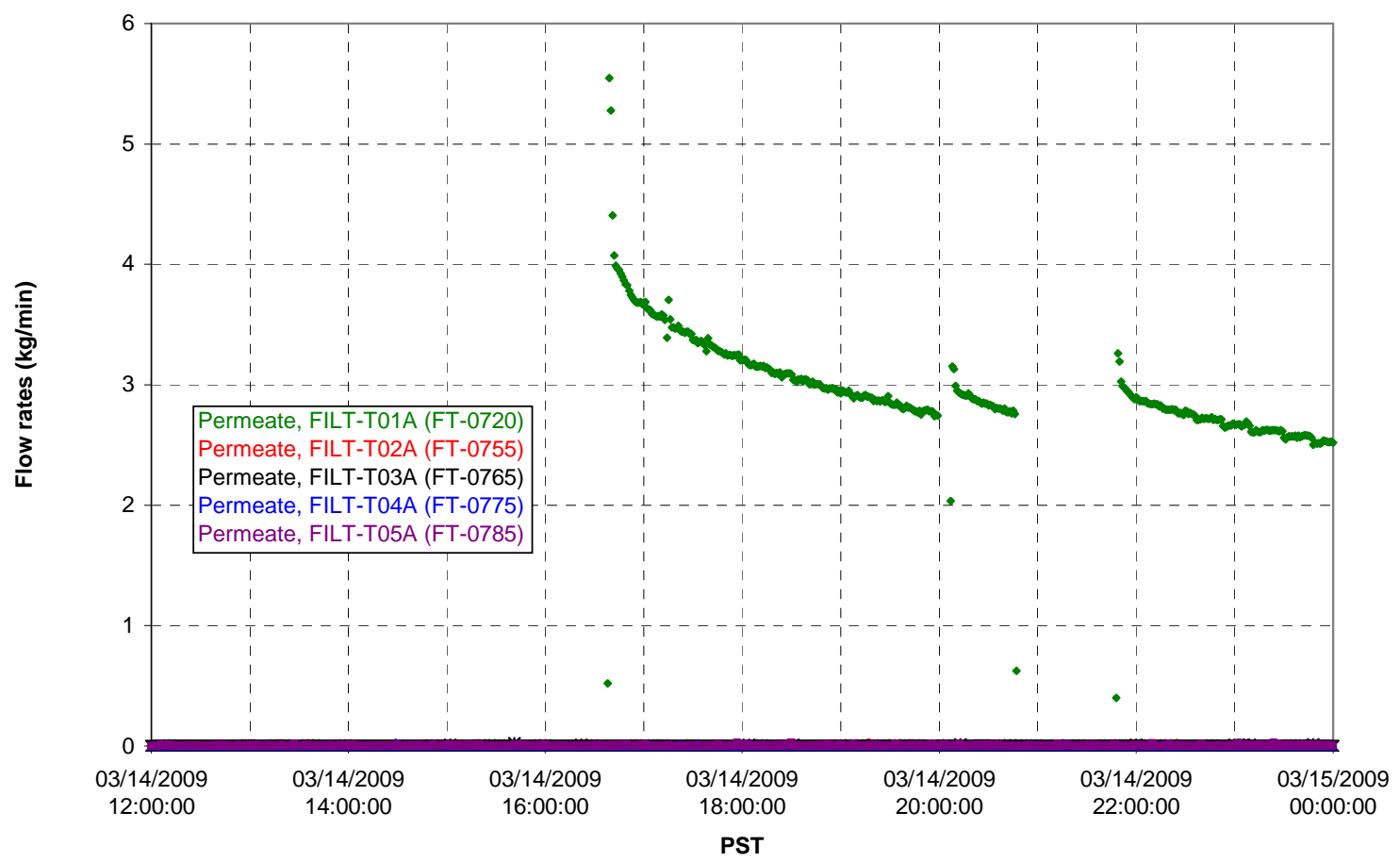


T02A Inner Temperature Tree

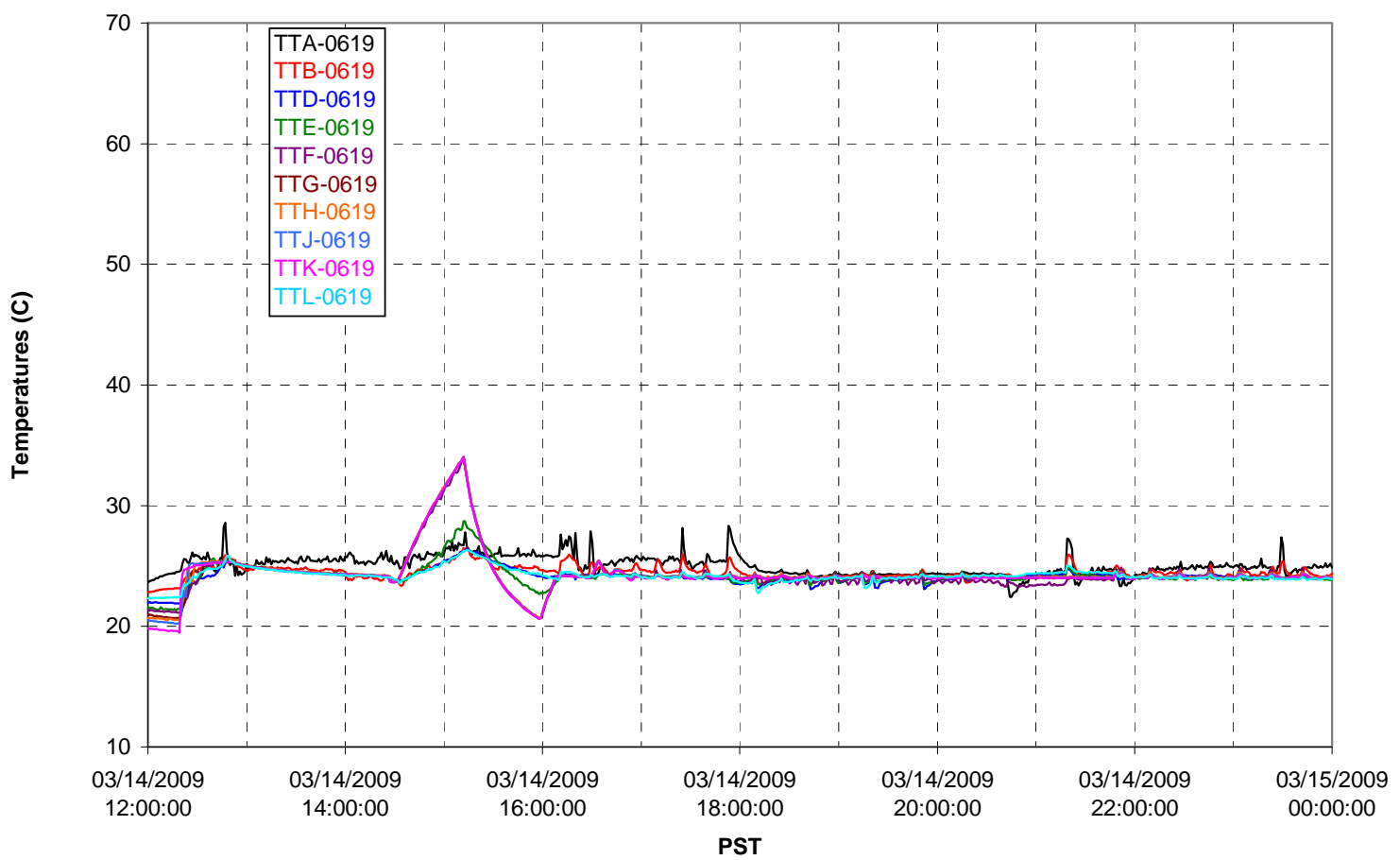

T02A Outer Temperature Tree

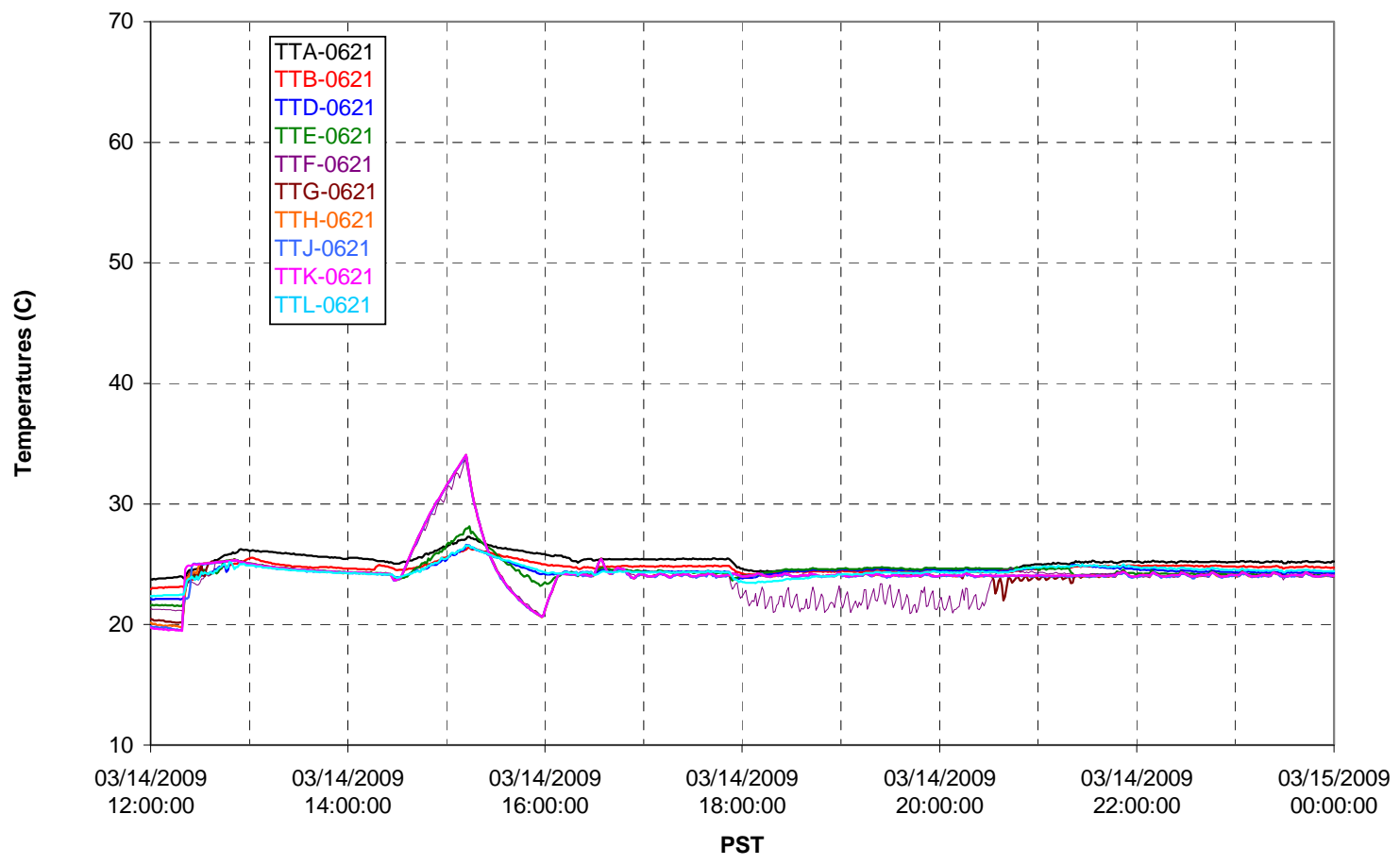


T02A temperatures

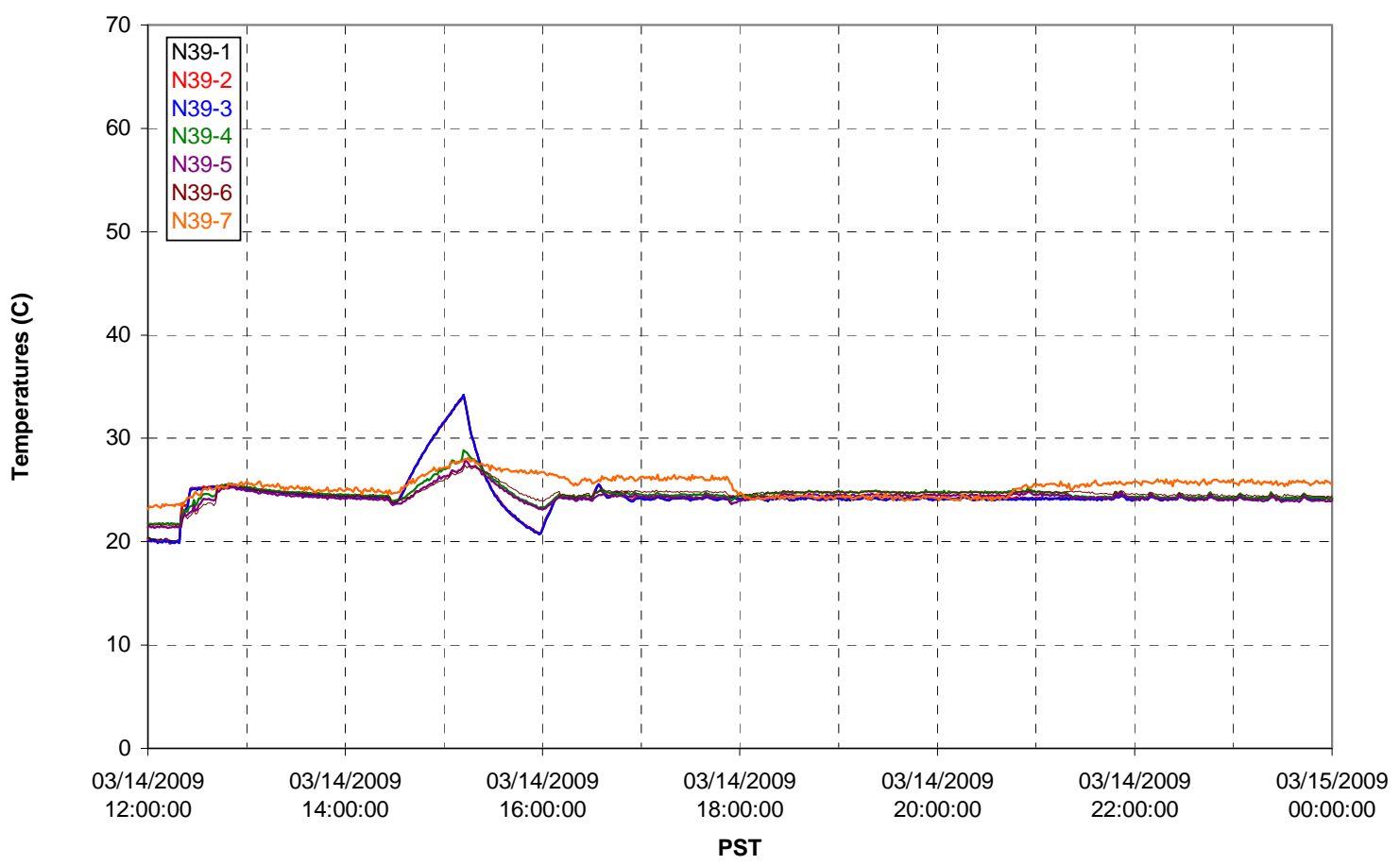


T02A temperatures

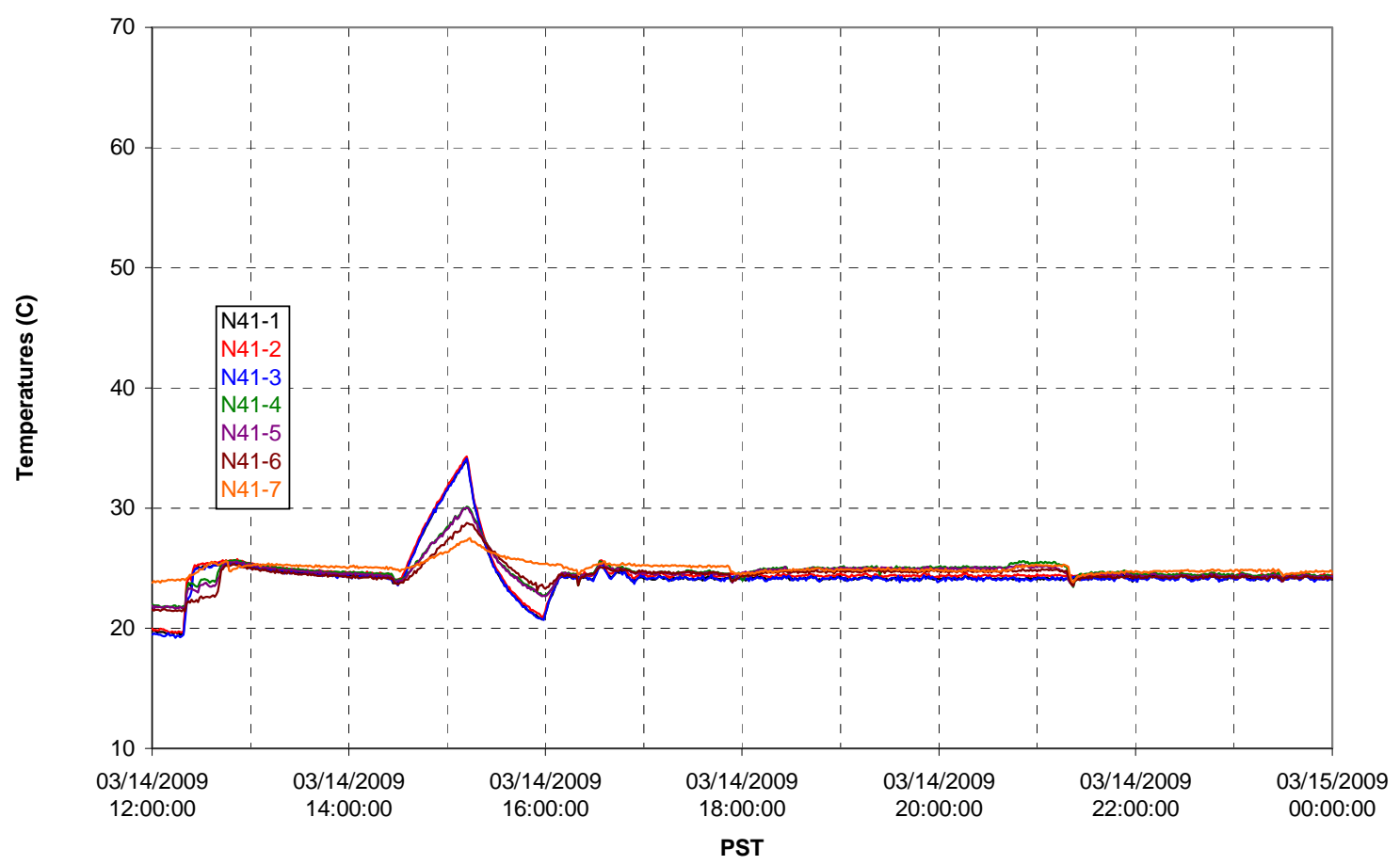

T02A temperatures

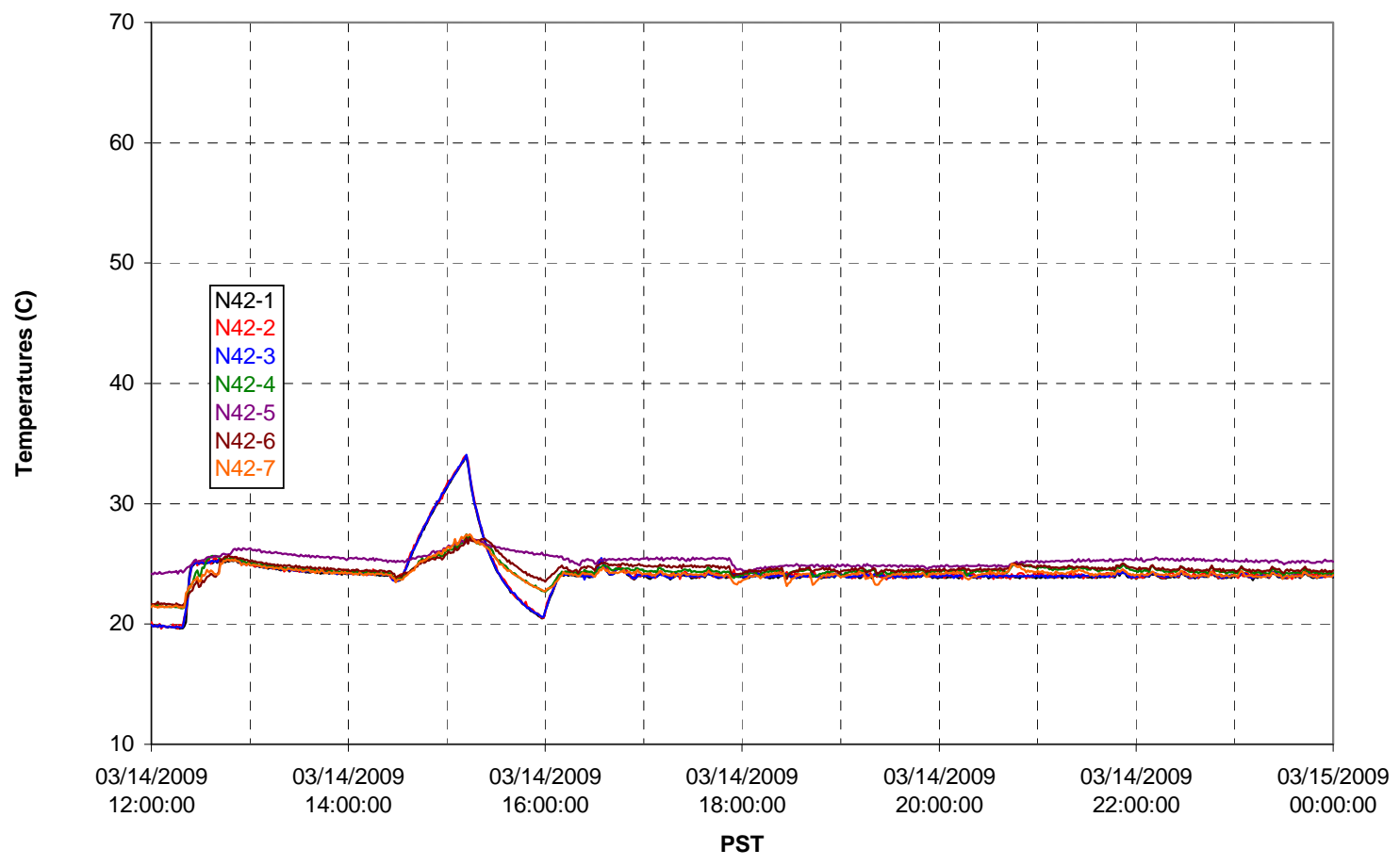


T02A temperatures

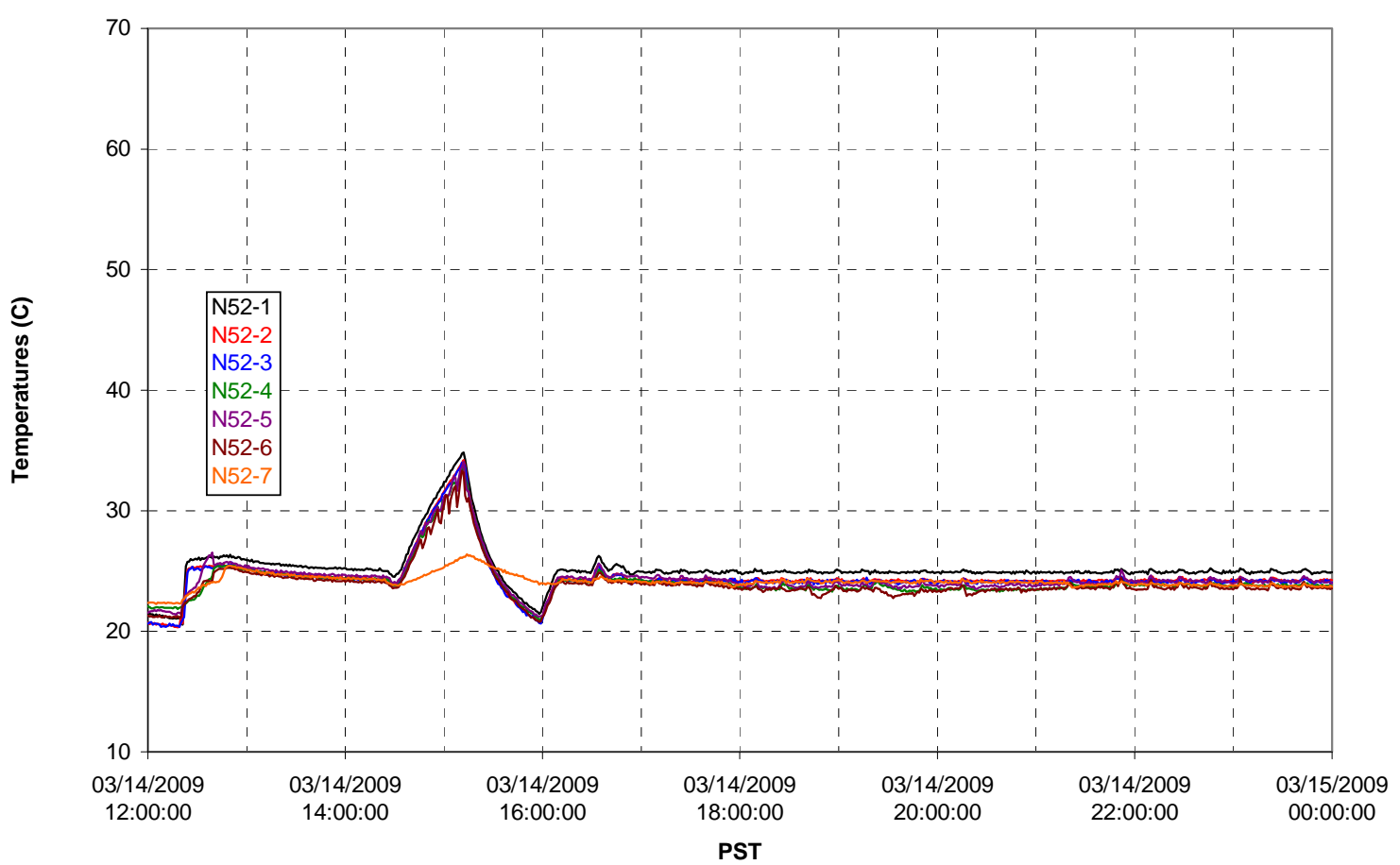

T02A Heating and Cooling

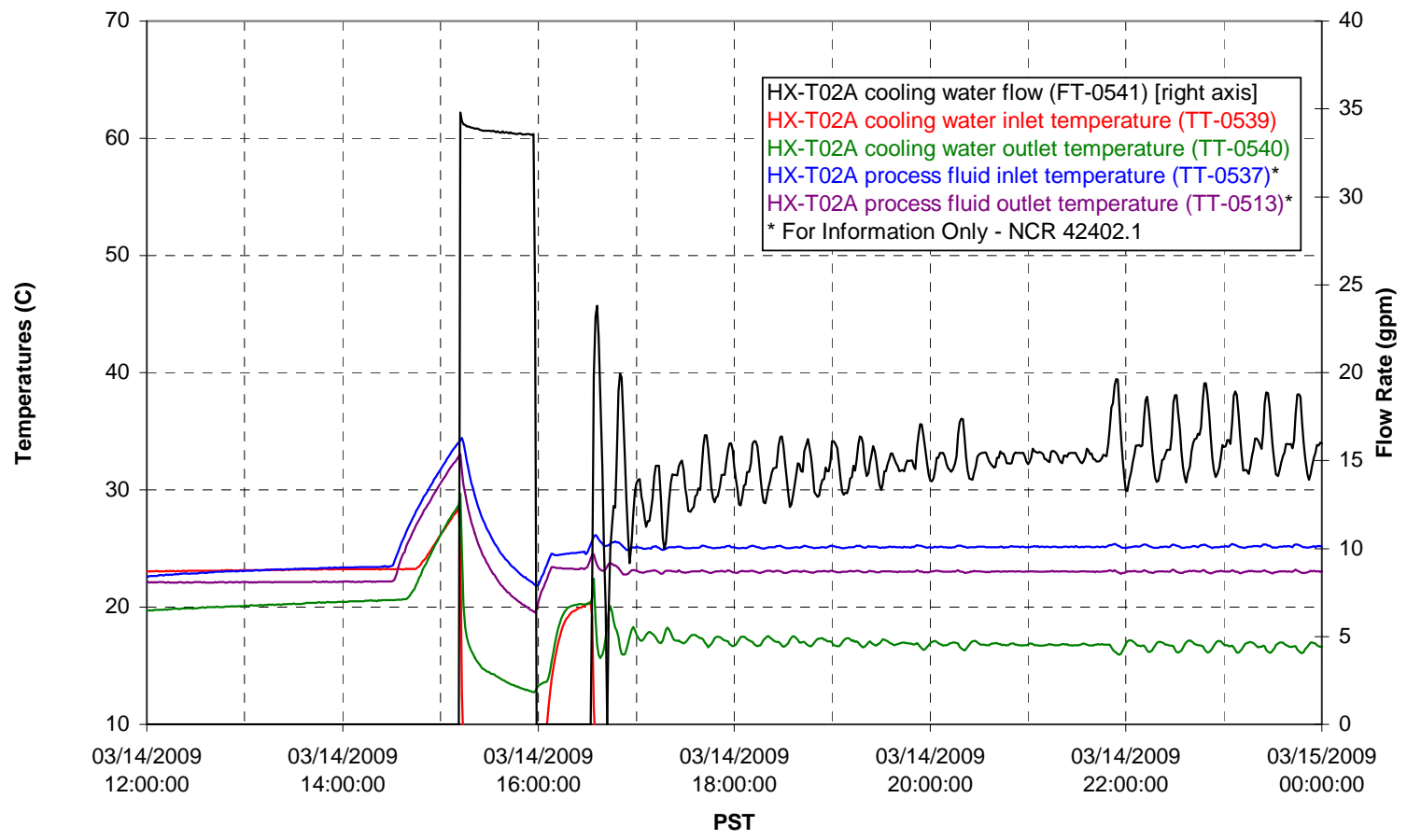




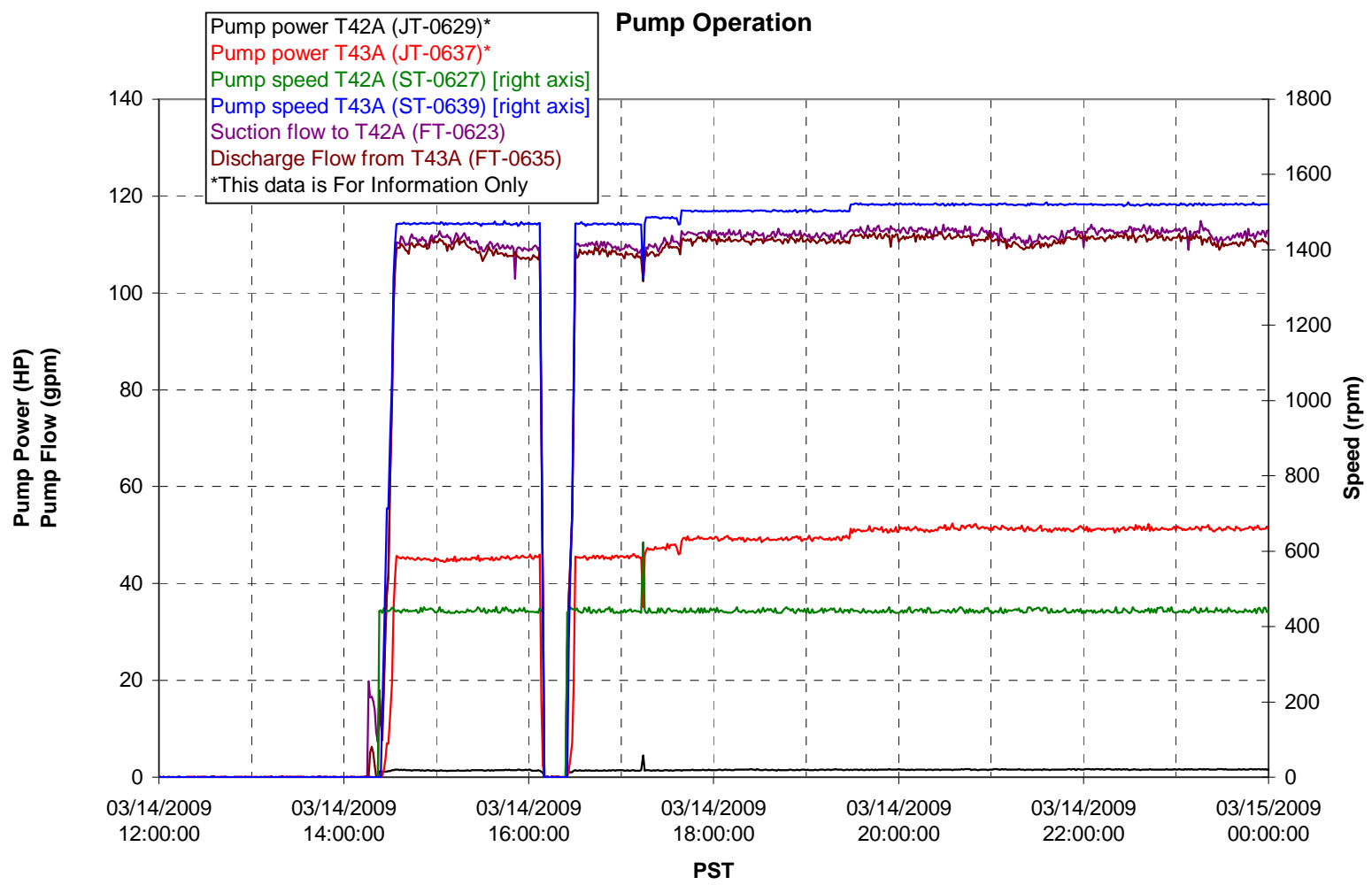

Pulsepot UFP-PP-T01A

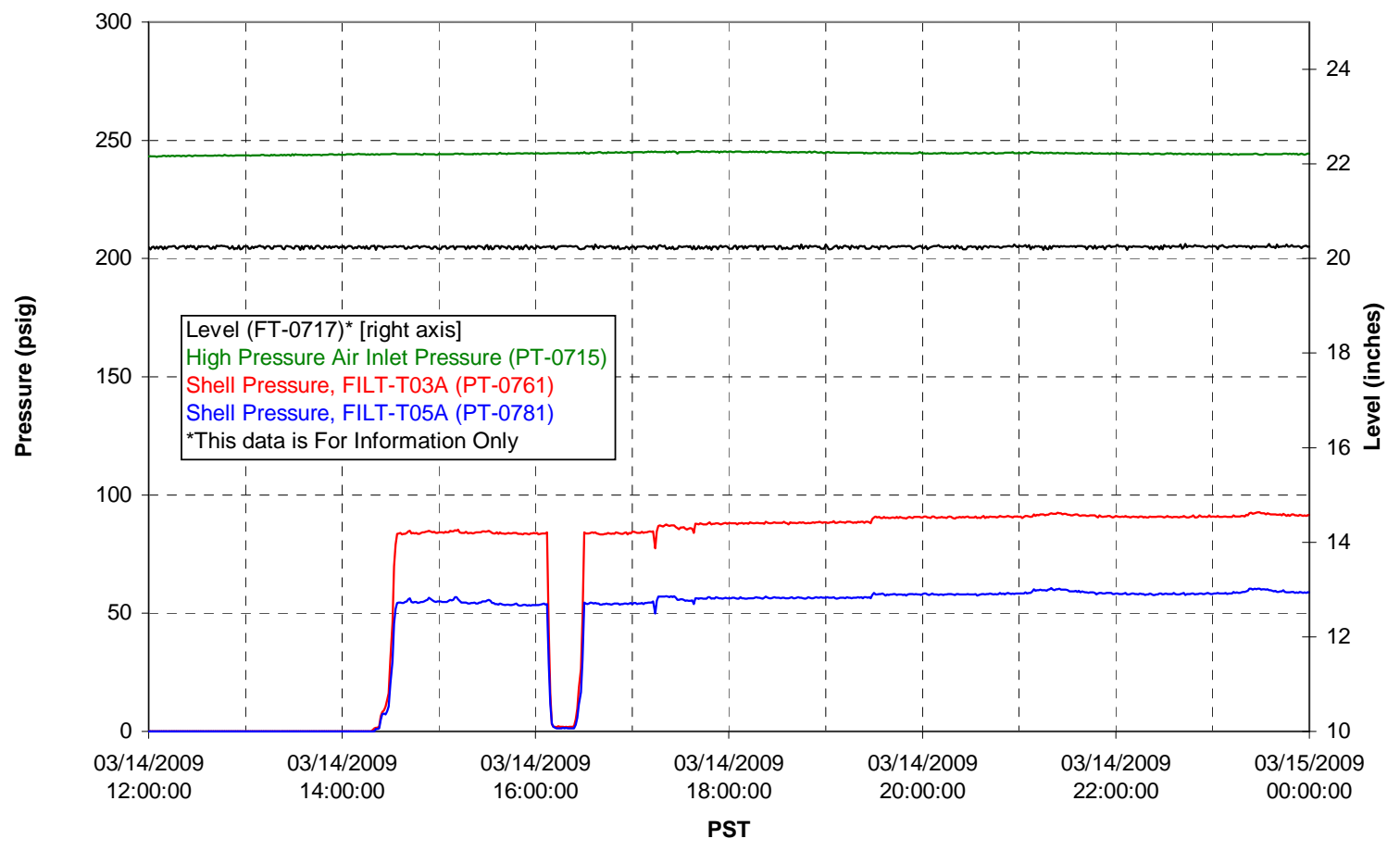


Pulsepot UFP-PP-T02A

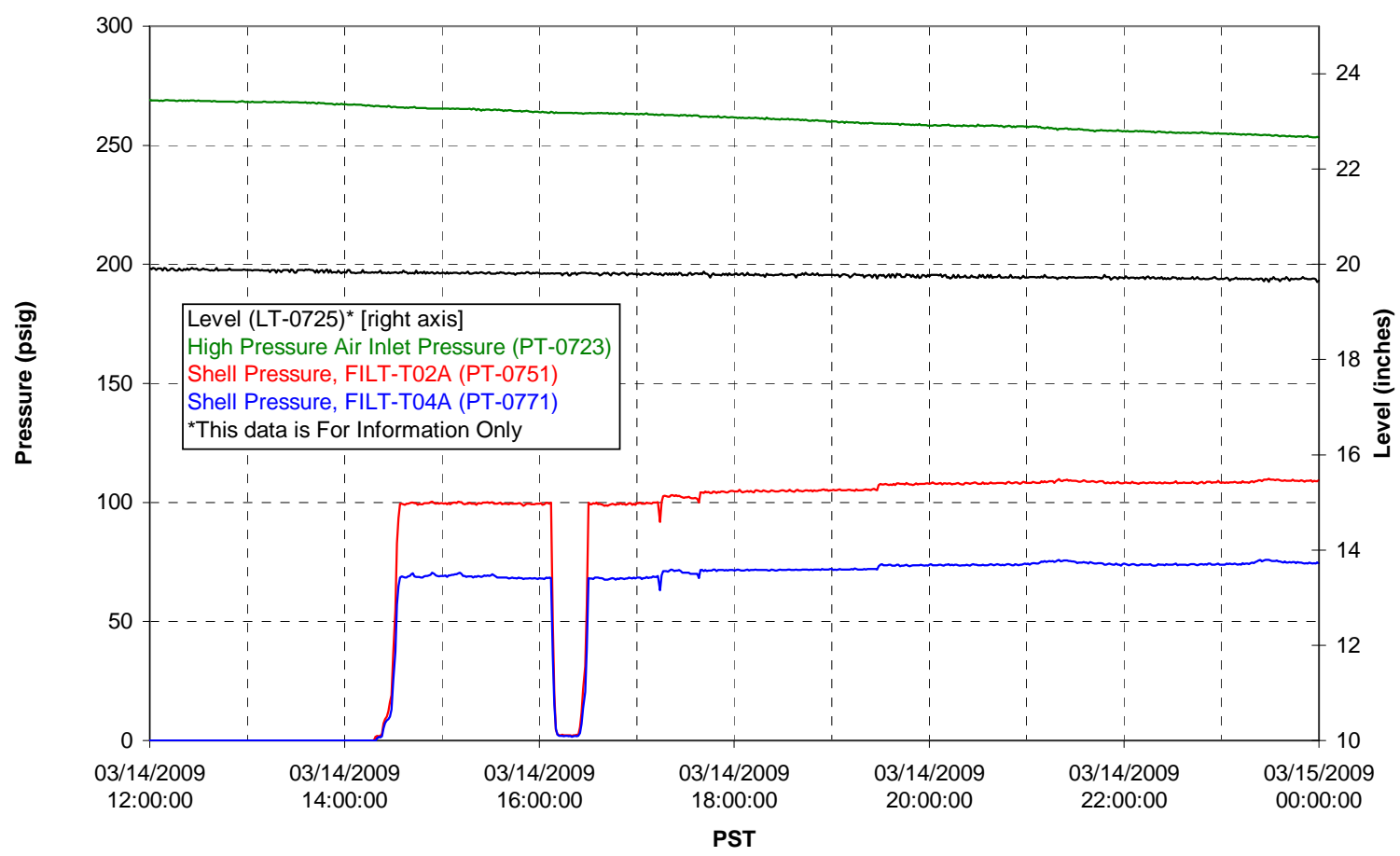

Pulsepot UFP-PP-T03A

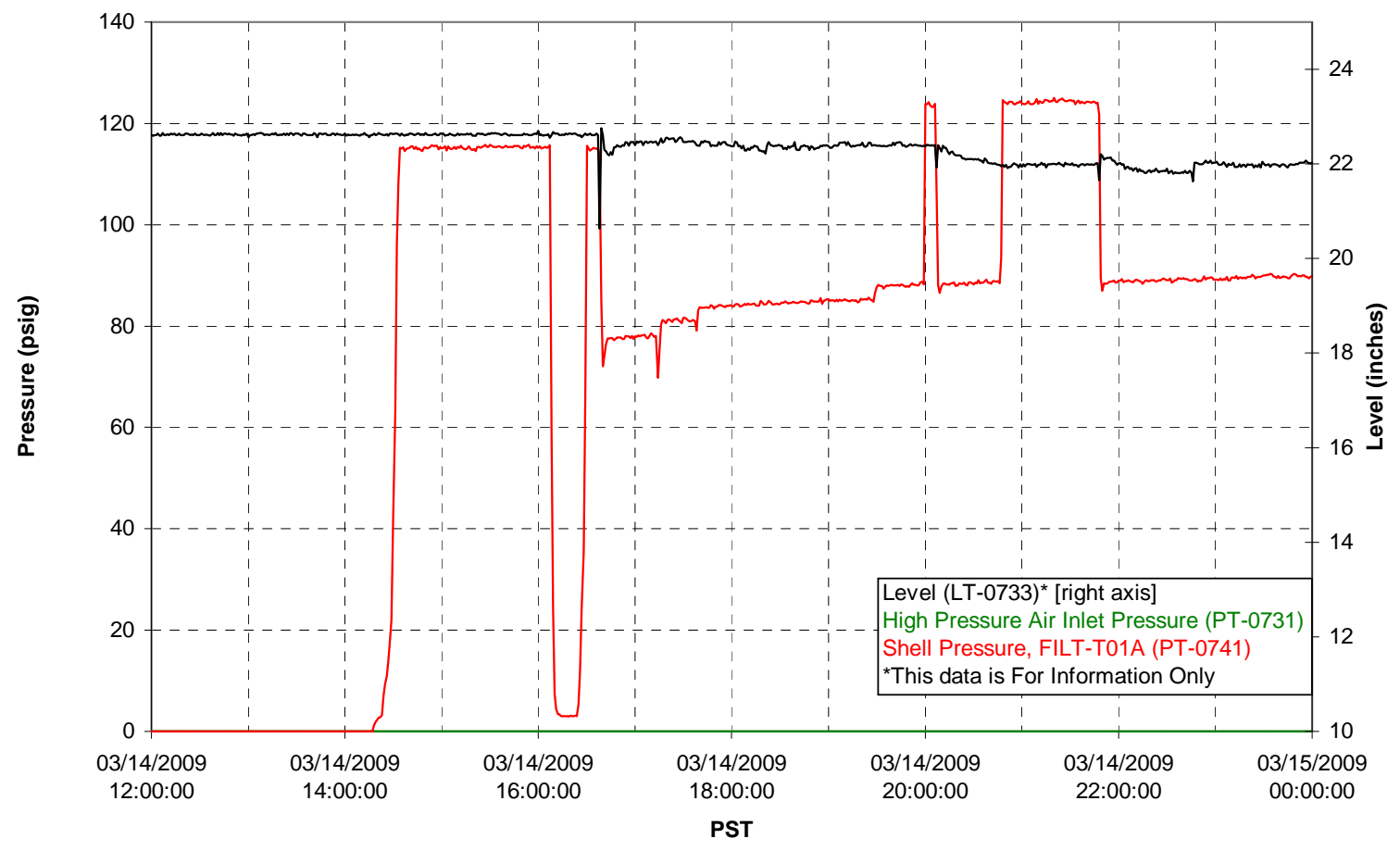


Pulsepot Levels

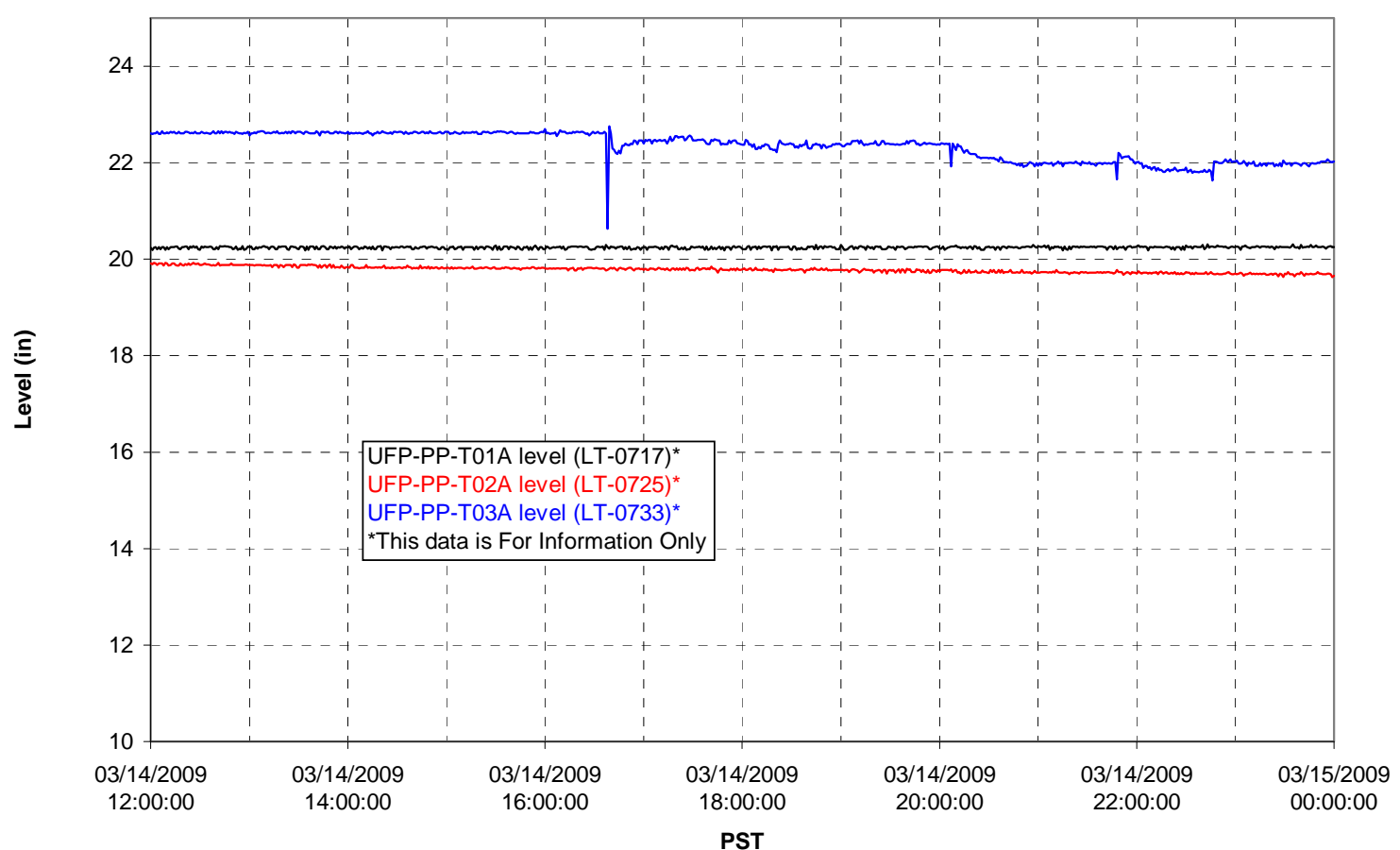

Filter UFP-FILT-T01A

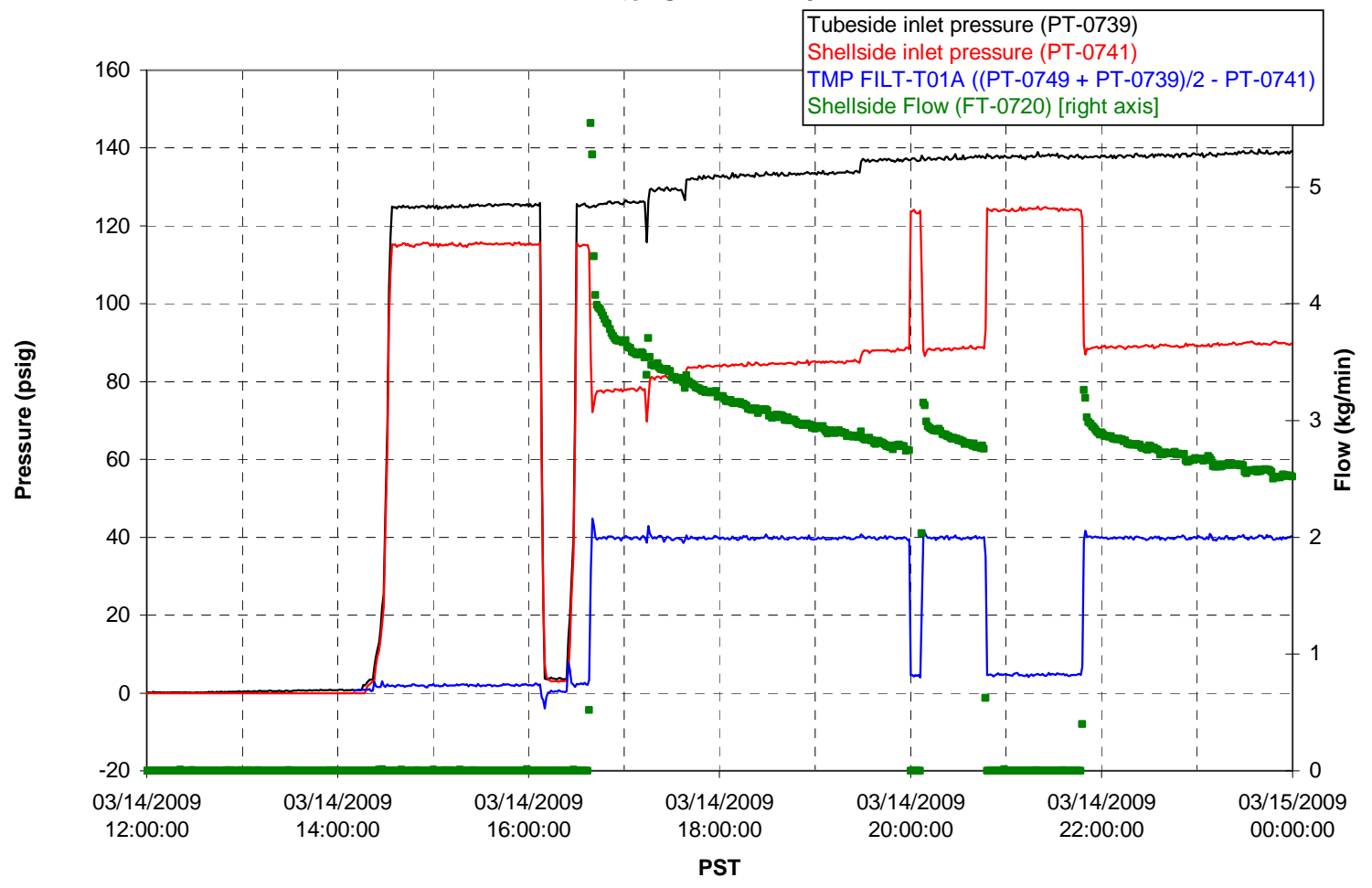


Filter UFP-FILT-T02A

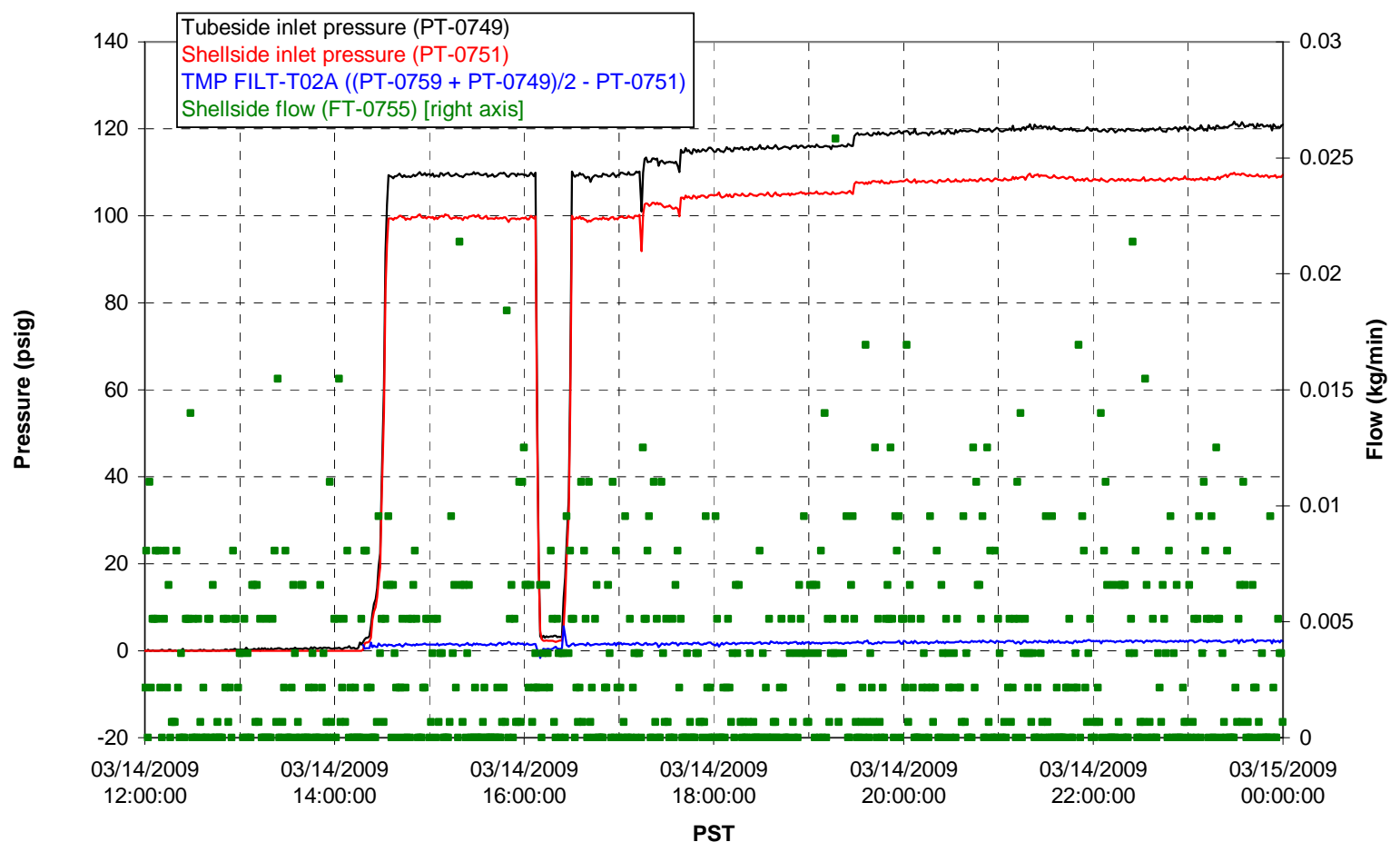

Filter UFP-FILT-T03A

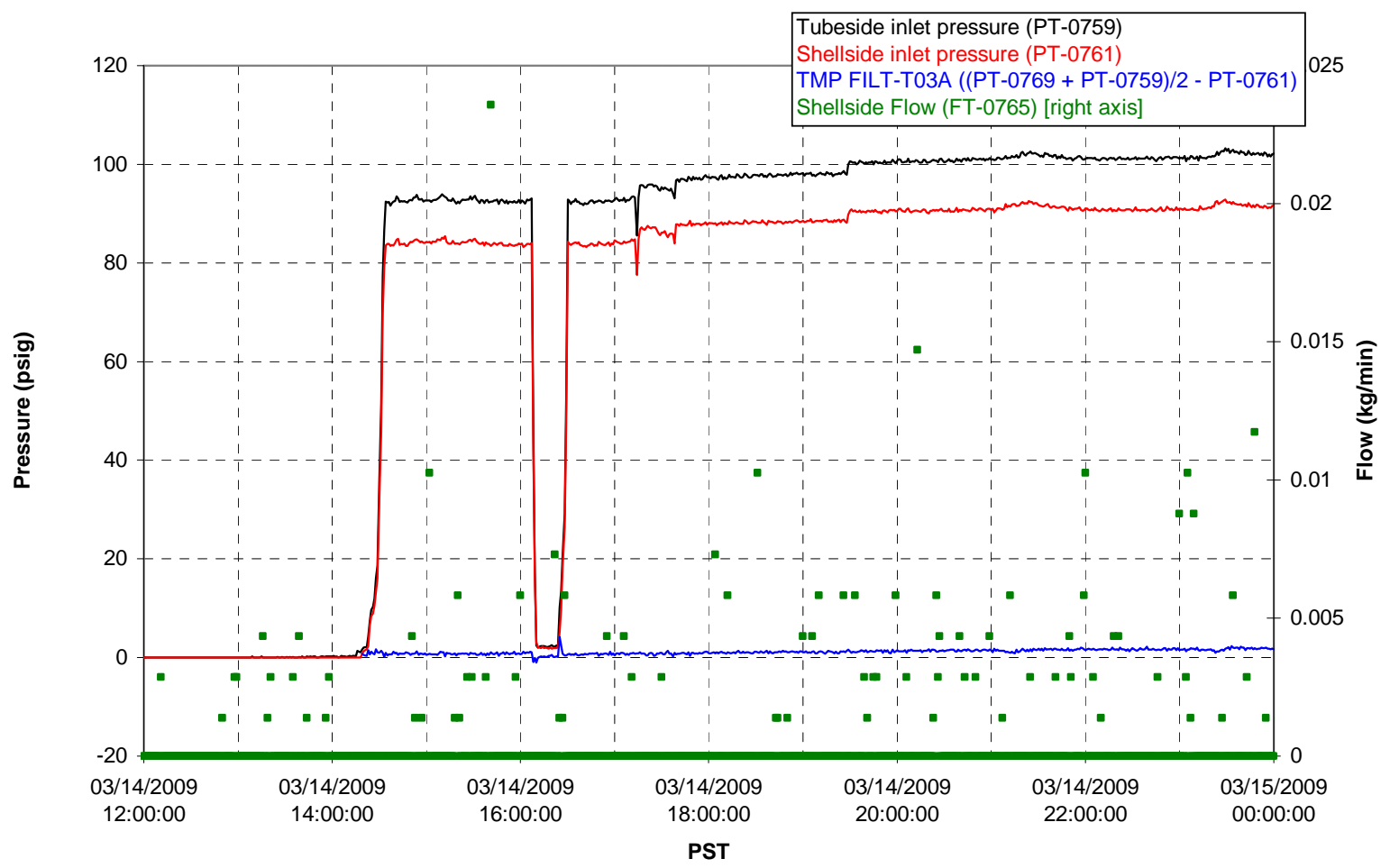


Filter UFP-FILT-T04A

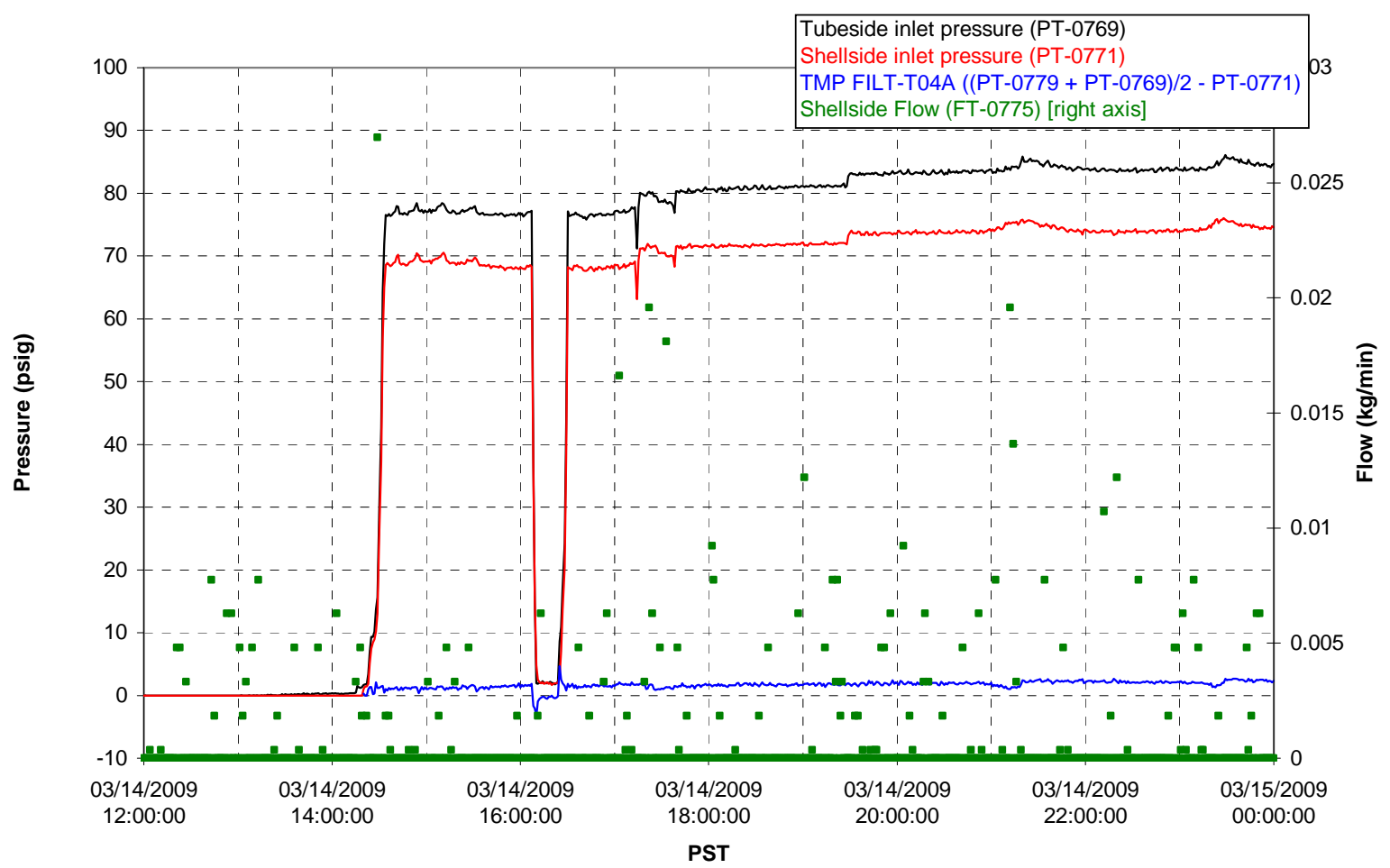

Filter UFP-FILT-T05A

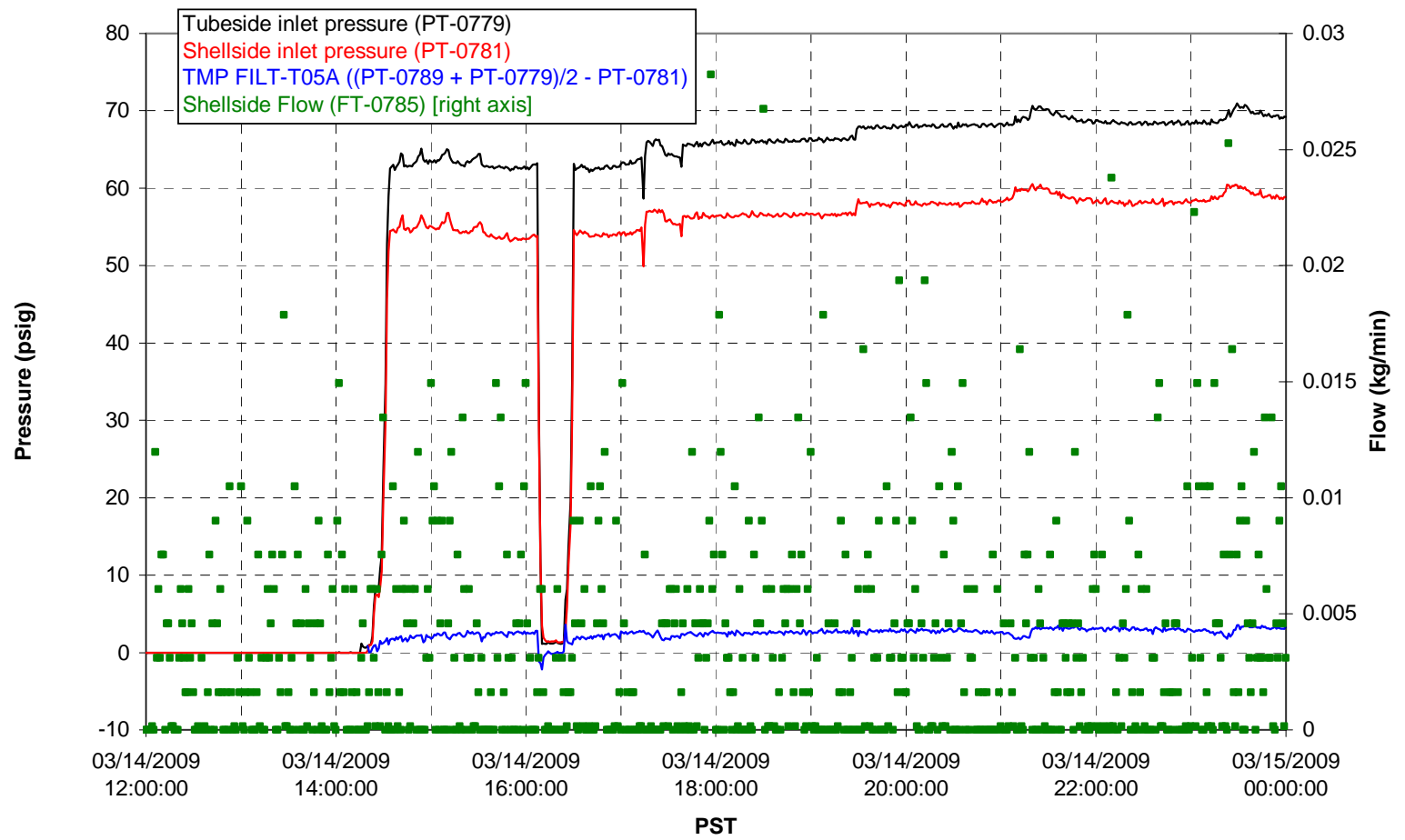



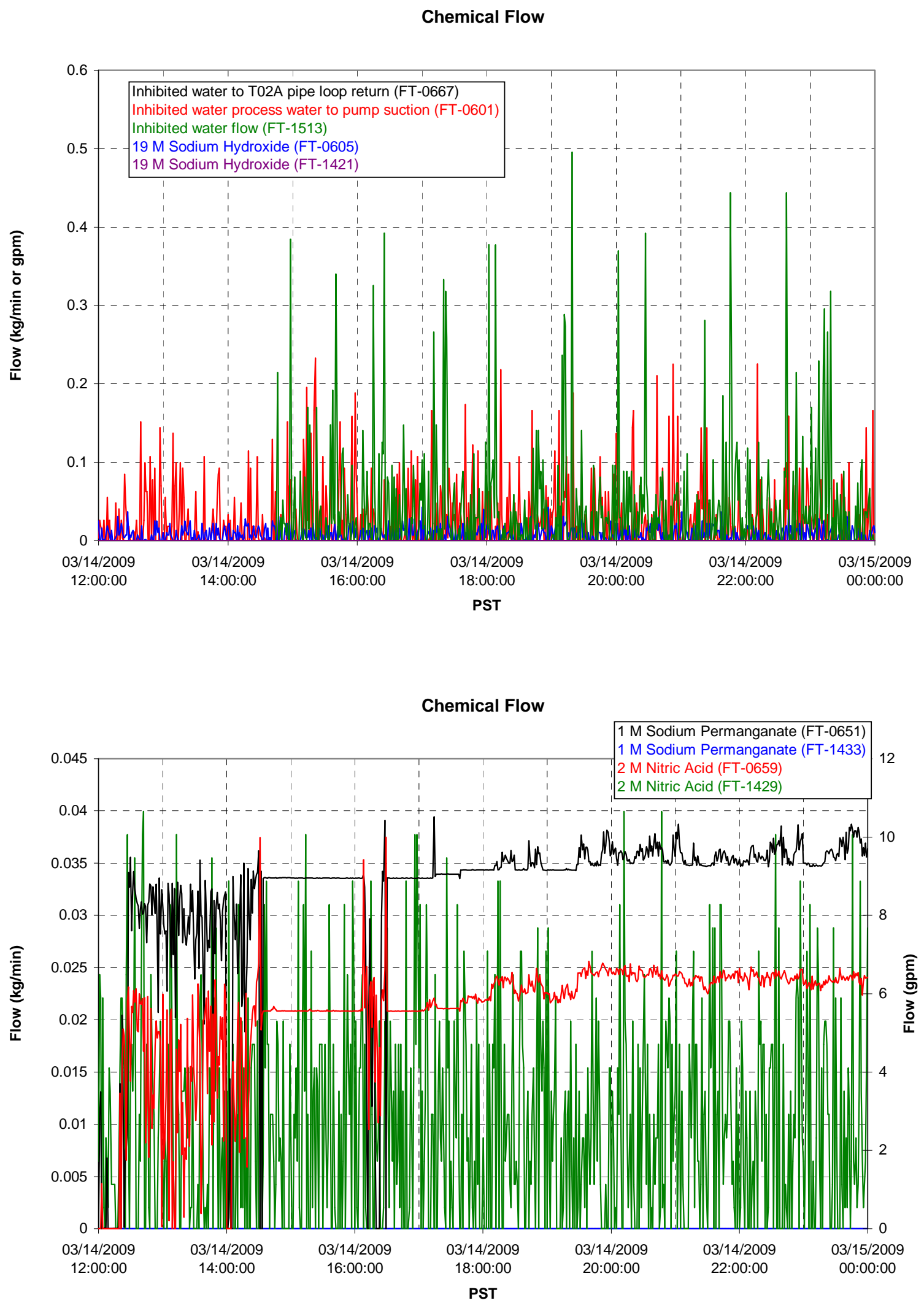


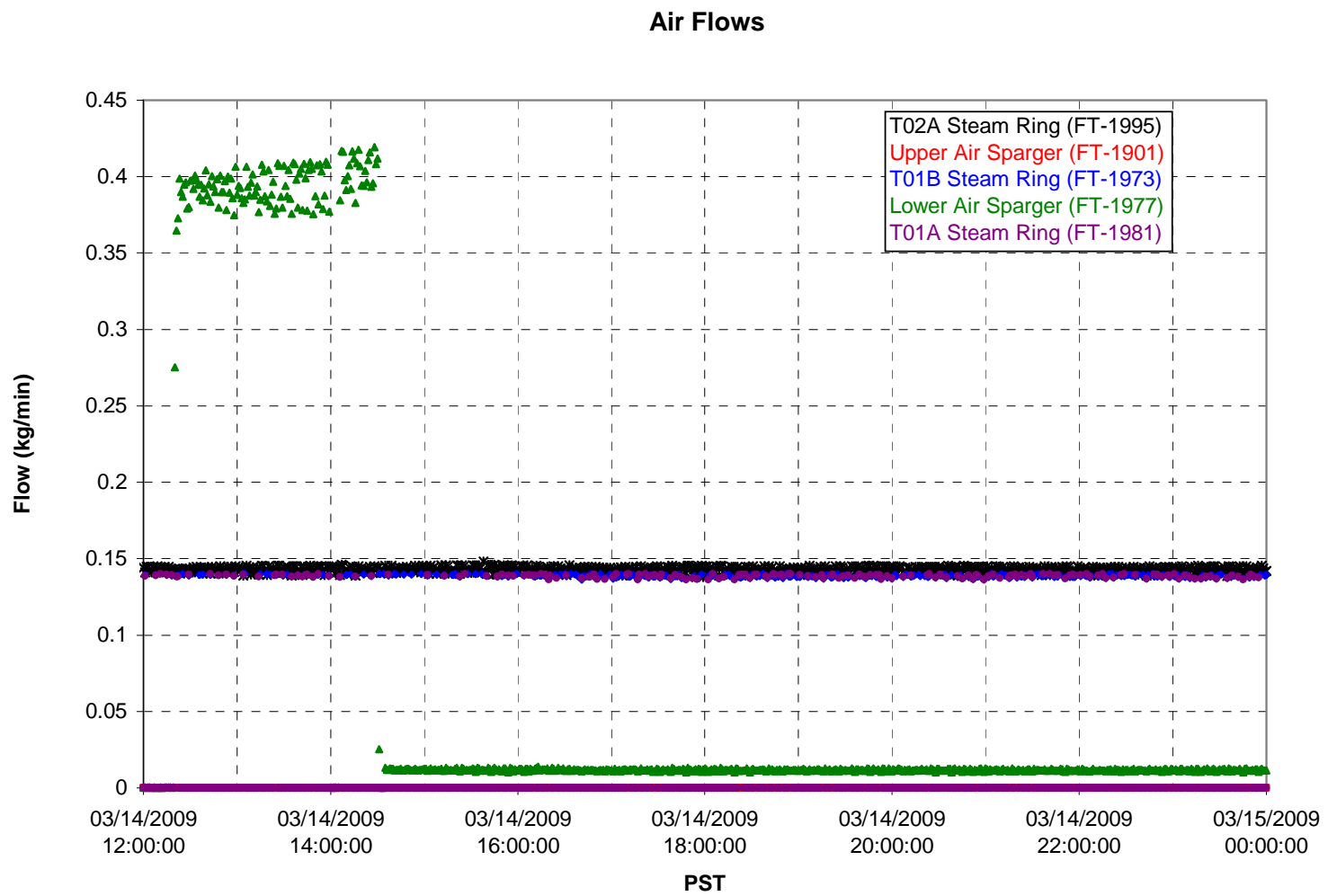

T02A Steam

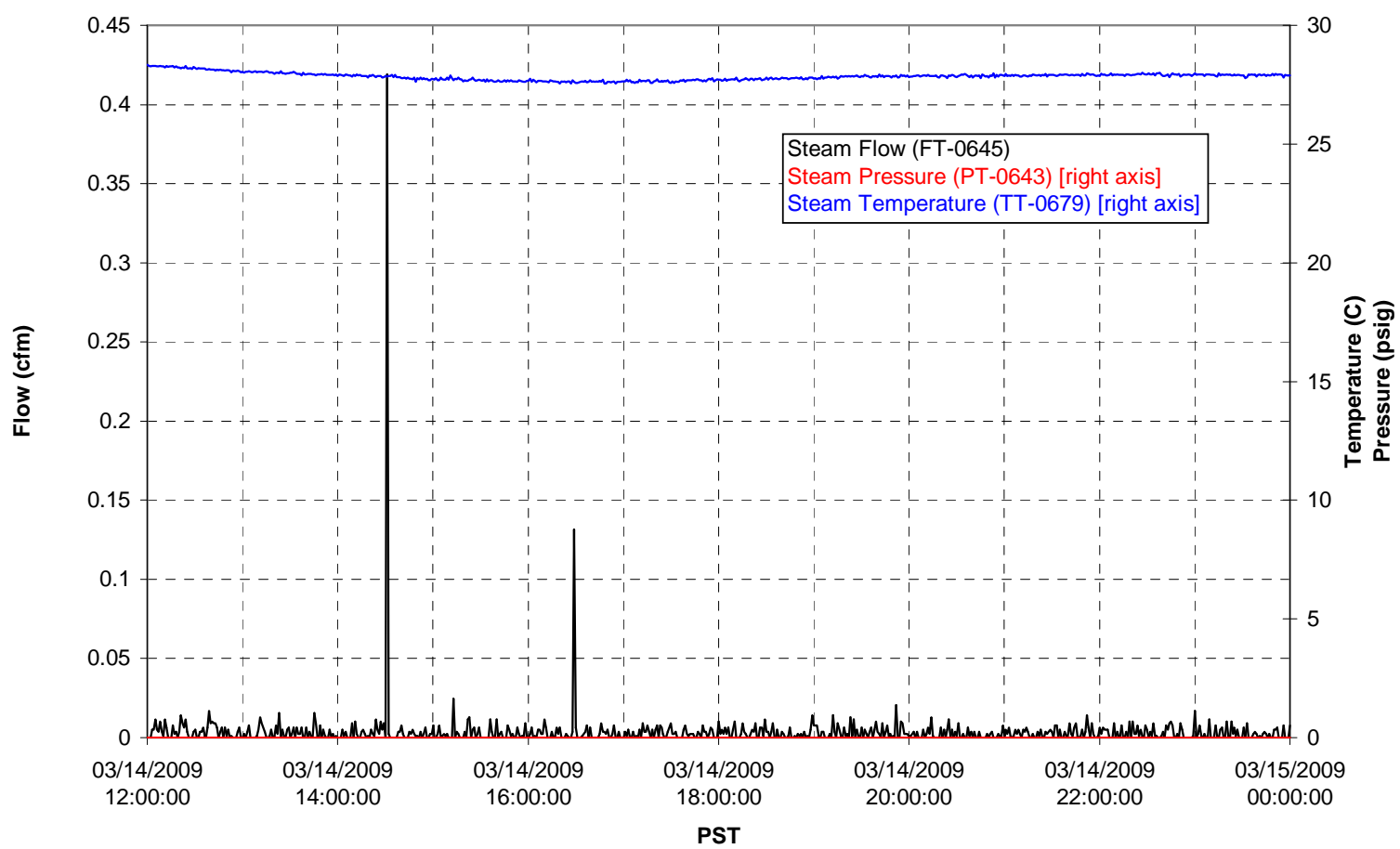


T01A Steam

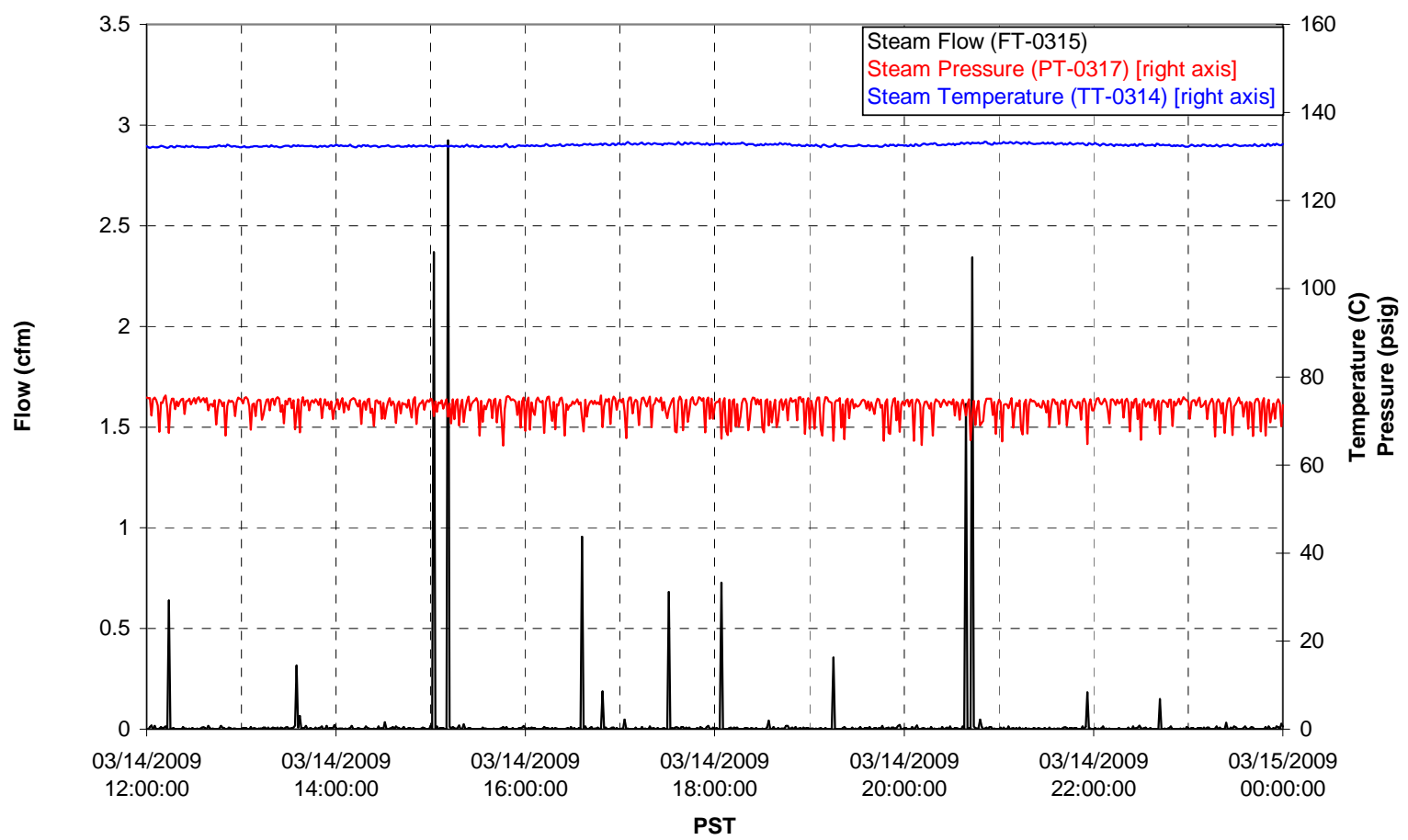

T01B Steam

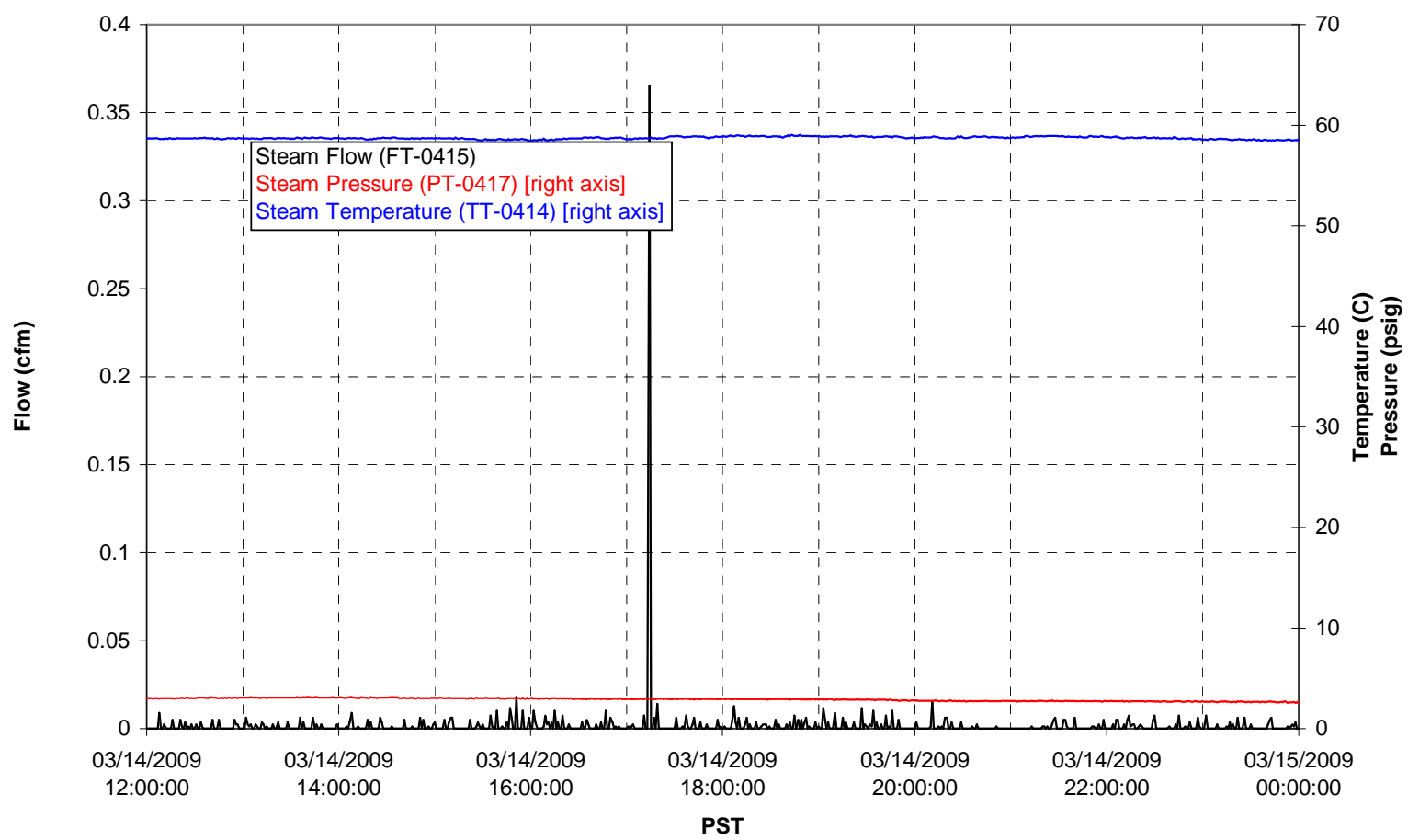




\section{Integrated Test B Data Plots \\ 03/15/09 00:00 - 03/15/09 12:00}


T01A level

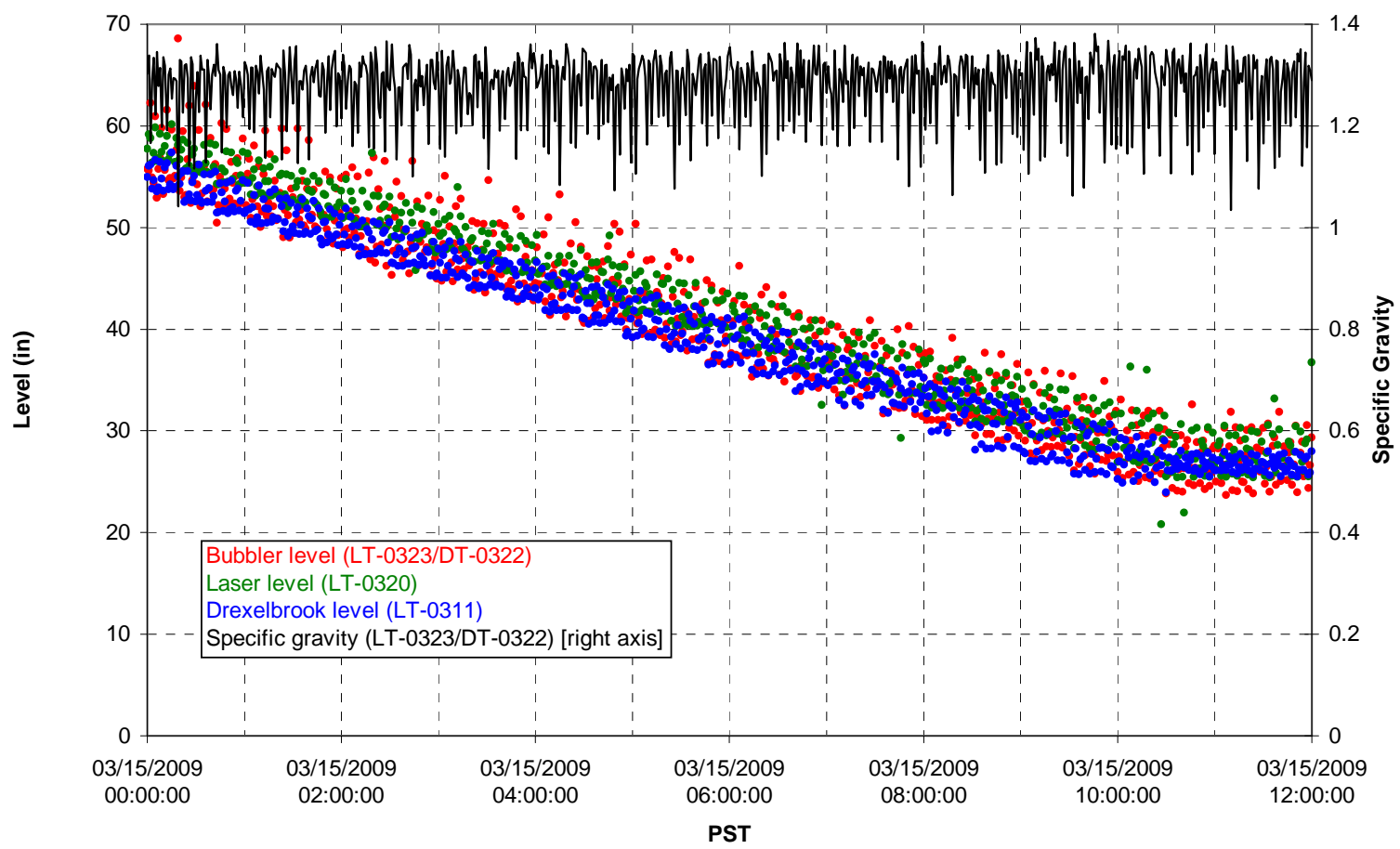

T01A temperatures

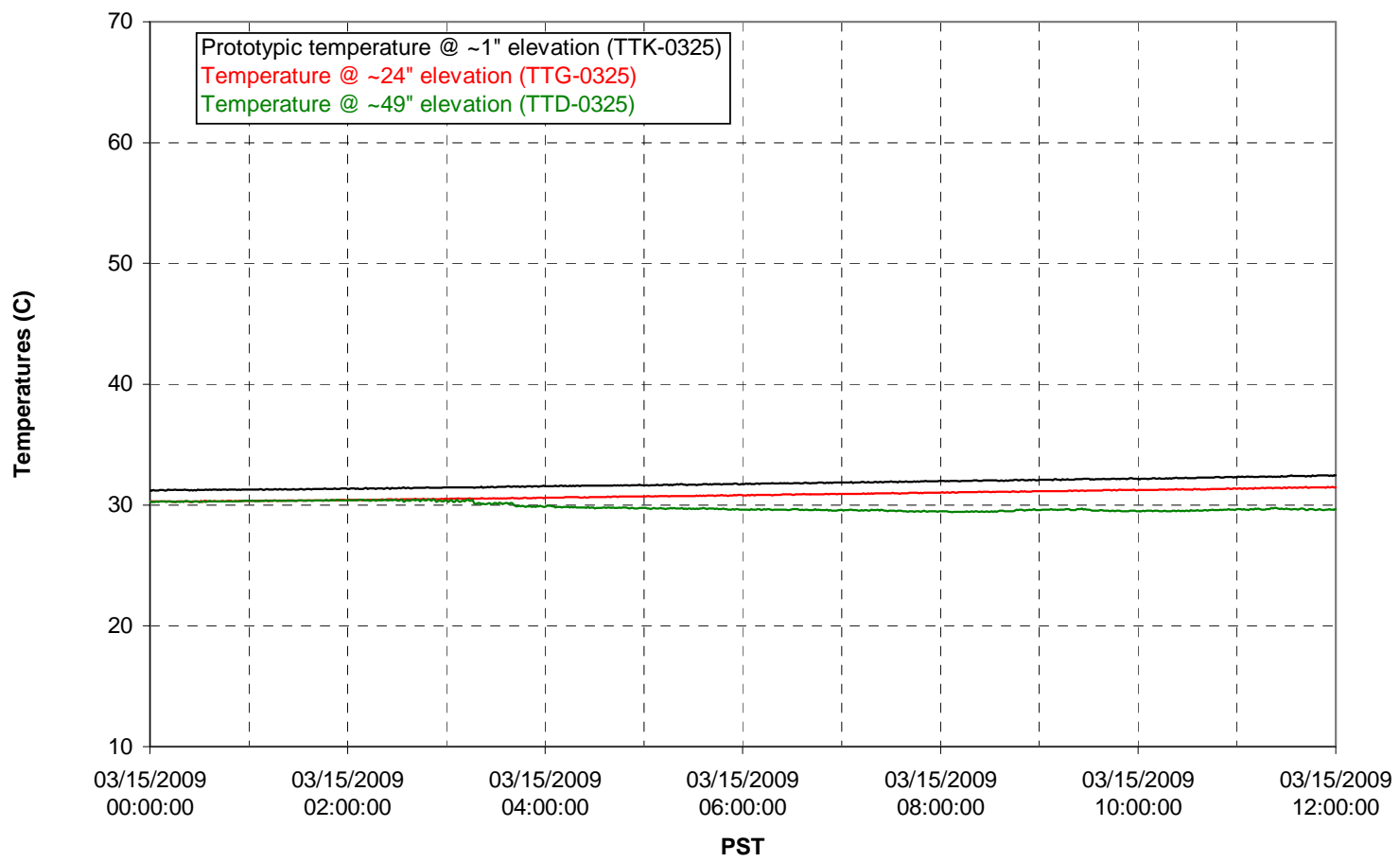


T01B level

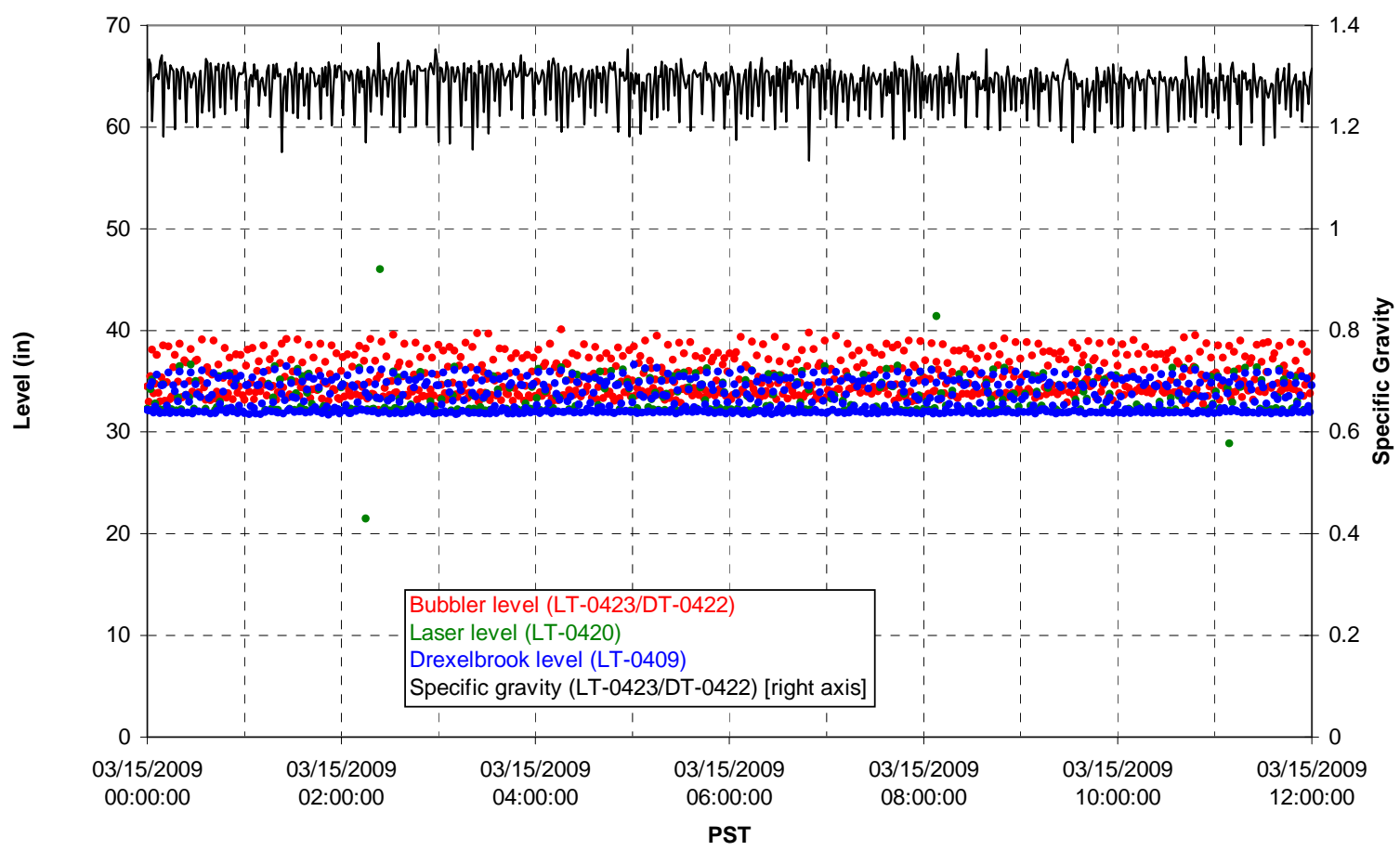

T01B temperatures

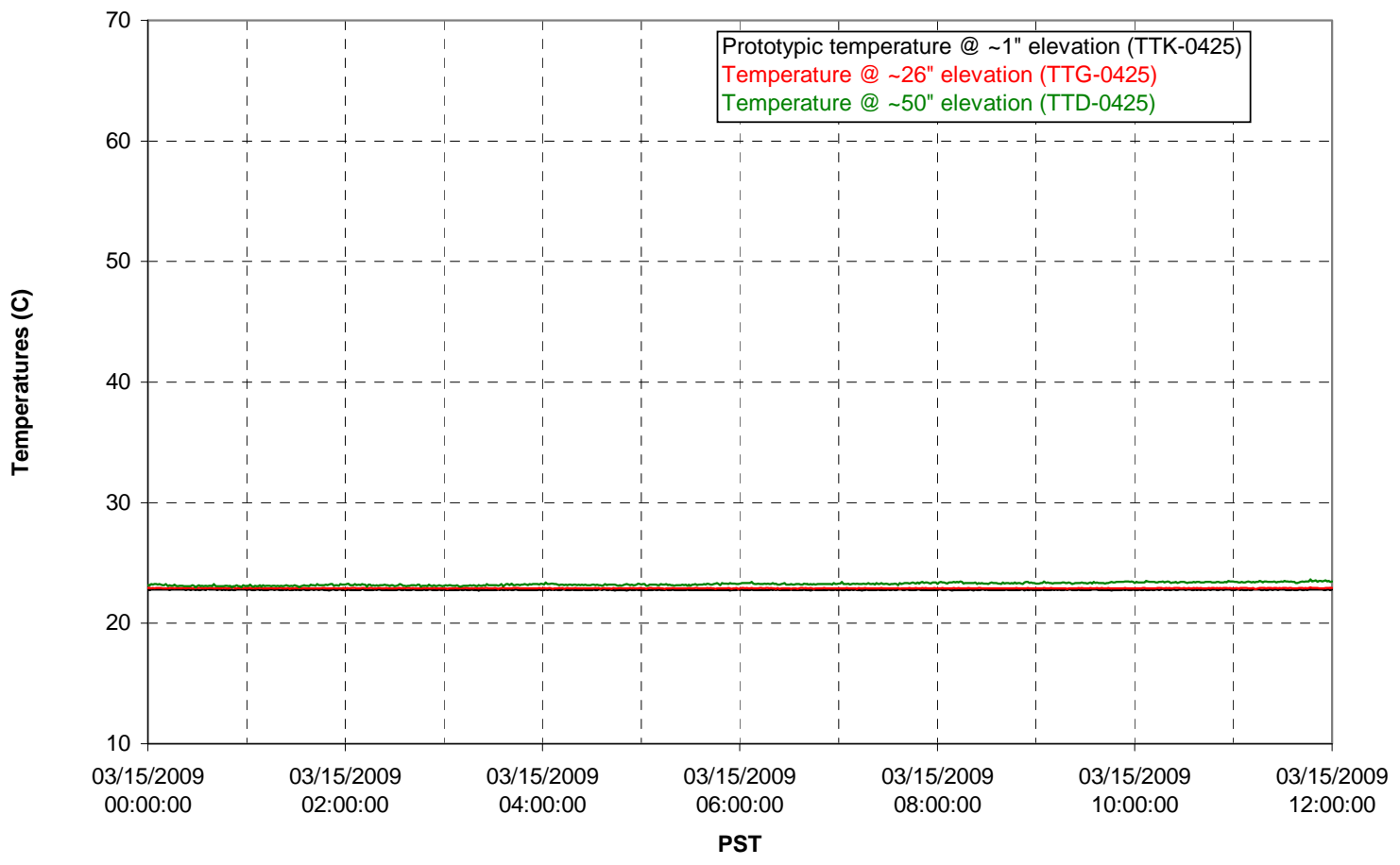


T02A level

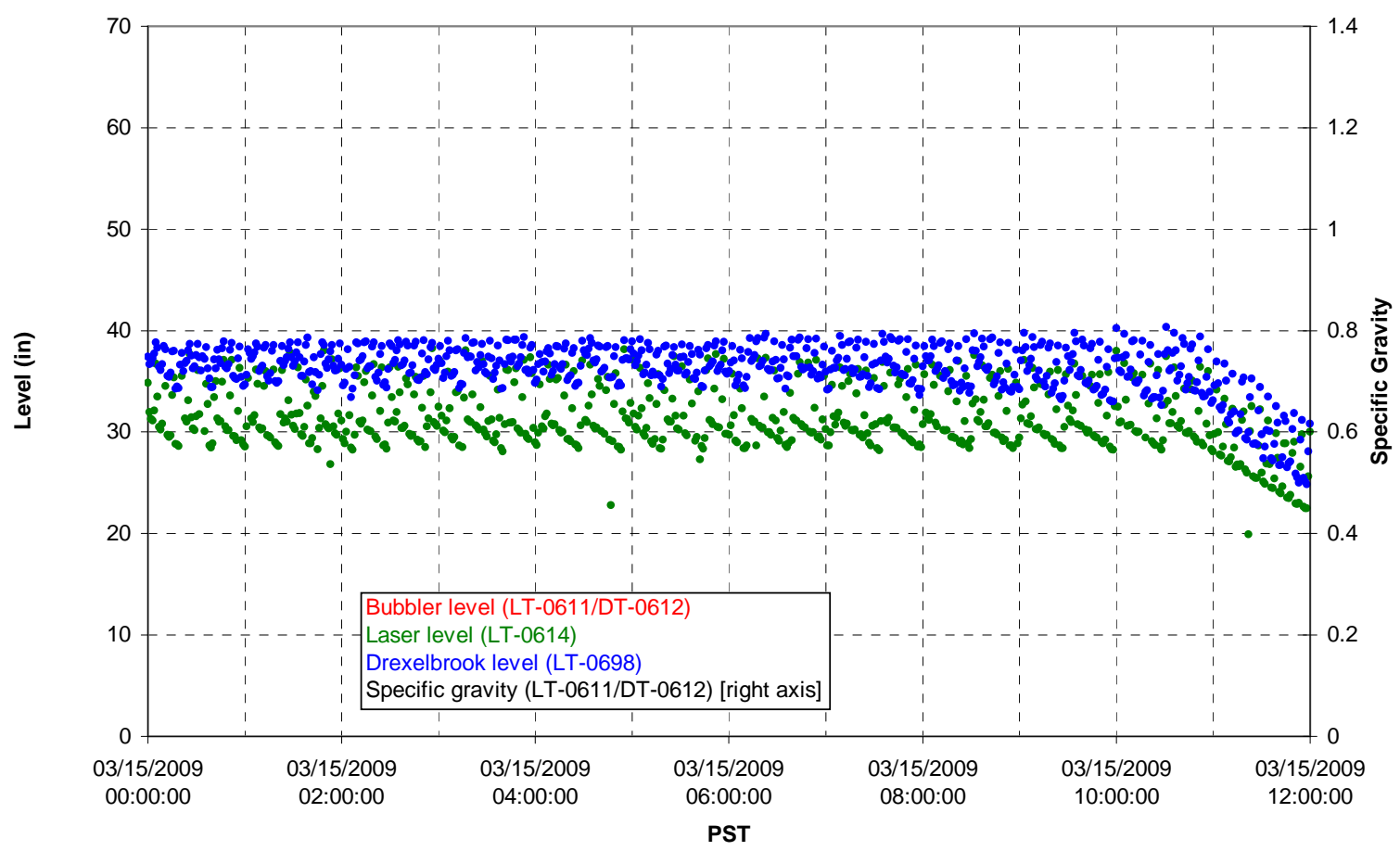

T02A temperatures

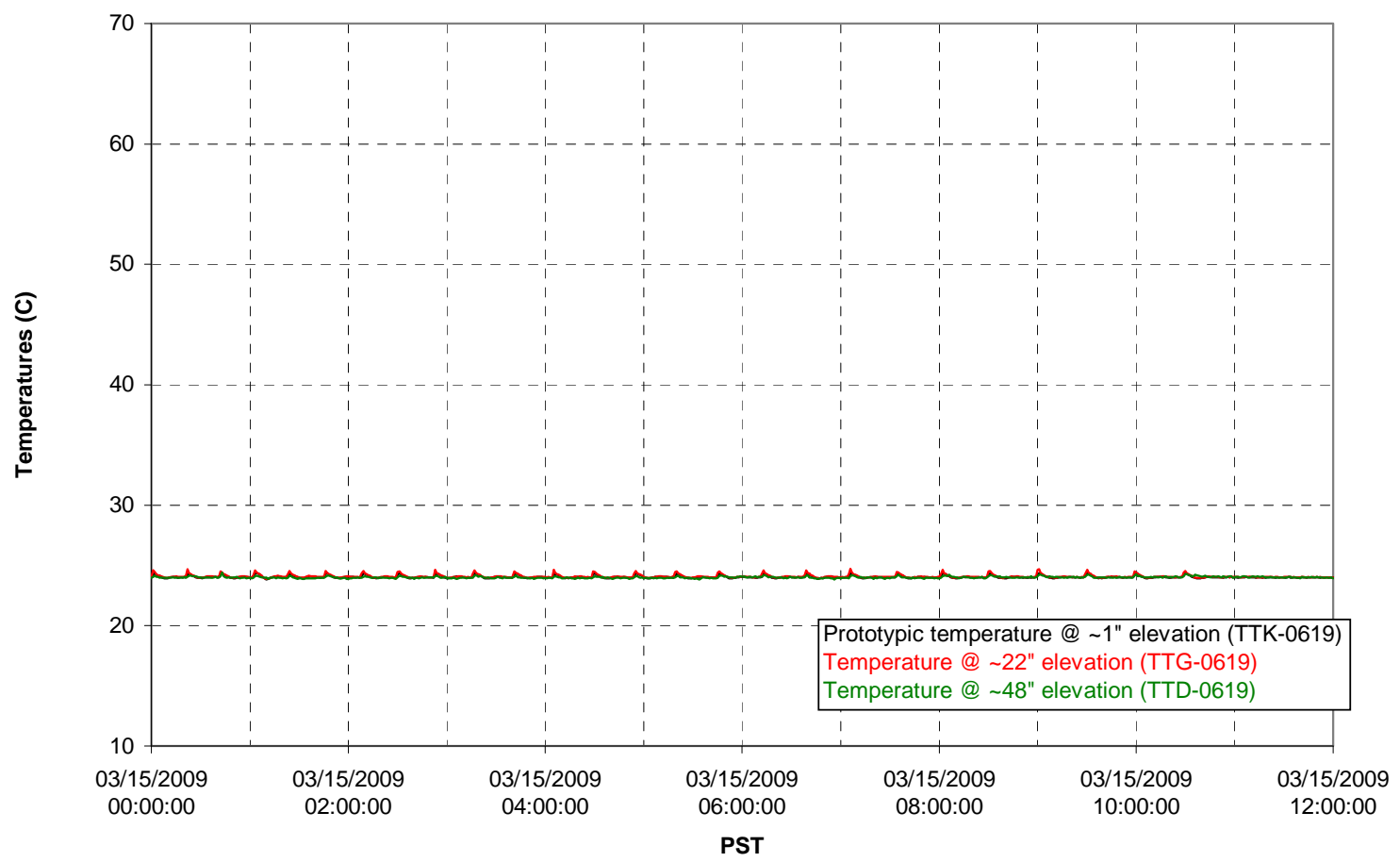


T02A and filter loop temperatures

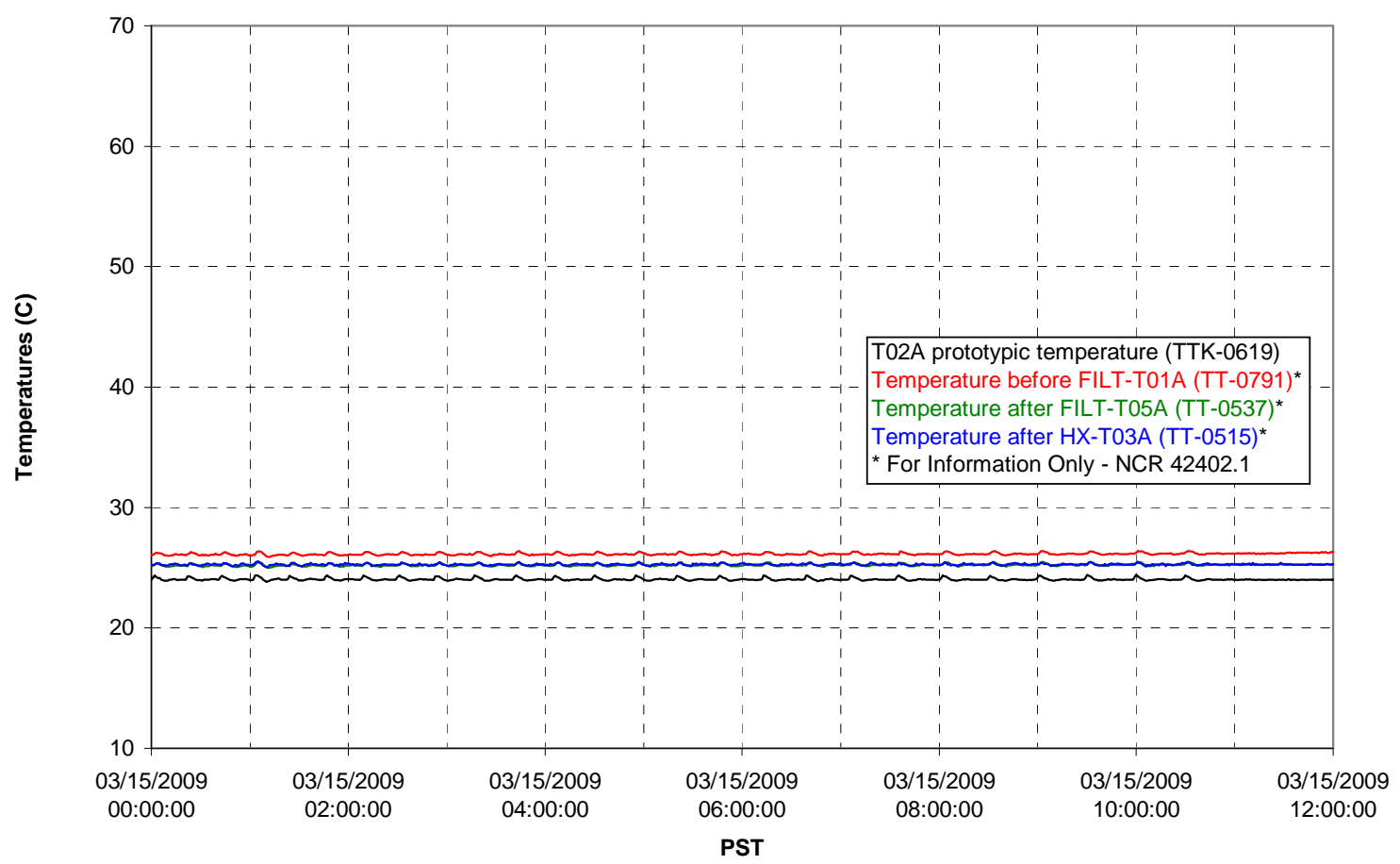

Pump Pressures and Flow

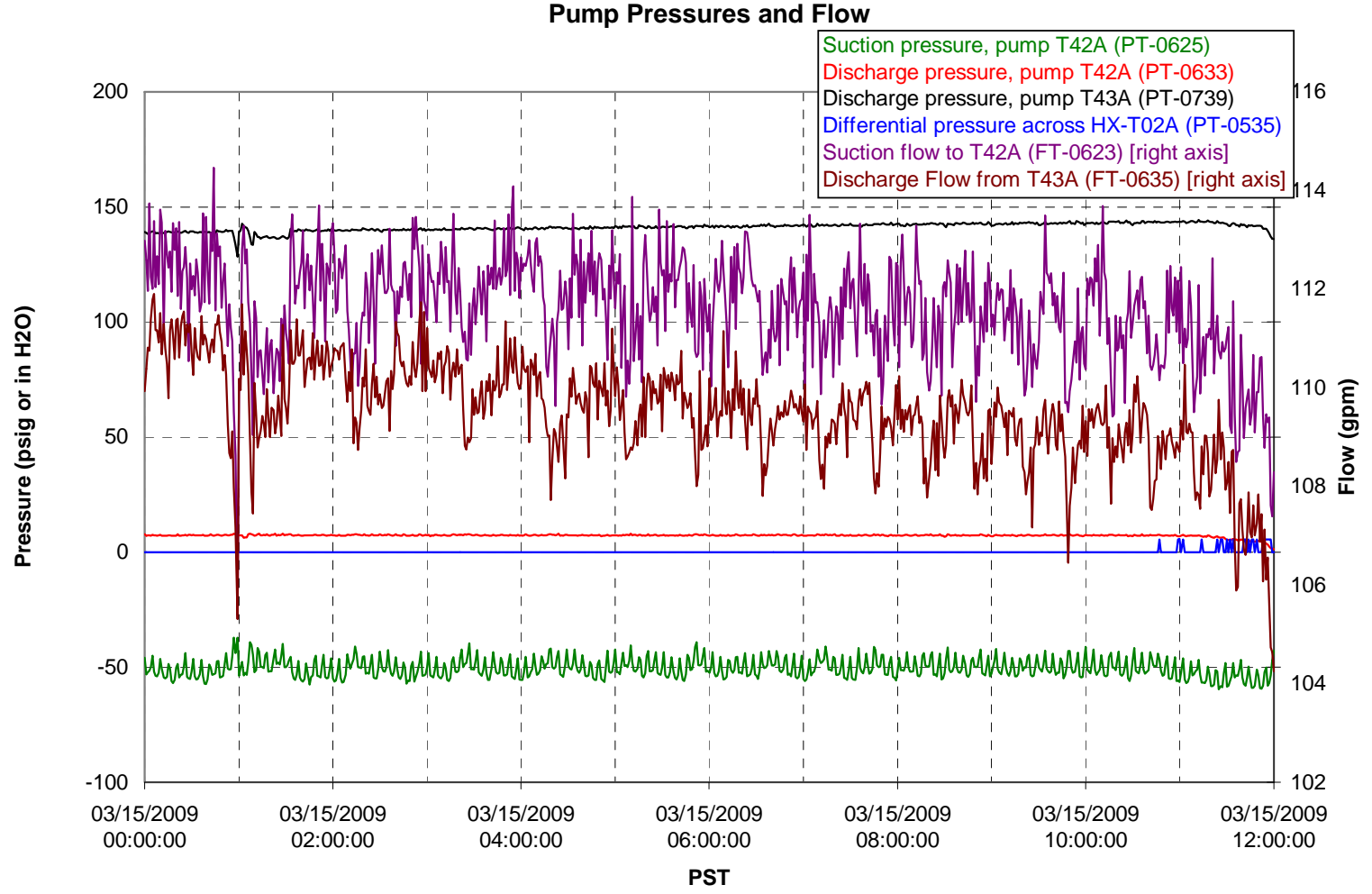




\section{Axial pressure drop}

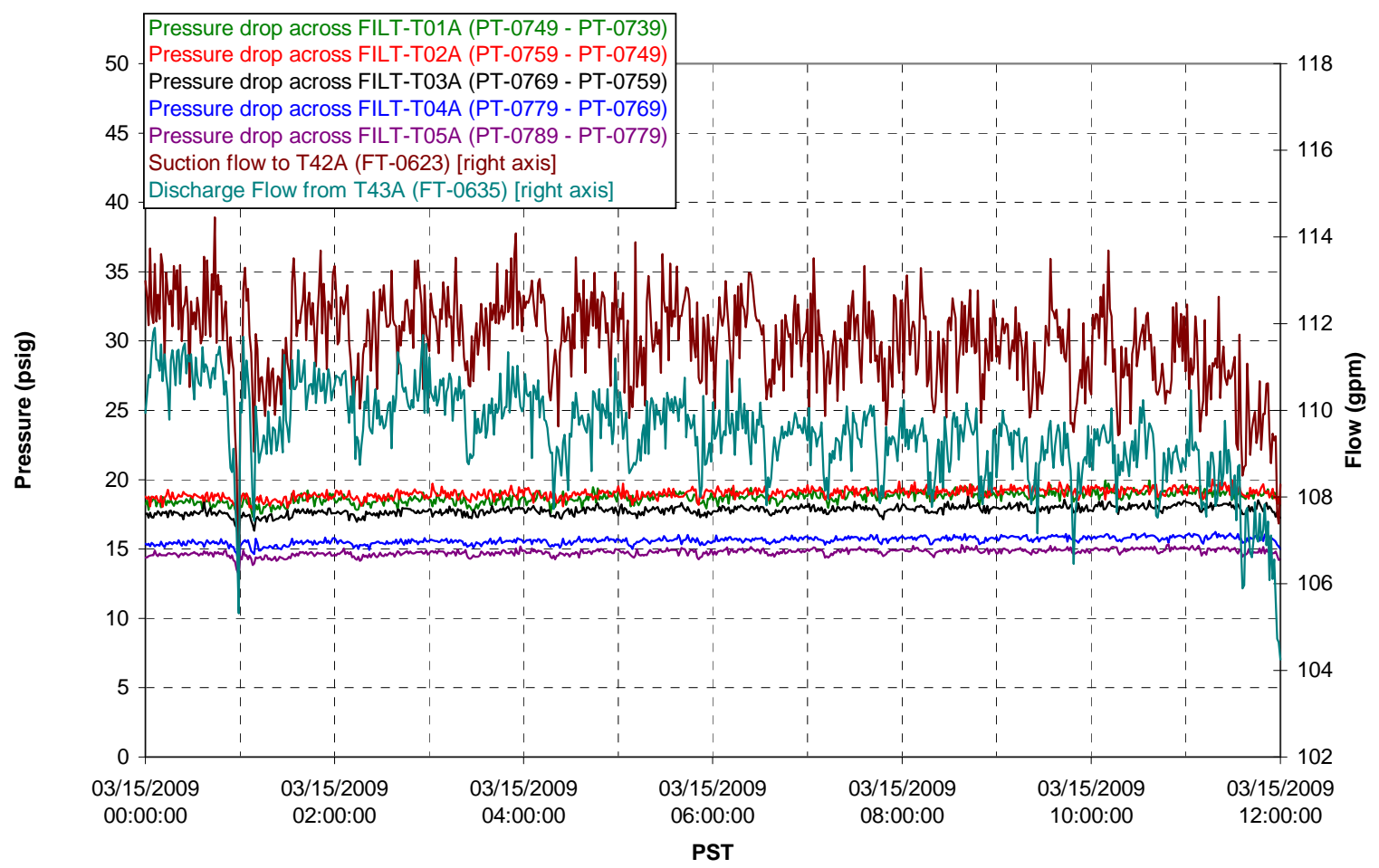

Permeate flow rates

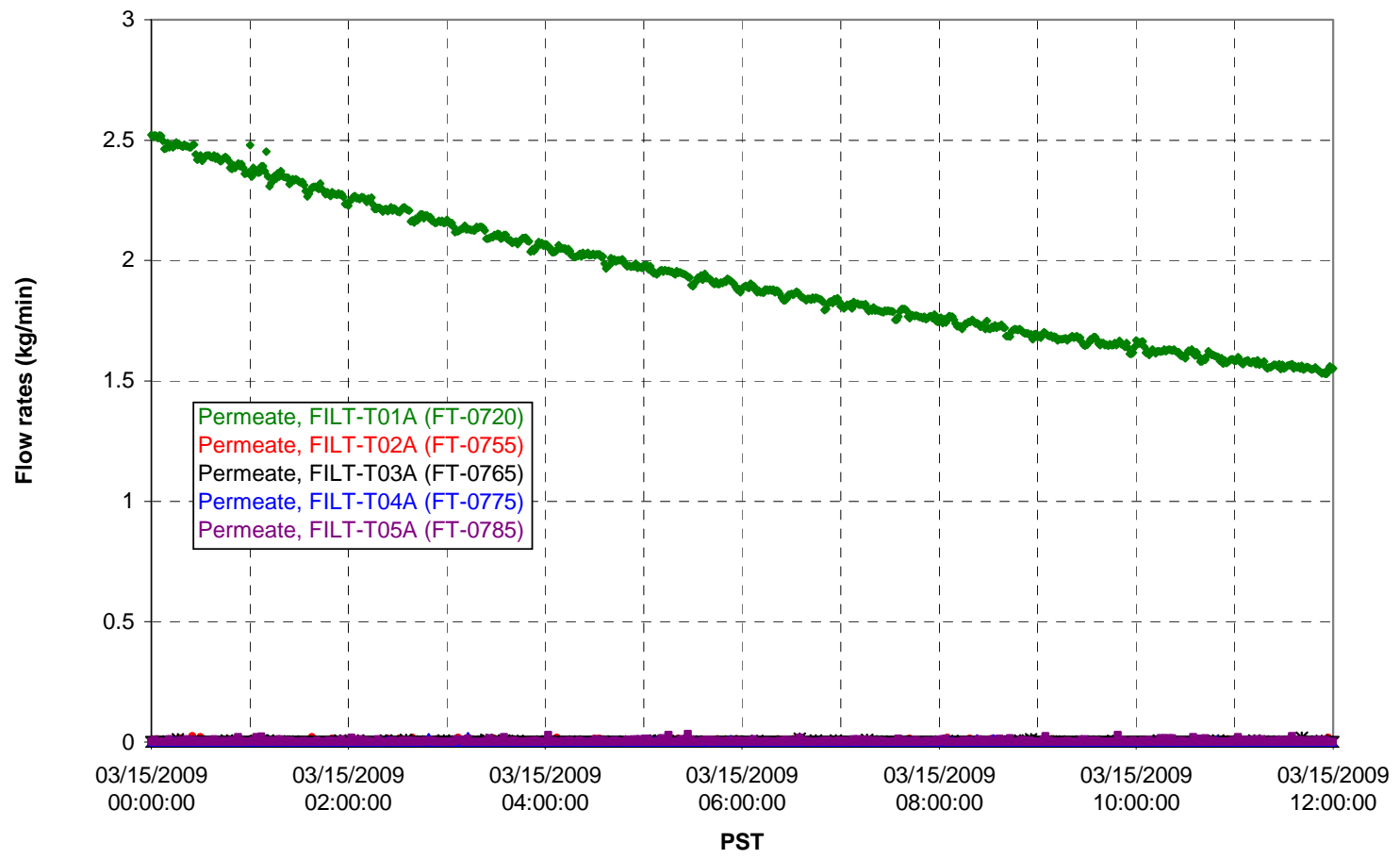


T02A Inner Temperature Tree

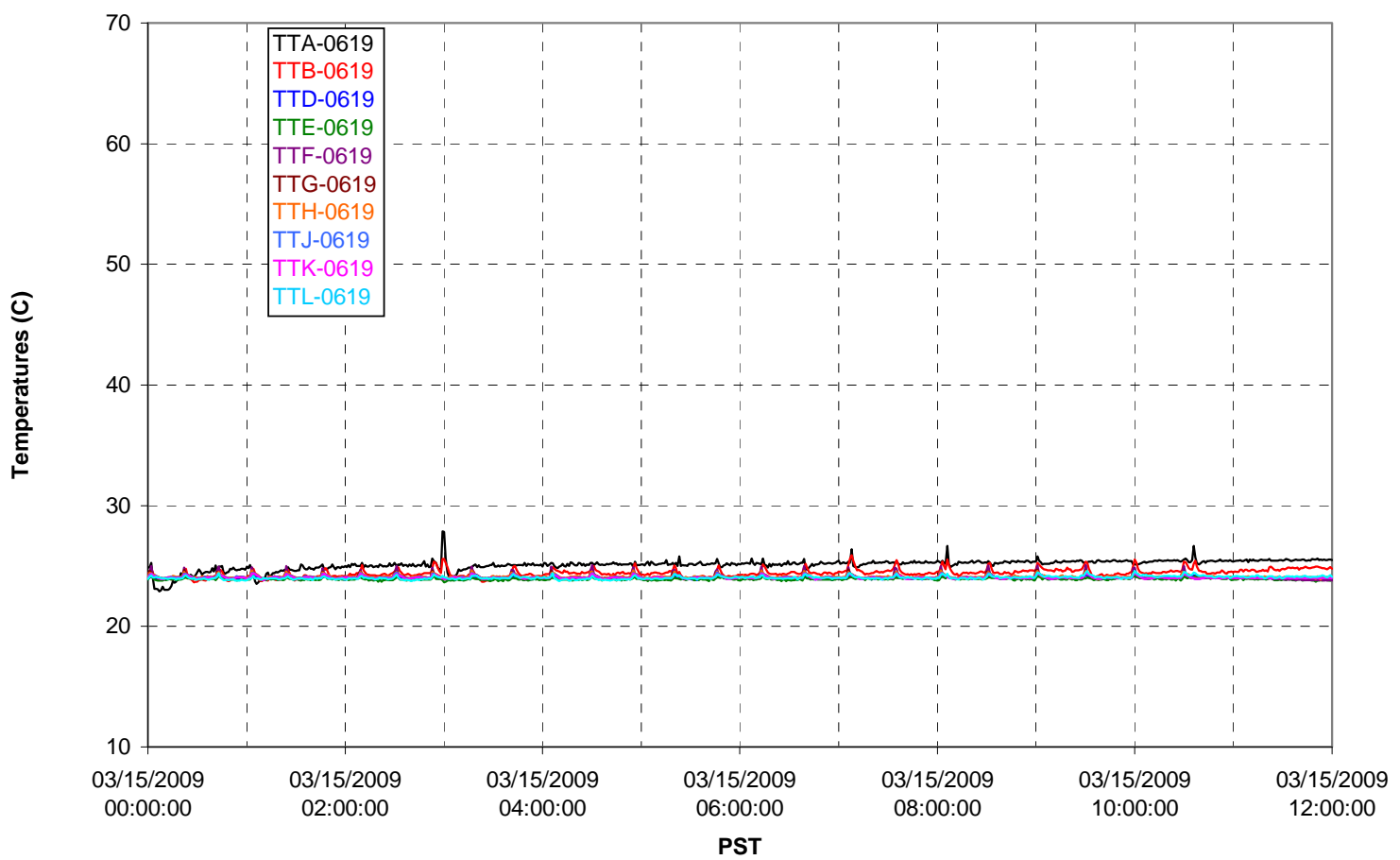

T02A Outer Temperature Tree

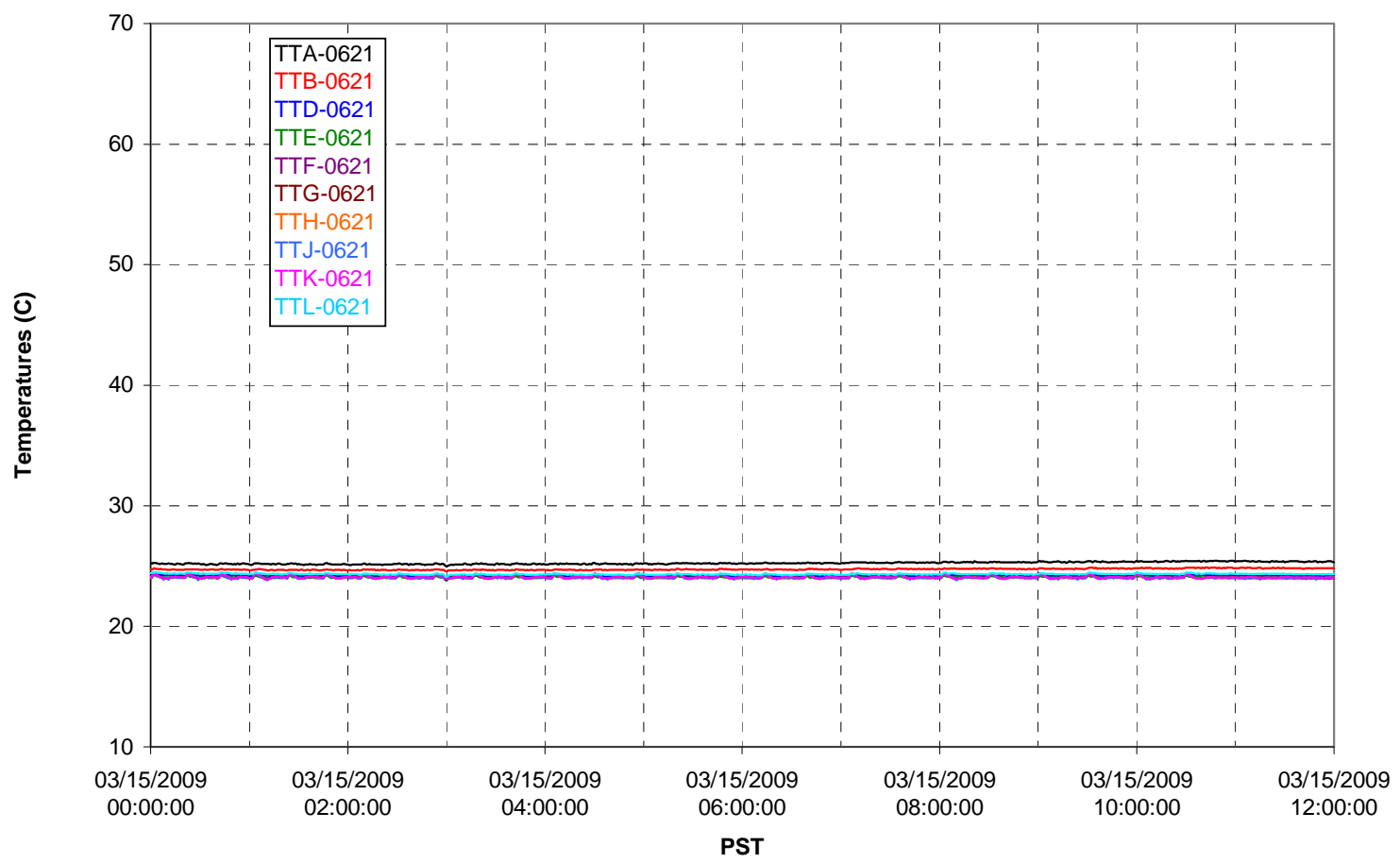


T02A temperatures

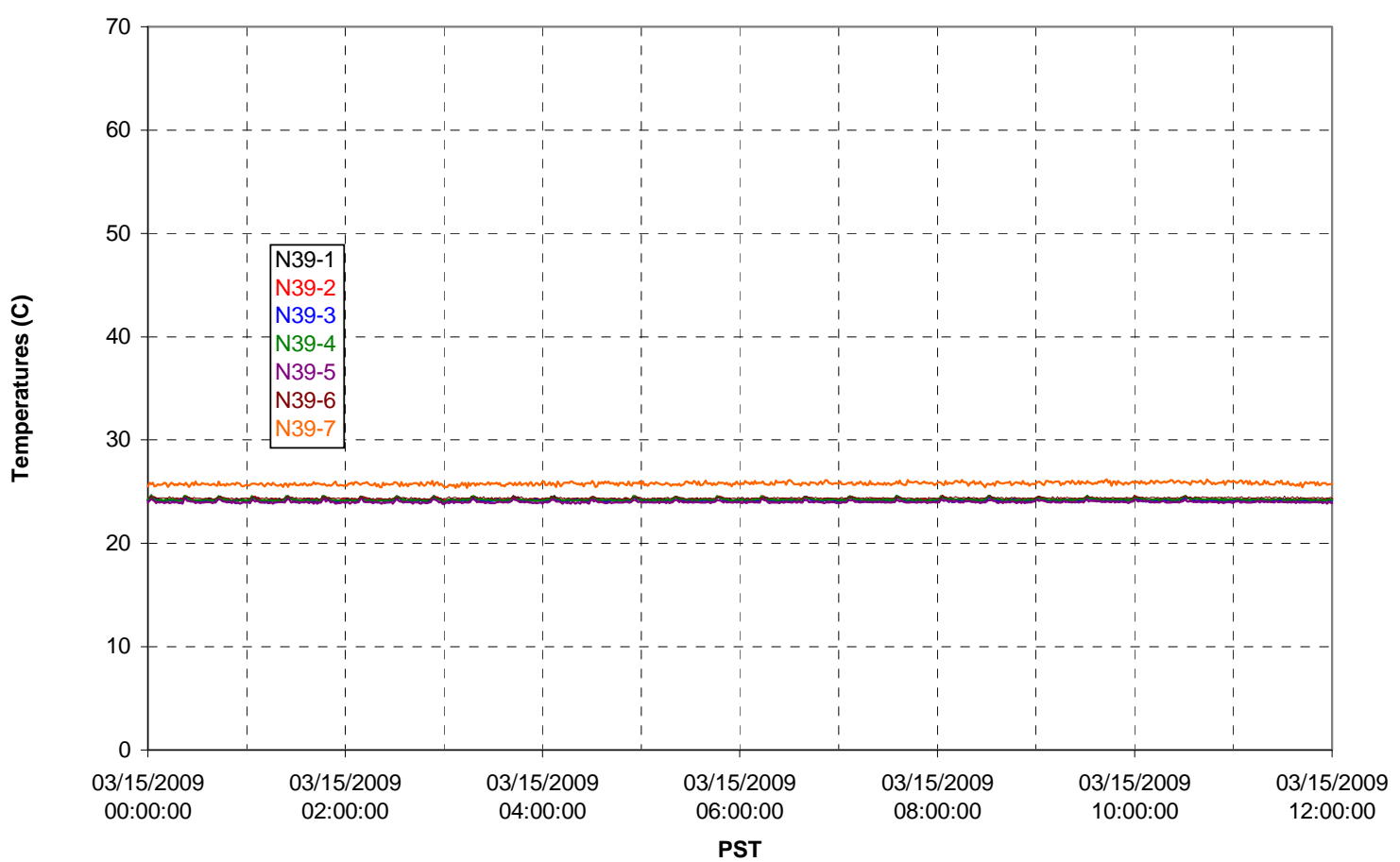

T02A temperatures

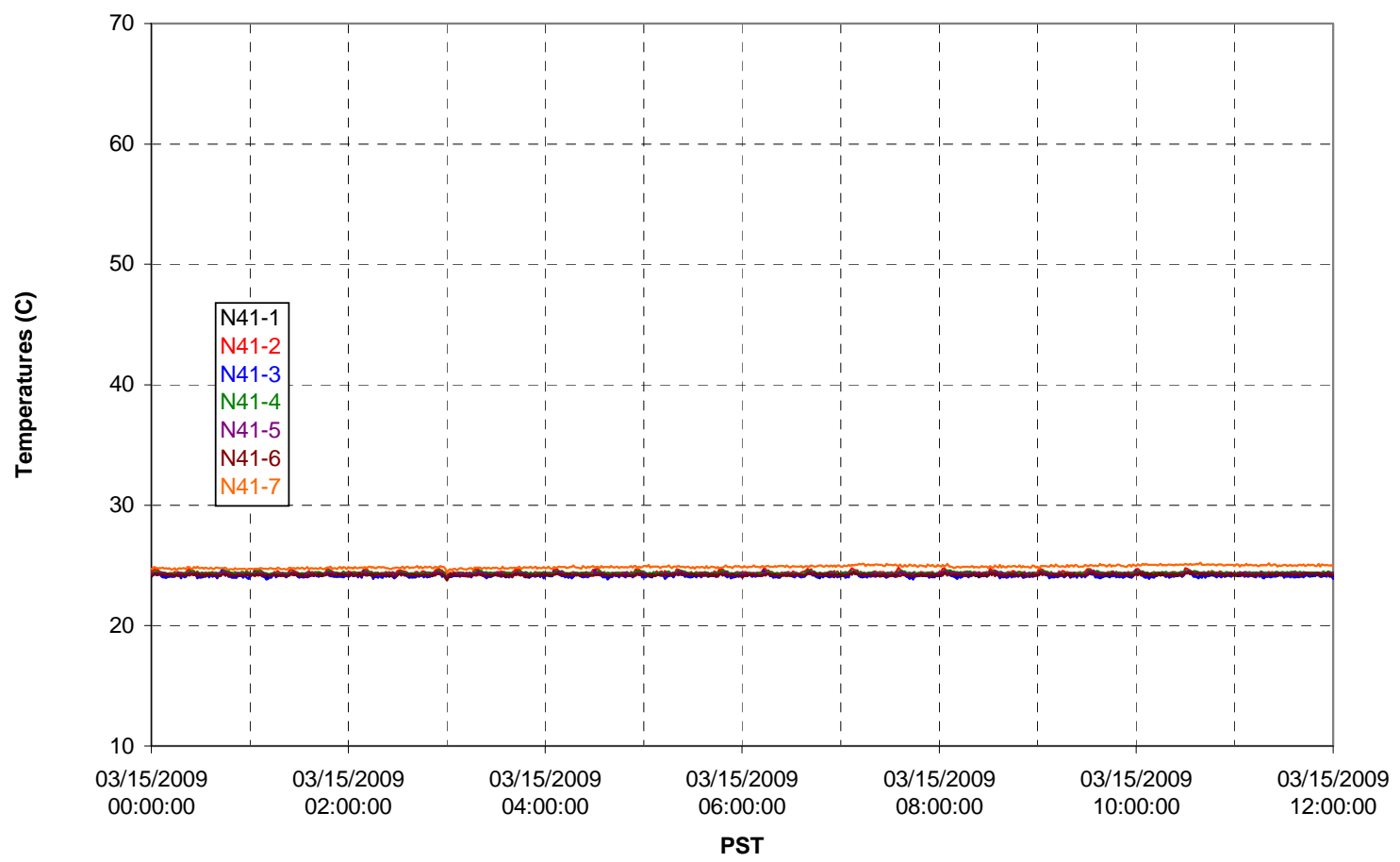


T02A temperatures

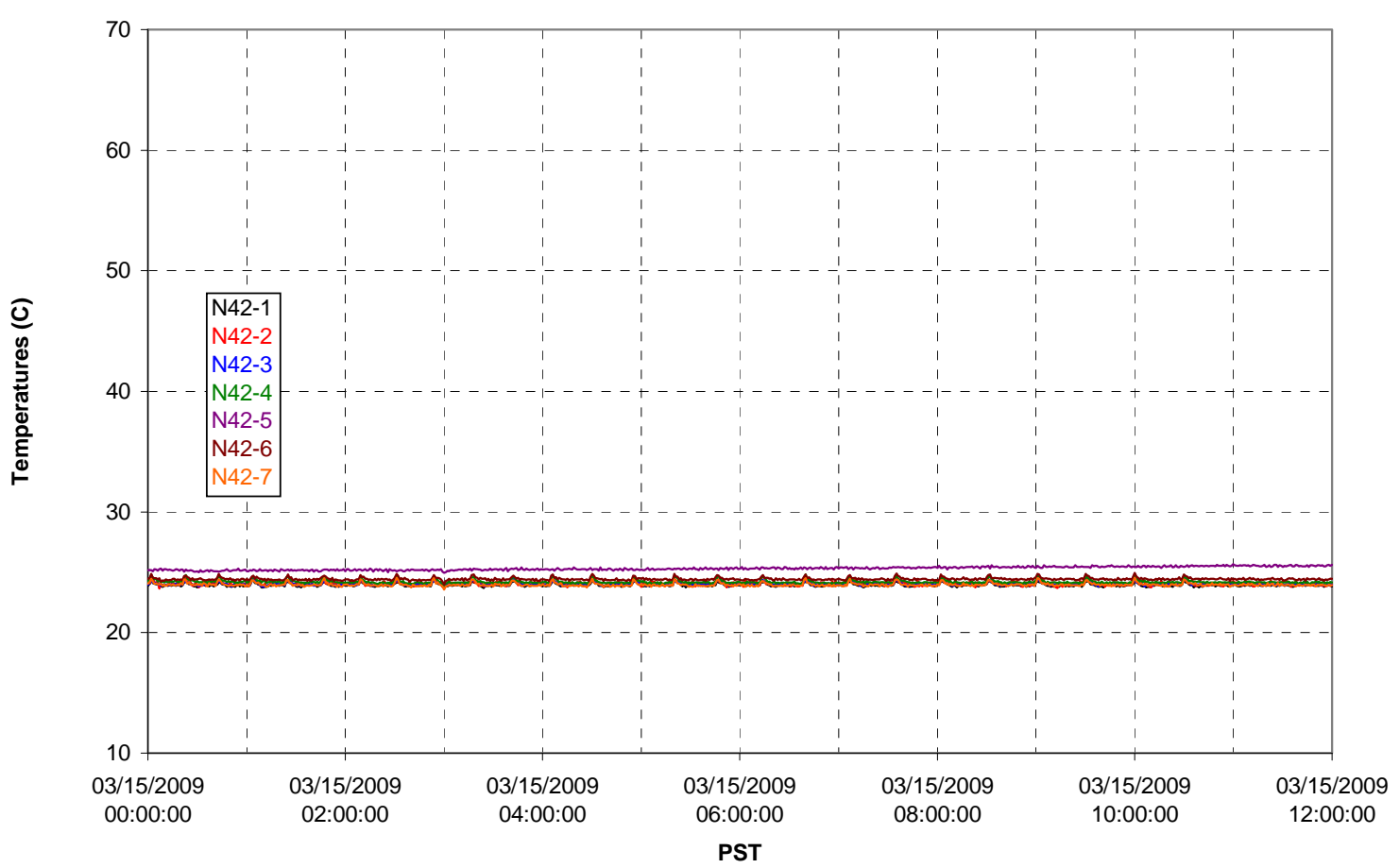

T02A temperatures

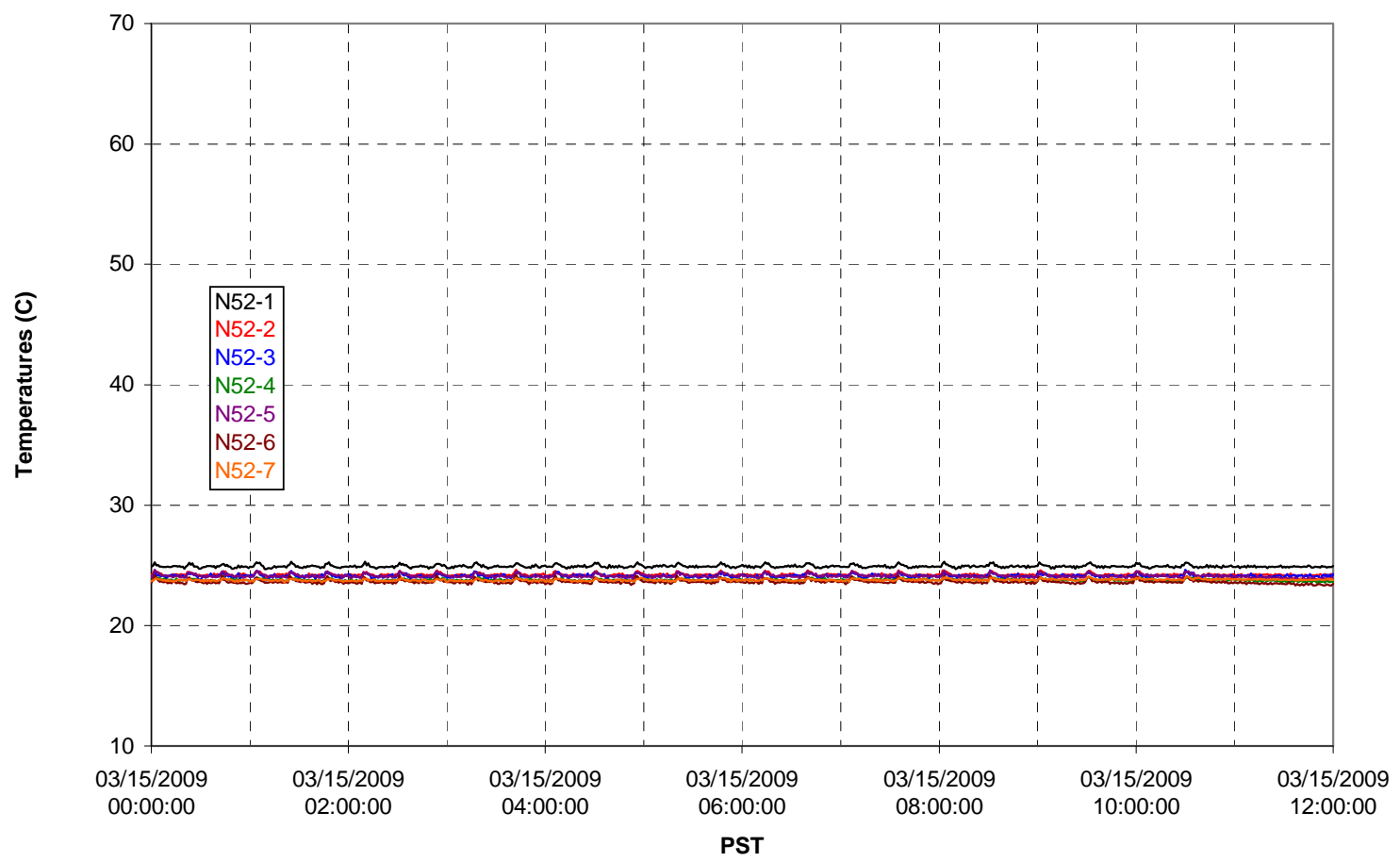


T02A Heating and Cooling

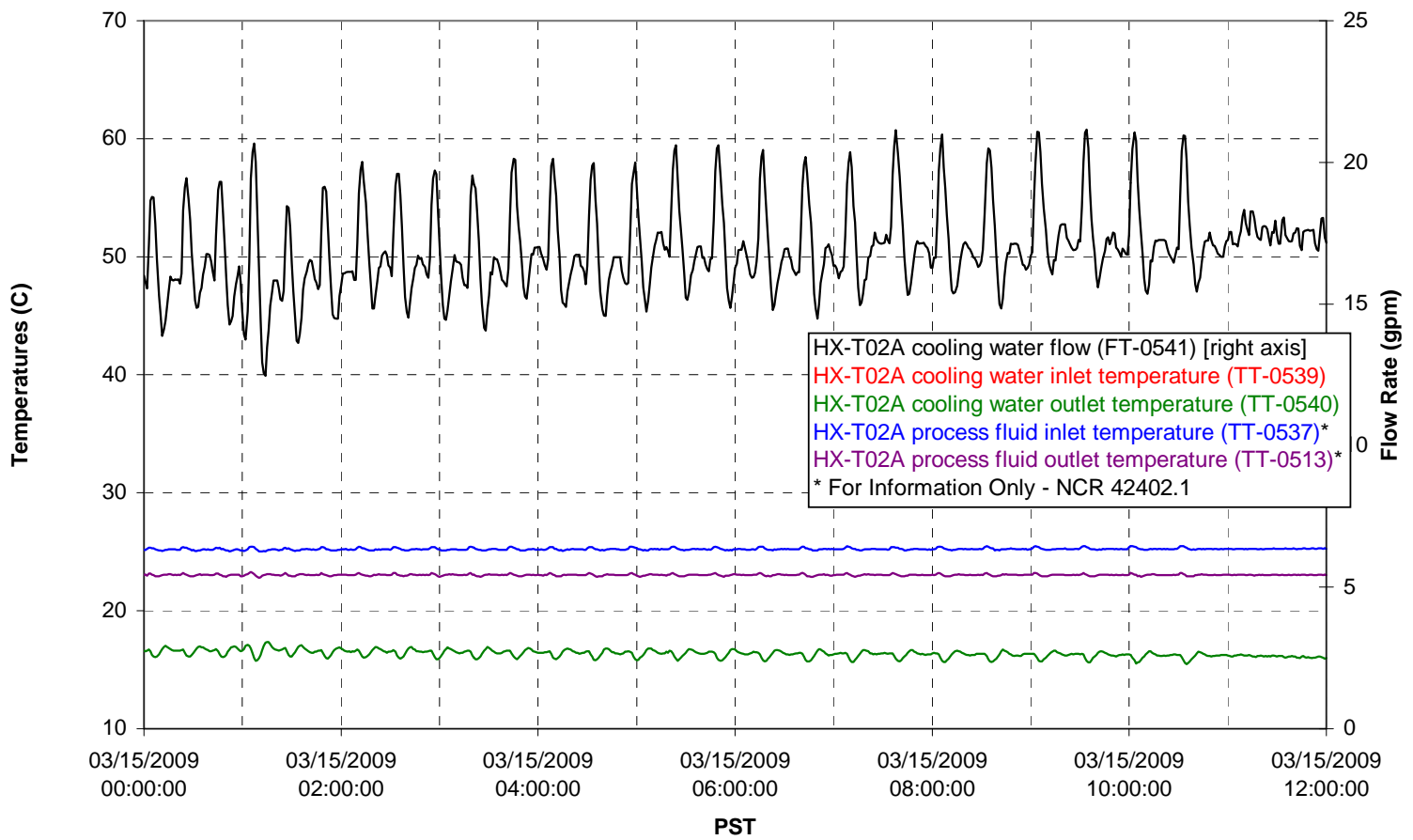

Pump Operation

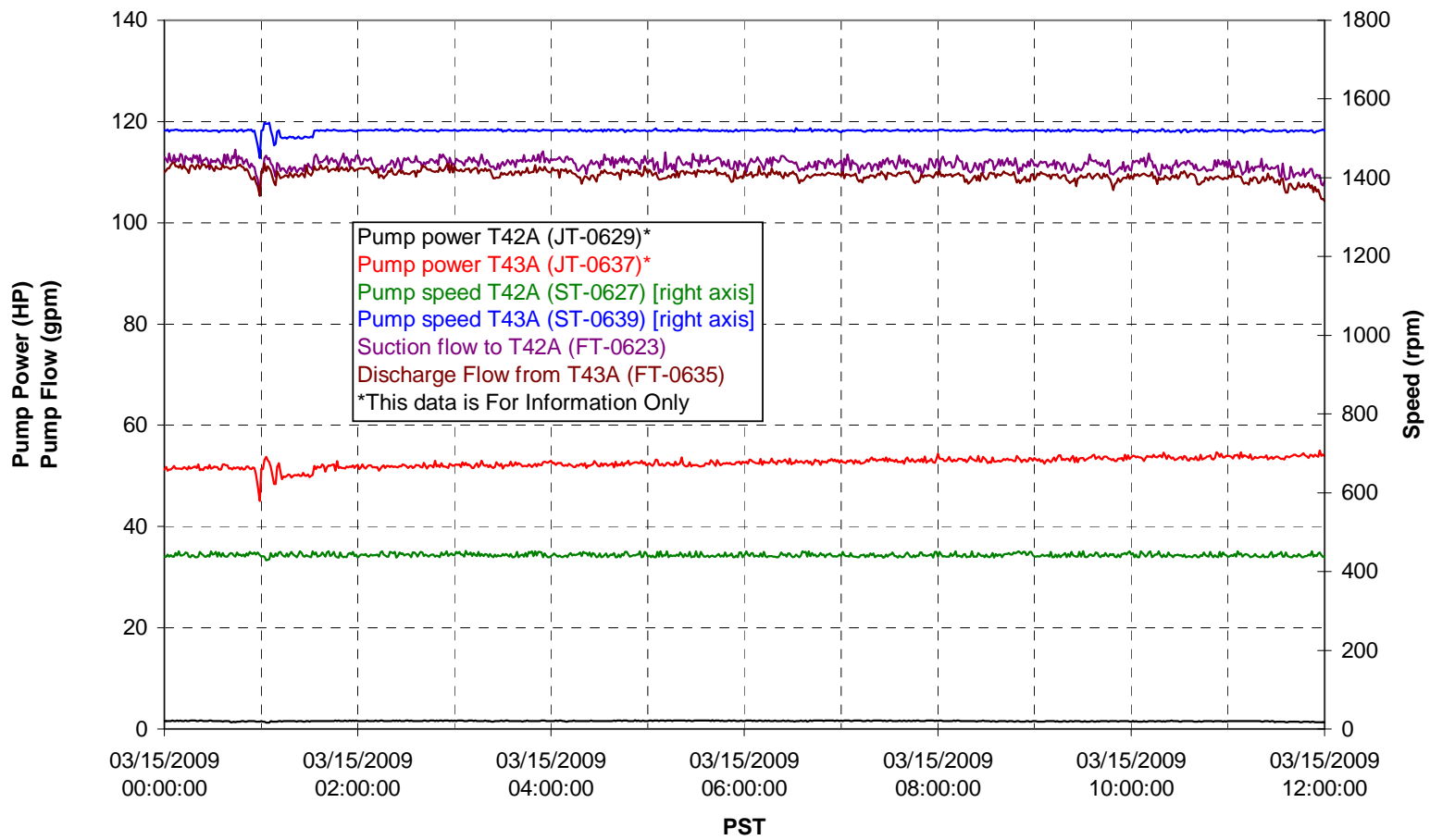


Pulsepot UFP-PP-T01A

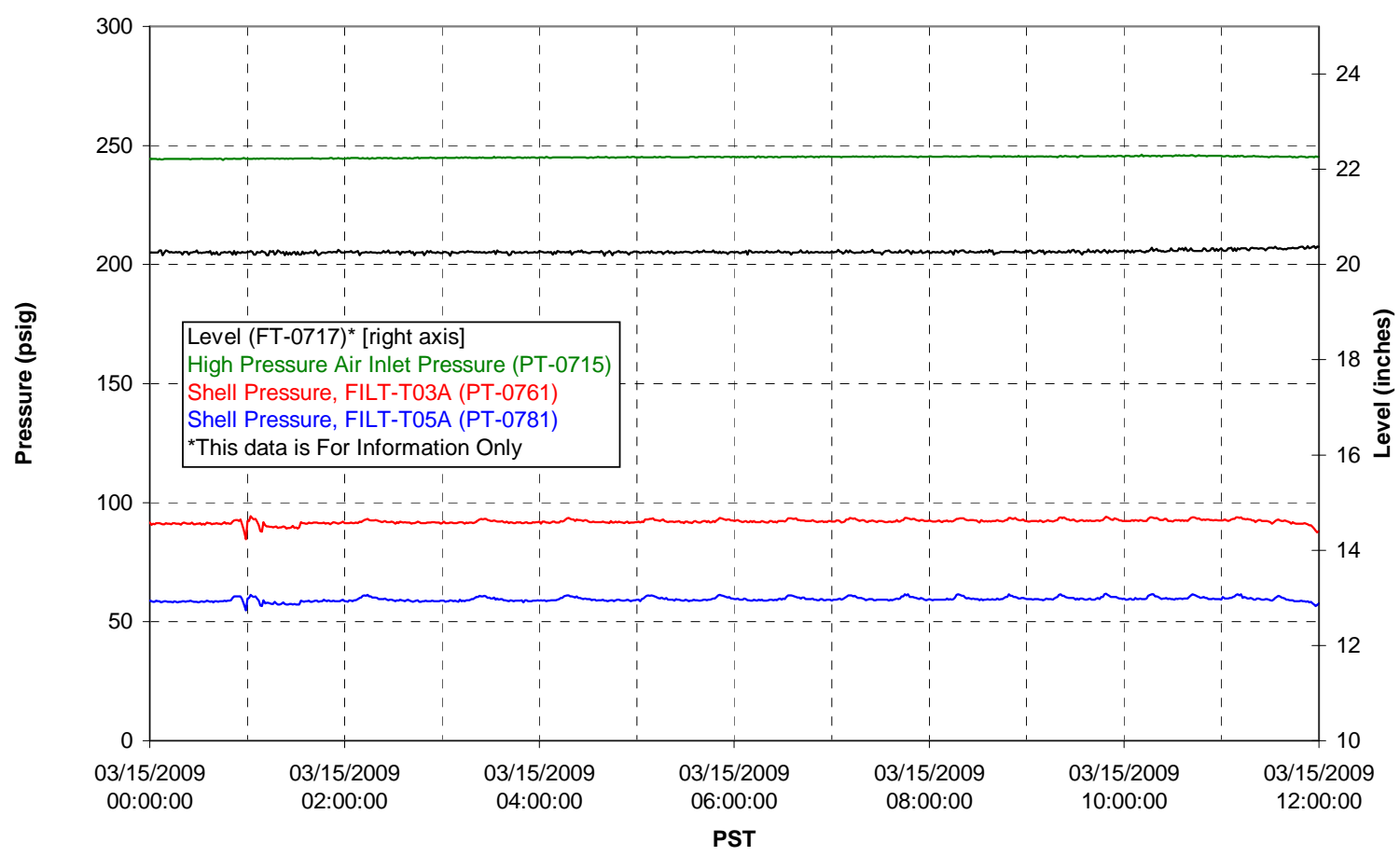

Pulsepot UFP-PP-T02A

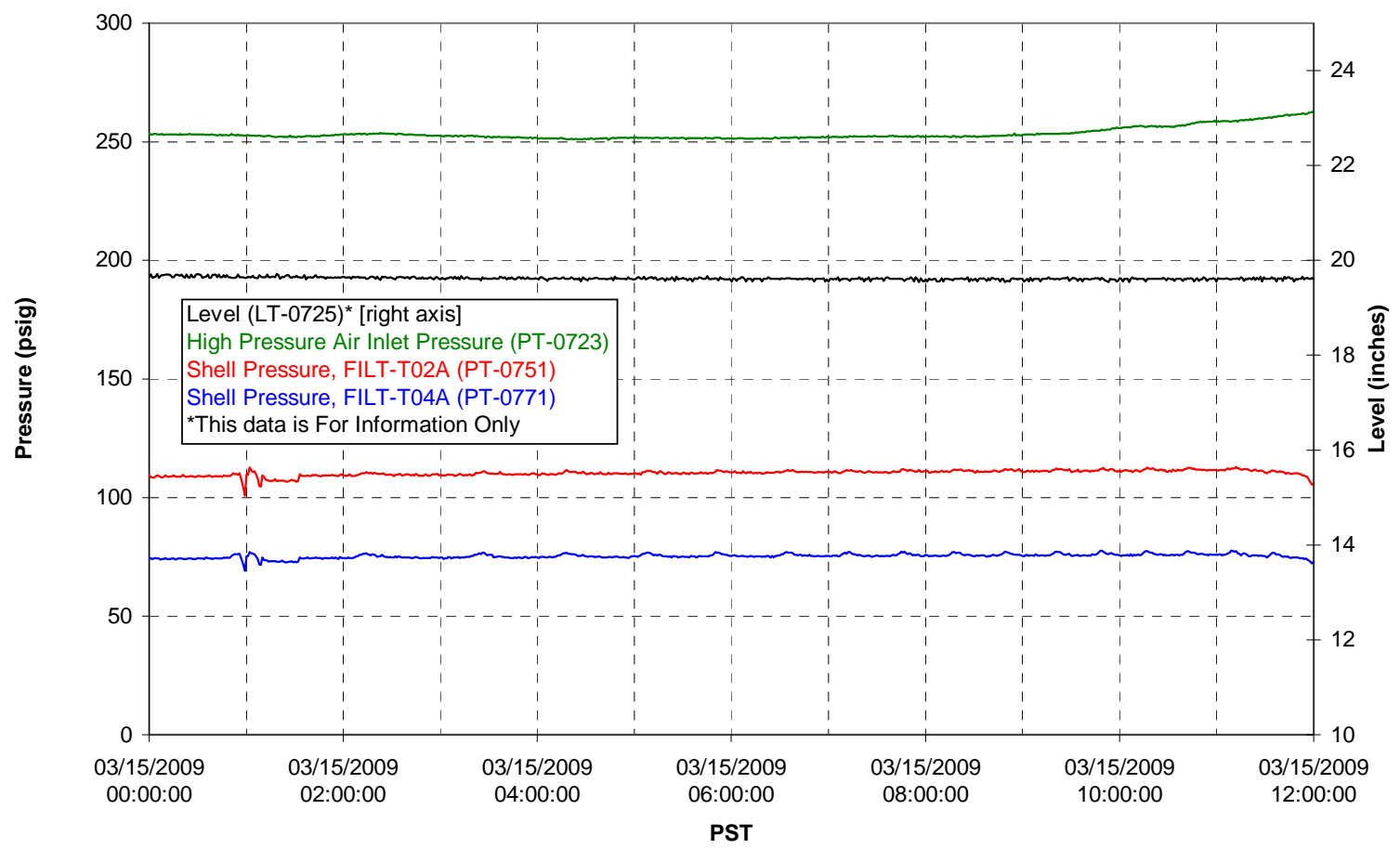


Pulsepot UFP-PP-T03A

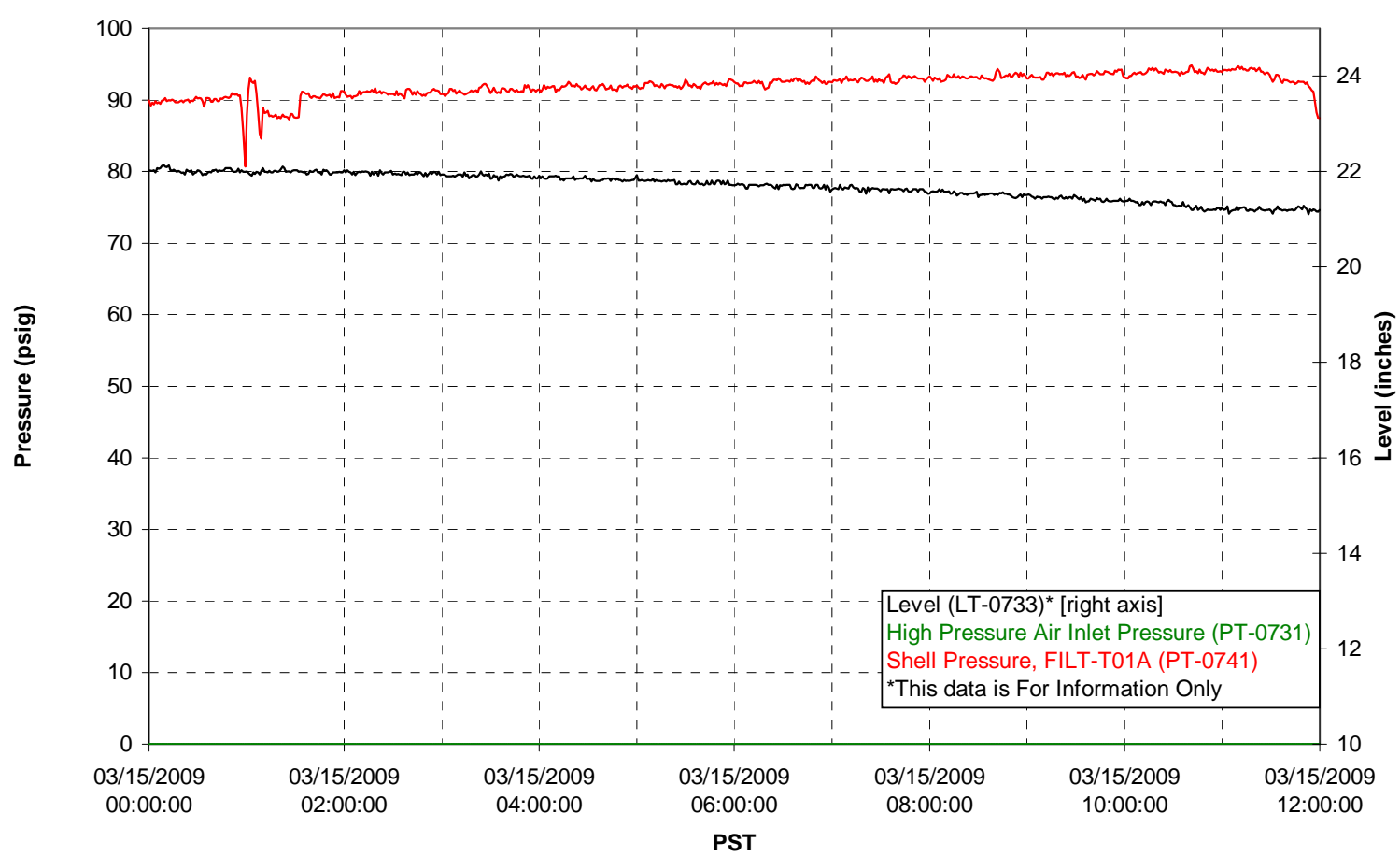

Pulsepot Levels

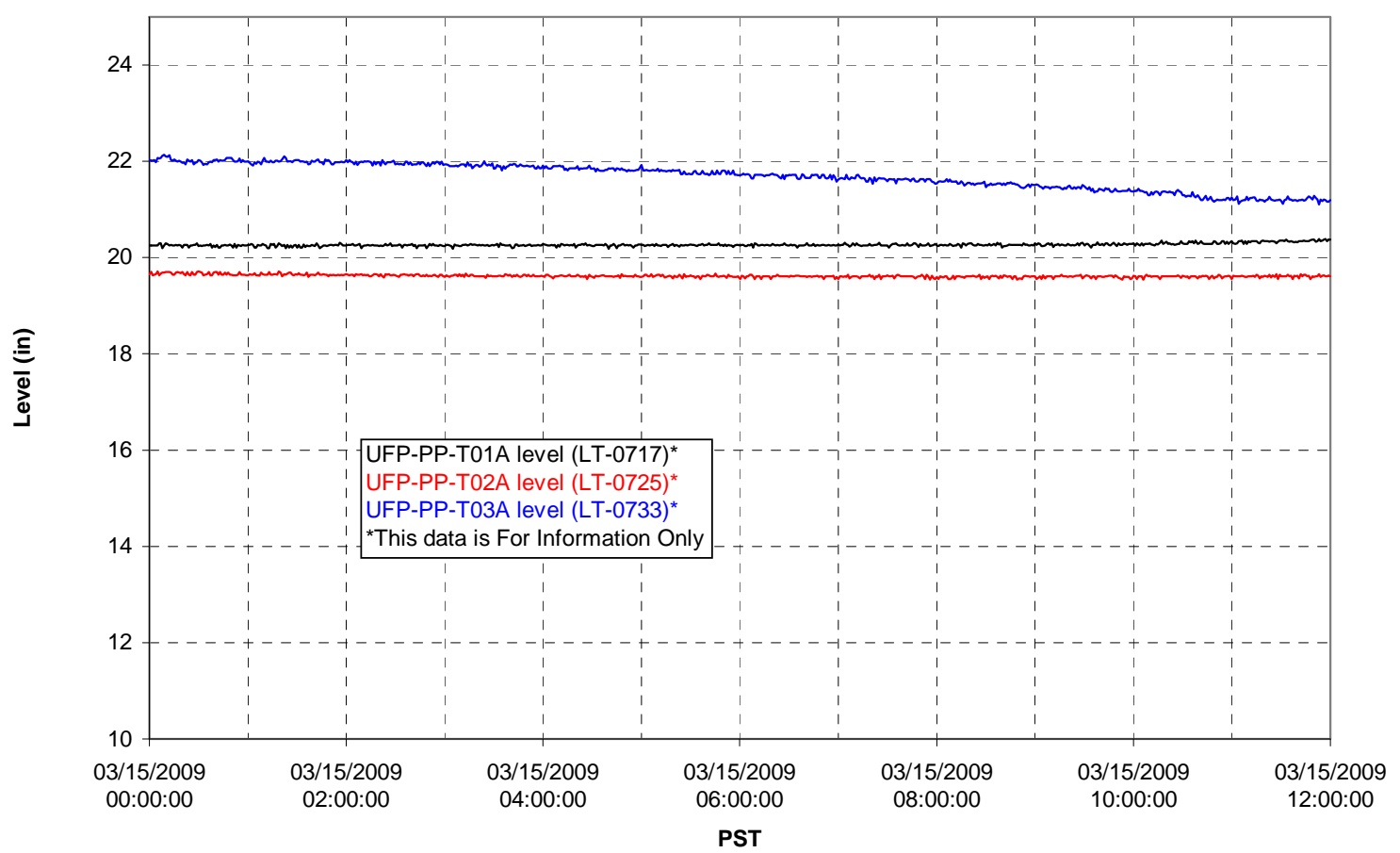


Filter UFP-FILT-T01A

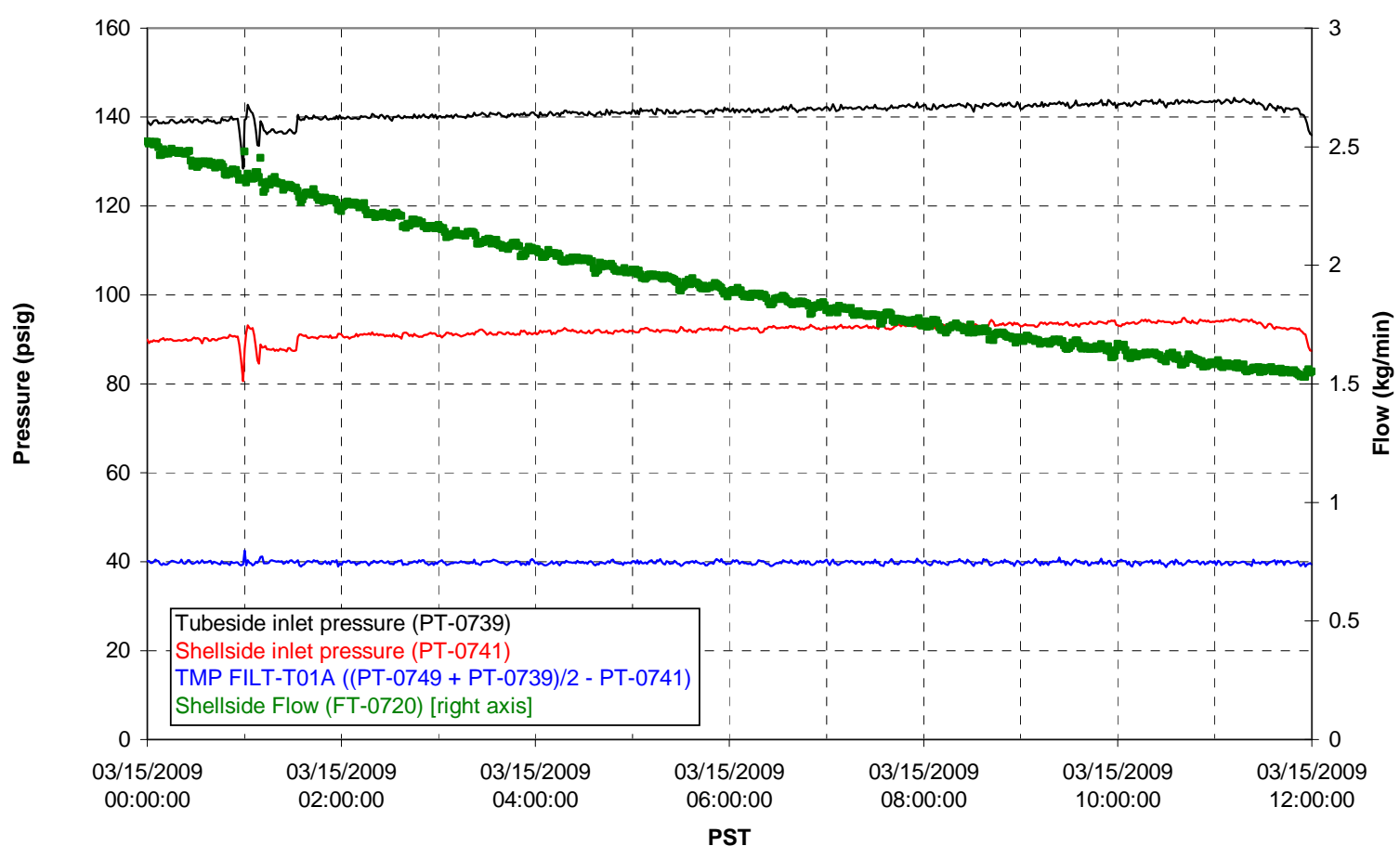

Filter UFP-FILT-T02A

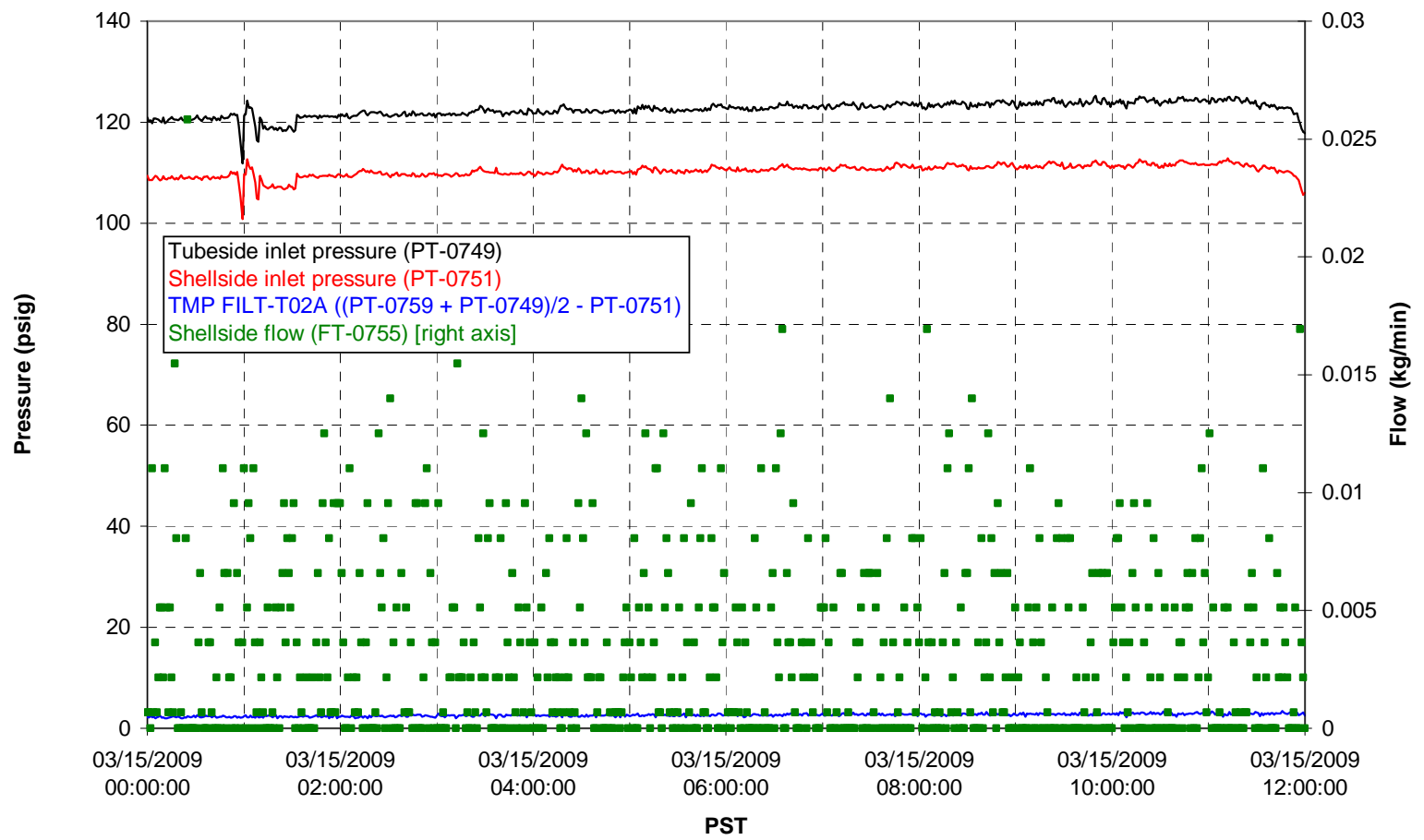


Filter UFP-FILT-T03A

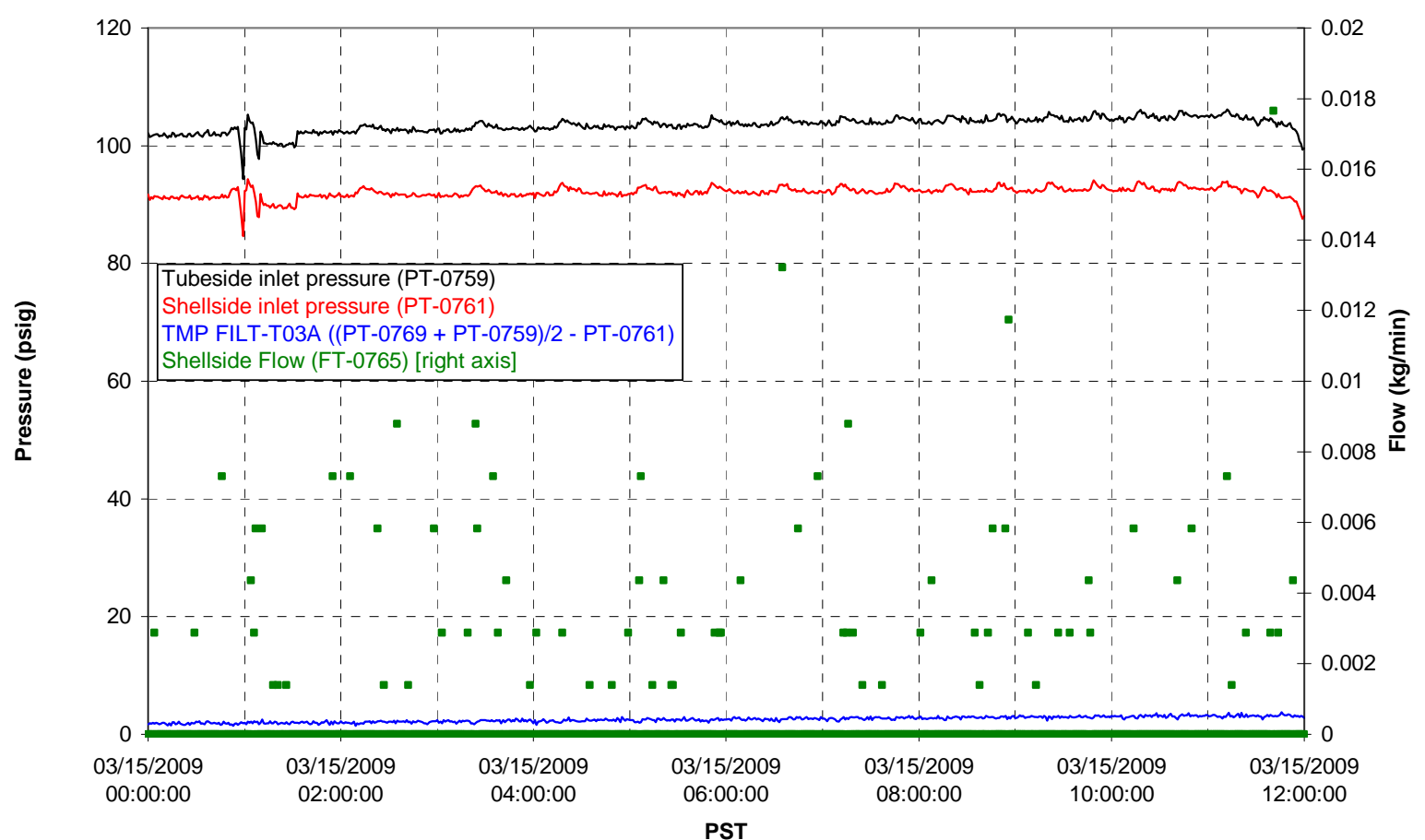

Filter UFP-FILT-T04A

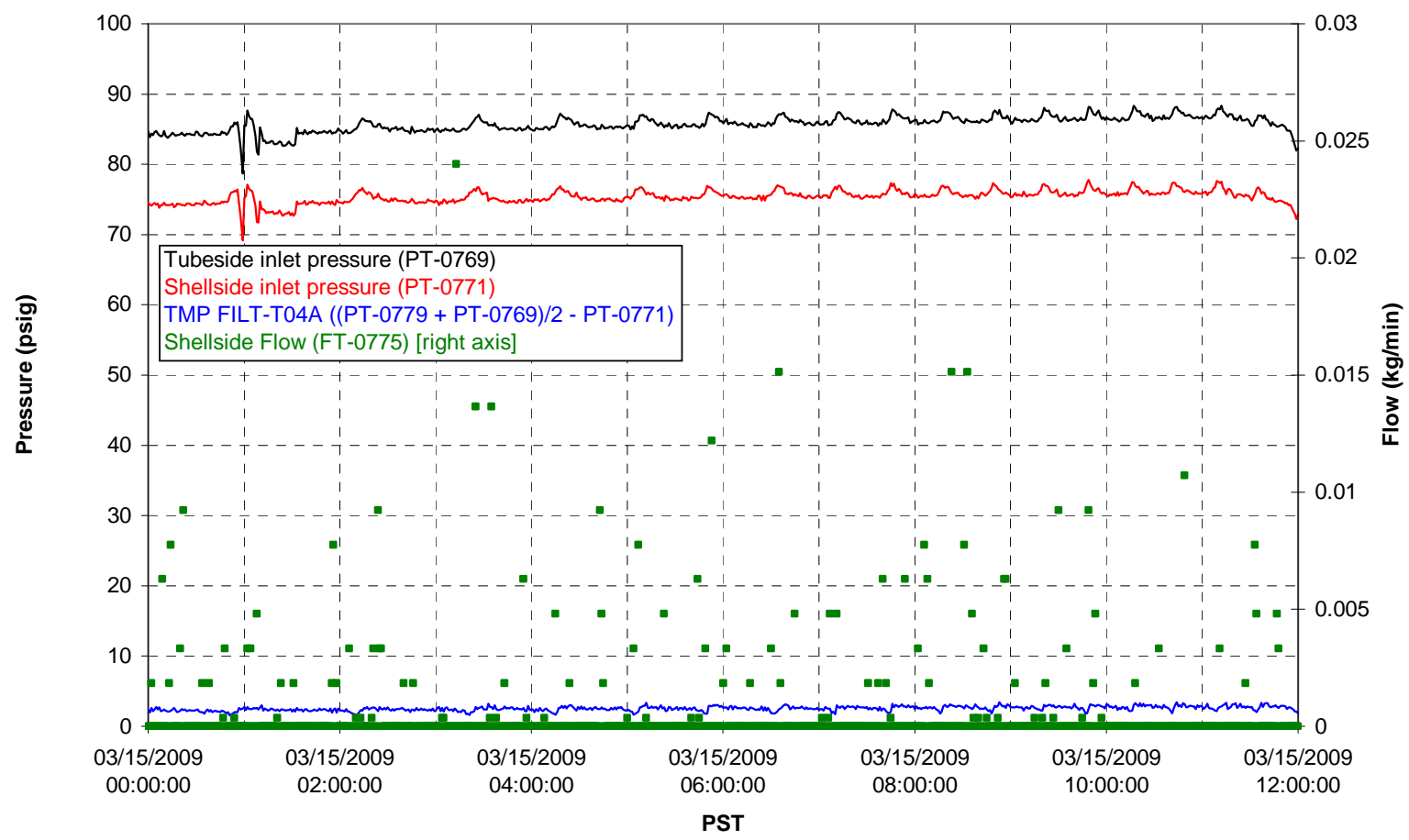


Filter UFP-FILT-T05A

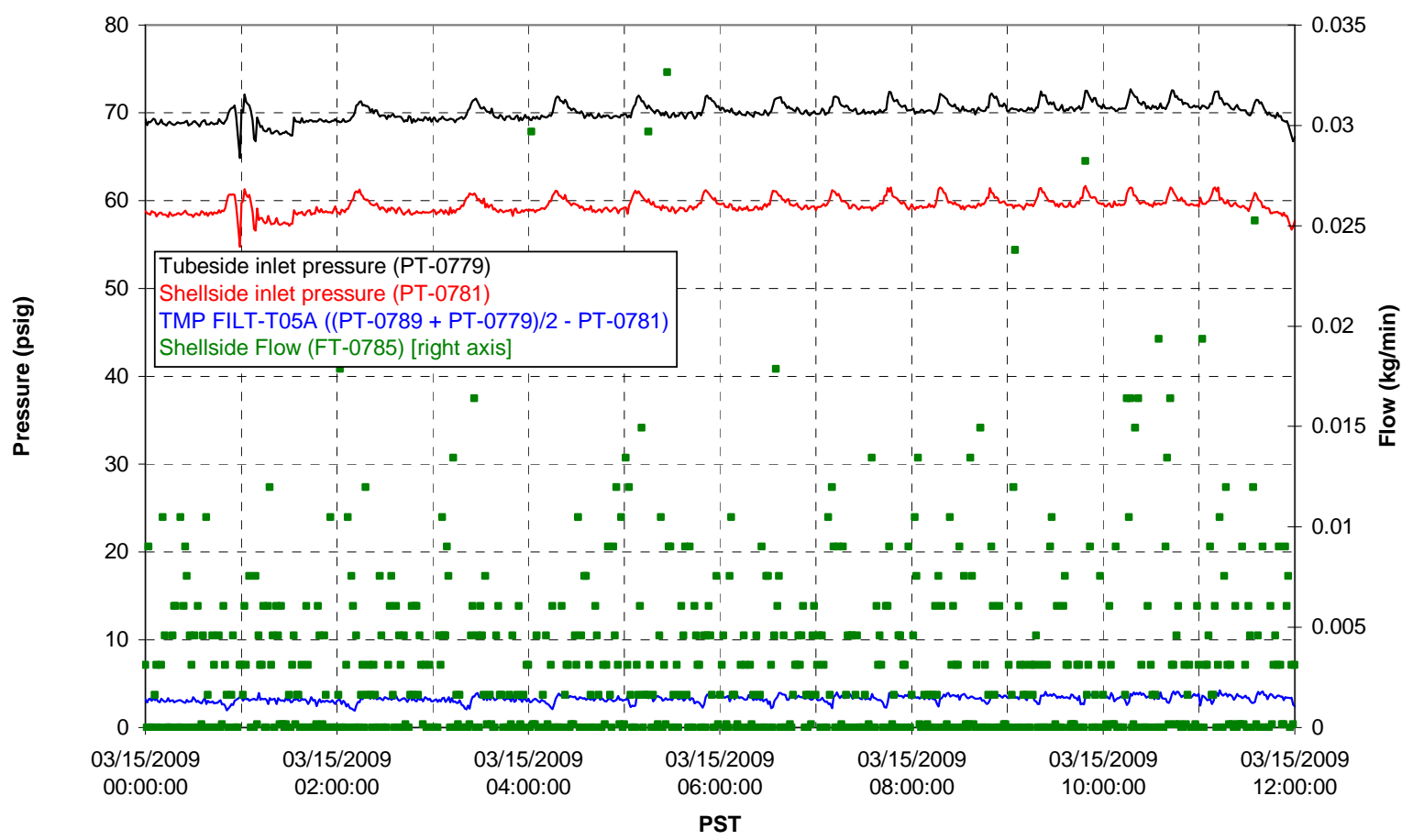

Chemical Flow

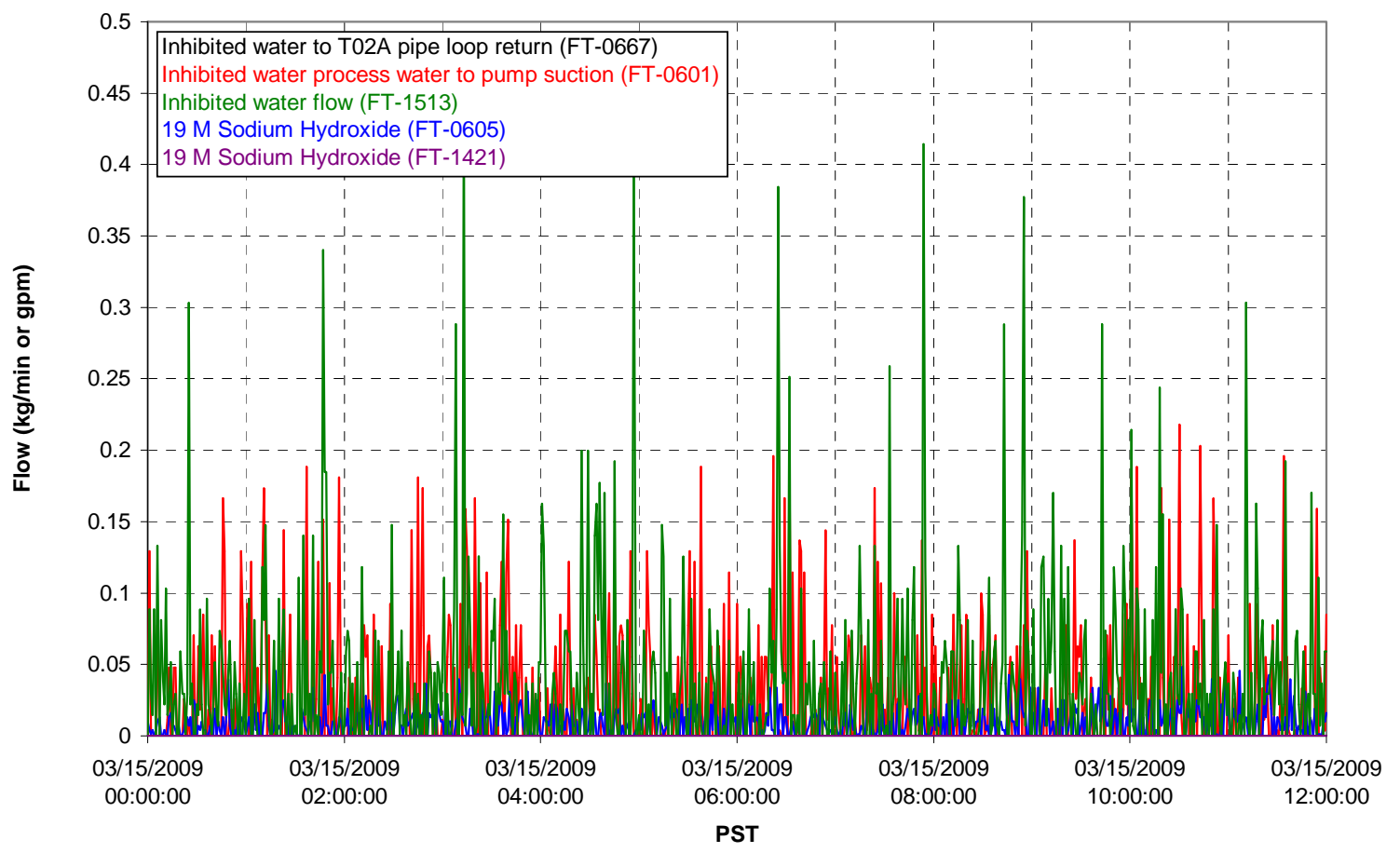




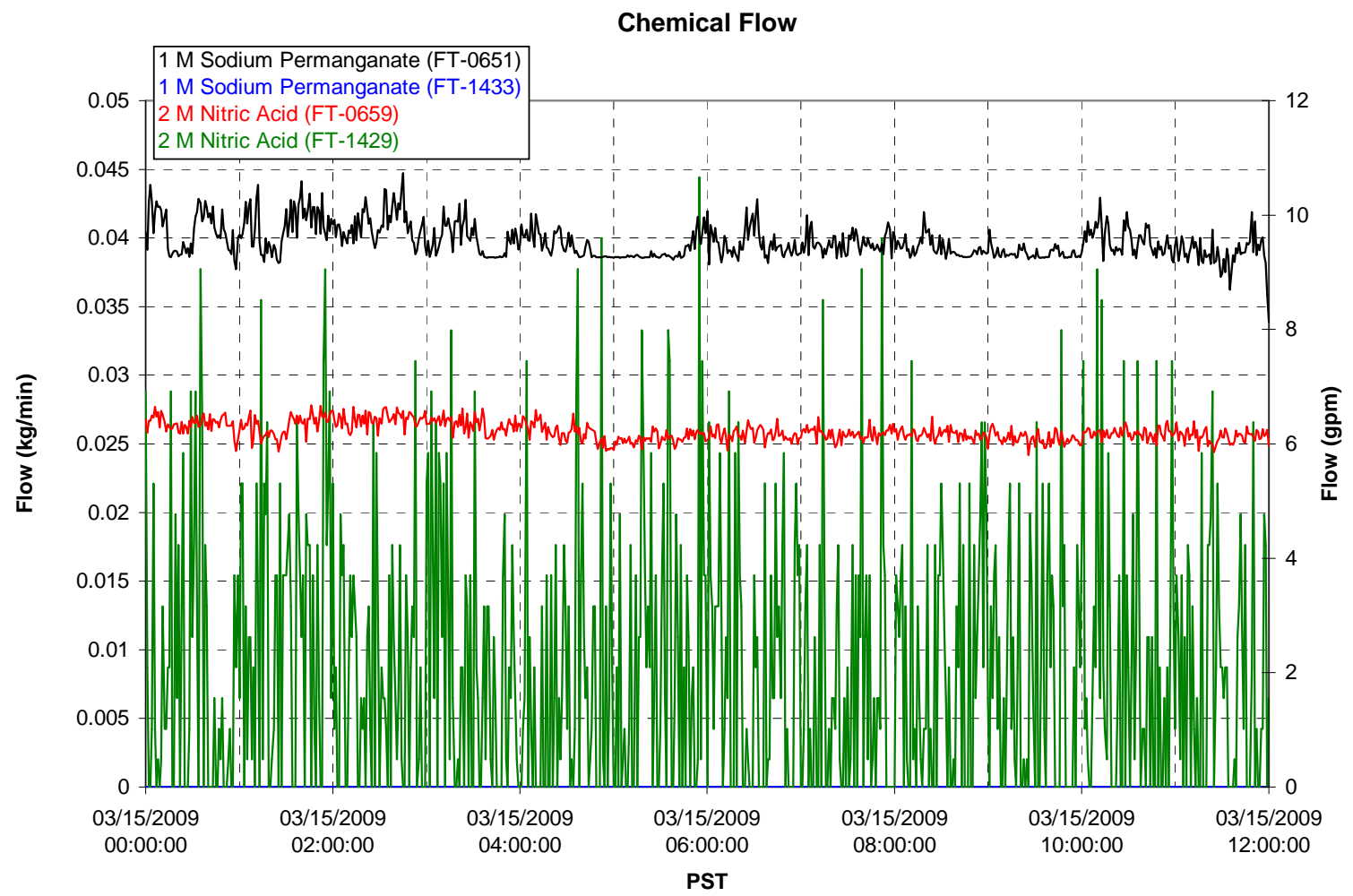

Air Flows

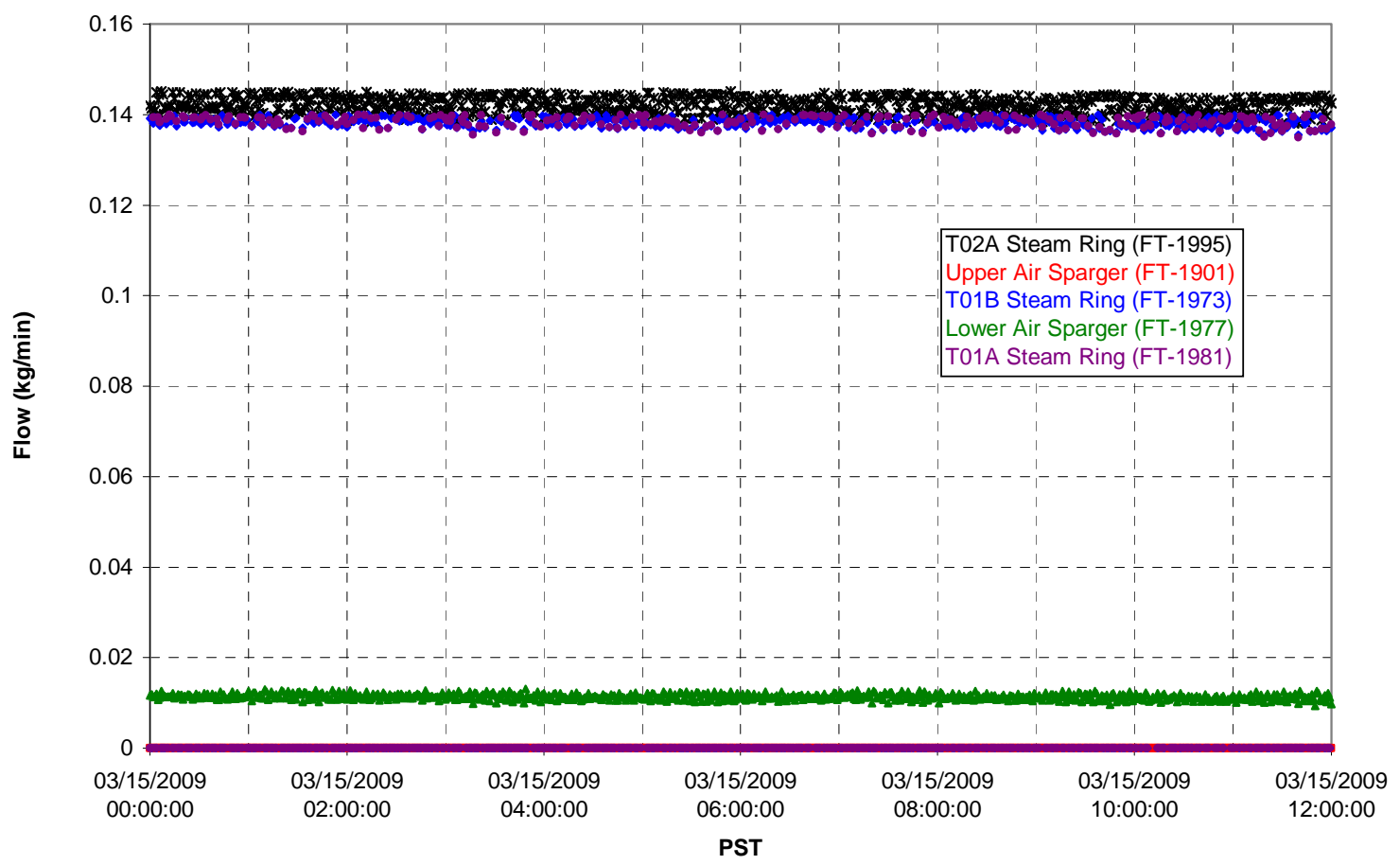


T02A Steam

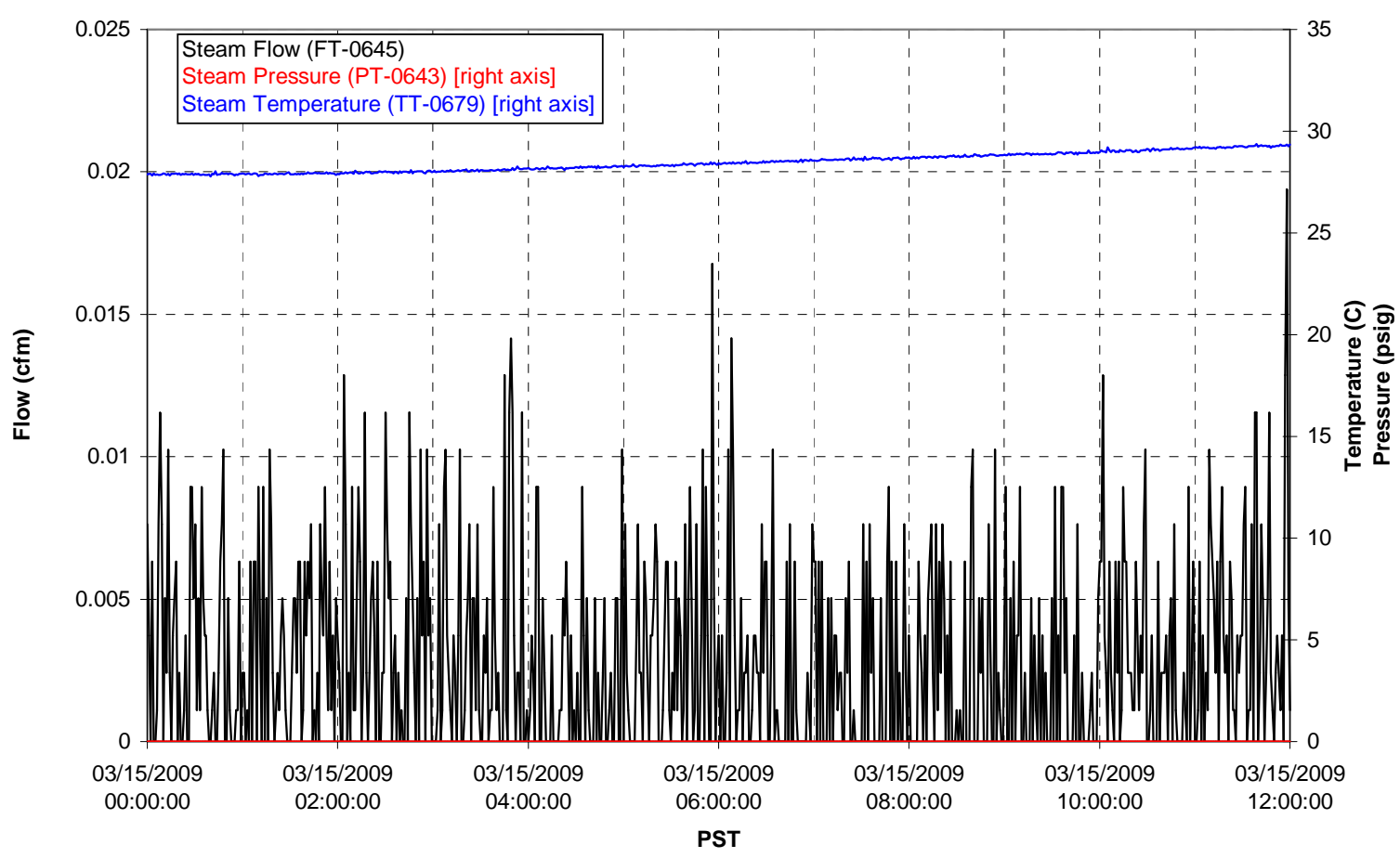

T01A Steam

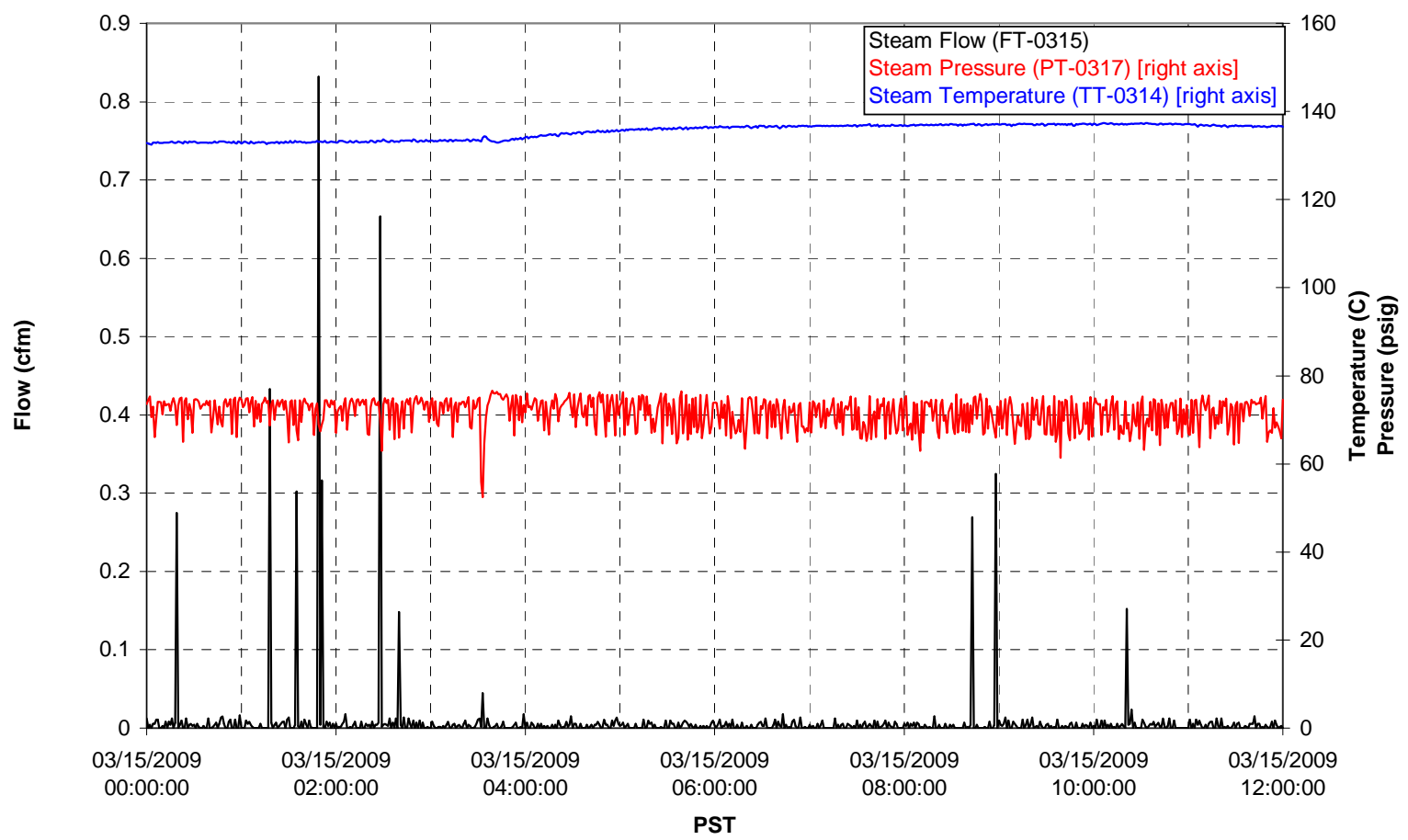


T01B Steam

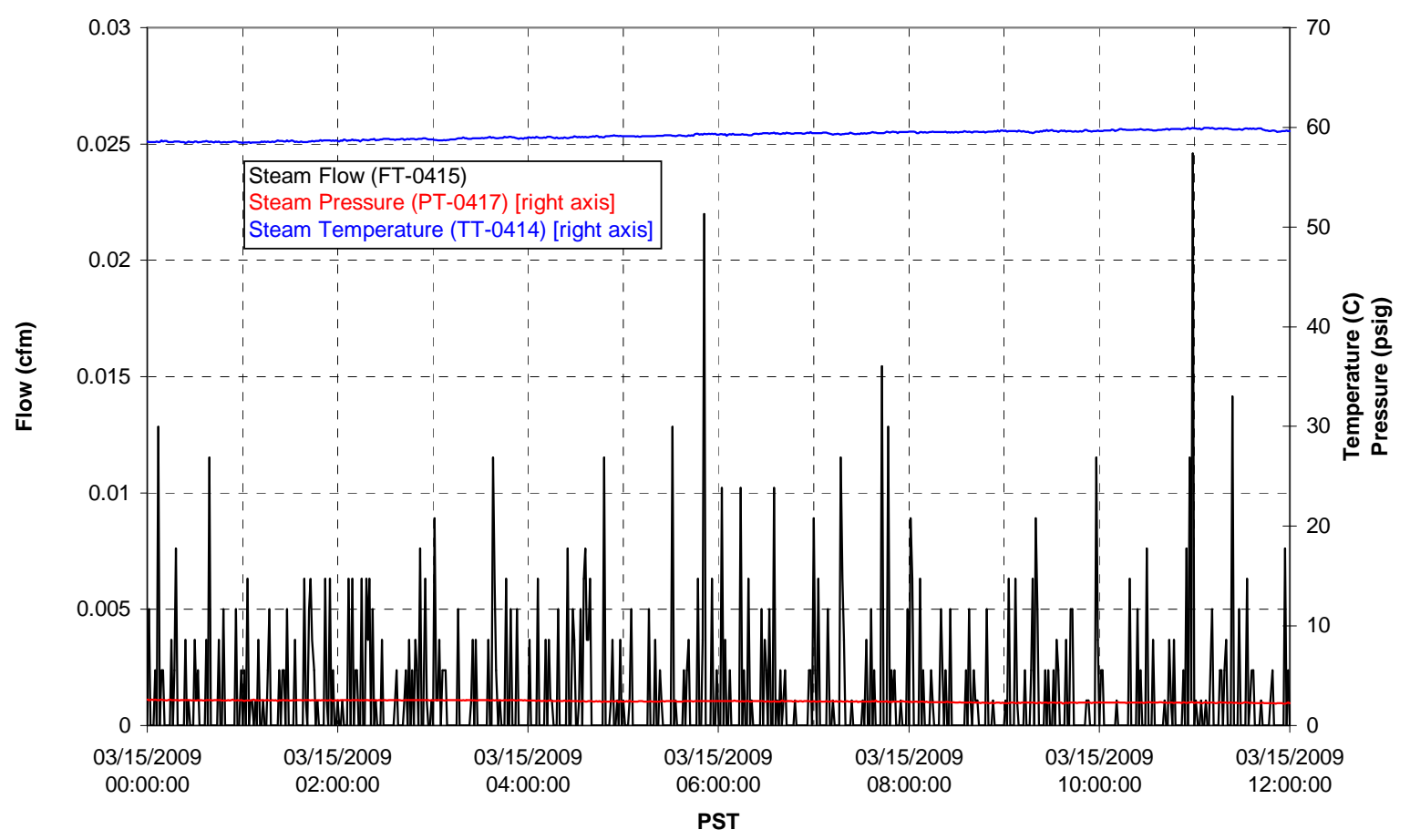




\section{Integrated Test B Data Plots \\ 03/15/09 12:00 - 03/16/09 00:00}


T01A level

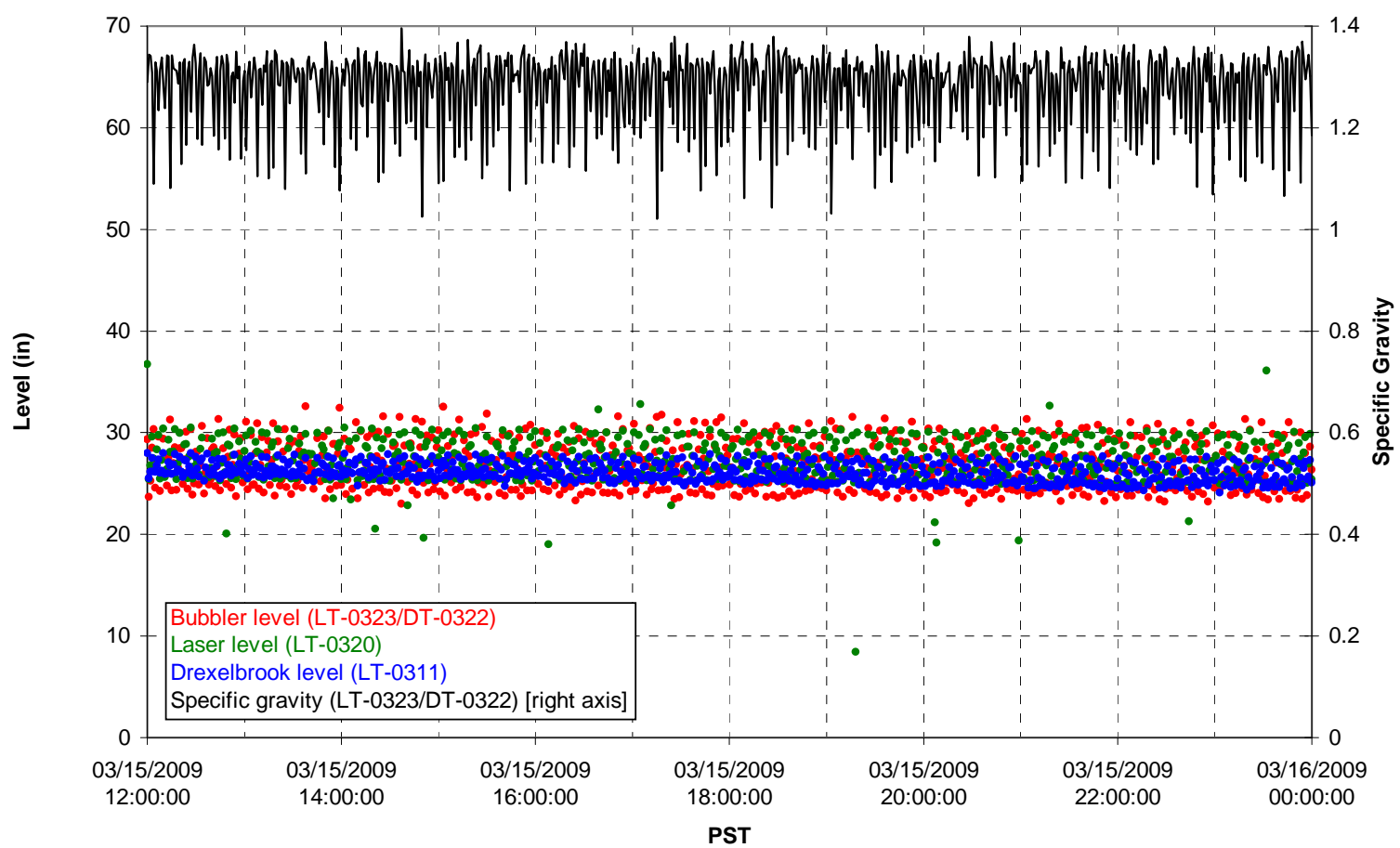

T01A temperatures

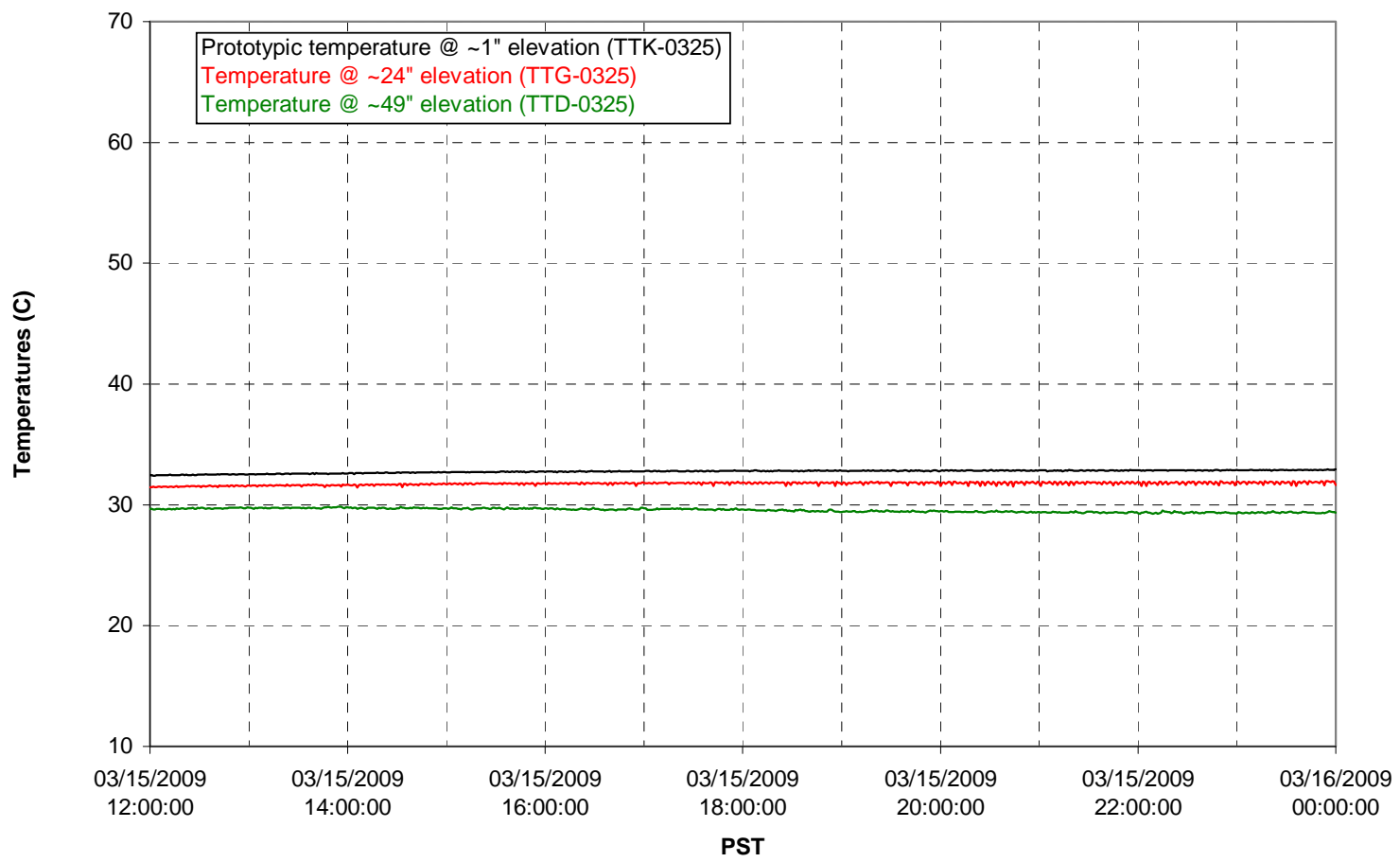


T01B level

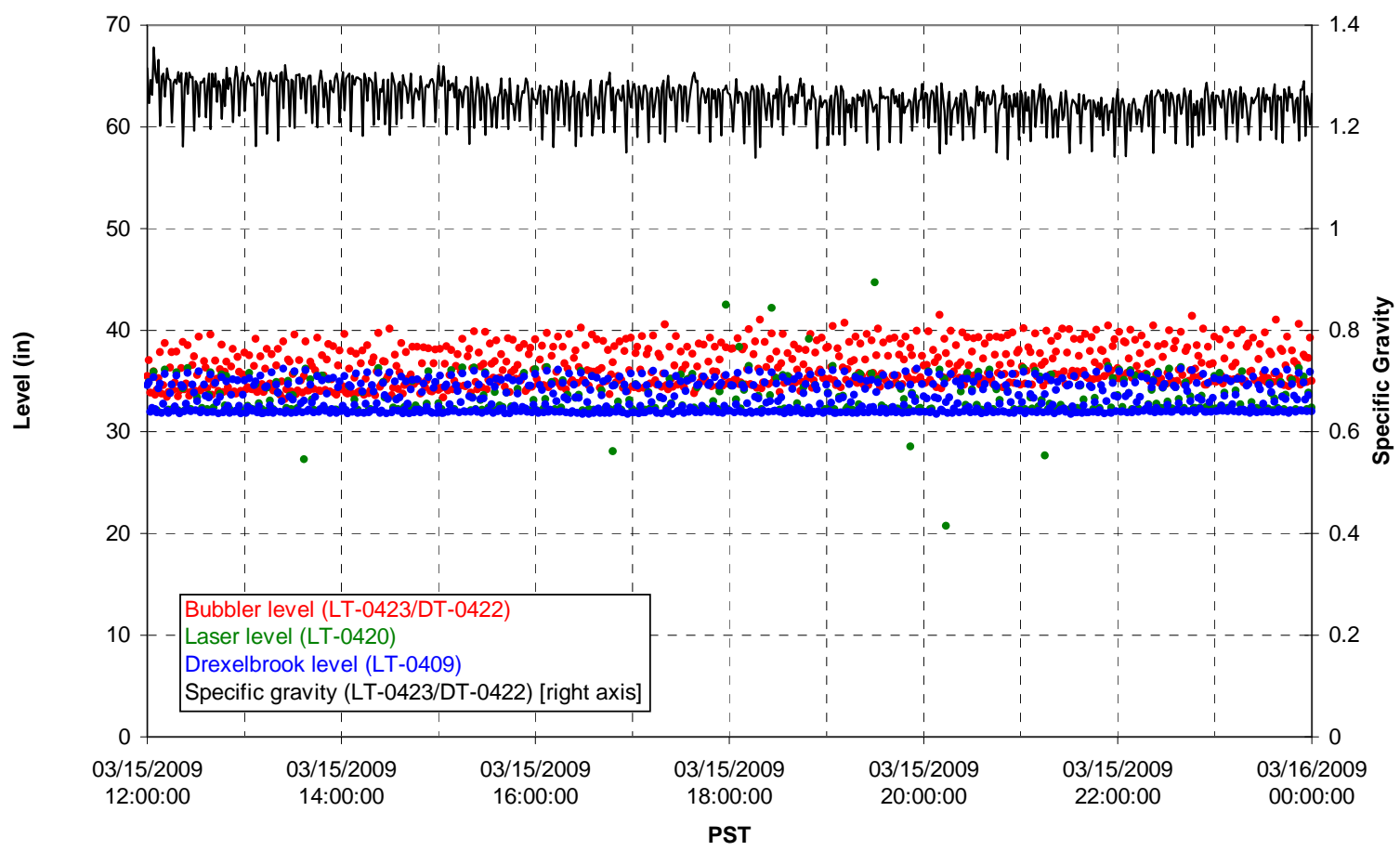

T01B temperatures

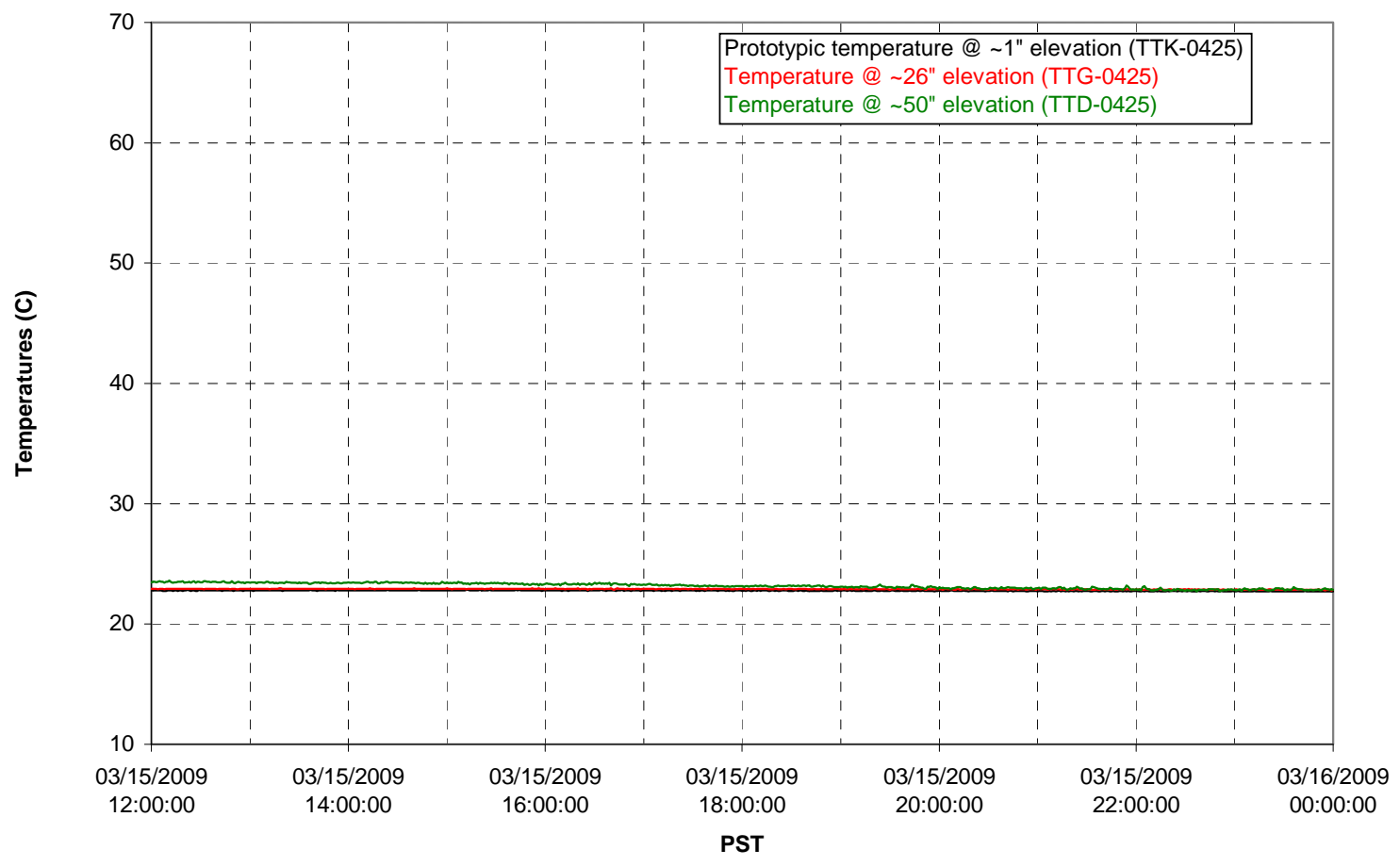


T02A level

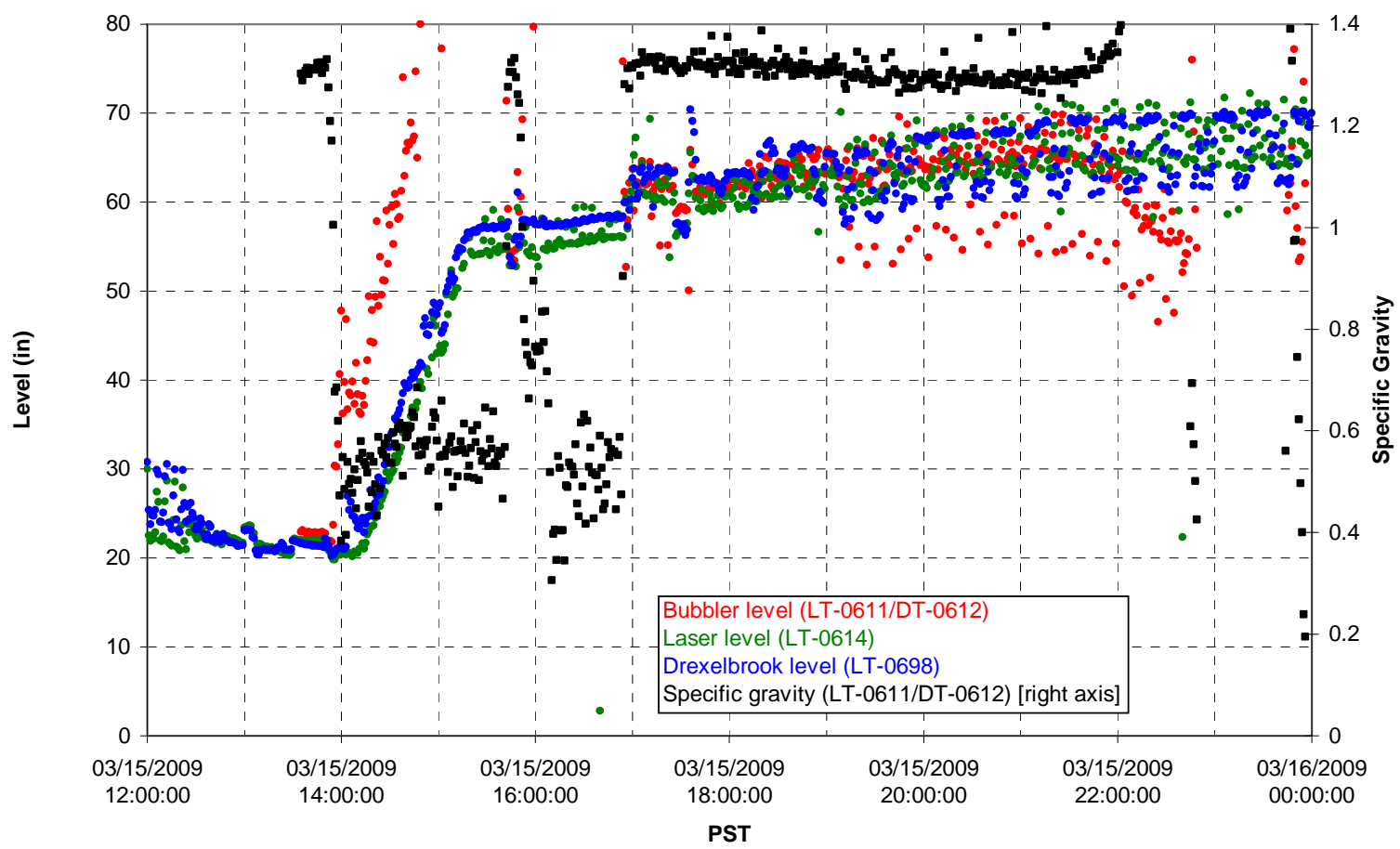

T02A temperatures

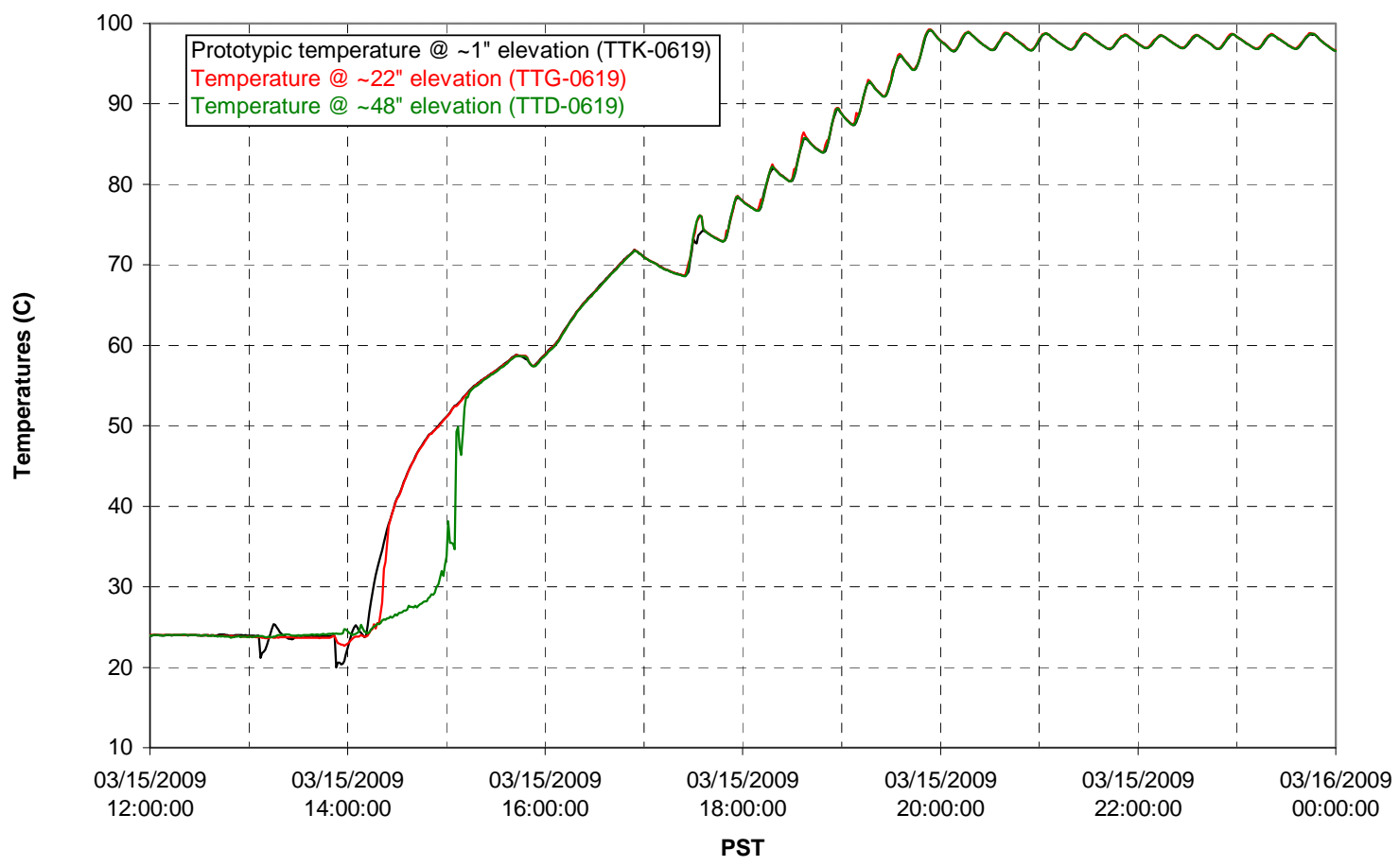


T02A and filter loop temperatures

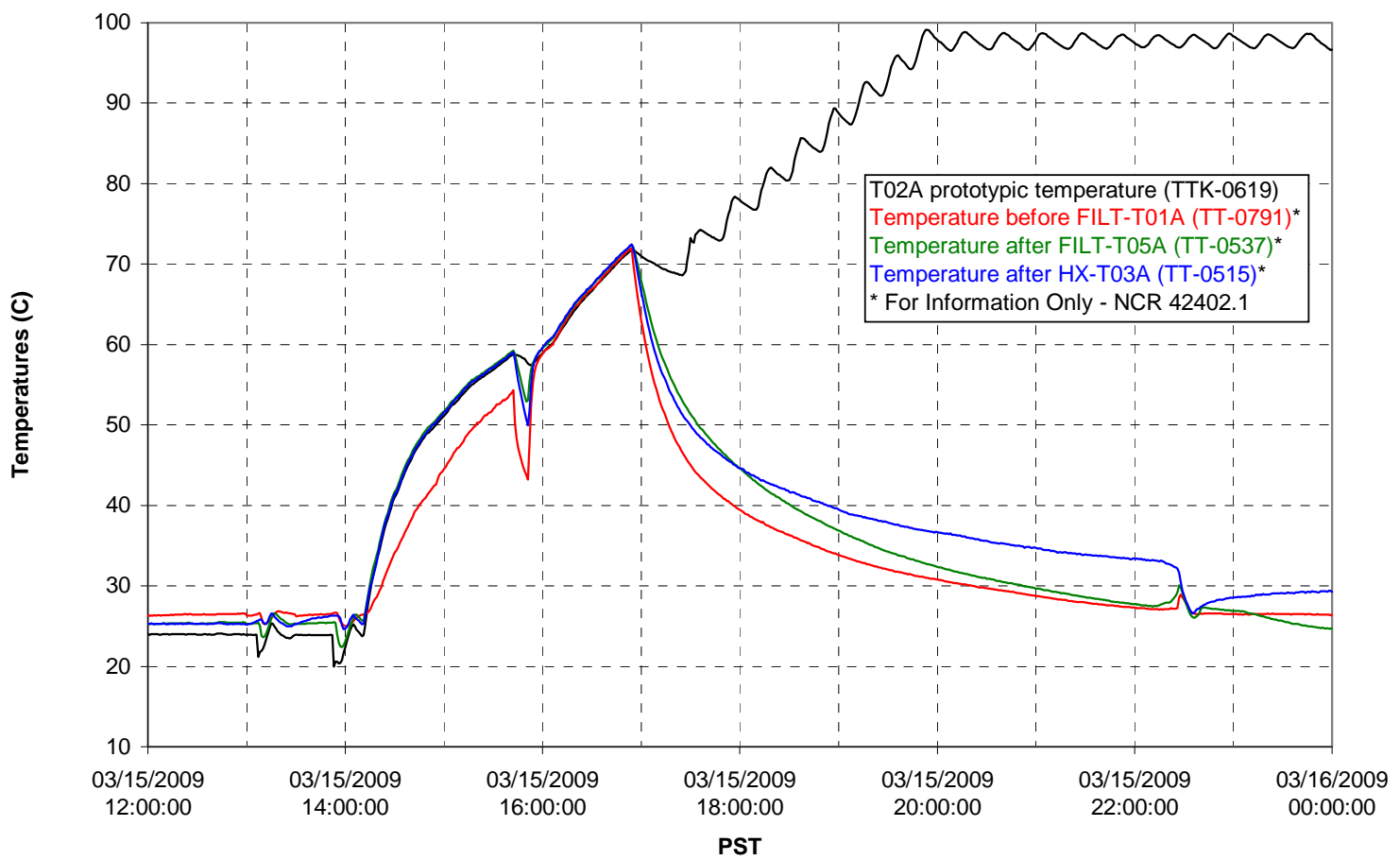

Pump Pressures and Flow

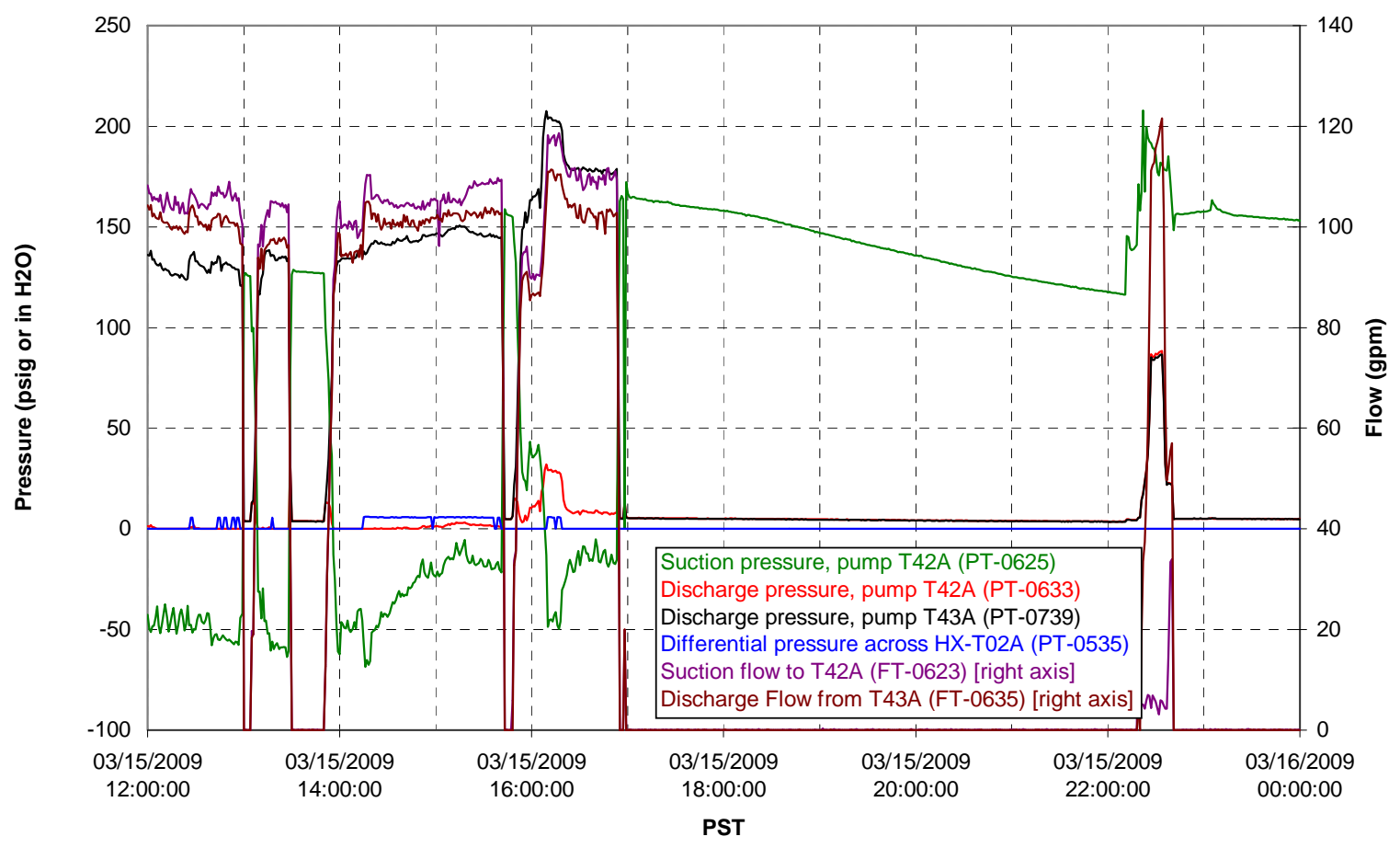


Axial pressure drop

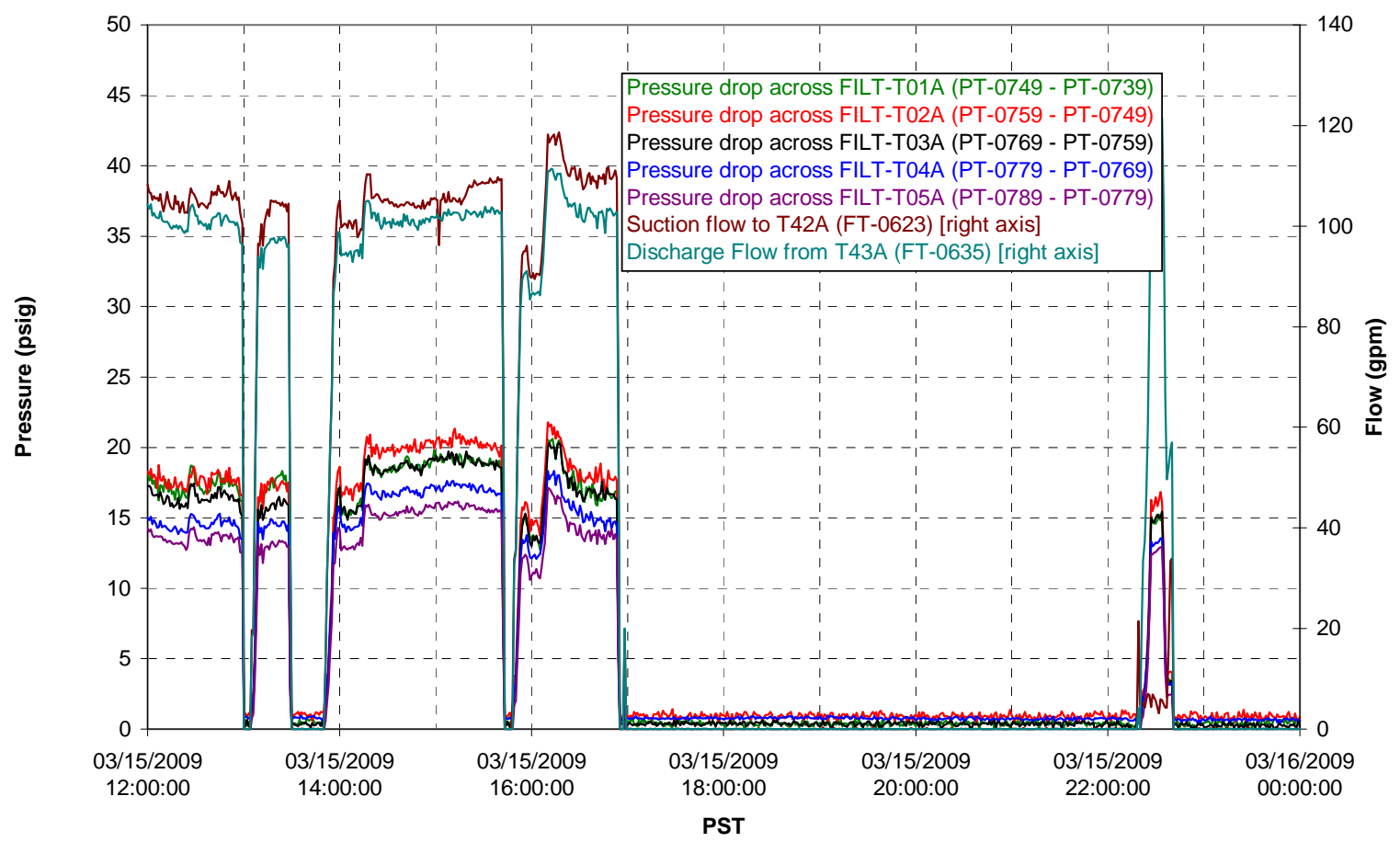

Permeate flow rates

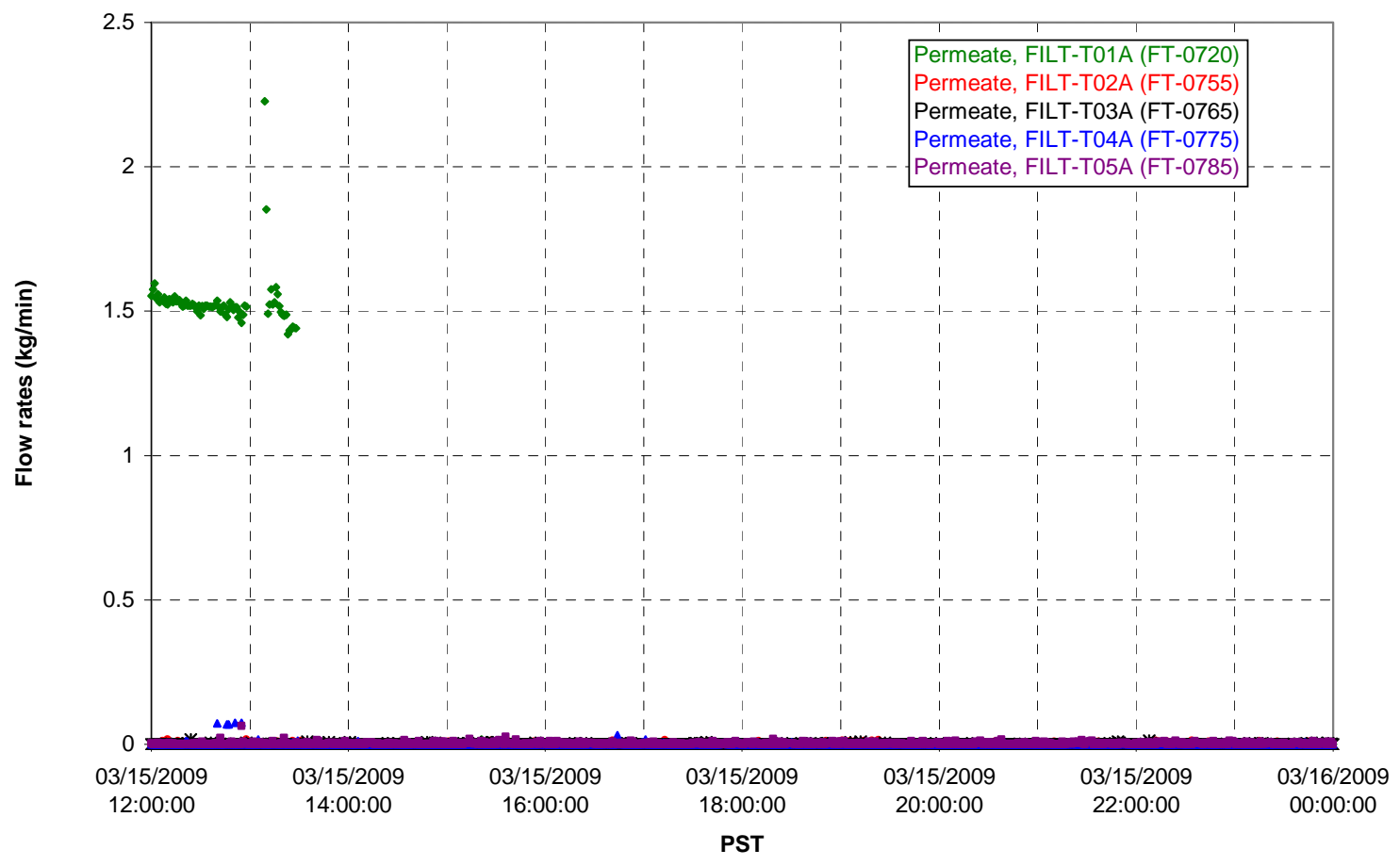


T02A Inner Temperature Tree

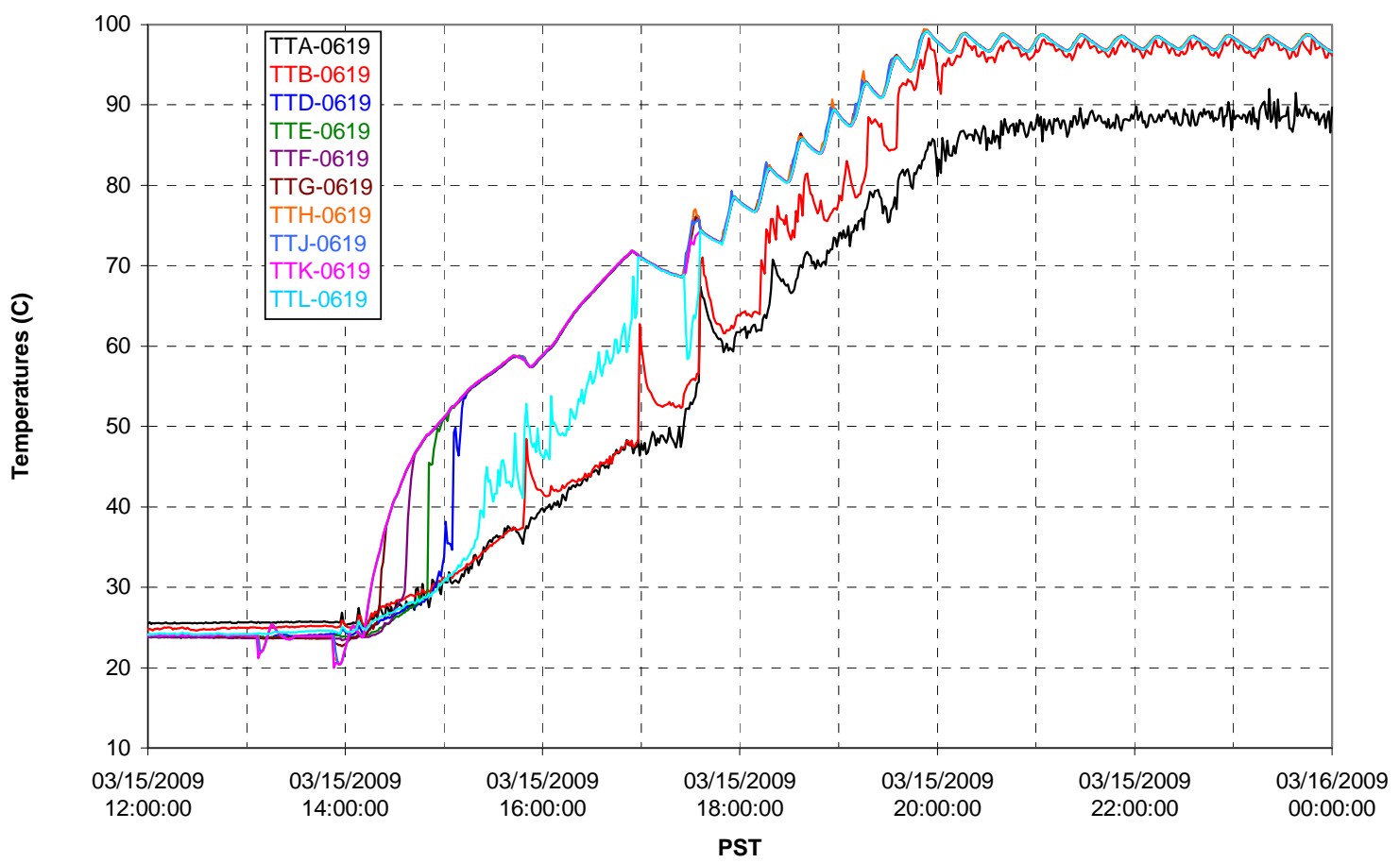

T02A Outer Temperature Tree

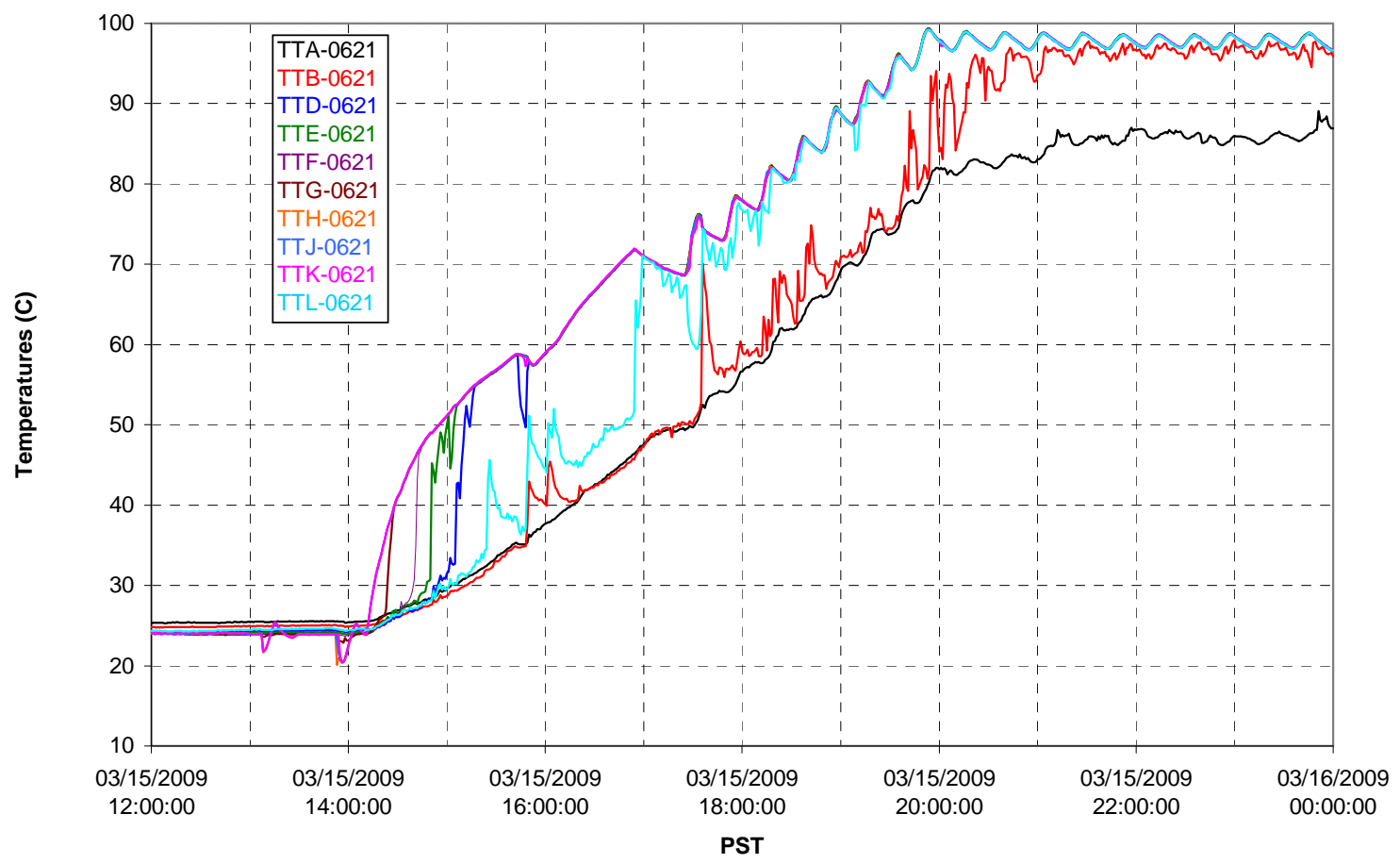


T02A temperatures

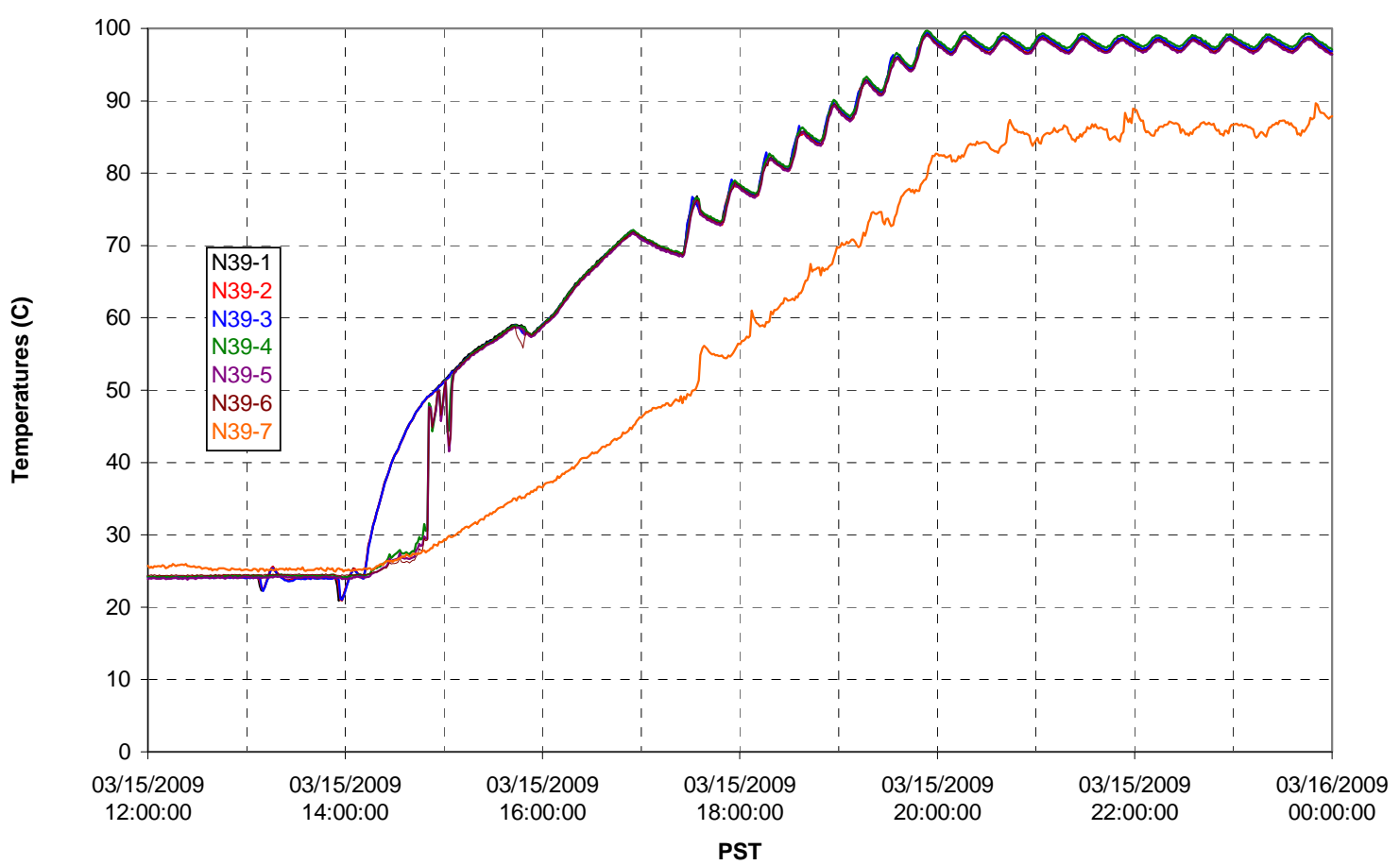

T02A temperatures

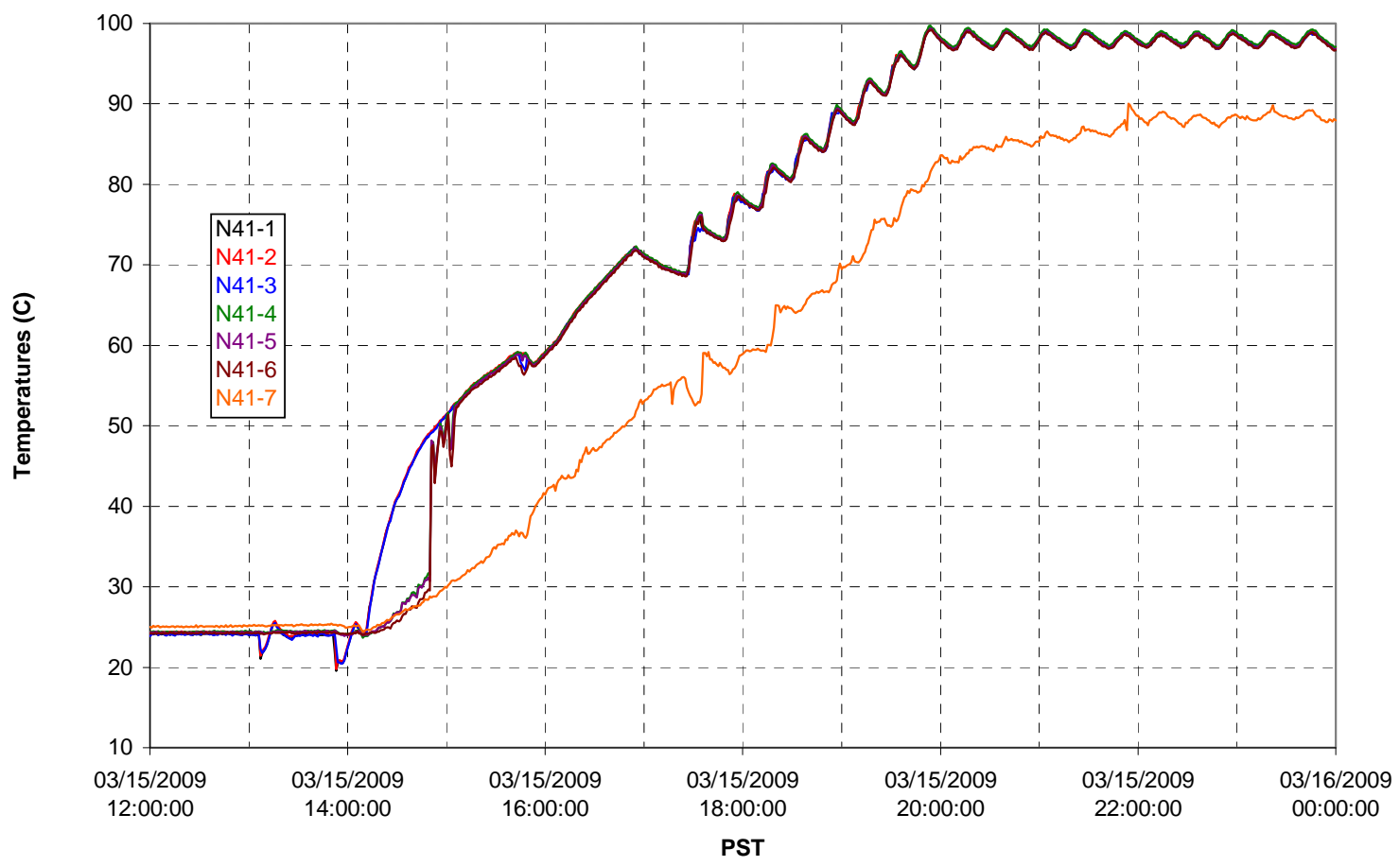


T02A temperatures

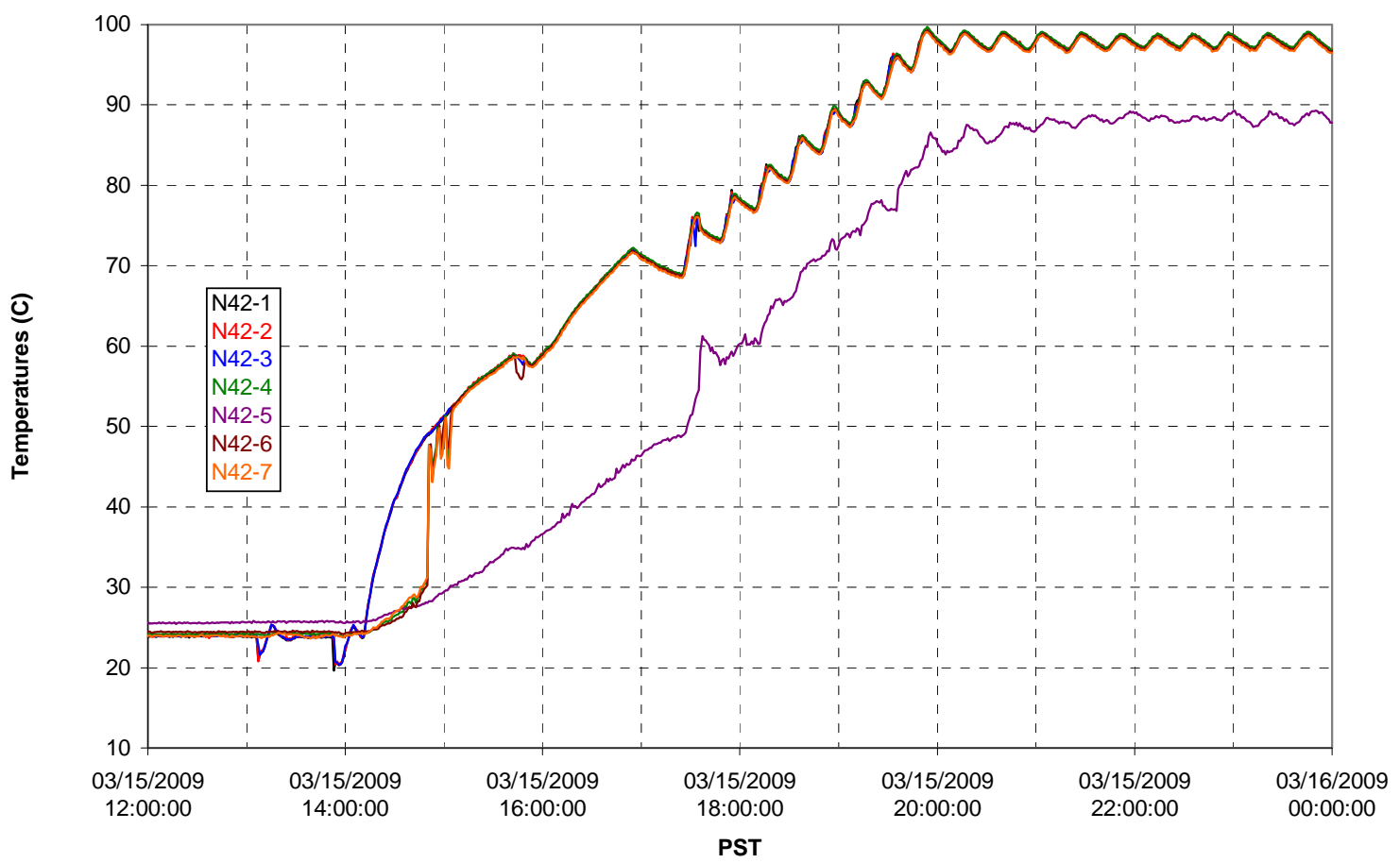

T02A temperatures

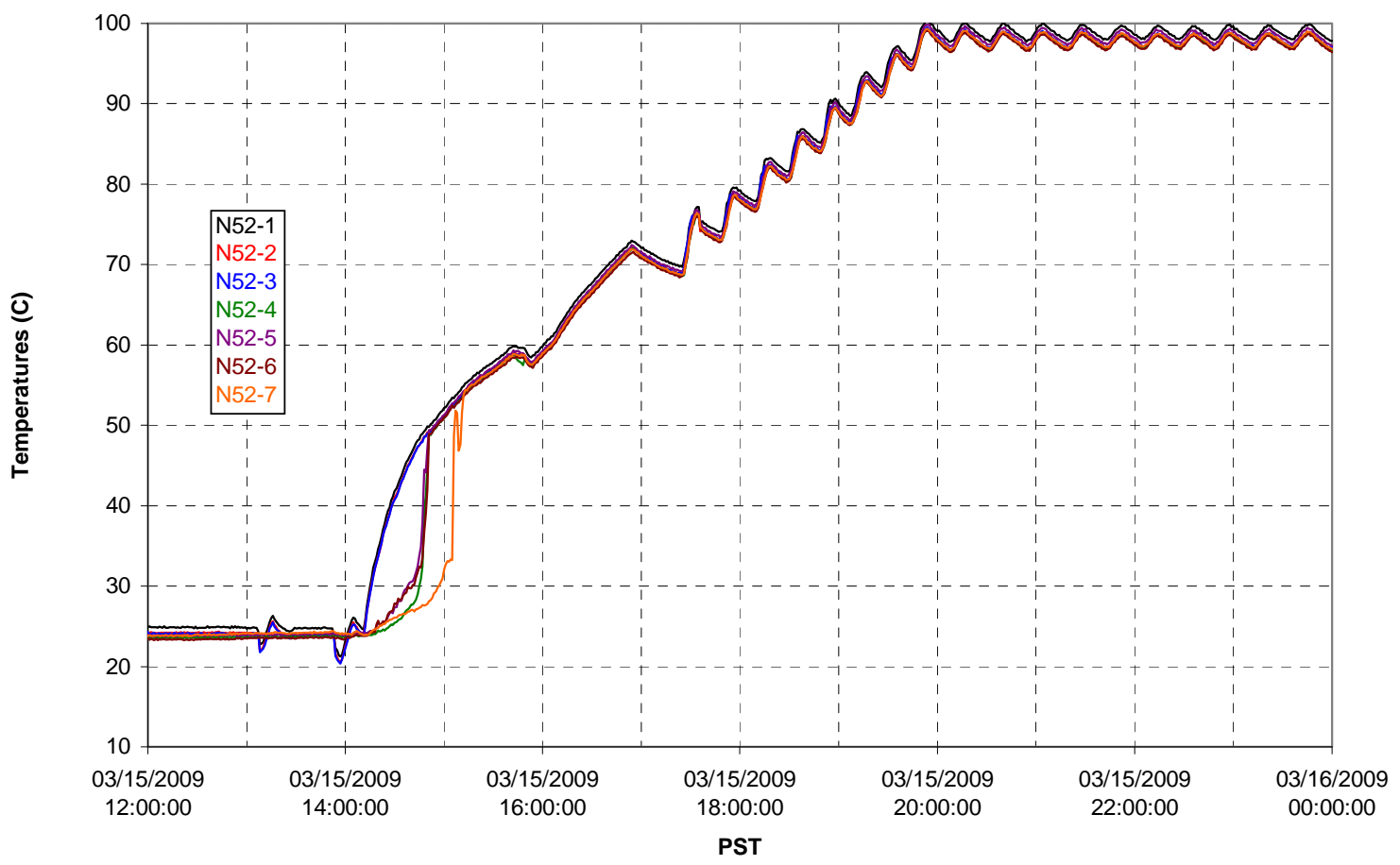


T02A Heating and Cooling

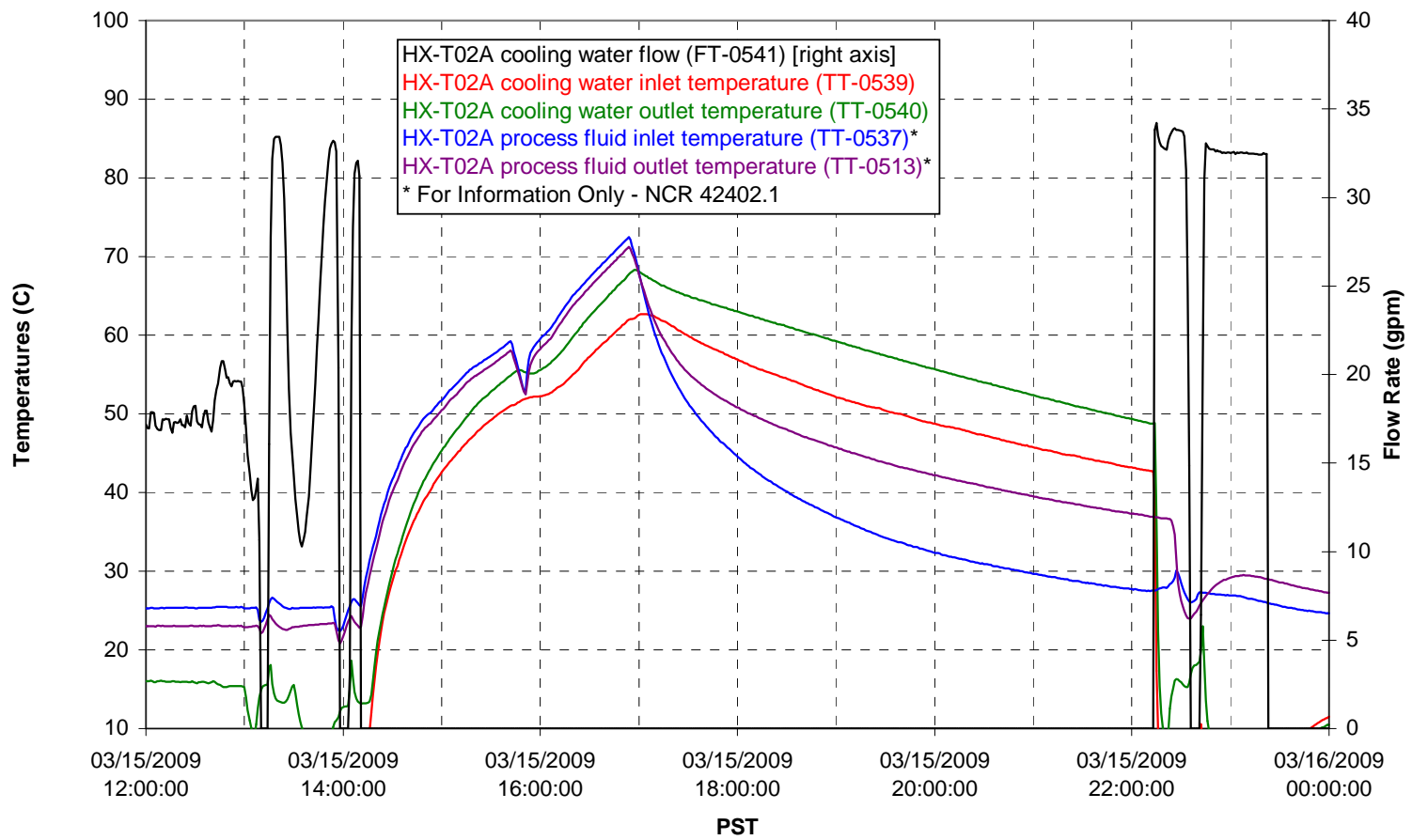

Pump Operation

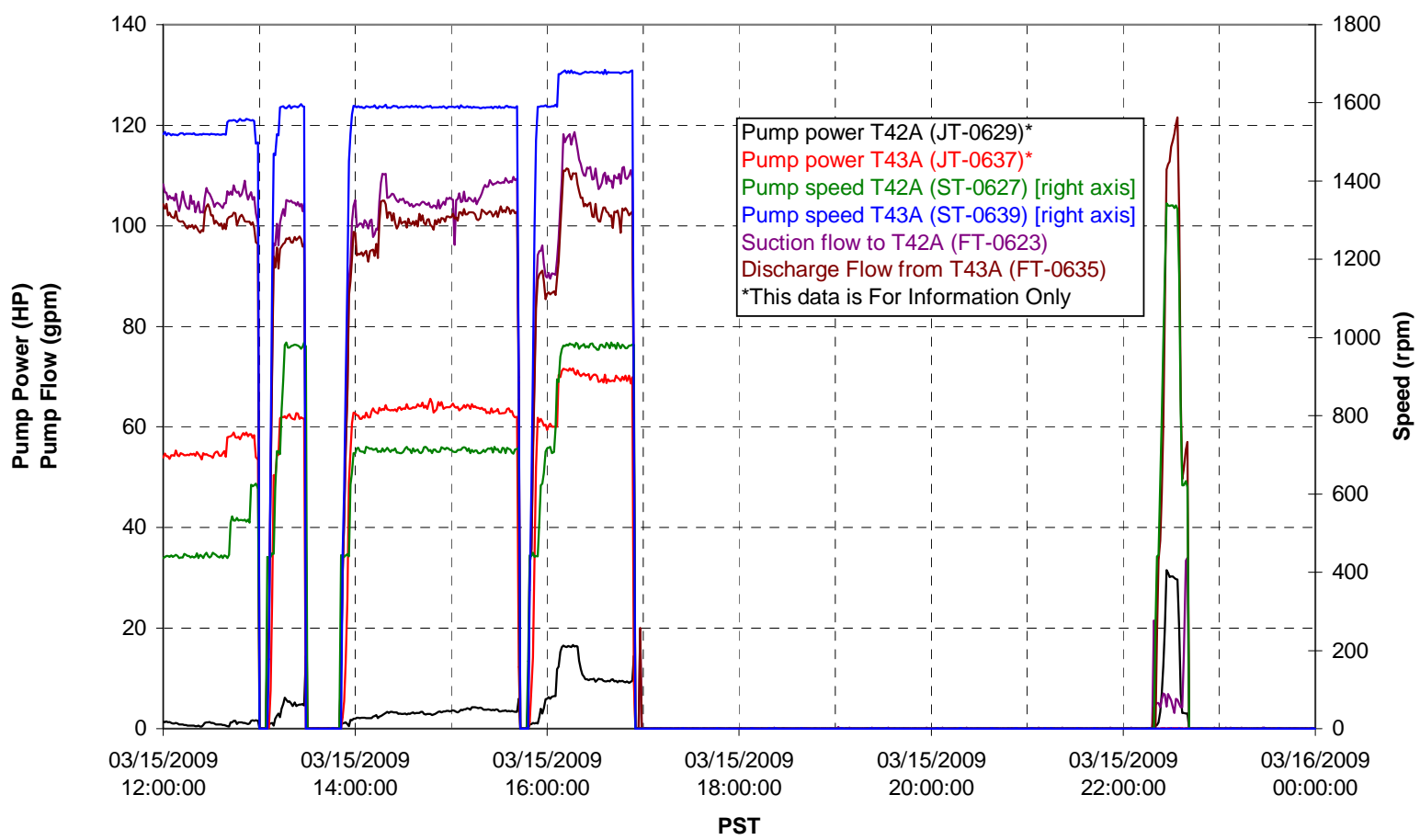


Pulsepot UFP-PP-T01A

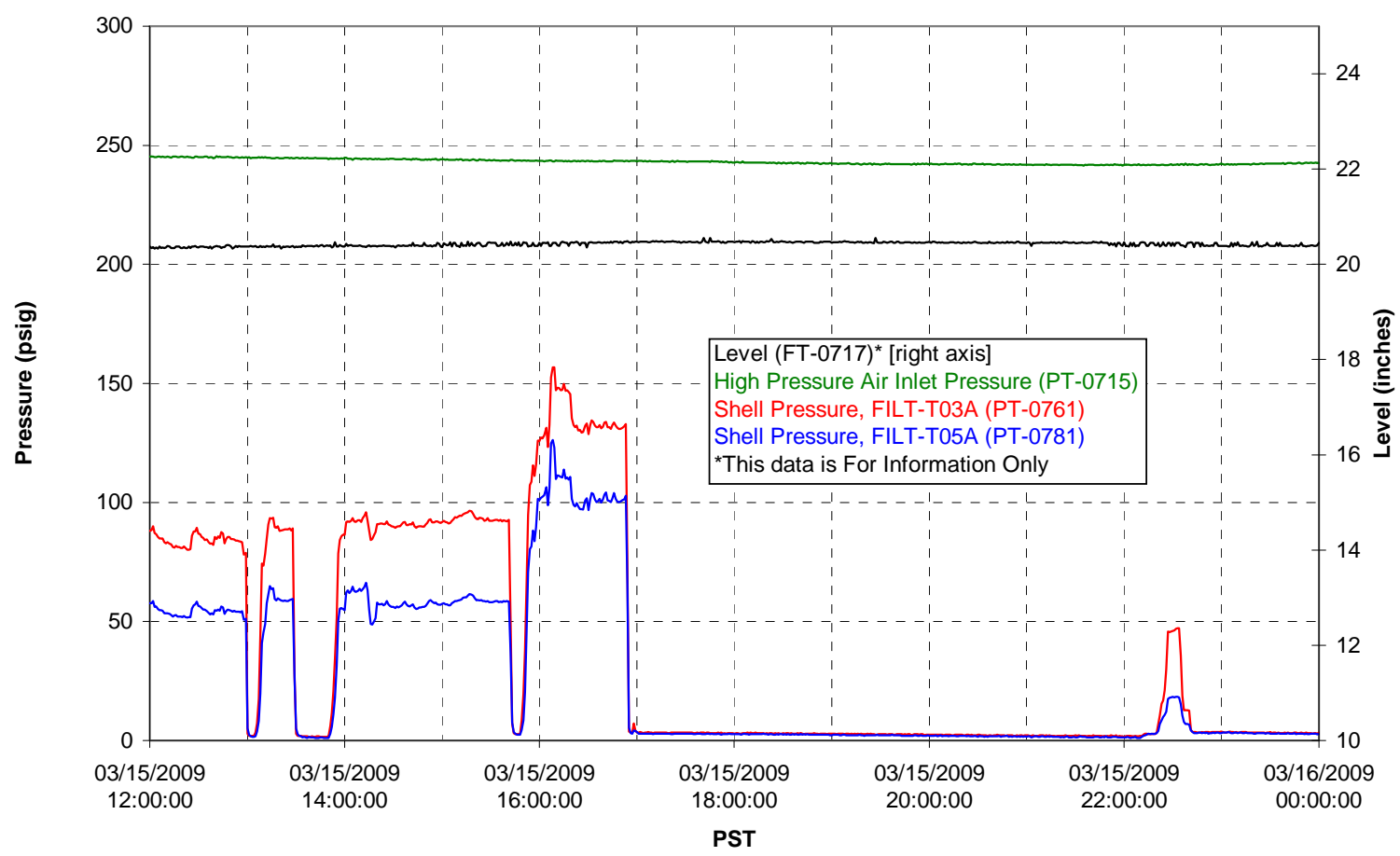

Pulsepot UFP-PP-T02A

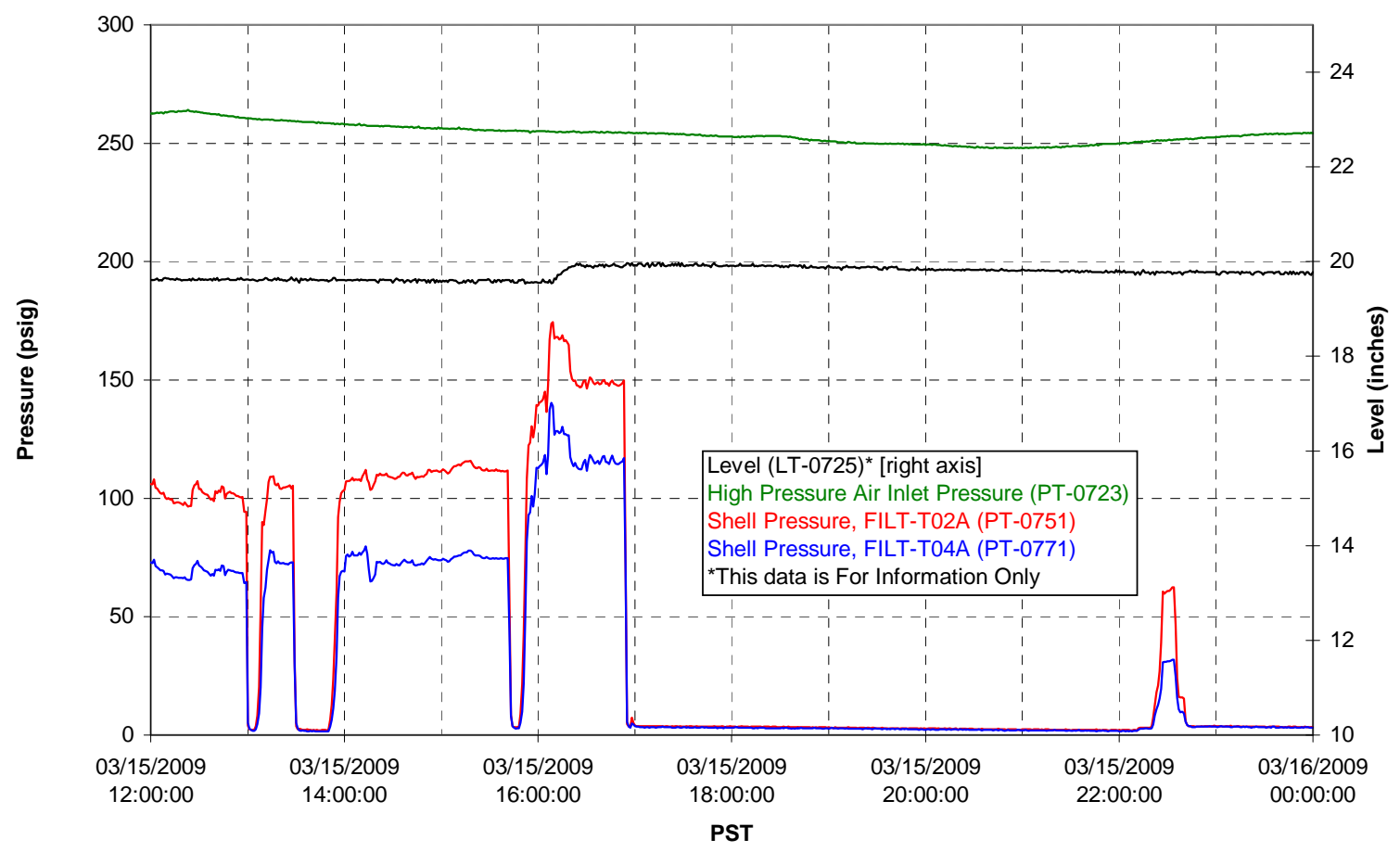


Pulsepot UFP-PP-T03A

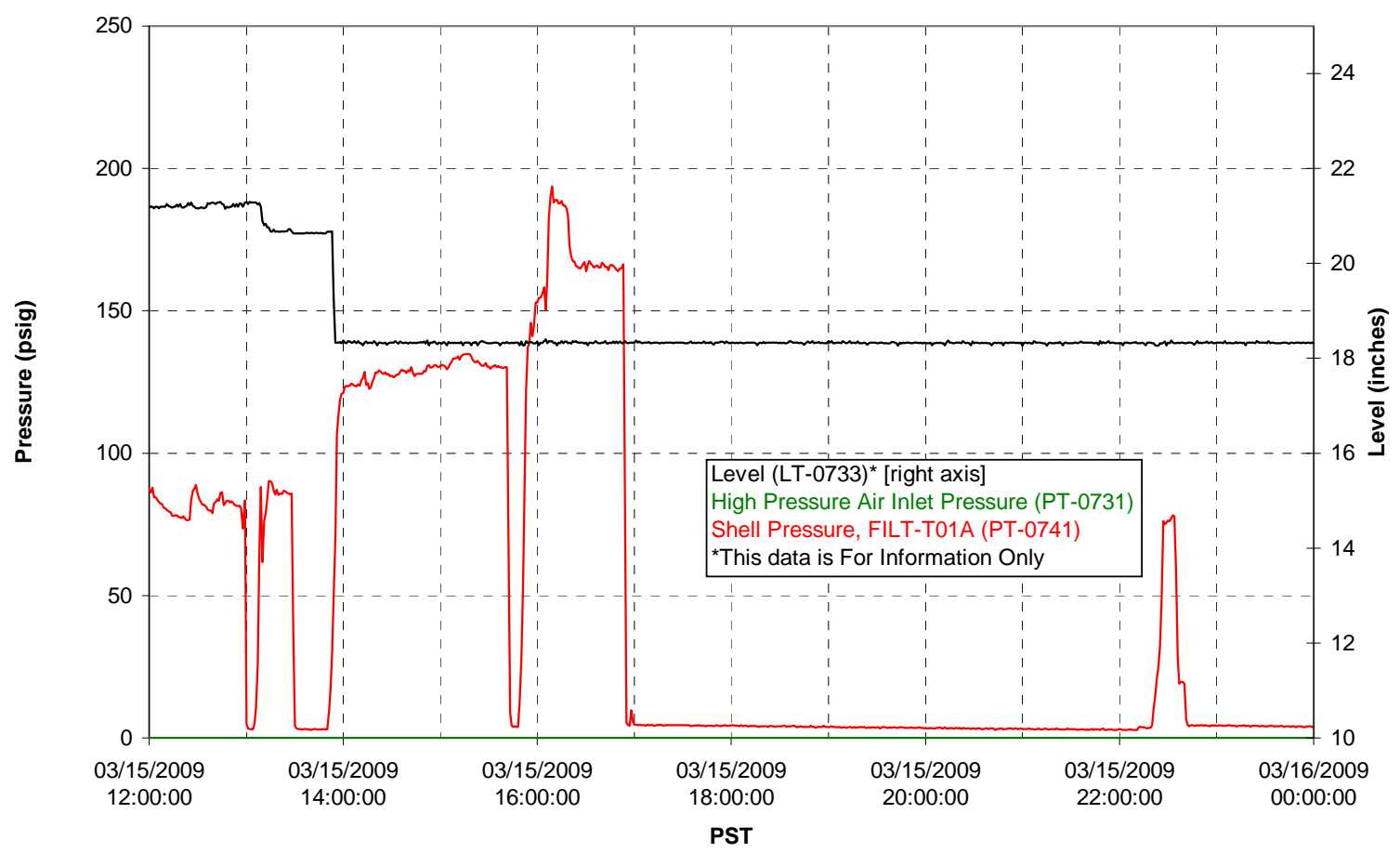

Pulsepot Levels

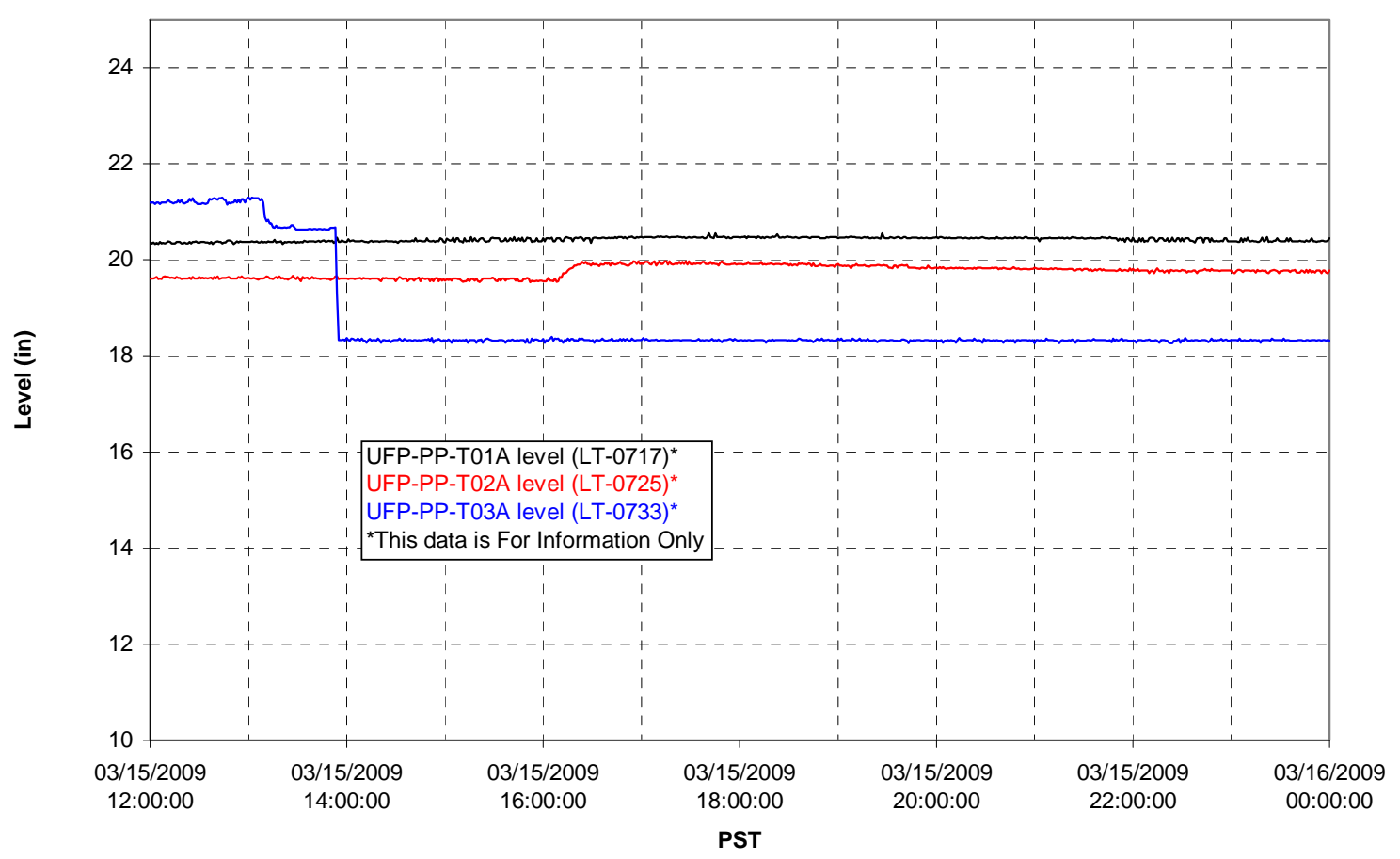


Filter UFP-FILT-T01A

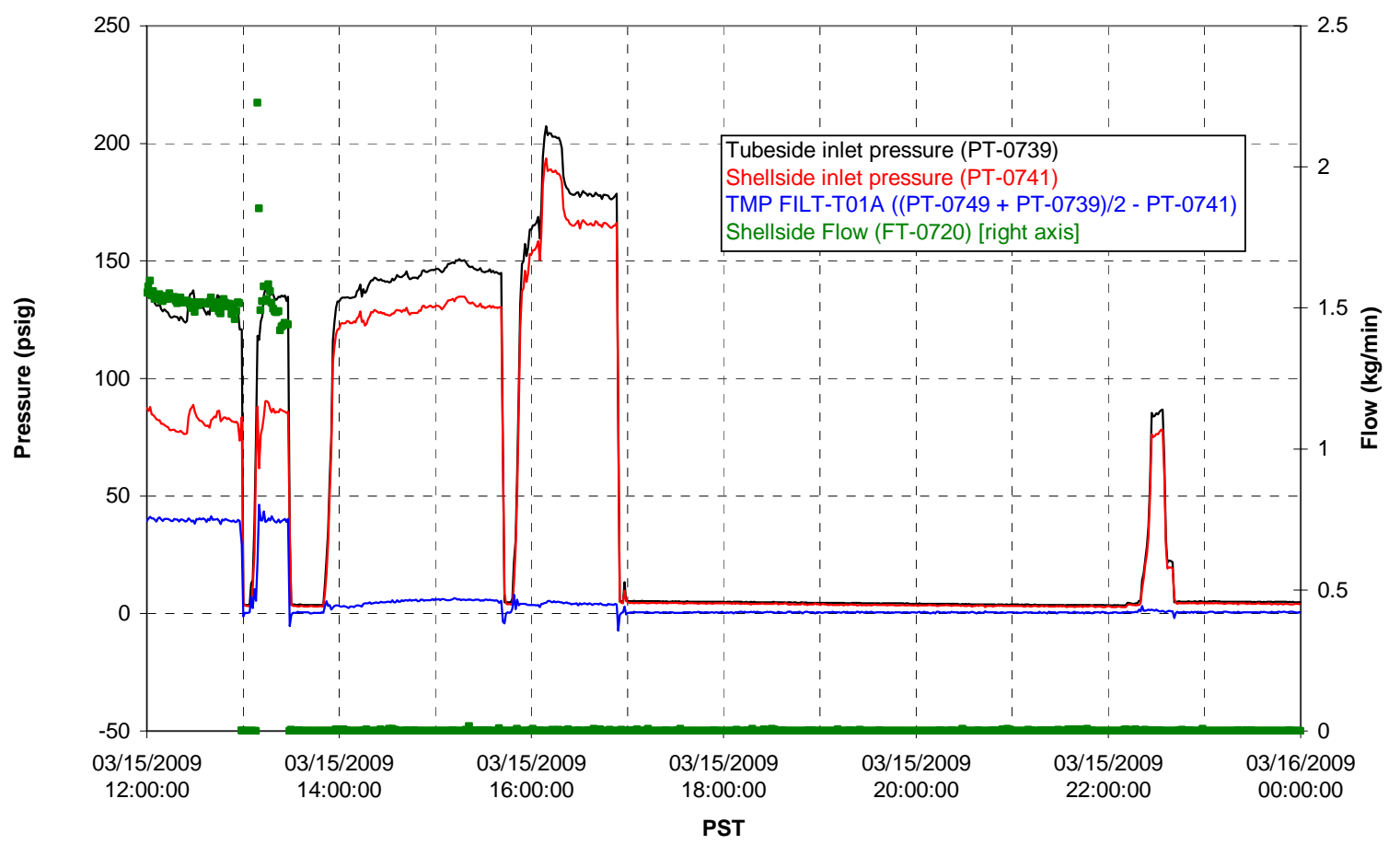

Filter UFP-FILT-T02A

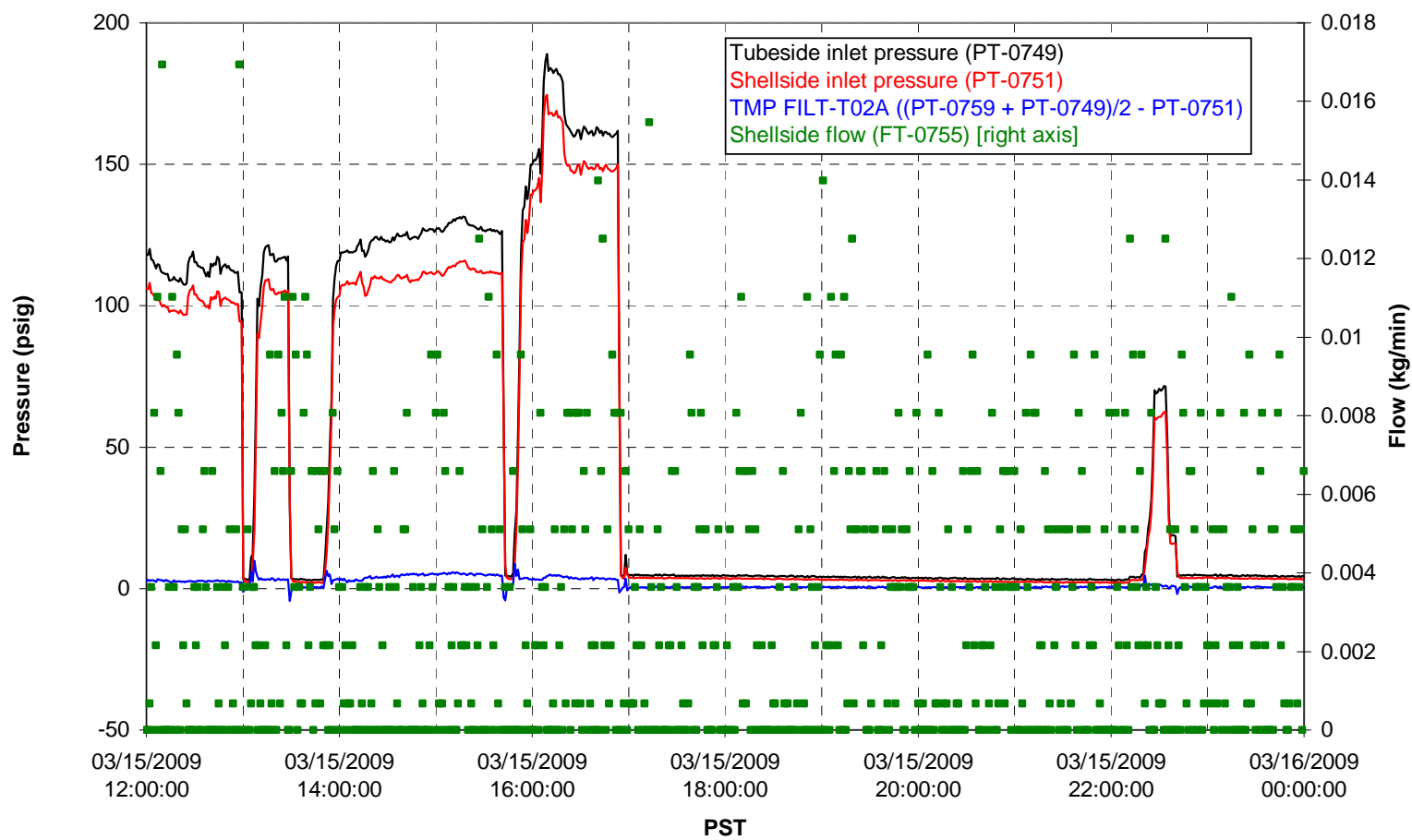


Filter UFP-FILT-T03A

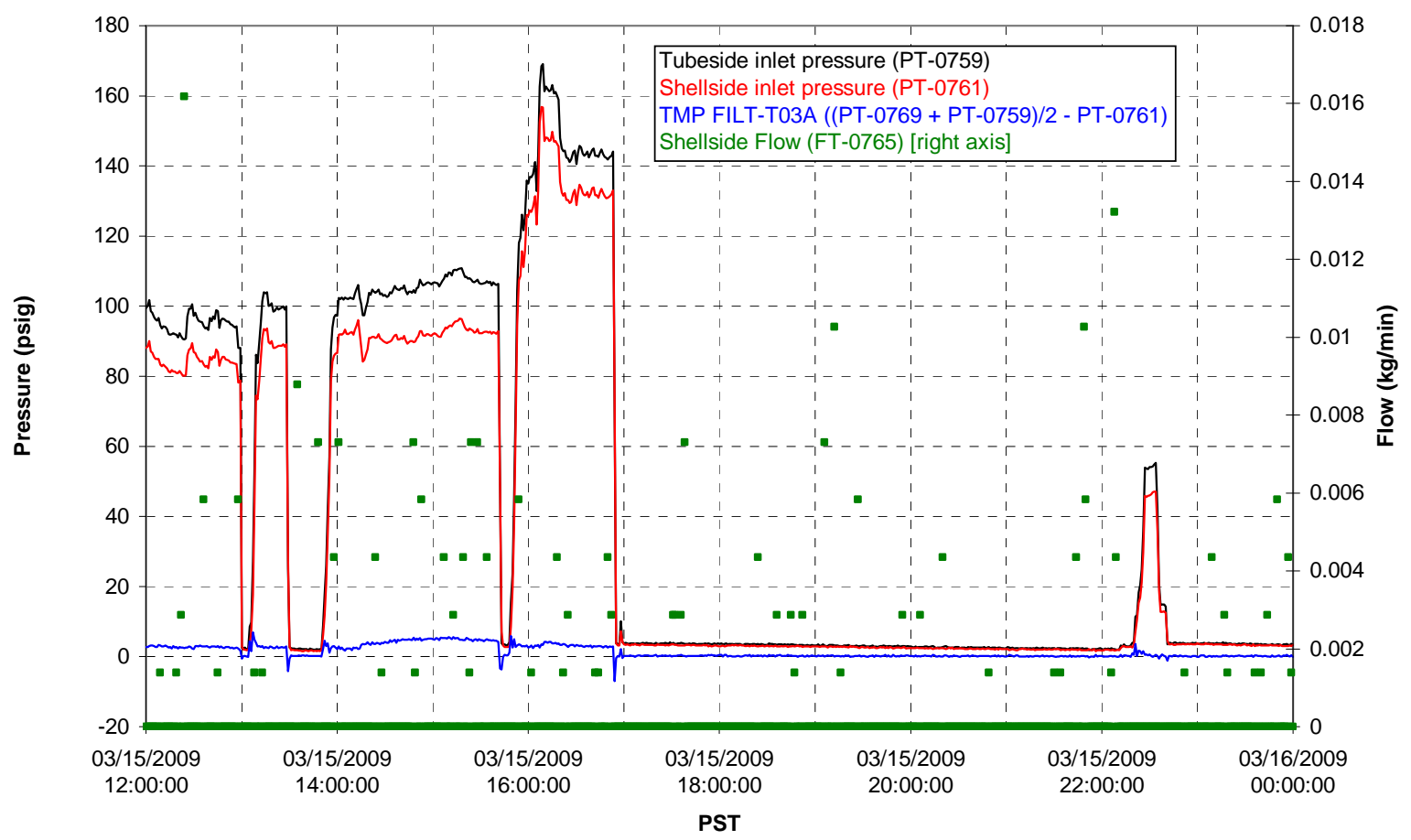

Filter UFP-FILT-T04A

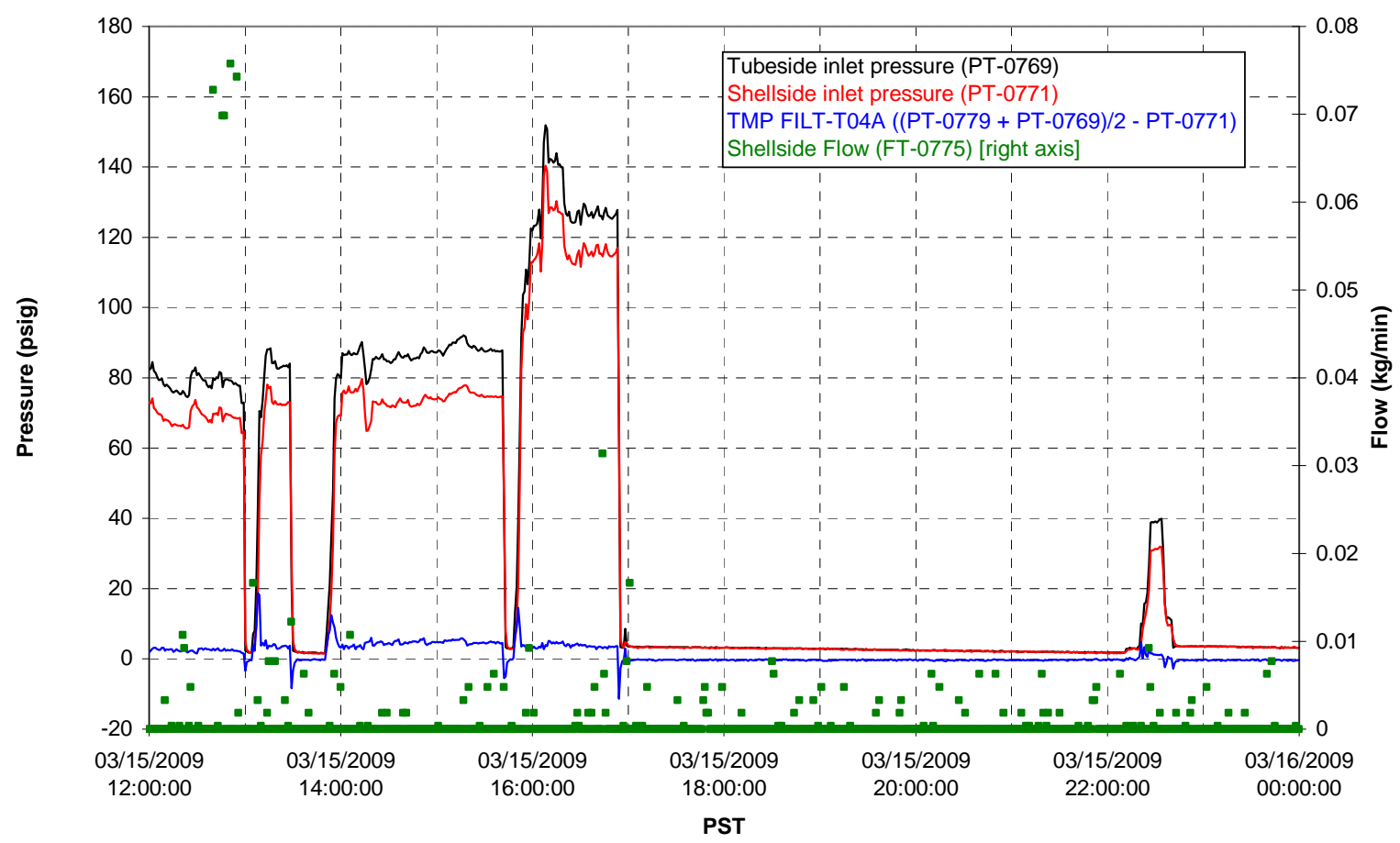


Filter UFP-FILT-T05A

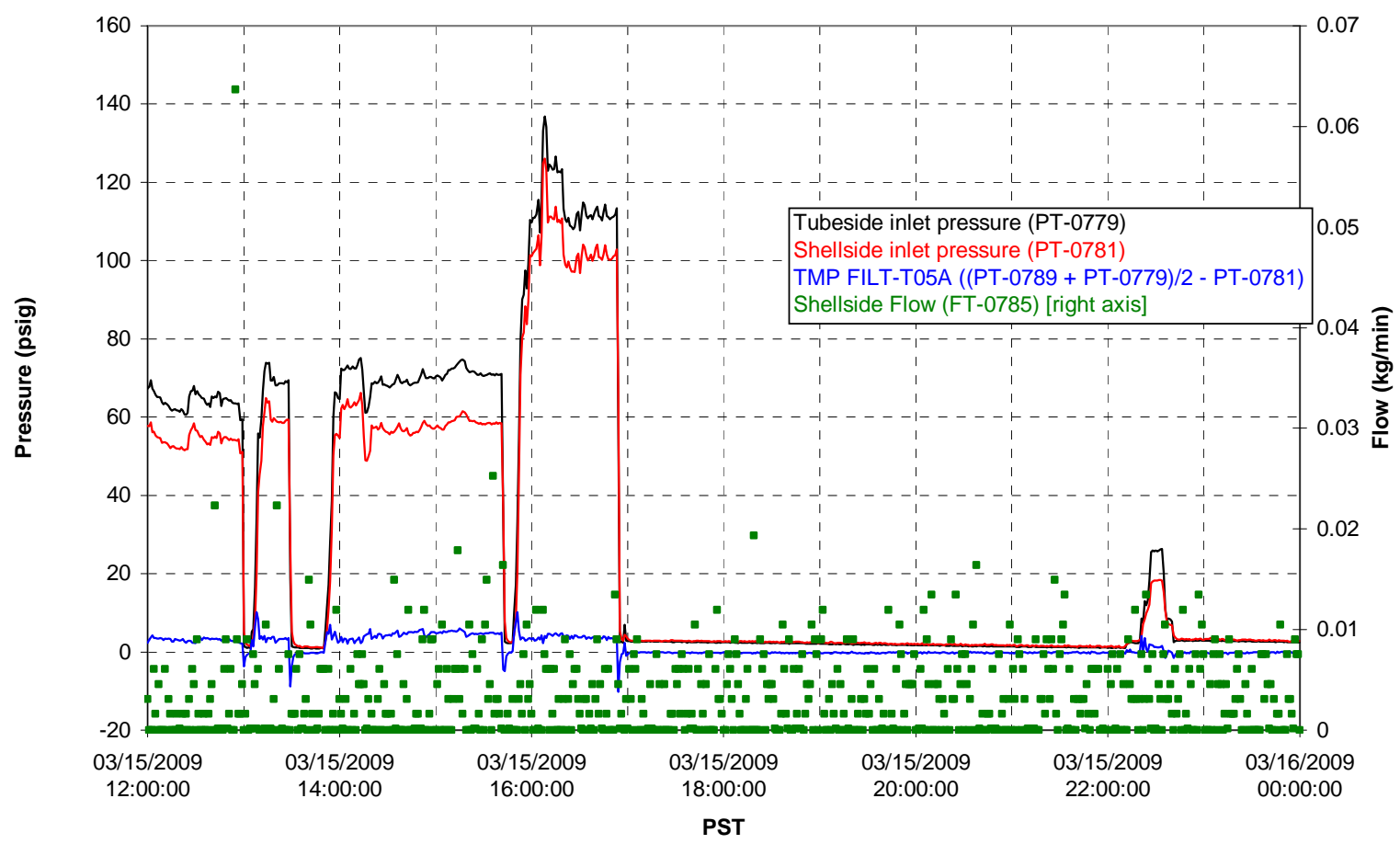

Chemical Flow

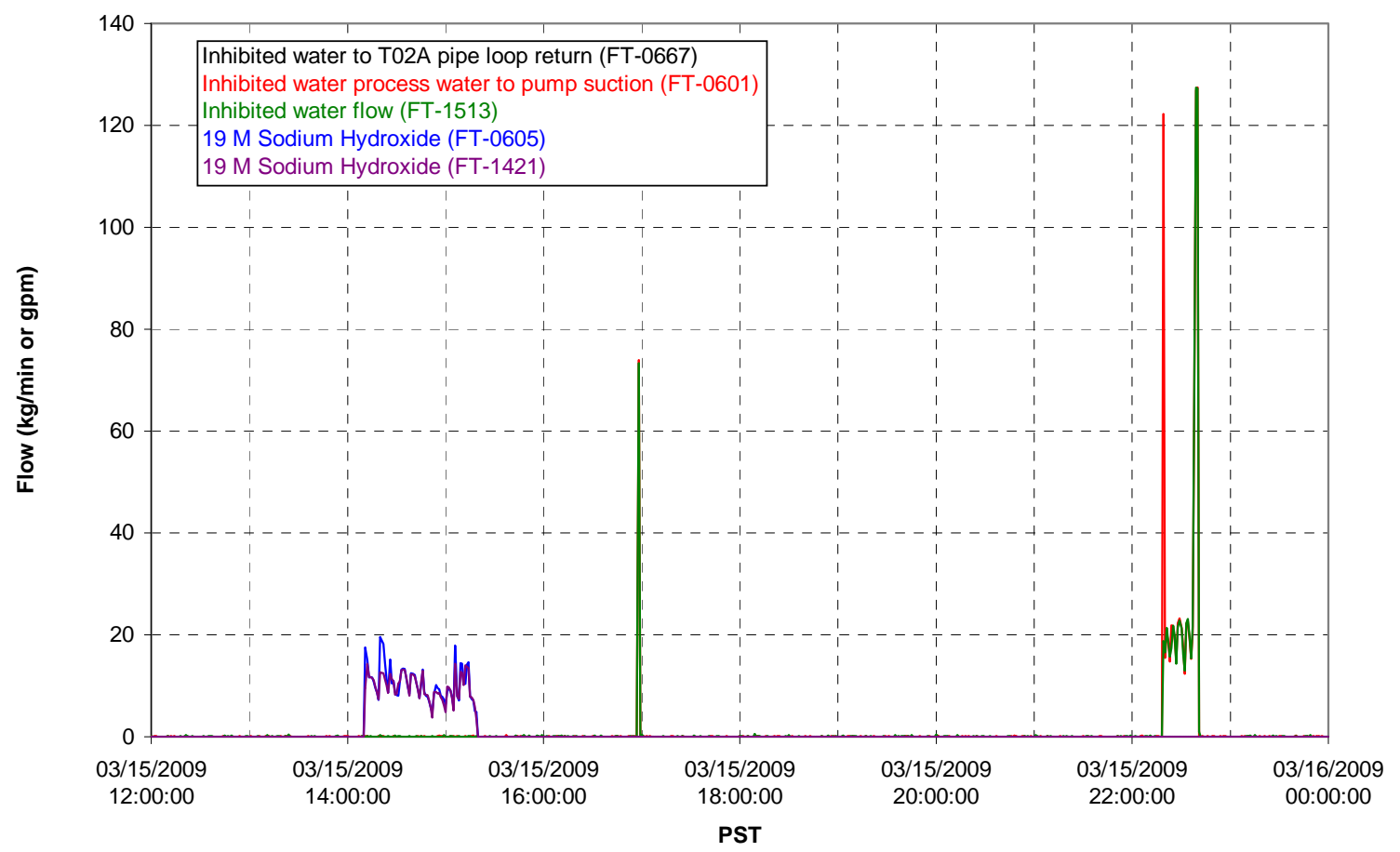




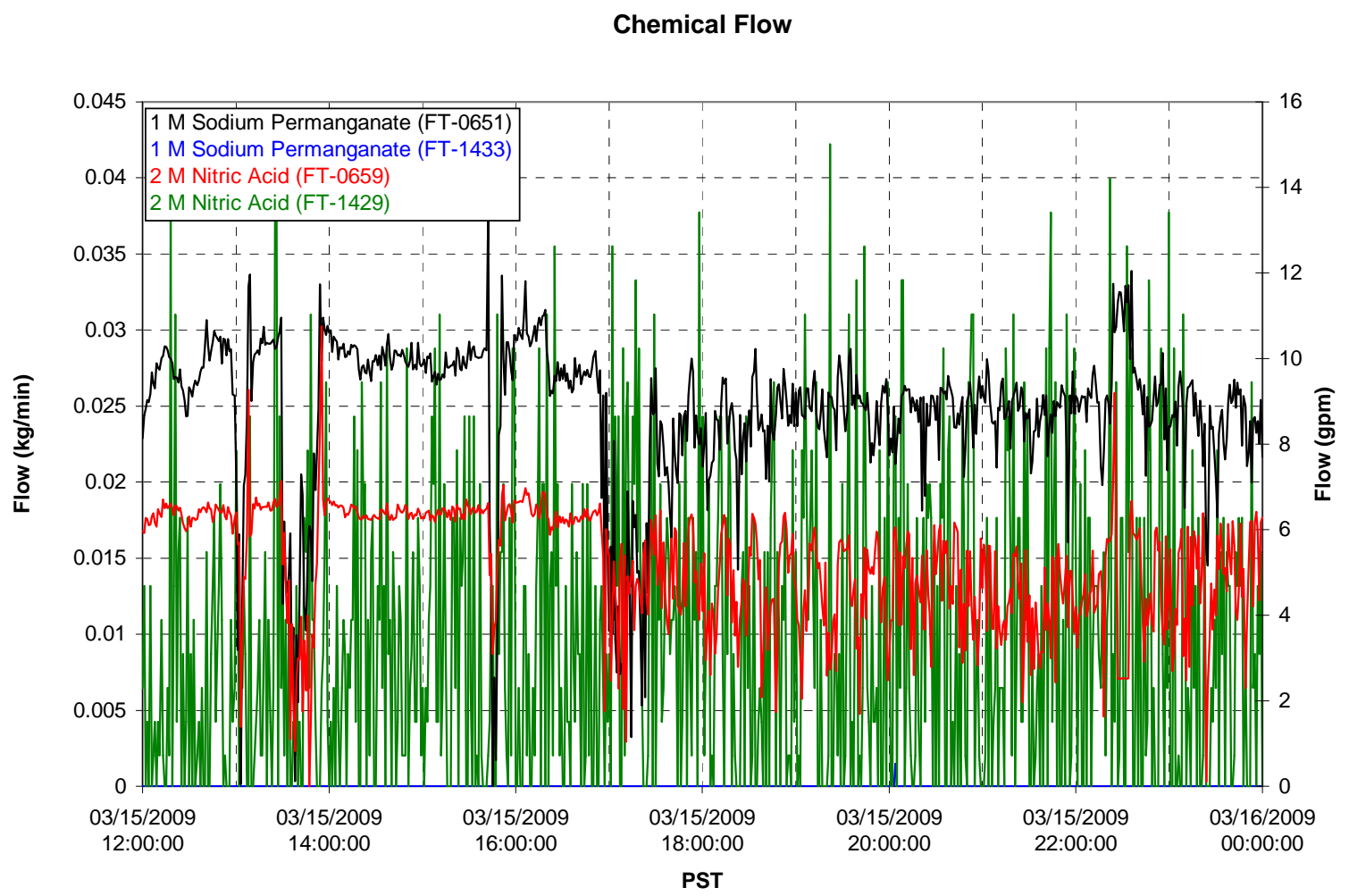

Air Flows

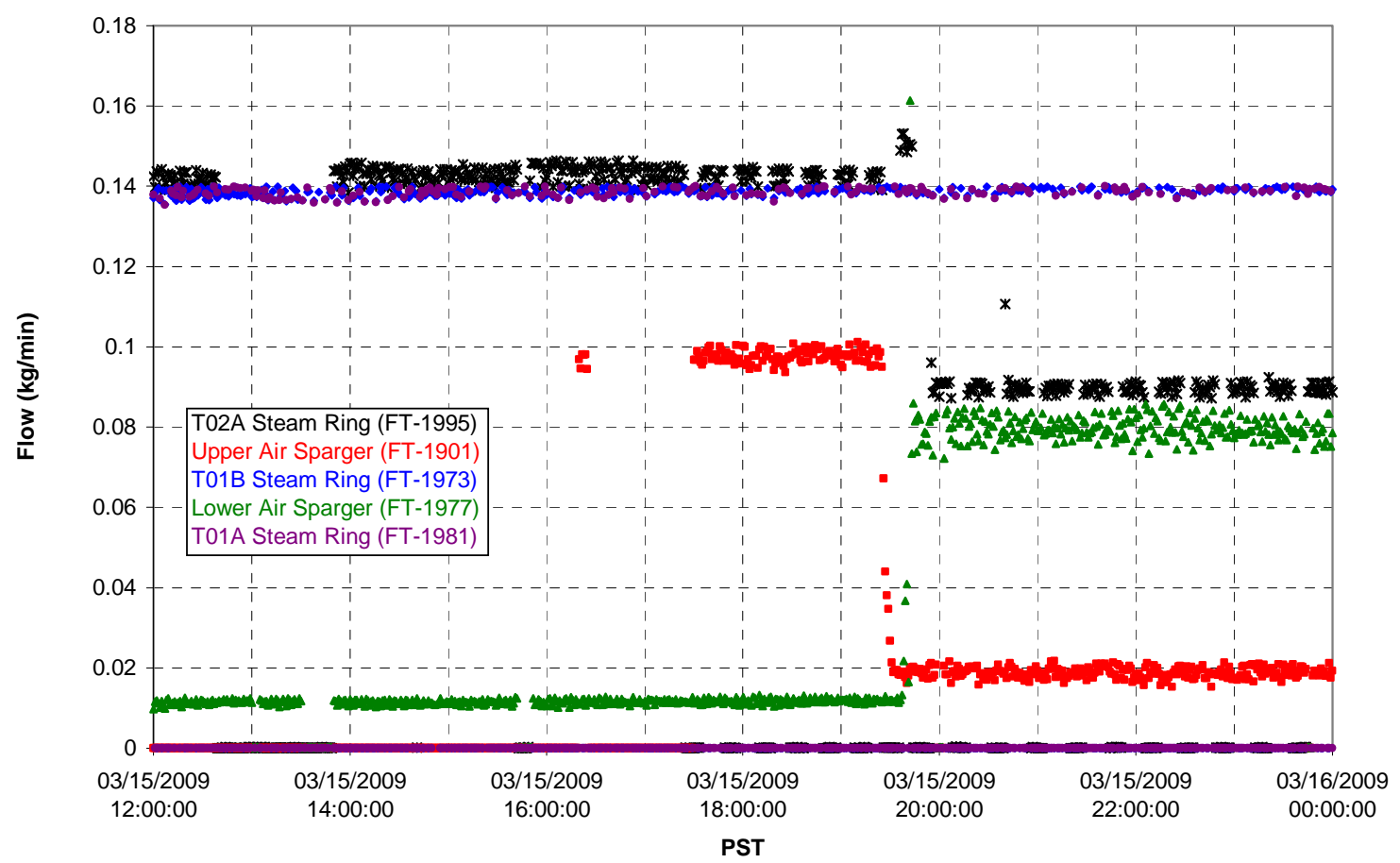


T02A Steam

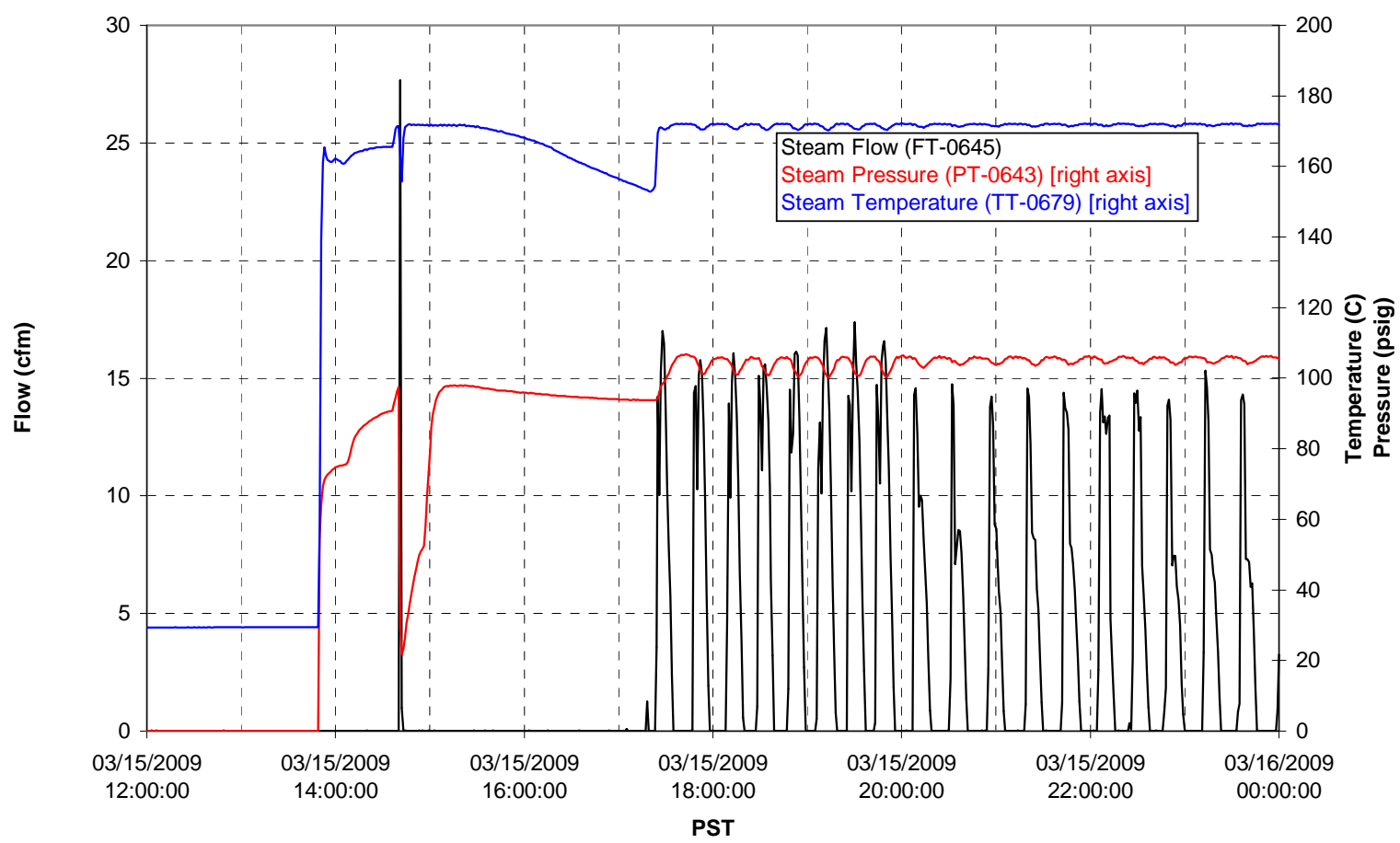

T01A Steam

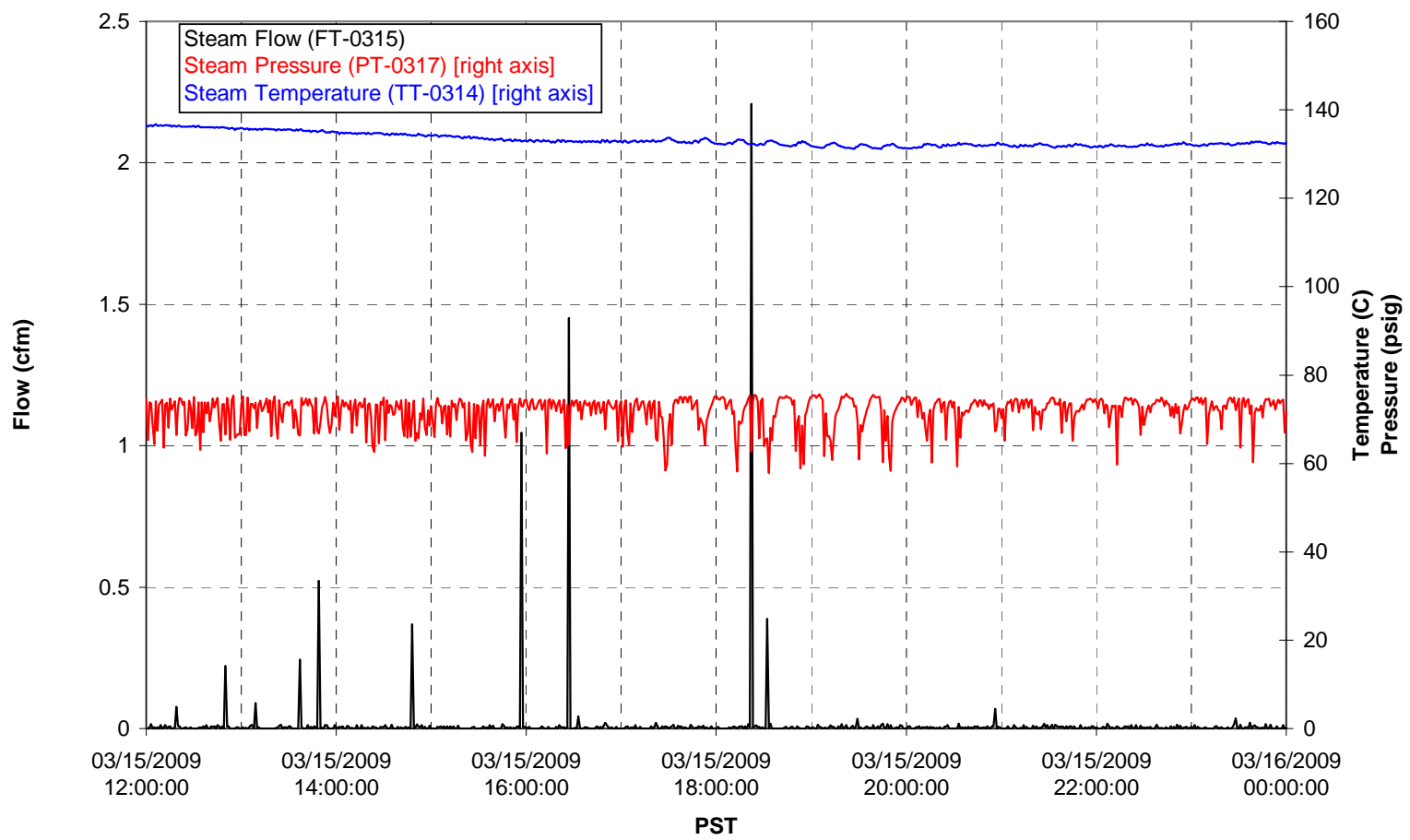


T01B Steam

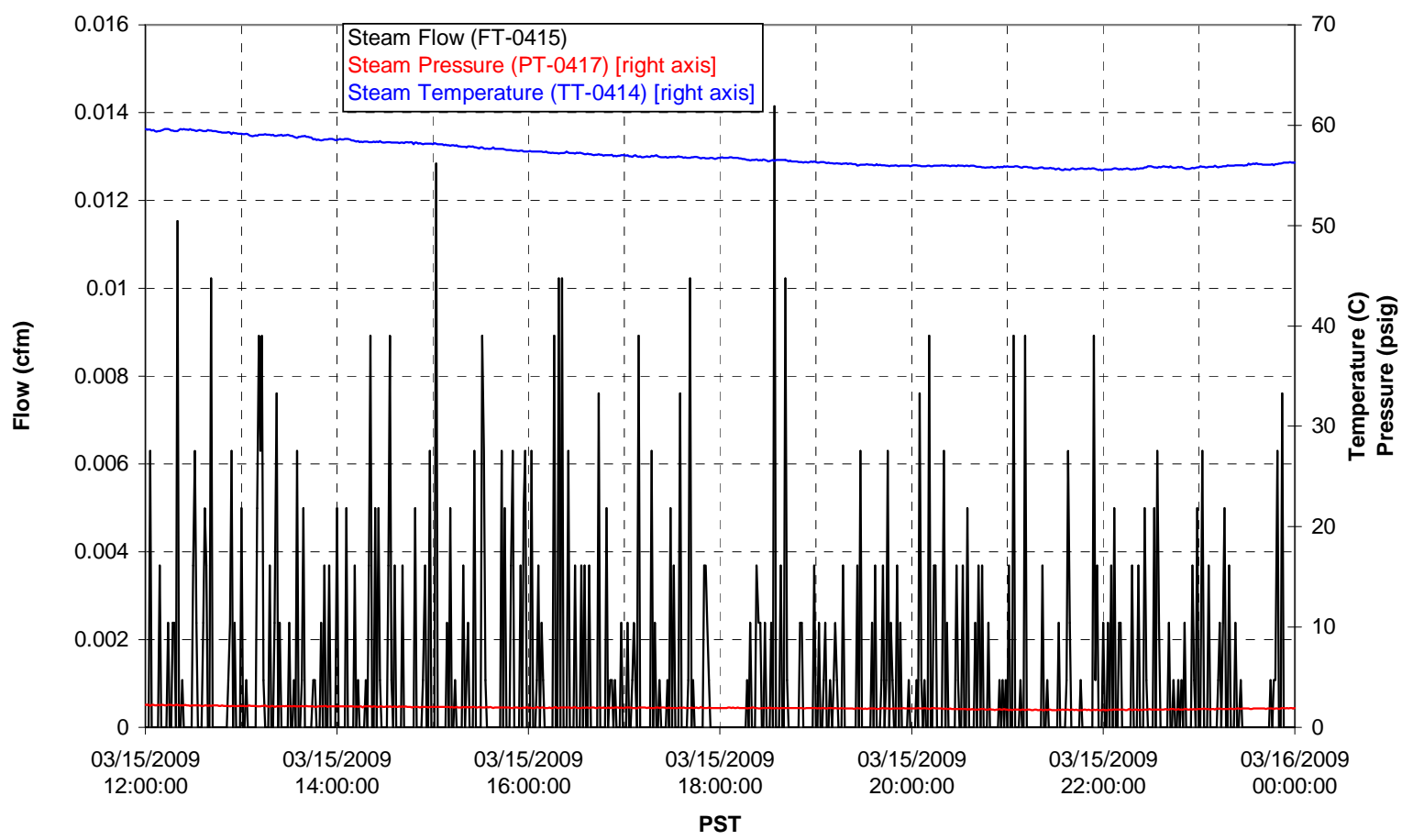




\section{Integrated Test B Data Plots \\ 03/16/09 00:00 - 03/16/09 12:00}


T01A level

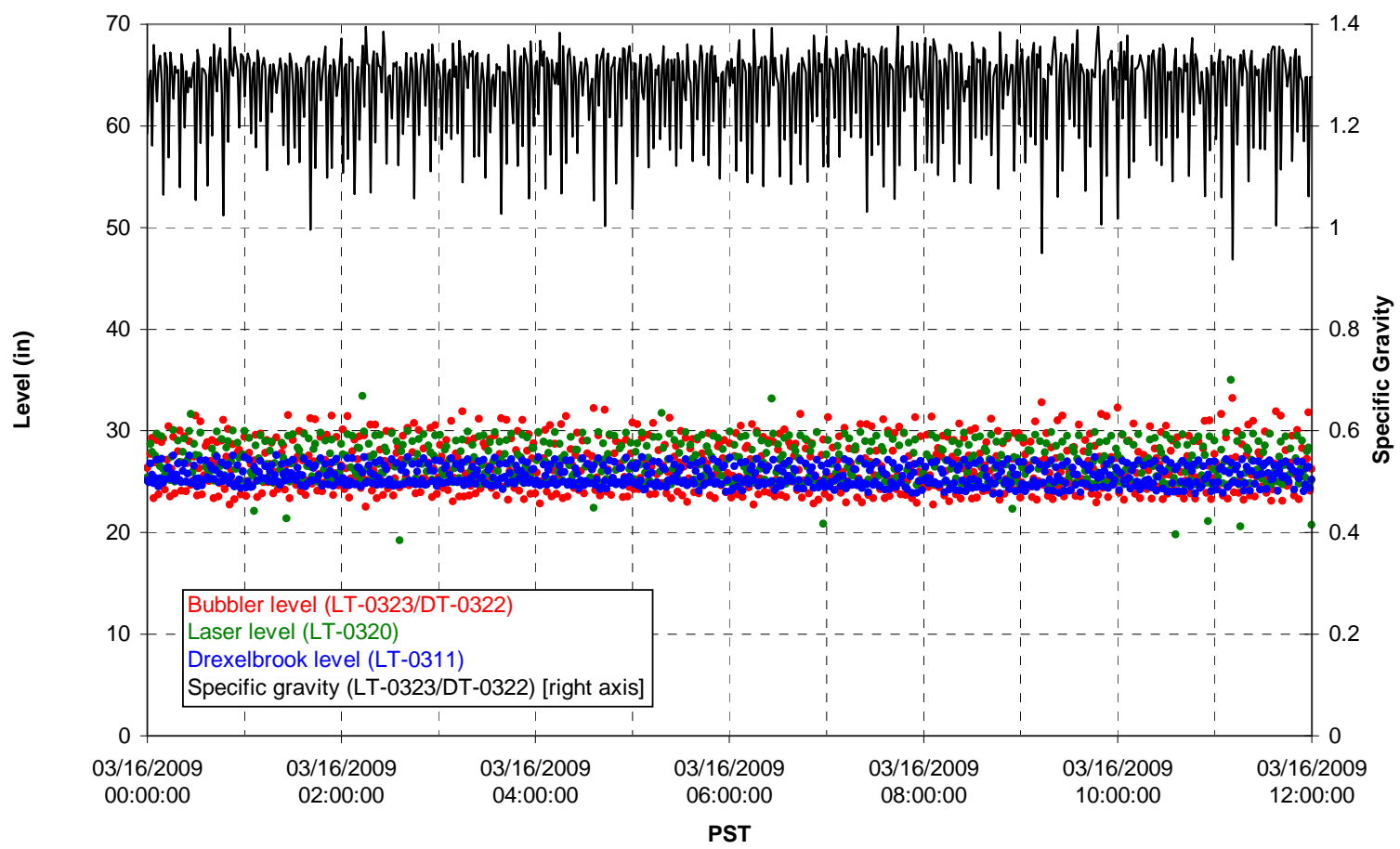

T01A temperatures

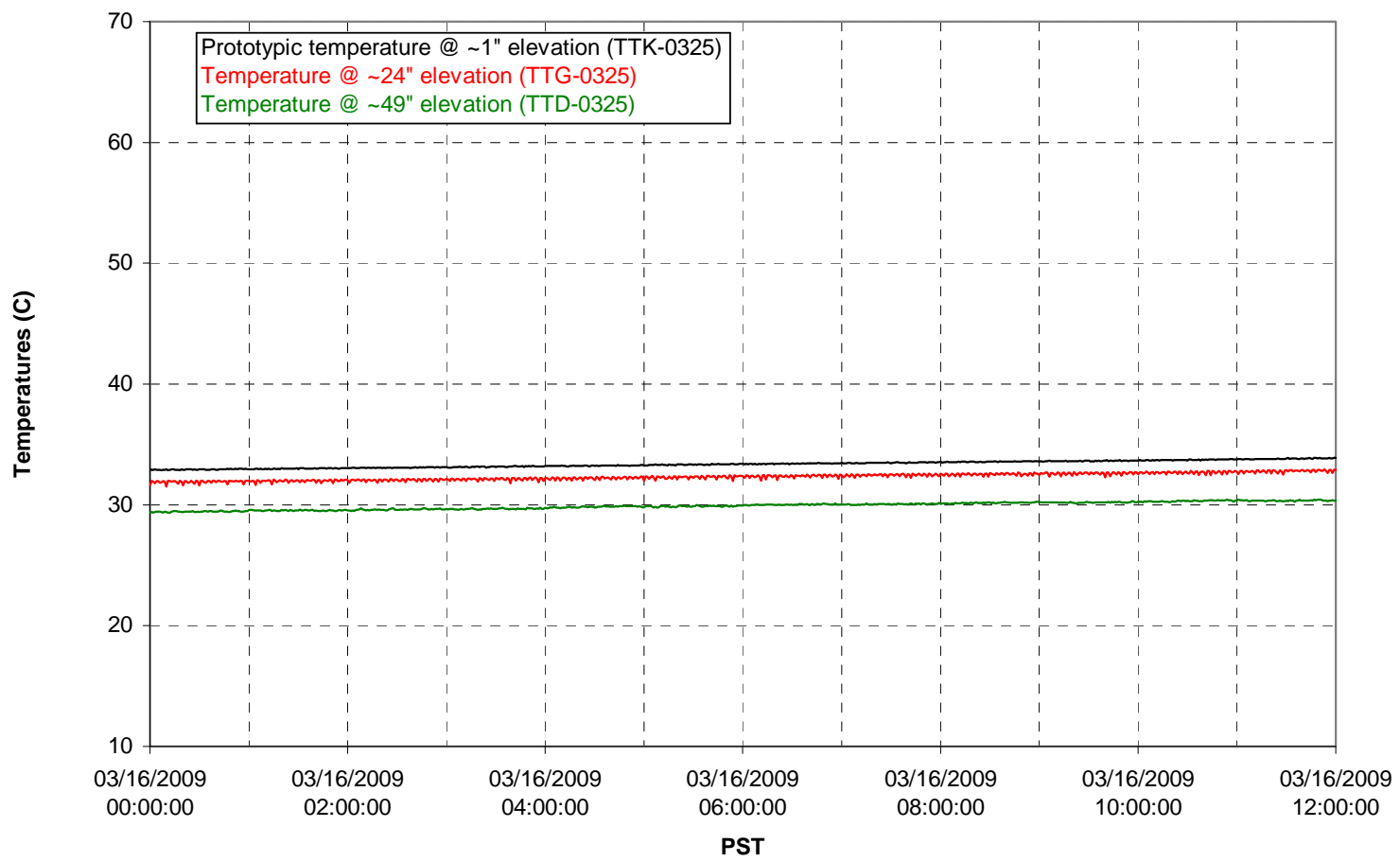


T01B level

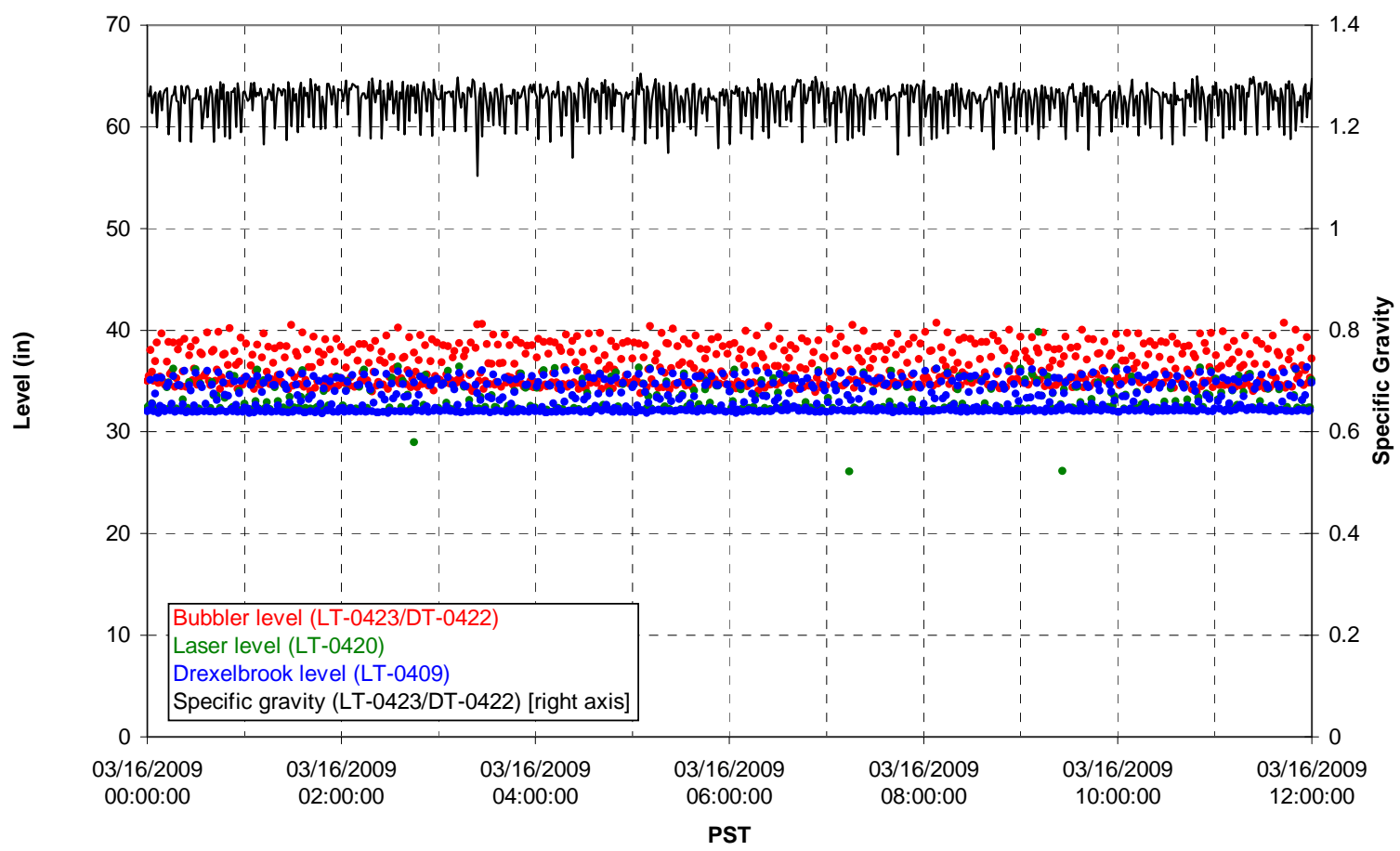

T01B temperatures

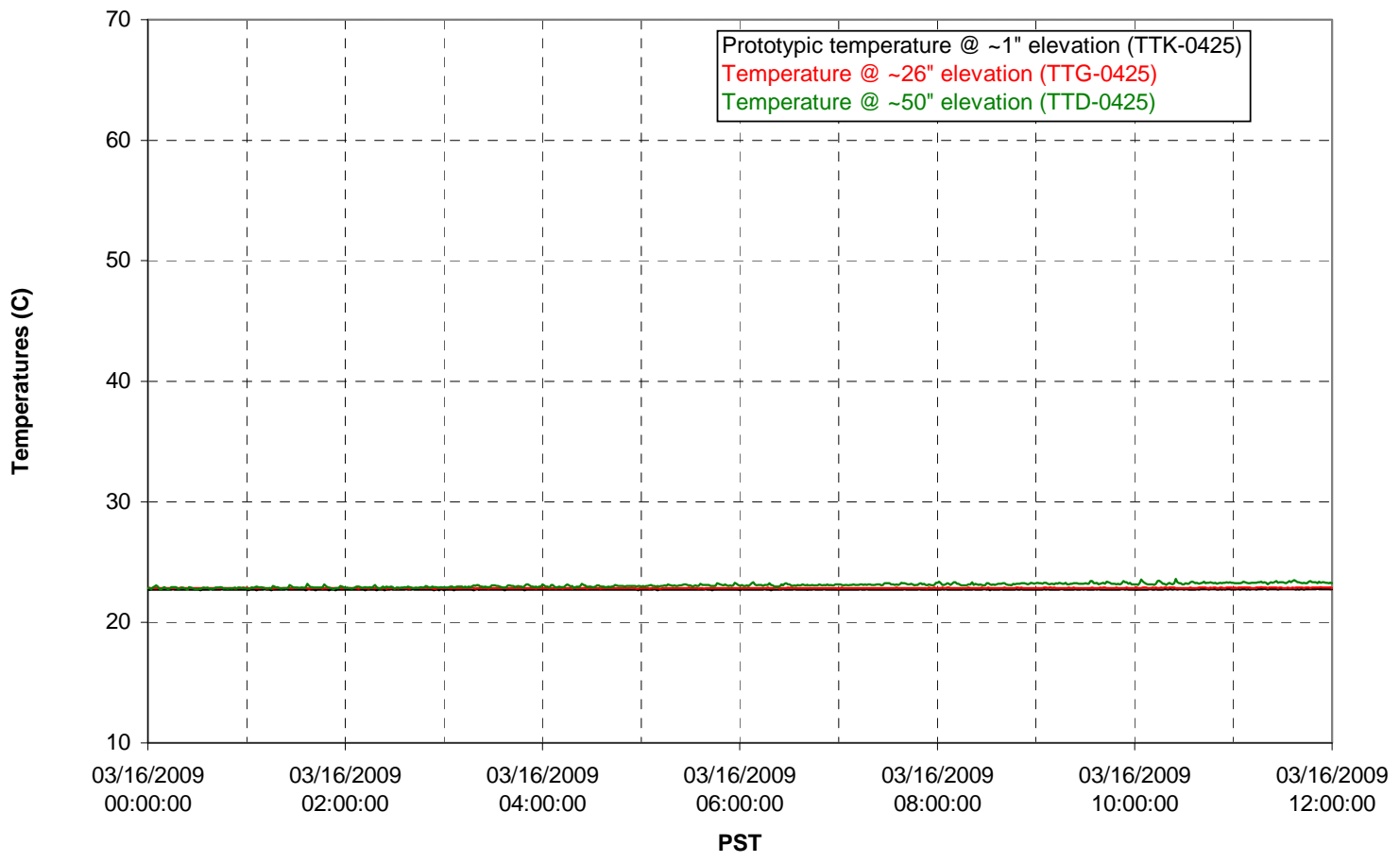


T02A level

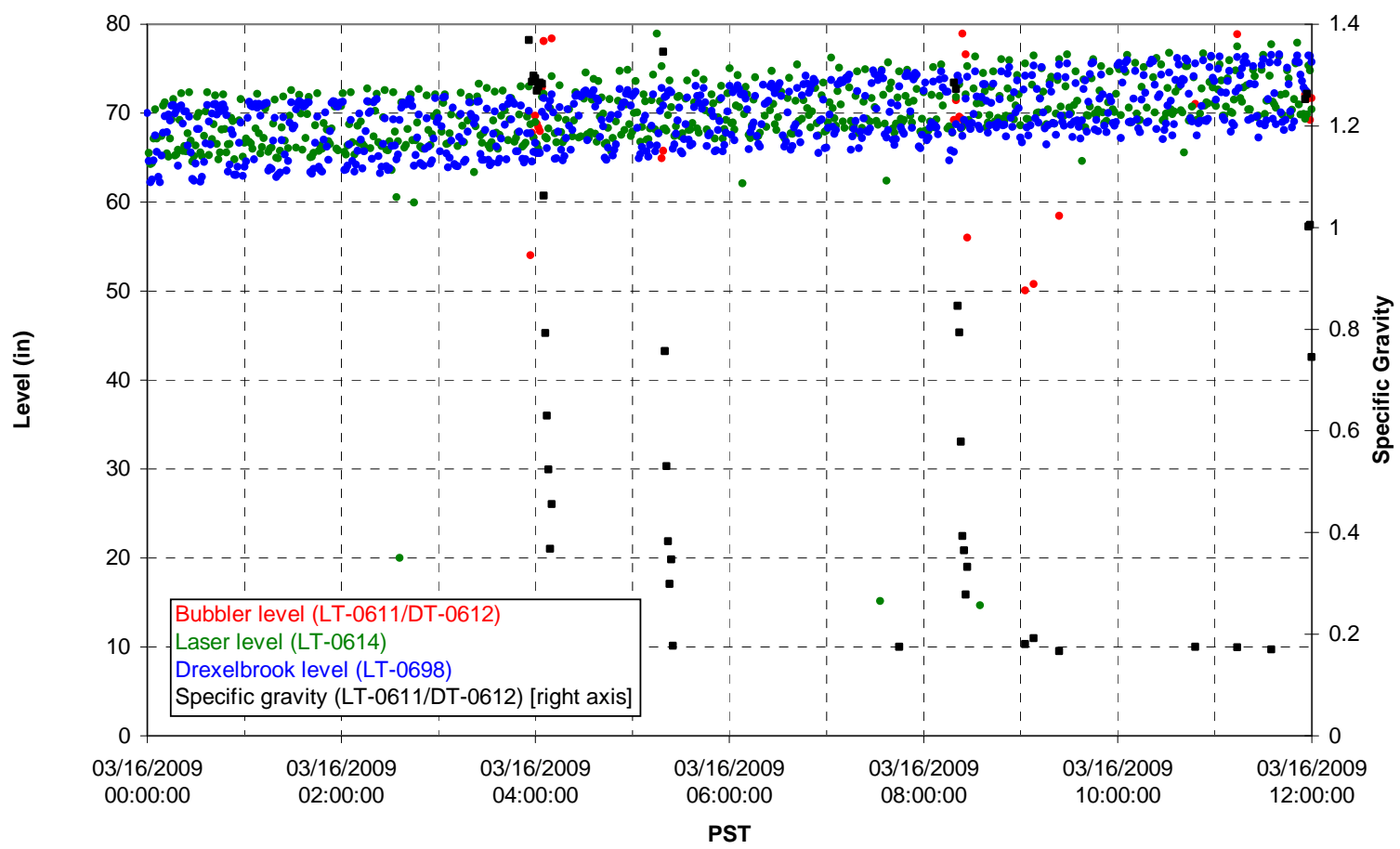

T02A temperatures

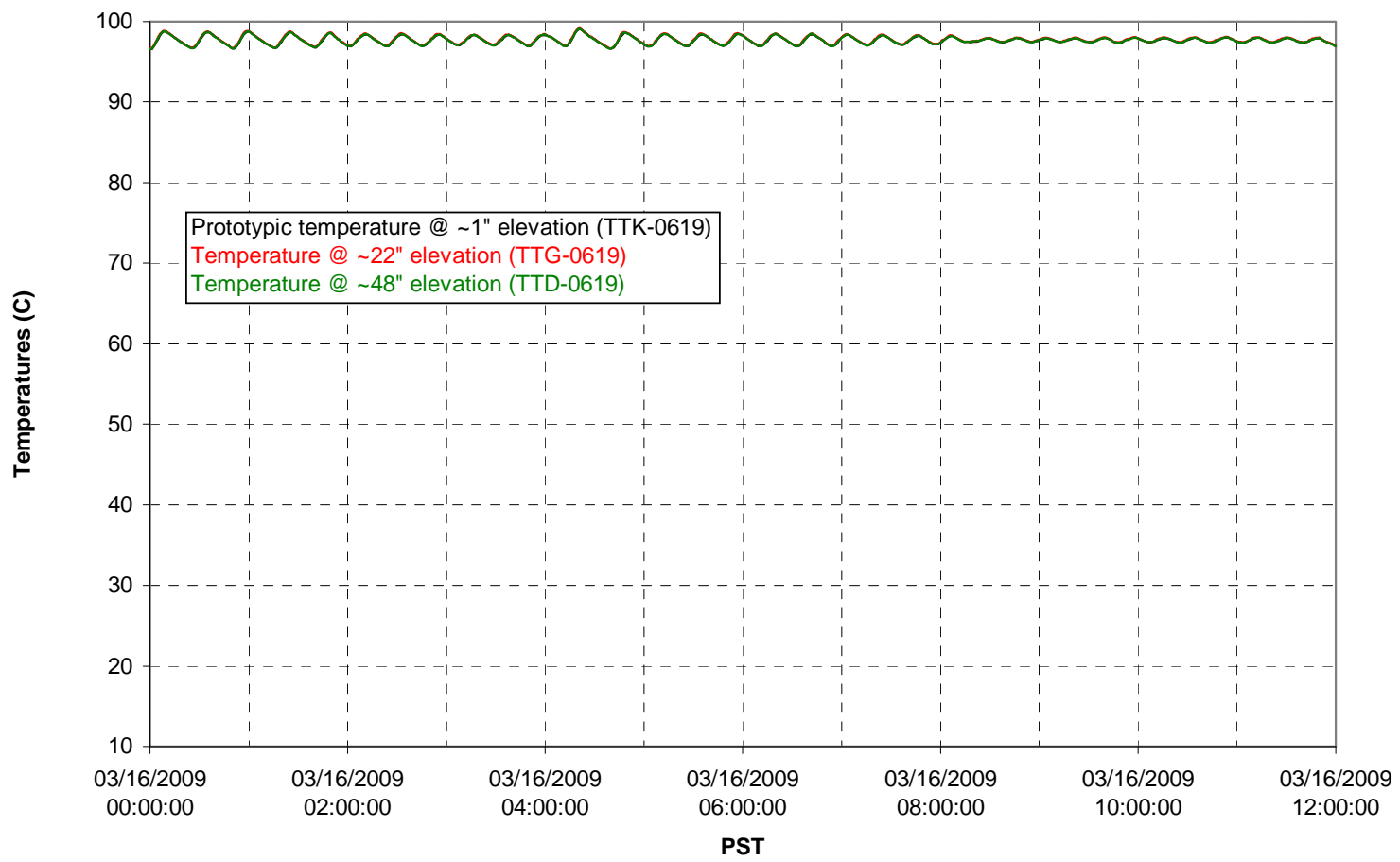


T02A and filter loop temperatures

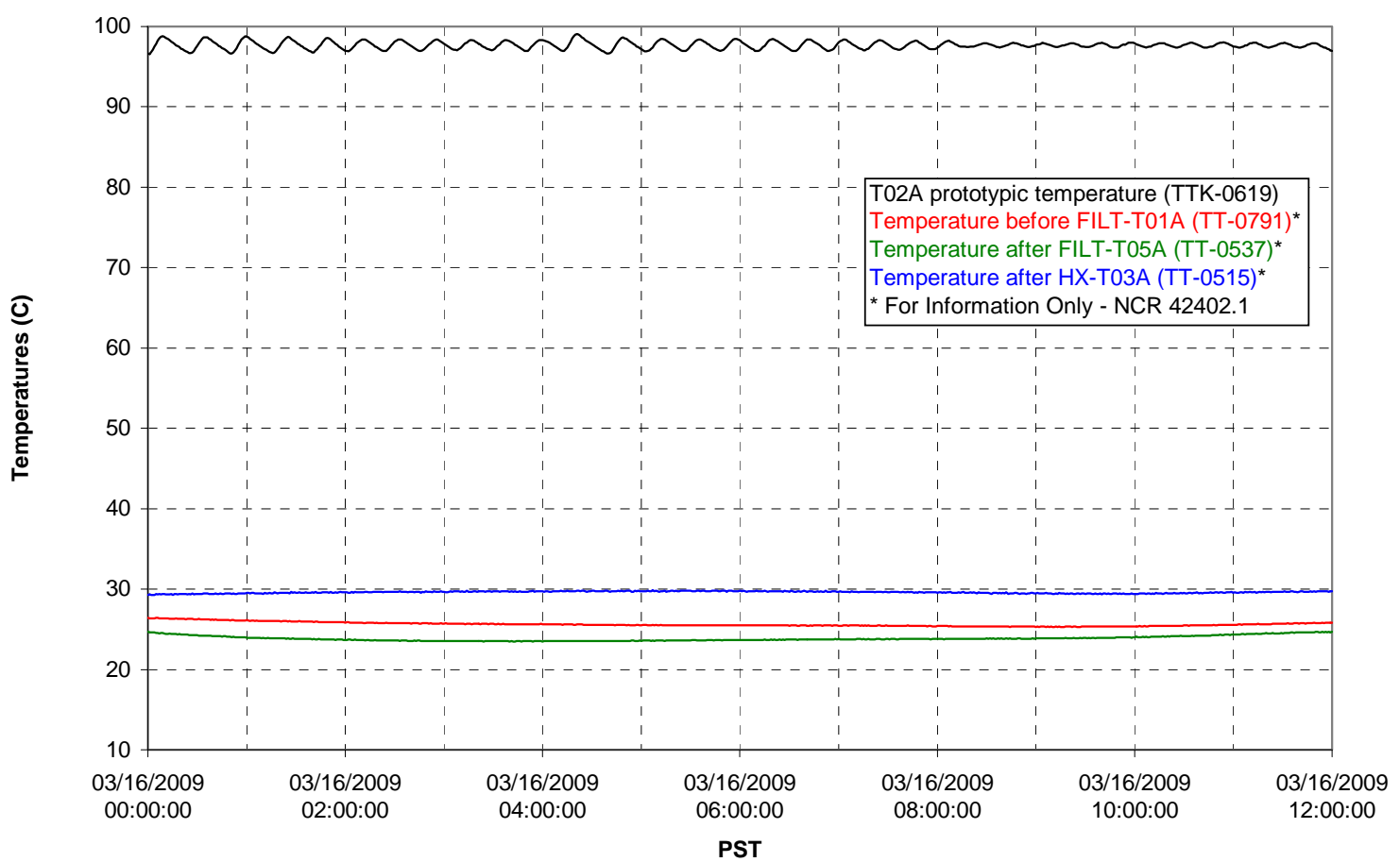

Pump Pressures and Flow

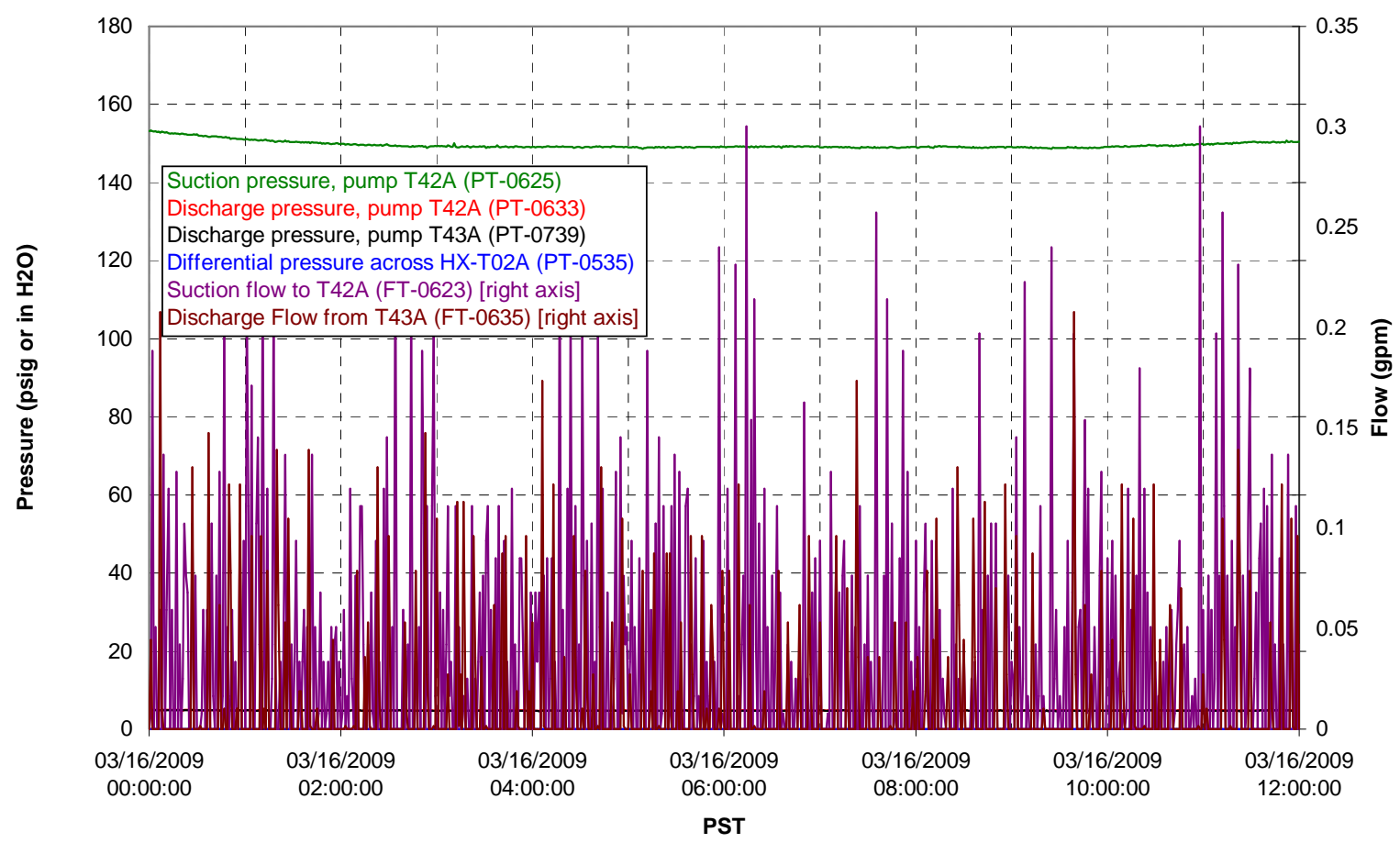


Axial pressure drop

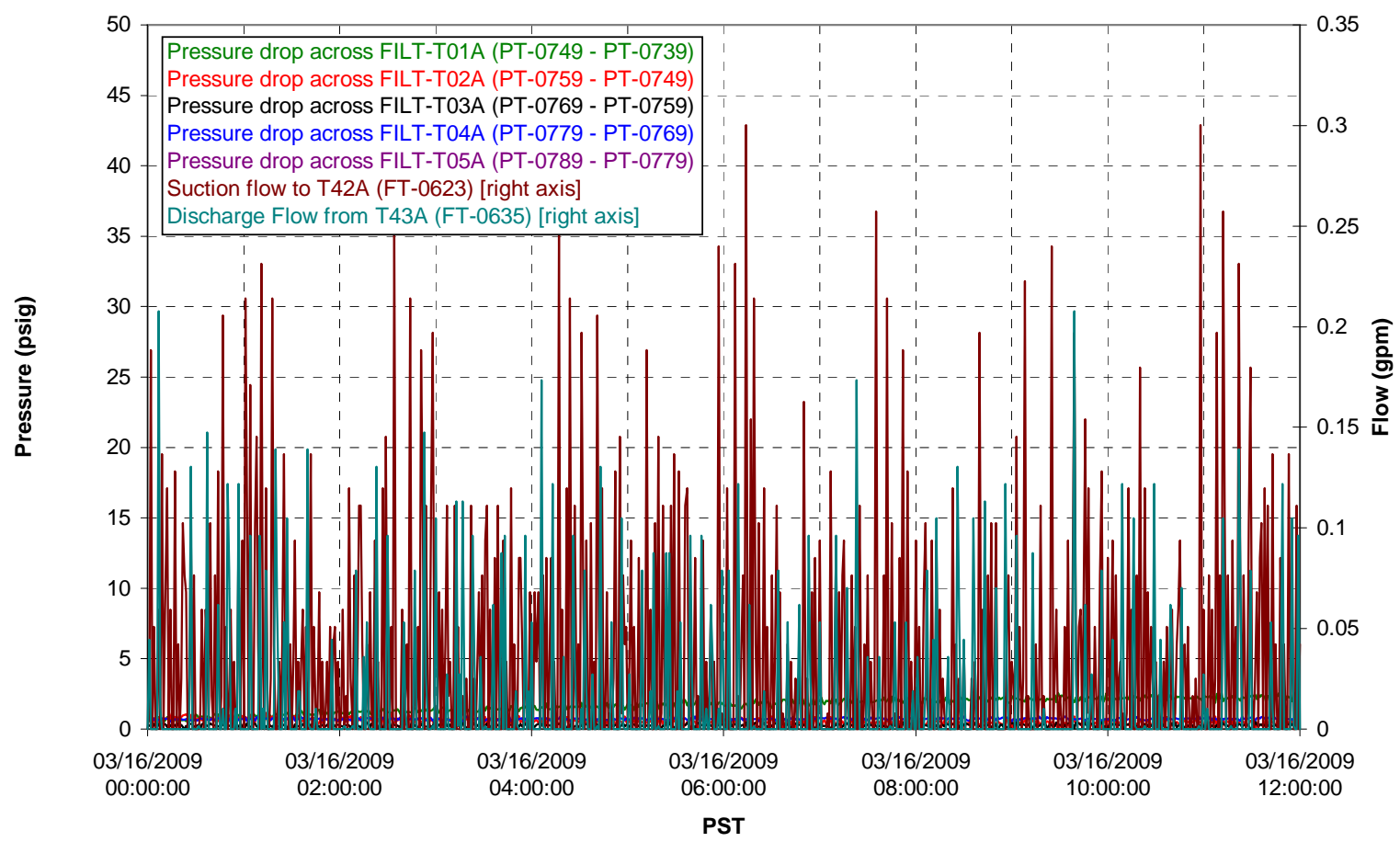

Permeate flow rates

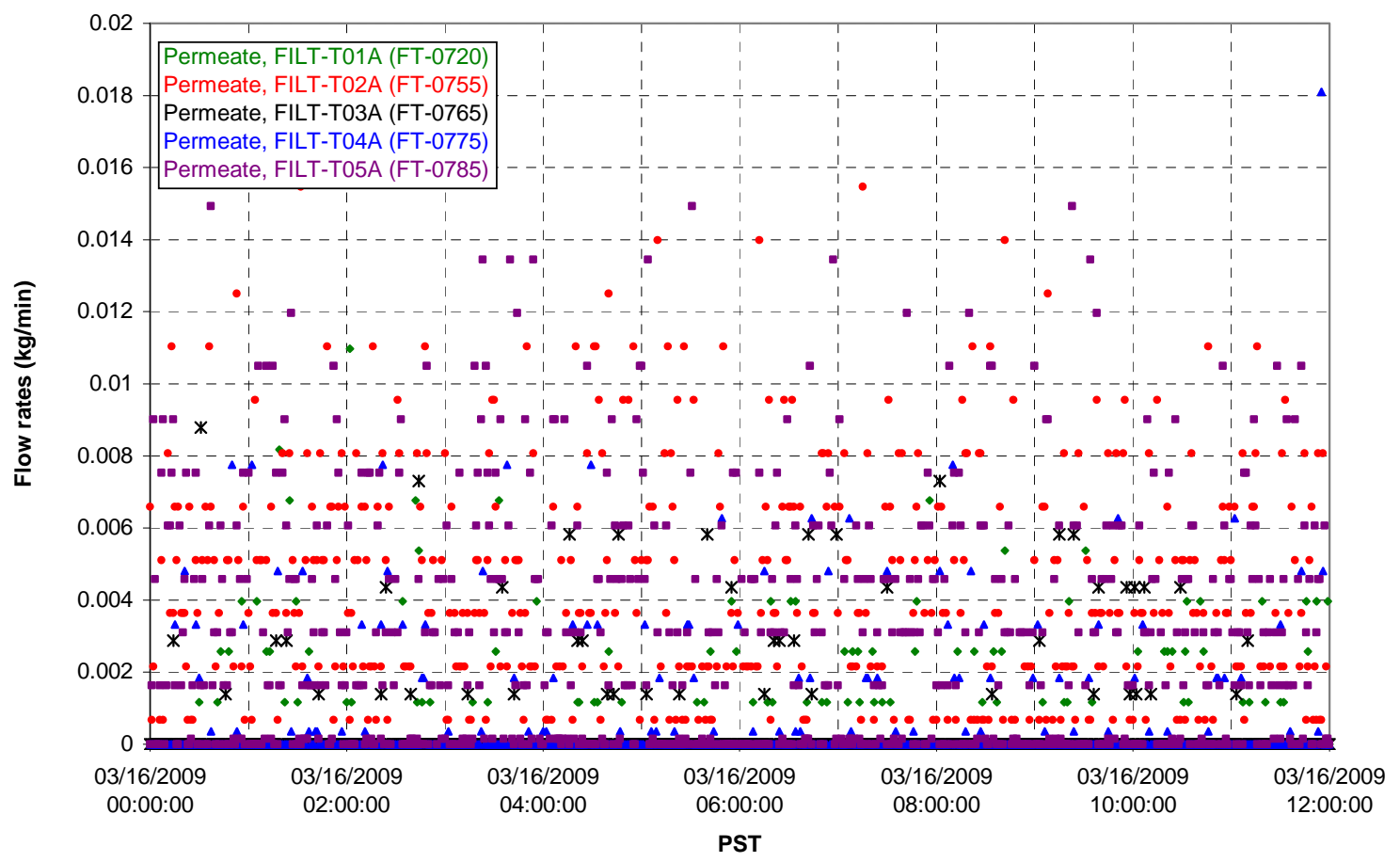


T02A Inner Temperature Tree

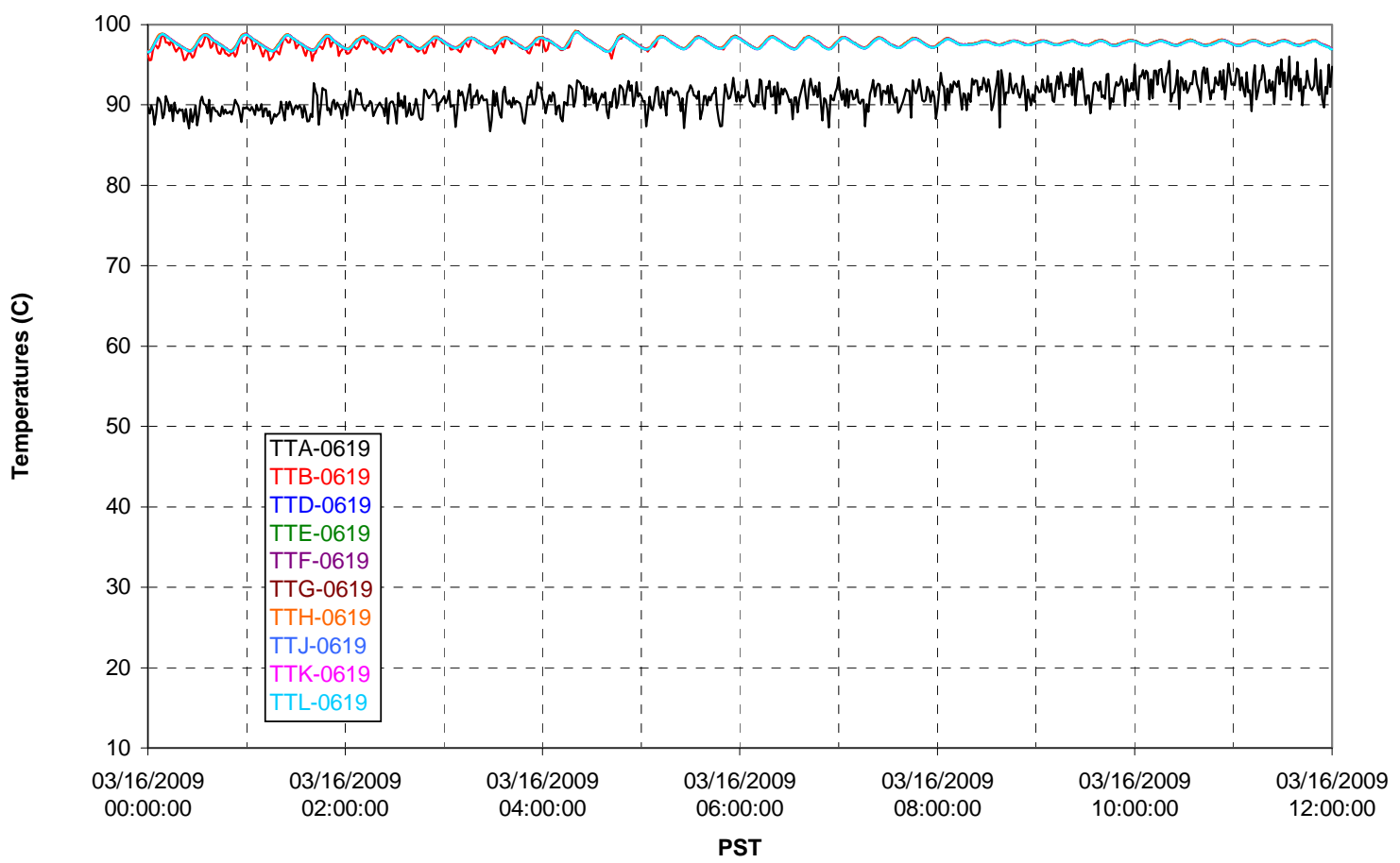

T02A Outer Temperature Tree

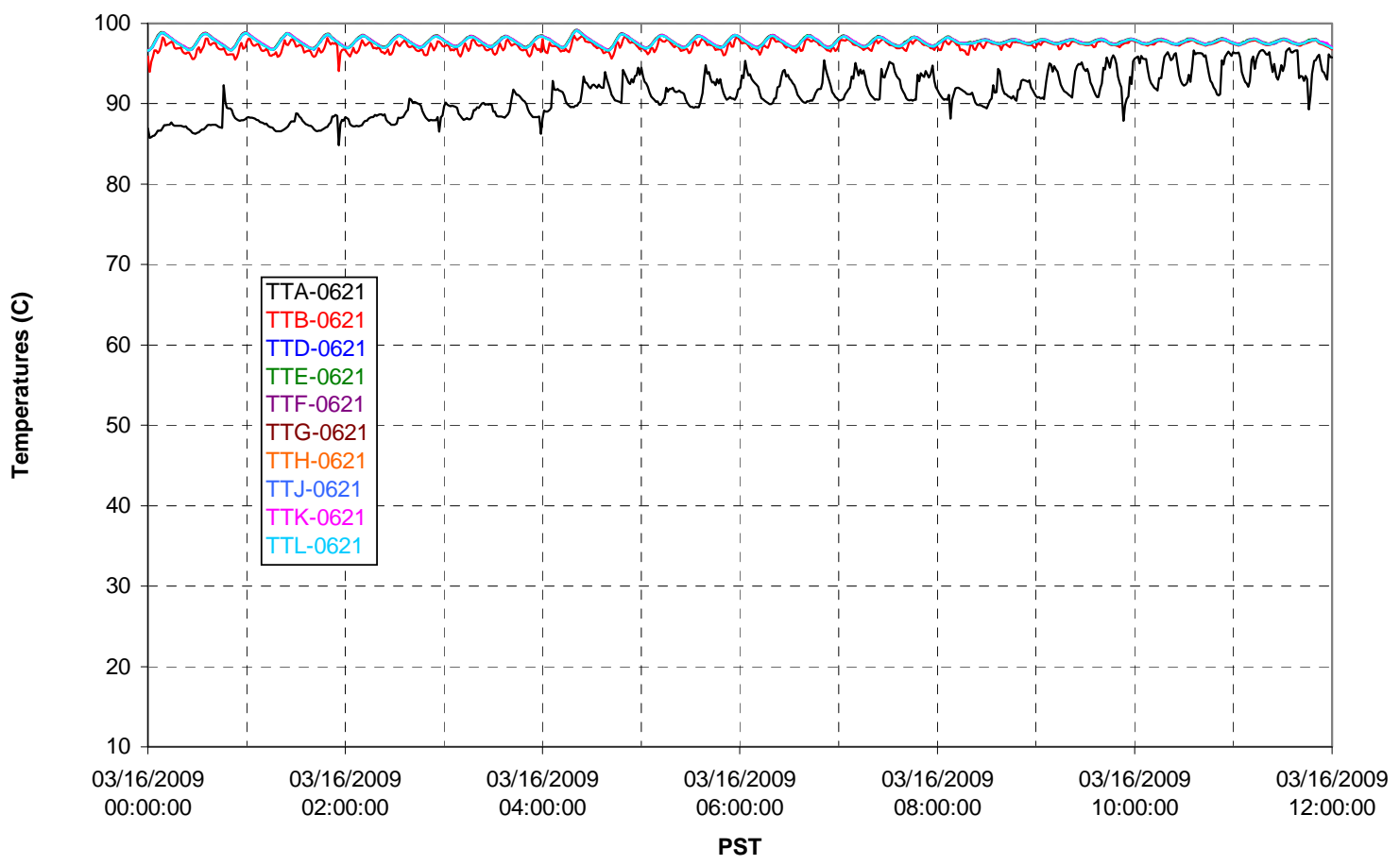


T02A temperatures

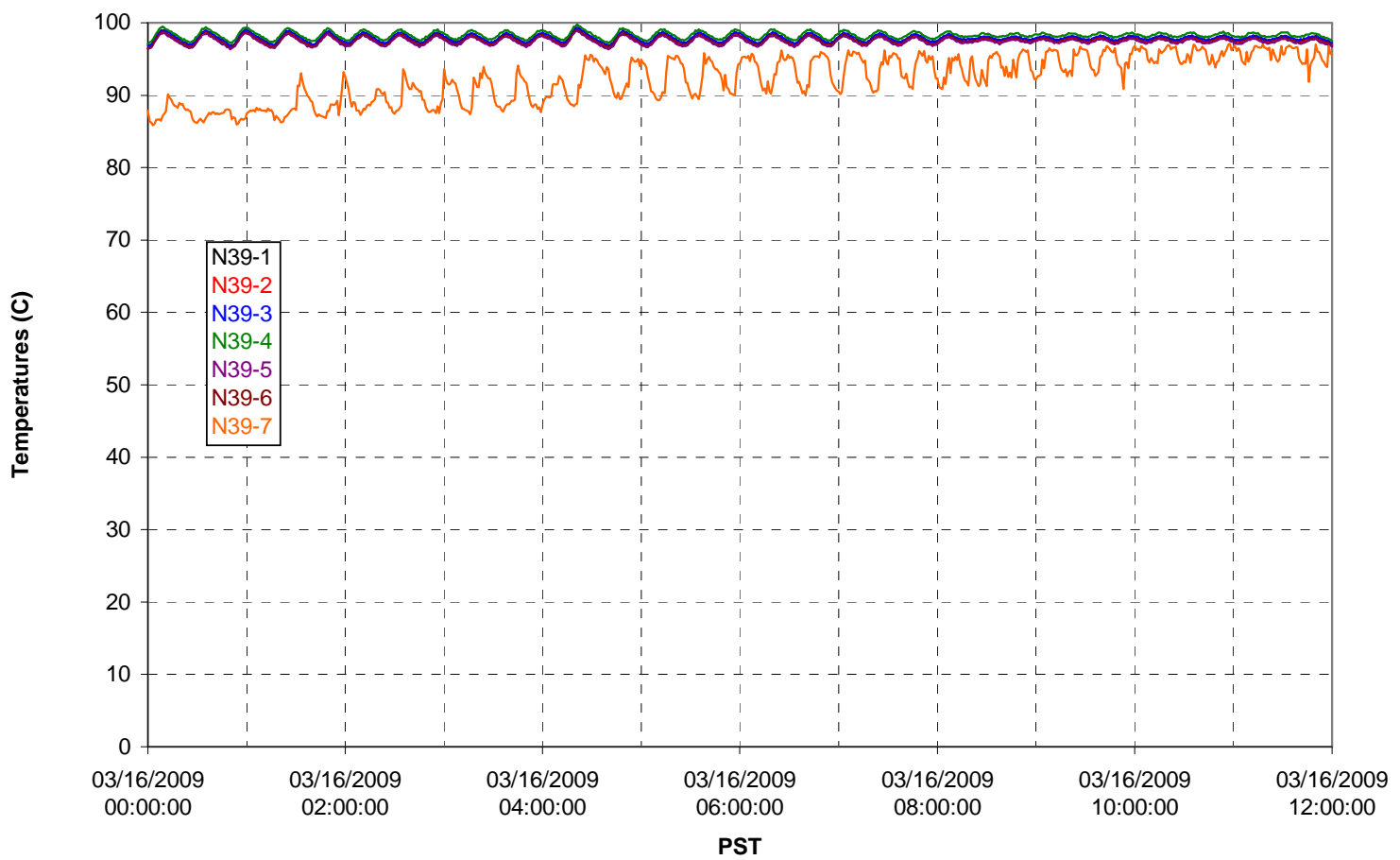

T02A temperatures

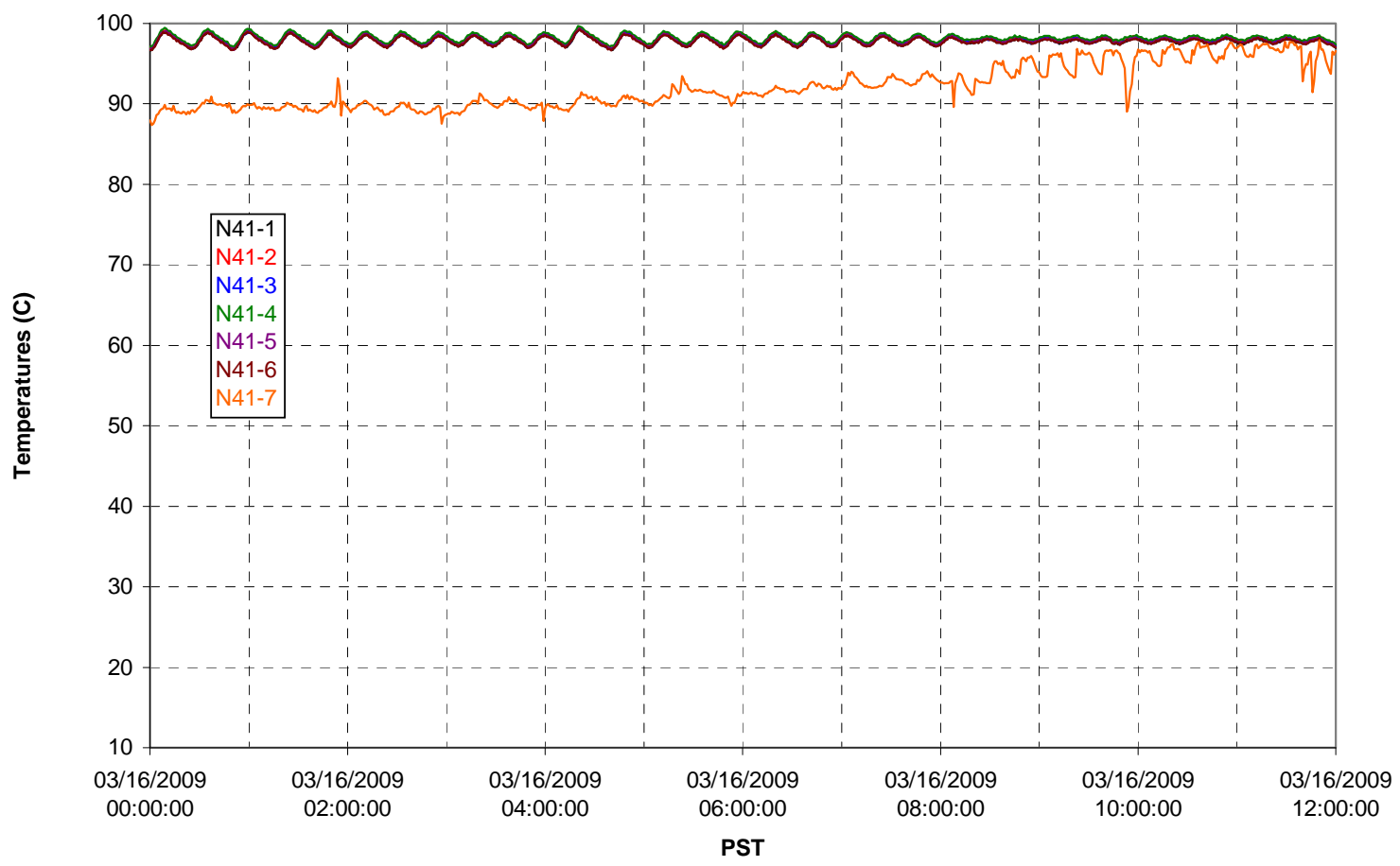


T02A temperatures

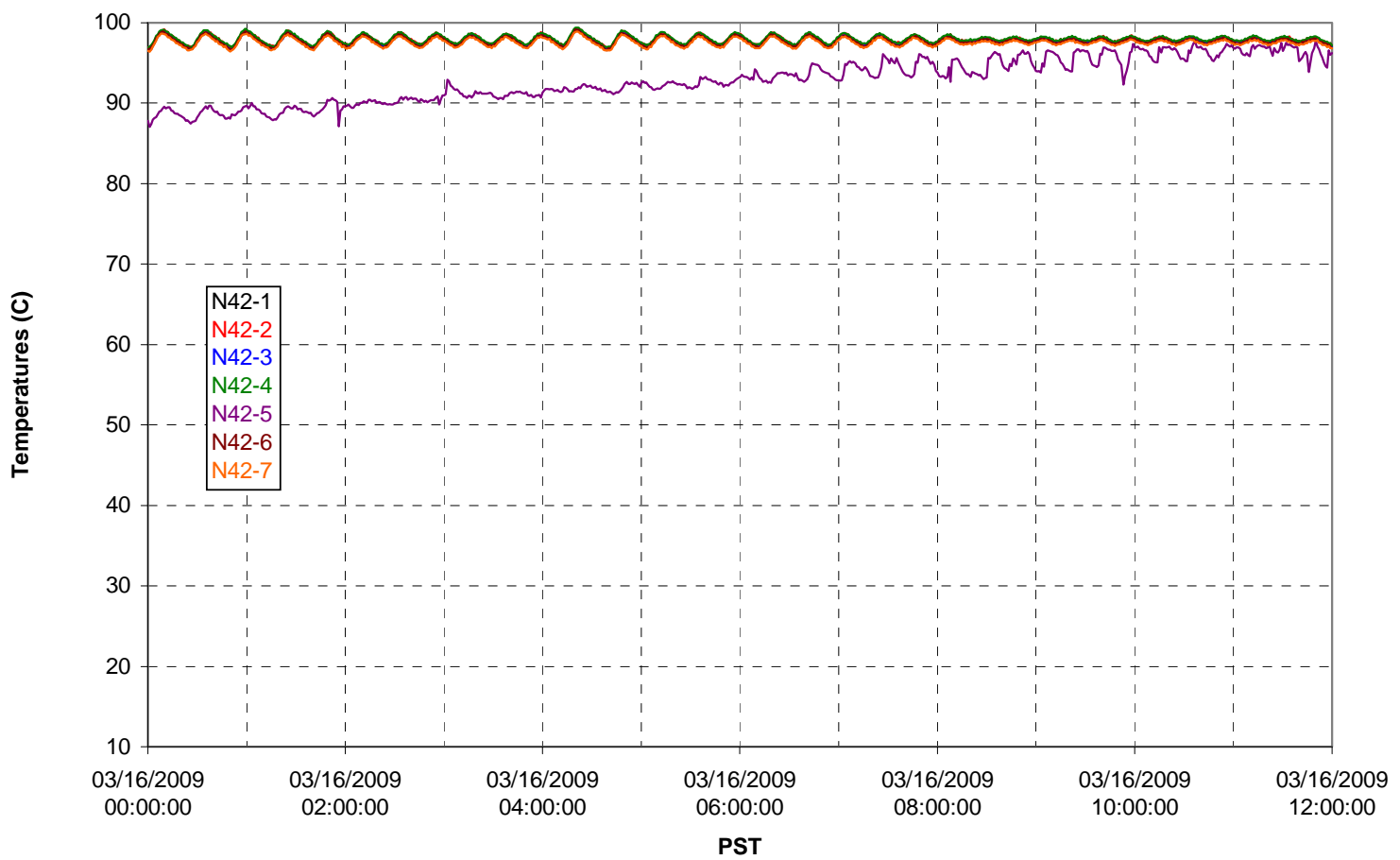

T02A temperatures

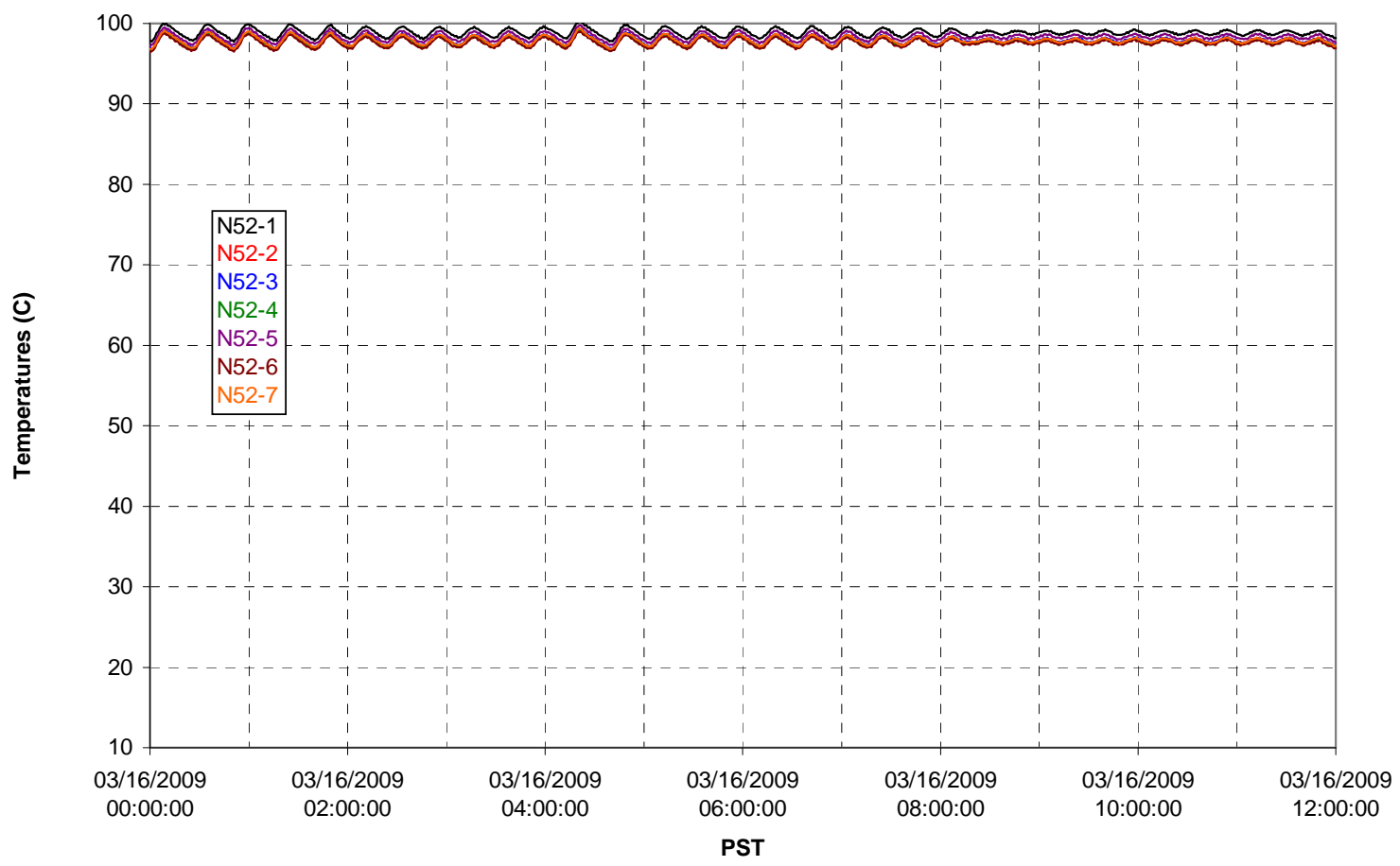


T02A Heating and Cooling

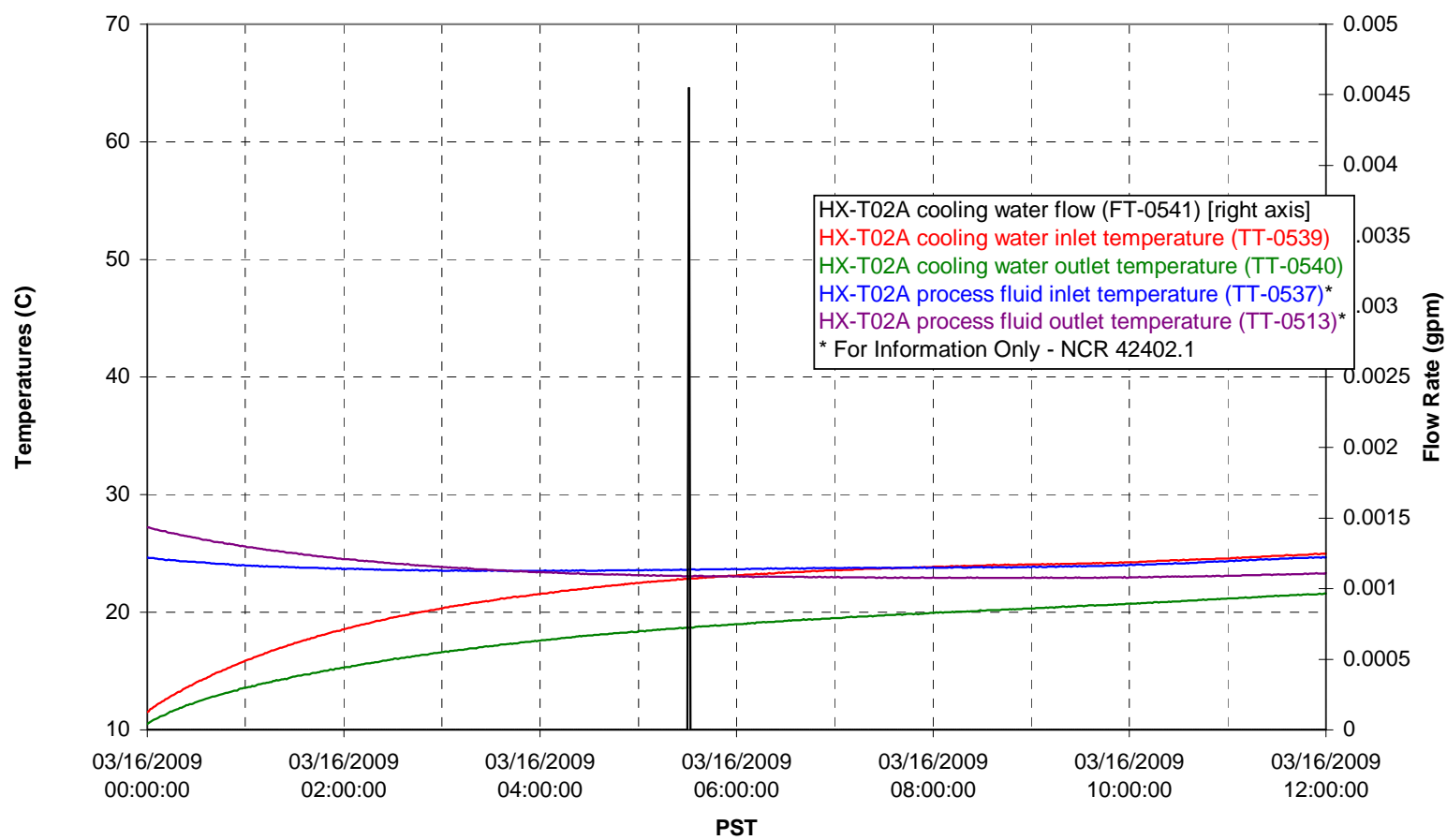

Pump Operation

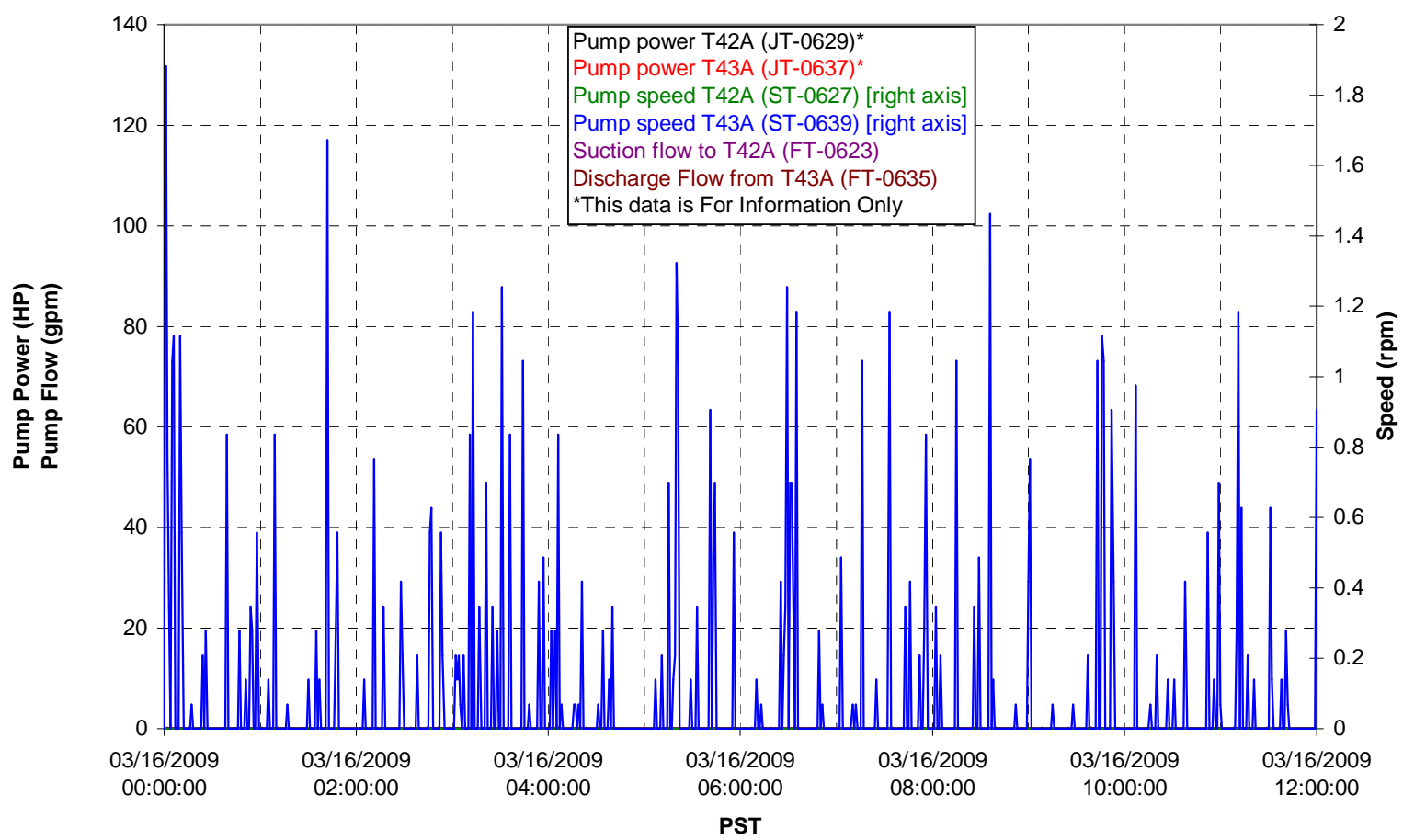


Pulsepot UFP-PP-T01A

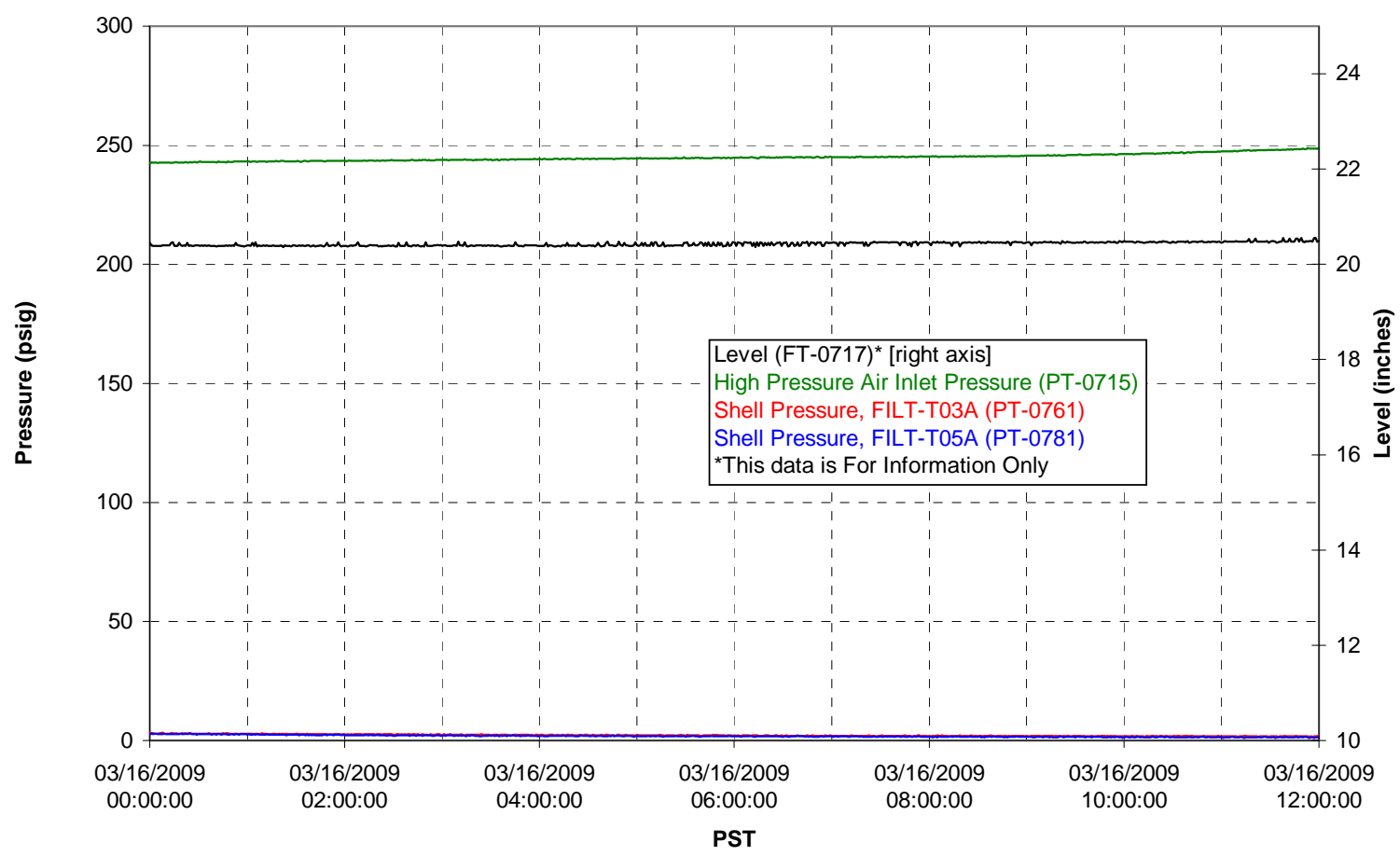

Pulsepot UFP-PP-T02A

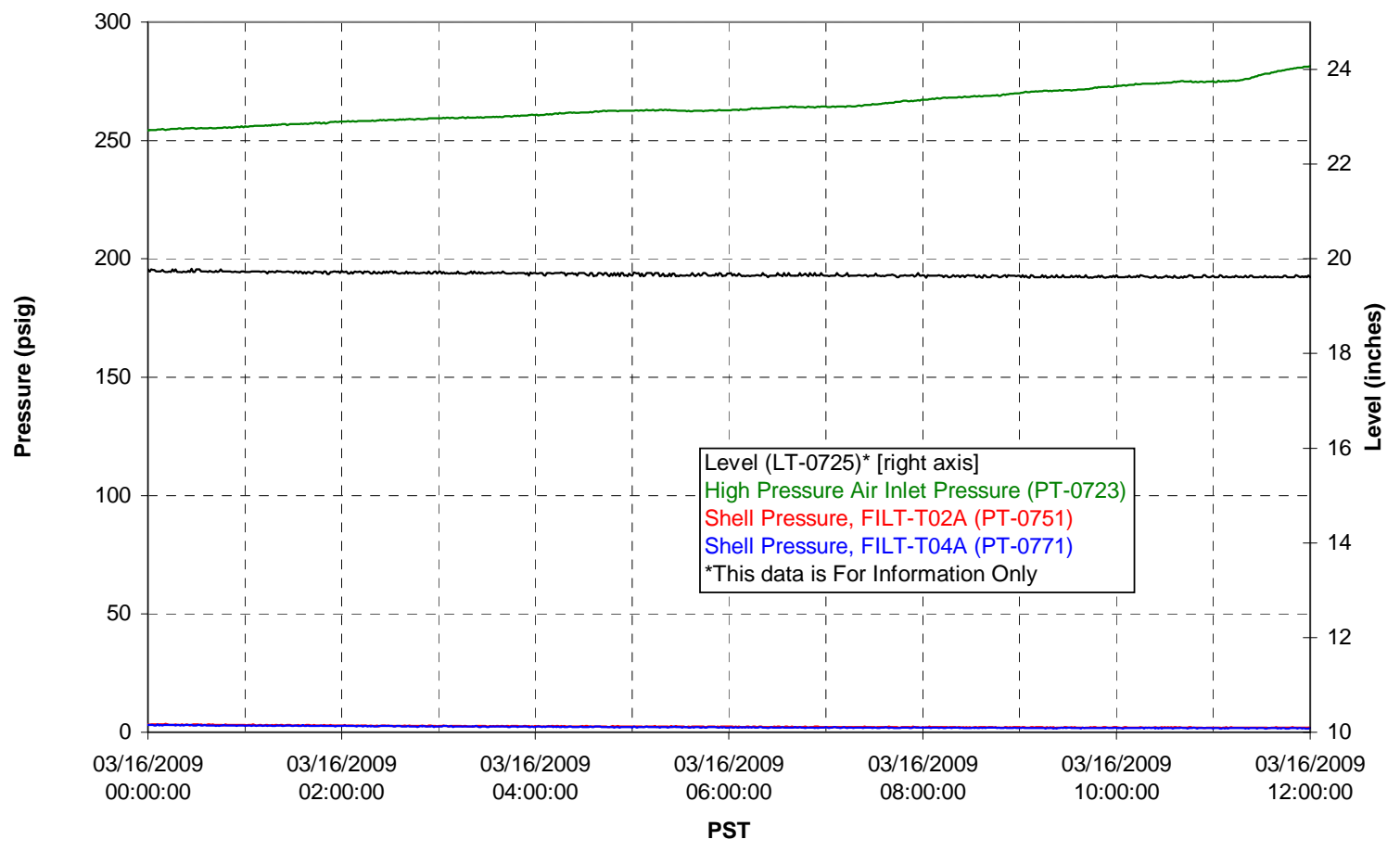


Pulsepot UFP-PP-T03A

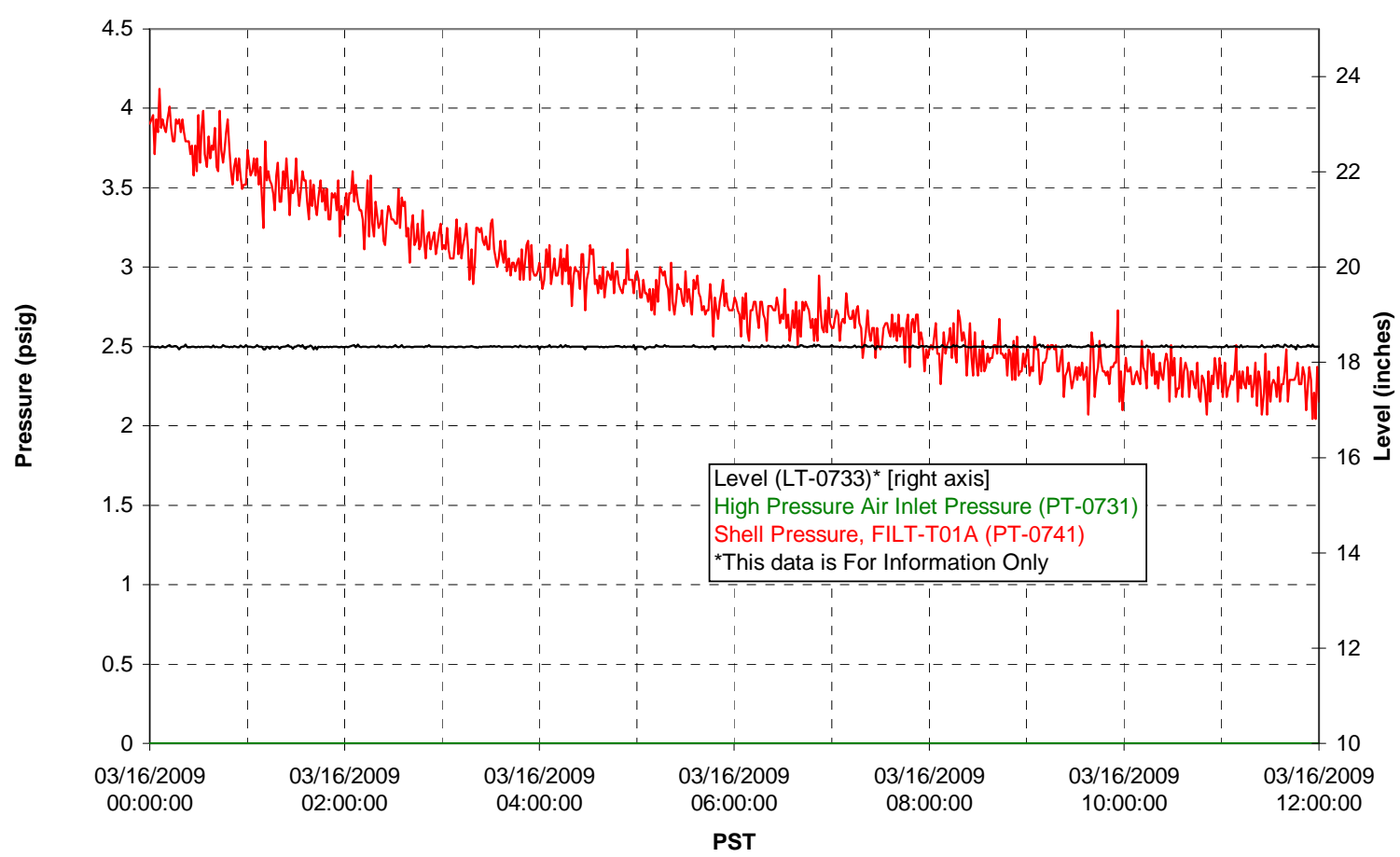

Pulsepot Levels

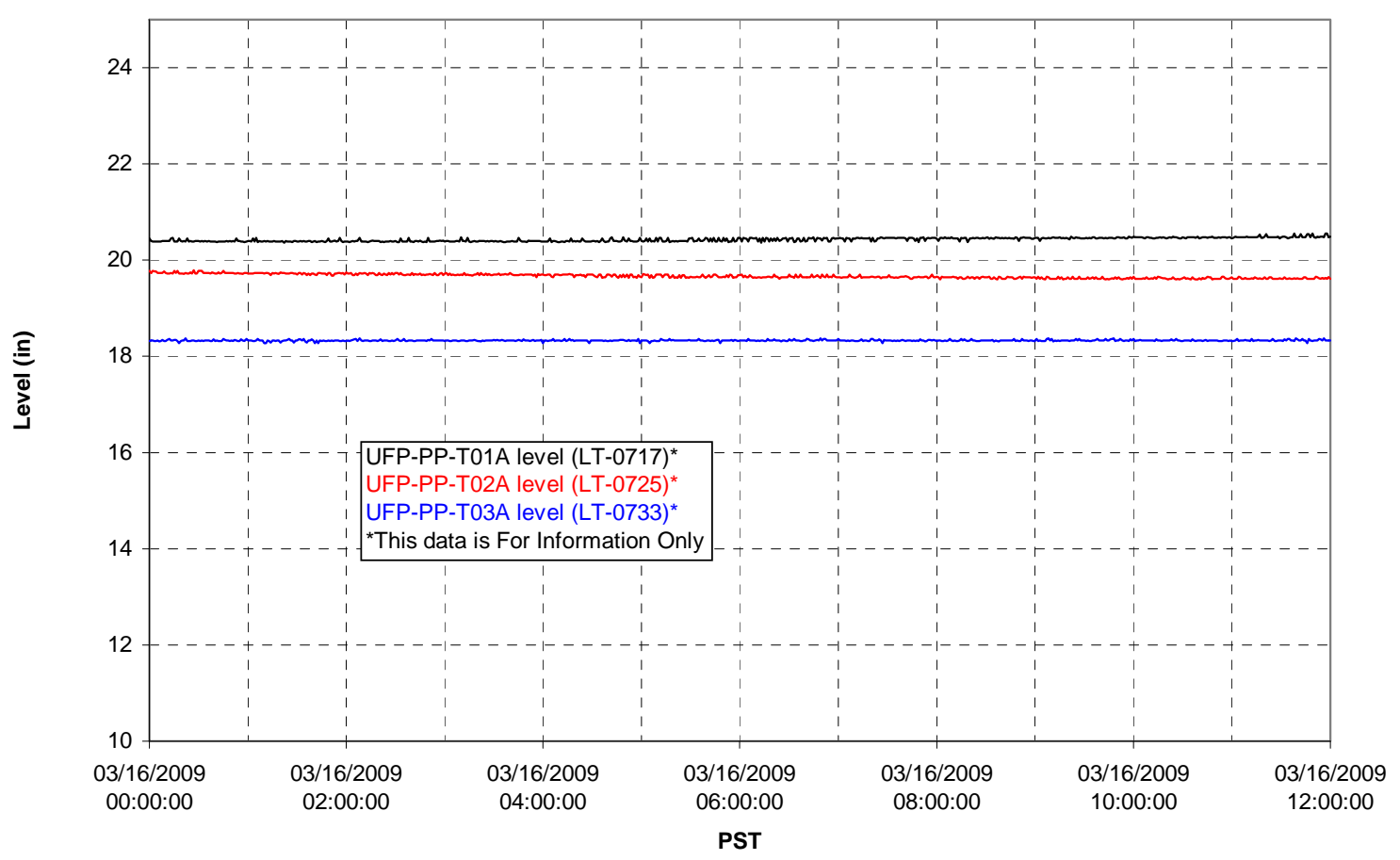


Filter UFP-FILT-T01A

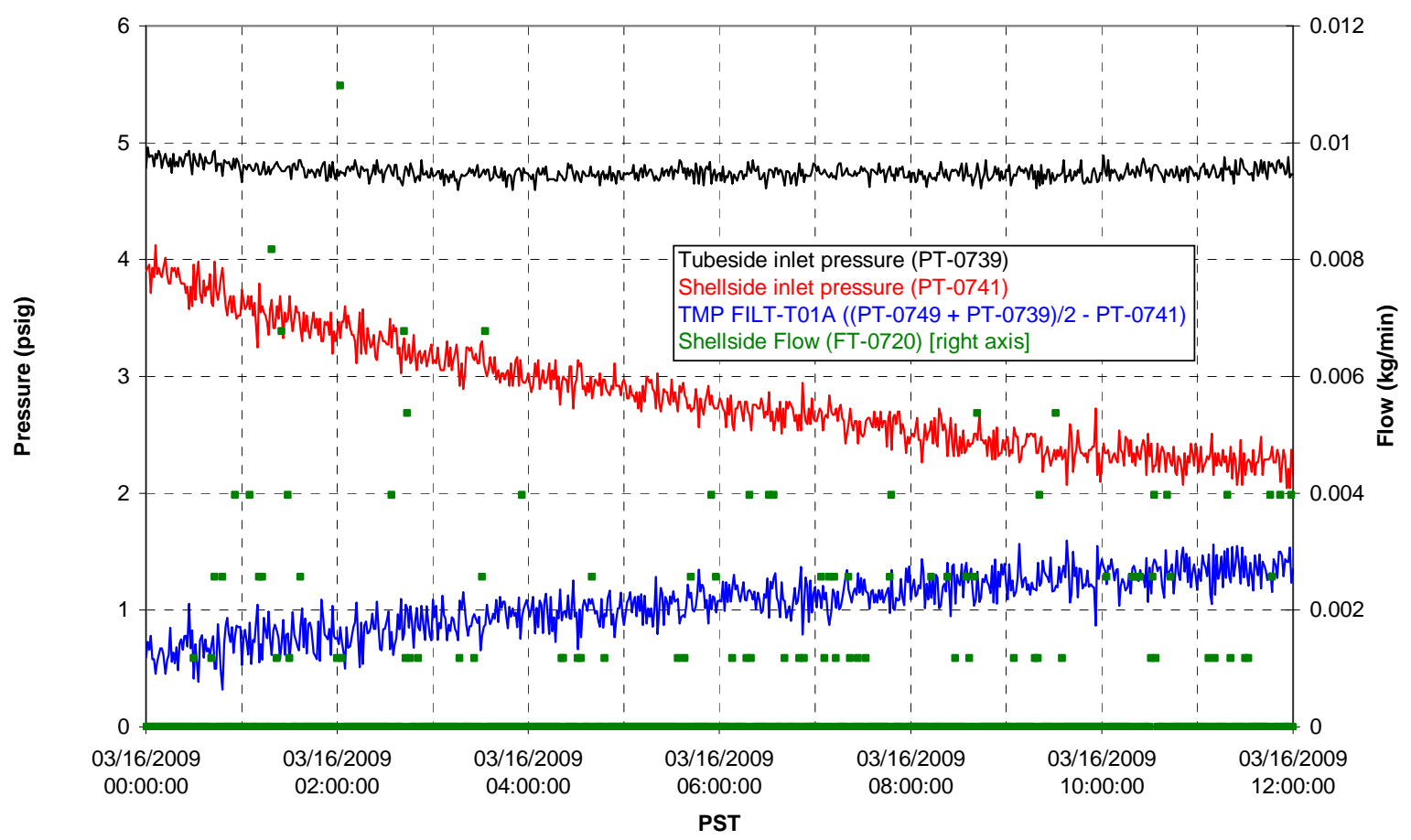

Filter UFP-FILT-T02A

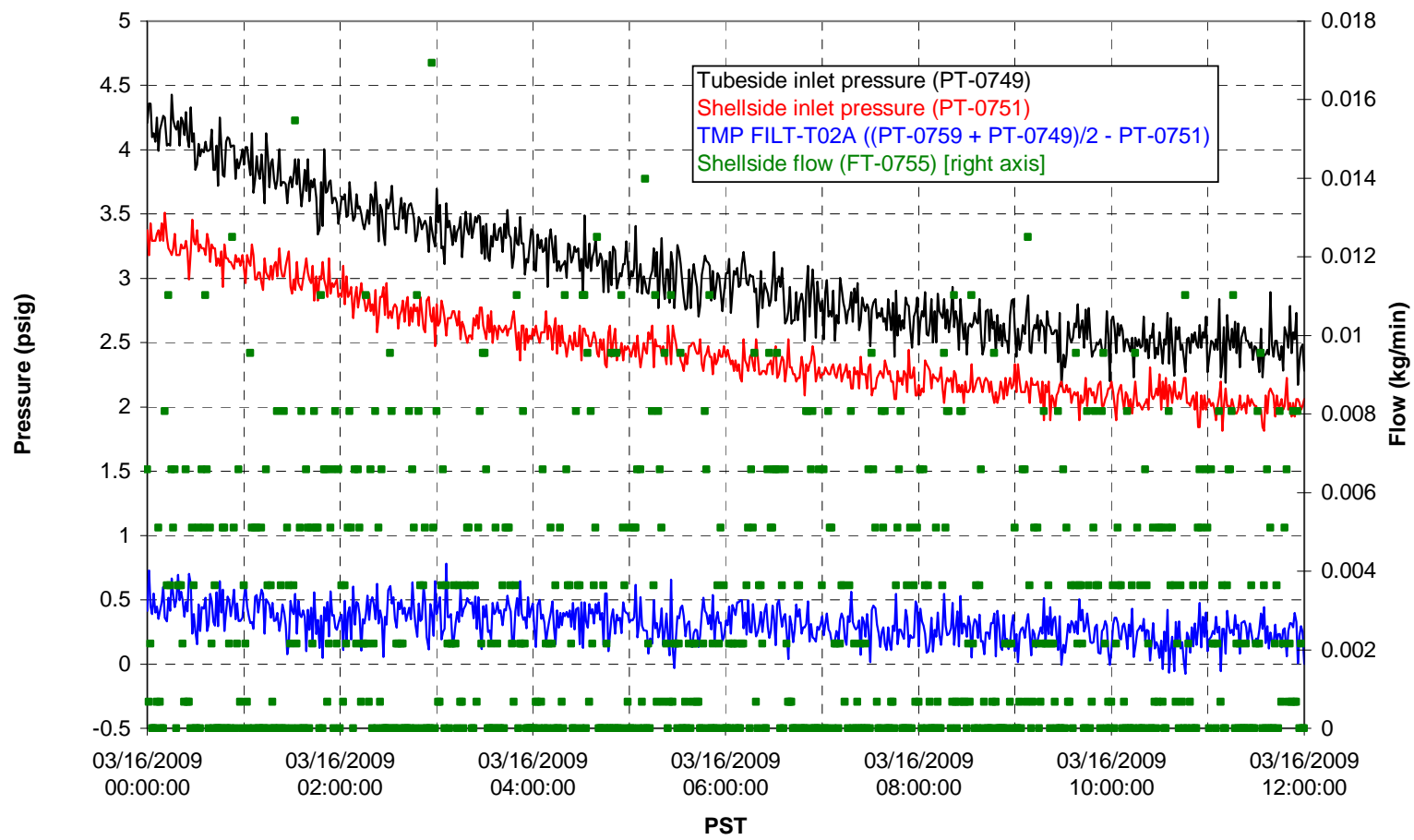


Filter UFP-FILT-T03A

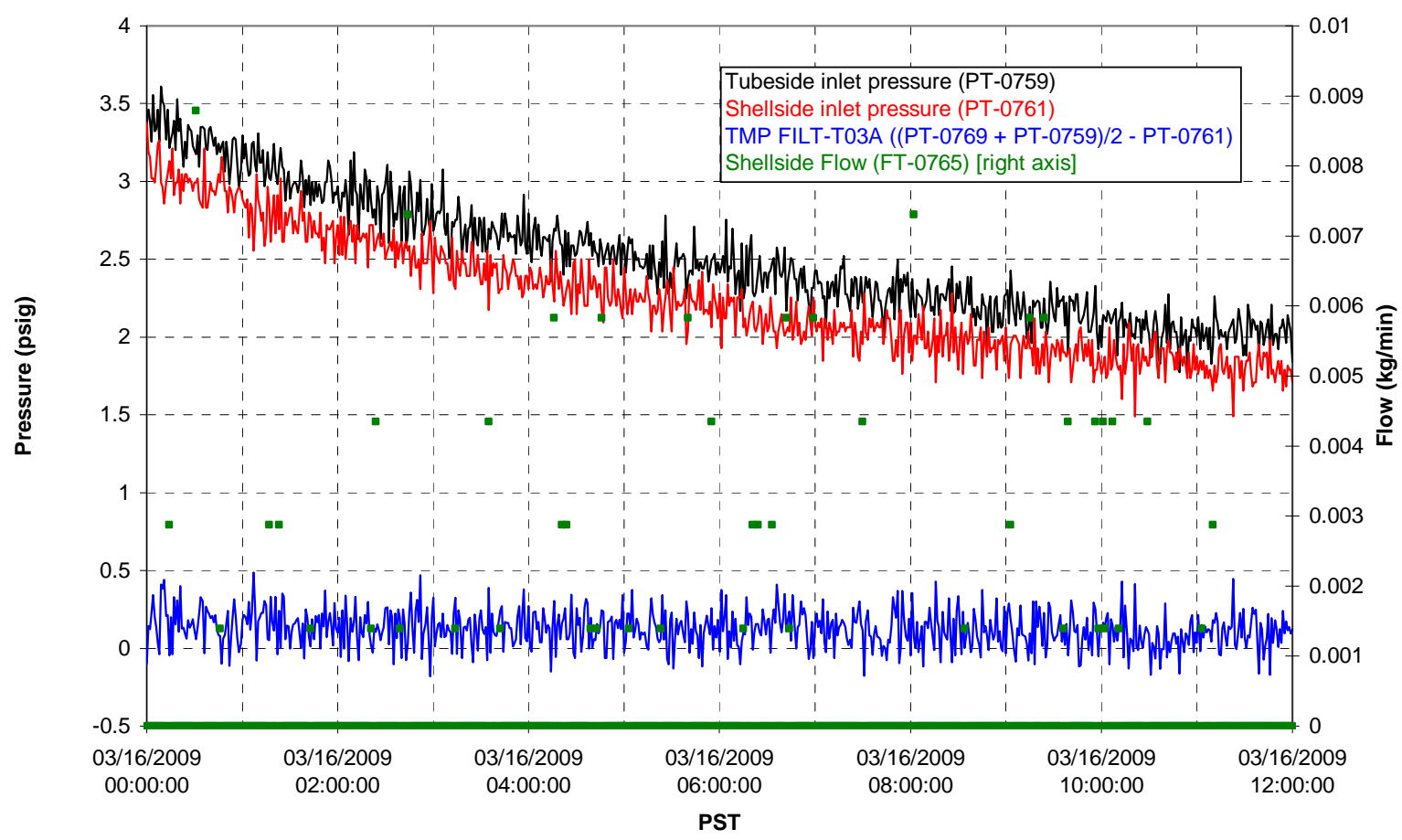


Filter UFP-FILT-T04A

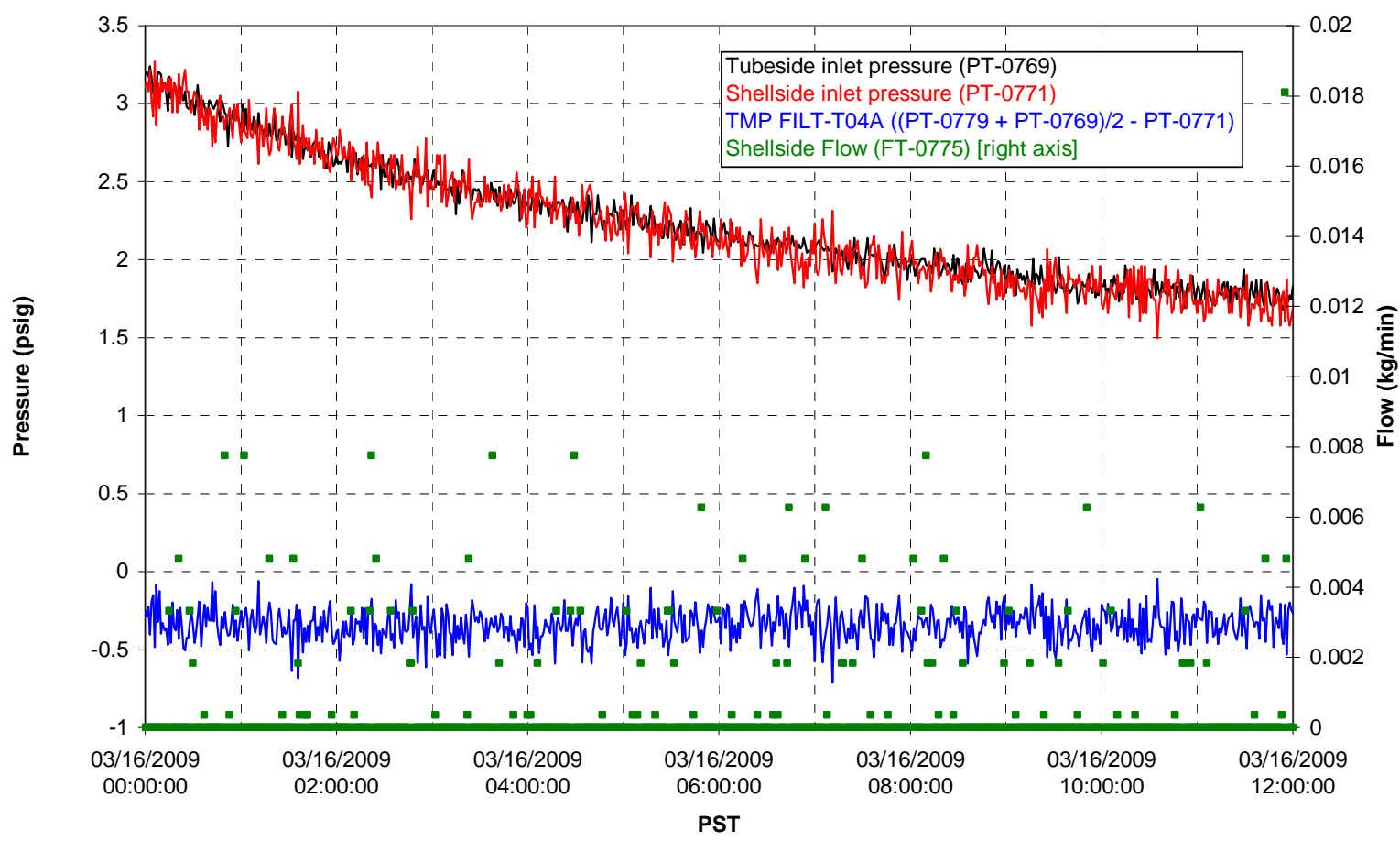

Filter UFP-FILT-T05A

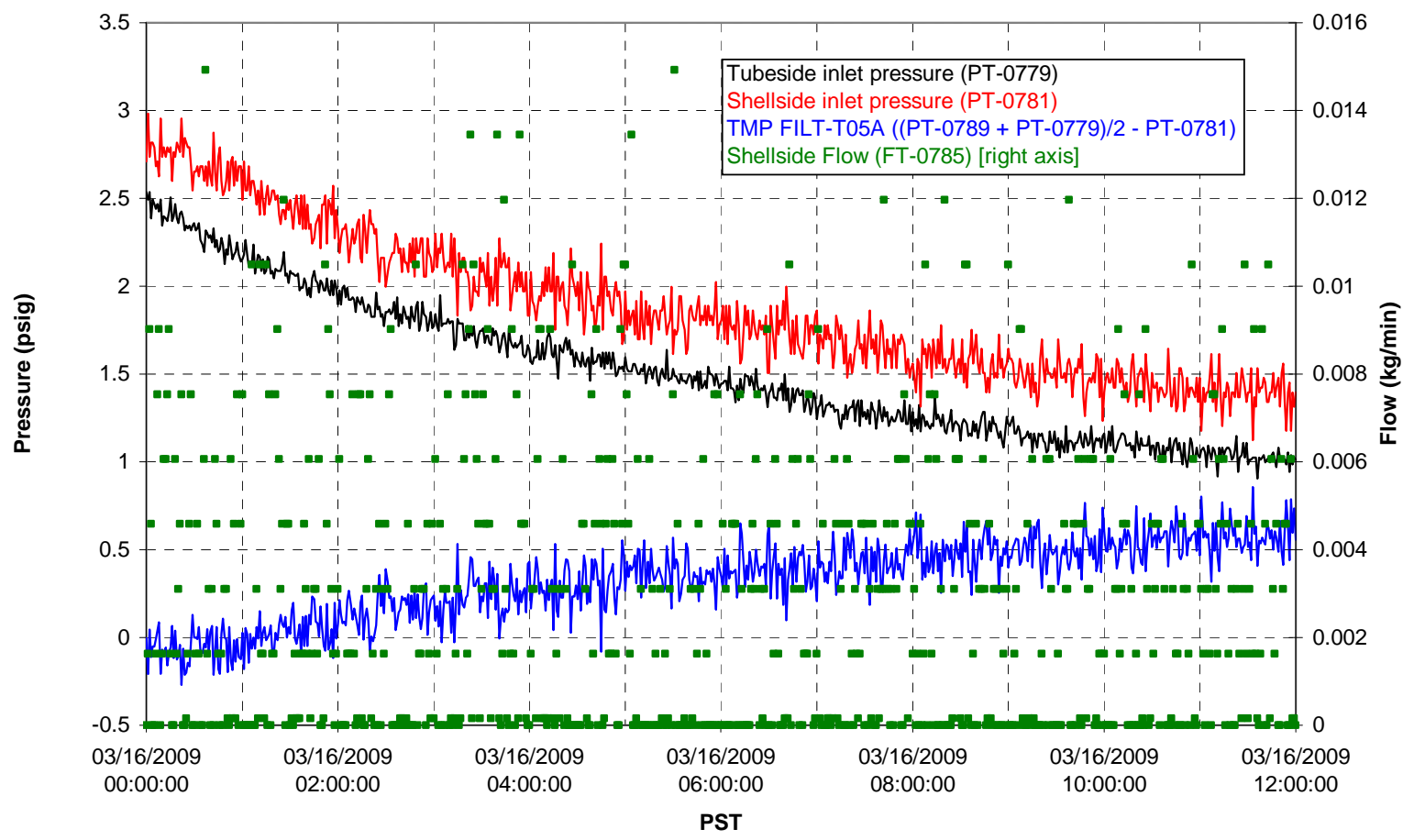



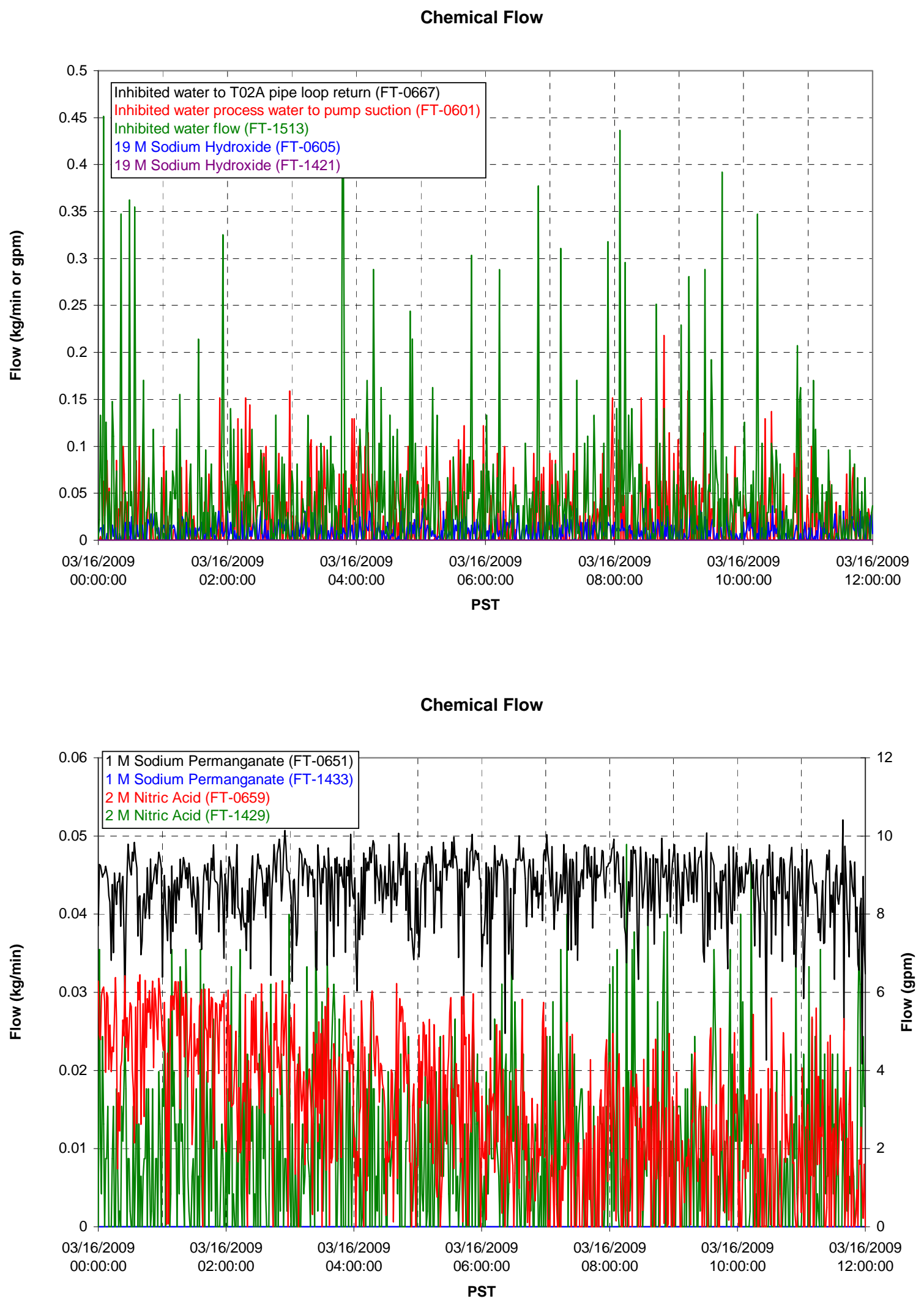
Air Flows

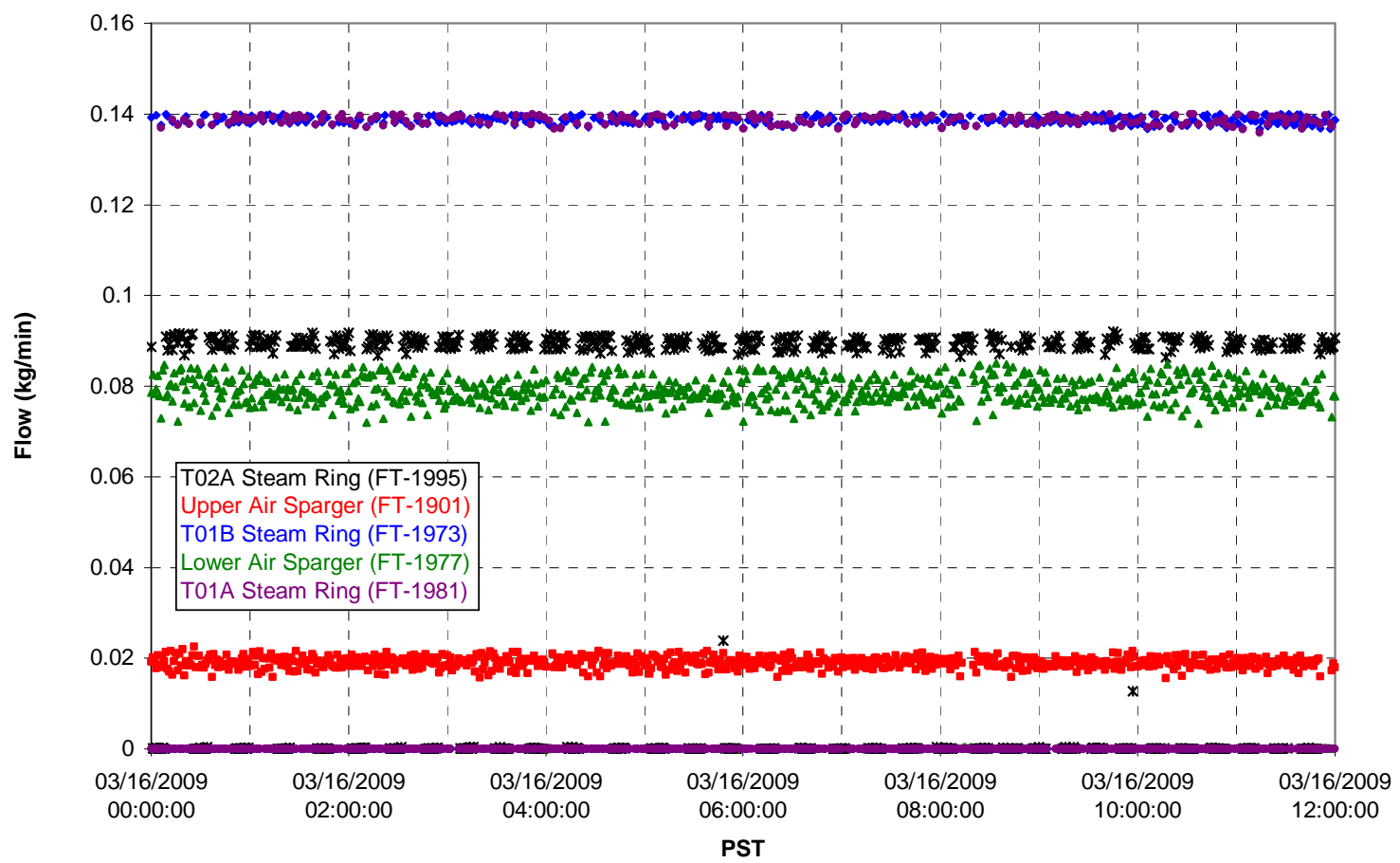

T02A Steam

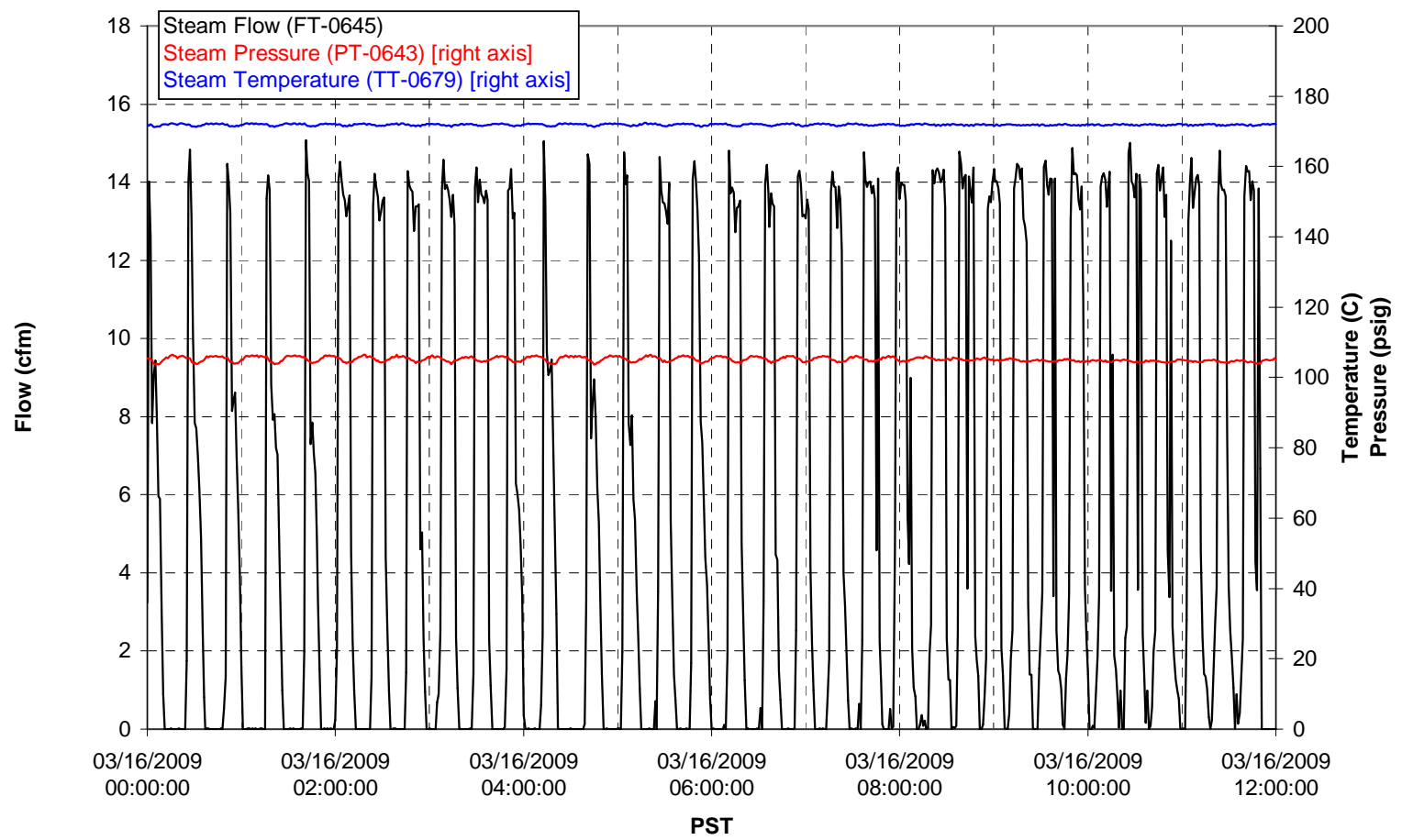


T01A Steam

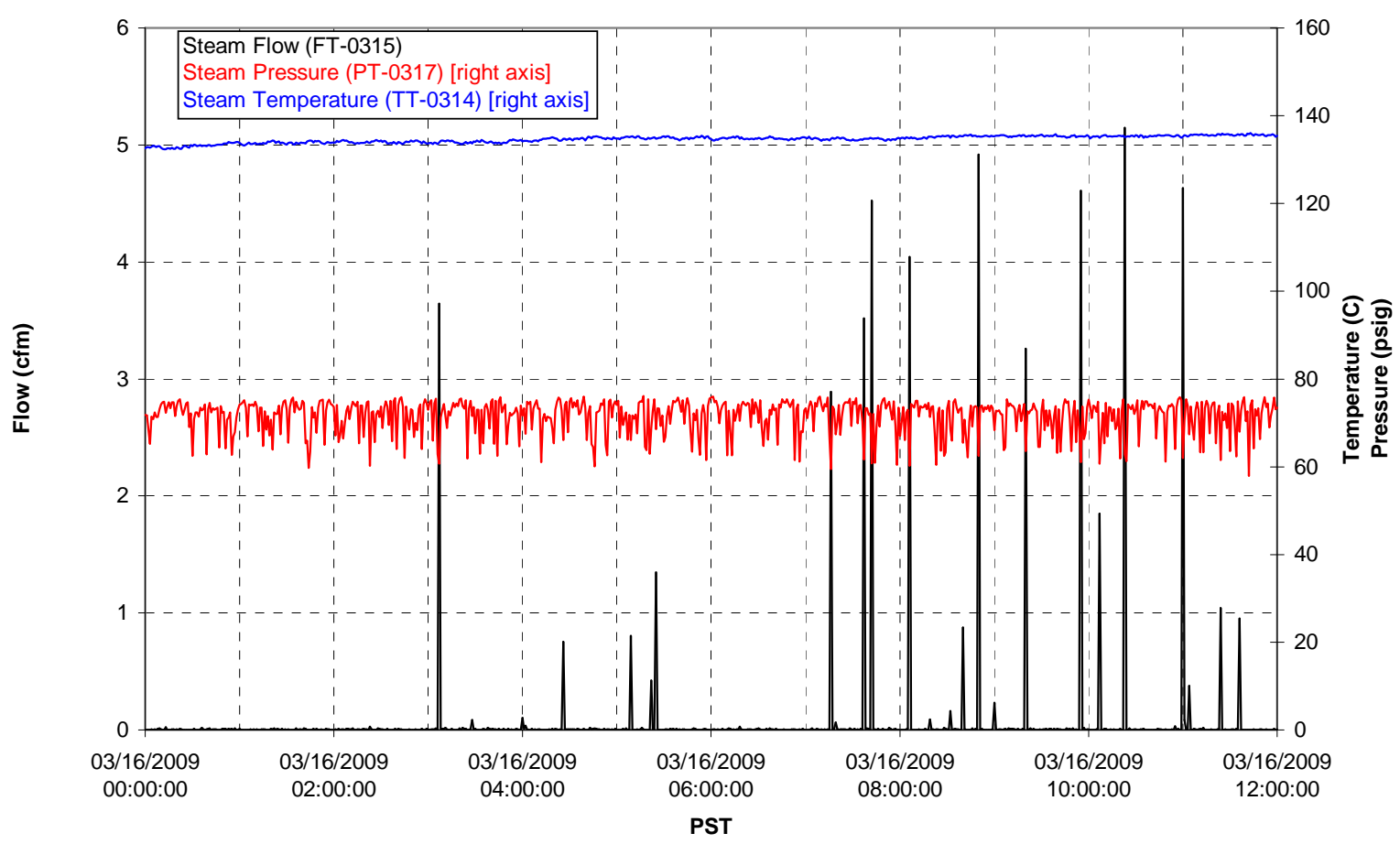

T01B Steam

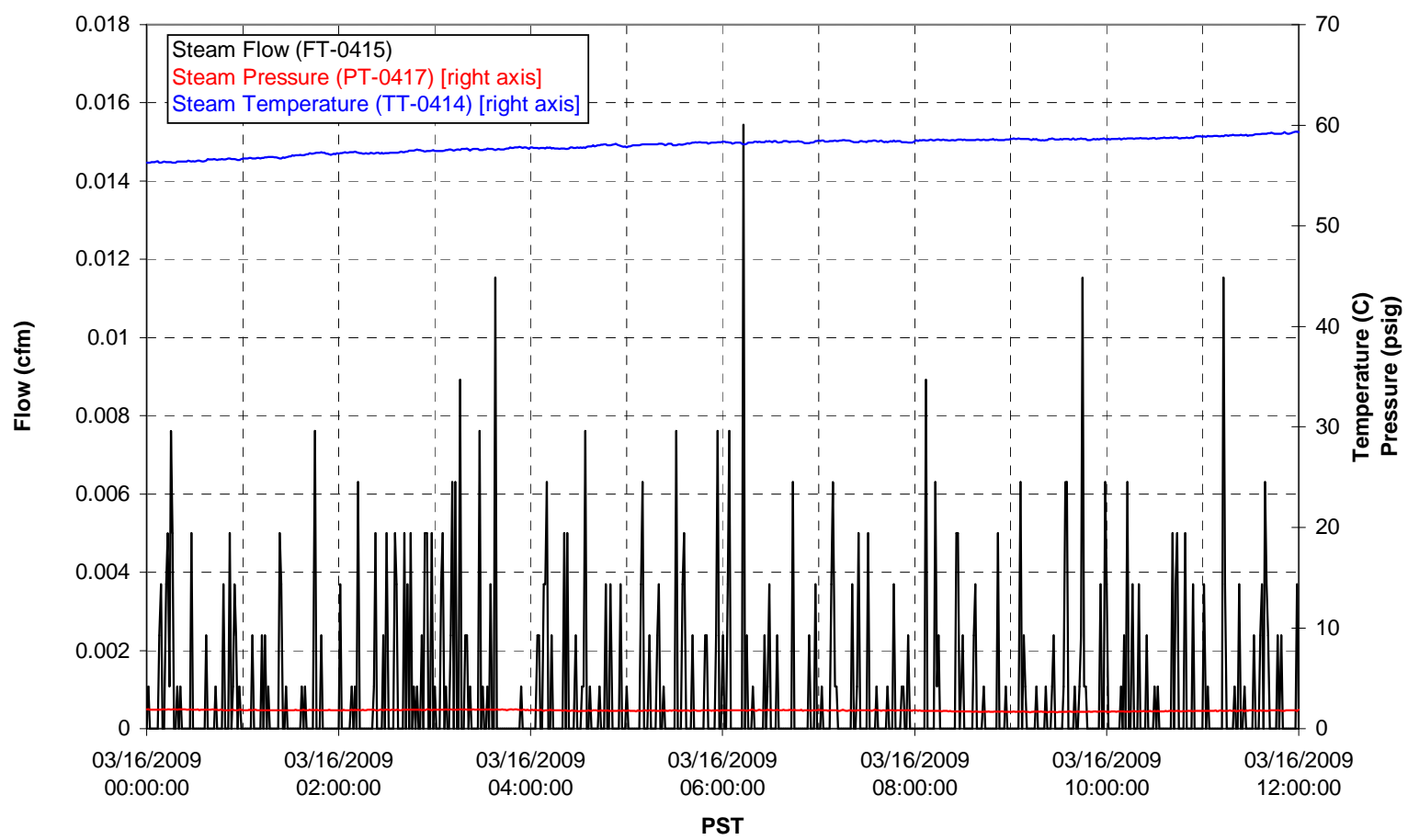




\section{Integrated Test B Data Plots \\ 03/16/09 12:00 - 03/17/09 00:00}


T01A level

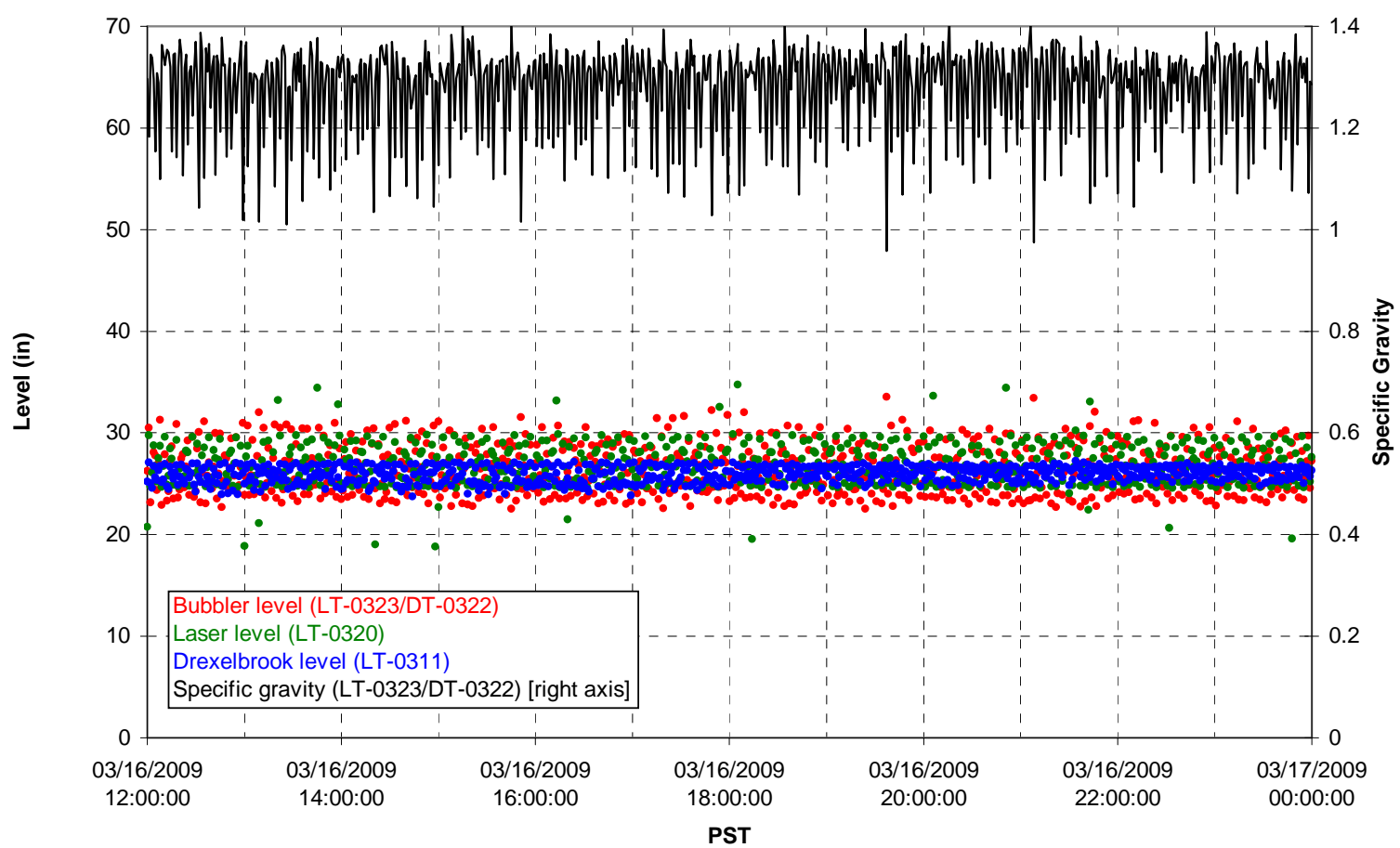

T01A temperatures

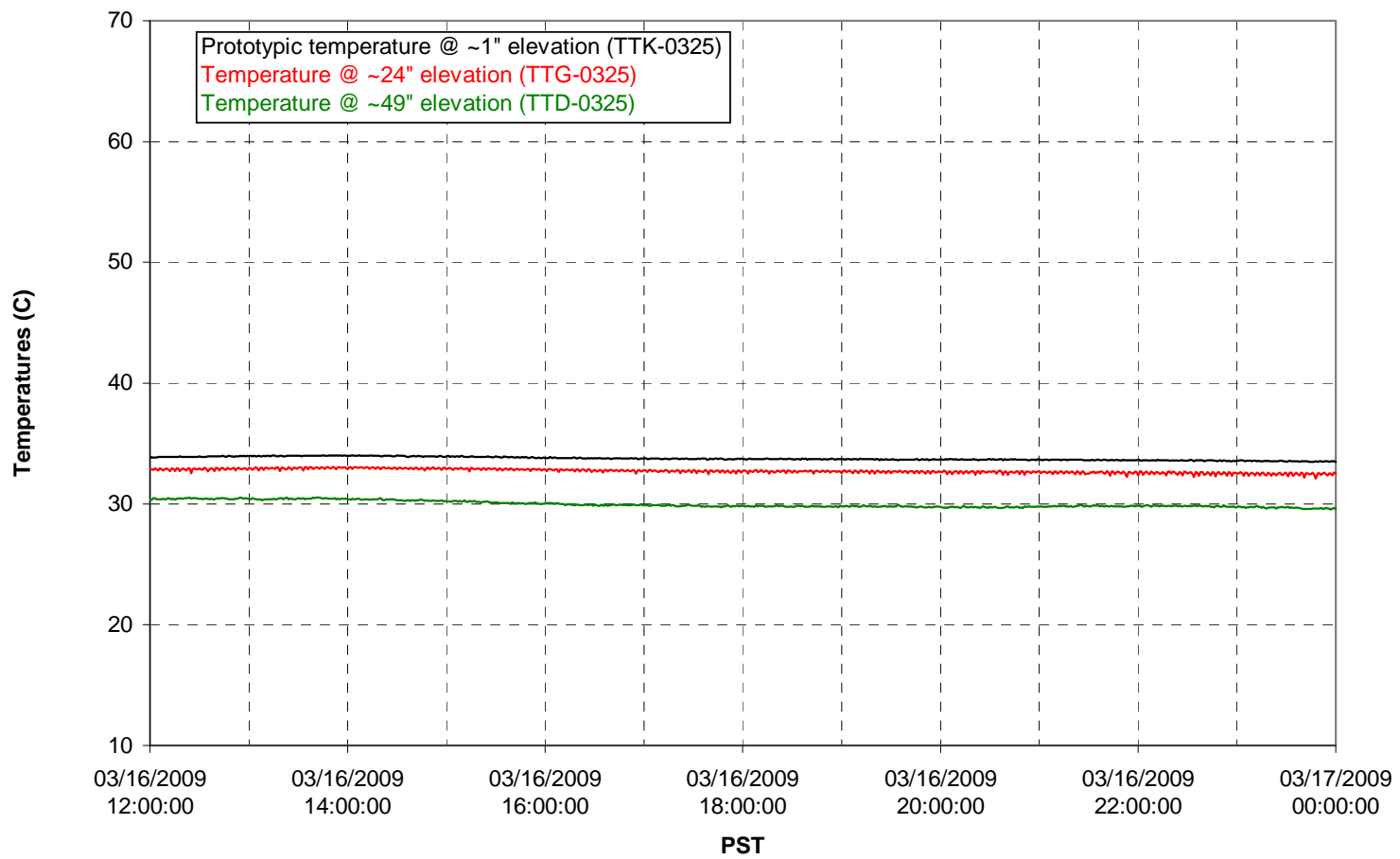


T01B level

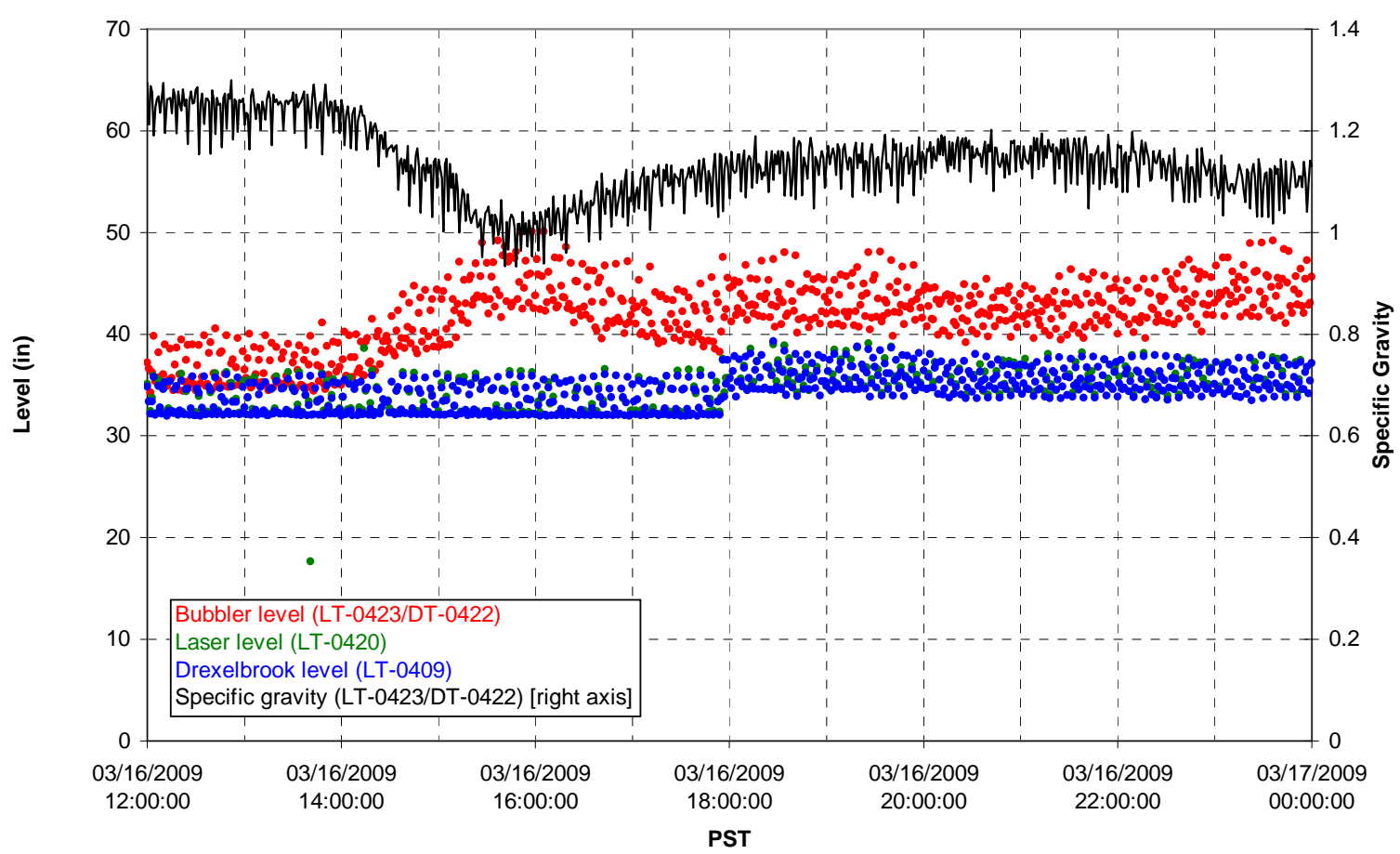

T01B temperatures

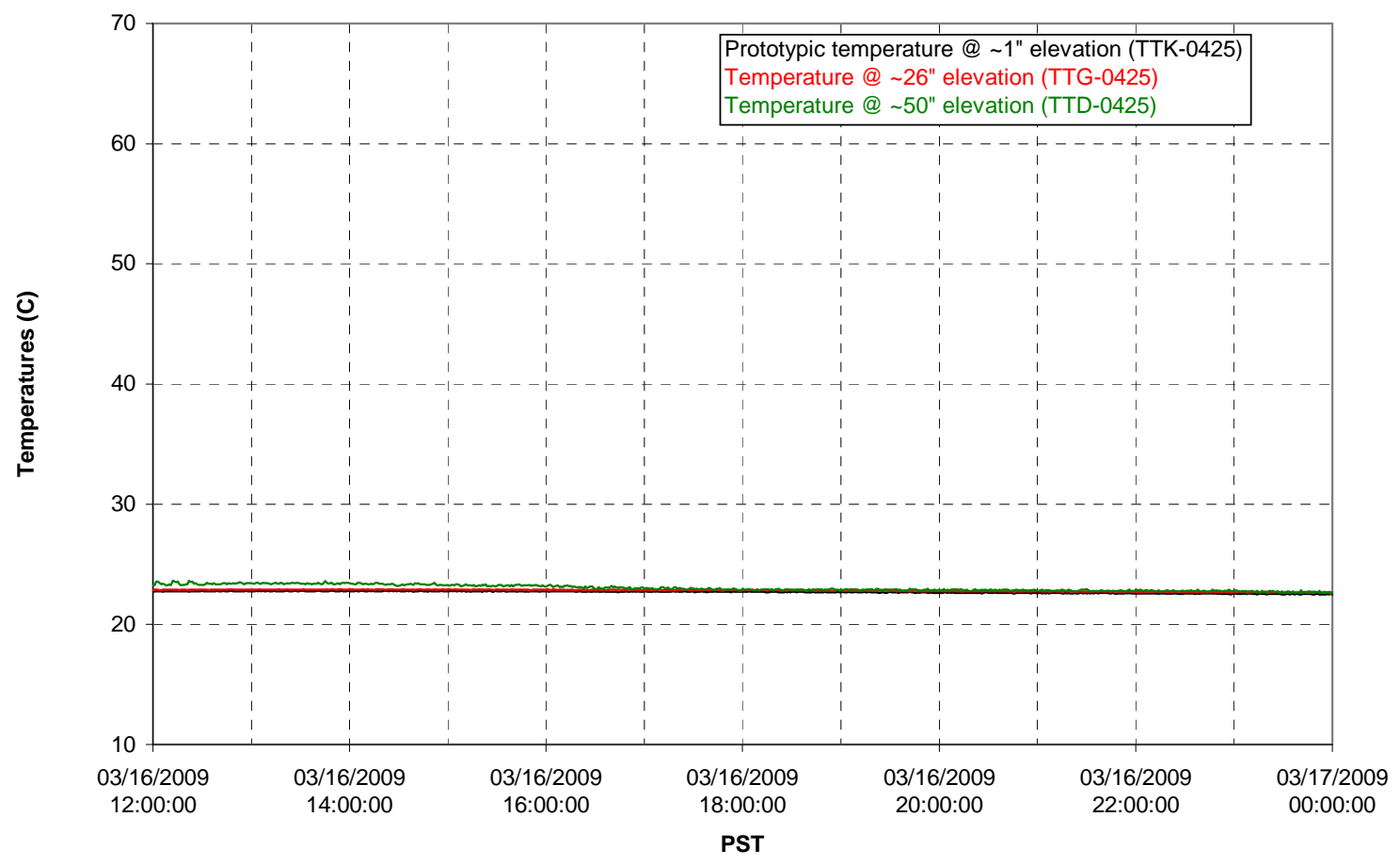


T02A level

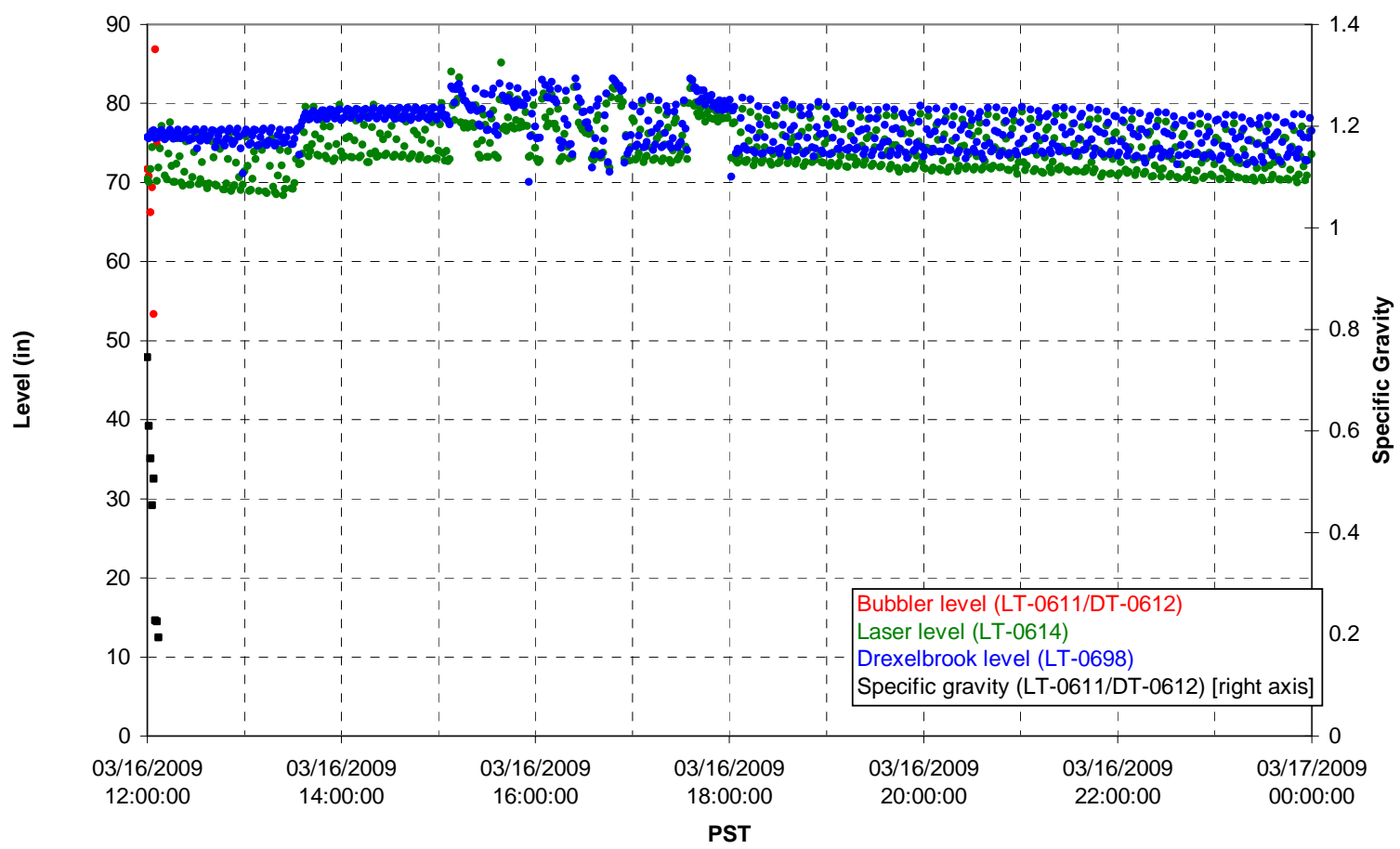

T02A temperatures

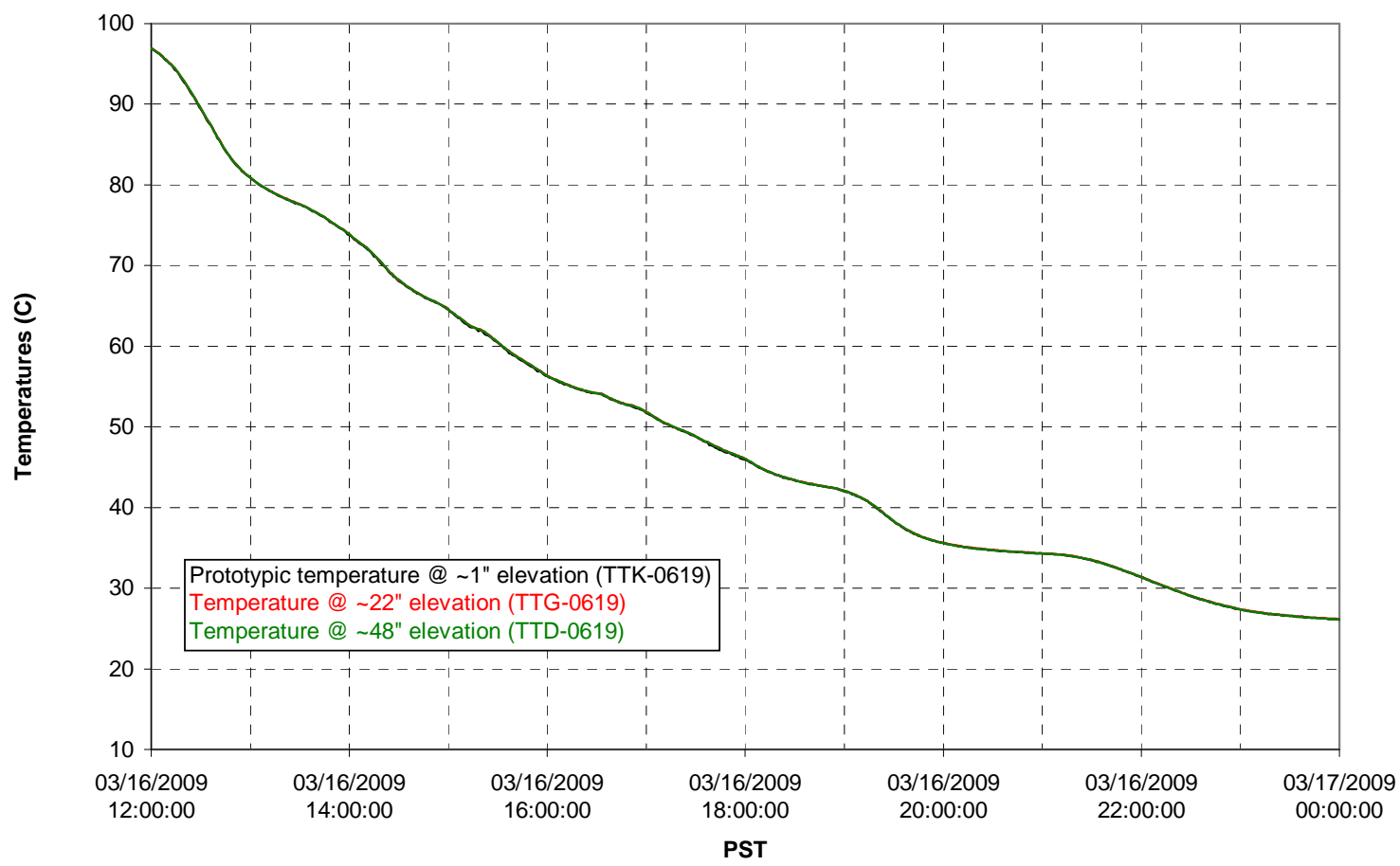


T02A and filter loop temperatures

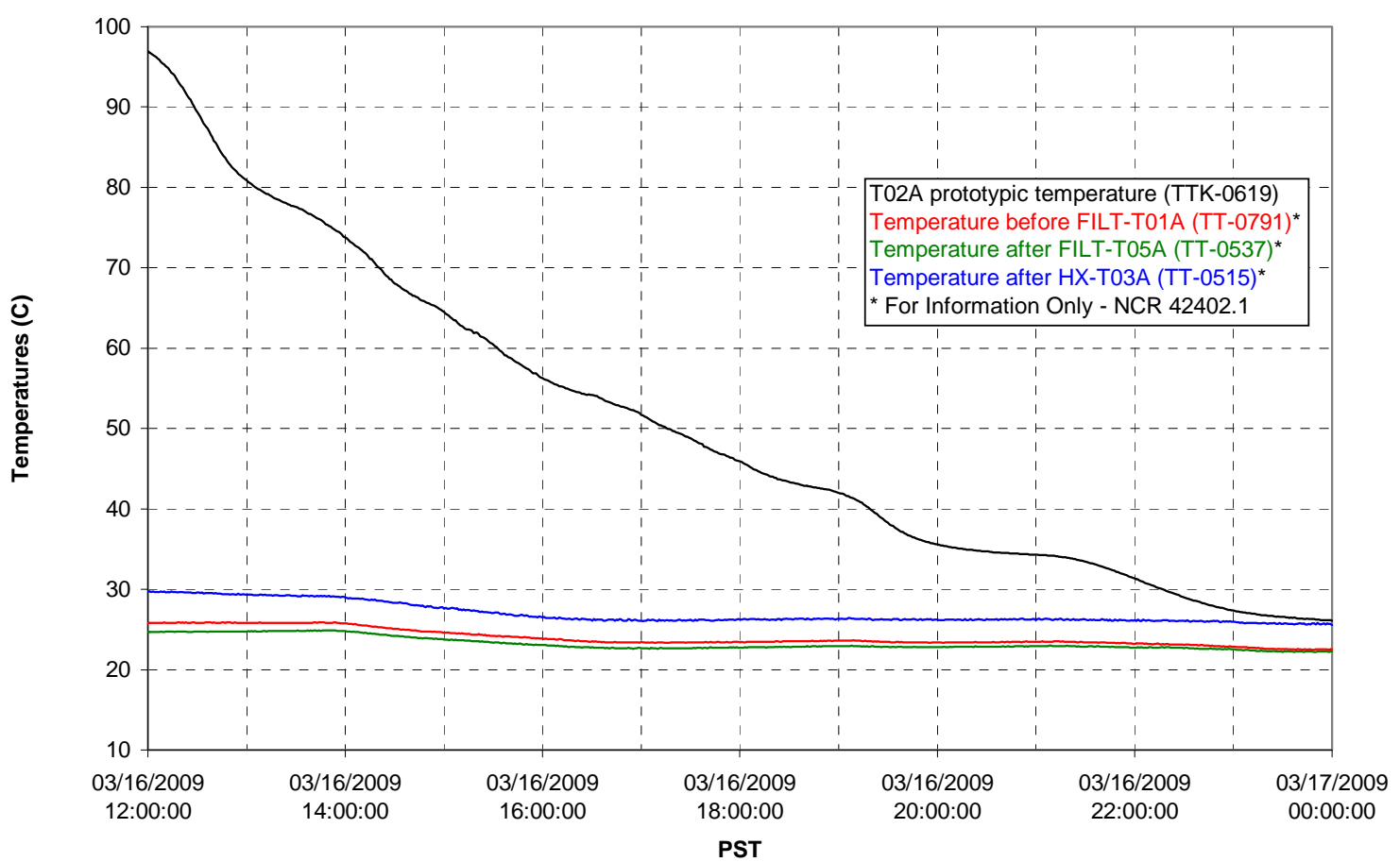

Pump Pressures and Flow

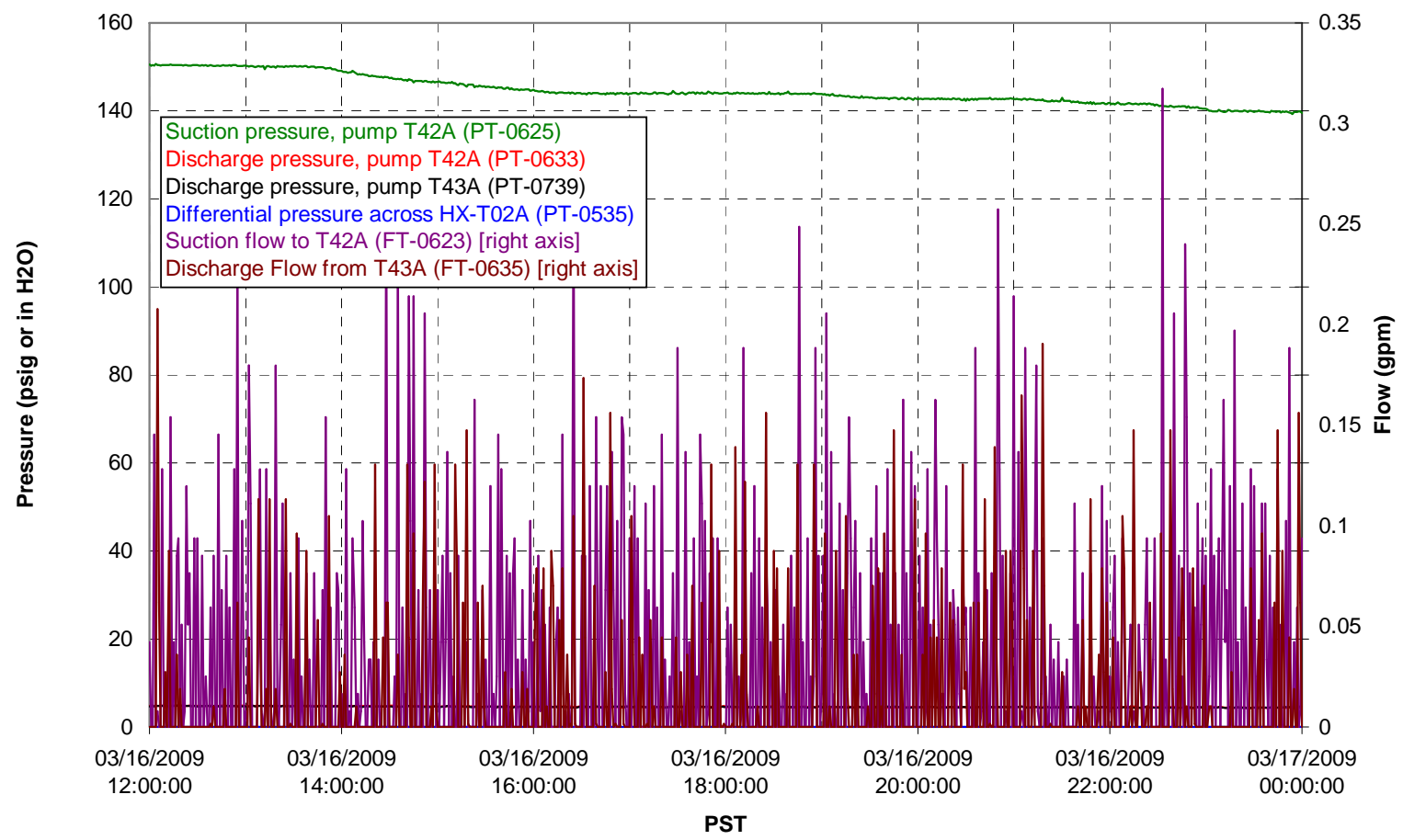


Axial pressure drop

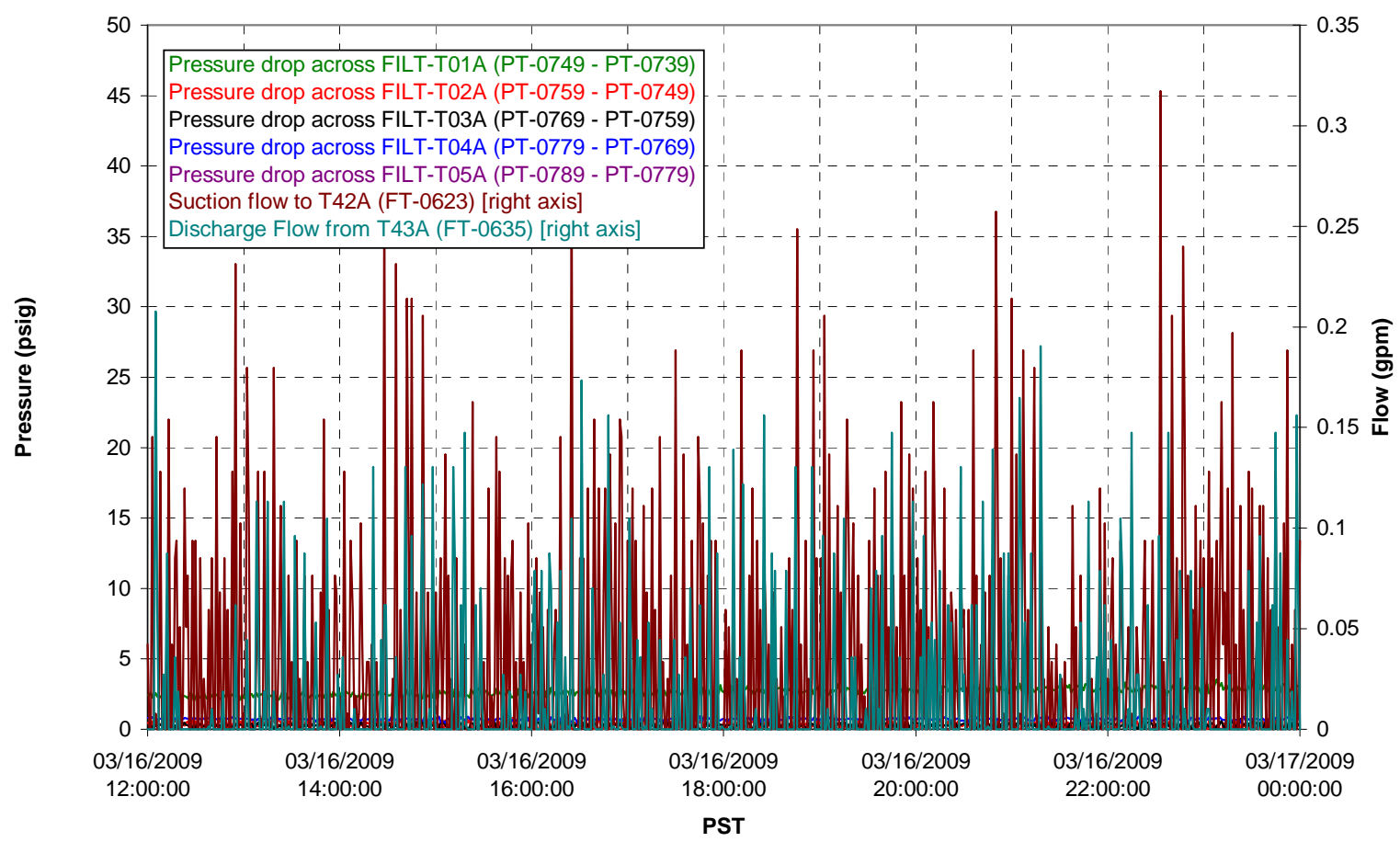

Permeate flow rates

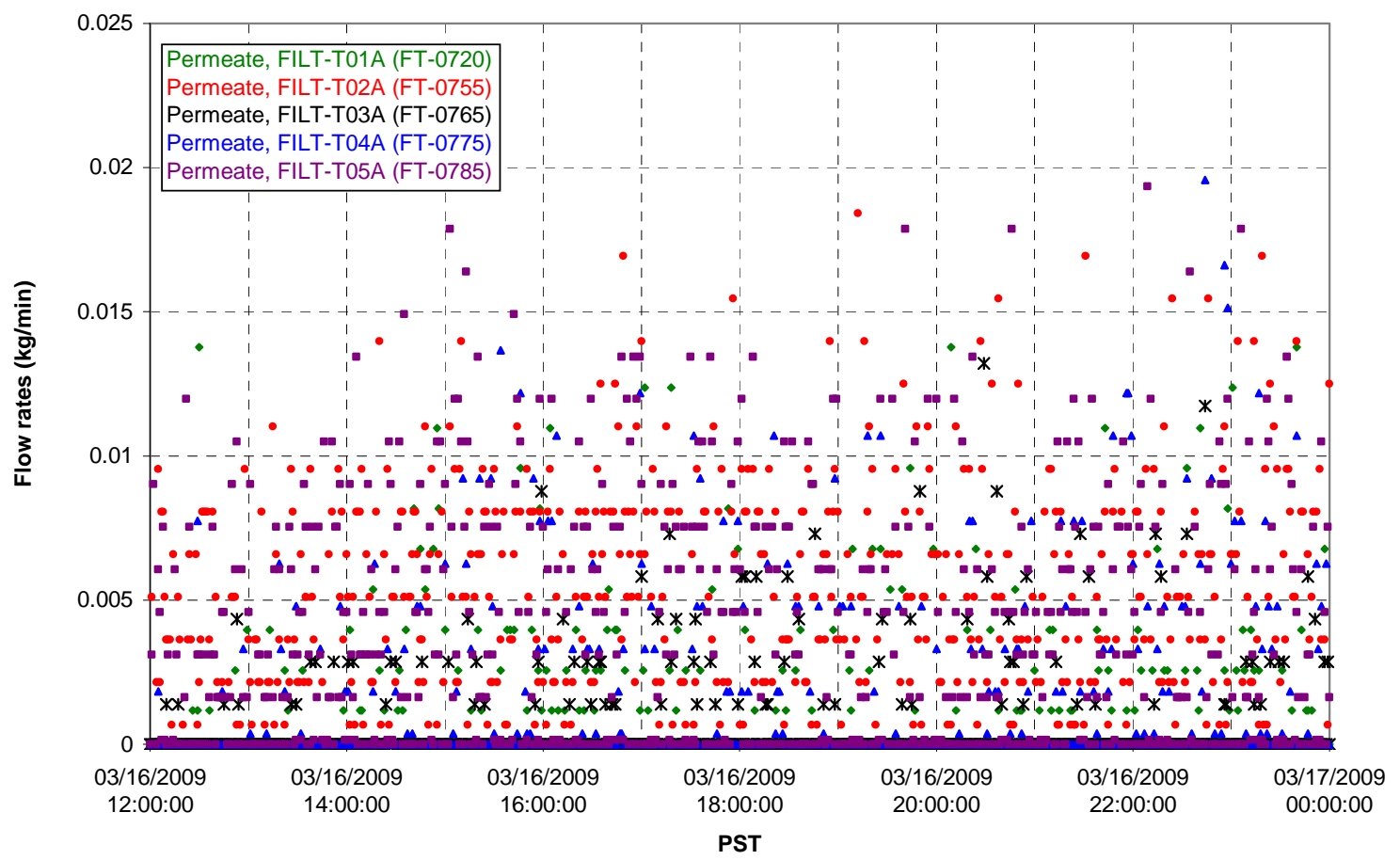


T02A Inner Temperature Tree

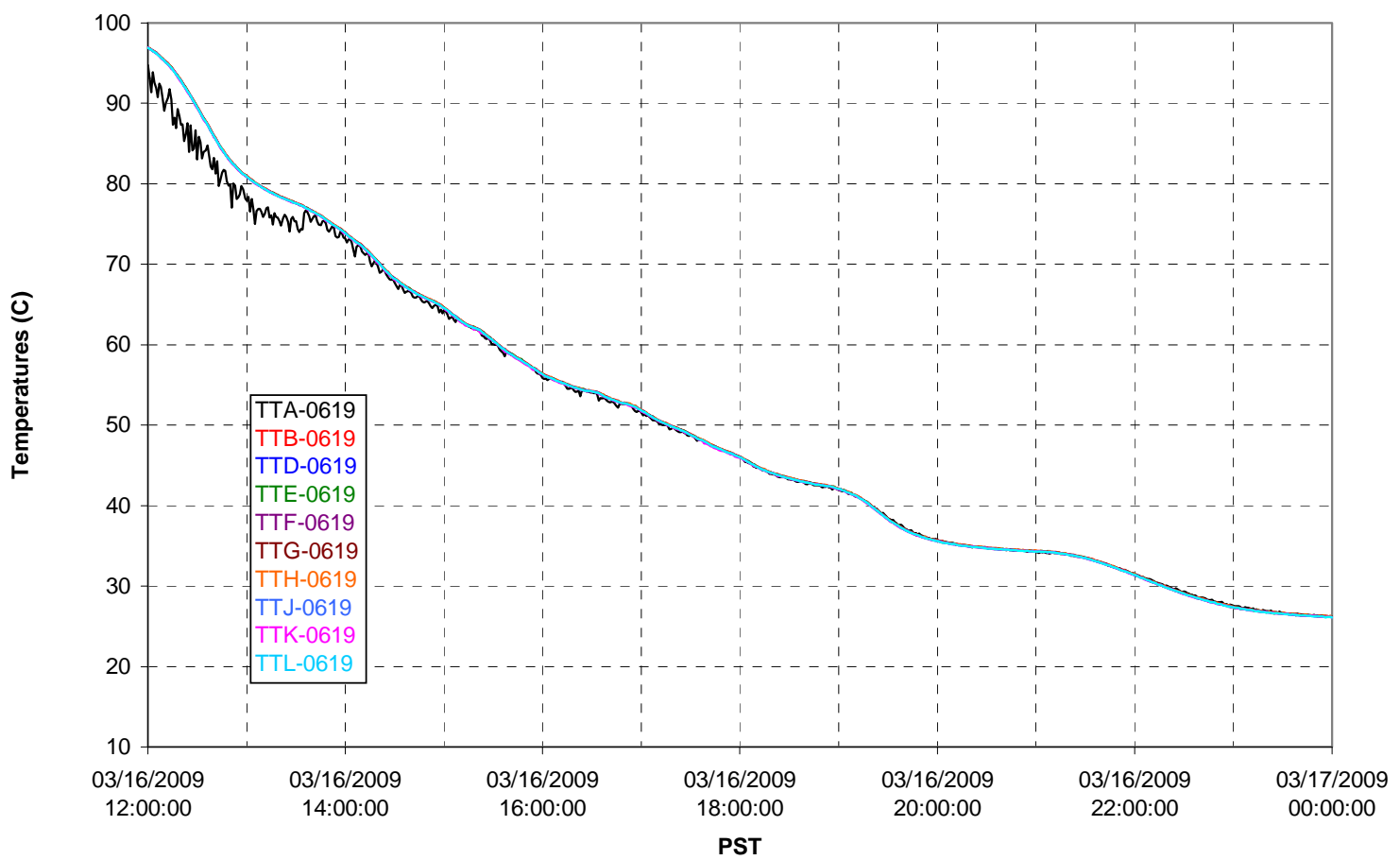

T02A Outer Temperature Tree

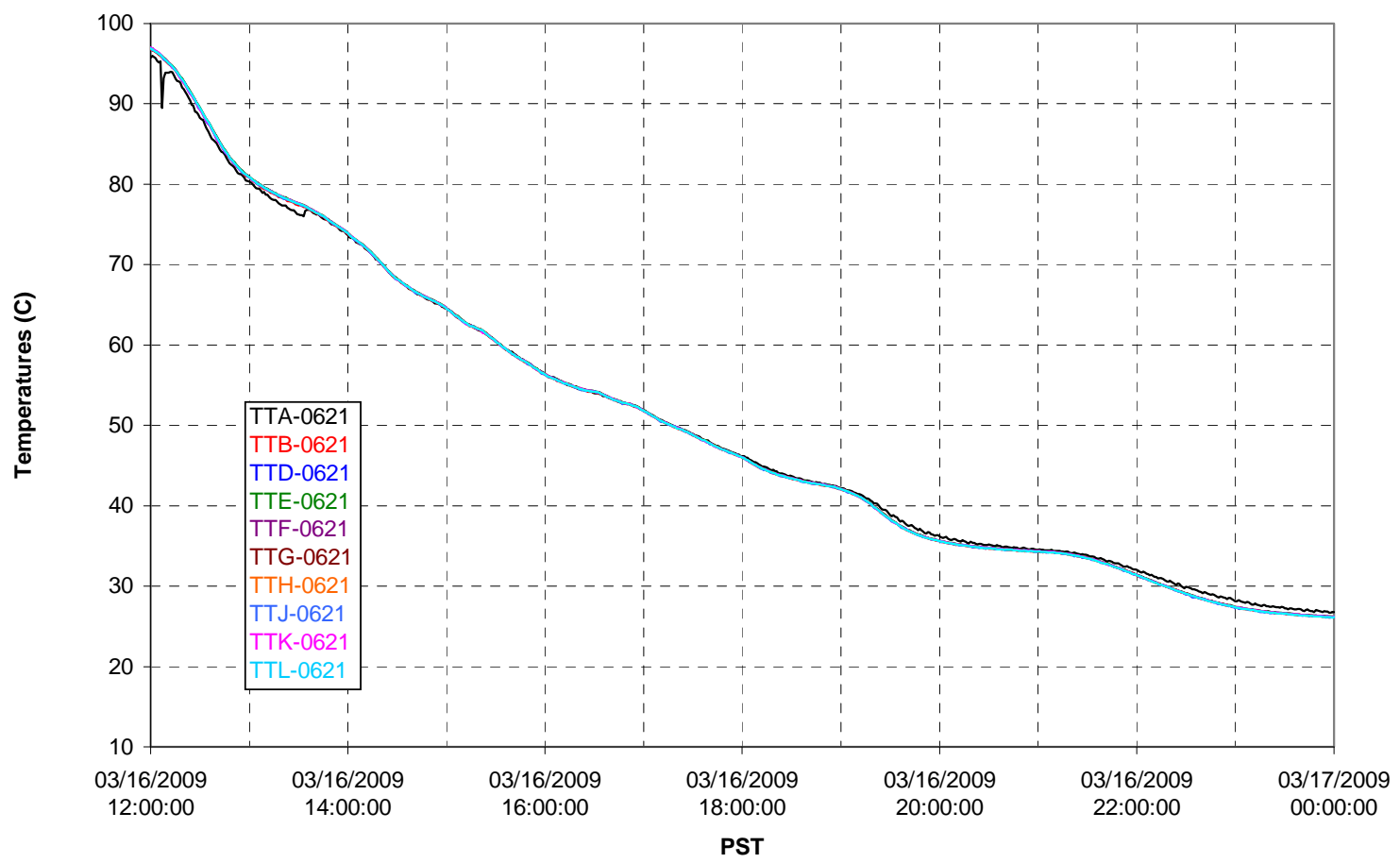


T02A temperatures

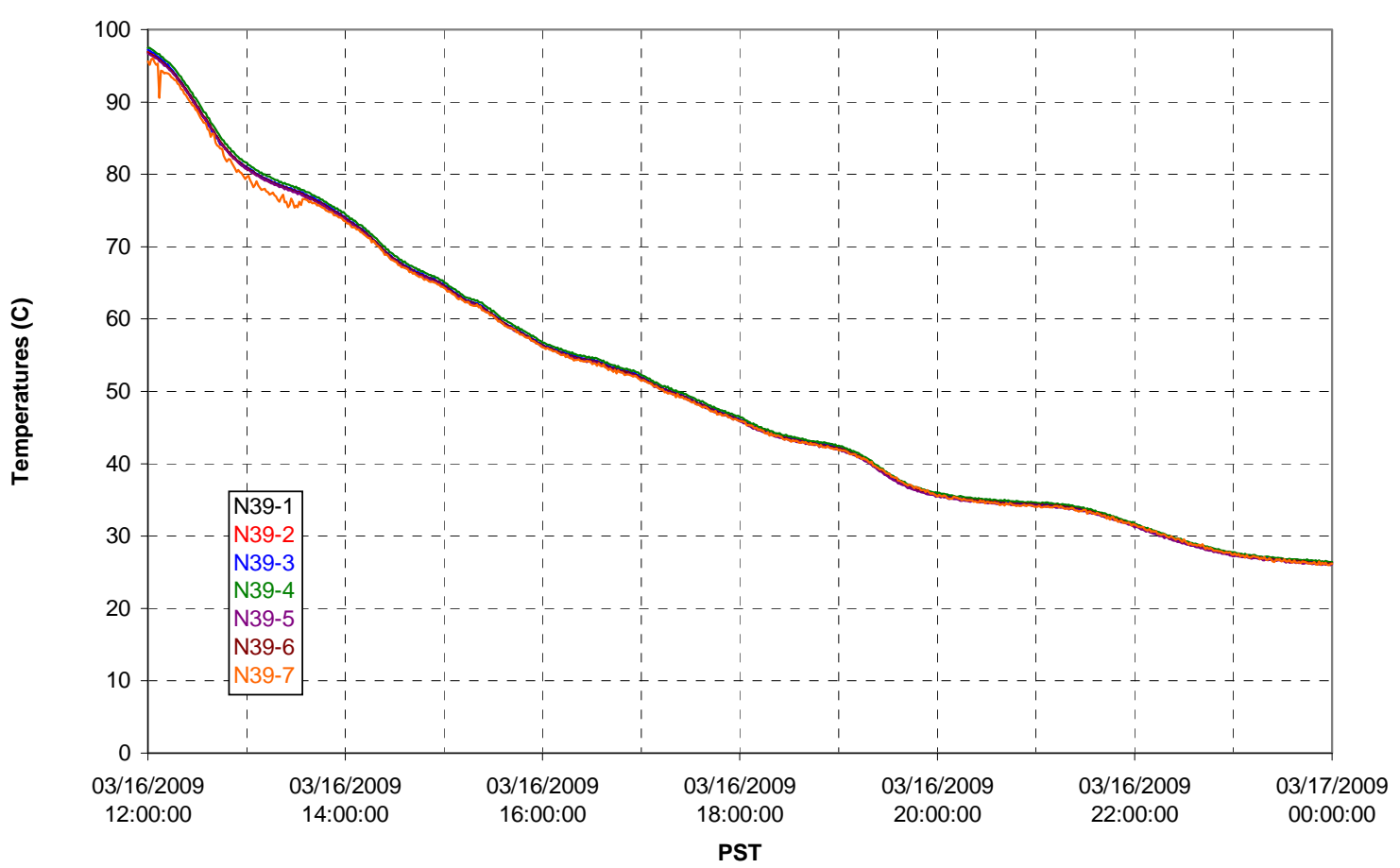

T02A temperatures

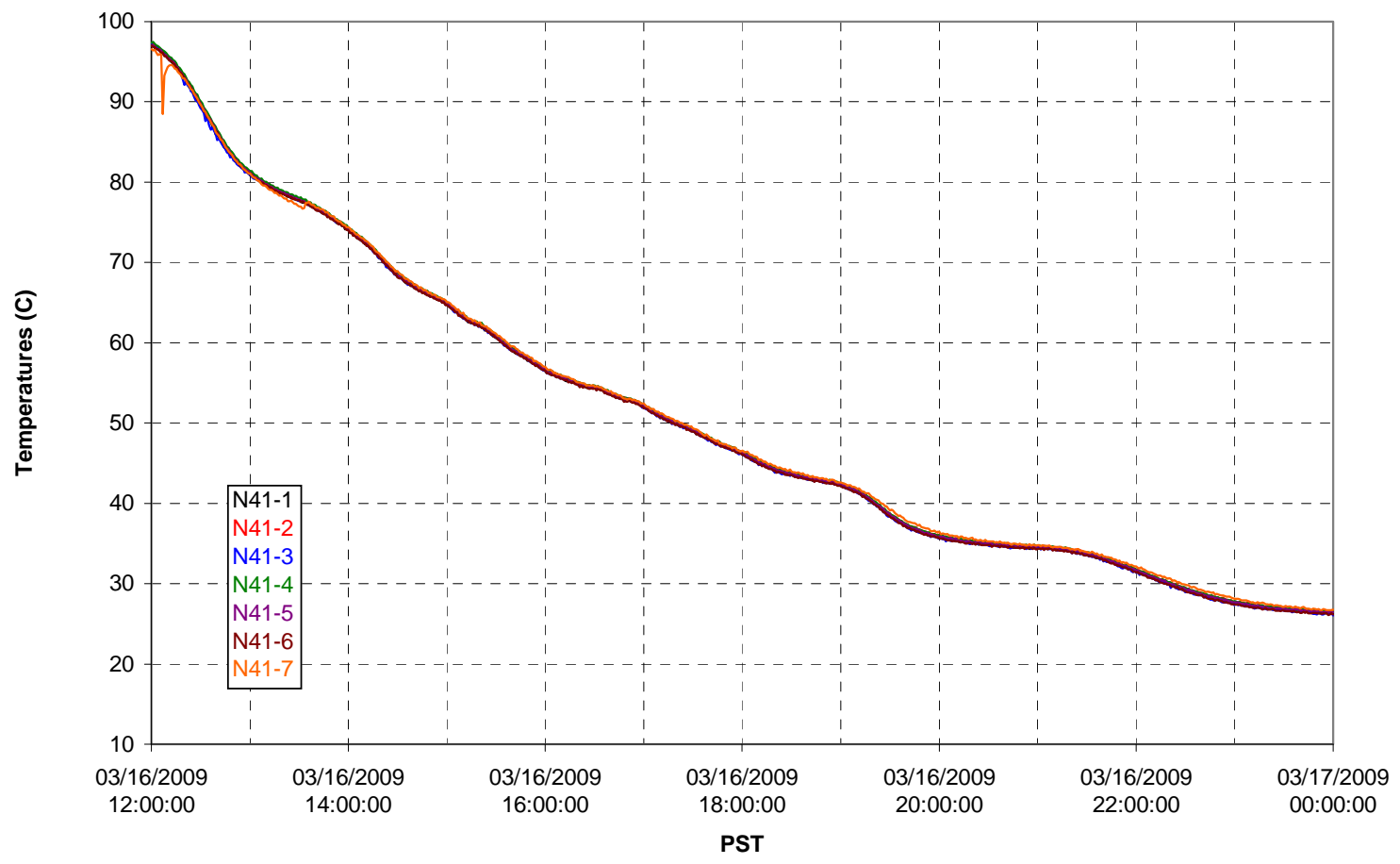


T02A temperatures

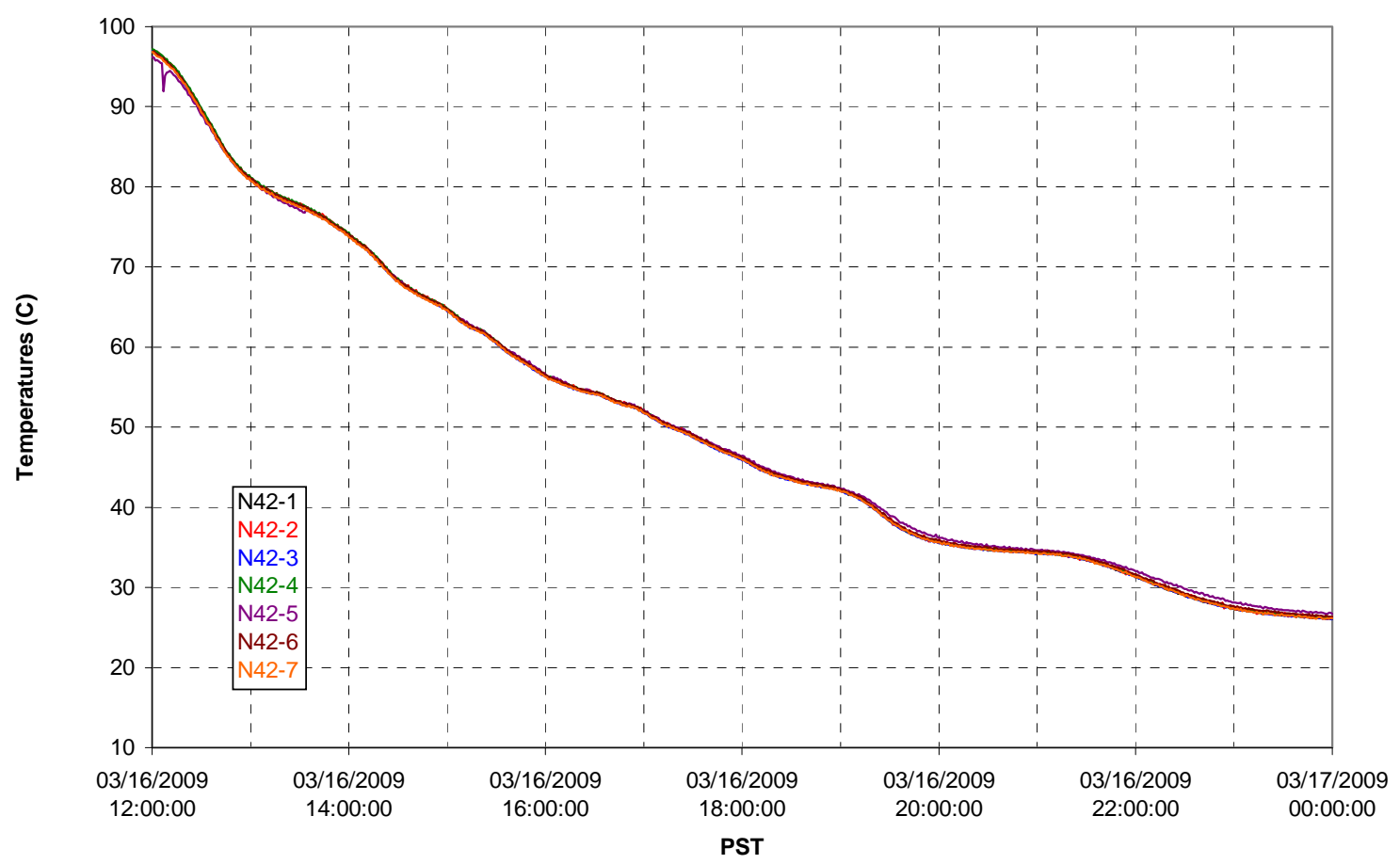

T02A temperatures

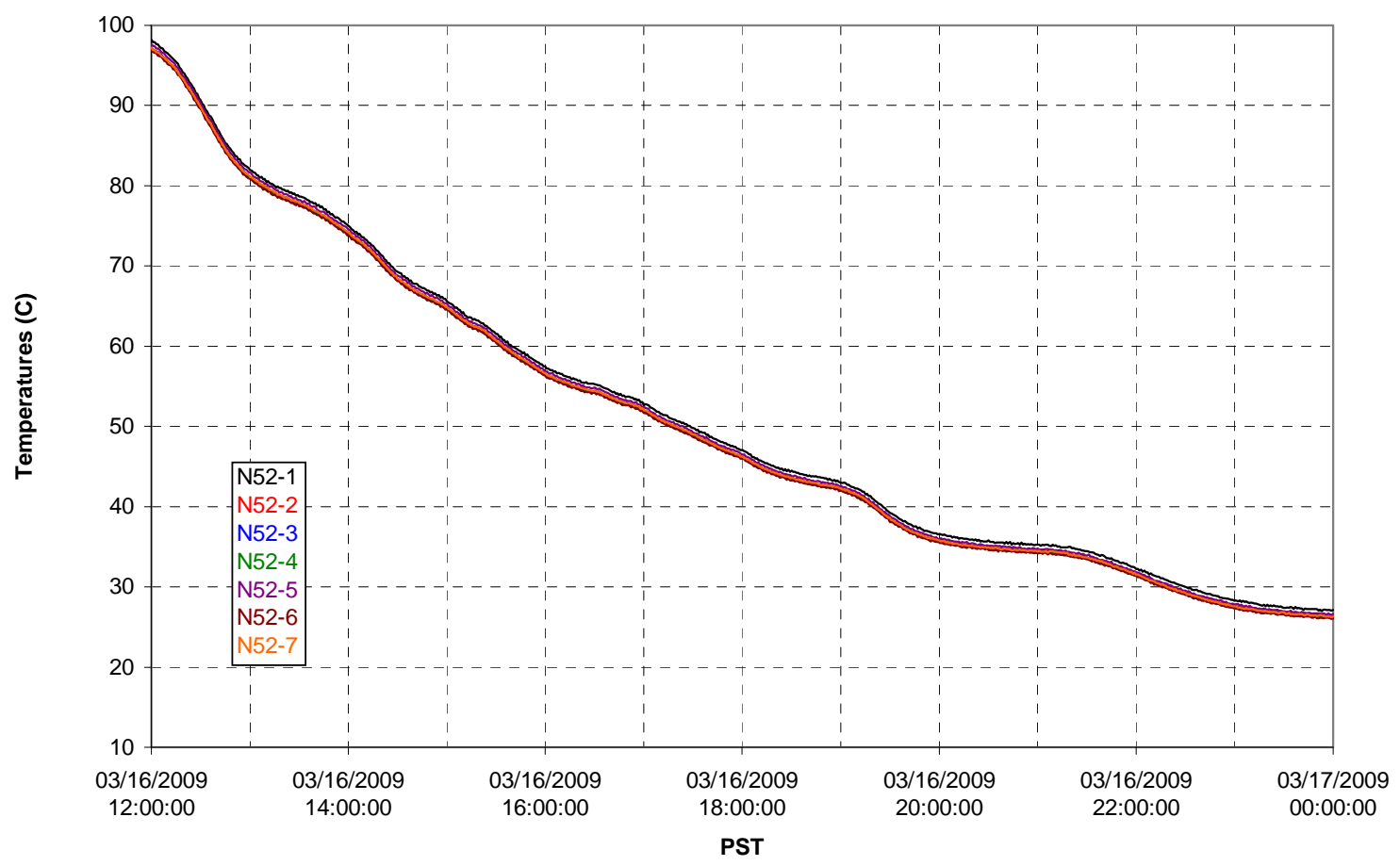


T02A Heating and Cooling

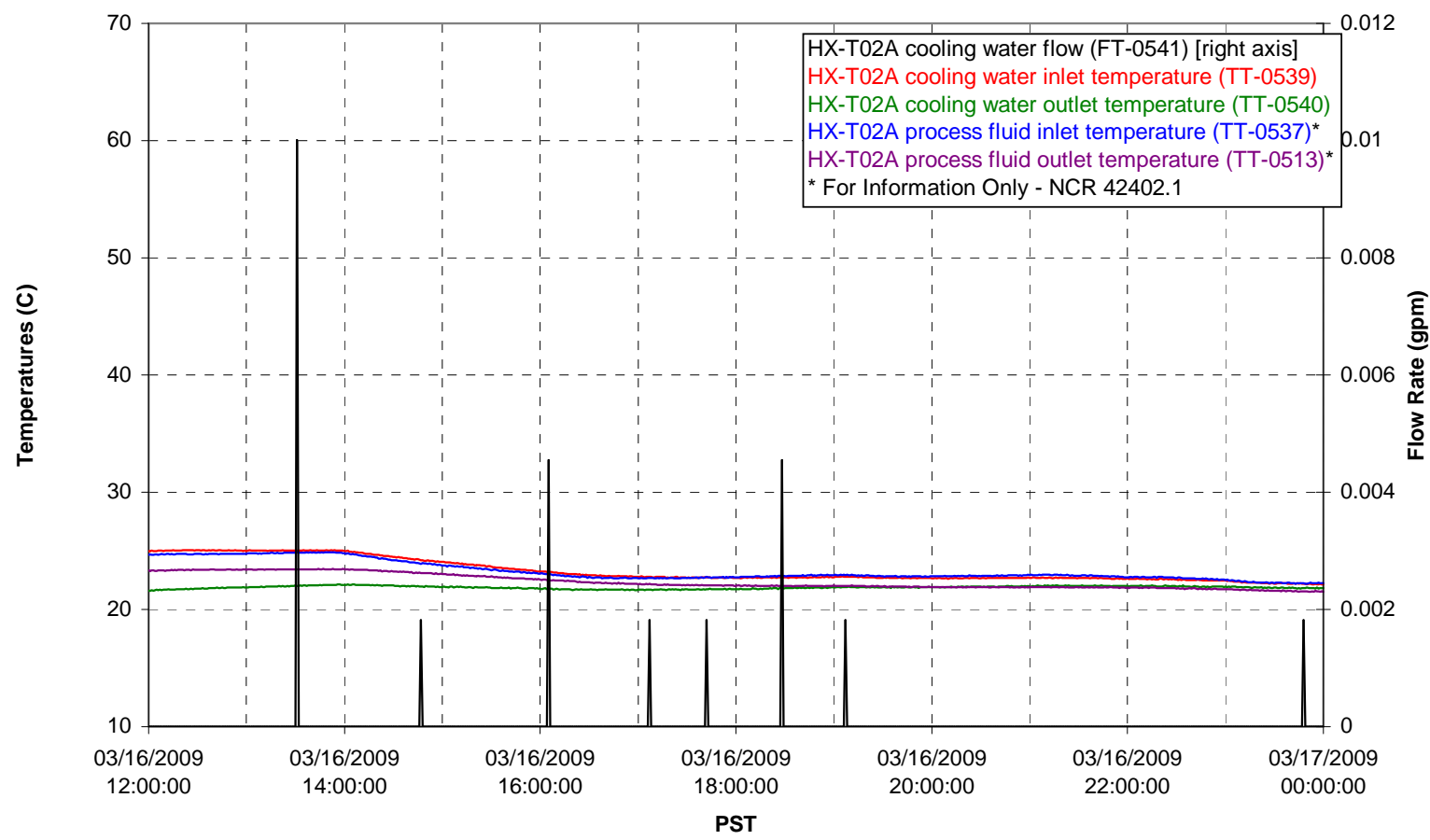

Pump Operation

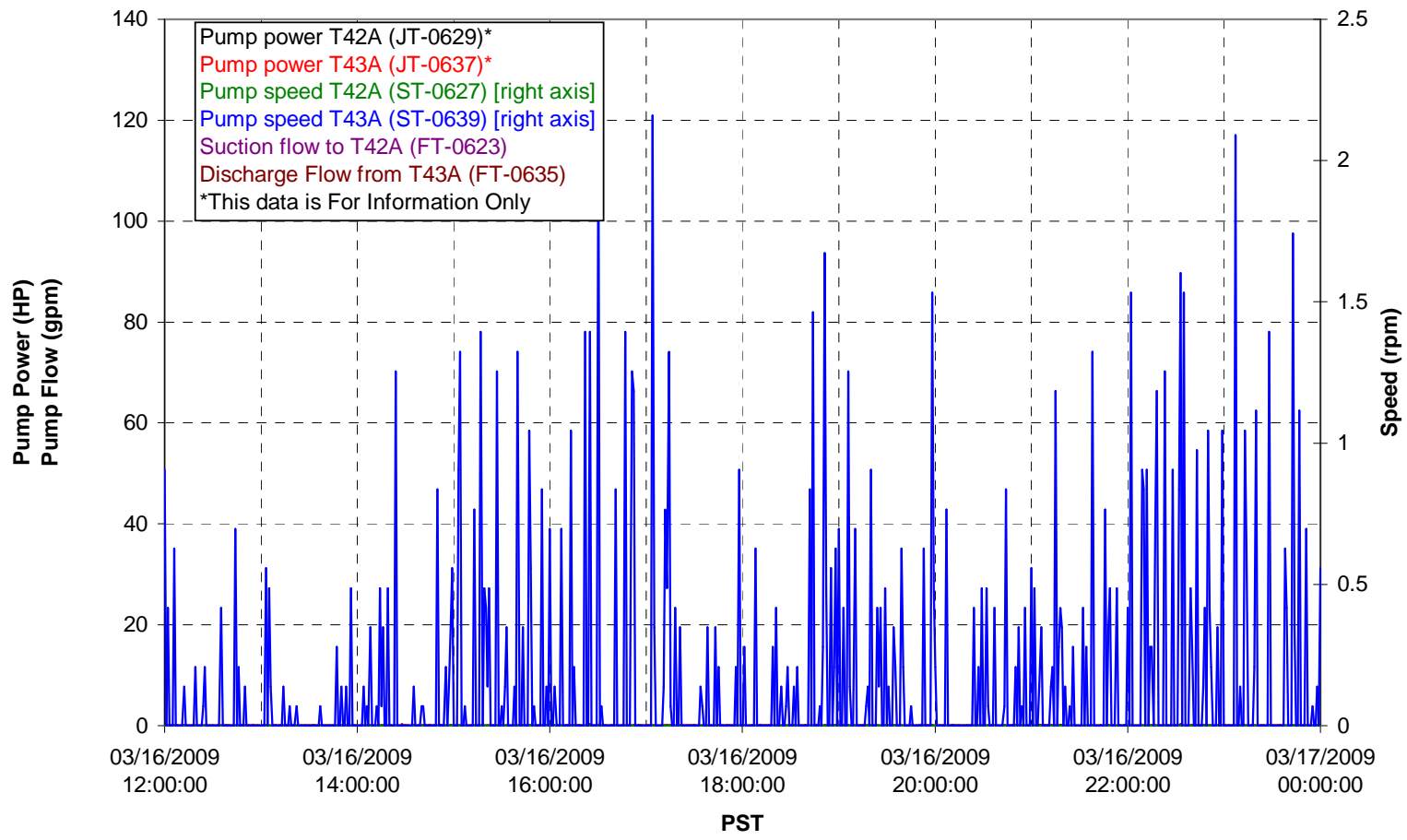


Pulsepot UFP-PP-T01A

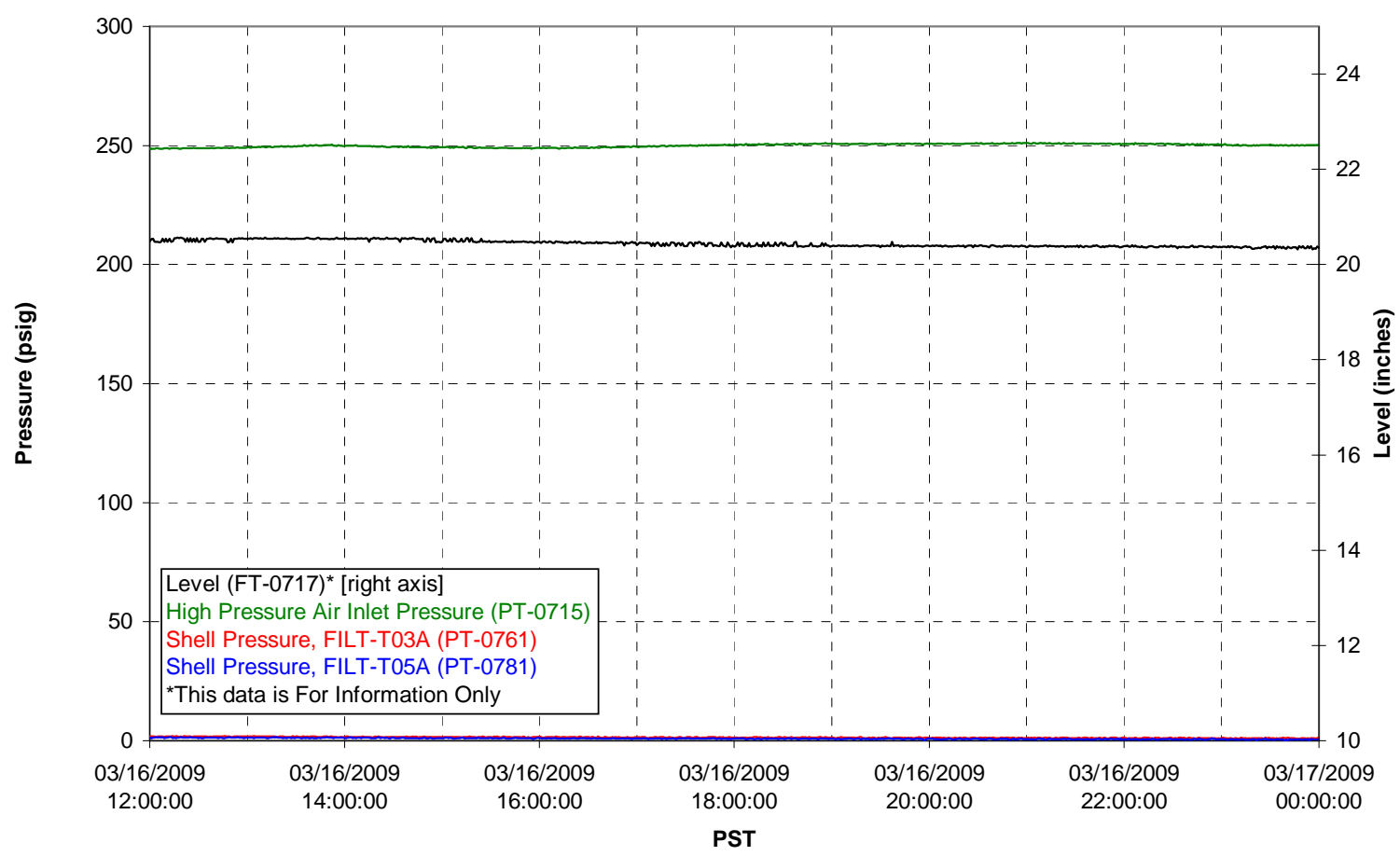

Pulsepot UFP-PP-T02A

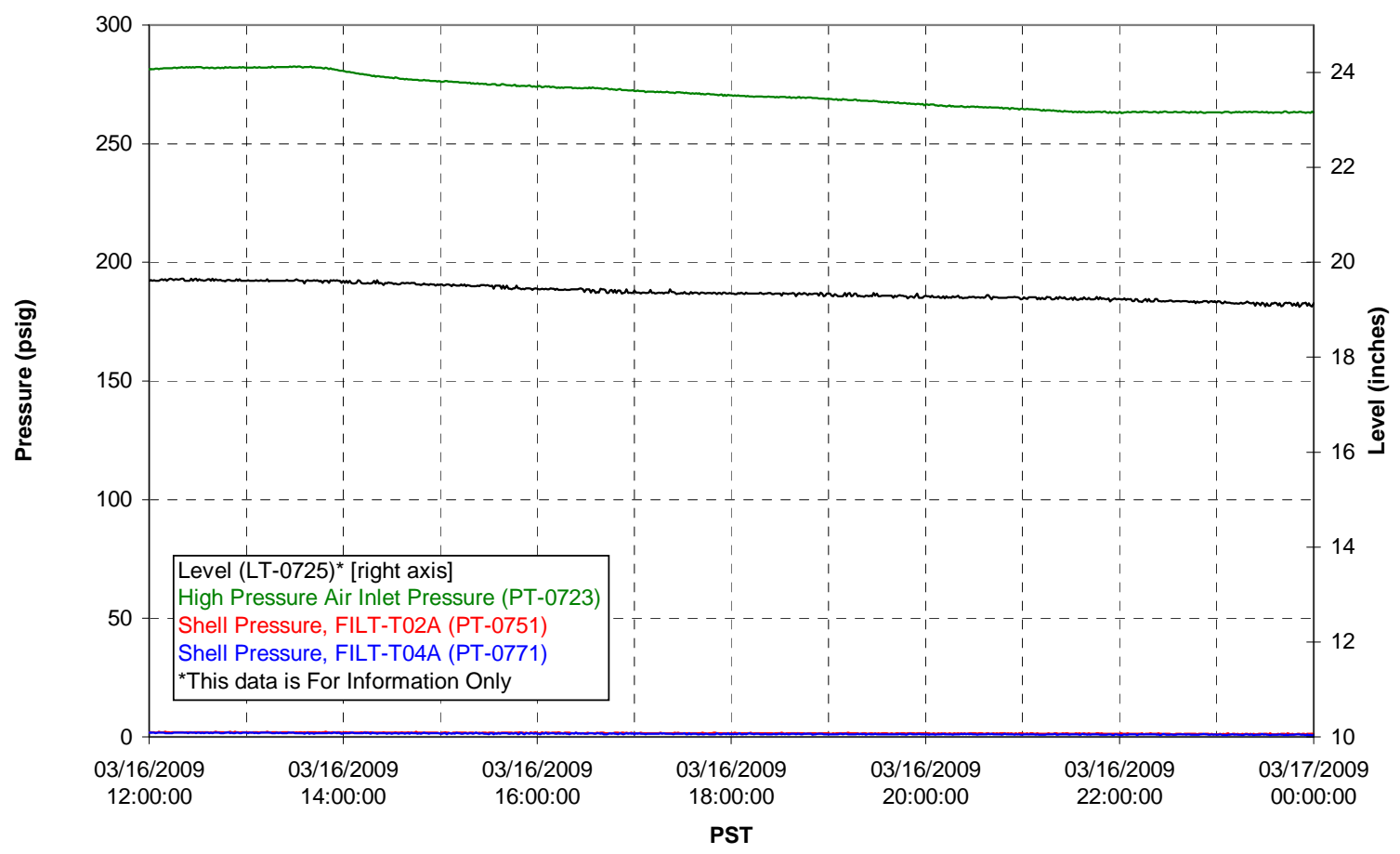


Pulsepot UFP-PP-T03A

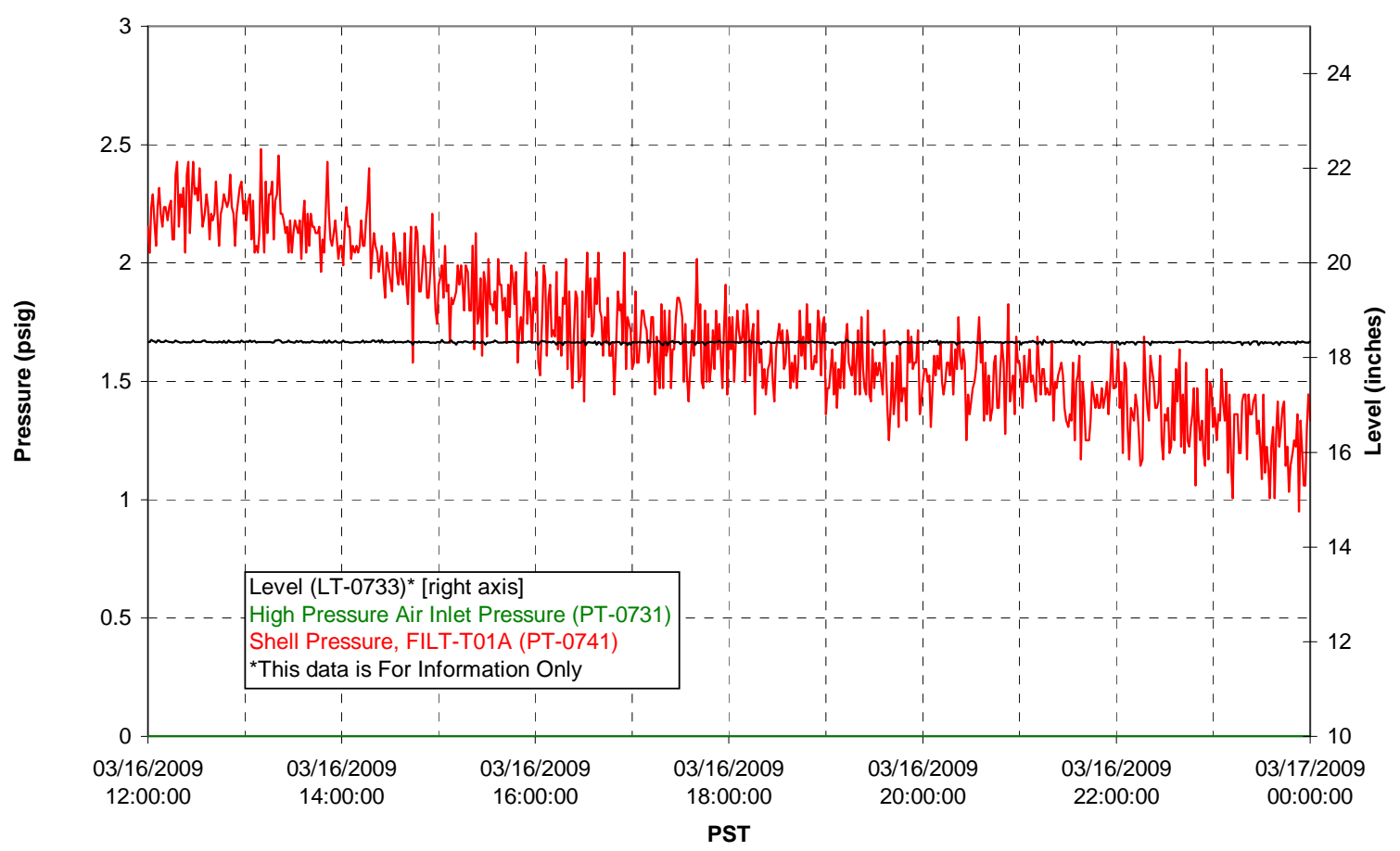

Pulsepot Levels

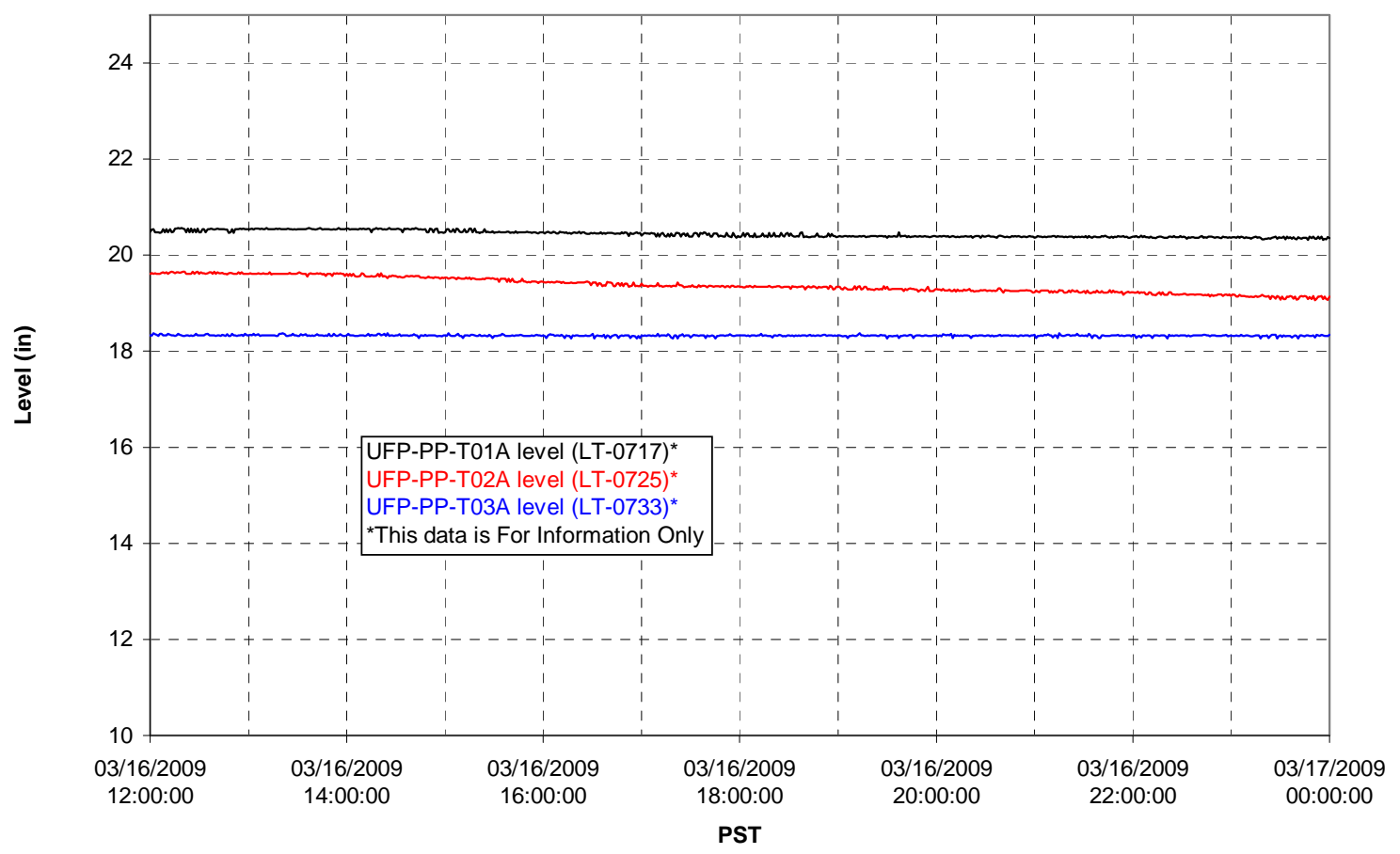


Filter UFP-FILT-T01A

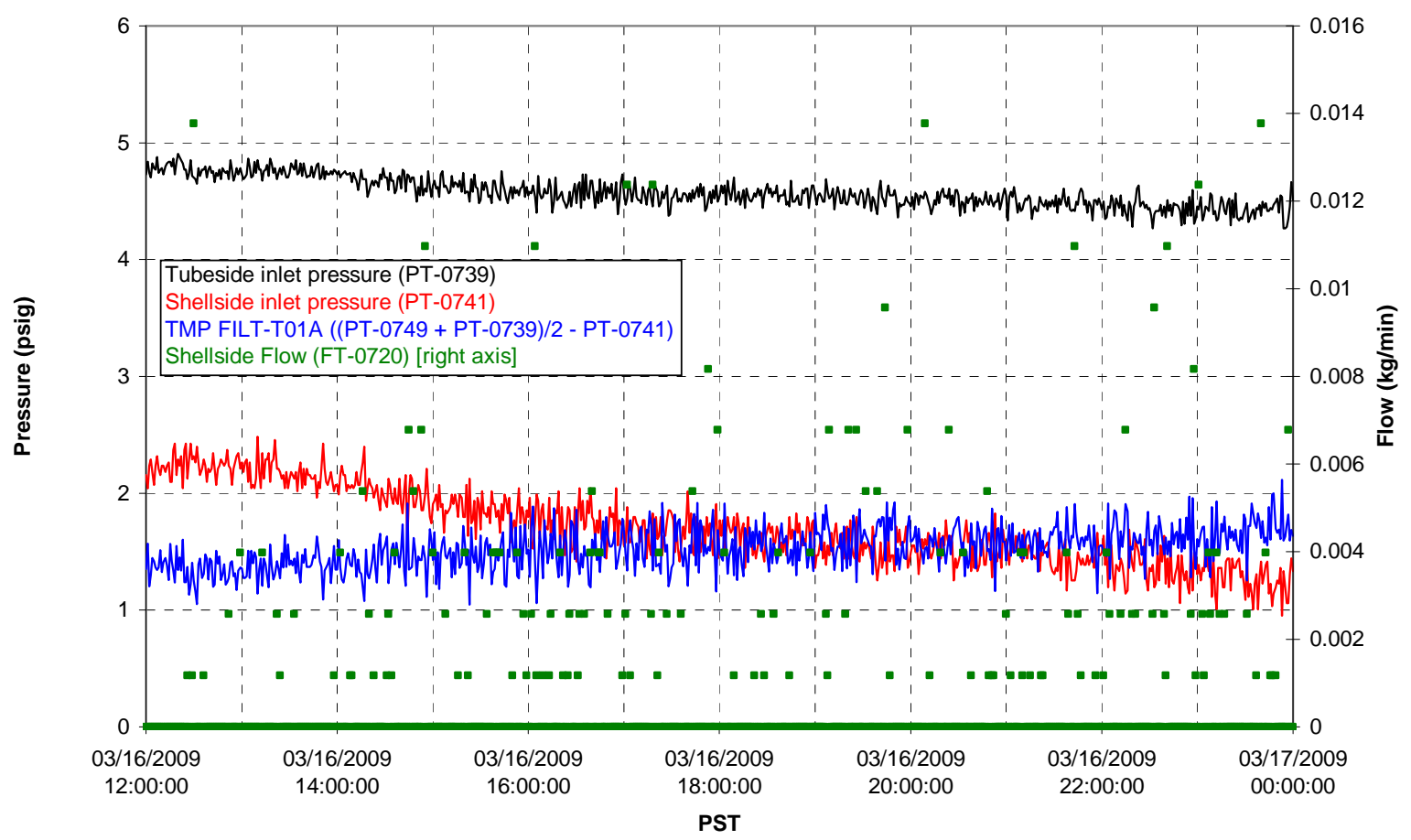

Filter UFP-FILT-T02A

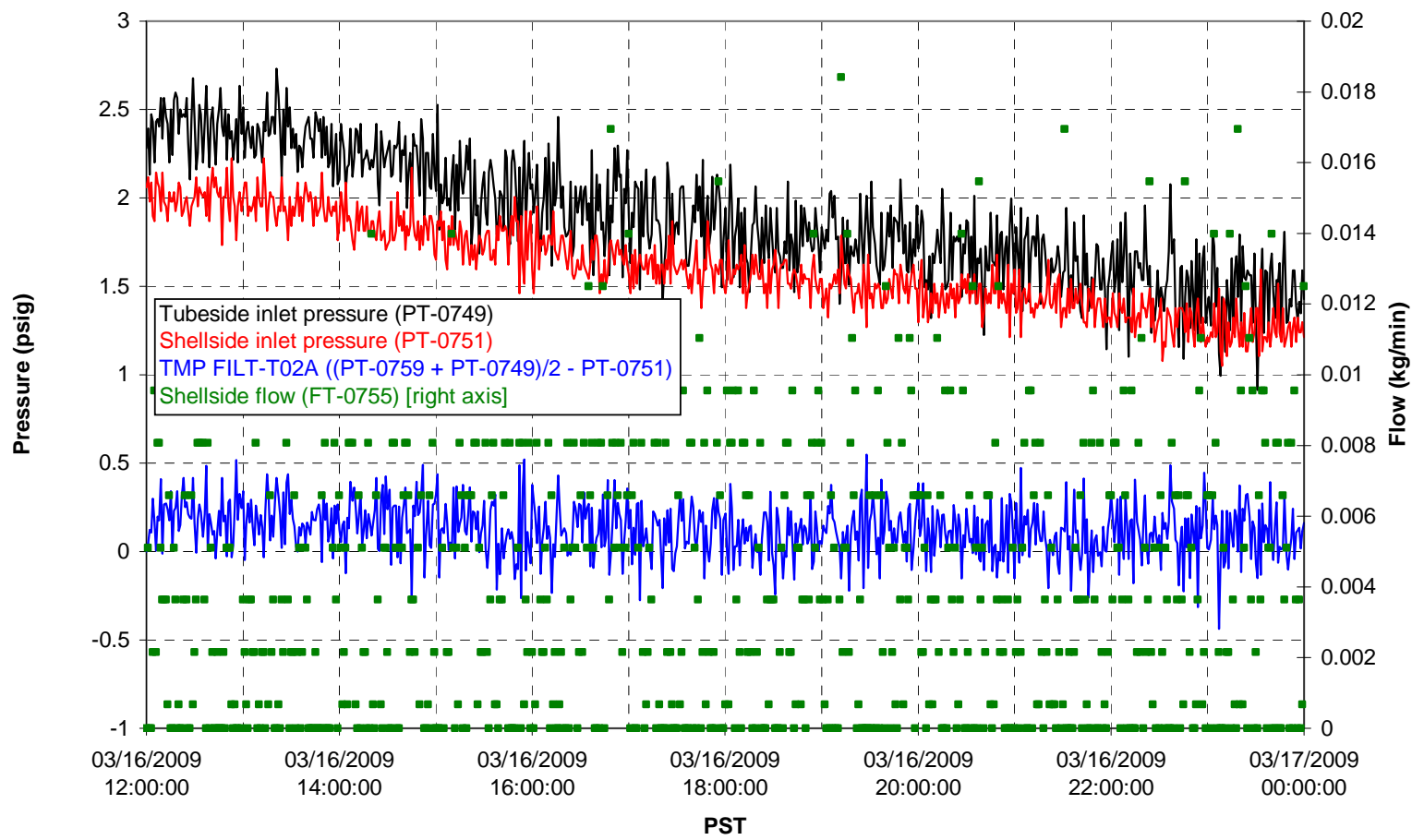


Filter UFP-FILT-T03A

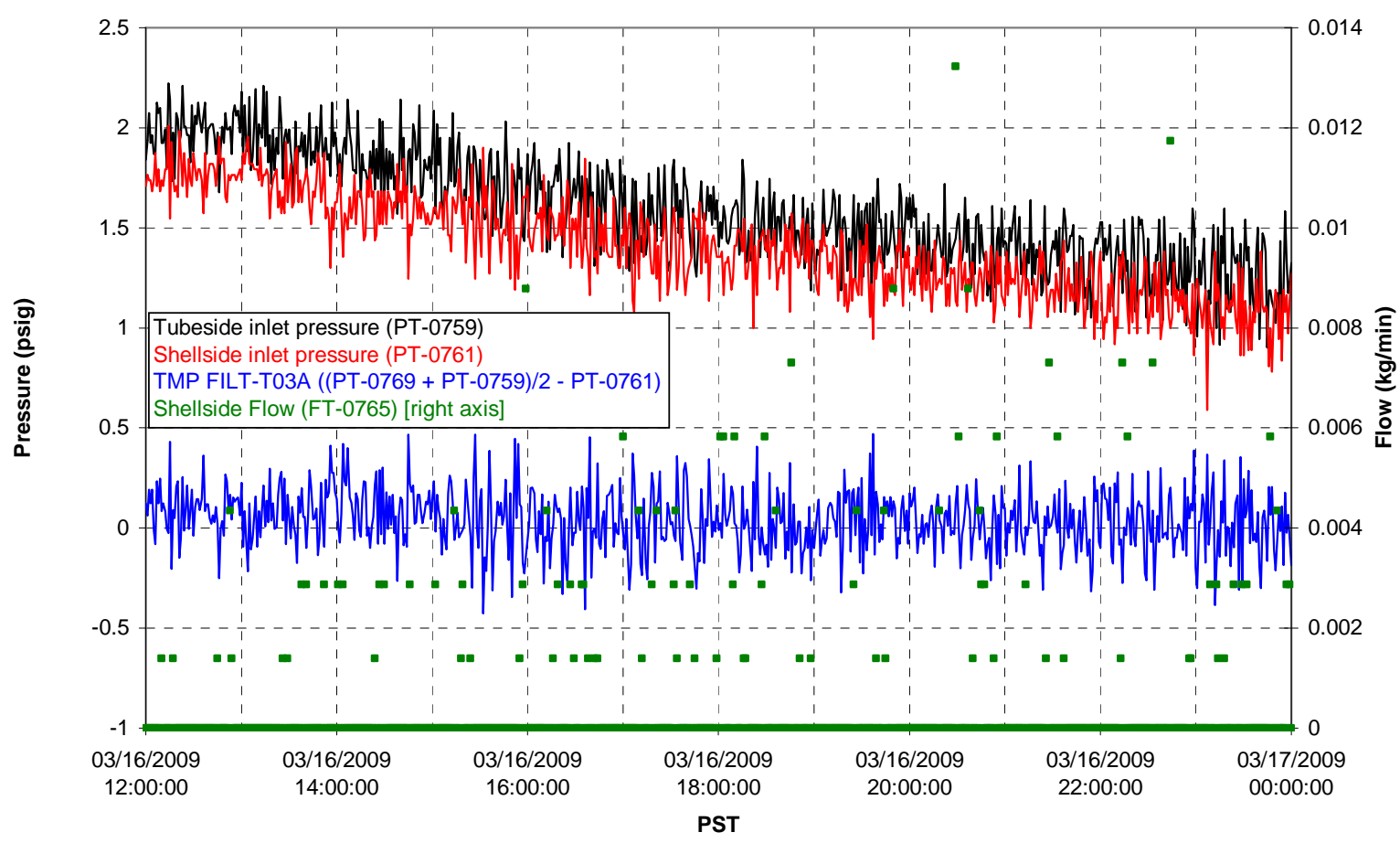

Filter UFP-FILT-T04A

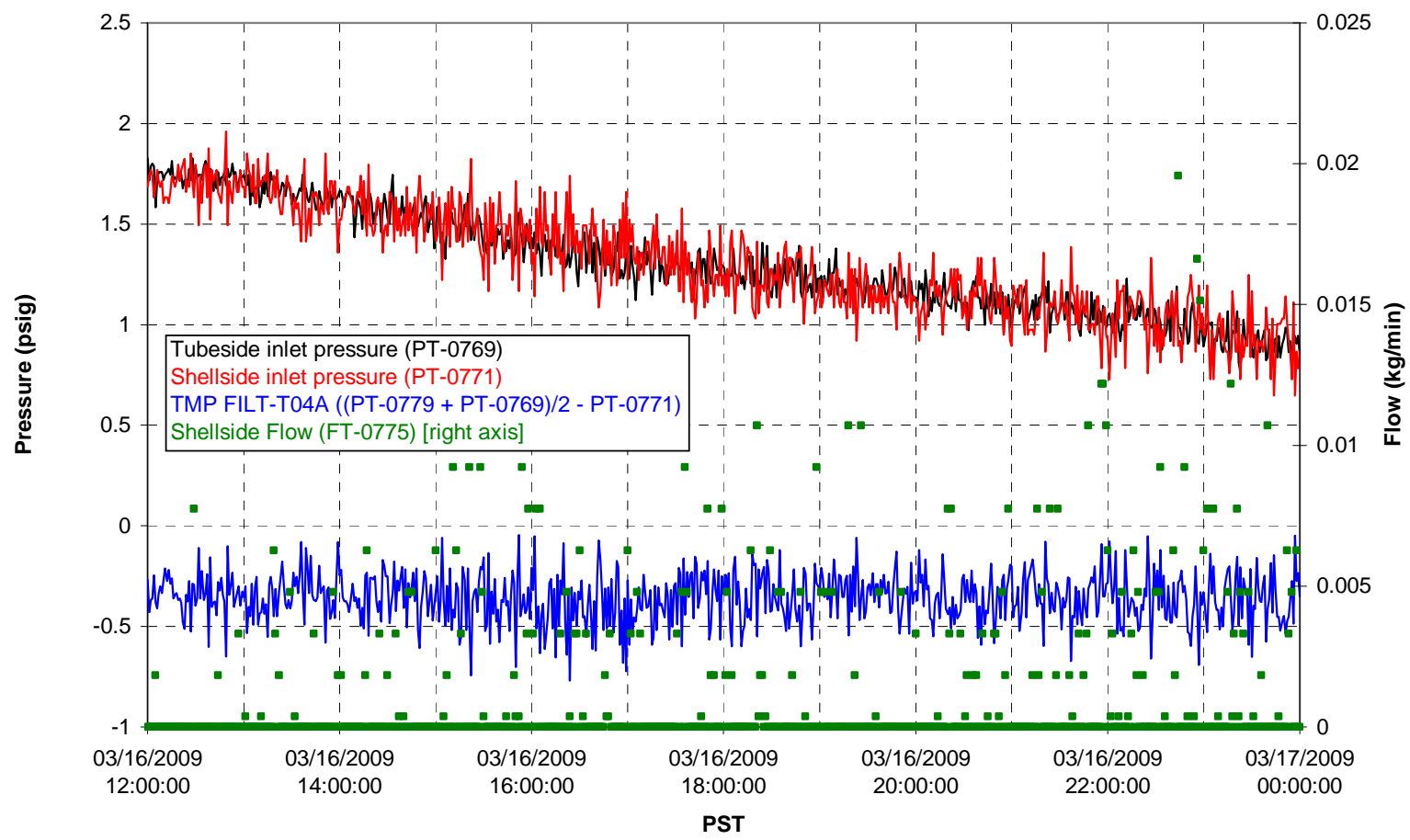


Filter UFP-FILT-T05A

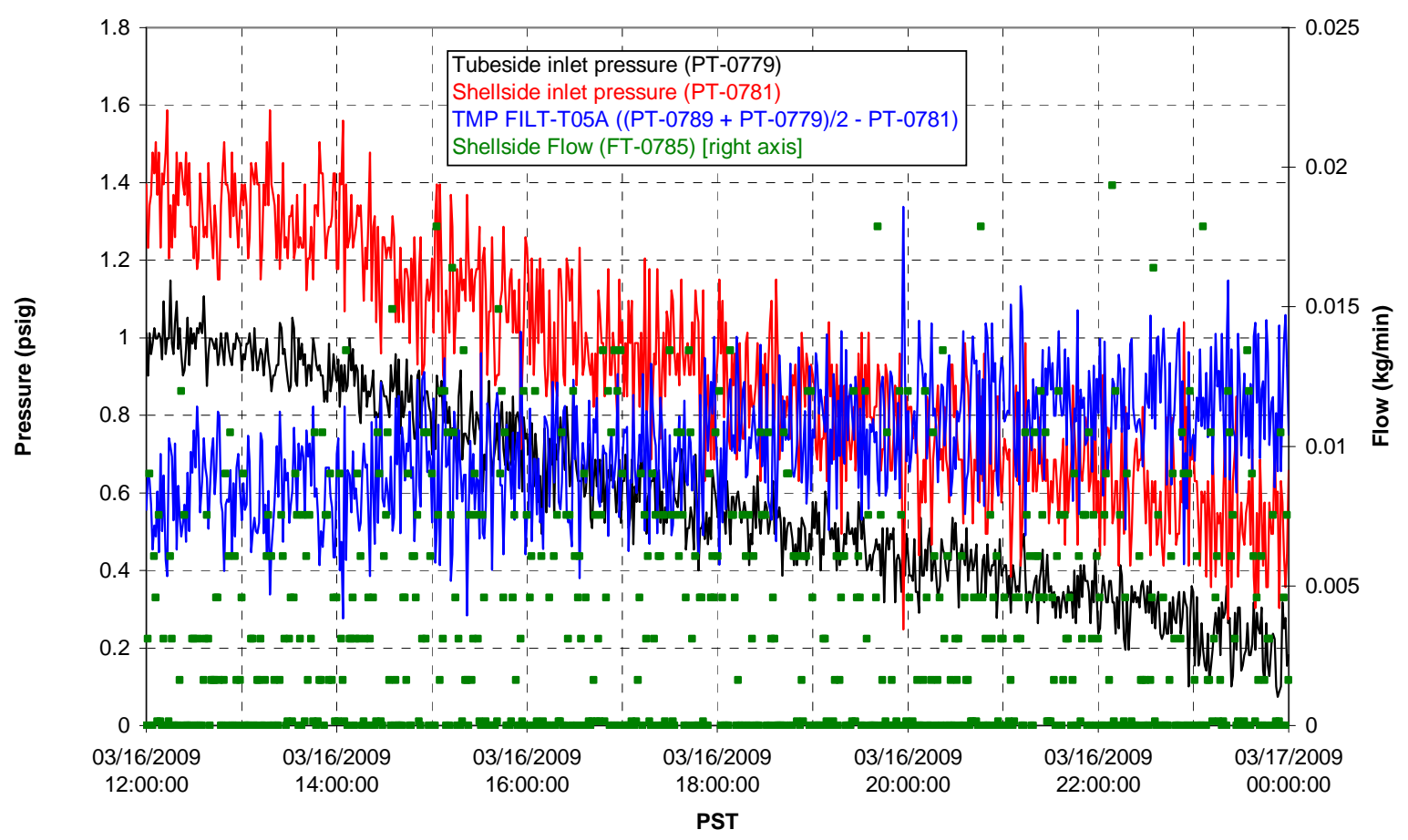

Chemical Flow

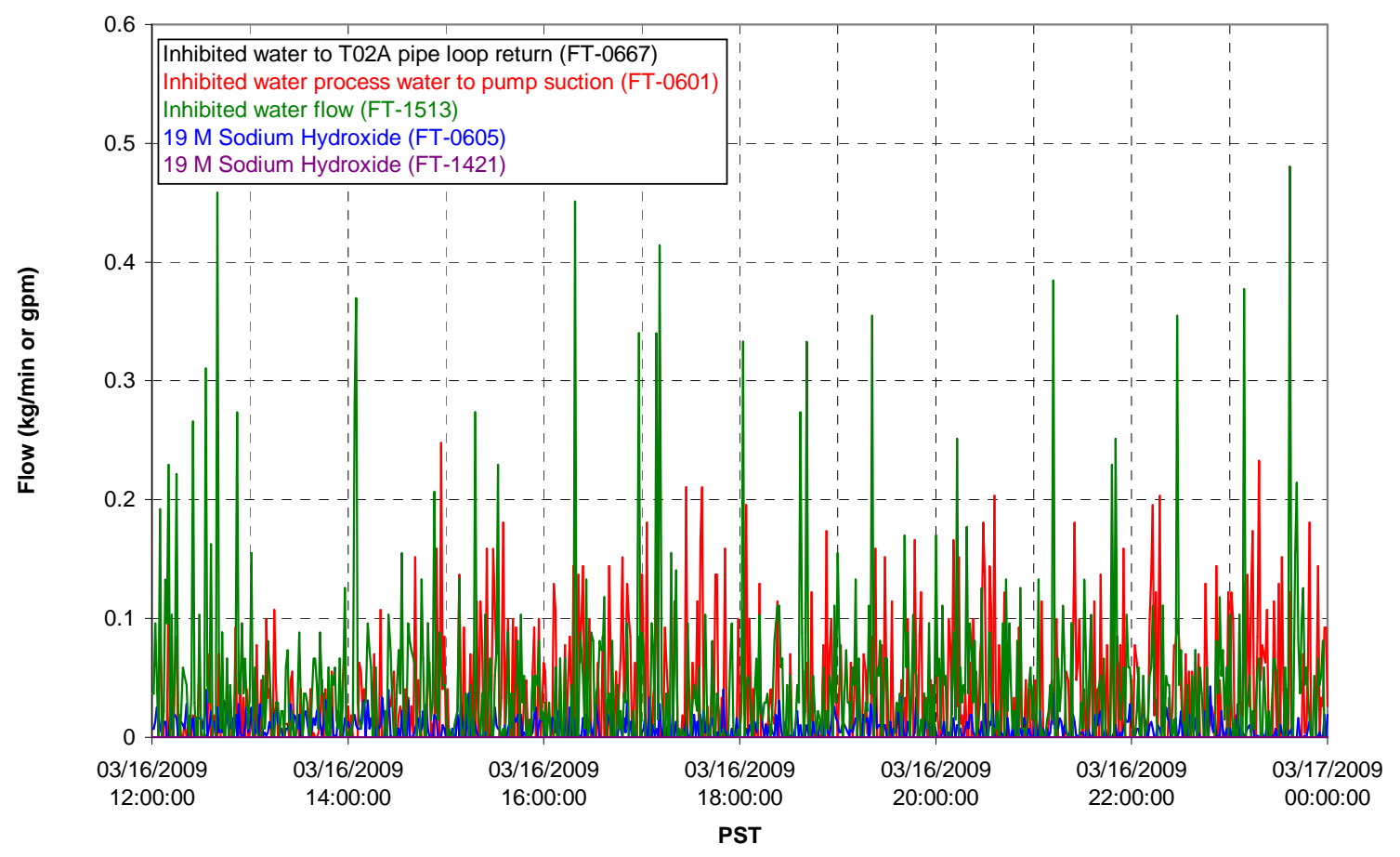




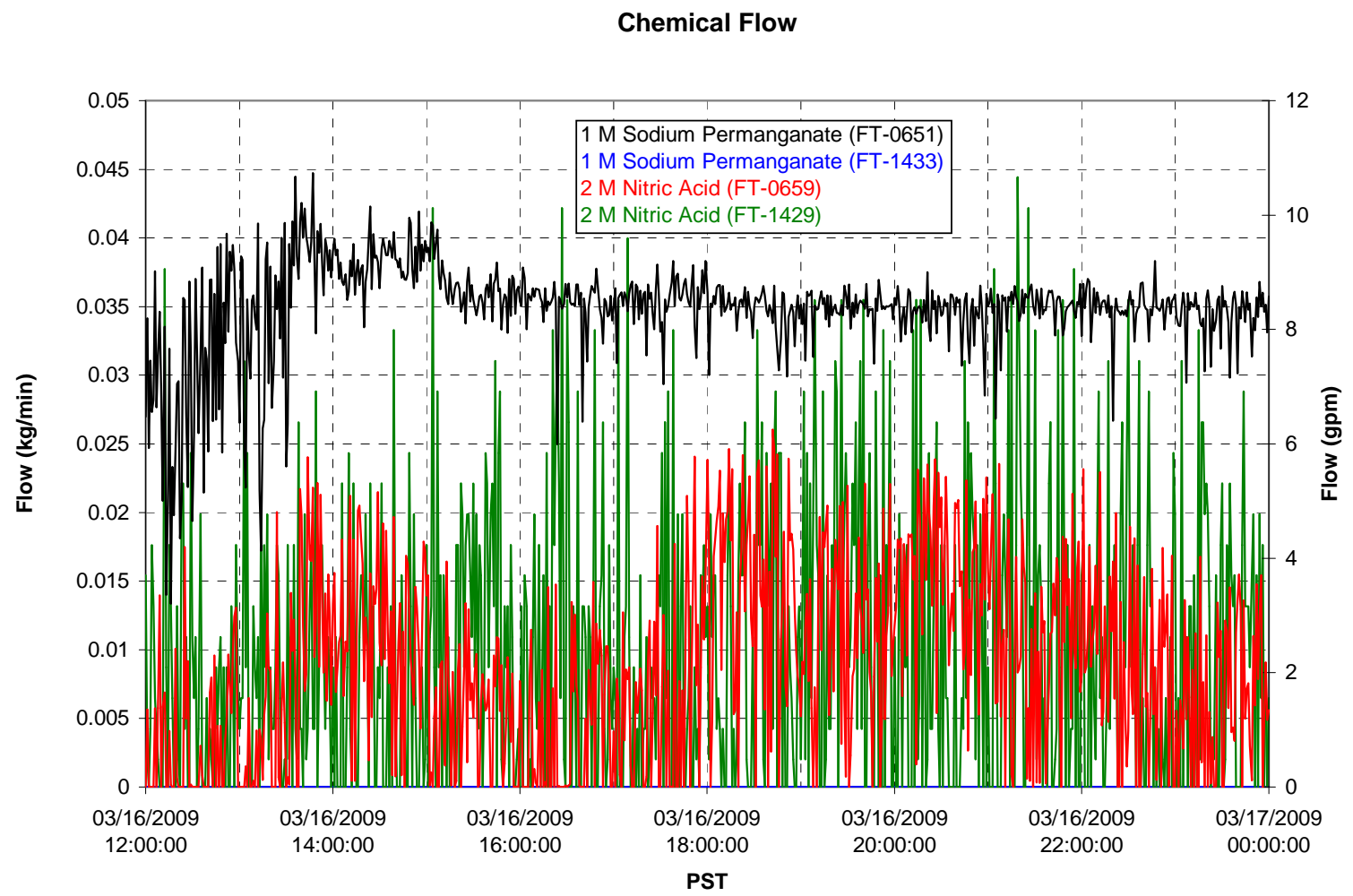

Air Flows

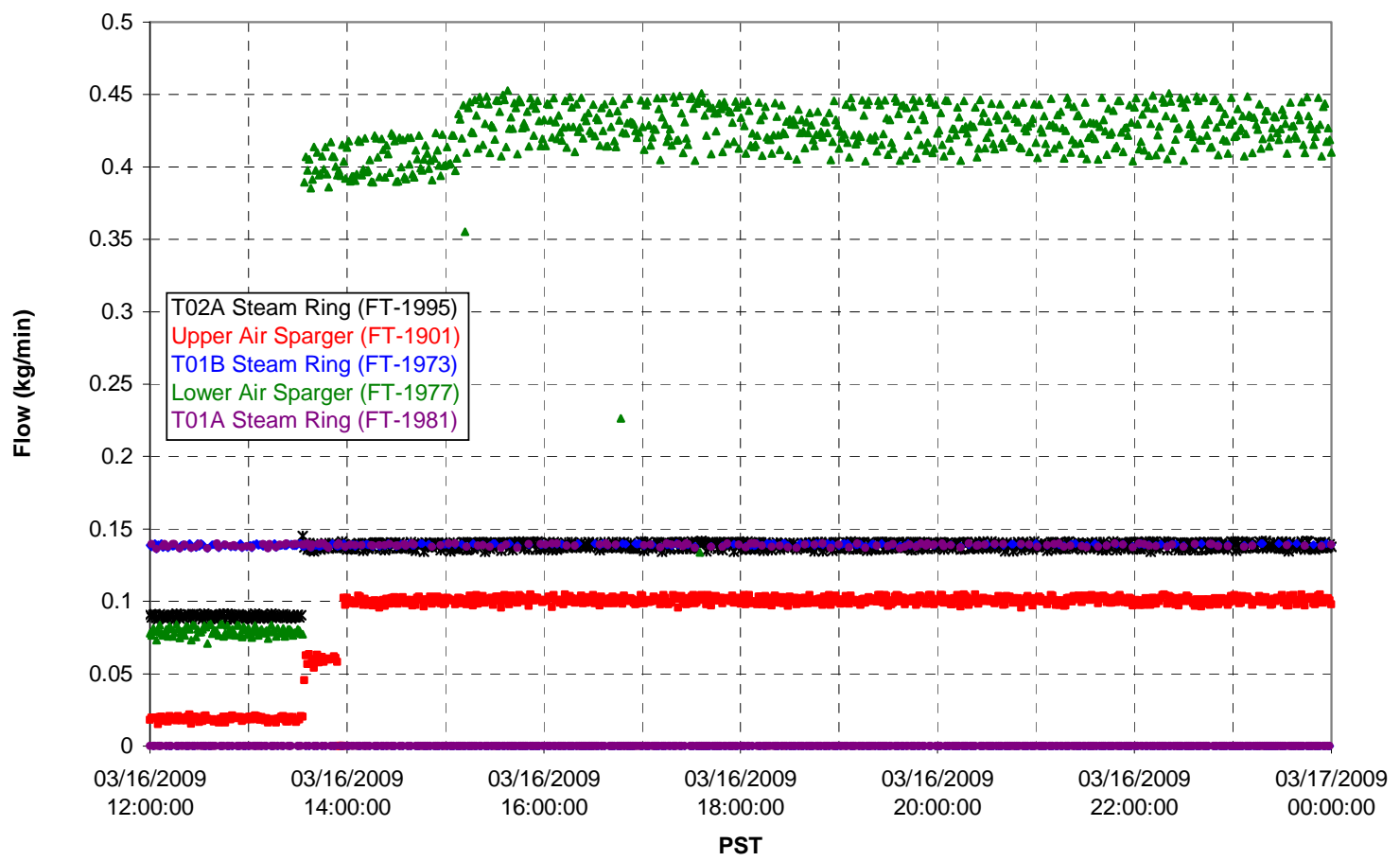


T02A Steam

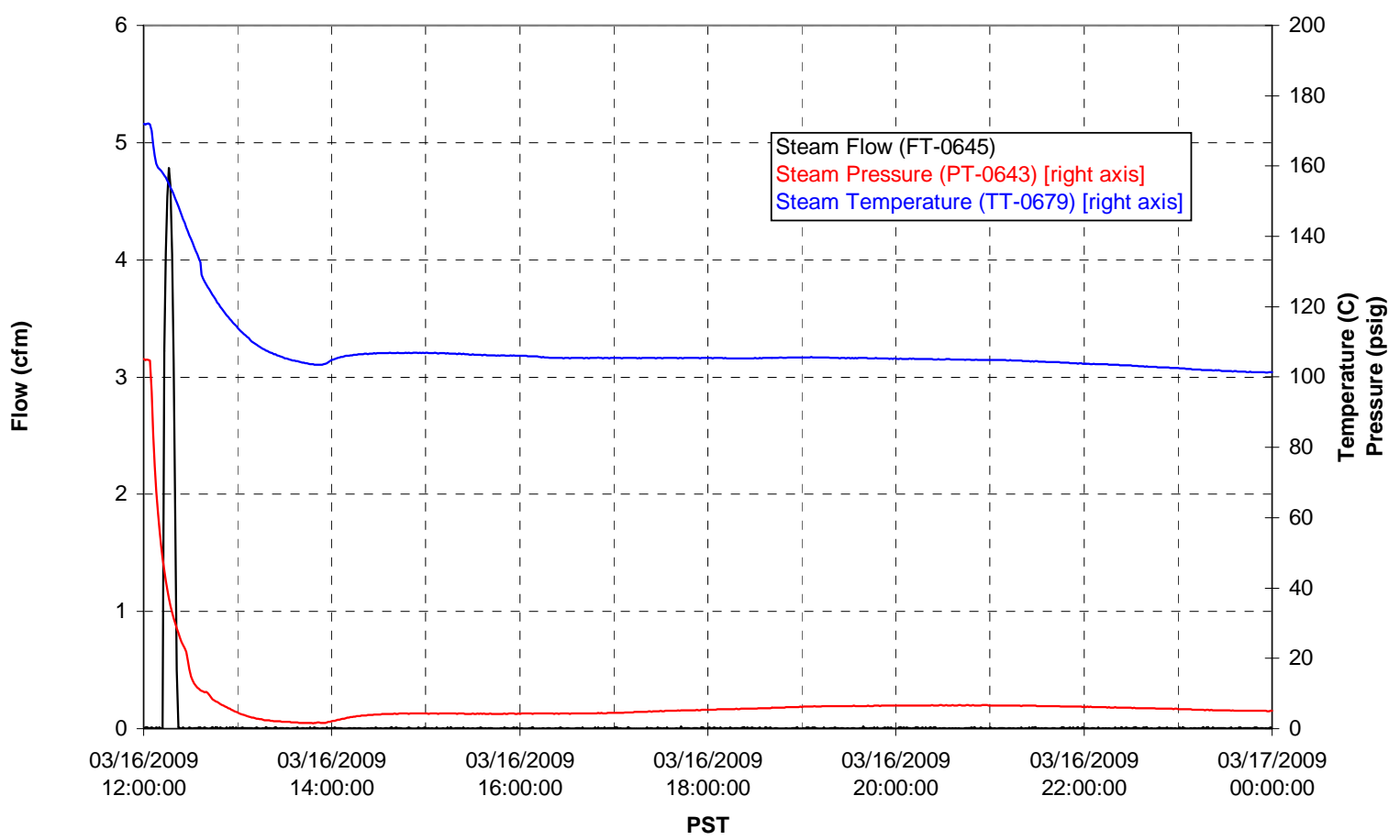

T01A Steam

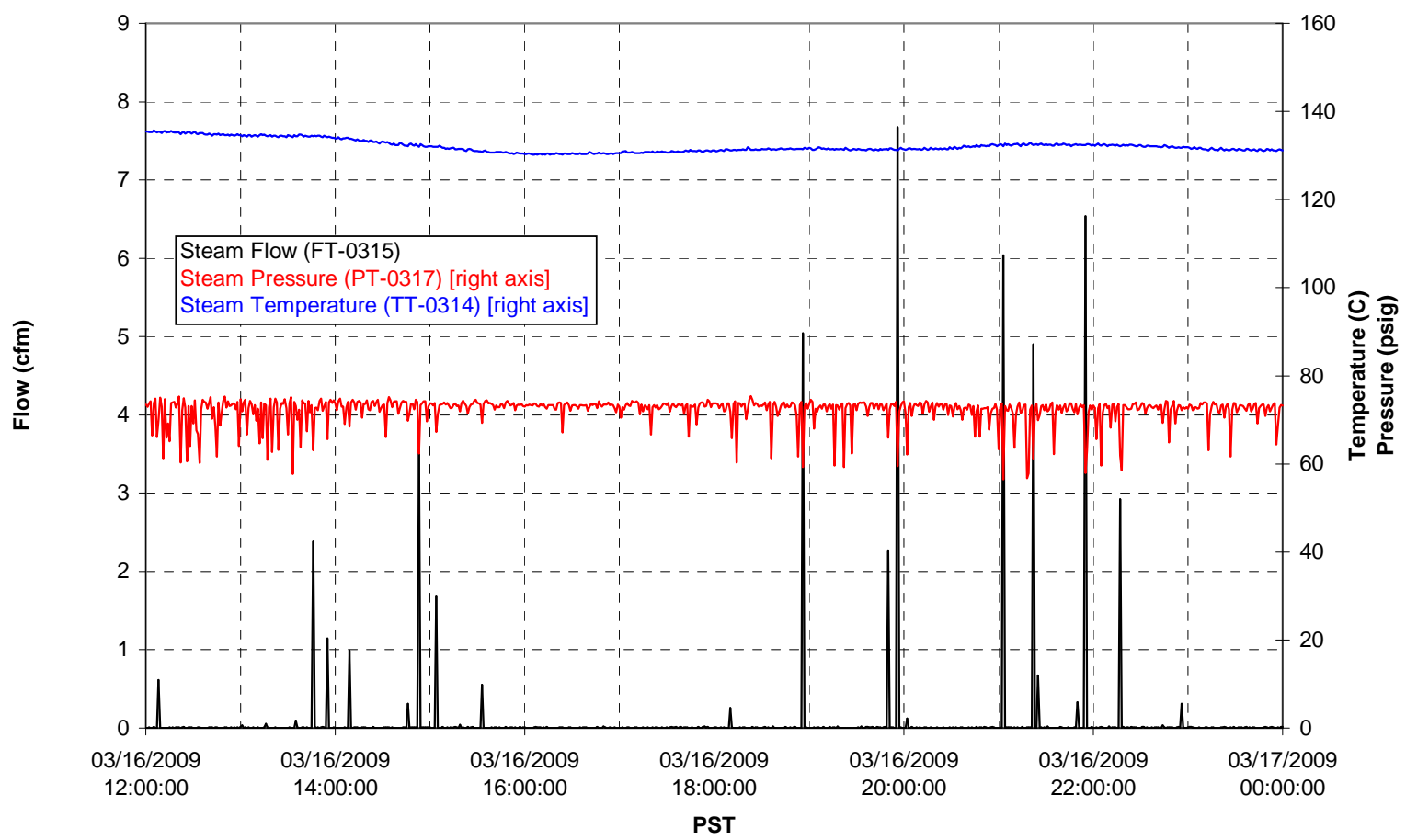


T01B Steam

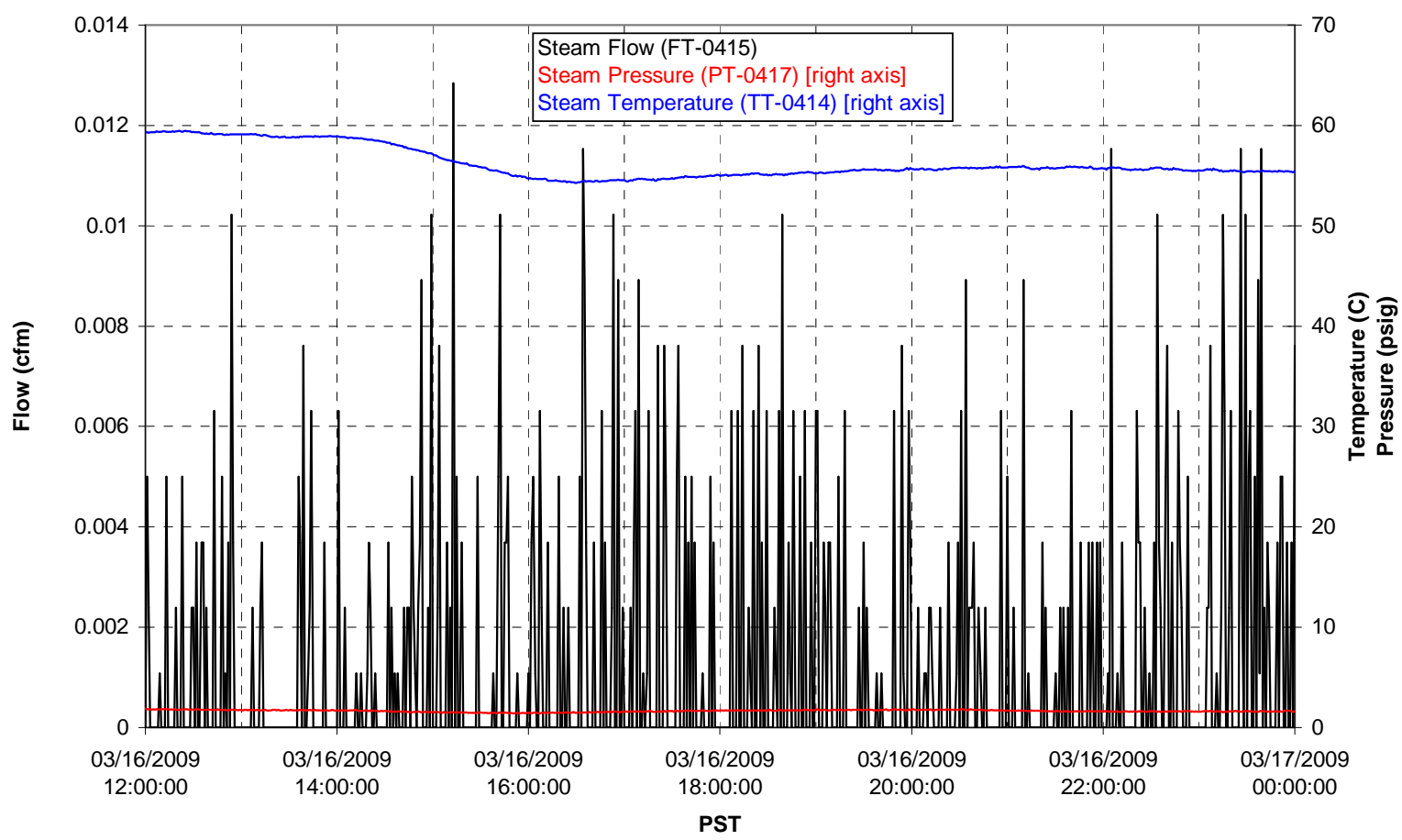




\section{Integrated Test B Data Plots 03/17/09 00:00 - 03/17/09 12:00}


T01A level

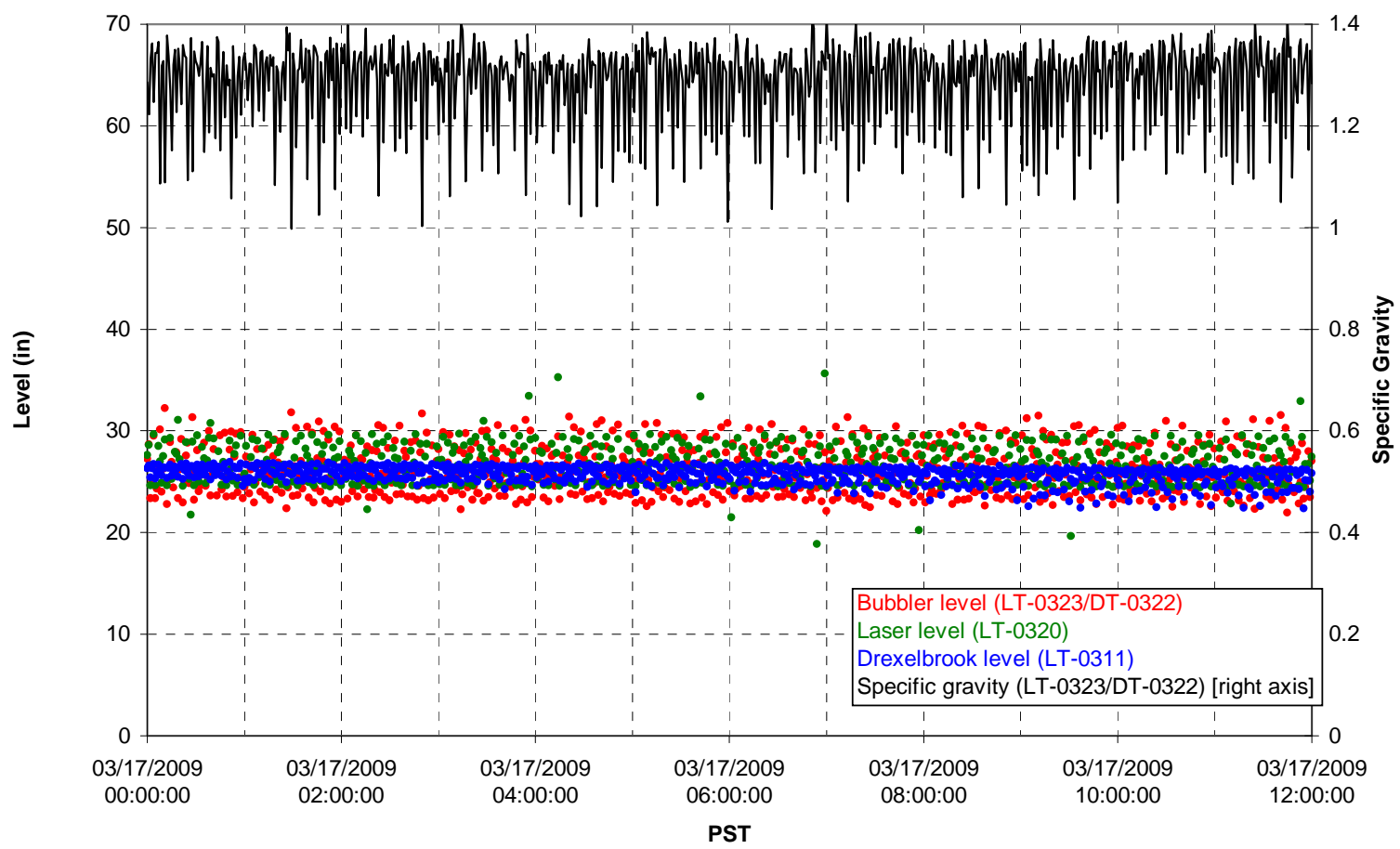

T01A temperatures

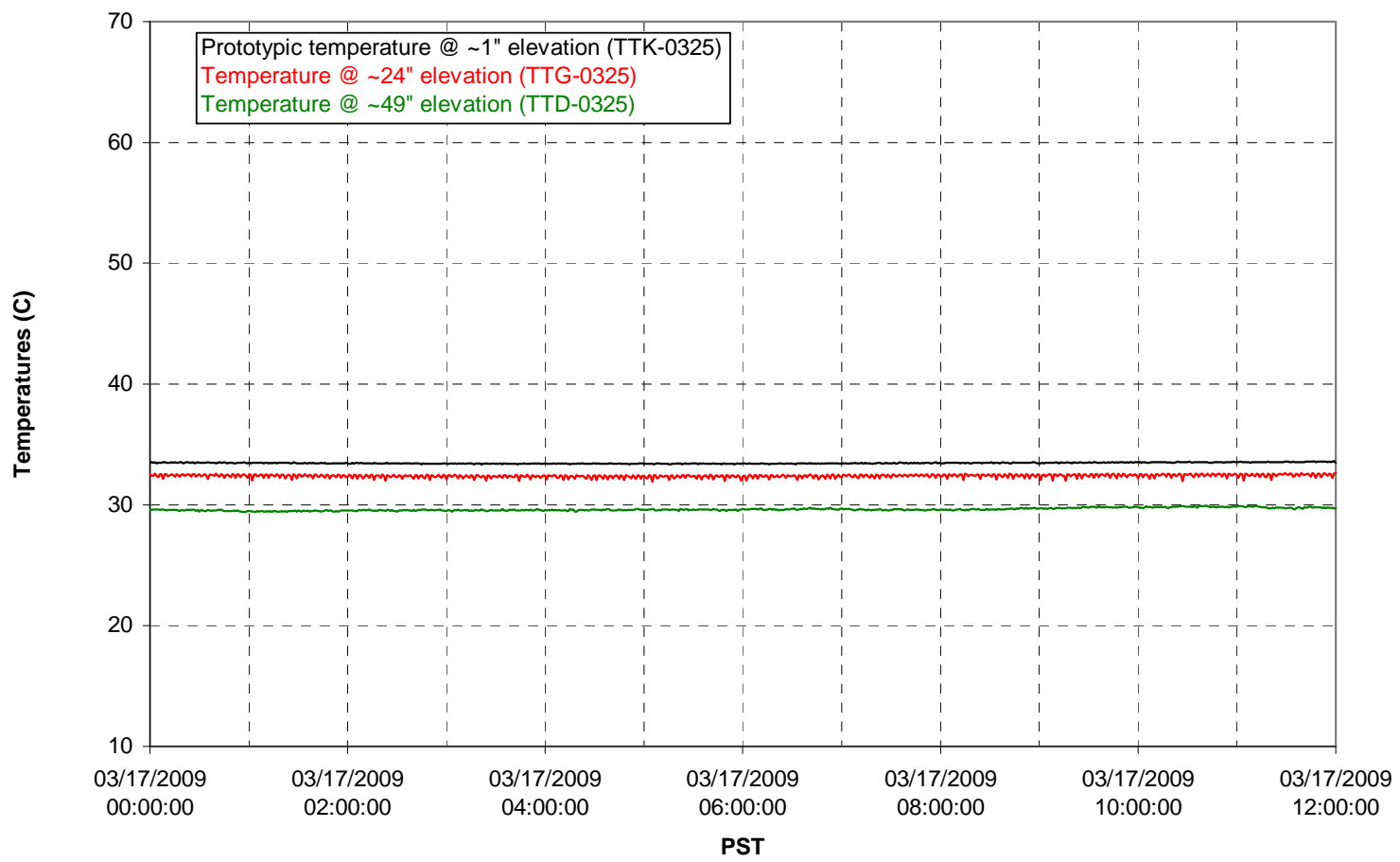


T01B level

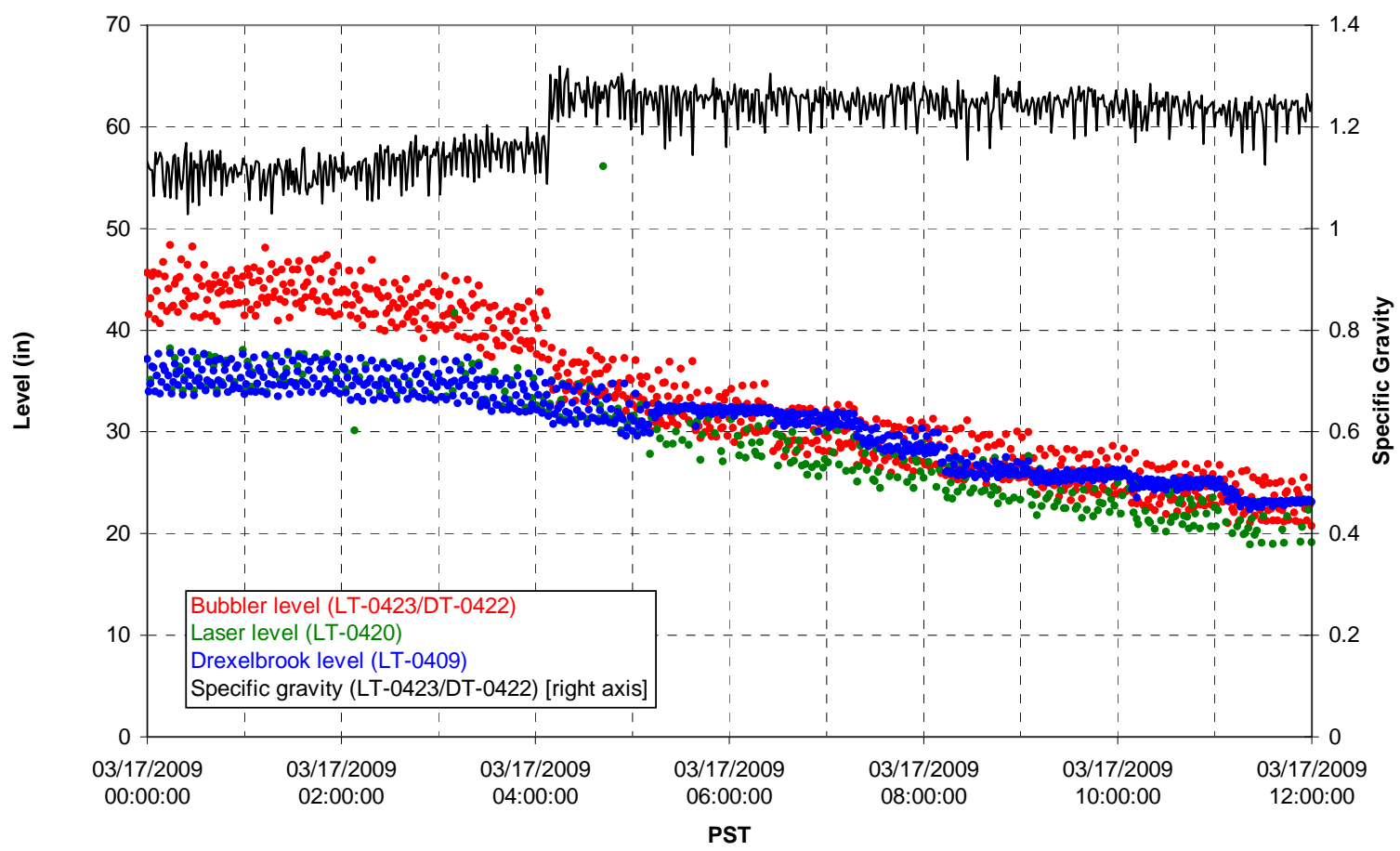

T01B temperatures

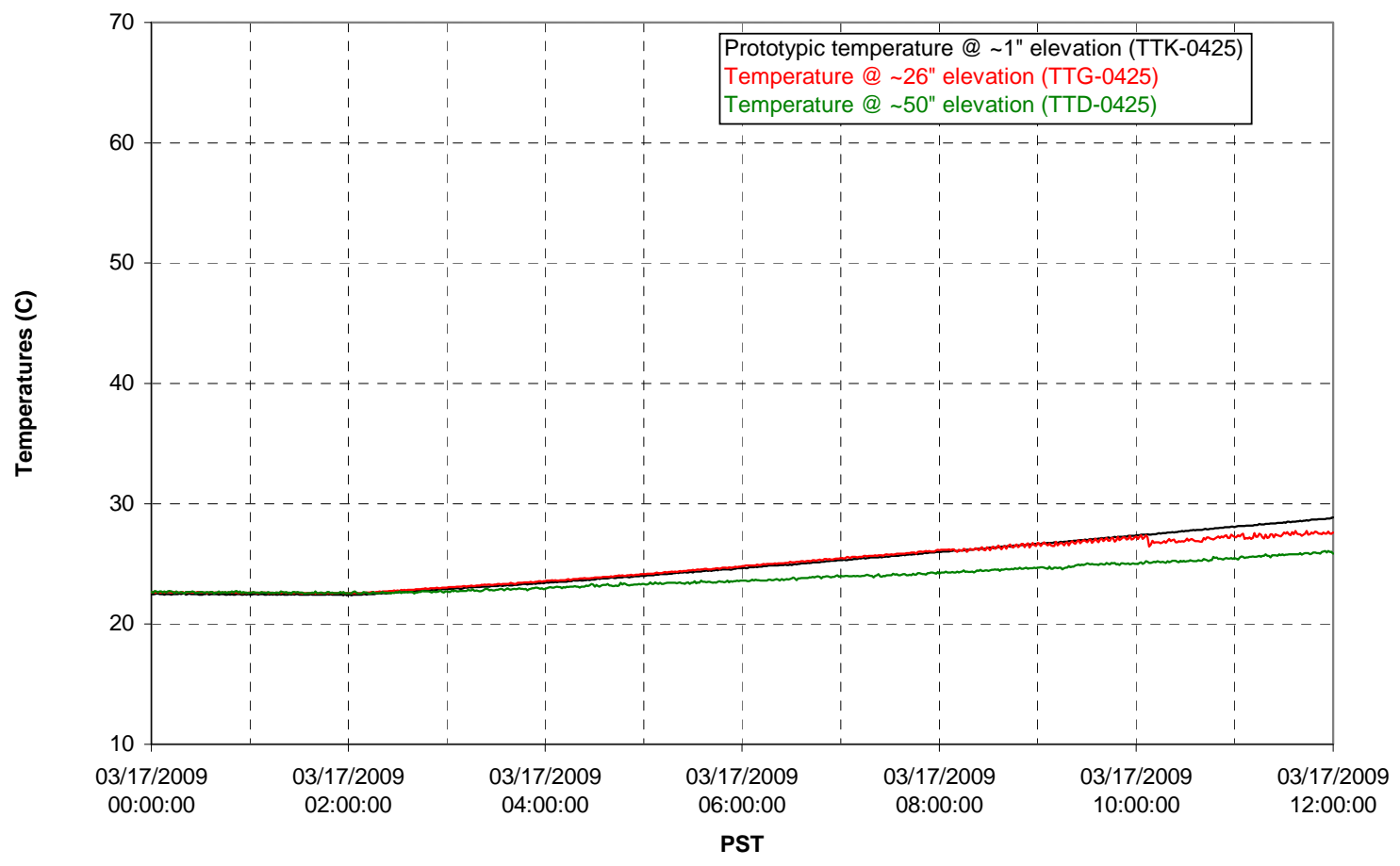


T02A level

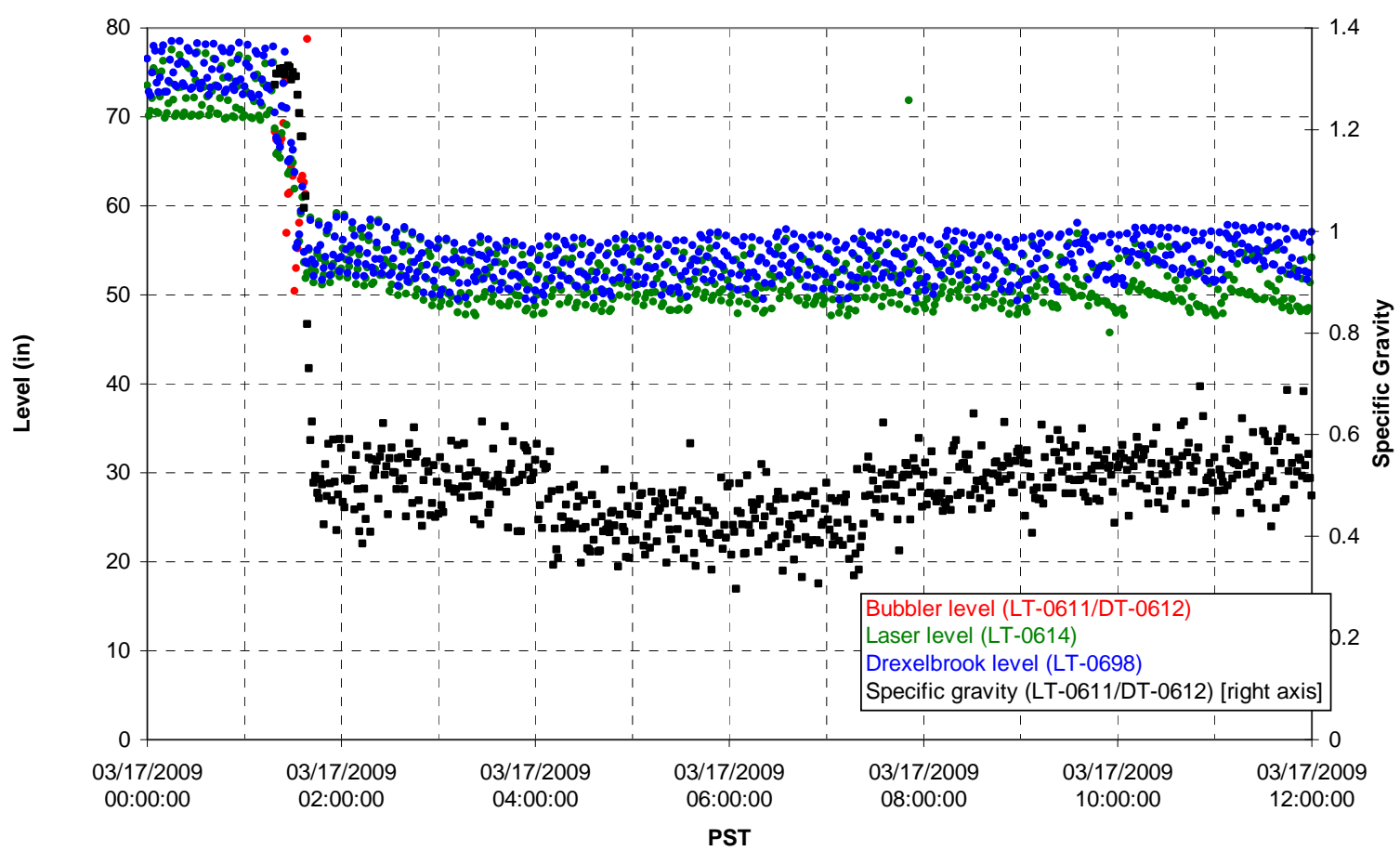

T02A temperatures

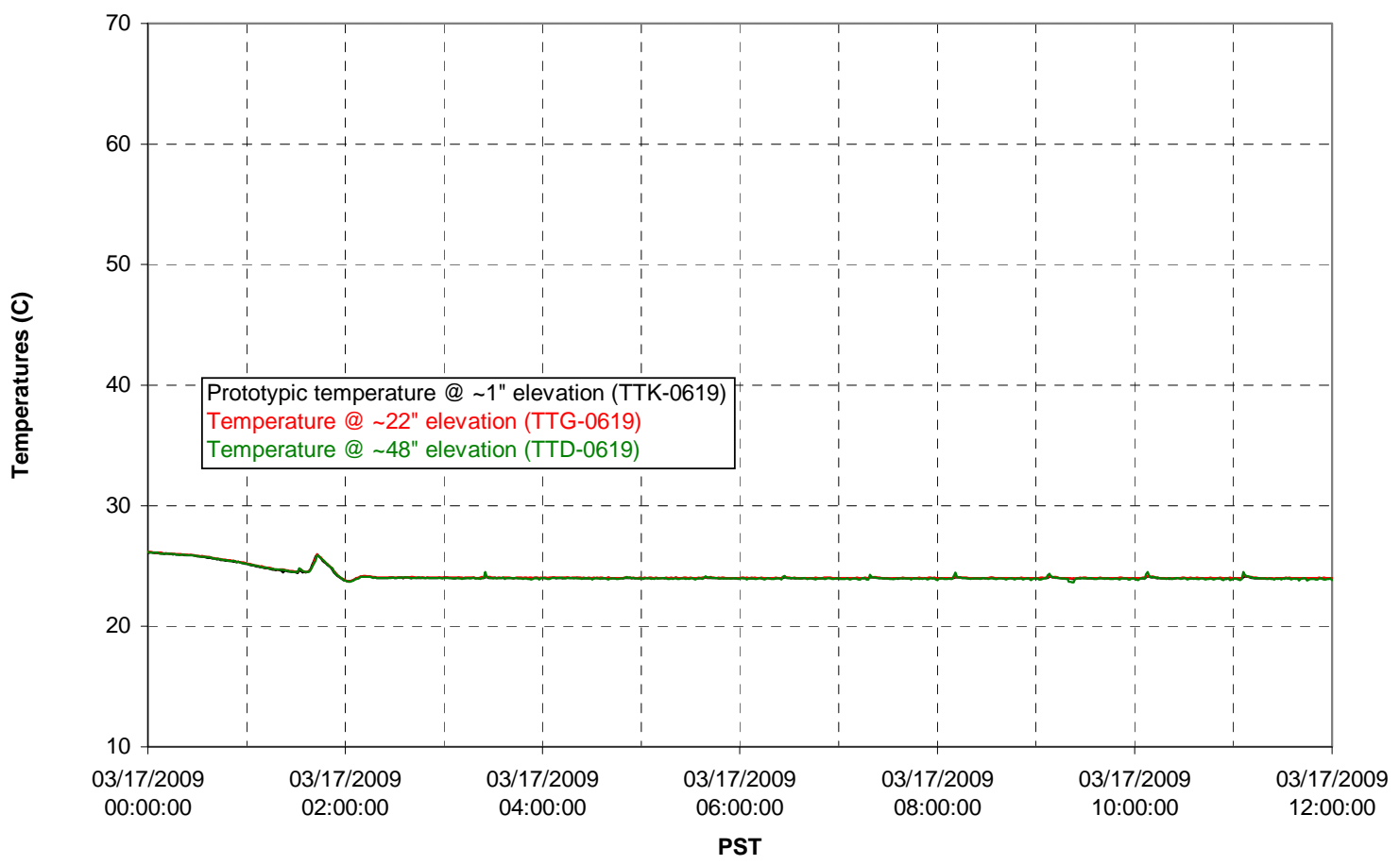


T02A and filter loop temperatures

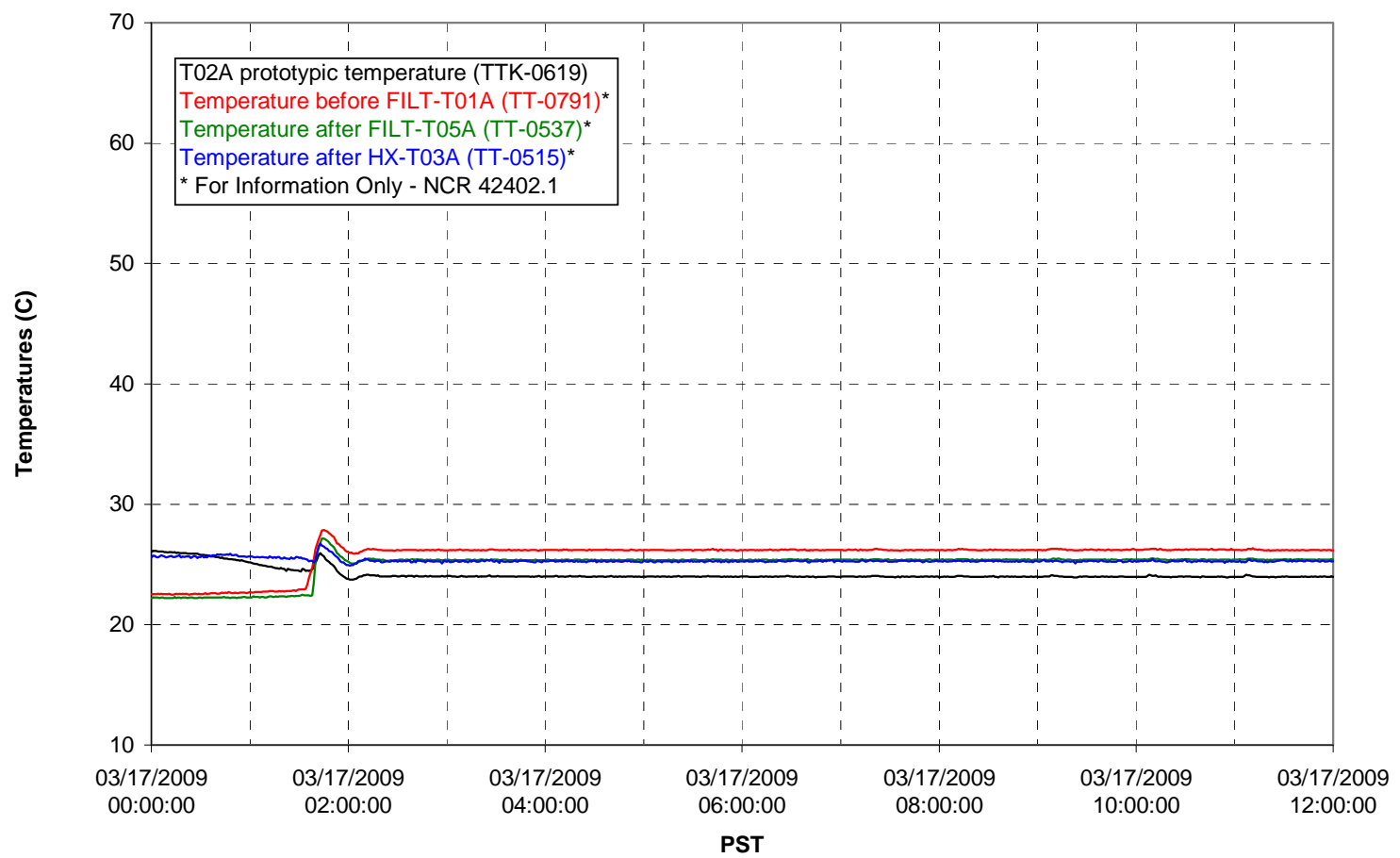

Pump Pressures and Flow

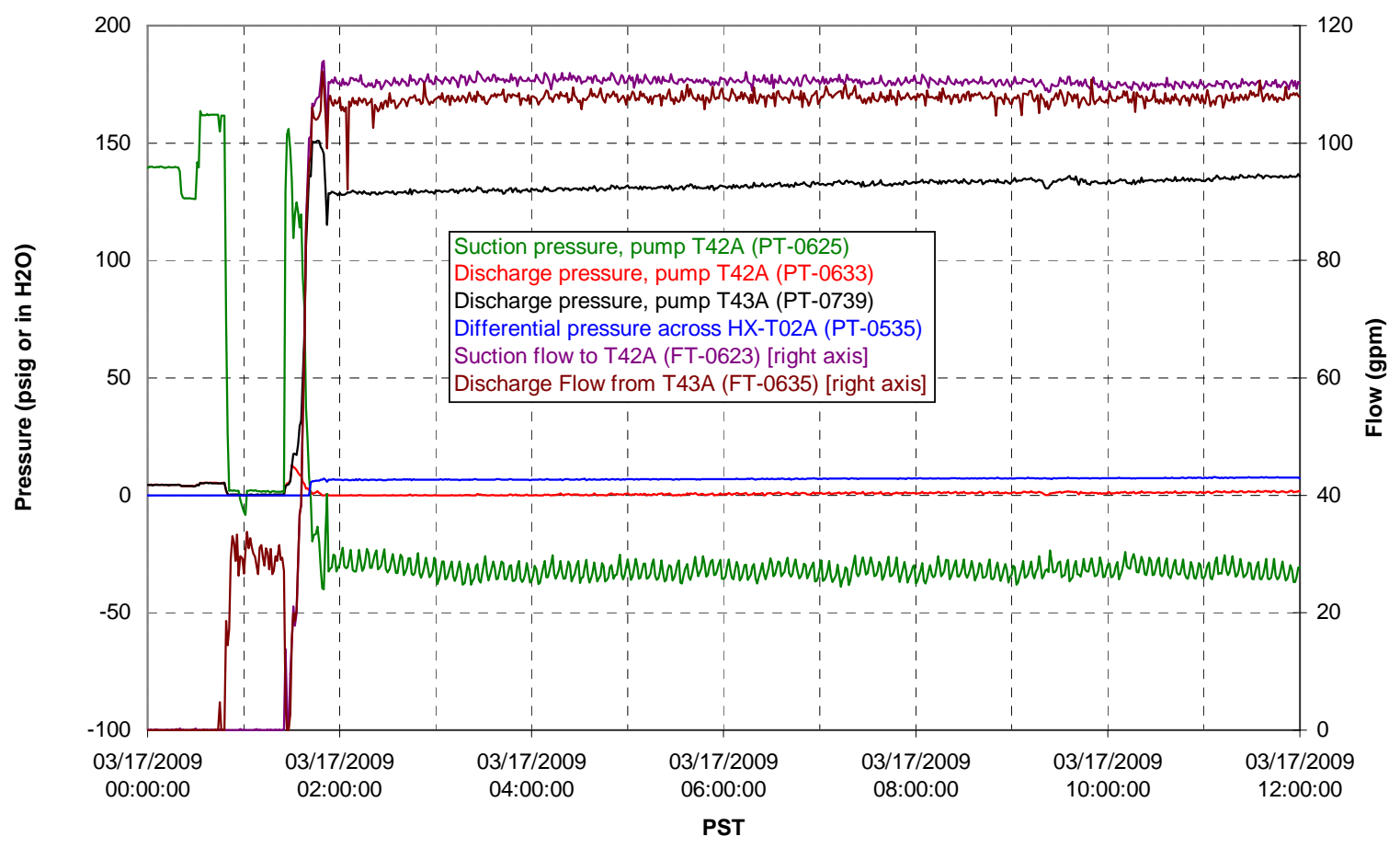


Axial pressure drop

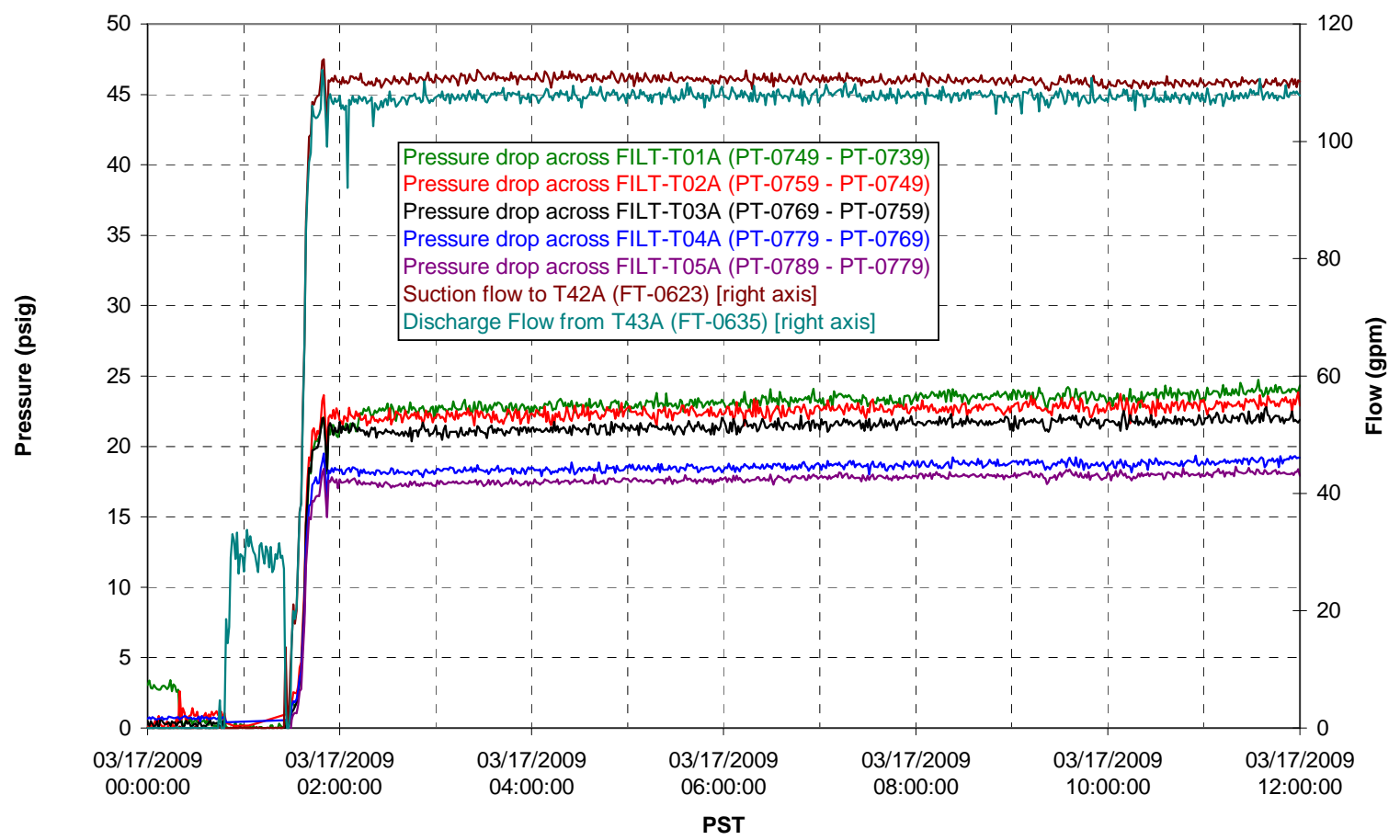

Permeate flow rates

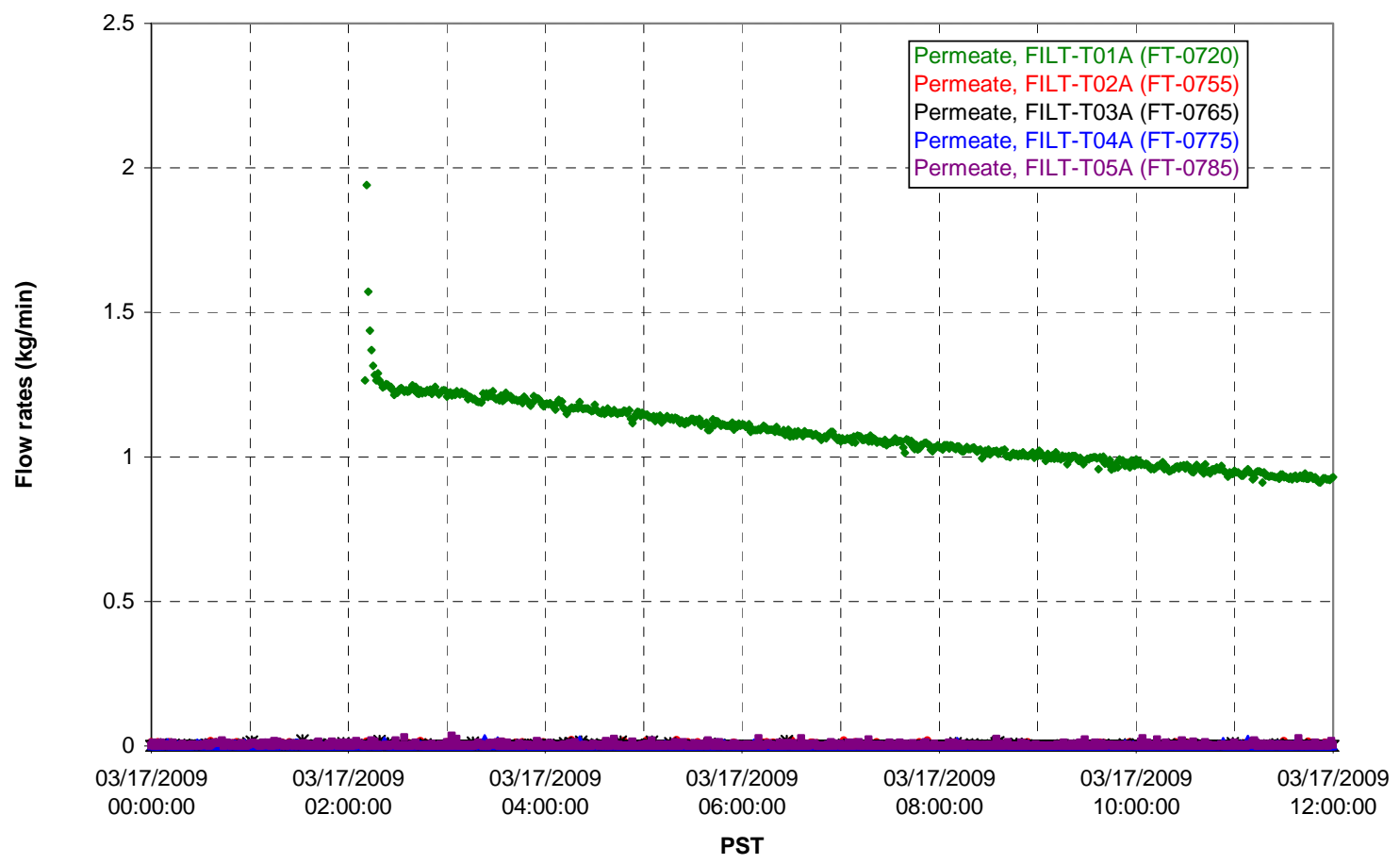


T02A Inner Temperature Tree

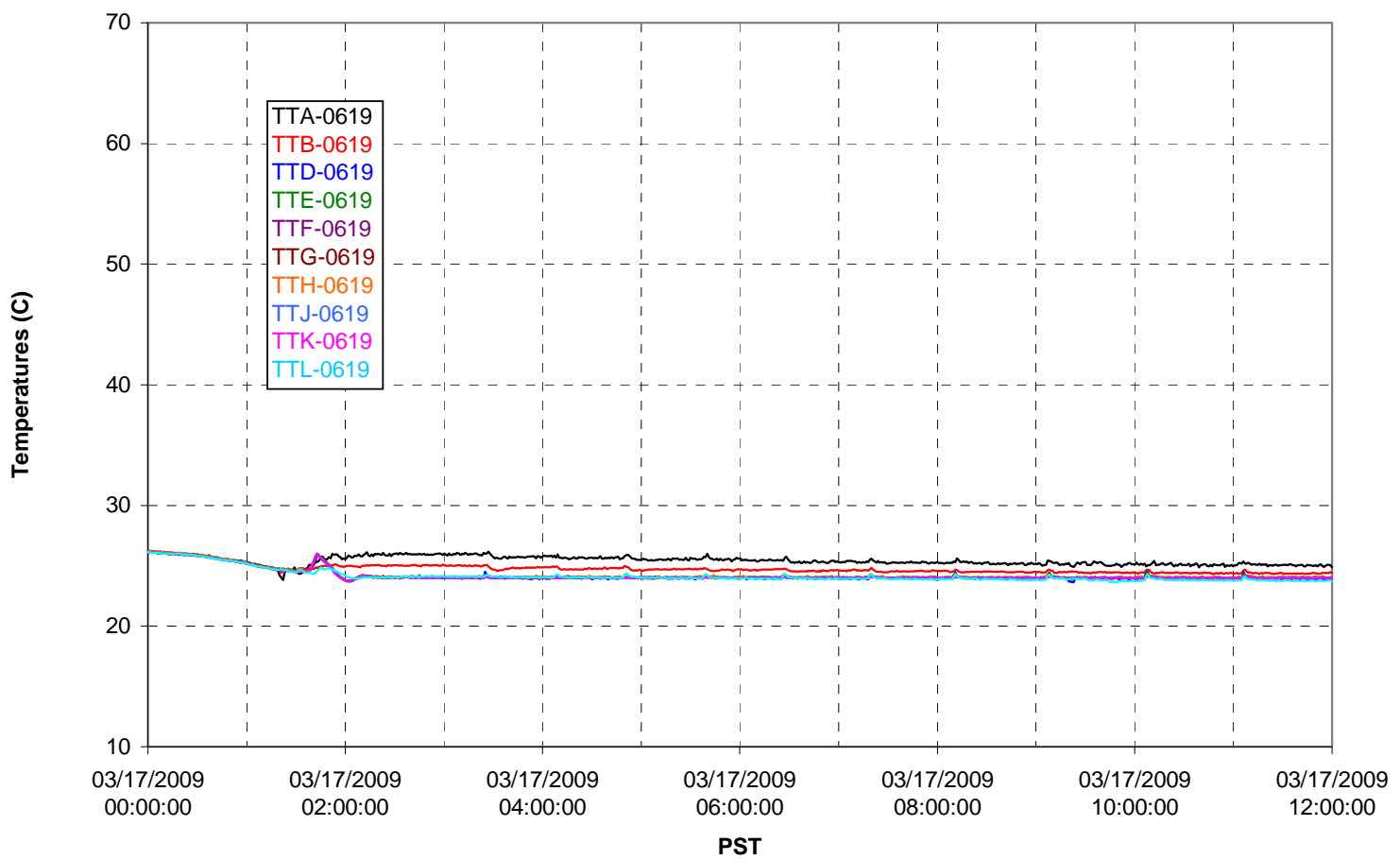

T02A Outer Temperature Tree

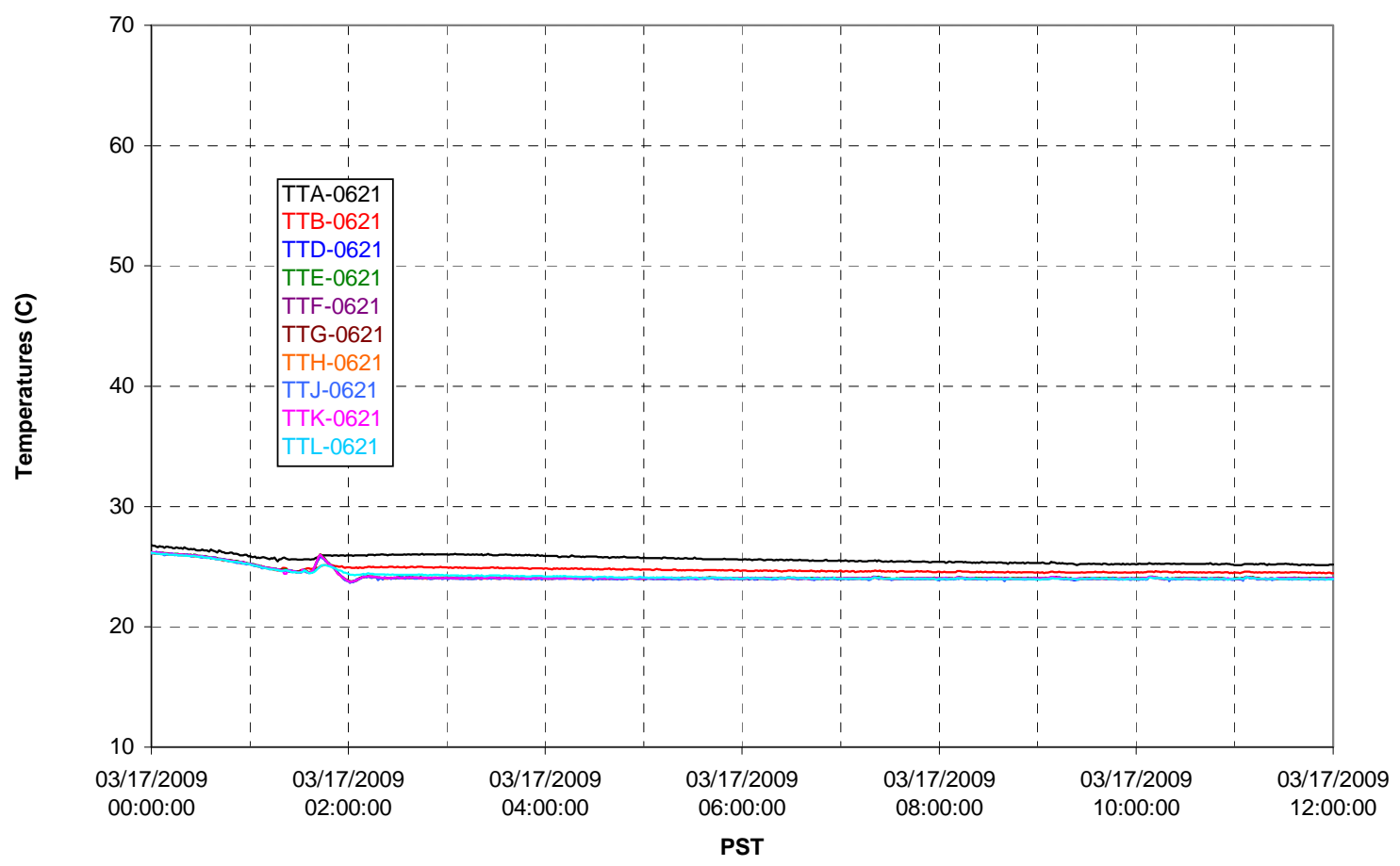


T02A temperatures

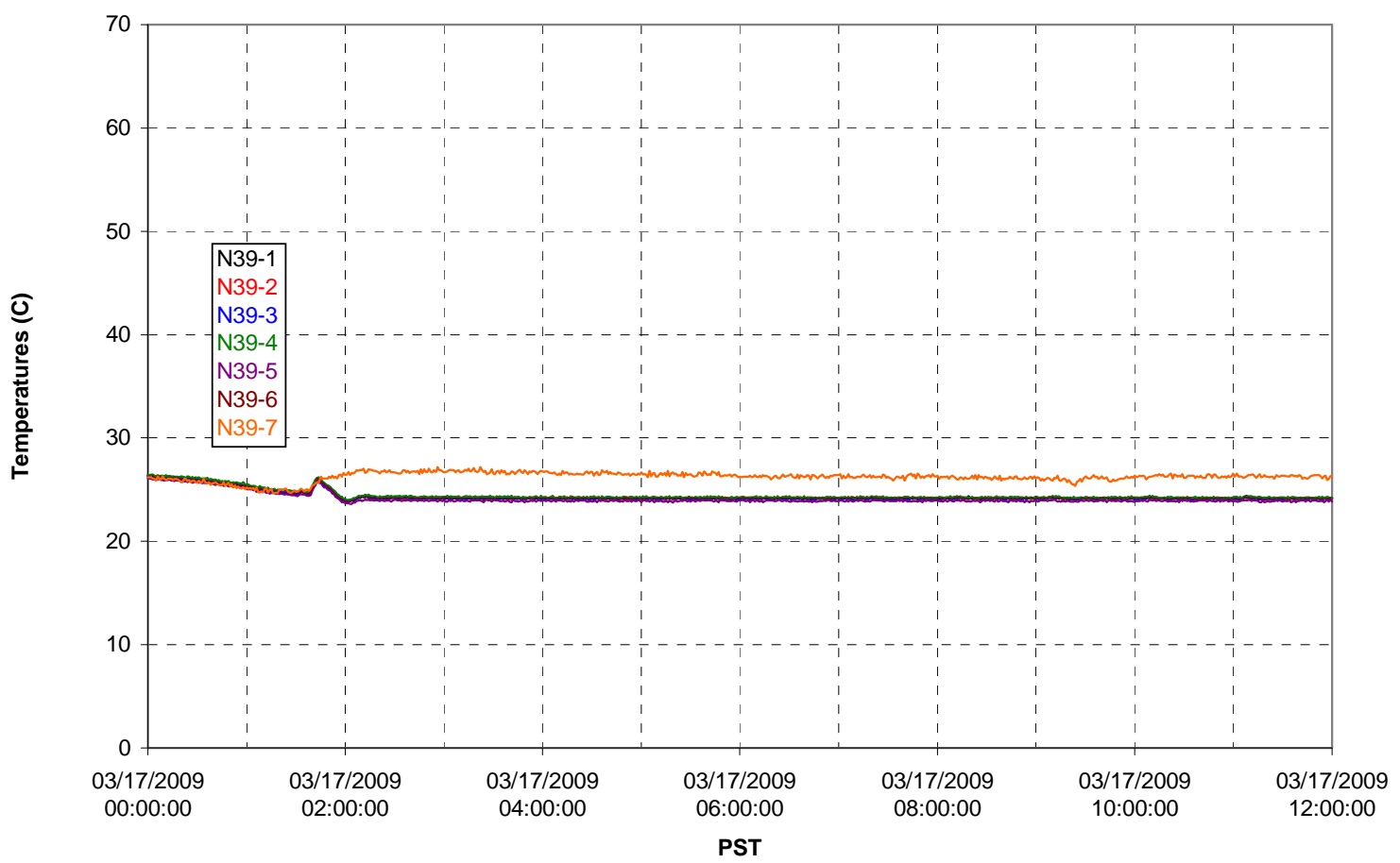

T02A temperatures

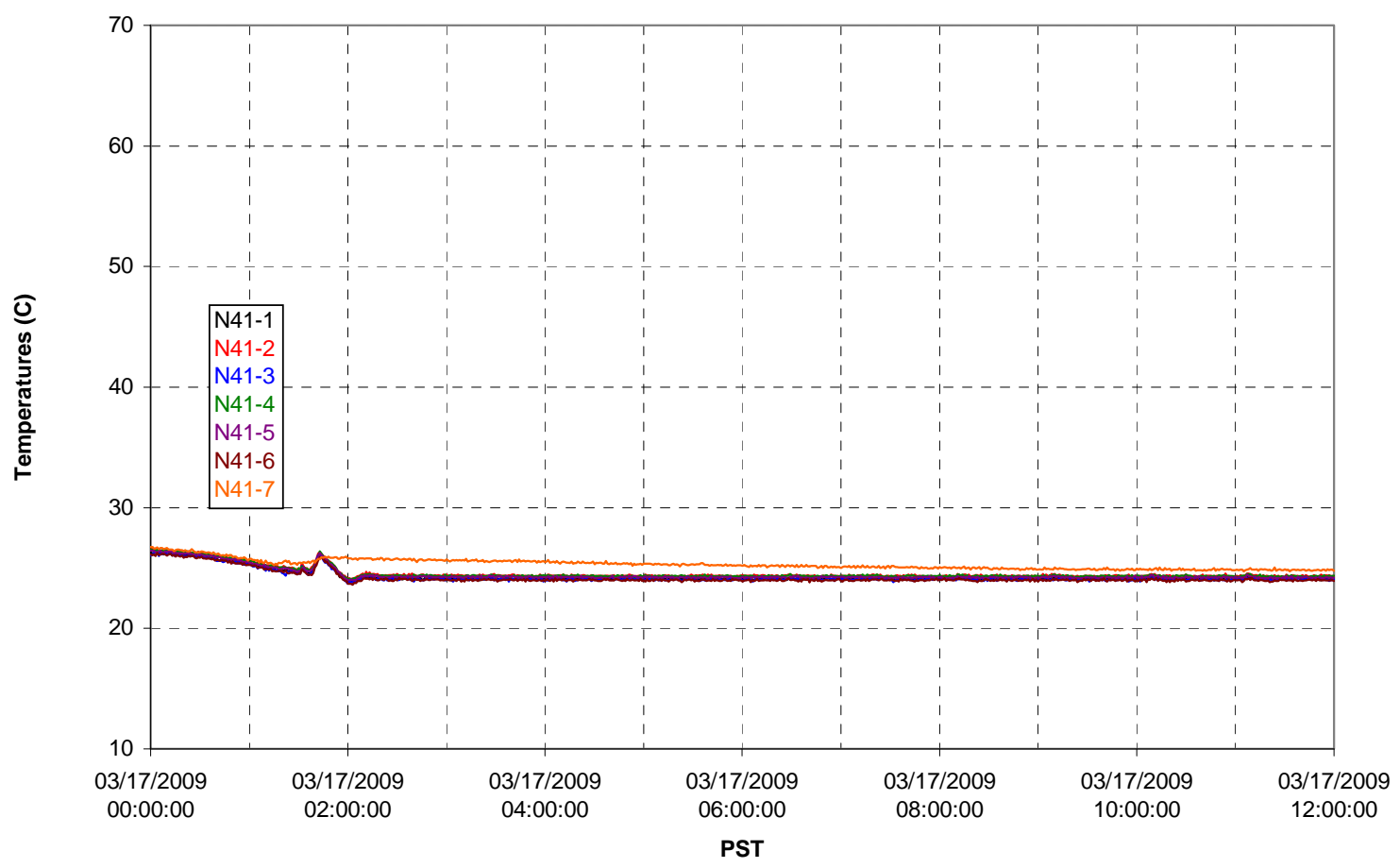


T02A temperatures

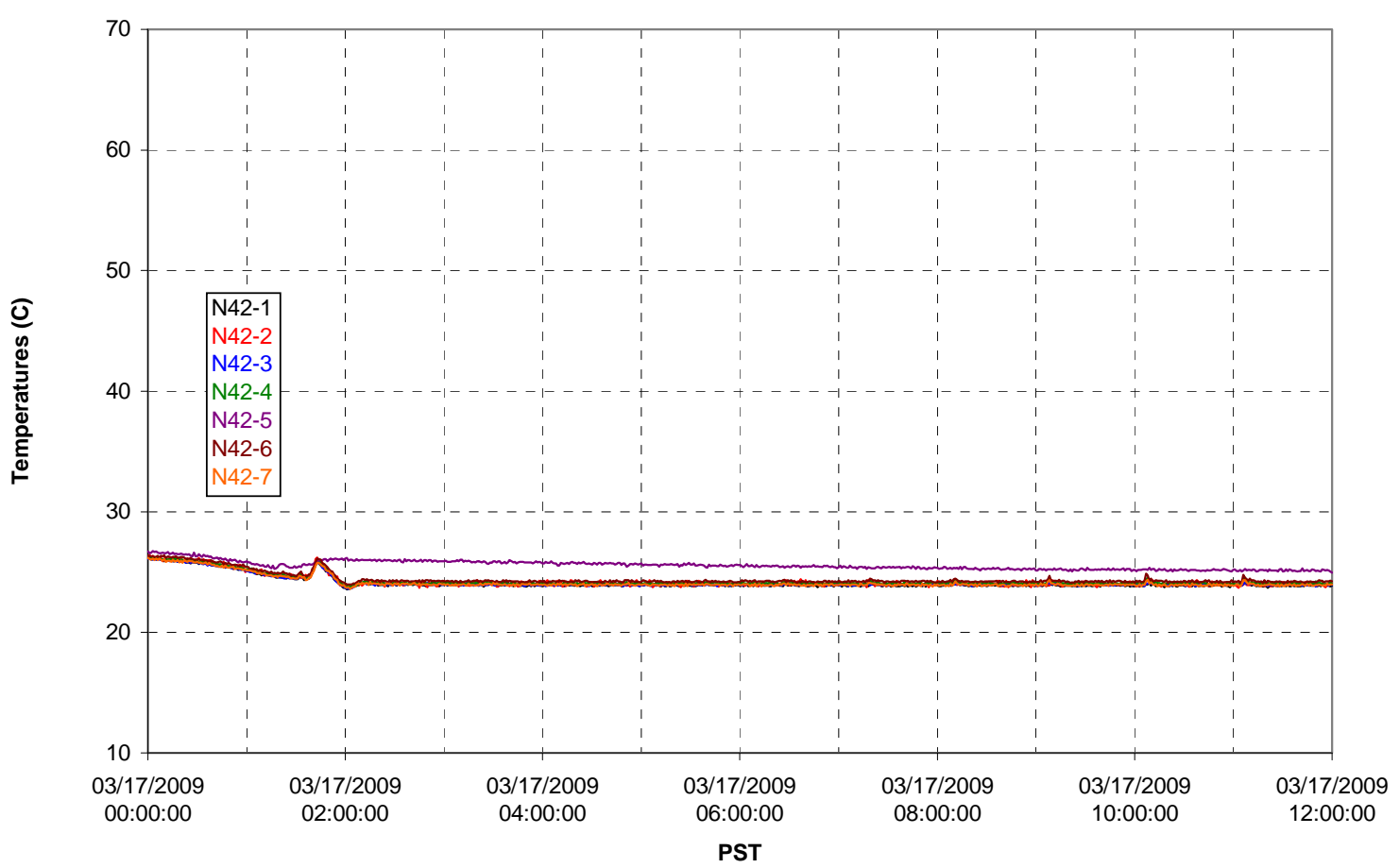

T02A temperatures

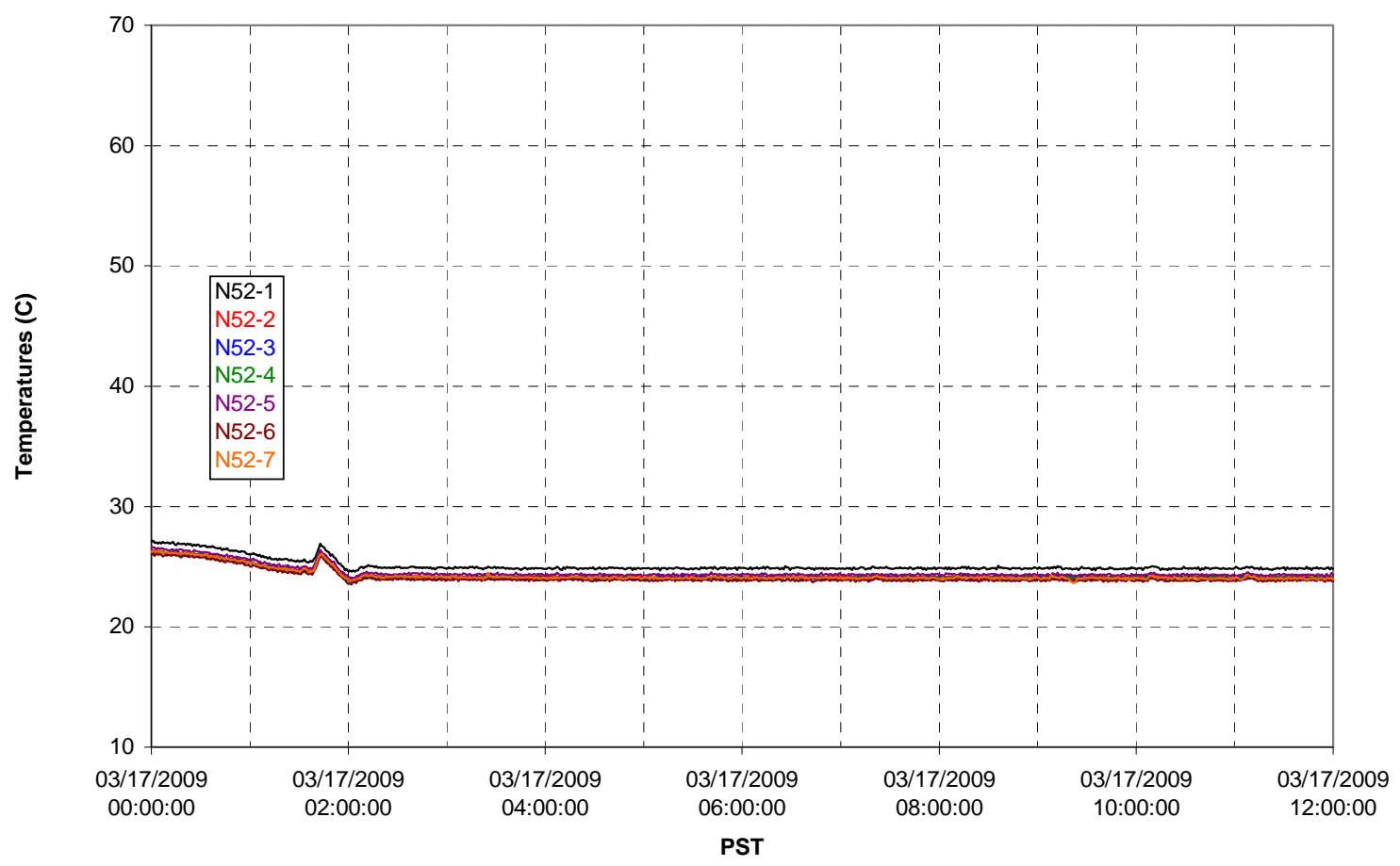


T02A Heating and Cooling

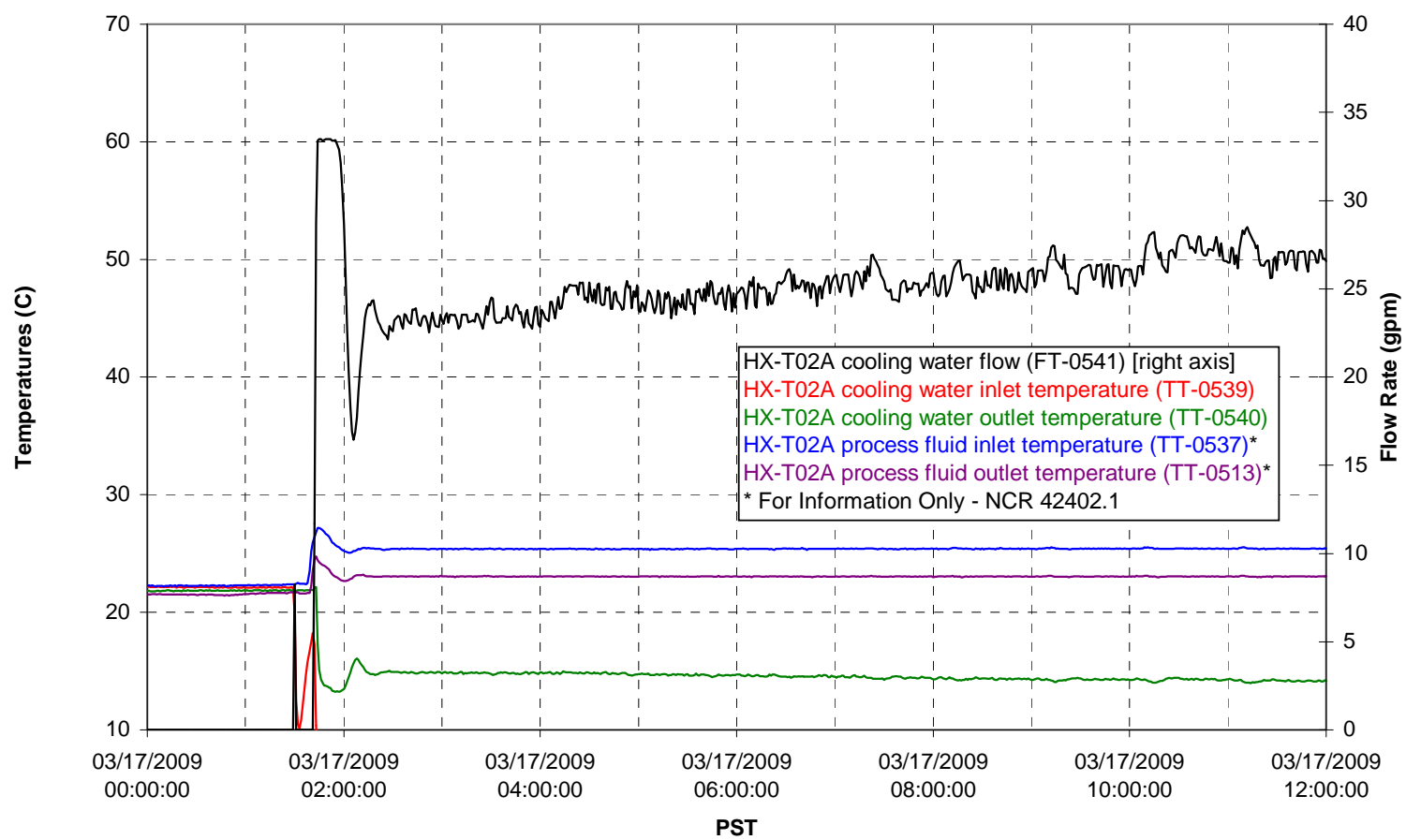

Pump Operation

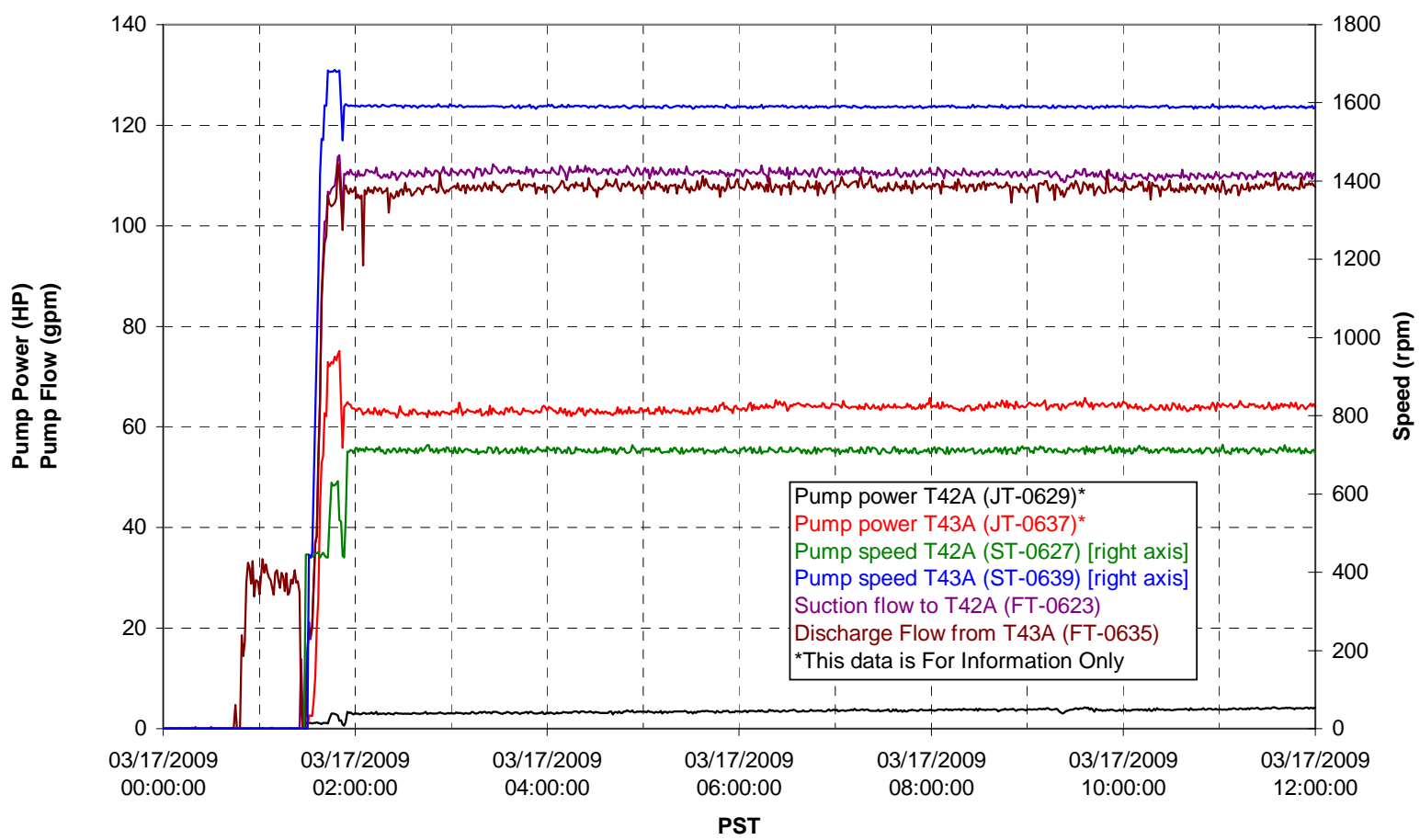


Pulsepot UFP-PP-T01A

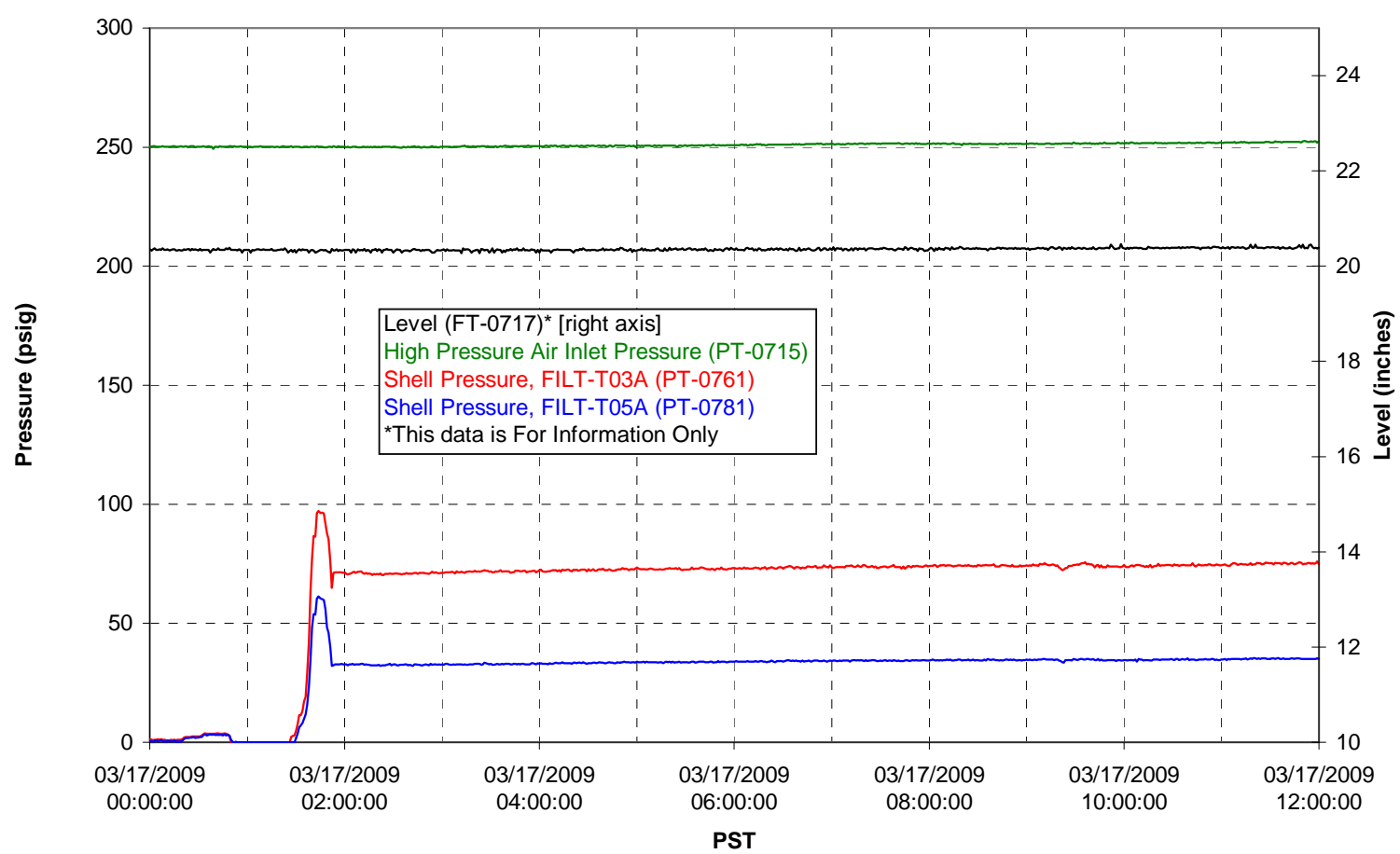

Pulsepot UFP-PP-T02A

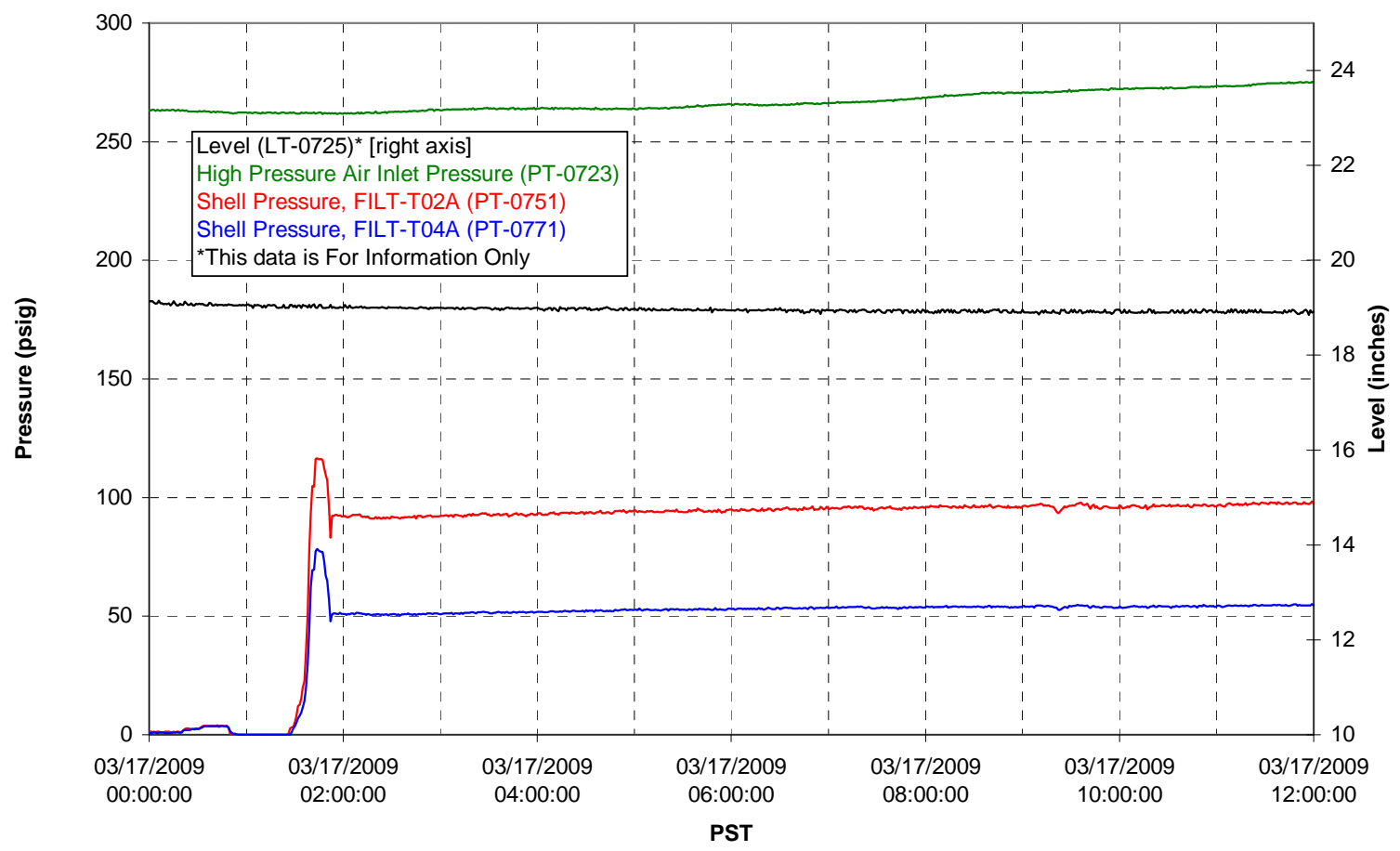


Pulsepot UFP-PP-T03A

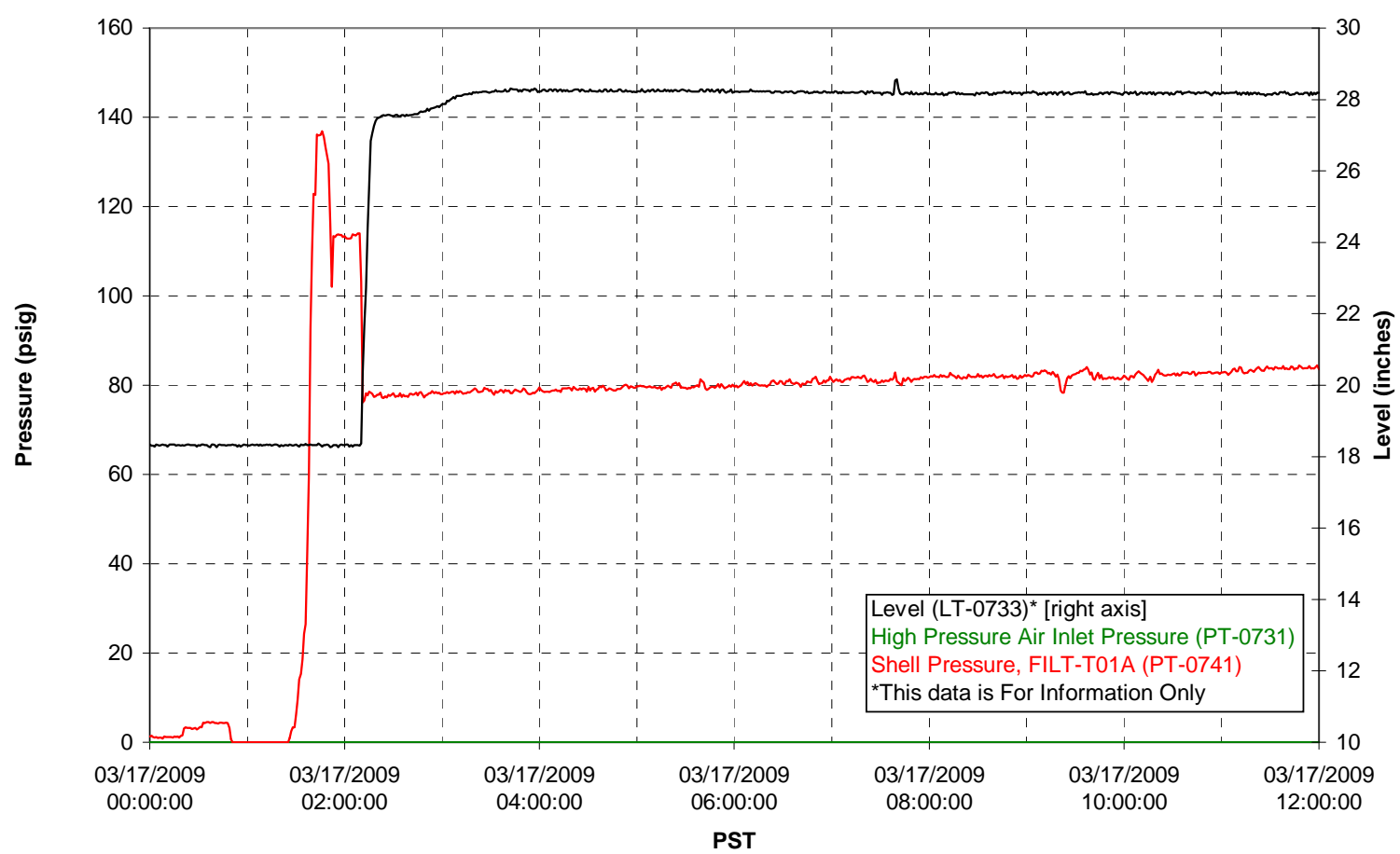

Pulsepot Levels

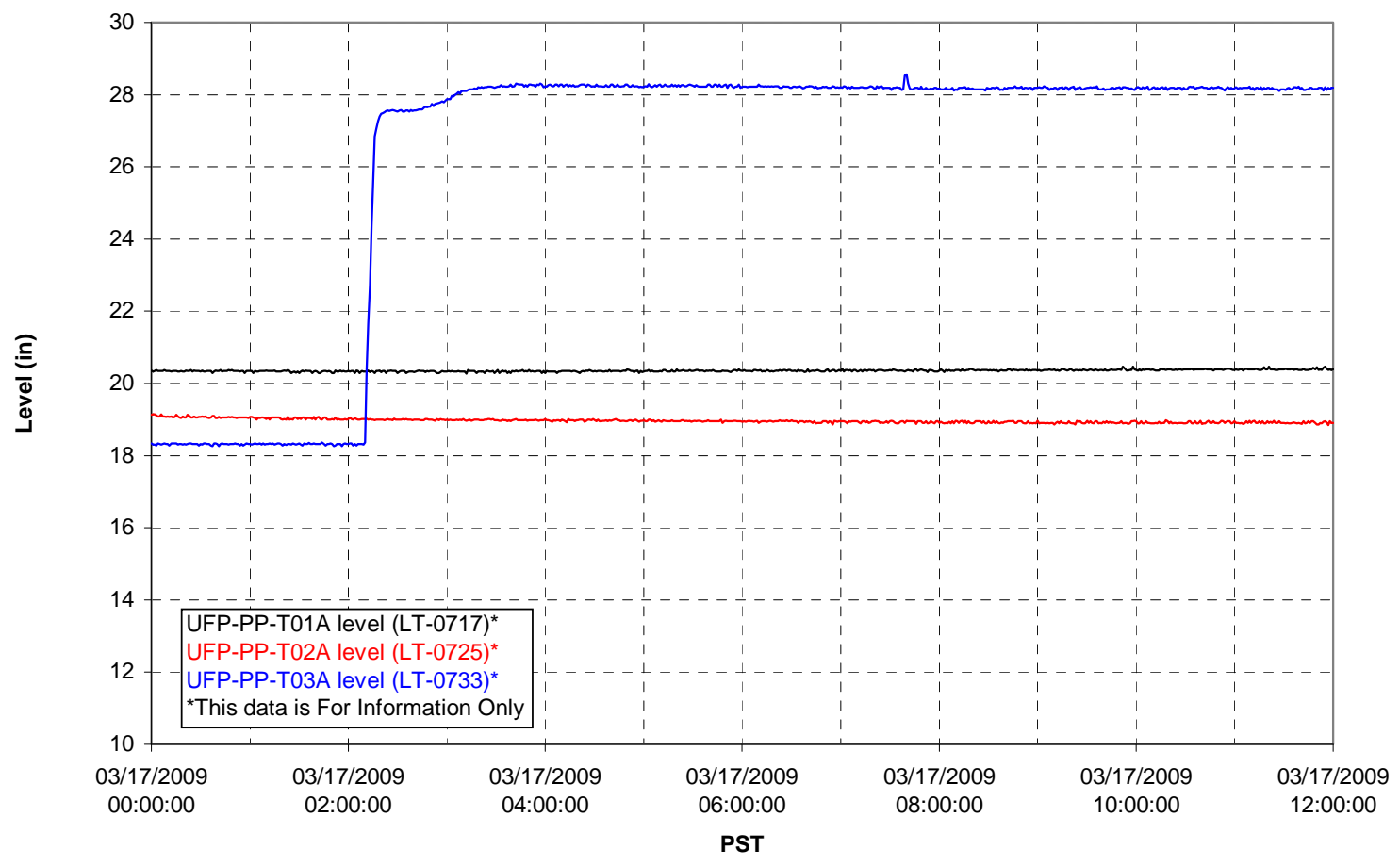


Filter UFP-FILT-T01A

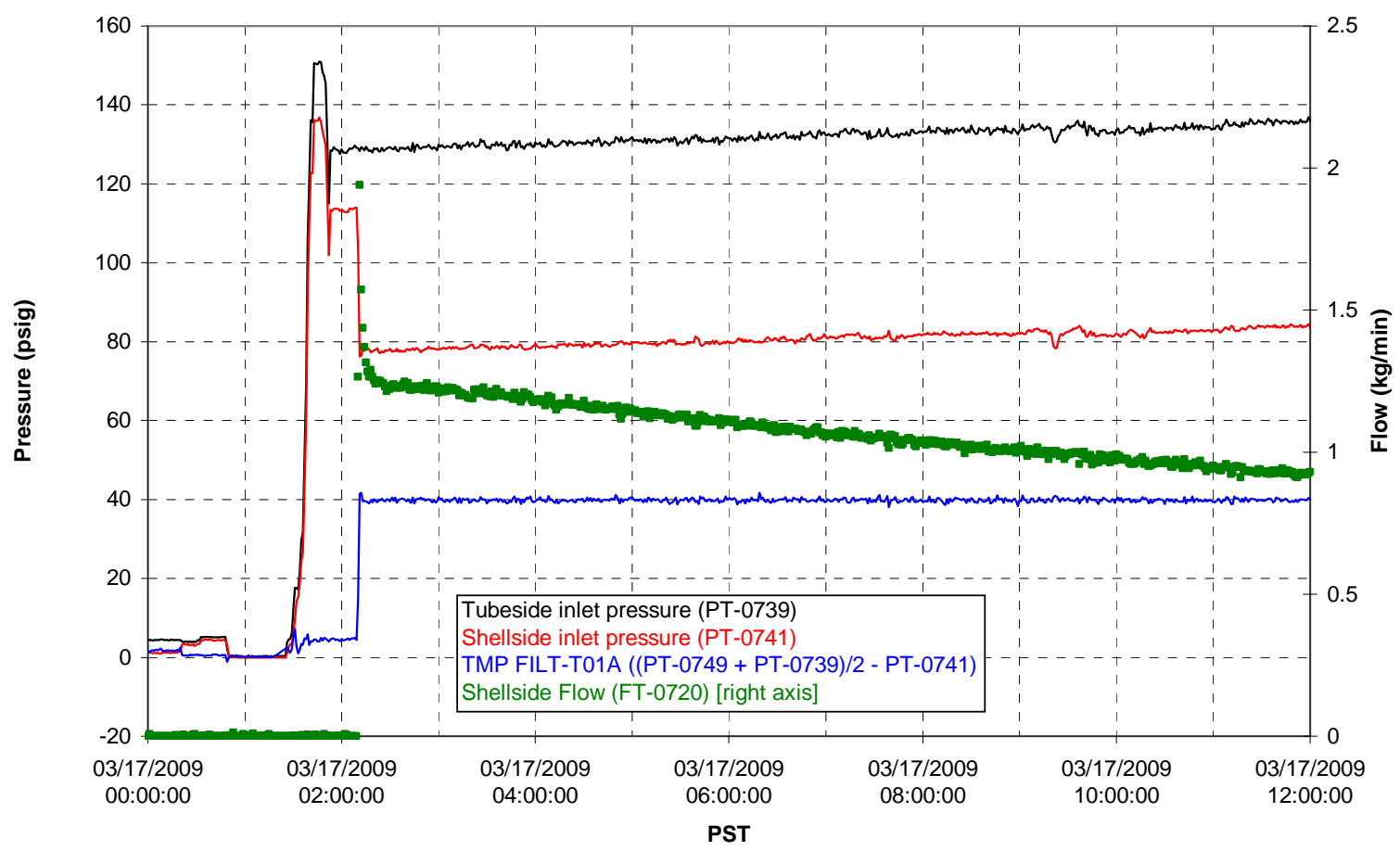

Filter UFP-FILT-T02A

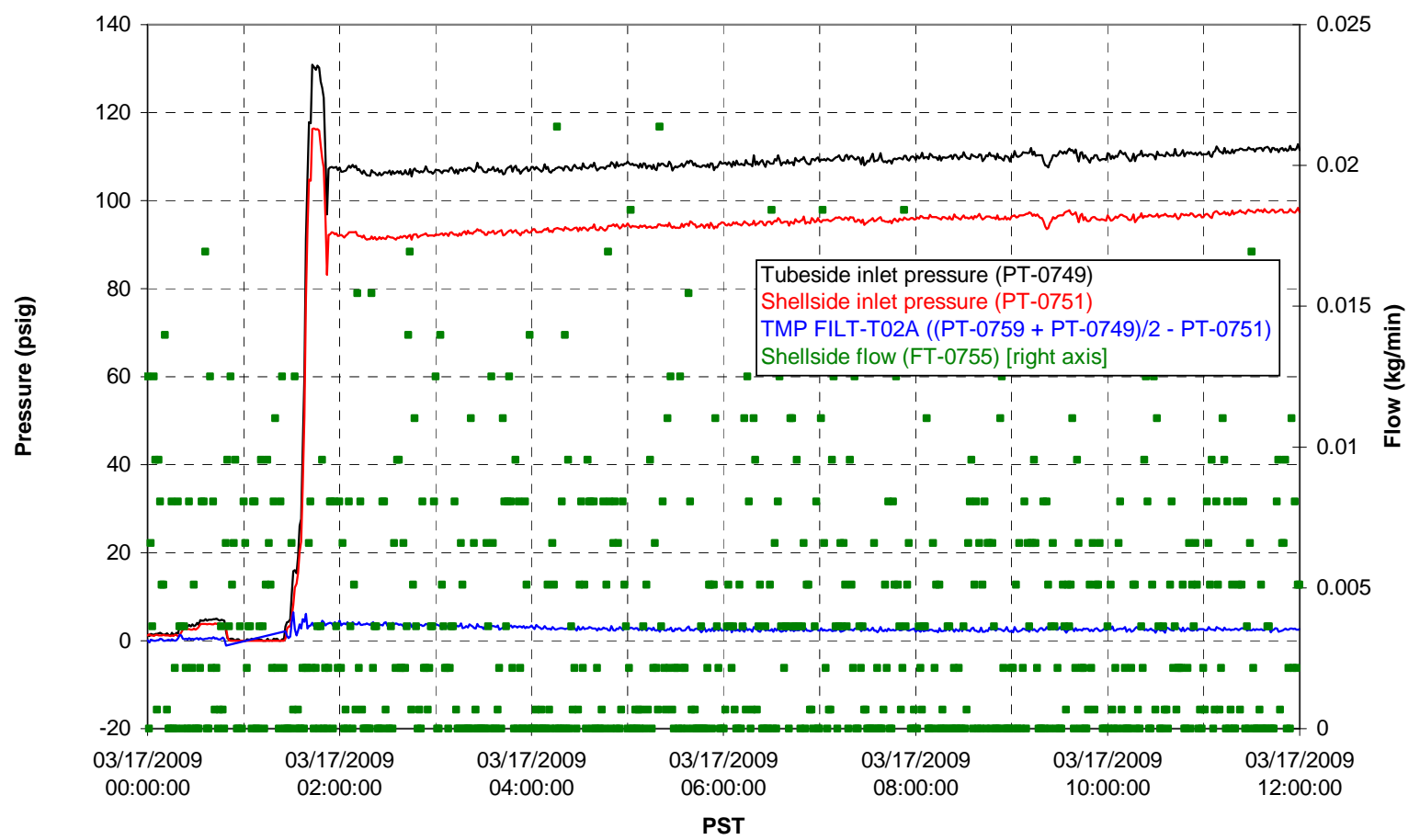


Filter UFP-FILT-T03A

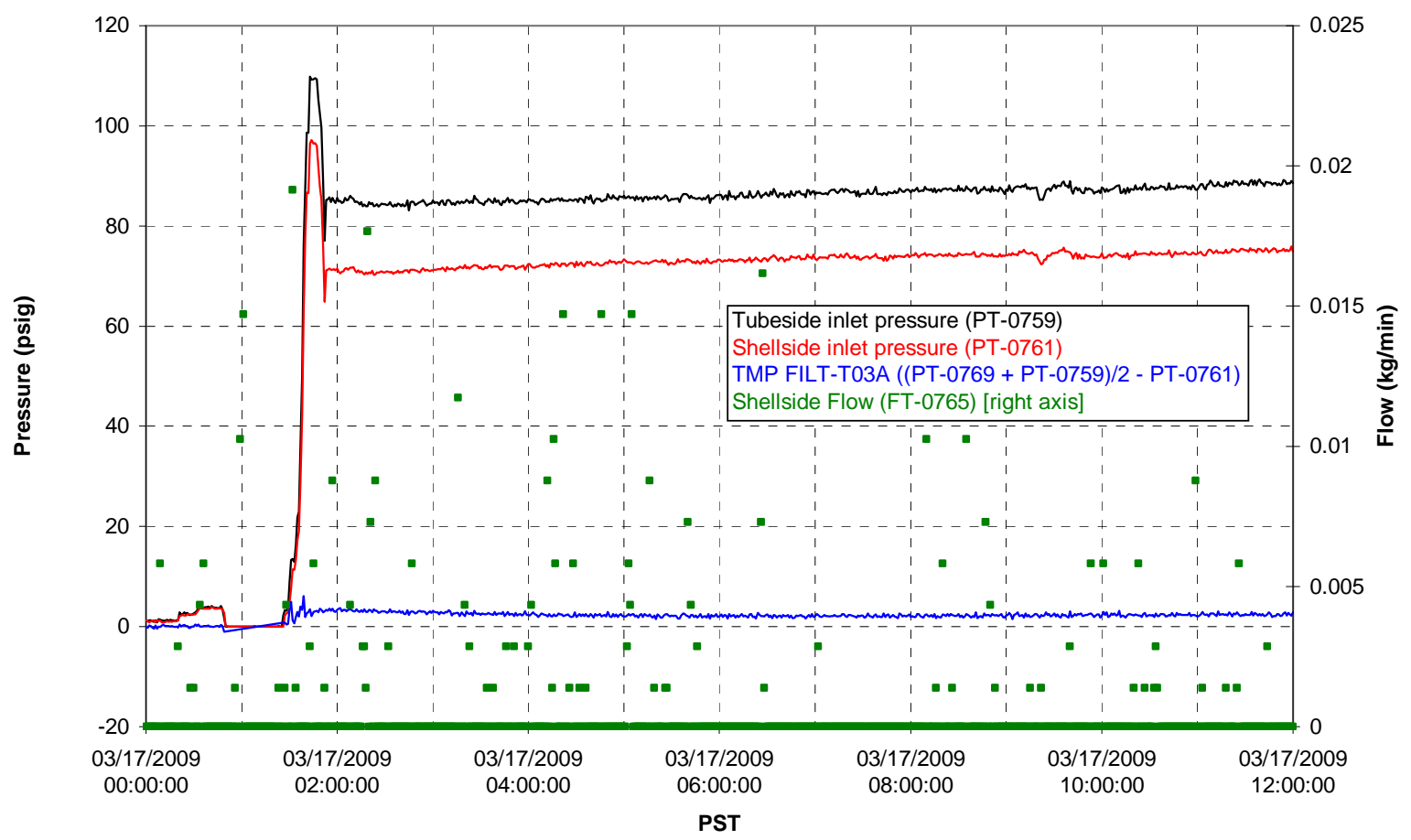

Filter UFP-FILT-T04A

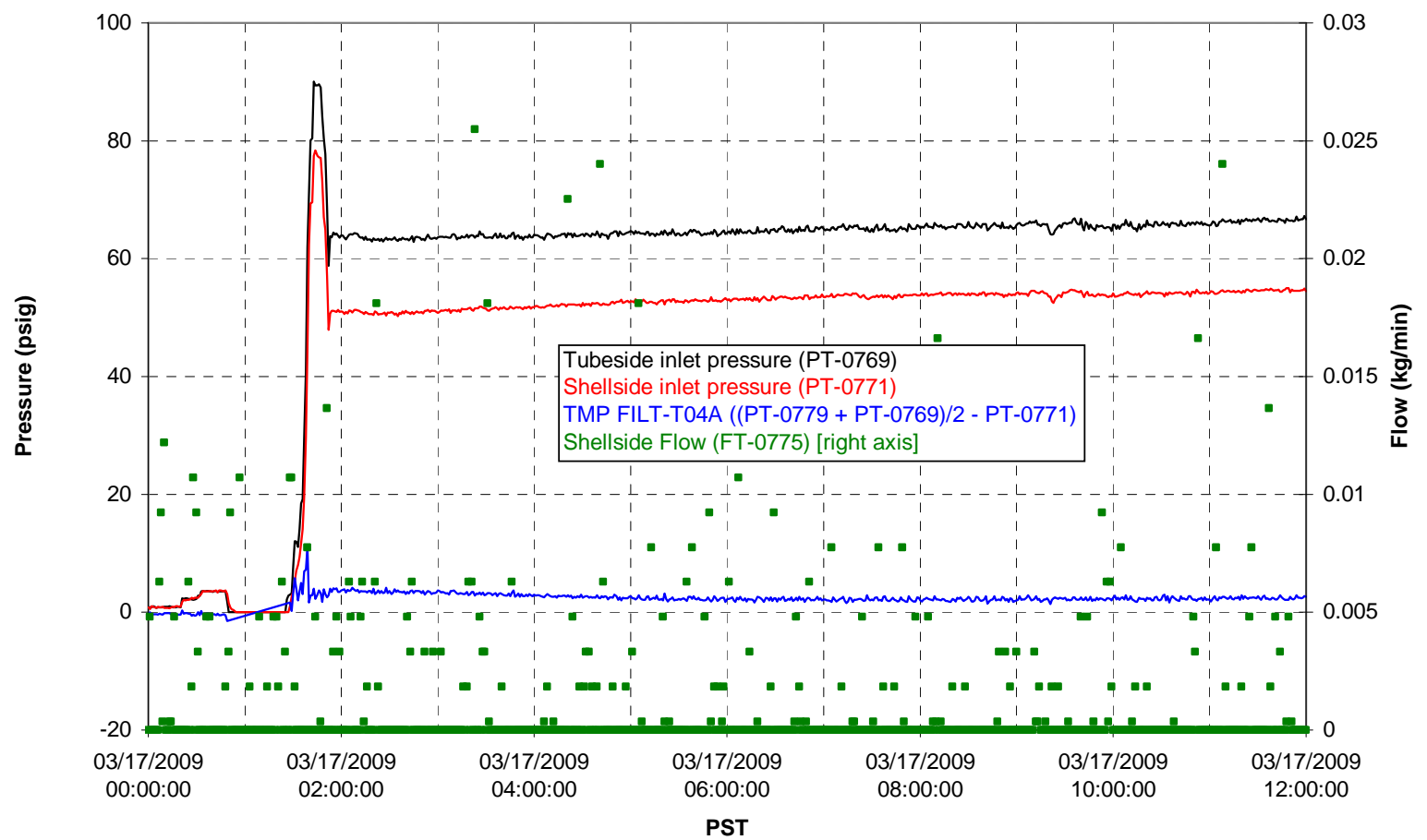


Filter UFP-FILT-T05A

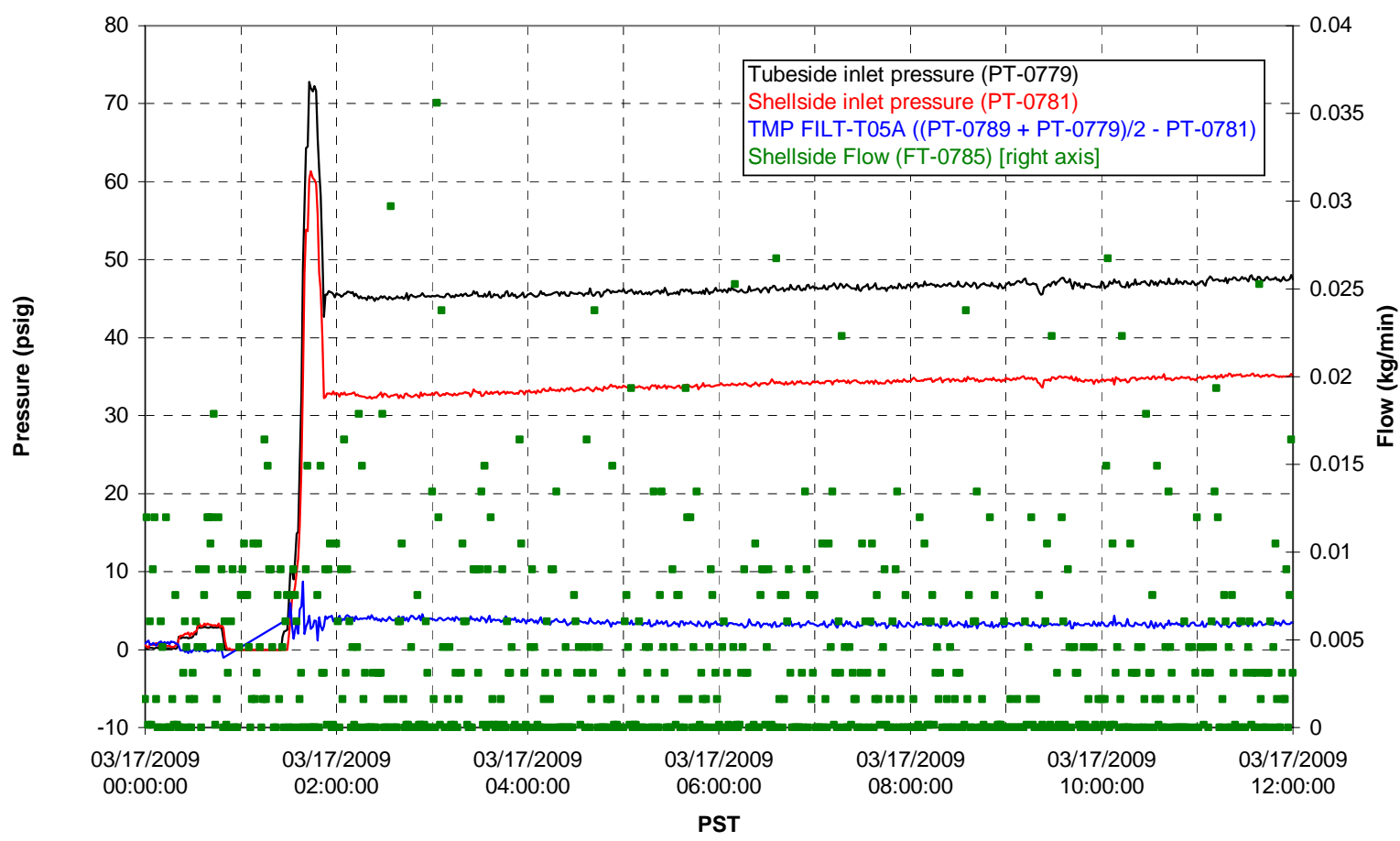

Chemical Flow

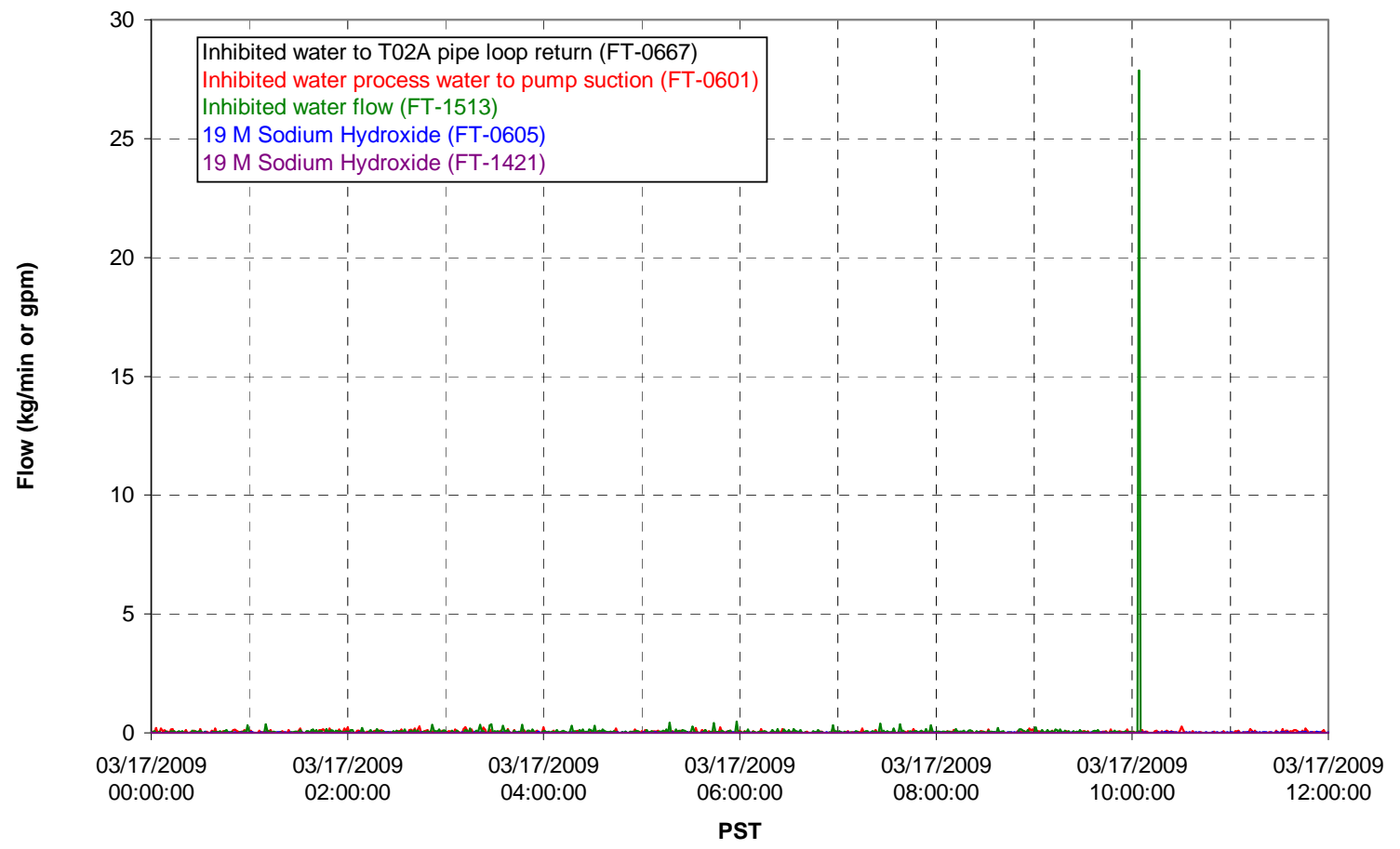




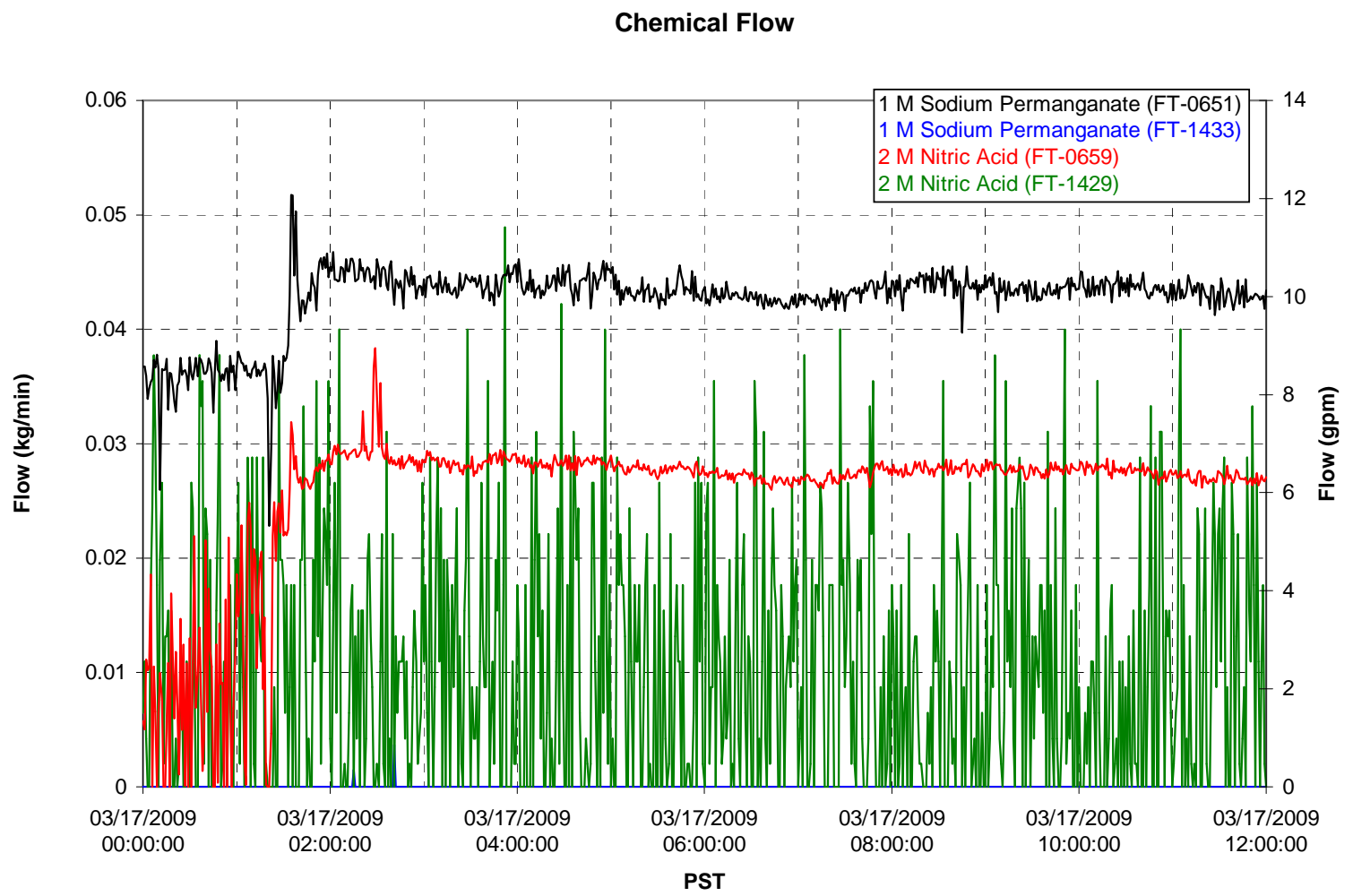

Air Flows

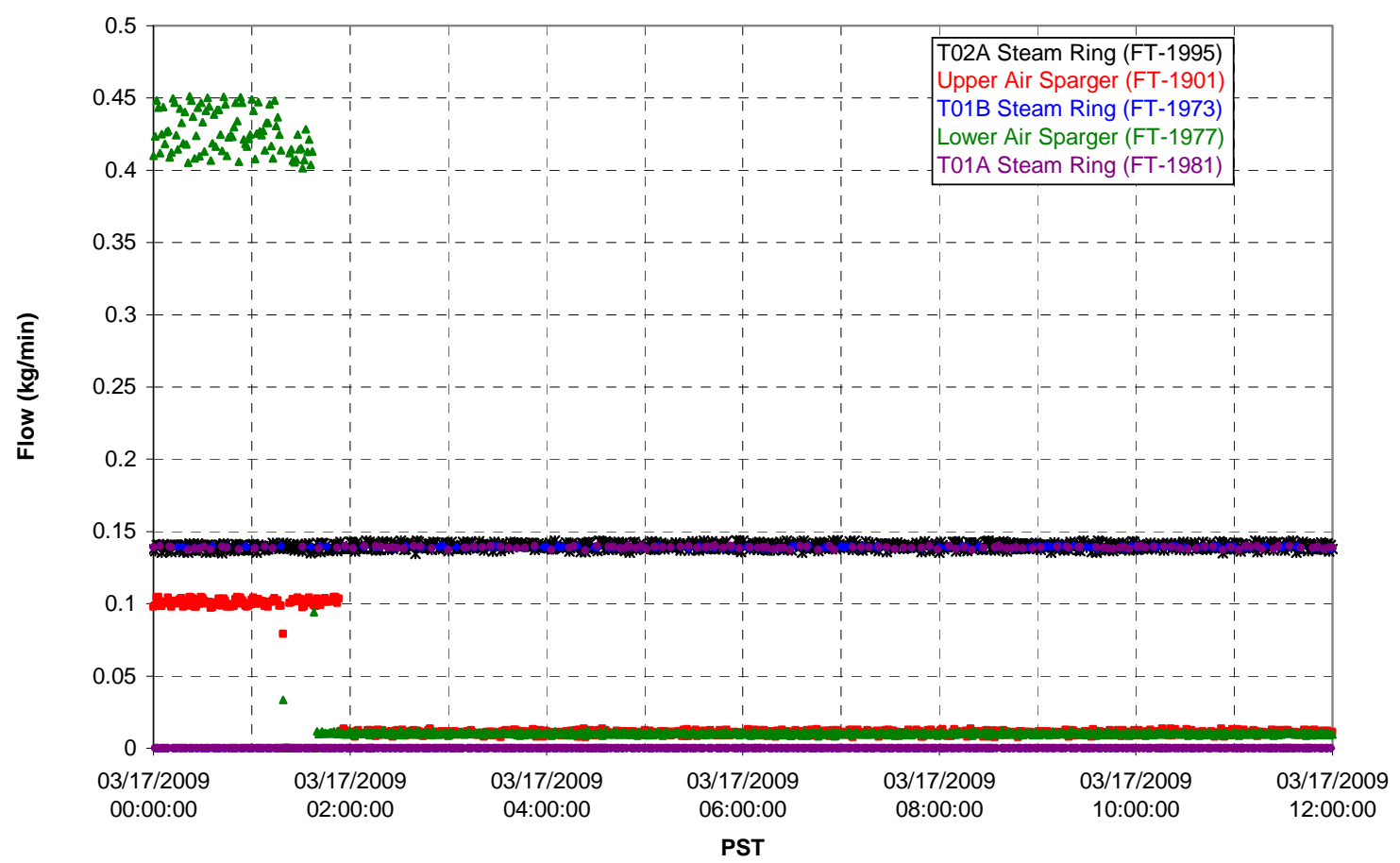


T02A Steam

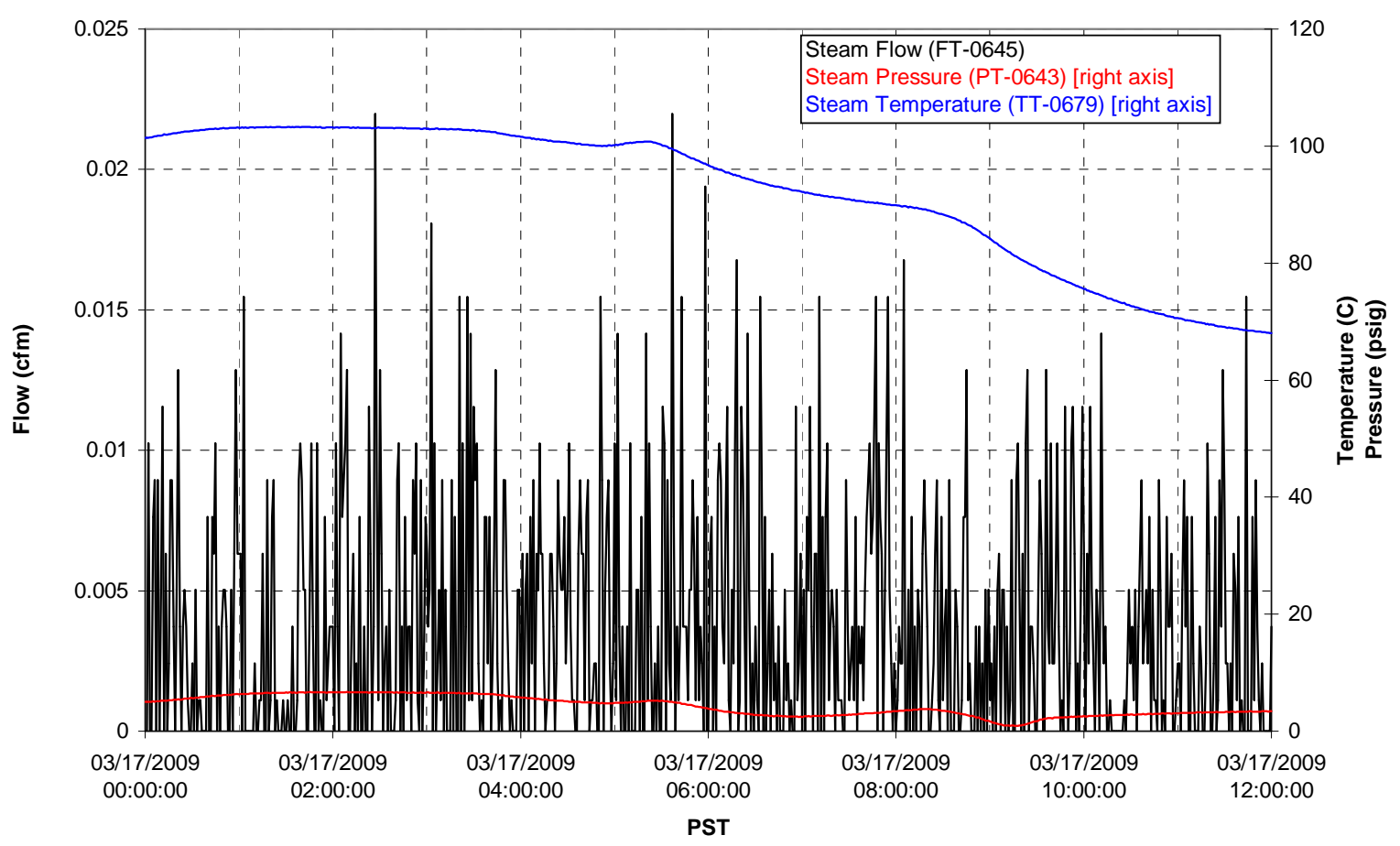

T01A Steam

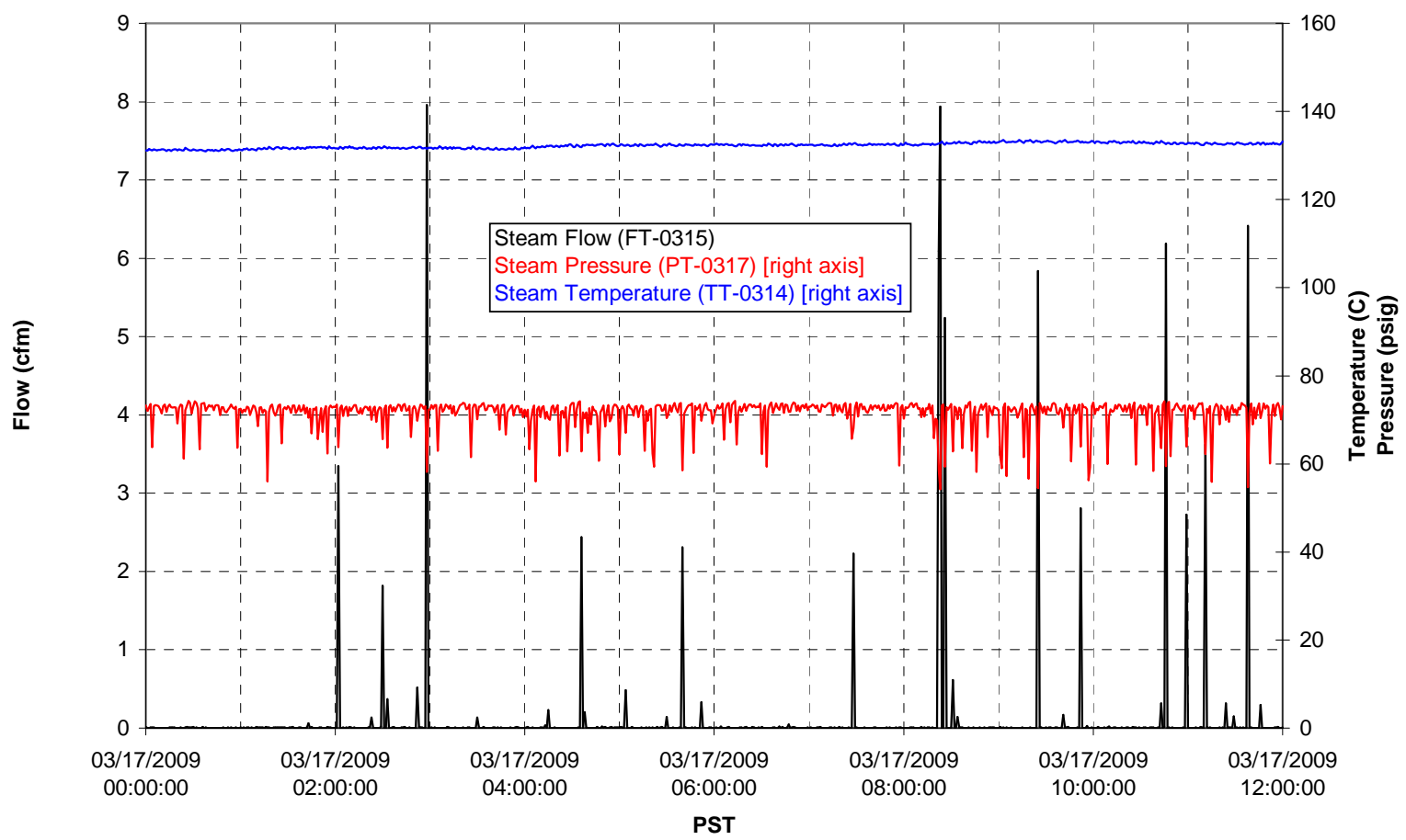


T01B Steam

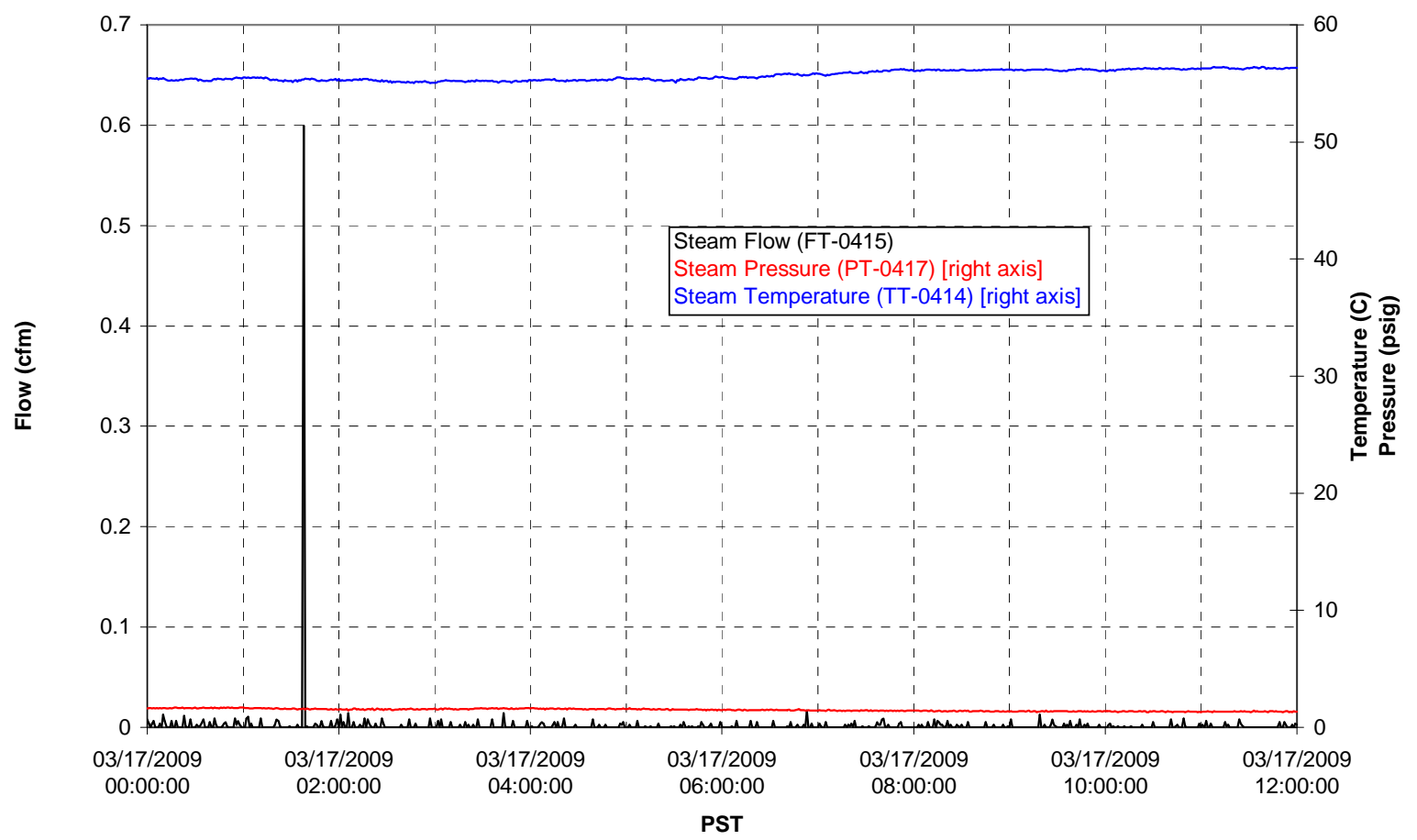




\section{Integrated Test B Data Plots \\ 03/17/09 12:00 - 03/18/09 00:00}


T01A level

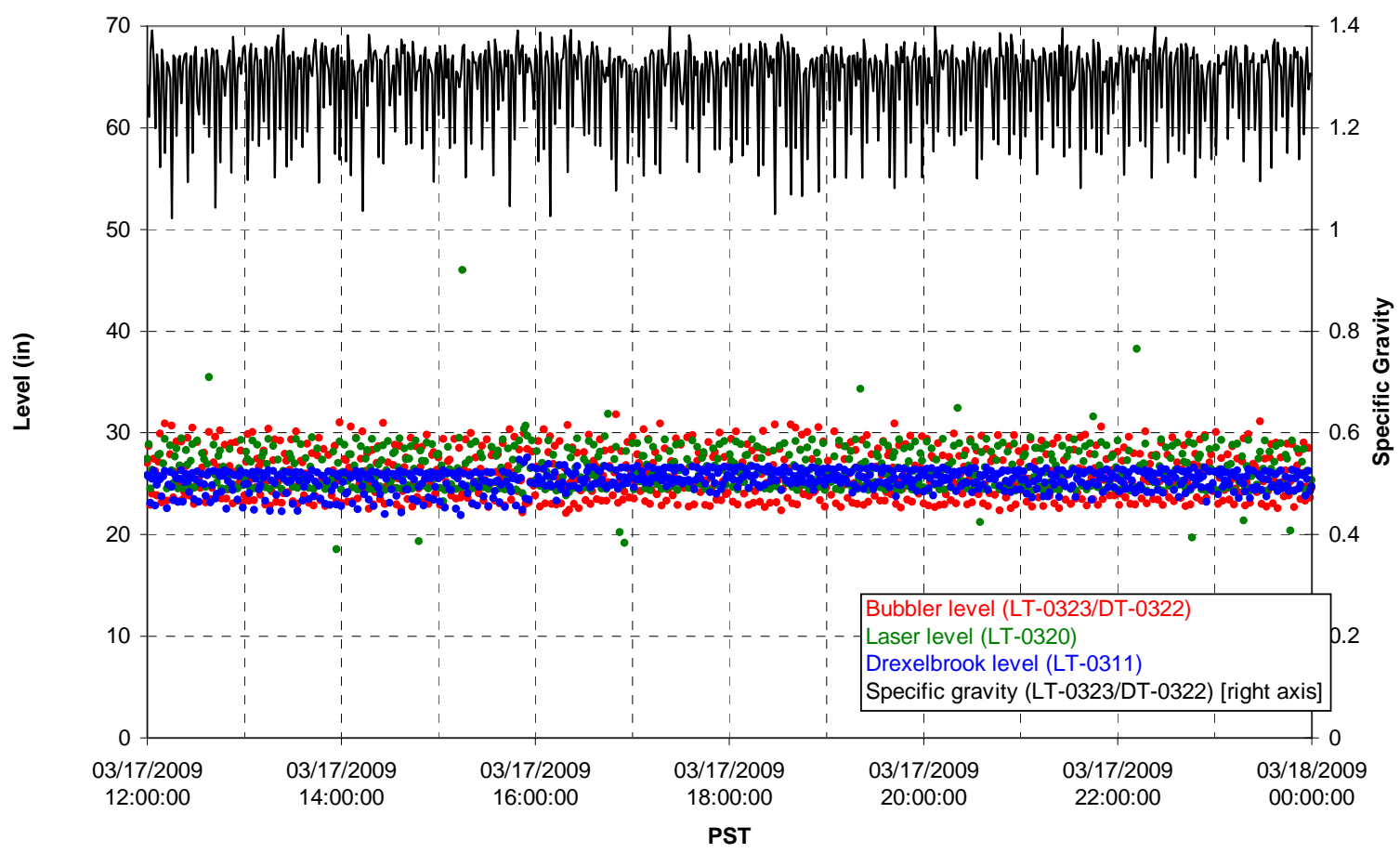

T01A temperatures

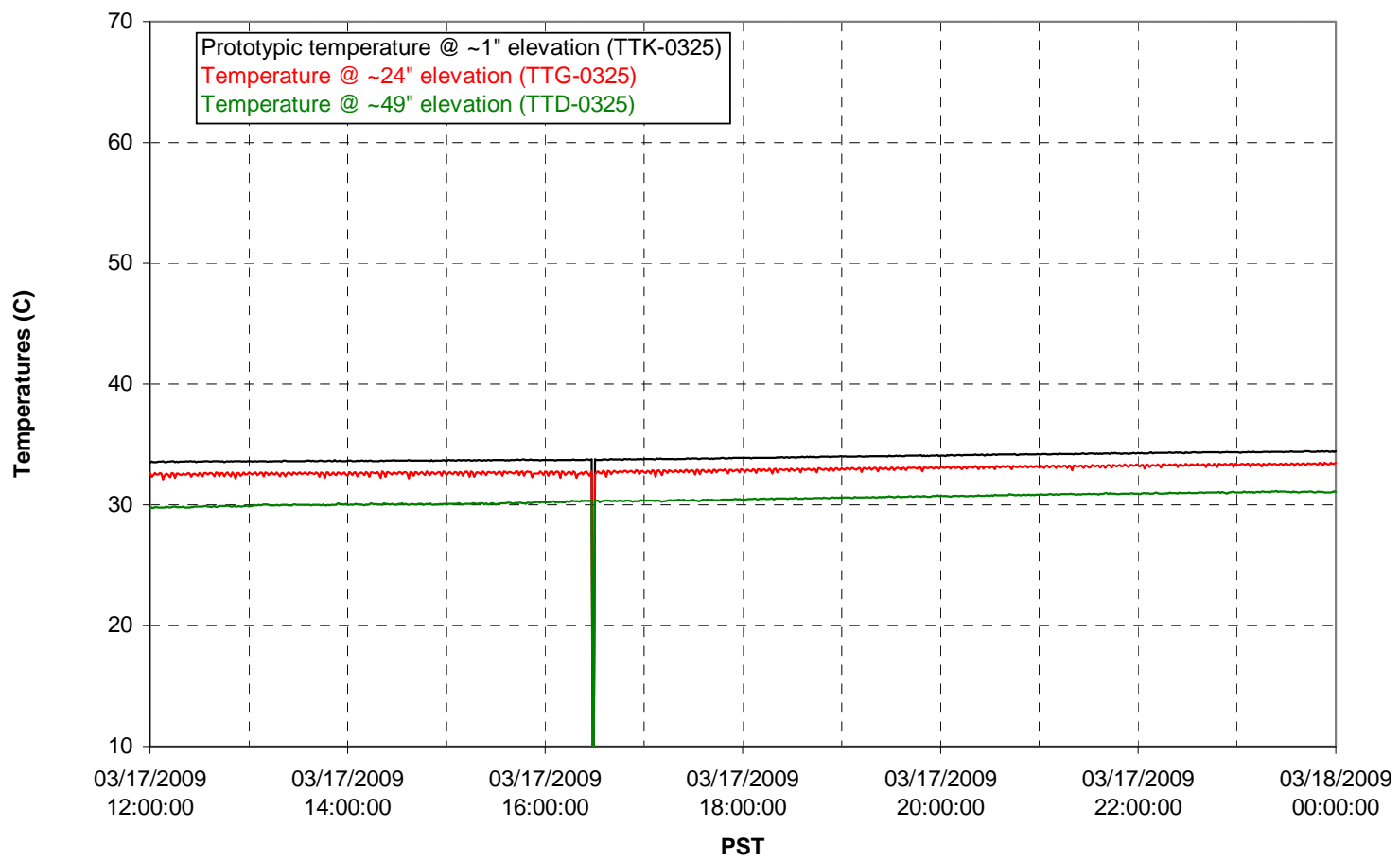


T01B level

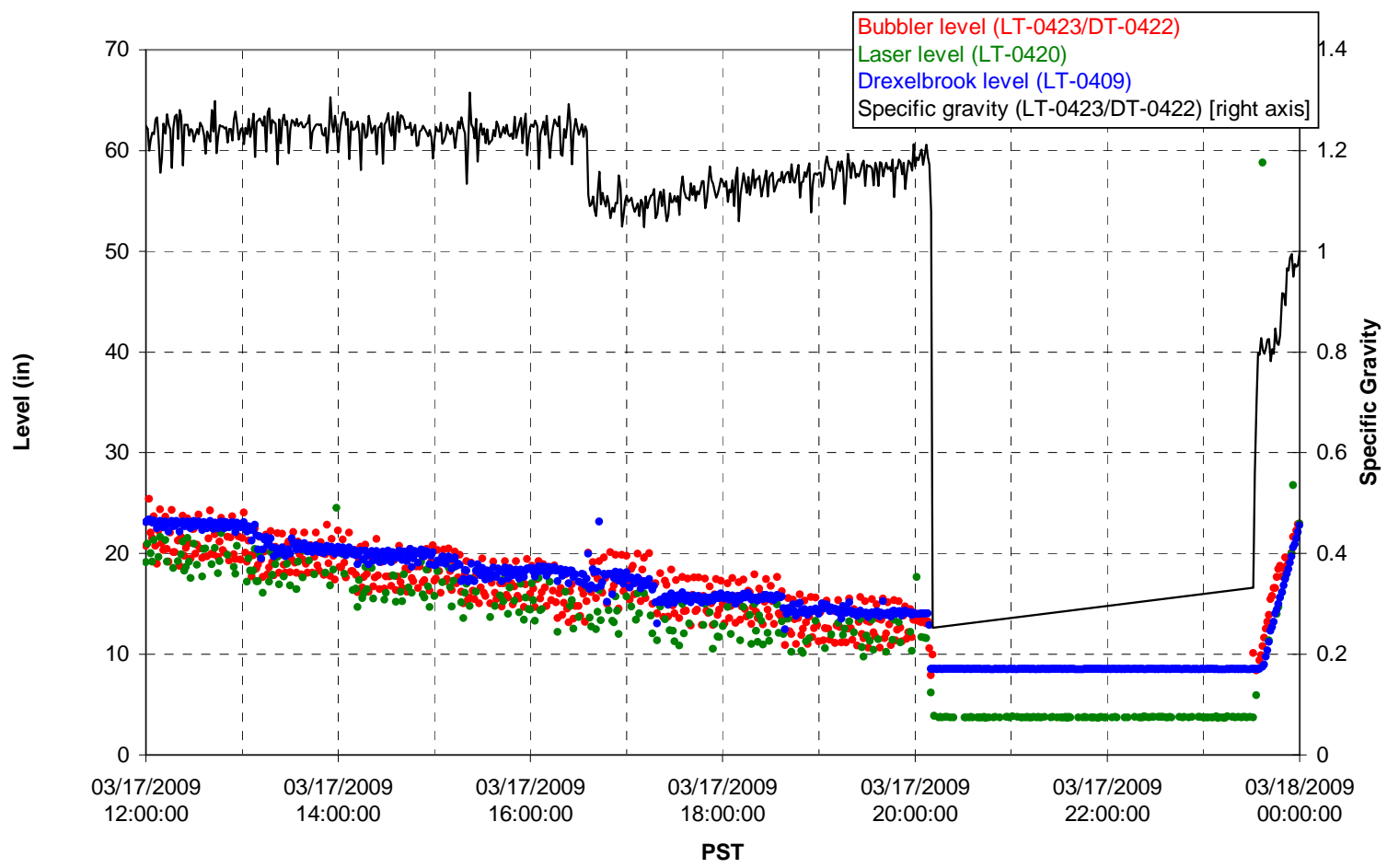

T01B temperatures

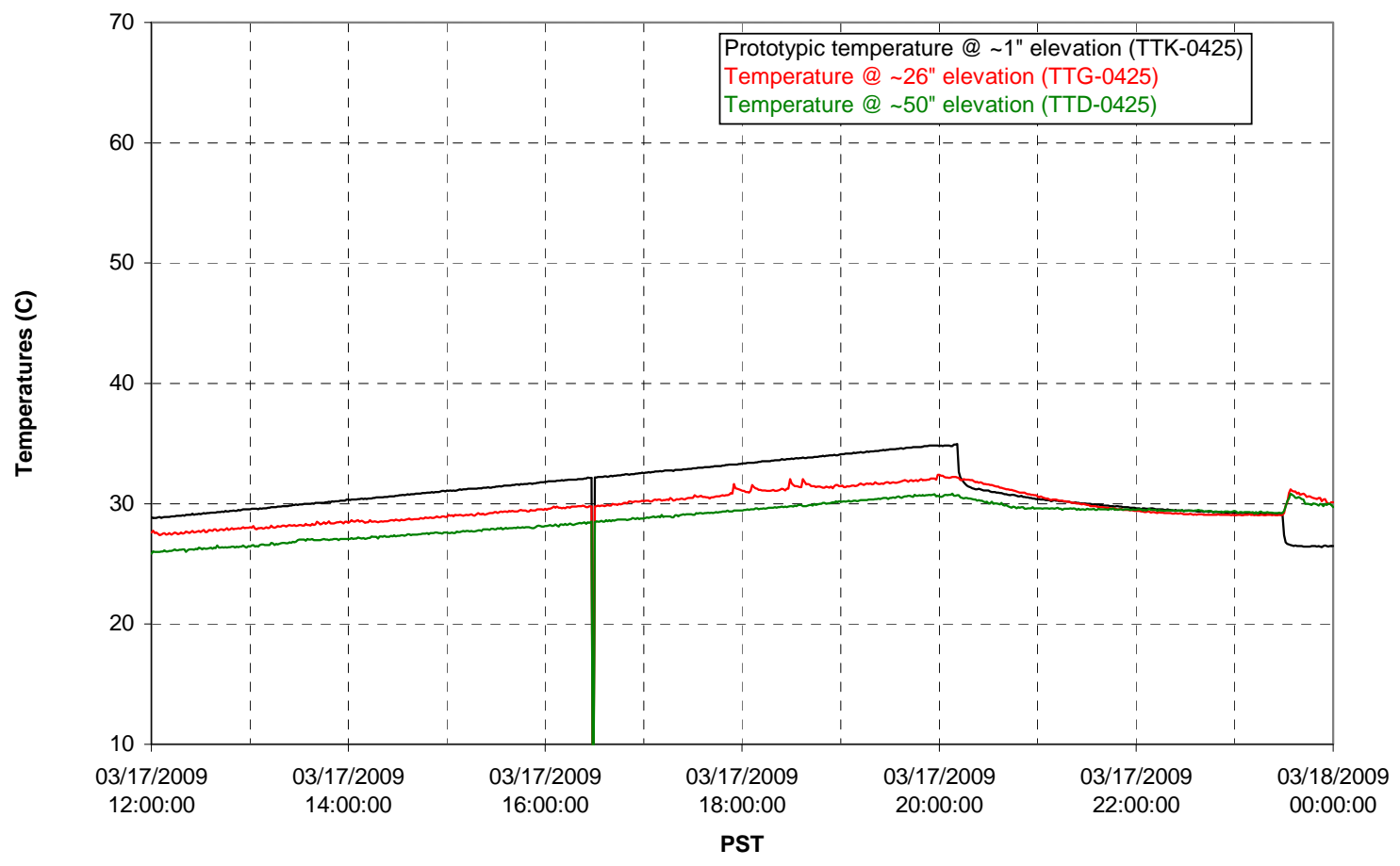


T02A level

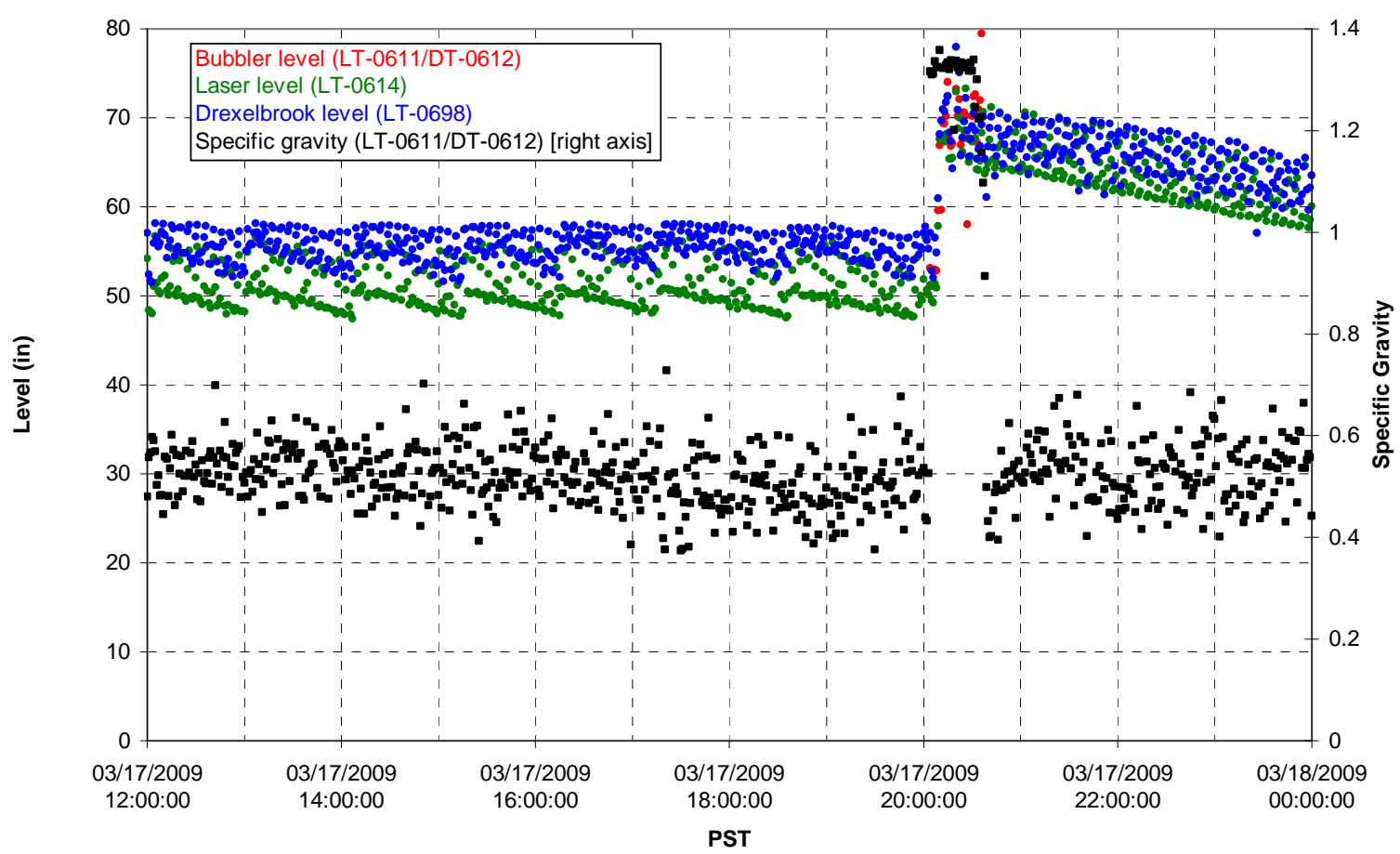

T02A temperatures

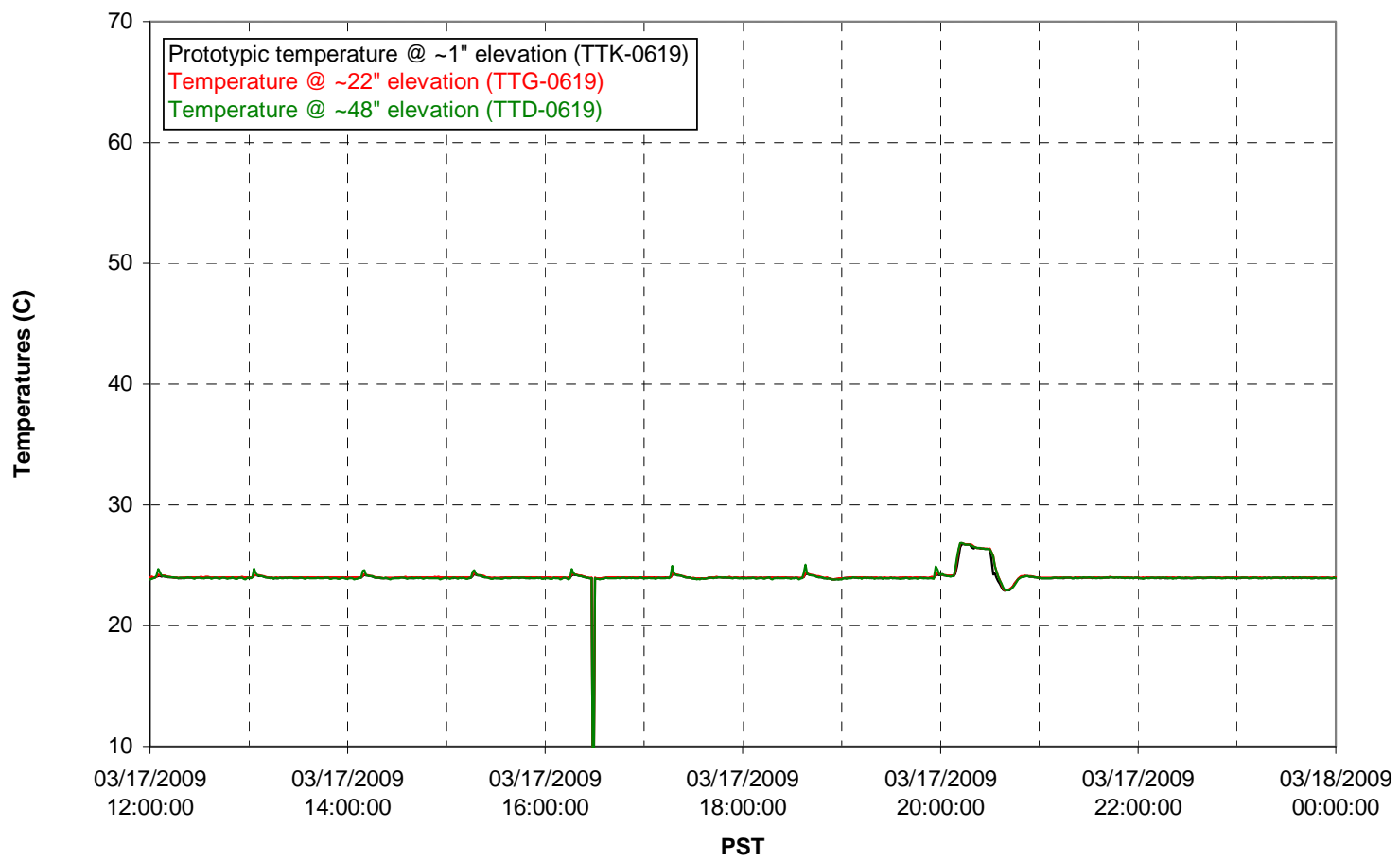


T02A and filter loop temperatures

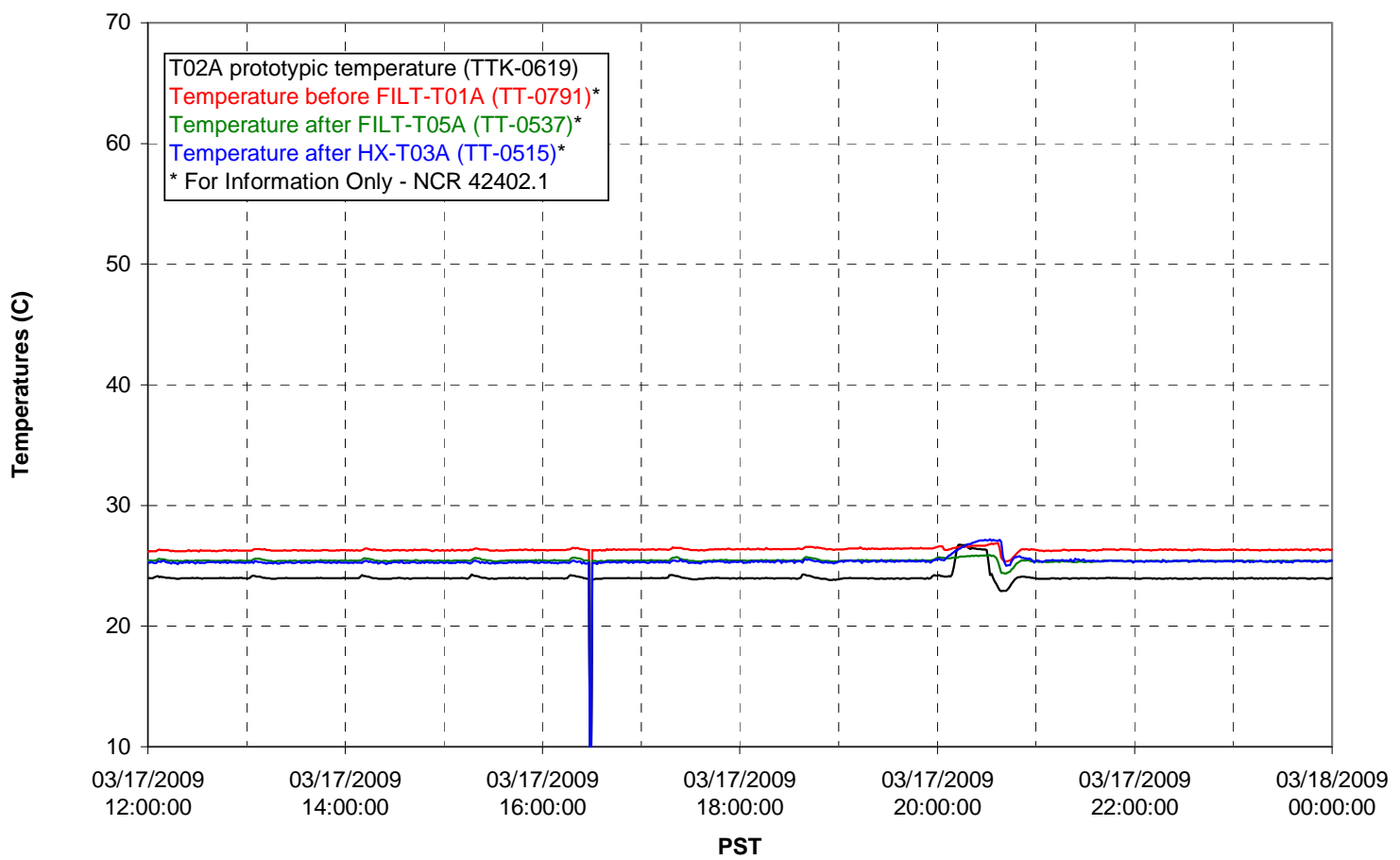

Pump Pressures and Flow

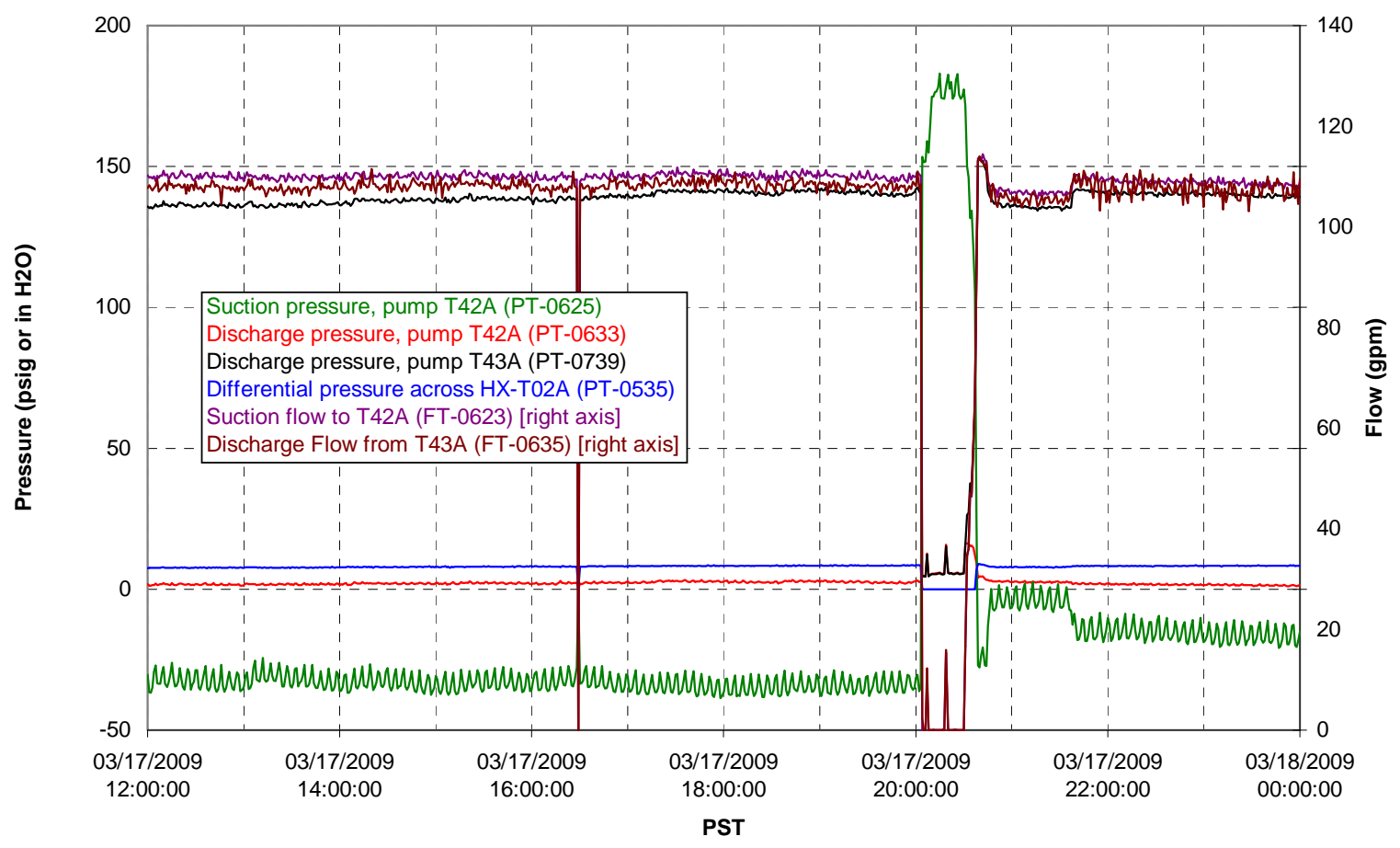


Axial pressure drop

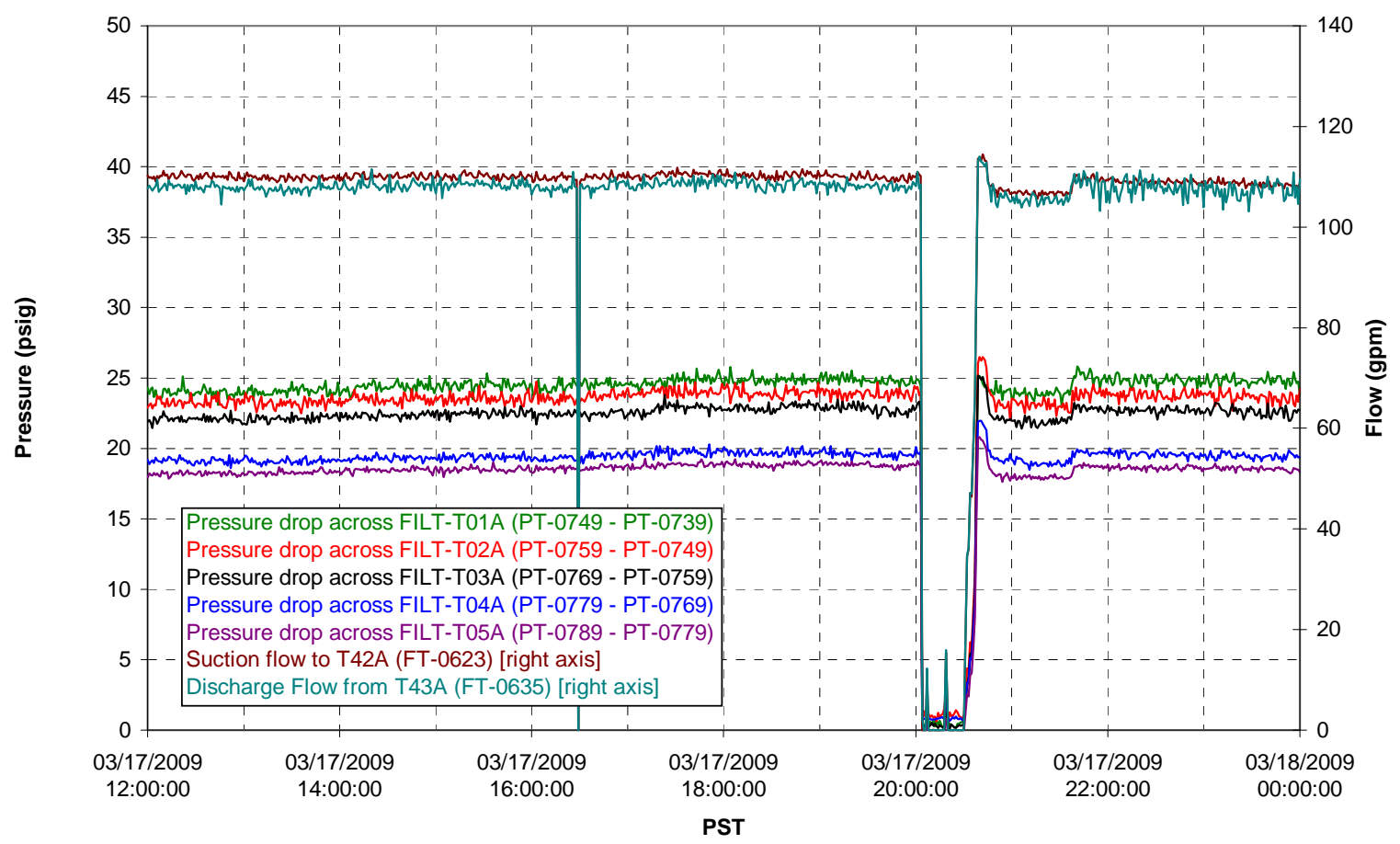

Permeate flow rates

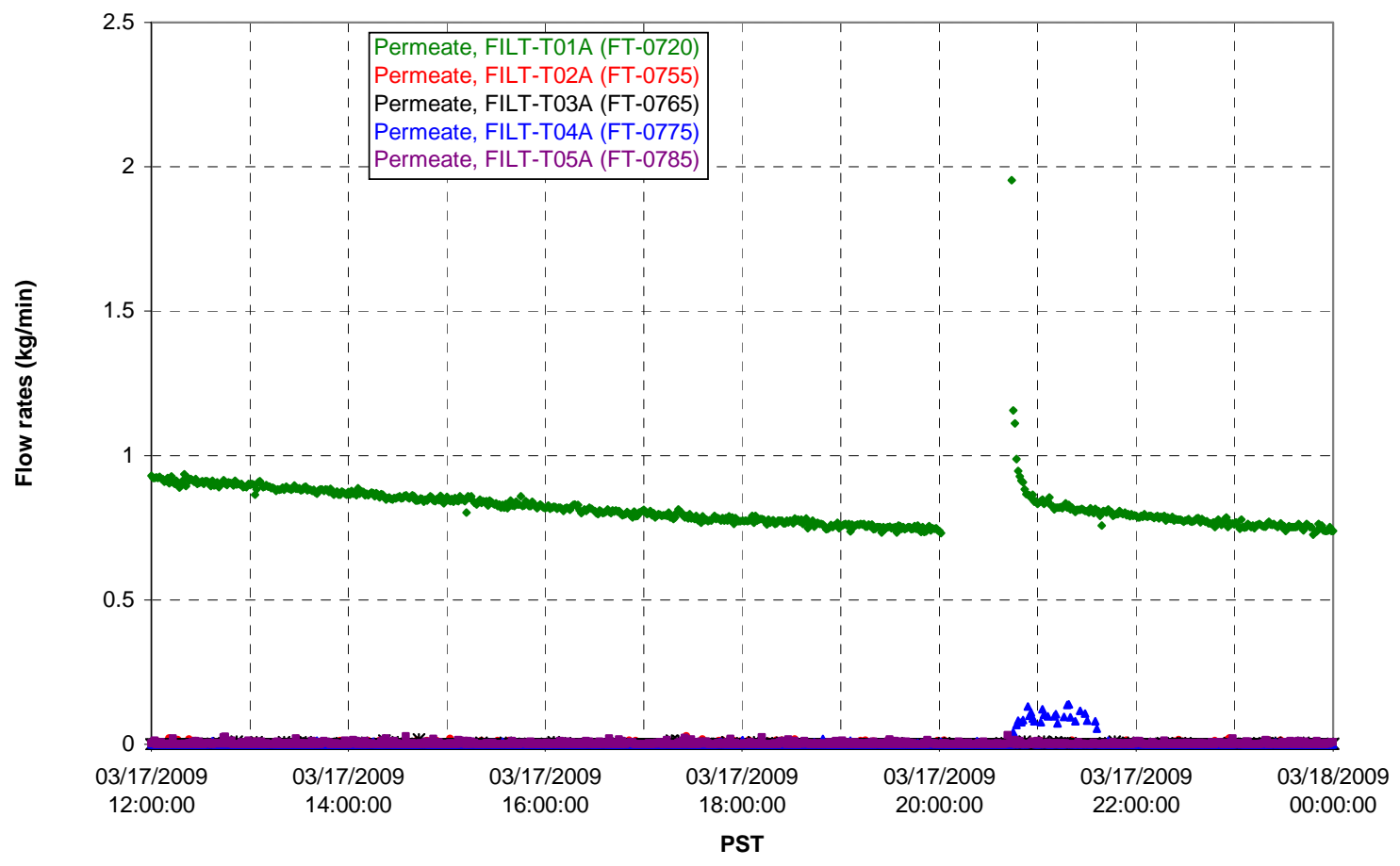


T02A Inner Temperature Tree

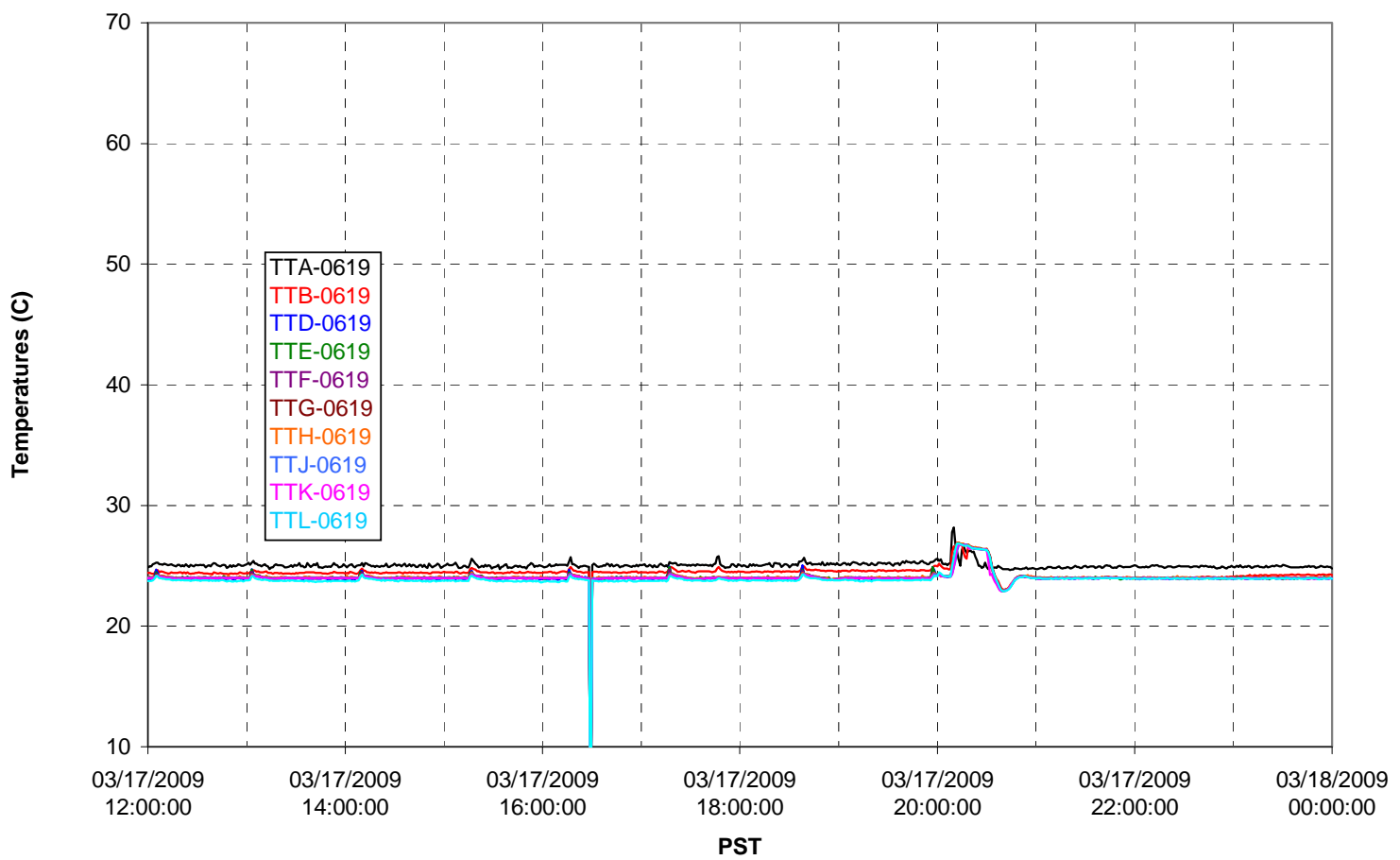

T02A Outer Temperature Tree

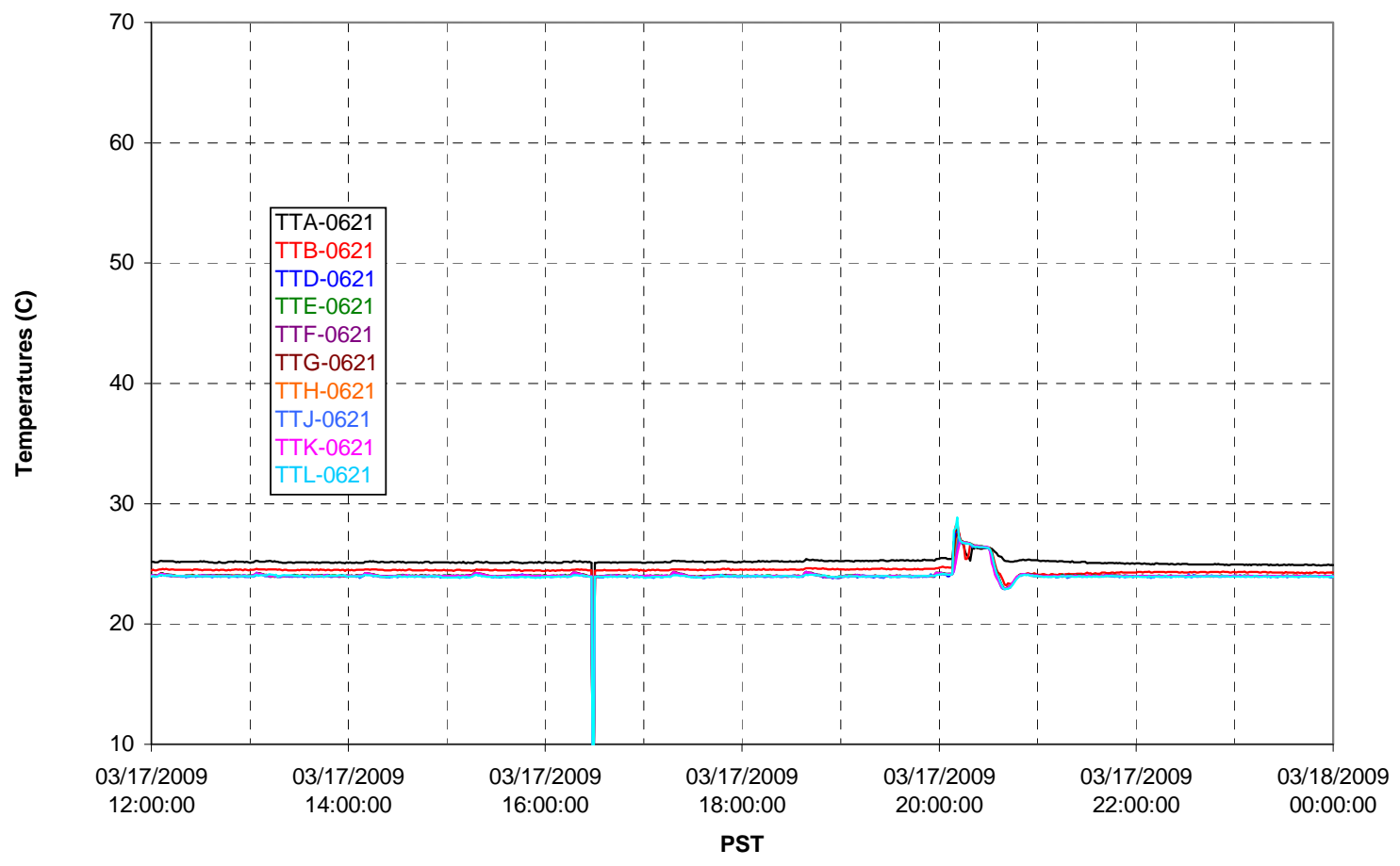


T02A temperatures

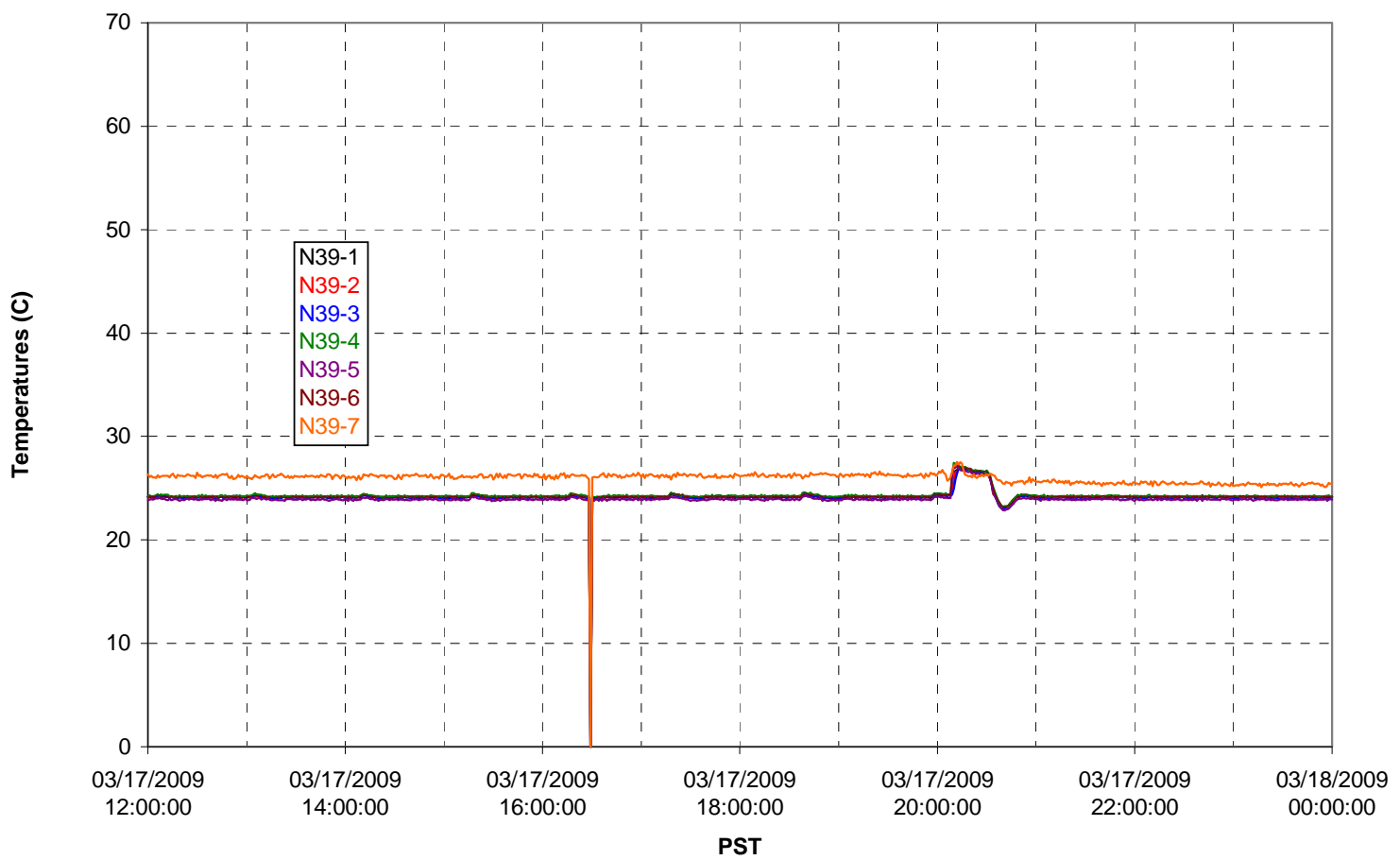

T02A temperatures

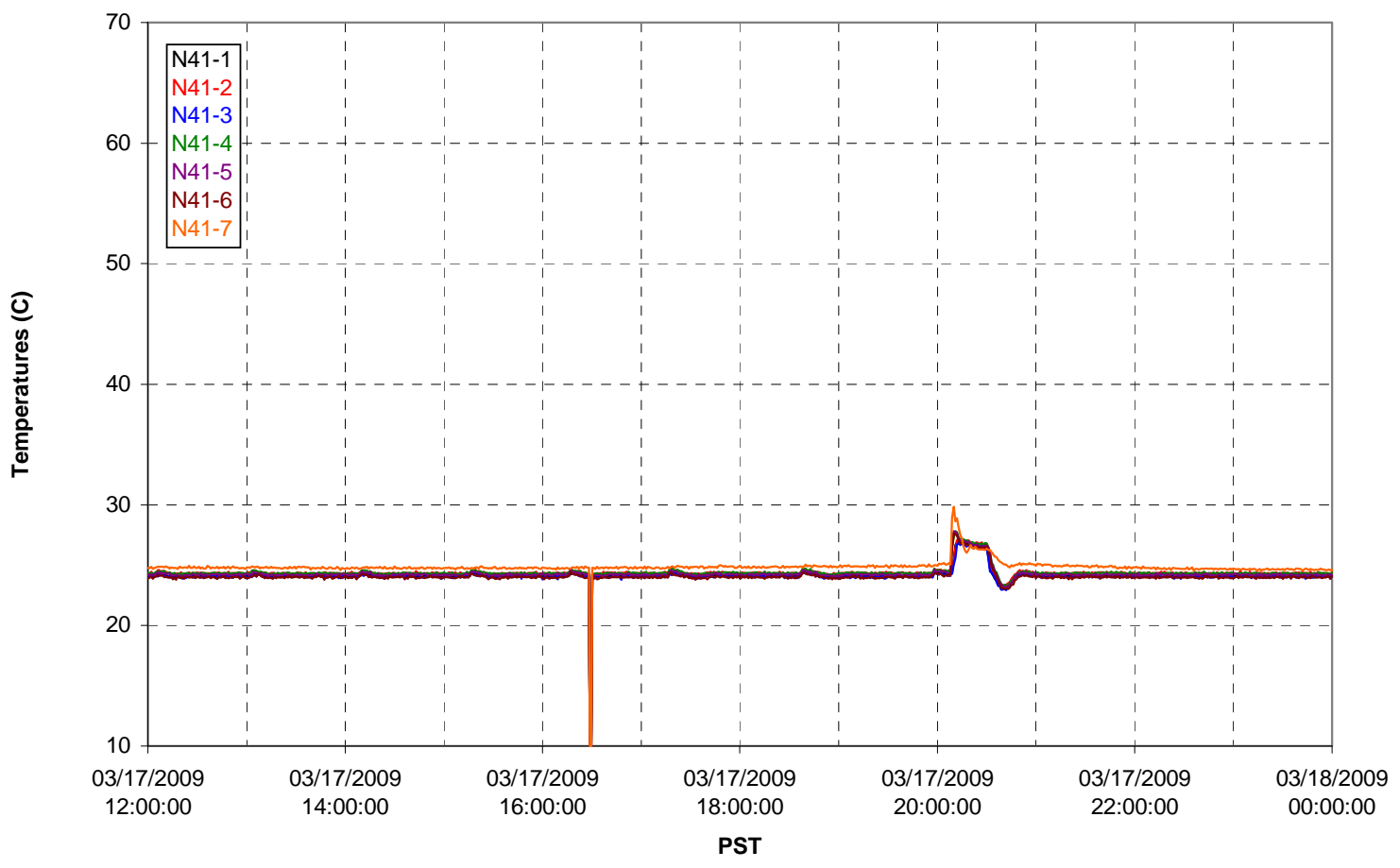


T02A temperatures

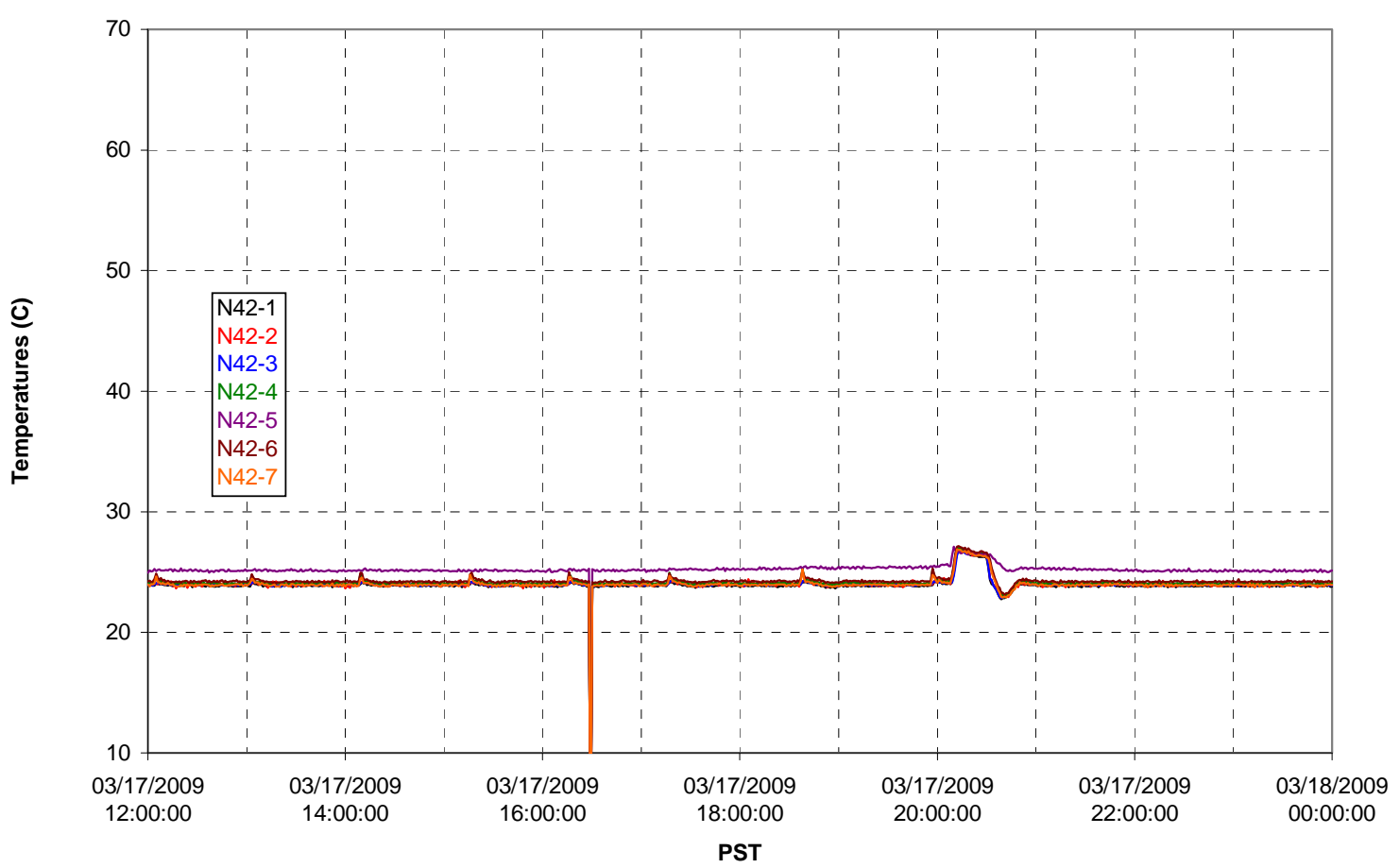

T02A temperatures

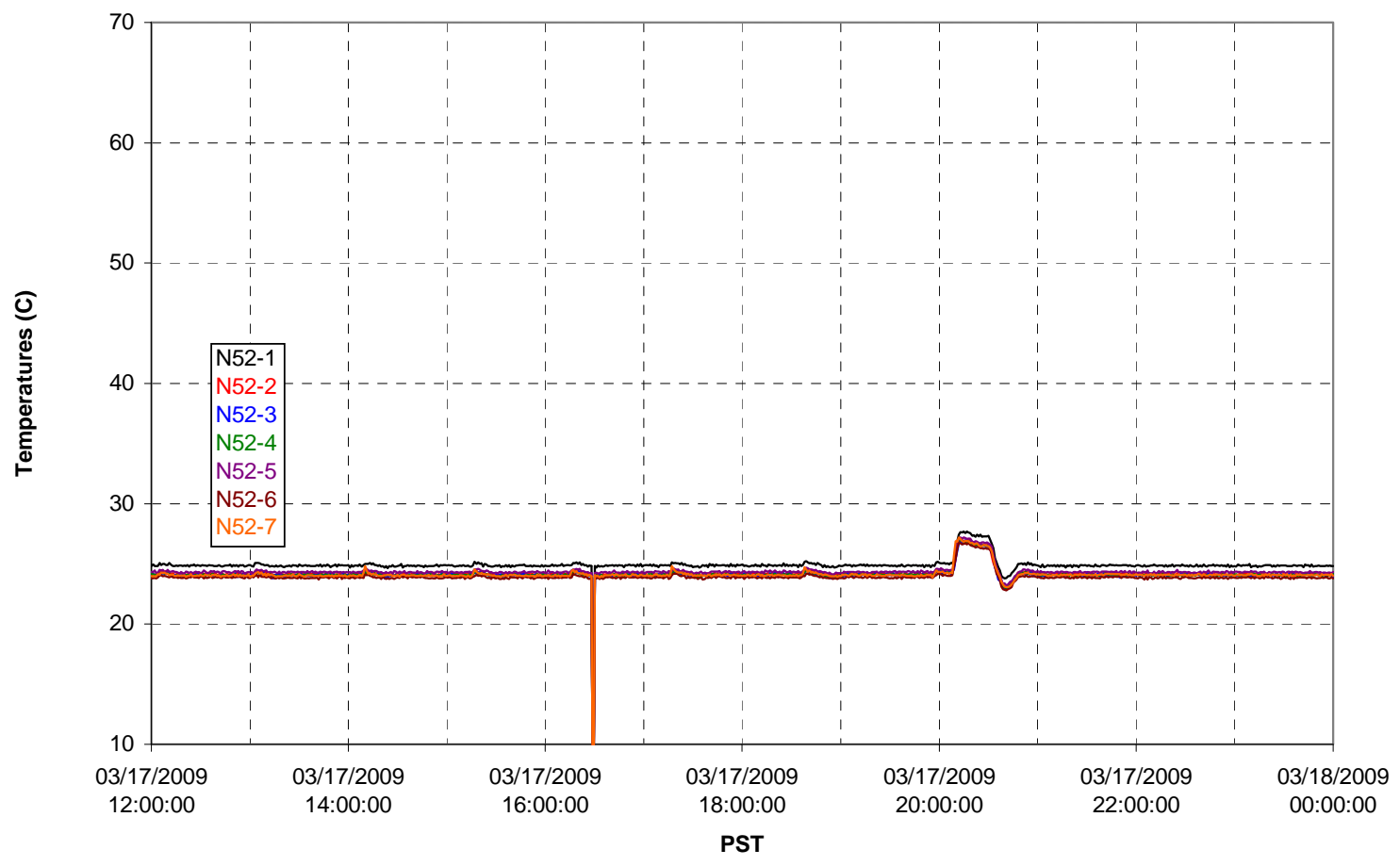


T02A Heating and Cooling

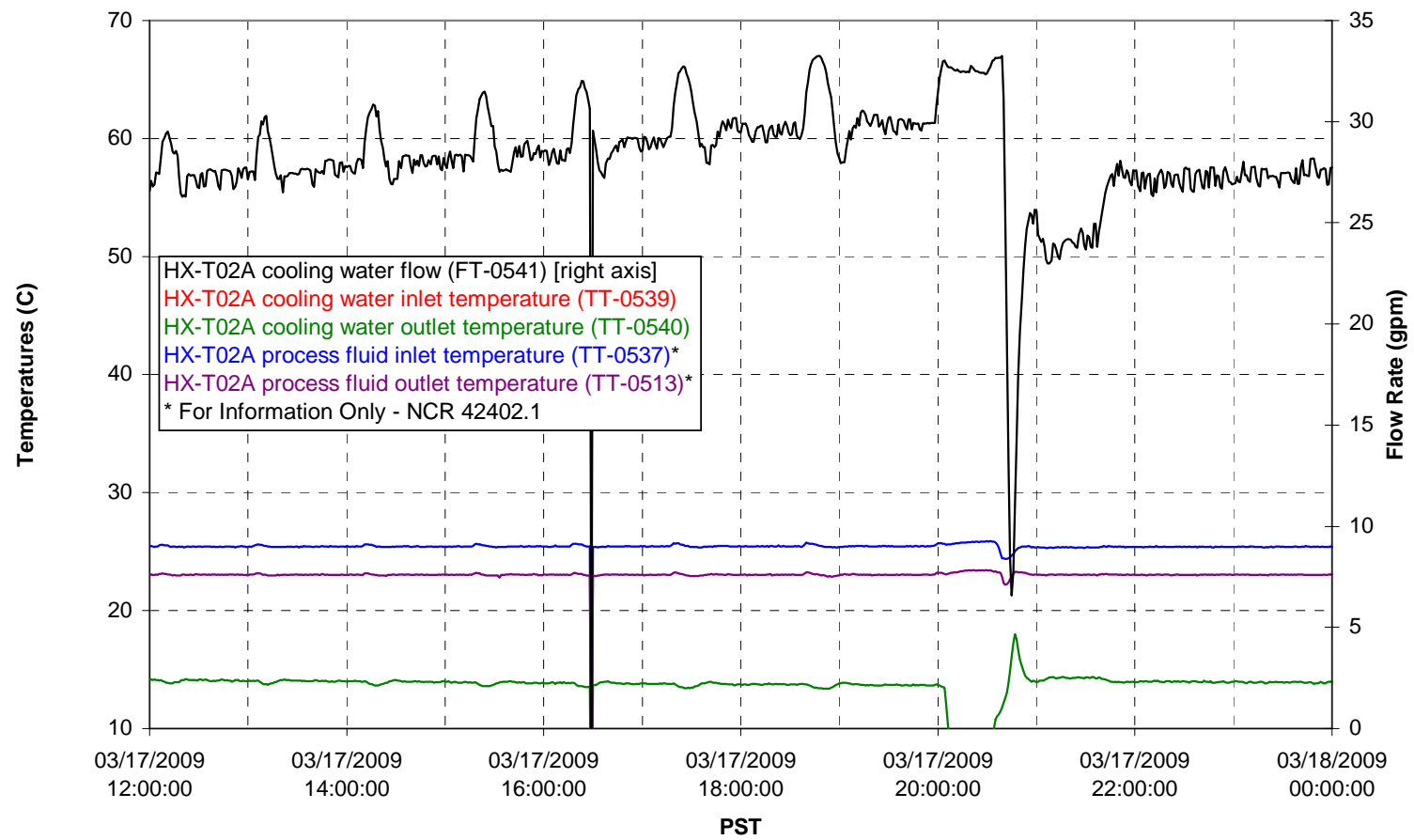


Pump Operation

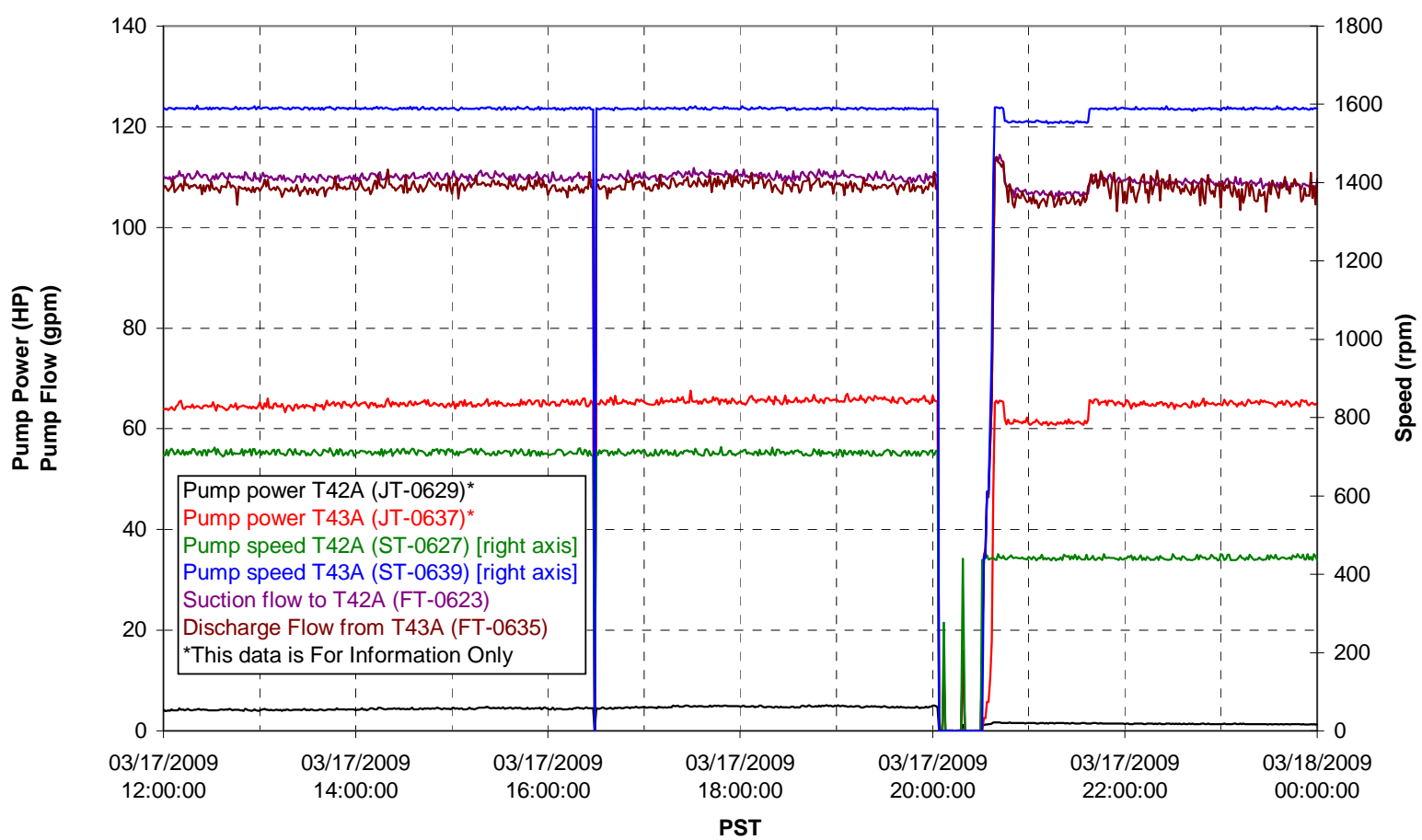

Pulsepot UFP-PP-T01A

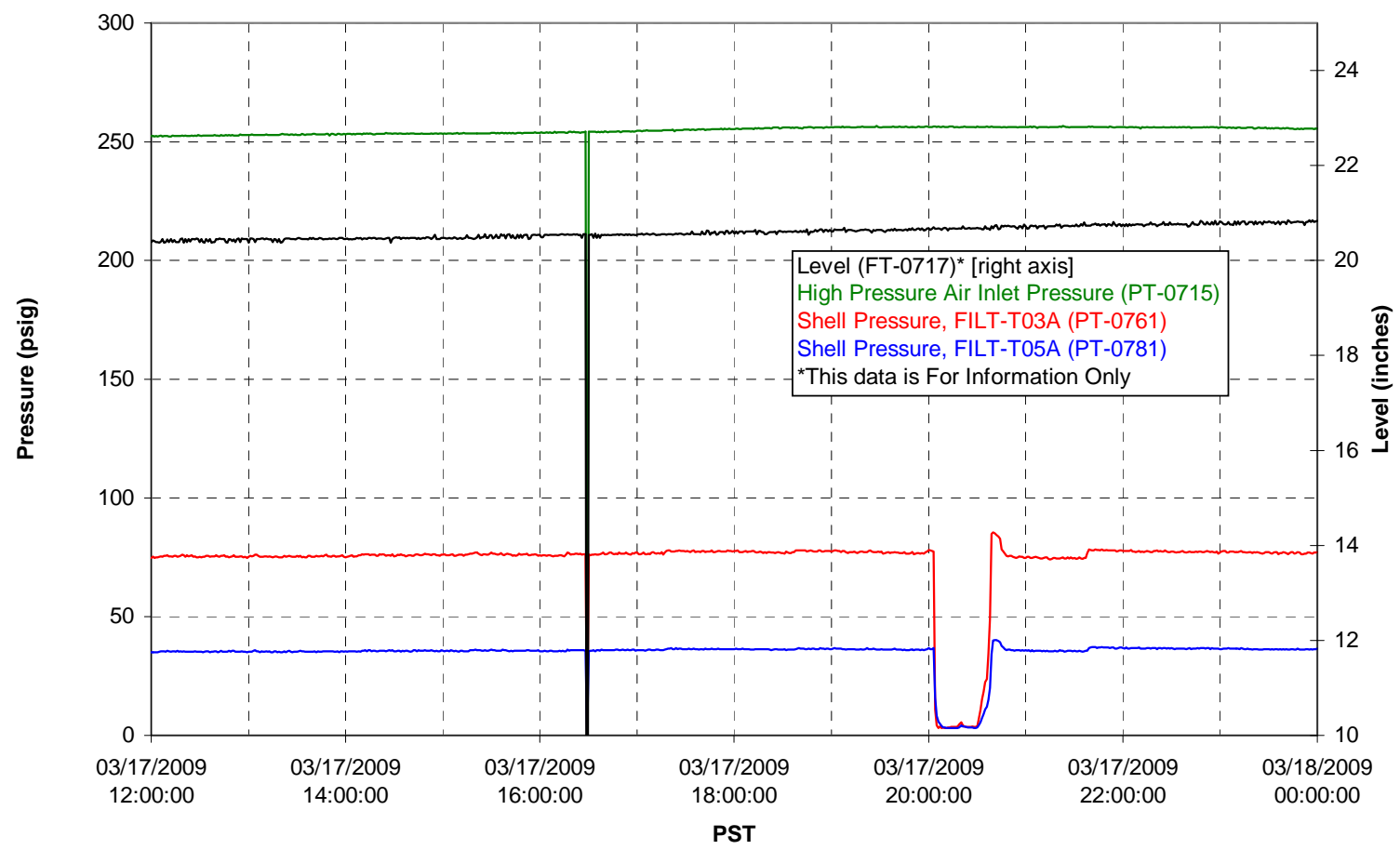


Pulsepot UFP-PP-T02A

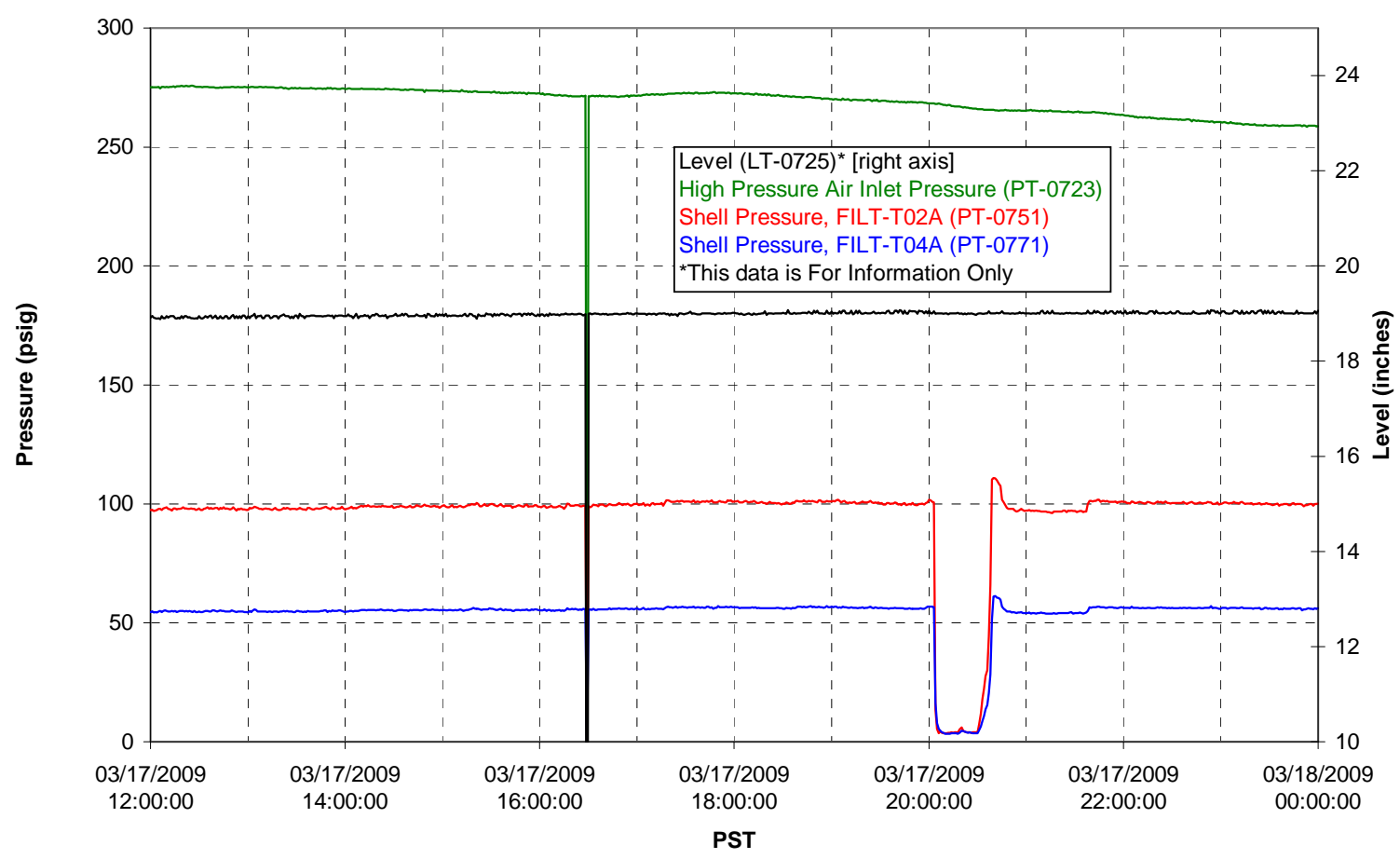

Pulsepot UFP-PP-T03A

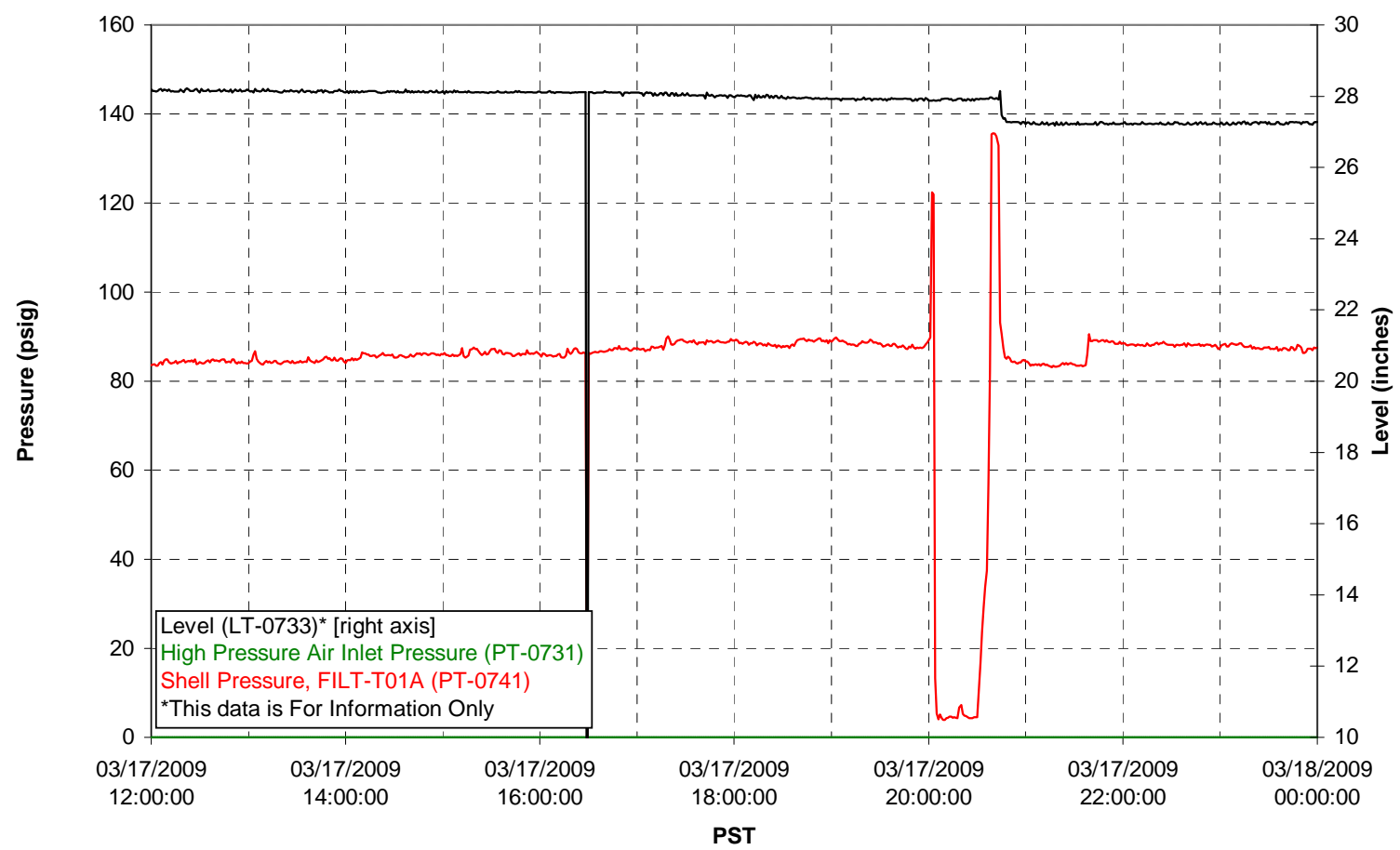


Pulsepot Levels

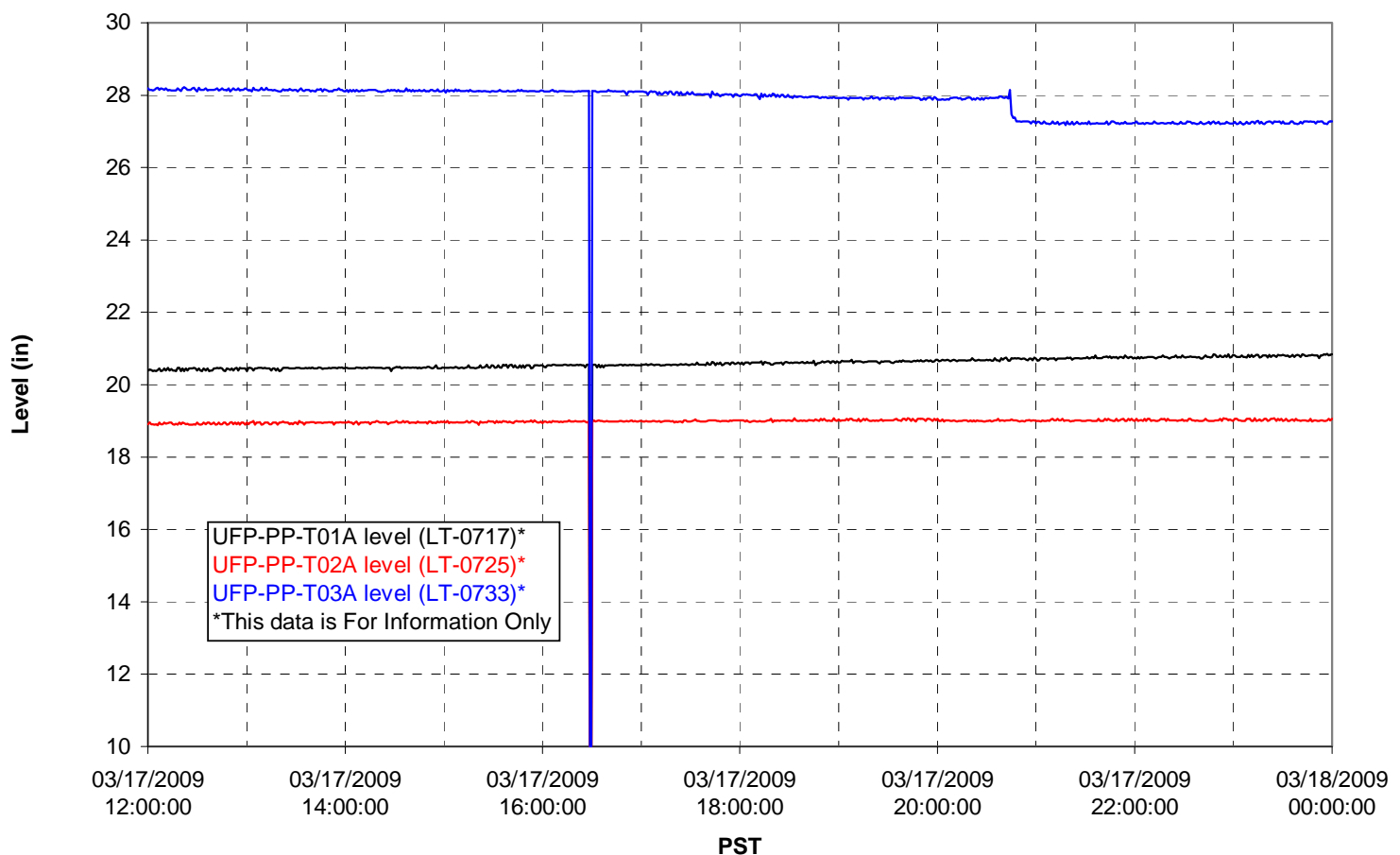

Filter UFP-FILT-T01A

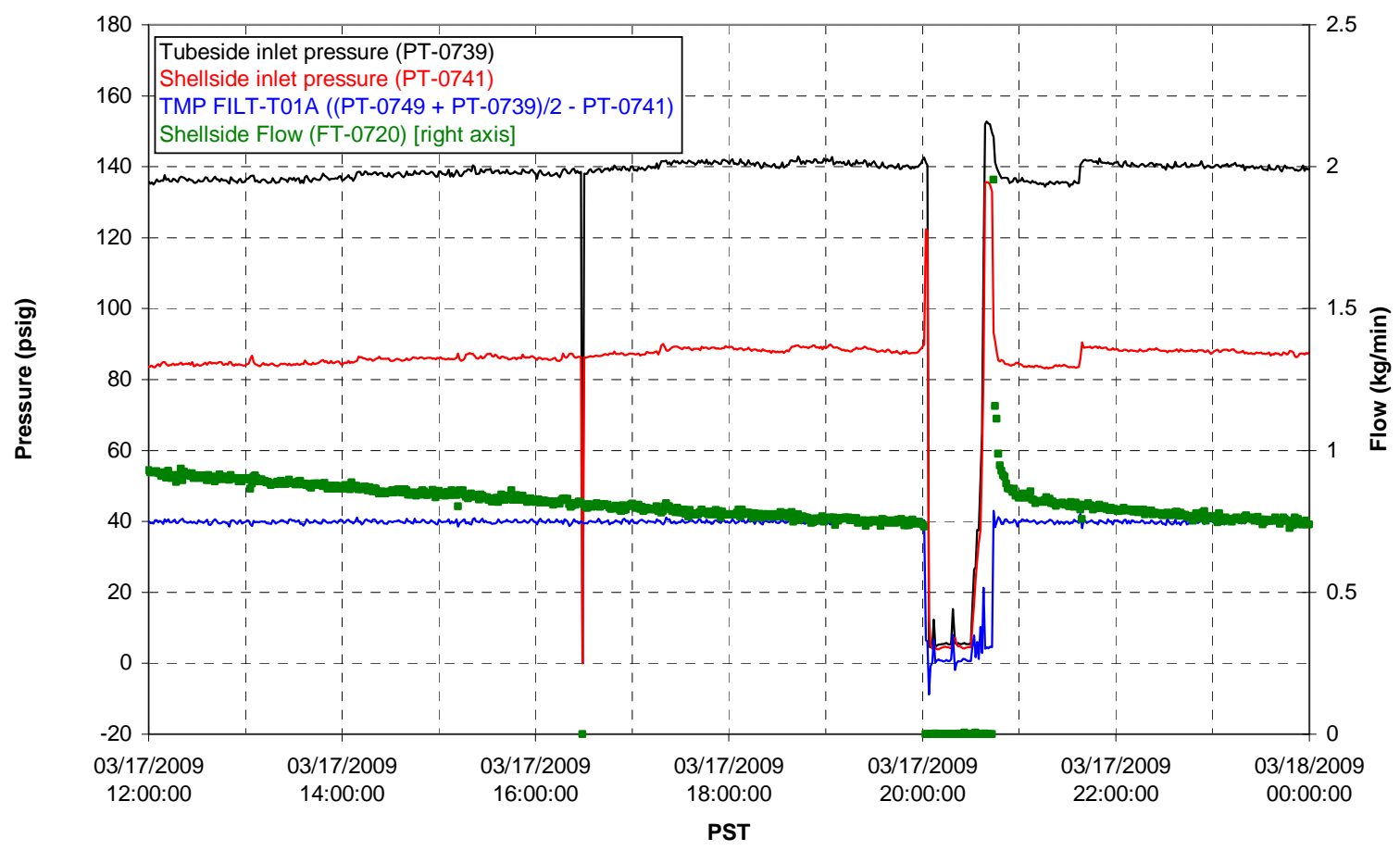


Filter UFP-FILT-T02A

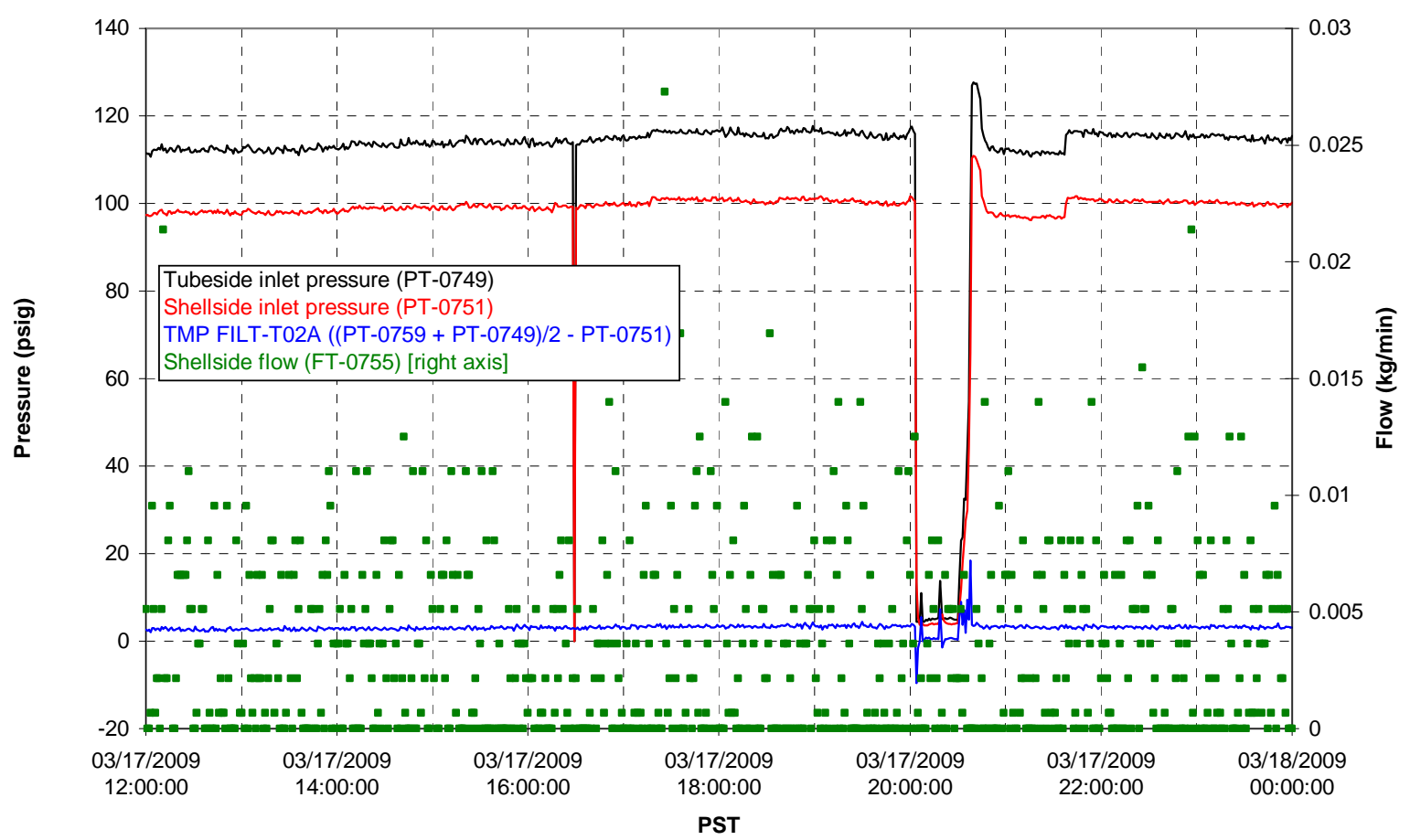

Filter UFP-FILT-T03A

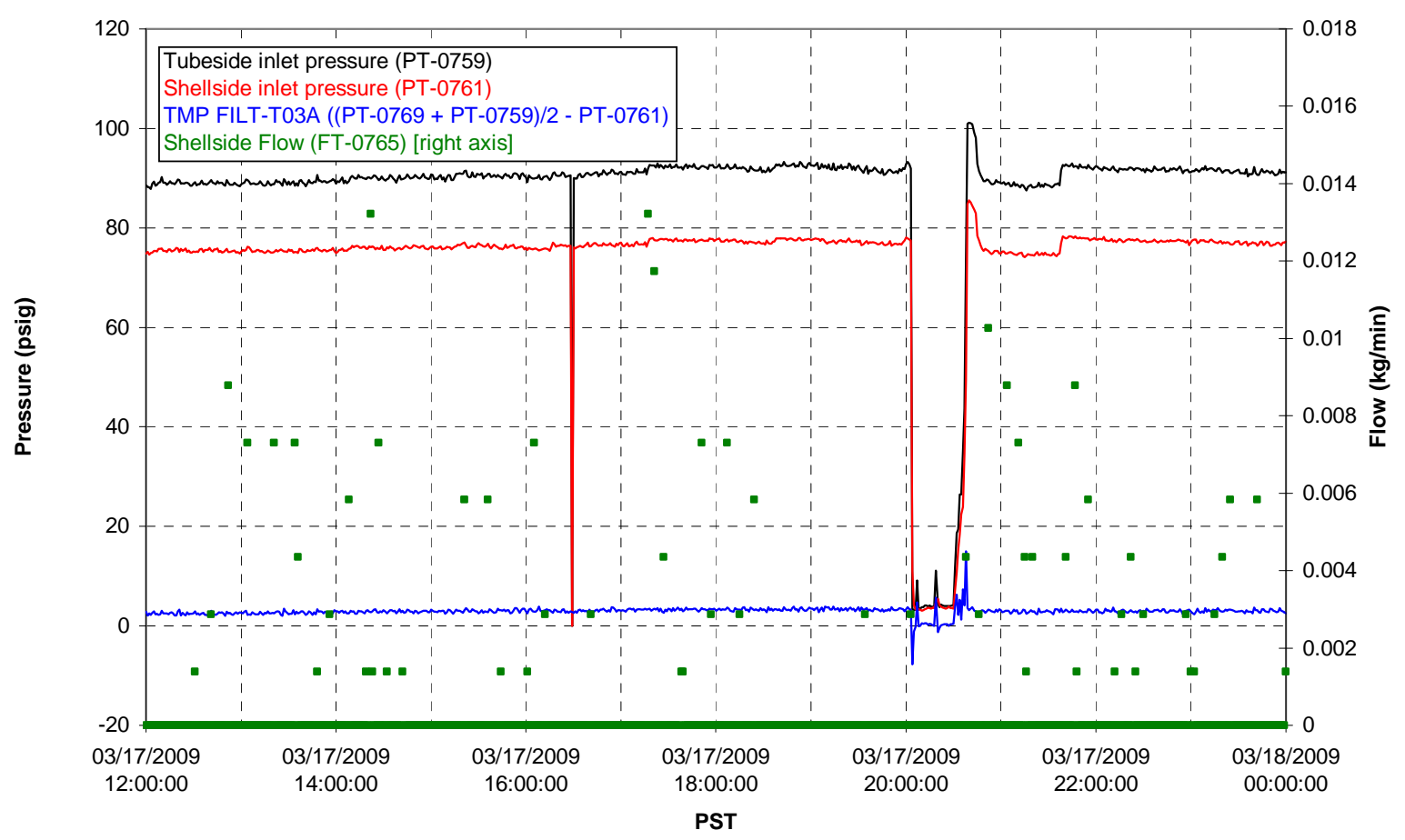


Filter UFP-FILT-T04A

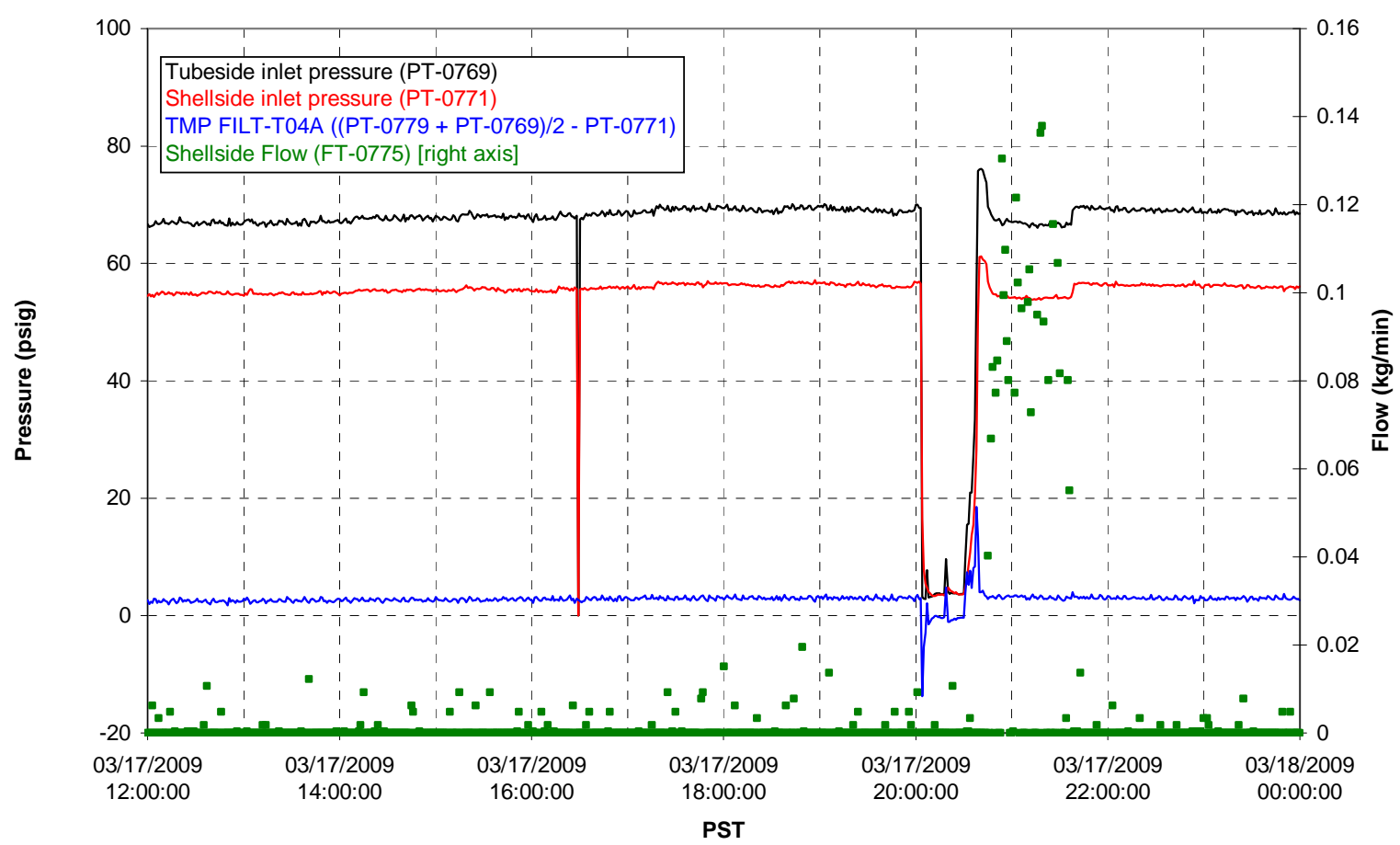

Filter UFP-FILT-T05A

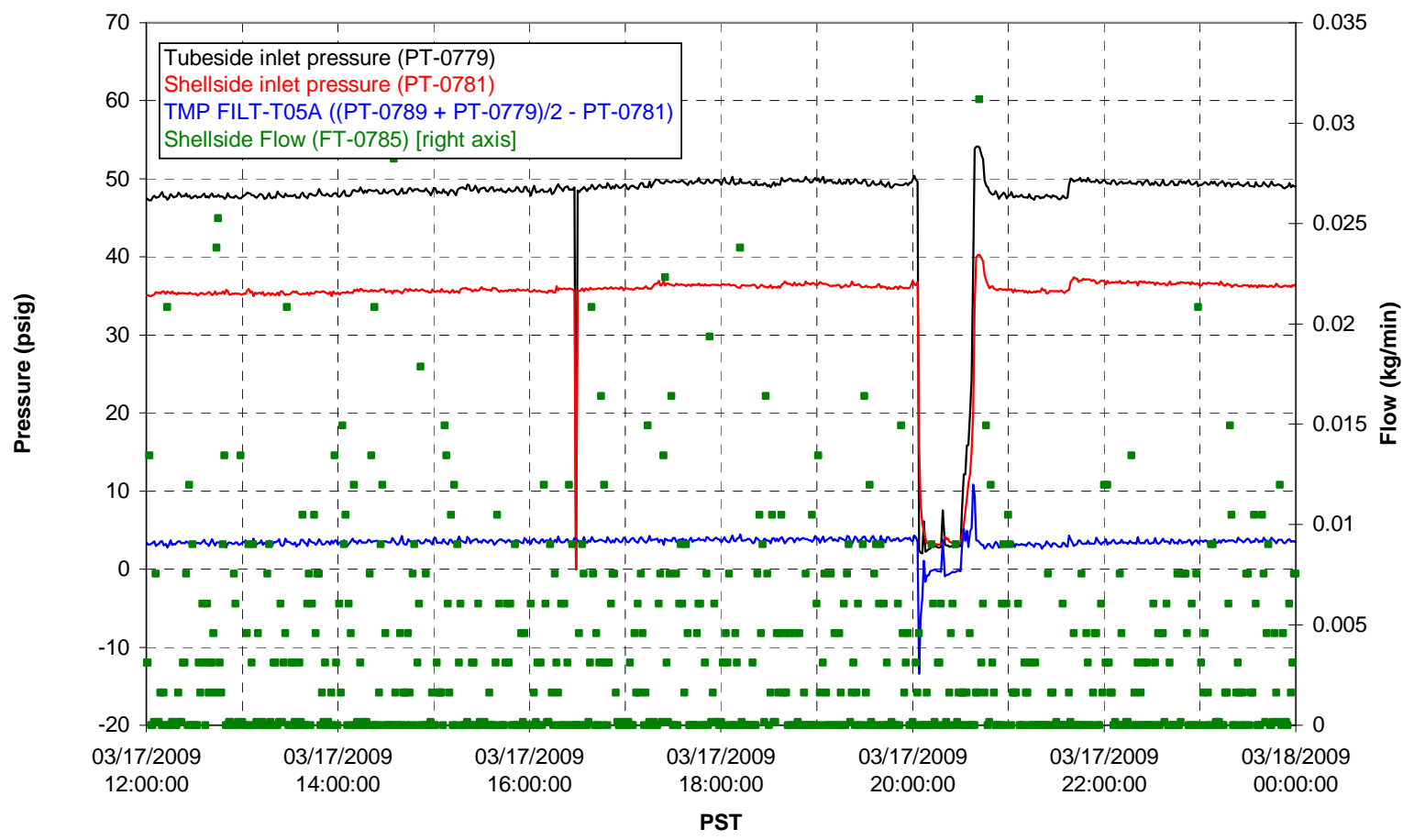




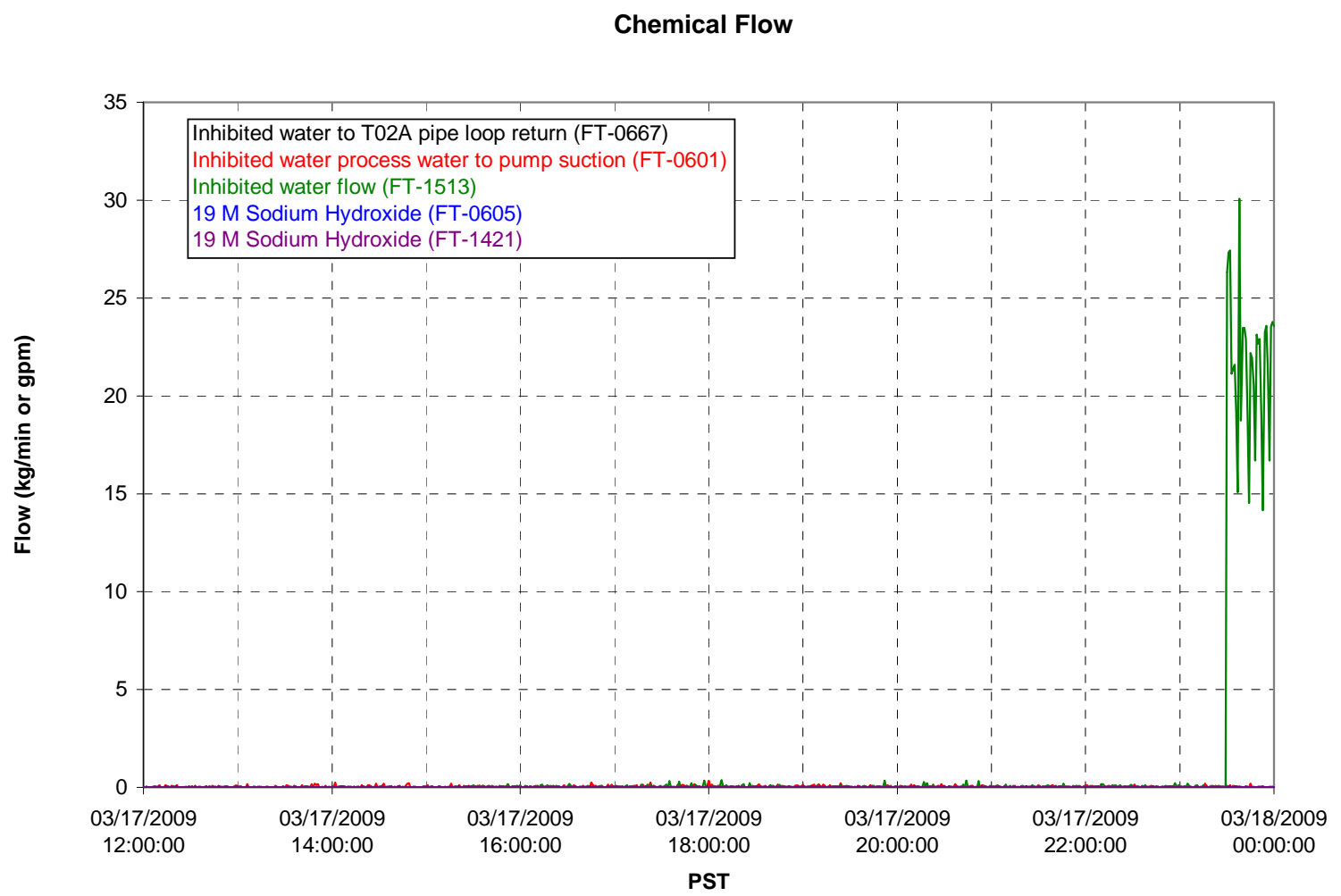

Chemical Flow

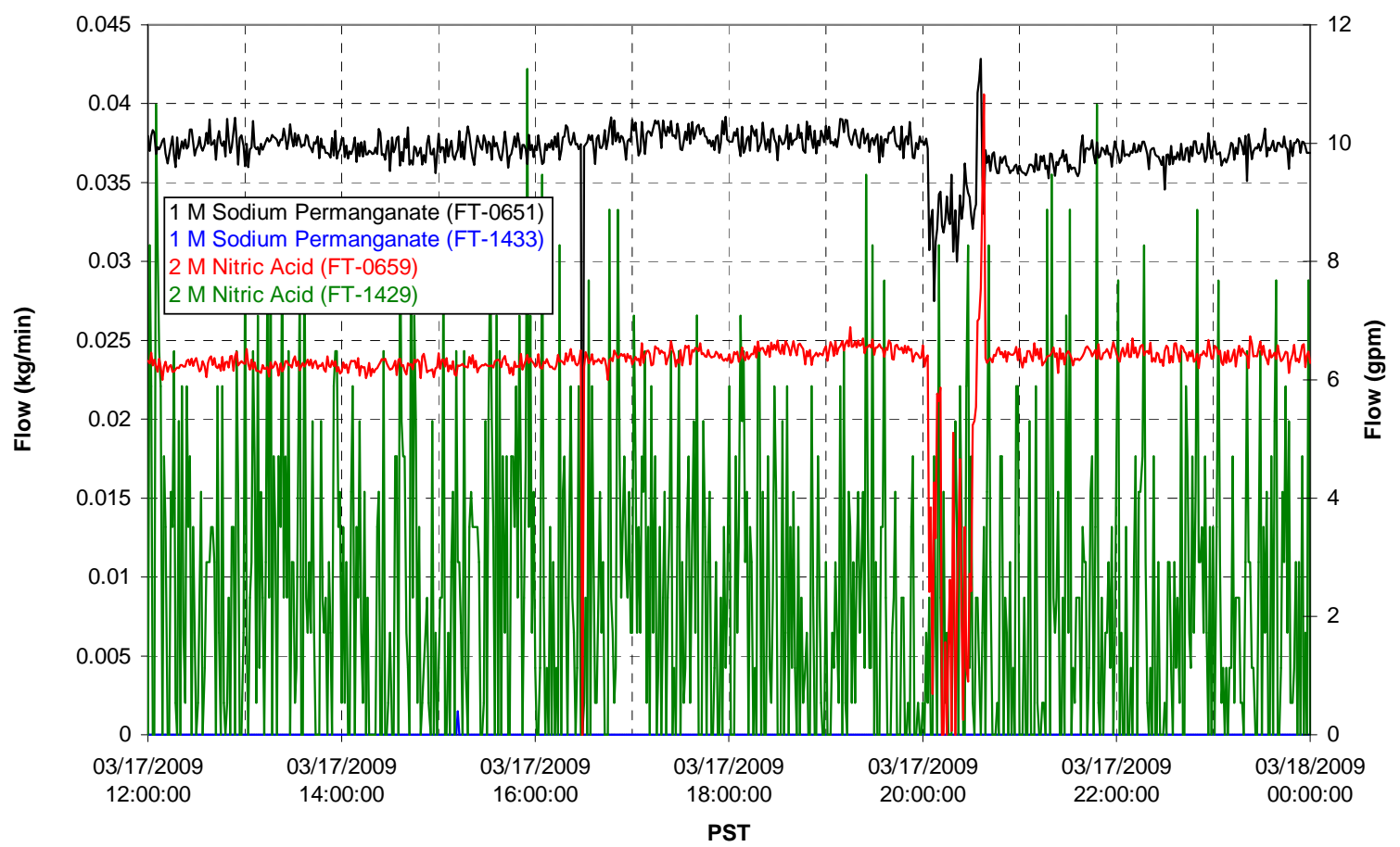




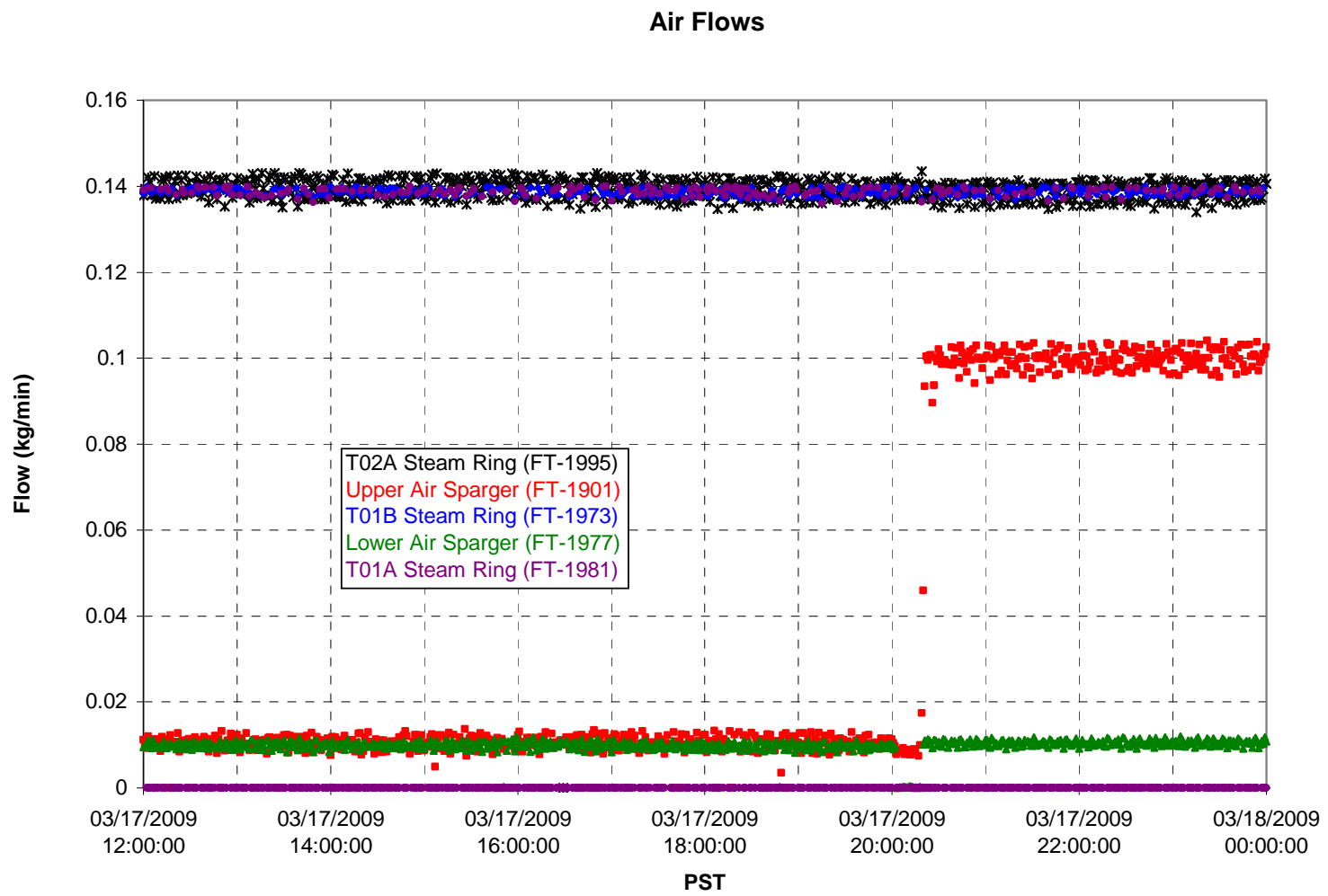

T02A Steam

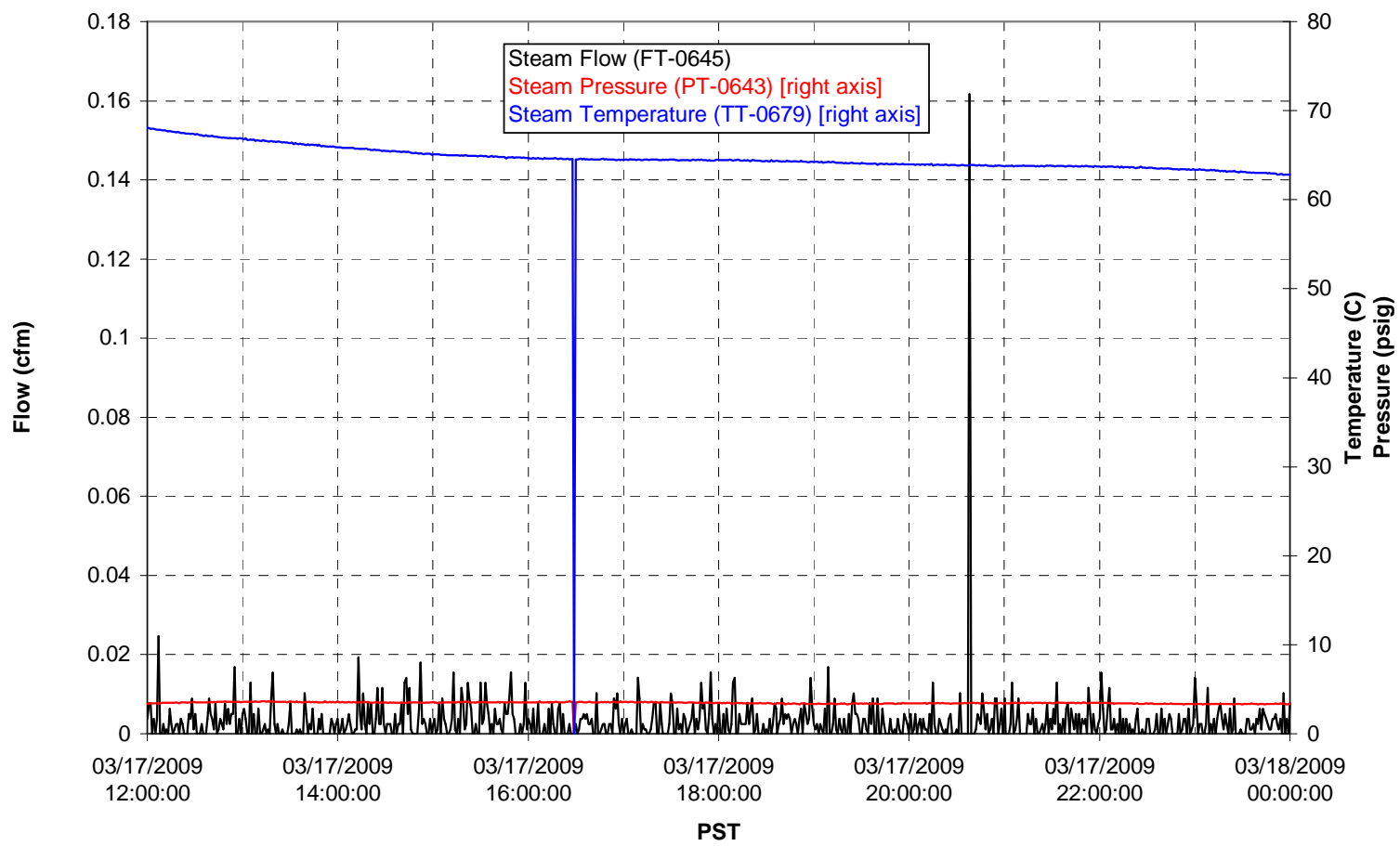


T01A Steam

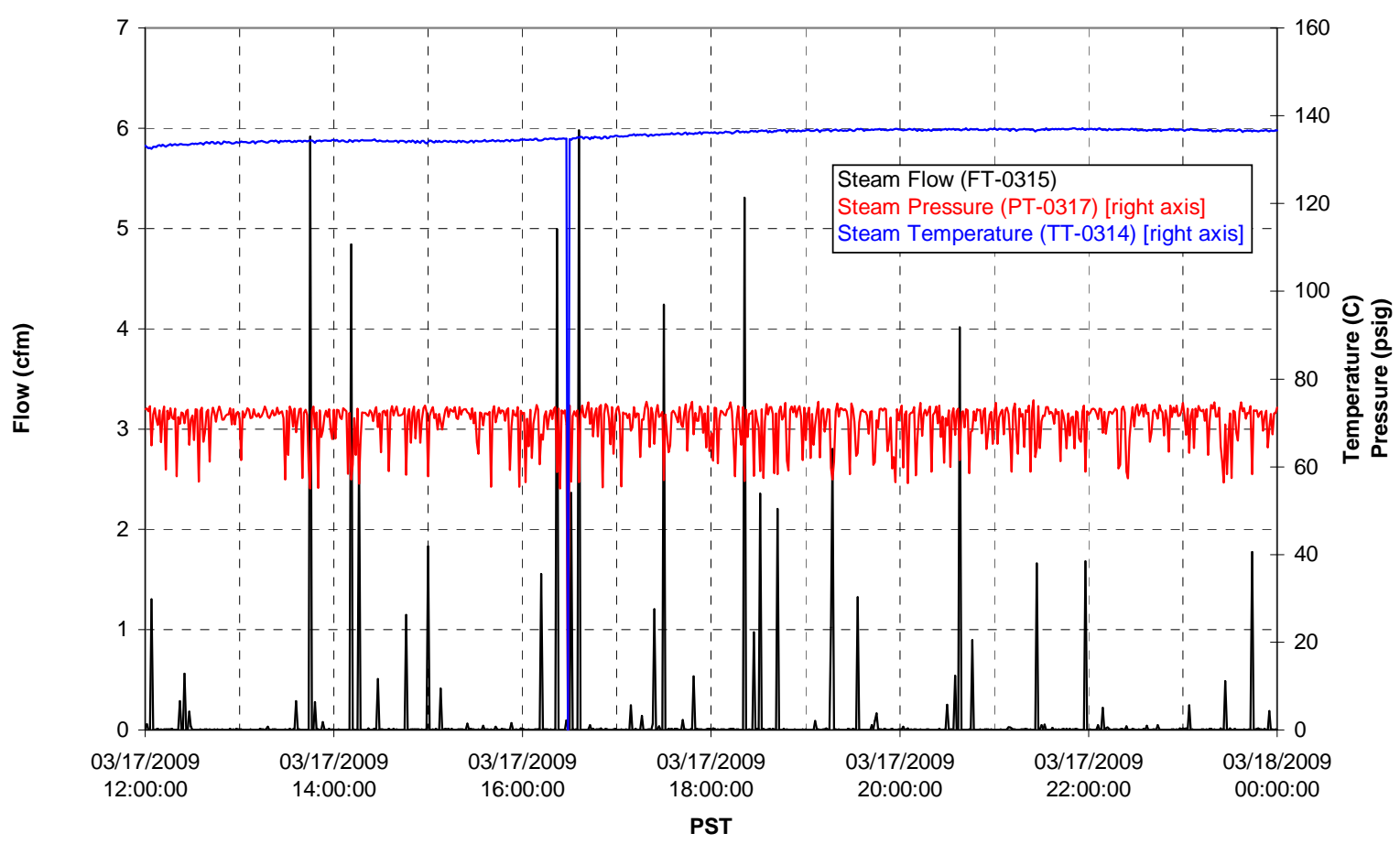

T01B Steam

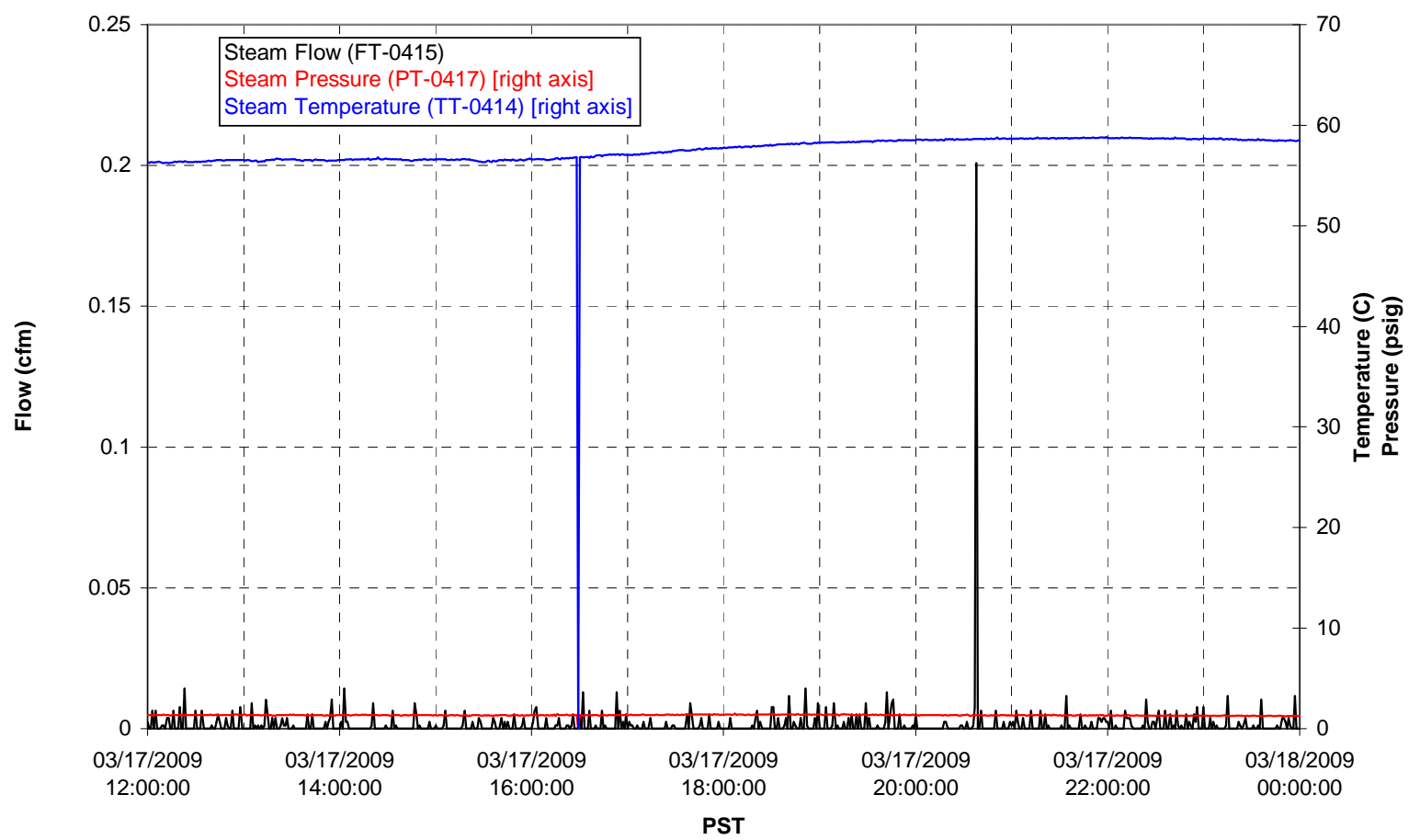




\section{Integrated Test B Data Plots 03/18/09 00:00 - 03/18/09 12:00}


T01A level

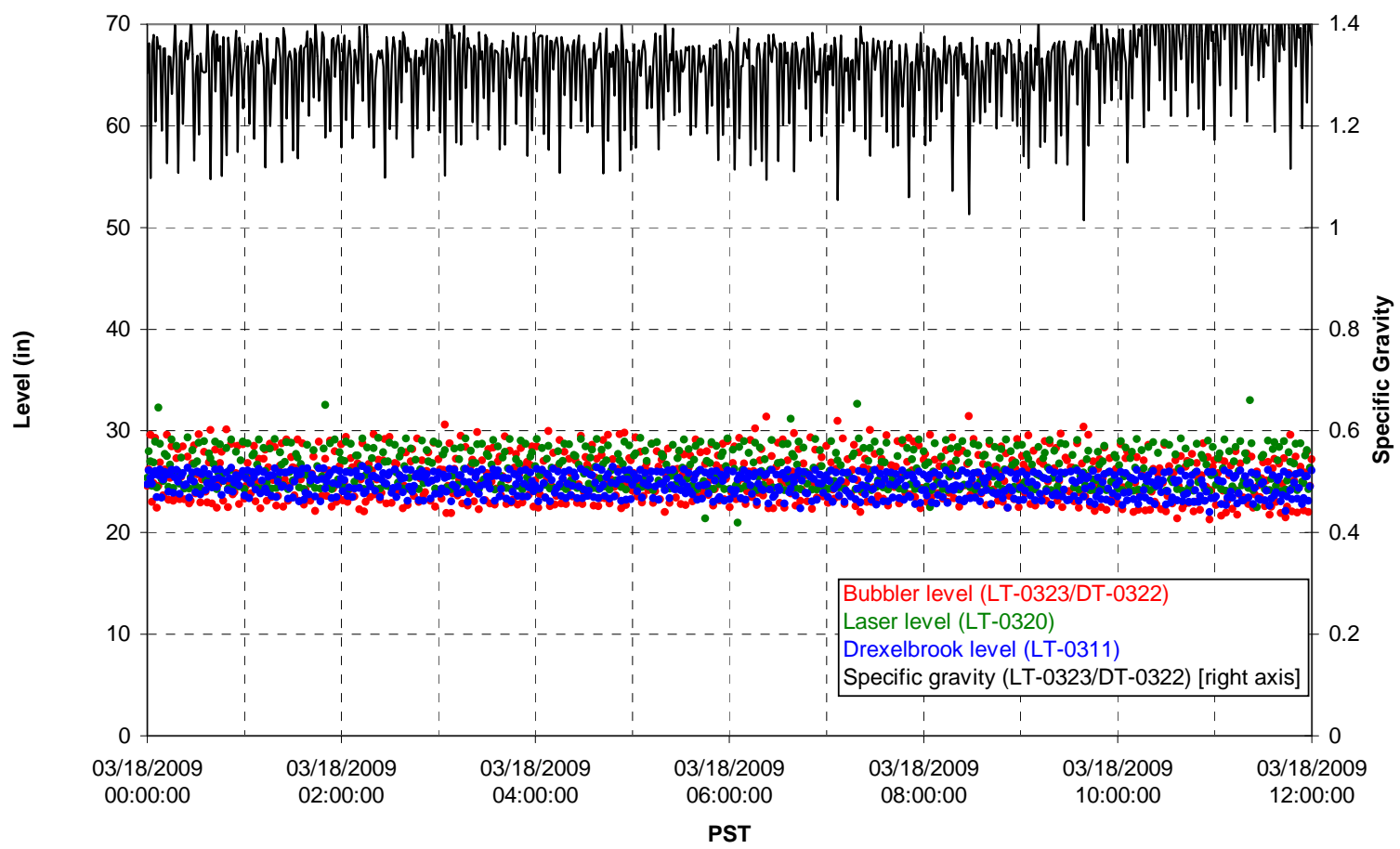

T01A temperatures

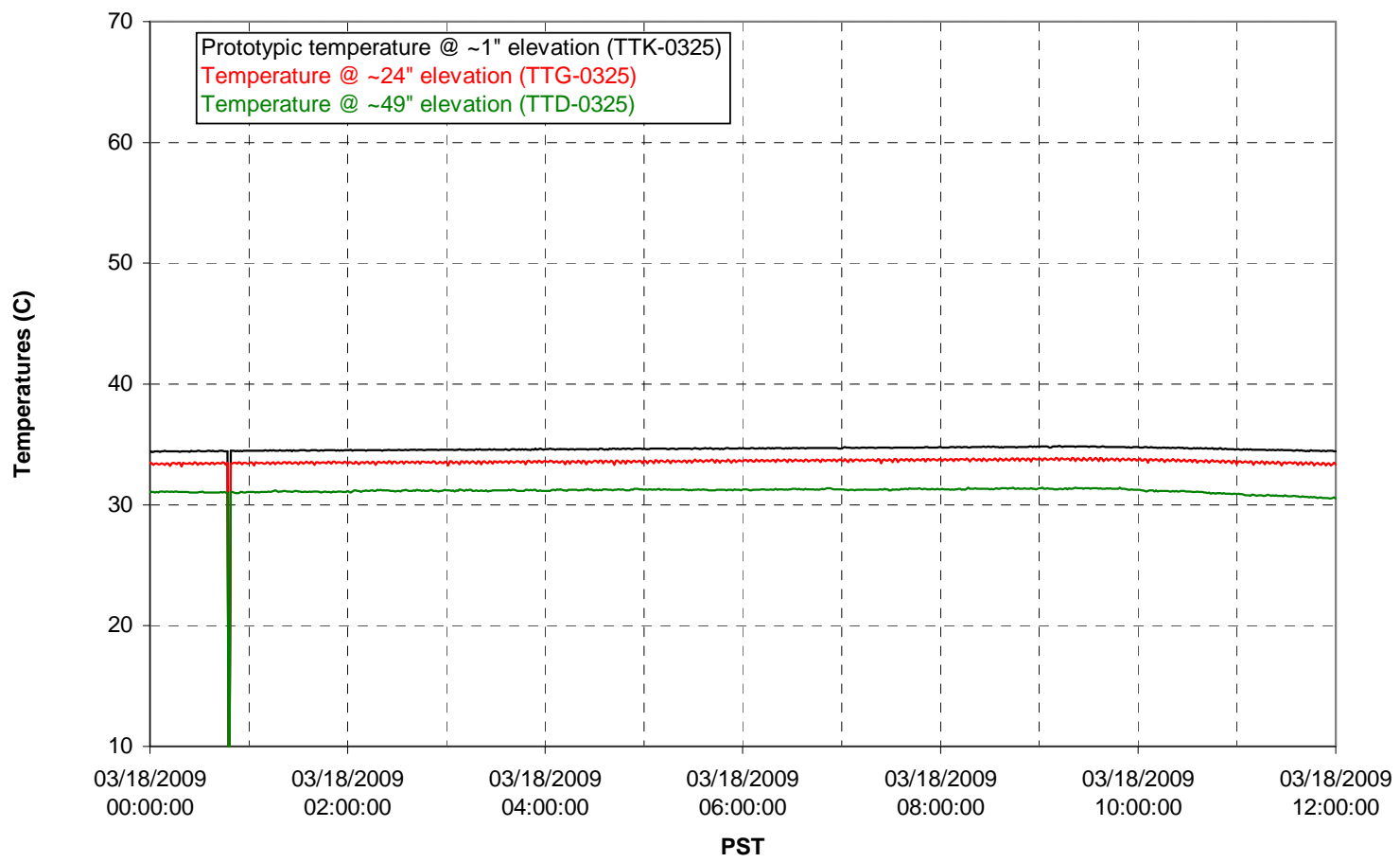


T01B level

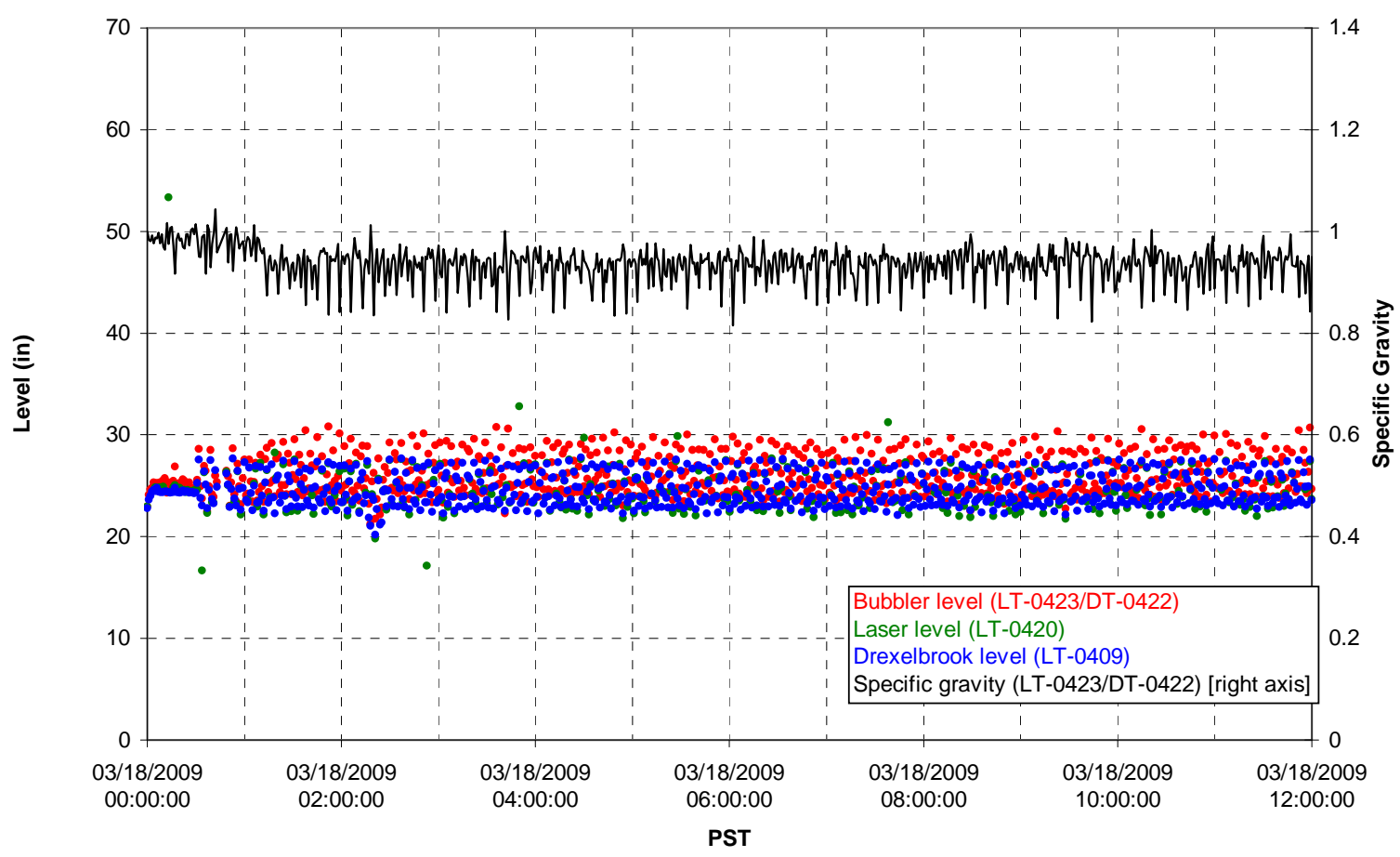

T01B temperatures

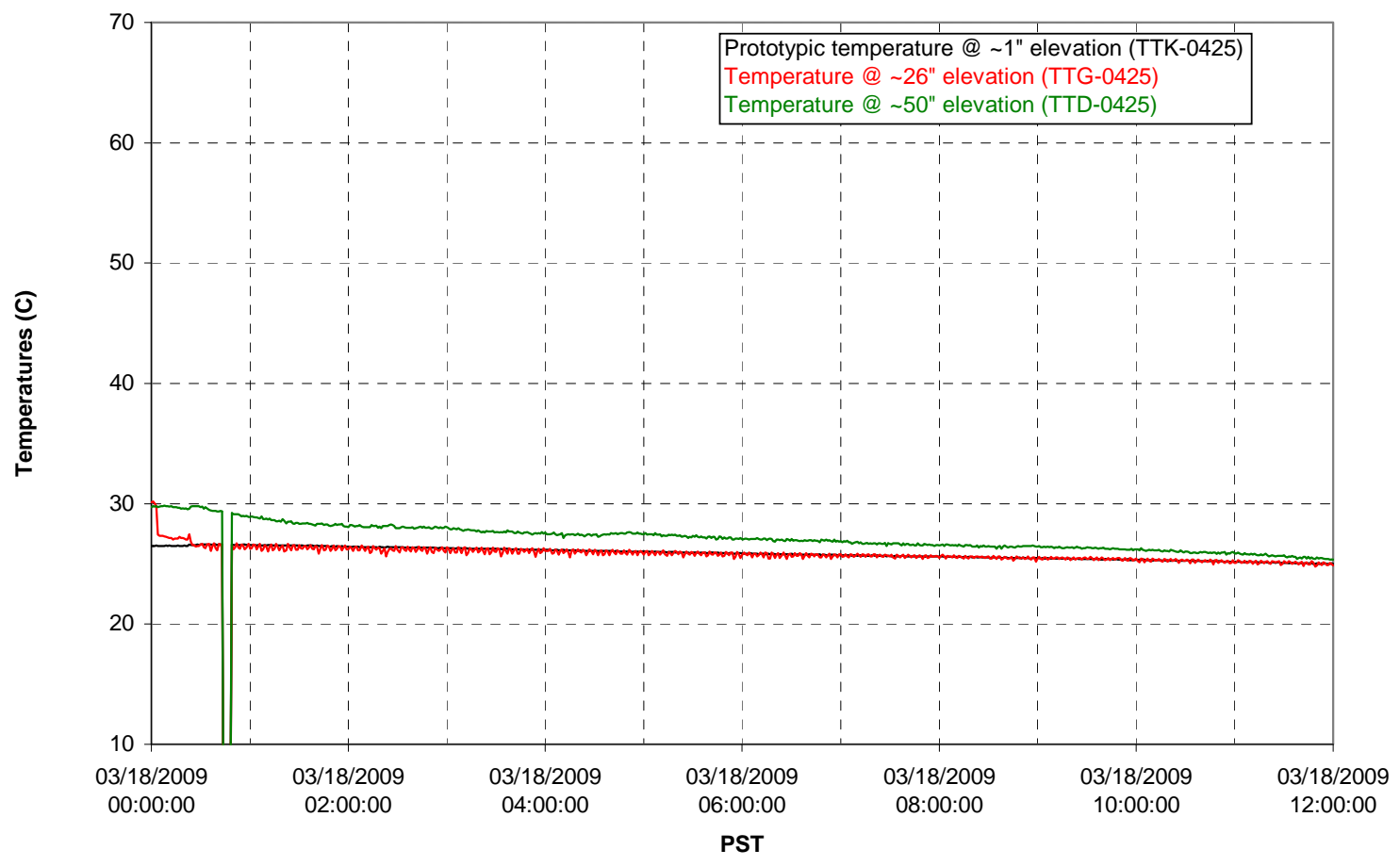


T02A level

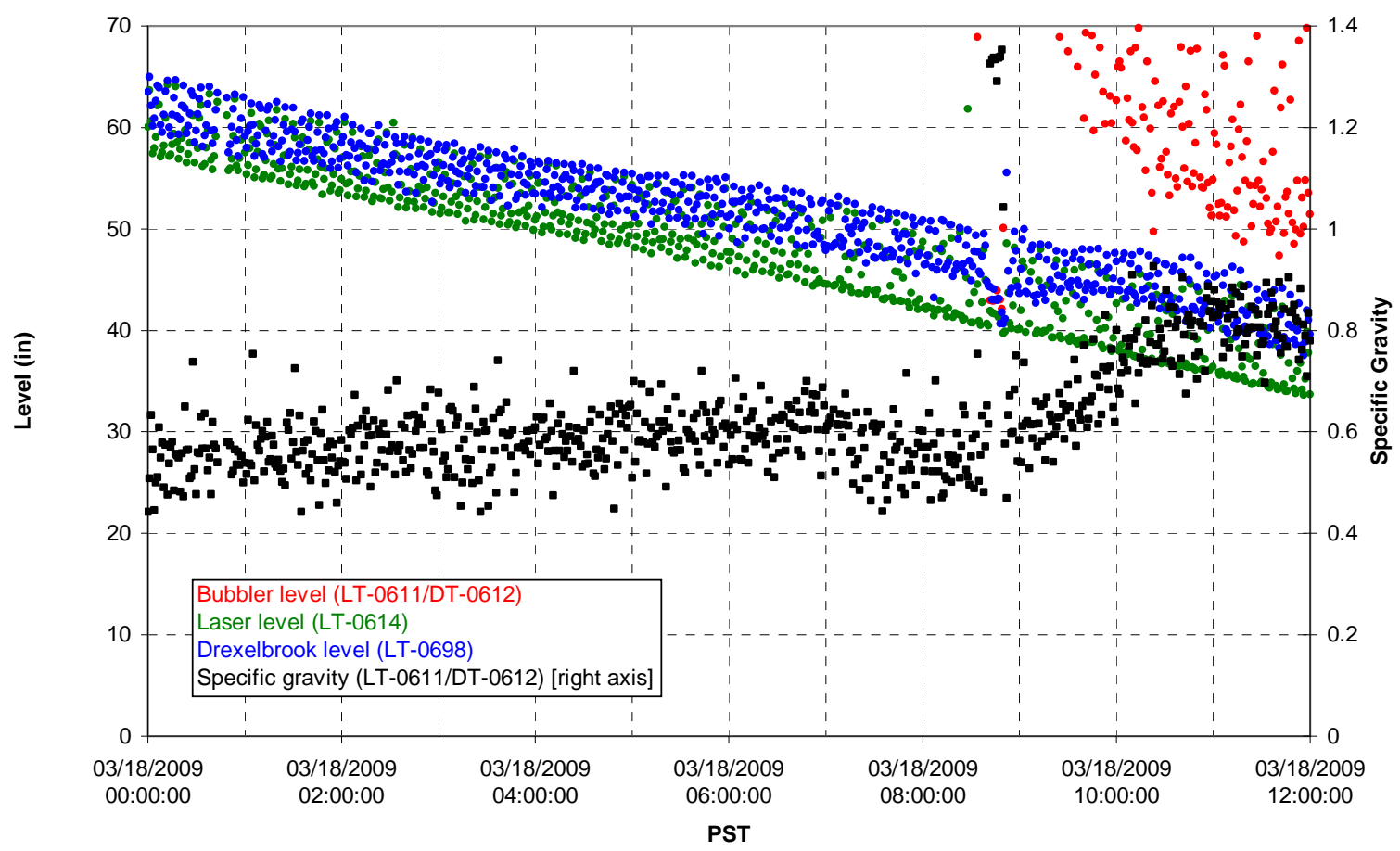

T02A temperatures

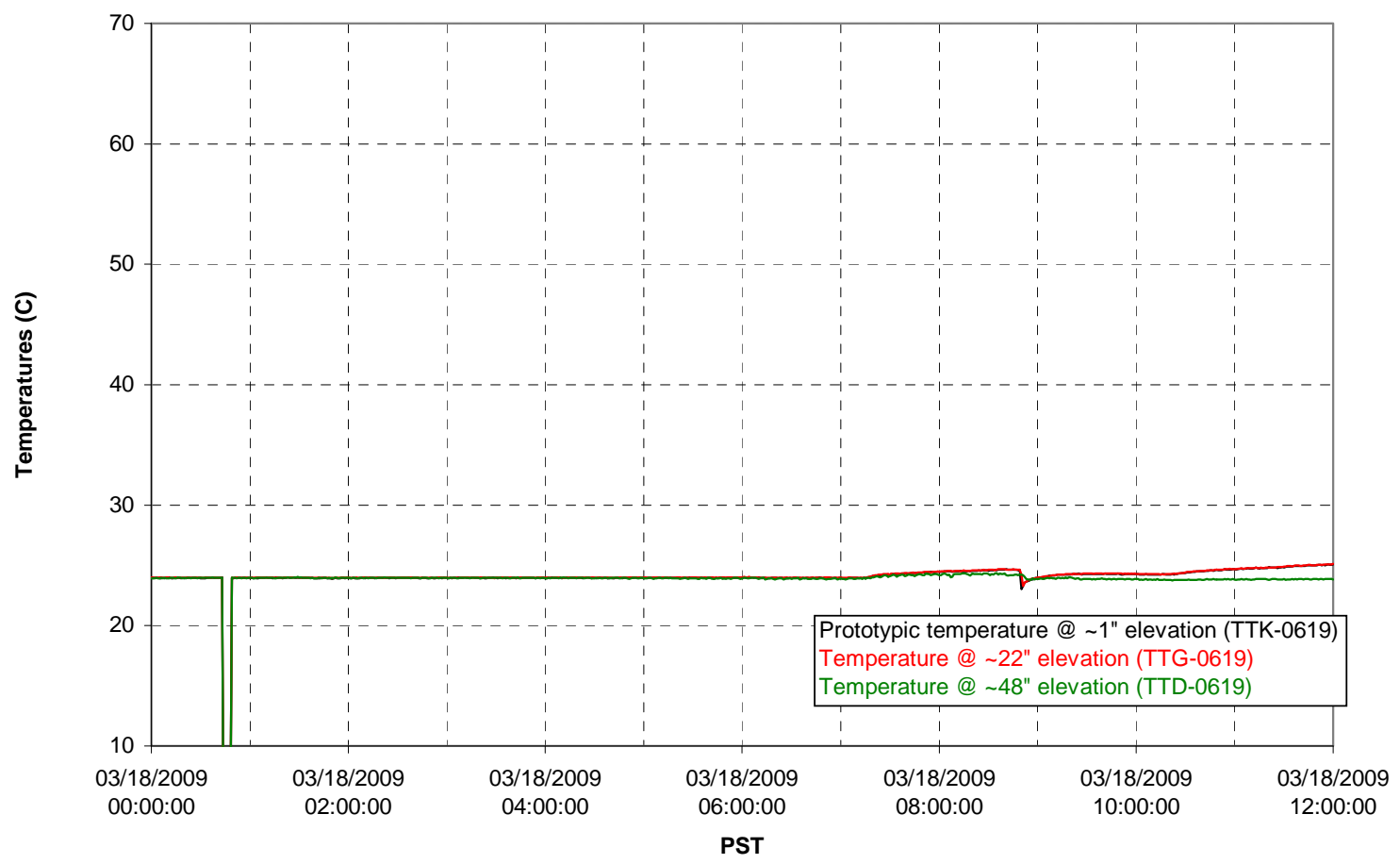


T02A and filter loop temperatures

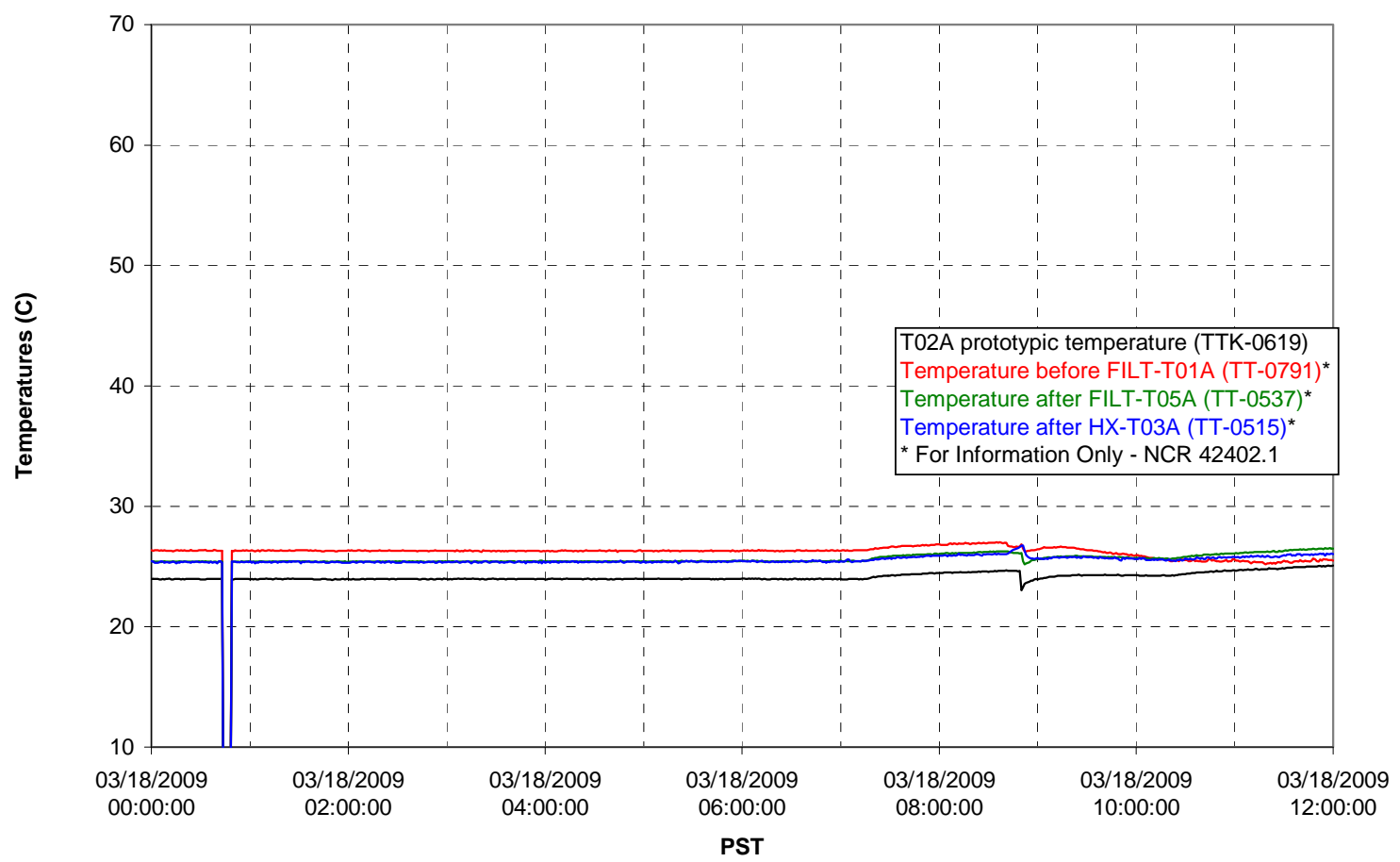

Pump Pressures and Flow

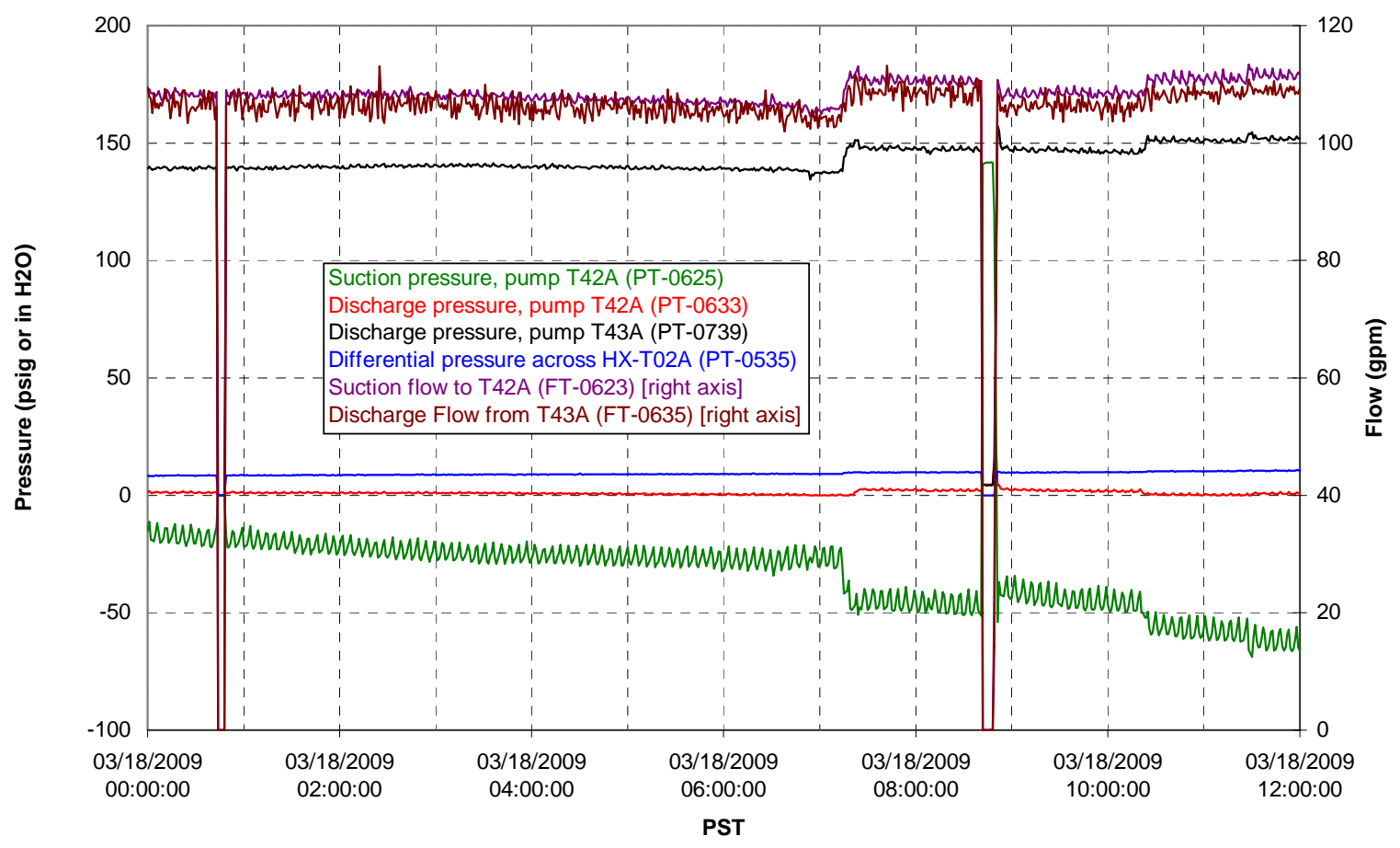


Axial pressure drop

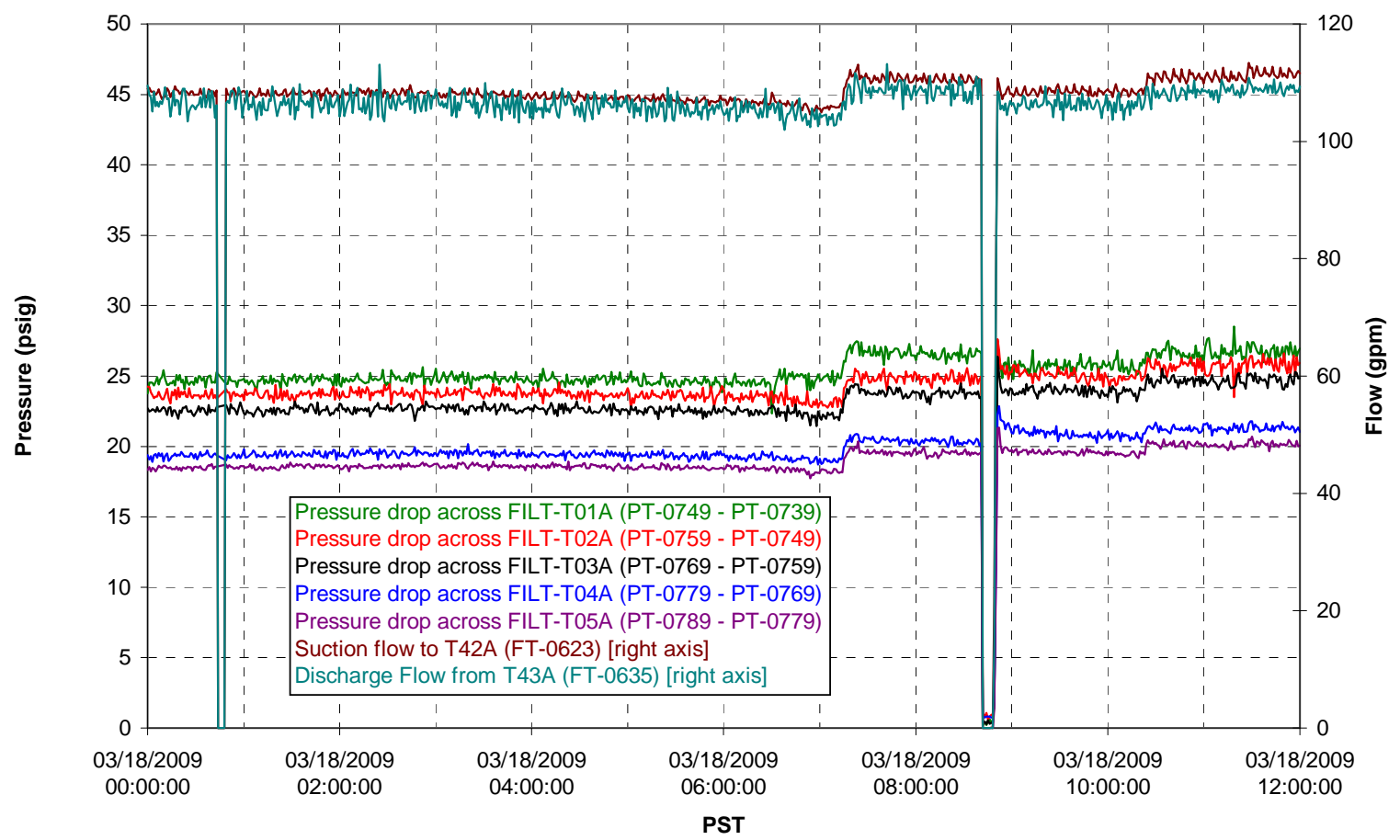

Permeate flow rates

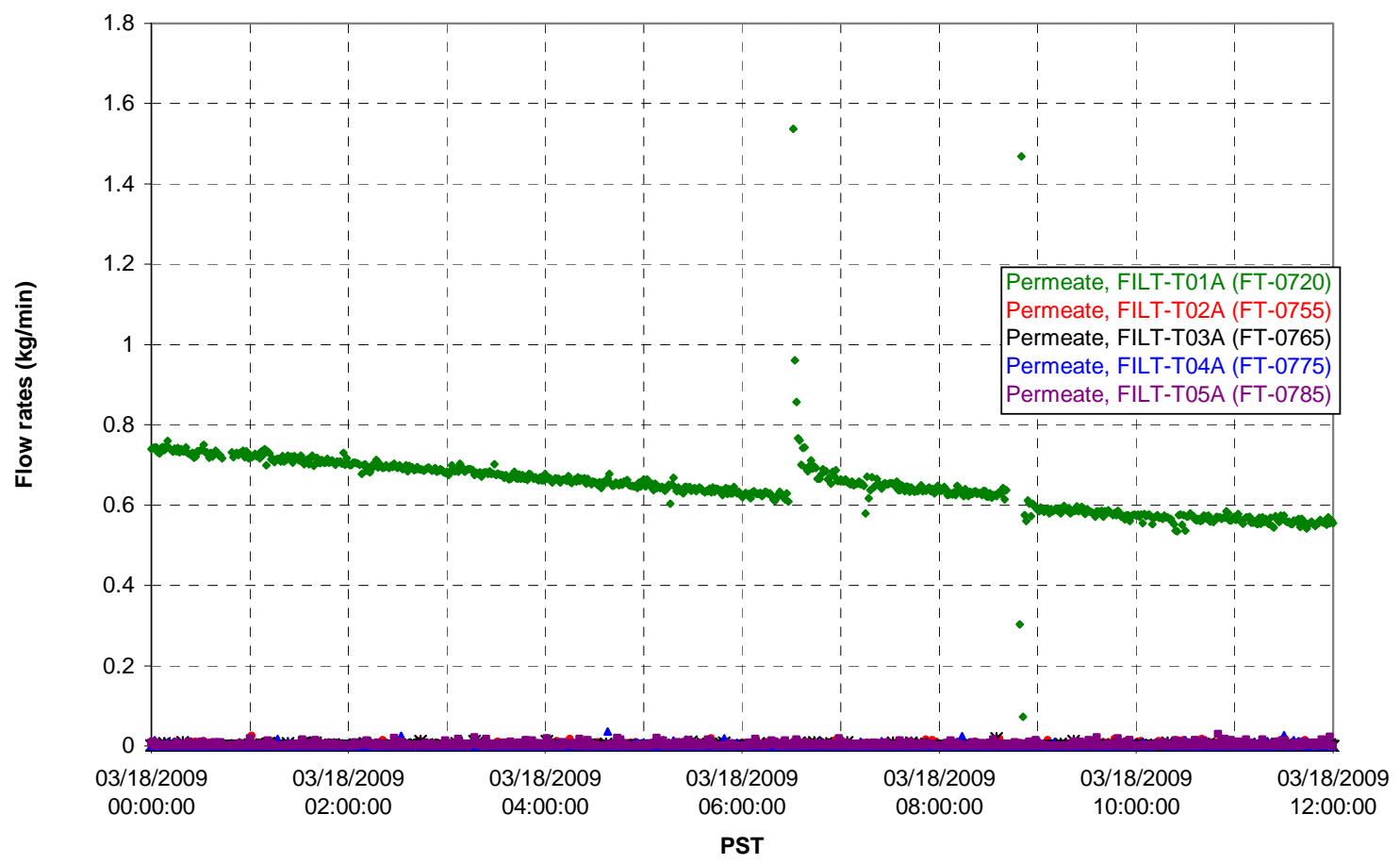


T02A Inner Temperature Tree

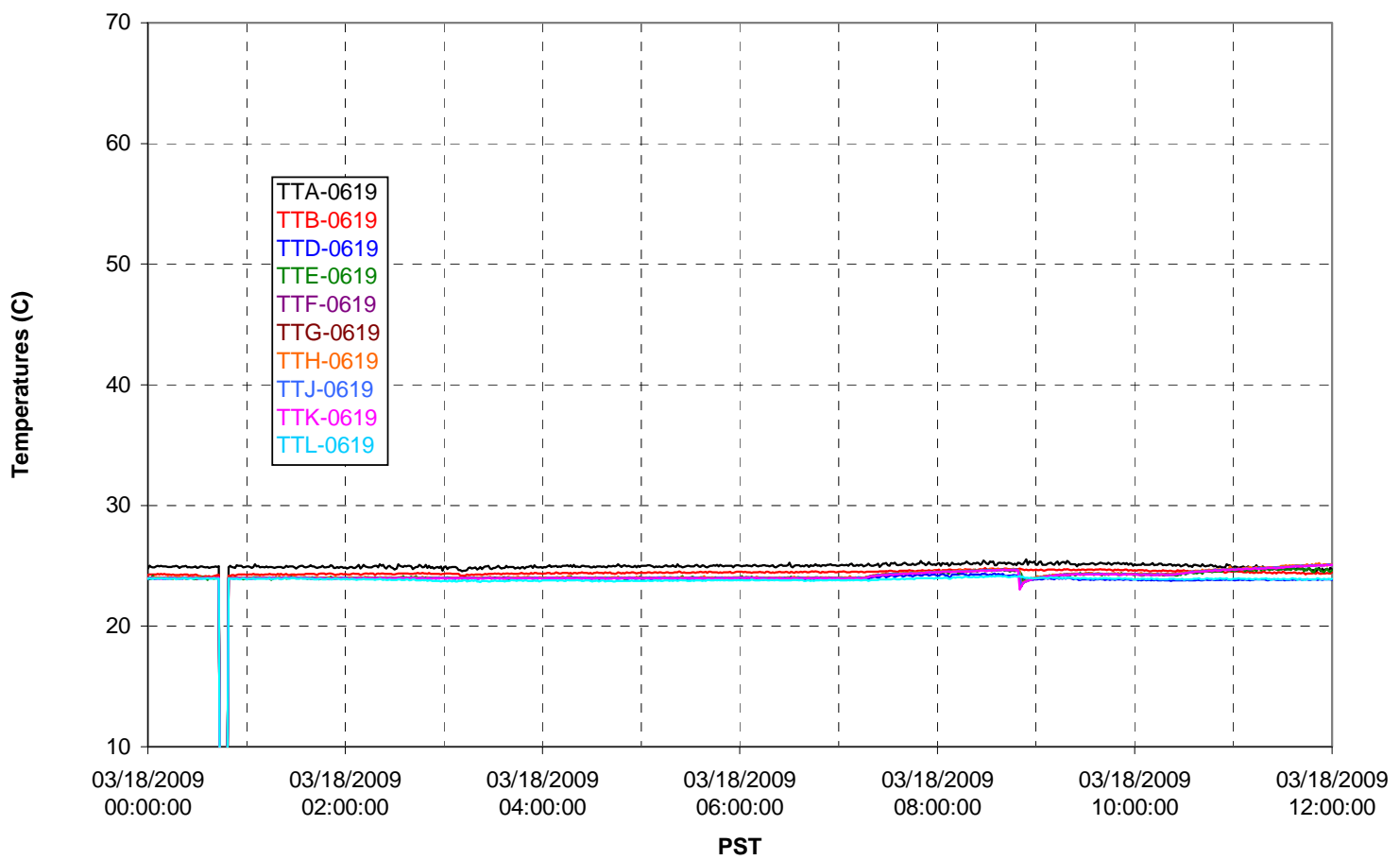

T02A Outer Temperature Tree

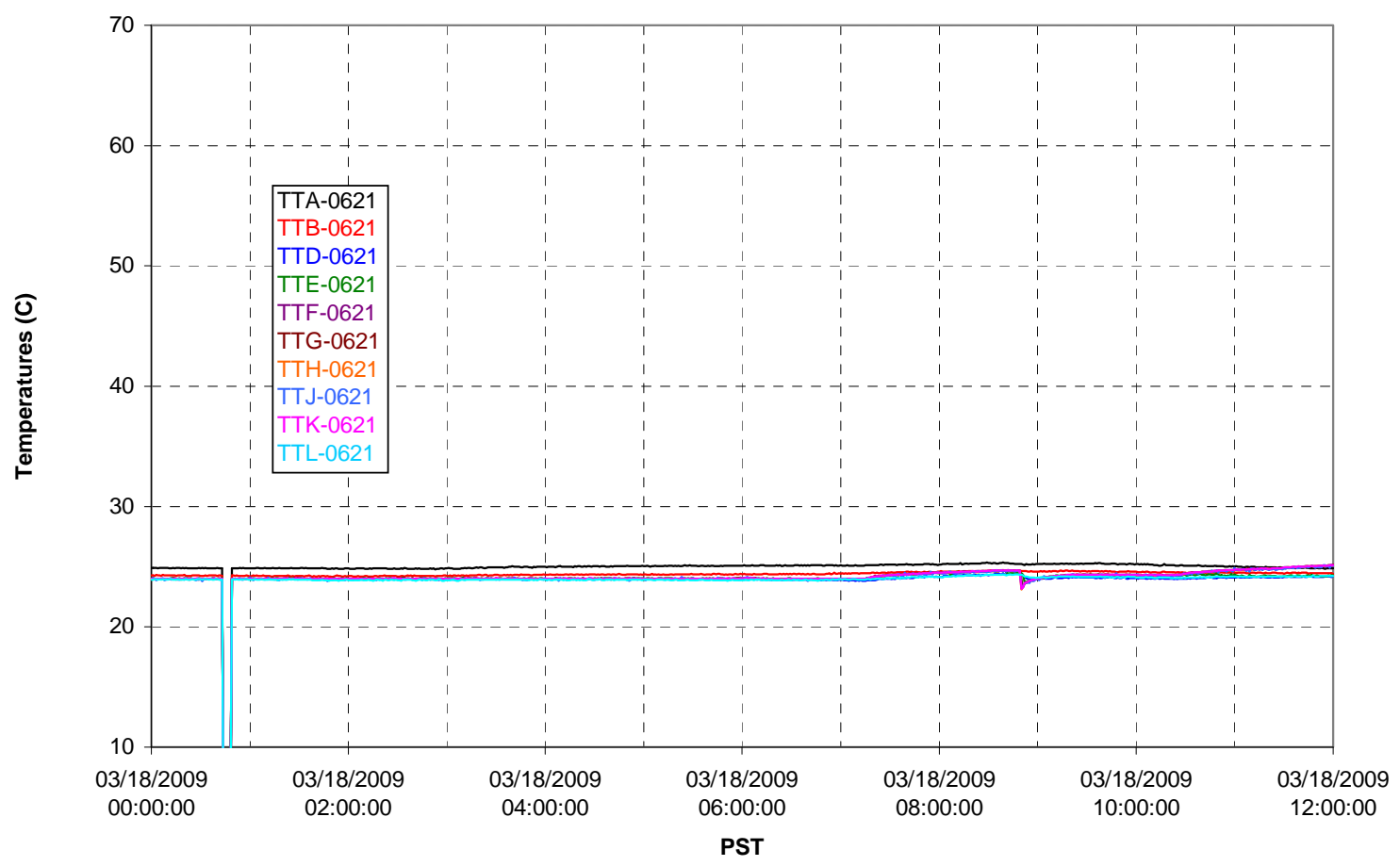


T02A temperatures

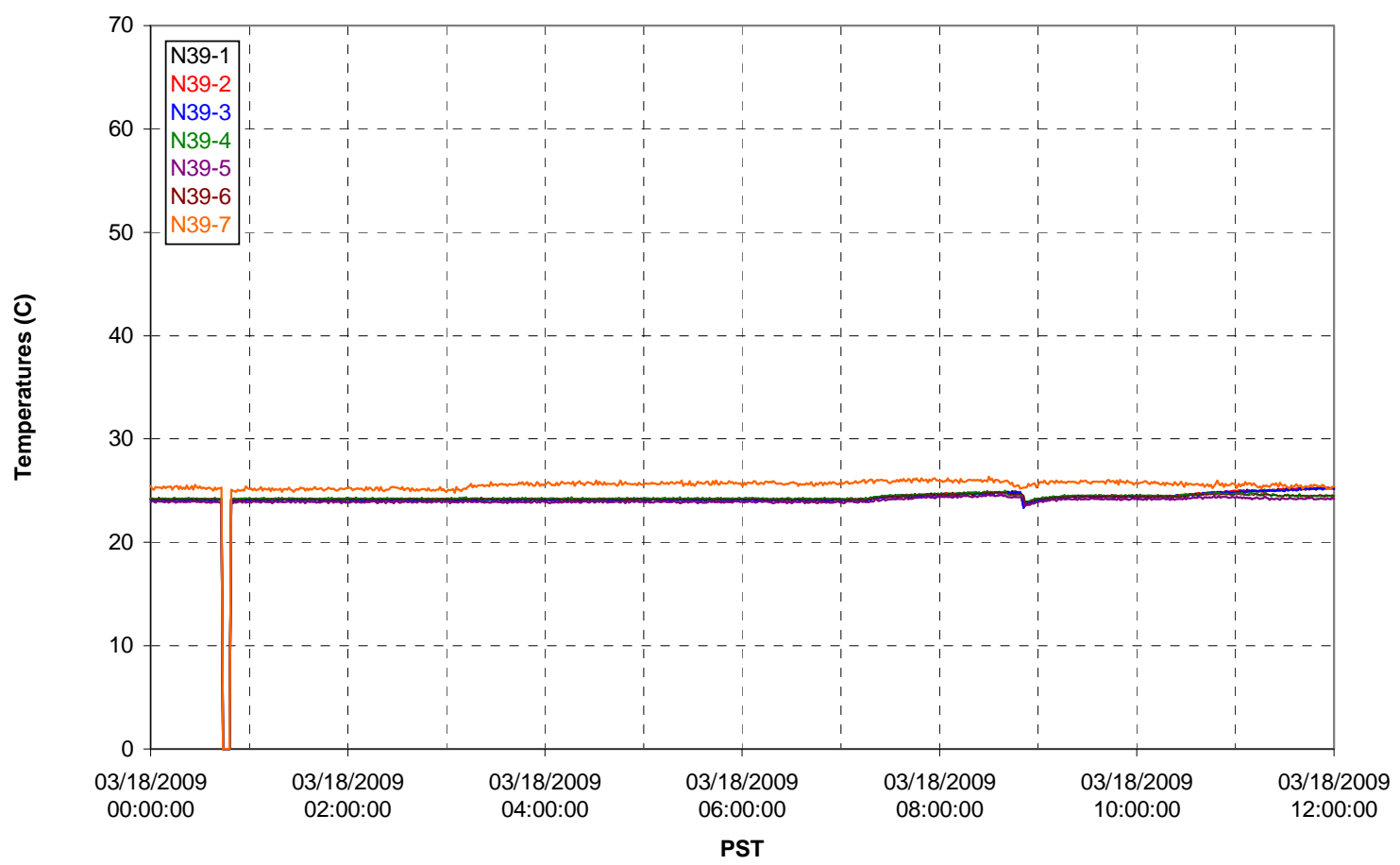

T02A temperatures

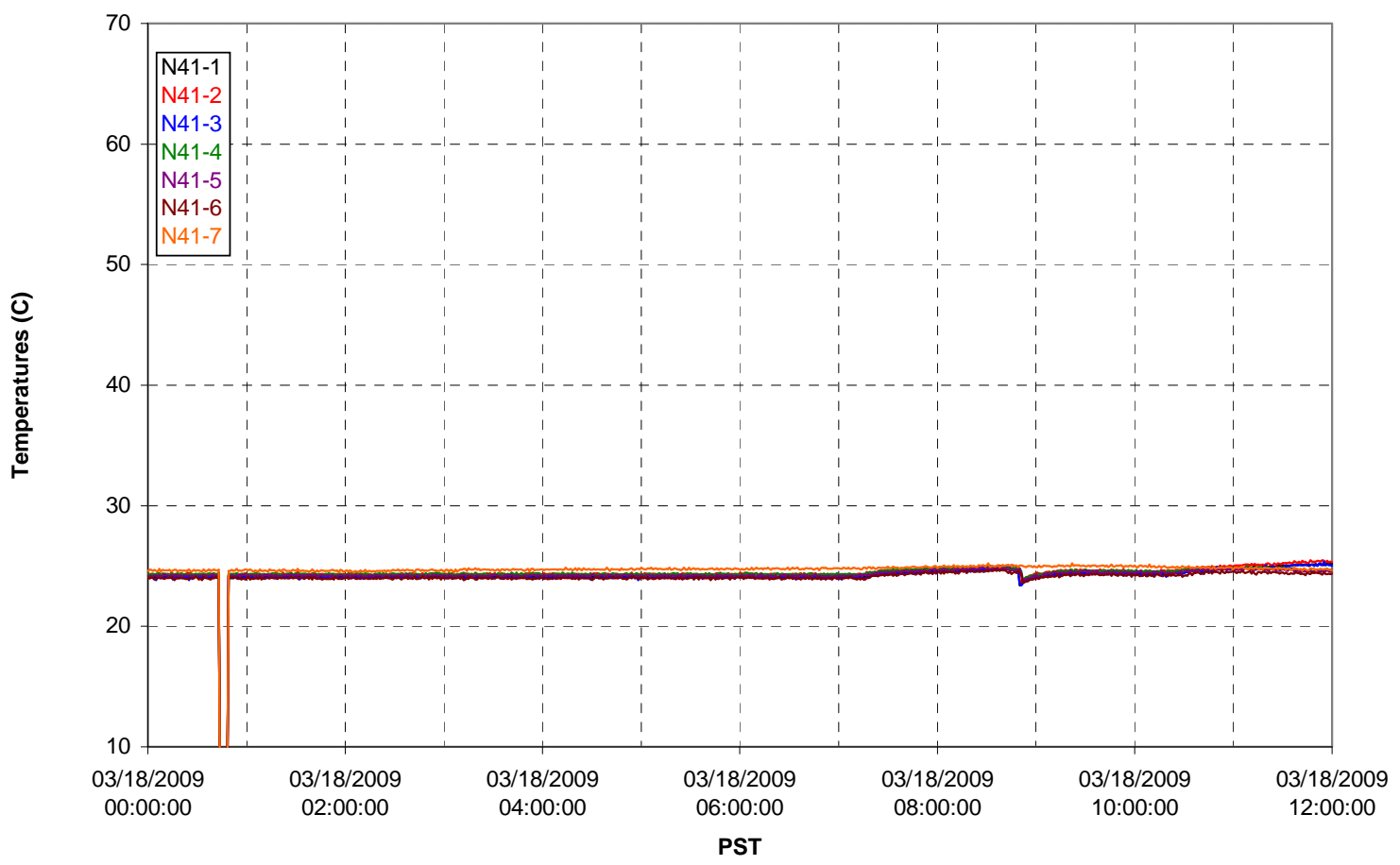


T02A temperatures

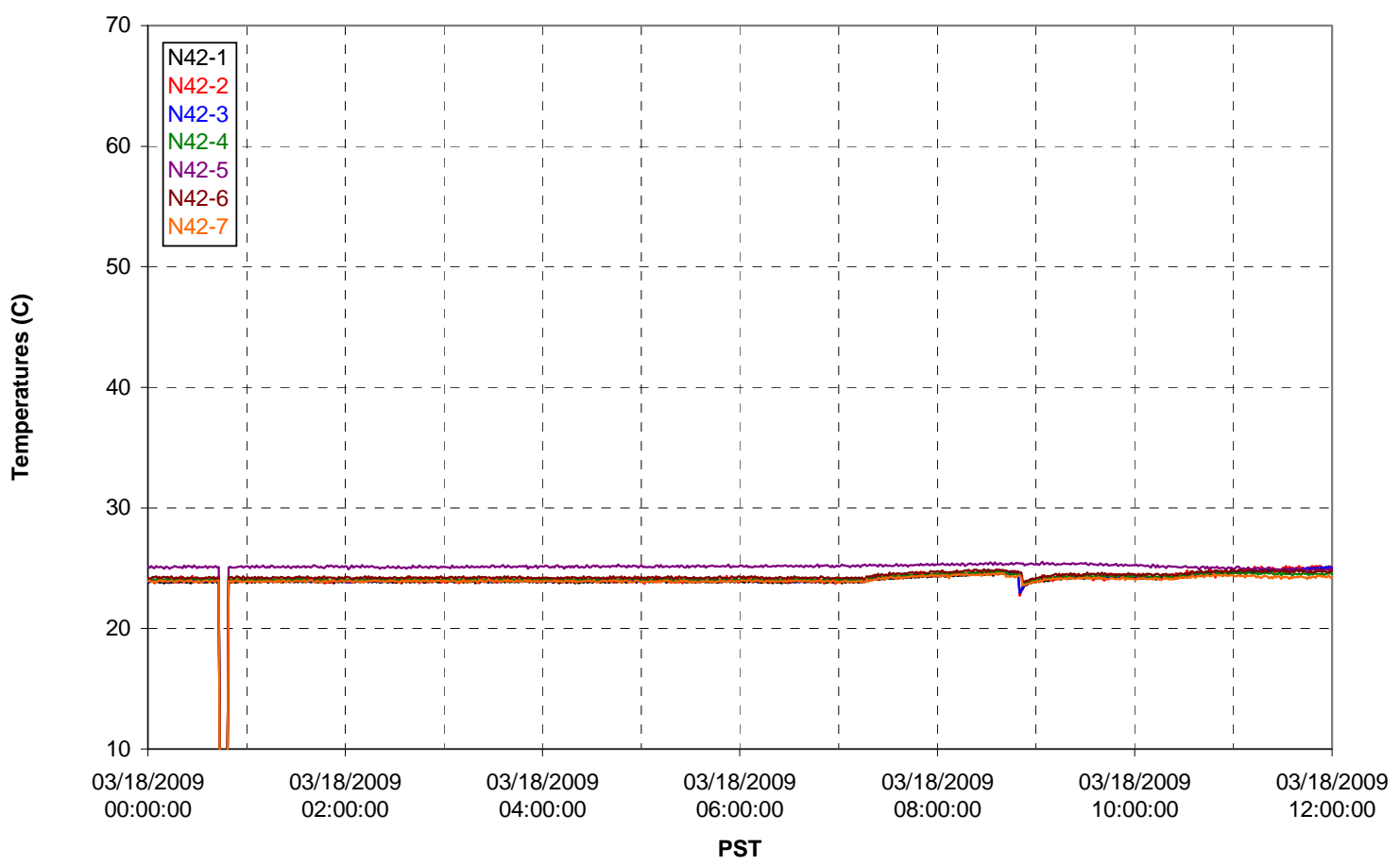

T02A temperatures

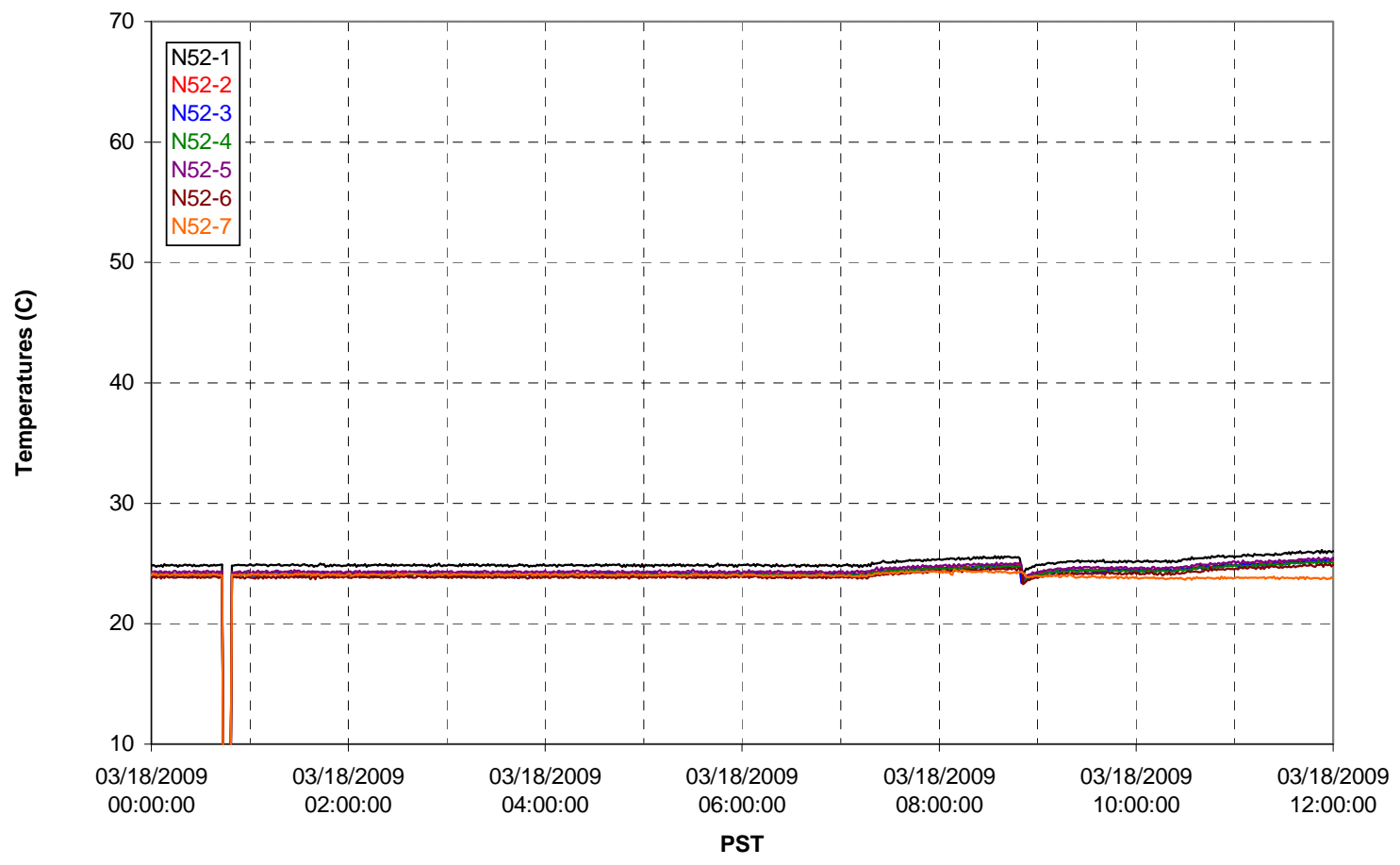


T02A Heating and Cooling
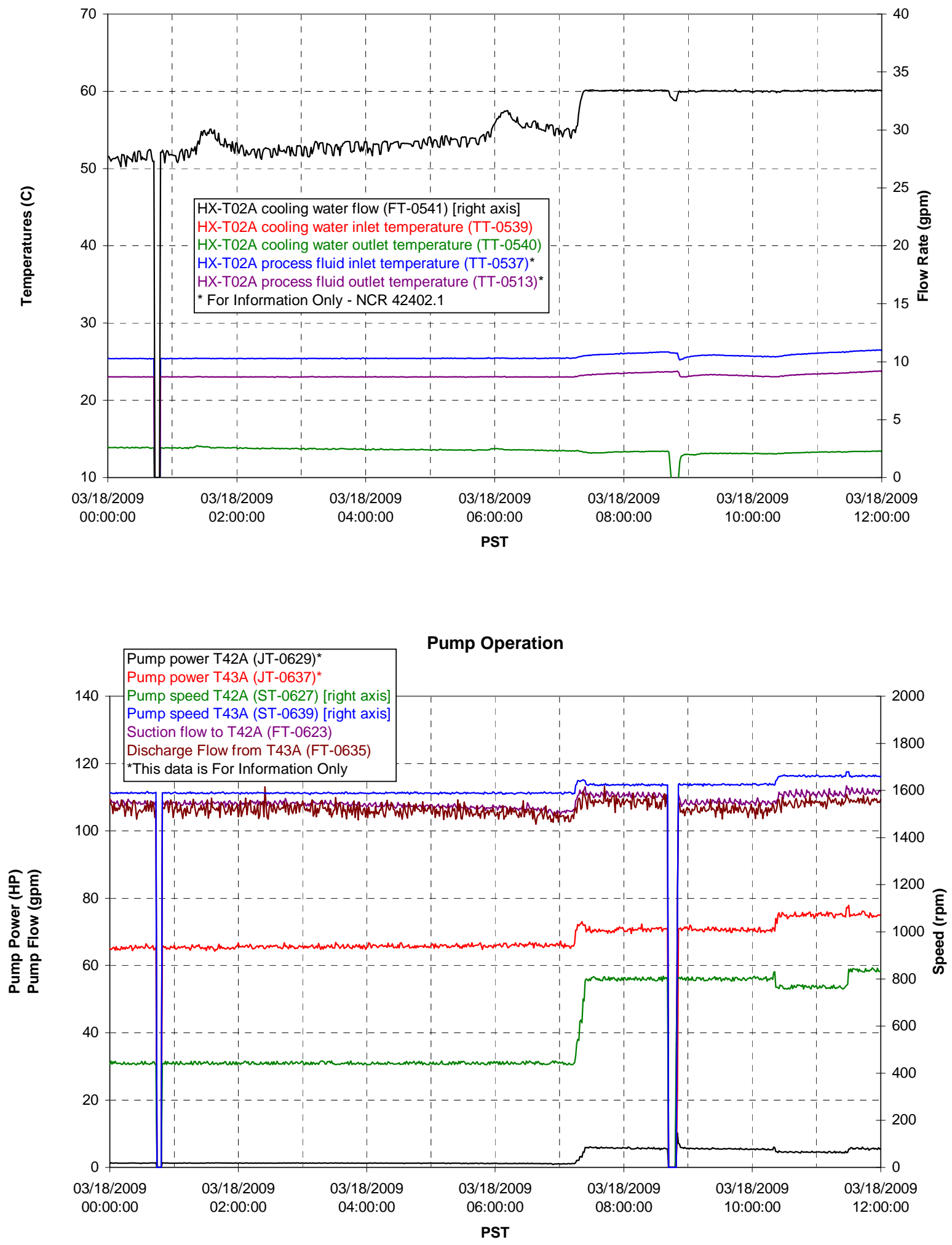
Pulsepot UFP-PP-T01A

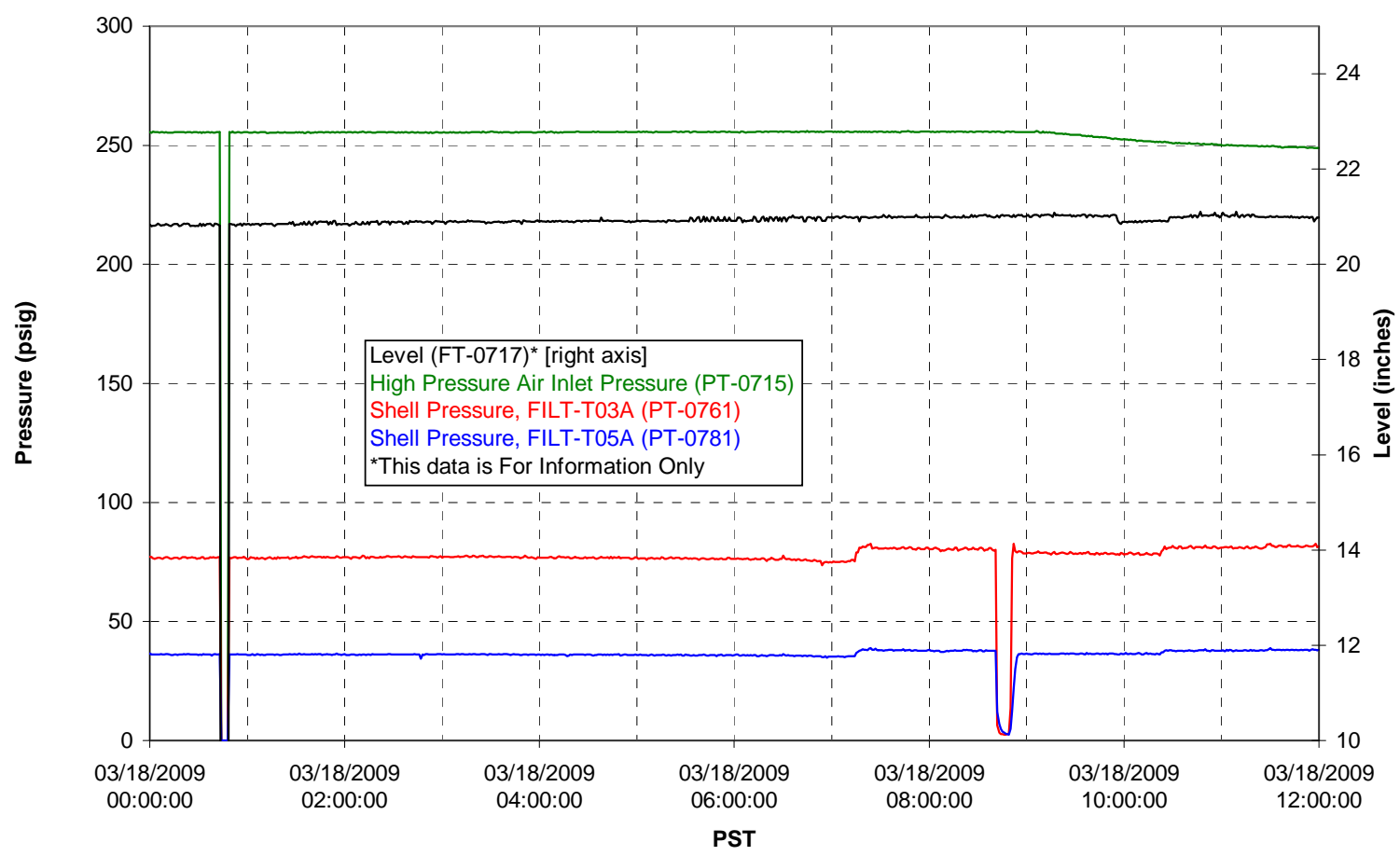

Pulsepot UFP-PP-T02A

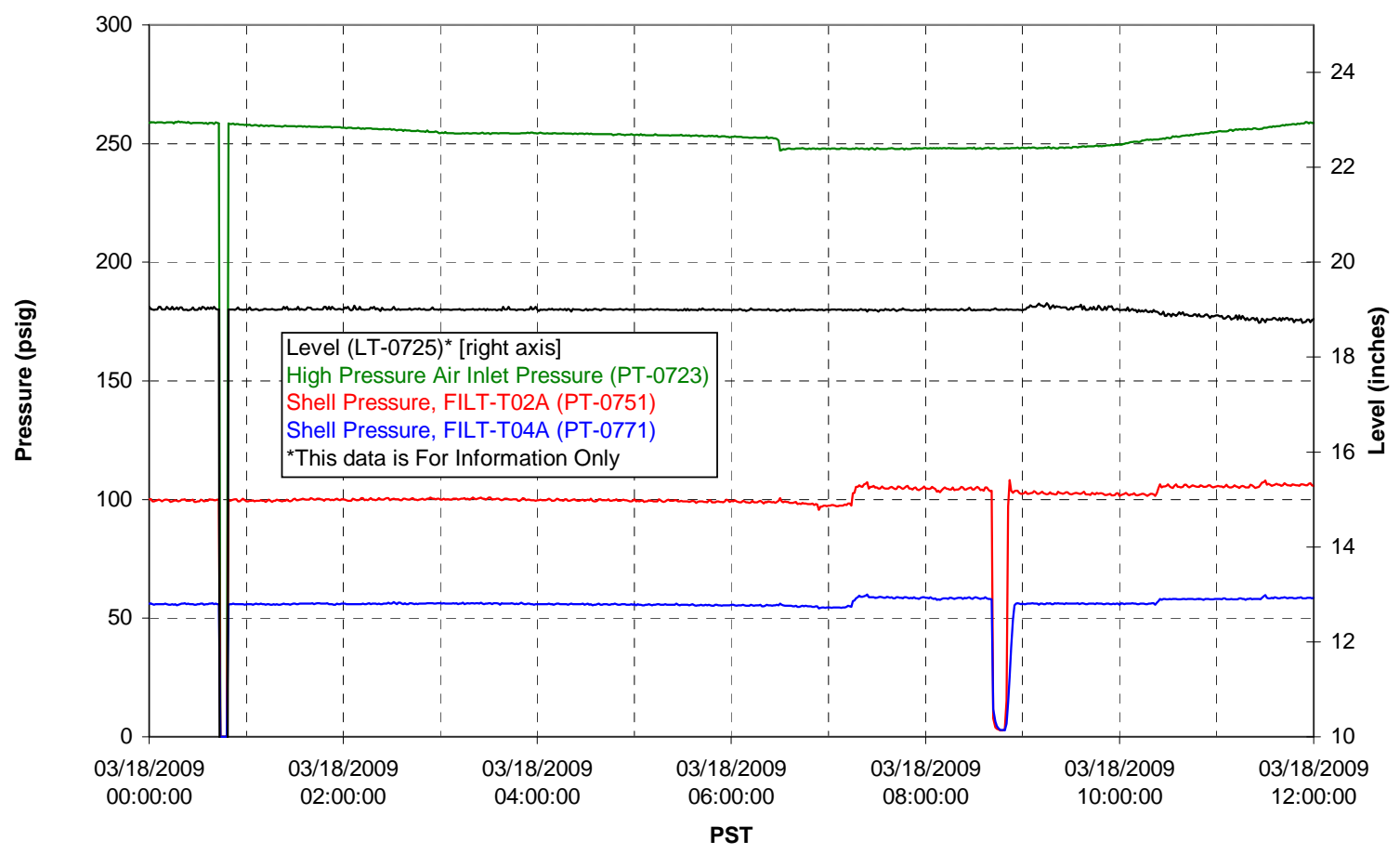


Pulsepot UFP-PP-T03A

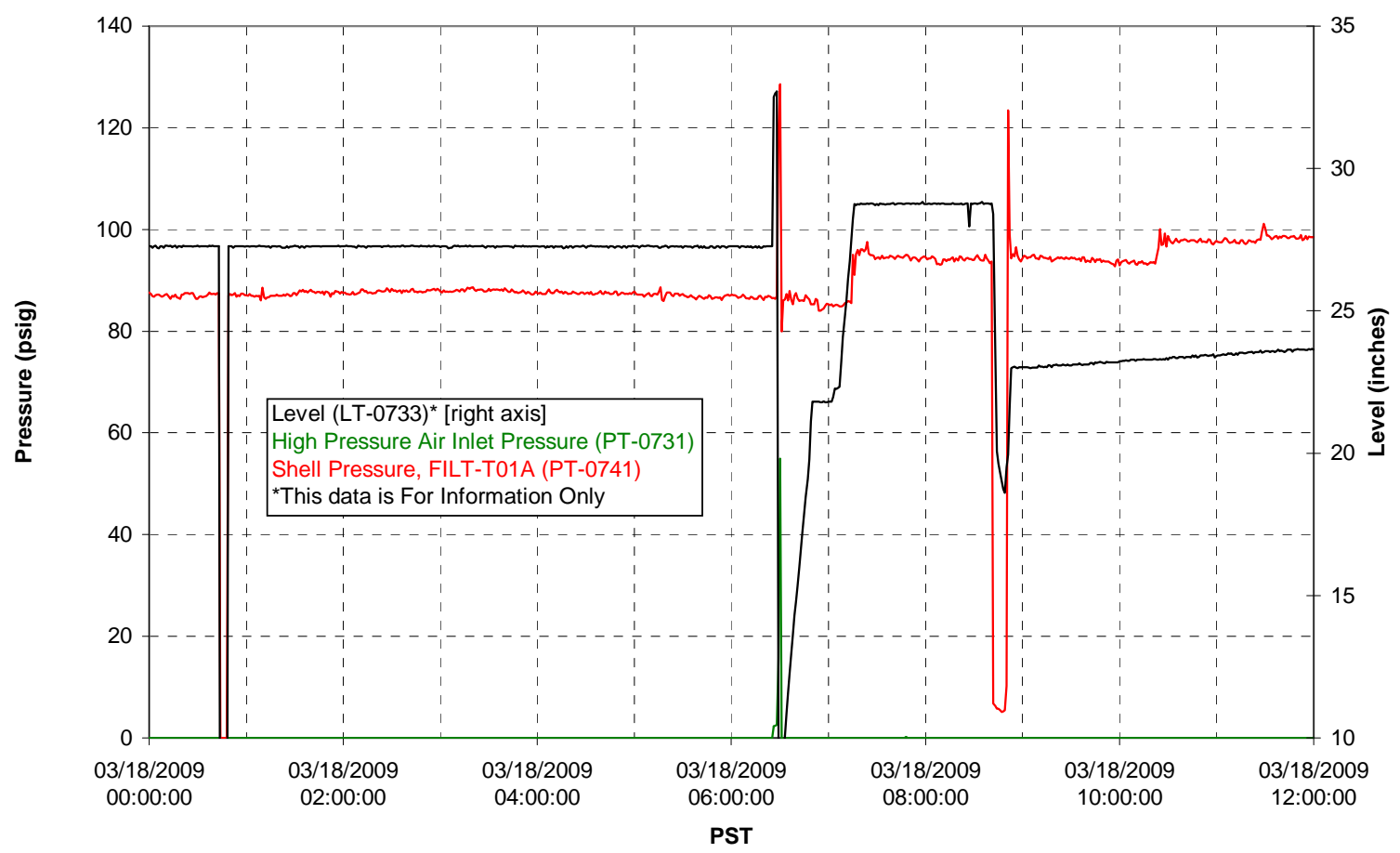

Pulsepot Levels

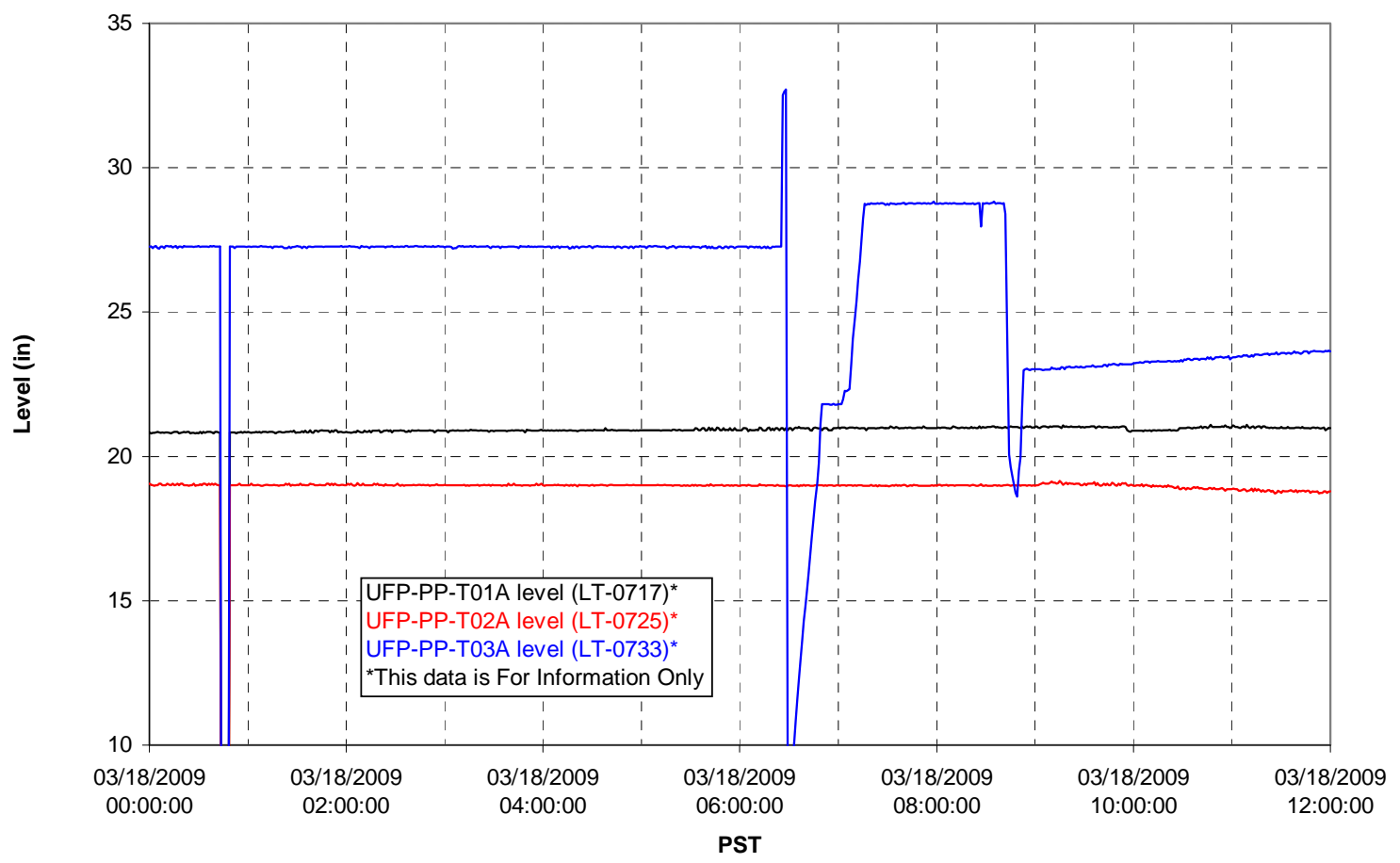


Filter UFP-FILT-T01A

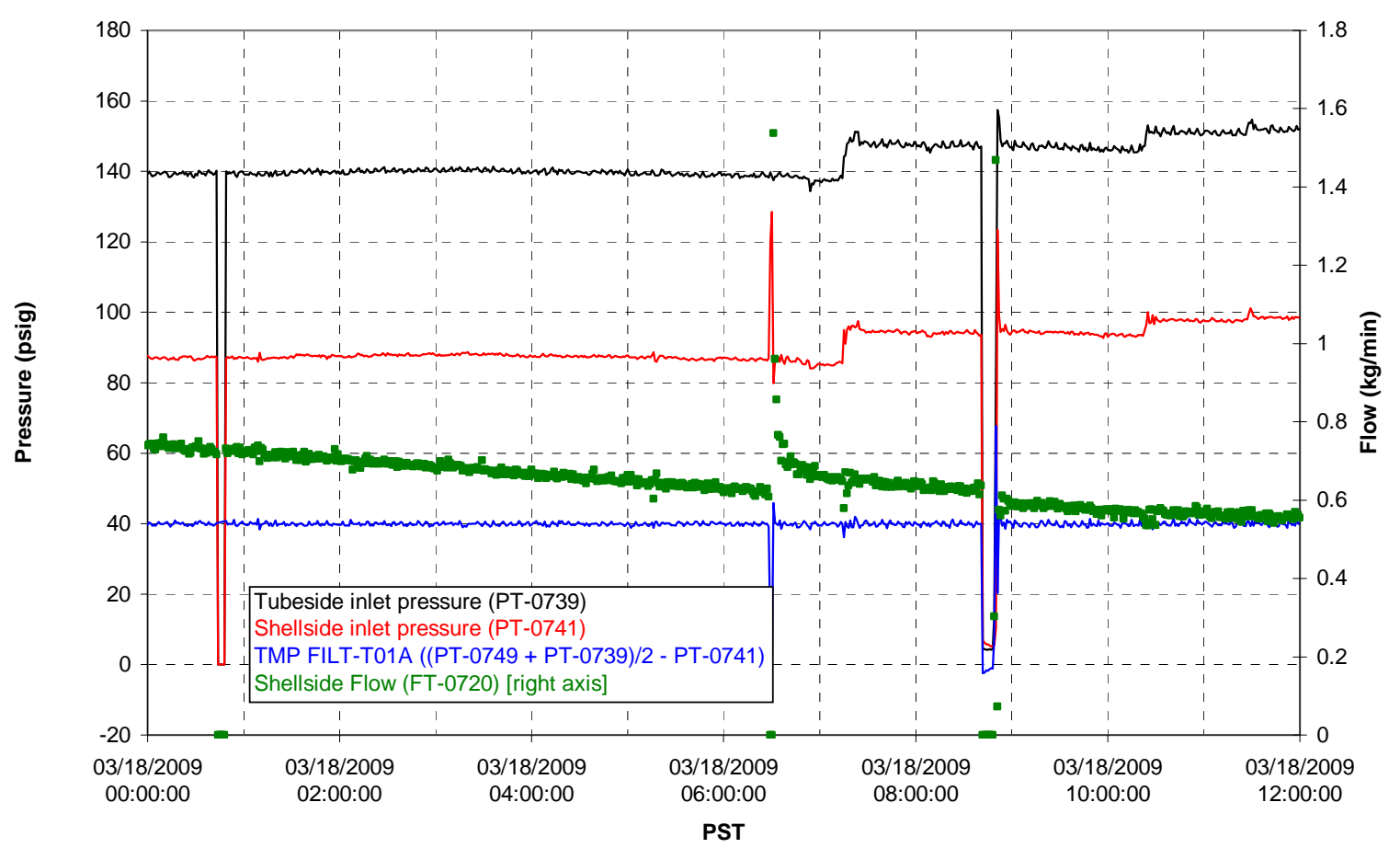

Filter UFP-FILT-T02A

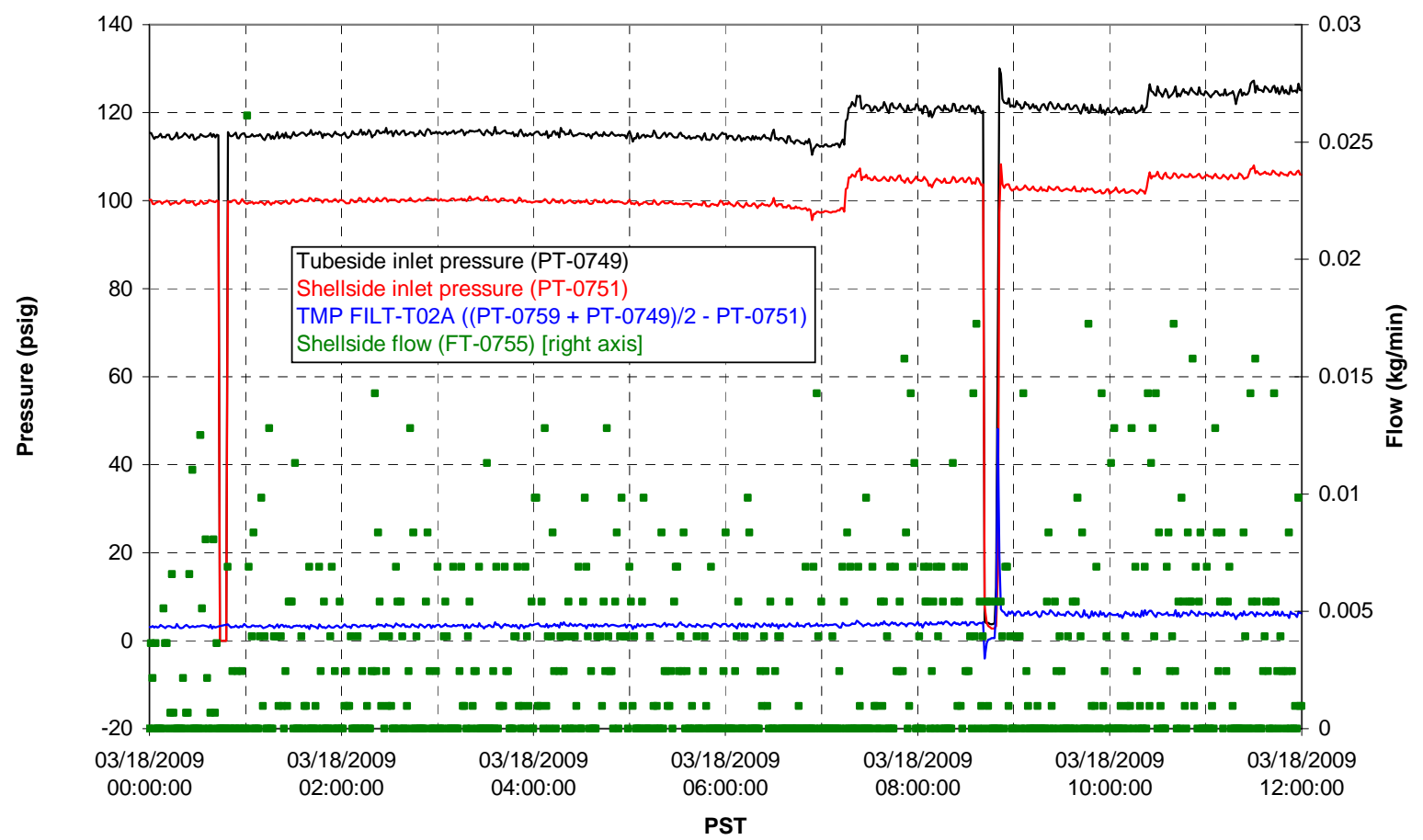


Filter UFP-FILT-T03A

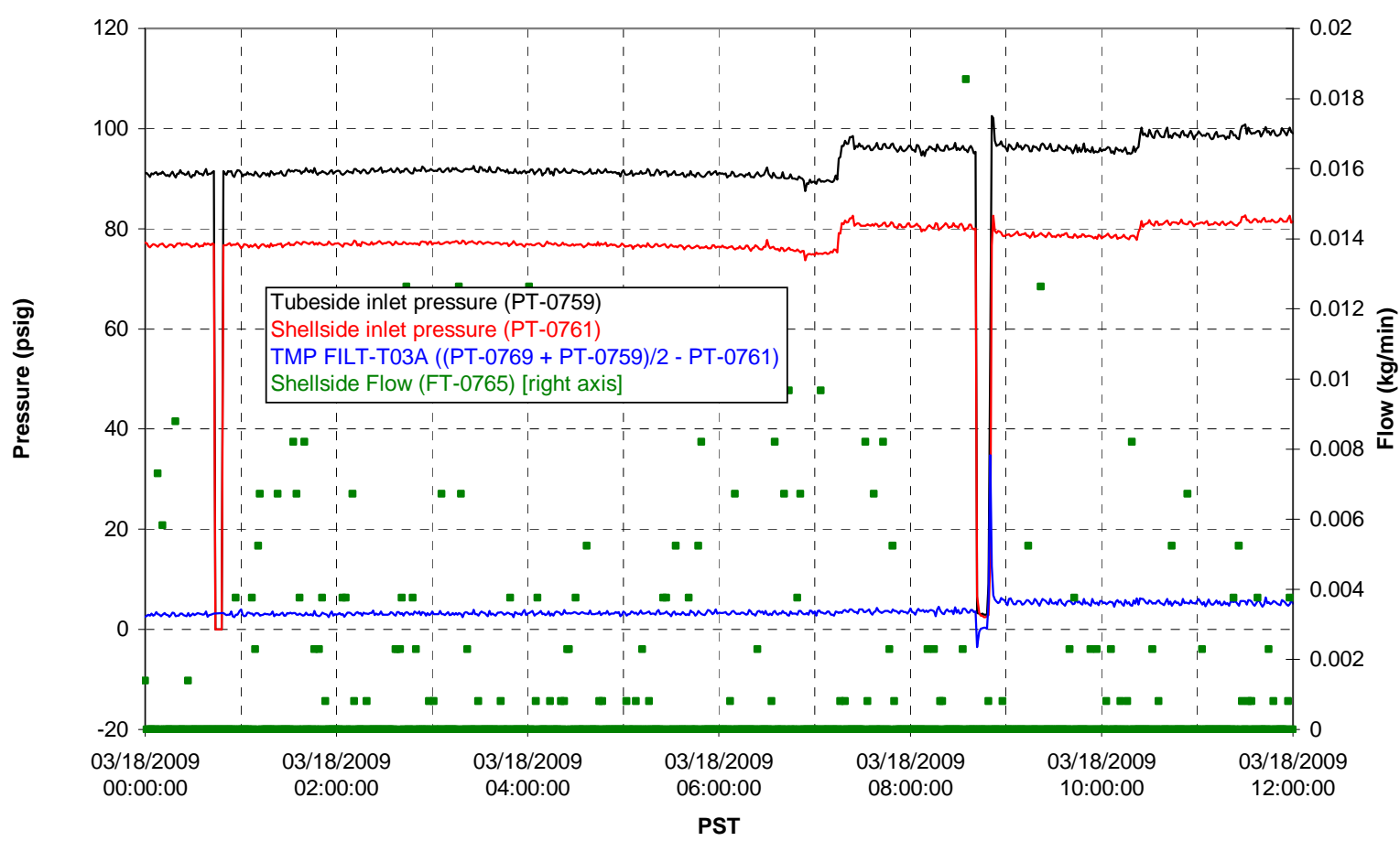

Filter UFP-FILT-T04A

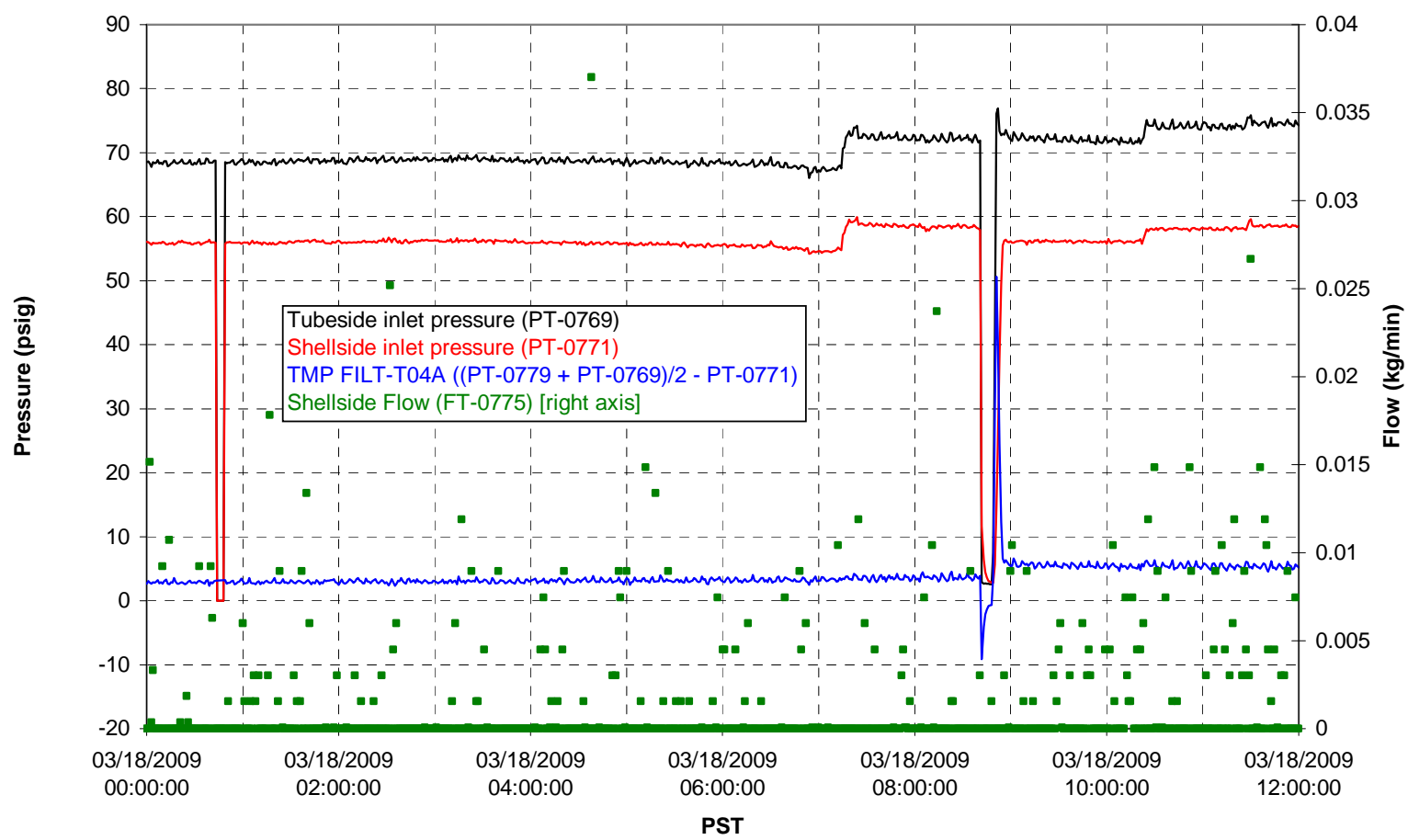


Filter UFP-FILT-T05A

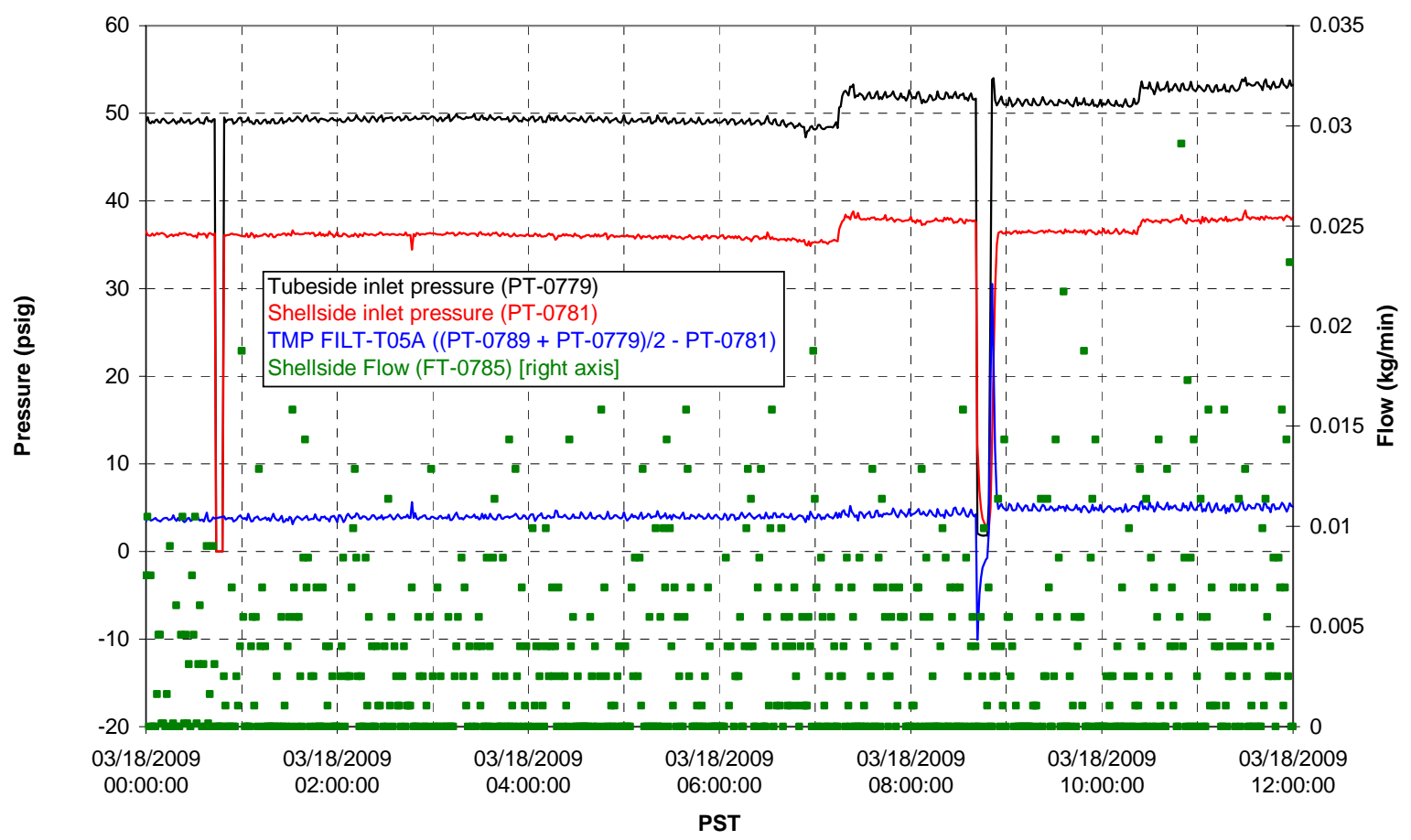

Chemical Flow

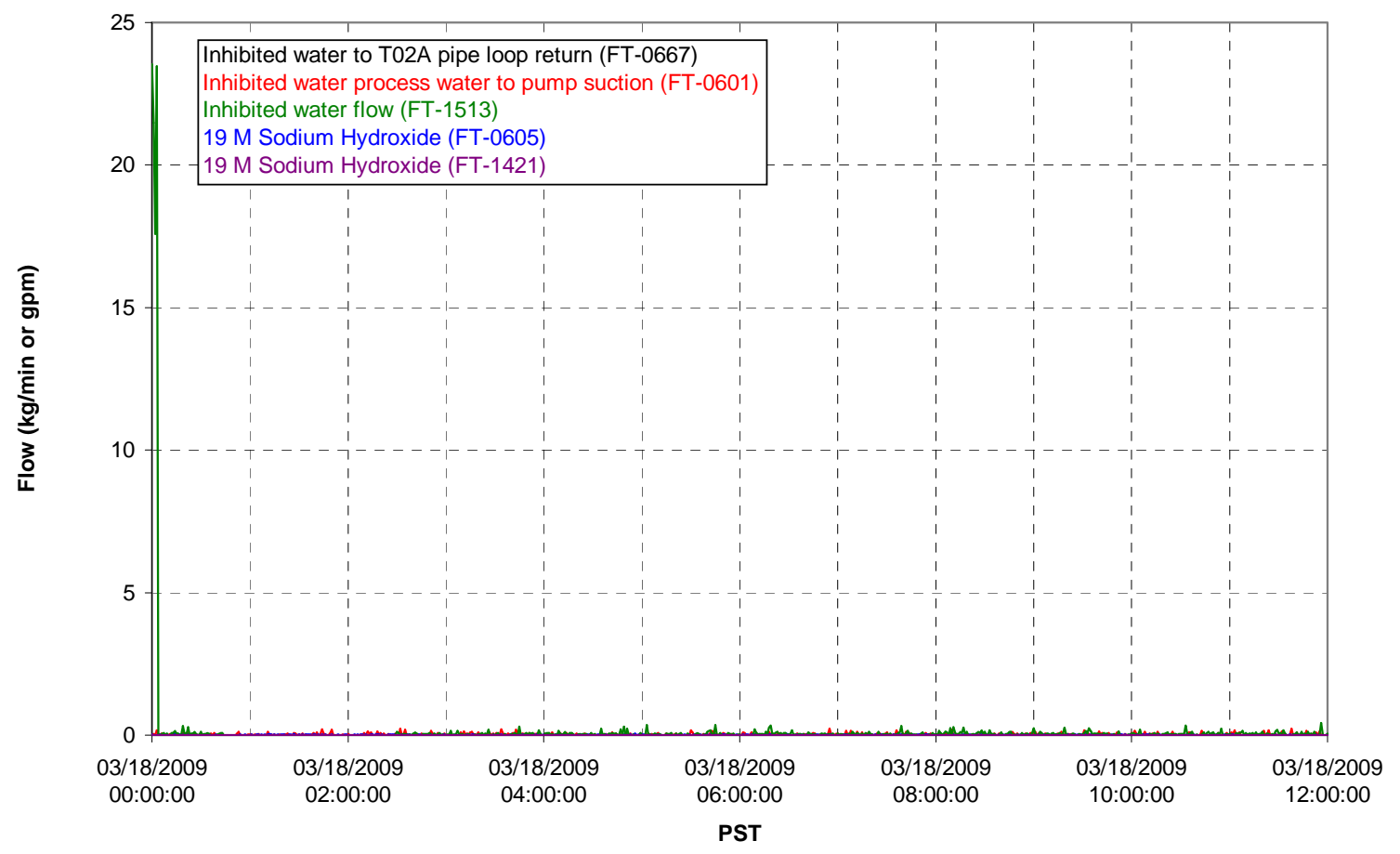


Chemical Flow

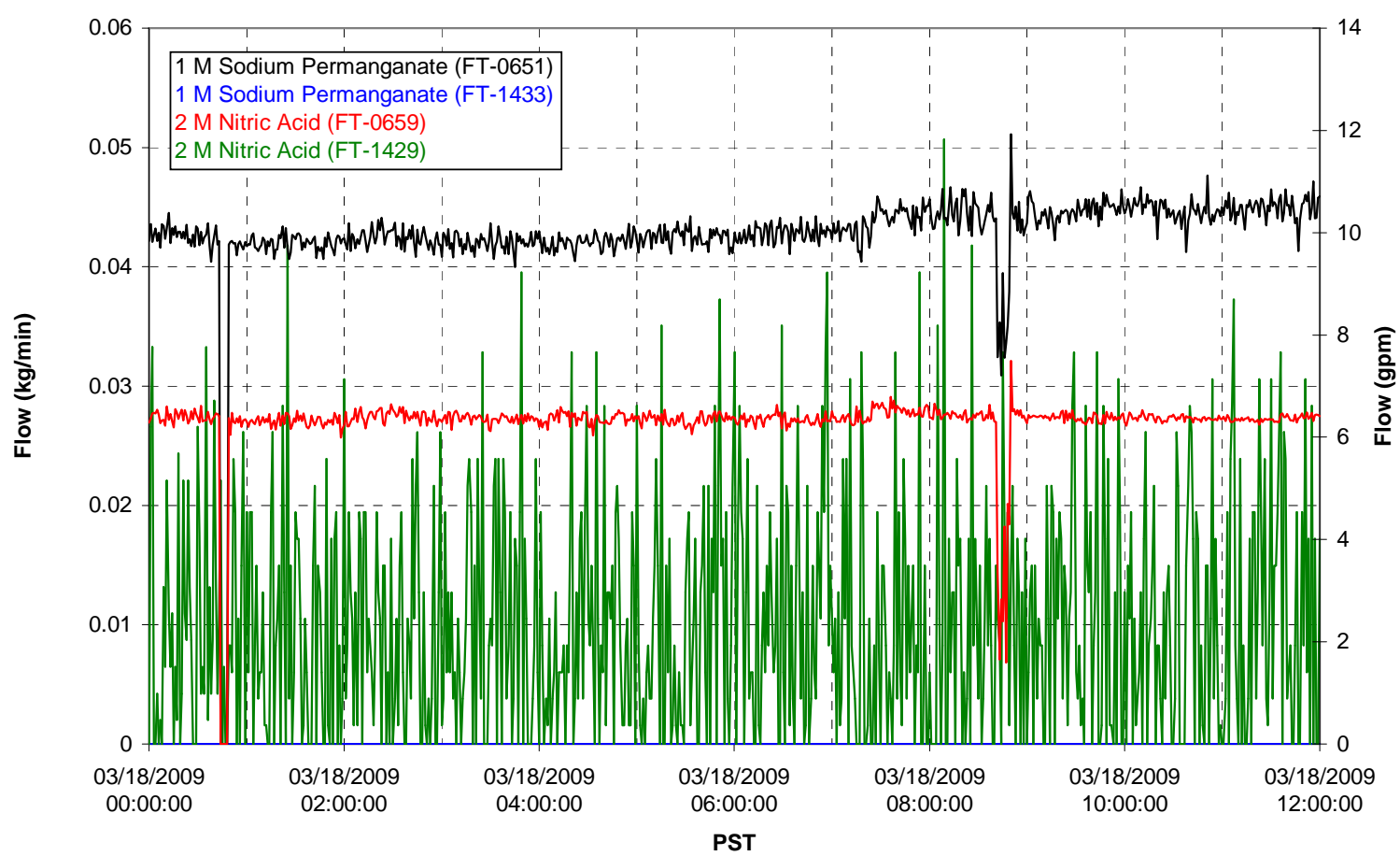

Air Flows

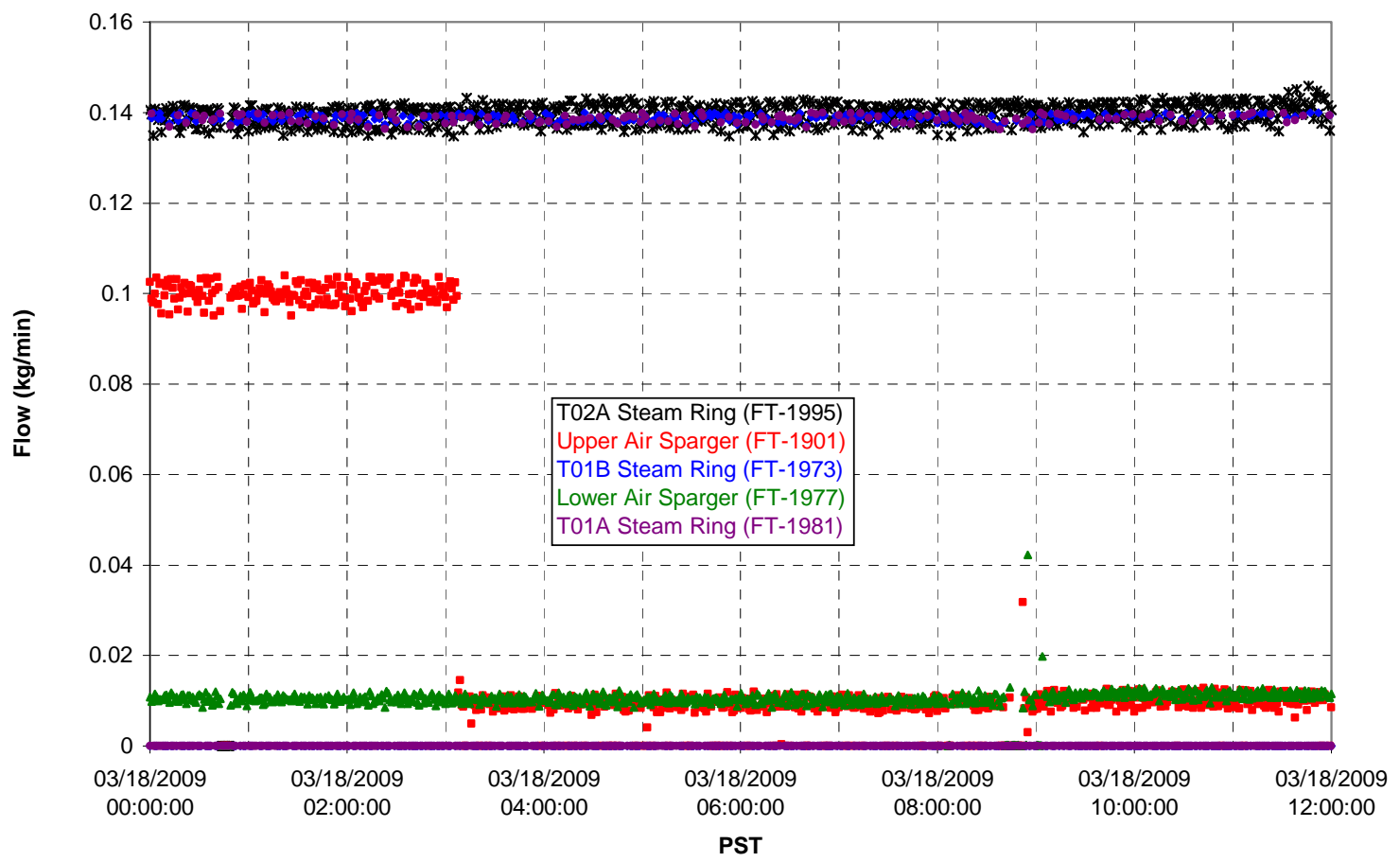


T02A Steam

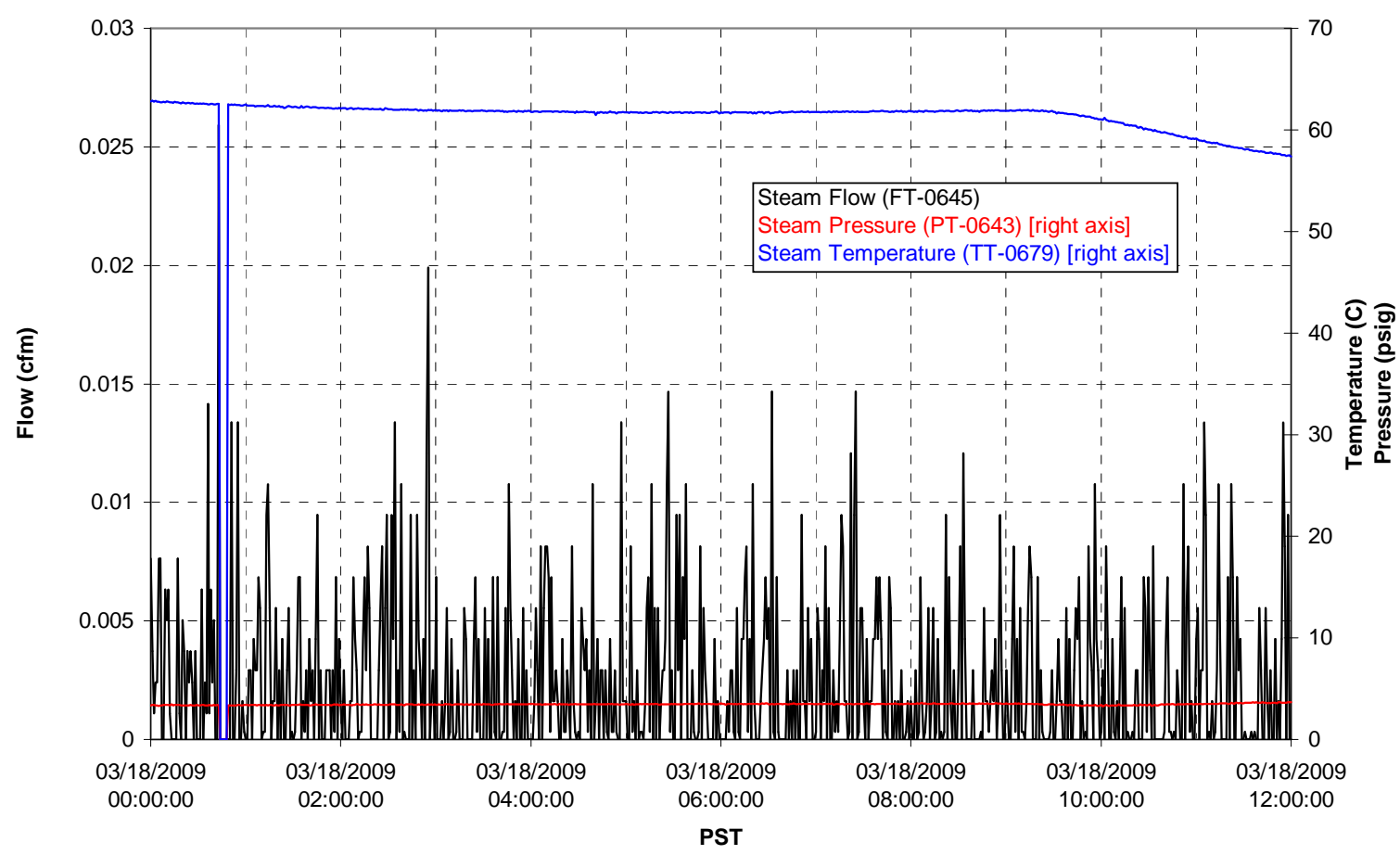

T01A Steam

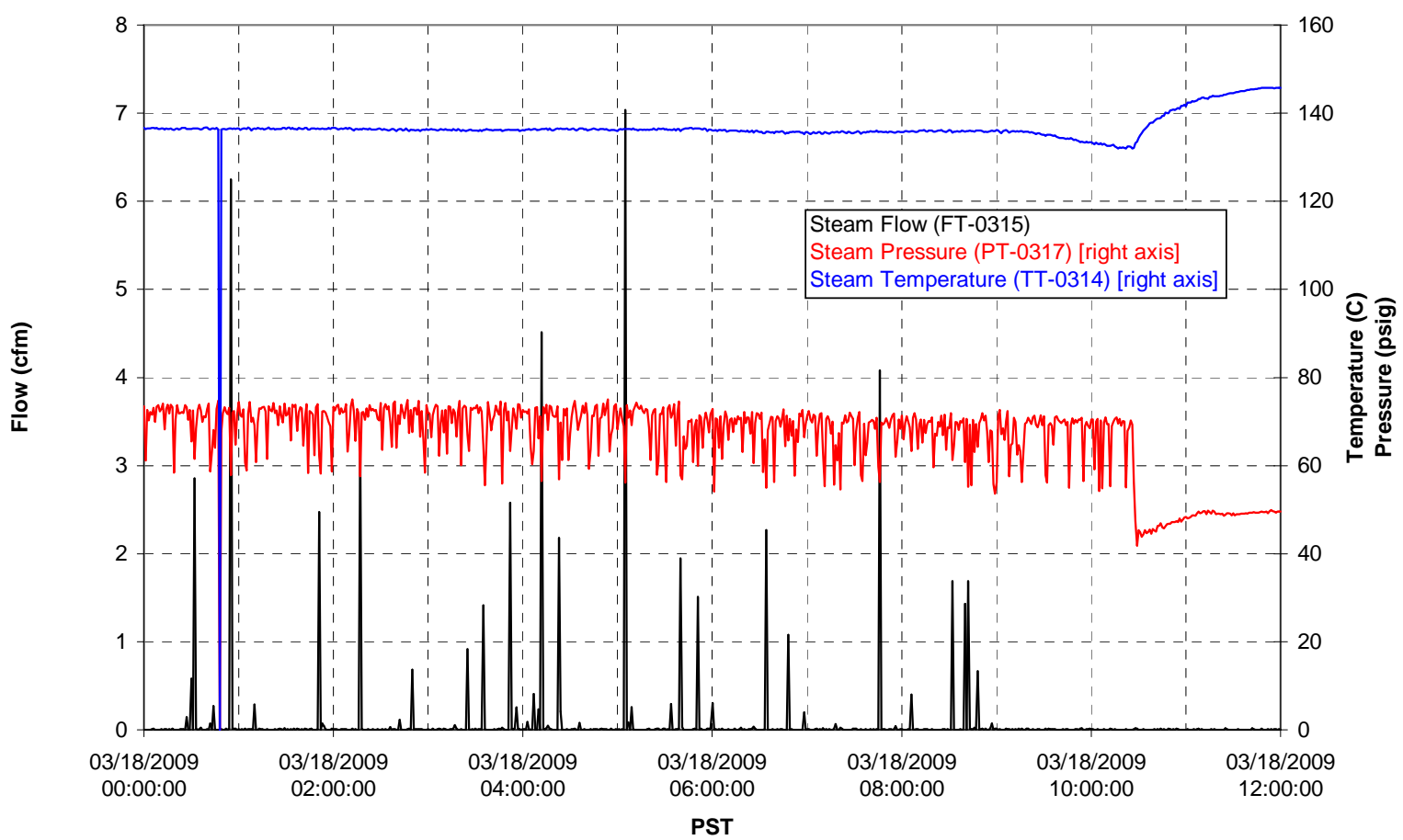


T01B Steam

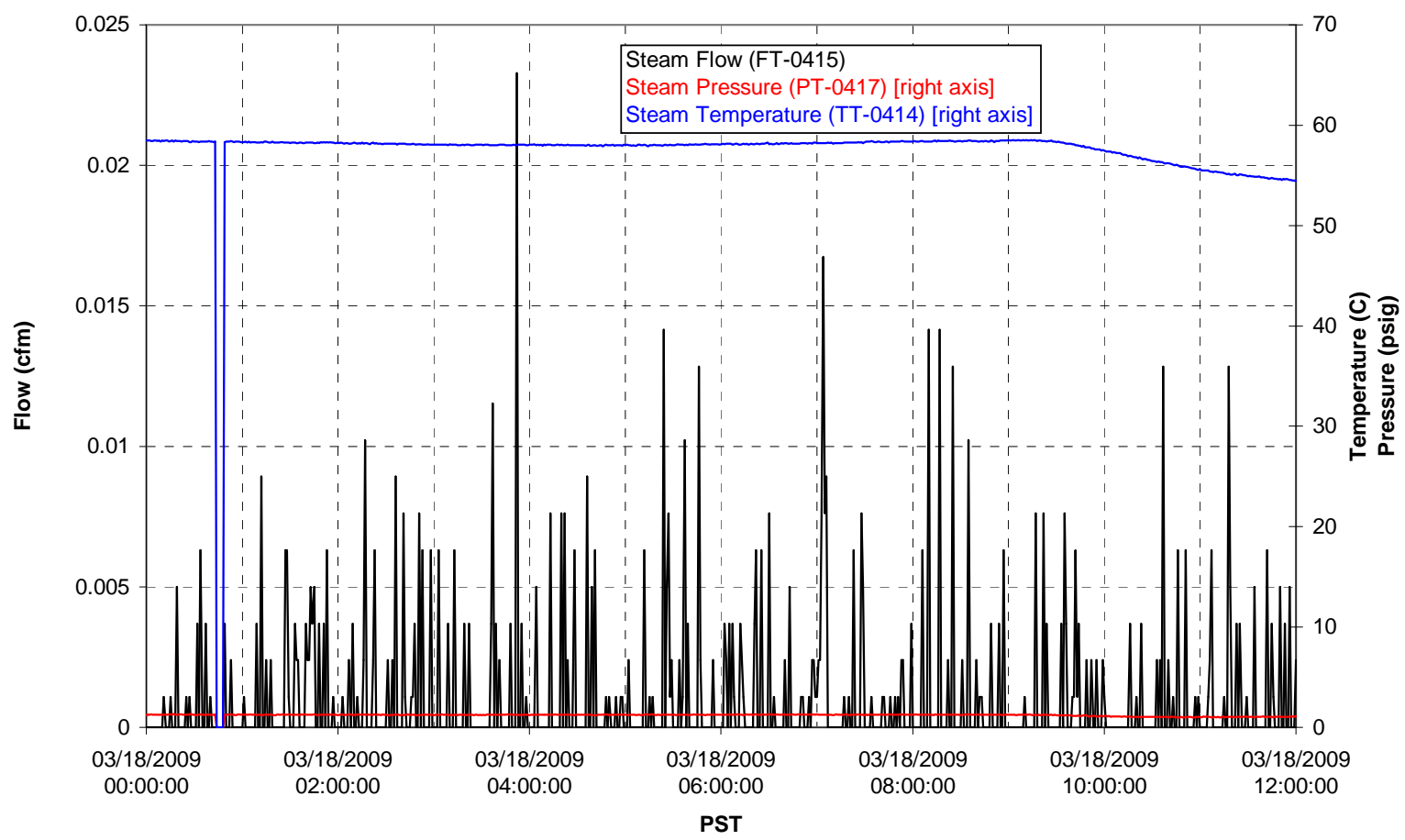




\section{Integrated Test B Data Plots \\ 03/18/09 12:00 - 03/19/09 00:00}


T01A level

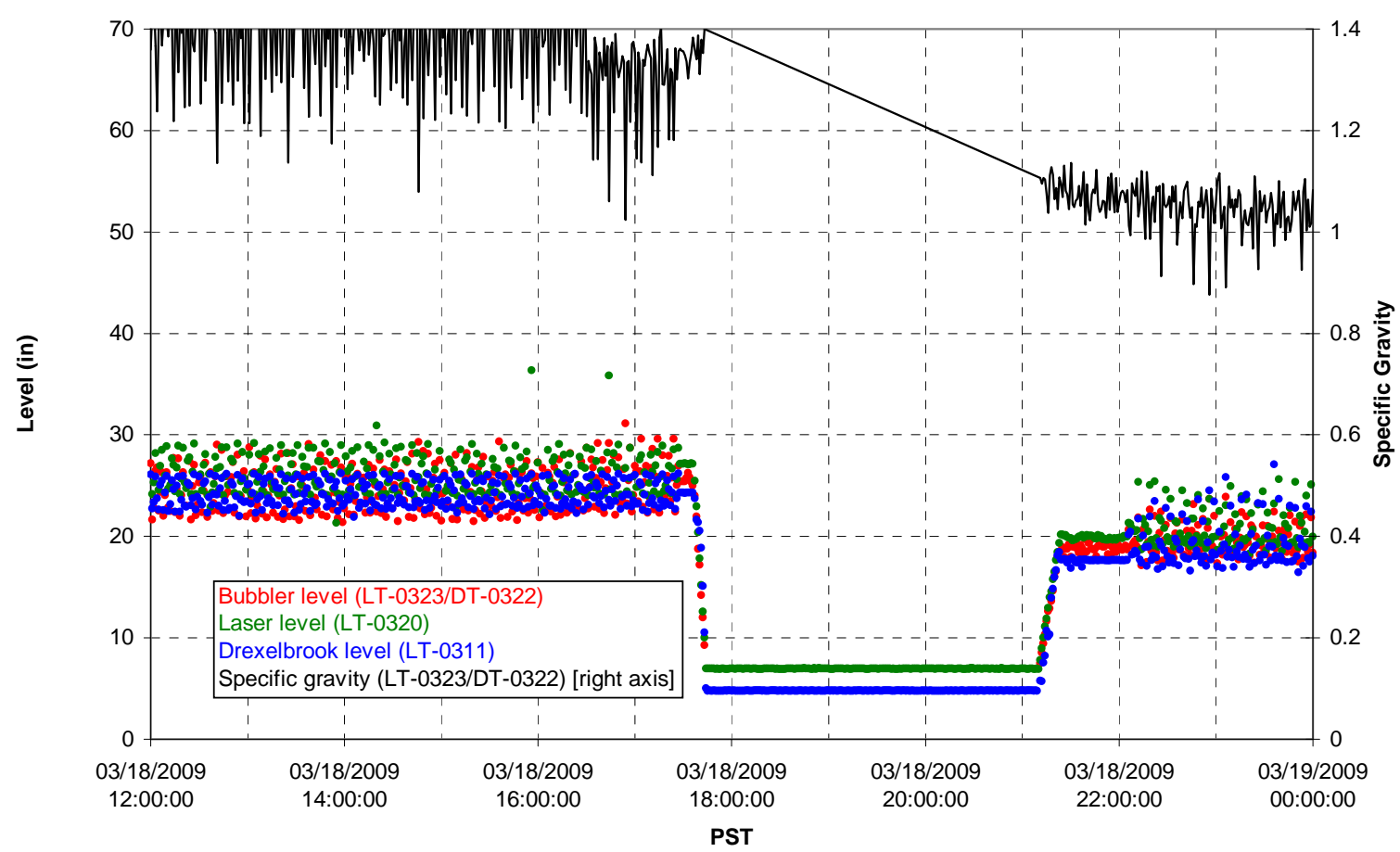

T01A temperatures

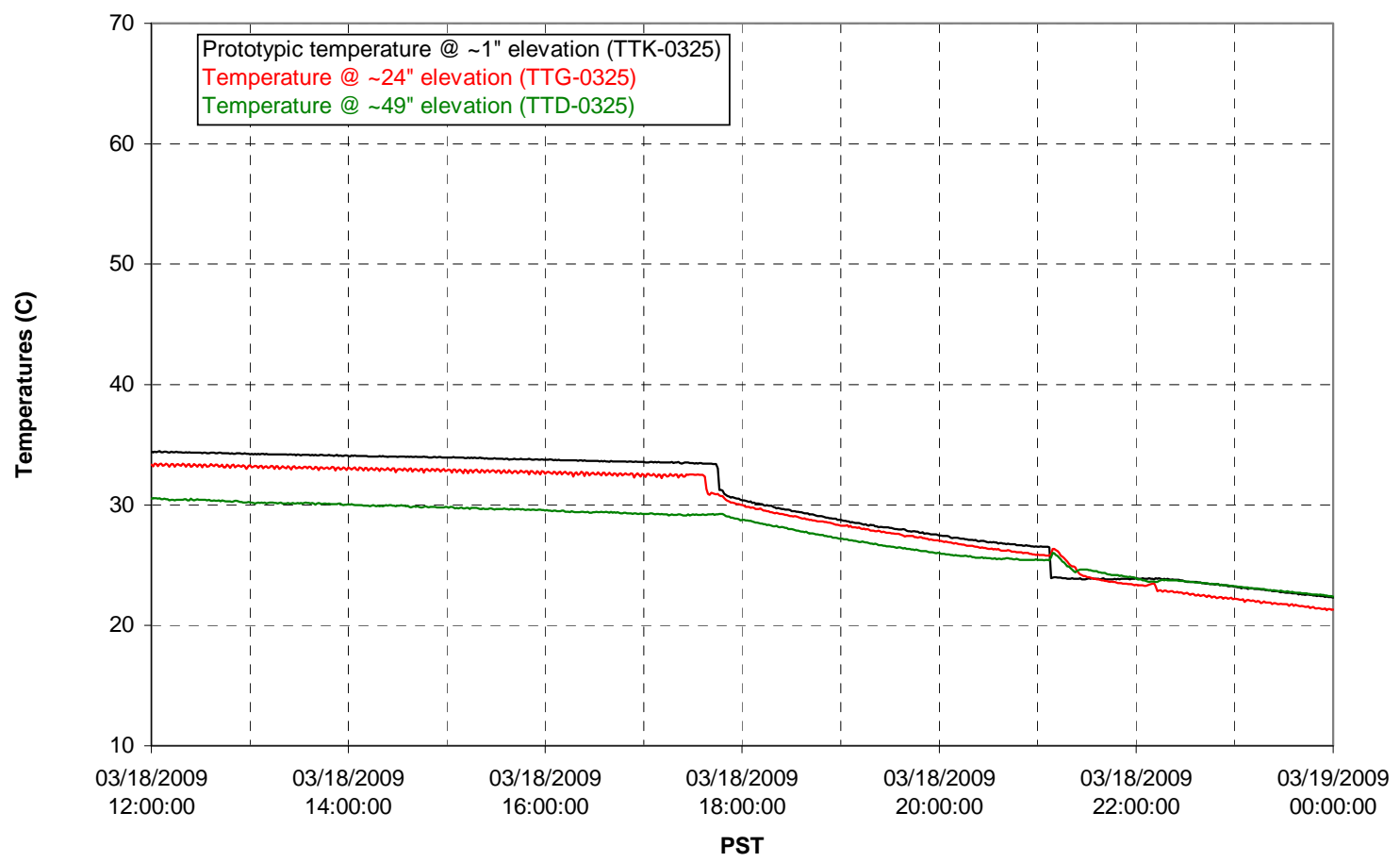


T01B level

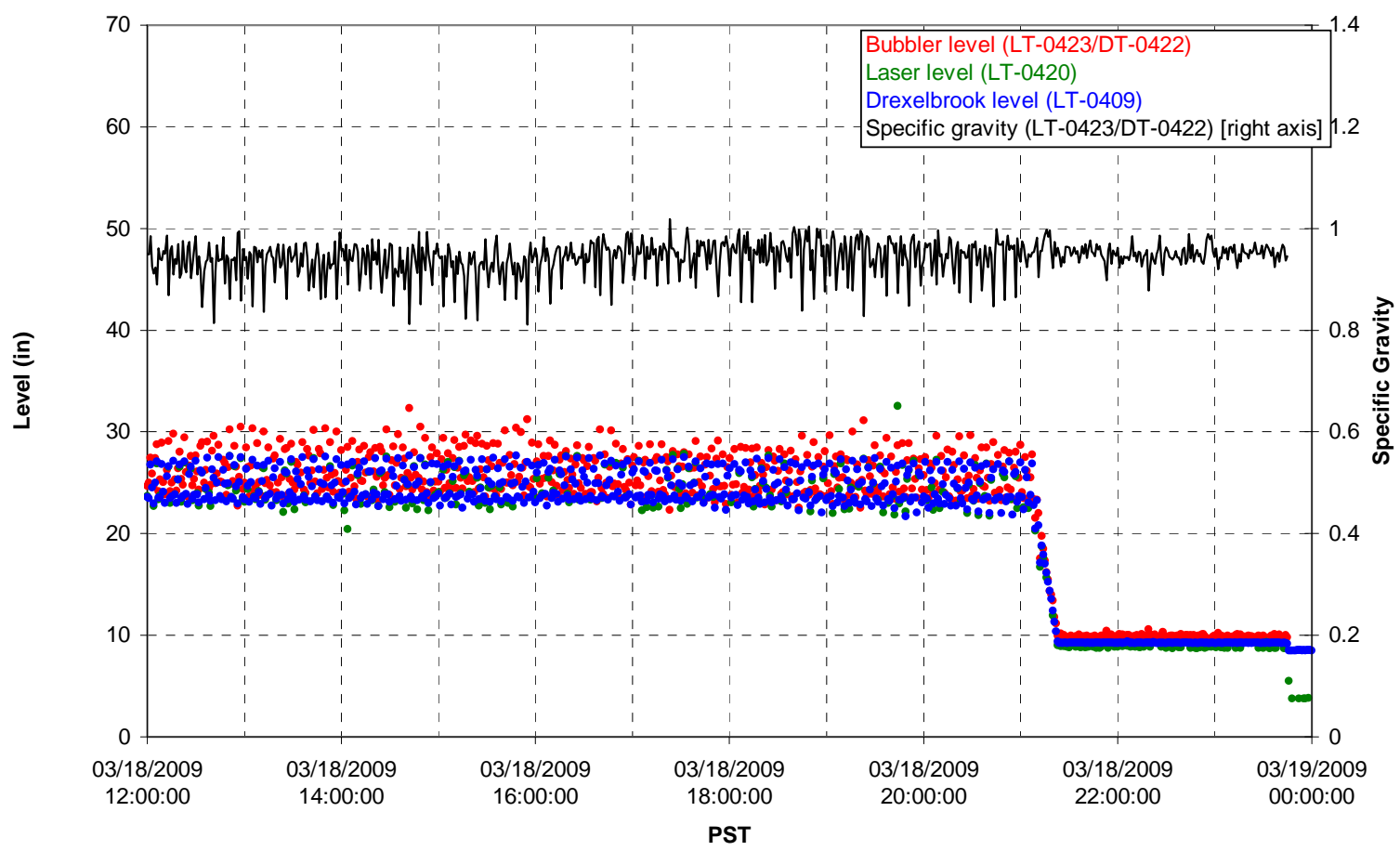

T01B temperatures

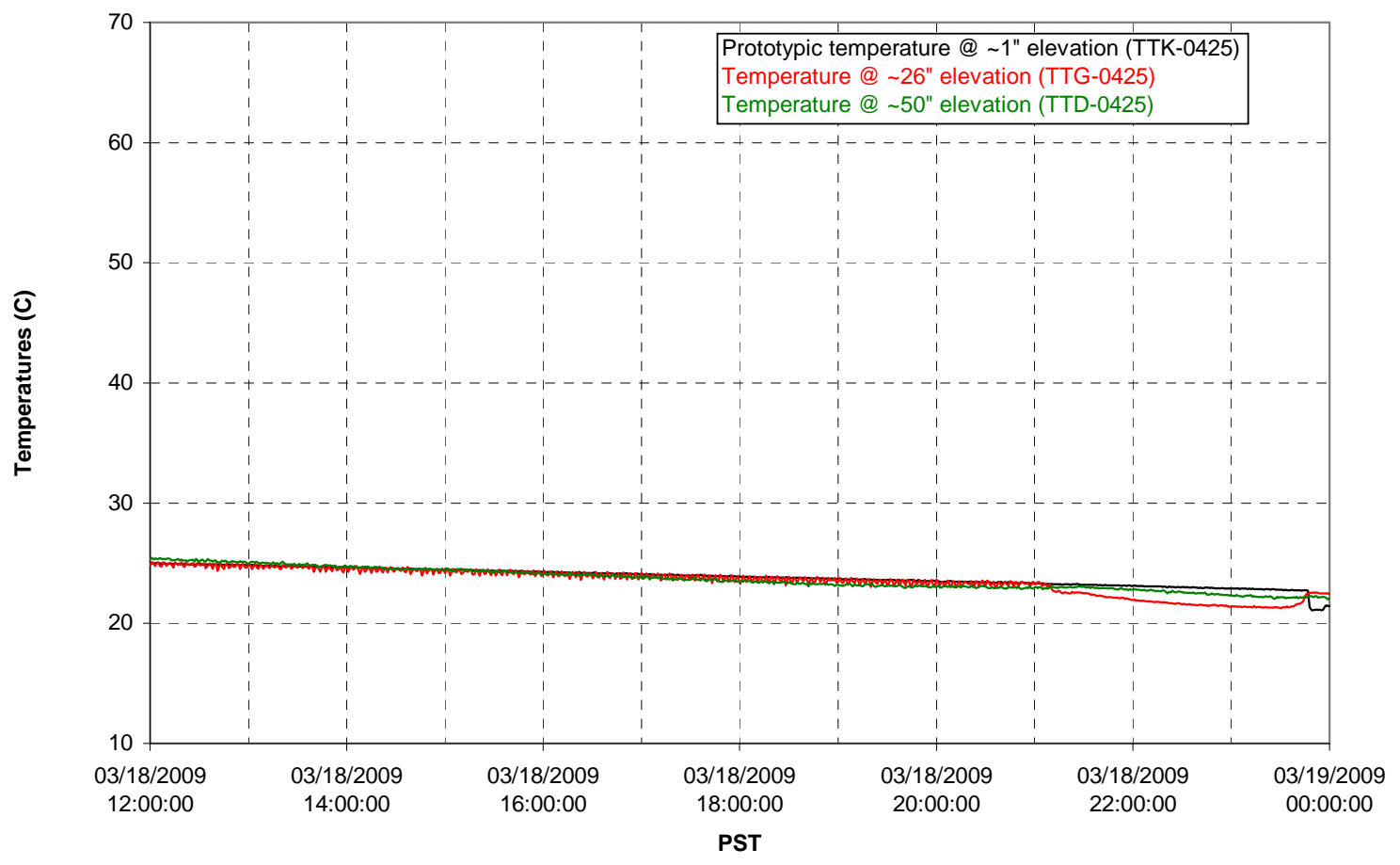


T02A level

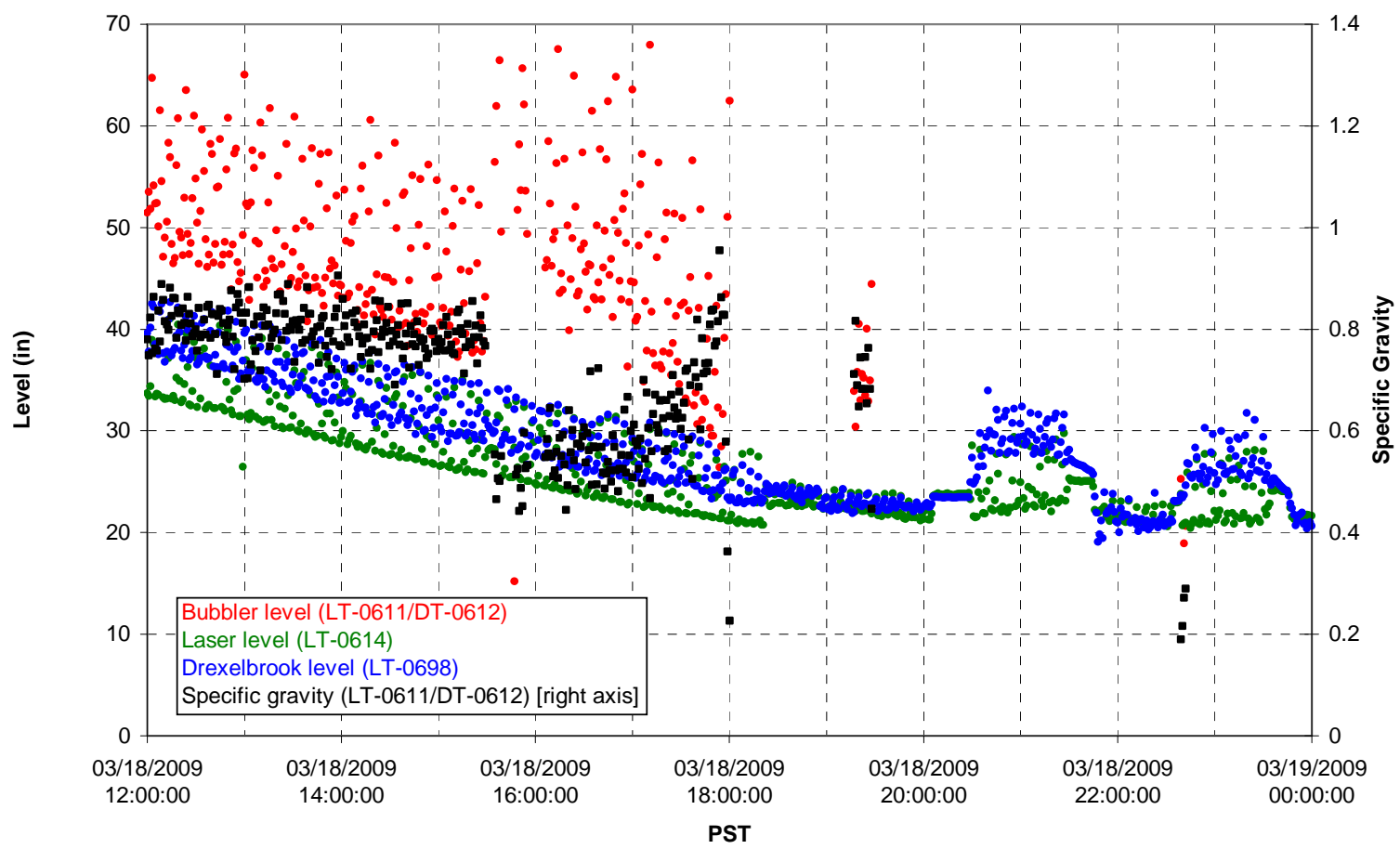

T02A temperatures

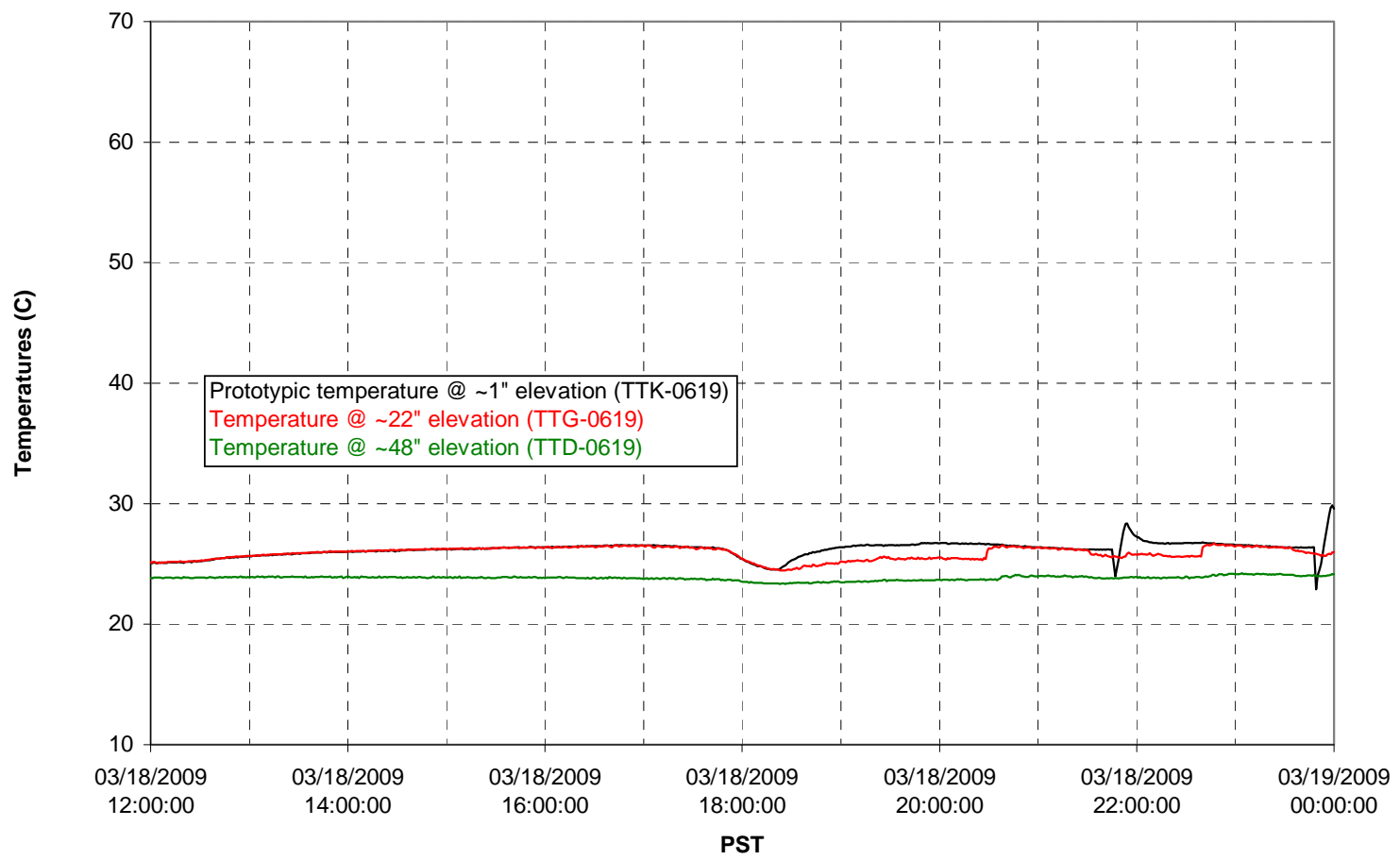


T02A and filter loop temperatures

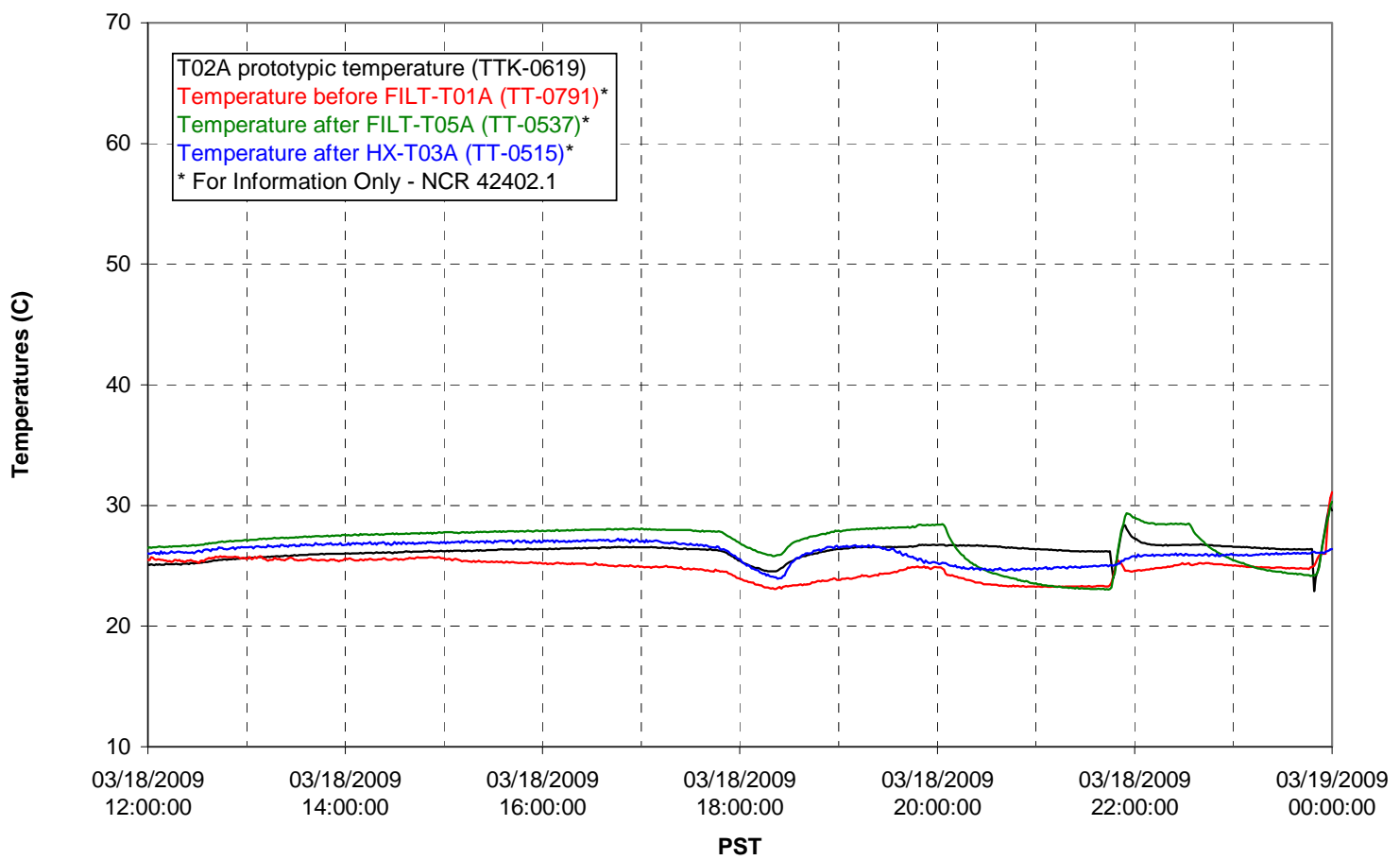

Pump Pressures and Flow

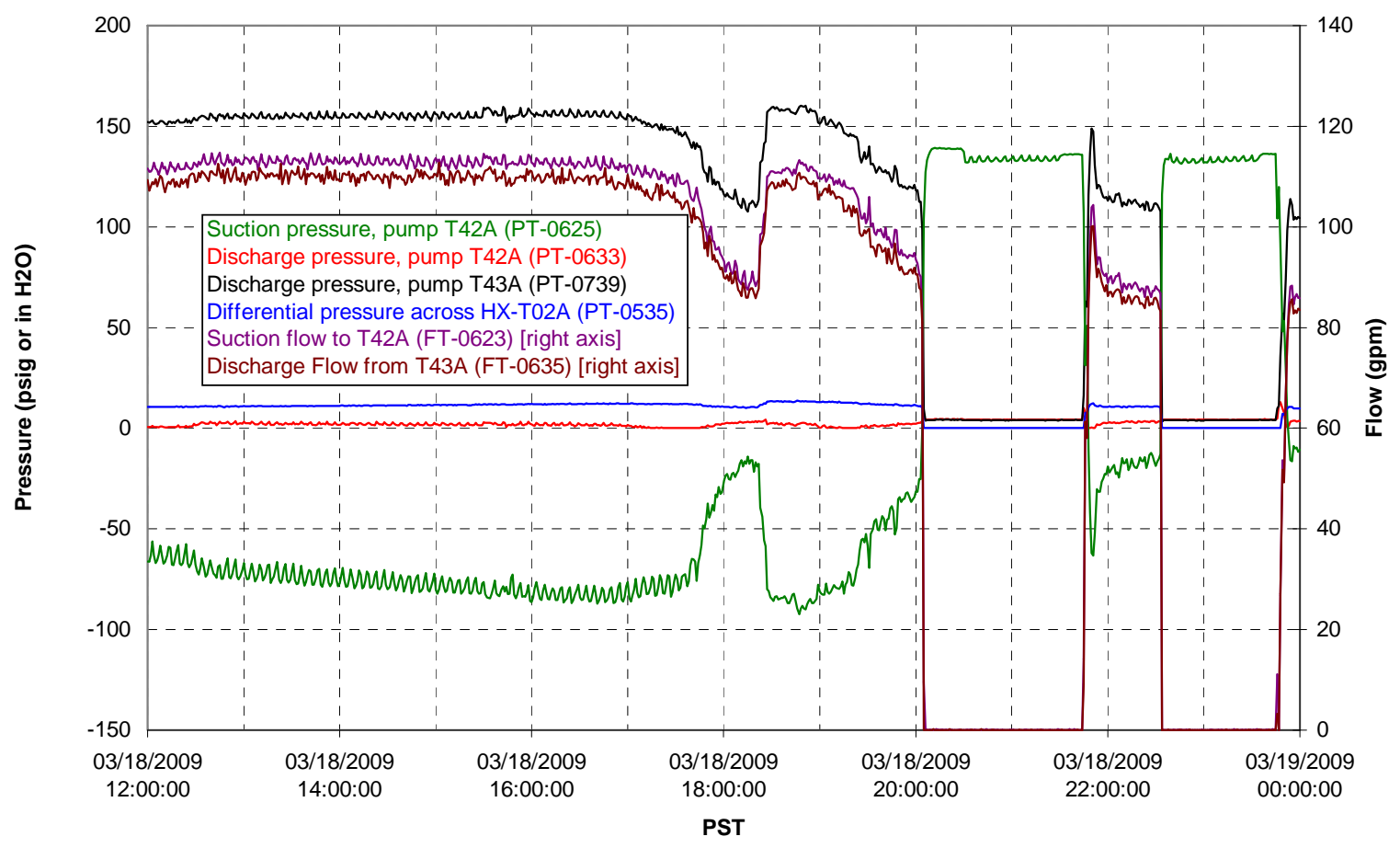


Axial pressure drop

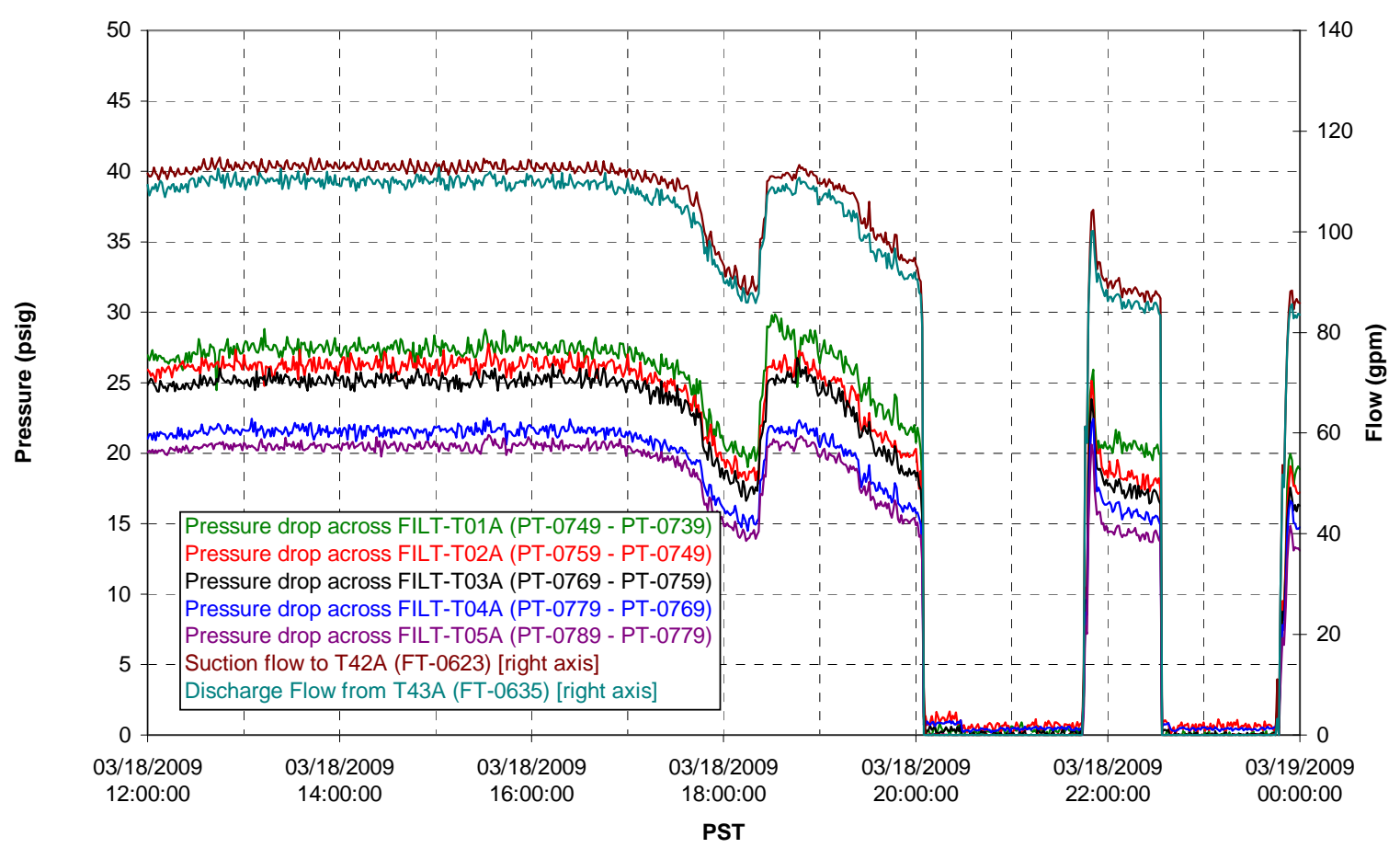

Permeate flow rates

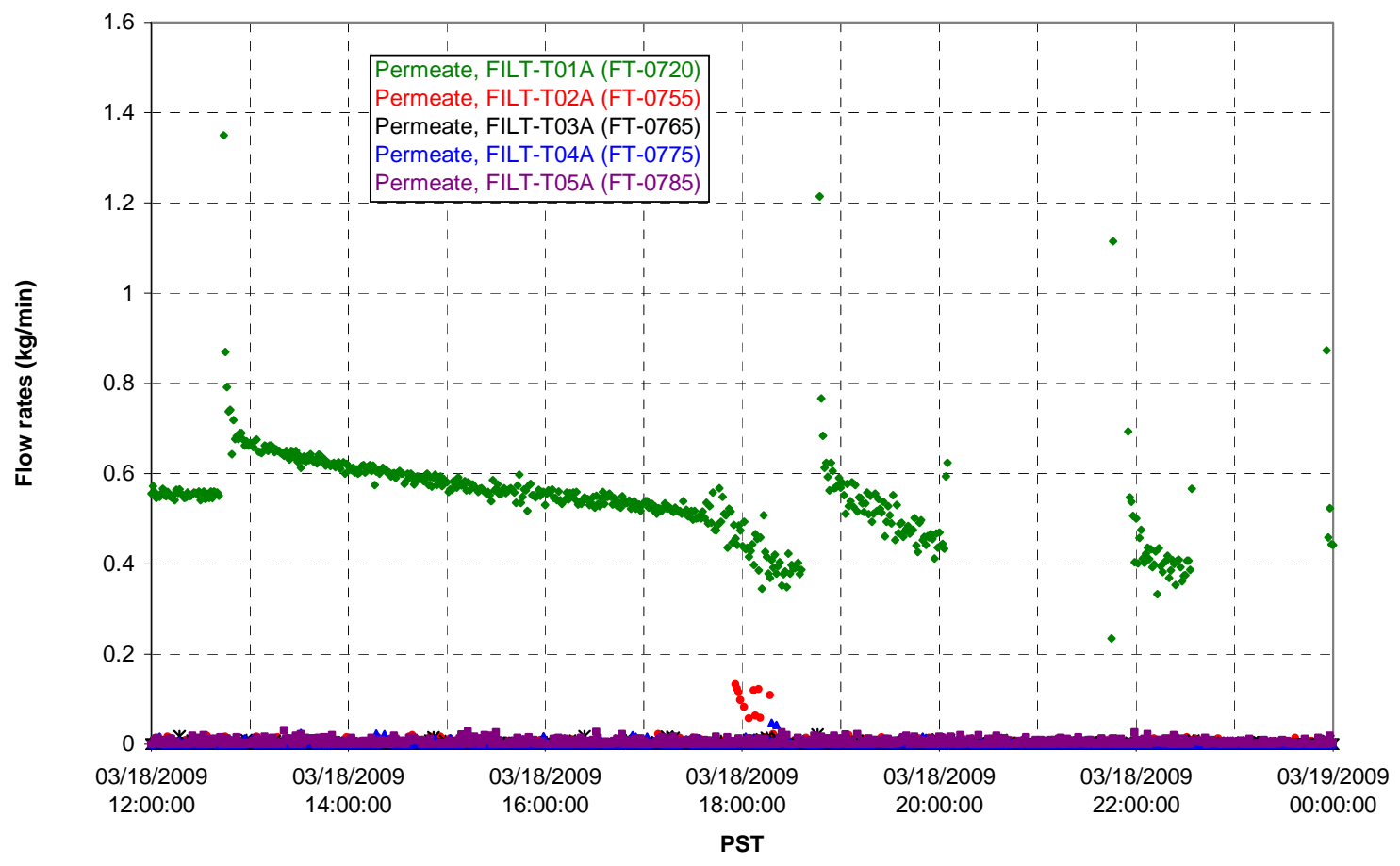


T02A Inner Temperature Tree

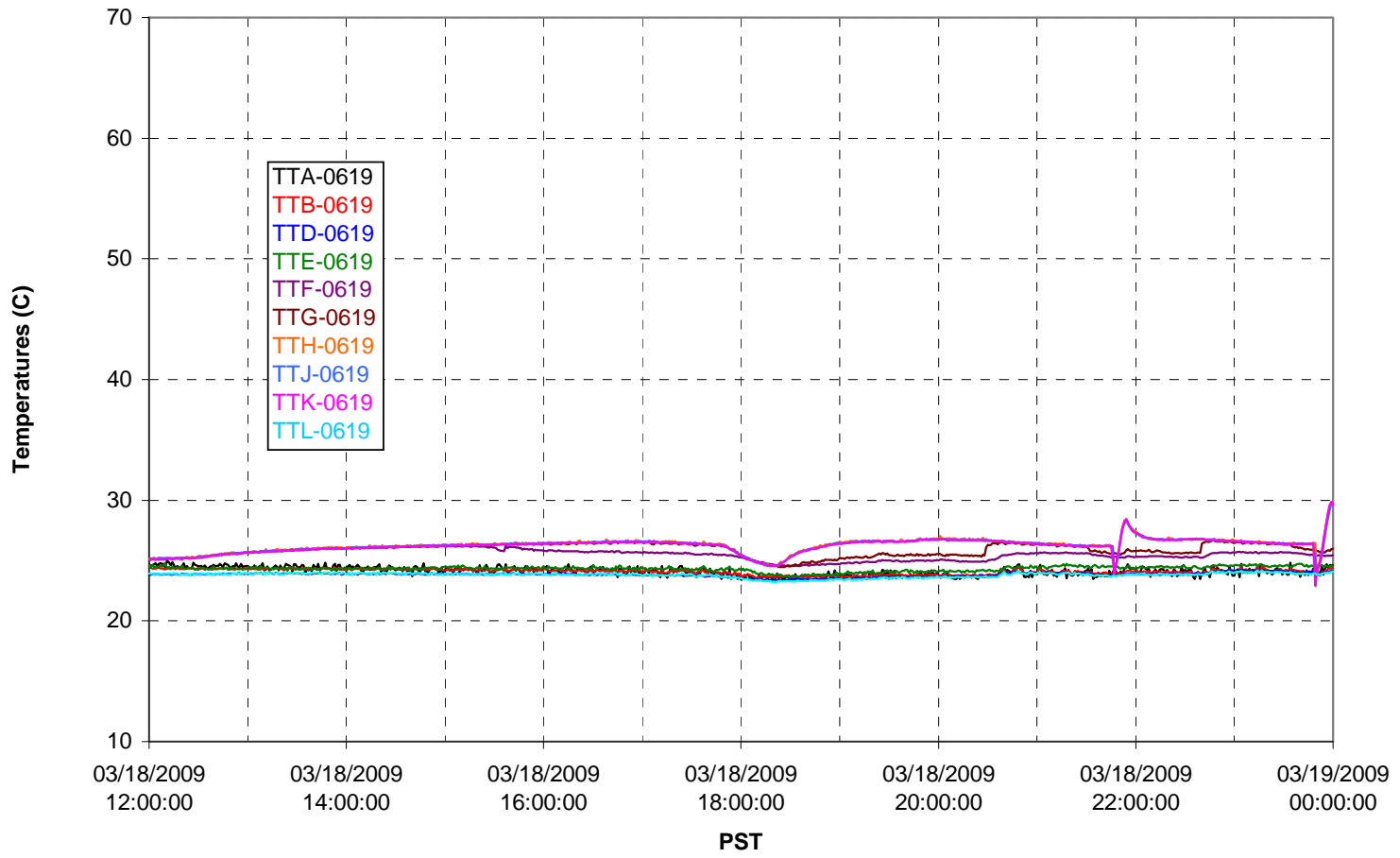

T02A Outer Temperature Tree

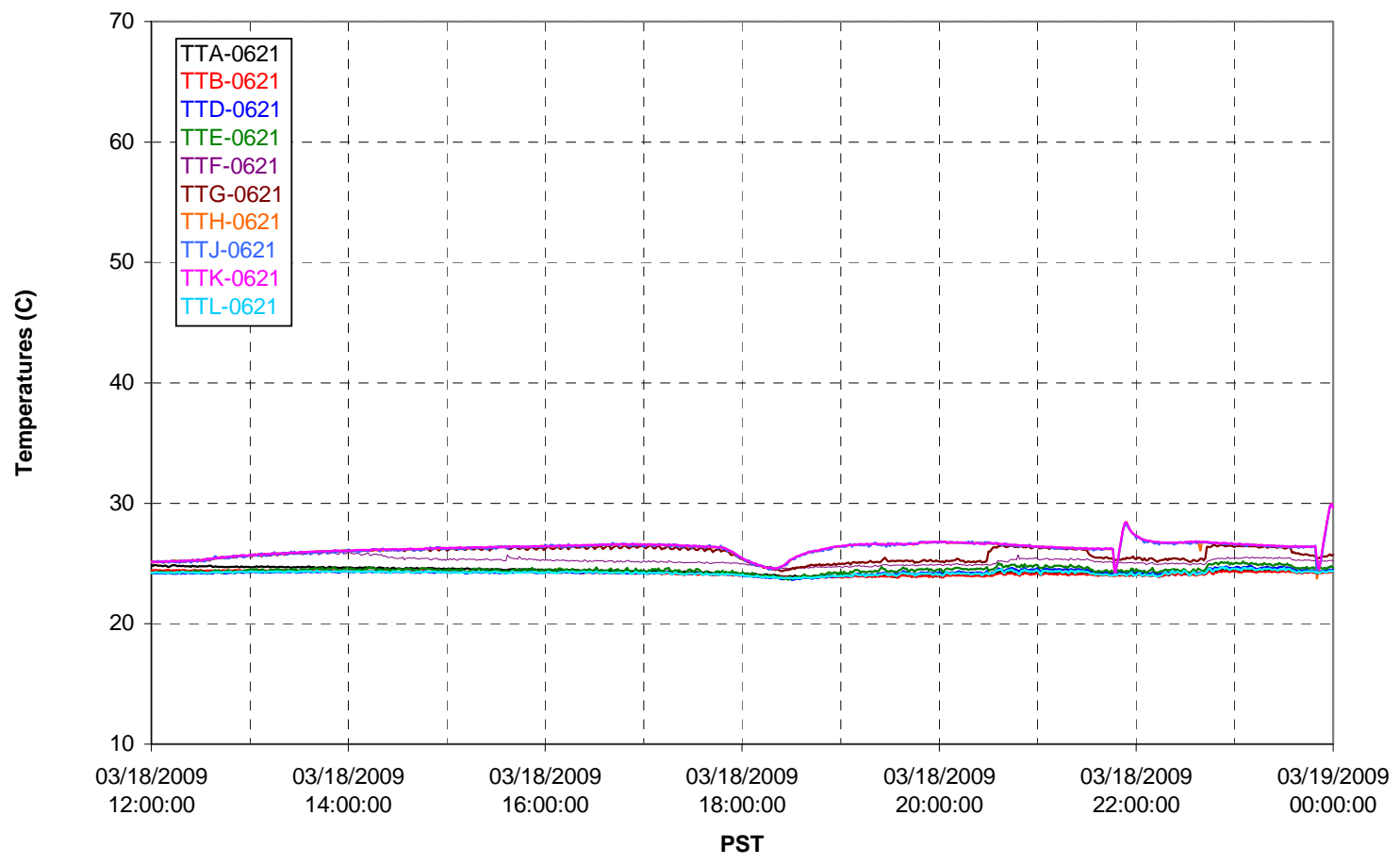


T02A temperatures

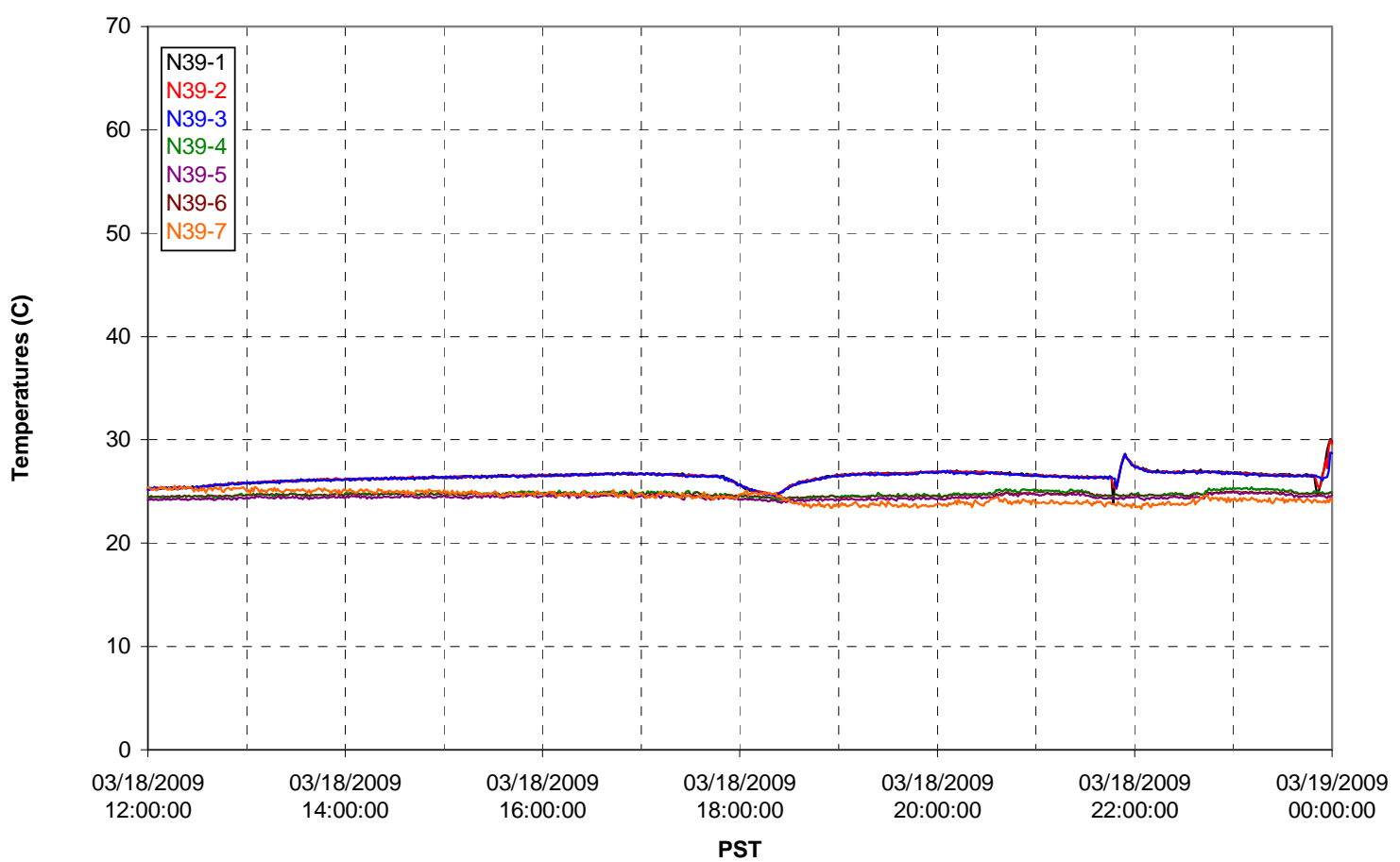

T02A temperatures

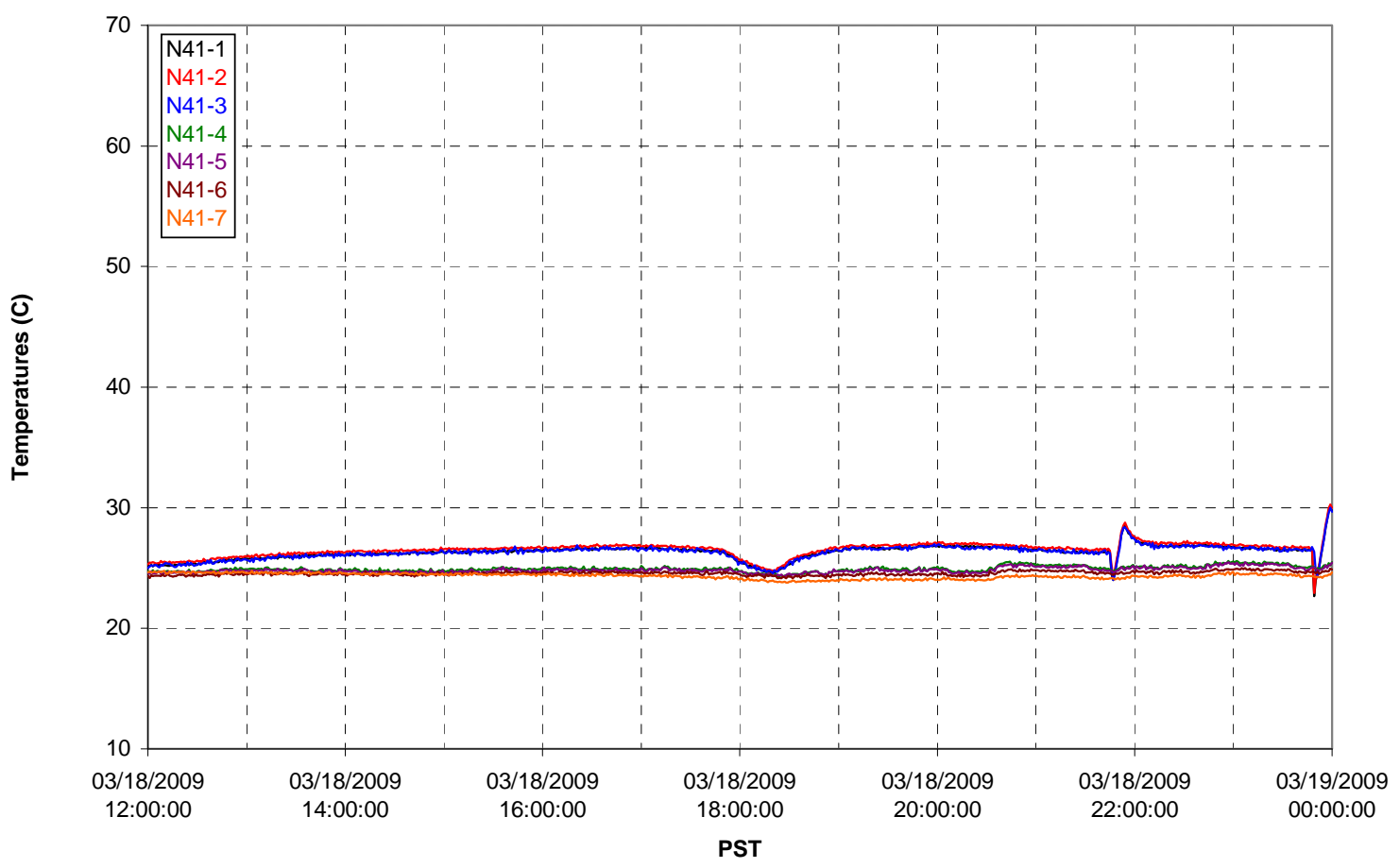


T02A temperatures

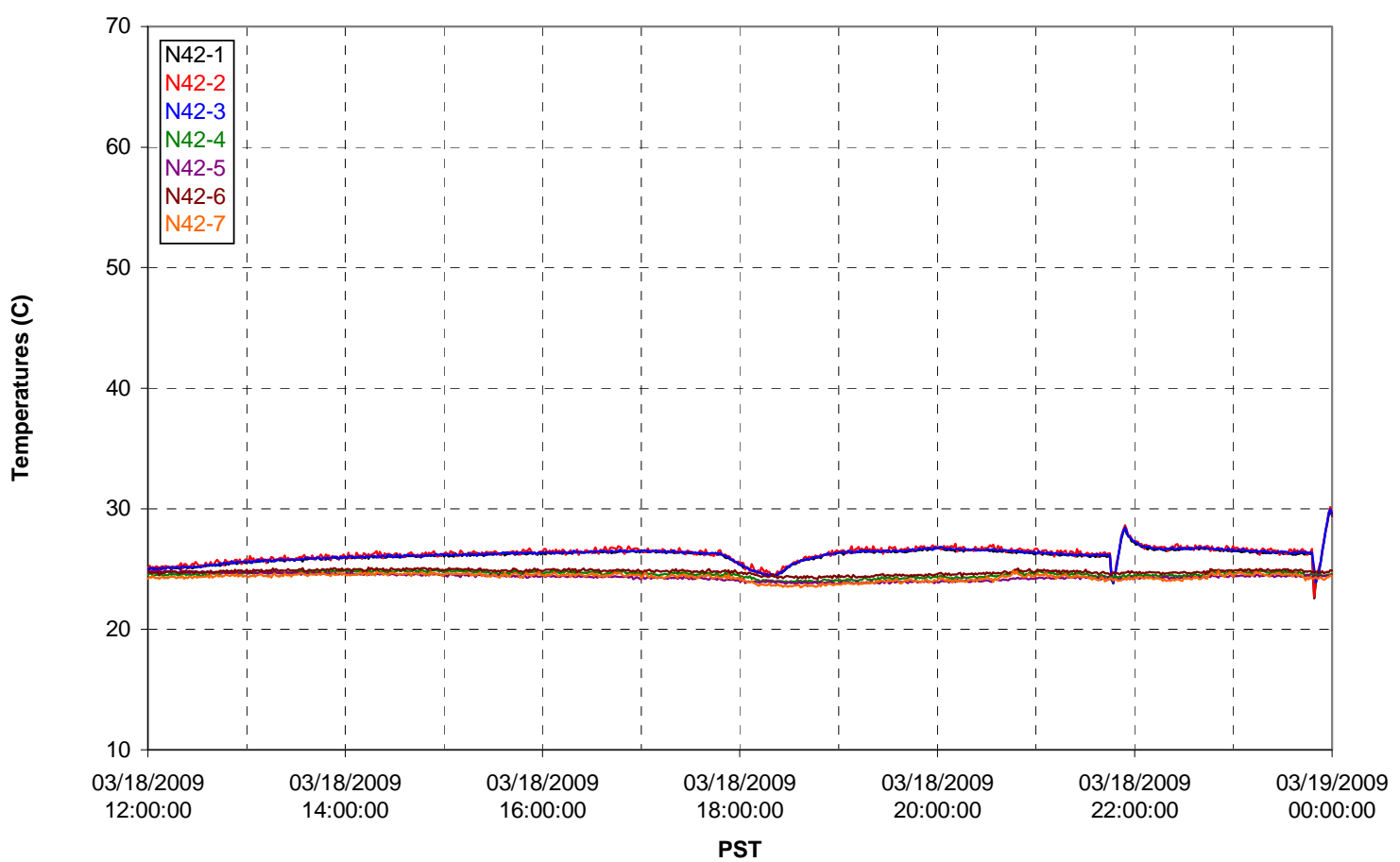

T02A temperatures

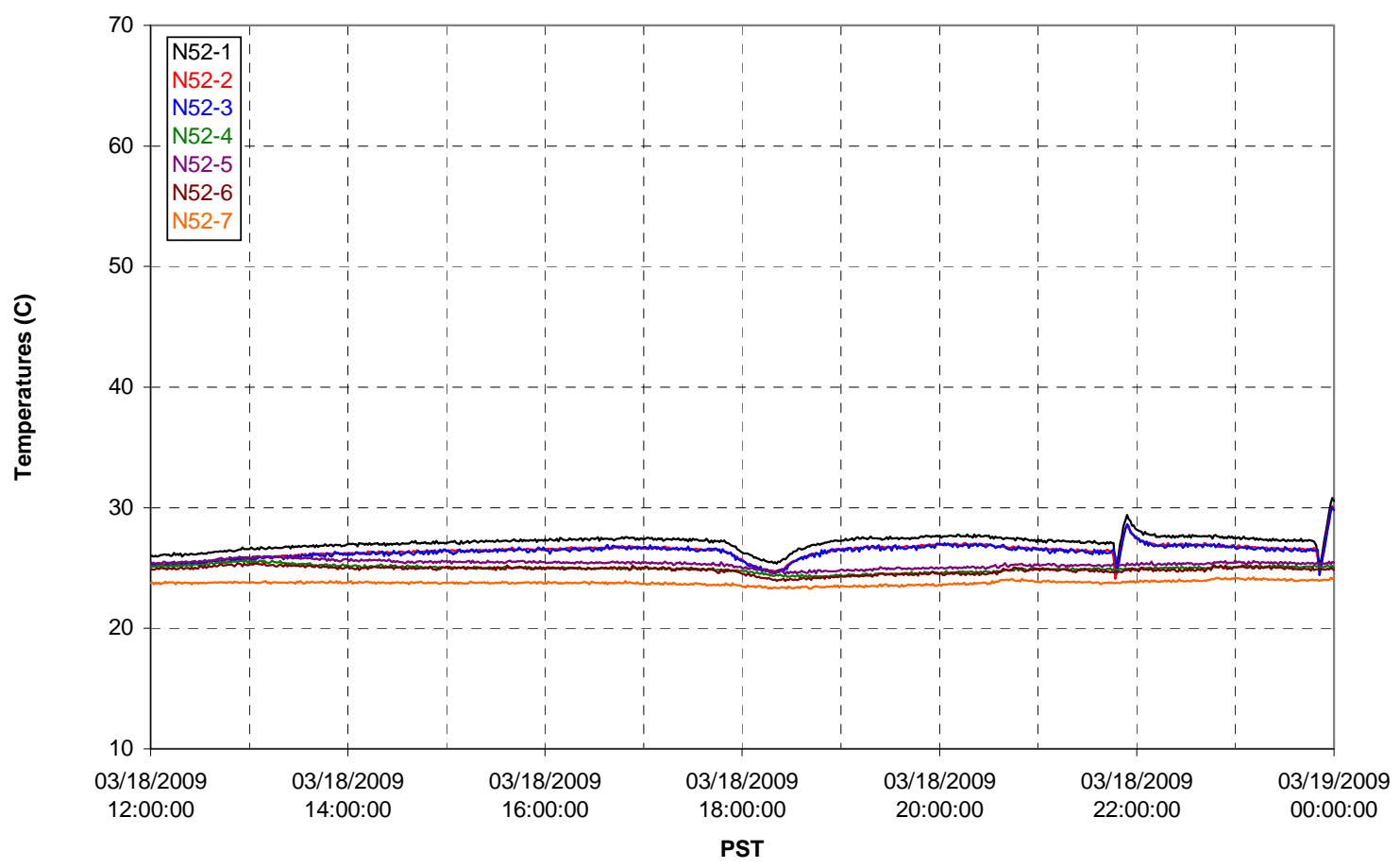


T02A Heating and Cooling

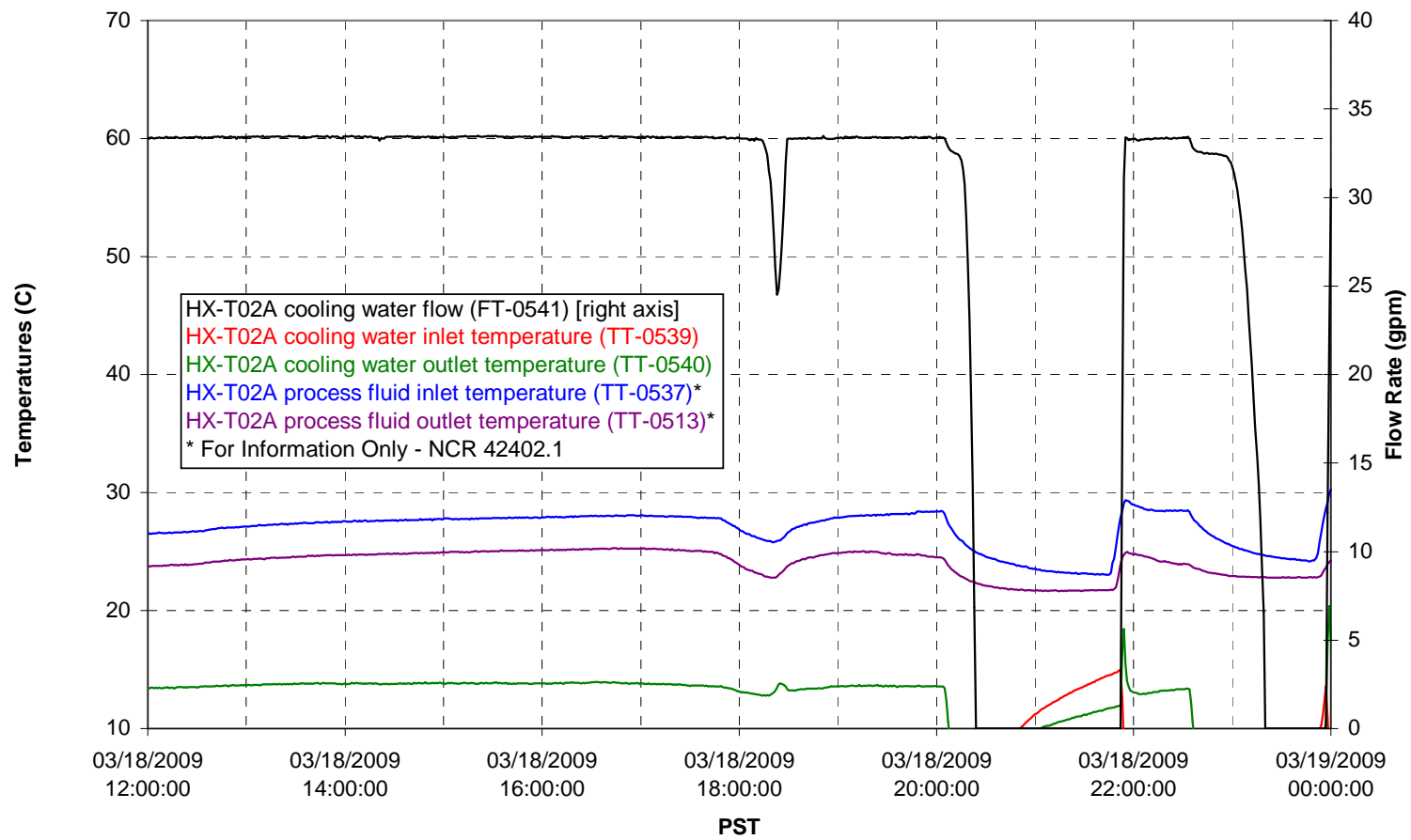

Pump Operation

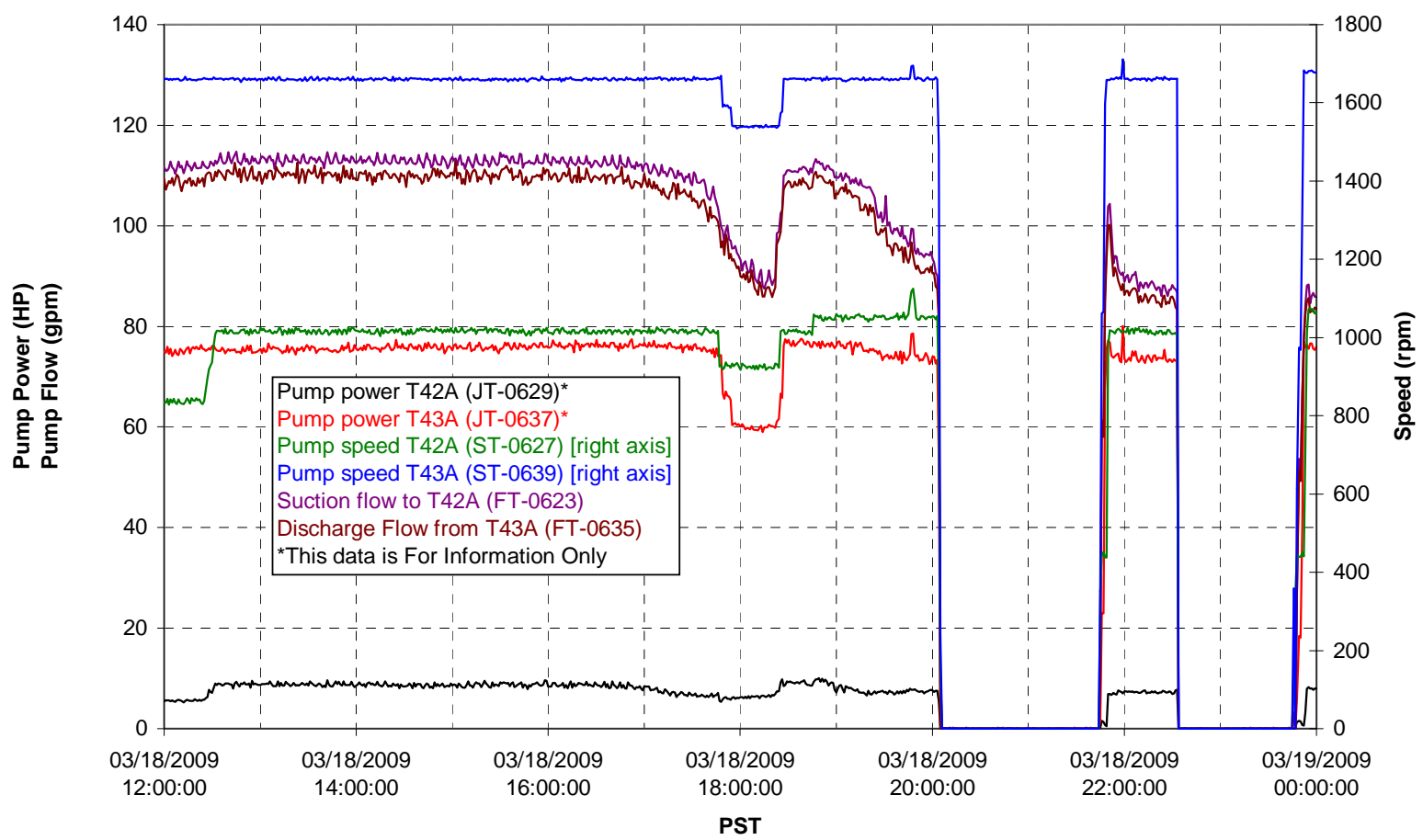


Pulsepot UFP-PP-T01A

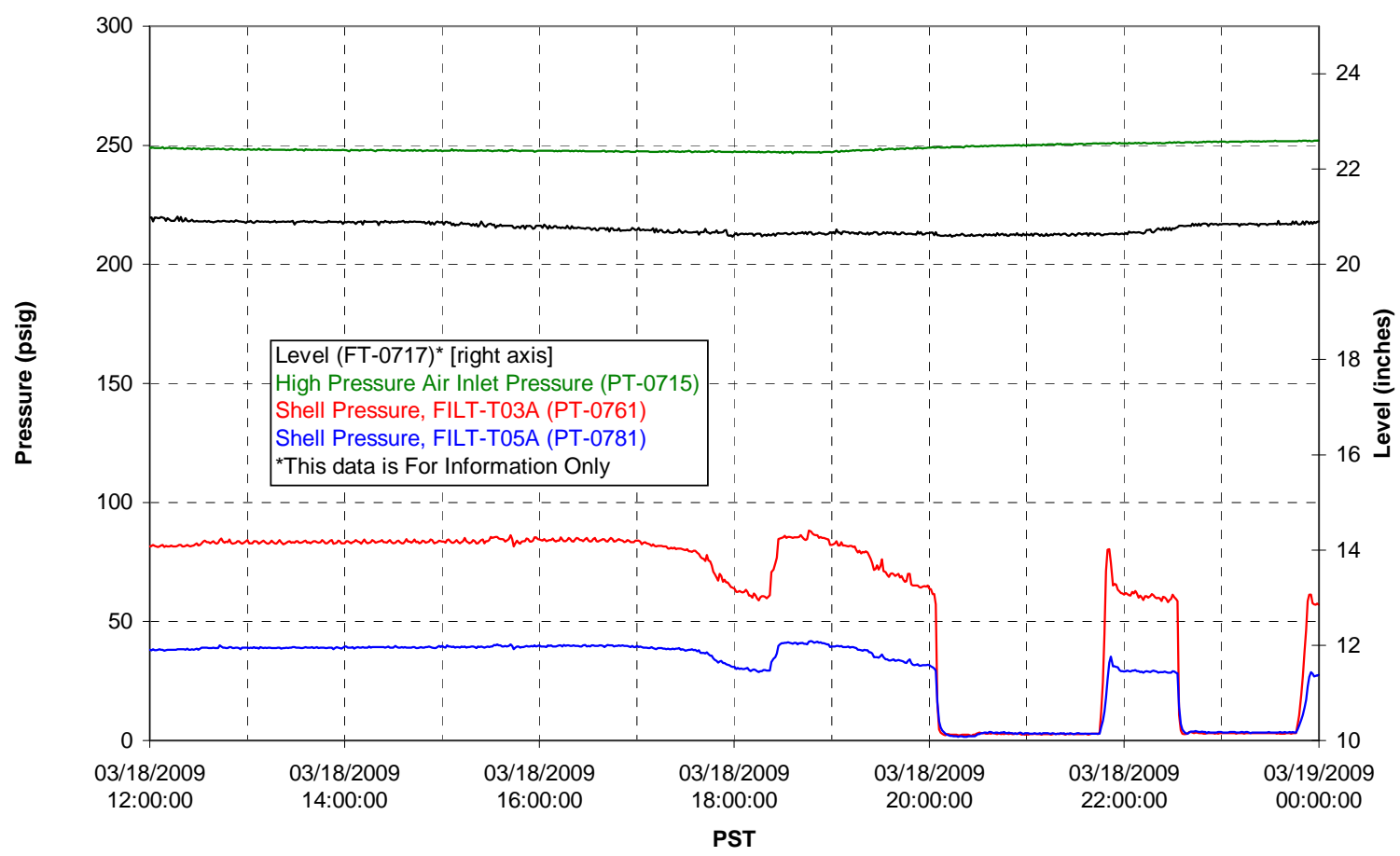

Pulsepot UFP-PP-T02A

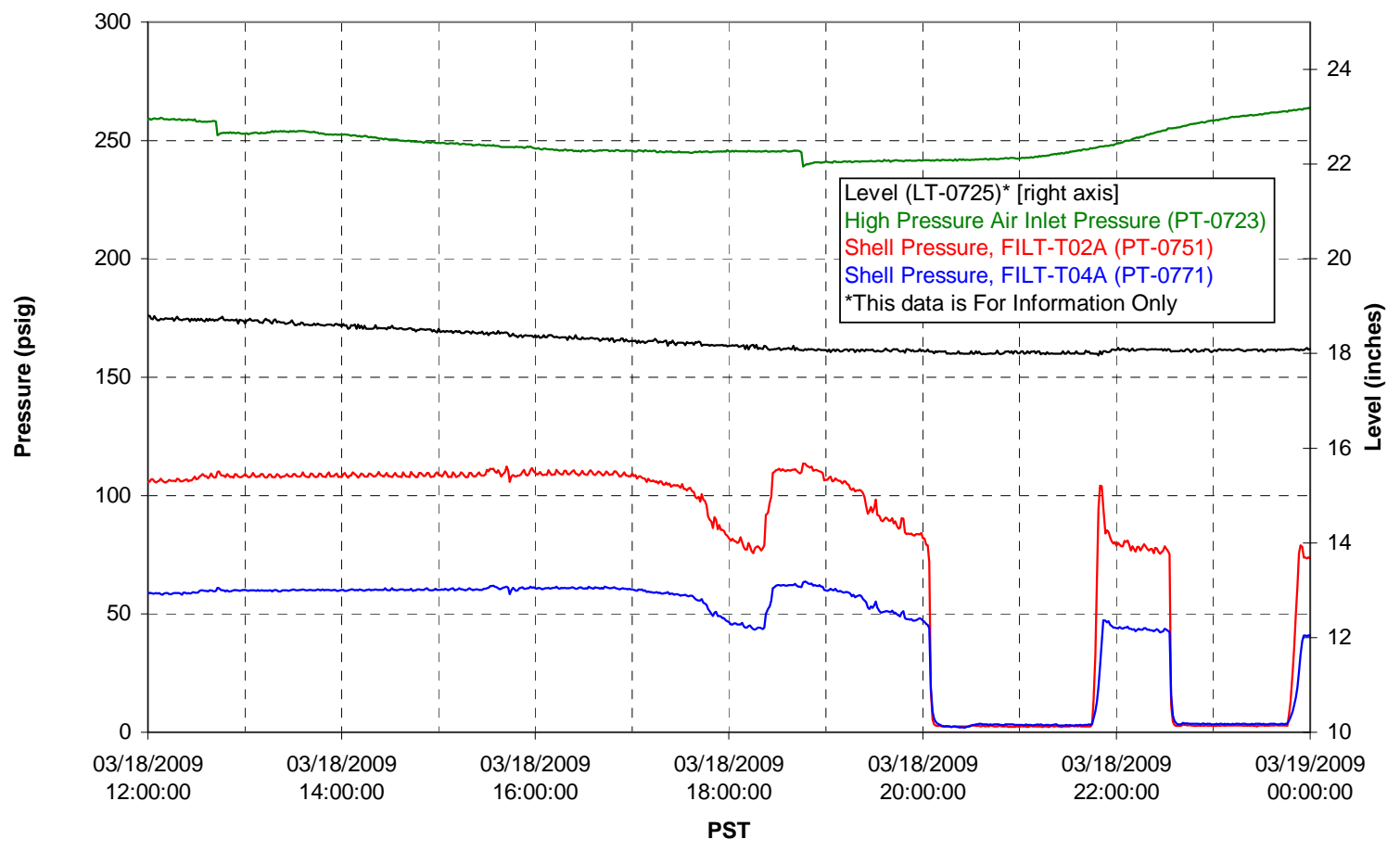


Pulsepot UFP-PP-T03A

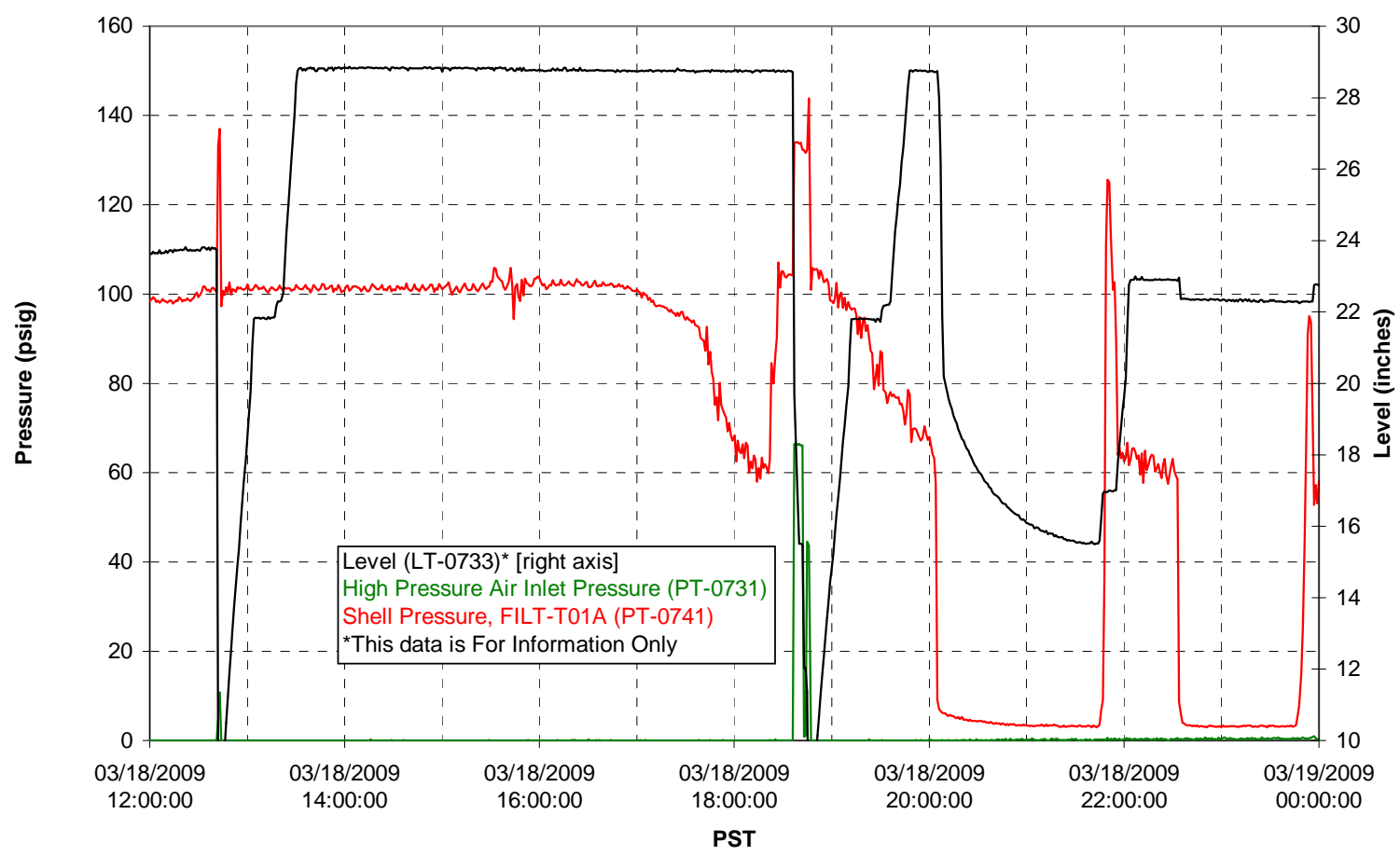

Pulsepot Levels

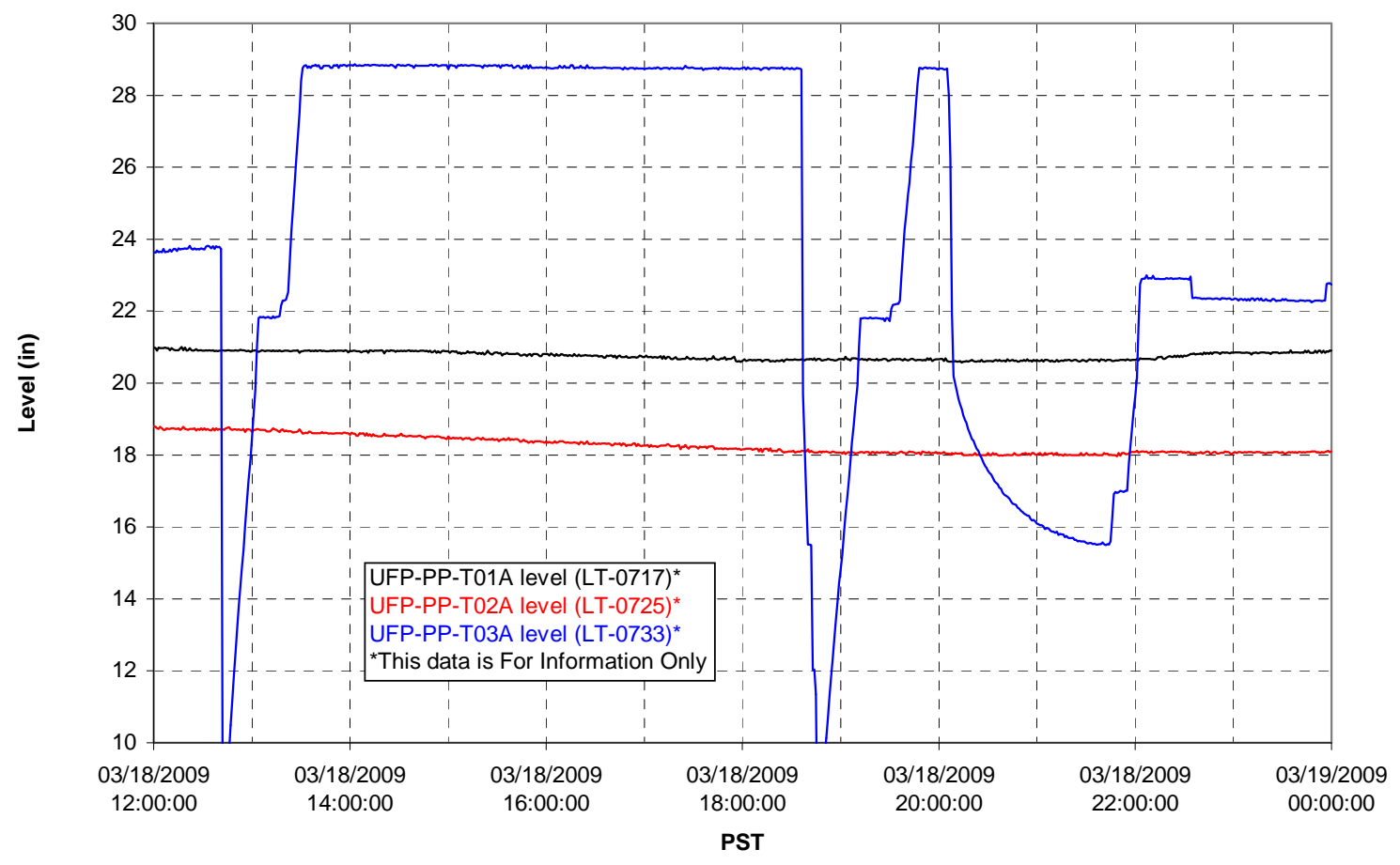


Filter UFP-FILT-T01A

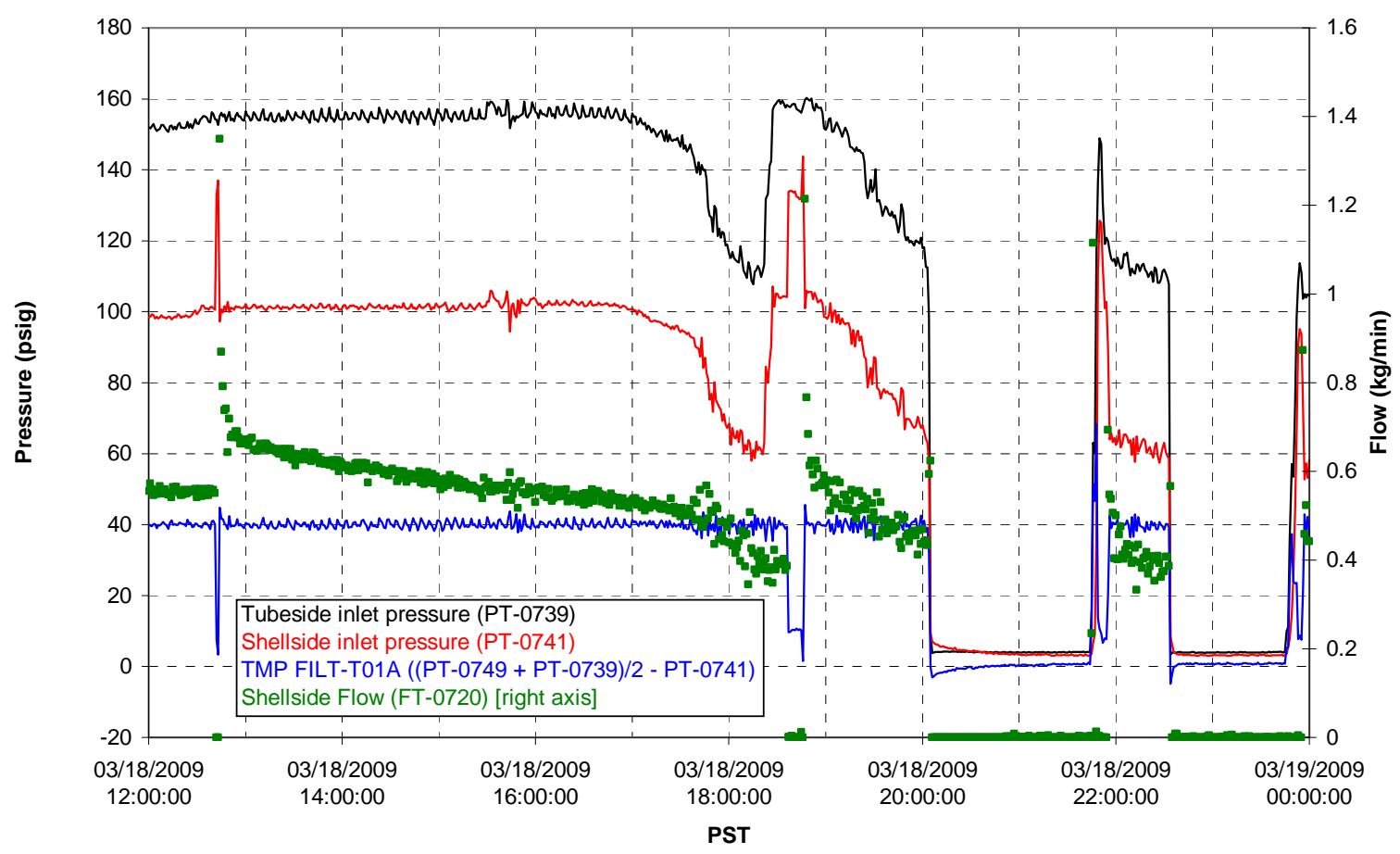

Filter UFP-FILT-T02A

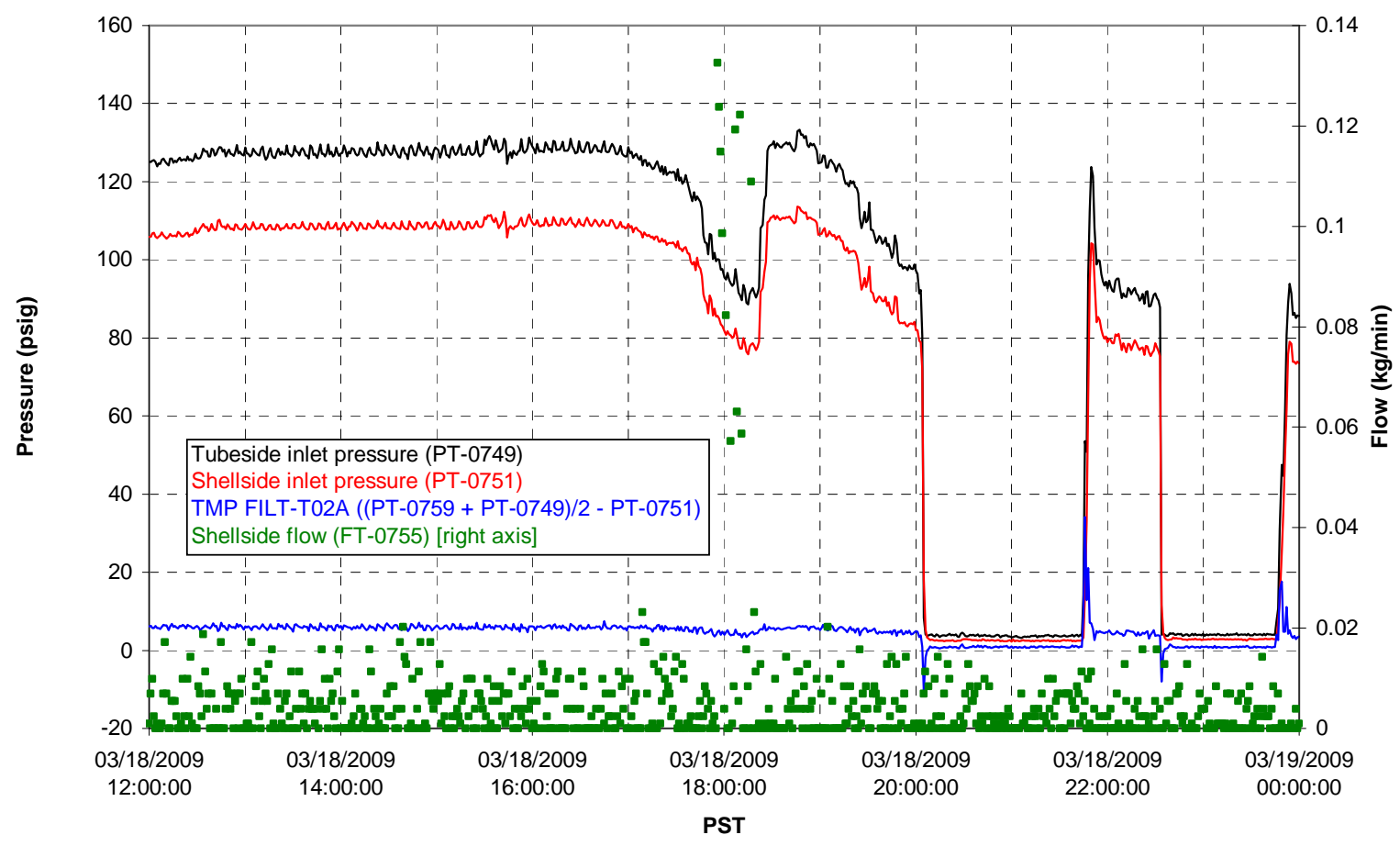


Filter UFP-FILT-T03A

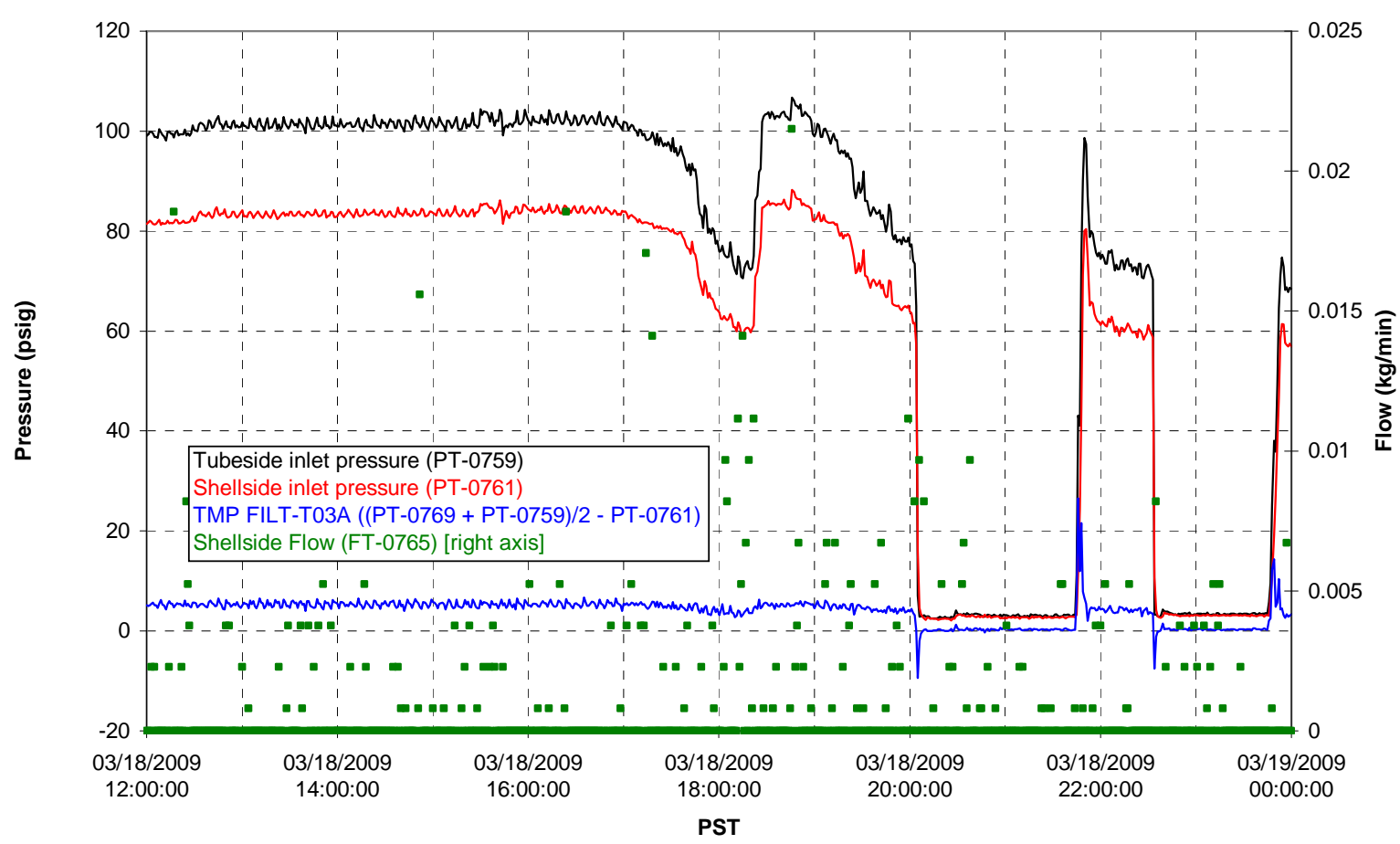

Filter UFP-FILT-T04A

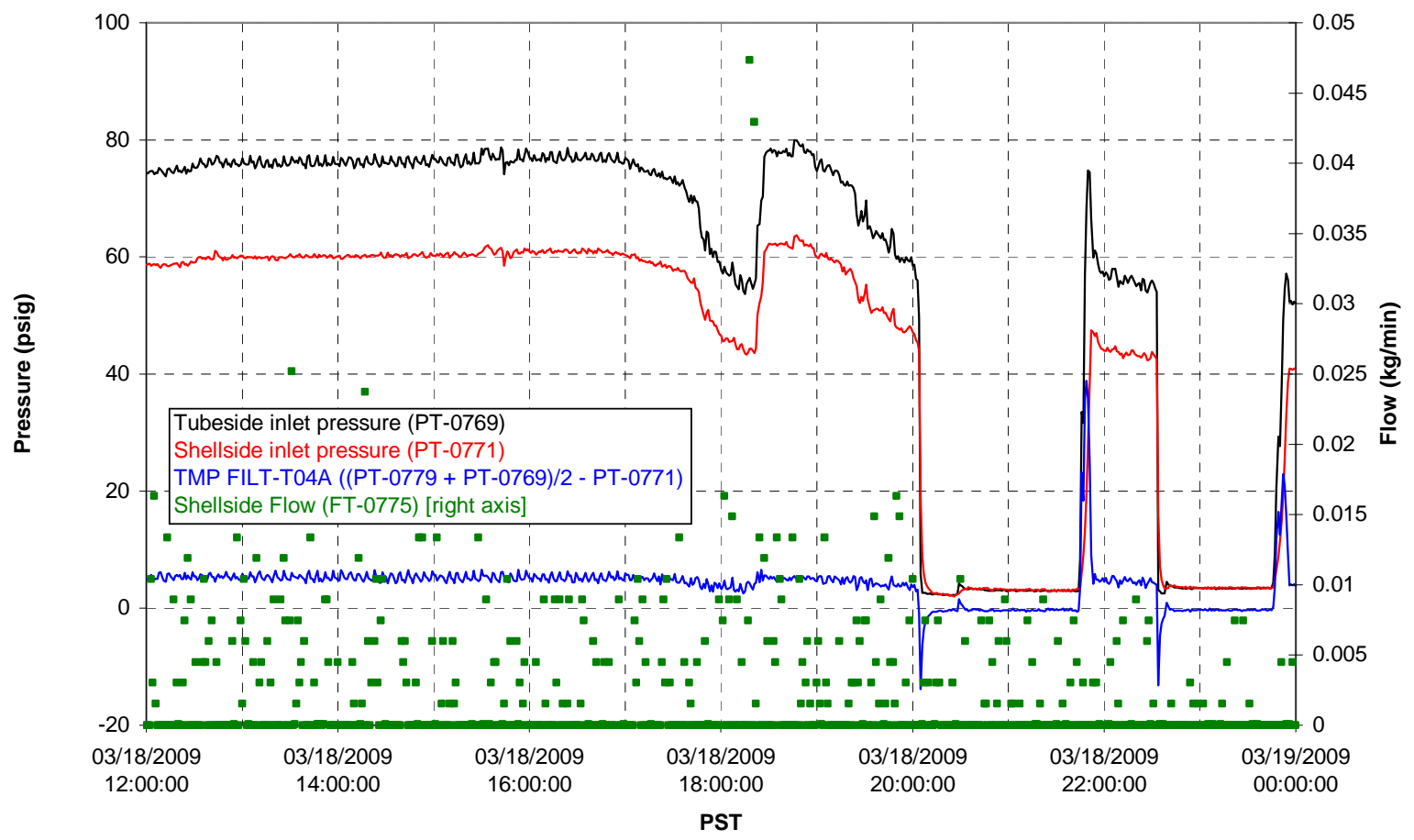


Filter UFP-FILT-T05A

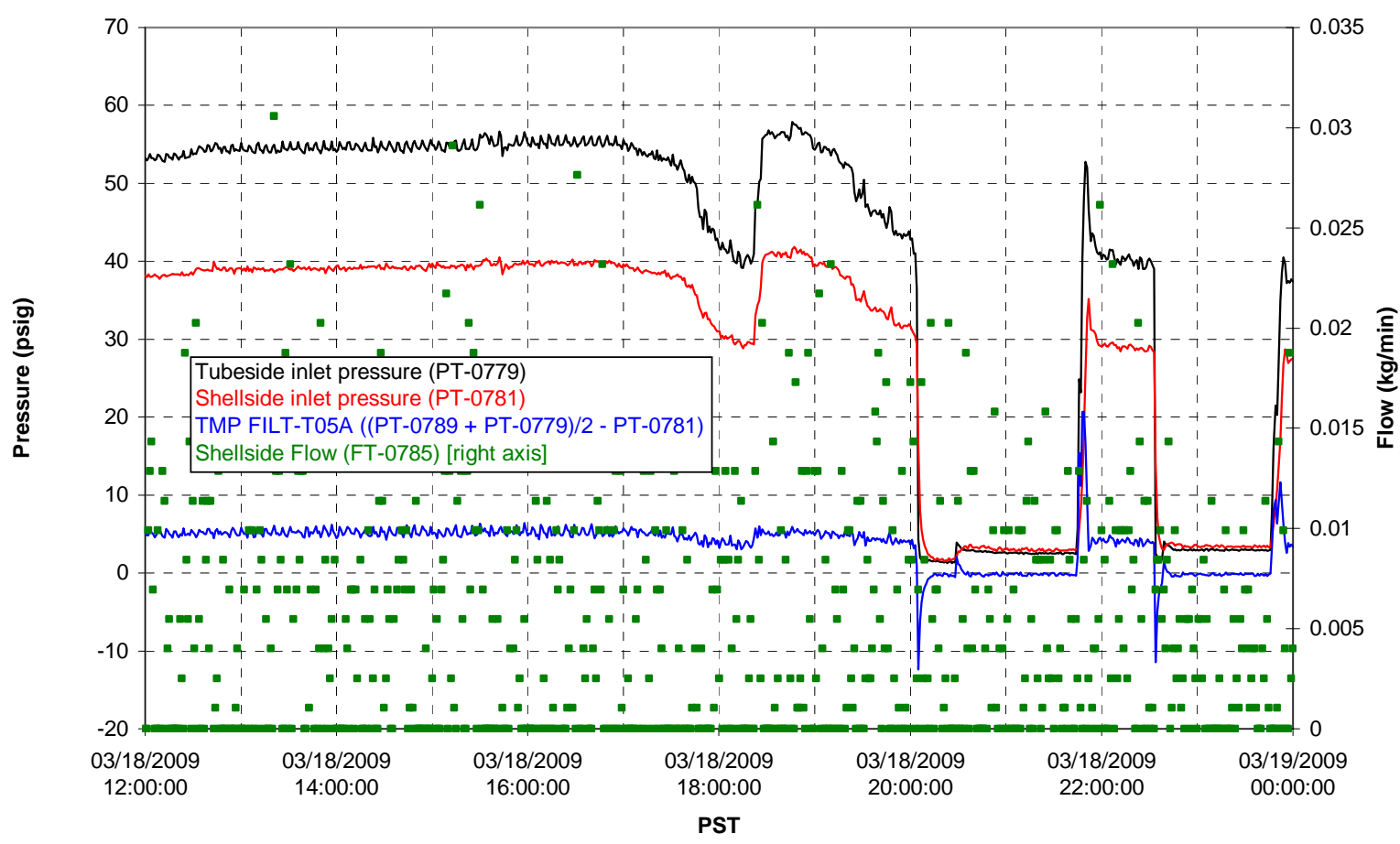

Chemical Flow

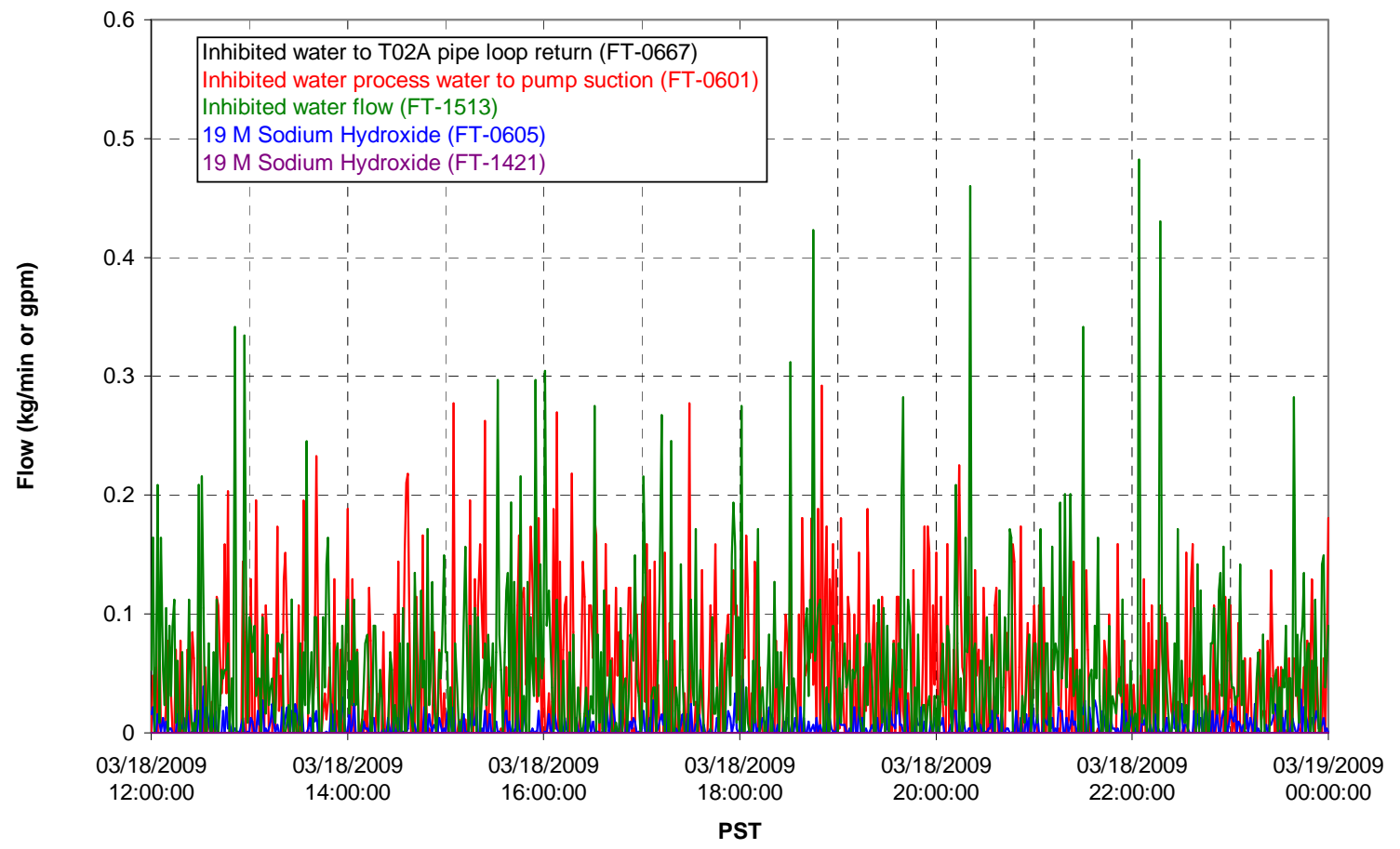




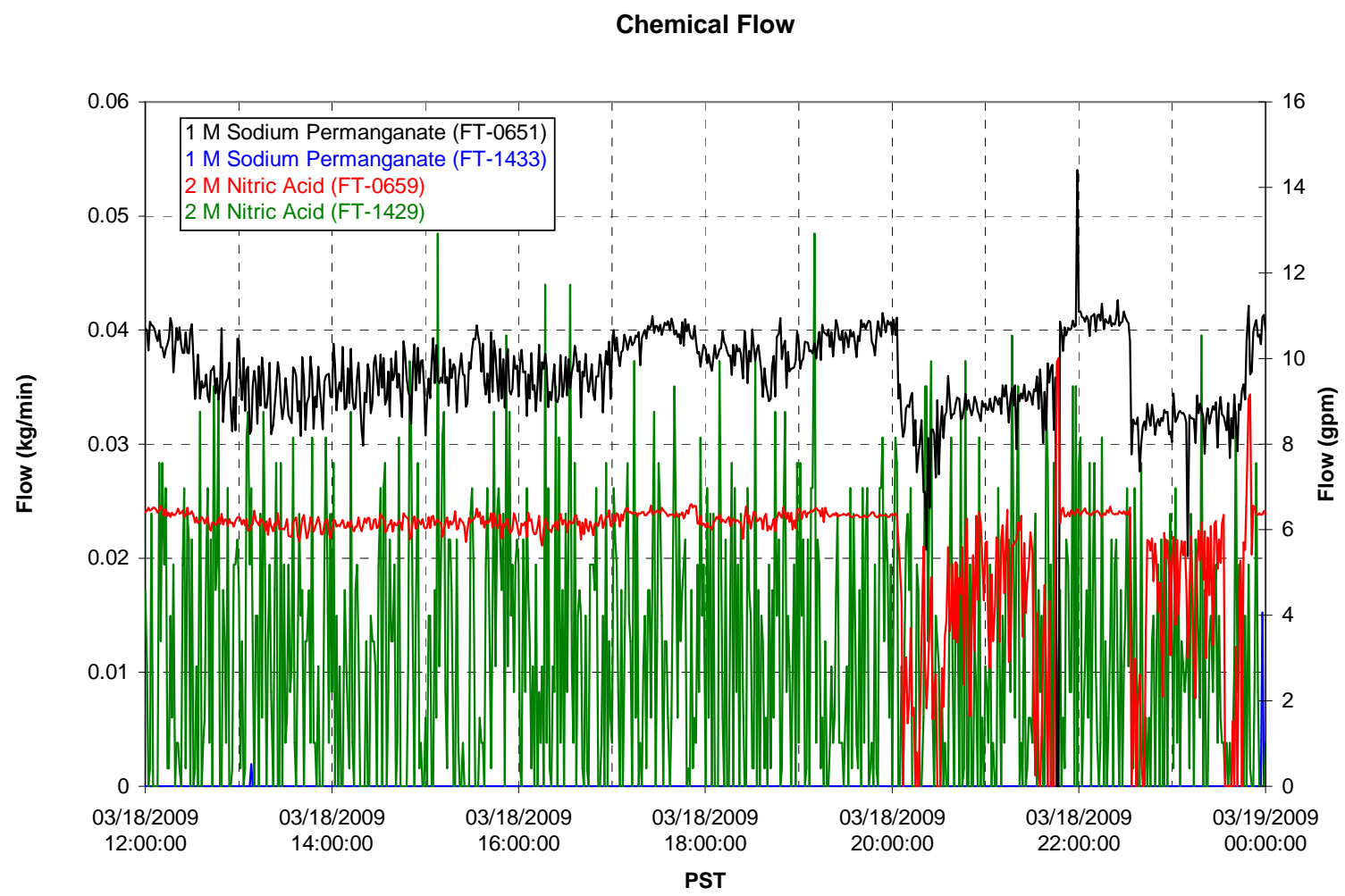

Air Flows

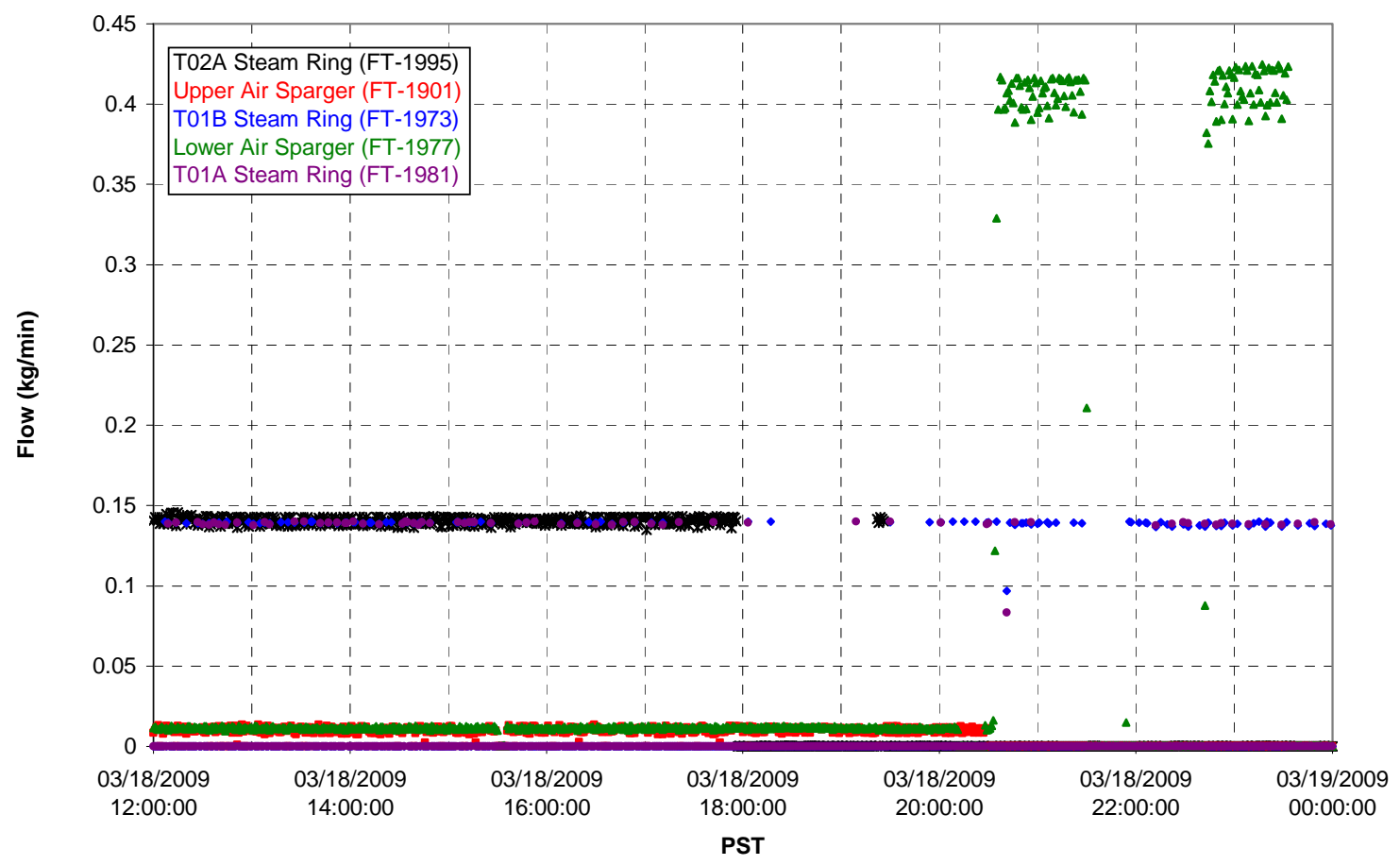


T02A Steam

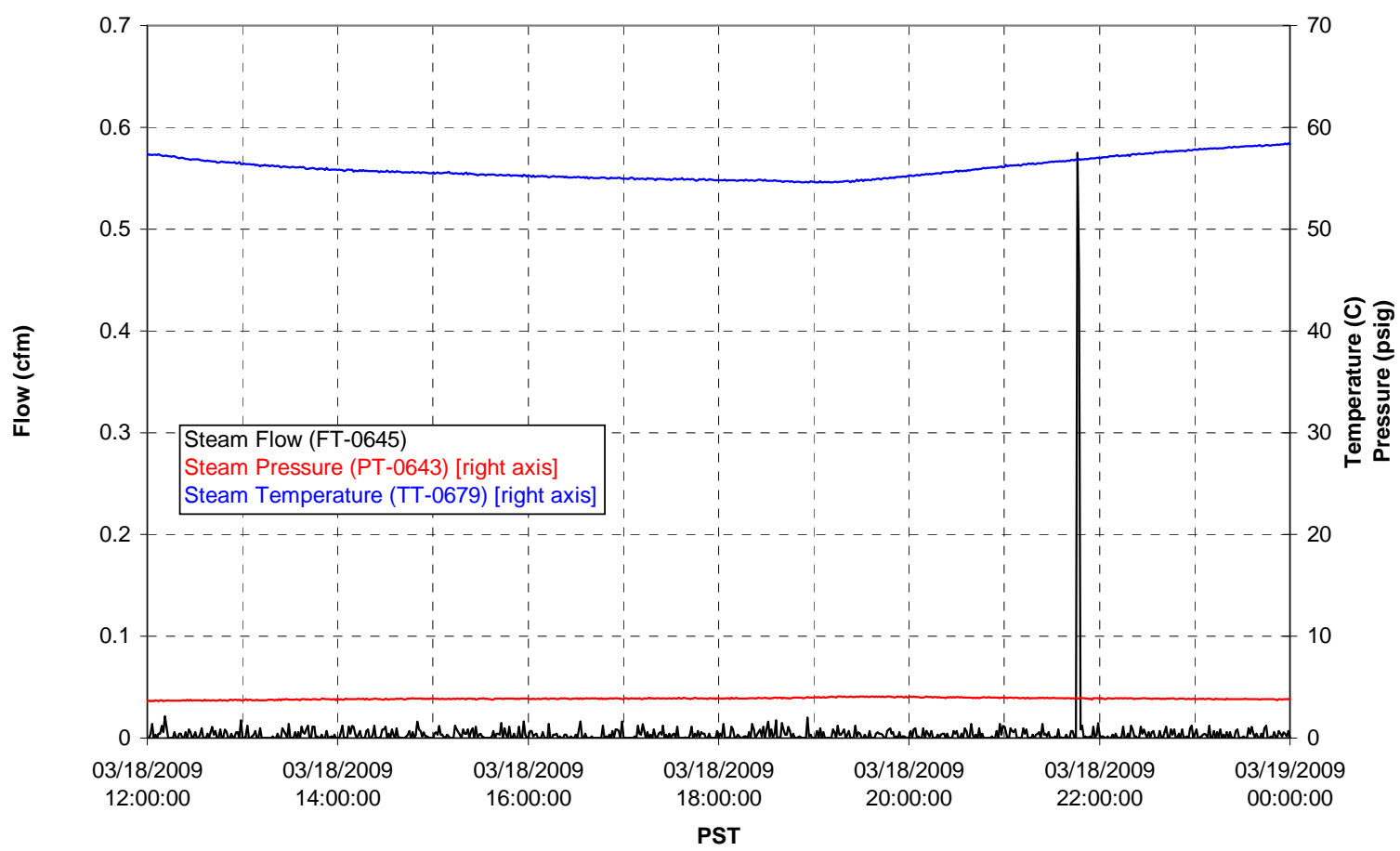

T01A Steam

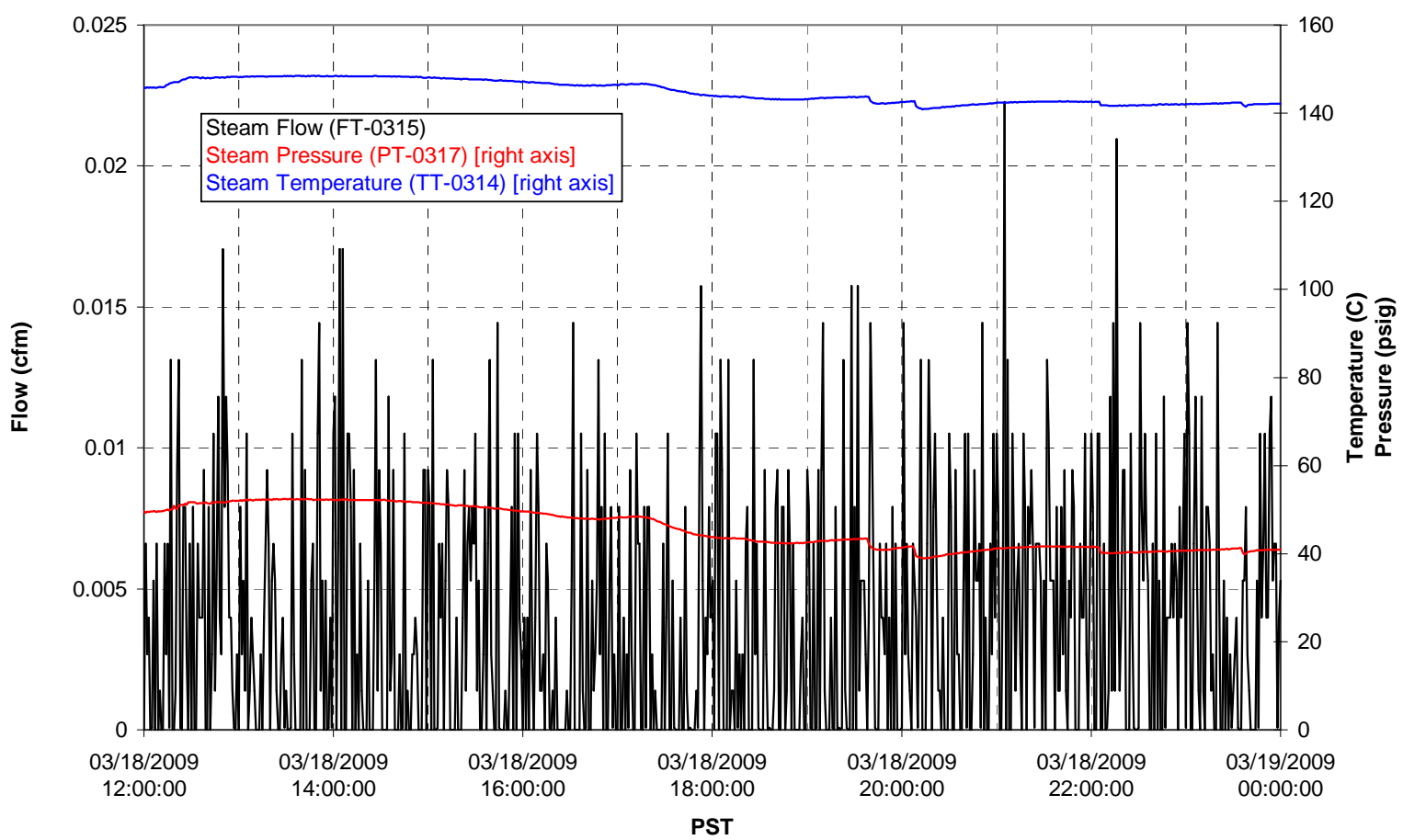


T01B Steam

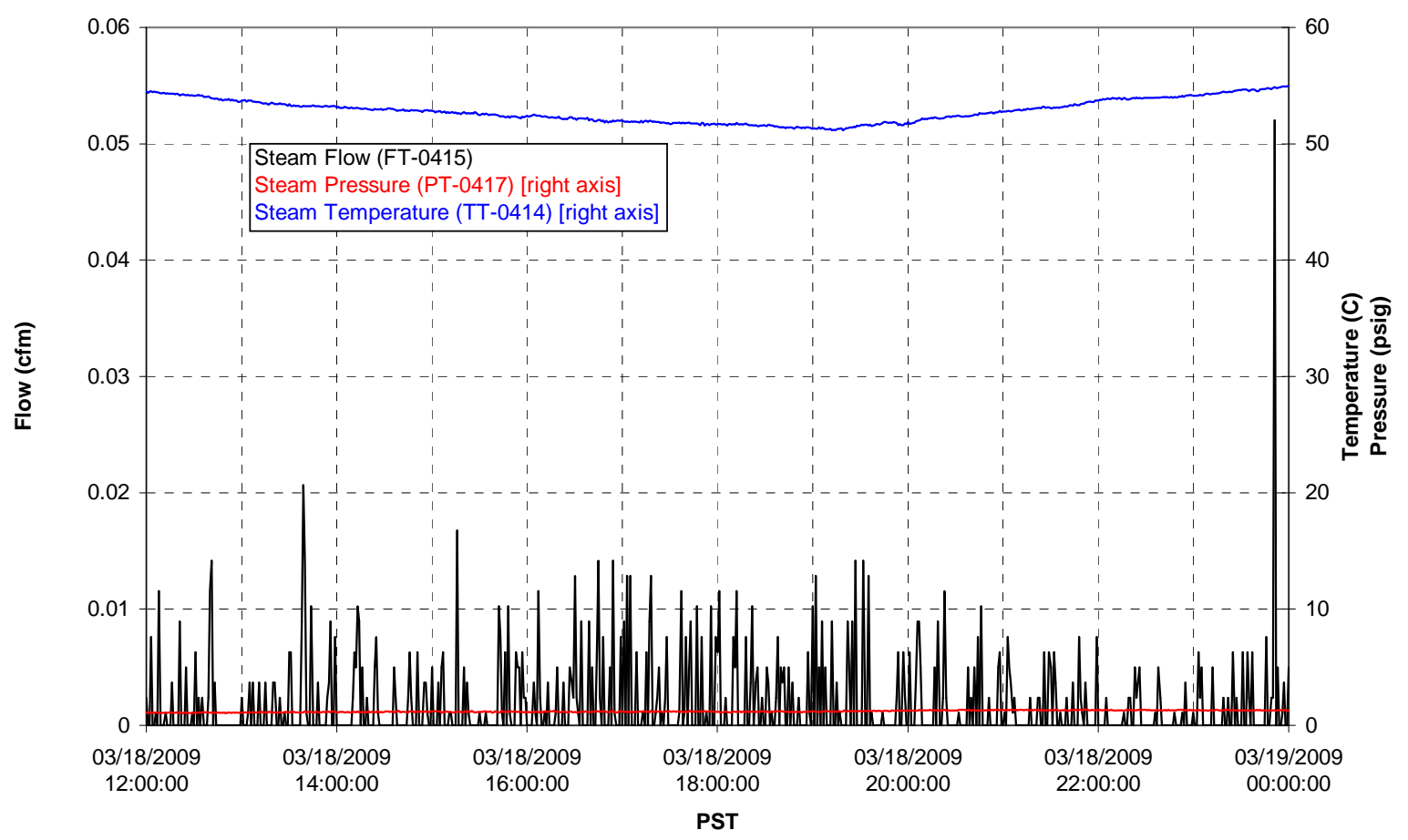




\section{Integrated Test B Data Plots \\ 03/19/09 00:00 - 03/19/09 12:00}


T01A level

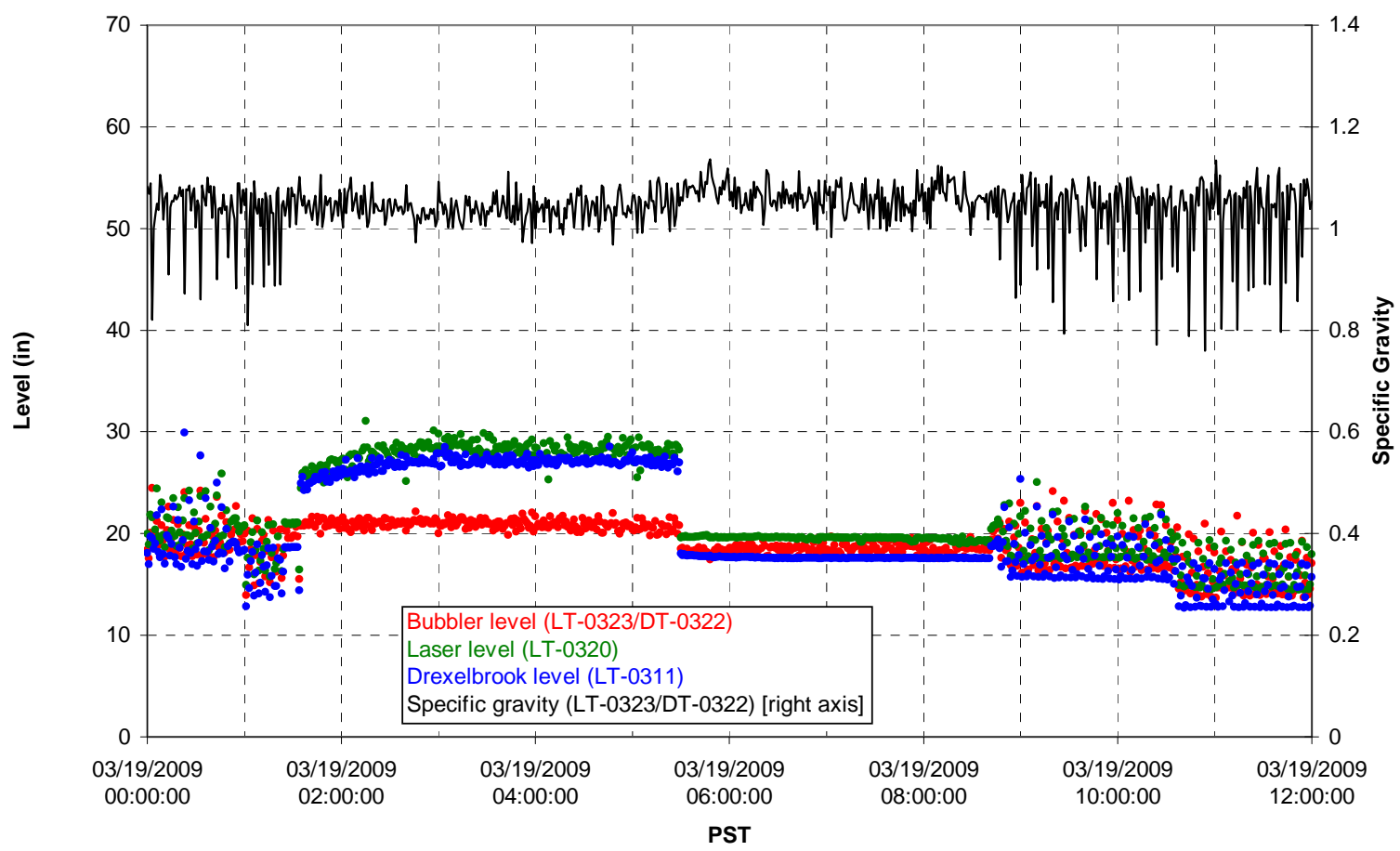

T01A temperatures

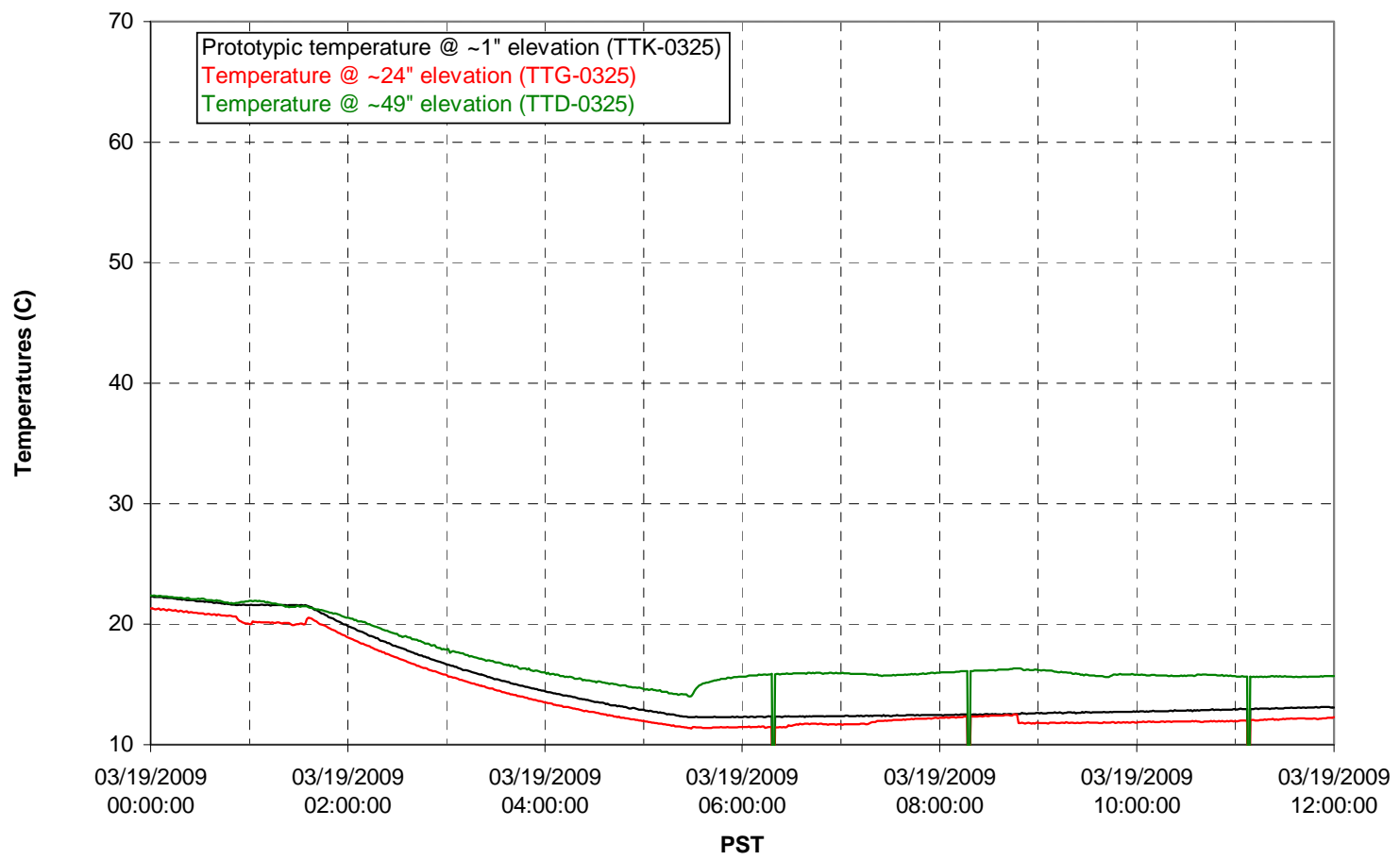


T01B level

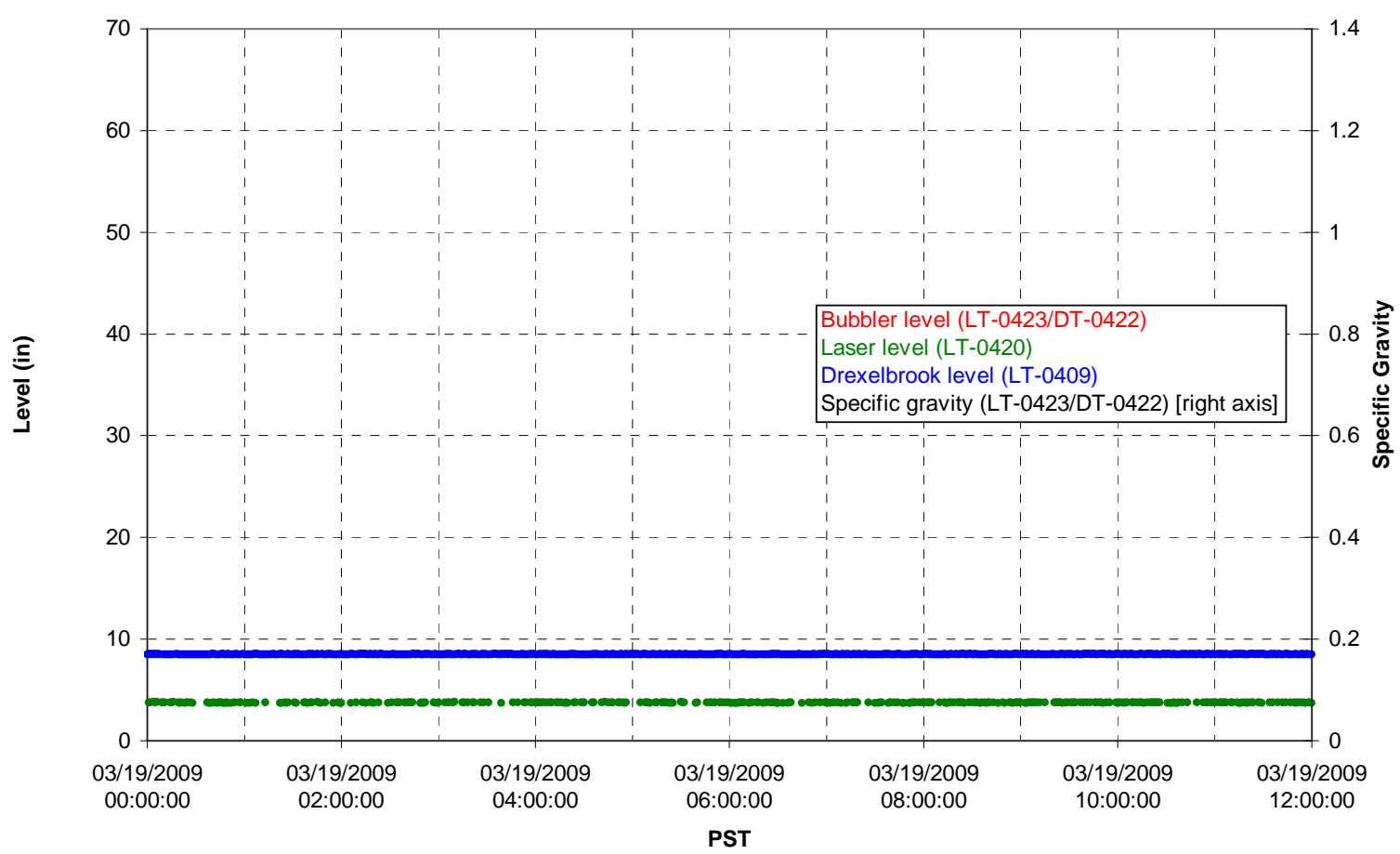

T01B temperatures

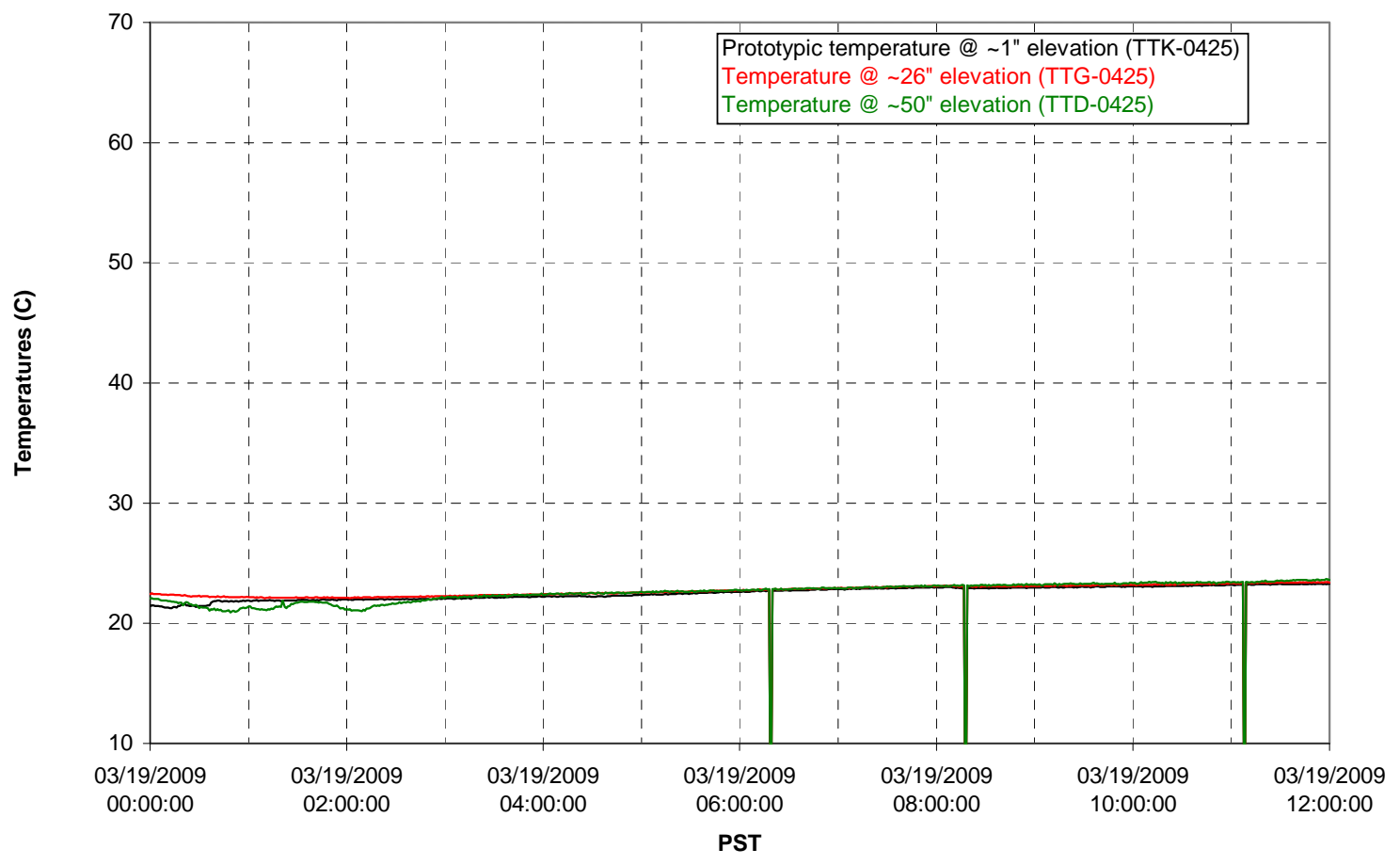


T02A level

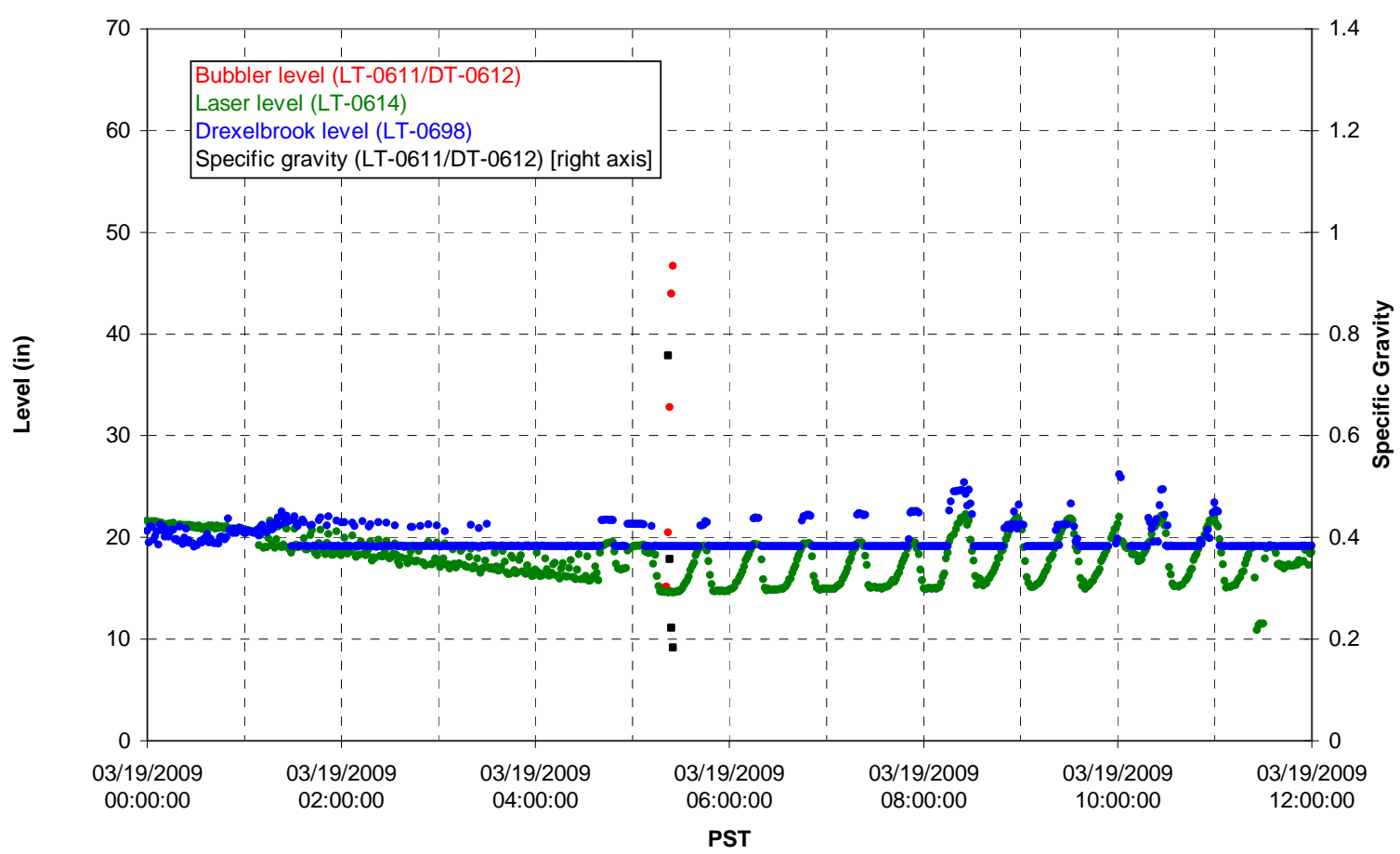

T02A temperatures

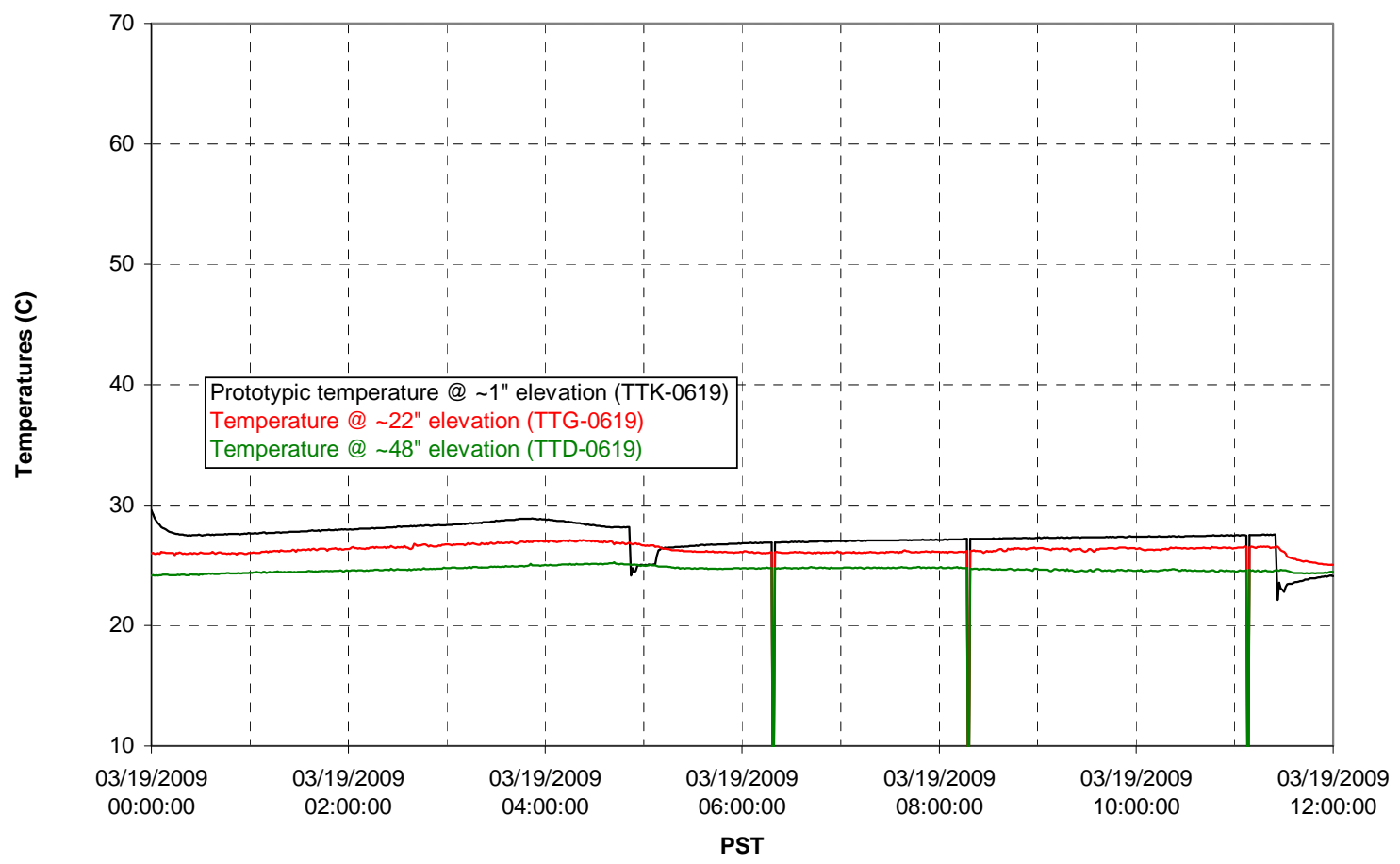


T02A and filter loop temperatures

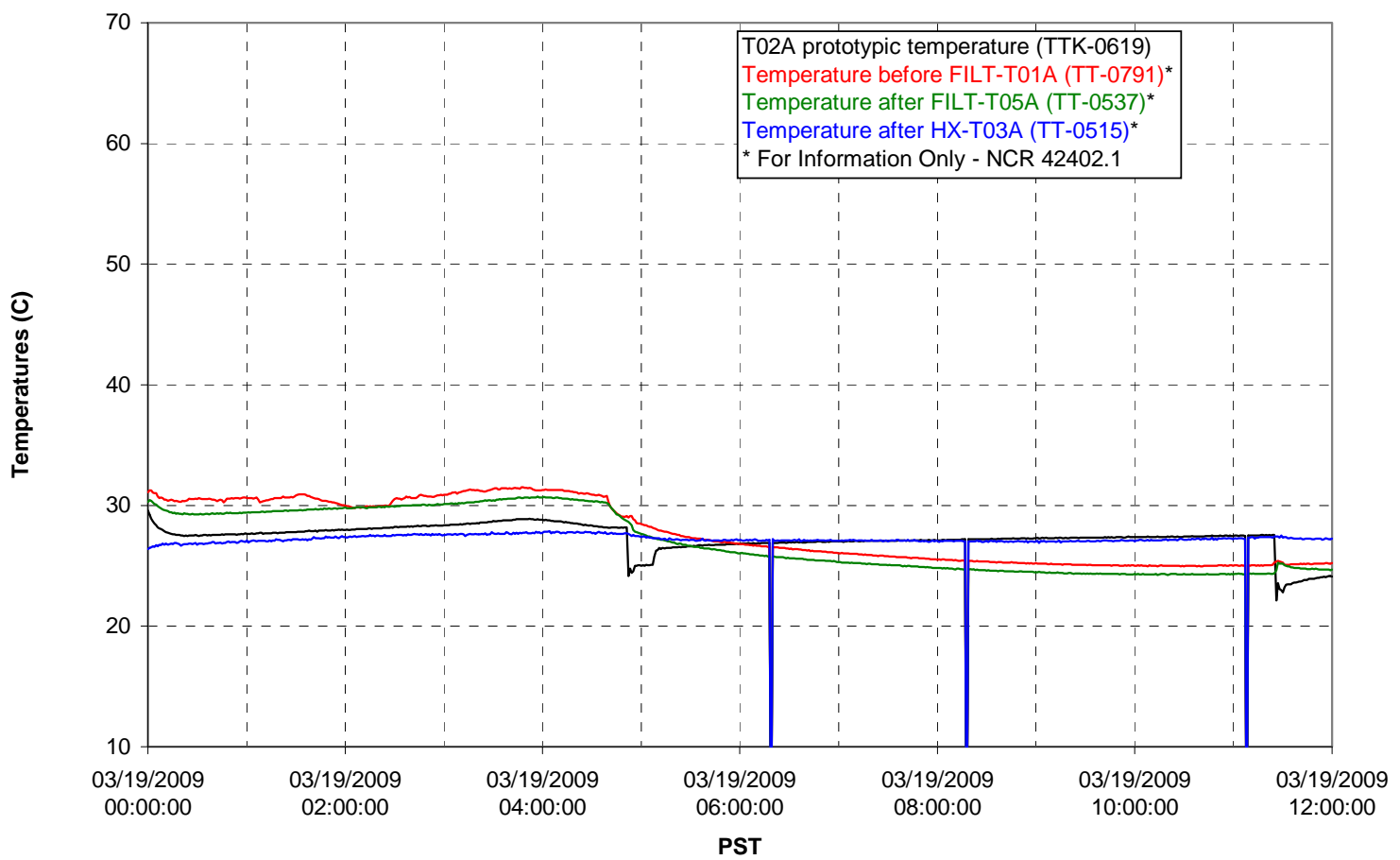

Pump Pressures and Flow

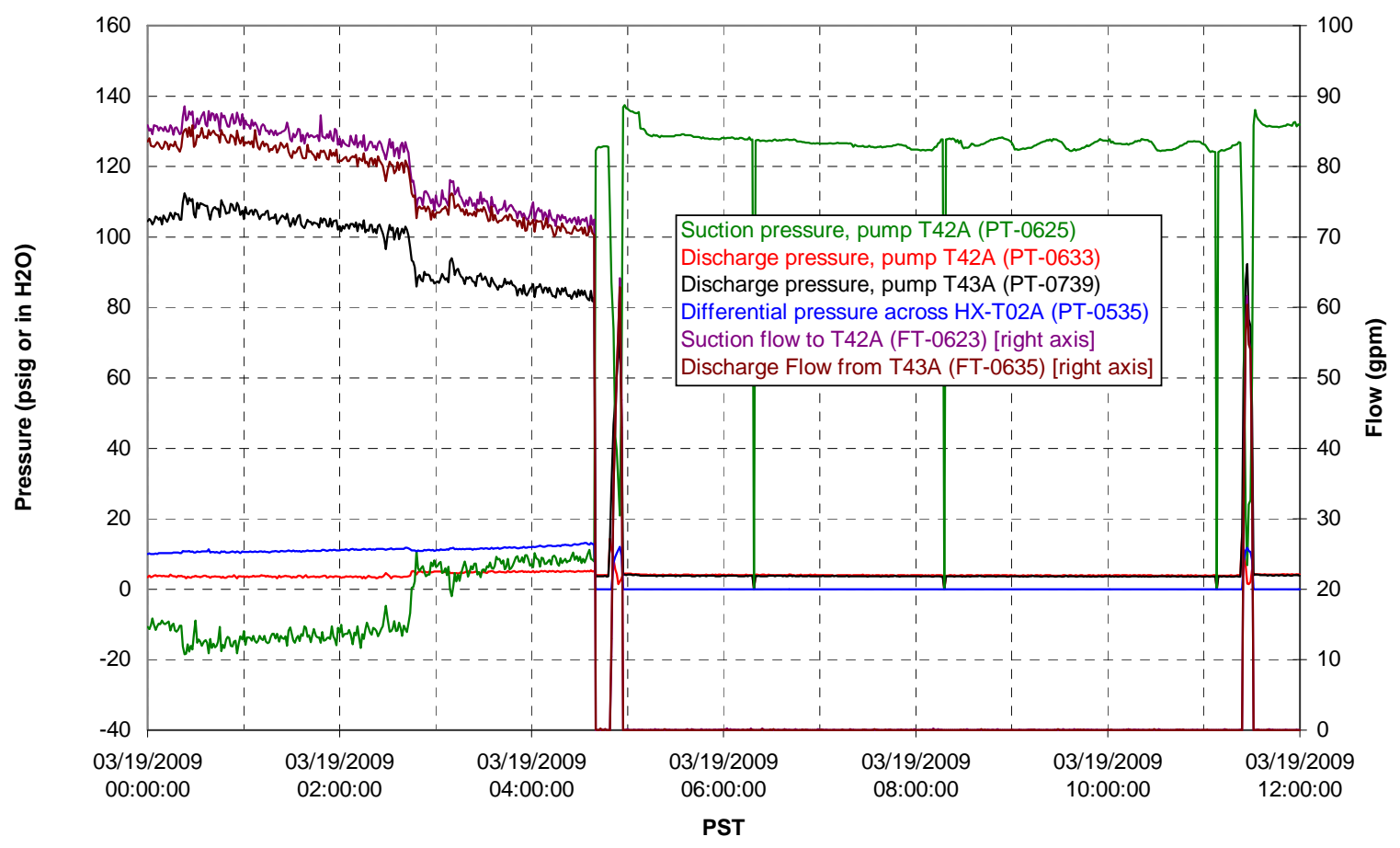




\section{Axial pressure drop}

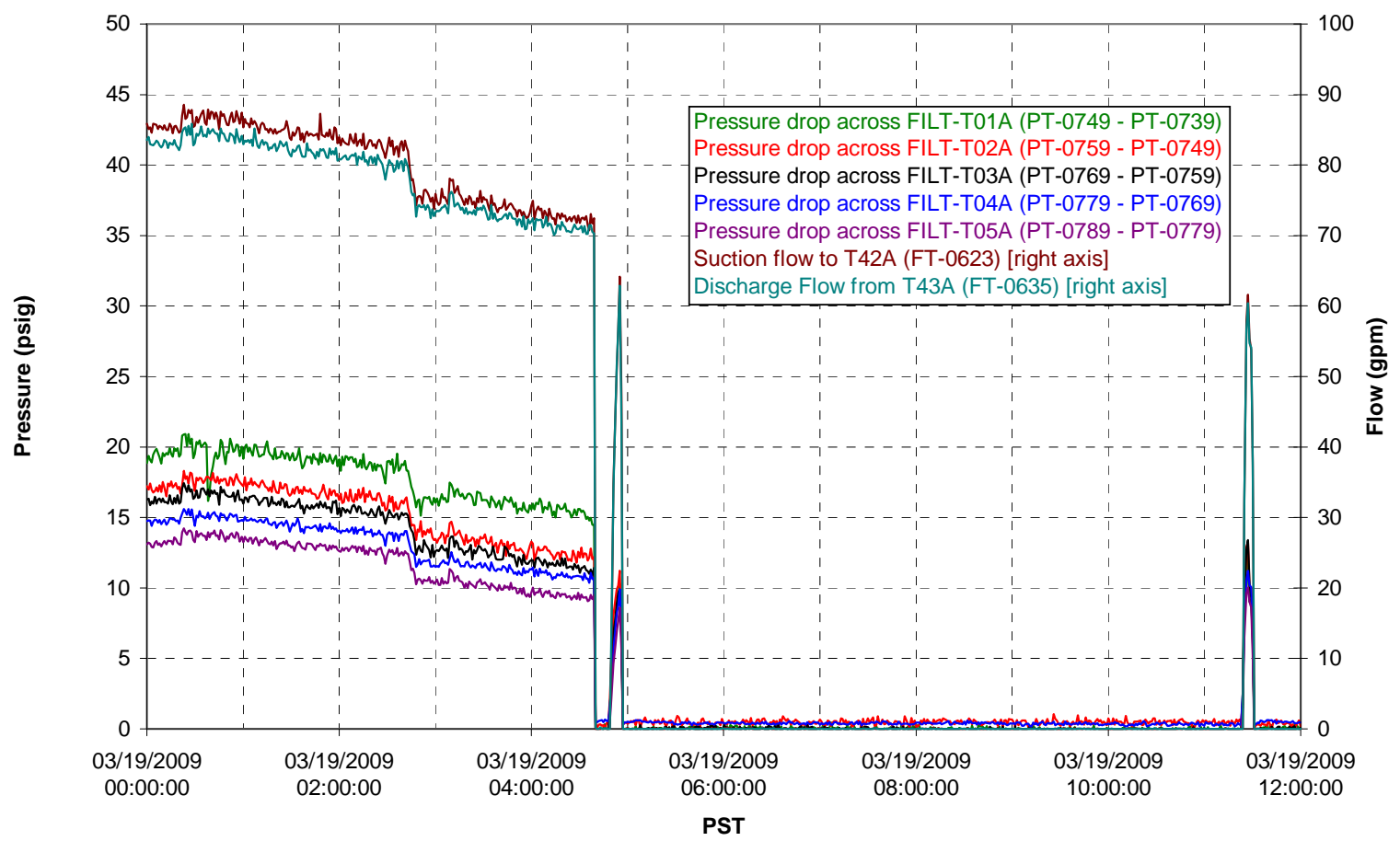

Permeate flow rates

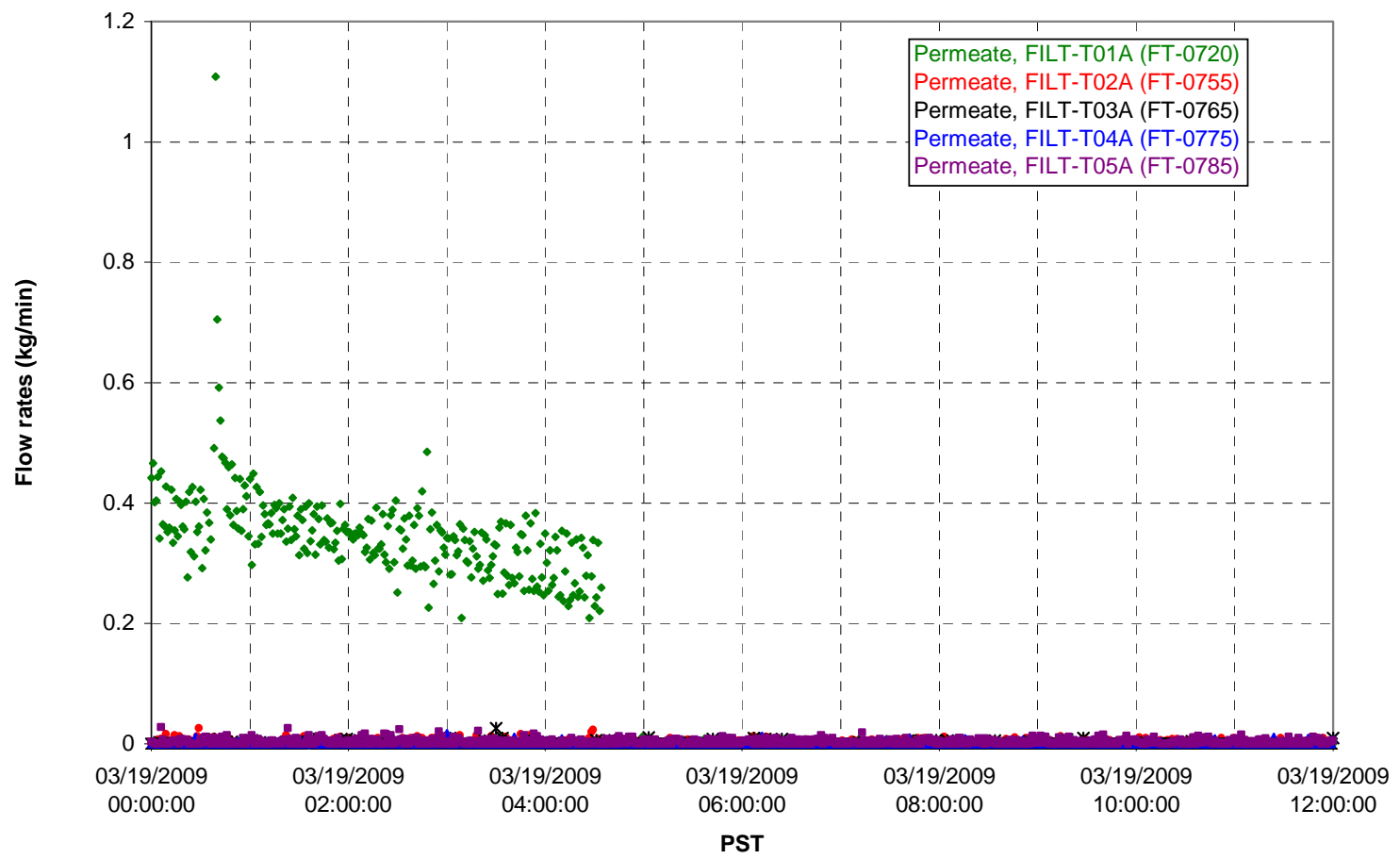


T02A Inner Temperature Tree

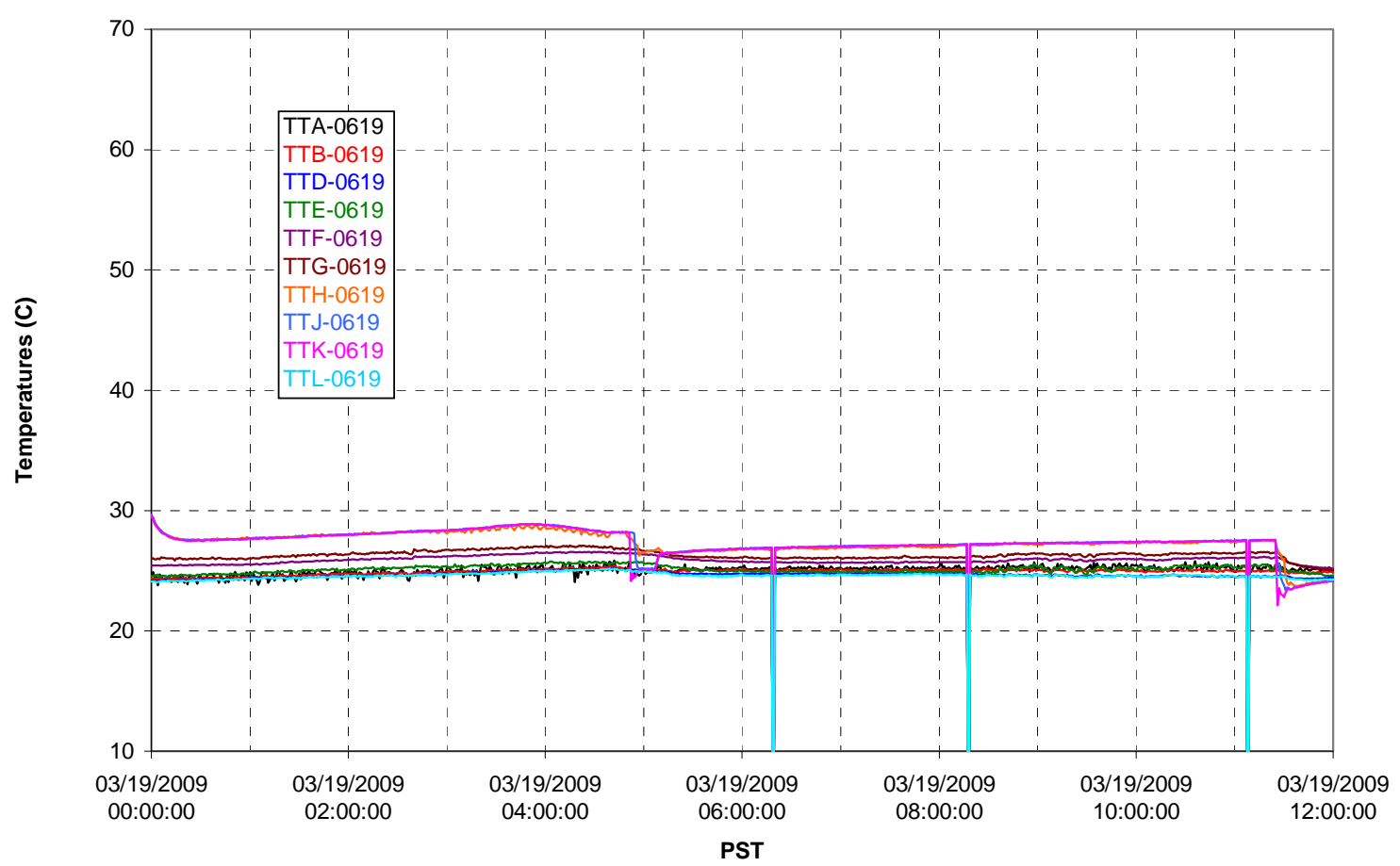

T02A Outer Temperature Tree

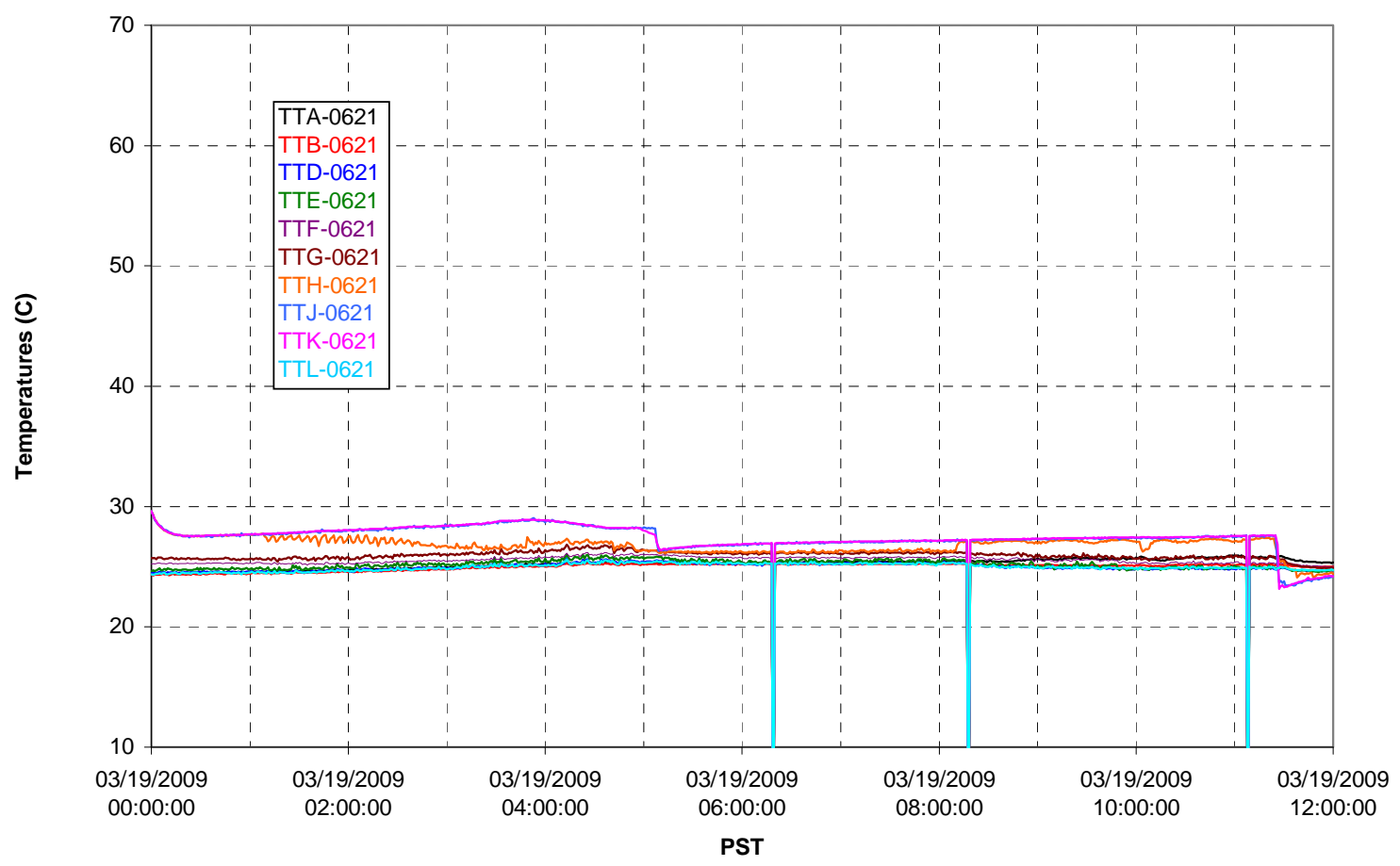


T02A temperatures

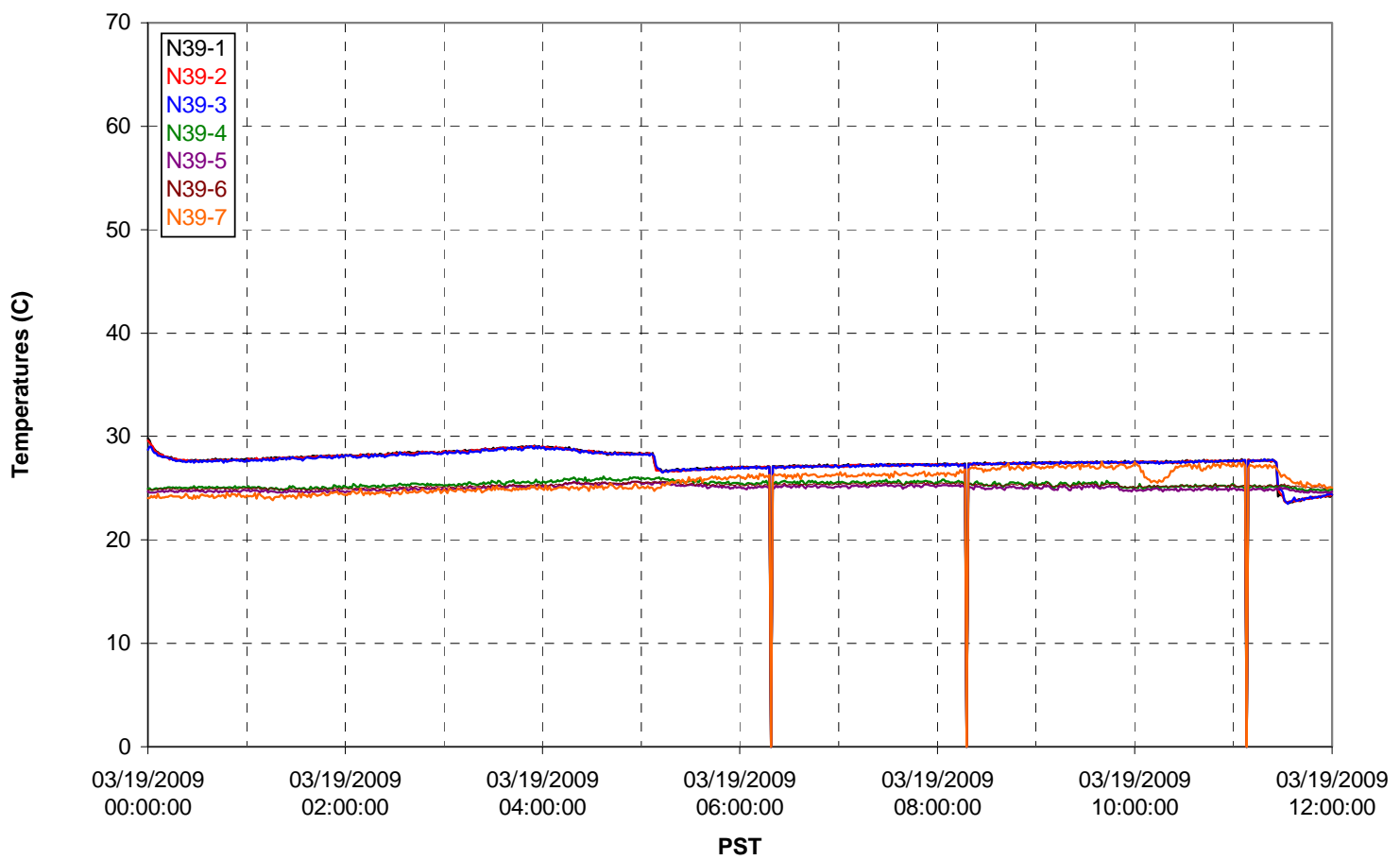

T02A temperatures

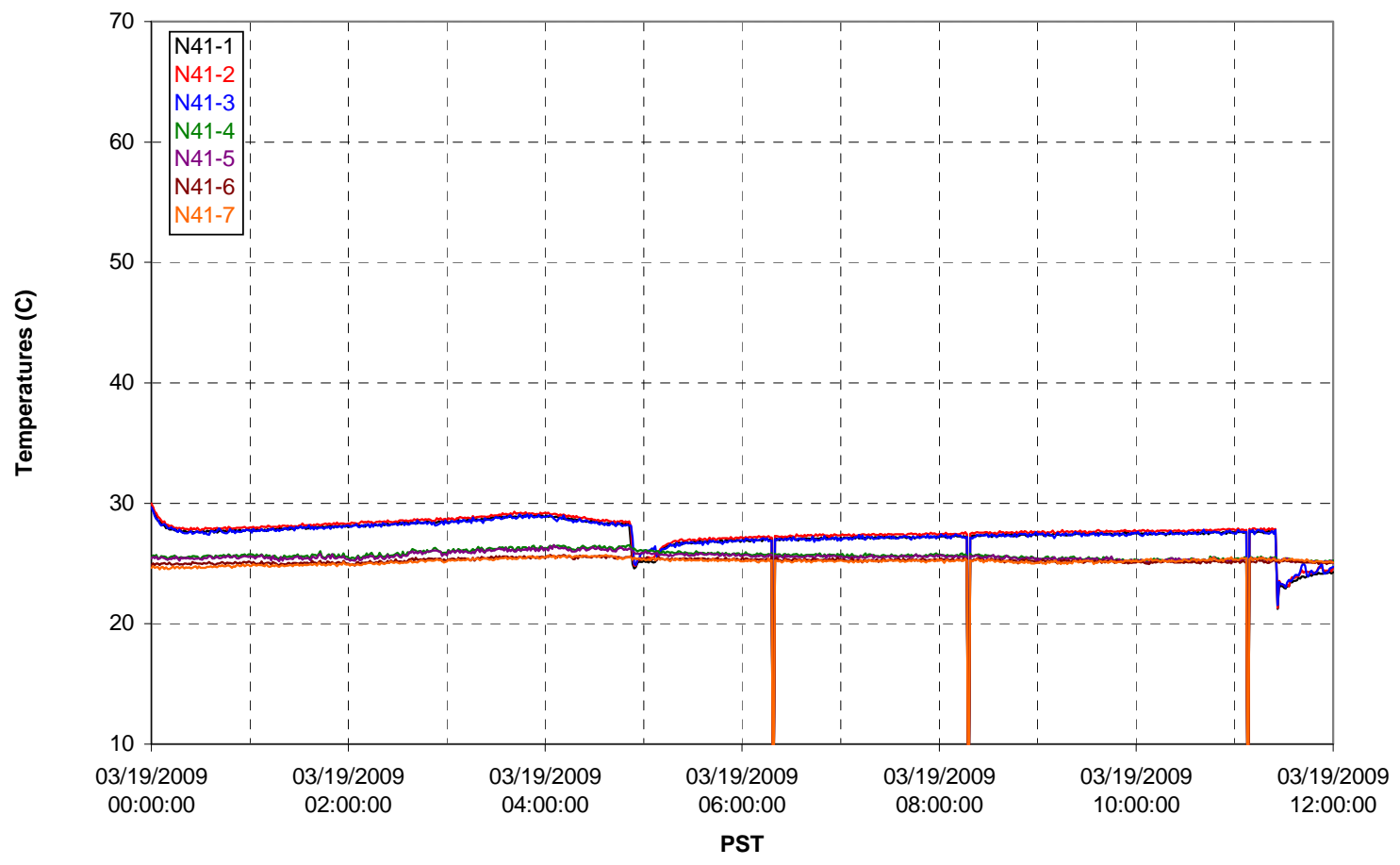


T02A temperatures

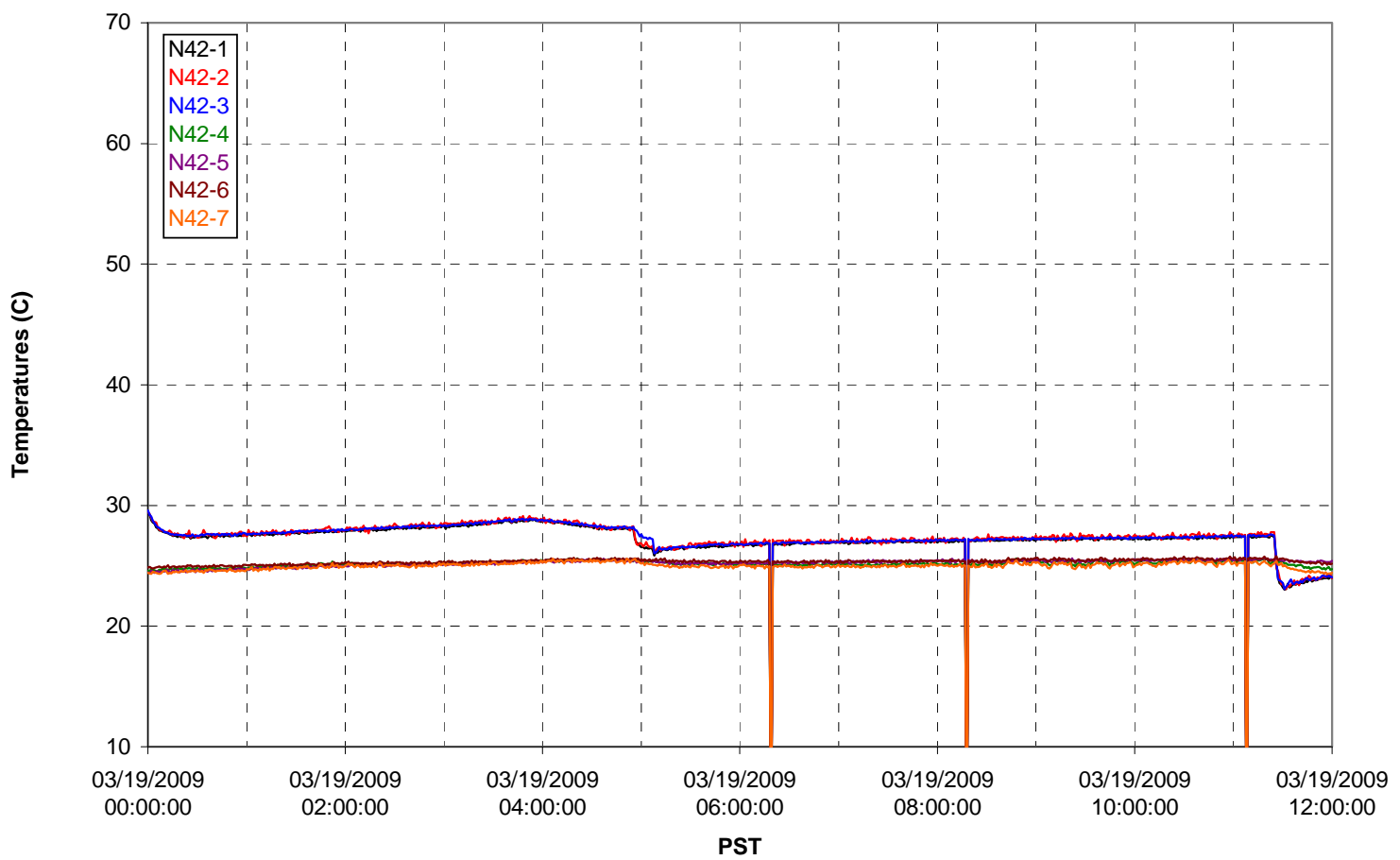

T02A temperatures

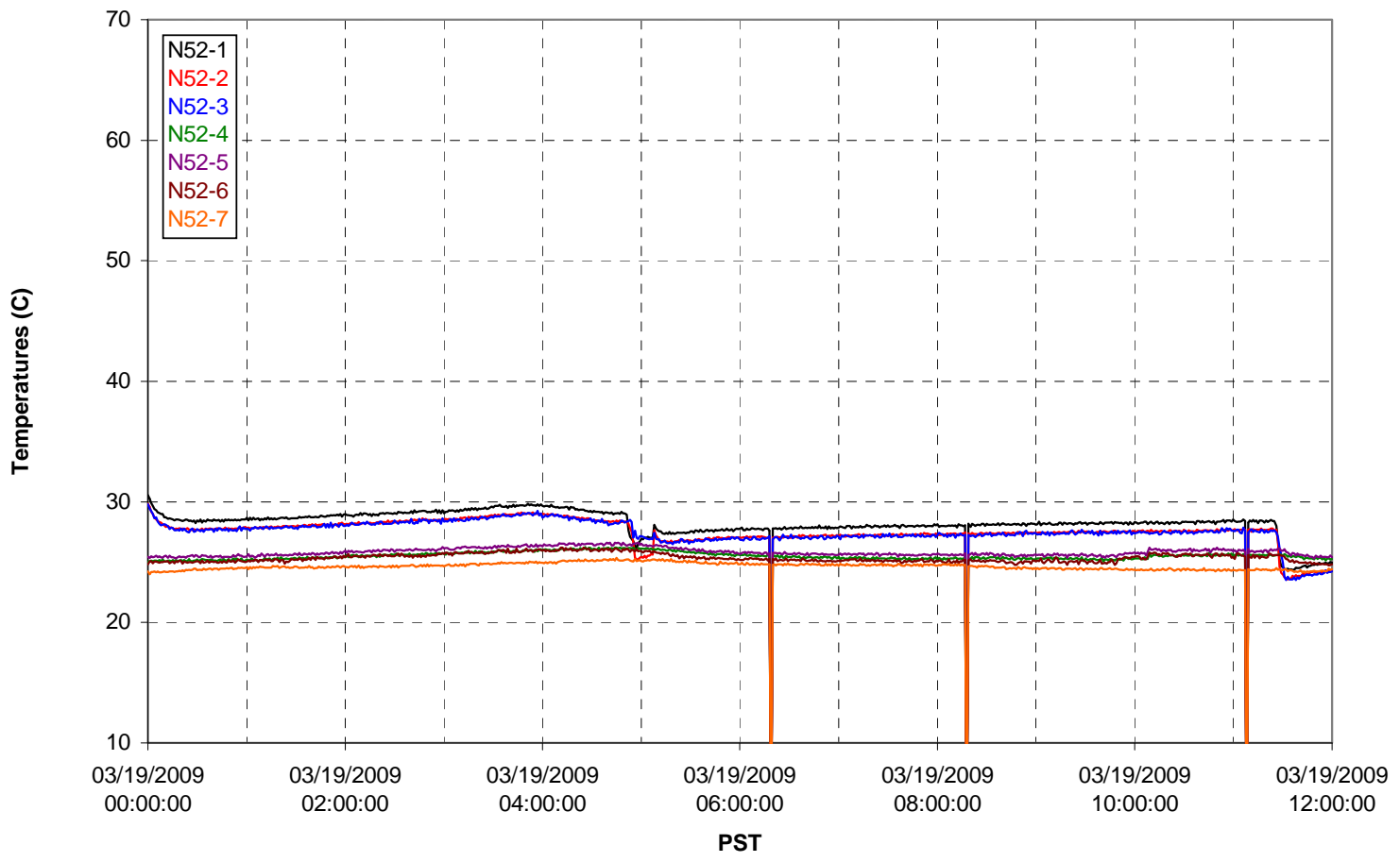


T02A Heating and Cooling

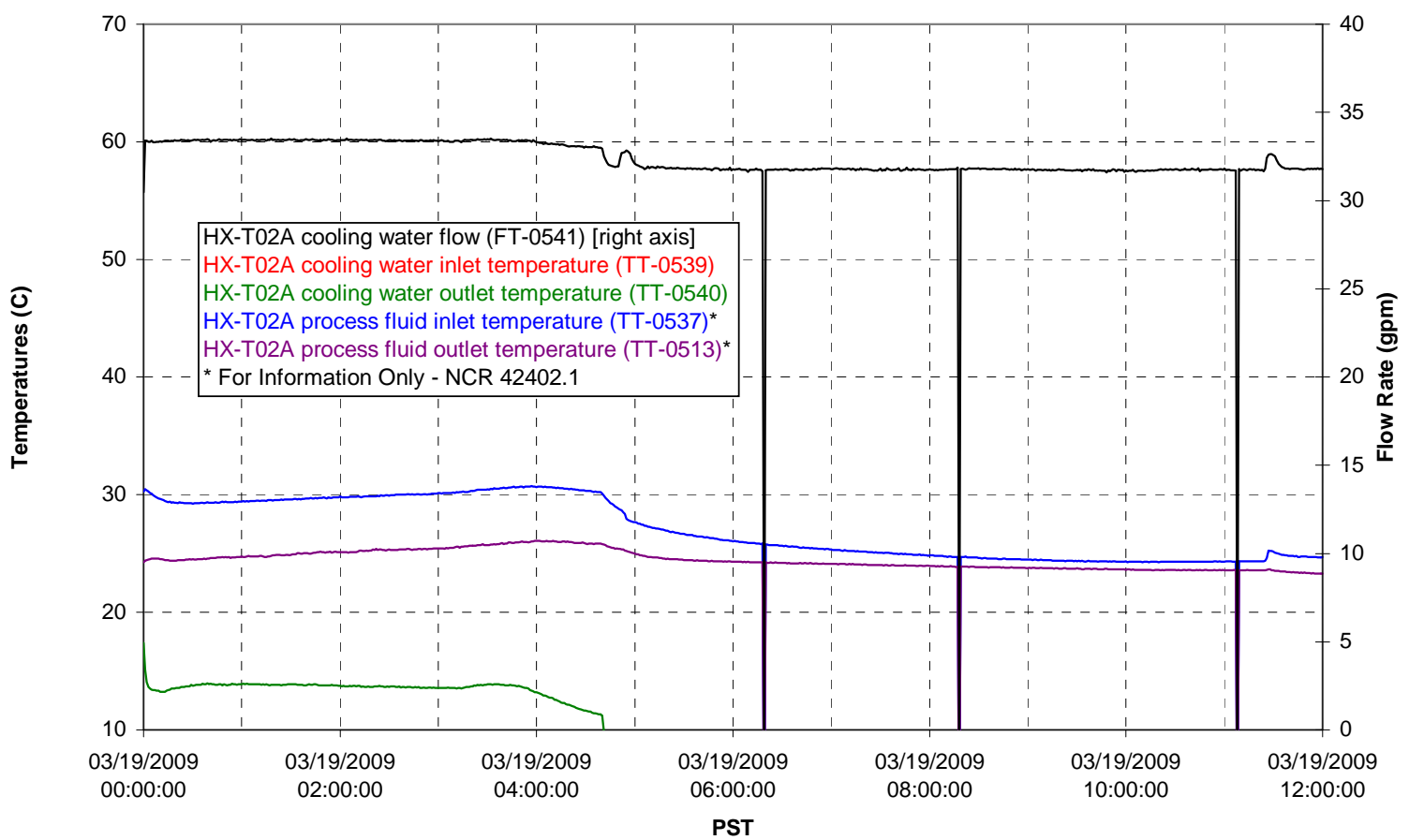

Pump Operation

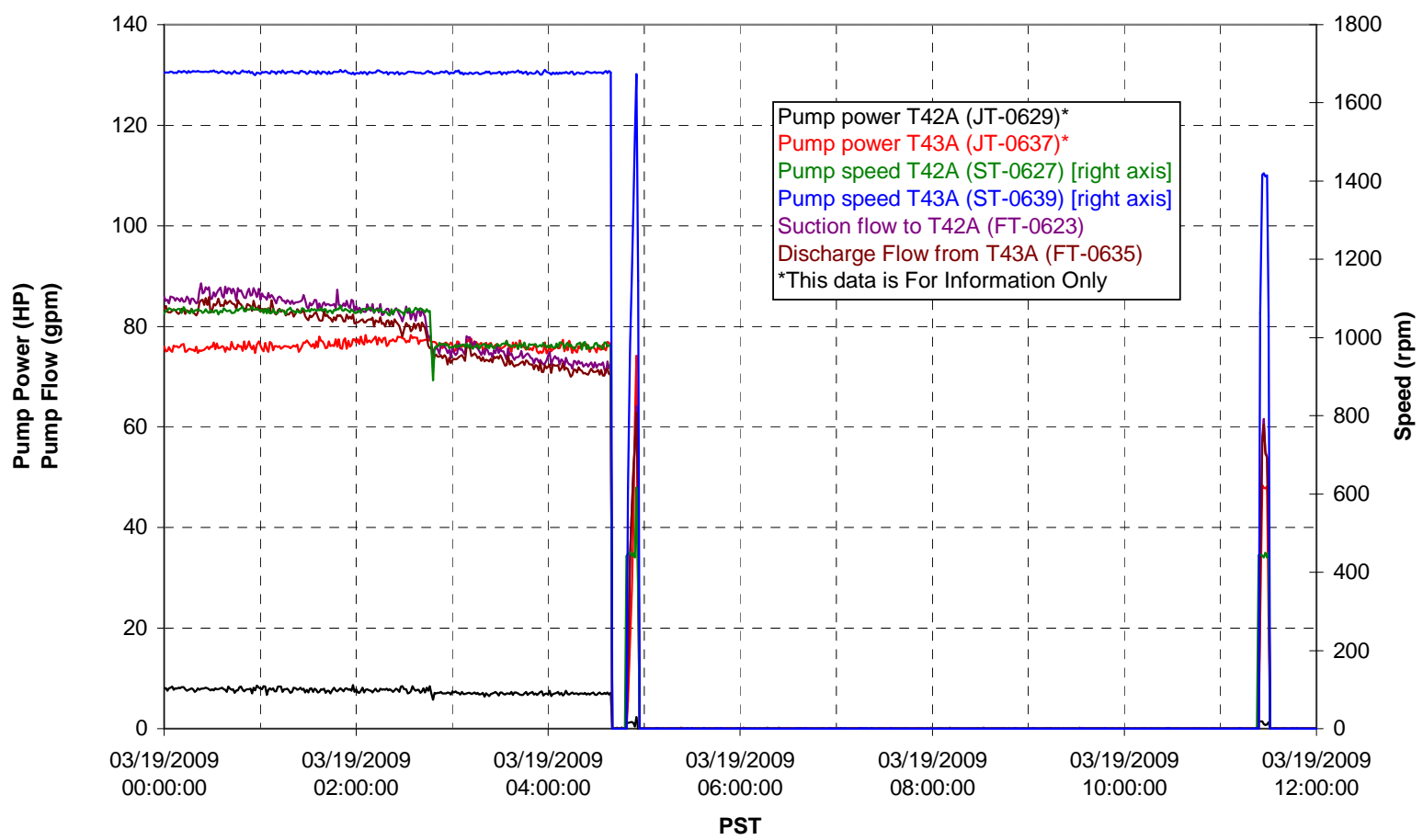


Pulsepot UFP-PP-T01A

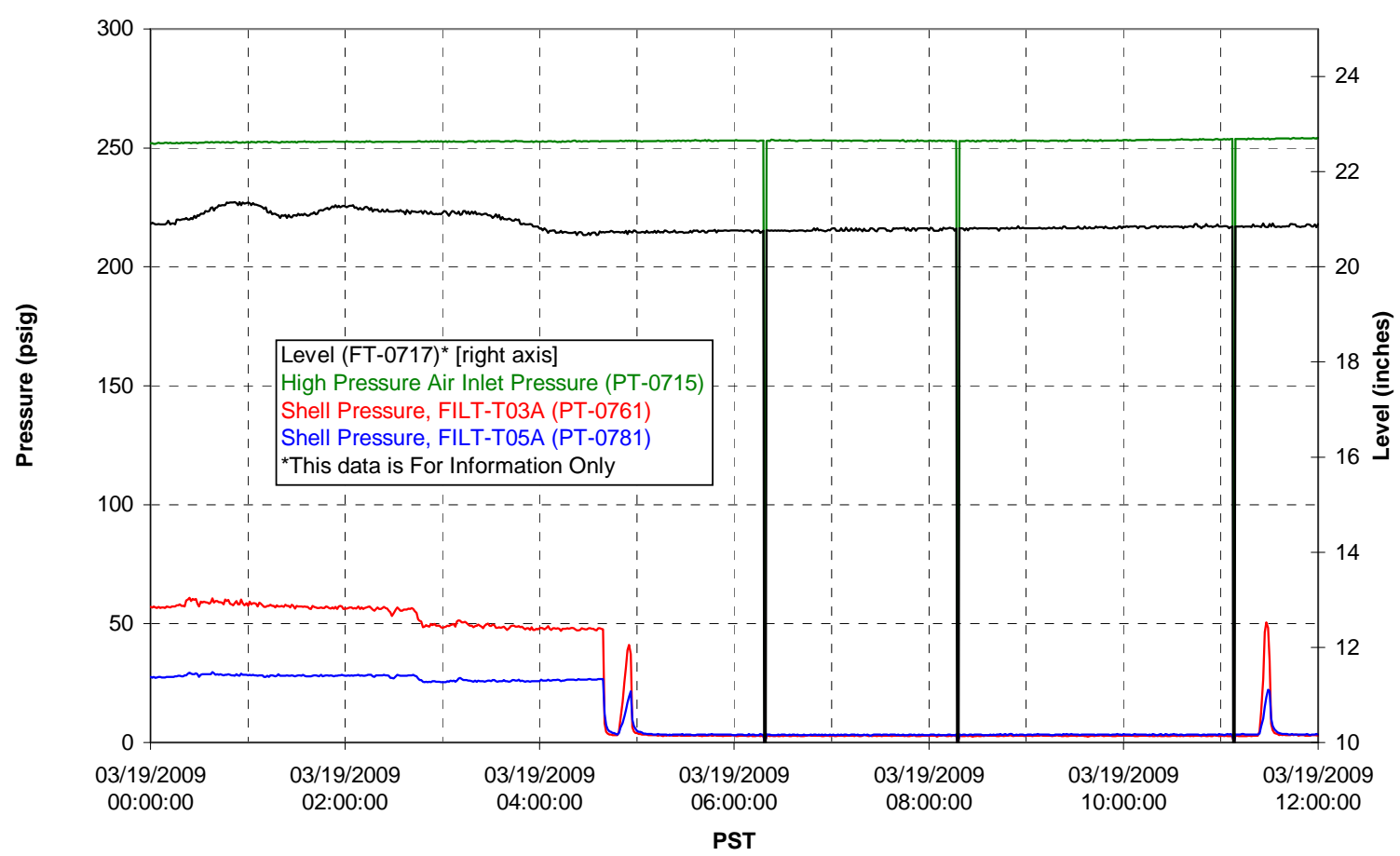

Pulsepot UFP-PP-T02A

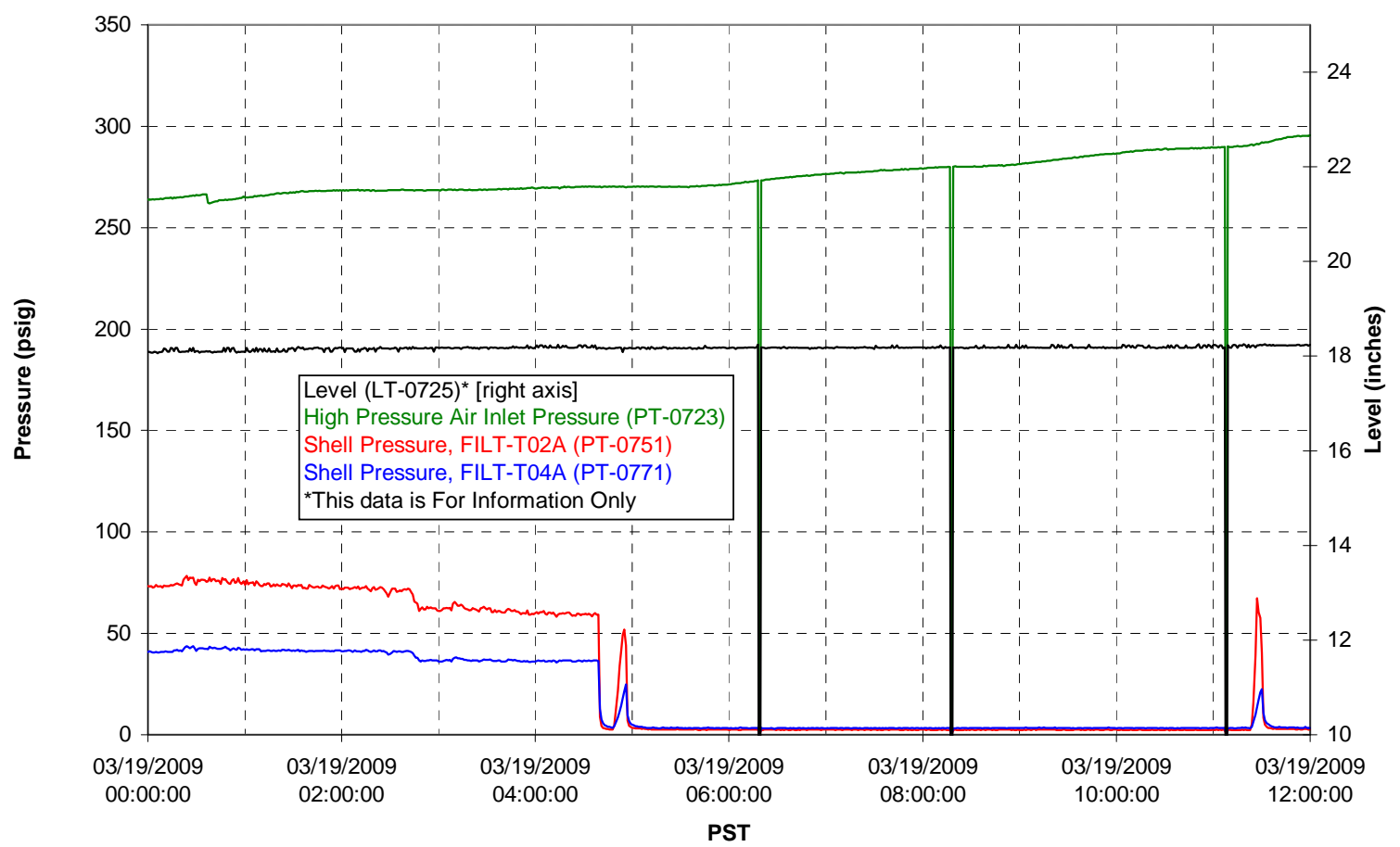




\section{Pulsepot UFP-PP-T03A}

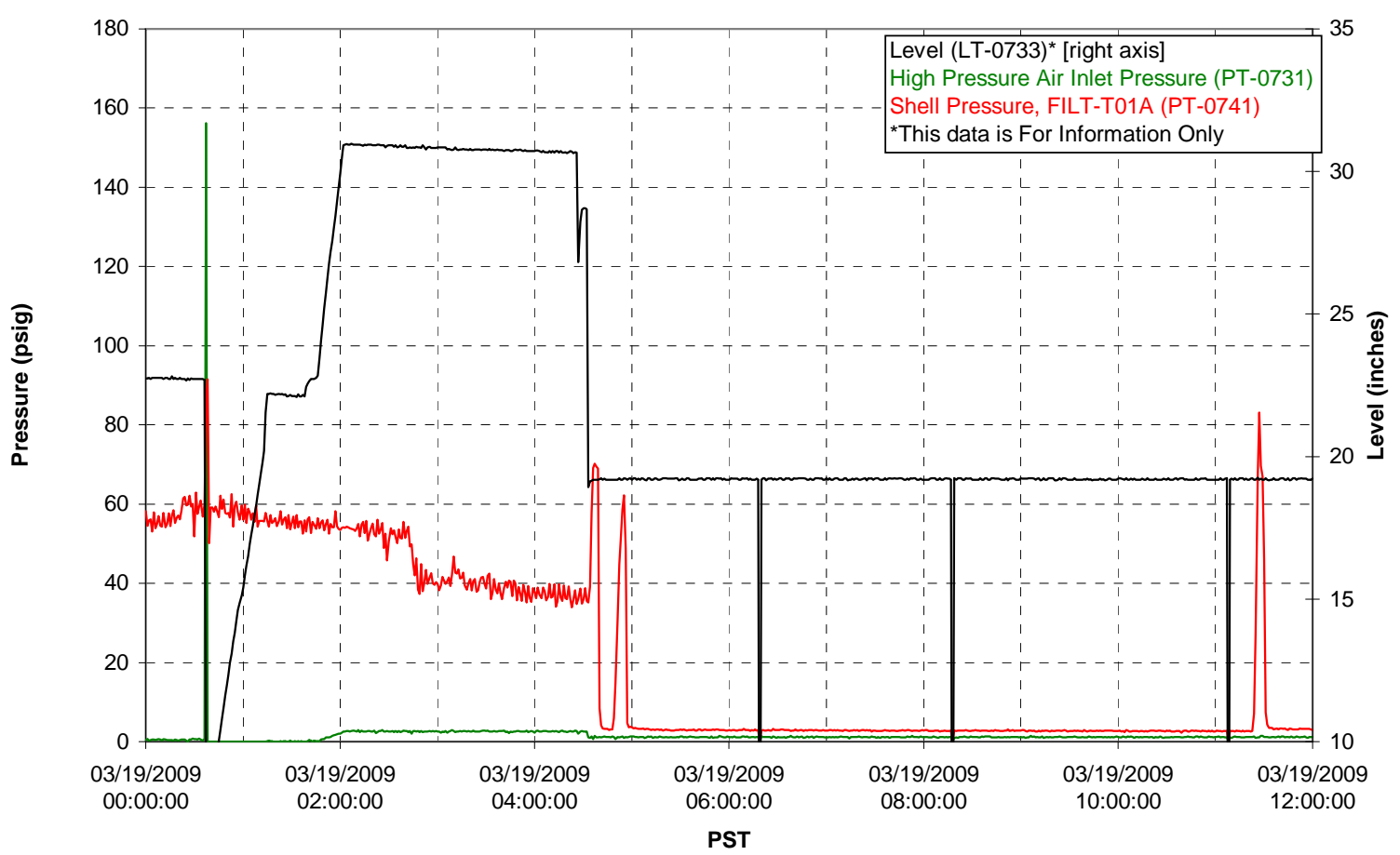

Pulsepot Levels

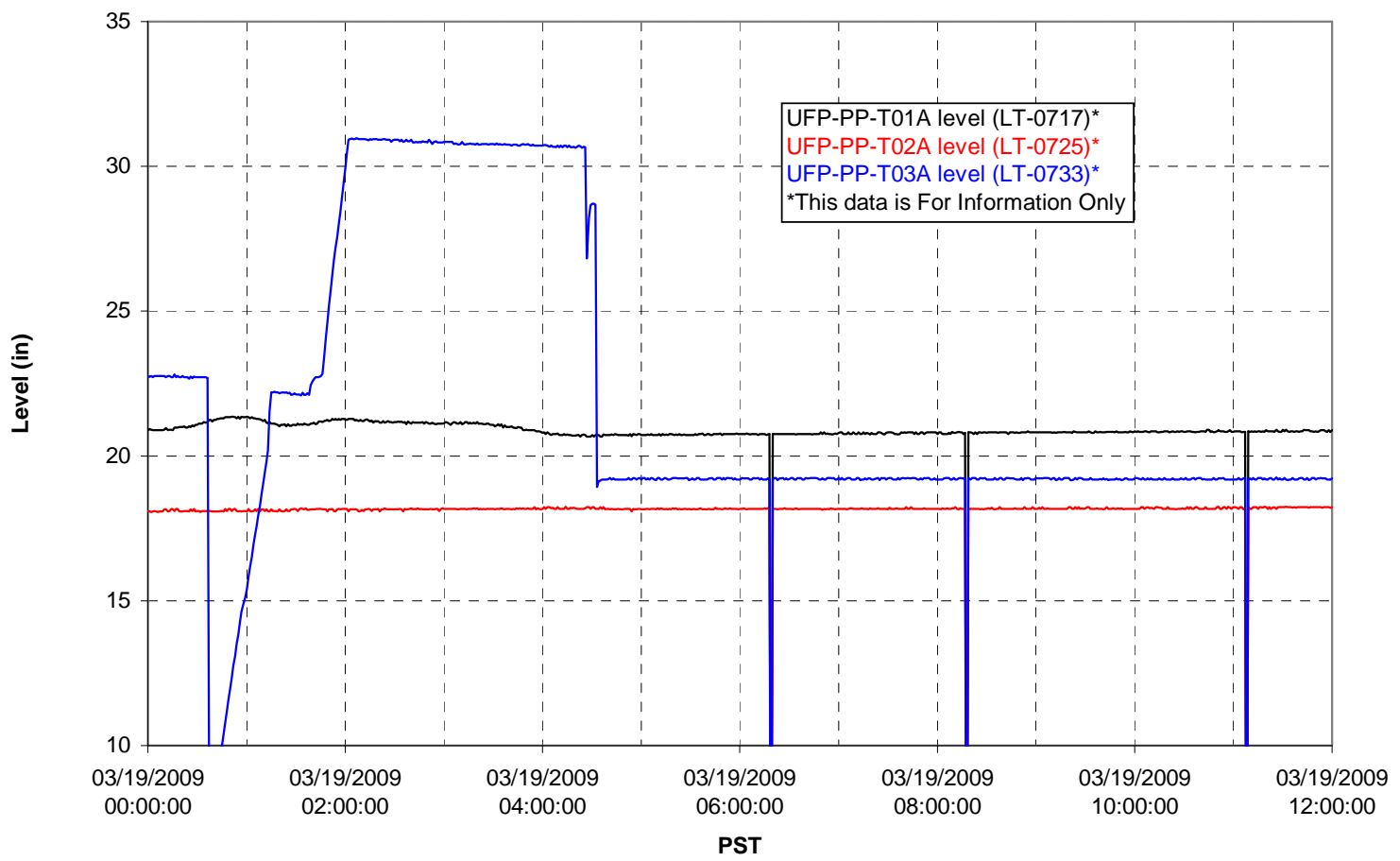


Filter UFP-FILT-T01A

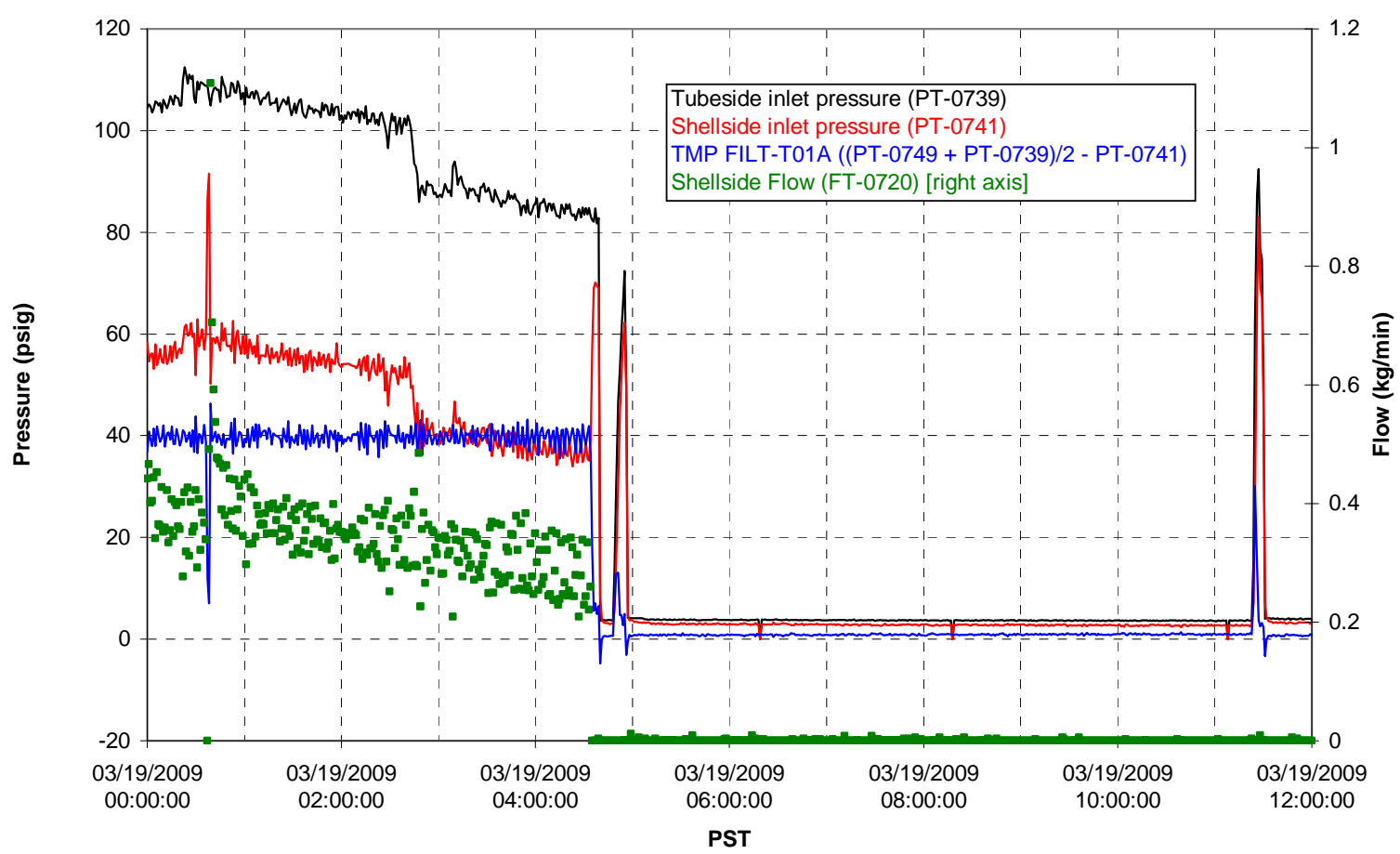

Filter UFP-FILT-T02A

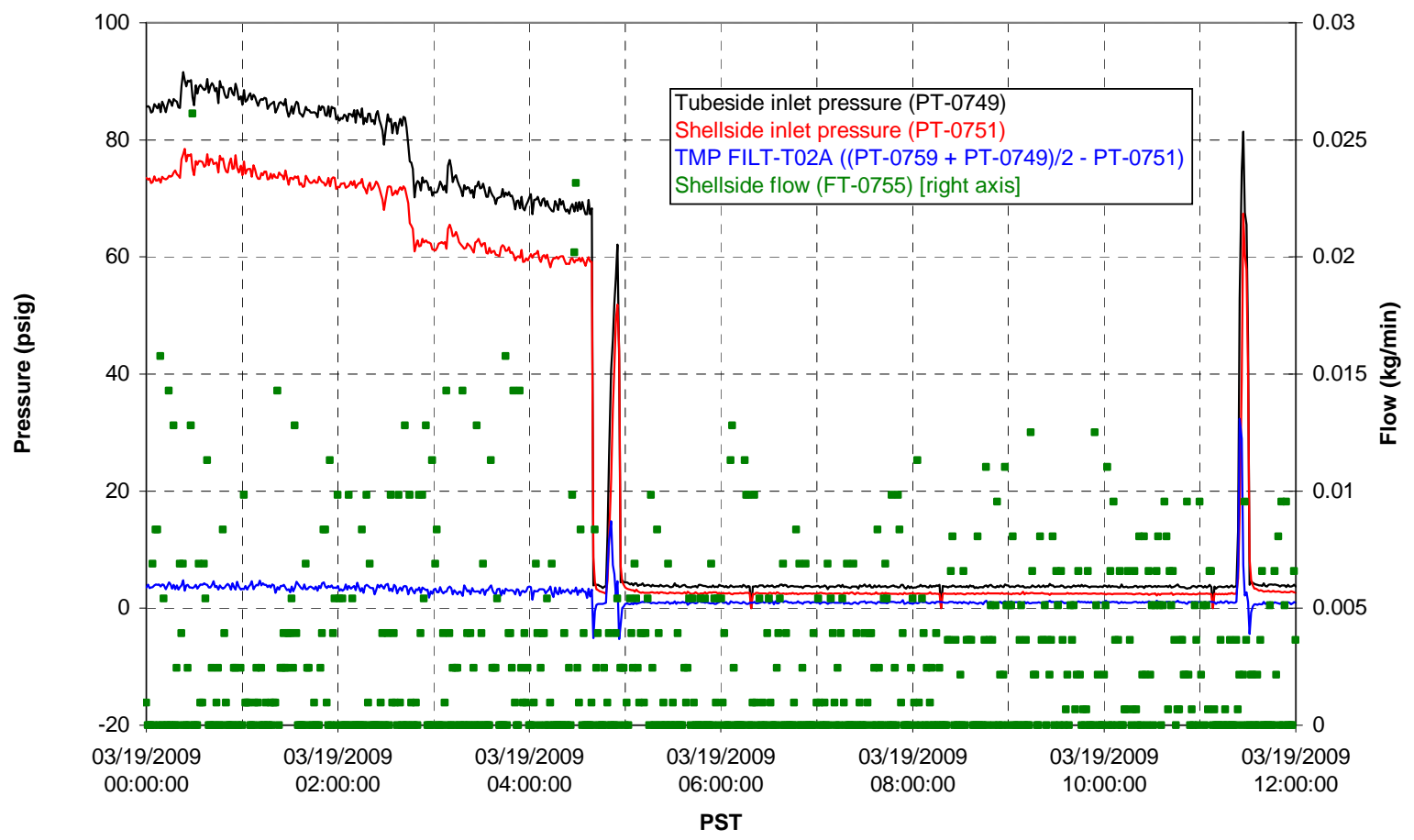


Filter UFP-FILT-T03A

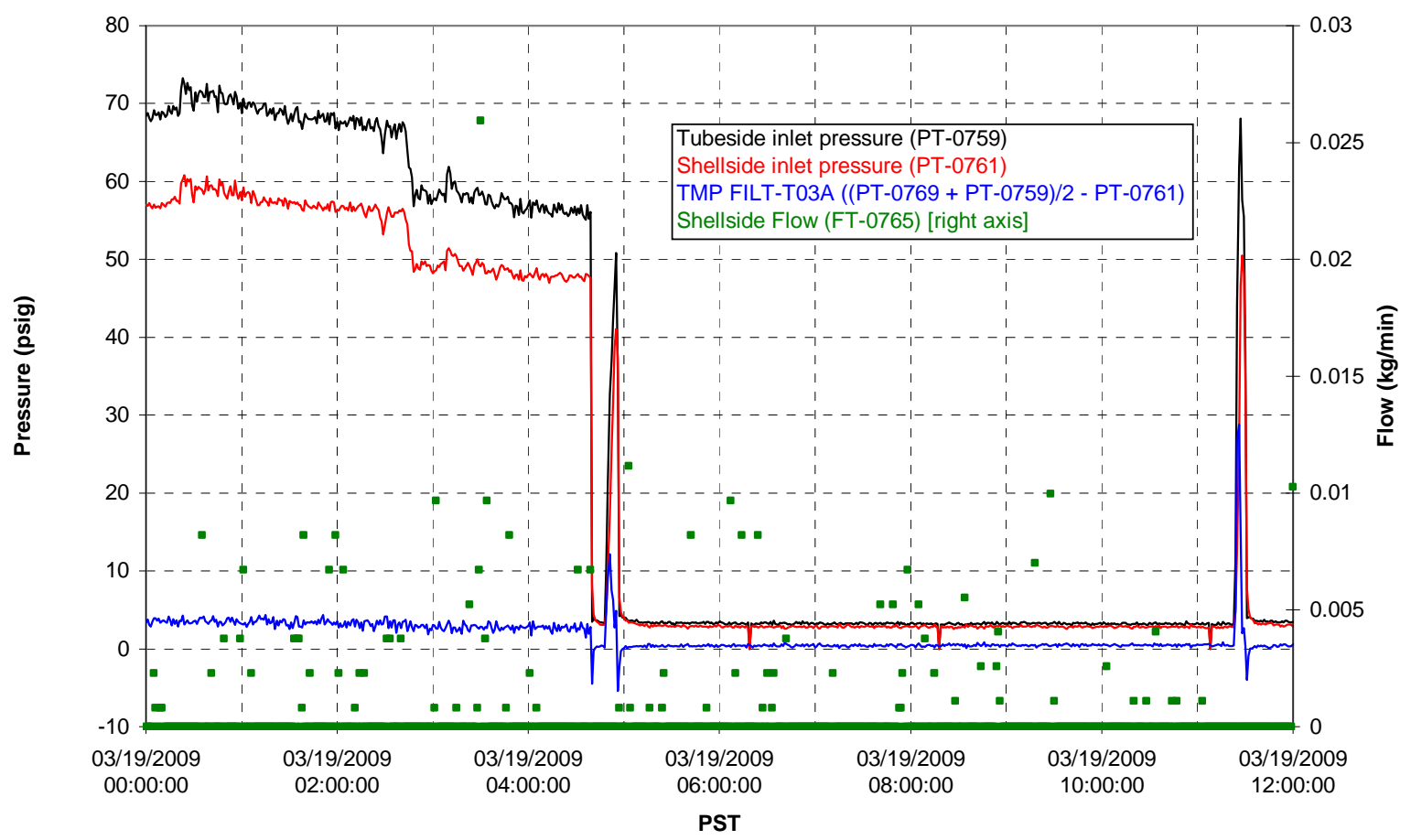

Filter UFP-FILT-T04A

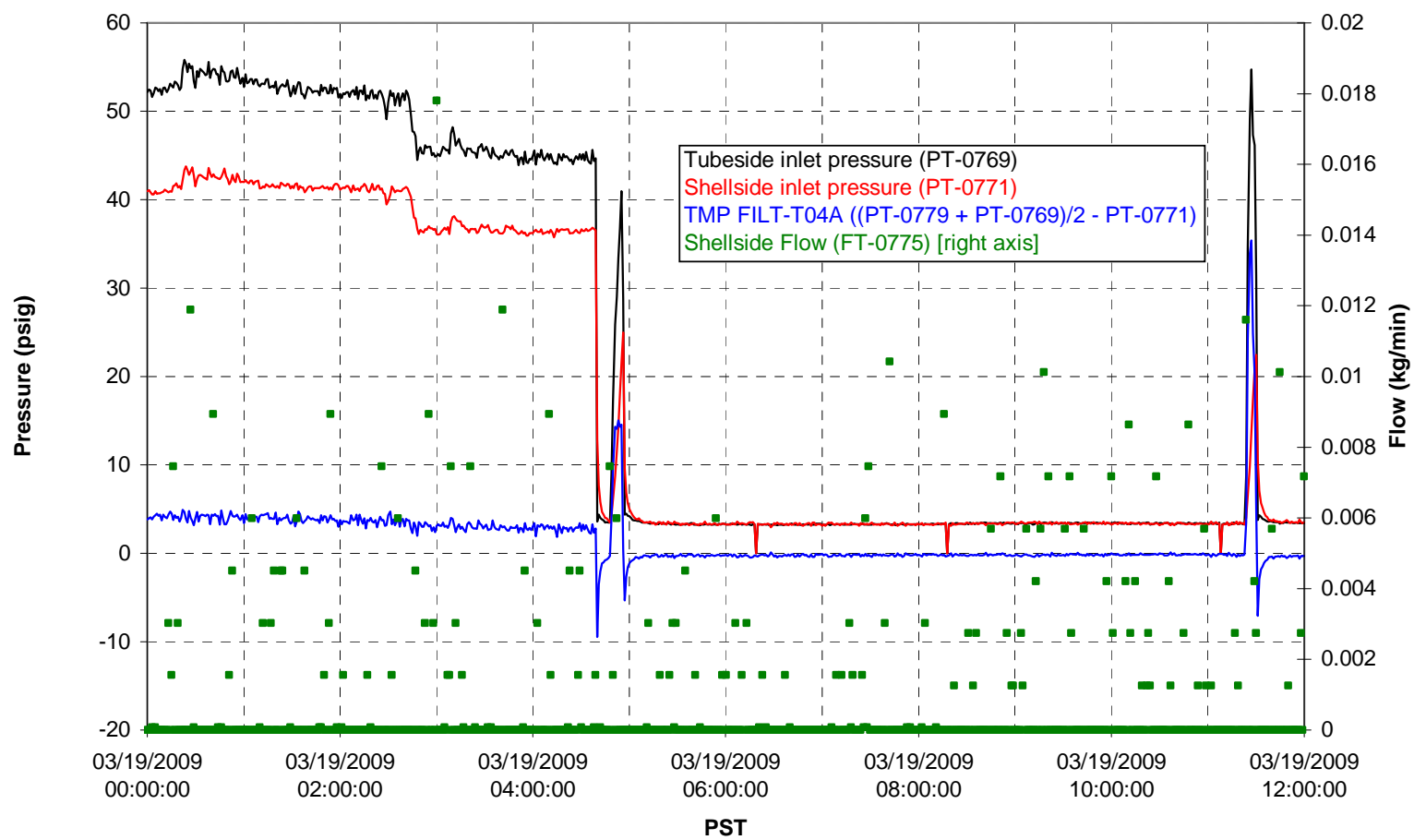


Filter UFP-FILT-T05A

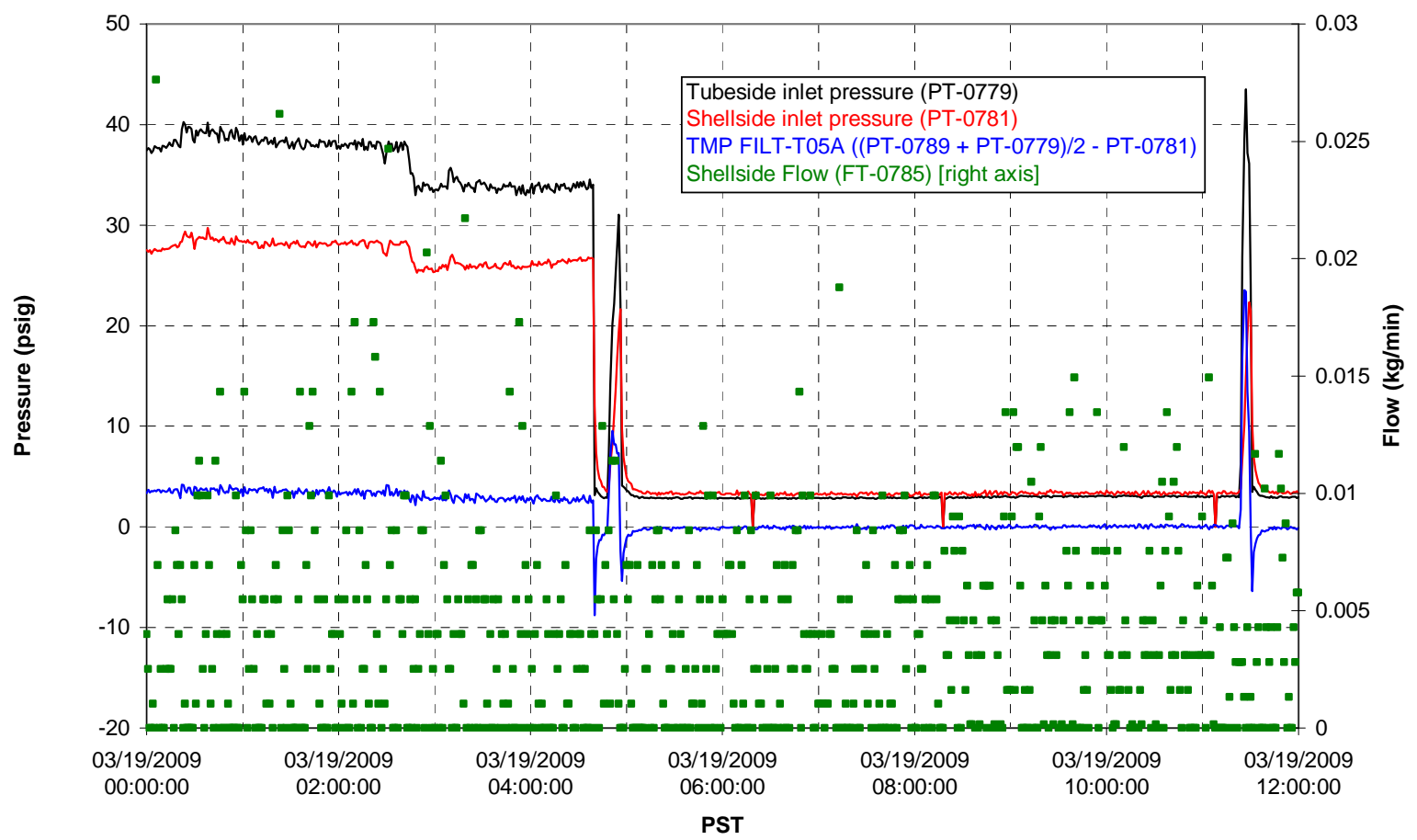

Chemical Flow

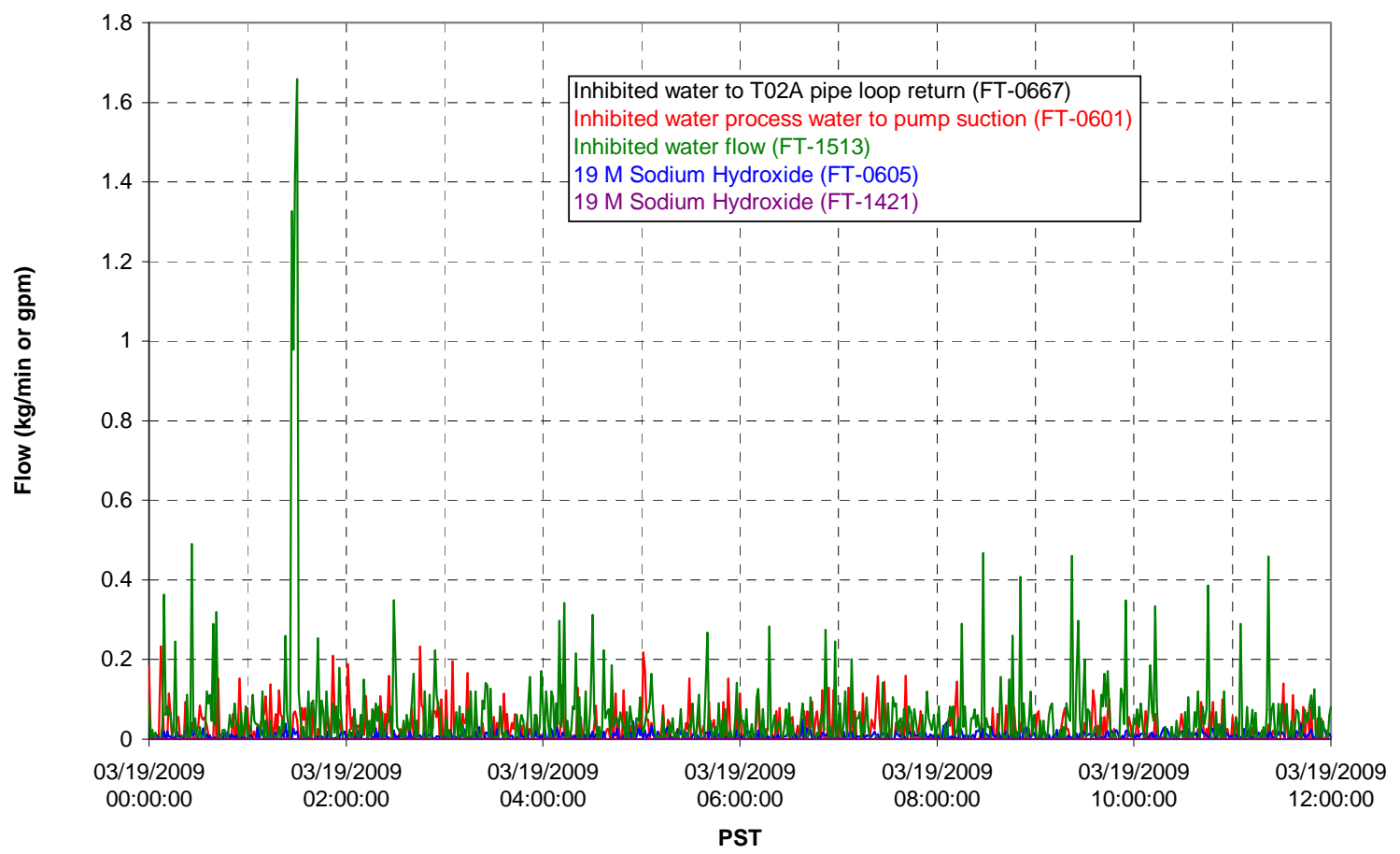




\section{Chemical Flow}

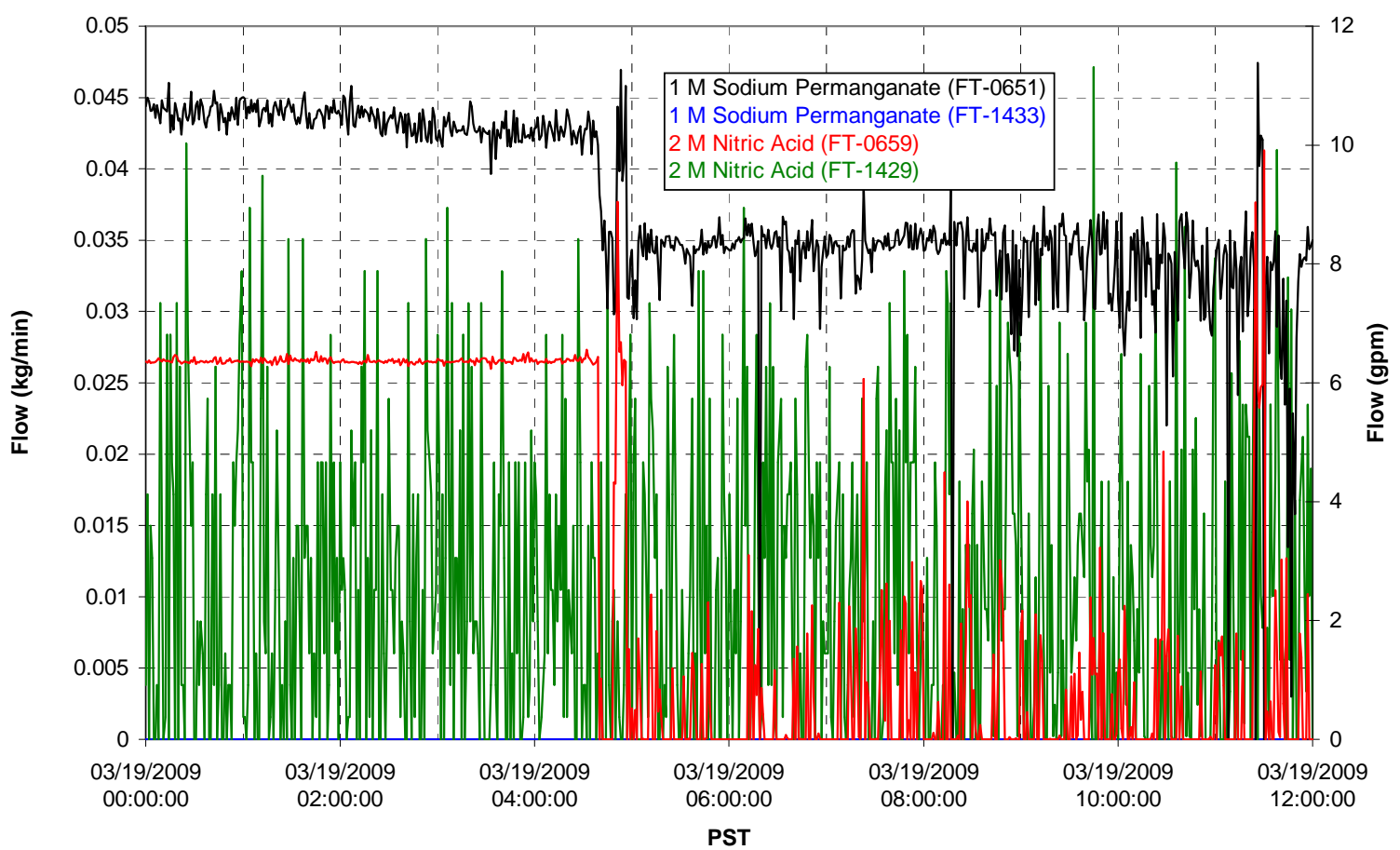

Air Flows

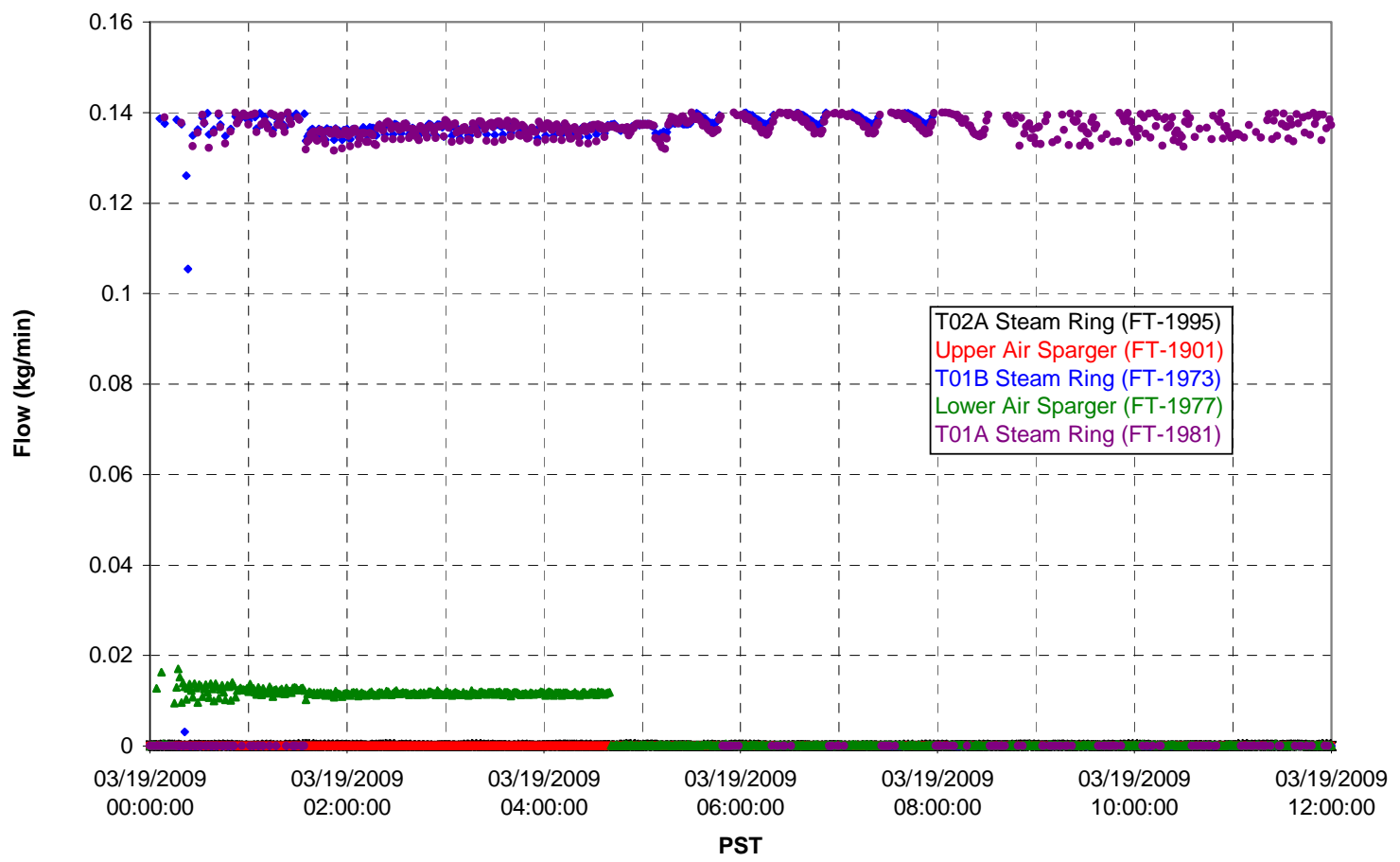


T02A Steam

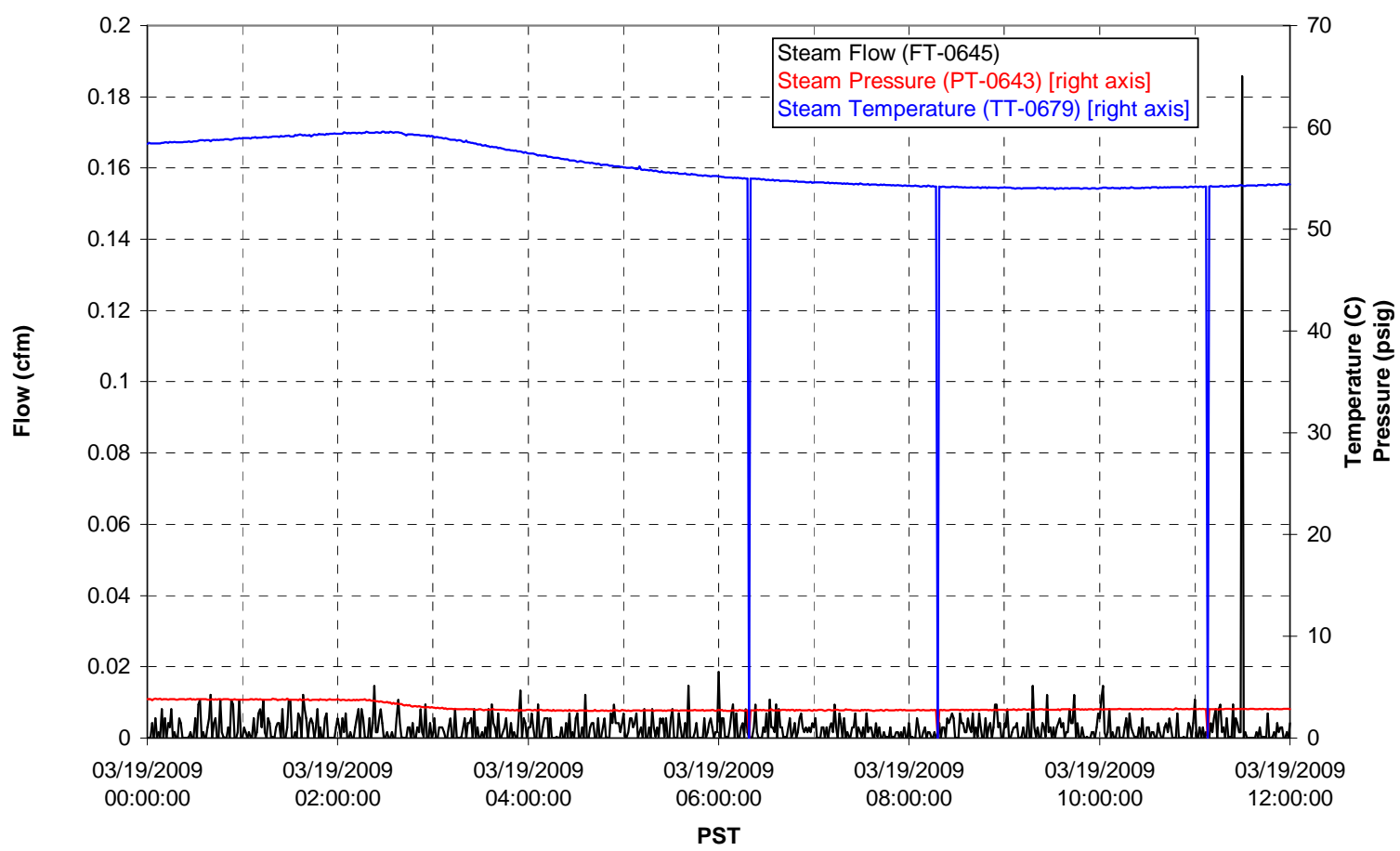

T01A Steam

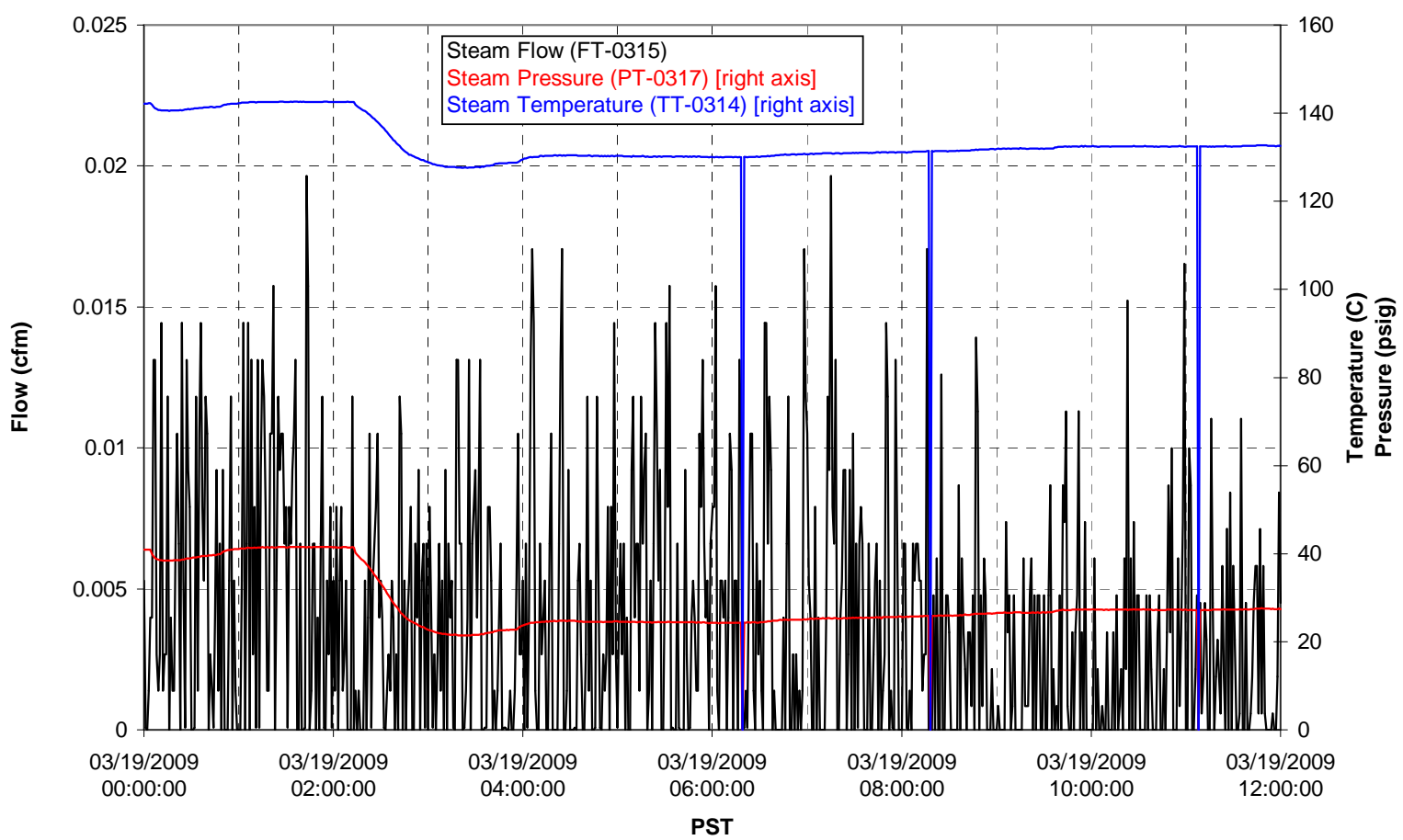


T01B Steam

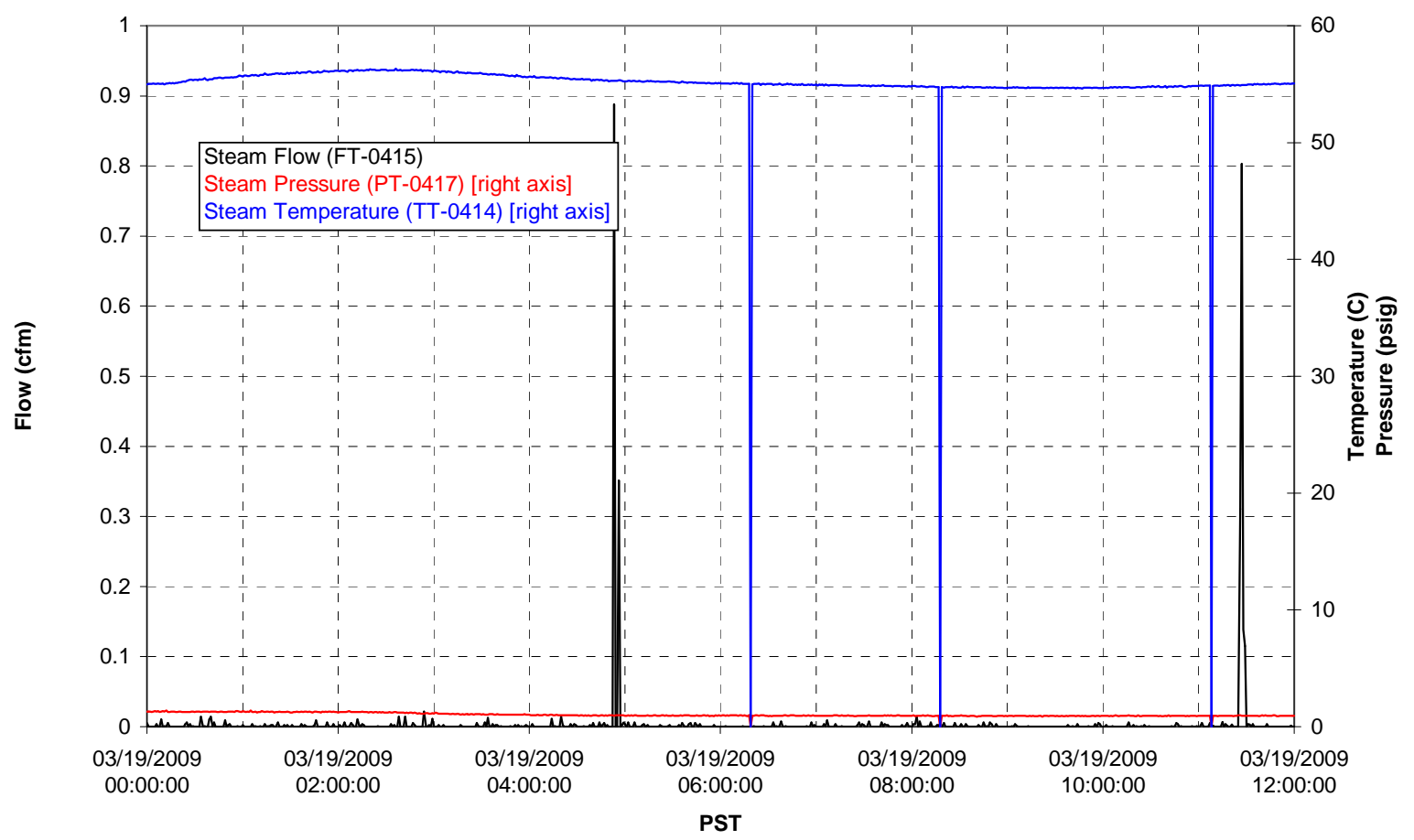




\section{Integrated Test B Data Plots \\ 03/19/09 12:00 - 03/20/09 00:00}


T01A level

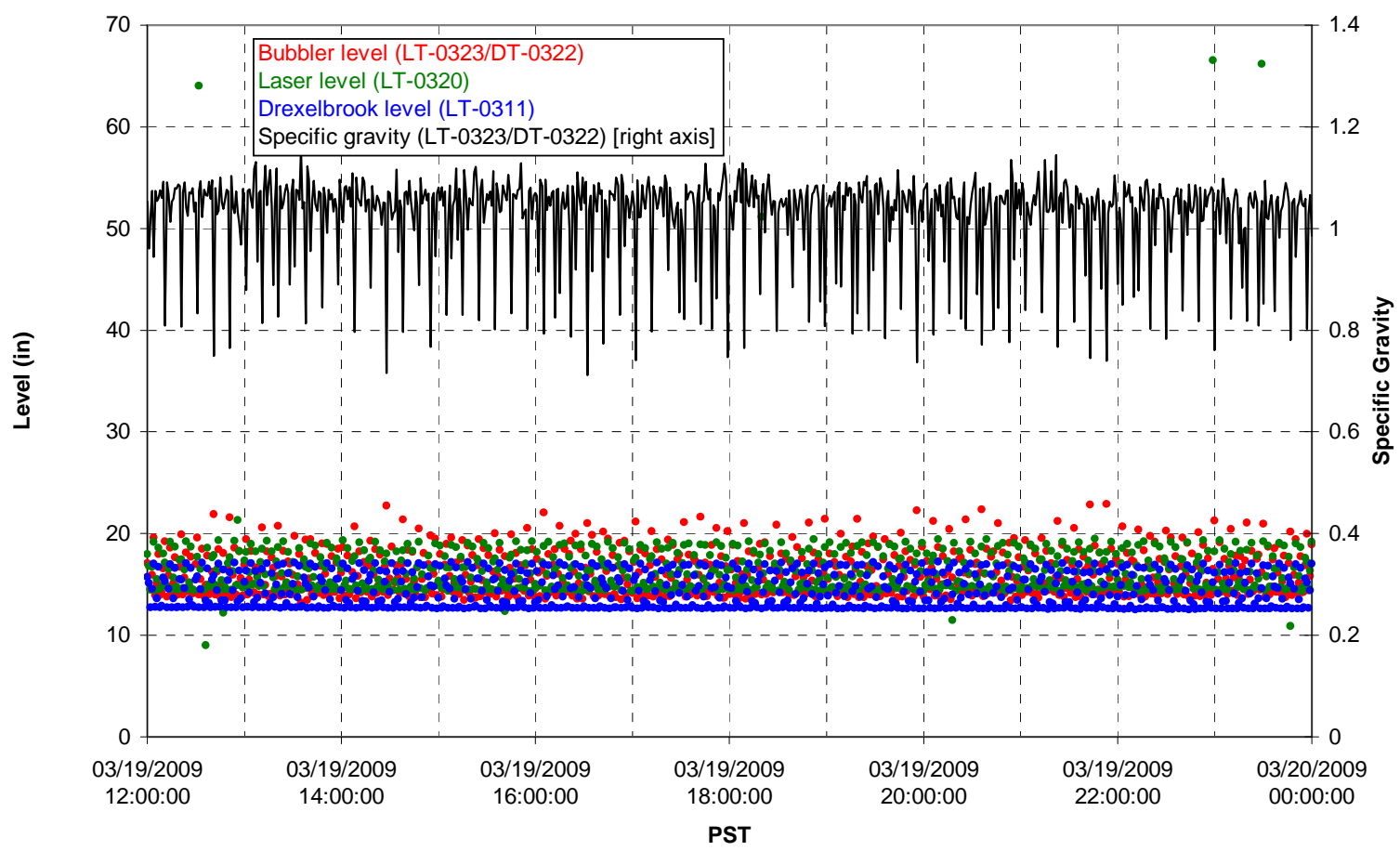

T01A temperatures

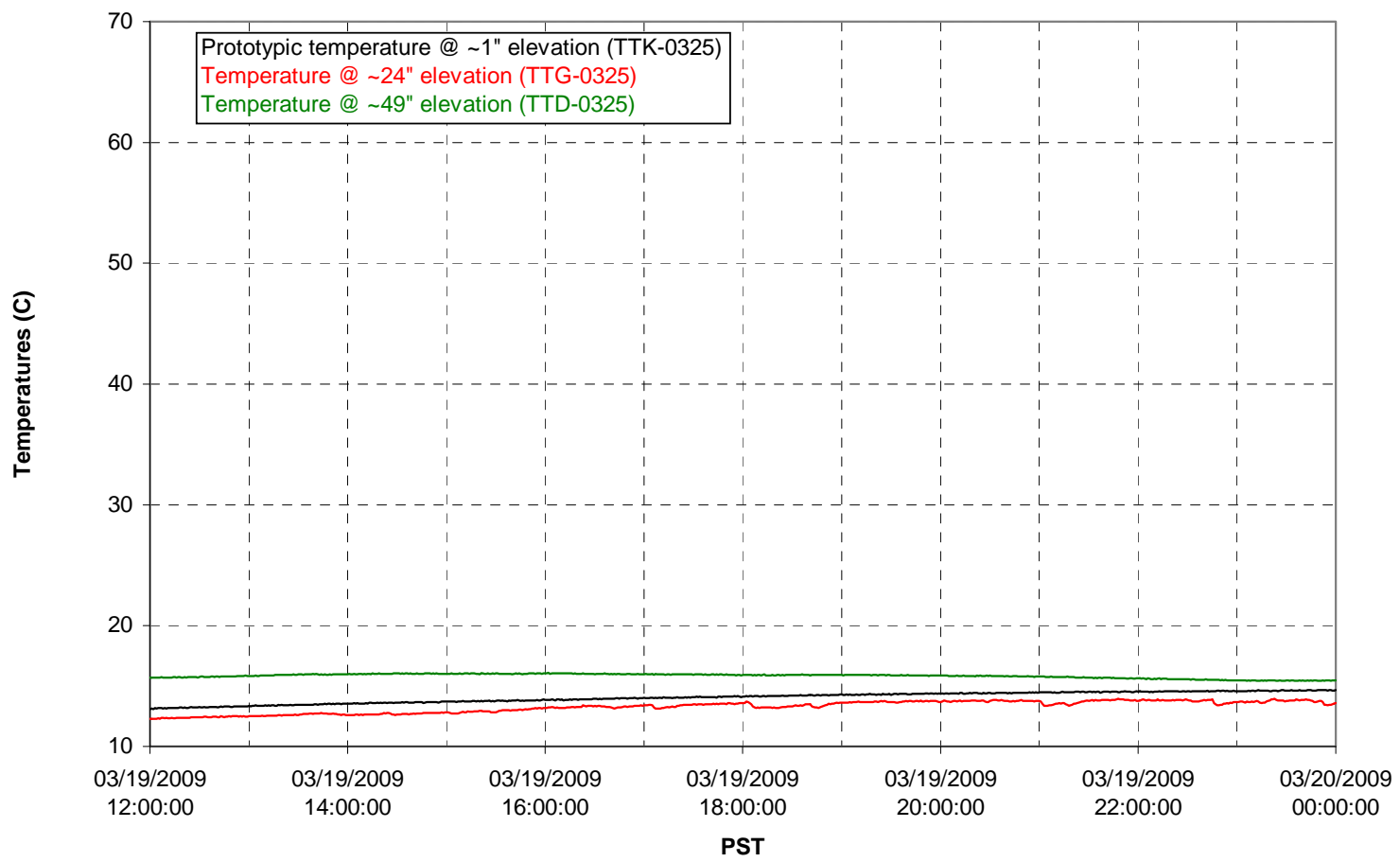


T01B level

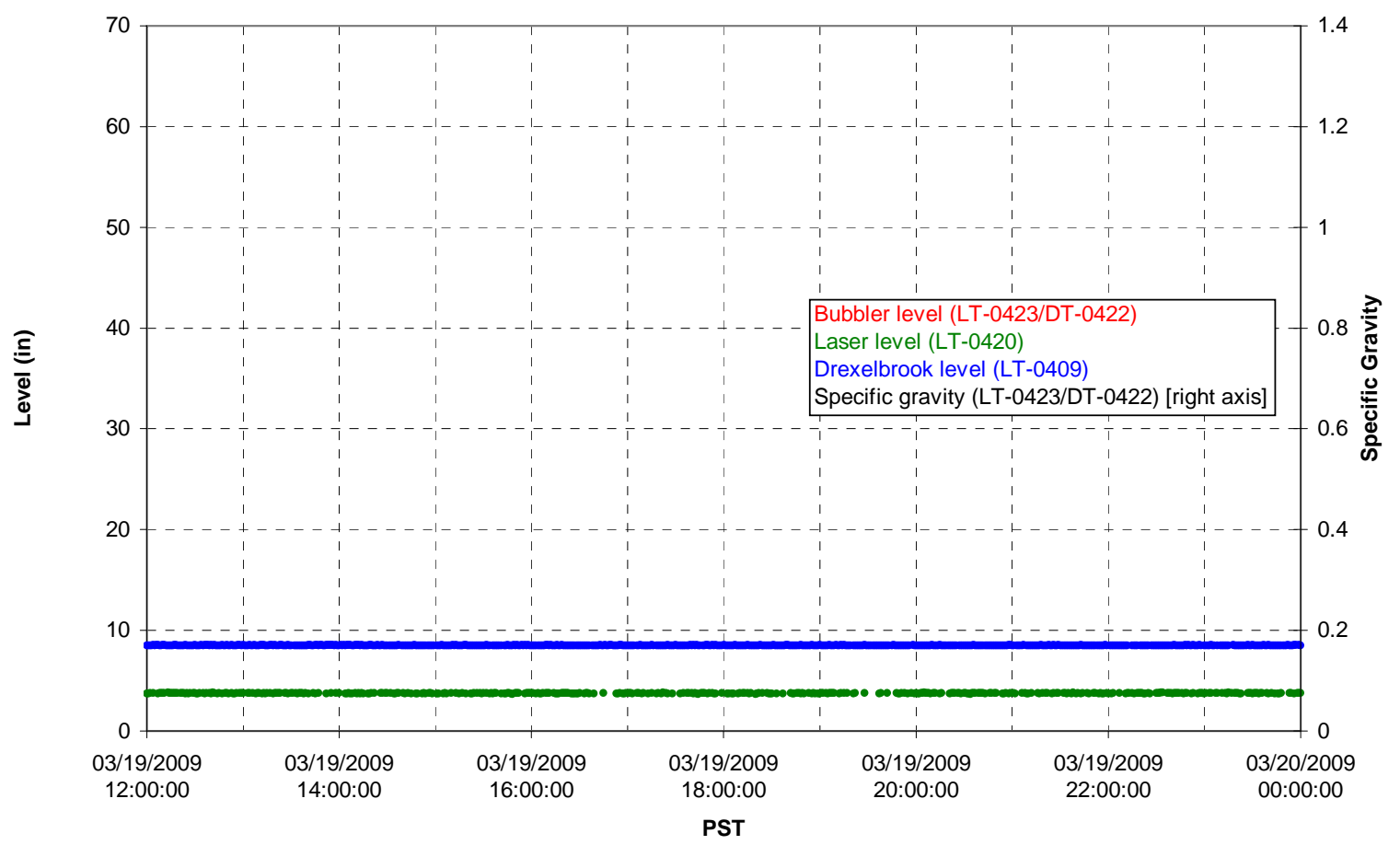

T01B temperatures

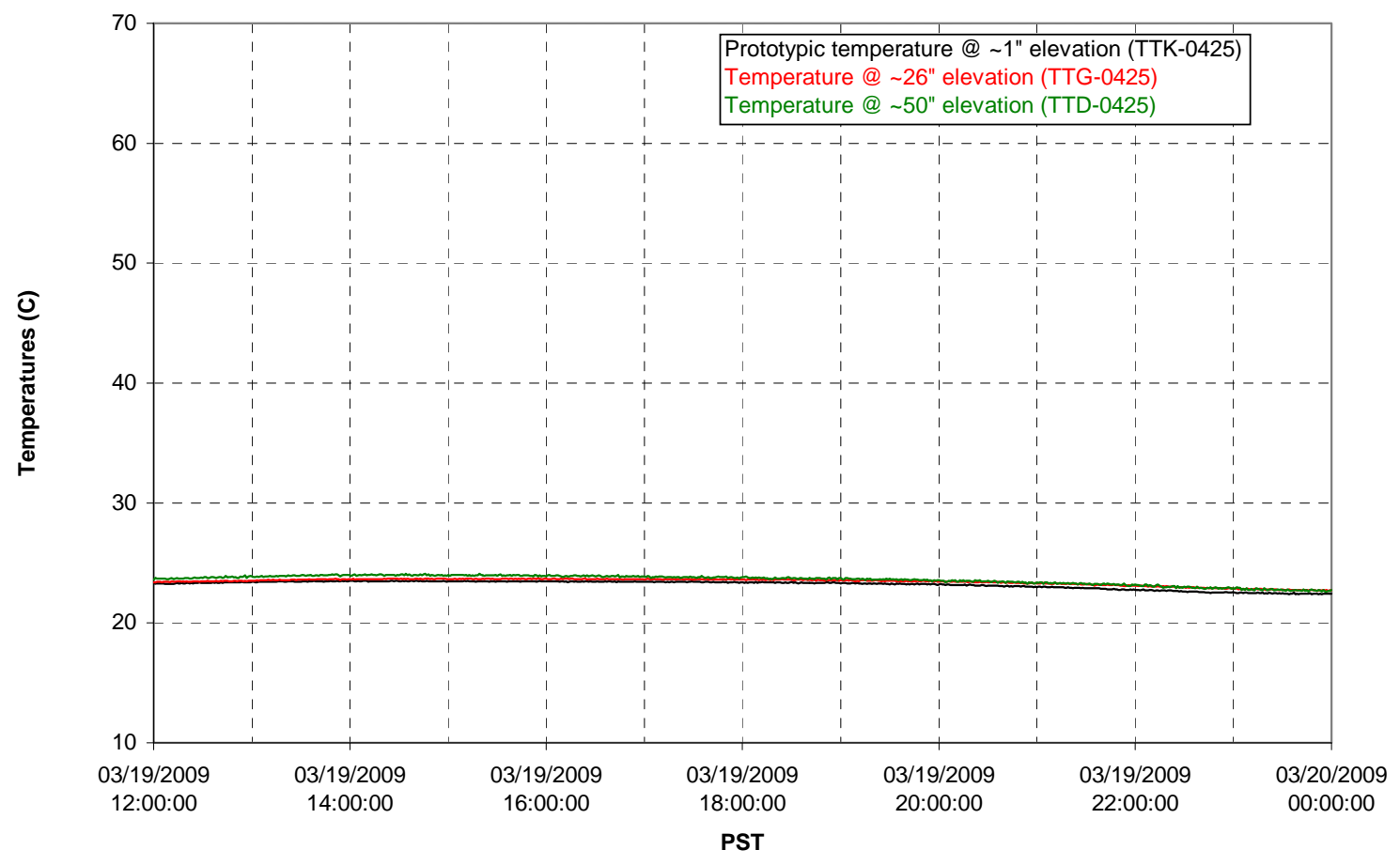


T02A level

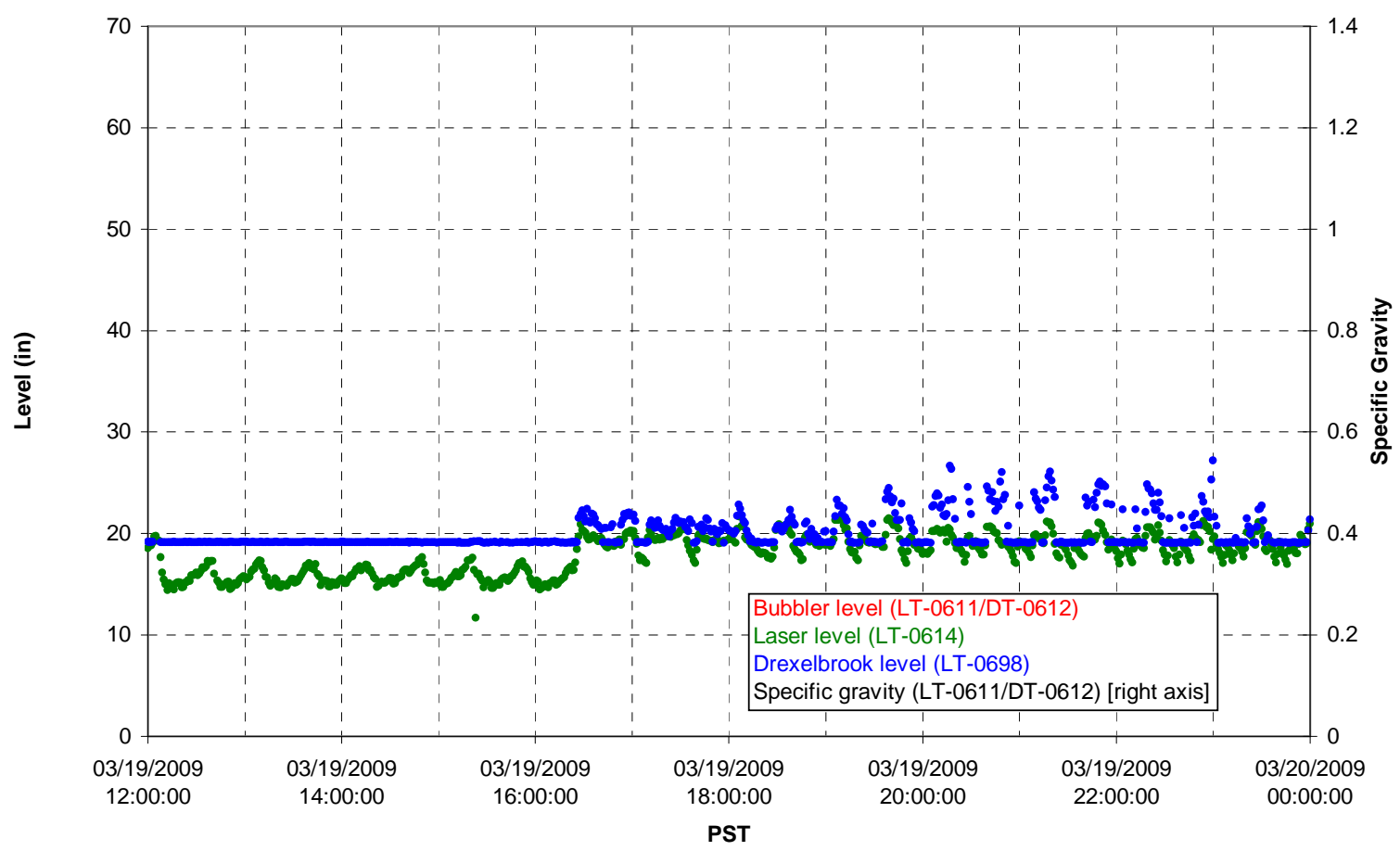

T02A temperatures

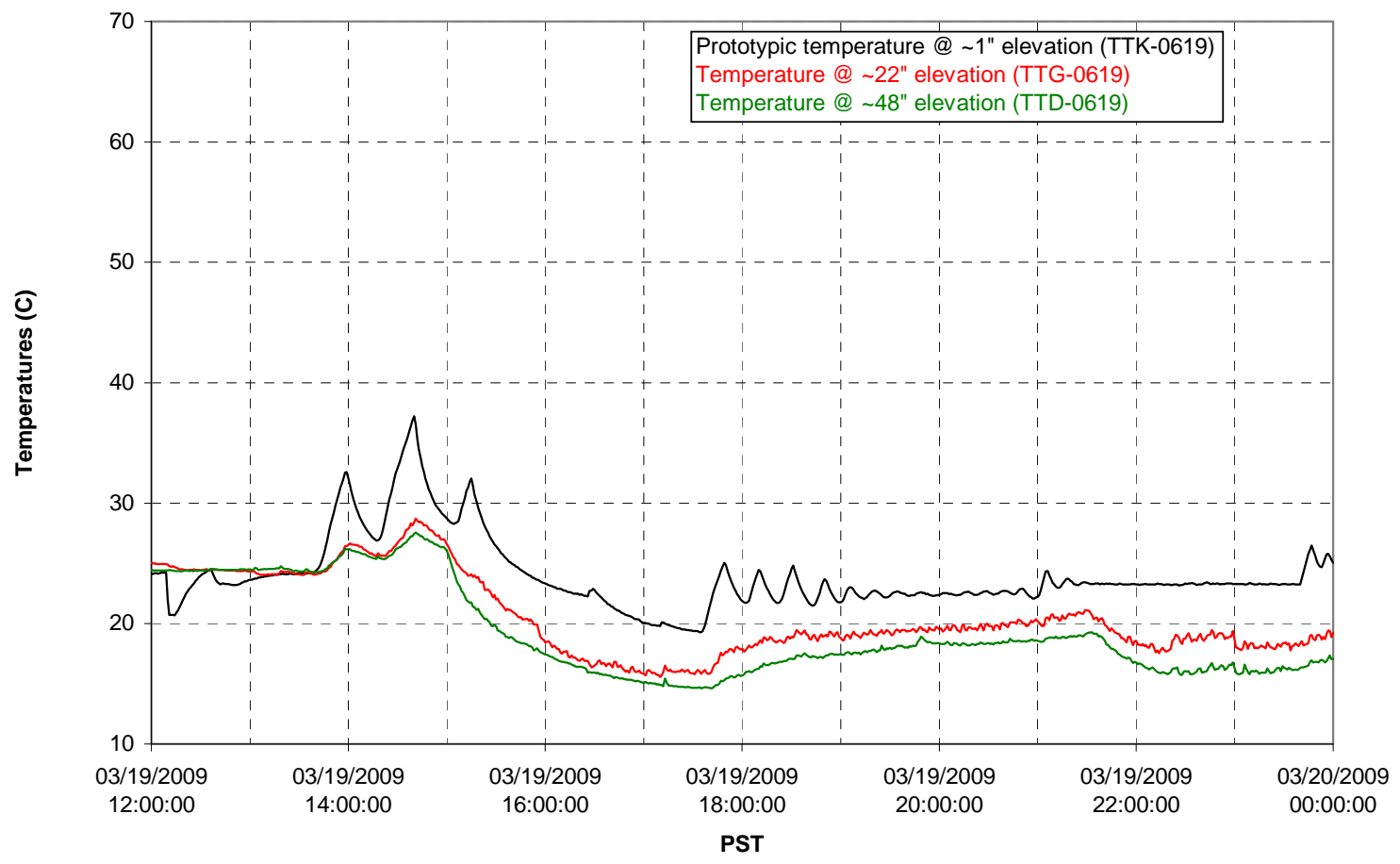


T02A and filter loop temperatures

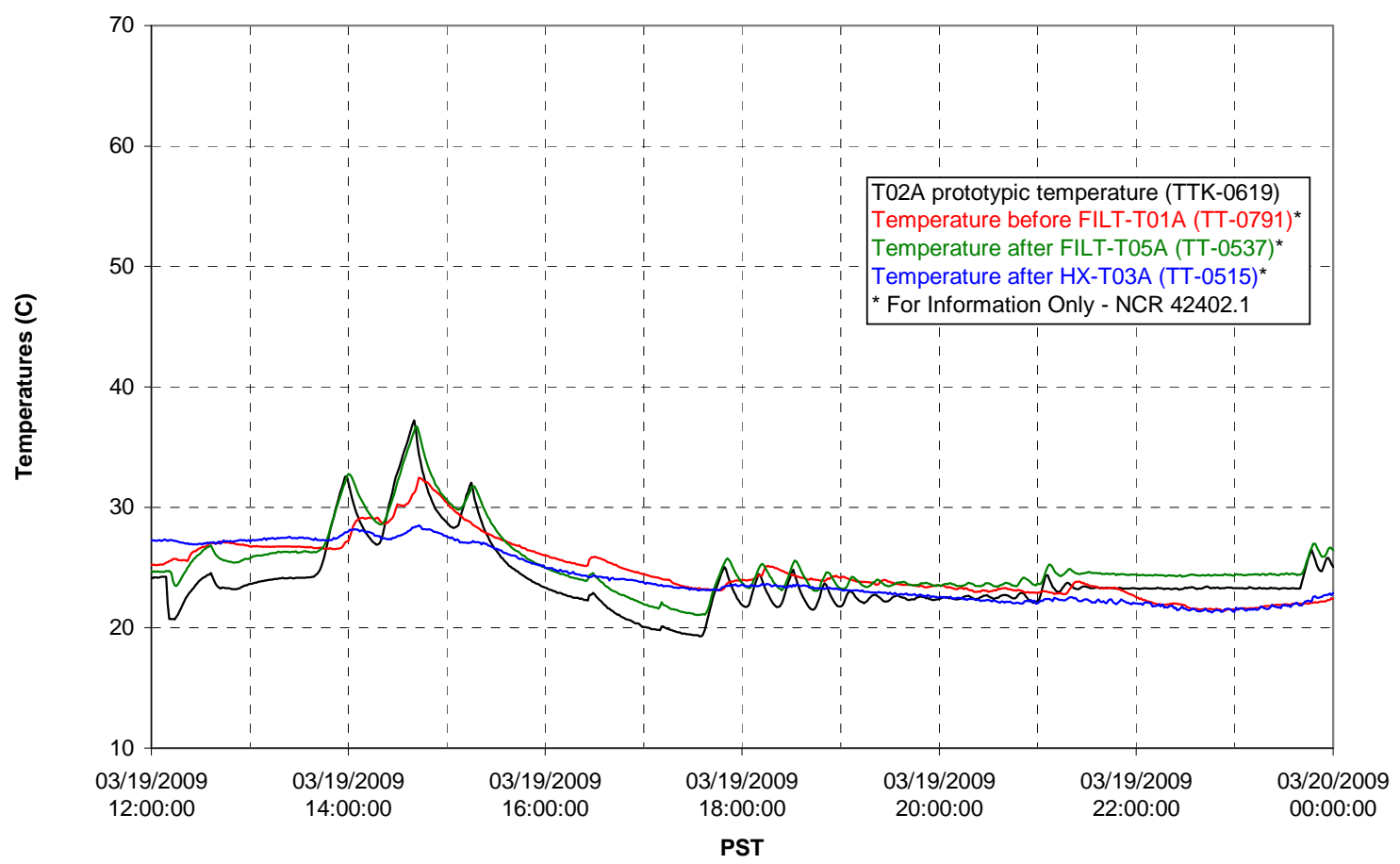

Pump Pressures and Flow

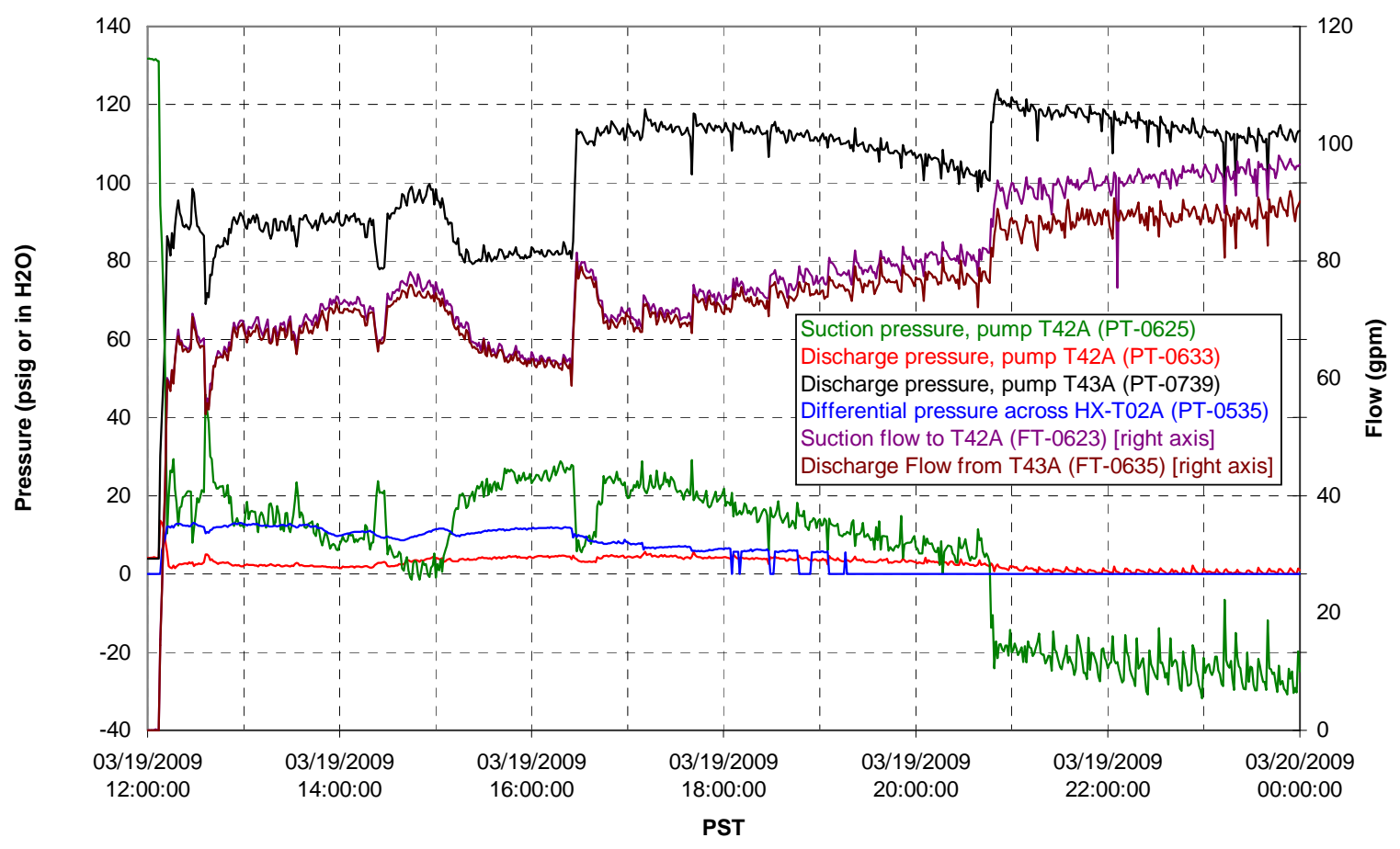




\section{Axial pressure drop}

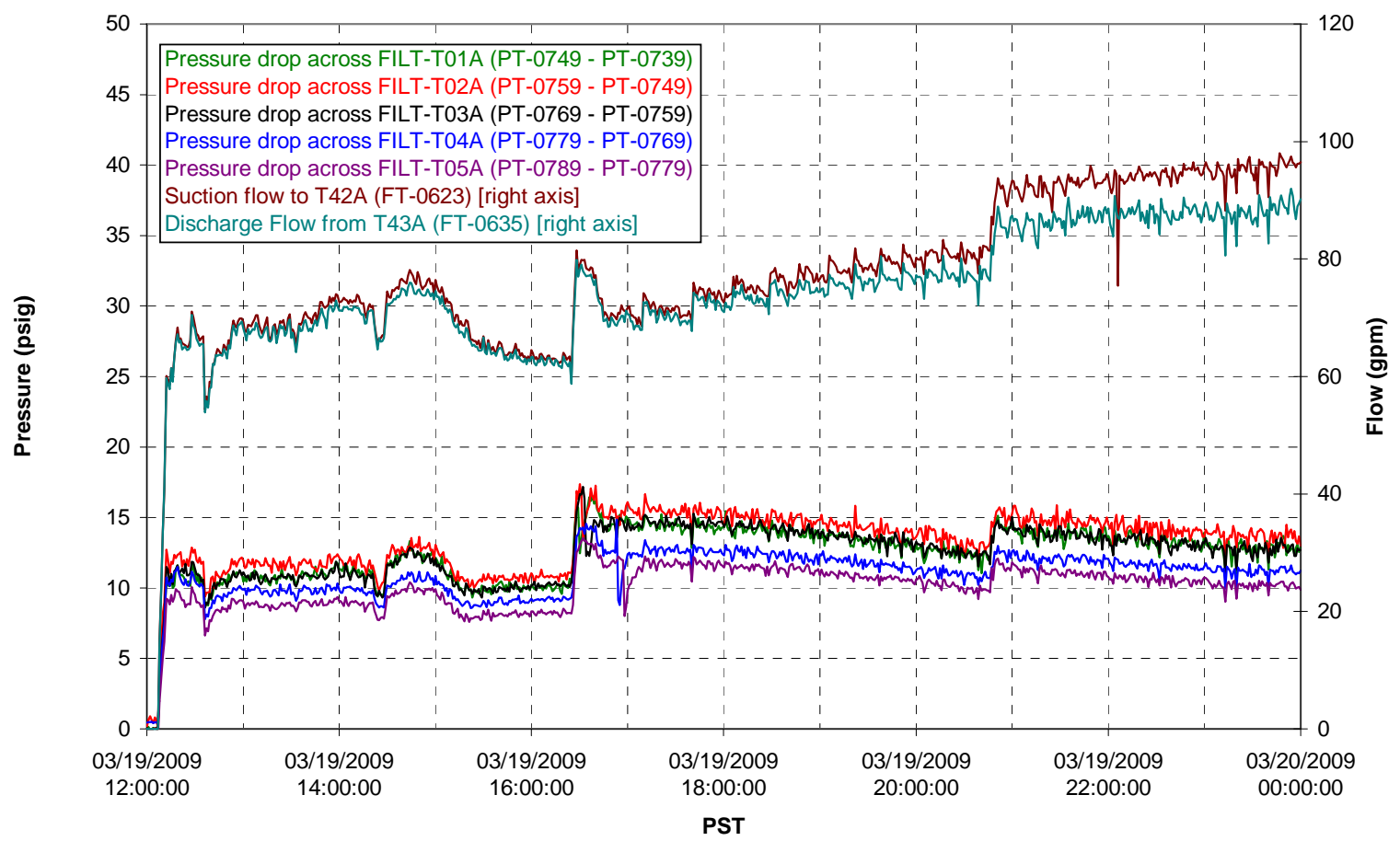

Permeate flow rates

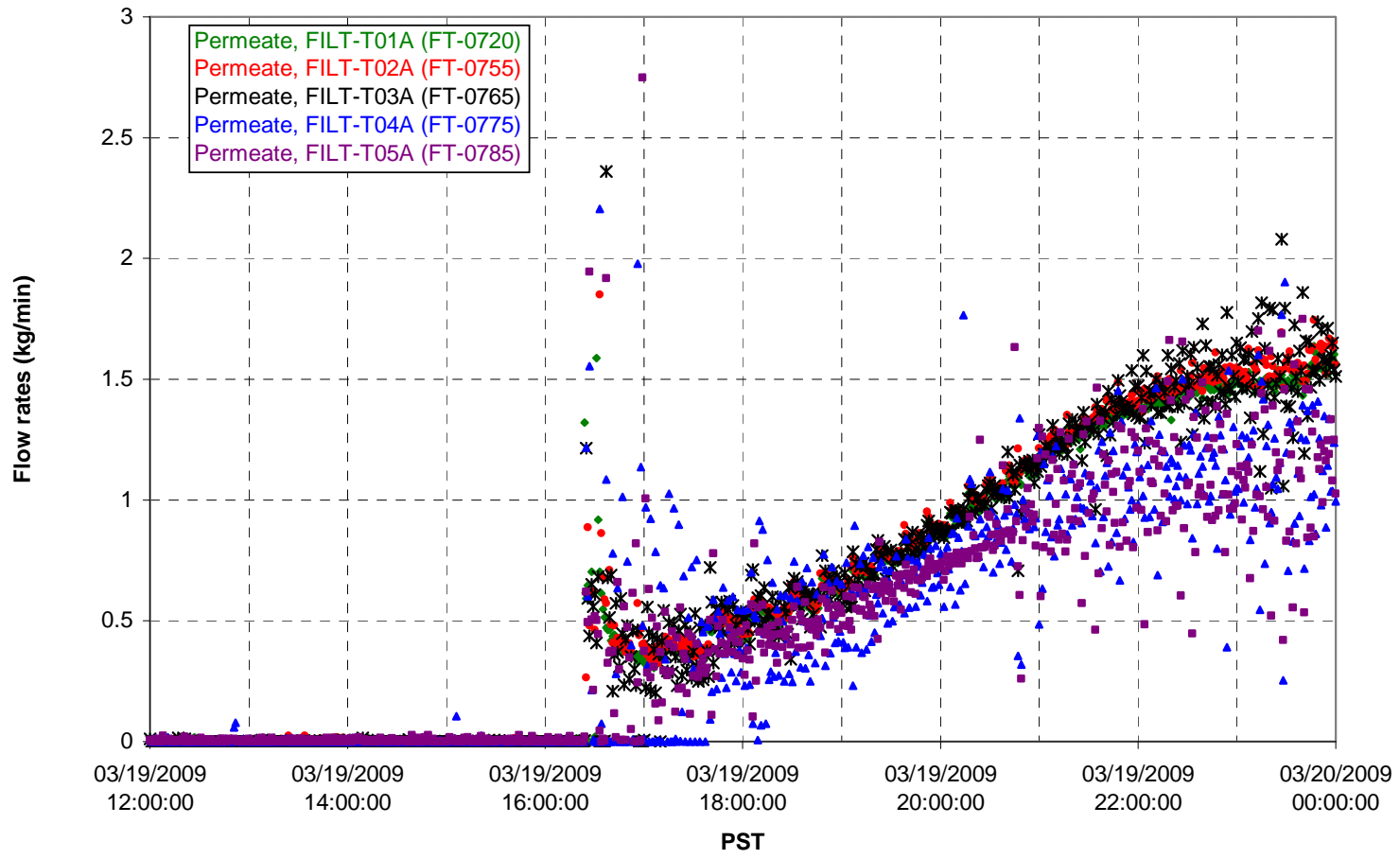


T02A Inner Temperature Tree

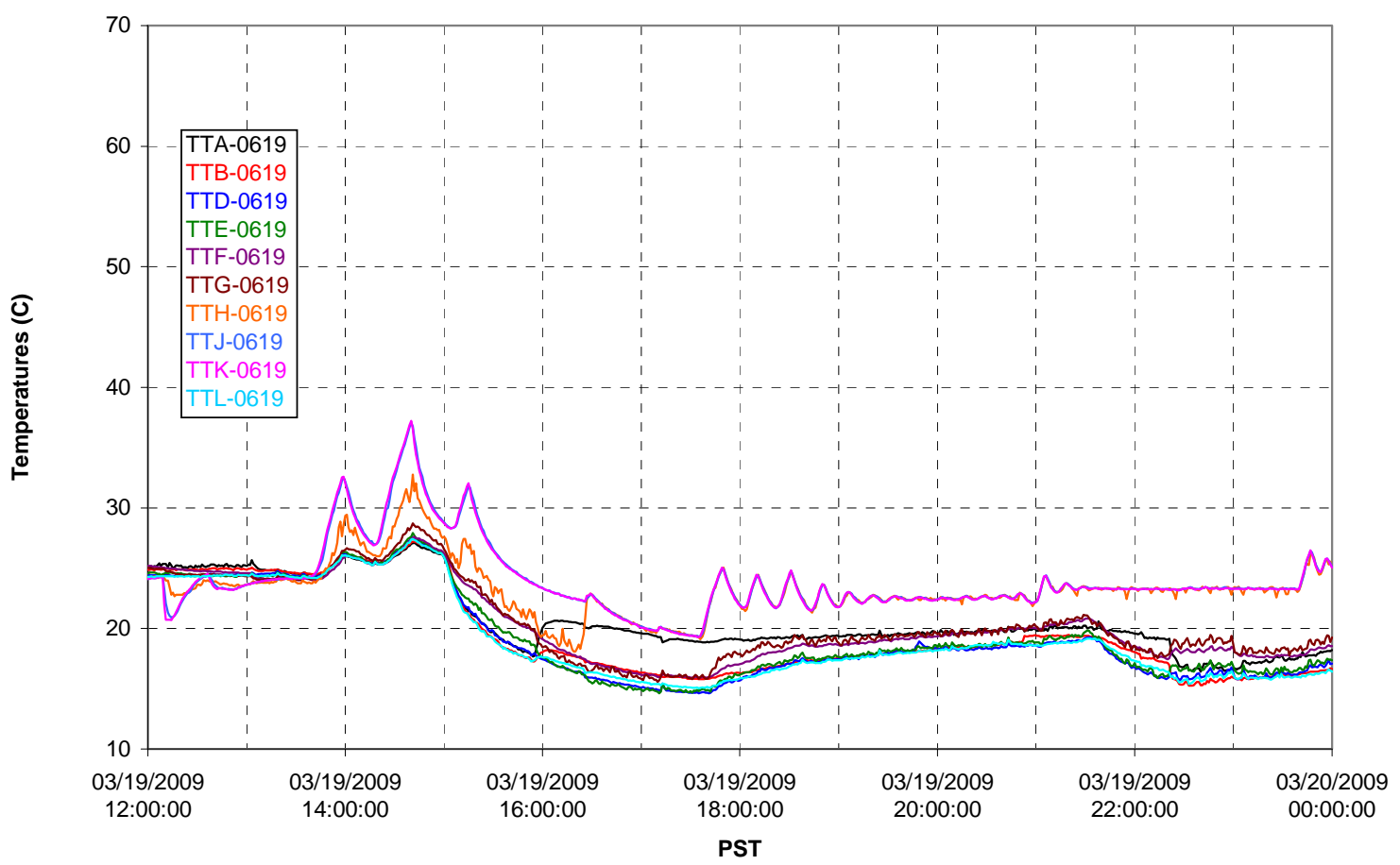

T02A Outer Temperature Tree

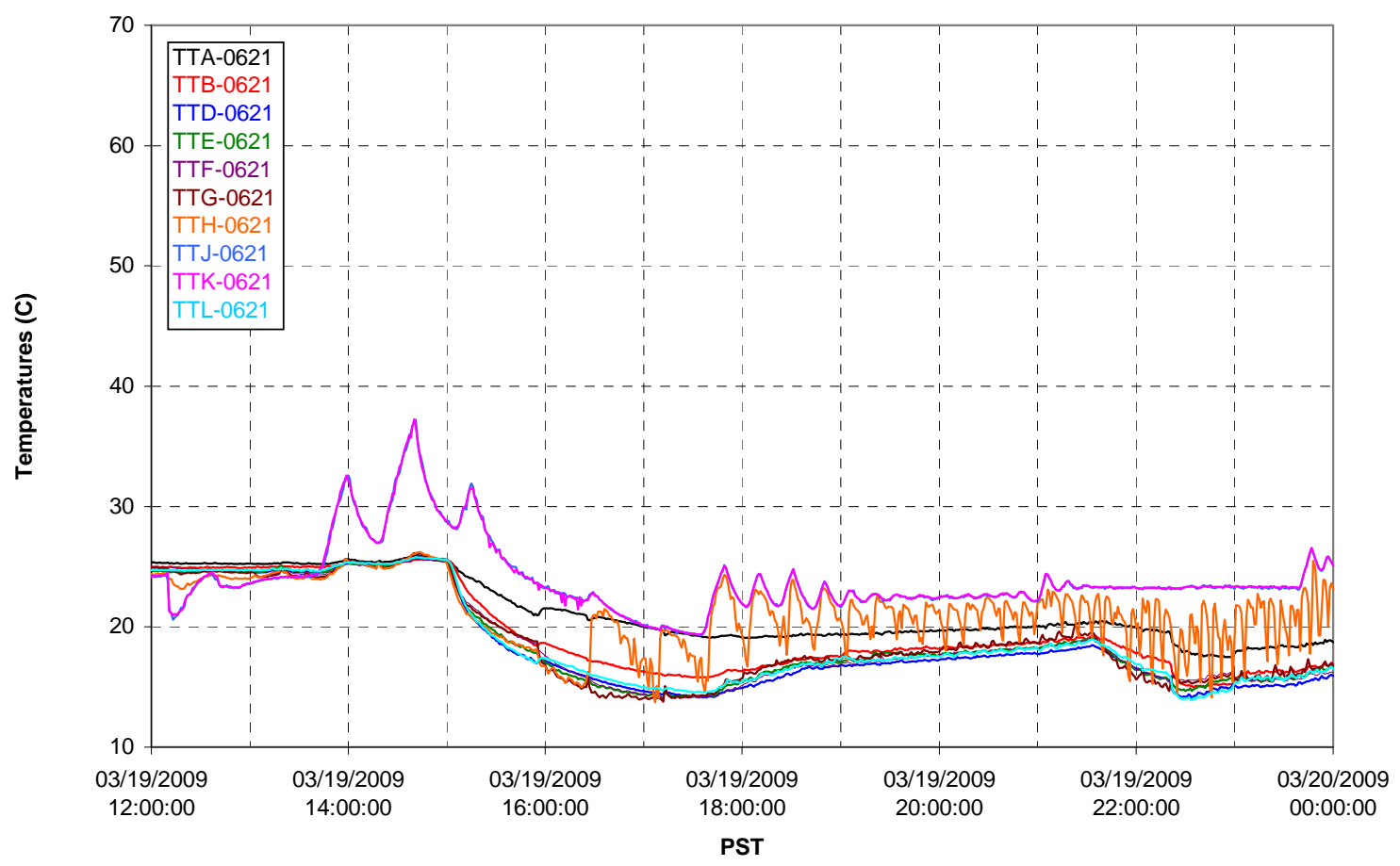


T02A temperatures

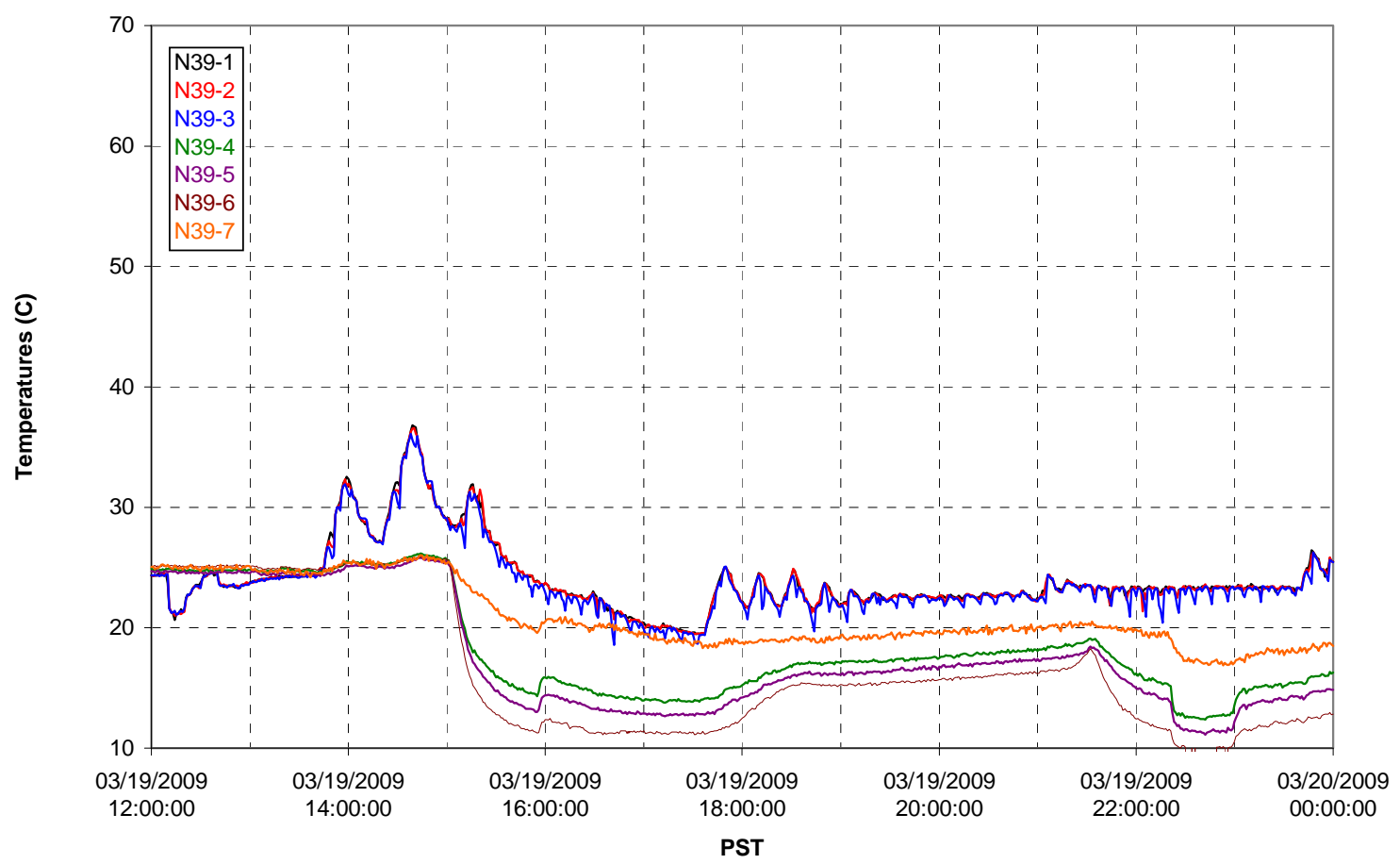

T02A temperatures

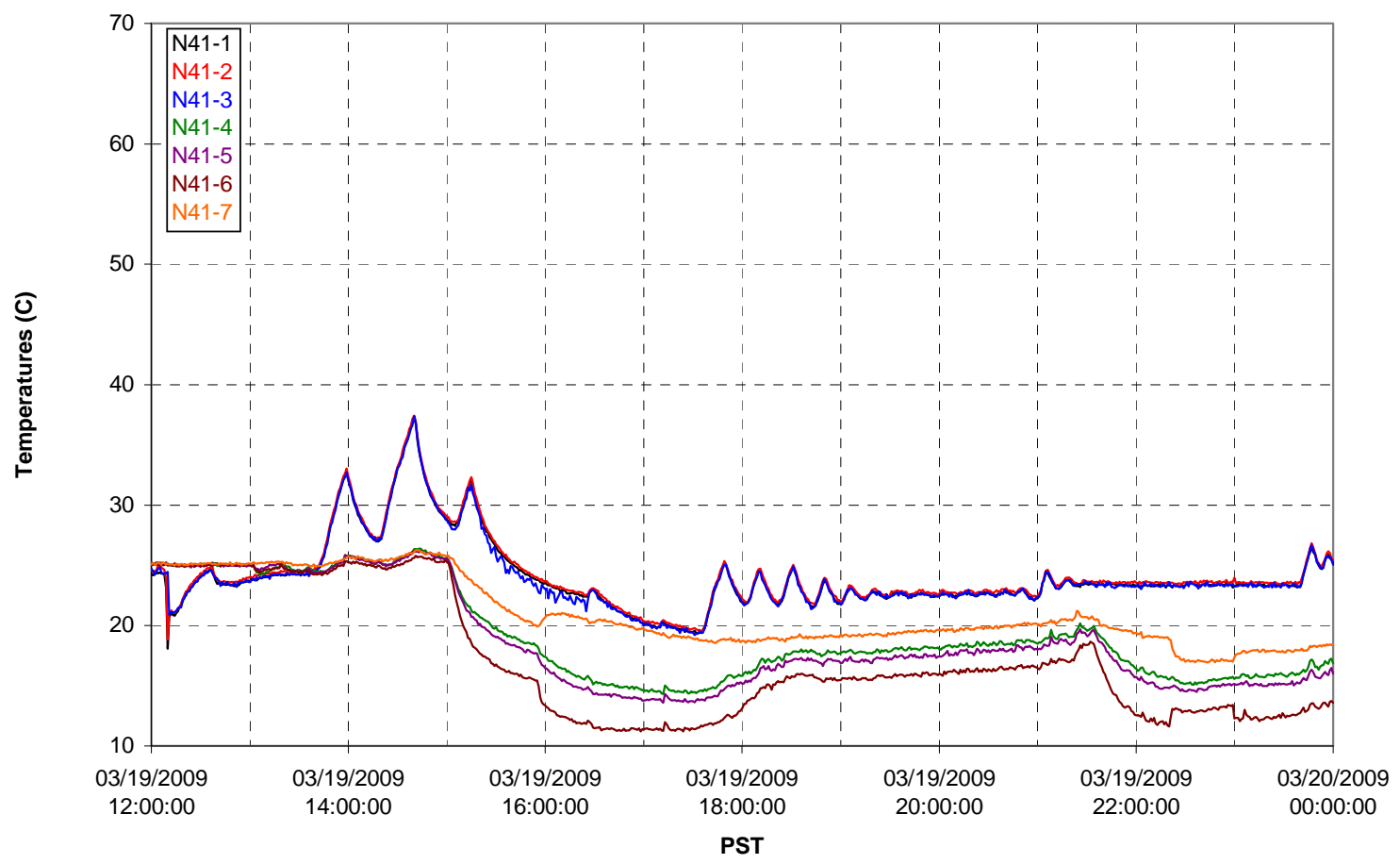


T02A temperatures

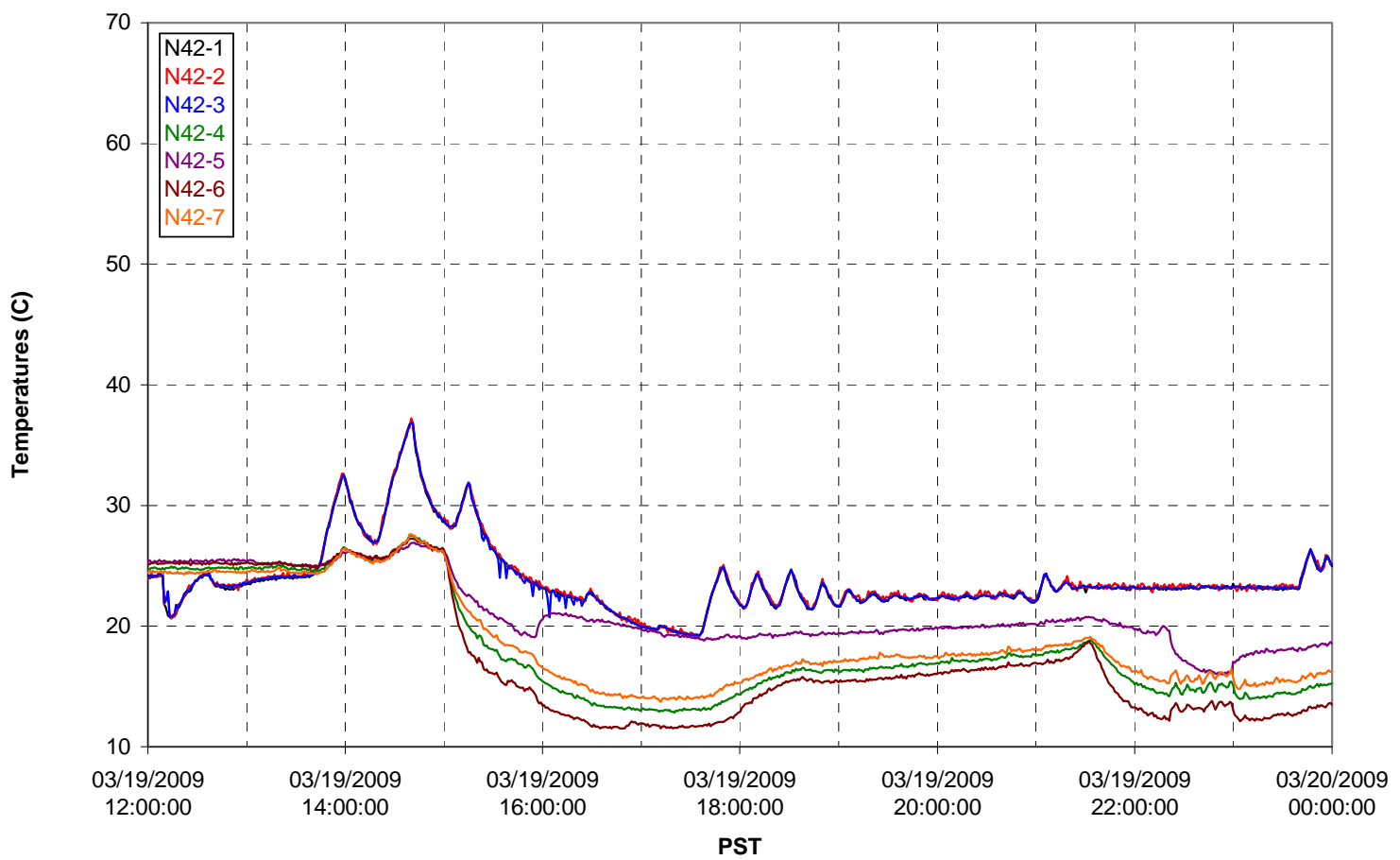

T02A temperatures

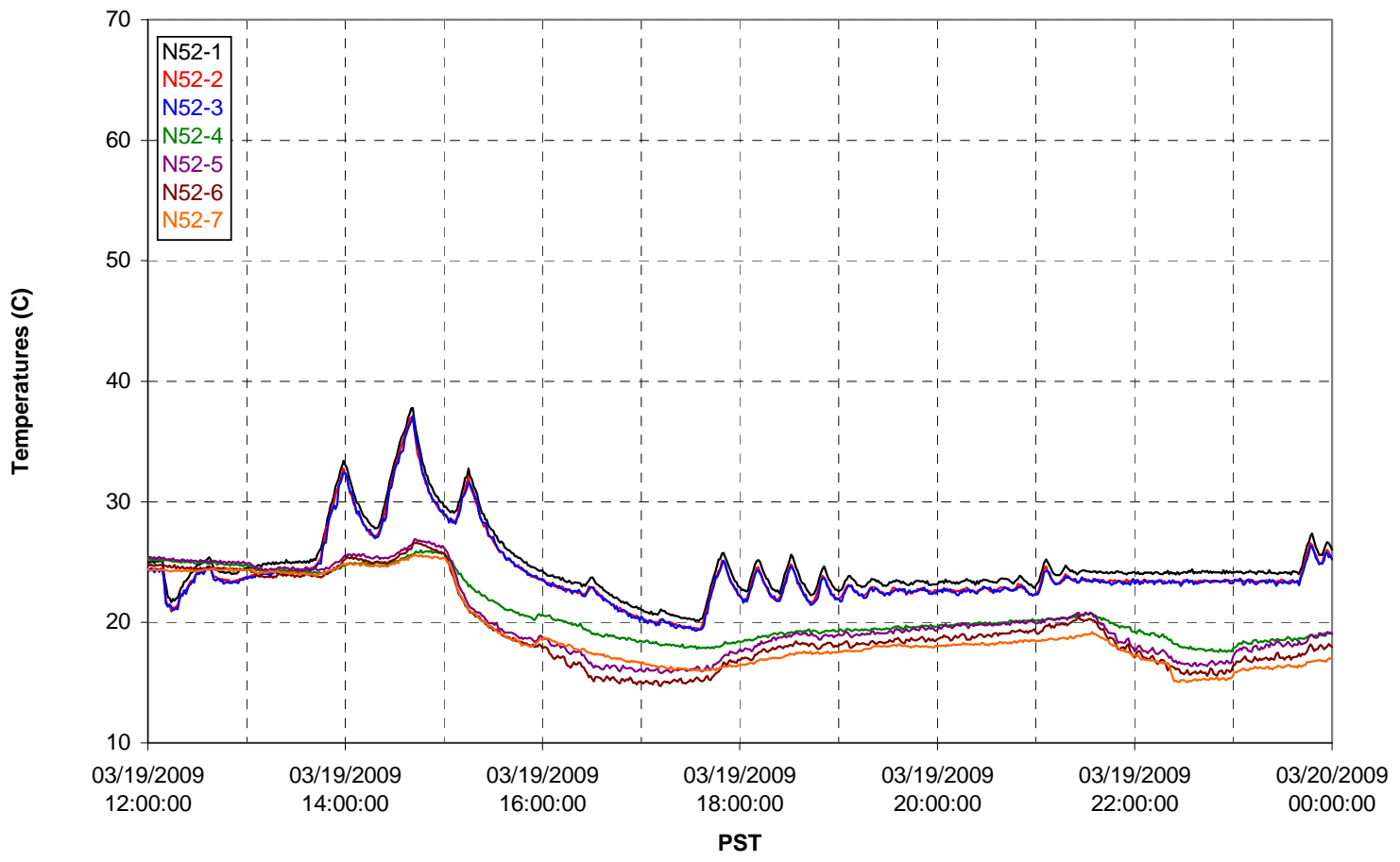


T02A Heating and Cooling

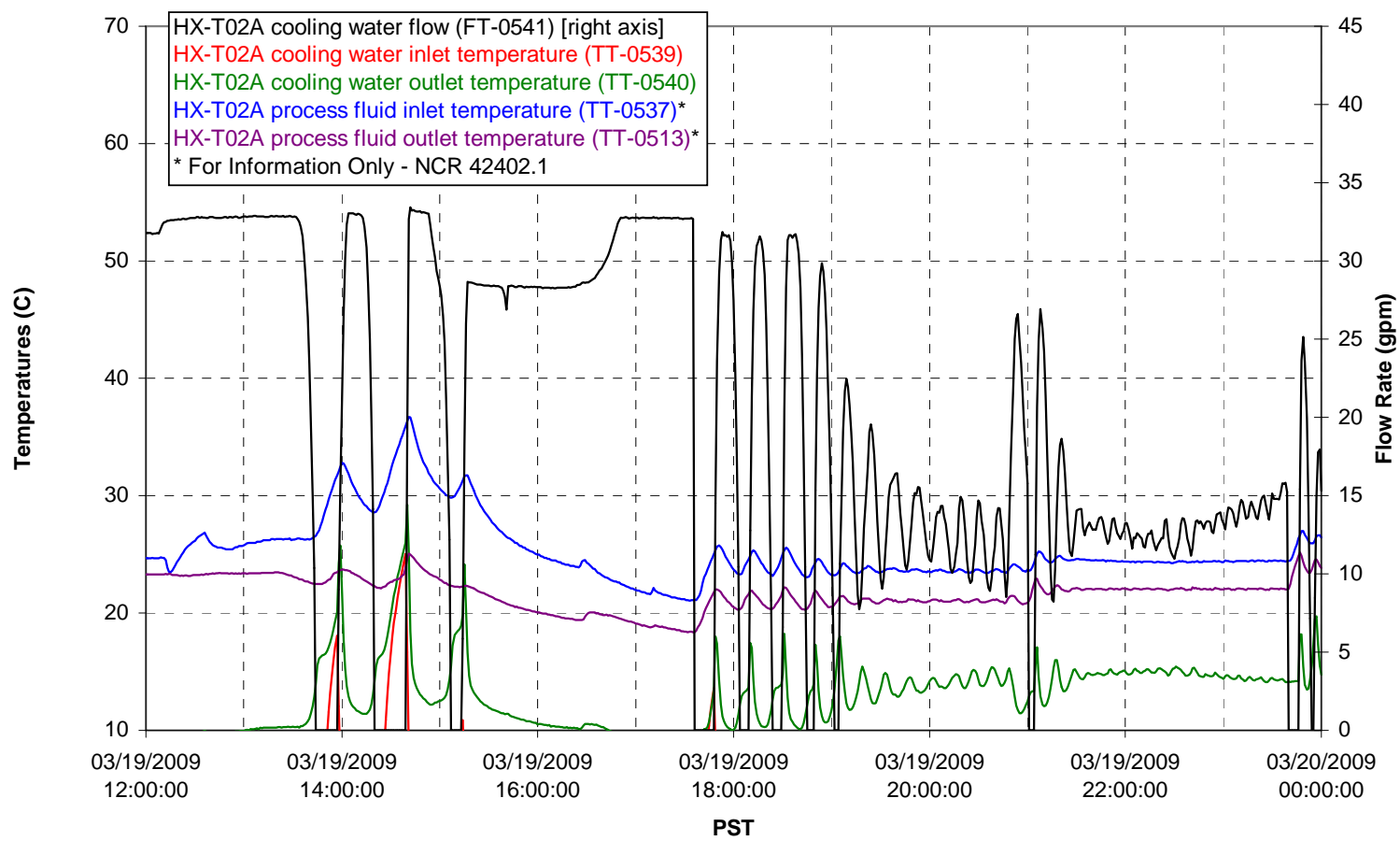

Pump Operation

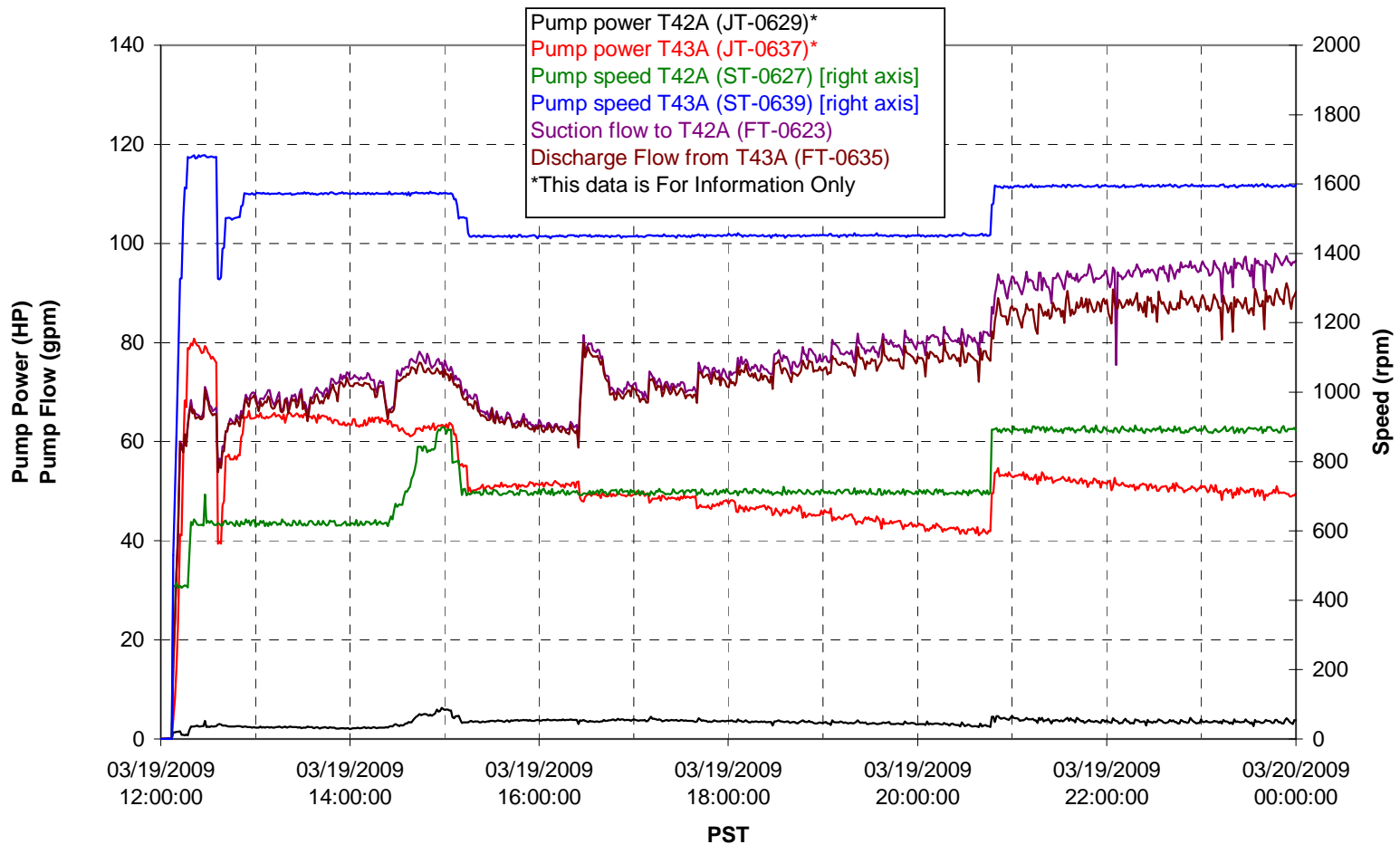


Pulsepot UFP-PP-T01A

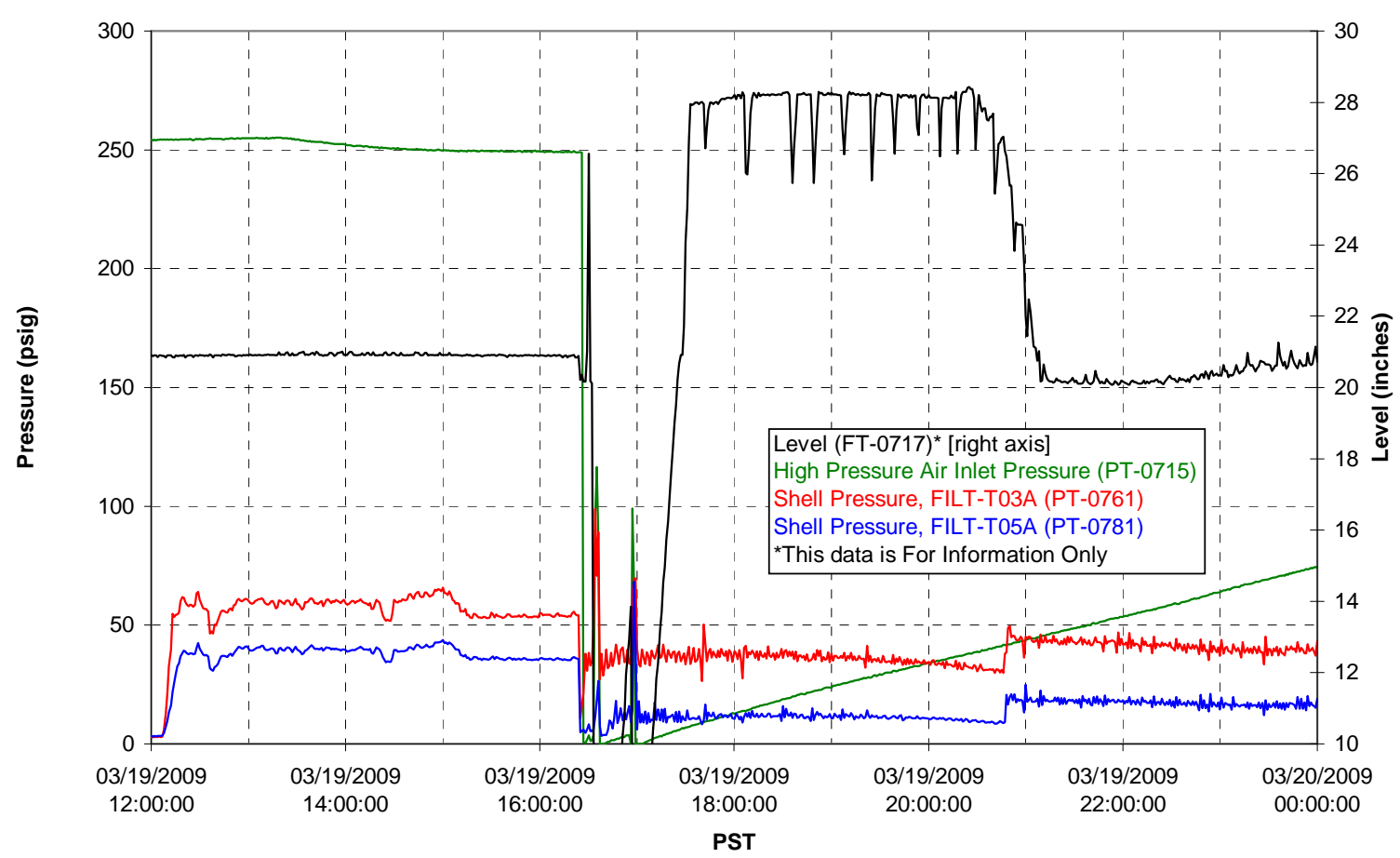

Pulsepot UFP-PP-T02A

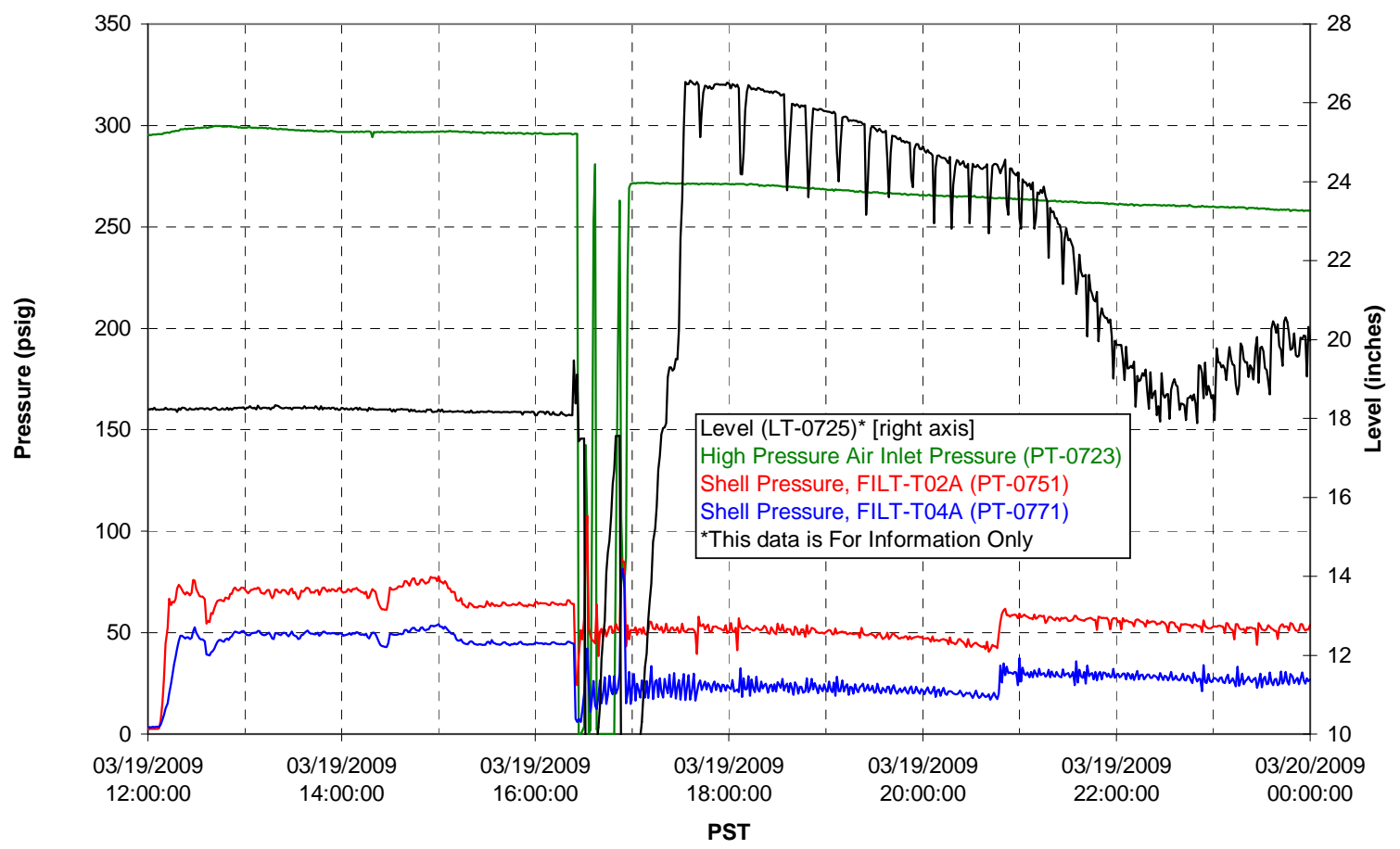


Pulsepot UFP-PP-T03A

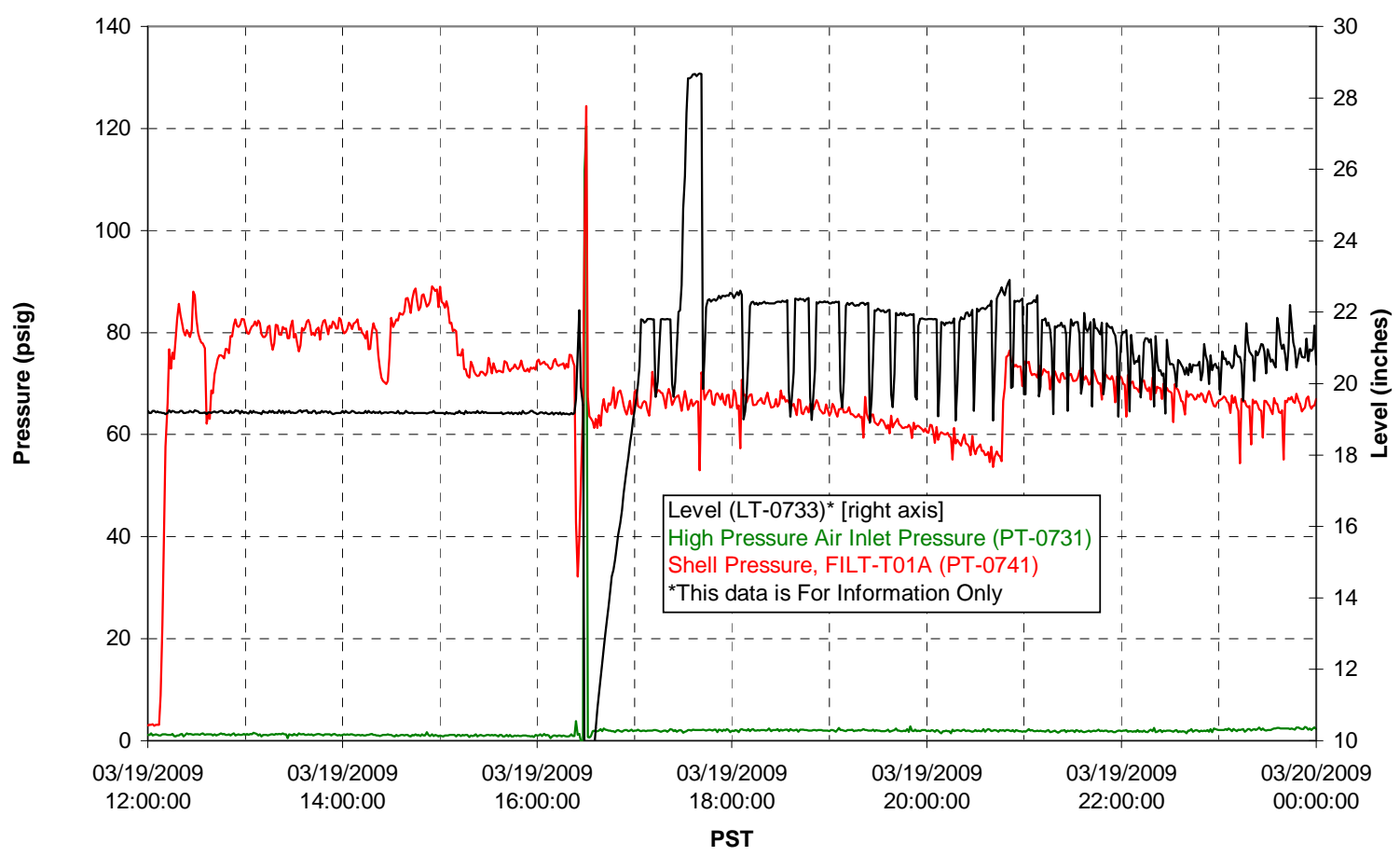

Pulsepot Levels

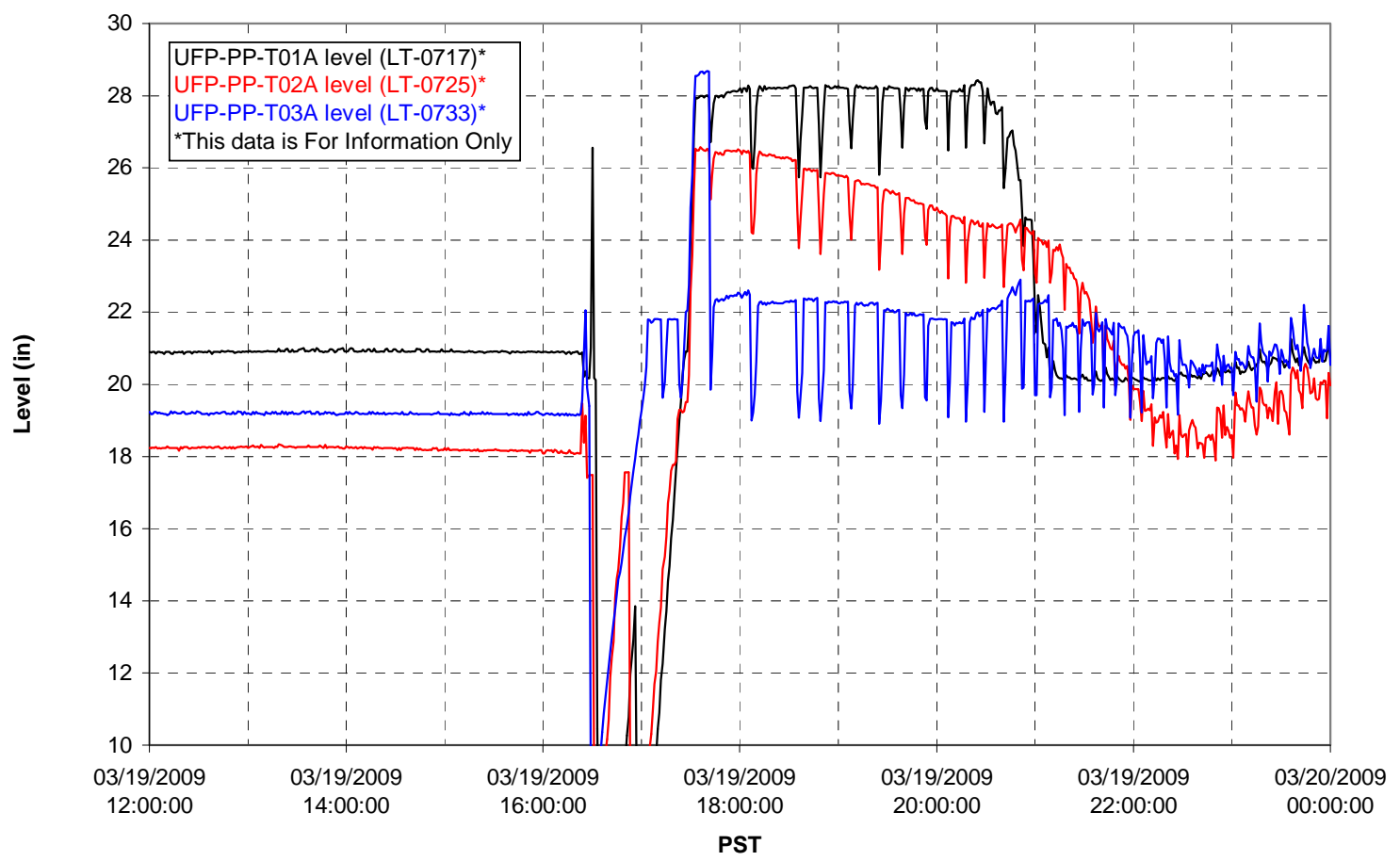


Filter UFP-FILT-T01A

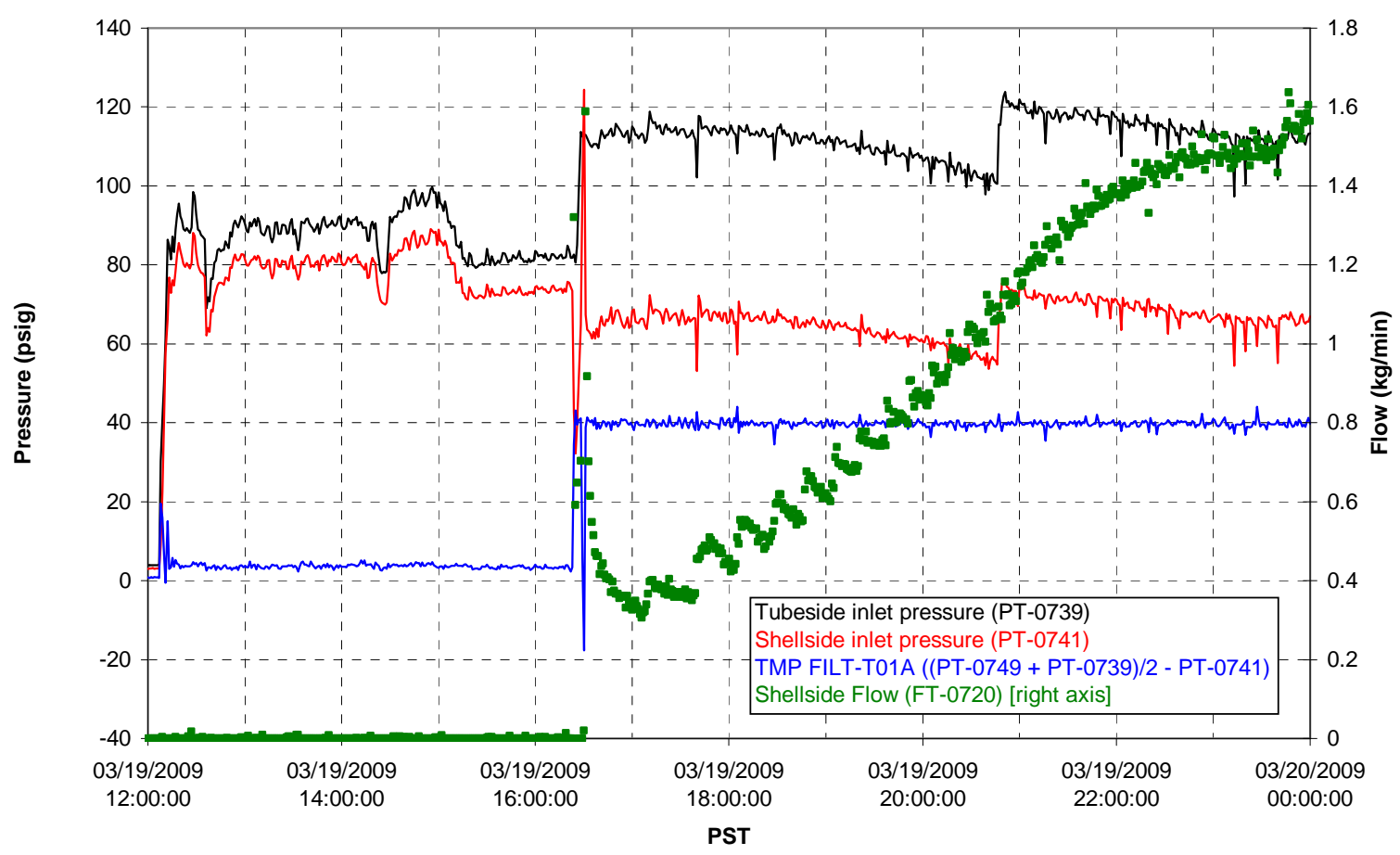

Filter UFP-FILT-T02A

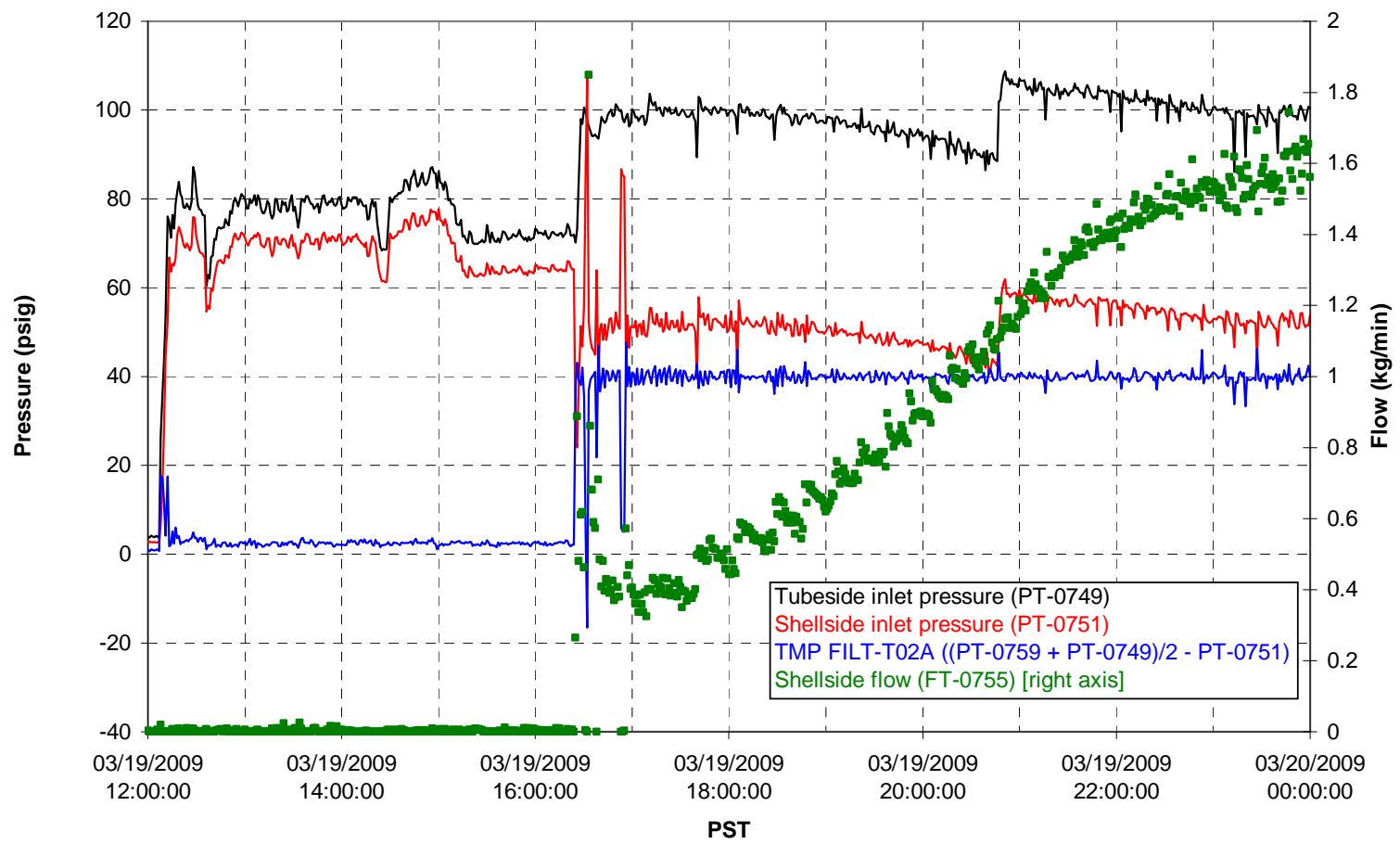


Filter UFP-FILT-T03A

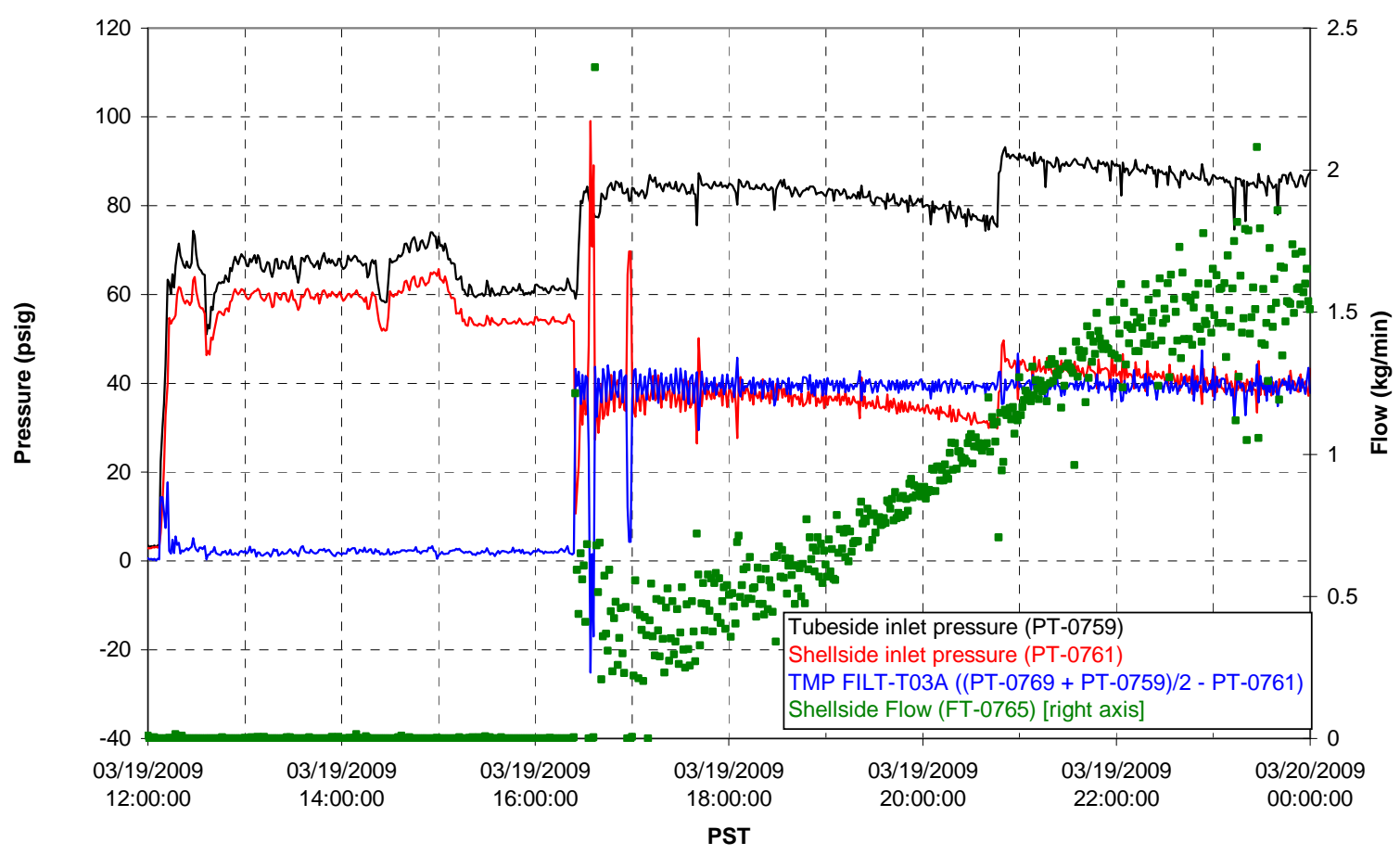

Filter UFP-FILT-T04A

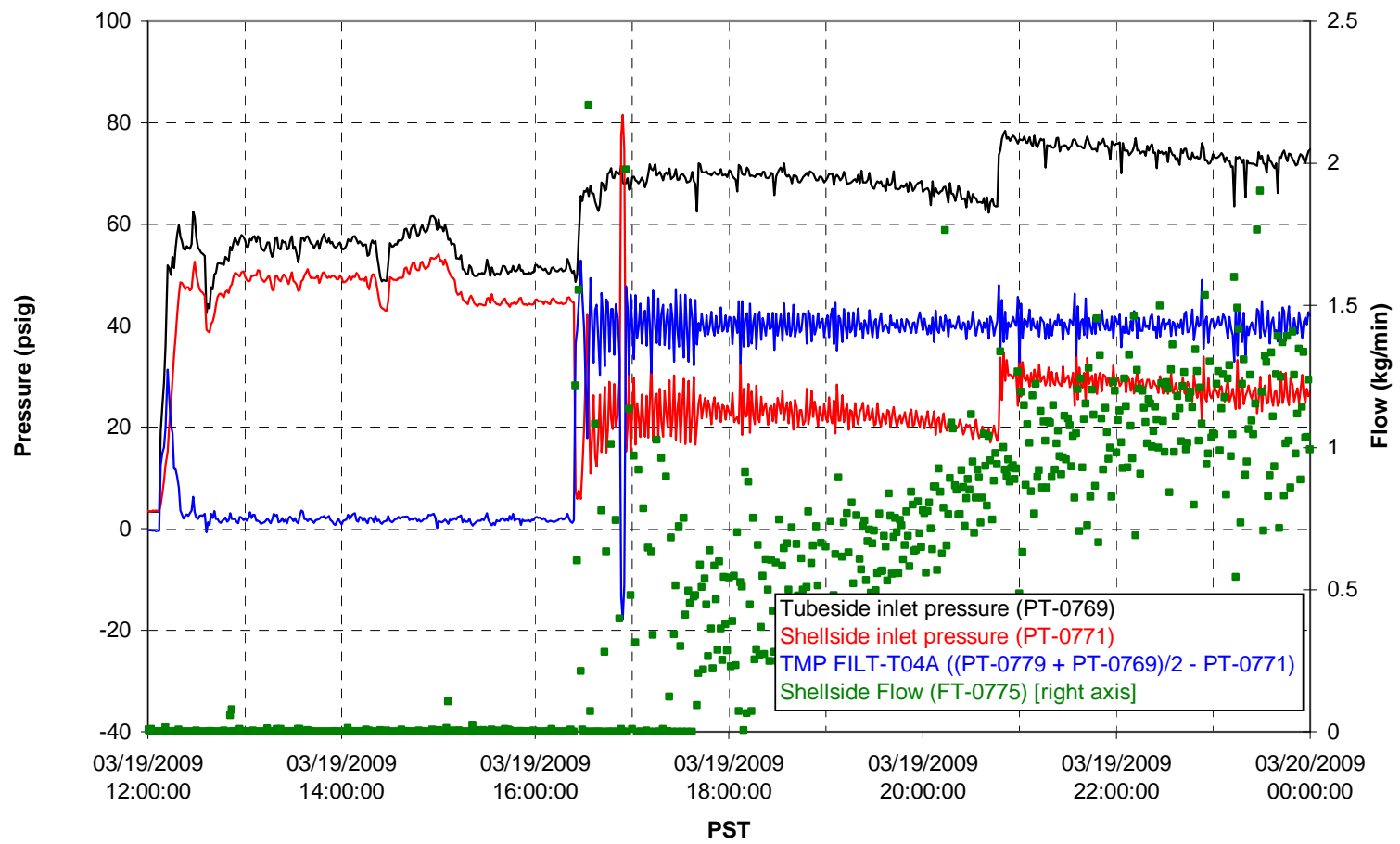


Filter UFP-FILT-T05A

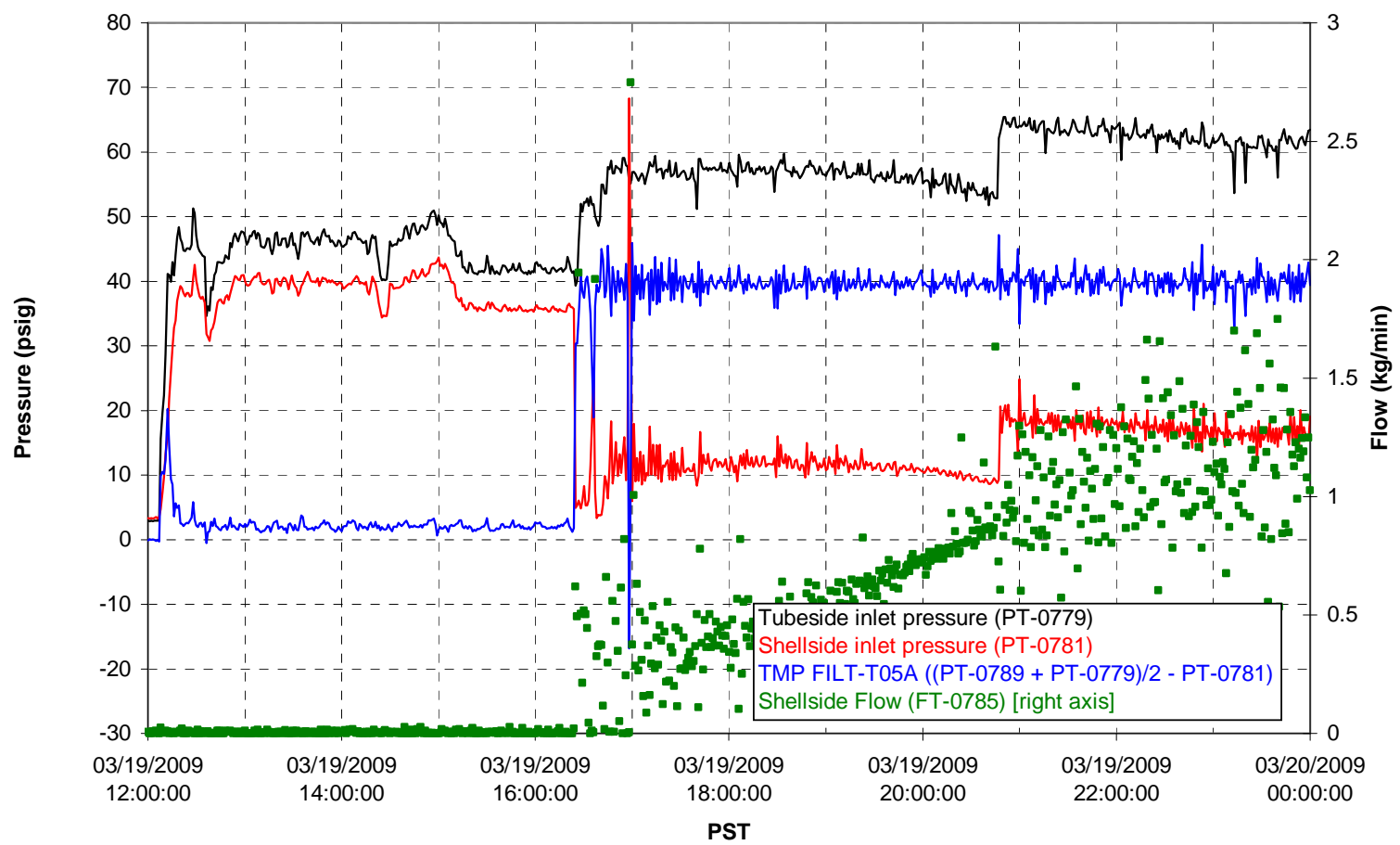



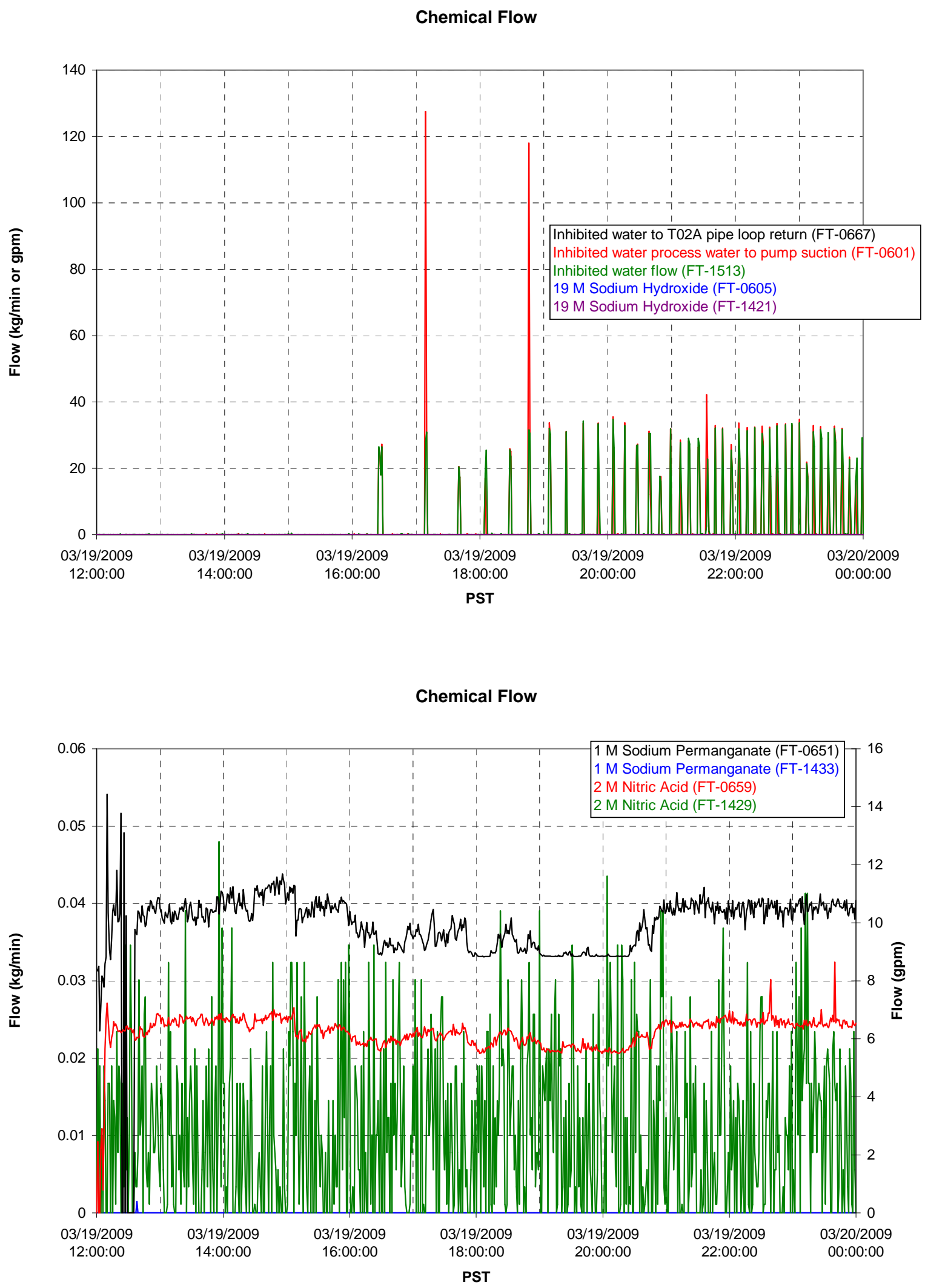


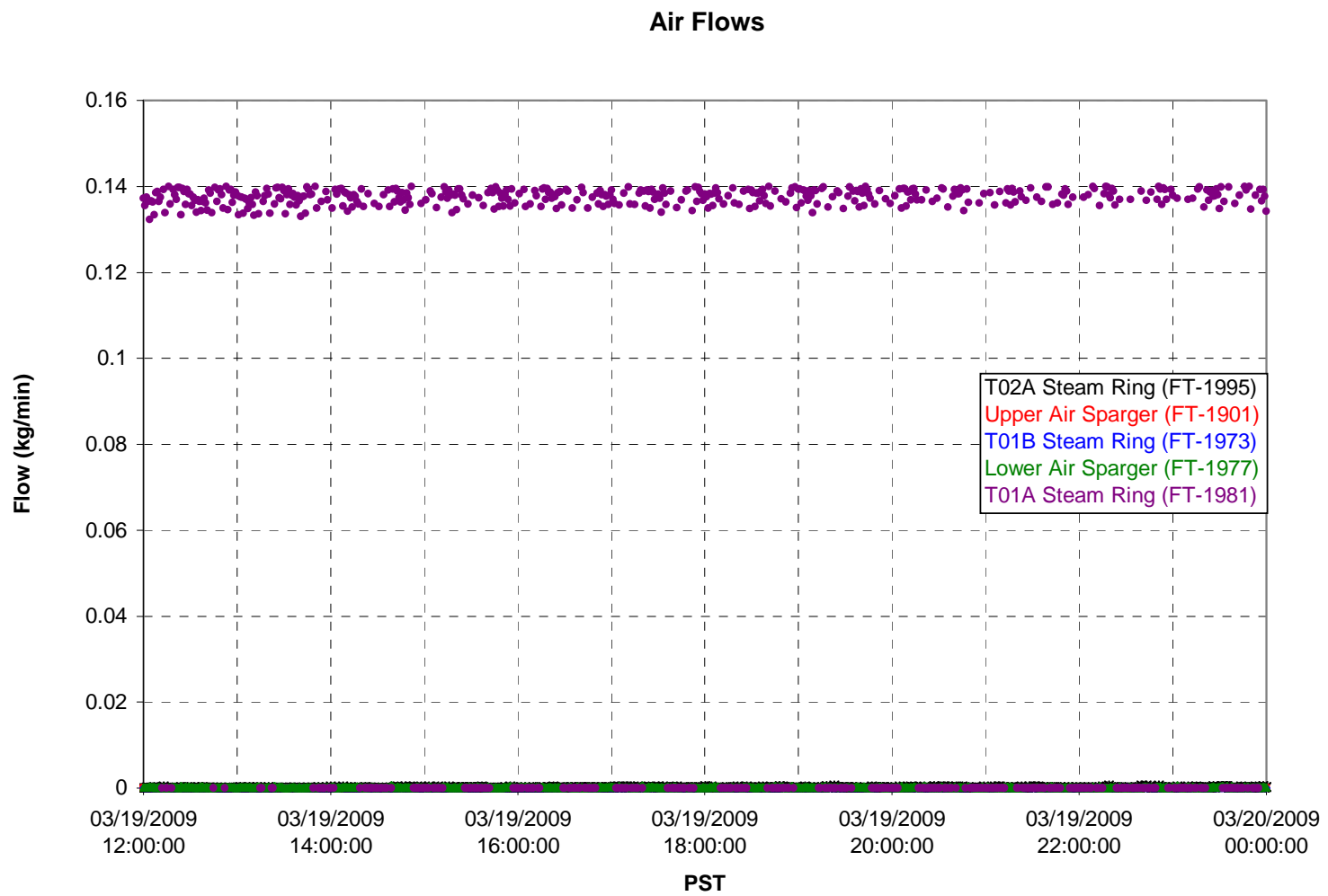

T02A Steam

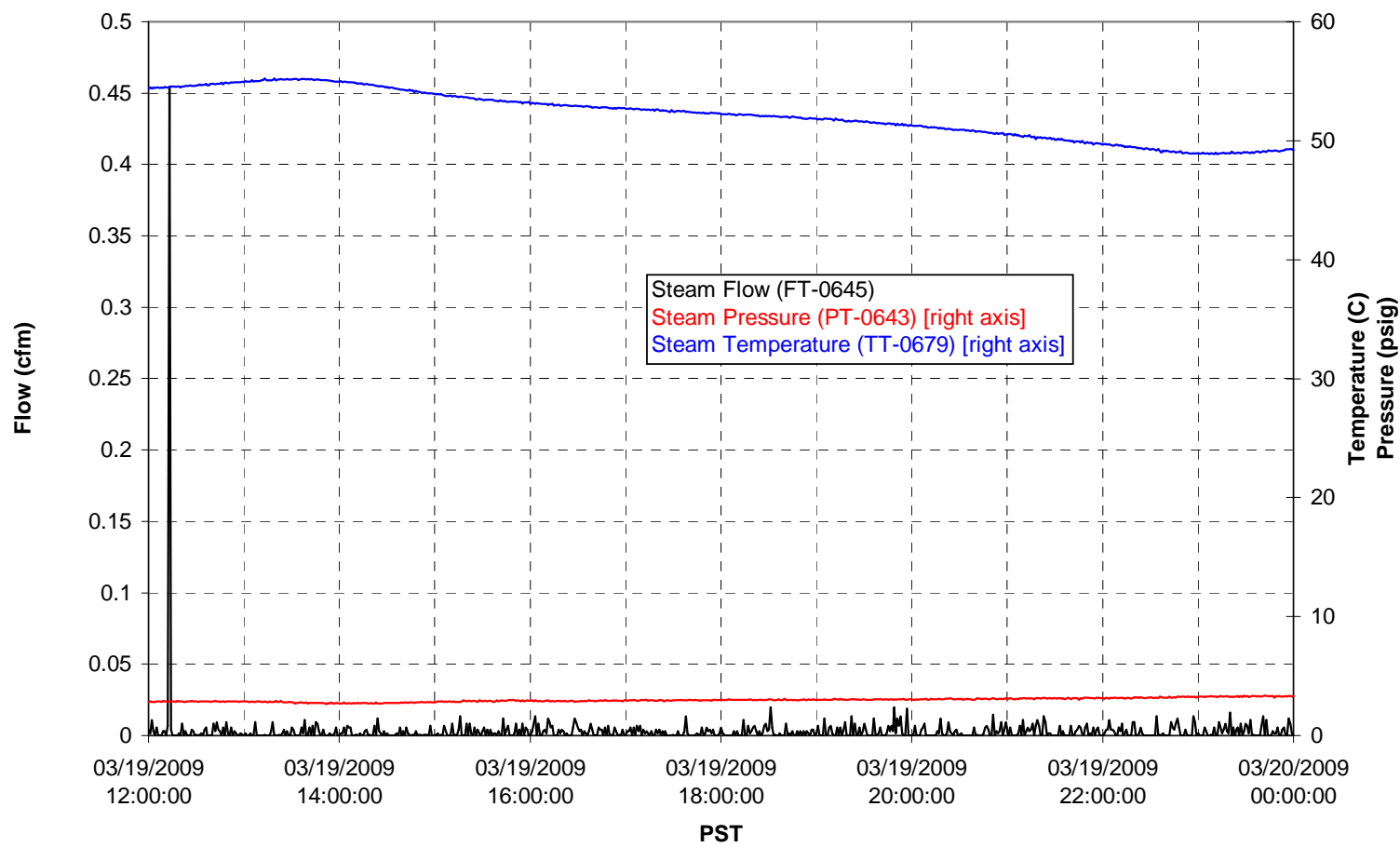


T01A Steam

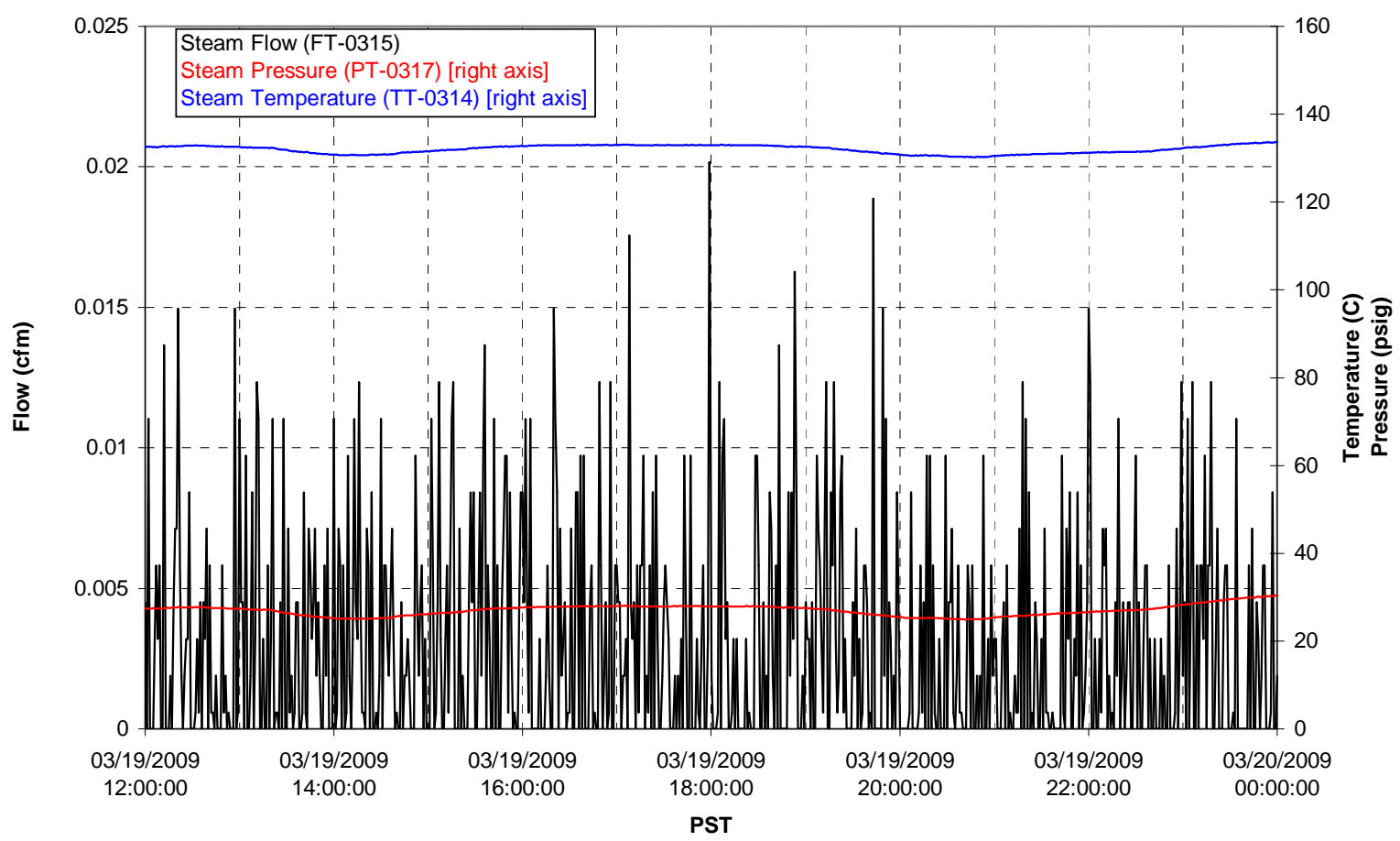

T01B Steam

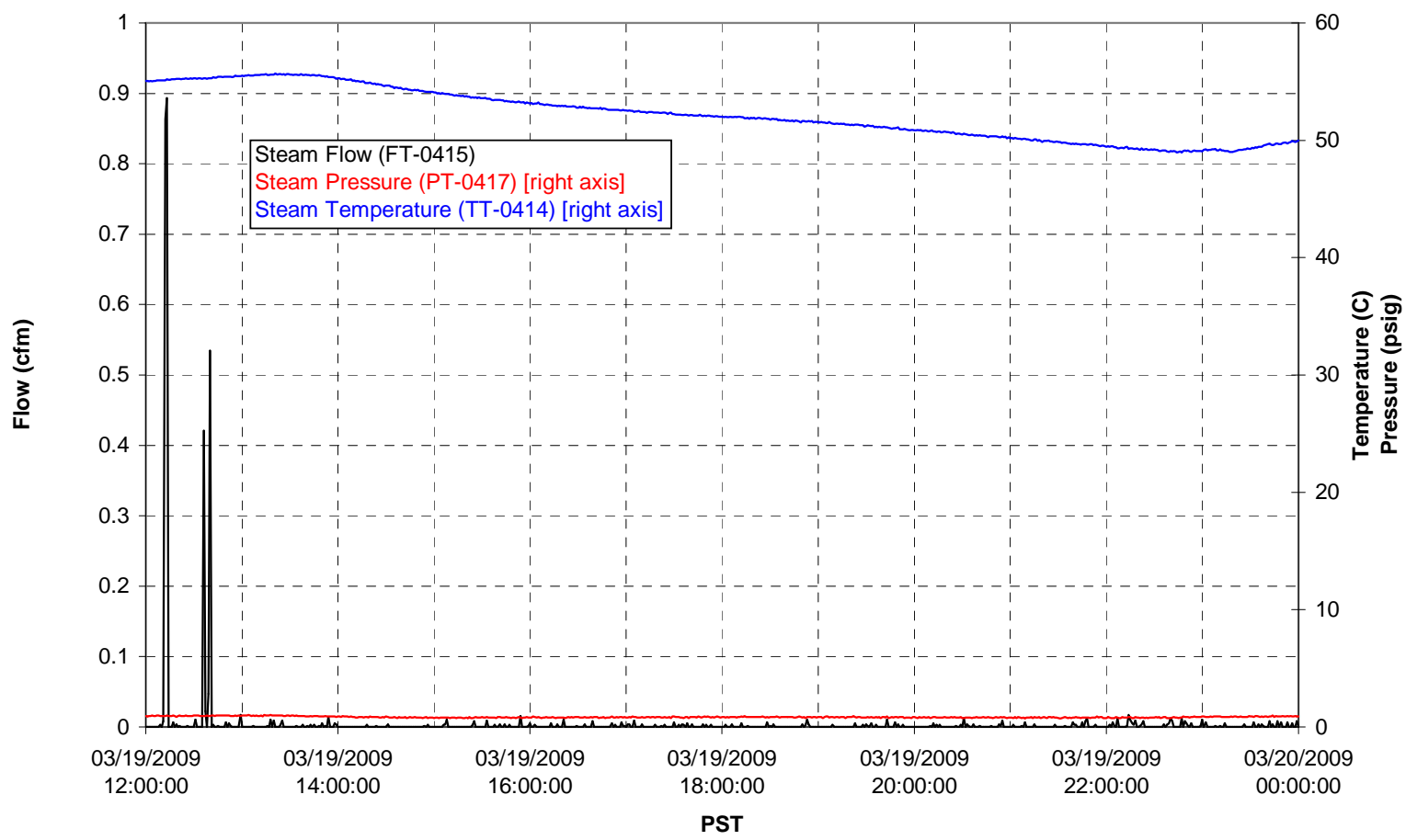




\section{Integrated Test B Data Plots 03/20/09 00:00 - 03/20/09 12:00}


T01A level

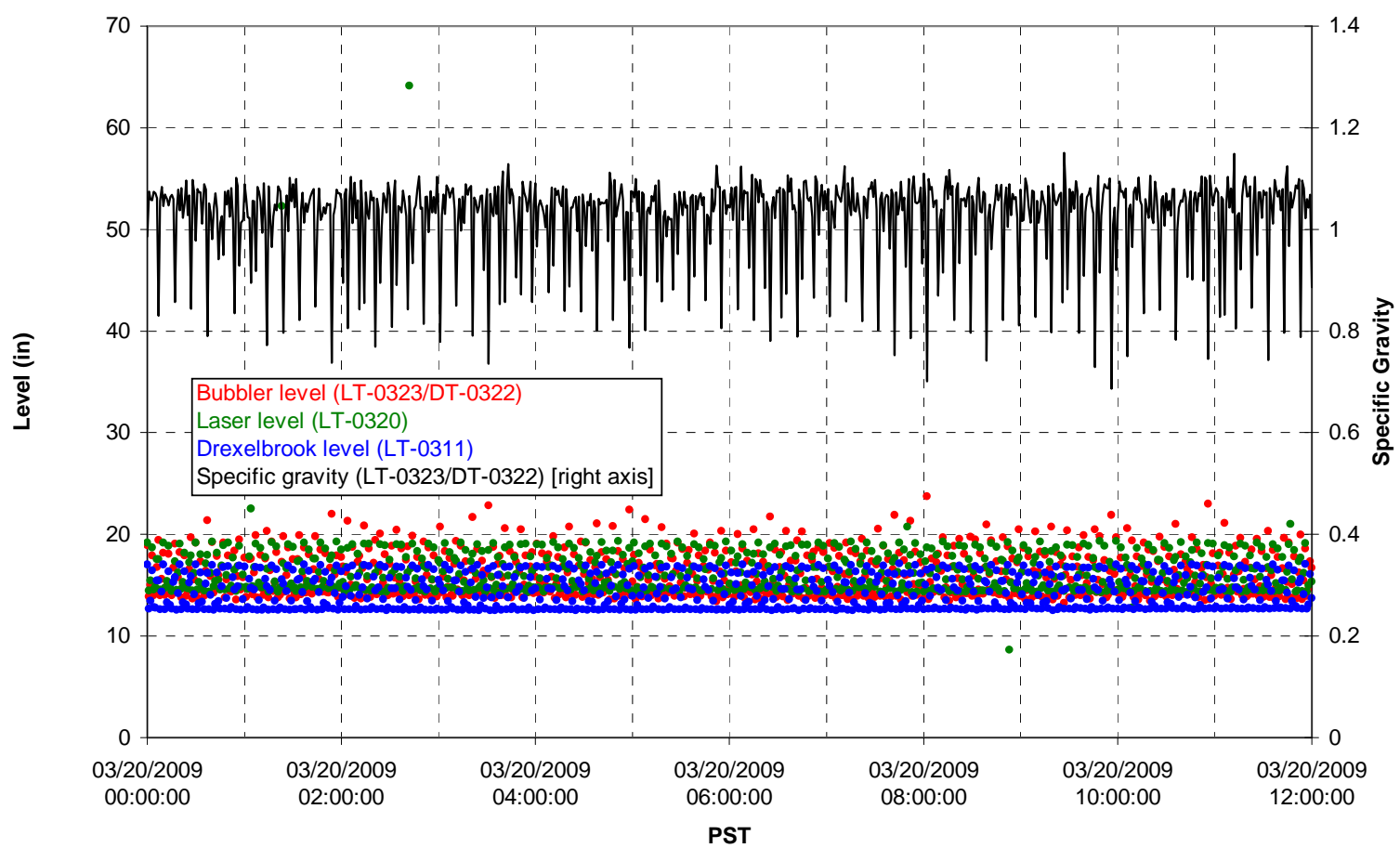

T01A temperatures

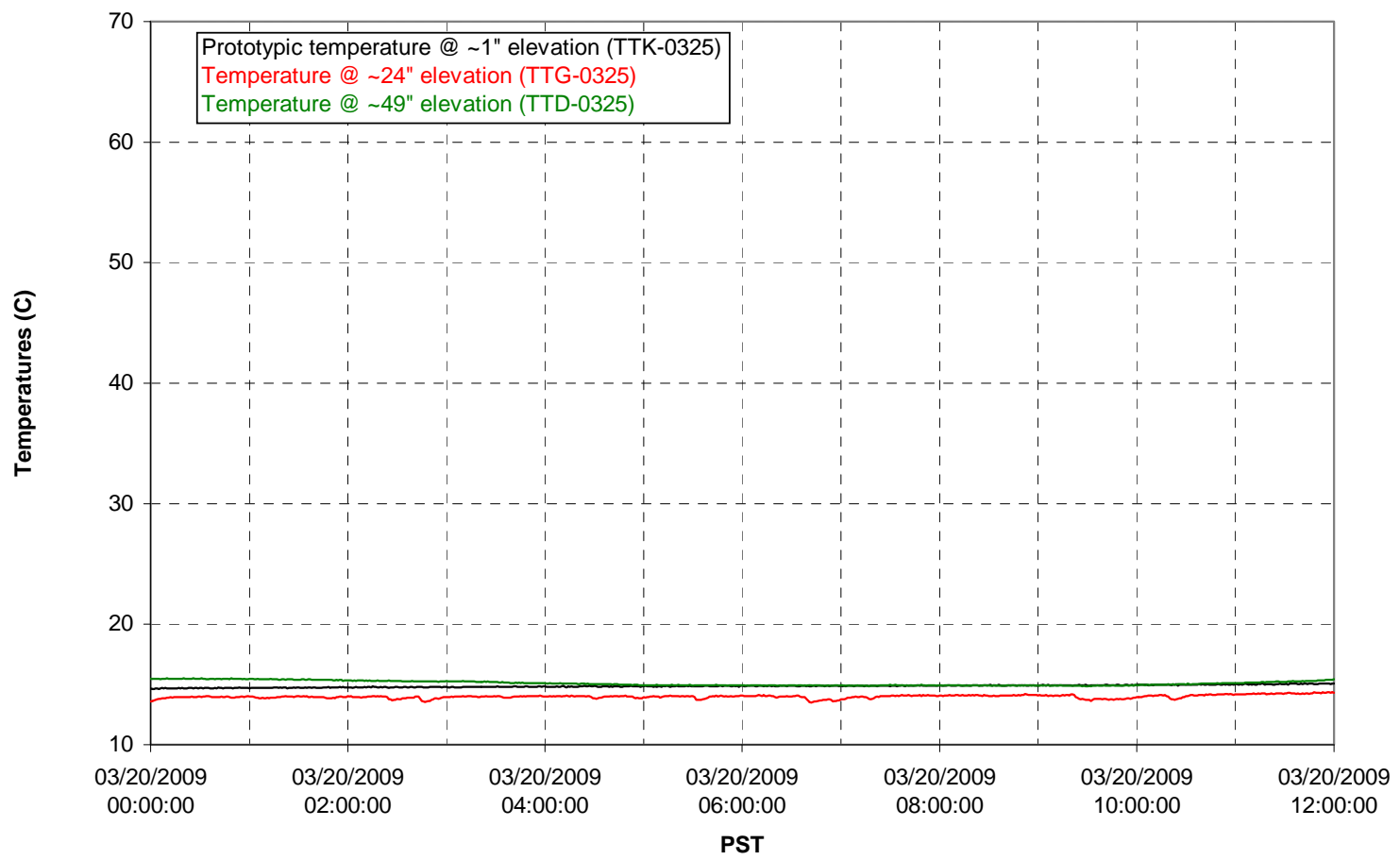


T01B level

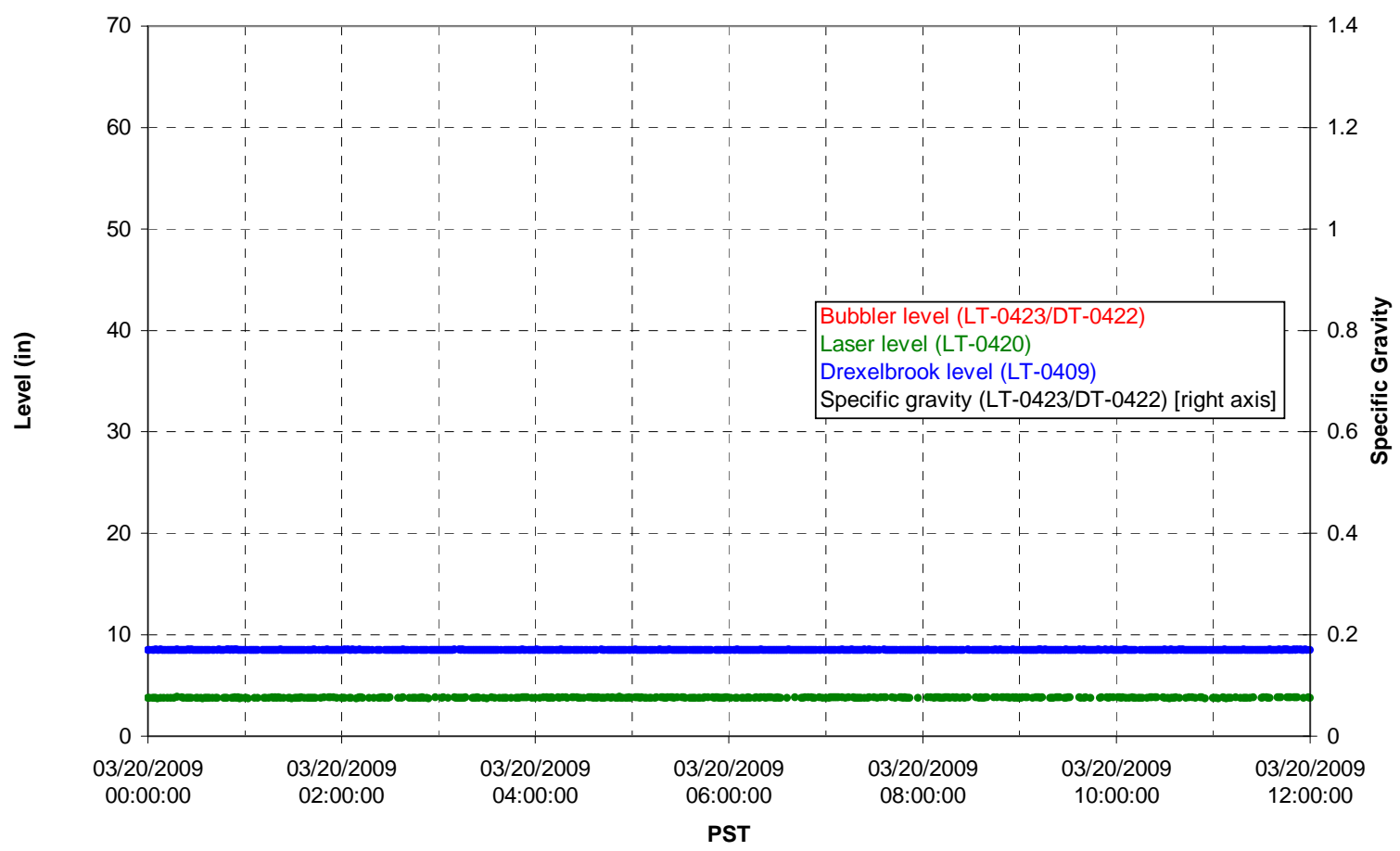

T01B temperatures

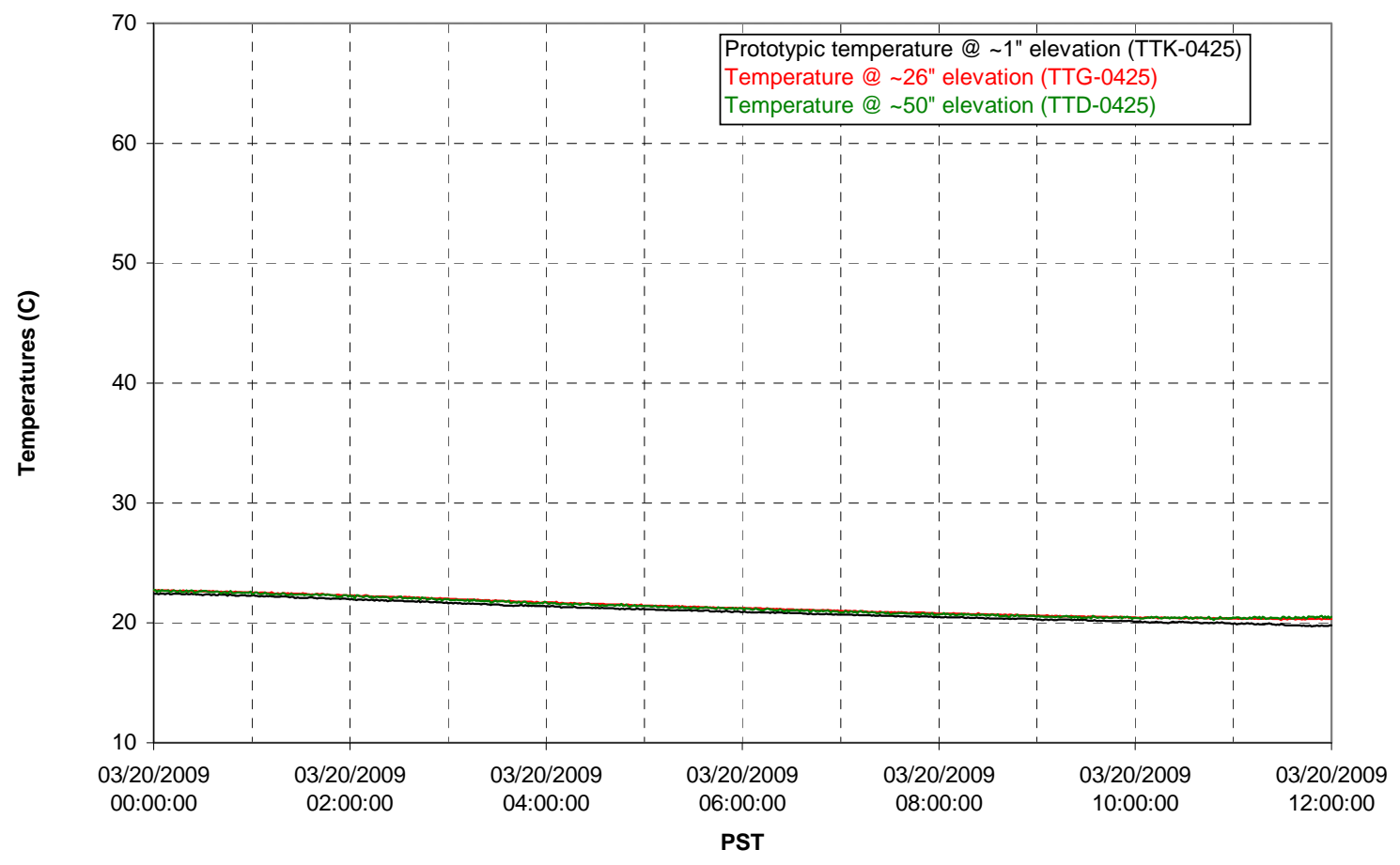


T02A level

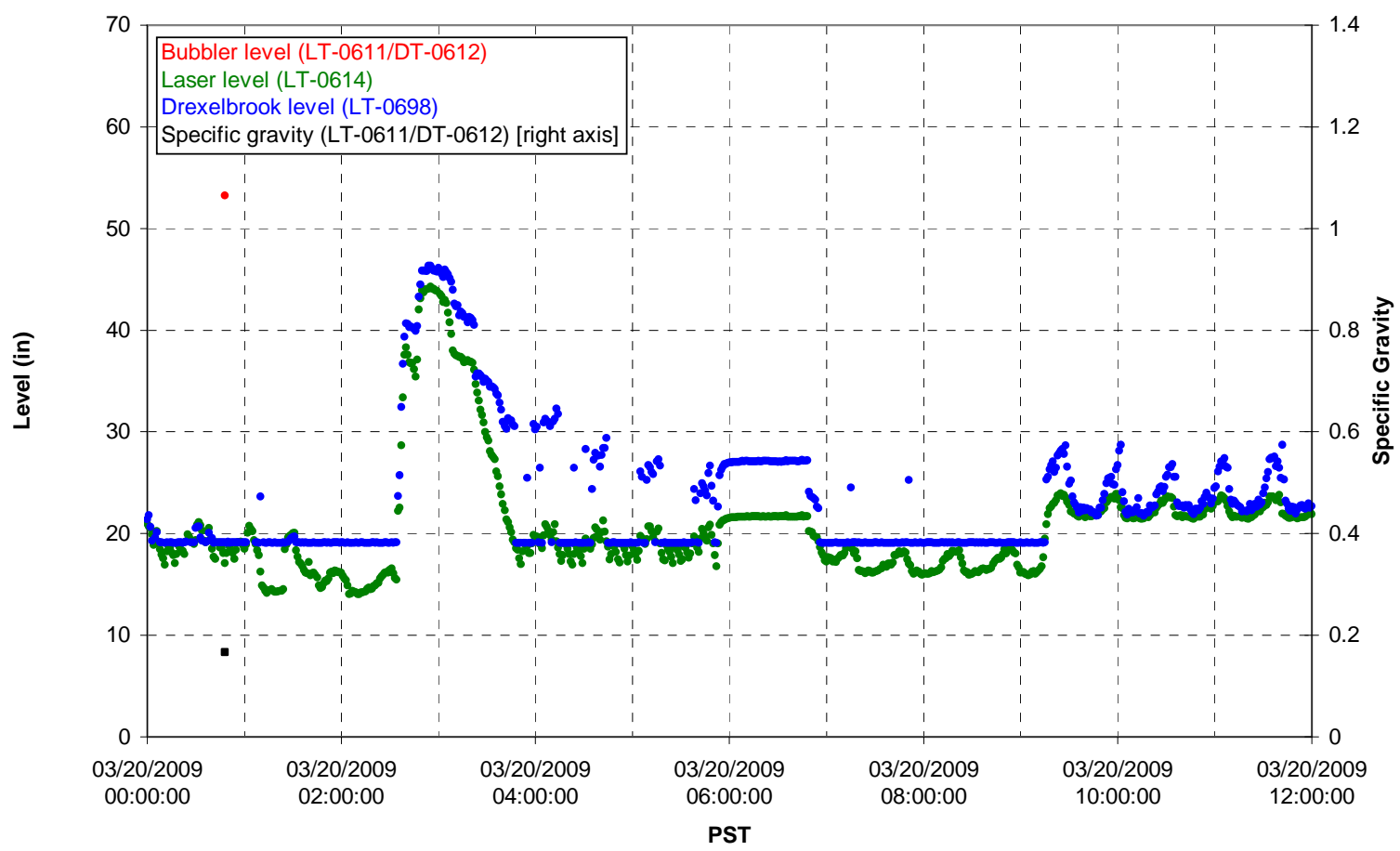

T02A temperatures

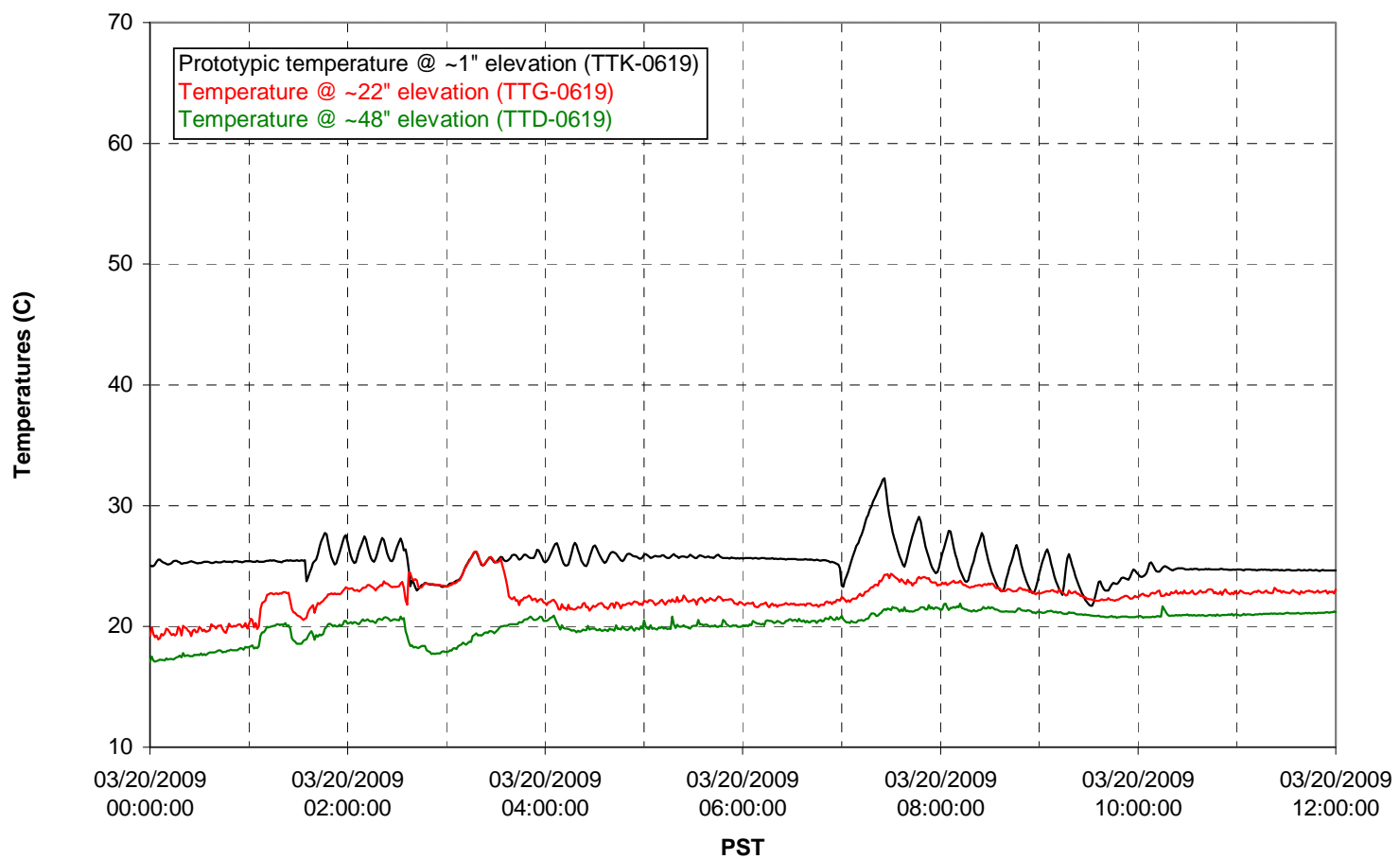


T02A and filter loop temperatures

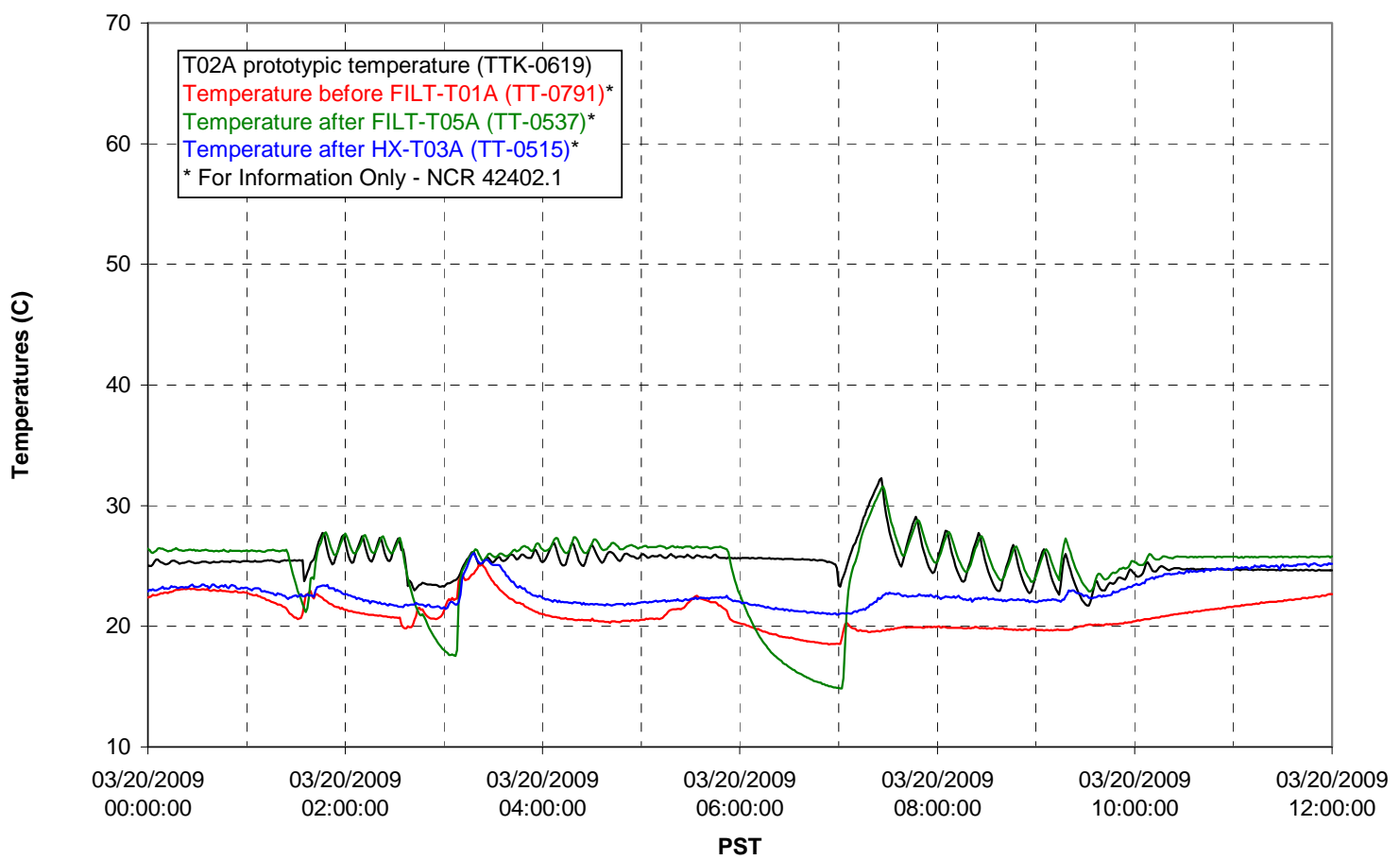

Pump Pressures and Flow

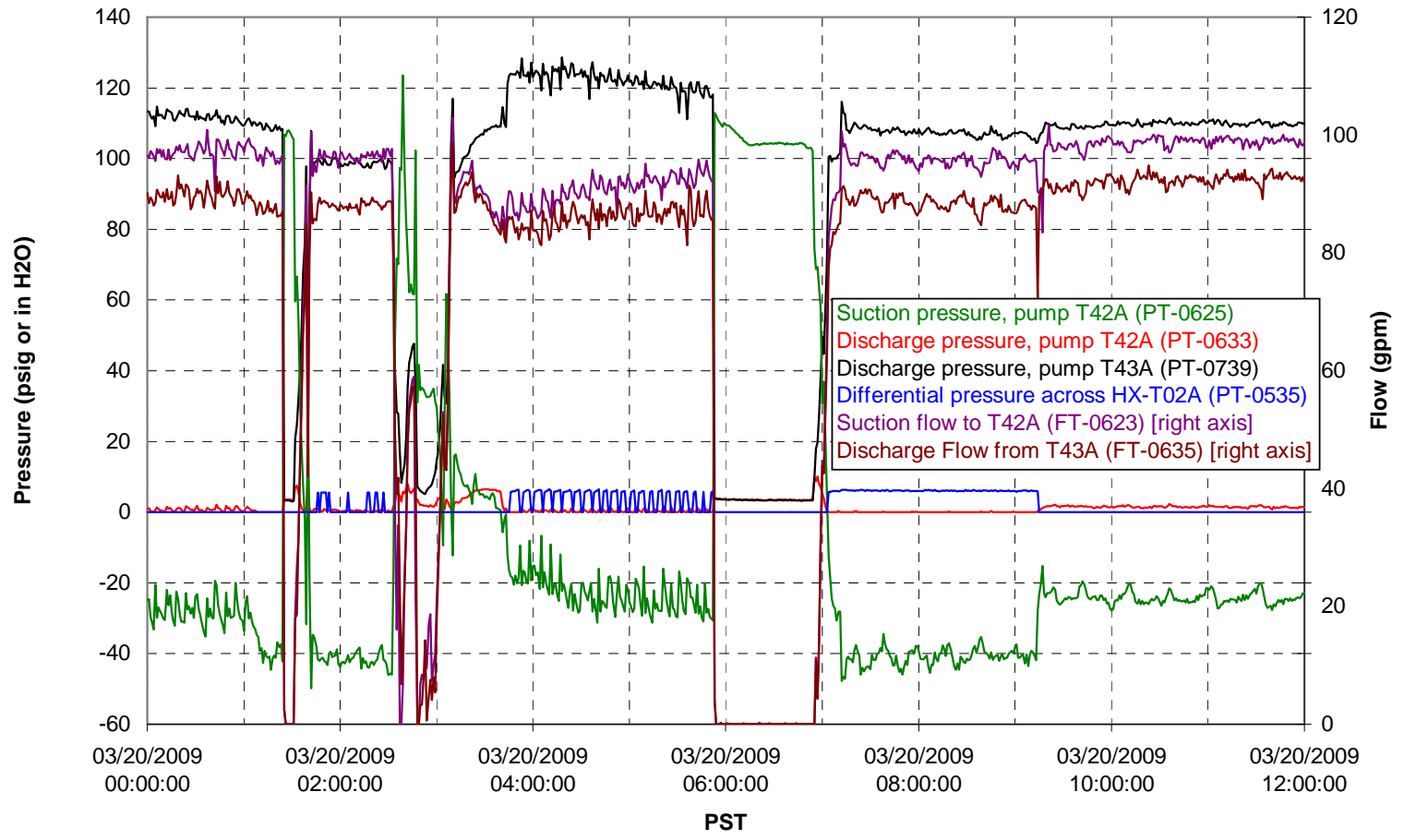


Axial pressure drop

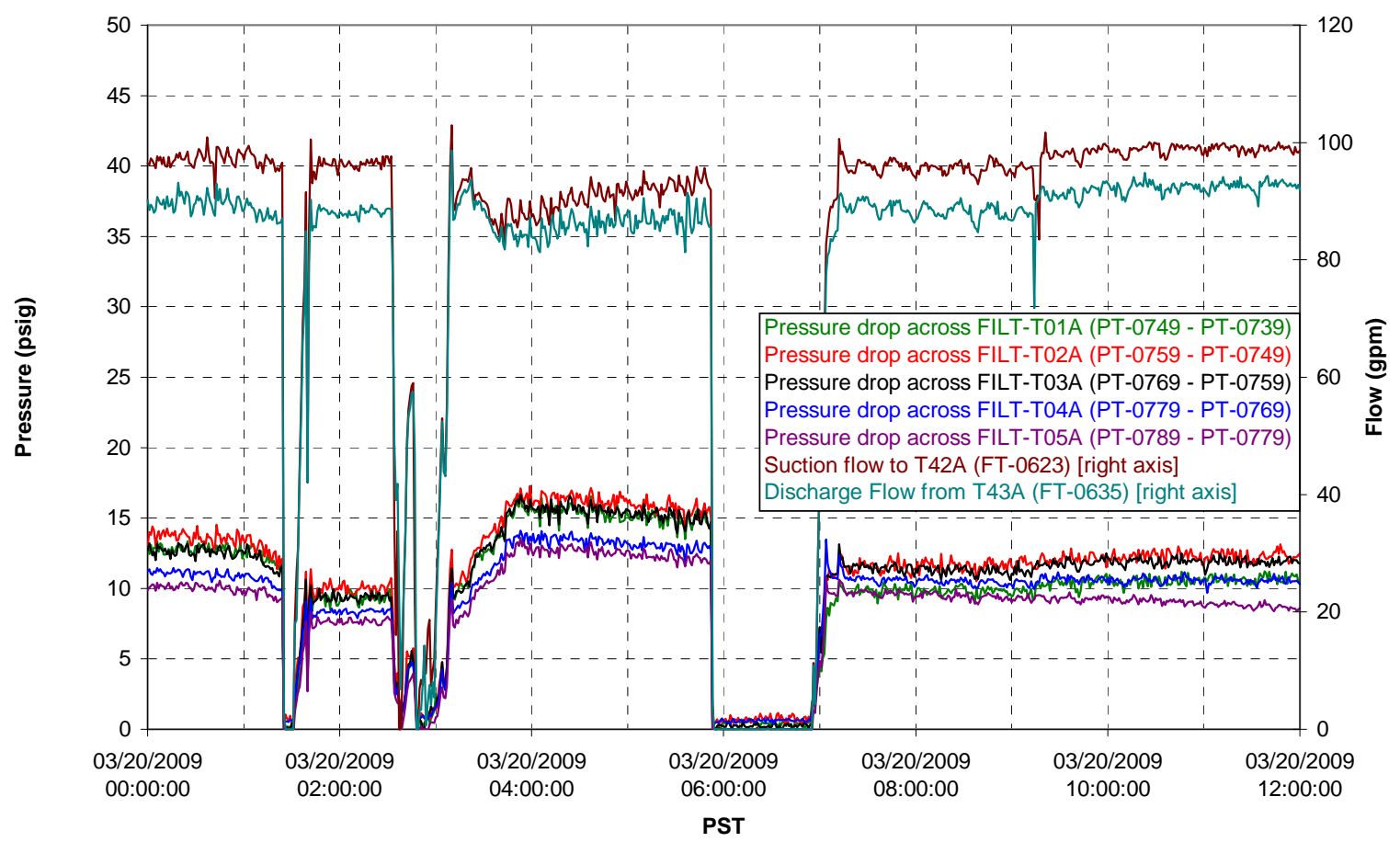

Permeate flow rates

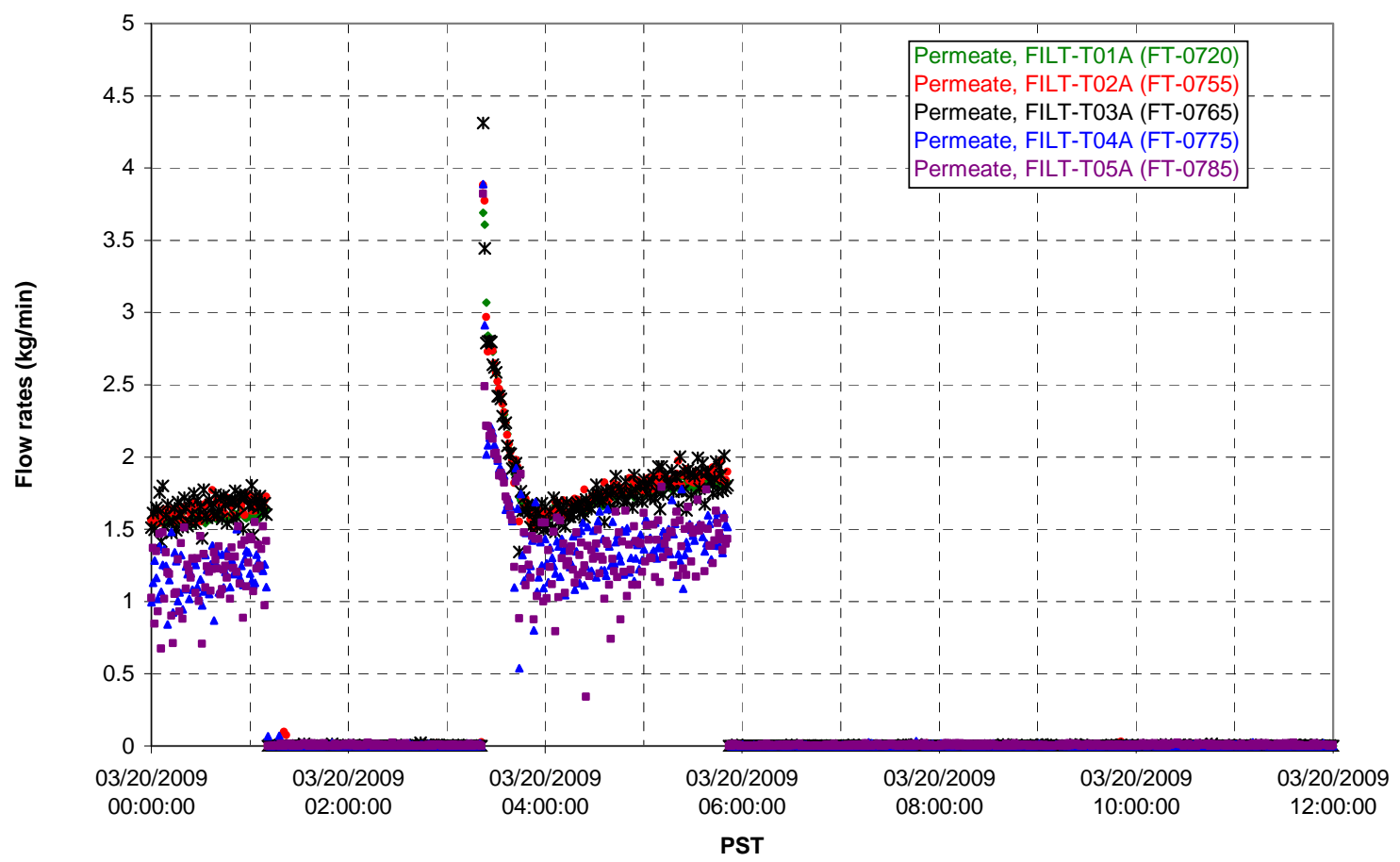


T02A Inner Temperature Tree

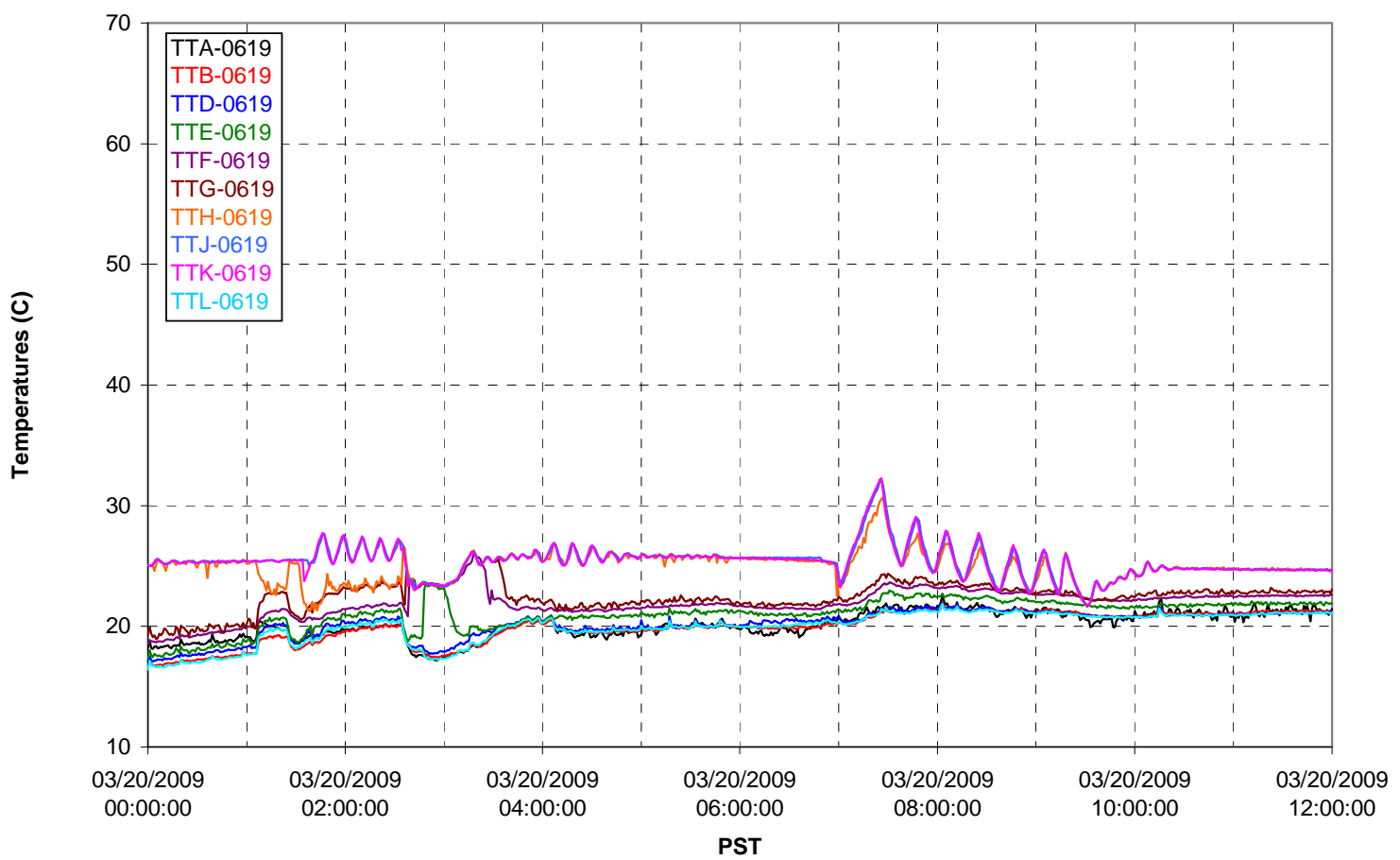

T02A Outer Temperature Tree

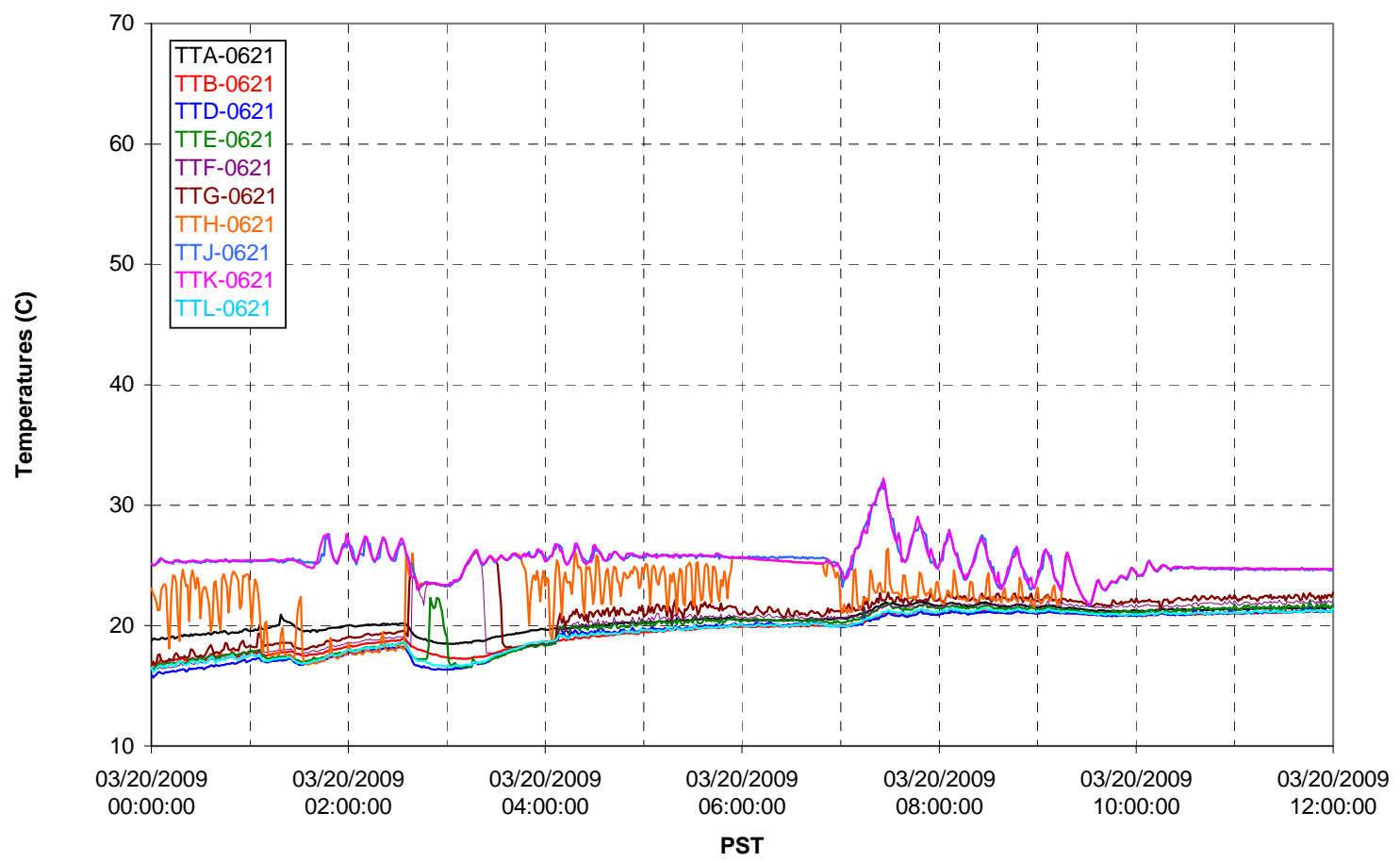


T02A temperatures

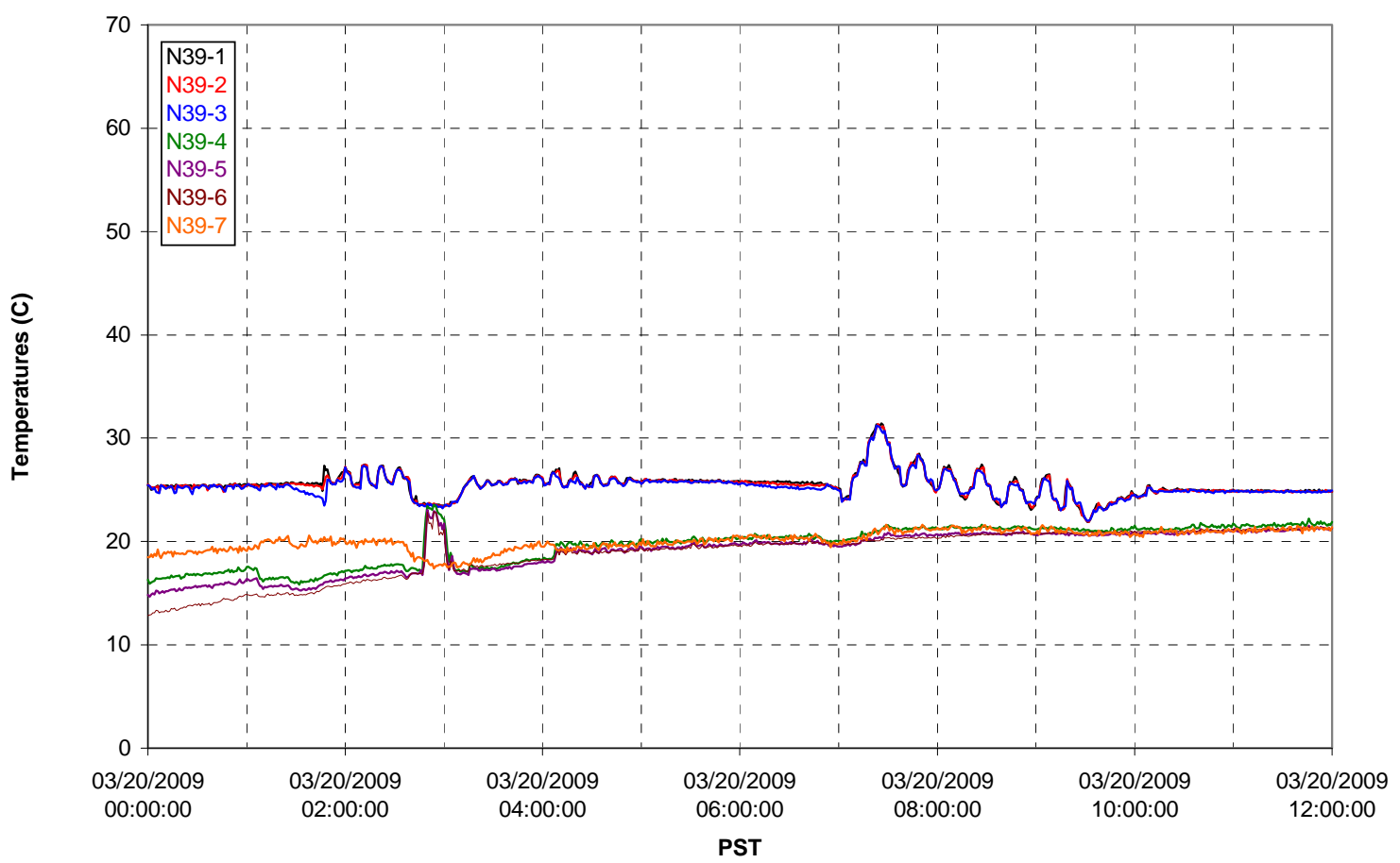

T02A temperatures

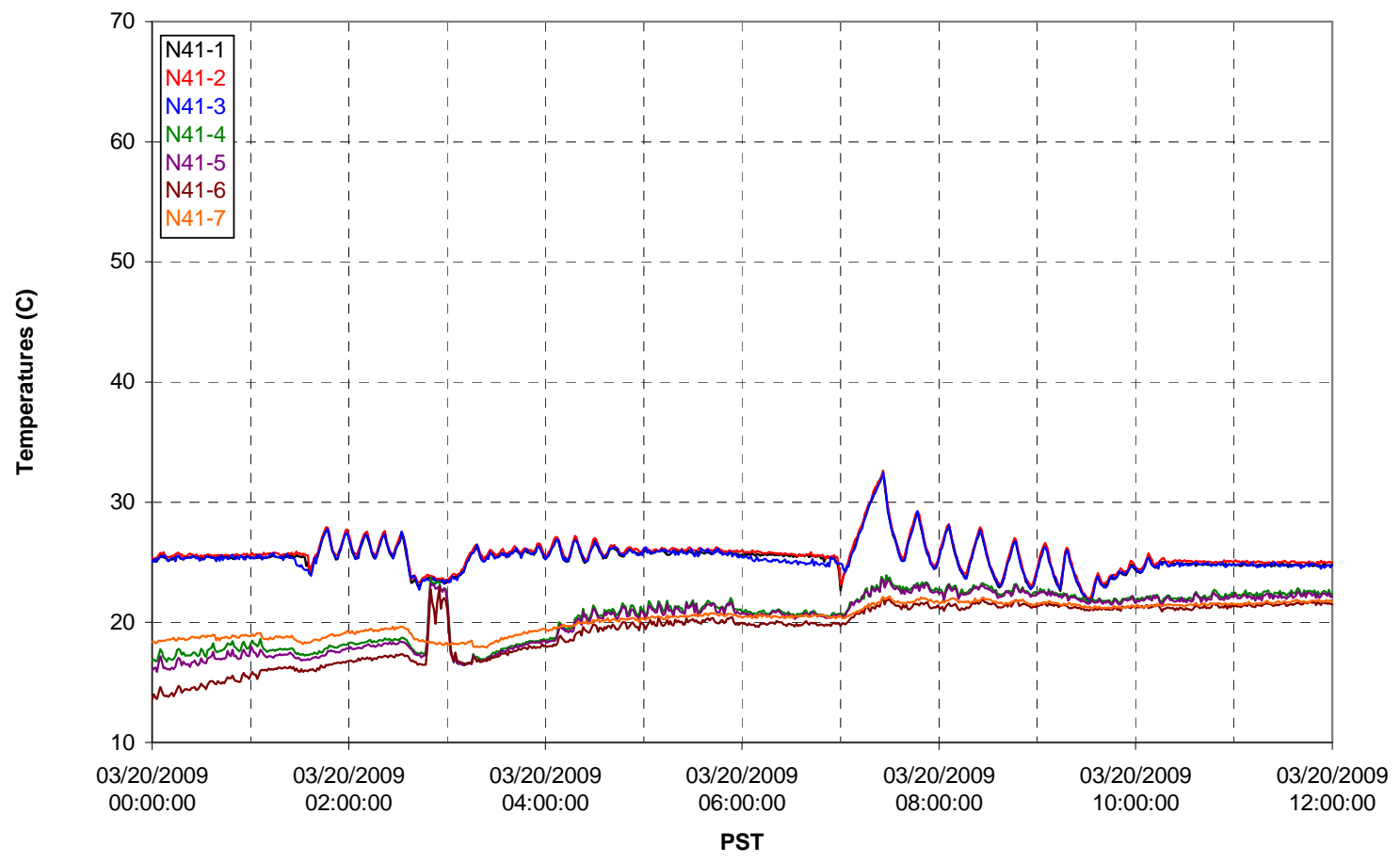


T02A temperatures

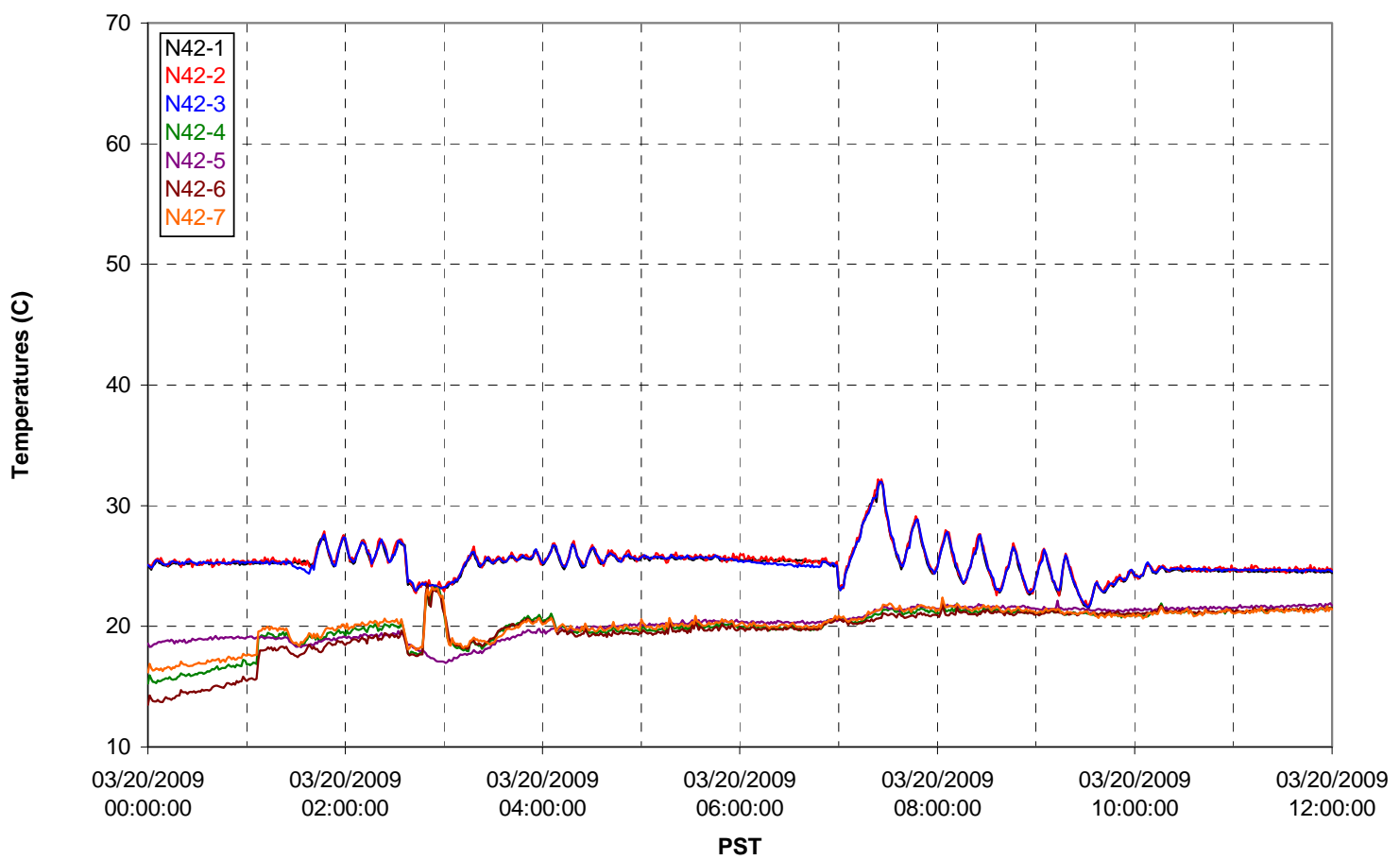

T02A temperatures

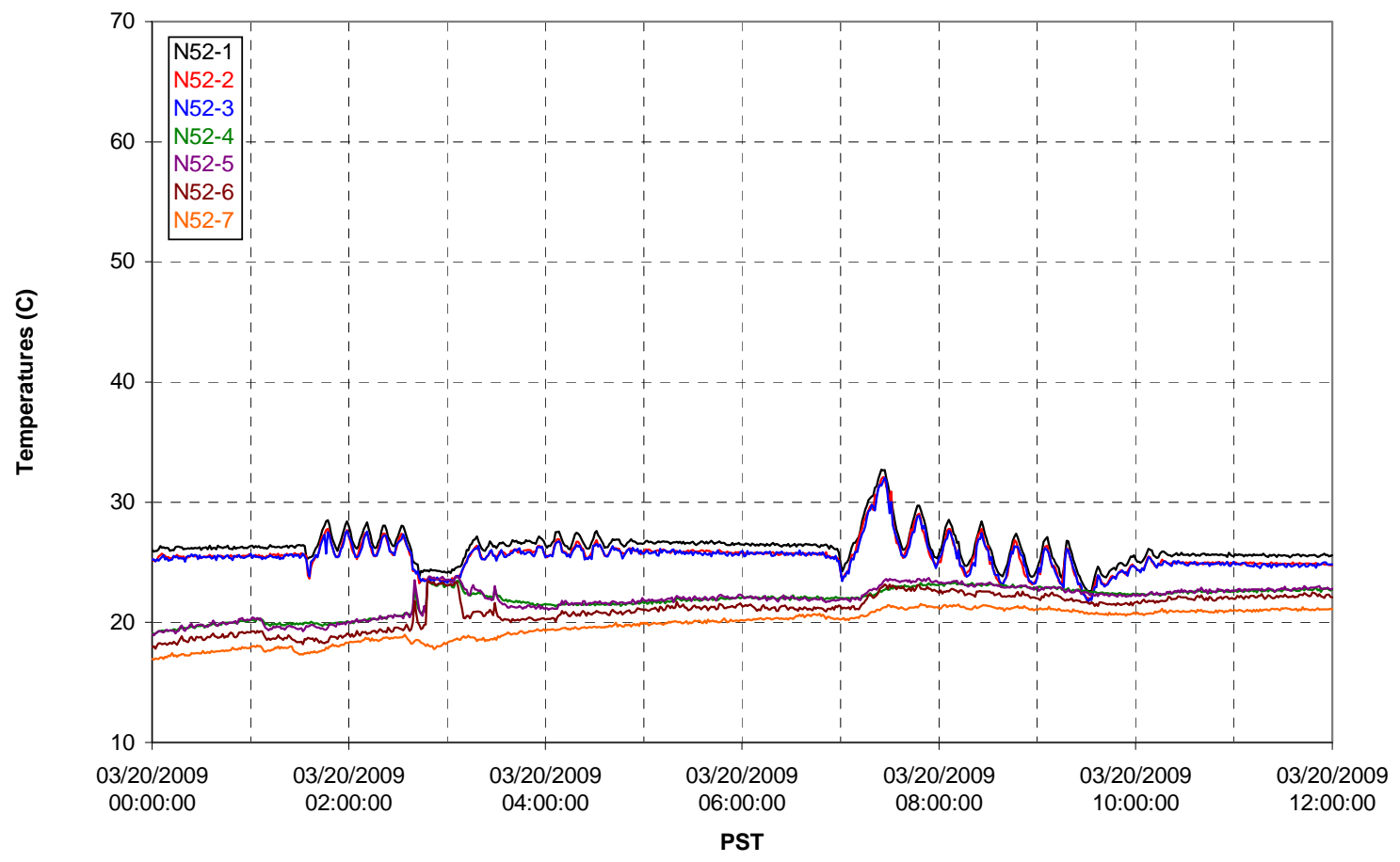


T02A Heating and Cooling
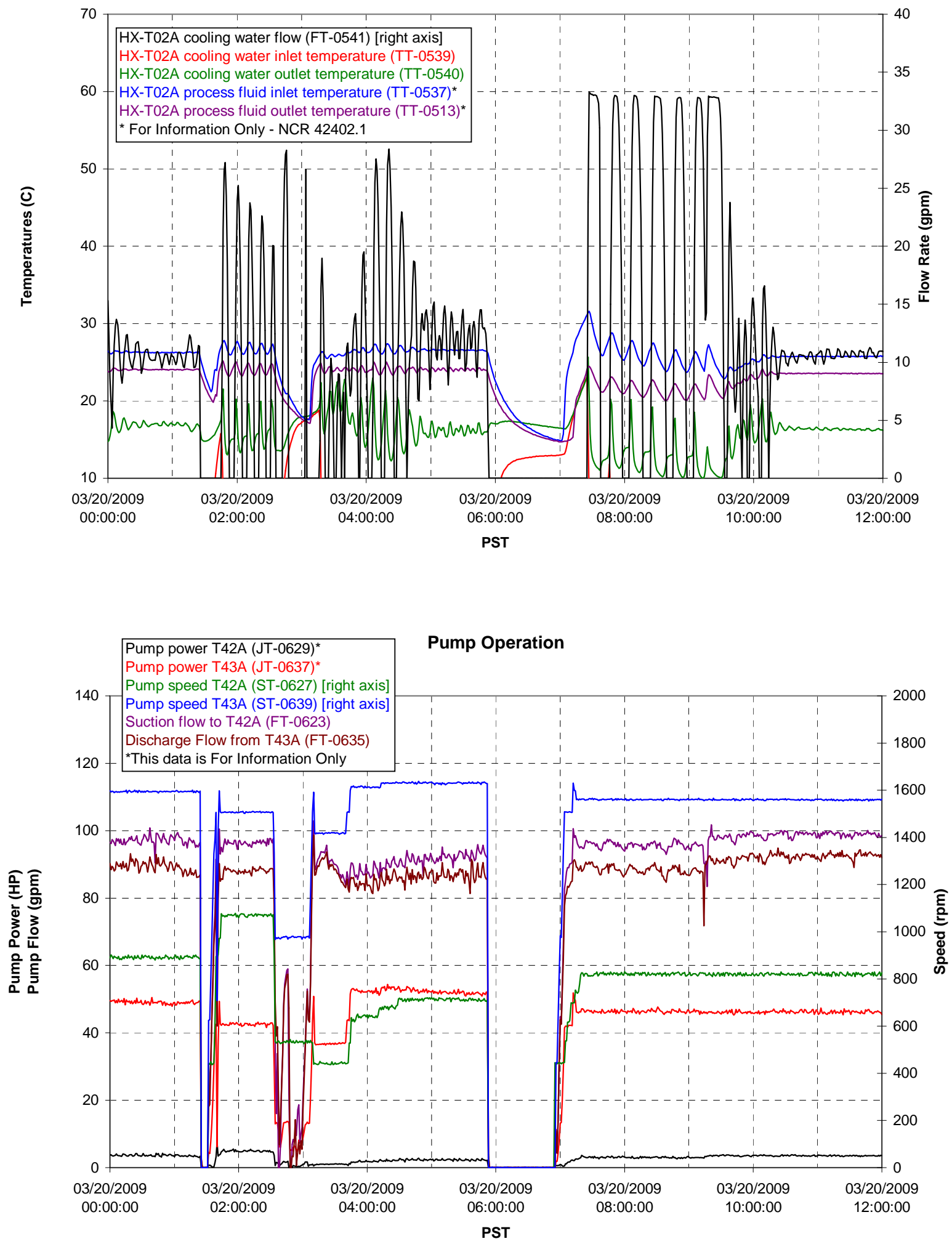
Pulsepot UFP-PP-T01A

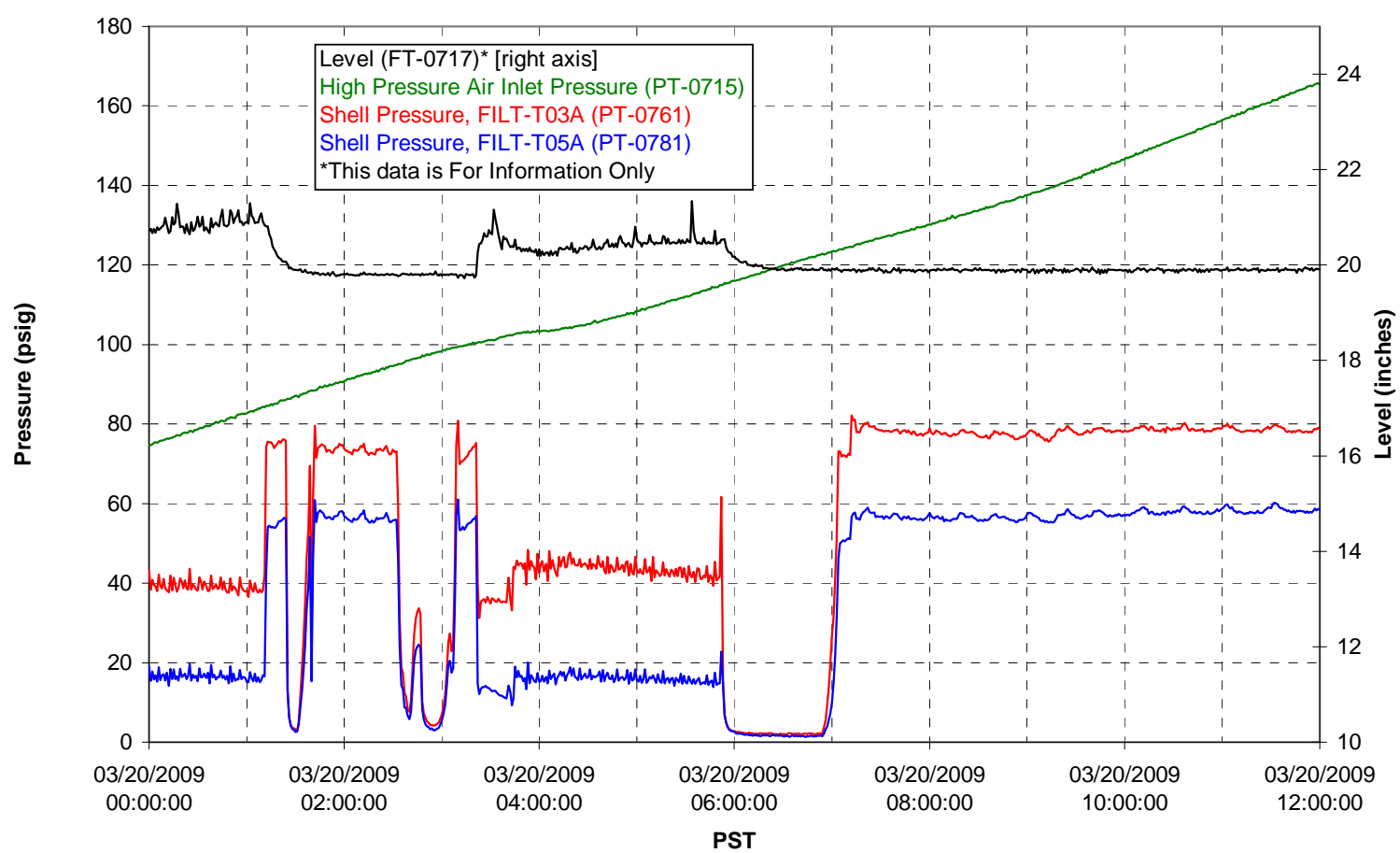

Pulsepot UFP-PP-T02A

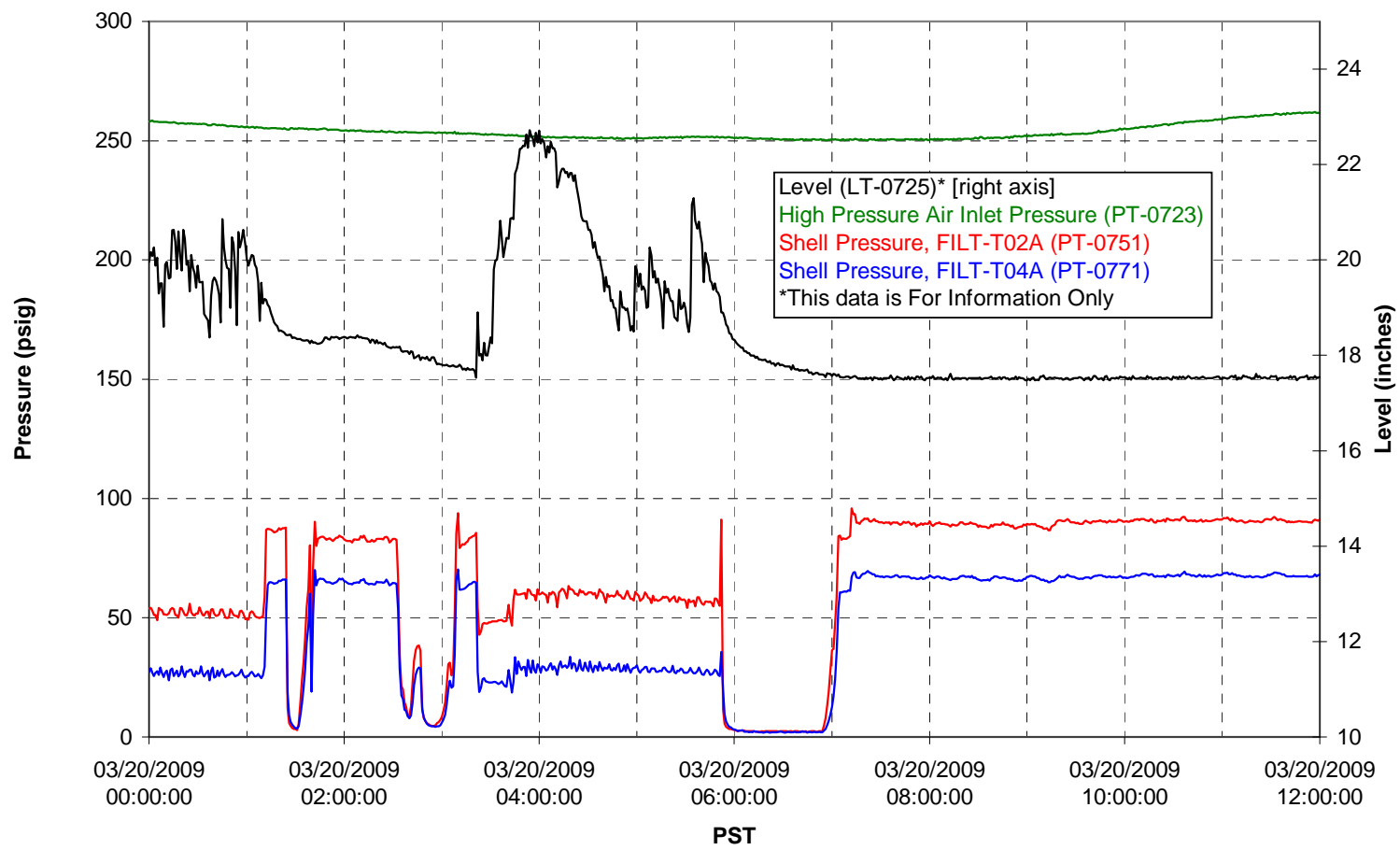


Pulsepot UFP-PP-T03A

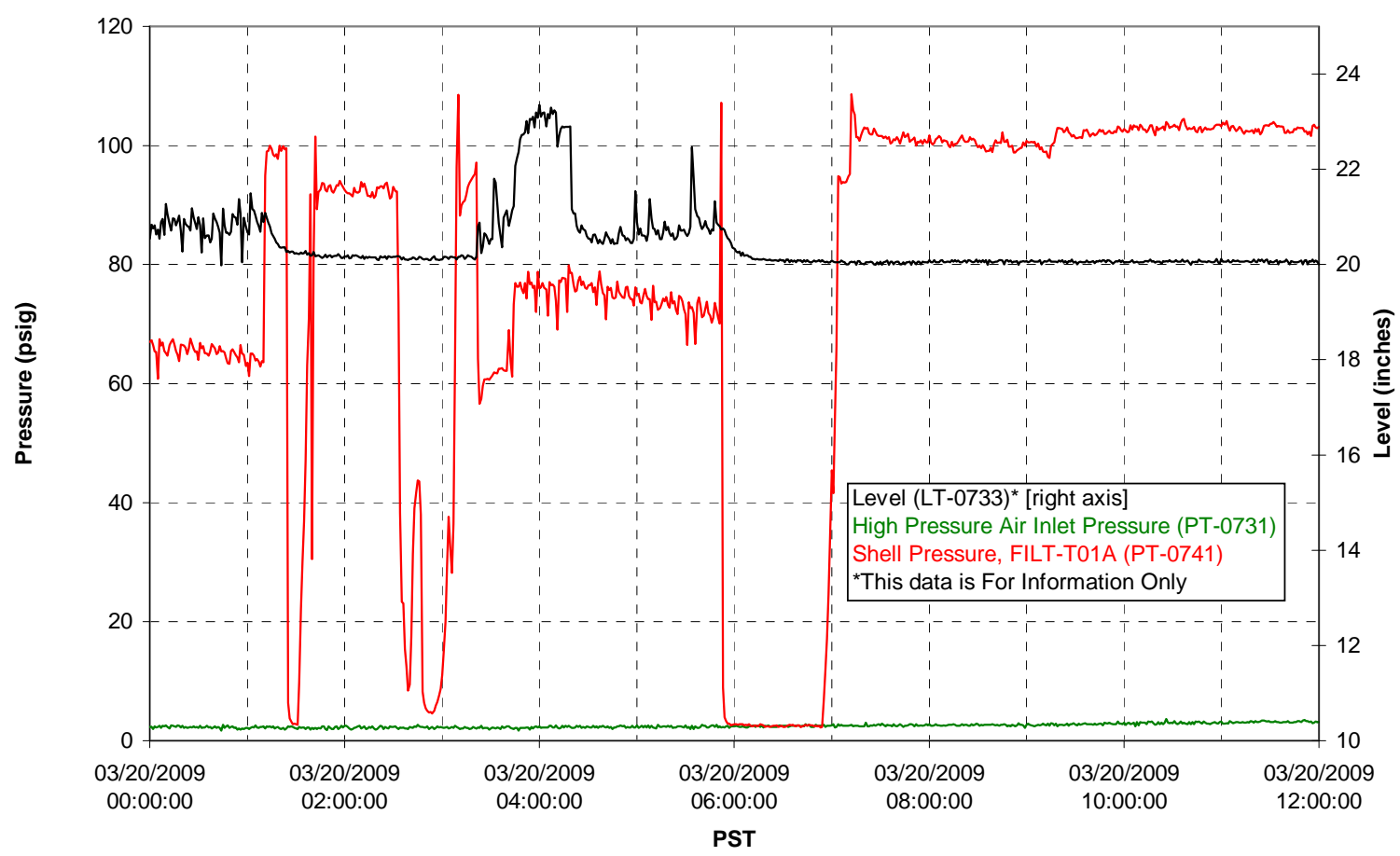

Pulsepot Levels

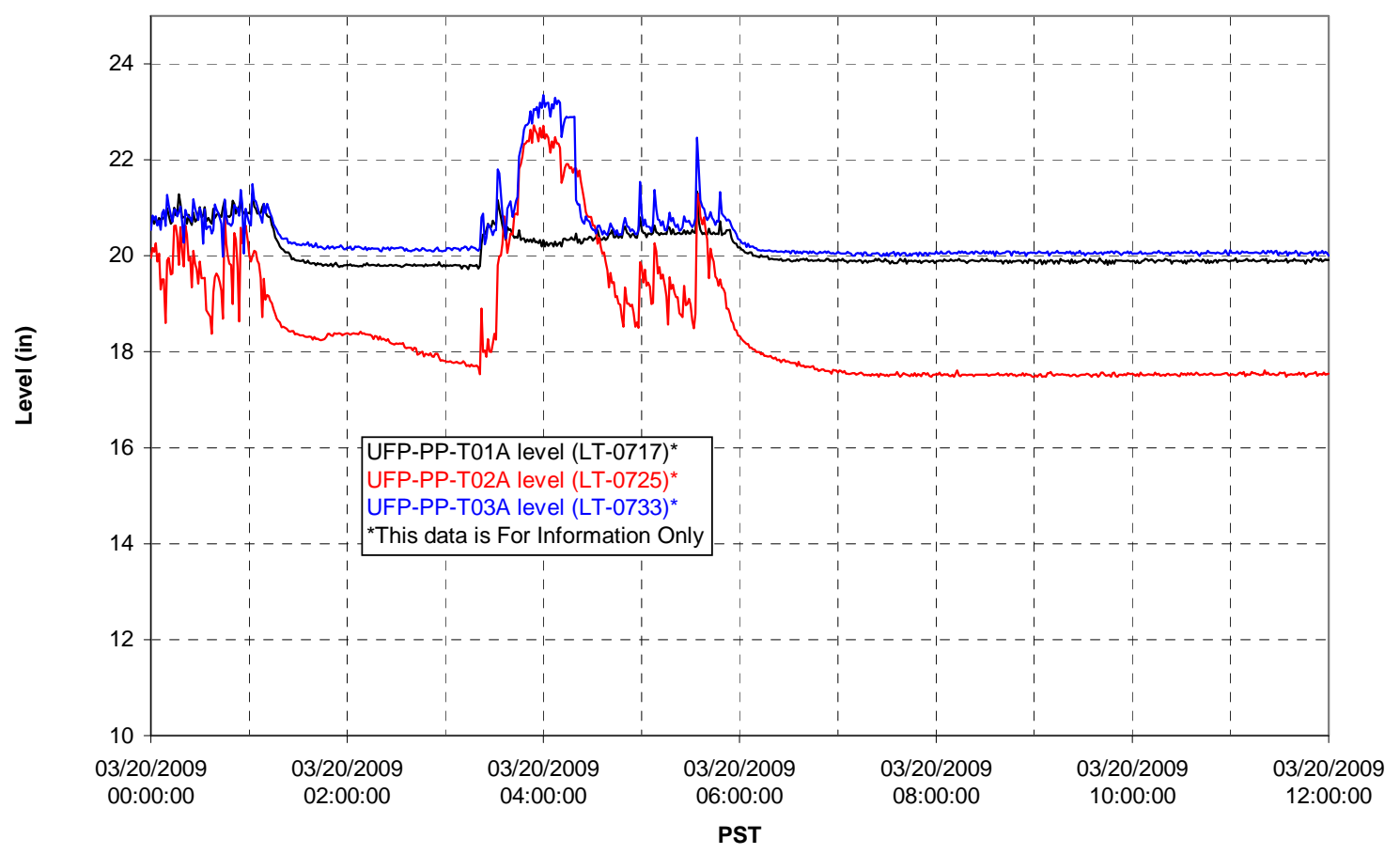


Filter UFP-FILT-T01A

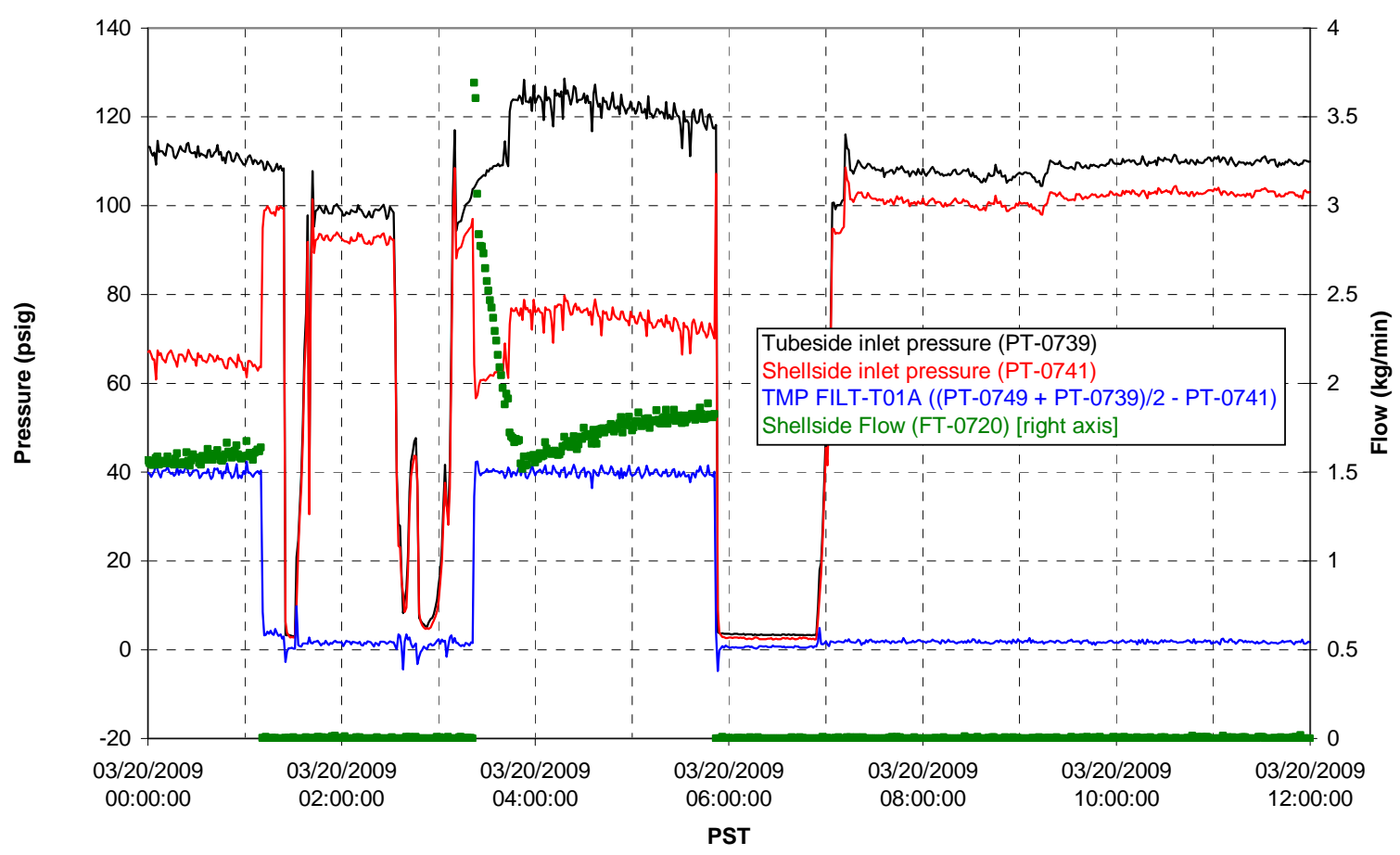

Filter UFP-FILT-T02A

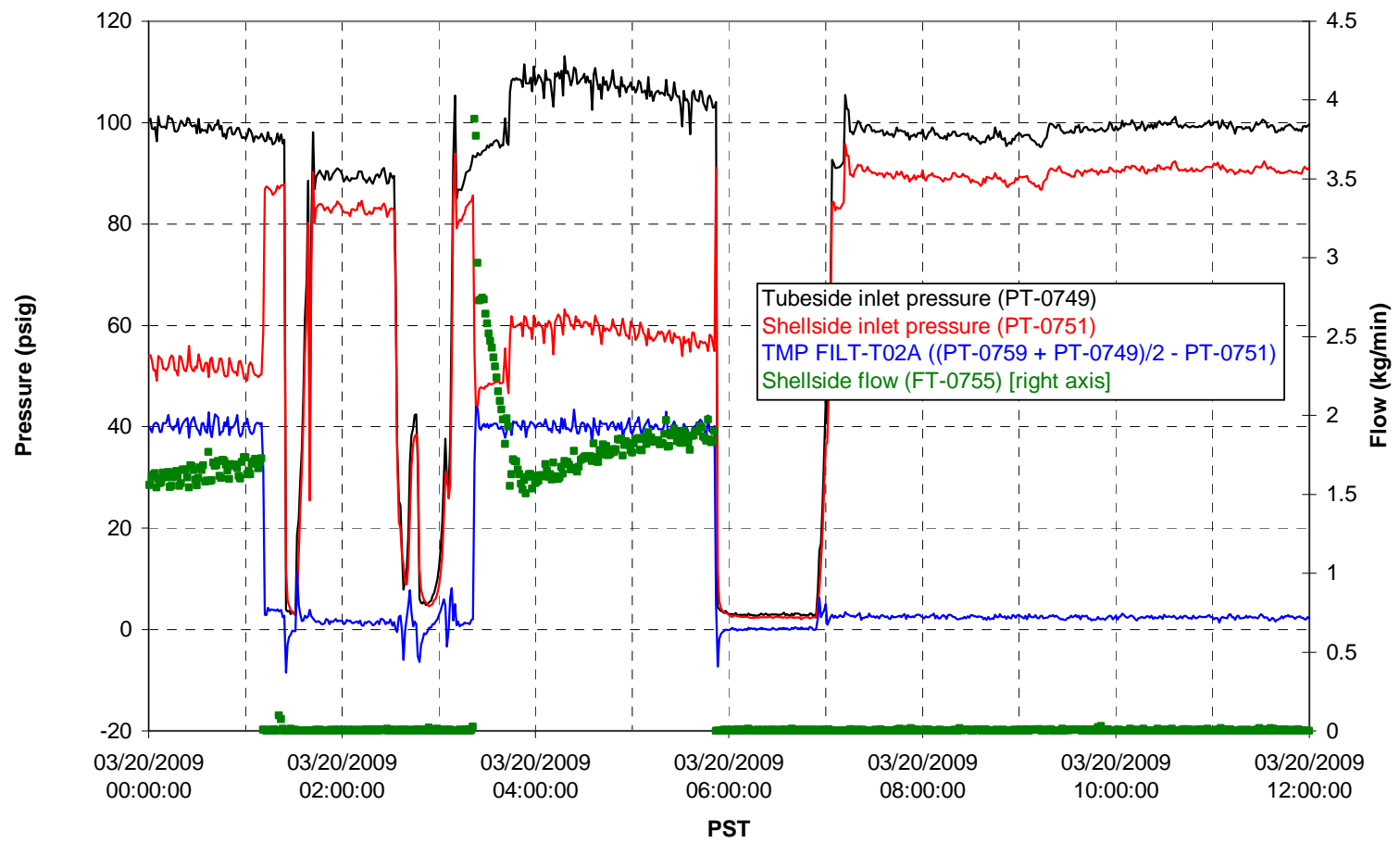


Filter UFP-FILT-T03A

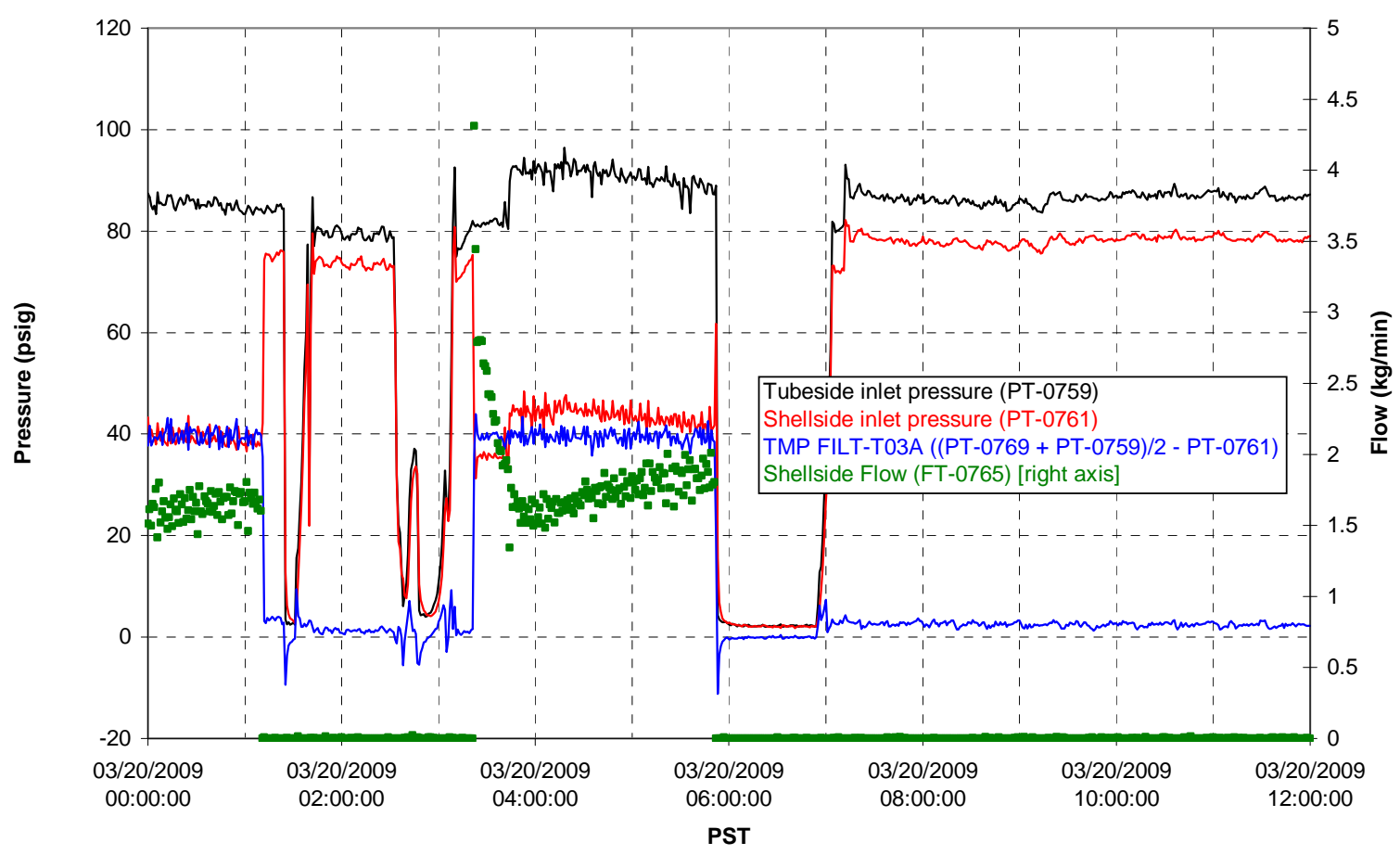

Filter UFP-FILT-T04A

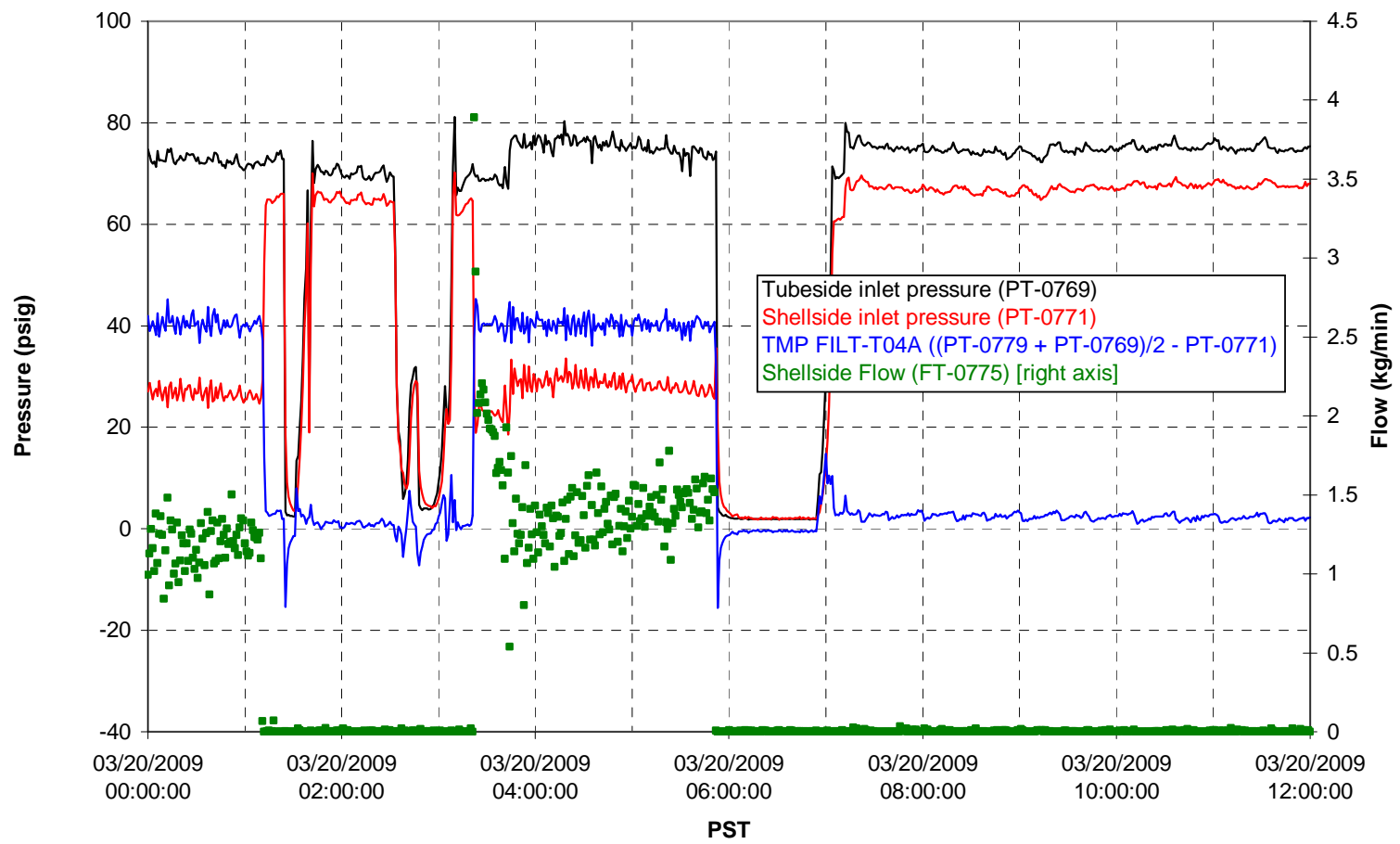


Filter UFP-FILT-T05A

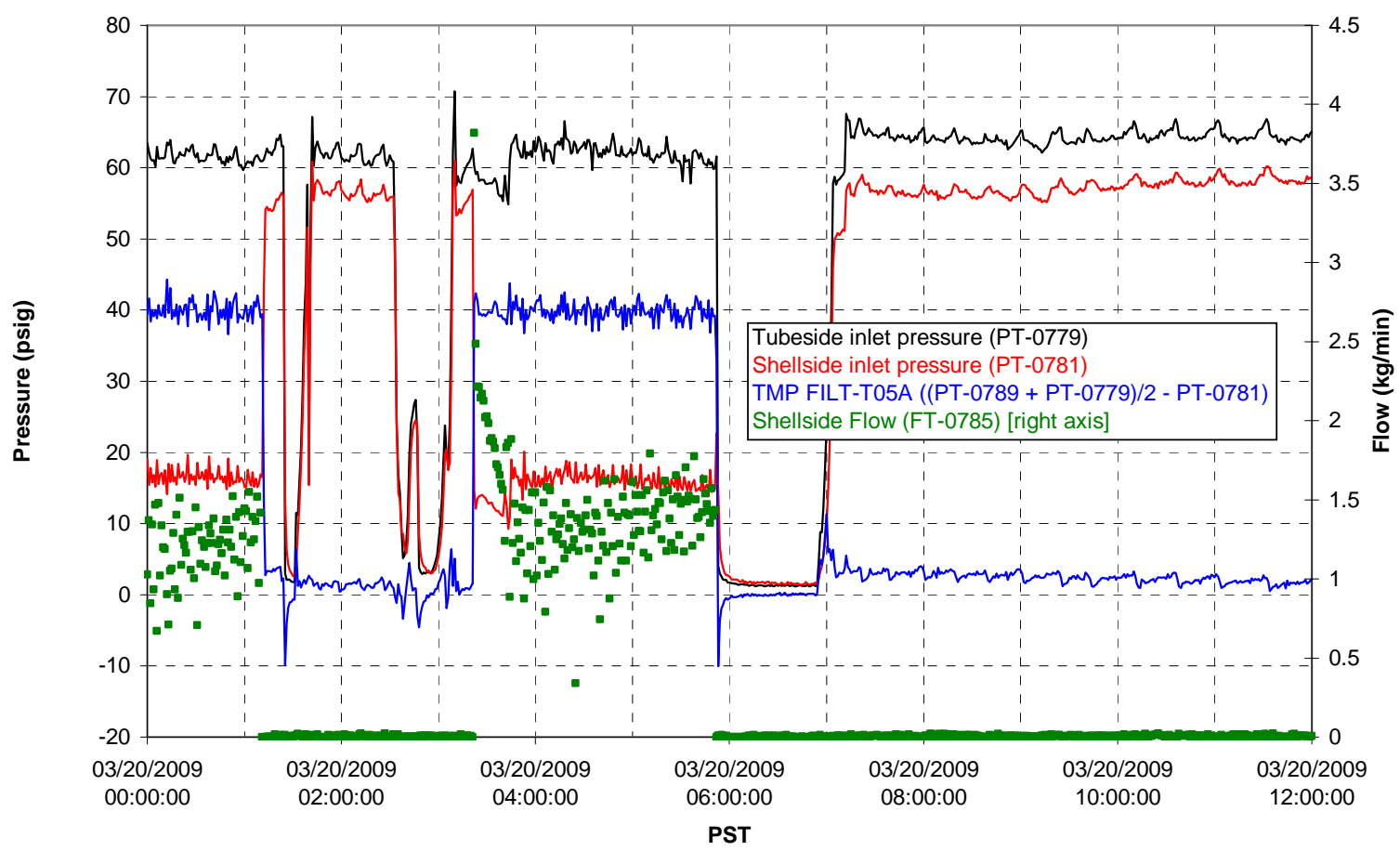

Chemical Flow

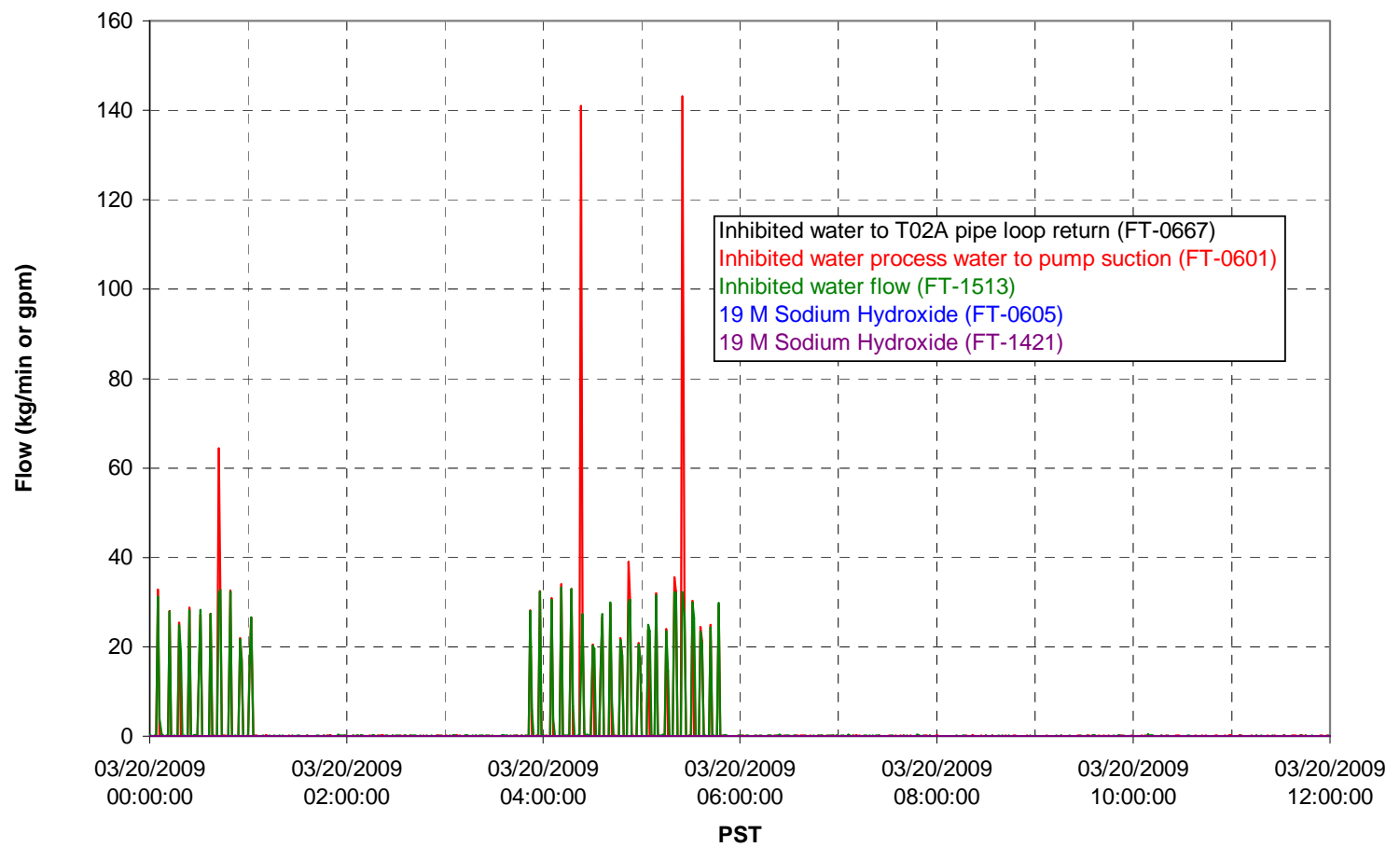




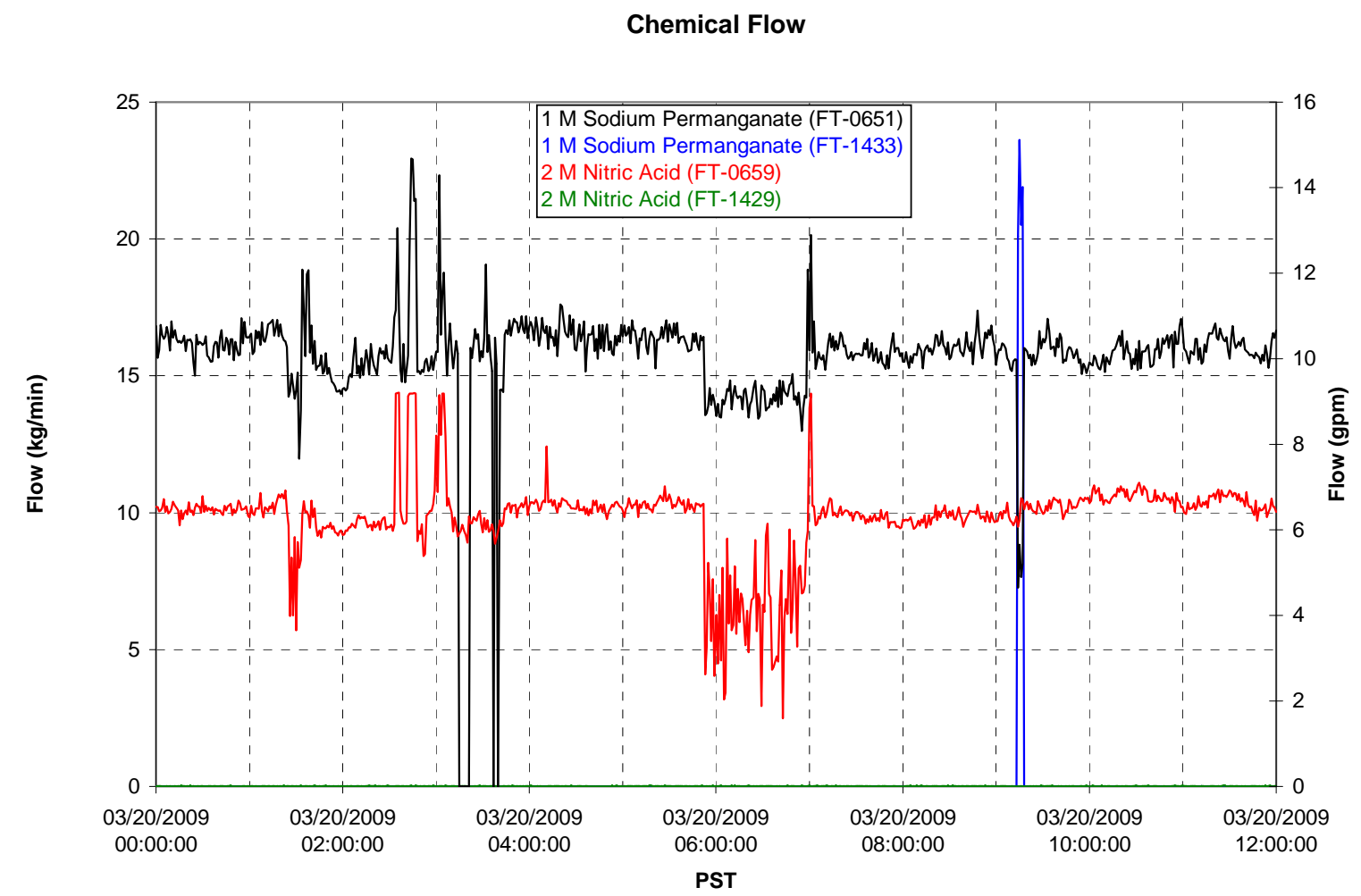

Air Flows

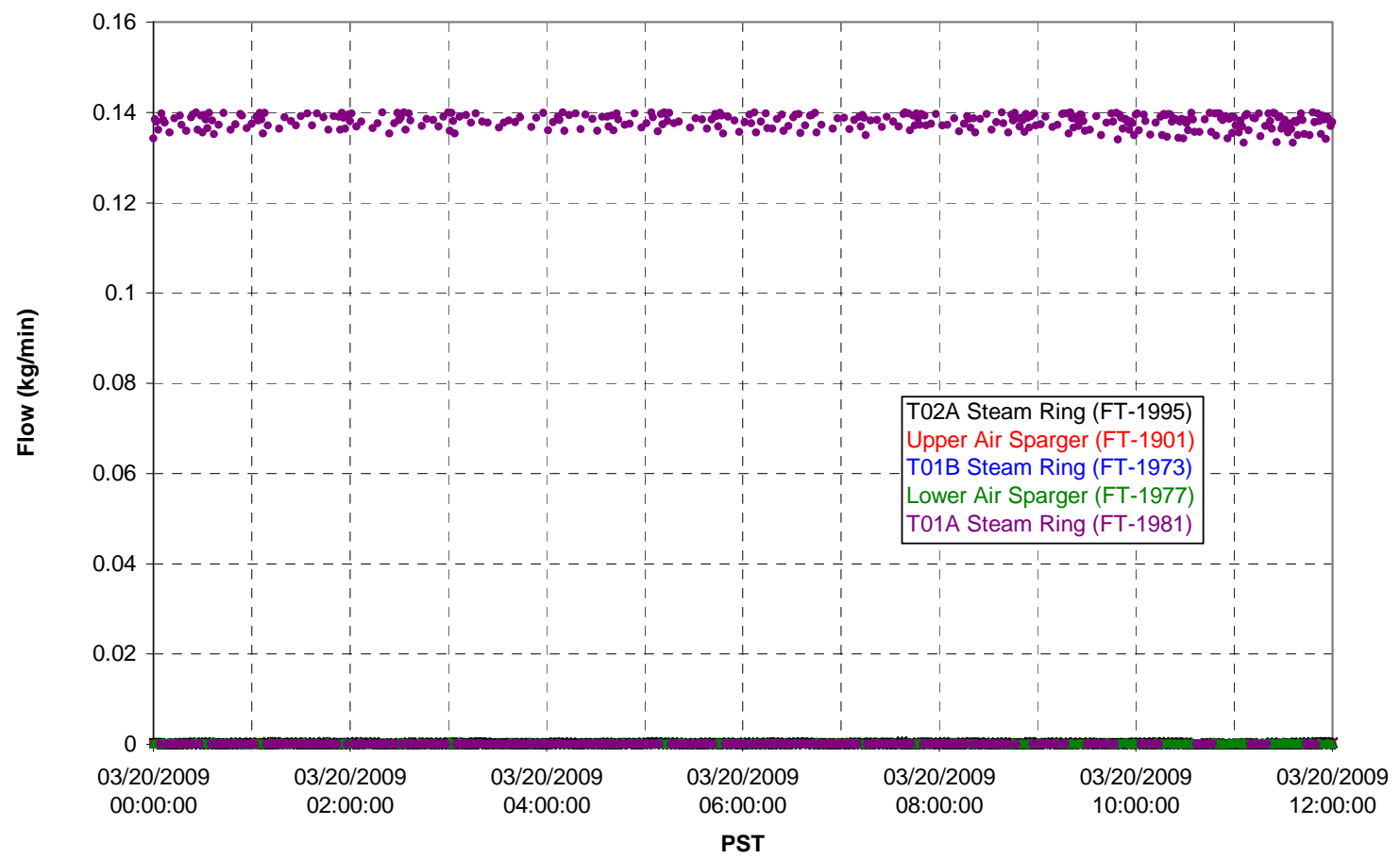


T02A Steam

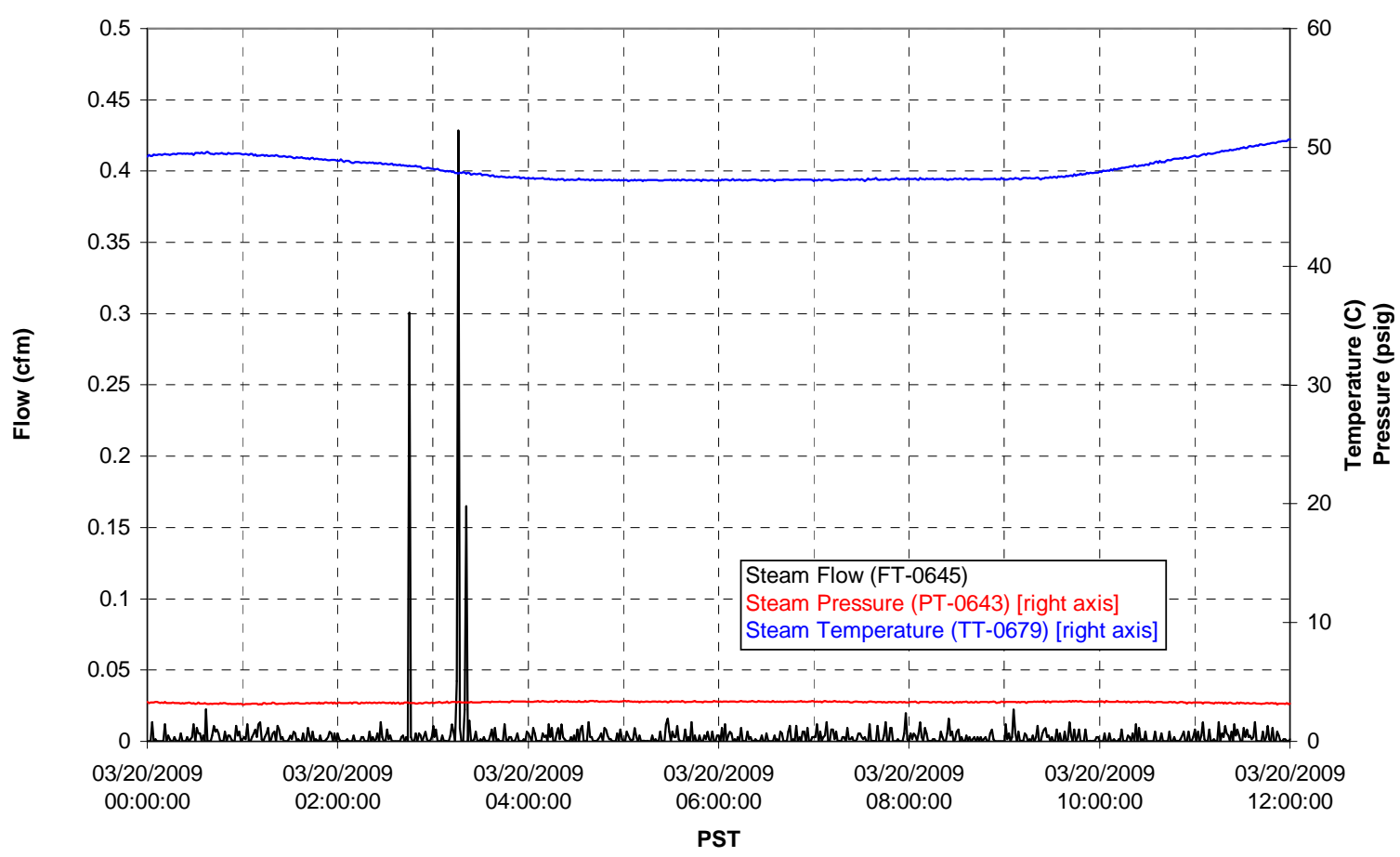

T01A Steam

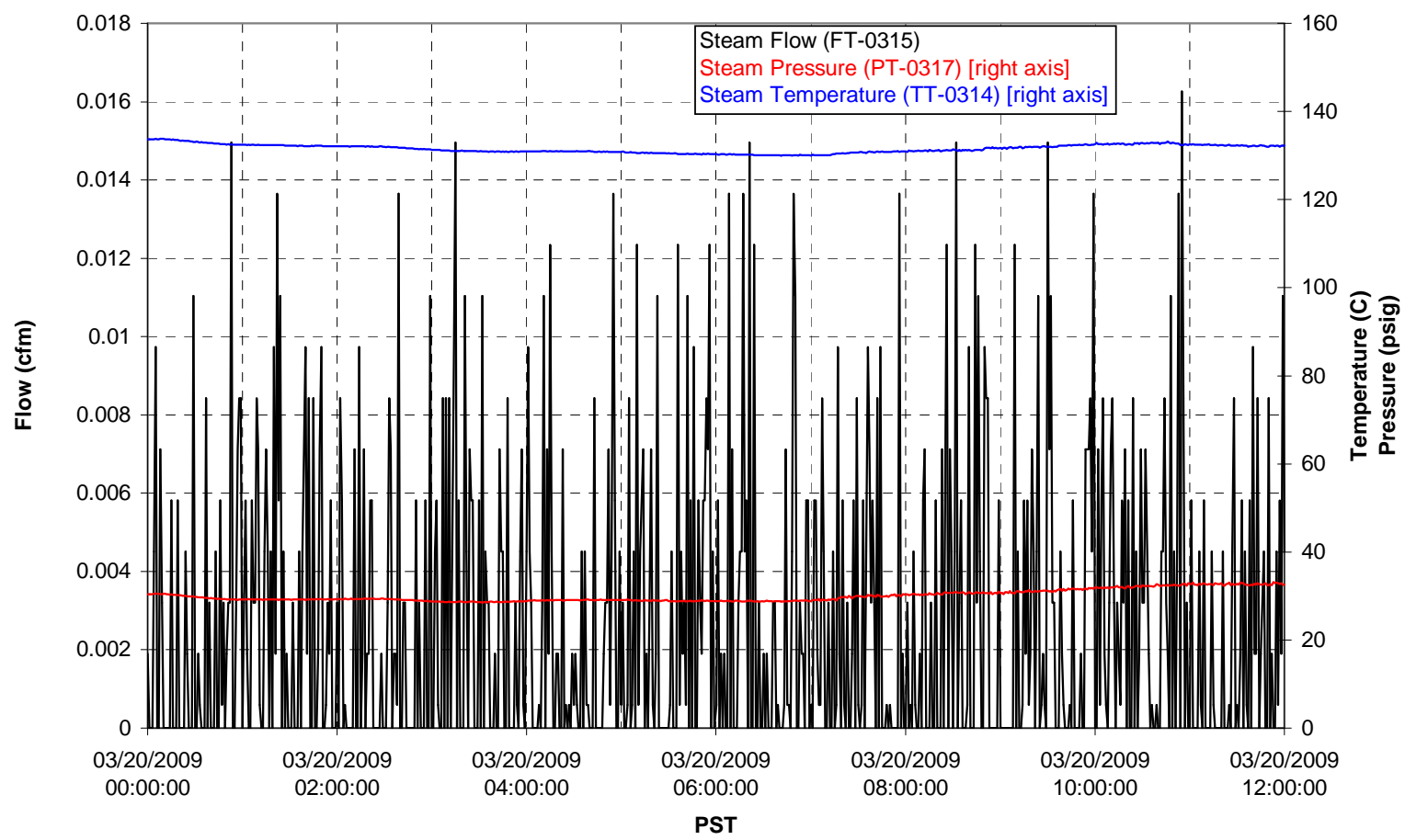


T01B Steam

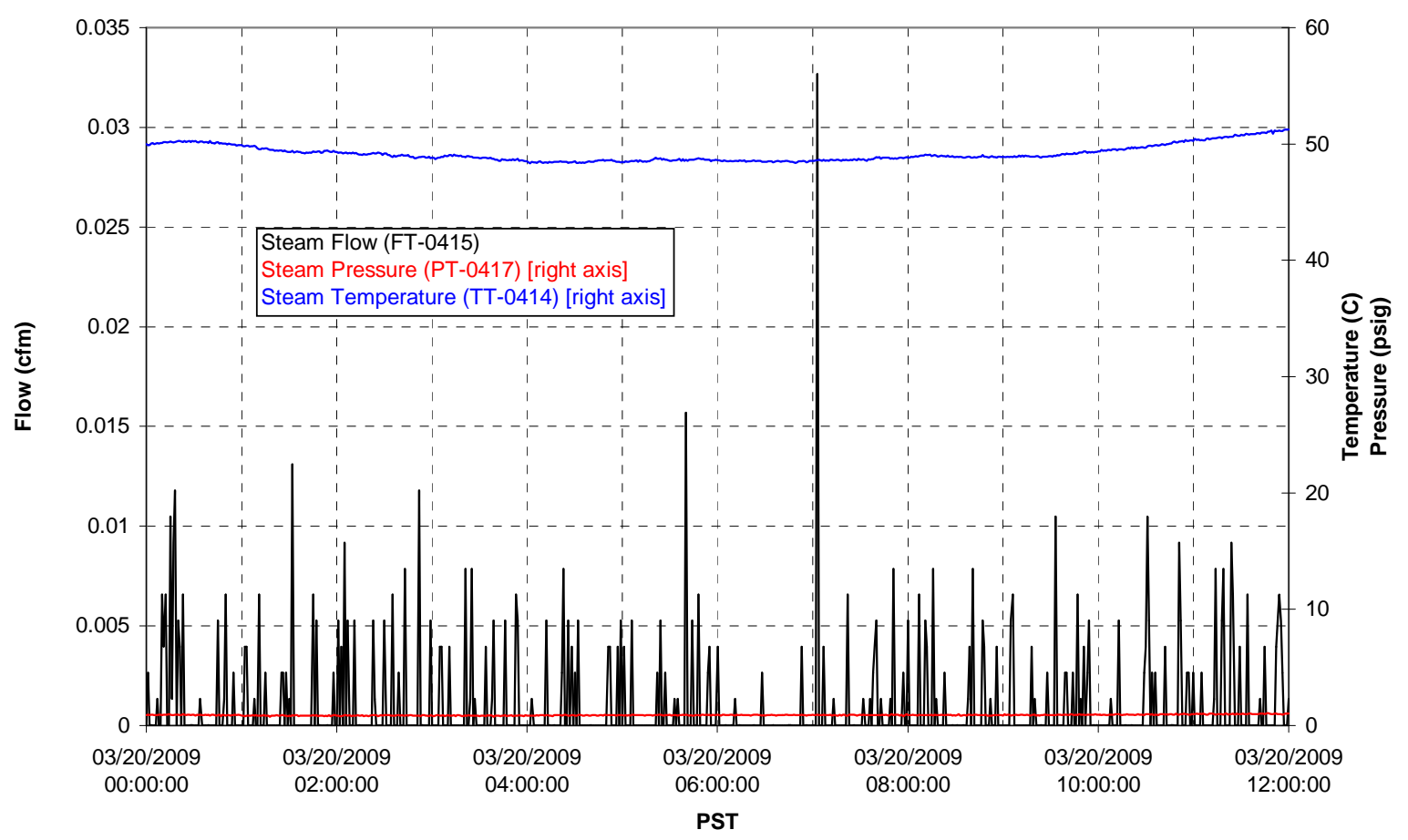




\section{Integrated Test B Data Plots 03/20/09 12:00 - 03/21/09 00:00}


T01A level

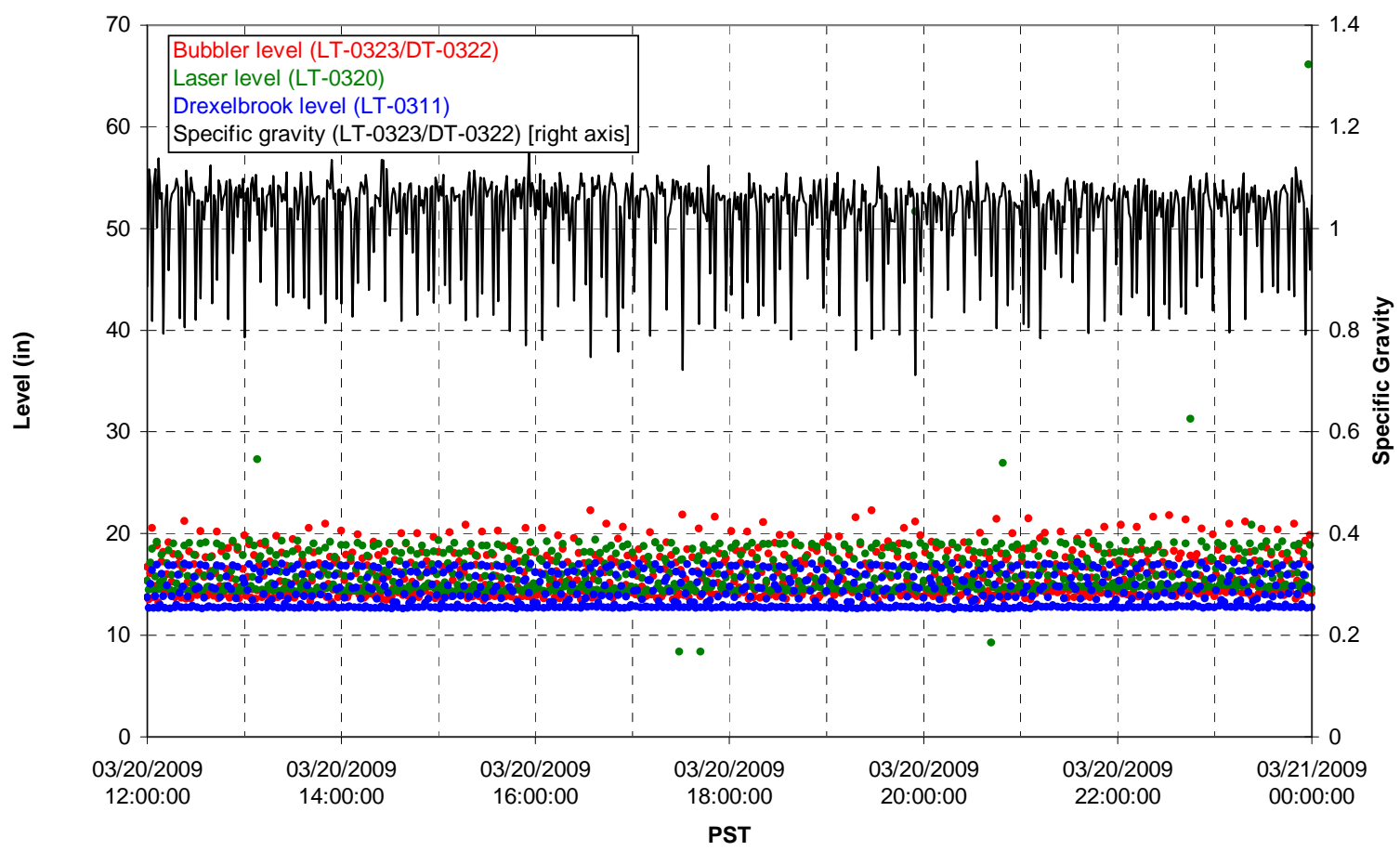

T01A temperatures

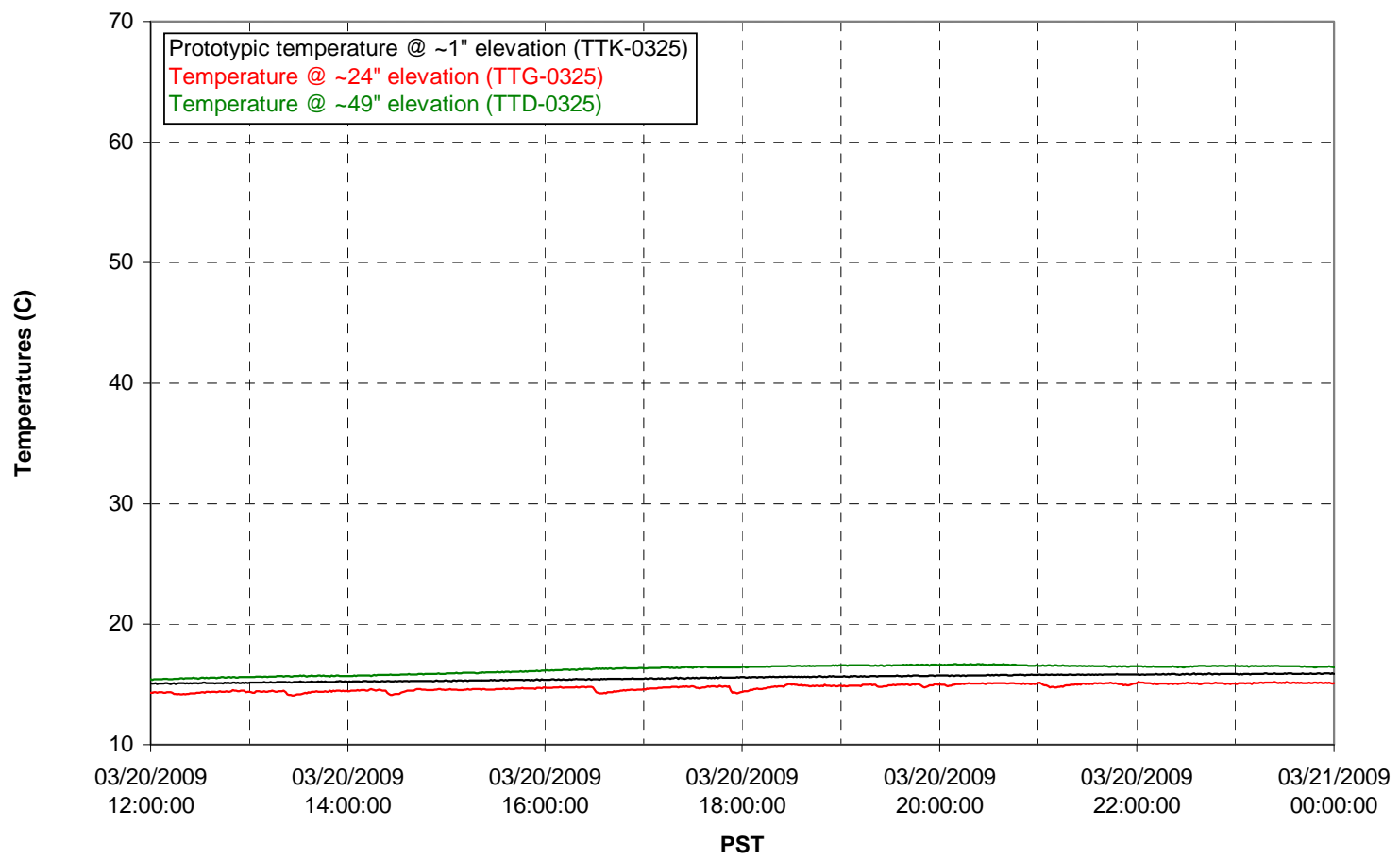


T01B level

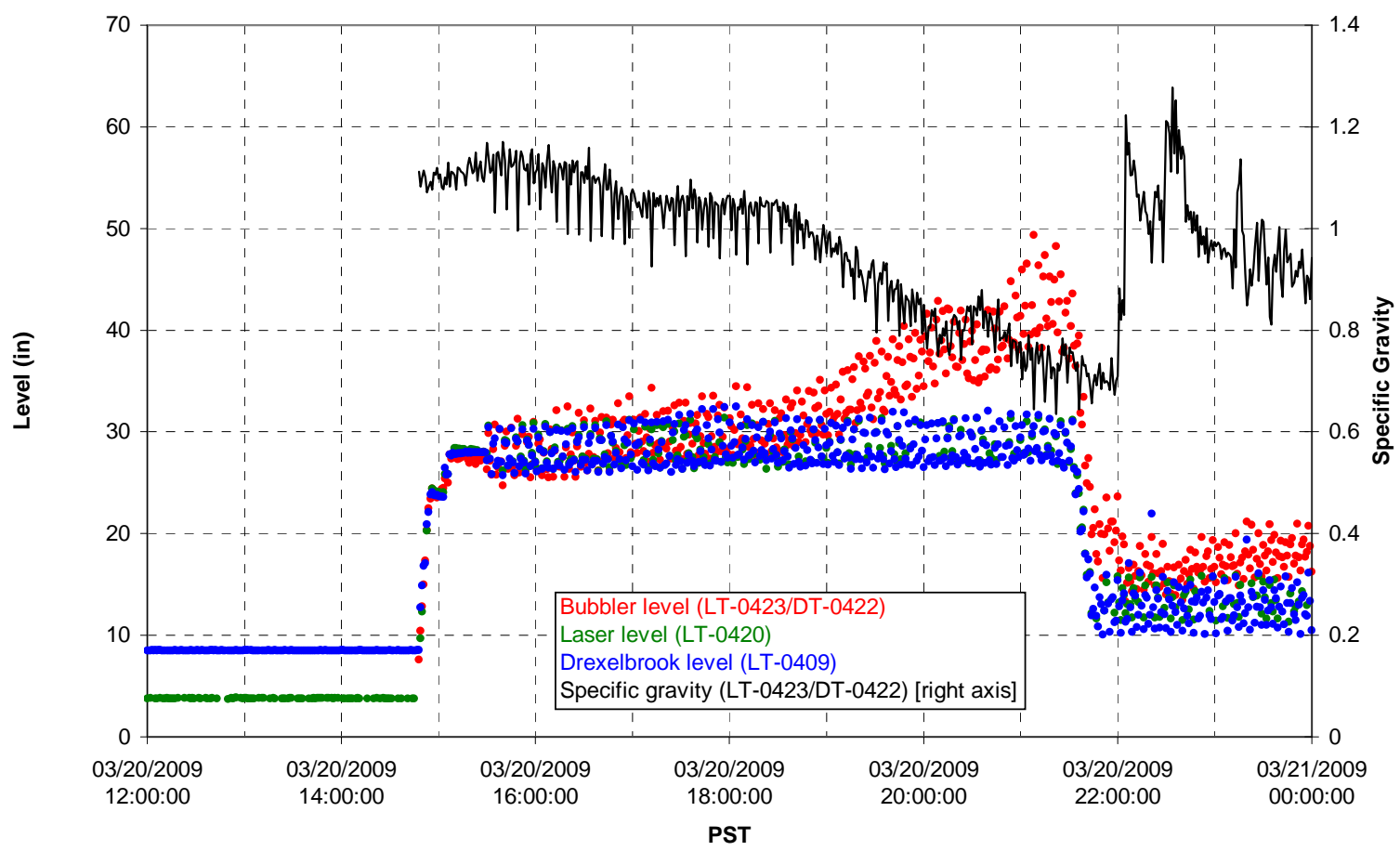

T01B temperatures

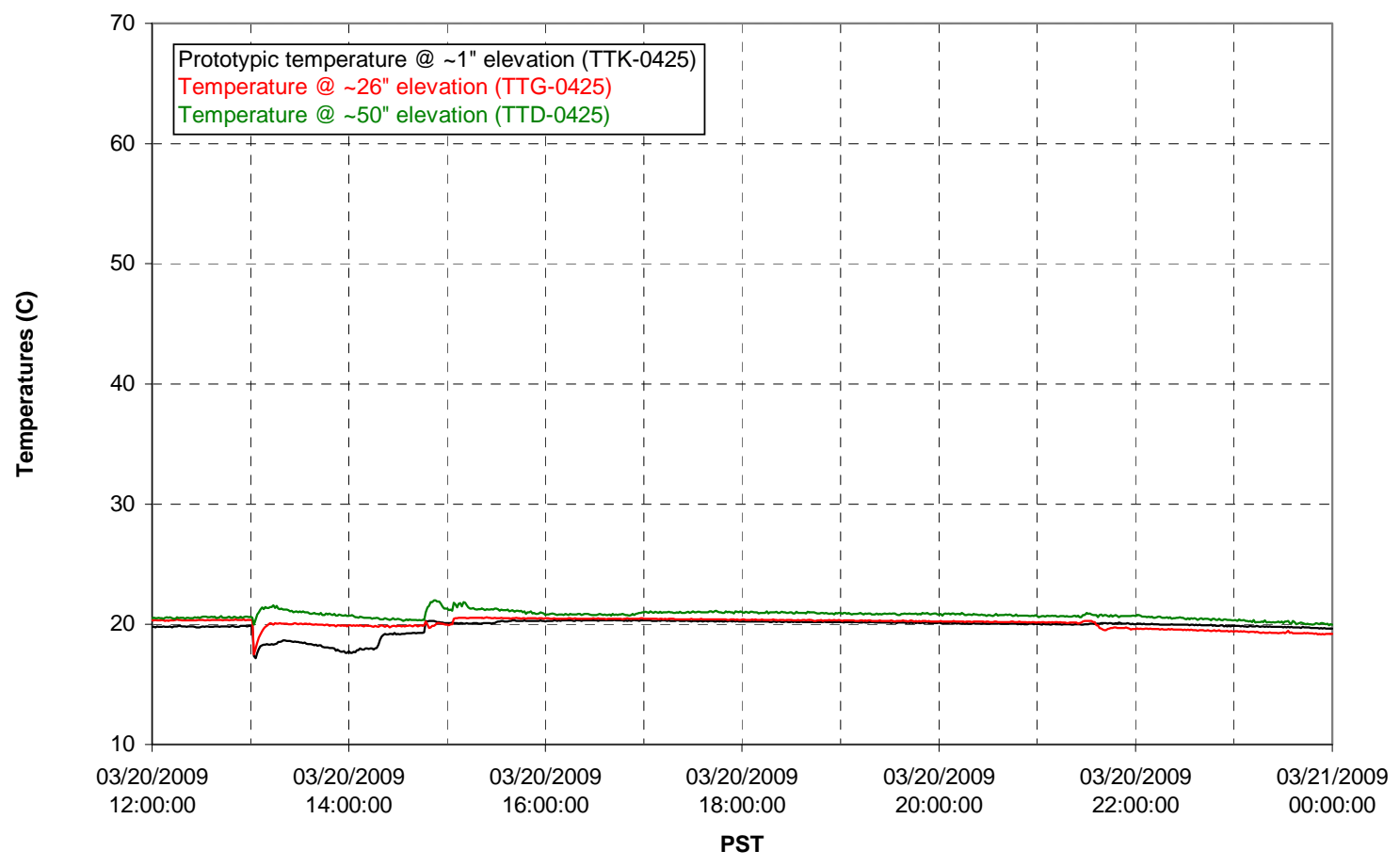


T02A level

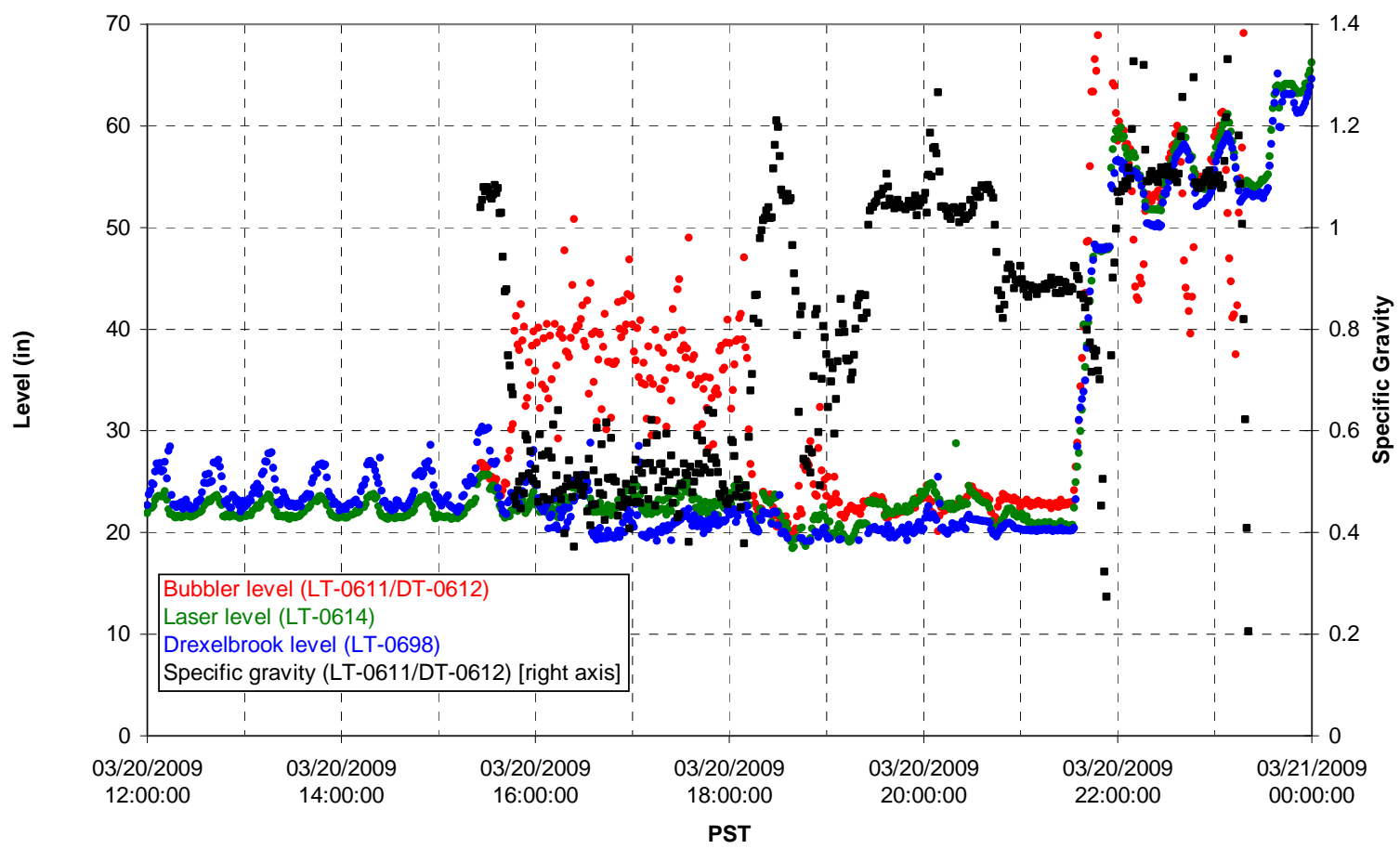

T02A temperatures

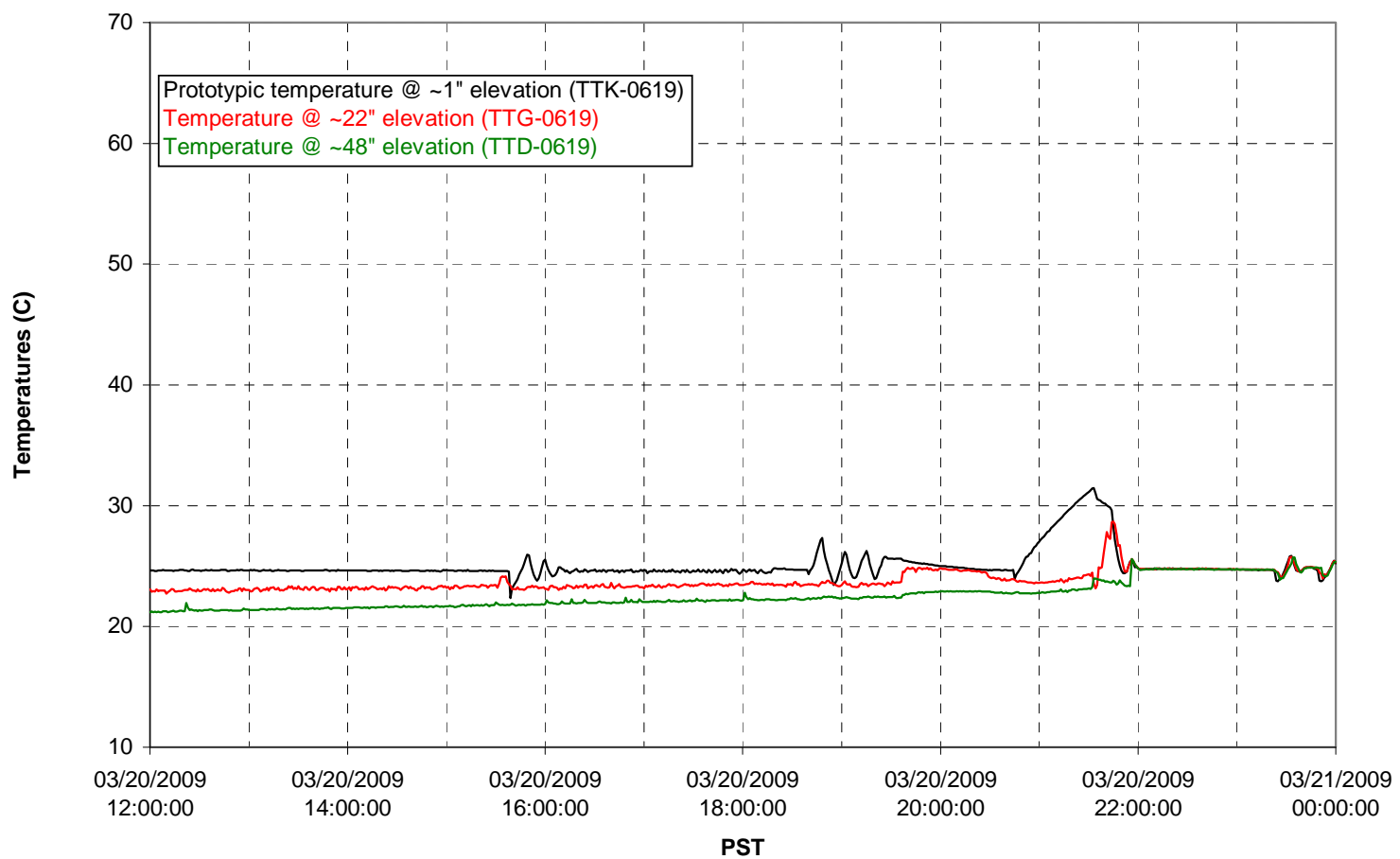


T02A and filter loop temperatures

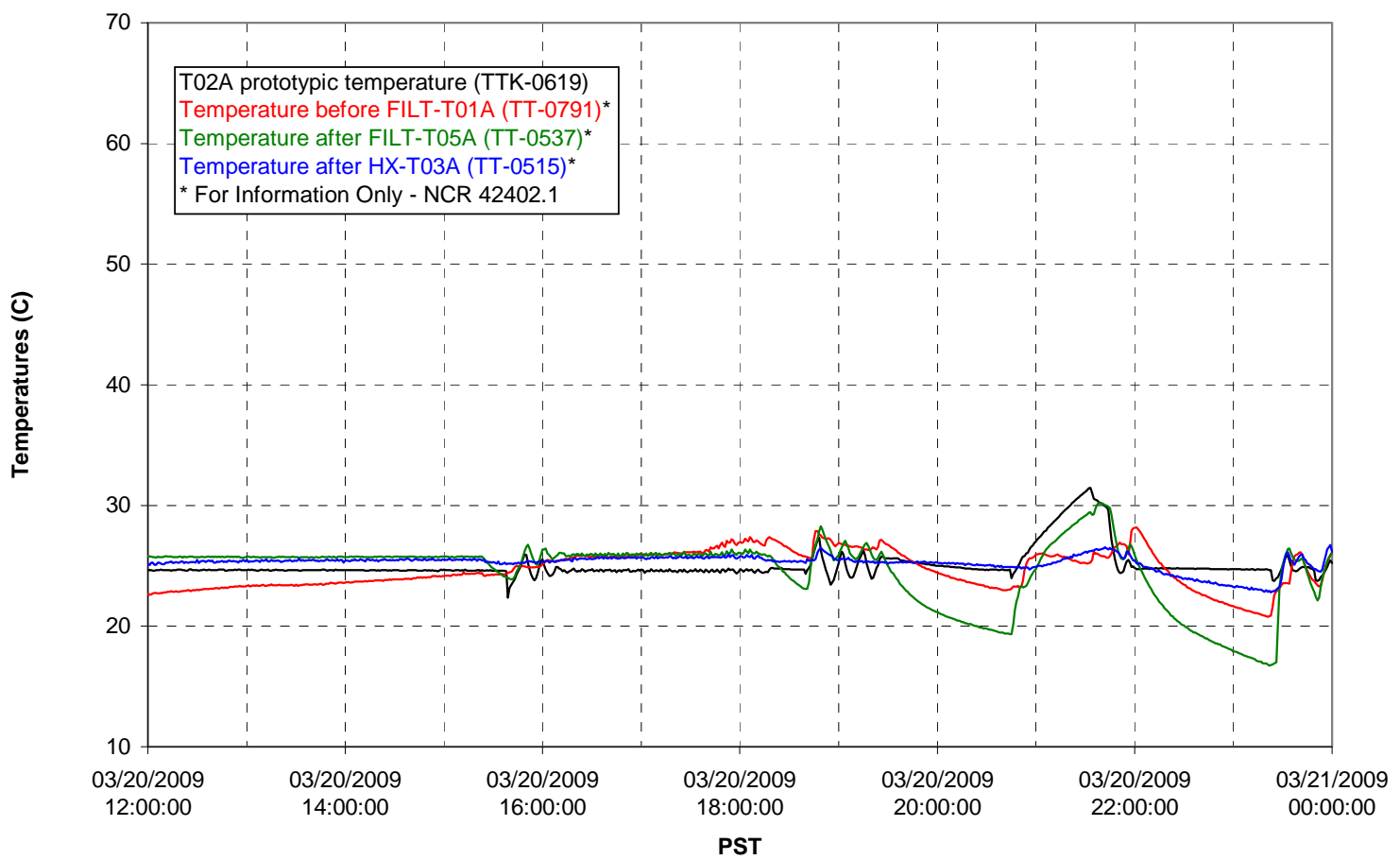

Pump Pressures and Flow

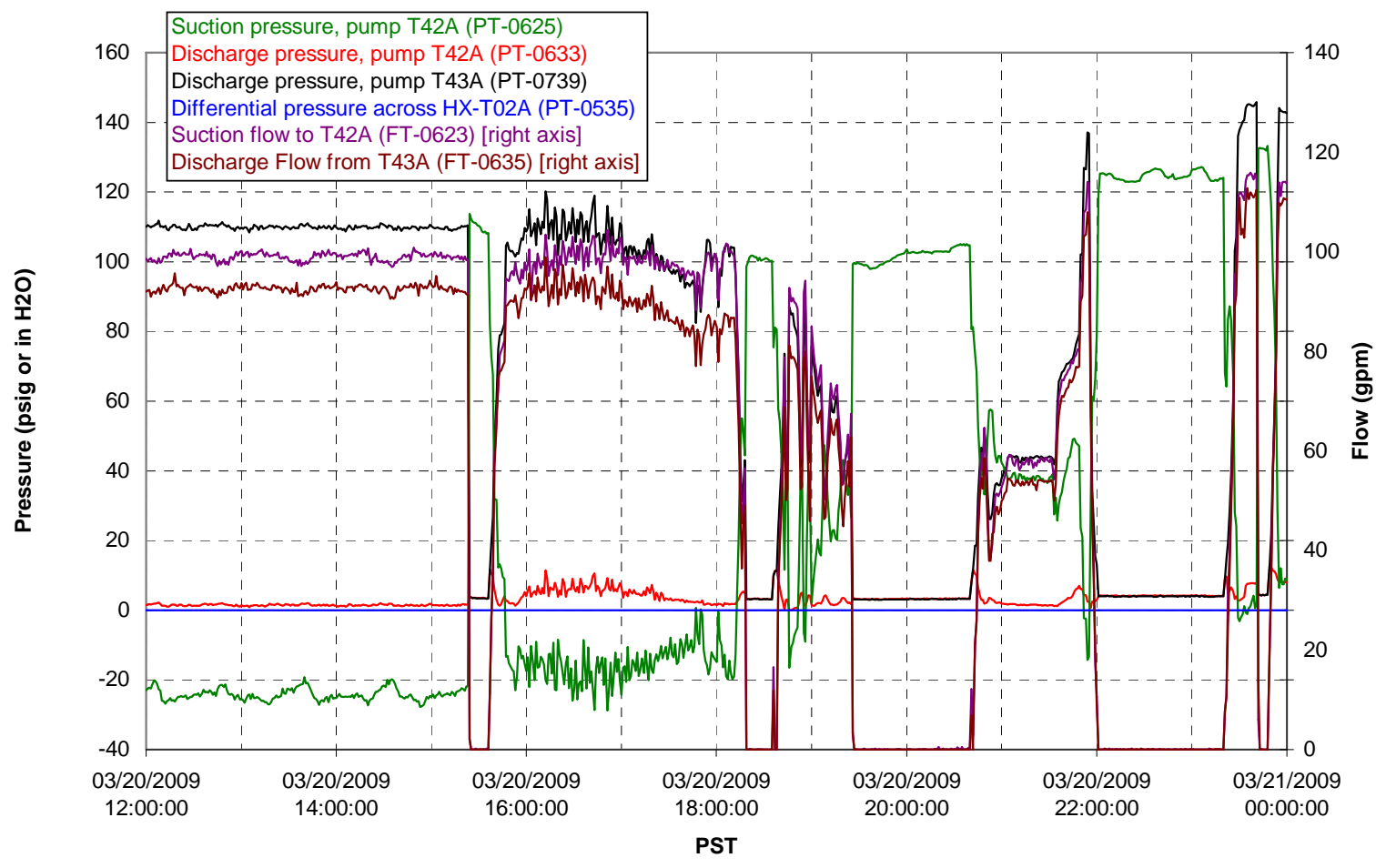




\section{Axial pressure drop}

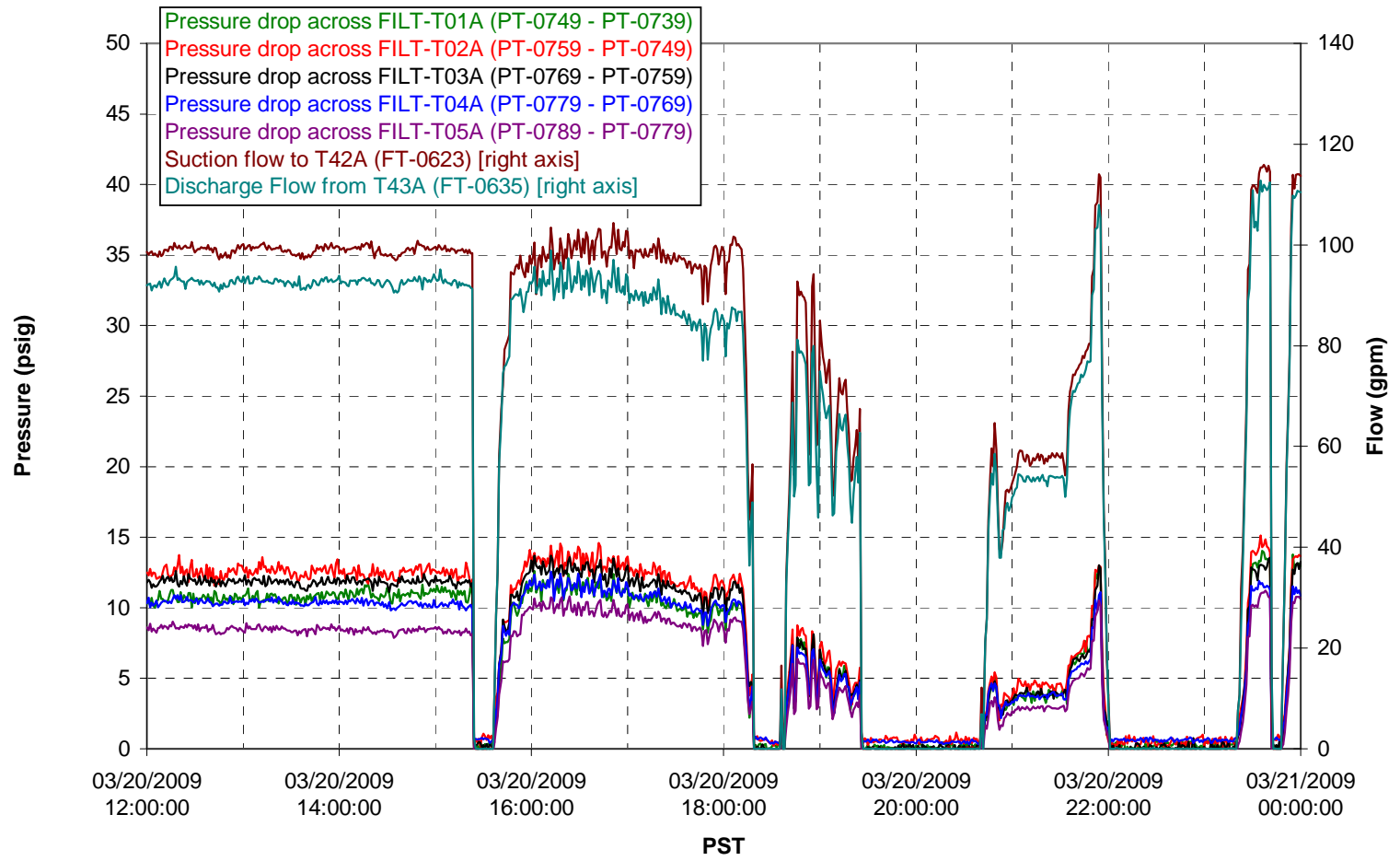

Permeate flow rates

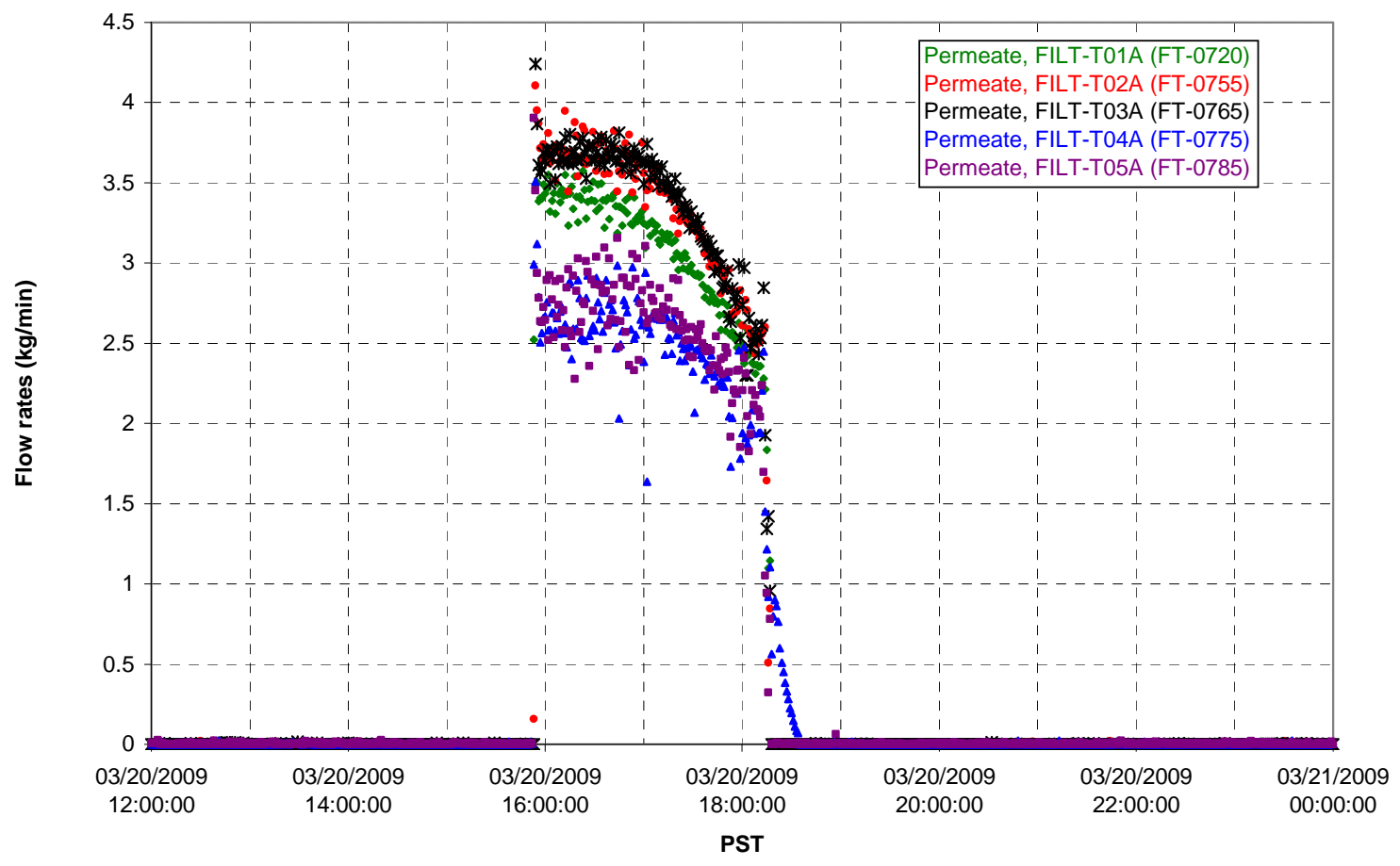


T02A Inner Temperature Tree

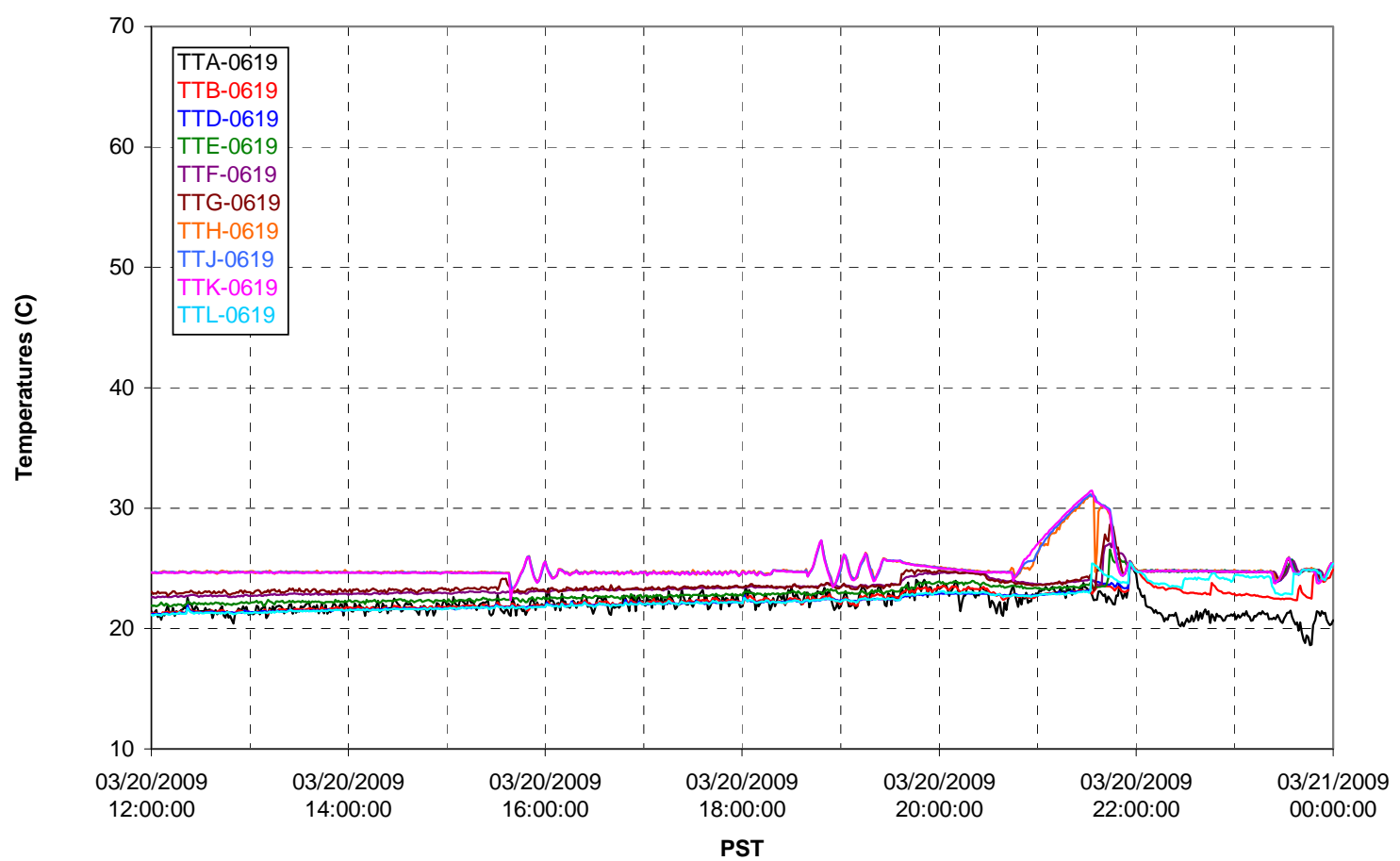

T02A Outer Temperature Tree

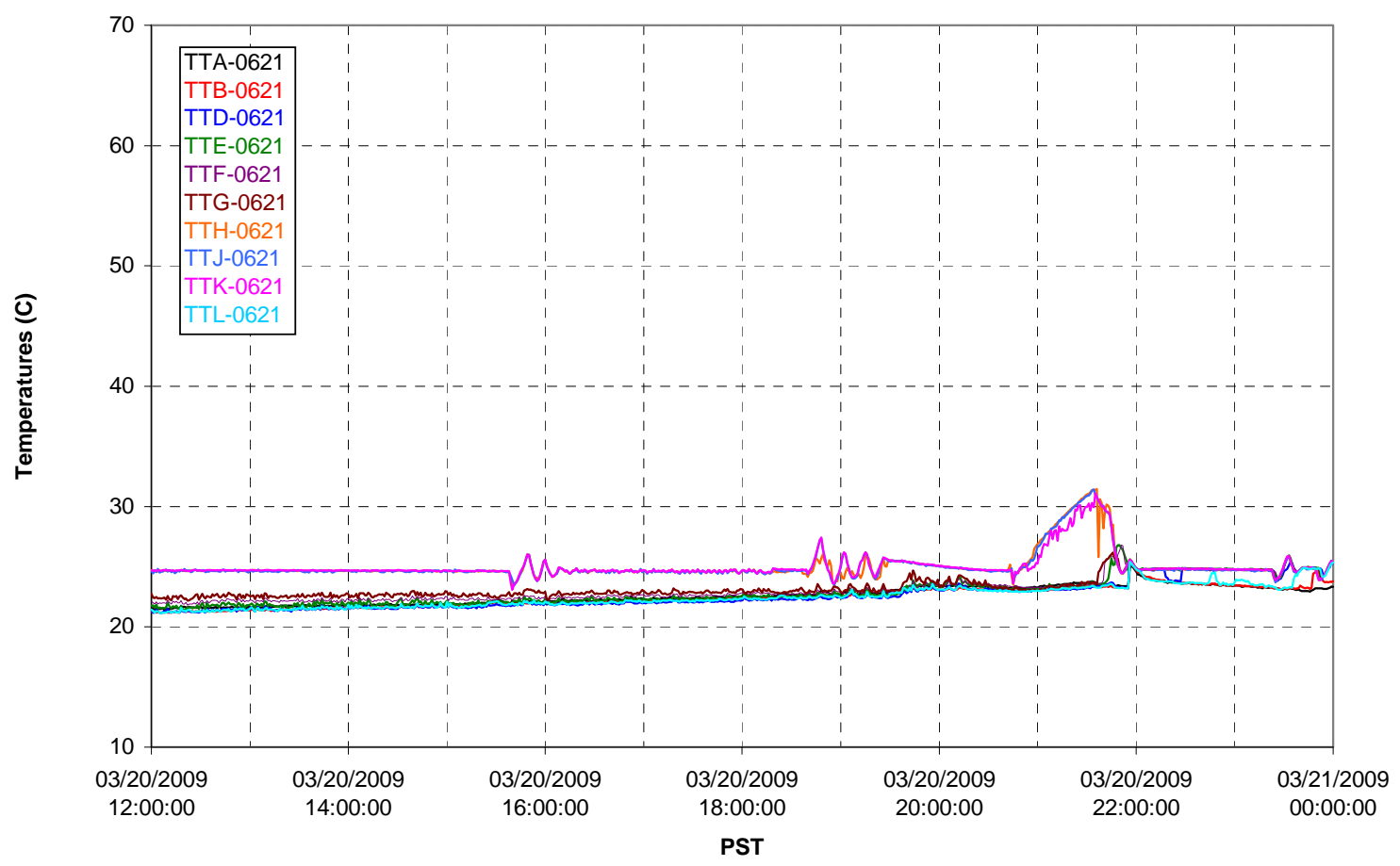


T02A temperatures

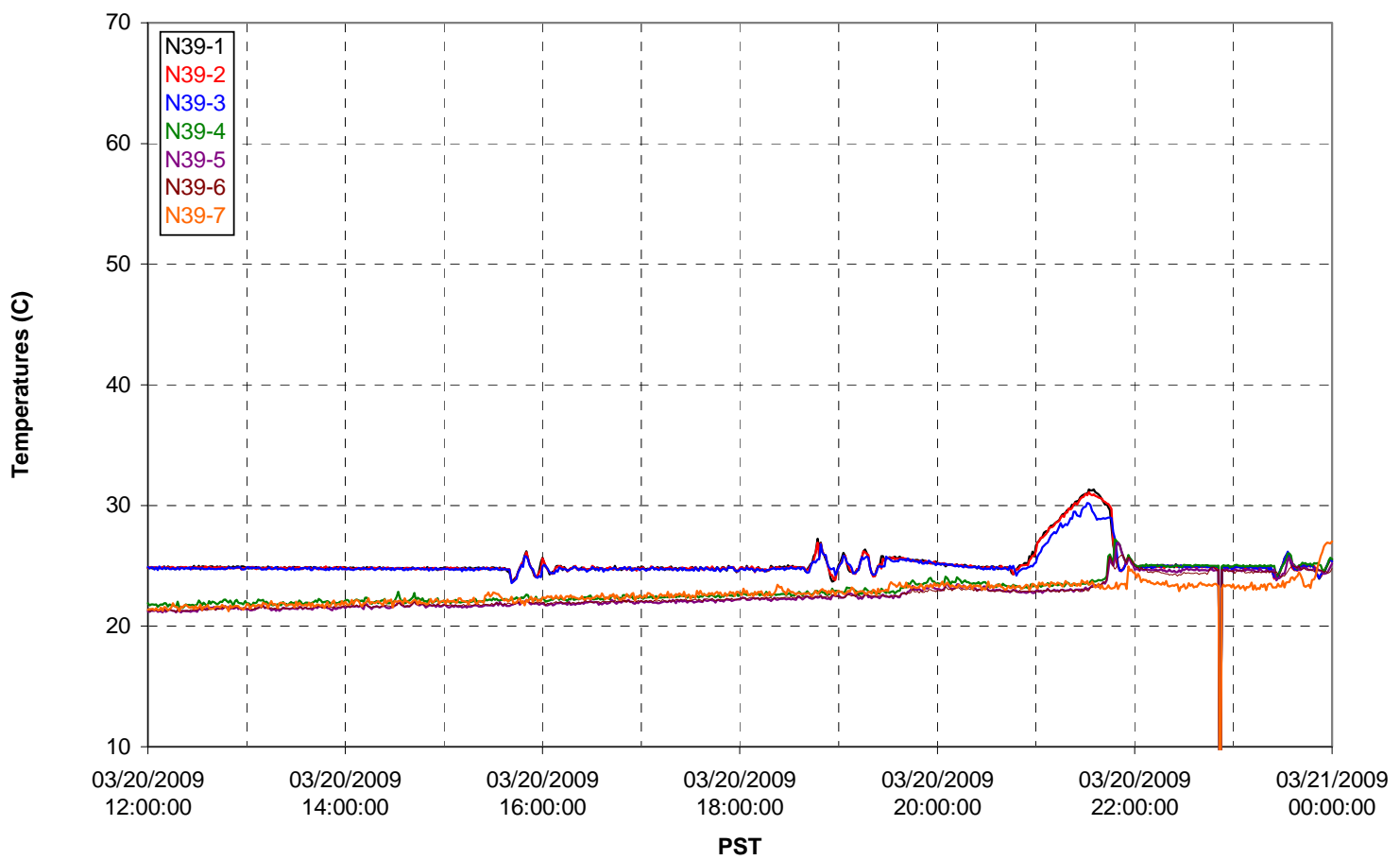

T02A temperatures

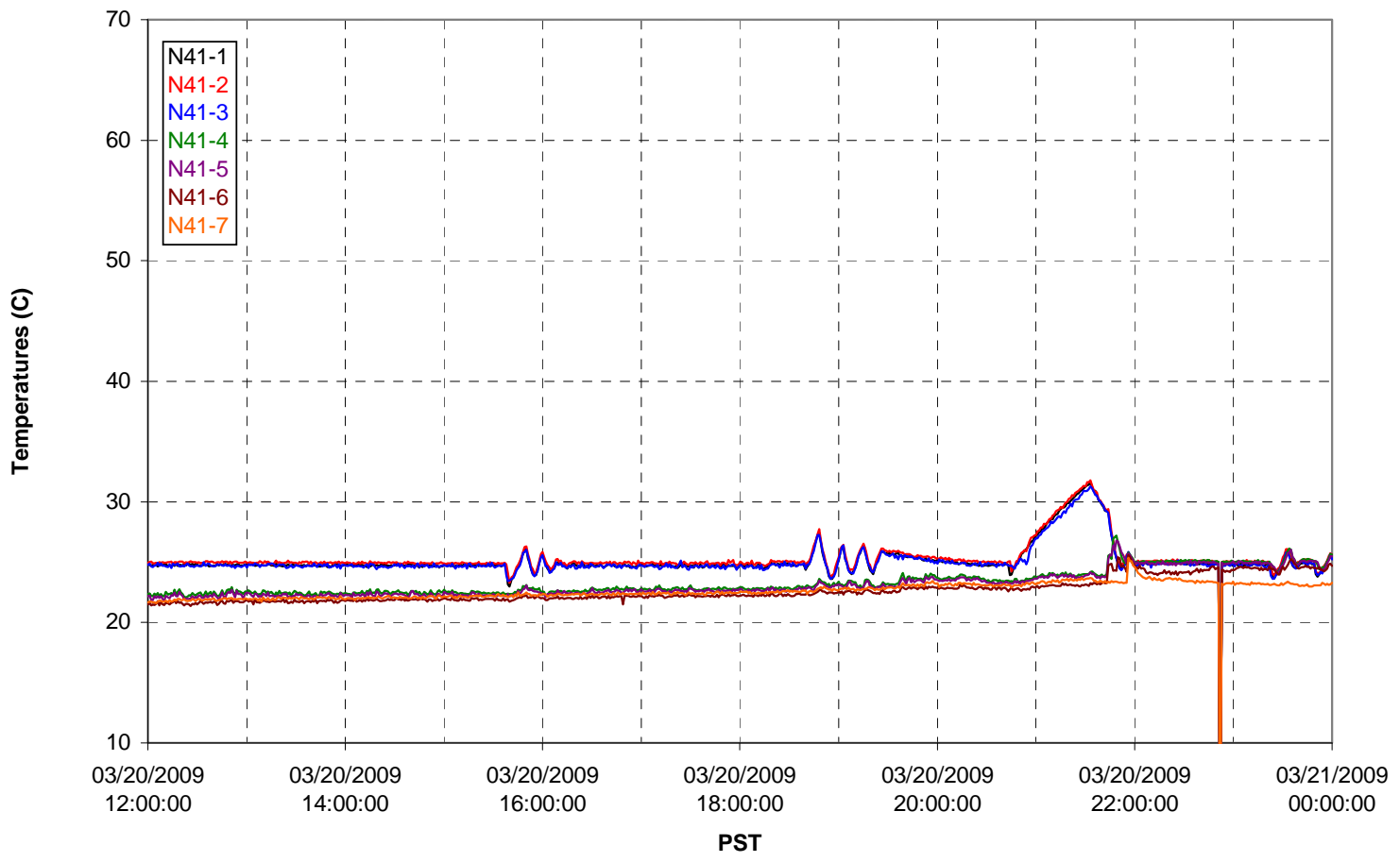


T02A temperatures

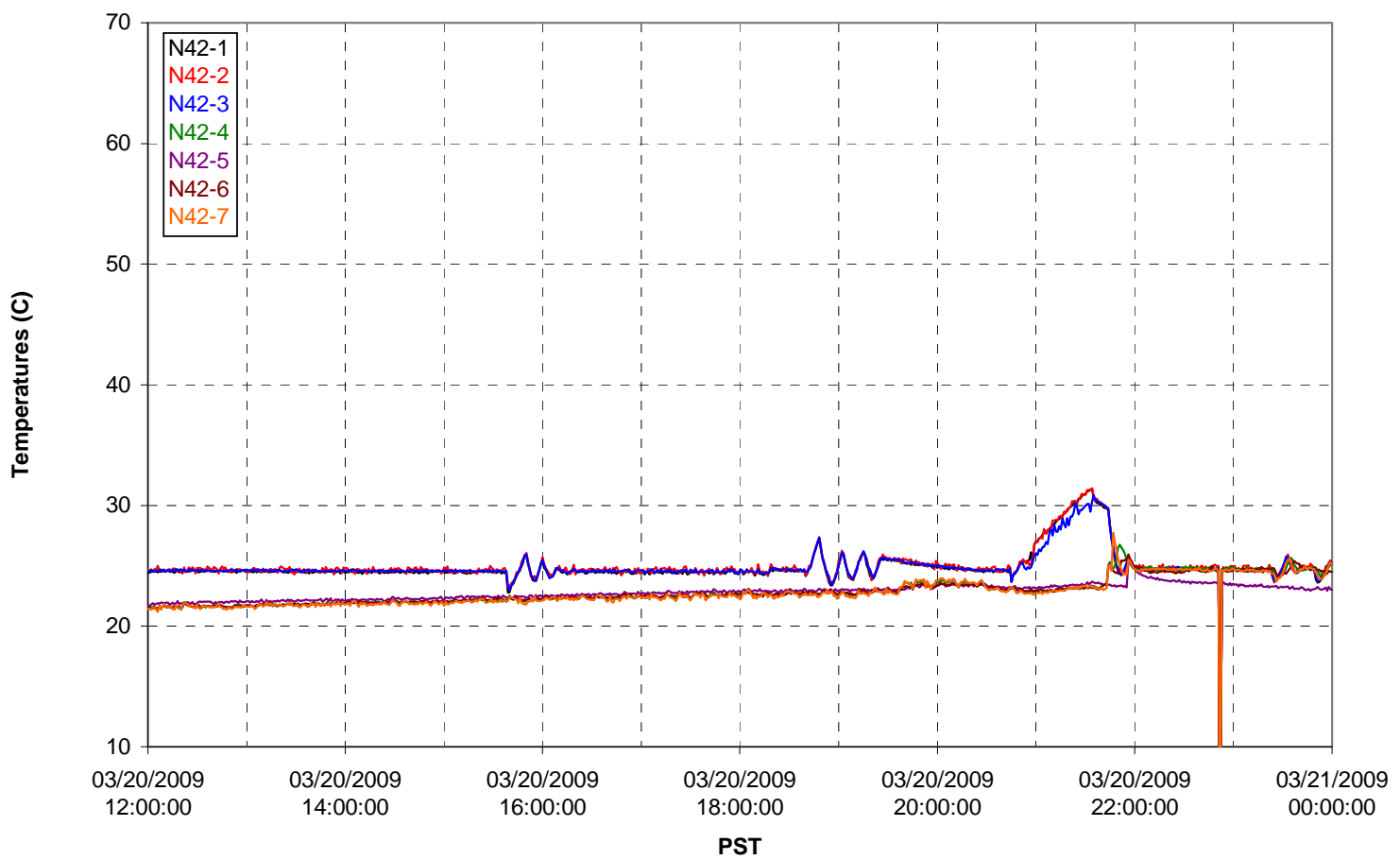

T02A temperatures

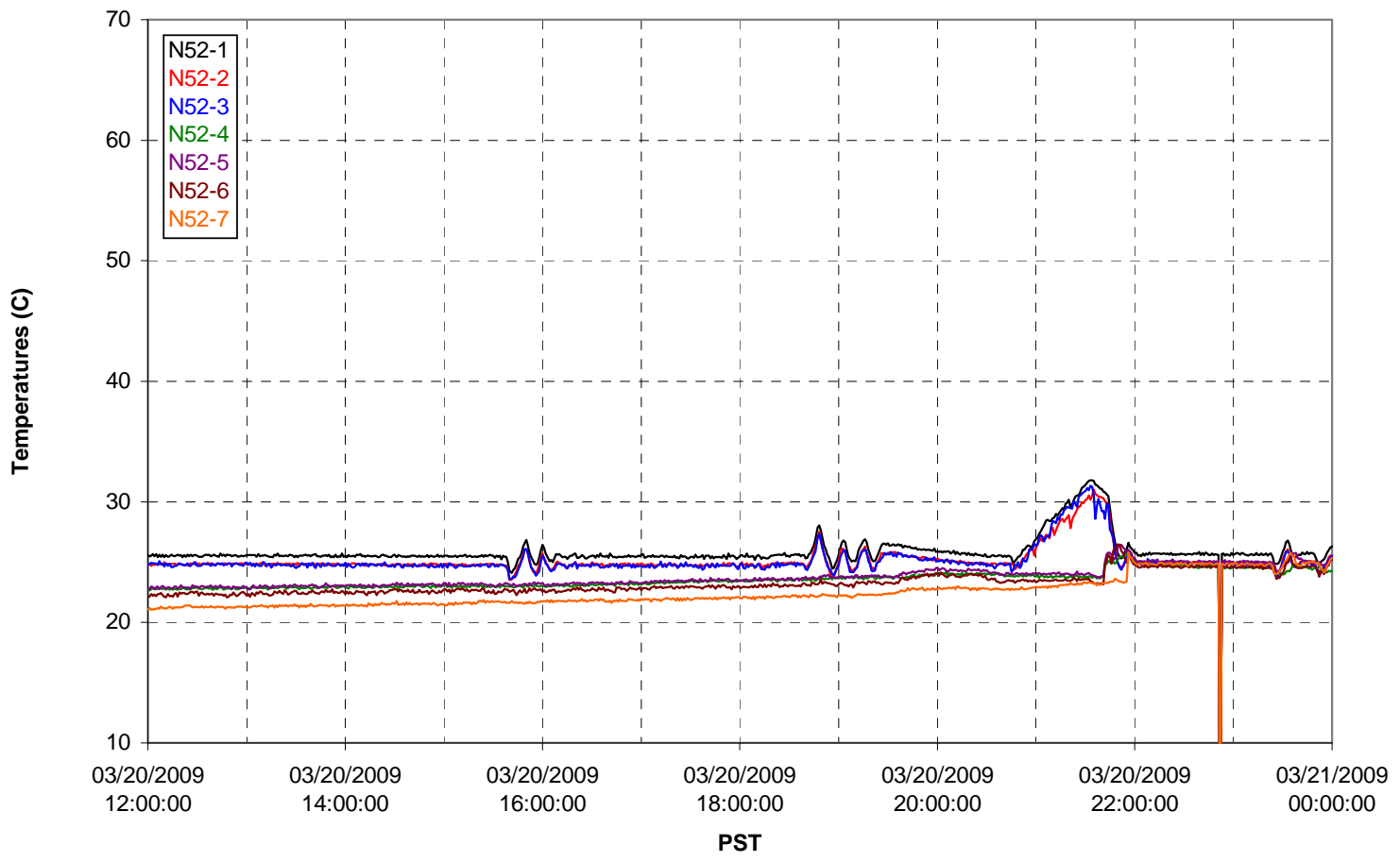


T02A Heating and Cooling
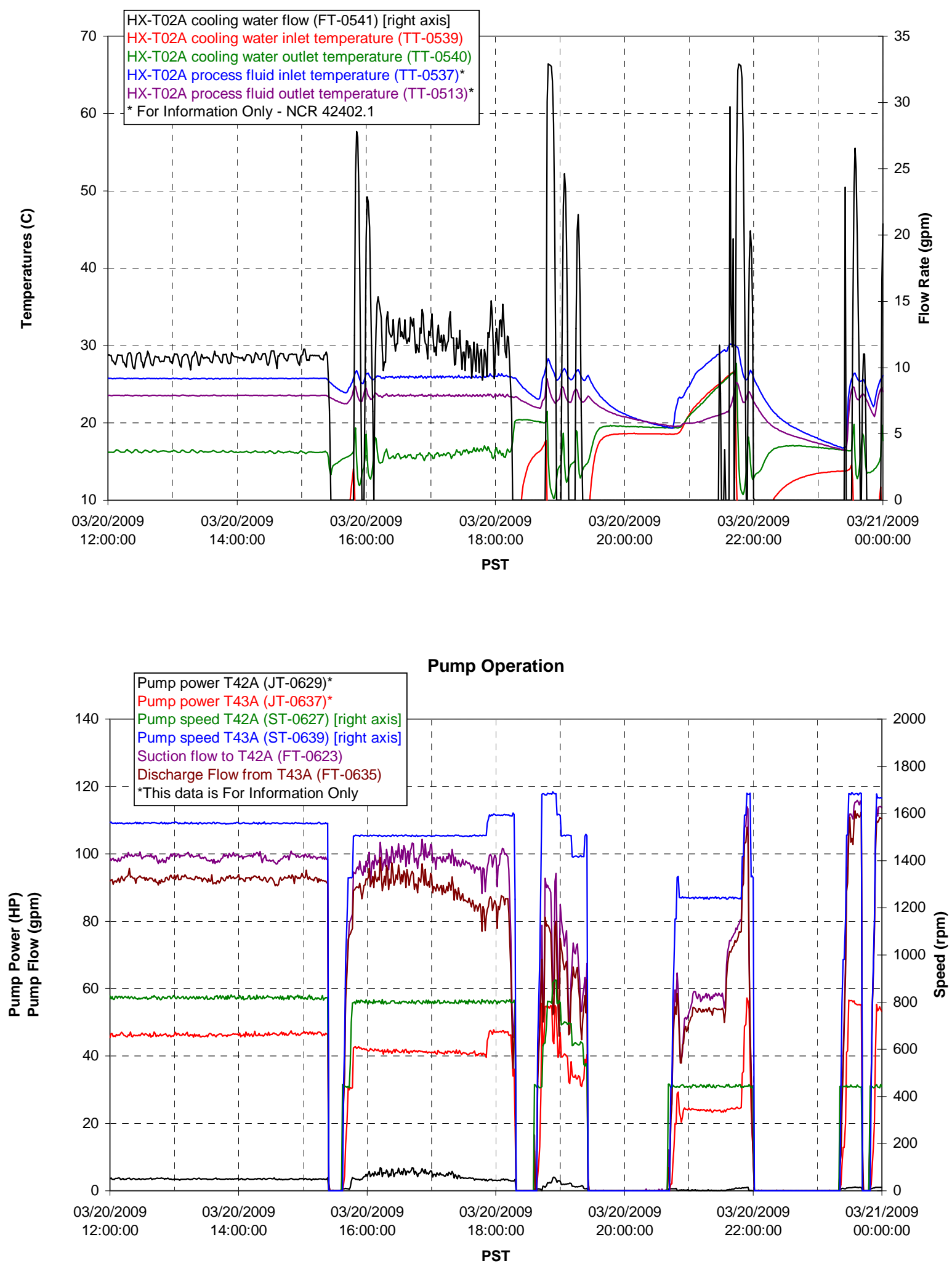
Pulsepot UFP-PP-T01A

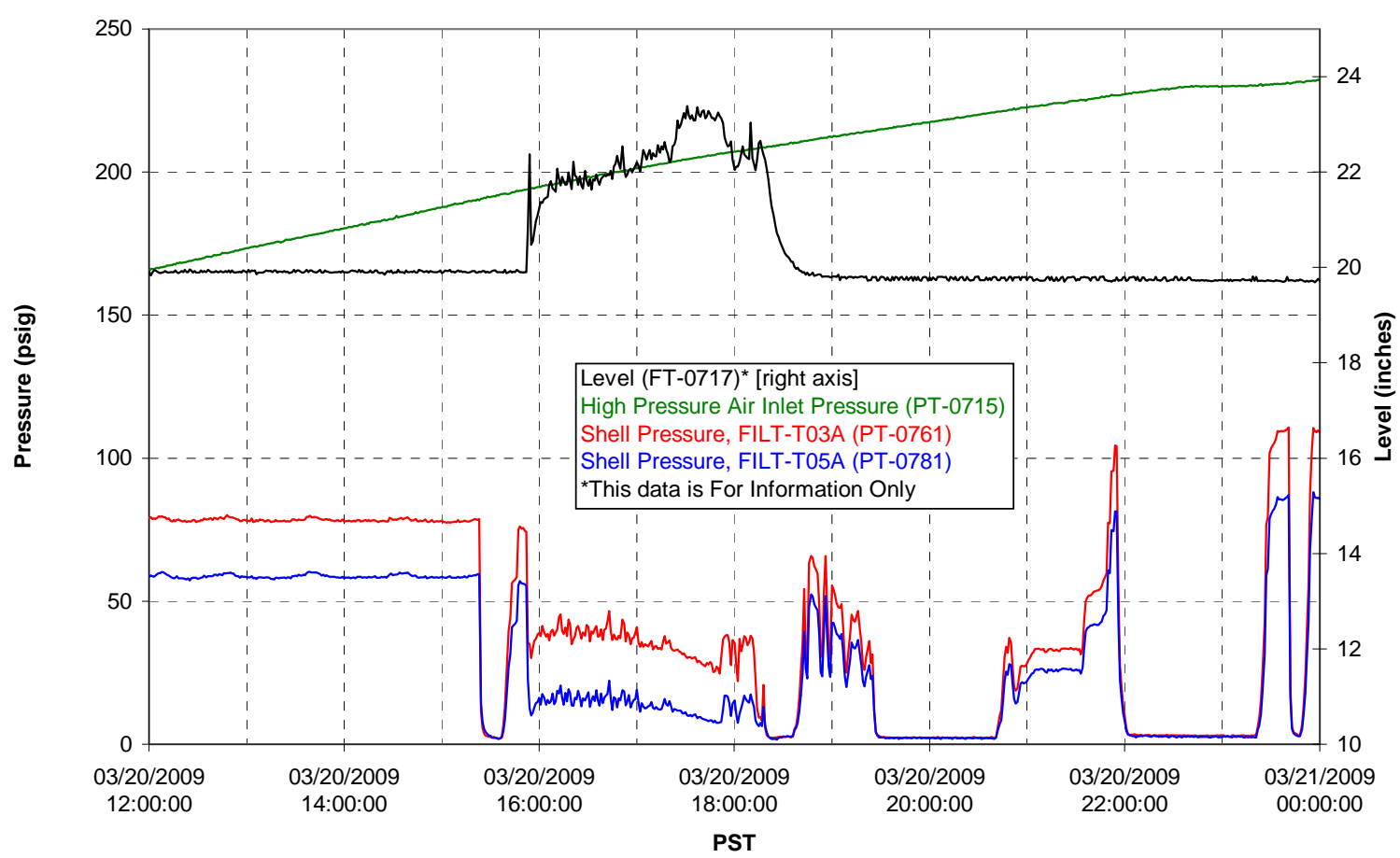

Pulsepot UFP-PP-T02A

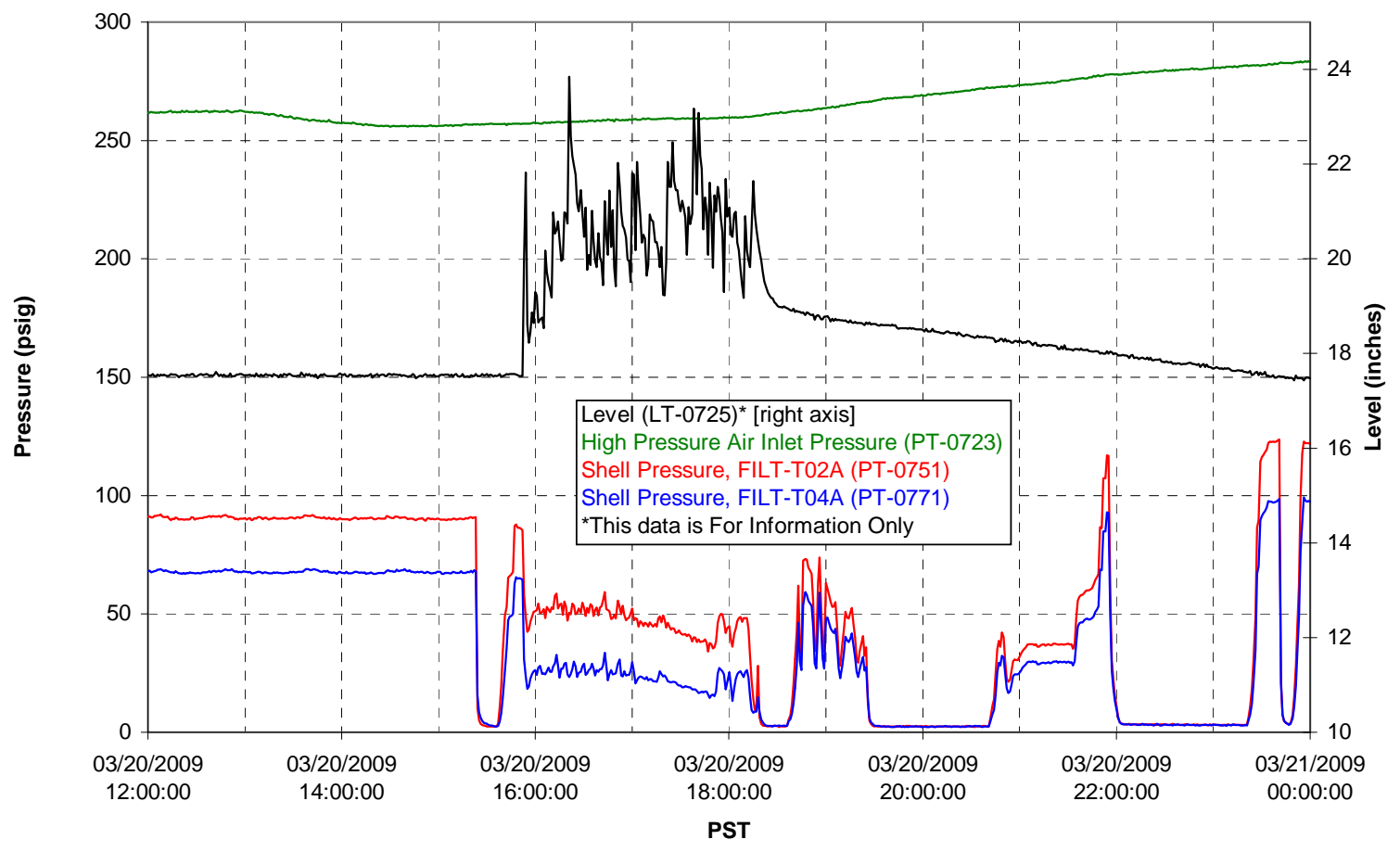


Pulsepot UFP-PP-T03A

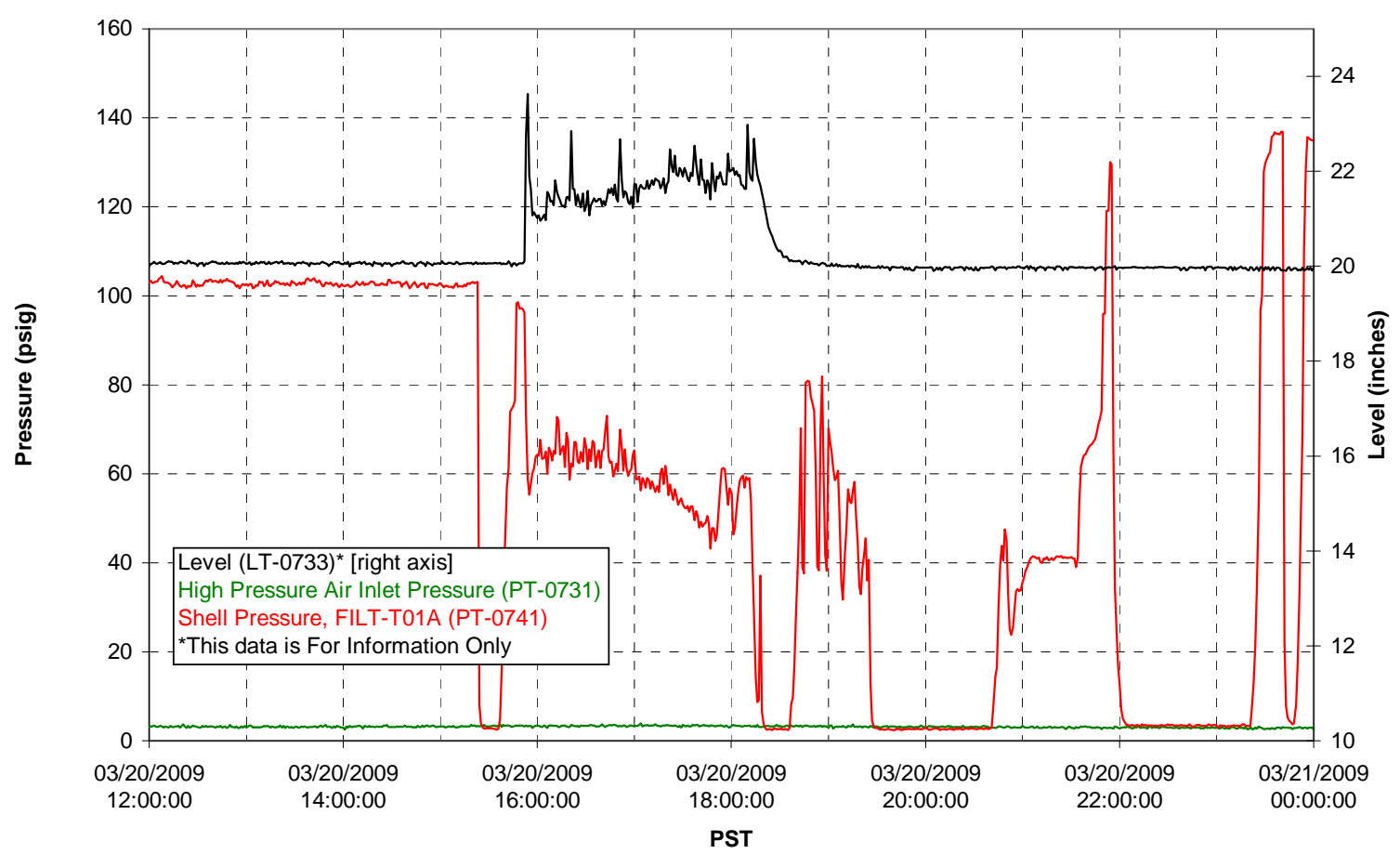

Pulsepot Levels

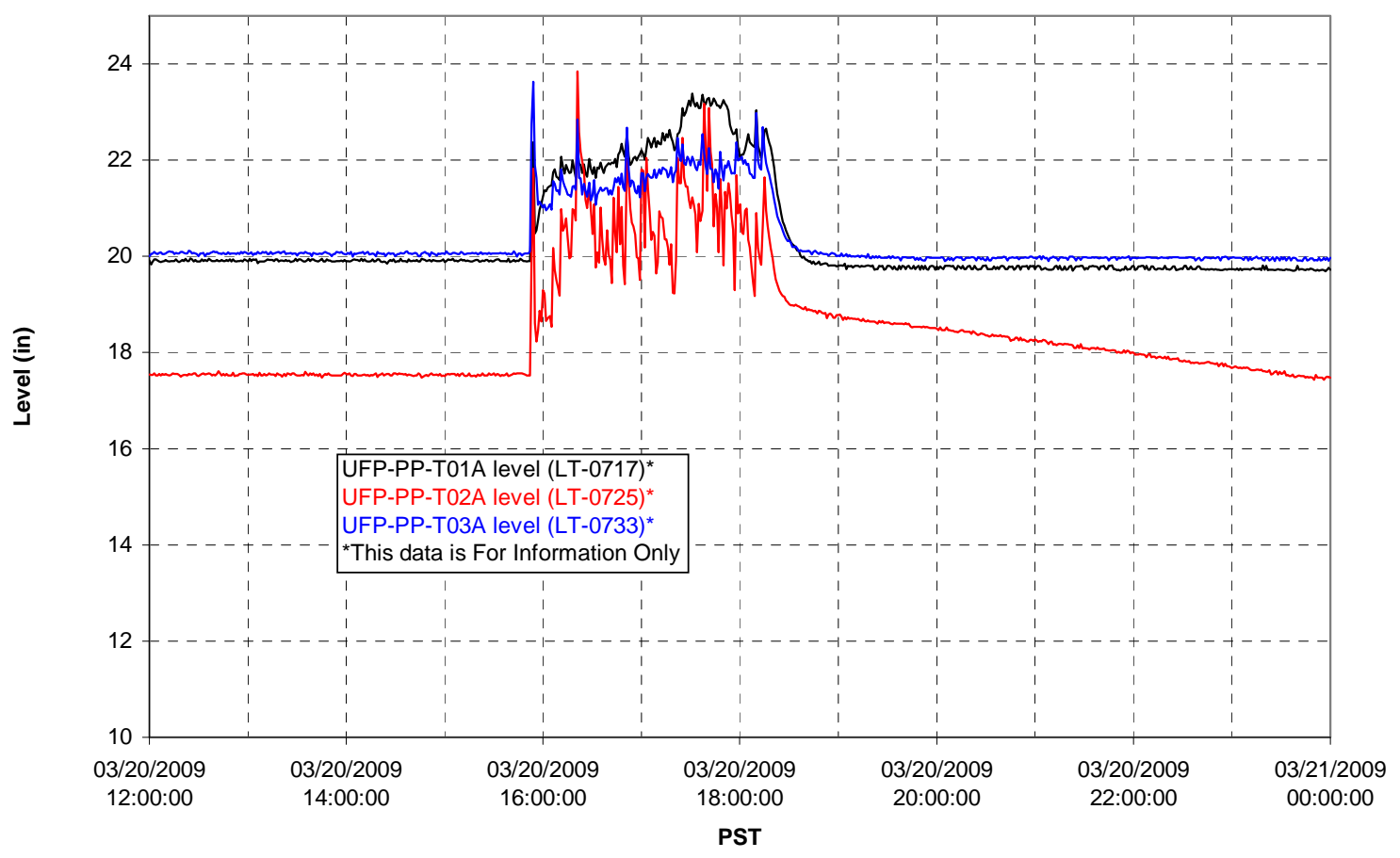


Filter UFP-FILT-T01A

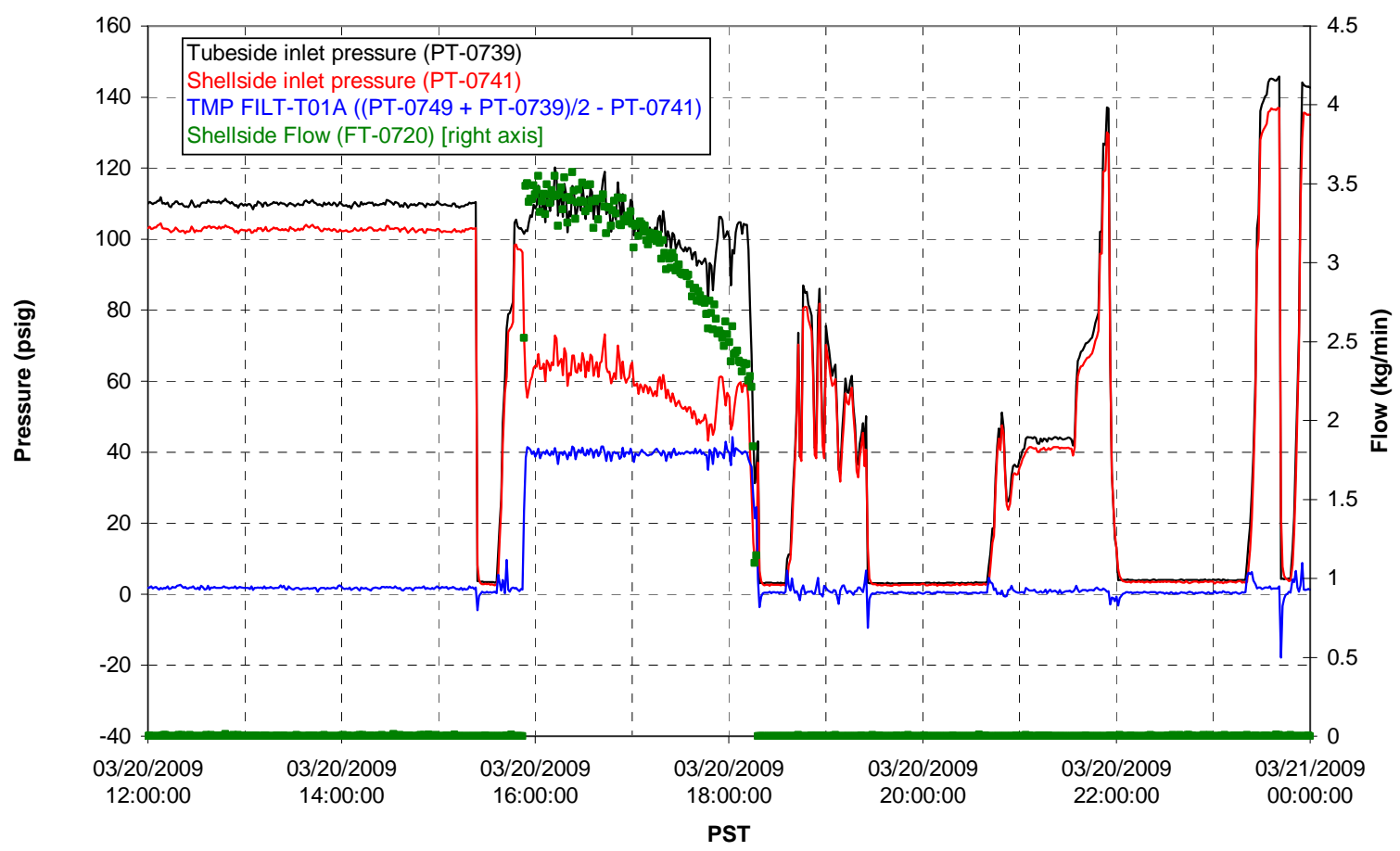

Filter UFP-FILT-T02A

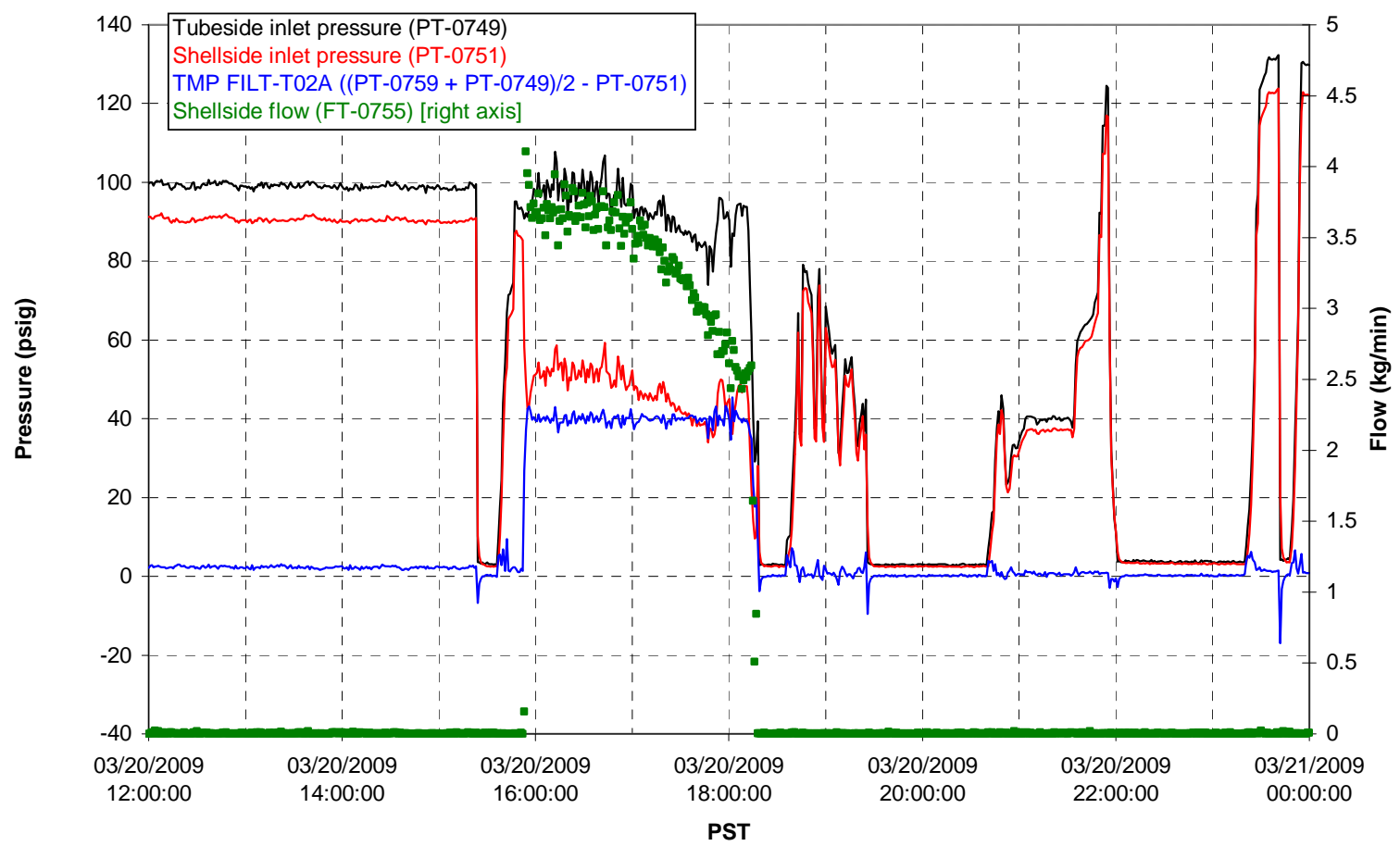


Filter UFP-FILT-T03A

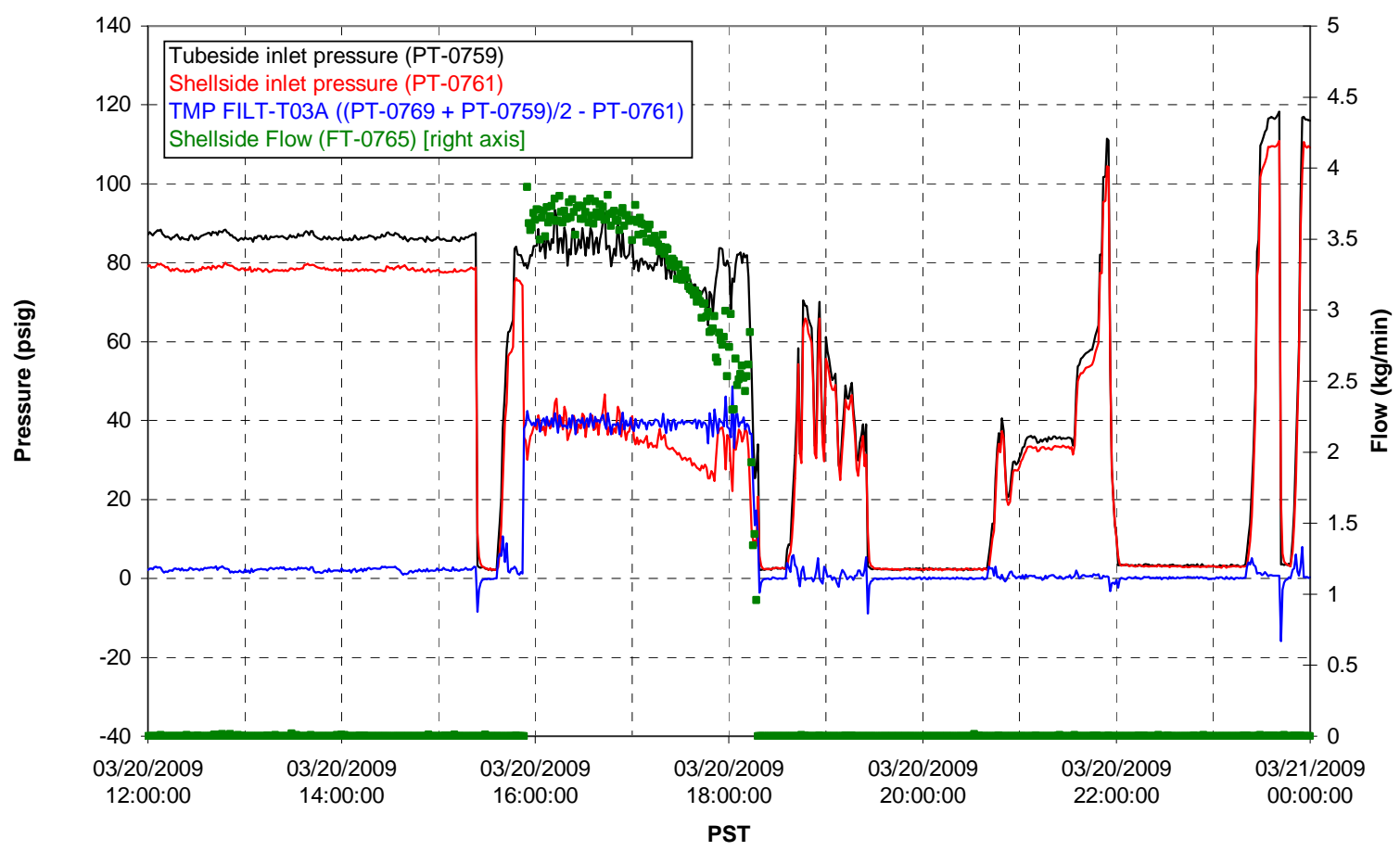

Filter UFP-FILT-T04A

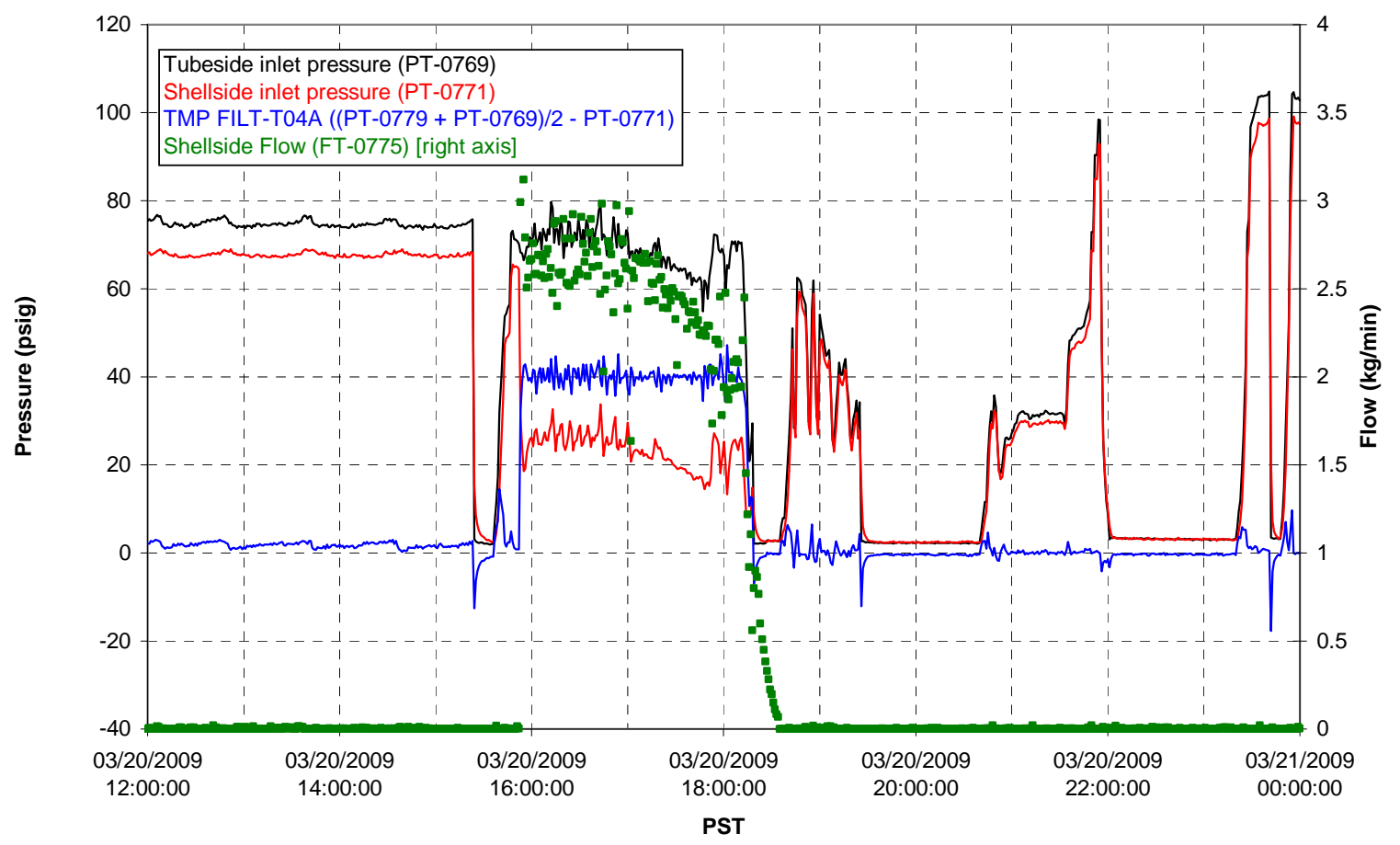


Filter UFP-FILT-T05A

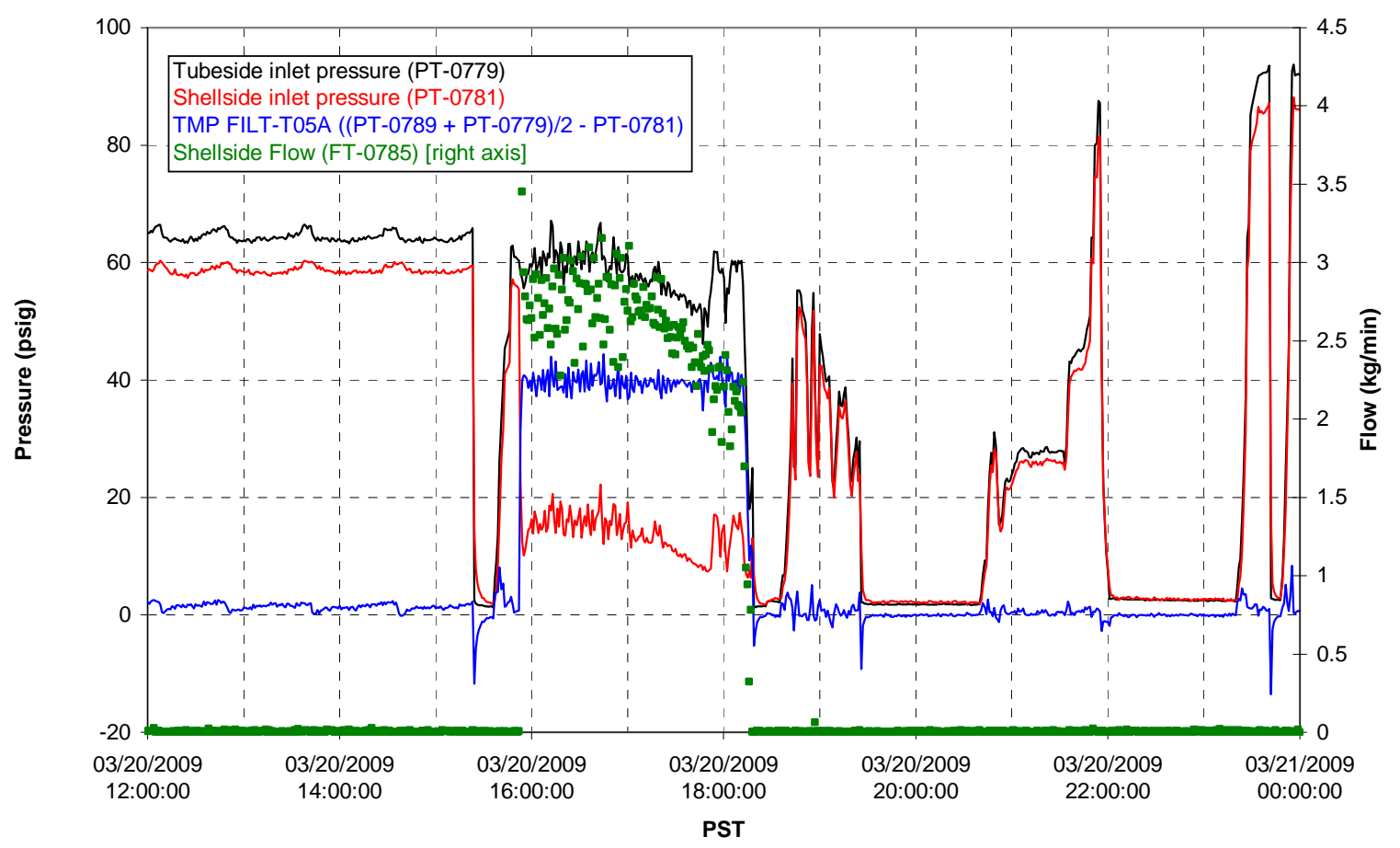

Chemical Flow

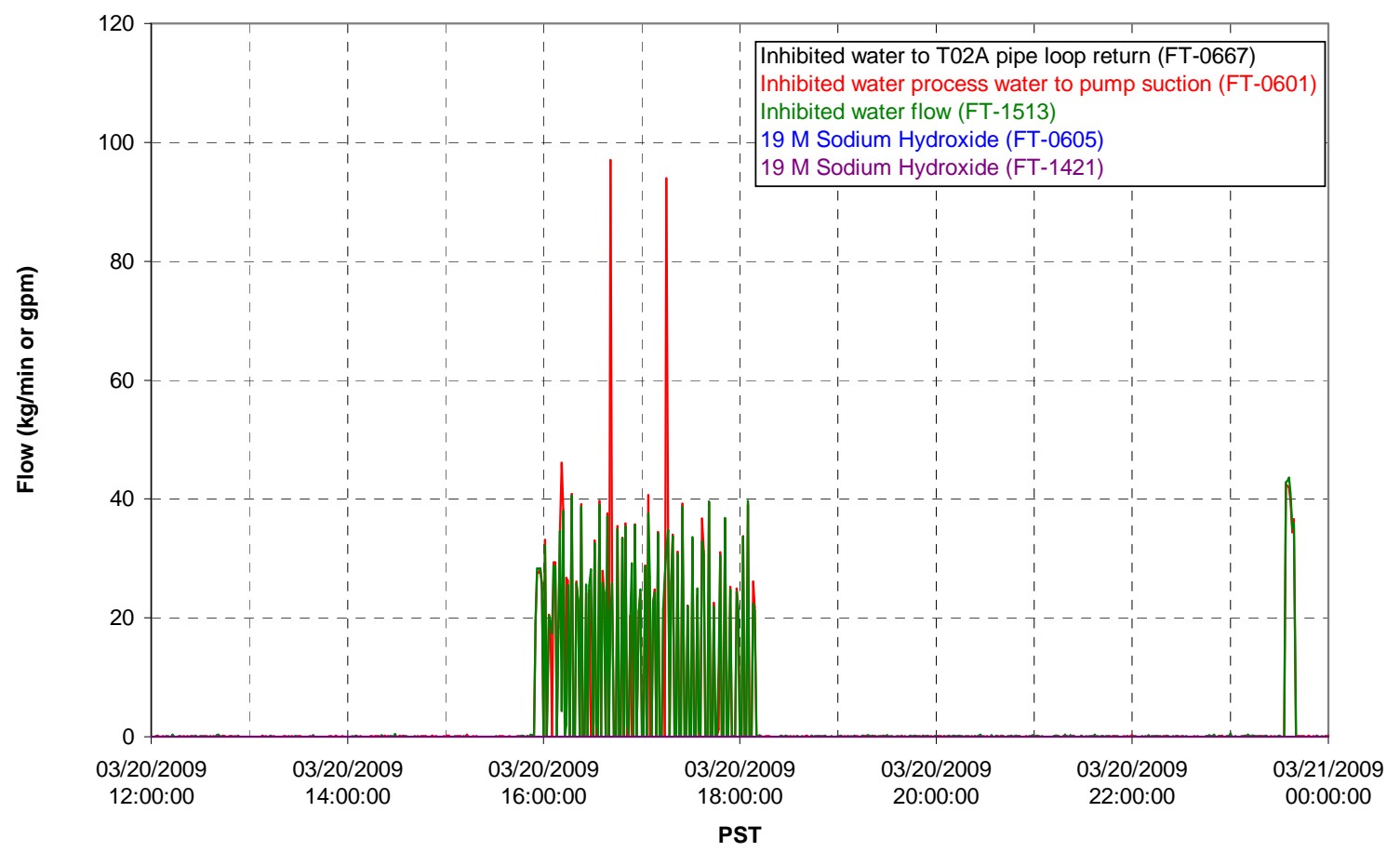




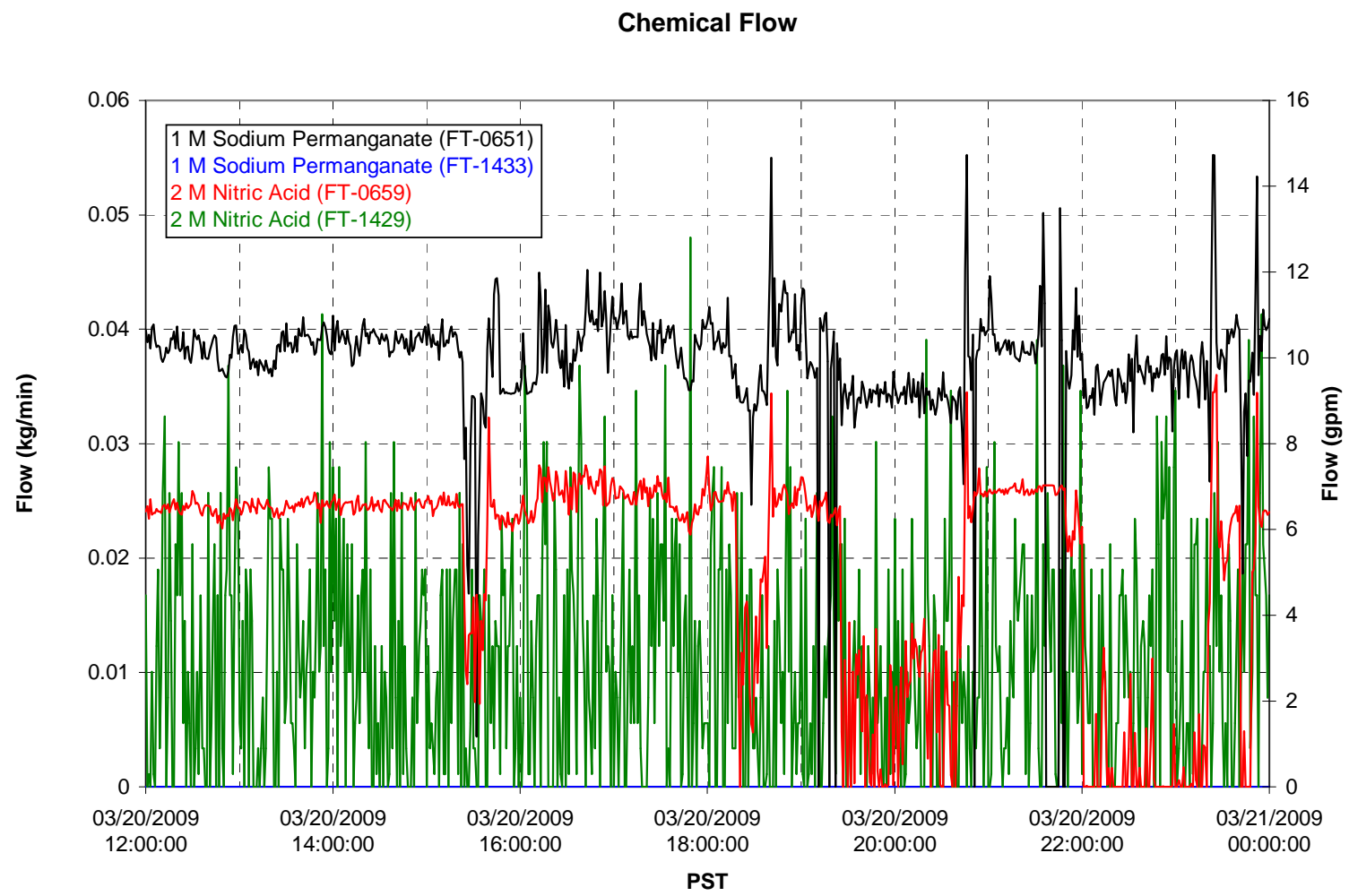

Air Flows

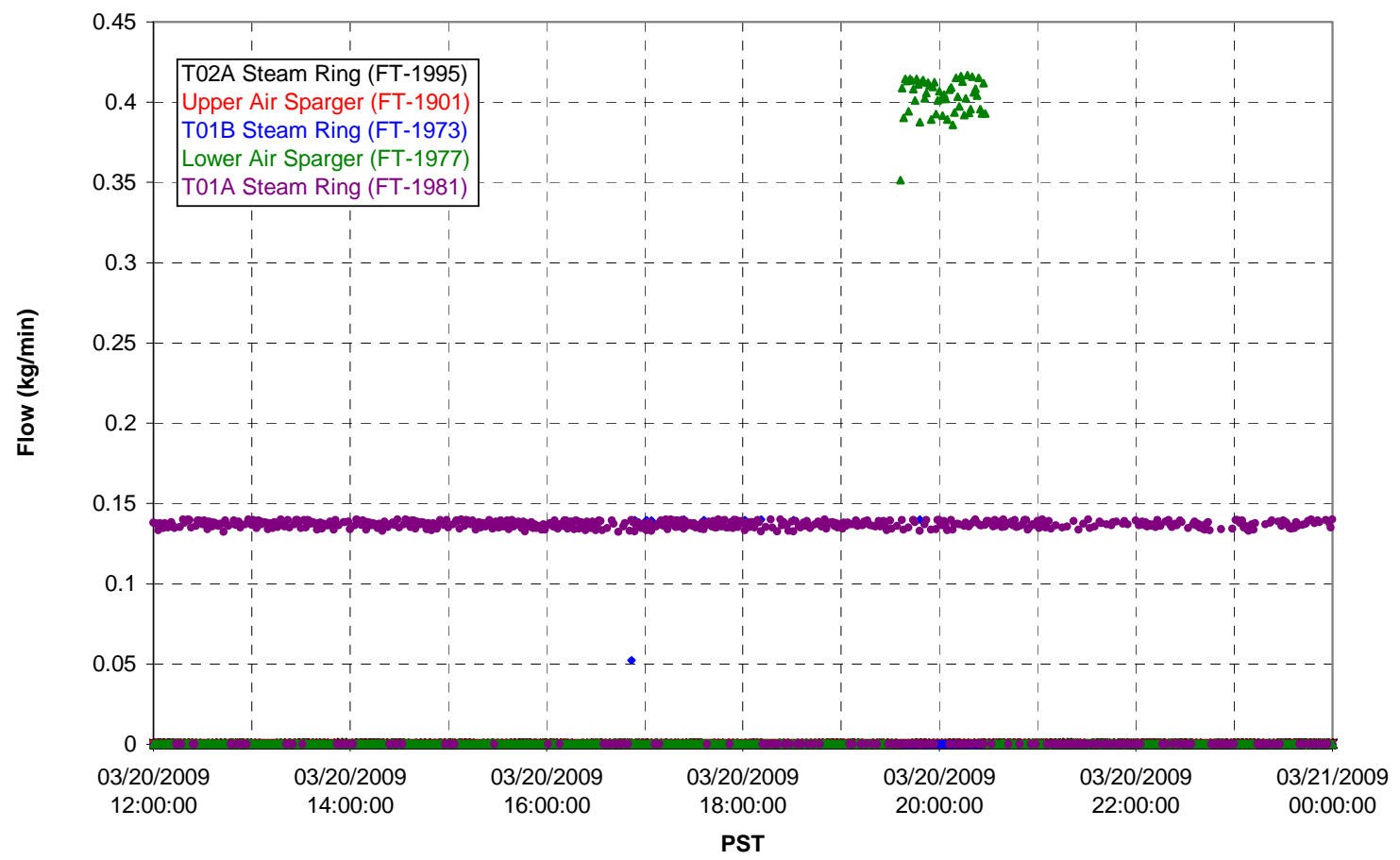


T02A Steam

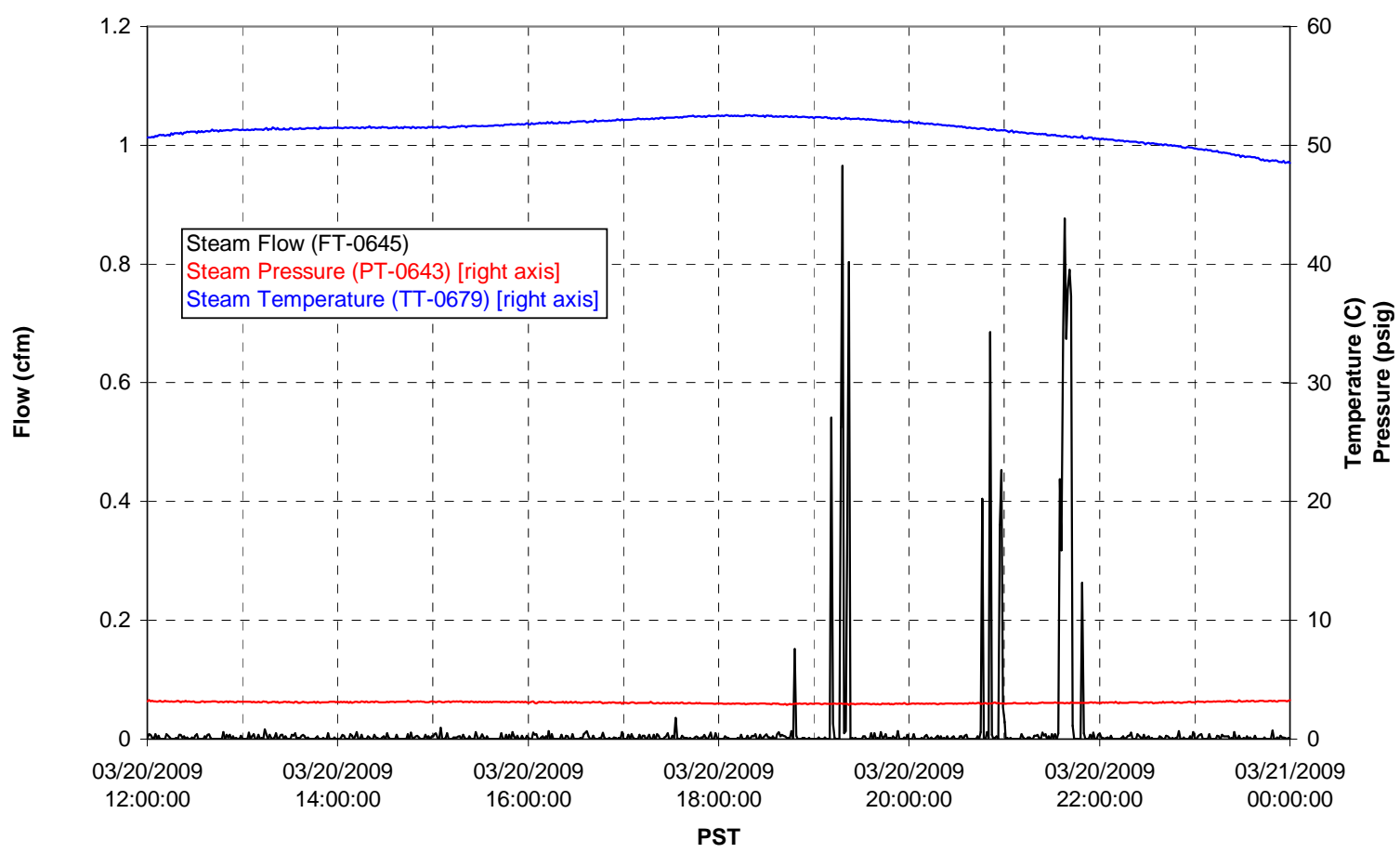

T01A Steam

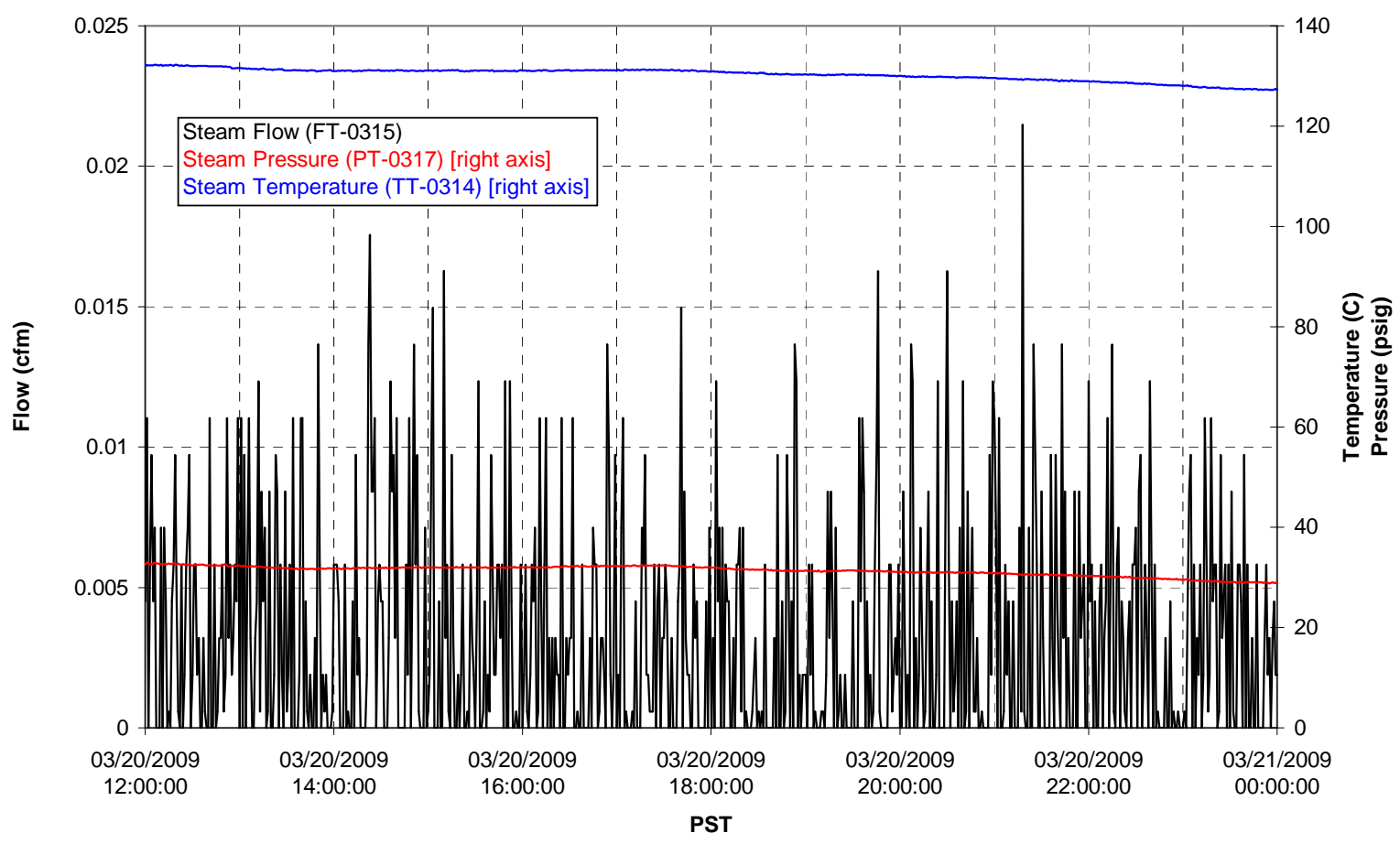


T01B Steam

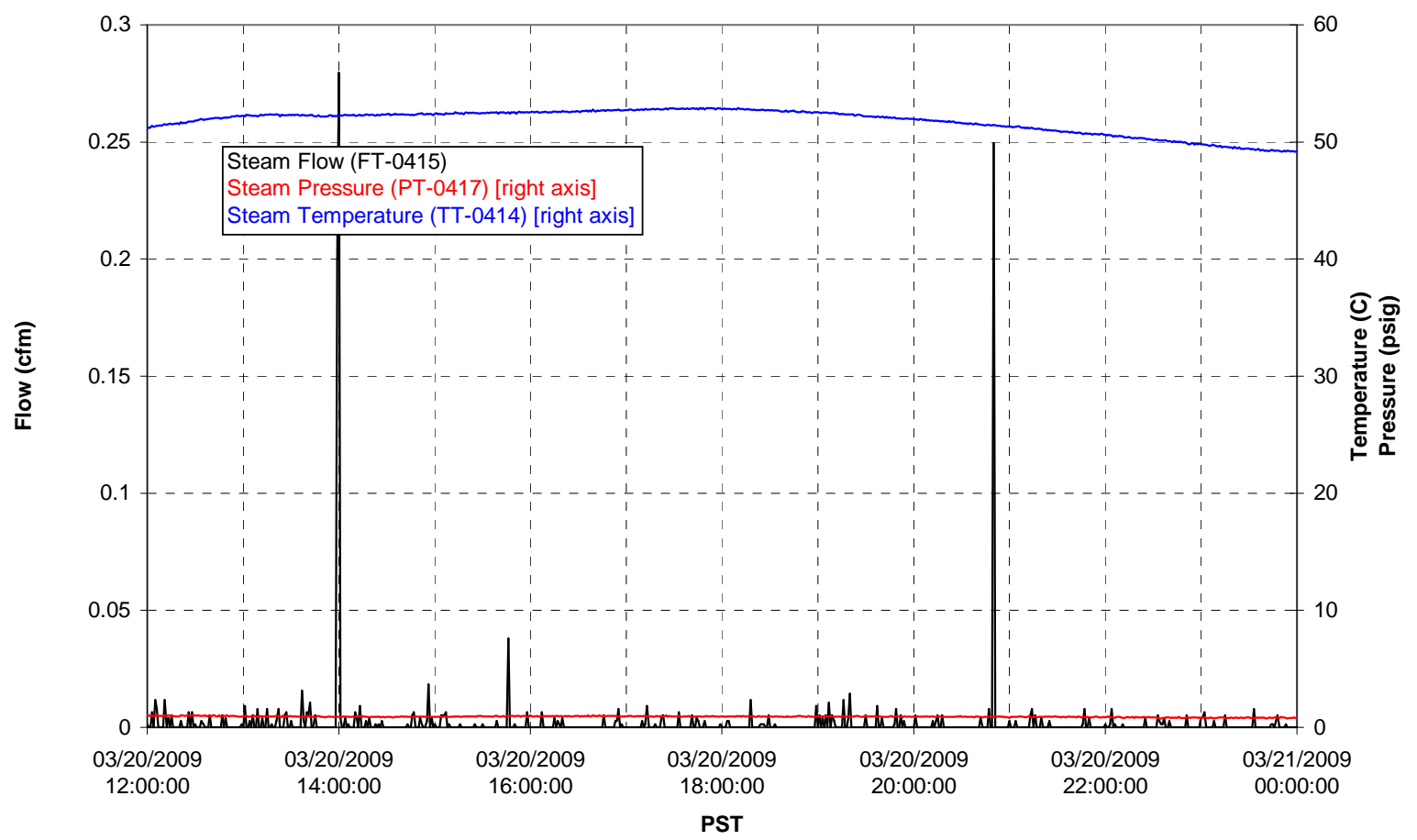



Appendix K

\section{Integrated Test B Summary}





\section{Appendix K: Integrated Test B Summary}

An abbreviated version of the Test Plan is used to outline Integrated Test B. Table K.1 summarizes test steps and key process conditions. It also provides approximate times steps were performed with major issues encountered identified.

Appendix K: Integrated Test B Summary

\begin{tabular}{|c|c|c|c|c|}
\hline Test Step & Test Step & Description/Purpose & Key Process Conditions & Notes \\
\hline B.2.1 & $\begin{array}{l}\text { Prepare and stage } \\
\text { simulant in } \\
\text { HLP-VSL-T22 }\end{array}$ & $\begin{array}{l}\text { Prepare sufficient quantity of Phase } 1 \\
\text { simulant in HLP-VSL-T22 to } \\
\text { complete tests. } \\
\text { Characterize simulant for specification } \\
\text { of all simulant-dependent process } \\
\text { parameters (e.g., batch volumes, } \\
\text { caustic and permanganate additions). } \\
\text { Note: This characterization may have } \\
\text { been completed at the start of } \\
\text { Functional testing. }\end{array}$ & $\begin{array}{l}\text { HLP-VSL-T22 agitator speed set so } \\
\text { vessel is well mixed; recirculate the } \\
\text { tank with the pump. }\end{array}$ & $\begin{array}{l}\text { Started: 00:40, 3/12/09 } \\
\text { Completed: 04:30,3/12/09 } \\
\text { Key process conditions: Met. Agitator VFD set to full speed, } 60 \\
\text { Hz. } \\
\text { Issues: Recirculation of HLP-VSL-T22 using HLP-PMP-T21 was } \\
\text { at } \sim 7.4 \text { gpm (versus } 30 \text { gpm targeted set point) to comply with } \\
\text { operations directive to keep pump speed low due to leaking seal. }\end{array}$ \\
\hline B.2.2 & $\begin{array}{l}\text { Transfer simulant } \\
\text { from } \\
\text { HLP-VSL-T22 } \\
\text { to } \\
\text { UFP-VSL-T01A }\end{array}$ & $\begin{array}{l}\text { Transfer prototypic quantity of } \\
\text { simulant from HLP-VSL-T22 to } \\
\text { UFP-VSL-T01A. } \\
\text { Add antifoam agent. }\end{array}$ & $\begin{array}{l}\text { HLP-VSL-T22 agitator speed set so } \\
\text { vessel is well mixed. } \\
\text { UFP-VSL-T01A initially empty or } \\
\text { with unleached heel. } \\
\text { Set transfer rate to confirm turbulent } \\
\text { flow in transfer pipes. } \\
\text { UFP-VSL-T01A PJM velocities and } \\
\text { cycle times match PTF PJM } \\
\text { power/volume. }\end{array}$ & $\begin{array}{l}\text { Batch } 1 \\
\text { Started: } 05: 11,3 / 12 / 09 \\
\text { Completed: } 11: 17,3 / 12 / 09 \\
\text { Key process conditions: } \\
\text { - } \quad \text { Agitator VFD in HLP-VSL-T22 set to full speed, } 60 \mathrm{~Hz} \text {. } \\
\text { UFP-VSL-T01A was empty and drained prior to simulant } \\
\text { transfer. } \\
\text { - Targeted simulant transfer rate achieved. } \\
\text { Targeted simulant transfer rate achieved. } \\
\text { PJM cycle time matched target. PJM parameters (drive } \\
\text { pressure and drive time) were adjusted to achieve a peak } \\
\text { average nozzle velocity of } 4.8 \text { m/s and } 80 \% \text { stroke length } \\
\text { in UFP-VSL-T01A. PJM-1605 (from tank } \\
\text { UFP-VSL-T01A) appeared to plug and was intentionally } \\
\text { overblew in an attempt to remove the plug. This } \\
\text { appeared to work for approximately } 1 \text { hour. However, } \\
\text { the PJM again plugged and would not clear by } \\
\text { overblowing. PJM-1605 was disabled. }\end{array}$ \\
\hline
\end{tabular}


Appendix K: Integrated Test B Summary

\begin{tabular}{|c|c|c|c|c|}
\hline Test Step & Test Step & Description/Purpose & Key Process Conditions & Notes \\
\hline & & & & $\begin{array}{l}\text { Batch } 2 \\
\text { Started: } 11: 31,3 / 13 / 09 \\
\text { Completed: } 13: 19,3 / 13 / 09 \\
\text { Key process conditions: } \\
\text { - } \quad \text { Agitator VFD in HLP-VSL-T22 set to full speed, } 60 \mathrm{~Hz} . \\
\text { - } \quad \text { sFP-VSL-T01A had unleached simulant heel prior to } \\
\text { - Targeted simulant transfer rate achieved. } \\
\text { - PJM cycle time } 35 \text { s, PJM drive stroke 30"; PJM nozzle } \\
\quad \text { velocity 4.8-5.9 seconds. }\end{array}$ \\
\hline B.2.3 & $\begin{array}{c}\text { Transfer } \\
\text { simulant from } \\
\text { UFP-VSL-T01A } \\
\text { to } \\
\text { UFP-VSL-T02A }\end{array}$ & $\begin{array}{l}\text { Transfer simulant from } \\
\text { UFP-VSL-T01A to UFP-VSL-T02A. } \\
\text { Monitor UFP-VSL-T01A for solids } \\
\text { settling that may impact transfer of } \\
\text { solids. }\end{array}$ & $\begin{array}{l}\text { UFP-VSL-T02A initially empty with } \\
\text { minimal IW heel or with heel that has } \\
\text { undergone caustic and oxidative } \\
\text { leaching. } \\
\text { Transfer at scale-time. } \\
\text { UFP-VSL-T01A PJM velocities and } \\
\text { cycle times match PTF PJM } \\
\text { power/volume. } \\
\text { UFP-VSL-T02A PJM velocities and } \\
\text { cycle times match PTF PJM } \\
\text { power/volume. }\end{array}$ & 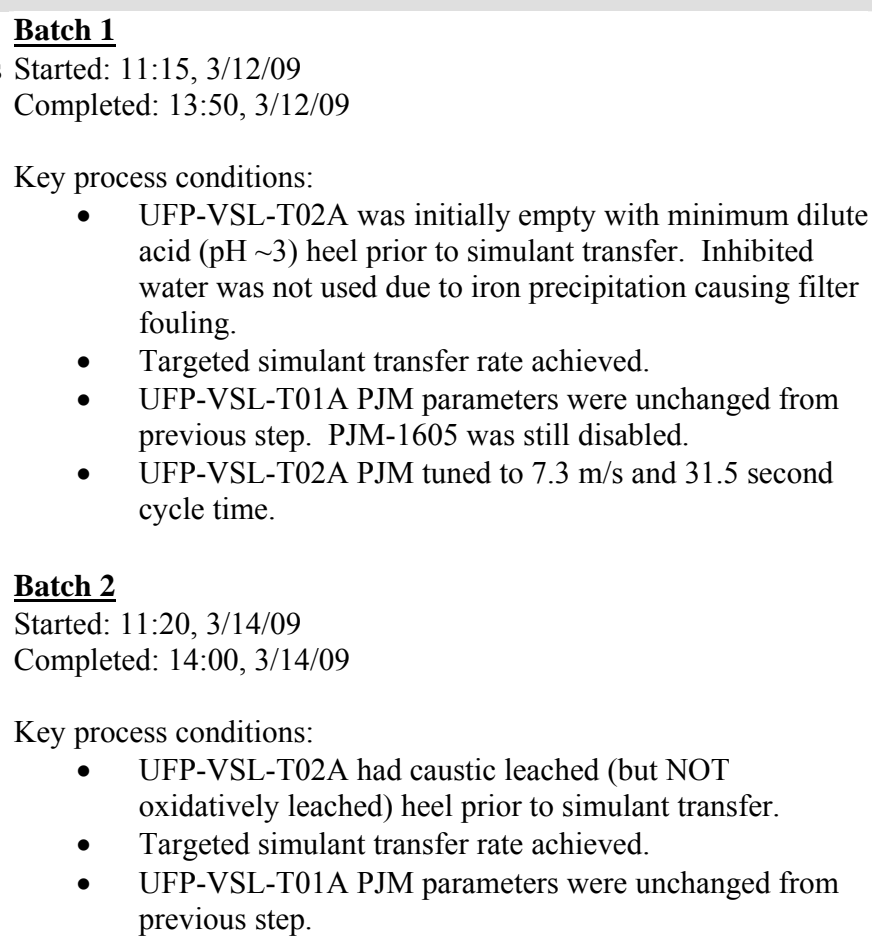 \\
\hline
\end{tabular}


Appendix K: Integrated Test B Summary

\begin{tabular}{|c|c|c|c|c|}
\hline Test Step & Test Step & Description/Purpose & Key Process Conditions & Notes \\
\hline & & & & $\begin{array}{l}\text { UFP-VSL-T02A PJM nozzle velocity and drive stroke } \\
\text { informally checked and nozzle velocities found to be too } \\
\text { high. "Drive pressures set to when Sato's tune } \\
\text { determined lower drive pressures because } \Delta \mathrm{L} / \Delta \mathrm{t} \text { nozzle } \\
\text { velocity appears too high. Lowered (drive) pressures } \\
\text { twice. Pressures are now }<6 \text { psi but } \Delta \mathrm{L} / \Delta \mathrm{t} \text { looks too } \\
\text { high!! Reluctant to reduce pressures again and deviate } \\
\text { from Sato tune (TI-WTP-PEP-066, p. 77). }\end{array}$ \\
\hline
\end{tabular}

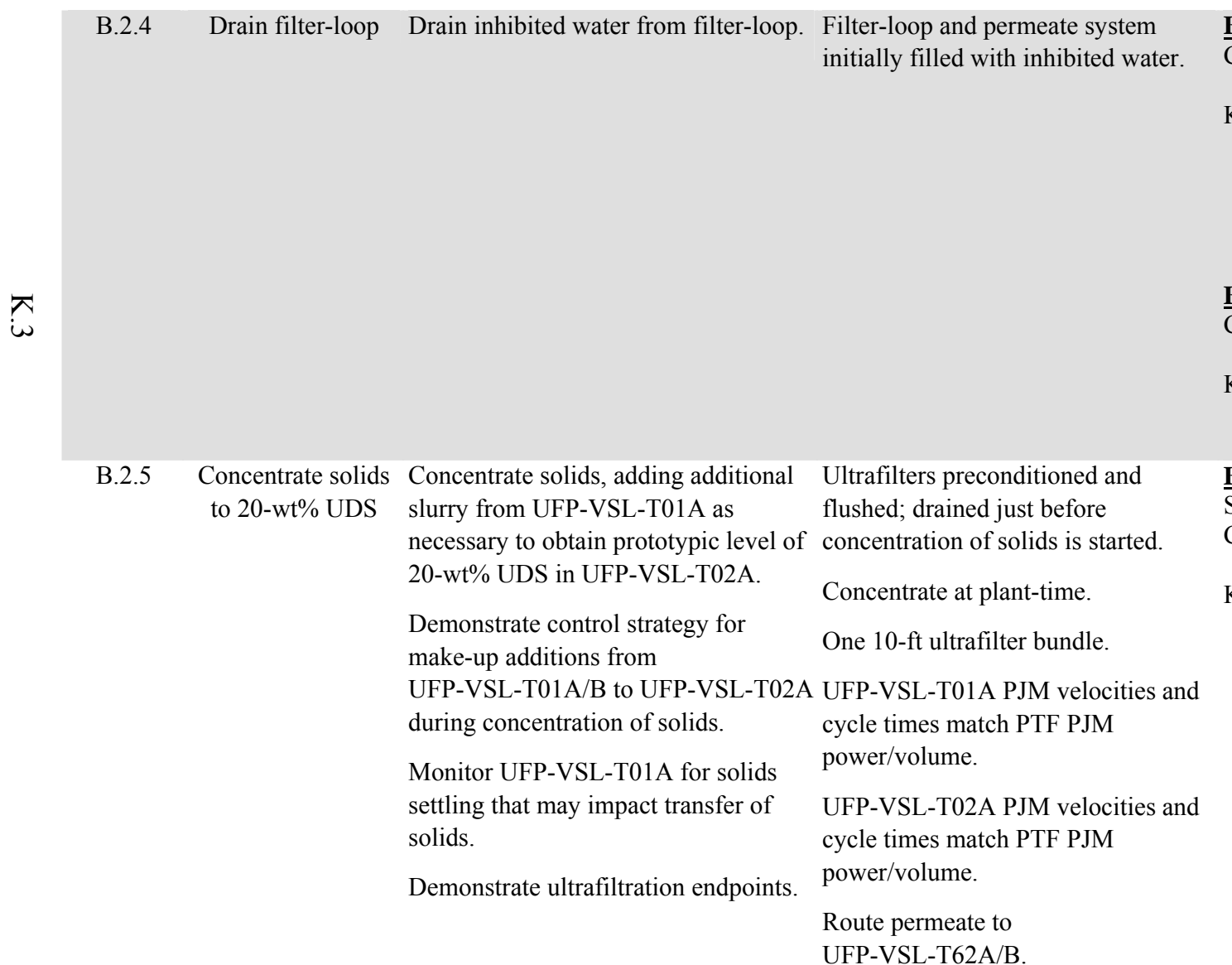

Completed: 13:30, 3/12/09

Key process conditions:

- The shell and tube side of the filter loop was initially filled with dilute acid ( $\mathrm{pH} \sim 3$ ) prior to draining. Inhibited water was not used due to iron precipitation causing filter fouling.

\section{Batch 2}

Completed: 11:42, 3/14/09

Key process conditions:

- Inhibited water drained from tube side of filter loop.

Batch 1

Started: 13:30, 3/12/09

Completed: 20:16,3/12/09

Key process conditions:

- Ultrafilters were not preconditioned prior to dewatering of Batch 1, as the filters had just been acid cleaned. The thinking was that the concentration of Batch 1 would serve as the preconditioning for the Batch 2 concentration.

- Concentration was not conducted at plant time. Concentration was conducted at scale time using all 5 ultrafilter bundles. (Note that Batch 2 was concentrated at plant time using 1 ultrafilter.)

- UFP-VSL-T01A PJM parameters were unchanged from previous step. PJM-1605 was still disabled.

- UFP-VSL-T02A PJM parameters were unchanged from 
Appendix K: Integrated Test B Summary

\begin{tabular}{|c|c|c|c|c|}
\hline Test Step & Test Step & Description/Purpose & Key Process Conditions & Notes \\
\hline
\end{tabular}

\section{Batch 2}

Started: 11:42, 3/14/09

Completed: 13:37, 3/15/09

\section{Key process conditions:}

- Ultrafilters were preconditioned through Batch 1 dewatering activities.

- Concentration was conducted at plant time using 1 ultrafilter bundle.

- $\quad$ UFP-VSL-T01A PJM parameters were unchanged from previous step.

- UFP-VSL-T02A PJM parameters were unchanged from previous step.

- Permeate was collected in UFP-VSL-T62A/B

\begin{tabular}{|c|c|c|c|}
\hline B.2.6 & $\begin{array}{c}\mathrm{NaOH} \\
\text { addition }\end{array}$ & $\begin{array}{l}\text { Add NaOH in-line to suction of } \\
\text { UFP-PMP-T42A. Use the pump heat } \\
\text { to continue heating to the target } \\
\text { temperature specified in the run sheet } \\
\text { if necessary. The heat exchanger } \\
\text { HX-T03A may also be used with Test } \\
\text { Director approval. } \\
\text { Use the UFP-HX-T02A to cool the } \\
\text { filter loop slurry to maintain } \\
\text { UFP-VSL-T02A temperature at or } \\
\text { below the temperature specified in the } \\
\text { run sheet, if necessary. } \\
\text { Flush filter-loop after caustic addition } \\
\text { is completed as specified in the run } \\
\text { sheet. }\end{array}$ & $\begin{array}{l}\text { Add caustic at scale-time. } \\
\text { UFP-VSL-T02A PJM velocities and } \\
\text { cycle times match PTF PJM } \\
\text { power/volume. }\end{array}$ \\
\hline
\end{tabular}

Issues:

DAS 1 did not record data from $01: 17$ to $06: 37$ on $3 / 14 / 09$.

Batch 1

Started: $21: 36,3 / 12 / 09$

Completed: $22 \cdot 38,3 / 12 / 09$

Key process conditions:

- Transfer of $720 \mathrm{~kg}$ of caustic was conducted with a manual control valve setting of $24 \%$ open, resulting in a transfer rate of $\sim 14 \mathrm{~kg} / \mathrm{min}$ versus target of $10.2 \mathrm{~kg} / \mathrm{min}$. Transfer using automatic control valve setting resulted in wild flow rate fluctuations.

- $\quad$ UFP-VSL-T02A PJM parameters were unchanged from previous step.

\section{Batch 2}

Started: 14:08, 3/15/09

Completed: 15:20, 3/15/09

\section{Key process conditions:}

- Transfer of $720 \mathrm{~kg}$ of caustic was conducted at a transfer rate of $\sim 10 \mathrm{~kg} / \mathrm{min}$ versus target of $10.2 \mathrm{~kg} / \mathrm{min}$. 
Appendix K: Integrated Test B Summary

\begin{tabular}{|c|c|c|c|}
\hline Test Step & Test Step & Key Process Conditions & Notes \\
\hline & & & $\begin{array}{l}\text { - UFP-VSL-T02A PJM parameters were unchanged from } \\
\text { previous step. }\end{array}$ \\
\hline B.2.7 & Flush filter-loop & $\begin{array}{l}\text { Flush slurry in filter-loop back into Flush at scale-time. } \\
\text { UFP-VSL-T02A with inhibited water. } \\
\text { Sample UFP-VSL-T02A to determine } \\
\text { if filter flush resulted in the addition of } \\
\text { significant amounts of flush water. }\end{array}$ & $\begin{array}{l}\text { Batch } 1 \\
\text { Started: } 23: 23,3 / 12 / 09 \\
\text { Completed: } 17: 55,3 / 13 / 09 \\
\text { Key process conditions: } \\
\text { - Flushed slurry in filter loop back into UFP-VSL-T02A } \\
\text { with } 74 \mathrm{~kg} \text { inhibited water. } \\
\text { - Sample UFP-VSL-T02A after filter flush was conducted. } \\
\text { - Re-configuration of the filter loop to by-pass UFP-VSL- } \\
\text { T02A and circulate flush water with UFP-PMP-T42A } \\
\text { and/or UFP-PMP-T43A was performed } 18 \text { hours after } \\
\text { the initial flush due to the following: 1) UFP-PMP-T43A } \\
\text { was leaking and consequently locked out pending } \\
\text { repair/evaluation; and 2) the filter loop bypass of UFP- } \\
\text { VSL-T02A was locked out by operations. } \\
\text { Filter loop flushed with second volume of inhibited water } \\
\text { (682 kg) Second draining and refilling of the filter loop } \\
\text { was not conducted per test instruction. }\end{array}$ \\
\hline
\end{tabular}

\section{Batch 2}

Started: 16:54, 3/15/09

Completed: 22:41, 3/15/09

Key process conditions

- $\quad$ Flushed slurry in filter-loop back into UFP-VSL-T02A with $74 \mathrm{~kg}$ inhibited water.

- Sample UFP-VSL-T02A after filter flush was conducted.

- Representative in-line sample of the filter-loop contents were taken.

Filter-loop flushed with second volume of inhibited water $(693 \mathrm{~kg})$ Second draining and refilling of the filter-loop was not conducted per Test Instruction. 
Appendix K: Integrated Test B Summary

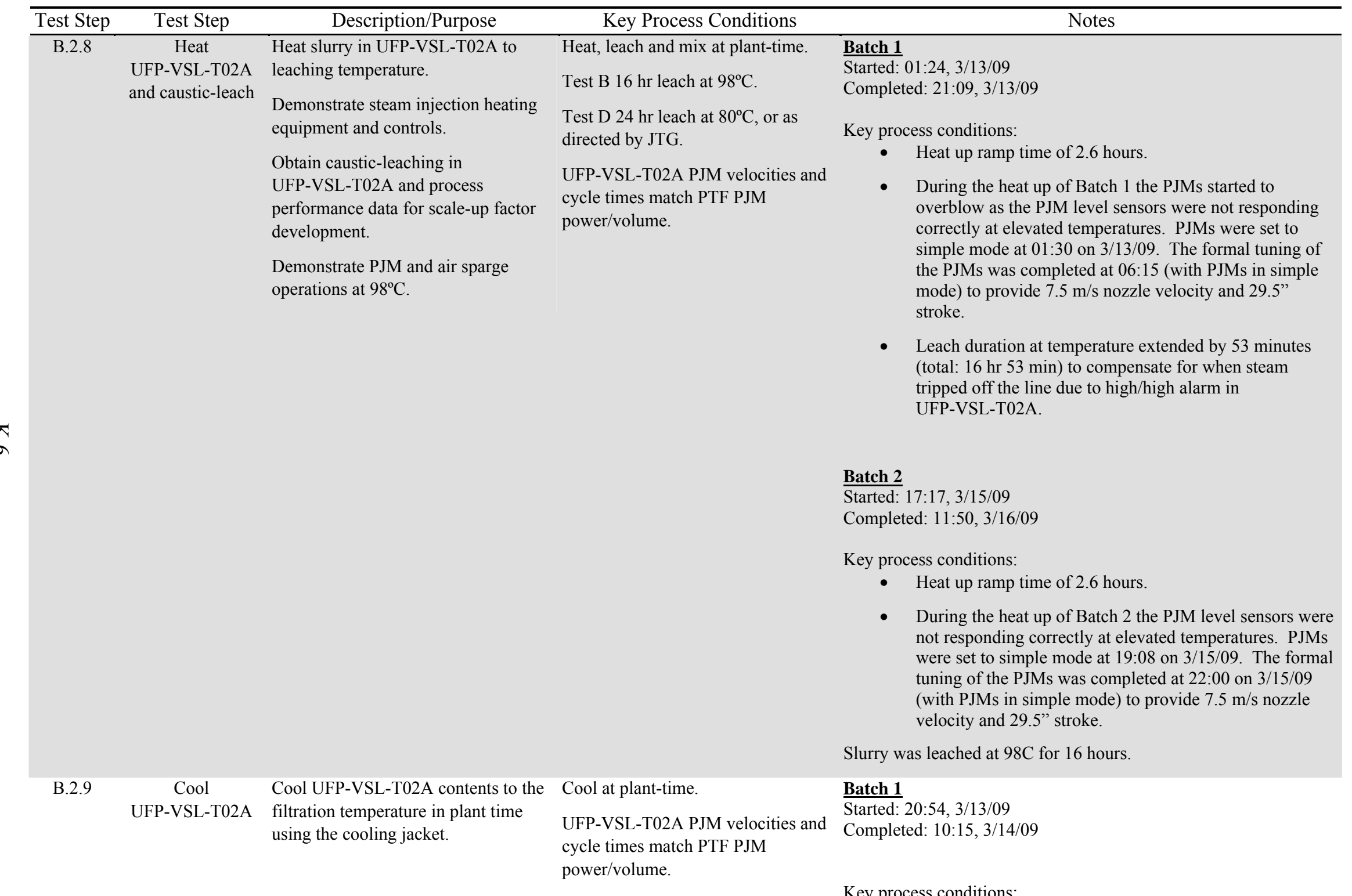

Key process conditions: 
Appendix K: Integrated Test B Summary

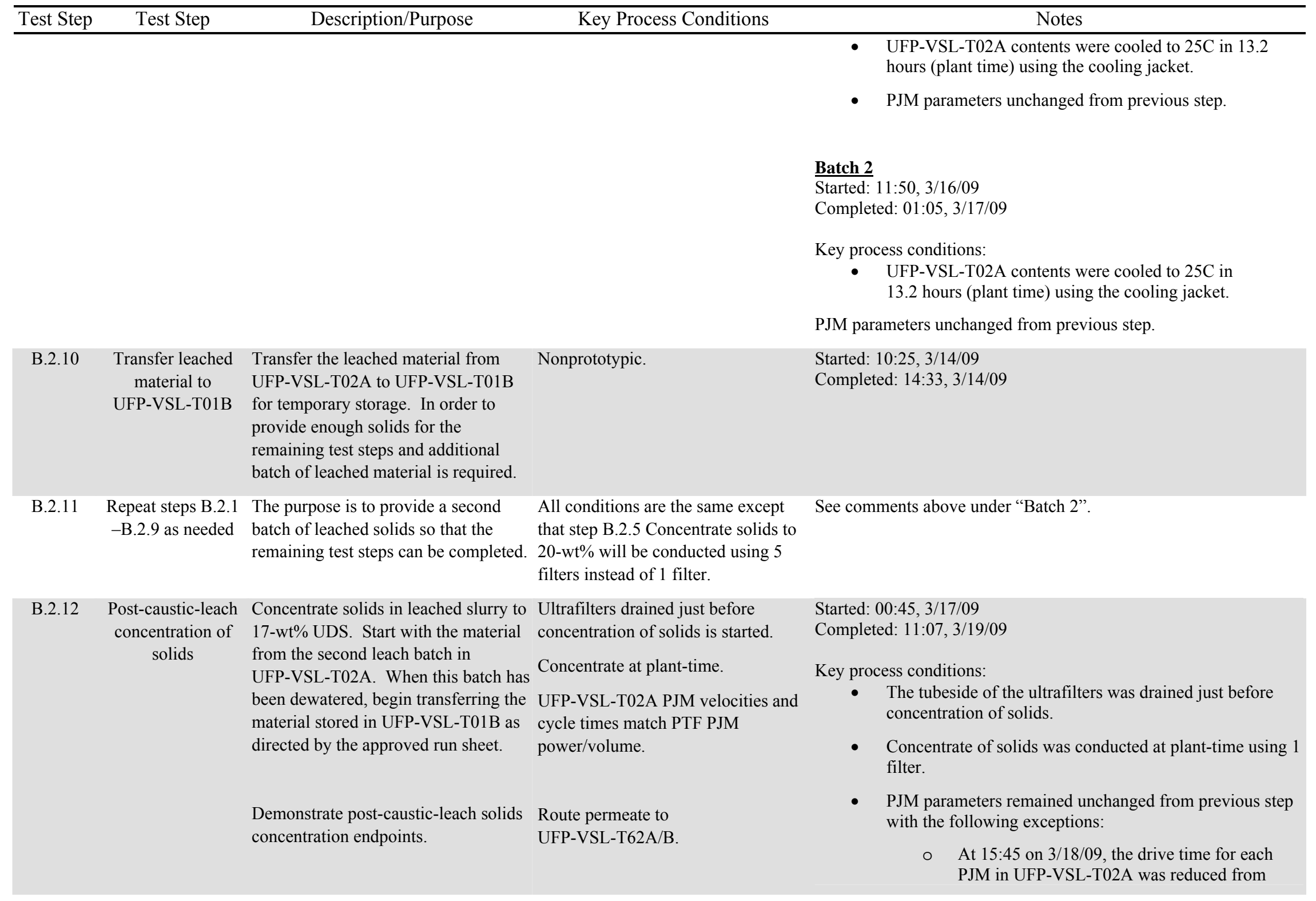


Appendix K: Integrated Test B Summary

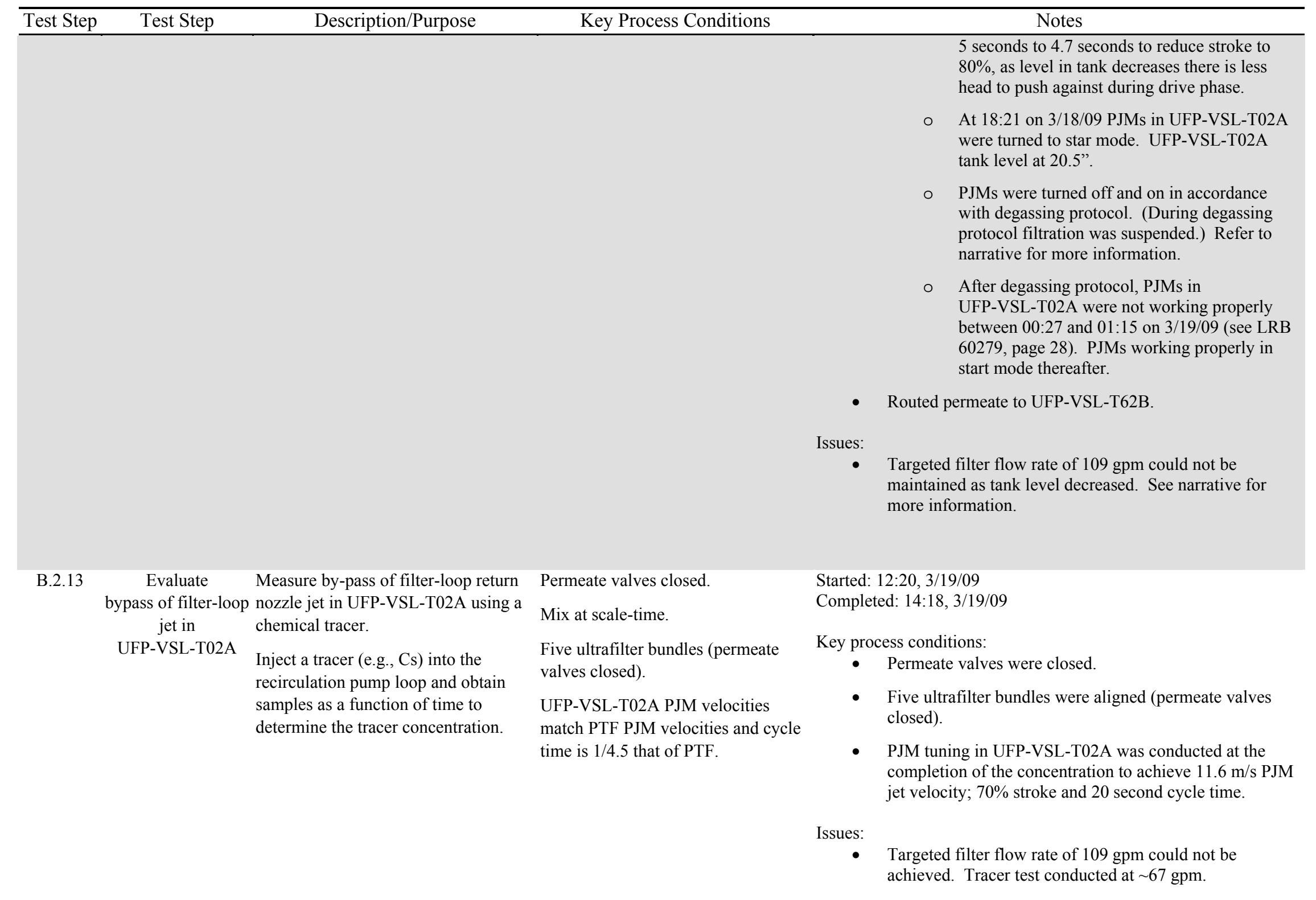


Appendix K: Integrated Test B Summary

\begin{tabular}{|c|c|c|c|c|}
\hline Test Step & Test Step & Description/Purpose & Key Process Conditions & Notes \\
\hline & & & & $\begin{array}{l}\text { - Spargers and steam ring air purge were off due to air } \\
\text { entrainment issues. }\end{array}$ \\
\hline B. 2.14 & $\begin{array}{l}\text { Post-caustic-leach } \\
\text { slurry wash }\end{array}$ & $\begin{array}{l}\text { Demonstrate post-caustic-leach slurry } \\
\text { wash to remove soluble solids and } \\
\text { achieve targeted hydroxide } \\
\text { concentration. } \\
\text { Measure filter flux and axial pressure } \\
\text { drop across filter bundles as functions } \\
\text { of solids content. } \\
\text { Monitor phase distribution of oxalate } \\
\text { and dissolved species to establish } \\
\text { washing efficiencies. }\end{array}$ & $\begin{array}{l}\text { Wash at scale-time. } \\
\text { Five ultrafilter bundles. } \\
\text { UFP-VSL-T02A PJM velocities } \\
\text { match PTF PJM velocities, and cycle } \\
\text { time is } 1 / 4.5 \text { that of PTF. } \\
\text { Route permeate to } \\
\text { UFP-VSL-T62A/B. } \\
\text { Close permeate valves at end of } \\
\text { wash. }\end{array}$ & $\begin{array}{l}\text { Started: } 16: 05,3 / 19 / 09 \\
\text { Completed: } 01: 19,3 / 20 / 09 \\
\text { Key process conditions: } \\
\text { - The slurry was washed at scale-time using five ultrafilter } \\
\text { bundles. } \\
\text { - PJM parameters were unchanged from the previous step; } \\
\text { however due to UFP-VSL-T02A tank level of 16.7”, } \\
\text { PJMs were operating under star mode. } \\
\text { - Routed permeate to UFP-VSL-T62B. } \\
\text { - Permeate valves were closed at the end of the wash. } \\
\text { Issues: } \\
\text { - Targeted filter flow rate of } 109 \text { gpm could not be } \\
\text { achieved. Slurry wash conducted at } ~ 75 \text { gpm. } \\
\text { - Steam ring air purge and spargers off per degassing } \\
\text { instructions. }\end{array}$ \\
\hline B.2.15 & $\begin{array}{l}\text { Chromium slurry } \\
\text { addition }\end{array}$ & $\begin{array}{l}\text { TEST B ONLY - Add chromium solids } \\
\text { (as a slurry in-line at suction of } \\
\text { UFP-PMP-T42A. Add AFA to } \\
\text { maintain to maintain a concentration } \\
\text { of } 350 \mathrm{ppm} \text {. }\end{array}$ & $\begin{array}{l}\text { Non-prototypic. } \\
\text { Permeate valves closed. } \\
\text { No change in mixing or pumping } \\
\text { parameters from Step B.2.12. }\end{array}$ & $\begin{array}{l}\text { Started: } 01: 19,3 / 20 / 09 \\
\text { Completed: } 05: 54,3 / 20 / 09 \\
\text { Chrome slurry was added and permeate was removed to reduce the } \\
\text { level in UFP-VSL-T02A to the targeted level of } 21.4 \text { ". Then the } \\
\text { slurry was washed with inhibited water. }\end{array}$ \\
\hline B.2.16 & $\begin{array}{l}\text { Wash and/or } \\
\text { reconcentrate slurry } \\
\text { solids }\end{array}$ & $\begin{array}{l}\text { TEST B ONLY - Wash and/or } \\
\text { reconcentrate slurry, if necessary, to } \\
\text { remove excess liquid and hydroxide } \\
\text { introduced by chromium solids slurry. }\end{array}$ & $\begin{array}{l}\text { Non-prototypic. } \\
\text { Concentrate and wash with } 5 \\
\text { ultrafilter bundles. }\end{array}$ & \\
\hline B.2.17 & Add permanganate & $\begin{array}{l}\text { Demonstrate in-line addition of } \\
\text { oxidative leach chemicals. }\end{array}$ & Add chemicals at scale-time. & $\begin{array}{l}\text { Started: 07:42, 3/19/09 } \\
\text { Completed: 09:17, 3/20/09 }\end{array}$ \\
\hline
\end{tabular}


Appendix K: Integrated Test B Summary

\begin{tabular}{|c|c|c|c|c|}
\hline Test Step & Test Step & Description/Purpose & Key Process Conditions & Notes \\
\hline & & & $\begin{array}{l}\text { UFP-VSL-T02A PJM velocities } \\
\text { match PTF PJM velocities, and cycle } \\
\text { time is } 1 / 4.5 \text { that of PTF. }\end{array}$ & $\begin{array}{l}\text { Key process conditions: } \\
\text { - Permanganate, } 87 \mathrm{~kg} \text {, was added at scale-time, } \\
20.8 \mathrm{~kg} / \mathrm{min} . \\
\text { - UFP-VSL-T02A PJM parameters unchanged from } \\
\text { previous step. }\end{array}$ \\
\hline B. 2.18 & $\begin{array}{l}\text { Oxidative- } \\
\text { leach }\end{array}$ & $\begin{array}{l}\text { Demonstrate oxidative leaching and } \\
\text { establish performance of Phase } 1 \\
\text { simulant in PEP. }\end{array}$ & $\begin{array}{l}\text { Leach and mix at plant-time. } \\
\text { UFP-VSL-T02A PJM velocities } \\
\text { match PTF PJM velocities, and cycle } \\
\text { time is } 1 / 4.5 \text { that of PTF. }\end{array}$ & $\begin{array}{l}\text { Started: } 09: 17,3 / 19 / 09 \\
\text { Completed: } 15: 17,3 / 20 / 09 \\
\text { Key process conditions: } \\
\text { - Oxidative leaching was conducted over } 6 \text { hours. } \\
\text { - PJM parameters unchanged from previous step. } \\
\text { Issues: }\end{array}$ \\
\hline B.2.19 & $\begin{array}{l}\text { Wash oxidatively } \\
\text { leached slurry }\end{array}$ & $\begin{array}{l}\text { Demonstrate post-oxidative-leach } \\
\text { slurry wash. } \\
\text { Monitor liquid fractions for } \\
\text { precipitation of solids. } \\
\text { Shut permeate valves at end of wash to } \\
\text { allow collection of final samples. }\end{array}$ & $\begin{array}{l}\text { Wash at scale-time. } \\
\text { Five ultrafilter bundles. } \\
\text { Add AFA as specified in the run } \\
\text { sheet. } \\
\text { UFP-VSL-T02A PJM velocities } \\
\text { match PTF PJM velocities, and cycle } \\
\text { time is } 1 / 4.5 \text { that of PTF. } \\
\text { Route permeate to } \\
\text { UFP-VSL-T62A/B. }\end{array}$ & $\begin{array}{l}\text { Started: } 15: 31,3 / 19 / 09 \\
\text { Completed: } 18: 17,3 / 20 / 09 \\
\text { Key process conditions: } \\
\text { - The slurry was washed at scale-time using five ultrafilter } \\
\text { bundles. } \\
\text { - Add AFA was added every } 3^{\text {rd }} \text { wash batch addition, as } \\
\text { specified in the run sheet. } \\
\text { - PJM parameters unchanged from previous step. } \\
\text { - } \quad \text { Permeate was routed to UFP-VSL-T62B. }\end{array}$ \\
\hline B.2.20 & $\begin{array}{l}\text { Concentrate washed } \\
\text { slurry }\end{array}$ & $\begin{array}{l}\text { Demonstrate final solids concentration } \\
\text { to } 20 \text {-wt } \% \text { UDS. } \\
\text { Demonstrate ultrafiltration end points. } \\
\text { Monitor liquid fractions for } \\
\text { precipitation of solids. }\end{array}$ & $\begin{array}{l}\text { Concentrate at scale-time. } \\
\text { Five ultrafilter bundles. } \\
\text { UFP-VSL-T02A PJM velocities } \\
\text { match PTF PJM velocities, and cycle } \\
\text { time is } 1 / 4.5 \text { that of PTF. } \\
\text { Route permeate to } \\
\text { UFP-VSL-T62A/B. }\end{array}$ & $\begin{array}{l}\text { Final concentration of slurry was conducted High-Solids Filter Test } \\
\text { in TI-WTP-PEP-065. }\end{array}$ \\
\hline
\end{tabular}


Appendix L

Physical Properties 



\section{Appendix L: Physical Properties}

This appendix contains abstractions from Test Data Packages. Interested readers are referred to the listed Test Data Packages listed in the title of each section for more information.

\section{L.1 Rheology (TDP-WTP-363)}




\begin{tabular}{|c|c|}
\hline Date: & $4 / 21 / 2009$ \\
\hline To: & Brad Johnson \\
\hline From: & Jaehun Chun \\
\hline Subject: & $\begin{array}{l}\text { Integrated Test B Rheology Data Report on } \\
\text { PEP Slurry March - April, } 2009\end{array}$ \\
\hline
\end{tabular}

Project No.

53569

Internal Distribution:

Specimens collected for the Integrated Test B were done under TI-WTP-PEP-066, Steps B.2.1, B.2.2, B.2.5, B.2.10, B.2.11, B.2.14, B.2.15, B.2.16, and B.2.17.

Multiple specimens of PEP slurry were delivered to APEL for rheological analysis between 3/18/09 3/23/09. Specimens were analyzed to determine either their flow curves (i.e., plots of shear stress vs. shear rate) or shear strengths. The analysis was done using an Anton Parr Rheometer MCR 301 SN80371304 (for flow curve) and Haake RS-600 (for shear strength). Rheometer calibration verifications were done 3 times for Anton Parr Rheometer MCR 301 SN80371304 and 1 time for Haake RS-600 during this time period by Maria Luna in accordance with QA procedure TPR-WTP-PEP-049. Two Newtonian standards with different viscosities were used to check the calibration of these systems: a 9.4 cP Brookfield Standard Lot \# 062408 and a 99.0 cP Brookfield Standard Lot \#071008. All verifications were within the $10 \%$ of the reported values.

Figures 1-4 are the calibration curves performed on the Anton Parr Rheometer MCR 301 SN80371304 with $9.4 \mathrm{cP}$ Brookfield Standards and the Haake RS-600 with $99.0 \mathrm{cP}$ Brookfield Standard, respectively. Note that the calibration for Haake RS-600 is only a performance check of the Haake RS-600 system, since the measurements performed on this system are shear strength measurements and Newtonian standards have a shear strength of zero.

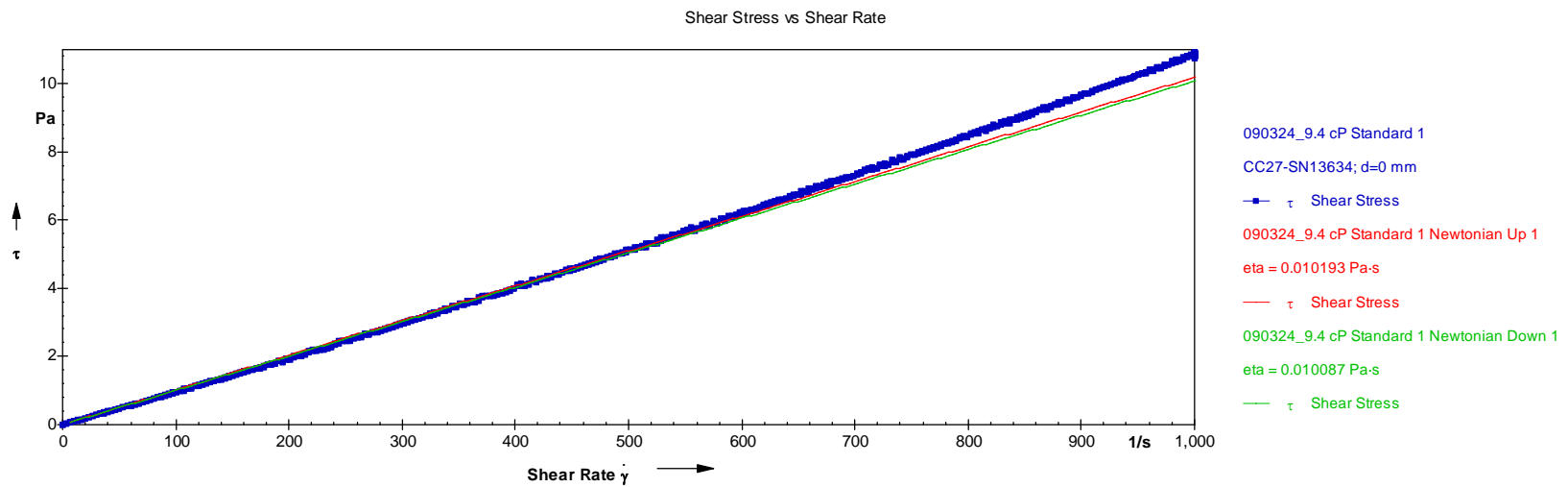

Figure 1. Flow curve measurement 1 of 3 of the $9.4 \mathrm{cP}$ Newtonian standard run on the Anton Paar rheometer 
Shear Stress vs Shear Rate

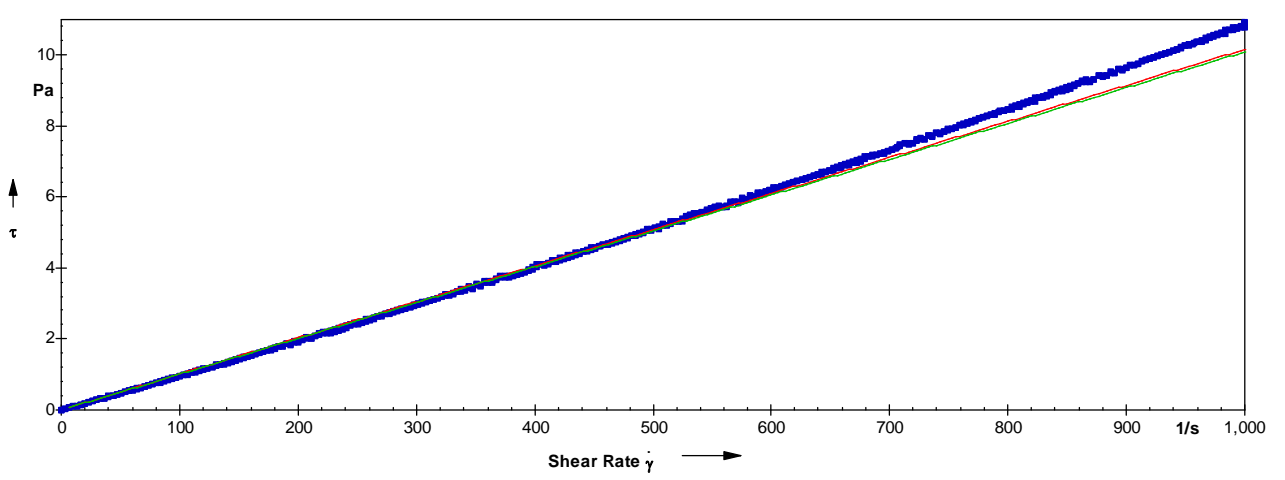

090331_9.4cP Standard CC27-SN13634; $d=0$ mm - $\tau$ Shear Stress

090331_9.4cP Standard 1 Newtonian Up 1 eta $=0.010165 \mathrm{~Pa} \cdot \mathrm{s}$

- $\tau$ Shear Stress

090331_9.4CP Standard 1 Newtonian Down 1

eta $=0.010092 \mathrm{~Pa} \cdot \mathrm{s}$

- $\tau$ Shear Stress

Figure 2. Flow curve measurement 2 of 3 of the $9.4 \mathrm{cP}$ Newtonian standard run on the Anton Paar rheometer

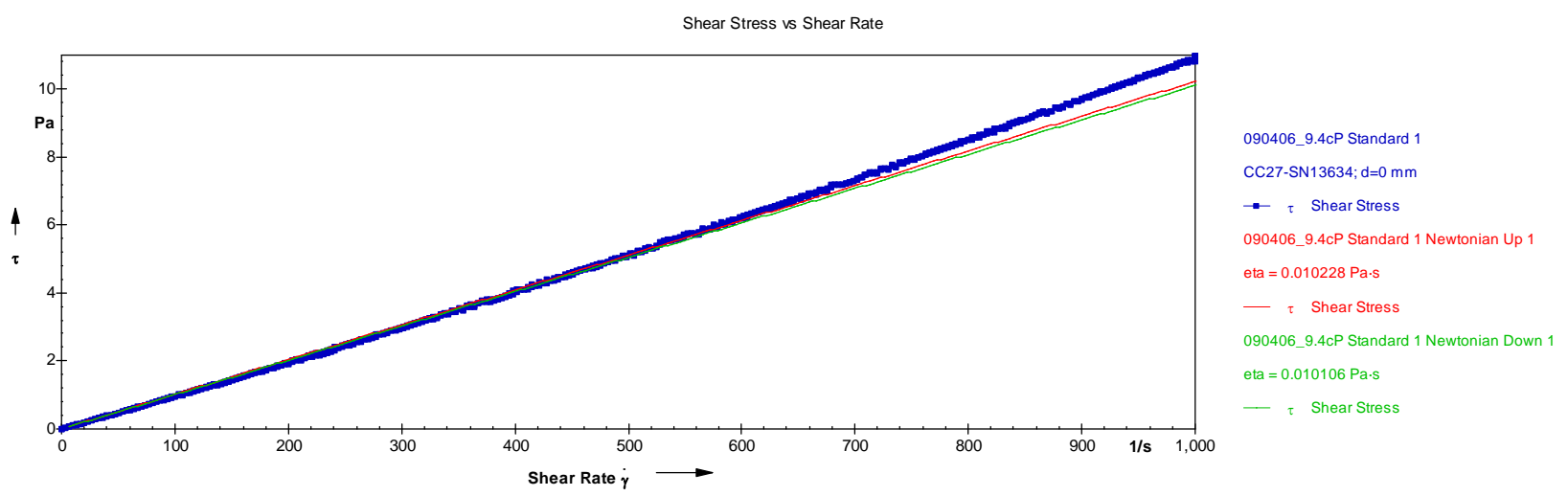

Figure 3. Flow curve measurement 3 of 3 of the $9.4 \mathrm{cP}$ Newtonian standard run on the Anton Paar rheometer

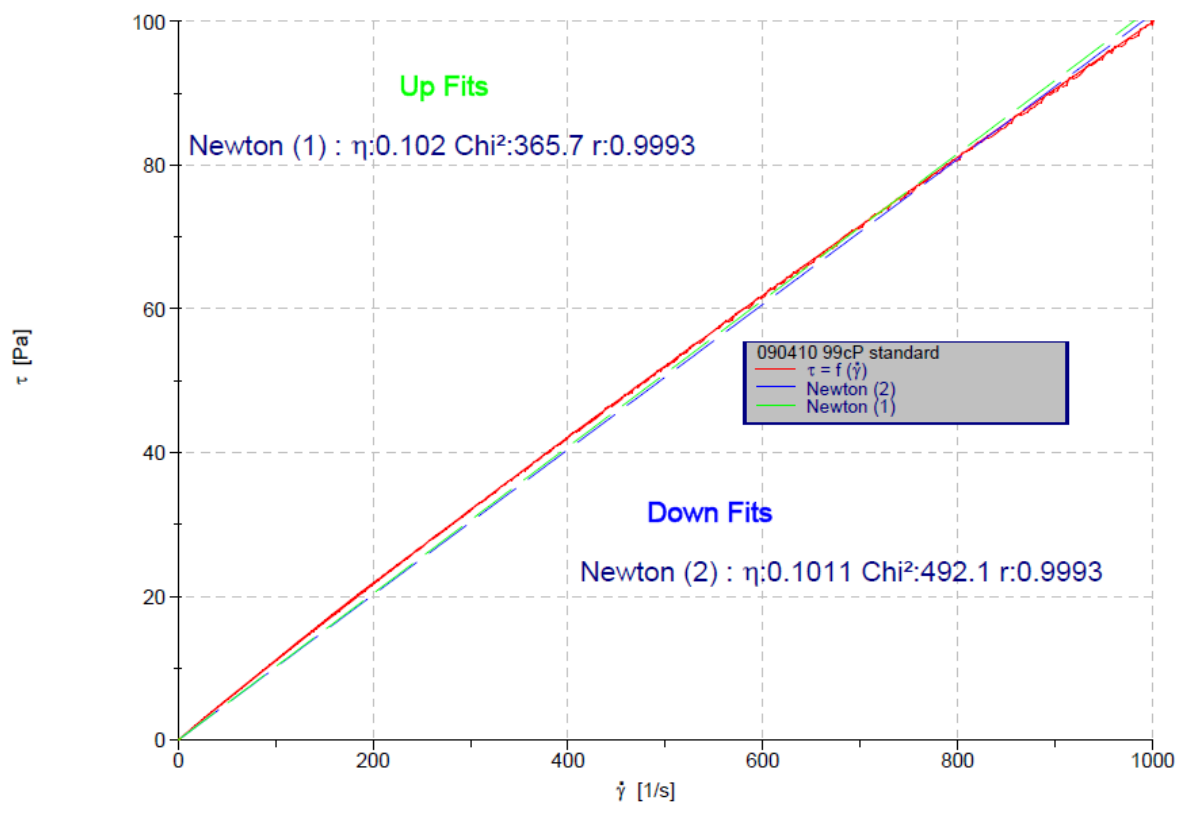

Figure 4. Flow curve of the $99.0 \mathrm{cP}$ Newtonian standard run on the Haake RS-600 rheometer 
The analysis was performed in accordance with procedure TPR-WTP-PEP-049 and the data were collected digitally using the RHEOPLUS/32 V3.21 21003751-33024 software (for flow curve) and RHEOWIN V2.96 software (for shear strength). The same software was used to operate the corresponding rheometers. For the flow curve measurement, the specimens were prepared for analysis by shaking the bottle before extracting the sample. In order to obtain supernatant from the slurry, the specimen was centrifuged at 1000 times $g$ for 1 hour. Experimental details were recorded in laboratory record book 60127.

Flow curve tests for both slurry and supernatant were done with the cup and bob sensor system CC27-SN13634. Shear stress was measured as a function of shear rate while the shear rate was ramped from 0 to 1000 1/sec over $5 \mathrm{~min}$, followed by a 1 minute hold at 1000 1/sec and a ramp down to $01 / \mathrm{sec}$ over 5 minutes. Each specimen was generally measured three times. An initial measurement ("FRESH RUN") was made on a fresh sample taken from the specimen, that same sample was then re-run a second time ("RERUN"). The rheometer was cleaned, and a third measurement ("REPEAT FRESH RUN") was made by taking a second aliquot from the specimen. Note that the sample, B-02AML-019-XX-4146-RHE4, was measured only two times, "FRESH RUN" and "RERUN", due to insufficient amount of the supernatant for the measurement. Samples were placed in the cup up to the designated fill line (about 8 to 10-mL). Settling cannot be prevented during the measurement if the particles are large and the viscosity low; however, no evidence of significant settling was seen in the data or the cup after testing.

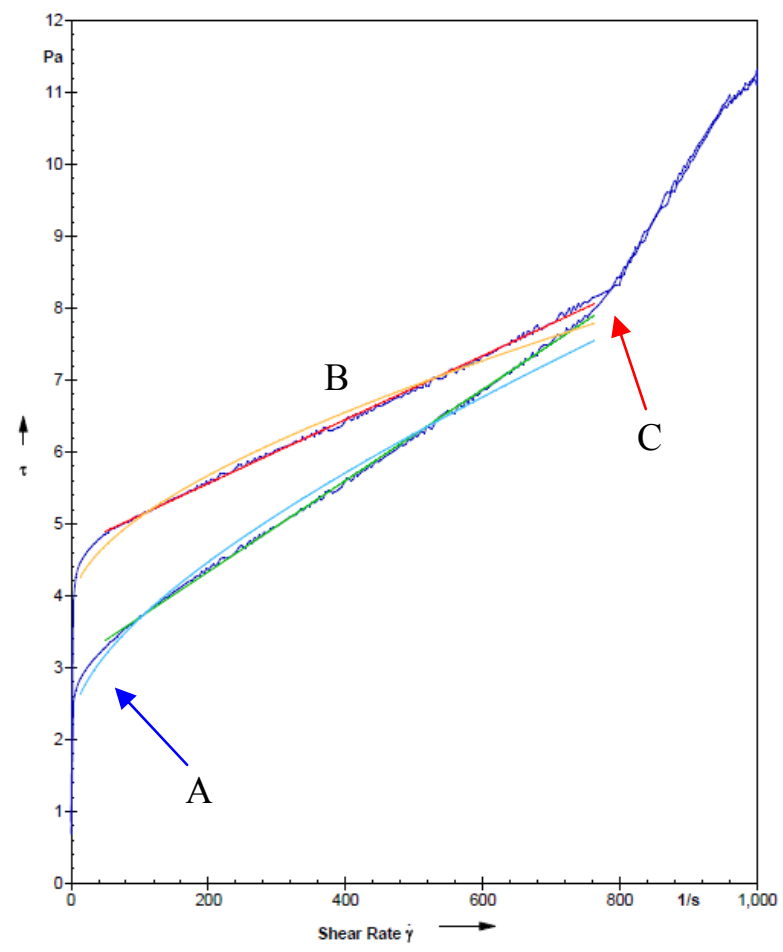

Figure 5. Typical rheogram of a slurry sample with low yield stress

Figure 5 is a typical rheogram of a slurry sample with low yield stress (sample B-02AOL-025-XX-4365-RHE4). There are three features that were typically characteristic of many slurry specimens: i) a yield stress at the onset of flow, (indicated by the blue arrow, "A"), ii) an approximately linear region where shear stress is linearly proportional to shear rate "B", and iii) a discontinuity where the slope changes and where there are more fluctuations in the data (starting about 
800 1/sec in Figure 5, indicated by the red arrow, "C"). The data at very low shear rates was not included in the analysis to avoid overweighting the Bingham plastic and Casson curve fit calculations performed by the RHEOPLUS/32 V3.21 21003751-33024 software. Slope changes and data fluctuation as shown in "C" above made it difficult to fit the data to a single mathematical expression because such behavior is not included in existing fitting models. Several different factors might cause such behavior: the flow instability associated with laminar Taylor vortices and other disturbances such as cavitation, minor settling, or the release of entrained gas. Previously it was presumed that these flow instabilities were associated with Taylor vortices. The data analysis team evaluated these instabilities and decided to perform curve fit analysis using only the data prior to the discontinuity (e.g., up to about $8001 / \mathrm{sec}$ as shown above).

A simple hydrodynamic theory may provide a reasonable estimate and physical insights for instability behavior. The hydrodynamic transition from laminar Couette flow to laminar Taylor vortices occurs when the Reynolds number of the experiment exceeds a geometrically determined critical Reynolds number, $\operatorname{Re}_{c}$. Assuming the infinite length of the fluid column, the critical Reynolds number in our case may be described by ${ }^{3}$

$$
\operatorname{Re}_{c}=41.2 \sqrt{\frac{\left(R_{o}+R_{i}\right)}{2 h}}
$$

where $R_{i}$ and $R_{o}$ denote bob radius and cup radius. Here $h$ denotes a gap between the bob and cup, which is $R_{o}-R_{i}$. The Reynolds number of the experiment, Re, can be defined by $\rho h \Omega R_{i} / \mu_{a p p}$ where $\rho, \Omega$, and $\mu_{a p p}$ represent the density of the sample, the angular velocity of the bob, and the apparent viscosity of the sample respectively. Using the geometries of the cup and bob sensor system CC27-SN13634, the critical Reynolds number is expected to be about 145 . For our experimental set up, $\operatorname{Re} \approx 112$ at $8001 / \mathrm{sec}$ using sample density and apparent viscosity values of $1.2 \mathrm{~g} / \mathrm{cm}^{3}$ and $10.5 \mathrm{cP}$, respectively. Considering that the formula for the critical Reynolds number is based on a Newtonian fluid, one can expect that two Reynolds numbers are comparable and the hydrodynamic transition to laminar Taylor vortices occurs around $8001 /$ sec. Consequently, the slope changes and data fluctuation as shown in "C" may be due to the onset of the laminar Taylor vortices although other disturbances are possibly coupled.

Applying this to the supernatant samples, one may expect that the transition occurs at a much smaller shear rate since the viscosity of the supernatant is typically 10 times less than that of the slurry sample, which was indeed observed in our tests; such transition occurs typically about 100 1/sec. Figure 6 shows a typical rheogram of a supernatant sample (sample B-02AOL-025-XX-4366-RHE4), indicating that the slope change and more fluctuation (indicated by the red arrow, "C") starts around $1201 /$ sec below which the curve fit analysis can be performed.

3. L. D. Landau and E. M. Lifshitz, "Course of Theoretical Physics - Volume 6: Fluid Mechanics," 2 ${ }^{\text {nd }}$ Ed., Butterworth-Heinemann, Oxford (1987). 


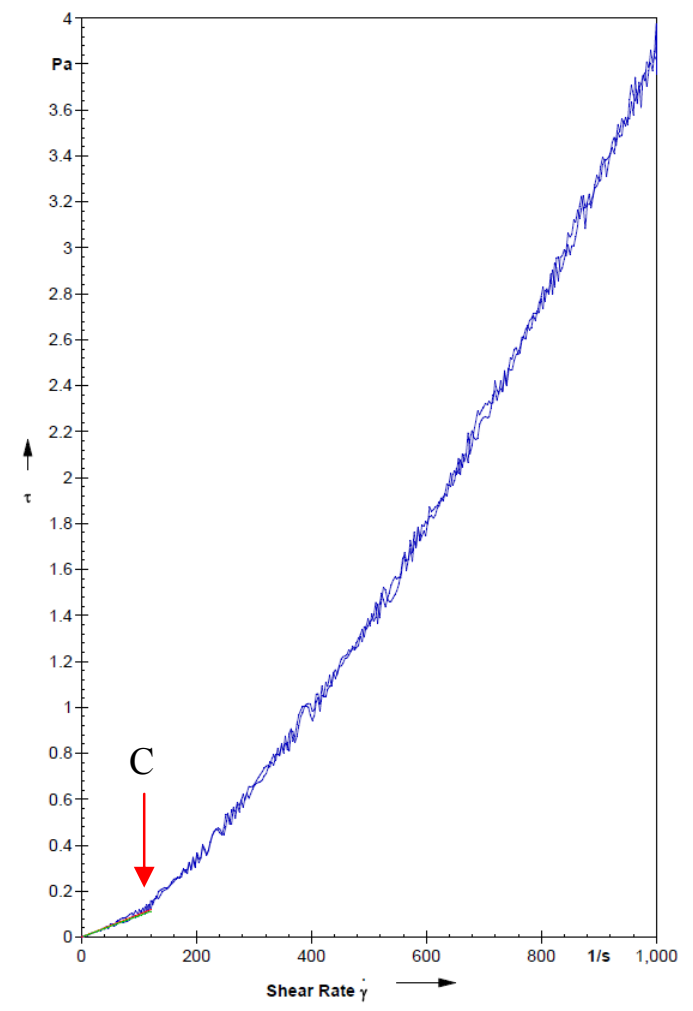

Figure 6. Typical rheogram of a supernatant sample

In the previous Shakedown Test and Integrated Test A, all slurry samples showed negligible or low yield stresses, typically 0-4 Pa (see TDP-WTP-355 and TDP-WTP-359 for details). Unlike slurry samples in both tests, there were several slurry samples with intermediate yield stresses in the Integrated Test B.

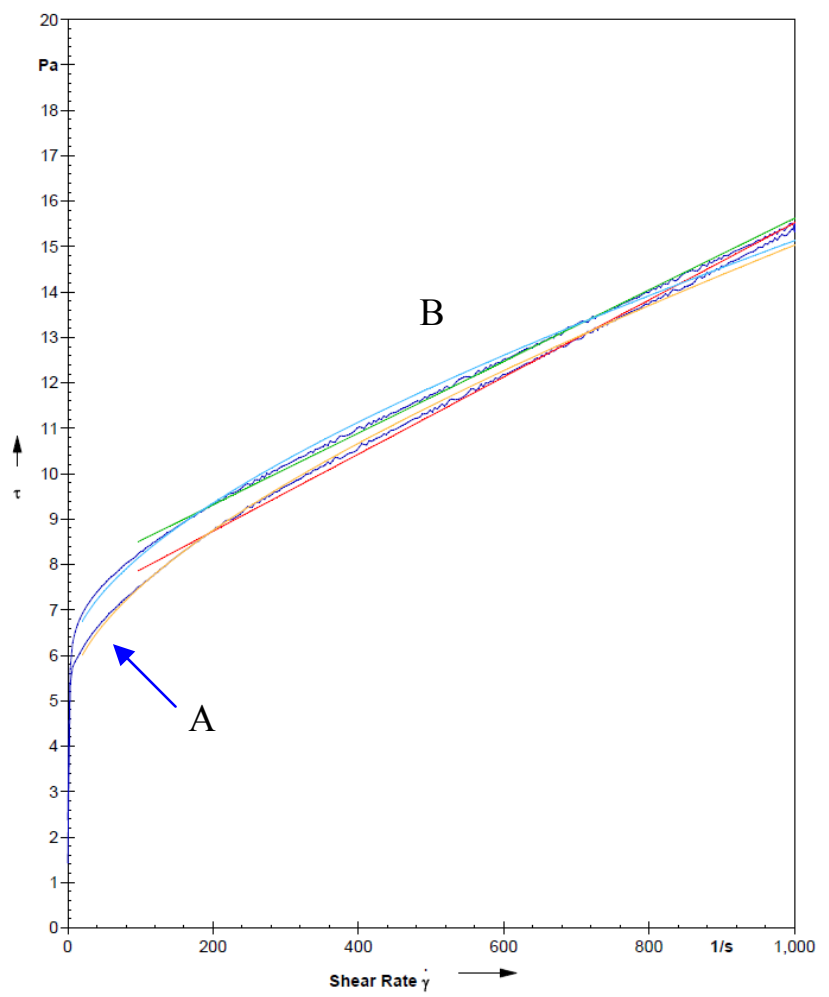

Figure 7. Typical rheogram of a slurry sample with intermediate yield stress 
Figure 7 is an example is a rheogram of a slurry sample with intermediate yield stress (sample B-02AOL-020-10-4194-RHE4). In this case, the slope change and more fluctuation were not observed in contrast to the previous two examples. It is mainly due to higher apparent viscosity, about $17-18 \mathrm{cP}$.

Following a similar methodology, one can calculate $\operatorname{Re} \approx 84$ even at $10001 / \mathrm{sec}$ using the density value of $1.2 \mathrm{~g} / \mathrm{cm}^{3}$, which is quite smaller than 145 of the critical Reynolds number. Thus, we do not expect any instability due to the onset of the laminar Taylor vortices.

The flow curves for the most of the samples did not show significant hysteresis and the data were fit with Newtonian, Bingham Plastic, and Casson flow-curve descriptors using the RHEOPLUS curve fit software, depending on the samples' rheological behavior. The software fits the entire range of data collected. However, as explained previously, a partial range of data was used to obtain fitting parameters for most of samples in the Integrated Test B because of the slope changes and/or fluctuations. The partial ranges of data were typically 50-700 1/sec (for low yield stress samples) and 50-1000 1/sec (for high yield stress samples) for slurries. 0-120 1/sec was typically used for supernatant samples.

For shear strength test, a $1.6 \times 1.6 \mathrm{~cm}$ vane shear spindle was used and an enough space (at least $1.6 \mathrm{~cm}$ away from the top solid bed and the side wall and bottom of a test container) was provided for proper measurements. A and $\mathrm{M}$ factors for the tests are $116568.602 \mathrm{~Pa} / \mathrm{N} \cdot \mathrm{m}$ and $1.0(1 / \mathrm{sec})(\mathrm{rad} / \mathrm{sec})$ respectively indicated in RHEOWIN V2.96 software. Settling times for the test with the same sample are 1, 2, 4, 8, 16, 32, and $72 \mathrm{hrs}$. For each settling time, shear stresses were collected as a function of duration time under a constant shear rate $(0.03 \mathrm{1} / \mathrm{sec})$ during 2 minutes. Then, a maximum shear stress was obtained for each settling time.

The following table is a brief summary of samples and their specifications (chronological order). Note that all measurements were done at $25^{\circ} \mathrm{C}$.

\begin{tabular}{|l|l|l|l|}
\hline Sample Name & $\begin{array}{l}\text { Date } \\
\text { received }\end{array}$ & $\begin{array}{l}\text { Date } \\
\text { Tested }\end{array}$ & Comments \\
\hline B-02AML-019-XX-4470-RHE4 & $03 / 18 / 09$ & $03 / 18 / 09$ & Bingham \\
\hline $\begin{array}{l}\text { B-02AOL-025-XX-4366-RHE4 } \\
\text { (supernatant) }\end{array}$ & $03 / 23 / 09$ & $03 / 26 / 09$ & Newtonian \\
\hline B-02AOL-025-XX-4365-RHE4 & $03 / 23 / 09$ & $03 / 24 / 09$ & Bingham \\
\hline B-02AOL-025-07-4348-RHE4 & $03 / 23 / 09$ & $03 / 24 / 09$ & Bingham \\
\hline $\begin{array}{l}\text { B-02AOL-024-06-4328-RHE4 } \\
\text { (supernatant) }\end{array}$ & $03 / 23 / 09$ & $03 / 26 / 09$ & Newtonian \\
\hline $\begin{array}{l}\text { B-02AOL-025-07-4349-RHE4 } \\
\text { (supernatant) }\end{array}$ & $03 / 23 / 09$ & $03 / 26 / 09$ & Newtonian \\
\hline $\begin{array}{l}\text { B-02AOL-020-10-4194-RHE4 } \\
\text { B-02AOL-020-XX-4216-RHE4 }\end{array}$ & $03 / 23 / 09$ & $03 / 24 / 09$ & Bingham \\
\hline $\begin{array}{l}\text { B-23/09 } \\
\text { B-02AOL-020-XX-4217-RHE4 } \\
\text { (supernatant) }\end{array}$ & $03 / 23 / 09$ & $03 / 26 / 09$ & Newtonian \\
\hline $\begin{array}{l}\text { B-02AOL-020-10-4195-RHE4 } \\
\text { (supernatant) }\end{array}$ & $03 / 23 / 09$ & $03 / 26 / 09$ & Newtonian \\
\hline B-02AML-019-XX-4145-RHE4 & $03 / 23 / 09$ & $03 / 25 / 09$ & Bingham \\
\hline $\begin{array}{l}\text { B-02AML-019-XX-4146-RHE4 } \\
\text { (supernatant) }\end{array}$ & $03 / 23 / 09$ & $04 / 06 / 09$ & Newtonian \\
\hline
\end{tabular}




\begin{tabular}{|l|l|l|l|}
\hline Sample Name & $\begin{array}{l}\text { Date } \\
\text { received }\end{array}$ & $\begin{array}{l}\text { Date } \\
\text { Tested }\end{array}$ & Comments \\
\hline B-02AOL-020-01-4165-RHE4 & $03 / 23 / 09$ & $04 / 01 / 09$ & Bingham \\
\hline A-02AOL-014-01-6011-RHE4 & $03 / 23 / 09$ & $04 / 01 / 09$ & Bingham \\
\hline $\begin{array}{l}\text { A-02AML-022-XX-2465-RHE4 } \\
\text { (supernatant) }\end{array}$ & $03 / 23 / 09$ & $04 / 01 / 09$ & Newtonian \\
\hline B-02AOL-023-XX-4290-RHE4 & $03 / 23 / 09$ & $04 / 06 / 09$ & Bingham \\
\hline B-02AML-005-XX-2950-RHE4 & $03 / 23 / 09$ & $04 / 06 / 09$ & Bingham \\
\hline B-02AML-005-XX-2923-RHE4 & $03 / 23 / 09$ & $04 / 06 / 09$ & Newtonian \\
\hline B-T22GM-002-XX-2904-RHE4 & $03 / 23 / 09$ & $04 / 10 / 09$ & Newtonian \\
\hline B-02AML-014-XX-4031-RHE4 & $03 / 23 / 09$ & $04 / 10 / 09$ & Bingham \\
\hline $\begin{array}{l}\text { B-02AML-019-XX-4129-RHE4 } \\
\text { (supernatant) }\end{array}$ & $03 / 23 / 09$ & $04 / 01 / 09$ & Newtonian \\
\hline B-02AML-019-XX-4128-RHE4 & $03 / 23 / 09$ & $04 / 10 / 09$ & $\begin{array}{l}\text { Very Slightly } \\
\text { Bingham }\end{array}$ \\
\hline $\begin{array}{l}\text { B-02AML-014-XX-4004-RHE4 } \\
\text { B-02AOL-023-XX-4288-RHE4 }\end{array}$ & $03 / 23 / 09$ & $04 / 10 / 09$ & Newtonian \\
\hline 4288-RHE4) & $03 / 09$ & - & $\begin{array}{l}\text { No Shear Strength } \\
\text { test is needed }\end{array}$ \\
\hline B-02AOL-023-XX-4289-RHE4 & $03 / 23 / 09$ & $04 / 17 / 09$ & Shear Strength \\
\hline
\end{tabular}

\section{Summary of Results}

One sample, B-02AOL-023-XX-4289-RHE4 (identical to B-02AOL-023-XX-4288-RHE4), was tested with a $1.6 \times 1.6 \mathrm{~cm}$ vane shear spindle (Haake RS-600). The sample showed a continuous increase in the maximum shear strength (within the experimental uncertainty) as the settling time increases and the complete asymptote was not observed due to insufficient settling time. Considering both the limited settling time and an asymptotic behavior as settling time approaches $72 \mathrm{hrs}$, the sample appear to be adequate for handling in the WTP (i.e., less than $30 \mathrm{~Pa}$ of the maximum shear strength). However, the shear strength measurement with a longer settling time might be needed to ensure an asymptotic behavior of the sample.

Some samples were run only as slurries and some only as supernatants as was needed for the matrix support for that part of the test plan. Most slurry samples were reasonably well fitted with the Bingham plastic model. All supernatants did not exhibit a yield stress and Newtonian fits appear to be the best fit. 


\begin{tabular}{|c|c|c|c|c|c|c|c|c|c|c|c|}
\hline & Rheology fits & $\begin{array}{c}\text { Bingham } \\
\text { Up }\end{array}$ & $\begin{array}{c}\text { Bingham } \\
\text { Up }\end{array}$ & $\begin{array}{l}\text { Bingham } \\
\text { Down }\end{array}$ & $\begin{array}{l}\text { Bingham } \\
\text { Down }\end{array}$ & $\begin{array}{c}\text { Casson } \\
\text { Up }\end{array}$ & $\begin{array}{c}\text { Casson } \\
\text { Up }\end{array}$ & $\begin{array}{l}\text { Casson } \\
\text { Down }\end{array}$ & $\begin{array}{l}\text { Casson } \\
\text { Down }\end{array}$ & $\begin{array}{c}\text { Newtonian } \\
\text { Up }\end{array}$ & $\begin{array}{l}\text { Newtonian } \\
\text { Down }\end{array}$ \\
\hline & & $\begin{array}{l}\text { Yield } \\
\text { Stress }\end{array}$ & Consistency & $\begin{array}{l}\text { Yield } \\
\text { Stress }\end{array}$ & Consistency & $\begin{array}{l}\text { Yield } \\
\text { Stress }\end{array}$ & Consistency & $\begin{array}{l}\text { Yield } \\
\text { Stress }\end{array}$ & Consistency & & \\
\hline \# & Sample names & $\operatorname{tau}(\mathrm{Pa})$ & eta $(\mathrm{Pa} \cdot \mathrm{sec})$ & $\operatorname{tau}(\mathrm{Pa})$ & eta $(\mathrm{Pa} \cdot \mathrm{sec})$ & $\operatorname{tau}(\mathrm{Pa})$ & eta $(\mathrm{Pa} \cdot \mathrm{sec})$ & $\operatorname{tau}(\mathrm{Pa})$ & eta $(\mathrm{Pa} \cdot \mathrm{sec})$ & eta $(\mathrm{Pa} \cdot \mathrm{sec})$ & eta $(\mathrm{Pa} \cdot \mathrm{sec})$ \\
\hline 1 & $\begin{array}{l}\text { B 02AML } 019 \text { XX } 4470 \\
\text { RHE } 4 \\
\text { Average }\end{array}$ & 2.357 & $2.153 \mathrm{E}-02$ & 1.963 & $2.143 \mathrm{E}-02$ & 0.661 & $1.604 \mathrm{E}-02$ & 0.534 & $1.624 \mathrm{E}-02$ & N/A & $\mathrm{N} / \mathrm{A}$ \\
\hline 2 & $\begin{array}{l}\text { B 02AOL } 025 \text { XX } 4366 \\
\text { RHE } 4 \\
\text { (supernatant) }\end{array}$ & & & & & & & & & & \\
\hline & Average & N/A & N/A & N/A & N/A & N/A & N/A & N/A & $\mathrm{N} / \mathrm{A}$ & $9.785 \mathrm{E}-04$ & $9.507 \mathrm{E}-04$ \\
\hline 3 & $\begin{array}{l}\text { B 02AOL } 025 \text { XX } 4365 \\
\text { RHE } 4 \\
\text { Average }\end{array}$ & 4.136 & 4.856E-03 & 2.991 & $6.296 \mathrm{E}-03$ & N/A & N/A & N/A & $\mathrm{N} / \mathrm{A}$ & $\mathrm{N} / \mathrm{A}$ & $\mathrm{N} / \mathrm{A}$ \\
\hline 4 & $\begin{array}{l}\text { B 02AOL } 025074348 \\
\text { RHE } 4\end{array}$ & & & & & & & & & & \\
\hline & Average & 4.708 & $5.181 \mathrm{E}-03$ & 4.081 & 5.991E-03 & N/A & N/A & N/A & $\mathrm{N} / \mathrm{A}$ & $\mathrm{N} / \mathrm{A}$ & $\mathrm{N} / \mathrm{A}$ \\
\hline 5 & $\begin{array}{l}\text { B 02AOL } 024064328 \\
\text { RHE } 4 \\
\text { (supernatant) }\end{array}$ & & & & & & & & & & \\
\hline & Average & N/A & $\mathrm{N} / \mathrm{A}$ & N/A & N/A & N/A & N/A & N/A & $\mathrm{N} / \mathrm{A}$ & $1.034 \mathrm{E}-03$ & $1.054 \mathrm{E}-03$ \\
\hline 6 & $\begin{array}{l}\text { B 02AOL } 025074349 \\
\text { RHE } 4 \\
\quad \text { (supernatant) }\end{array}$ & & & & & & & & & & \\
\hline 7 & $\begin{array}{l}\text { Average } \\
\text { B 02AOL } 020104194 \\
\text { RHE } 4\end{array}$ & N/A & $\mathrm{N} / \mathrm{A}$ & N/A & N/A & N/A & N/A & $\mathrm{N} / \mathrm{A}$ & $\mathrm{N} / \mathrm{A}$ & $9.664 \mathrm{E}-04$ & $9.916 \mathrm{E}-04$ \\
\hline & Average & 7.280 & 8.351E-03 & 7.802 & 7.925E-03 & 5.171 & $2.620 \mathrm{E}-03$ & 5.744 & $2.267 \mathrm{E}-03$ & $\mathrm{~N} / \mathrm{A}$ & $\mathrm{N} / \mathrm{A}$ \\
\hline 8 & $\begin{array}{l}\text { B 02AOL } 020 \text { XX } 4216 \\
\text { RHE } 4\end{array}$ & & & & & & & & & & \\
\hline & Average & 10.758 & $8.368 \mathrm{E}-03$ & 11.600 & $7.632 \mathrm{E}-03$ & 8.387 & $2.009 \mathrm{E}-03$ & 9.277 & $1.655 \mathrm{E}-03$ & $\mathrm{~N} / \mathrm{A}$ & $\mathrm{N} / \mathrm{A}$ \\
\hline
\end{tabular}




\begin{tabular}{|c|c|c|c|c|c|c|c|c|c|c|c|}
\hline & Rheology fits & $\begin{array}{c}\text { Bingham } \\
\text { Up }\end{array}$ & Bingham & $\begin{array}{l}\text { Bingham } \\
\text { Down }\end{array}$ & $\begin{array}{l}\text { Bingham } \\
\text { Down }\end{array}$ & $\begin{array}{l}\text { Casson } \\
\text { Up }\end{array}$ & $\begin{array}{l}\text { Casson } \\
\text { Up }\end{array}$ & $\begin{array}{l}\text { Casson } \\
\text { Down }\end{array}$ & $\begin{array}{l}\text { Casson } \\
\text { Down }\end{array}$ & $\begin{array}{l}\text { Newtonian } \\
\text { Up..... }\end{array}$ & $\begin{array}{c}\text { Newtonian } \\
\text { Down }\end{array}$ \\
\hline & & $\begin{array}{l}\text { Yield } \\
\text { Stress }\end{array}$ & Consistency & $\begin{array}{l}\text { Yield } \\
\text { Stress }\end{array}$ & Consistency & $\begin{array}{l}\text { Yield } \\
\text { Stress }\end{array}$ & Consistency & $\begin{array}{l}\text { Yield } \\
\text { Stress }\end{array}$ & Consistency & & \\
\hline \# & Sample names & $\operatorname{tau}(\mathrm{Pa})$ & eta $(\mathrm{Pa} \cdot \mathrm{sec})$ & $\operatorname{tau}(\mathrm{Pa})$ & eta $(\mathrm{Pa} \cdot \mathrm{sec})$ & tau $(\mathrm{Pa})$ & eta $(\mathrm{Pa} \cdot \mathrm{sec})$ & tau $(\mathrm{Pa})$ & eta $(\mathrm{Pa} \cdot \mathrm{sec})$ & eta $(\mathrm{Pa} \cdot \mathrm{sec})$ & eta $(\mathrm{Pa} \cdot \mathrm{sec})$ \\
\hline 9 & $\begin{array}{l}\text { B 02AOL } 020 \text { XX } 4217 \\
\text { RHE } 4 \\
\quad \text { (supernatant) } \\
\text { Average }\end{array}$ & $\mathrm{N} / \mathrm{A}$ & N/A & $\mathrm{N} / \mathrm{A}$ & N/A & N/A & N/A & N/A & N/A & $1.029 \mathrm{E}-03$ & $1.047 \mathrm{E}-03$ \\
\hline 10 & $\begin{array}{l}\text { B 02AOL } 020104195 \\
\text { RHE } 4 \\
\quad \text { (supernatant) }\end{array}$ & & & & & & & & & & \\
\hline & Average & N/A & $\mathrm{N} / \mathrm{A}$ & $\mathrm{N} / \mathrm{A}$ & N/A & $\mathrm{N} / \mathrm{A}$ & N/A & $\mathrm{N} / \mathrm{A}$ & N/A & $1.168 \mathrm{E}-03$ & $1.172 \mathrm{E}-03$ \\
\hline 11 & $\begin{array}{l}\text { B 02AML } 019 \text { XX } 4145 \\
\text { RHE } 4 \\
\text { Average }\end{array}$ & 9.172 & $3.001 \mathrm{E}-02$ & 7.594 & $3.035 \mathrm{E}-02$ & 4.287 & $1.677 \mathrm{E}-02$ & 3.381 & $1.773 \mathrm{E}-02$ & $\mathrm{~N} / \mathrm{A}$ & $\mathrm{N} / \mathrm{A}$ \\
\hline 12 & $\begin{array}{l}\text { B 02AML } 019 \text { XX } 4146 \\
\text { RHE } 4 \\
\quad \text { (supernatant) } \\
\text { Average }\end{array}$ & N/A & N/A & $\mathrm{N} / \mathrm{A}$ & N/A & $\mathrm{N} / \mathrm{A}$ & N/A & N/A & N/A & $8.874 \mathrm{E}-03$ & $8.853 \mathrm{E}-03$ \\
\hline 13 & $\begin{array}{l}\text { B 02AOL } 020014165 \\
\text { RHE } 4 \\
\text { Average }\end{array}$ & 9.223 & $3.005 \mathrm{E}-02$ & 7.587 & $3.046 \mathrm{E}-02$ & 4.252 & $1.694 \mathrm{E}-02$ & 3.380 & $1.779 \mathrm{E}-02$ & $\mathrm{~N} / \mathrm{A}$ & $\mathrm{N} / \mathrm{A}$ \\
\hline 14 & $\begin{array}{l}\text { A 02AOL } 014016011 \\
\text { RHE } 4 \\
\text { Average }\end{array}$ & 11.891 & $3.488 \mathrm{E}-02$ & 9.516 & $3.556 \mathrm{E}-02$ & 5.853 & $1.866 \mathrm{E}-02$ & 4.456 & $2.002 \mathrm{E}-02$ & $\mathrm{~N} / \mathrm{A}$ & $\mathrm{N} / \mathrm{A}$ \\
\hline 15 & $\begin{array}{l}\text { A 02AML } 022 \text { XX } 2465 \\
\text { RHE } 4 \\
\quad \text { (supernatant) } \\
\text { Average }\end{array}$ & $\mathrm{N} / \mathrm{A}$ & N/A & $\mathrm{N} / \mathrm{A}$ & N/A & N/A & N/A & $\mathrm{N} / \mathrm{A}$ & N/A & $9.975 \mathrm{E}-04$ & $9.990 \mathrm{E}-04$ \\
\hline 16 & $\begin{array}{l}\text { B 02AOL } 023 \text { XX } 4290 \\
\text { RHE } 4 \\
\text { Average }\end{array}$ & 12.250 & $7.255 \mathrm{E}-03$ & 11.887 & 7.684E-03 & 9.261 & $1.725 \mathrm{E}-03$ & 9.566 & $1.598 \mathrm{E}-03$ & $\mathrm{~N} / \mathrm{A}$ & $\mathrm{N} / \mathrm{A}$ \\
\hline
\end{tabular}




\begin{tabular}{|c|c|c|c|c|c|c|c|c|c|c|c|}
\hline & Rheology fits & $\begin{array}{l}\text { Bingham } \\
\text { Up }\end{array}$ & $\begin{array}{l}\text { Bingham } \\
\text { Up }\end{array}$ & $\begin{array}{l}\text { Bingham } \\
\text { Down }\end{array}$ & $\begin{array}{c}\text { Bingham } \\
\text { Down }\end{array}$ & $\begin{array}{c}\text { Casson } \\
\text { Up. }\end{array}$ & $\begin{array}{c}\text { Casson } \\
\text { Up. }\end{array}$ & $\begin{array}{l}\text { Casson } \\
\text { Down }\end{array}$ & $\begin{array}{l}\text { Casson } \\
\text { Down }\end{array}$ & $\begin{array}{c}\text { Newtonian } \\
\text { Up. }\end{array}$ & $\begin{array}{l}\text { Newtonian } \\
\text { Down }\end{array}$ \\
\hline & & $\begin{array}{l}\text { Yield } \\
\text { Stress }\end{array}$ & Consistency & $\begin{array}{l}\text { Yield } \\
\text { Stress }\end{array}$ & Consistency & $\begin{array}{l}\text { Yield } \\
\text { Stress }\end{array}$ & Consistency & $\begin{array}{l}\text { Yield } \\
\text { Stress }\end{array}$ & Consistency & & \\
\hline \# & Sample names & $\operatorname{tau}(\mathrm{Pa})$ & eta $(\mathrm{Pa} \cdot \mathrm{sec})$ & tau $(\mathrm{Pa})$ & eta $(\mathrm{Pa} \cdot \mathrm{sec})$ & tau $(\mathrm{Pa})$ & eta $(\mathrm{Pa} \cdot \mathrm{sec})$ & tau $(\mathrm{Pa})$ & eta $(\mathrm{Pa} \cdot \mathrm{sec})$ & eta $(\mathrm{Pa} \cdot \mathrm{sec})$ & eta $(\mathrm{Pa} \cdot \mathrm{sec})$ \\
\hline
\end{tabular}

17 B 02AML 005 XX 2950

RHE 4

Average

$2.916 \quad 1.004 \mathrm{E}-02$

$2.553 \quad 1.056 \mathrm{E}-02$

$1.634 \quad 4.860 \mathrm{E}-03$

$1.345 \quad 5.493 \mathrm{E}-03$

N/A

N/A

18 B 02AML 005 XX 2923 RHE 4

Average

N/A

N/A

N/A

N/A

N/A

N/A

N/A

N/A 4.845E-03 4.718E-03

19 B T22GM 002 XX 2904 RHE 4

Average

N/A

N/A N/A

N/A

N/A

N/A

N/A

N/A 4.188E-03 4.089E-03

20 B 02AML 014 XX 4031 RHE 4

Average

$2.610 \quad 1.050 \mathrm{E}-02$

$2.310 \quad 1.102 \mathrm{E}-02$

$1.374 \quad 5.435 \mathrm{E}-03$

$1.145 \quad 6.067 \mathrm{E}-03$

N/A

N/A

21 B 02AML 019 XX 4129

RHE 4

(supernatant)

Average

N/A N/A

N/A

N/A

N/A

N/A

N/A $\quad 8.685 E-03 \quad 8.628 E-03$

22 B 02AML 019 XX 4128

RHE 4

Average

$0.244 \quad 1.431 \mathrm{E}-02$

$0.199 \quad 1.401 \mathrm{E}-02$

$0.007 \quad 1.419 \mathrm{E}-02$

$0.002 \quad 1.423 \mathrm{E}-02 \quad 1.517 \mathrm{E}-02 \quad 1.475 \mathrm{E}-02$

23 B 02AML 014 XX 4004 RHE 4

Average

N/A N/A N/A

N/A N/A

N/A

N/A 5.299E-03 5.182E-03 


\section{L.2 Heat Capacity (TDP-WTP-372 and TDP-WTP-368)}




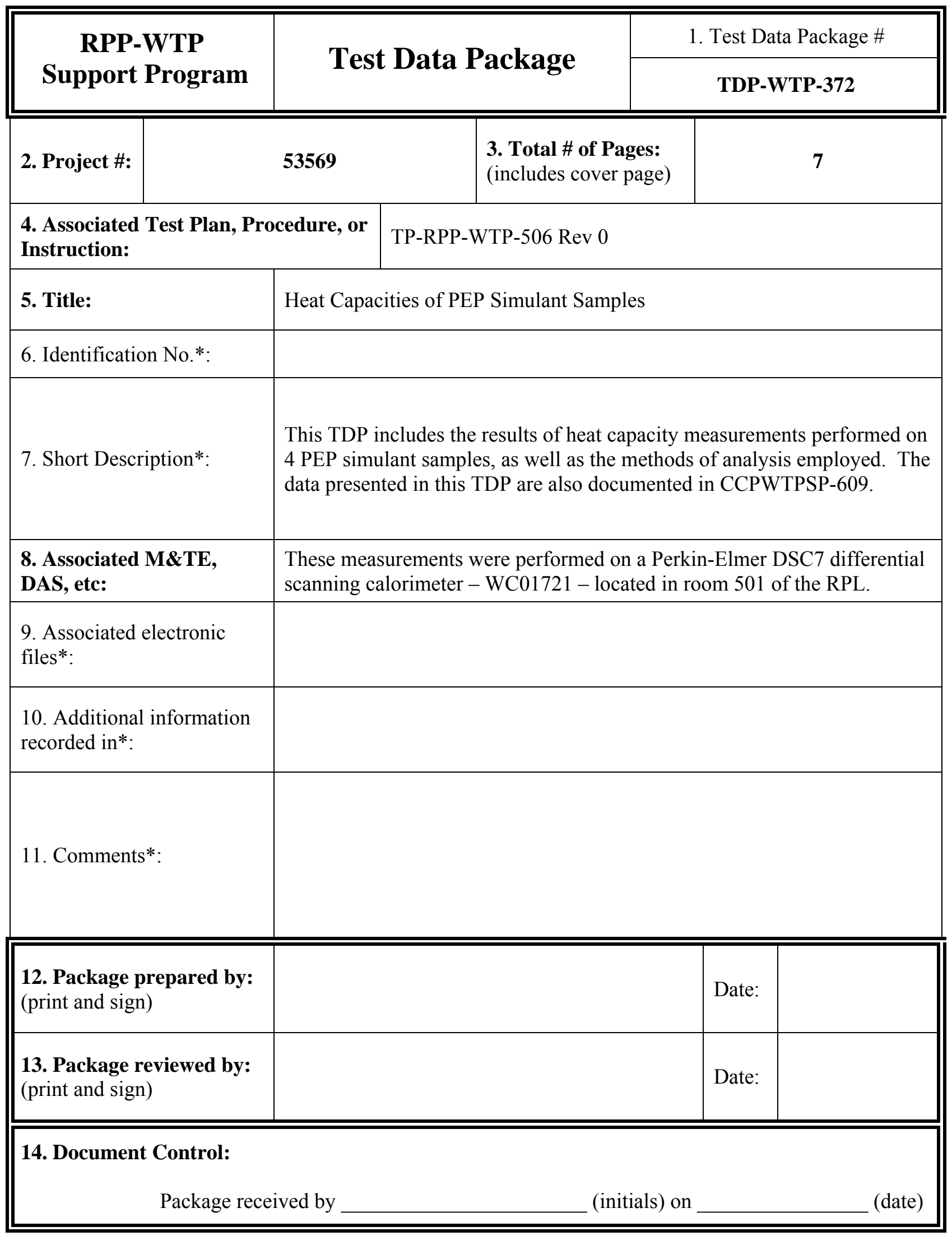




\section{PEP Slurry Sample Heat Capacity Report \\ Analysis}

This report presents results of heat capacity measurements for four PEP simulant slurry samples delivered via Chain of Custody form numbers WTP-COC-063-048 on 03/13/2009 and WTP-COC-063-051 on 03/16/2009. The samples provided were:

- B 02AML 005 XX 2949 HTC 3

- B 02AML 007 XX 2965 HTC 3

- B 02AML 008 BT 2972 HTC 3

- B 02AML 008002988 HTC 3

Three nominal $30 \mathrm{mg}$ subsamples were taken from each of these samples as they were vigorously stirred using a pipette with an enlarged tip to assure that the slurry samples were representative. We analyzed each slurry sample in triplicate, recognizing that obtaining a $30 \mathrm{mg}$ representative sample is challenging.

A Perkin-Elmer DSC7 differential scanning calorimeter (DSC) was used to determine the heat capacity of each subsample at temperatures between $20^{\circ} \mathrm{C}$ and $95^{\circ} \mathrm{C}$. The DSC7 was temperature- and enthalpy-calibrated using a gallium standard (NIST SRM 2234) (m.p. $29.8^{\circ} \mathrm{C}$ ) and/or an indium standard (NIST SRM 2232) (m.p. $156.6^{\circ} \mathrm{C}$ ).

The method used for heat capacity measurement was as provided in the DSC7 operating manual. In this method, the heat capacity of the empty sample pan is measured to provide the "baseline"; the sample pan was a gold "volatile" sample pan designed to contain volatile samples such as the water in these PEP slurries. The heat capacity of a reference or standard material such as sapphire or high purity water is then determined; the purity of these water standards was determined by measuring their electrical conductivity. Finally the heat capacity of the sample is determined. The measured heat capacity of the sample is adjusted to remove the contribution of the empty pan. We further adjusted the reported heat capacity by applying a measured response factor for the high purity water.

Optimally the heat capacity measurement approach would include analyzing the standard or reference material in the same container as the sample would be analyzed; however, because analysis of these slurry samples require a hermetic seal which cannot be broken and resealed, a different but nearly equivalent gold volatile sample pan is used for the water. In addition, we characterized a sapphire standard to demonstrate instrument performance but unfortunately, the heat capacity of sapphire $\left(0.8 \mathrm{~J} / \mathrm{g}{ }^{\circ} \mathrm{C}\right)$ is significantly lower than that of the PEP slurries $(\sim 3.5 \mathrm{~J} / \mathrm{g})$ and does not provide an adequate calibration adjustment.

\section{Results}

The expected results for the measurements can be generated by considering what the theoretical heat capacity would be for slurries with solid fraction in the range that are expected in the samples. The slurry is mostly water, which at $20^{\circ} \mathrm{C}$ is known to have a specific heat of $4.1818 \mathrm{~J} / \mathrm{g}^{\circ} \mathrm{C}$ [1]. It is also known that at $20^{\circ} \mathrm{C}$, the heat capacity of boehmite is roughly $0.9 \mathrm{~J} / \mathrm{g}^{\circ} \mathrm{C}$ [2]. So for slurries with mass fraction of $10 \%$, $20 \%$, and $30 \%$ boehmite in water, the theoretical heat capacities would be, respectively:

- $4.1818 \mathrm{~J} / \mathrm{g}^{\circ} \mathrm{C} * .9+0.9 \mathrm{~J} / \mathrm{g}^{\circ} \mathrm{C} * .1=3.85 \mathrm{~J} / \mathrm{g}^{\circ} \mathrm{C}$,

- $4.1818 \mathrm{~J} / \mathrm{g}^{\circ} \mathrm{C} * .80+0.9 \mathrm{~J} / \mathrm{g}^{\circ} \mathrm{C} * .2=3.52 \mathrm{~J} / \mathrm{g}^{\circ} \mathrm{C}$, and 
- $4.1818 \mathrm{~J} / \mathrm{g}^{\circ} \mathrm{C} * .7+0.9 \mathrm{~J} / \mathrm{g}^{\circ} \mathrm{C} * .3=\mathbf{3 . 2 0} \mathrm{J} / \mathrm{g}^{\circ} \mathrm{C}$.

Table 1 summarizes the results from these analyses by providing the mean measured heat capacities with their standard deviations. In general, the measured heat capacities are consistent with our expectations of hear capacities in the 3.2 to $3.8 \mathrm{~J} / \mathrm{g}^{\circ} \mathrm{C}$ assuming solids contents ranging from 10 to 30 -wt $\%$. We used only those analyses found to be internally self-consistent, not erratic and as-such, we discarded one measurement for sample B 02AML 005 XX 2949 HTC 3, one measurement for sample.

B 02AML 007 XX 2965 HTC 3, and one from B 02AML 008 BT 2972 HTC 3, but replaced it with a re-analysis.

Table 1. Results of Heat Capacity Measurements

\begin{tabular}{|l|r|r|r|r|}
\hline Temperature & $\begin{array}{l}\text { B 02AML 005 XX } \\
\text { 2949 HTC 3 } \\
\mathrm{C}_{\mathrm{p}}\left(\mathrm{J} / \mathrm{g}^{\circ} \mathrm{C}\right)\end{array}$ & $\begin{array}{l}\text { B 02AML 007 XX } \\
2965 \mathrm{HTC} 3 \\
\mathrm{C}_{\mathrm{p}}\left(\mathrm{J} / \mathrm{g}^{\circ} \mathrm{C}\right)\end{array}$ & $\begin{array}{l}\text { B 02AML 008 BT } \\
2972 \mathrm{HTC} 3 \\
\mathrm{C}_{\mathrm{p}}\left(\mathrm{J} / \mathrm{g}^{\circ} \mathrm{C}\right)\end{array}$ & $\begin{array}{l}\text { B 02AML 008 00 } \\
\text { 2988 HTC 3 } \\
\mathrm{C}_{\mathrm{p}}\left(\mathrm{J} / \mathrm{g}{ }^{\circ} \mathrm{C}\right)\end{array}$ \\
\hline $35^{\circ} \mathrm{C}$ & $2.98 \pm 0.00$ & $3.21 \pm 0.01$ & $3.23 \pm 0.03$ & $3.22 \pm 0.09$ \\
\hline $40^{\circ} \mathrm{C}$ & $2.97 \pm 0.00$ & $3.22 \pm 0.01$ & $3.25 \pm 0.02$ & $3.23 \pm 0.09$ \\
\hline $45^{\circ} \mathrm{C}$ & $2.97 \pm 0.00$ & $3.24 \pm 0.01$ & $3.26 \pm 0.03$ & $3.23 \pm 0.09$ \\
\hline $50^{\circ} \mathrm{C}$ & $2.96 \pm 0.02$ & $3.25 \pm 0.00$ & $3.26 \pm 0.03$ & $3.23 \pm 0.09$ \\
\hline $55^{\circ} \mathrm{C}$ & $2.96 \pm 0.02$ & $3.25 \pm 0.00$ & $3.27 \pm 0.02$ & $3.24 \pm 0.08$ \\
\hline $60^{\circ} \mathrm{C}$ & $2.96 \pm 0.02$ & $3.26 \pm 0.00$ & $3.28 \pm 0.02$ & $3.23 \pm 0.09$ \\
\hline $65^{\circ} \mathrm{C}$ & $2.95 \pm 0.03$ & $3.27 \pm 0.00$ & $3.28 \pm 0.02$ & $3.23 \pm 0.09$ \\
\hline $70^{\circ} \mathrm{C}$ & $2.95 \pm 0.04$ & $3.28 \pm 0.00$ & $3.29 \pm 0.02$ & $3.23 \pm 0.08$ \\
\hline $75^{\circ} \mathrm{C}$ & $2.95 \pm 0.04$ & $3.28 \pm 0.01$ & $3.30 \pm 0.02$ & $3.23 \pm 0.08$ \\
\hline $80^{\circ} \mathrm{C}$ & $2.96 \pm 0.05$ & $3.29 \pm 0.01$ & $3.29 \pm 0.02$ & $3.23 \pm 0.08$ \\
\hline $85^{\circ} \mathrm{C}$ & $2.97 \pm 0.05$ & $3.29 \pm 0.02$ & $3.30 \pm 0.02$ & $3.22 \pm 0.08$ \\
\hline $90^{\circ} \mathrm{C}$ & $2.98 \pm 0.05$ & $3.30 \pm 0.02$ & $3.30 \pm 0.02$ & $3.22 \pm 0.08$ \\
\hline
\end{tabular}

The results from each sample measurement are presented in graphical form in Figures 1-4. In general, the heat capacity will increase nominally linearly as a function of temperature. In general, the average measured heat capacities do increase linearly with temperature, although some deviations do exist. 


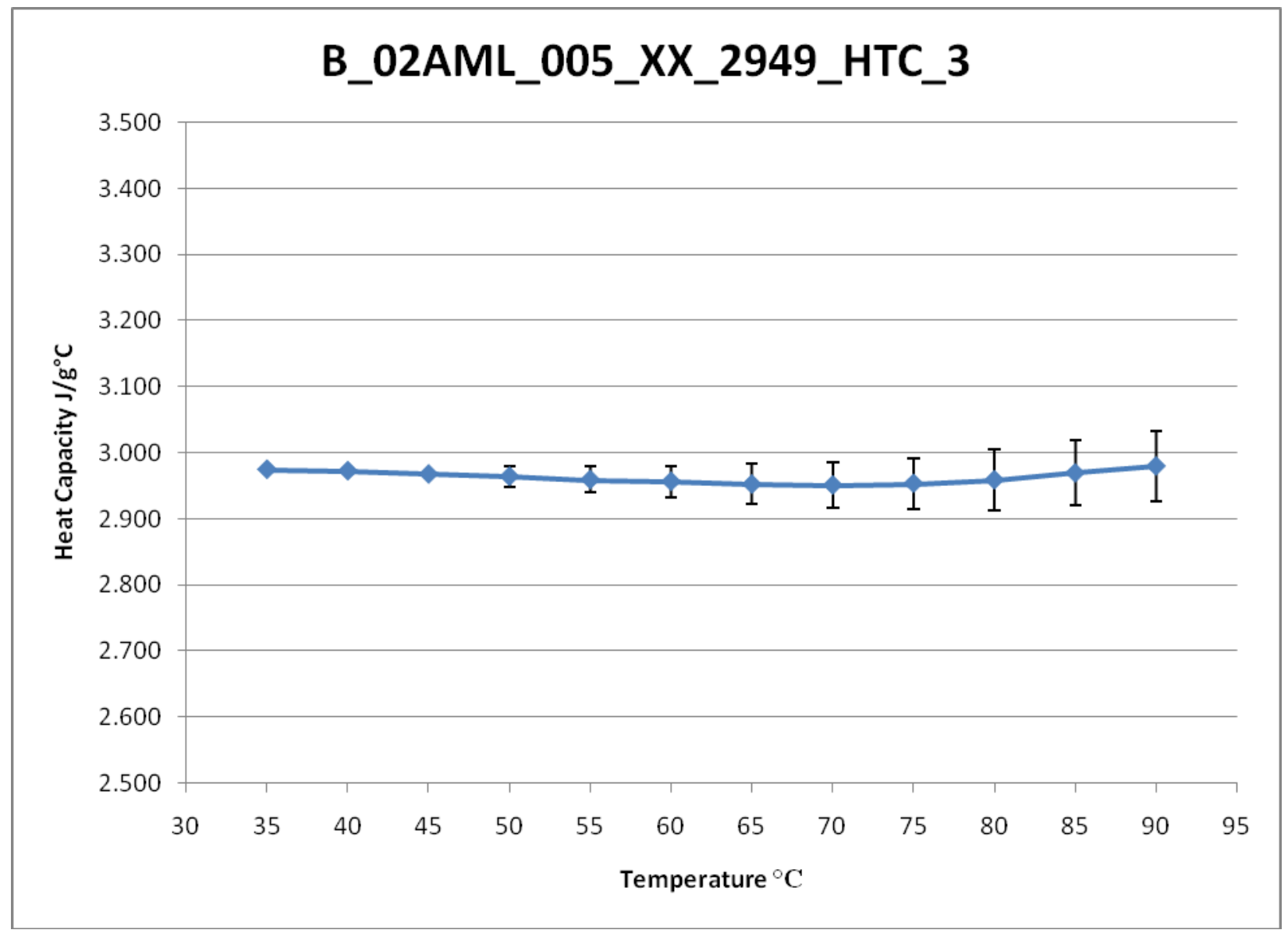

Figure 1. Heat Capacity Curve for Sample B_02AML_005_XX_2949_HTC_3 


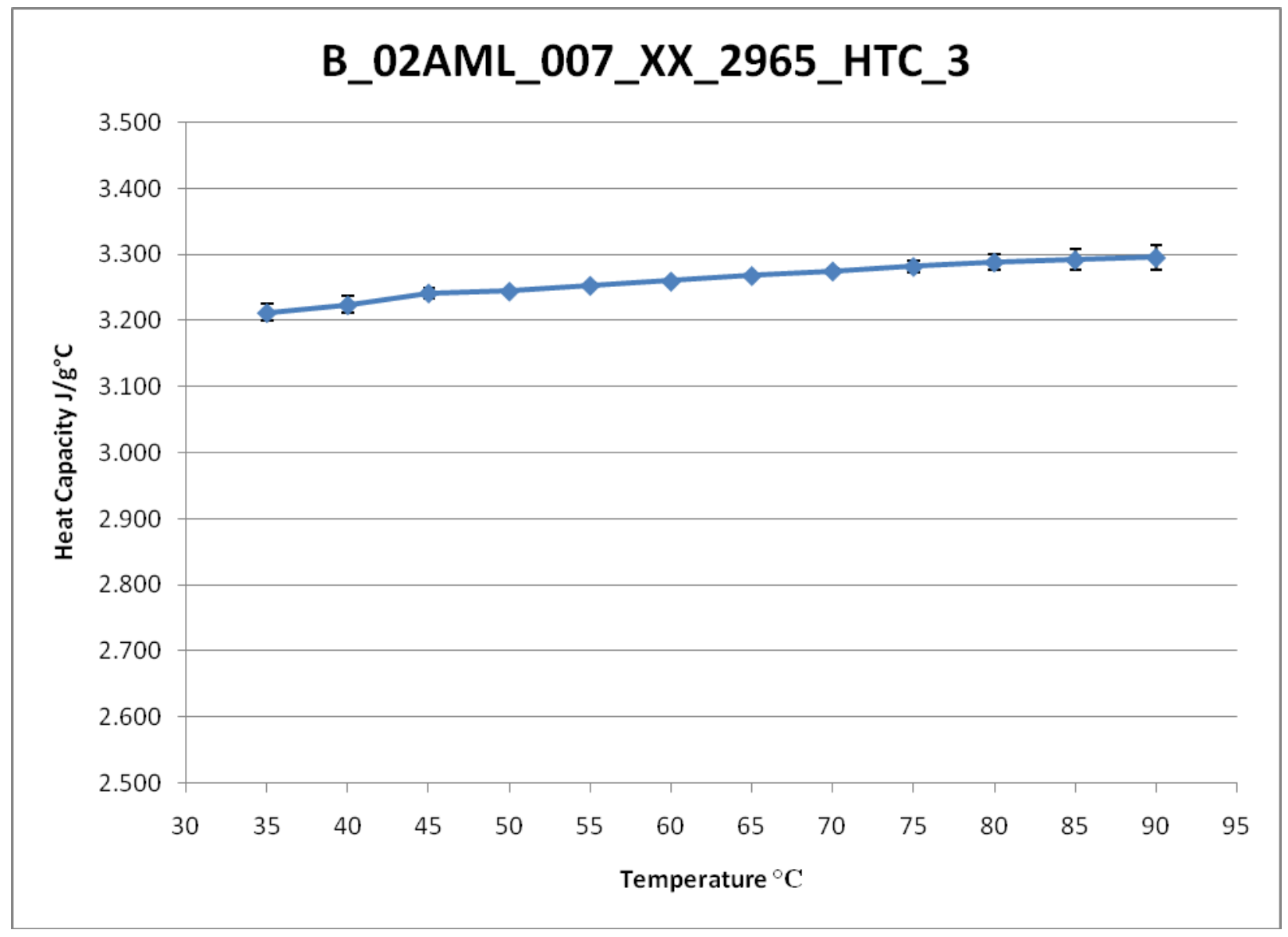

Figure 2. Heat Capacity Curve for Sample B_02AML_007_XX_2695_HTC_3 


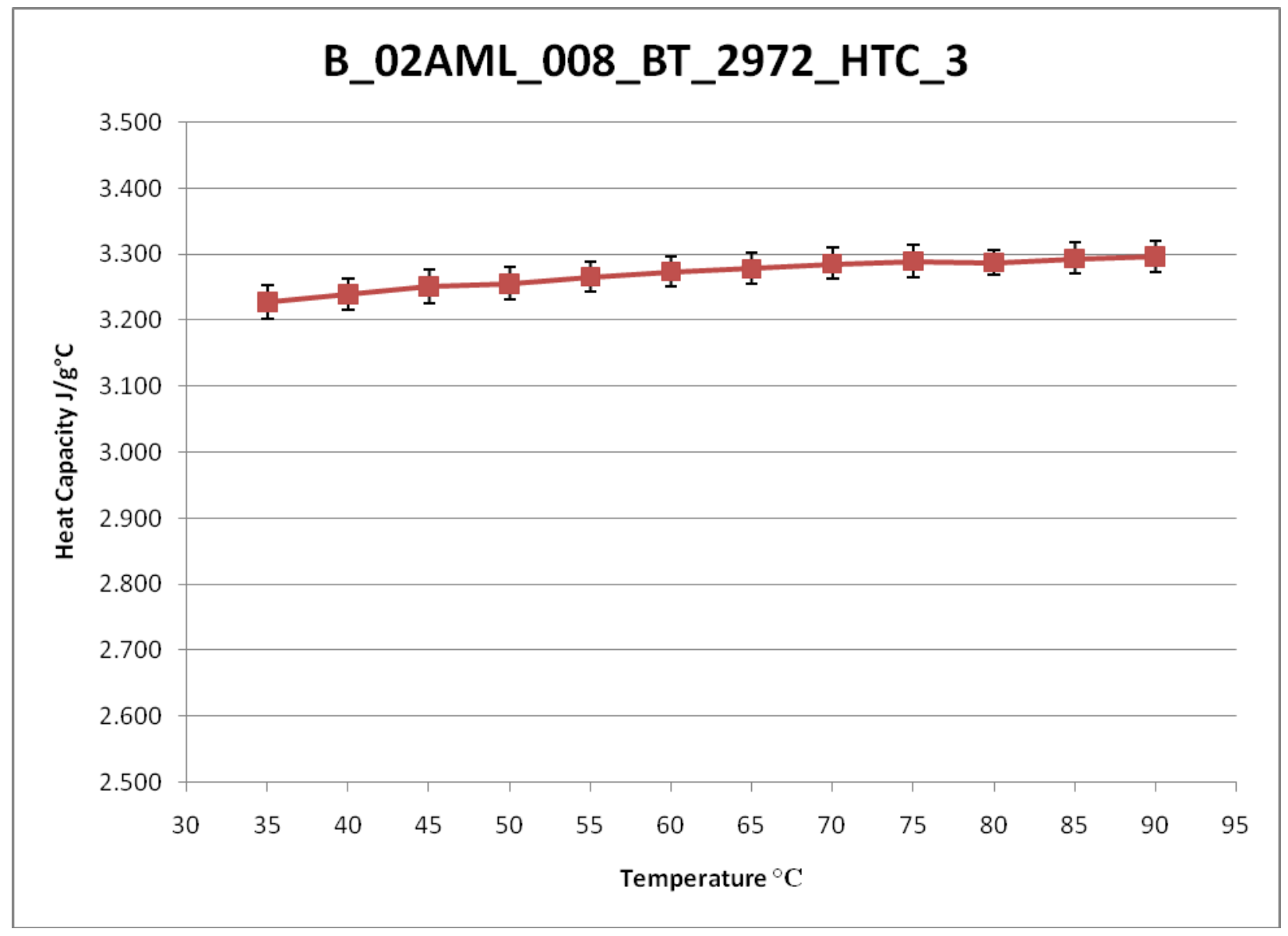

Figure 3. Heat Capacity Curve for Sample B_02AML_008_BT_2972_HTC_3 


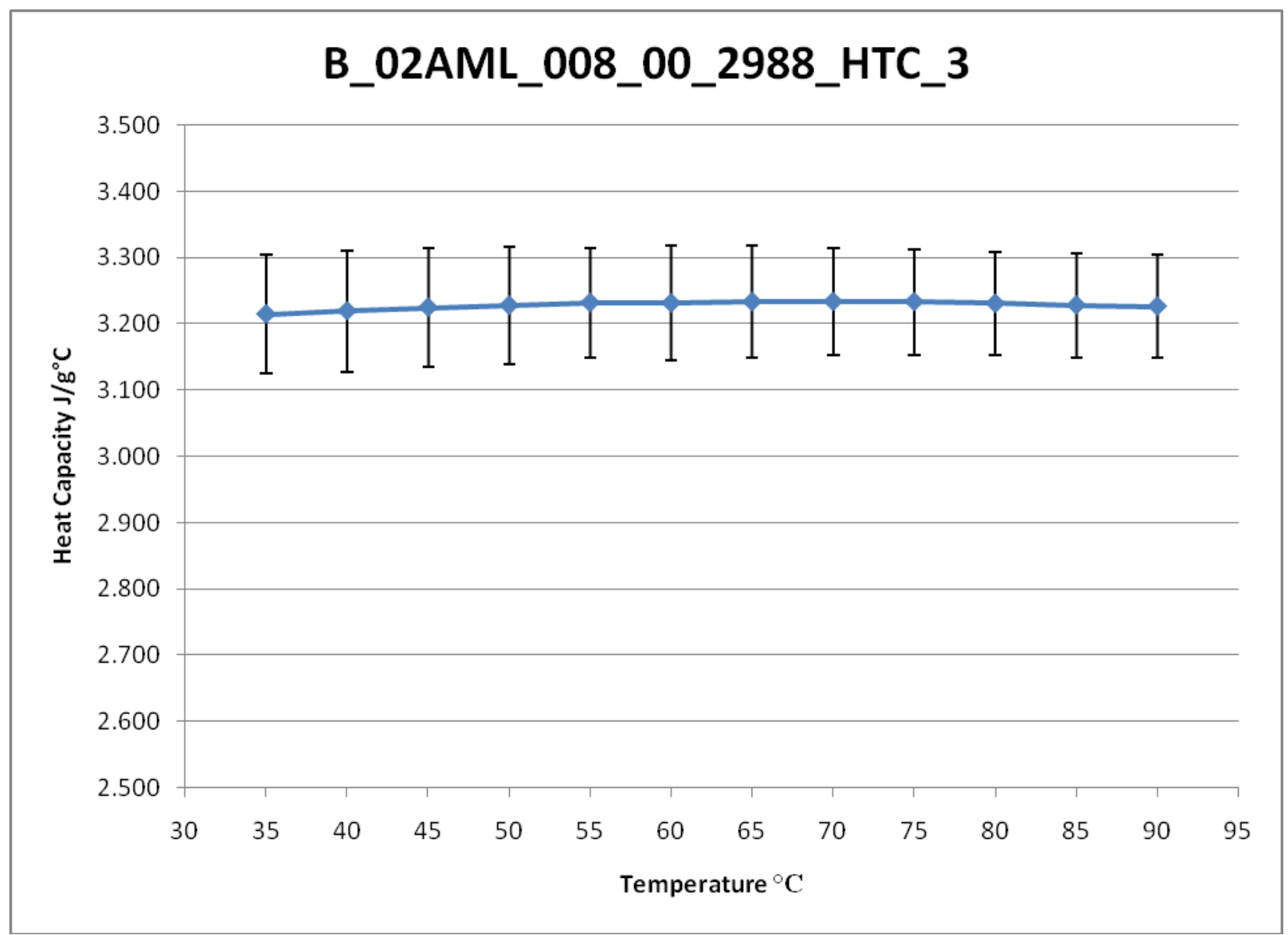

Figure 4. Heat Capacity Curve for Sample B_02AML_008_00_2988_HTC_3

\section{Conclusions:}

The measured heat capacity values for the PEP slurry samples discussed in this report are well within the expected range.

\section{References:}

[1] Lide, D. "CRC Handbook of Chemistry and Physics," $89^{\text {th }}$ Edition, 2008-2009, 6-2.

[2] Hemingway, B.S., Robie, R.A., Apps, J.A., "Revised values for the thermodynamic properties of boehmite, $\mathrm{AlO}(\mathrm{OH})$, and related species and phases in the system Al-H-O," American Mineralogist, Volume 76, pages 445-457, 1991. 


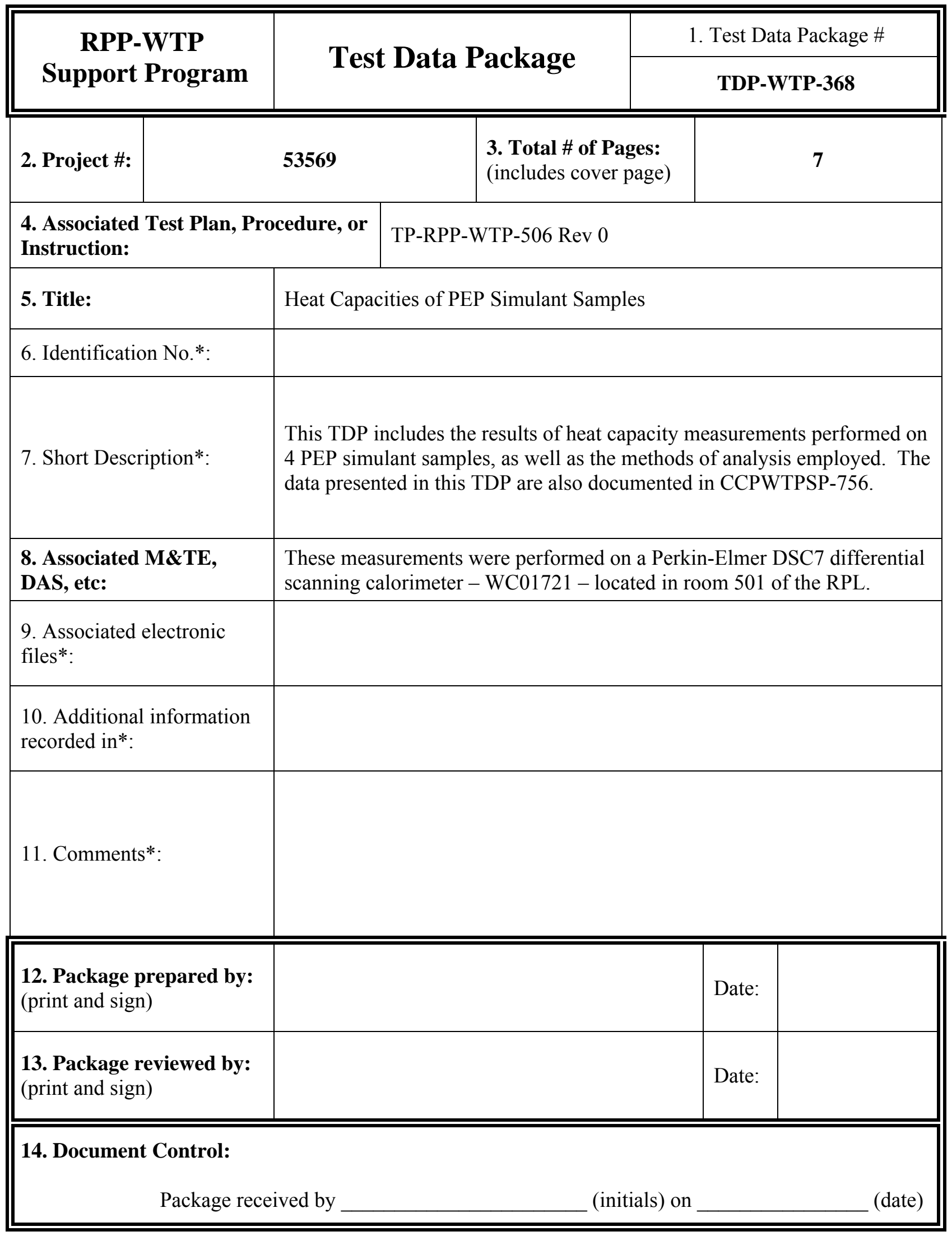




\section{PEP Slurry Sample Heat Capacity Report \\ Analysis}

This report presents results of heat capacity measurements for four PEP simulant slurry samples delivered via Chain of Custody form numbers WTP-COC-063-020 on 02/02/2009 and WTP-COC-063-024 on 02/03/2009. The samples provided were:

\section{- A 01AIM 002 XX 1519 HTC 3}

- A 01AIM 003001538 HTC 3

- A 01AIM 003 BT 1526 HTC 3

- B FRPGM 001 XX 2706 HTC 3

Three nominal $30 \mathrm{mg}$ subsamples were taken from each of these samples as they were vigorously stirred using a pipette with an enlarged tip to assure that the slurry samples were representative. We analyzed each slurry sample in triplicate, recognizing that obtaining a $30 \mathrm{mg}$ representative sample is challenging.

A Perkin-Elmer DSC7 differential scanning calorimeter (DSC) was used to determine the heat capacity of each subsample at temperatures between $20^{\circ} \mathrm{C}$ and $95^{\circ} \mathrm{C}$. The DSC7 was temperature- and enthalpy-calibrated using a gallium standard (NIST SRM 2234) (m.p. 29.8 ${ }^{\circ} \mathrm{C}$ ) and/or an indium standard (NIST SRM 2232) (m.p. $156.6^{\circ} \mathrm{C}$ ).

The method used for heat capacity measurement was as provided in the DSC7 operating manual. In this method, the heat capacity of the empty sample pan is measured to provide the "baseline"; the sample pan was a gold "volatile" sample pan designed to contain volatile samples such as the water in these PEP slurries. The heat capacity of a reference or standard material such as sapphire or high purity water is then determined; the purity of these water standards was determined by measuring their electrical conductivity. Finally the heat capacity of the sample is determined. The measured heat capacity of the sample is adjusted to remove the contribution of the empty pan. We further adjusted the reported heat capacity by applying a measured response factor for the high purity water.

Optimally the heat capacity measurement approach would include analyzing the standard or reference material in the same container as the sample would be analyzed; however, because analysis of these slurry samples require a hermetic seal which cannot be broken and resealed, a different but nearly equivalent gold volatile sample pan is used for the water. In addition, we characterized a sapphire standard to demonstrate instrument performance but unfortunately, the heat capacity of sapphire $\left(0.8 \mathrm{~J} / \mathrm{g}{ }^{\circ} \mathrm{C}\right)$ is significantly lower than that of the PEP slurries $(\sim 3.5 \mathrm{~J} / \mathrm{g})$ and does not provide an adequate calibration adjustment.

\section{Results}

The expected results for the measurements can be generated by considering what the theoretical heat capacity would be for slurries with solid fraction in the range that are expected in the samples. The slurry is mostly water, which at $20^{\circ} \mathrm{C}$ is known to have a specific heat of $4.1818 \mathrm{~J} / \mathrm{g}^{\circ} \mathrm{C}$ [1]. It is also known that at $20^{\circ} \mathrm{C}$, the heat capacity of boehmite is roughly $0.9 \mathrm{~J} / \mathrm{g}^{\circ} \mathrm{C}$ [2]. So for slurries with mass fraction of $10 \%$, $20 \%$, and $30 \%$ boehmite in water, the theoretical heat capacities would be, respectively:

- $4.1818 \mathrm{~J} / \mathrm{g}^{\circ} \mathrm{C} * .9+0.9 \mathrm{~J} / \mathrm{g}^{\circ} \mathrm{C} * .1=3.85 \mathrm{~J} / \mathrm{g}^{\circ} \mathrm{C}$,

- $\quad 4.1818 \mathrm{~J} / \mathrm{g}^{\circ} \mathrm{C} * .80+0.9 \mathrm{~J} / \mathrm{g}^{\circ} \mathrm{C} * .2=\mathbf{3 . 5 2} \mathrm{J} / \mathrm{g}^{\circ} \mathrm{C}$, and 


\section{- $\quad 4.1818 \mathrm{~J} / \mathrm{g}^{\circ} \mathrm{C} * .7+0.9 \mathrm{~J} / \mathrm{g}^{\circ} \mathrm{C} * .3=\mathbf{3 . 2 0} \mathrm{J} / \mathbf{g}^{\circ} \mathrm{C}$.}

Table 1 summarizes the results from these analyses by providing the mean measured heat capacities with their standard deviations. In general, the measured heat capacities are consistent with our expectations of hear capacities in the 3.2 to $3.8 \mathrm{~J} / \mathrm{g}^{\circ} \mathrm{C}$ assuming solids contents ranging from 10 to 30 -wt $\%$. We used only those analyses found to be internally self-consistent, not erratic and as-such, we discarded one measurement for sample A_01AIM_002_XX_1519_HTC_3, one measurement for sample A_01AIM_003_BT_1526_HTC_3 but replaced it with a reanalysis, and one measurement for Sample A_01AIM_003_00_1538_HTC_3.

Table 1. Results of Heat Capacity Measurements

\begin{tabular}{|l|r|r|r|r|}
\hline Temperature & $\begin{array}{l}\text { A 01AIM 002 XX } \\
-1519 \text { HTC 3 } \\
\mathrm{C}_{\mathrm{p}}\left(\mathrm{J} / \mathrm{g}^{\circ} \mathrm{C}\right)\end{array}$ & $\begin{array}{l}\text { A 01AIM 003 00- } \\
\text { 1538 HTC 3 } \\
\mathrm{C}_{\mathrm{p}}\left(\mathrm{J} / \mathrm{g}^{\circ} \mathrm{C}\right)\end{array}$ & $\begin{array}{l}\text { A 01AIM 003 BT- } \\
\text { 1526 HTC 3 } \\
\mathrm{C}_{\mathrm{p}}\left(\mathrm{J} / \mathrm{g}^{\circ} \mathrm{C}\right)\end{array}$ & $\begin{array}{l}\text { B FRPGM 001 } \\
\text { XX- 2706 HTC 3 } \\
\mathrm{C}_{\mathrm{p}}\left(\mathrm{J} / \mathrm{g}^{\circ} \mathrm{C}\right)\end{array}$ \\
\hline $35^{\circ} \mathrm{C}$ & $3.32 \pm 0.01$ & $3.46 \pm 0.00$ & $3.33 \pm 0.09$ & $3.33 \pm 0.02$ \\
\hline $40^{\circ} \mathrm{C}$ & $3.32 \pm 0.02$ & $3.48 \pm 0.01$ & $3.35 \pm 0.09$ & $3.33 \pm 0.02$ \\
\hline $45^{\circ} \mathrm{C}$ & $3.34 \pm 0.01$ & $3.49 \pm 0.01$ & $3.36 \pm 0.09$ & $3.33 \pm 0.02$ \\
\hline $50^{\circ} \mathrm{C}$ & $3.33 \pm 0.01$ & $3.51 \pm 0.02$ & $3.36 \pm 0.09$ & $3.34 \pm 0.02$ \\
\hline $55^{\circ} \mathrm{C}$ & $3.34 \pm 0.00$ & $3.51 \pm 0.03$ & $3.37 \pm 0.09$ & $3.34 \pm 0.02$ \\
\hline $60^{\circ} \mathrm{C}$ & $3.35 \pm 0.01$ & $3.53 \pm 0.04$ & $3.39 \pm 0.09$ & $3.34 \pm 0.02$ \\
\hline $65^{\circ} \mathrm{C}$ & $3.36 \pm 0.02$ & $3.54 \pm 0.05$ & $3.40 \pm 0.09$ & $3.34 \pm 0.02$ \\
\hline $70^{\circ} \mathrm{C}$ & $3.37 \pm 0.03$ & $3.54 \pm 0.05$ & $3.41 \pm 0.09$ & $3.35 \pm 0.02$ \\
\hline $75^{\circ} \mathrm{C}$ & $3.38 \pm 0.04$ & $3.55 \pm 0.06$ & $3.43 \pm 0.10$ & $3.35 \pm 0.02$ \\
\hline $80^{\circ} \mathrm{C}$ & $3.39 \pm 0.06$ & $3.56 \pm 0.07$ & $3.43 \pm 0.10$ & $3.35 \pm 0.02$ \\
\hline $85^{\circ} \mathrm{C}$ & $3.40 \pm 0.07$ & $3.58 \pm 0.09$ & $3.44 \pm 0.10$ & $3.35 \pm 0.03$ \\
\hline $90^{\circ} \mathrm{C}$ & $3.40 \pm 0.08$ & $3.59 \pm 0.10$ & $3.45 \pm 0.10$ & $3.36 \pm 0.03$ \\
\hline
\end{tabular}

The results from each sample measurement are presented in graphical form in Figures 1-4. In general, the heat capacity will increase nominally linearly as a function of temperature. In general, the average measured heat capacities do increase linearly with temperature, although some deviations do exist. 


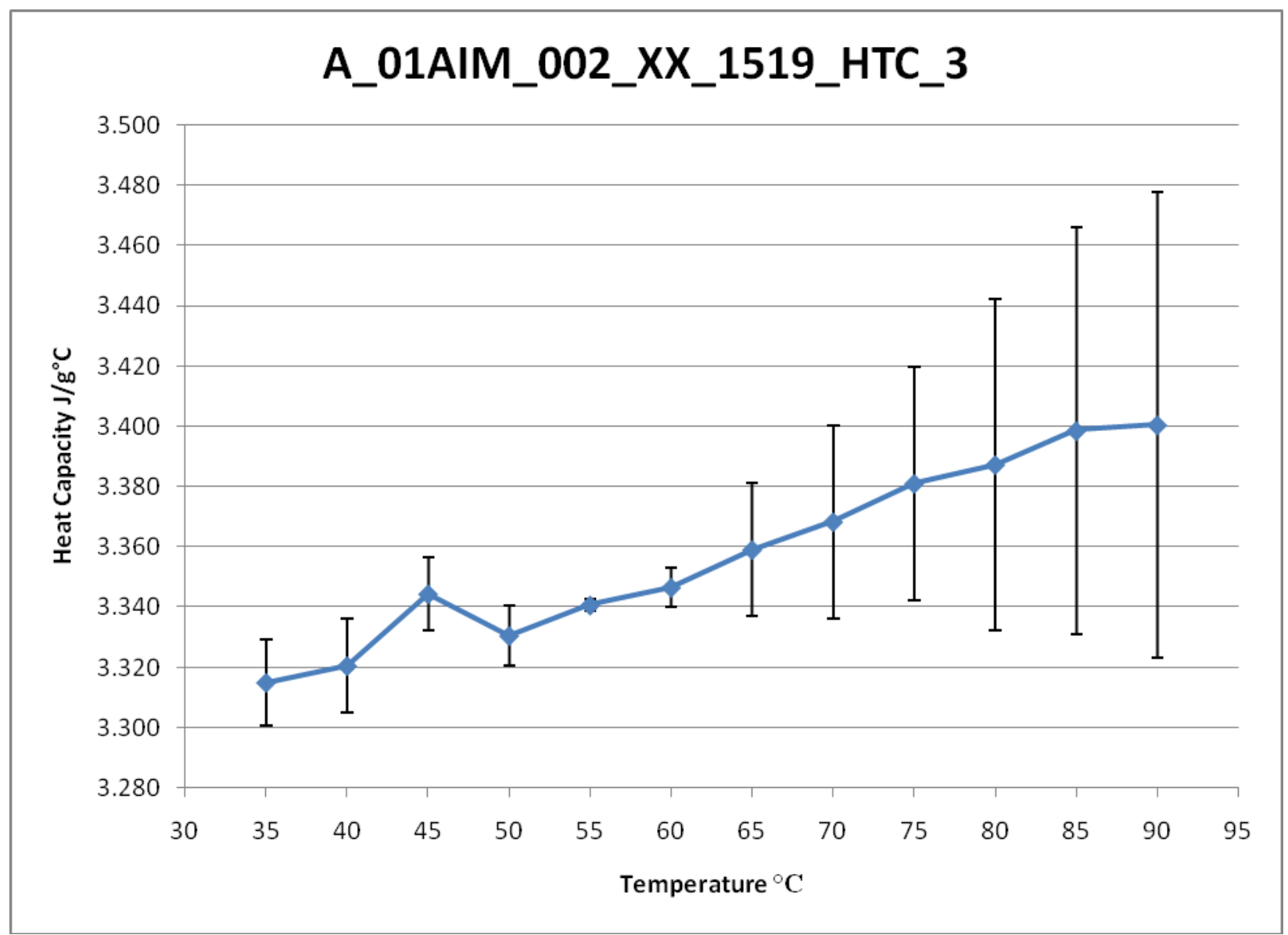

Figure 5. Heat Capacity Curve for Sample A_01AIM_002_XX_1519_HTC_3 


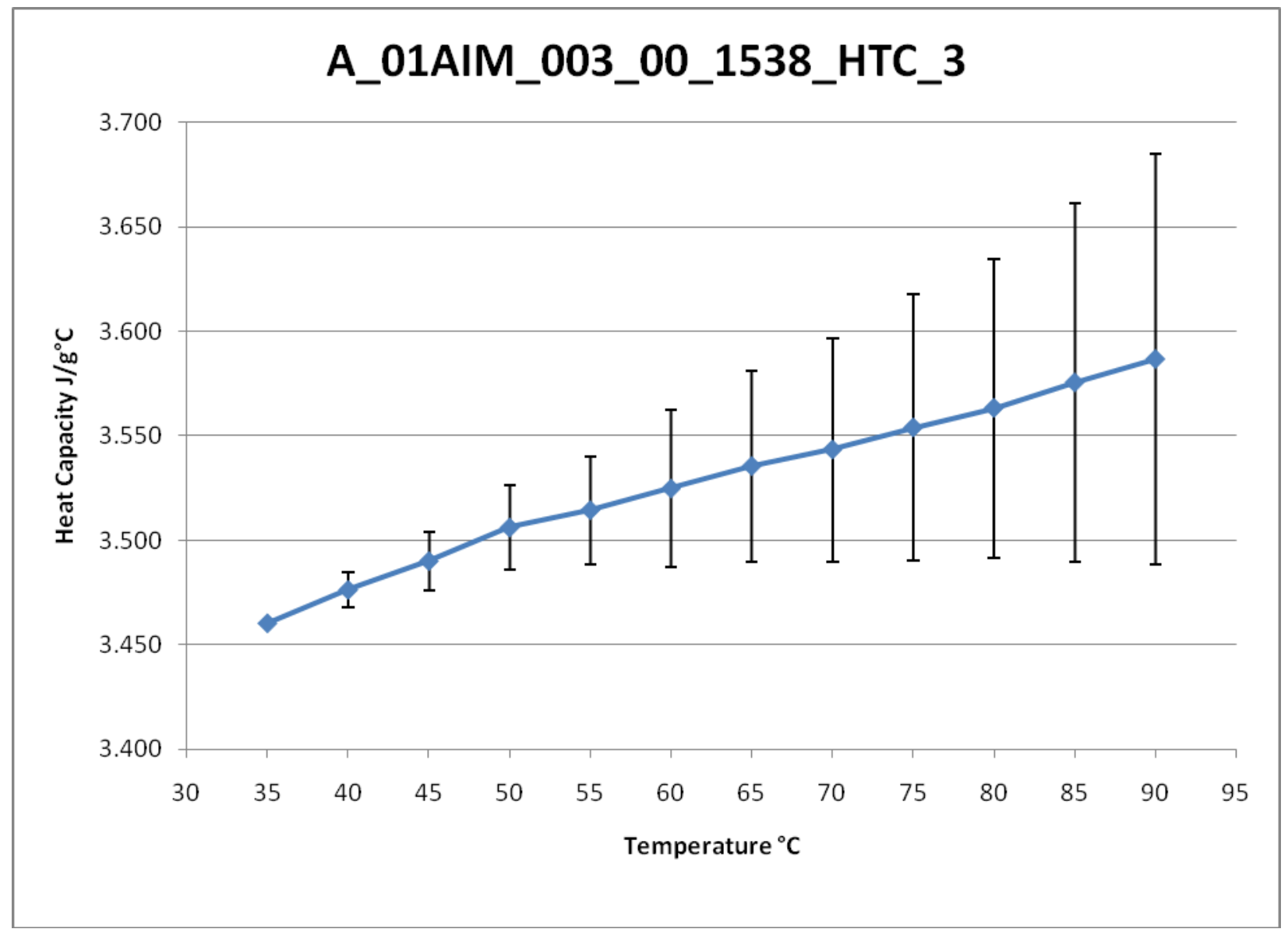

Figure 6. Heat Capacity Curve for Sample A_01AIM_003_00_1538_HTC_3 


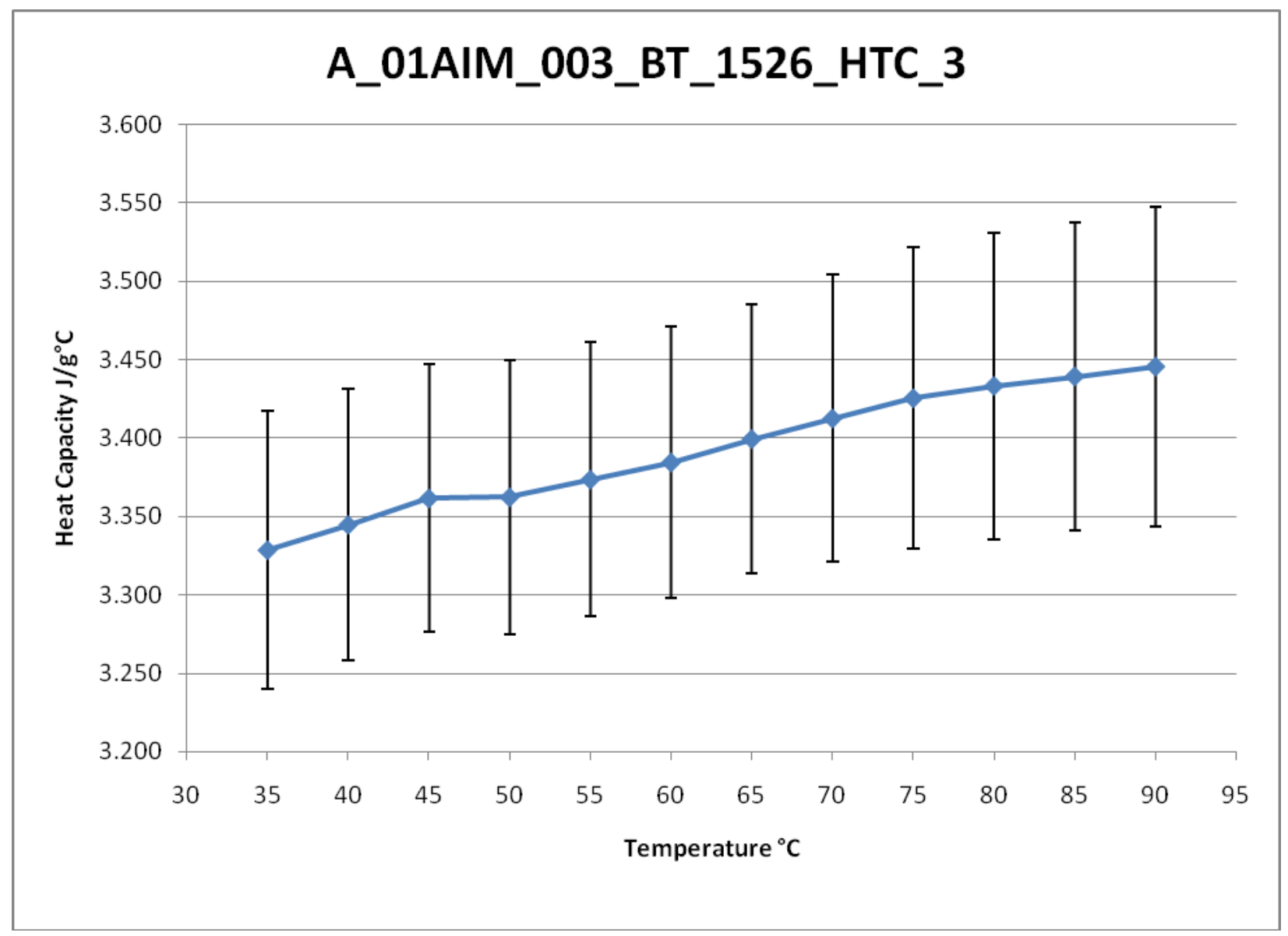

Figure 7. Heat Capacity Curve for Sample A_01AIM_003_BT_1526_HTC_3 


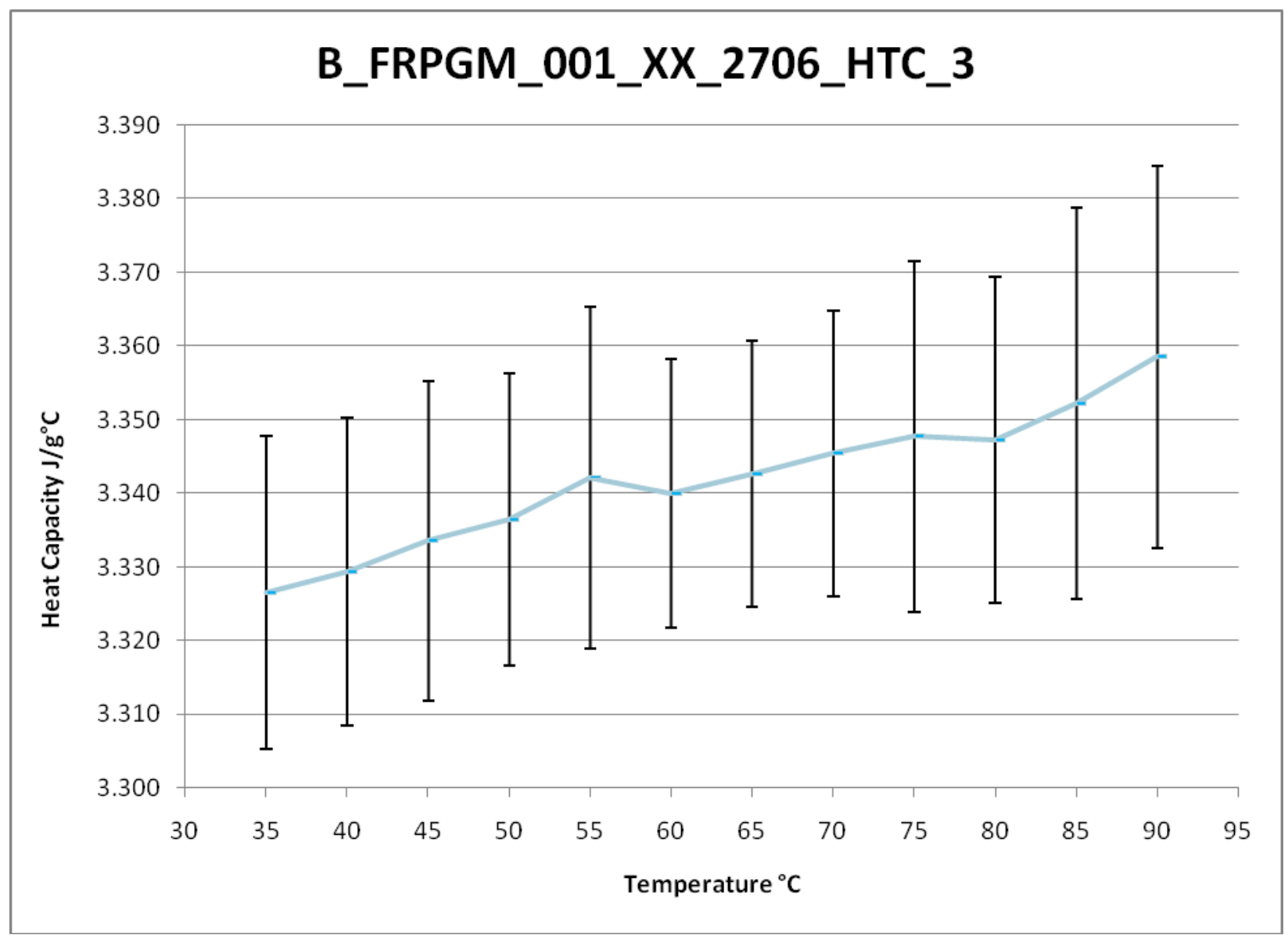

Figure 8. Heat Capacity Curve for Sample B_FRPGM_001_XX_2706_HTC_3

\section{Conclusions}

The measured heat capacity values for the PEP slurry samples discussed in this report are well within the expected range.

\section{References}

[1] Lide, D. "CRC Handbook of Chemistry and Physics," $89^{\text {th }}$ Edition, 2008-2009, 6-2.

[2] Hemingway, B.S., Robie, R.A., Apps, J.A., "Revised values for the thermodynamic properties of boehmite, $\mathrm{AlO}(\mathrm{OH})$, and related species and phases in the system Al-H-O," American Mineralogist, Volume 76, pages 445-457, 1991. 


\section{L.3 Scanning Electron Microscopy (TDP-WTP-356)}




\begin{tabular}{|c|c|c|}
\hline Date: & March 19, 2009 & Project No.: \\
\hline To: & Ofelia Bredt & Internal \\
\hline From: & Brian Riley & Distribution: \\
\hline Subject: & $\begin{array}{l}\text { PEP SEM Analyses for Integrated Test A, } \\
\text { TI-065 }\end{array}$ & \\
\hline
\end{tabular}

Two specimens were received for scanning electron microscopy (SEM) examinations in support of work being performed at the Pretreatment Engineering Platform (PEP) during the course of Integrated Test A. The morphologies of these specimens were analyzed using a JEOL 5900 SEM in accordance with procedure APEL-102-SEM, Rev. 1.

\section{Sample Preparation}

Once received from PEP, the specimens were cleaned of nitrates. This cleaning process included 3 cycles of adding $10-\mathrm{mL}$ of fresh $0.01 \mathrm{M} \mathrm{NaOH}$ to the centrifuge tube, vortexing for 3 sets of 15 seconds, centrifuging at $4500 \mathrm{rpm}$ for 10 minutes, decanting and then adding $10-\mathrm{mL}$ of fresh $0.01 \mathrm{M} \mathrm{NaOH}$. These washing steps were recorded in laboratory record book BNW-60176.

After the last rinse, the $\mathrm{NaOH}$ and the pellet were vortexed, a small volume of the slurry was drawn up using a pipette, and a drop was placed on the top of an aluminum SEM sample stub. It was then placed in an oven at approximately $100^{\circ} \mathrm{C}$ to dry overnight. Once it was dry, it was coated with a thin layer of $\mathrm{Au} / \mathrm{Pd}$ using the Polaron Range plasma sputter coater in APEL/102. The Au/Pd layer was deposited using $800 \mathrm{~V}$ and $10 \mathrm{~mA}$ for $180 \mathrm{~s}$. The coated specimen was placed in an SEM sample holder and was placed in the SEM for analysis.

\section{Analysis}

Scanning electron micrographs were collected for informational purposes only using the Scan 4 option with $160 \mathrm{~s}$ collection times using the settings as seen below in Table 1. See Table 2 for information regarding the person that performed the analysis as well as the date of analysis. Images at the four magnifications listed in Table 1 were compiled in a $2 \times 2$ arrangement to show a macroscopic to microscopic view of the particles. See APPENDIX A: Micrograph Collages.

\section{Results}

The micrographs collected for this report compare the morphology of undissolved solids in the PEP slurry at the beginning of the PEP treatment process (B_FRPGM_001_XX_2713_XSP_4_B; "B_FRPGM" for short) and after having completed the oxidative leaching step (A_02AML_021_XX_2460_XSP_4; "A_02AML" for short). The differences between these specimens were readily apparent. Specimen B_FRPGM appeared to be single phase, and consisted of particles that were somewhat tabular and faceted, with sizes ranging from 1 to $10-\mu \mathrm{m}$ (due to similar, average atomic numbers for boehmite and gibbsite, there is not a readily apparent difference in contrast or brightness between them when imaged in an SEM). Specimen A_02AML appeared to consist of two kinds of particles that were in somewhat of a bimodal size distribution. The smaller particles were approximately $180 \mathrm{~nm}$ in apparent diameter, and were equiaxed. The larger particles had distinct facets, and were approximately $1-\mu \mathrm{m}$ in apparent diameter. Morphologically, the larger particles appeared similar to the particles in B_FRPGM. Based on 
XRD results from a separate report, specimen B_FRPGM consisted of 51.6-wt $\%$ boehmite and 32.1-wt\% gibbsite (balance was amorphous), and specimen A_02AML consisted of $38.4 \mathrm{wt} \%$ boehmite, and 1.1-wt $\%$ hematite (balance was amorphous). Thus, the micron-scale, faceted particles in A_02AML might be residual, crystalline boehmite (orthorhombic crystal structure) that remained after the treatment process, whereas the sub-micron, equiaxed particles could be the large fraction of amorphous material ( $\sim 80 \%$, as indicated by XRD analysis) which was the product from the PEP process. More definitive chemical information about these different particles is possible using energy dispersive spectroscopy, which is available, if desired. One can conclude that the PEP treatment process had a dramatic impact on the size, shape, composition, and the atomic structure (crystalline vs amorphous) of the undissolved solids in the slurry that was tested.

Table 1. Parameters Used for Capturing Images

\begin{tabular}{|l|l|l|l|l|l|}
\hline Sample ID & $\begin{array}{l}\text { Voltage } \\
(\mathrm{KV})\end{array}$ & $\begin{array}{l}\text { Spot } \\
\text { Size }\end{array}$ & Detector & $\begin{array}{l}\text { Working } \\
\text { Distance } \\
(\mathrm{mm})\end{array}$ & Magnification \\
\hline B_FRPGM_001_XX_2713_XSP_4_B & 20 & 30 & SEI & 12 & $1,000 \times$ \\
\hline B_FRPGM_001_XX_2713_XSP_4_B & 20 & 30 & SEI & 12 & $2,500 \times$ \\
\hline B_FRPGM_001_XX_2713_XSP_4_B & 20 & 25 & SEI & 12 & $5,000 \times$ \\
\hline B_FRPGM_001_XX_2713_XSP_4_B & 20 & 25 & SEI & 12 & $10,000 \times$ \\
\hline A_02AML_021_XX_2460_XSP_4 & 15 & 24 & BSE & 10 & $1,000 \times$ \\
\hline A_02AML_021_XX_2460_XSP_4 & 15 & 24 & BSE & 10 & $2,500 \times$ \\
\hline A_02AML_021_XX_2460_XSP_4 & 15 & 24 & BSE & 10 & $5,000 \times$ \\
\hline A_02AML_021_XX_2460_XSP_4 & 15 & 24 & BSE & 10 & $10,000 \times$ \\
\hline
\end{tabular}

Table 2. Analysis Information

\begin{tabular}{|l|l|l|l|}
\hline Sample ID & $\begin{array}{l}\text { Date of } \\
\text { Analysis }\end{array}$ & $\begin{array}{l}\text { Analysis } \\
\text { Performed By }\end{array}$ & Signature \\
\hline B_FRPGM_001_XX_2713_XSP_4_B & $03 / 05 / 2009$ & Brian Riley & \\
\hline A_02AML_021_XX_2460_XSP_4 & $03 / 18 / 2009$ & Brad Johnson & \\
& & & \\
\hline
\end{tabular}




\section{APPENDIX A: Micrograph Collages}

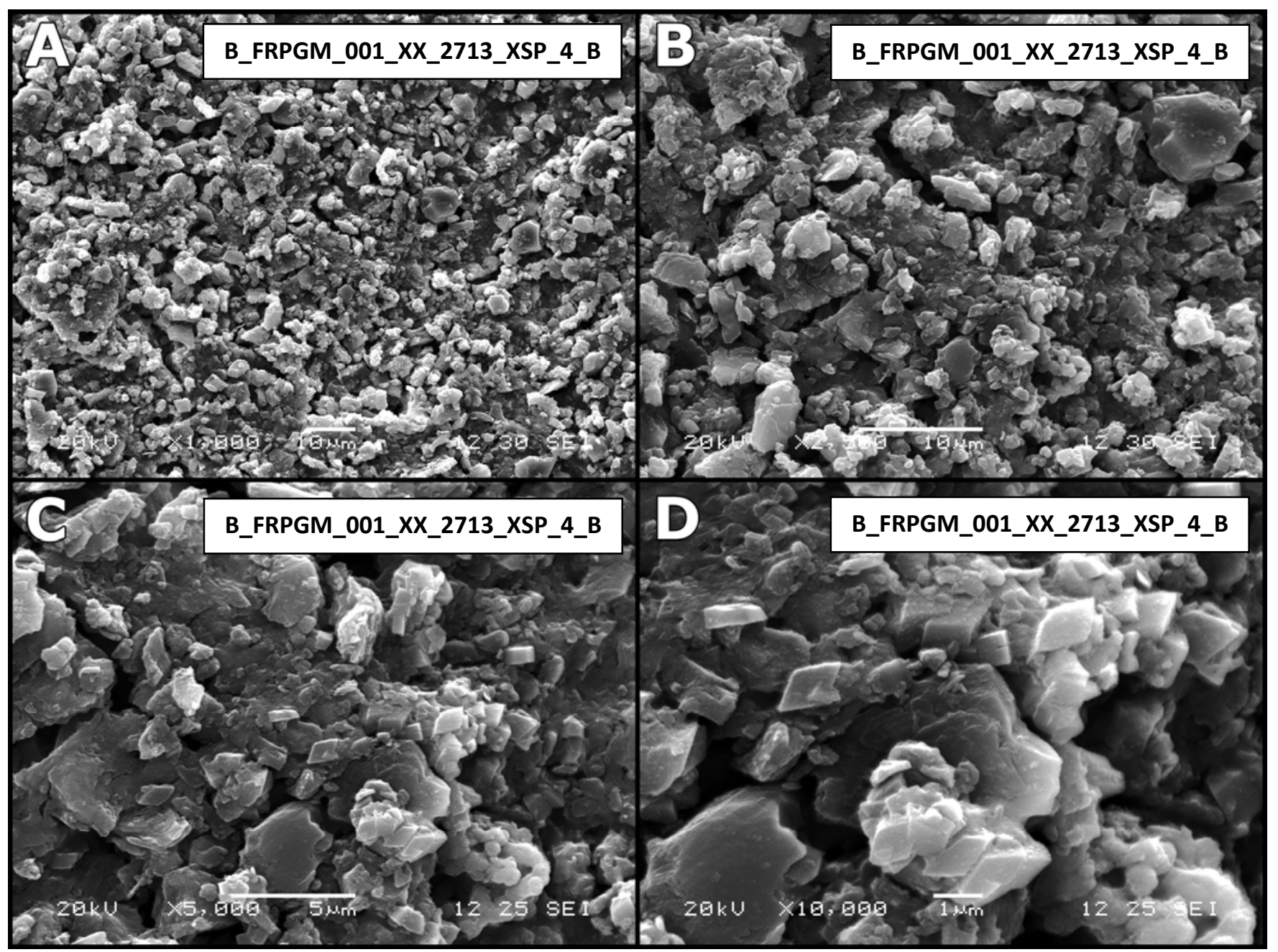

SEM micrographs from Pretreatment Engineering Platform (PEP) specimen

B FRPGM 001_XX 2713 XSP 4 B. The specimen, while in the original 50-mL centrifuge tube, was rinsed with $0.01 \mathrm{M} \mathrm{NaOH}$, vortexed, centrifuged (4500 rpm for 10 minutes), and decanted $3 \times$ to clean off the nitrates. After the final rinse, the contents of the tube were vortexed in order to resuspend the pellet, and a drop of the suspended particles was placed on an Al SEM stub and placed in an oven at approximately $105^{\circ} \mathrm{C}$ to dry overnight. Once dry, the specimen was coated with $\mathrm{Au} / \mathrm{Pd}$ and analyzed using the APEL/102 SEM (JEOL 5900).

Figure 9. Scanning Electron Micrographs Collected for Informational Purposes Only on B_FRPGM_001_XX_2713_XSP_4_B at Different Magnifications 


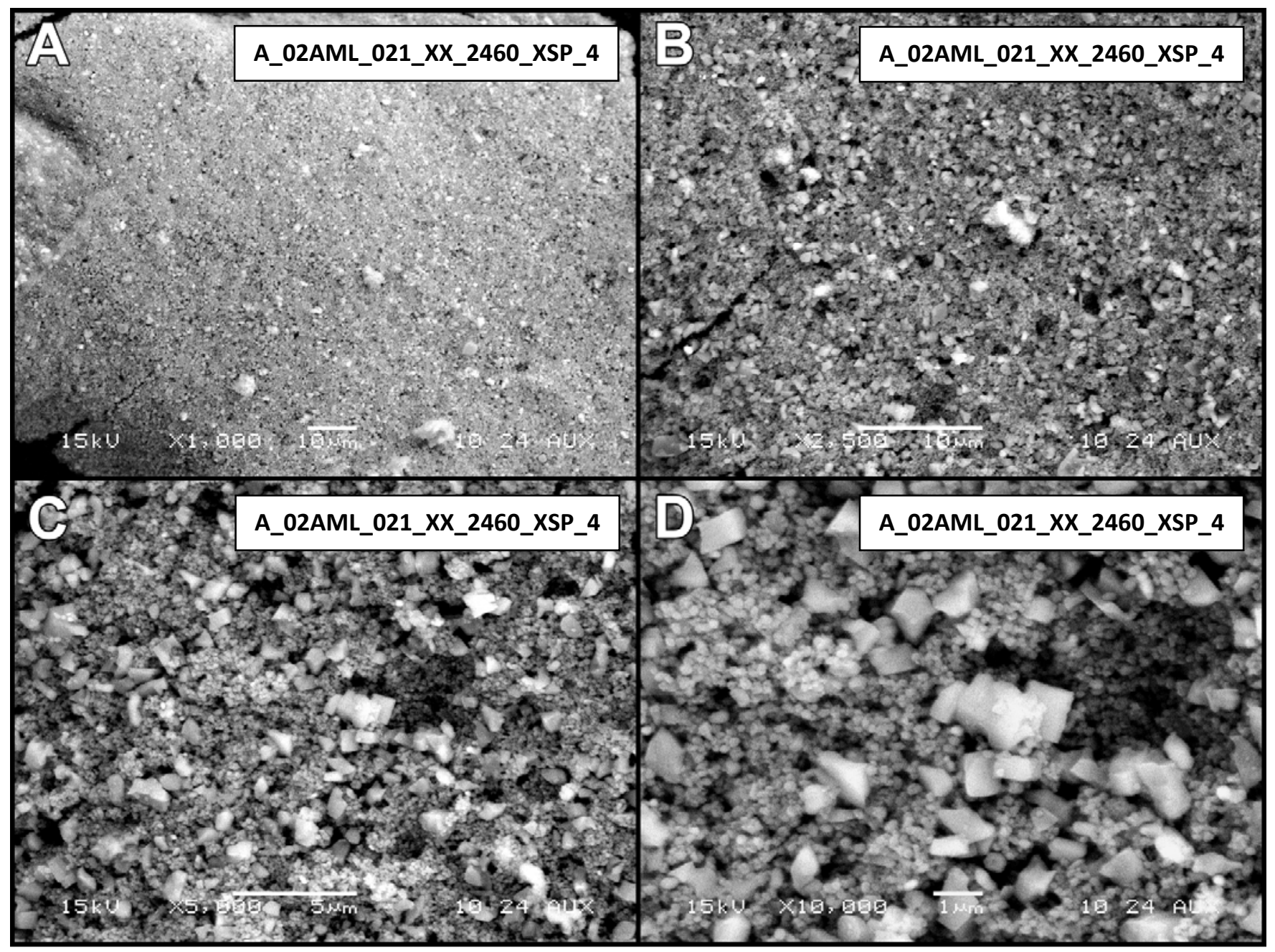

SEM micrographs from Pretreatment Engineering Platform (PEP) specimen

A_02AML_021_XX_2460_XSP_4. The specimen, while in the original 50-mL centrifuge tube, was rinsed with $0.01 \mathrm{M} \mathrm{NaOH}$, vortexed, centrifuged (4500 rpm for 10 minutes), and decanted $3 \times$ to clean off the nitrates. After the final rinse, the contents of the tube were vortexed in order to resuspend the pellet, and a drop of the suspended particles was placed on an Al SEM stub and placed in an oven at approximately $105^{\circ} \mathrm{C}$ to dry overnight. Once dry, the specimen was coated with Au/Pd and analyzed using the APEL/102 SEM (JEOL 5900).

Figure 10. Scanning Electron Micrographs Collected for Informational Purposes Only on A_02AML_021_XX_2460_XSP_4 at Different Magnifications 


\section{L.4 X-Ray Diffraction (WTP-RPT-357)}

Date: $\quad$ February 06, 2009

To: $\quad$ O. Bredt

From J. V. Crum

Subject: XRD Examination of: B_FRPGM_001_XX_2713_XSP_4_B

Analyst:

Reviewer:

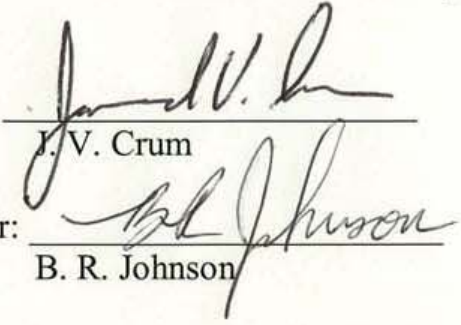

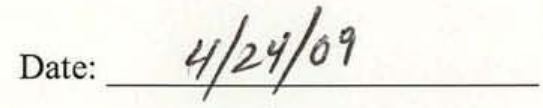

Date:

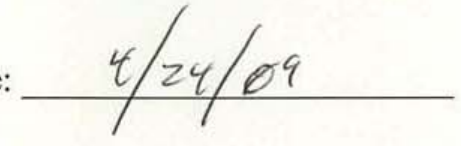

XRD data from the samples identified in Table I have been examined for identification of crystalline phases present.

Table I: Samples Examined in this Series

\begin{tabular}{|c|c|c|c|c|}
\hline Identification & XRD File $\mathrm{ID}^{(\mathrm{a})}$ & $\begin{array}{l}\text { Count Time per } \\
\text { Step (Seconds) }\end{array}$ & $\begin{array}{l}\text { Number of } \\
\text { Replications }\end{array}$ & Internal Standard \\
\hline $\begin{array}{c}\text { B_FRPGM_001_XX_2713 } \\
\text { XSP 4 4 B }\end{array}$ & 031309A11 & 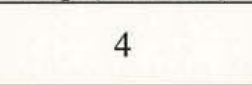 & 1 & $4.93 \mathrm{wt} \% \mathrm{TiO}_{2}$ \\
\hline
\end{tabular}

(a) The letter appended to the XRD File ID identifies the first sample in the replication series. The letter has been dropped in subsequent text to indicate that the referenced file is the summation of data from replicate runs.

Sample analysis was carried out by the Pacific Northwest National Laboratory Advanced Processing and Application Group. The XRD instrument used was the Scintag PAD V X-ray Diffractometer, Property Number WD33356, located in lab 102 in the APEL building. The data range for the sample was $5^{\circ}$ to $80^{\circ}$ 2 -theta, with a step size of $0.04^{\circ} 2$-theta and count time 4.0 seconds per step. Copper Ka X-rays were used. The X-ray tube operating conditions were $45 \mathrm{kV}$ and $40 \mathrm{~mA}$.

Sample 031309A11 was prepared with SRM 674b, $\mathrm{TiO}_{2}$ internal standard. The dried powder was placed into a tungsten carbide milling chamber and milled for $2 \mathrm{~min}$ in the angstrom shaker mill. The $\mathrm{TiO}_{2}$ standard was then spiked in at $4.93 \mathrm{wt} \%$ with the dried powder and milled again for $2 \mathrm{~min}$ to insure a homogenous mixture of the two powders. The sample was then mounted into standard sample holder.

Phase identification was done by use of the JADE search match routines (version 6.0, Materials Data Inc.) with comparison to the ICDD (International Centre for Diffraction Data) data base PDF-2 release 1999, which includes the ICSD (Inorganic Crystal Structure Database maintained by Fachinformationszentrum (FIZ), Karlsruhe, Germany). Searches were restricted to the PDF and ICSD Inorganic sections.

The phase identification plots are shown using "stick figures" to indicate the phases present; peak positions and line intensities. The phase showing the greatest peak intensity is shown at the top of the stick figures display (except the internal standard, if used, is always the topmost). Phases present at lower peak area/height are shown in decreasing order down the display. 
The identified phases, ranked by relative peak intensities, are given below for each sample. Figure 1 shows the raw summed pattern. The background fitted pattern, along with the identified phases, are shown in figures 2,3 , and 4 .

\section{B FRPGM 001 XX 2713 XSP 4 B, File: 031309A11}

- Rutile, $\mathrm{TiO}_{2}$, card\# 21-1276

- Boehmite, syn, $\mathrm{AlO}(\mathrm{OH})$, card\# 21-1307, Excellent overall fit. Peak locations and intensities are a good match to the pattern.

- Gibbsite, $\mathrm{Al}(\mathrm{OH})_{3}$, card\# 60-2702, Good fit.

- Amorphous material, $\sim 36^{\circ} 2 \theta$.

The pattern was also examined using RIQAS (release 4.0.0.26, 6/10/2002, Materials Data Inc.) rietveld analysis software. The phases identified above were input into the analysis along with a cubic spline function for the background and an amorphous hump at $\sim 36^{\circ} 2 \theta$, with fitted pattern shown in figure 5 .

The results of the analysis for the crystalline phases are:

- Boehmite, $\mathrm{AlO}(\mathrm{OH}), 51.6 \mathrm{wt} \%$, with a crystallite size of $414 \AA$

- Gibbsite, $\mathrm{Al}(\mathrm{OH})_{3}, 32.1 \mathrm{wt} \%$ with a crystallite size of $160 \AA$

- The balance of the material is amorphous

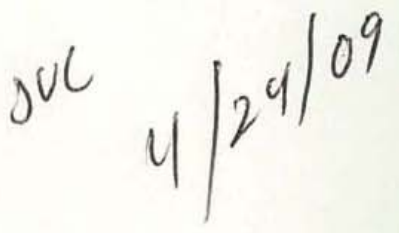




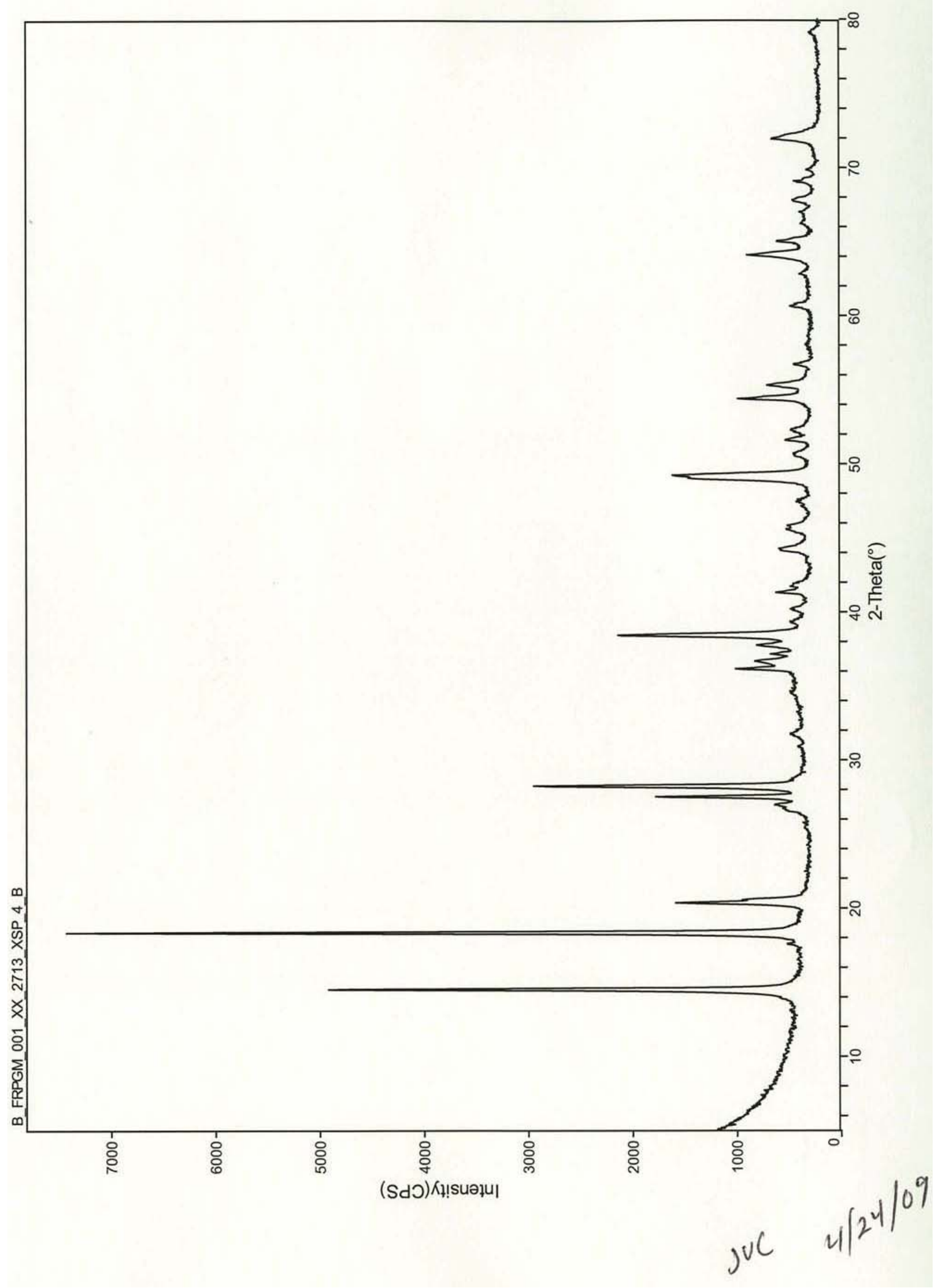




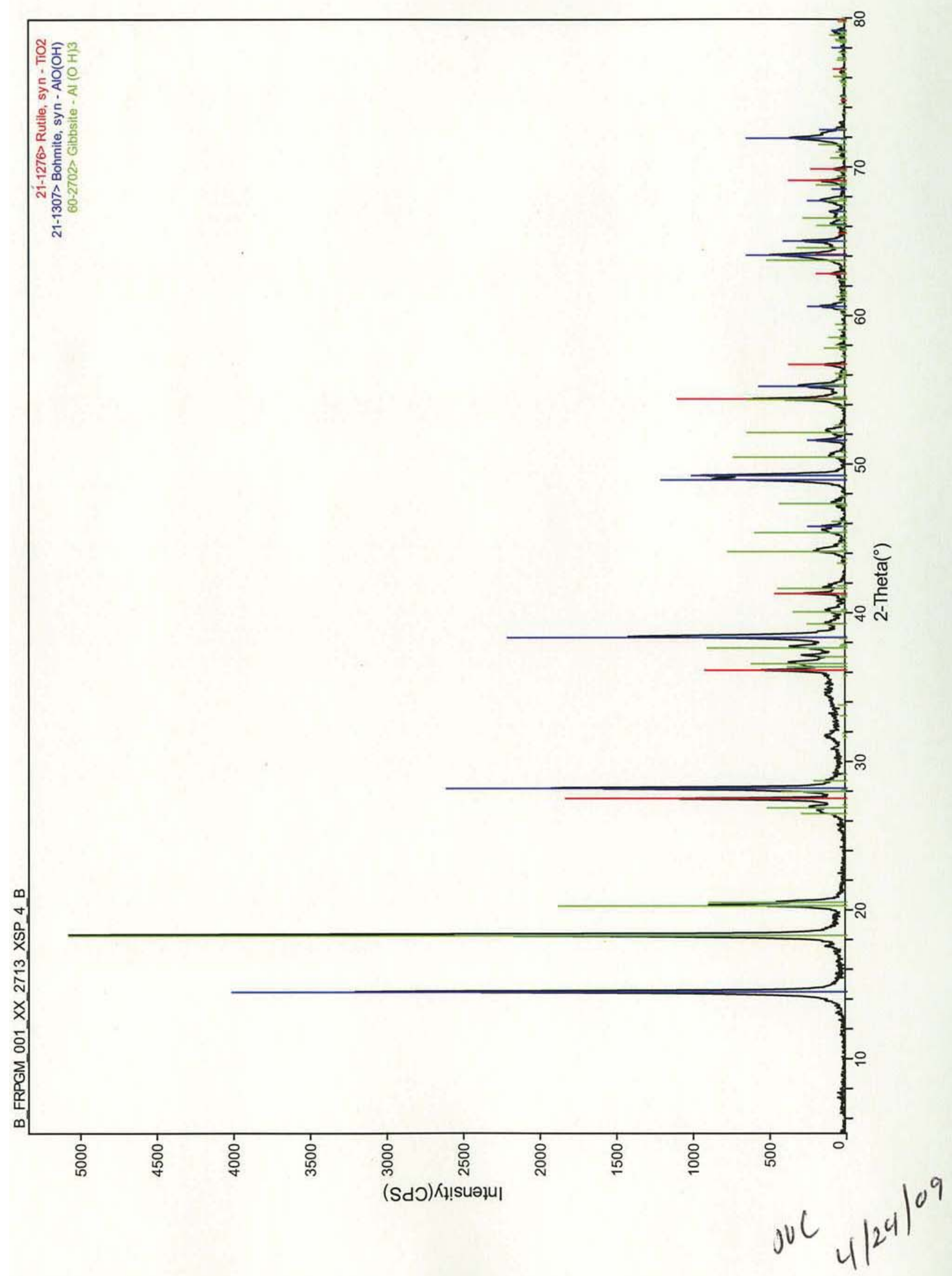




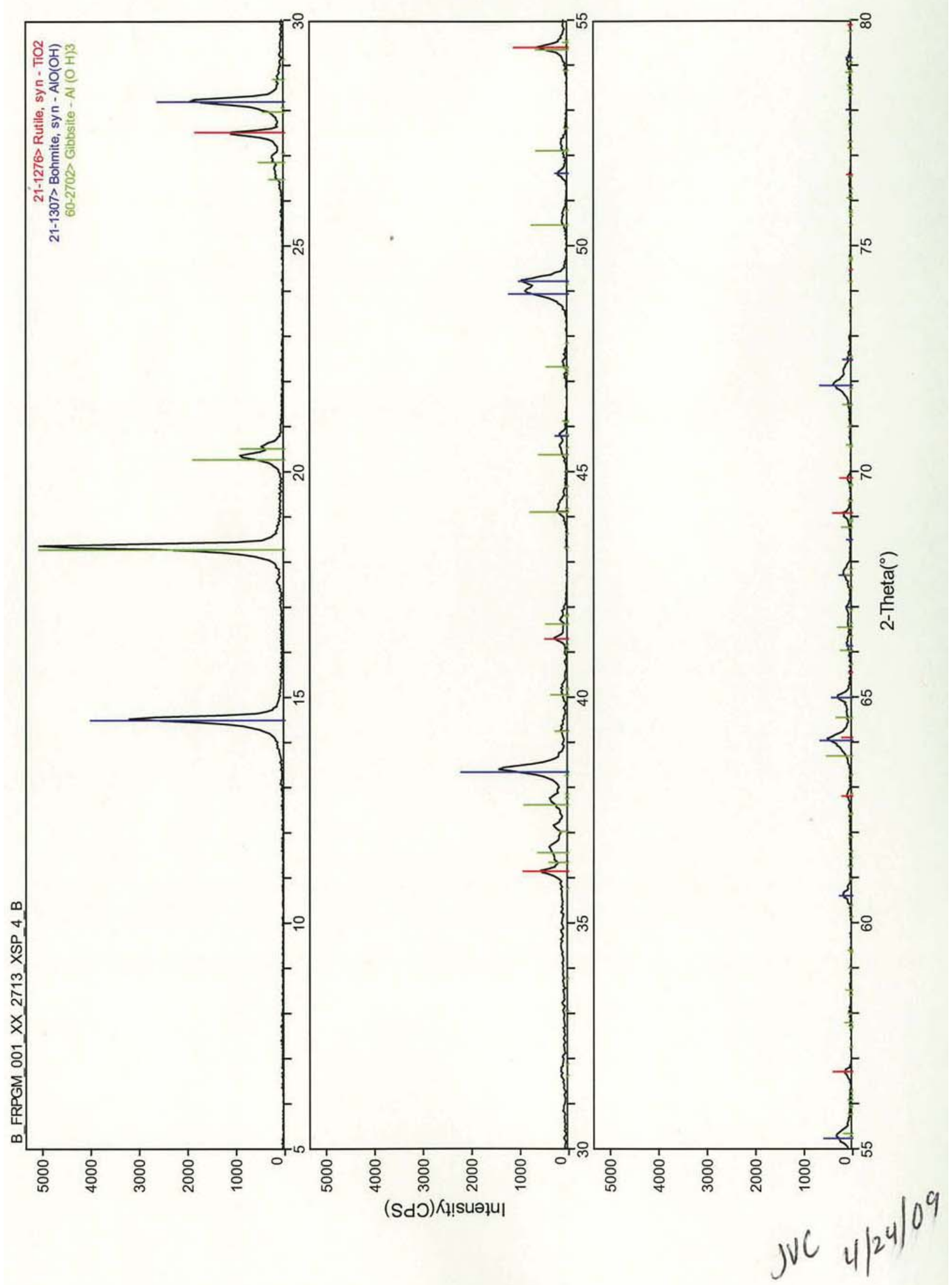




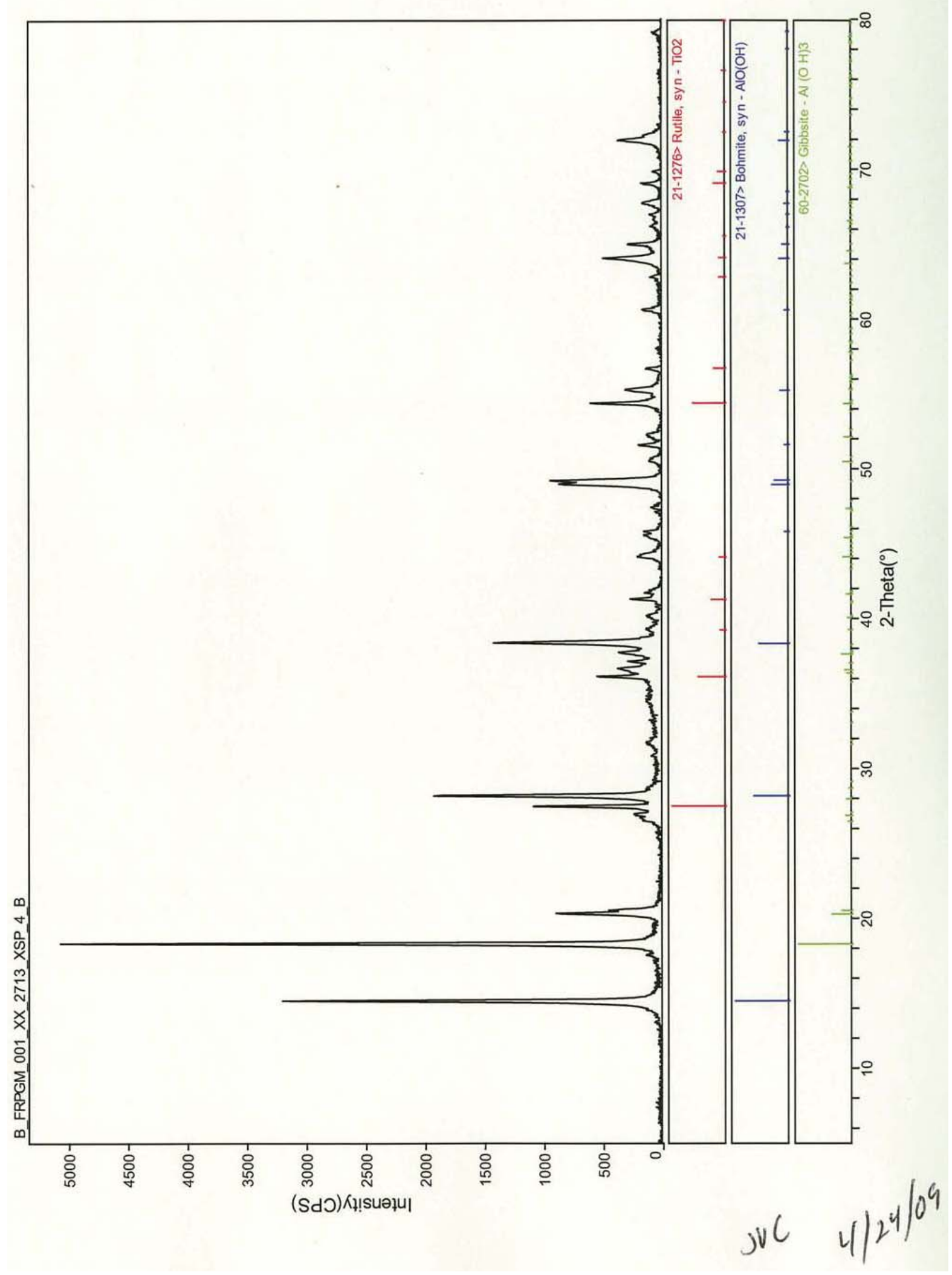




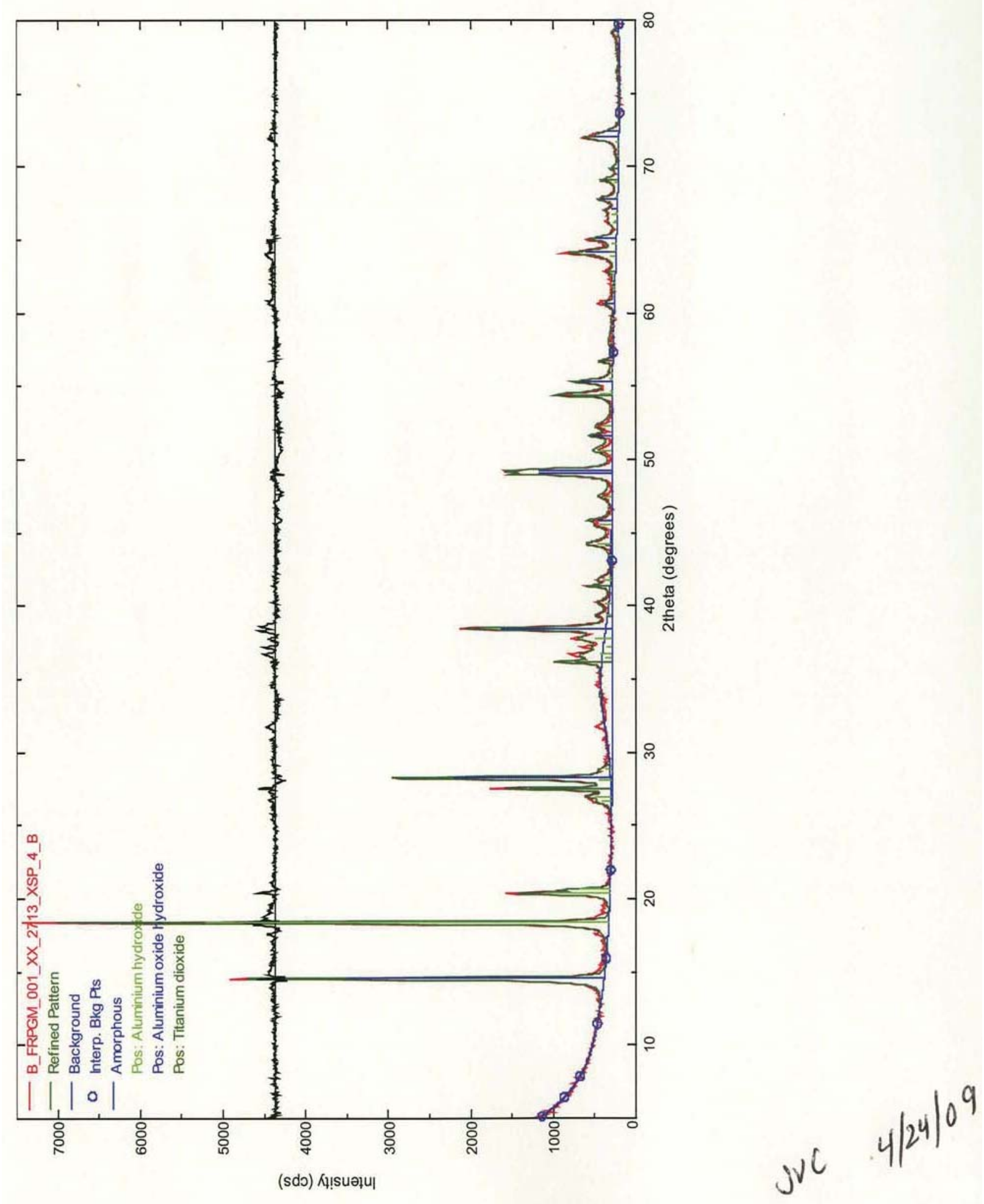


Appendix A8

ICDD cards of interest for this sample 
- $\mathrm{PDF} \# 21-1276: \mathrm{QM}=$ Common $(+) ; \mathrm{d}=$ Other/Unknown; $\mathrm{I}=($ Unknown $)$

Rutile, syn

$\mathrm{TiO}_{2}$

Radiation $=\mathrm{CuKa} 1 \quad$ Lambda $=1.5406$ Filter $=$

Calibration $=\quad \mathrm{d}$-Cutoff $=\quad \mathrm{I} / \mathrm{Ic}(\mathrm{RIR})=3.4$

Ref: Level-1 PDF

Tetragonal, $\mathrm{P} 42 / \mathrm{mnm}(136) \quad \mathrm{Z}=2 \quad \mathrm{mp}=$

CELL: $4.5933 \times 4.5933 \times 2.9592<90.0 \times 90.0 \times 90.0>\quad$ P.S $=$

$\operatorname{Density}(\mathrm{c})=4.23$ Density $(\mathrm{m})=\quad \mathrm{Mwt}=\mathrm{Vol}=62.4$

Ref: Ibid.

Strong Line: $3.25 / \mathrm{X} \quad 1.69 / 6 \quad 2.49 / 5 \quad 2.19 / 3 \quad 1.62 / 2 \quad 1.36 / 2 \quad 0.82 / 1 \quad 1.35 / 1$

- PDF\#21-1307: $\mathrm{QM}=$ Common $(+)$; $\mathrm{d}=$ Other/Unknown; $\mathrm{I}=($ Unknown)

Boehmite, syn

$\mathrm{AlO}(\mathrm{OH})$

Radiation $=$ CuKa1 $\quad$ Lambda $=1.5406$ Filter $=$

Calibration $=\quad \mathrm{d}$-Cutoff $=\quad \mathrm{I} / \mathrm{Ic}(\mathrm{RIR})=$

Ref: Level-1 PDF

Orthorhombic, $\operatorname{Amam}(63) \quad \mathrm{Z}=4 \quad \mathrm{mp}=$

CELL: $3.7 \times 12.227 \times 2.868<90.0 \times 90.0 \times 90.0>\quad$ P.S $=$

Density $(\mathrm{c})=3.07$ Density $(\mathrm{m})=\quad \mathrm{Mwt}=\quad \mathrm{Vol}=129.8$

Ref: Ibid.

Strong Line: $6.11 / \mathrm{X} \quad 3.16 / 7 \quad 2.35 / 6 \quad 1.86 / 3 \quad 1.85 / 3 \quad 1.45 / 2 \quad 1.31 / 2 \quad 1.66 / 1$

- $\mathrm{PDF} \# 60-2702: \mathrm{QM}=$ Calculated; $\mathrm{d}=\mathrm{O}$ ther/Unknown; $\mathrm{I}=($ Unknown $)$

Gibbsite

$\mathrm{Al}(\mathrm{O} \mathrm{H})_{3}$

Radiation $=\mathrm{CuKa} 1 \quad$ Lambda $=1.5406$ Filter $=$

Calibration $=\quad \mathrm{d}$-Cutoff $=\quad \mathrm{I} / \mathrm{Ic}(\mathrm{RIR})=1.67$

Ref: Calculated from FIZ\#6162 (ICSD @08/01/01) by Jade 6.x

Monoclinic, $\mathrm{P} 21 / \mathrm{n}(14) \quad \mathrm{Z}=8 \quad \mathrm{mp}=$

CELL: $8.684 \times 5.078 \times 9.736<90.0 \times 94.54 \times 90.0>$ P.S $=$

Density $(\mathrm{c})=2.421$ Density $(\mathrm{m})=\quad \mathrm{Mwt}=\quad \mathrm{Vol}=428.0$

Ref: Ibid.

Strong Line: $4.85 / \mathrm{X} \quad 4.38 / 4 \quad 2.39 / 2 \quad 4.33 / 2 \quad 2.05 / 2 \quad 1.81 / 1 \quad 1.69 / 1 \quad 1.75 / 1$

NOTE: Refinement of the crystal structure of gibbsite, $\mathrm{Al}(\mathrm{O} \mathrm{H})_{3}$, Zeitschrift fuer Kristallographie, Kristallgeometrie, Kristallphysik, Kristallchemie (-144,1977) [ZEKGA] 139 (1974) 129-135, Saalfeld H, Wedde M, Dx=2.4.

M PDF 33-18.

$\mathrm{R}=0.032$. 
Appendix M

\section{Integrated Test B Operational Process Sheet (Run Sheet)}





\section{Appendix M: Integrated Test B Operational Process Sheet (Run Sheet)}

The Run Sheet section of the Test Instruction (TI-WTP-PEP-066) provided the LTE target or setpoint values for key process operations. The LTE filled in the actual value during testing from available data. Often these data were from the HMI, which is not NQA-1 qualified. Data recorded were a snapshot in time rather than an average. The following is a reproduction of the run sheet section of the Test Instruction with the "actual value" column filled in from various data sources. Data provided in the "actual value" column are generally from the original Test Instruction as filled in by the LTE. In cases where certified data were available as a result of data evaluation efforts, these data were used instead. The data source is provided in the "comments" column, with the following definitions:

HMI - The LTE obtained the value from the HMI screen, which does not provide certified calibrated readings. Reading taken as a snapshot in time with no averaging applied.

VFD - The LTE obtained this value from the VFD readout, which was not calibrated.

Local flowmeter-The steam ring air purge and the air sparge values were read locally from the instrument (Coriolis gas flowmeter). These local readouts were calibrated.

Test Instruction-The value or state was recorded on the test instruction. The timing of events, number of events (e.g., wash batches), equipment line ups, and AFA additions were documented in this manner and provide the best available record.

CCP-XXX - If a CCP is cited as the data source, the value was evaluated and checked in the listed CCP.

WTP-RPT-XXX - If a report is cited as the data source, the value was evaluated and checked via a CCP traceable through the report referenced.

The run sheet (Table M.1) is provided for information only. 
Table M.1. Integrated Test B Run Sheet

\begin{tabular}{|c|c|c|c|c|c|c|c|}
\hline & $\begin{array}{l}\text { Run } \\
\text { Sheet } \\
\text { Step }\end{array}$ & Operation & $\begin{array}{c}\text { Target or } \\
\text { Setpoint } \\
\text { Value }\end{array}$ & $\begin{array}{c}\text { Range } \\
(-/+)\end{array}$ & Units & $\begin{array}{l}\text { Actual } \\
\text { Value }\end{array}$ & Comments \\
\hline \multirow{4}{*}{$\begin{array}{l}\text { Simulant } \\
\text { storage in HLP- } \\
\text { VSL-T22 } \\
\text { (Section 7.1) }\end{array}$} & \multirow[t]{4}{*}{ A } & HLP-VSL-T22 Operation & & & & & \\
\hline & & Agitator speed & 60 & $-5 /+5$ & $\mathrm{~Hz}$ & 60 & Data Source: VFD \\
\hline & & $\begin{array}{l}\text { Minimum simulant volume } \\
\text { required for test }\end{array}$ & 1,600 & N/A & gal & $\sim 3000$ & Data Source: HMI \\
\hline & & & & & & & $\begin{array}{l}\text { Value does NOT include nominal } 500 \text { gal } \\
\text { heel of HLP-VSL-T } 22 .\end{array}$ \\
\hline \multirow{14}{*}{$\begin{array}{c}\text { Transfer from } \\
\text { HLP-VSL-T22 } \\
\text { to UFP-VSL- } \\
\text { T01A (Section } \\
7.2)\end{array}$} & \multirow[t]{14}{*}{$\mathrm{B}$} & Total transfer volume (endpoint) & 593 & $-5 /+5$ & gal & 593.2 & $\begin{array}{l}\text { Data Source: HMI } \\
\text { Assumes no previous heel in } \\
\text { UFP-VSL-T01A. Level should be } \sim 67 \text { in. }\end{array}$ \\
\hline & & Transfer rate & 7.4 & $-2 /+2$ & gpm & 8.2 & Data Source: HMI \\
\hline & & UFP-VSL-T01A Operation & & & & & \\
\hline & & $\begin{array}{l}\text { Level to initiate steam ring air } \\
\text { purge and adjust flow rate }\end{array}$ & $<15$ & N/A & in. & 0 & Data Source: HMI \\
\hline & & & & & & & $\begin{array}{l}\text { Steam ring air purge should be on before } \\
\text { the rising simulant level reaches the ring. }\end{array}$ \\
\hline & & Steam ring air purge flow rate & 0.20 & $-0.02 /+0.02$ & $\mathrm{~kg} / \mathrm{min}$ & 0.20 & Data Source: Local flow meter \\
\hline & & & & & & & $6.1 \mathrm{scfm}$ below $90^{\circ} \mathrm{C}$ in UFP-VSL-T01A \\
\hline & & Level to initiate PJM operation & 15 & $-2 /+10$ & in. & 24 & Data Source: HMI \\
\hline & & PJM jet velocity & 4.8 & $-0.3 /+0.3$ & $\mathrm{~m} / \mathrm{s}$ & 4.7 & Data Source: CCP-901 \\
\hline & & PJM stroke $(80 \%)$ & 29.0 & $-3 /+3$ & in. & 28.6 & Data Source: CCP-901 \\
\hline & & PJM cycle time & 35 & $-1 /+1$ & s & 35.2 & Data Source: CCP-901 \\
\hline & & Level to add antifoam agent (AFA) & 15 & $-2 /+10$ & in. & 15 & Data Source: HMI \\
\hline & & $\begin{array}{l}\text { Volume of AFA to add to } \\
\text { UFP-VSL-T01A }\end{array}$ & 1,000 & $-10 /+10$ & $\mathrm{~mL}$ & 1000 & Data Source: Test Instruction \\
\hline & & & & & & & This volume is for undiluted AFA. \\
\hline \multirow{5}{*}{$\begin{array}{l}\text { Initial transfer } \\
\text { from UFP-VSL- } \\
\text { T01A to UFP- } \\
\text { VSL-T02A } \\
\text { (Section 7.3) }\end{array}$} & \multirow[t]{5}{*}{$\mathrm{C}$} & Transfer volume (endpoint) & 220 & $-3 /+3$ & gal & 220 & Data Source: HMI \\
\hline & & & & & & & UFP-VSL-T02A level should be $\sim 52$ in. \\
\hline & & Transfer rate & 6.9 & $-1 /+1$ & gpm & 6.9 & Data Source: HMI \\
\hline & & UFP-VSL-T02A Operation & & & & & \\
\hline & & Level to initiate PJM operation & 12 & $-2 /+10$ & in. & 57 & Data Source: HMI \\
\hline
\end{tabular}


Table M.1. Integrated Test B Run Sheet

\begin{tabular}{|c|c|c|c|c|c|c|c|}
\hline & $\begin{array}{l}\text { Run } \\
\text { Sheet } \\
\text { Step }\end{array}$ & Operation & $\begin{array}{l}\text { Target or } \\
\text { Setpoint } \\
\text { Value } \\
\end{array}$ & $\begin{array}{c}\text { Range } \\
(-/+)\end{array}$ & Units & $\begin{array}{c}\text { Actual } \\
\text { Value }\end{array}$ & Comments \\
\hline \multirow{13}{*}{ 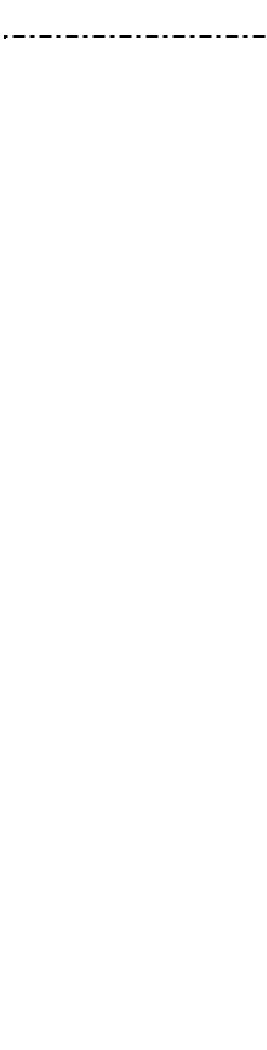 } & & & & & & & $\begin{array}{l}12 \text { in. is the UFP-VSL-T02A low mixing } \\
\text { level. }\end{array}$ \\
\hline & & PJM jet velocity & 7.3 & $-0.4 /+0.4$ & $\mathrm{~m} / \mathrm{s}$ & 7.1 & Data Source: CCP-901 \\
\hline & & PJM stroke $(80 \%)$ & 31.5 & $-3 /+3$ & in. & 31.5 & Data Source: CCP-901 \\
\hline & & PJM cycle time & 33 & $-1 /+1$ & $\mathrm{~s}$ & 33.2 & Data Source: CCP-901 \\
\hline & & $\begin{array}{l}\text { Level to adjust steam ring air purge } \\
\text { rotameter }\end{array}$ & $<15$ & N/A & in. & 0 & Data Source: HMI \\
\hline & & Steam ring air purge flow rate & 0.14 & $-0.02 /+0.02$ & $\mathrm{~kg} / \mathrm{min}$ & 0.14 & Data Source: Local flow meter \\
\hline & & & & & & & $\begin{array}{l}4.1 \mathrm{scfm} \text { in UFP-VSL-T02A whenever the } \\
\text { temperature is below } 90^{\circ} \mathrm{C} \text {. }\end{array}$ \\
\hline & & $\begin{array}{l}\text { Level to initiate and adjust lower } \\
\text { air spargers to full flow }\end{array}$ & 12 & $-2 /+10$ & in. & 27 & Data Source: HMI \\
\hline & & & & & & & $\begin{array}{l}\text { Full flow to lower spargers is required } \\
\text { whenever level is above lower mixing level } \\
\text { and filter-loop pumps are off. }\end{array}$ \\
\hline & & $\begin{array}{l}\text { Total lower air sparger flow rate } \\
\text { (for } 5 \text { sparge tubes) }\end{array}$ & 0.40 & $-0.05 /+0.05$ & $\mathrm{~kg} / \mathrm{min}$ & 0.40 & Data Source: Local flow meter \\
\hline & & & & & & & $\begin{array}{l}\text { Total of } 12 \mathrm{scfm} \text { for the } 5 \text { sparge tubes, or } \\
\sim 2.4 \mathrm{scfm} \text { for each sparge tube whenever } \\
\text { filter-loop pumps are not running. }\end{array}$ \\
\hline & & Upper air sparger flow rate & OFF & N/A & N/A & Off & Data Source: Test Instruction \\
\hline & & & & & & & $\begin{array}{l}\text { Upper air sparger is not turned on until } \\
\text { UFP-VSL-T02A level reaches } 52 \text { in. }\end{array}$ \\
\hline \multirow{5}{*}{$\begin{array}{l}\text { Concentrate } \\
\text { solids to } \\
\text { 20-wt\% in UFP- } \\
\text { VSL-T02A } \\
\text { (Section } 7.4 \text { - } \\
7.5 \text { ) }\end{array}$} & $\mathrm{D}$ & $\begin{array}{l}\text { UFP-VSL-T02A level to initiate } \\
\text { make-up batch transfers }\end{array}$ & 28.1 & $-1 /+3$ & in. & 28.1 & Data Source: HMI \\
\hline & & Make-up batch volume & 11 & $-1 /+1$ & gal & 11 & Data Source: HMI \\
\hline & & $\begin{array}{l}\text { Total number of make-up batches } \\
\text { (endpoint) }\end{array}$ & 47 & $0 /+0$ & & 47 & Data Source: Test Instruction \\
\hline & & & & & & & $\begin{array}{l}\text { Concentrate a total of } 737 \text { gal of } \sim 5-w t \% \\
\text { simulant to make } 173 \text { gal of } 20-w t \% \text { slurry. }\end{array}$ \\
\hline & & Make-up batch flow rate & 6.9 & $-1 /+1$ & gpm & 5.4 & Data Source: CCP-887 \\
\hline
\end{tabular}


Table M.1. Integrated Test B Run Sheet

\begin{tabular}{|c|c|c|c|c|c|c|c|}
\hline & $\begin{array}{l}\text { Run } \\
\text { Sheet } \\
\text { Step }\end{array}$ & Operation & $\begin{array}{l}\text { Target or } \\
\text { Setpoint } \\
\text { Value }\end{array}$ & $\begin{array}{c}\text { Range } \\
(-/+)\end{array}$ & Units. & $\begin{array}{l}\text { Actual } \\
\text { Value }\end{array}$ & Comments \\
\hline & & UFP-VSL-T02A Operation & & & & & \\
\hline & & $\begin{array}{l}\text { Total lower air sparger flow rate } \\
\text { (total for } 5 \text { sparge tubes, when } \\
\text { below } 90^{\circ} \mathrm{C} \text { ) }\end{array}$ & 0.010 & $\begin{array}{c}- \\
0.005 /+0.00 \\
5\end{array}$ & $\mathrm{~kg} / \mathrm{min}$ & 0.01 & $\begin{array}{l}\text { Data Source: Local flow meter } \\
\sim 3.7 \text { scfh for each sparge tube whenever } \\
\text { filter-loop pumps are running and the } \\
\text { temperature is below } 90^{\circ} \mathrm{C} \text {. }\end{array}$ \\
\hline & & Upper air sparger flow rate & OFF & N/A & N/A & Off & Data Source: Test Instruction \\
\hline & & & & & & & $\begin{array}{l}\text { Upper air sparger is not turned on until } \\
\text { UFP-VSL-T02A level reaches } 52 \text { in. }\end{array}$ \\
\hline & & Ultrafiltration Loop Operation & & & & & - \\
\hline & & $\begin{array}{l}\text { Number of filter bundles } \\
\text { (by-pass UFP_FILT-T02A-T05A) }\end{array}$ & 5 & $-0 /+0$ & & 5 & Data Source: Test Instruction \\
\hline & & Filter-loop flow rate & 109 & $-10 /+10$ & gpm & 109 & Data Source: HMI \\
\hline & & Transmembrane pressure & 40 & $-4 /+4$ & psi & 40 & Data Source: HMI \\
\hline & & & & & & & $\begin{array}{l}\text { Lower range not applicable if back } \\
\text { pressure valve is } 100 \% \text { open. }\end{array}$ \\
\hline & & $\begin{array}{l}\text { Temperature at inlet of UFP-FILT- } \\
\text { T01A }\end{array}$ & 25 & $-2 /+2$ & ${ }^{\circ} \mathrm{C}$ & 25 & Data Source: HMI \\
\hline \multirow{8}{*}{$\begin{array}{l}\text { Transfer from } \\
\text { HLP-VSL-T22 } \\
\text { to UFP-VSL- } \\
\text { T01A (1st refill) } \\
\text { (Section 7.4) }\end{array}$} & \multirow[t]{8}{*}{$\mathrm{E}$} & $\begin{array}{l}\text { Level in UFP-VSL-T01A to initiate } \\
1 \text { st refill from HLP-VSL-T22 }\end{array}$ & 28 & $-10 /+1$ & in. & 25 & Data Source: HMI \\
\hline & & & & & & & $\begin{array}{l}\text { This level should be reached after the } \\
\text { initial transfer and } \sim 14 \text { make-up batch } \\
\text { transfers. }\end{array}$ \\
\hline & & 1st refill transfer volume (endpoint) & 369 & $-10 /+10$ & gal & 369 & Data Source: HMI \\
\hline & & Refill transfer rate & 7.4 & $-1 /+1$ & gpm & 7.4 & Data Source: HMI \\
\hline & & & & & & & $\begin{array}{l}\text { This is to match plant Reynolds number of } \\
6,500\end{array}$ \\
\hline & & UFP-VSL-T01A Operation & & & & & \\
\hline & & $\begin{array}{l}\text { Volume of AFA to add to UFP- } \\
\text { VSL-T01A }\end{array}$ & 624 & $-10 /+10$ & $\mathrm{~mL}$ & 624 & Data Source: Test Instruction \\
\hline & & & & & & & This volume is for undiluted AFA. \\
\hline Add caustic to & $\mathrm{F}$ & $19 \mathrm{M}$ caustic to add (endpoint) & 720 & $-5 /+5$ & $\mathrm{~kg}$ & 721 & Data Source: WTP-RPT-197 \\
\hline
\end{tabular}


Table M.1. Integrated Test B Run Sheet

\begin{tabular}{|c|c|c|c|c|c|c|c|}
\hline & $\begin{array}{l}\text { Run } \\
\text { Sheet } \\
\text { Step }\end{array}$ & Operation & $\begin{array}{l}\text { Target or } \\
\text { Setpoint } \\
\text { Value } \\
\end{array}$ & $\begin{array}{c}\text { Range } \\
(-/+) \\
\end{array}$ & Units & $\begin{array}{l}\text { Actual } \\
\text { Value }\end{array}$ & Comments \\
\hline \multirow{13}{*}{$\begin{array}{l}\text { UFP-VSL- } \\
\text { T02A (Section } \\
7.6)\end{array}$} & & Caustic addition rate & 10.2 & $-0.5 /+0.5$ & $\mathrm{~kg} / \mathrm{min}$ & 11.5 & Data Source: CCP-887 \\
\hline & & & & & & & $\begin{array}{l}1.8 \mathrm{gpm} \text {. Target applies to average transfer } \\
\text { rate. }\end{array}$ \\
\hline & & Tank level after caustic addition & 49.5 & $-2 /+2$ & in. & & Not recorded in Test Instruction \\
\hline & & $\begin{array}{l}\text { Volume of antifoam to add to } \\
\text { UFP-VSL-T02A }\end{array}$ & 330 & $-10 /+10$ & $\mathrm{~mL}$ & 330 & Data Source: Test Instruction \\
\hline & & & & & & & $\begin{array}{l}\text { Antifoam is added here to account for } \\
\text { caustic and steam additions. This value is } \\
\text { the undiluted AFA volume. }\end{array}$ \\
\hline & & Ultrafiltration Loop Operation & & & & & \\
\hline & & Filter-loop flow rate & 109 & $-10 /+10$ & gpm & 109 & Data Source: HMI \\
\hline & & Permeate valves & CLOSED & & & Closed & Data Source: Test Instruction \\
\hline & & Maximum filter-loop temperature & 71 & N/A & $\mathrm{C}$ & 71 & Data Source: HMI \\
\hline & & UFP-VSL-T02A Operation & & & & & \\
\hline & & Level to initiate upper air sparge & 50 & $-5 /+5$ & & 50 & Data Source: HMI \\
\hline & & Upper air sparger flow rate & 0.10 & $-0.03 /+0.03$ & $\mathrm{~kg} / \mathrm{min}$ & 0.1 & Data Source: Local flow meter \\
\hline & & & & & & & $\begin{array}{l}3.0 \mathrm{scfm} \text {. This applies whenever the level } \\
\text { is above } 52 \mathrm{in} \text {. and temperature is below } \\
90^{\circ} \mathrm{C} \text {. }\end{array}$ \\
\hline \multirow{6}{*}{$\begin{array}{l}\text { Initial heat-up } \\
\text { of UFP-VSL- } \\
\text { T02A (Section } \\
7.6)\end{array}$} & G & $\begin{array}{l}\text { Initial heat-up temperature } \\
\text { (endpoint) }\end{array}$ & 71 & $-1 /+1$ & $\mathrm{C}$ & 72 & Data Source: CCP-996 \\
\hline & & Initial heat-up time & $<2$ & N/A & $\mathrm{hr}$ & $<2$ & Data Source: Test Instruction \\
\hline & & & & & & & $\begin{array}{l}\text { Starts after } 19 \mathrm{M} \text { caustic addition is } \\
\text { complete. }\end{array}$ \\
\hline & & Ultrafiltration Loop Operation & & & & & \\
\hline & & Filter-loop flow rate & 109 & $-10 /+10$ & gpm & 109 & Data Source: HMI \\
\hline & & Permeate valves & CLOSED & & & Closed & Data Source: Test Instruction \\
\hline $\begin{array}{l}\text { Back flush } \\
\text { filter-loop to } \\
\text { UFP-VSL- }\end{array}$ & $\mathrm{H}$ & $\begin{array}{l}\text { Inhibited water addition for filter } \\
\text { loop back-flush to UFP-VSL-T02A } \\
\text { (endpoint) }\end{array}$ & 74 & $-5 /+5$ & $\mathrm{~kg}$ & 74 & Data Source: HMI \\
\hline T02A (Section & & Flush water addition rate & maximum & & & -- & Not recorded. \\
\hline
\end{tabular}


Table M.1. Integrated Test B Run Sheet

\begin{tabular}{|c|c|c|c|c|c|c|c|}
\hline & $\begin{array}{l}\text { Run } \\
\text { Sheet } \\
\text { Step }\end{array}$ & Operation & $\begin{array}{l}\text { Target or } \\
\text { Setpoint } \\
\text { Value }\end{array}$ & $\begin{array}{c}\text { Range } \\
(-/+)\end{array}$ & Units & $\begin{array}{l}\text { Actual } \\
\text { Value }\end{array}$ & Comments \\
\hline \multirow[t]{10}{*}{$7.6)$} & & & $\begin{array}{l}\text { achievable } \\
\text { flow }\end{array}$ & & & & $\begin{array}{l}\text { The pump capacity is } \sim 35 \mathrm{gpm} \\
(132 \mathrm{~kg} / \mathrm{min}) \text {; observed to be } \sim 22 \mathrm{gpm} \\
(83 \mathrm{~kg} / \mathrm{min}) \text { in November } 2008 \text {. }\end{array}$ \\
\hline & & Tank level after loop flush & 53.5 & $-2 /+2$ & in. & 60 & Data Source: HMI \\
\hline & & UFP-VSL-T02A Operation & & & & & \\
\hline & & $\begin{array}{l}\text { Steam ring air purge flow rate } \\
\text { (when below } 90^{\circ} \mathrm{C} \text { ) }\end{array}$ & 0.012 & $-0.02 /+0.02$ & $\mathrm{~kg} / \mathrm{min}$ & 0.012 & Data Source: Local flow meter \\
\hline & & & & & & & $\begin{array}{l}4.1 \mathrm{scfm} \text { in UFP-VSL-T02A whenever the } \\
\text { temperature is below } 90^{\circ} \mathrm{C} \text {. }\end{array}$ \\
\hline & & $\begin{array}{l}\text { Total lower air sparger flow rate } \\
\text { (total for } 5 \text { sparge tubes, when } \\
\text { below } 90^{\circ} \mathrm{C} \text { ) }\end{array}$ & 0.40 & $-0.05 /+0.05$ & $\mathrm{~kg} / \mathrm{min}$ & 0.40 & $\begin{array}{l}\text { Data Source: Local flow meter } \\
\text { Total of } 12 \mathrm{scfm} \text { for the } 5 \text { sparge tubes, or } \\
\sim 2.4 \mathrm{scfm} \text { for each sparge tube whenever } \\
\text { filter-loop pumps are not running and the } \\
\text { temperature is below } 90^{\circ} \mathrm{C} \text {. }\end{array}$ \\
\hline & & $\begin{array}{l}\text { Upper air sparger flow rate (when } \\
\text { below } 90^{\circ} \mathrm{C} \text { ) }\end{array}$ & 0.10 & $-0.03 /+0.03$ & $\mathrm{~kg} / \mathrm{min}$ & 0.10 & Data Source: Local flow meter \\
\hline & & & & & & & $\begin{array}{l}3 \mathrm{scfm} \text {. This applies whenever the level is } \\
\text { above } 52 \mathrm{in} \text {. and temperature is below } \\
90^{\circ} \mathrm{C} \text {. }\end{array}$ \\
\hline & & Ultrafiltration Loop Operation & & & & & \\
\hline & & $\begin{array}{l}\text { Filter-loop pumps UFP-PMP-T42A } \\
\text { and T43A }\end{array}$ & OFF & & & Off & Data Source: Test Instruction \\
\hline \multirow{8}{*}{$\begin{array}{l}\text { Final heat-up of } \\
\text { UFP-VSL- } \\
\text { T02A (Section } \\
7.6)\end{array}$} & \multirow[t]{8}{*}{$\mathrm{I}$} & Target final temperature (endpoint) & 98 & $-2 /+2$ & $\mathrm{C}$ & 98 & Data Source: CCP-996 \\
\hline & & Heat-up time & 2.6 & $-0.5 /+0.5$ & $\mathrm{hr}$ & 2.6 & Data Source: CCP-996 \\
\hline & & UFP-VSL-T02A Operation & & & & & \\
\hline & & PJM jet velocity & 7.3 & $-0.4 /+0.4$ & $\mathrm{~m} / \mathrm{s}$ & 7.5 & Data Source: NQA-1 calculation \\
\hline & & PJM stroke $(80 \%)$ & 31.5 & $-3 /+3$ & in. & 29.7 & Data Source: NQA-1 calculation \\
\hline & & PJM cycle time & 33 & $-1 /+1$ & $\mathrm{~s}$ & 33.2 & Data Source: NQA-1 calculation \\
\hline & & $\begin{array}{l}\text { Temperature limit to adjust air } \\
\text { sparger and steam ring air purge } \\
\text { rates }\end{array}$ & 90 & $-2 /+2$ & $\mathrm{C}$ & -- & Not recorded \\
\hline & & $\begin{array}{l}\text { Steam ring air purge flow rate } \\
\text { (when above } 90^{\circ} \mathrm{C} \text { ) }\end{array}$ & 0.09 & $-0.02 /+0.02$ & $\mathrm{~kg} / \mathrm{min}$ & 0.09 & $\begin{array}{l}\text { Data Source: CCP-996 } \\
2.6 \mathrm{scfm} \text { above } 90^{\circ} \mathrm{C} \text { in UFP-VSL-T02A }\end{array}$ \\
\hline
\end{tabular}


Table M.1. Integrated Test B Run Sheet

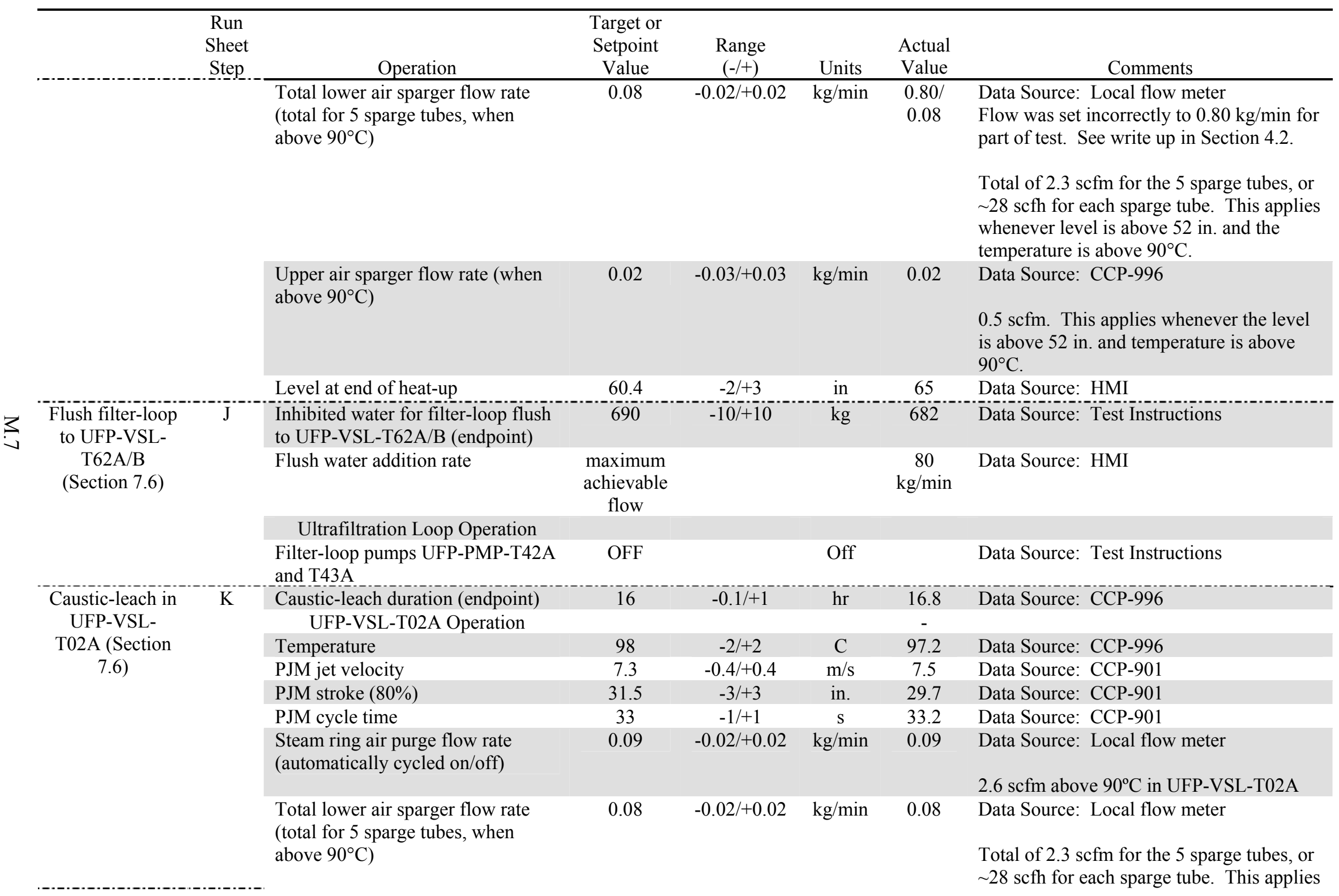


Table M.1. Integrated Test B Run Sheet

\begin{tabular}{|c|c|c|c|c|c|c|c|}
\hline & $\begin{array}{l}\text { Run } \\
\text { Sheet } \\
\text { Step }\end{array}$ & Operation & $\begin{array}{l}\text { Target or } \\
\text { Setpoint } \\
\text { Value }\end{array}$ & $\begin{array}{c}\text { Range } \\
(-/+)\end{array}$ & Units & $\begin{array}{l}\text { Actual } \\
\text { Value }\end{array}$ & Comments \\
\hline & & & & & & & $\begin{array}{l}\text { whenever level is above } 52 \text { in. and the } \\
\text { temperature is above } 90^{\circ} \mathrm{C} \text {. }\end{array}$ \\
\hline & & $\begin{array}{l}\text { Upper air sparger flow rate (when } \\
\text { above } 90^{\circ} \mathrm{C} \text { ) }\end{array}$ & 0.02 & $-0.03 /+0.03$ & $\mathrm{~kg} / \mathrm{min}$ & 0.02 & Data Source: Local flow meter \\
\hline & & & & & & & $\begin{array}{l}0.5 \mathrm{scfm} \text {. This applies whenever the level } \\
\text { is above } 52 \text { in. and temperature is above } \\
90^{\circ} \mathrm{C} \text {. }\end{array}$ \\
\hline & & Level at end of caustic-leach & 65.2 & $-5 /+5$ & in. & 73.1 & Data Source: HMI \\
\hline \multirow{10}{*}{$\begin{array}{l}\text { Cool-down of } \\
\text { UFP-VSL- } \\
\text { T02A (Section } \\
7.7)\end{array}$} & \multirow[t]{10}{*}{$\mathrm{L}$} & Final cool-down temperature & 25 & $-2 /+2$ & $\mathrm{C}$ & 25 & Data Source: HMI \\
\hline & & $\begin{array}{l}\text { Time to reach final cool-down } \\
\text { temperature }\end{array}$ & 13.2 & $-1 /+1$ & $\mathrm{hr}$ & 13.2 & Data Source: Test Instructions \\
\hline & & UFP-VSL-T02A Operation & & & & & \\
\hline & & $\begin{array}{l}\text { Temperature for air spargers and } \\
\text { steam ring air purge rate adjustment }\end{array}$ & 90 & $-2 /+2$ & $\mathrm{C}$ & 88 & Data Source: HMI \\
\hline & & $\begin{array}{l}\text { Steam ring air purge flow rate } \\
\text { (when below } 90^{\circ} \mathrm{C} \text { ) }\end{array}$ & 0.14 & $-0.02 /+0.02$ & $\mathrm{~kg} / \mathrm{min}$ & 0.14 & Data Source: Local flow meter \\
\hline & & & & & & & $\begin{array}{l}4.1 \mathrm{scfm} \text { in UFP-VSL-T02A whenever the } \\
\text { temperature is below } 90^{\circ} \mathrm{C} \text {. }\end{array}$ \\
\hline & & $\begin{array}{l}\text { Total lower air sparger flow rate } \\
\text { (total for } 5 \text { sparge tubes, when }\end{array}$ & 0.40 & $-0.05 /+0.05$ & $\mathrm{~kg} / \mathrm{min}$ & 0.40 & Data Source: Local flow meter \\
\hline & & below $\left.90^{\circ} \mathrm{C}\right)$ & & & & & $\begin{array}{l}\text { Total of } 12 \mathrm{scfm} \text { for the } 5 \text { sparge tubes, or } \\
2.4 \mathrm{scfm} \text { for each sparge tube whenever } \\
\text { filter-loop pumps are not running and the } \\
\text { temperature is below } 90^{\circ} \mathrm{C} \text {. }\end{array}$ \\
\hline & & $\begin{array}{l}\text { Upper air sparger flow rate (when } \\
\text { below } 90^{\circ} \mathrm{C} \text { ) }\end{array}$ & 0.10 & $-0.03 /+0.03$ & $\mathrm{~kg} / \mathrm{min}$ & 0.10 & Data Source: Local flow meter \\
\hline & & & & & & & $\begin{array}{l}3 \mathrm{scfm} \text {. This applies whenever the level is } \\
\text { above } 52 \mathrm{in} \text {. and temperature is below } \\
90^{\circ} \mathrm{C} \text {. }\end{array}$ \\
\hline \multirow{2}{*}{$\begin{array}{l}\text { Transfer from } \\
\text { UFP-VSL- } \\
\text { T02A to UFP- } \\
\text { VSL-T01B } \\
\text { (Section } 7.8 \text { ) }\end{array}$} & \multirow[t]{2}{*}{ M } & Transfer volume (endpoint) & ALL & $\mathrm{N} / \mathrm{A}$ & & All & Data Source: Test Instruction \\
\hline & & Transfer rate & $\begin{array}{l}\text { No } \\
\text { targeted } \\
\text { rate }\end{array}$ & & gpm & & $\begin{array}{l}\text { Transfer at maximum rate to keep solids } \\
\text { suspended. }\end{array}$ \\
\hline
\end{tabular}


Table M.1. Integrated Test B Run Sheet

\begin{tabular}{|c|c|c|c|c|c|c|c|}
\hline & $\begin{array}{l}\text { Run } \\
\text { Sheet } \\
\text { Step. }\end{array}$ & Operation & $\begin{array}{l}\text { Target or } \\
\text { Setpoint } \\
\text { Value }\end{array}$ & $\begin{array}{c}\text { Range } \\
(-/+)\end{array}$ & Units & $\begin{array}{l}\text { Actual } \\
\text { Value }\end{array}$ & Comments \\
\hline & & $\begin{array}{l}\text { Total lower air sparger flow rate } \\
\text { (total for } 5 \text { sparge tubes) }\end{array}$ & 0.40 & $-0.05 /+0.05$ & $\mathrm{~kg} / \mathrm{min}$ & 0.40 & $\begin{array}{l}\text { Data Source: Local flow meter } \\
\text { Total of } 12 \mathrm{scfm} \text { for the } 5 \text { sparge tubes, or } \\
2.4 \mathrm{scfm} \text { for each sparge tube, whenever } \\
\text { filter-loop pumps are off. }\end{array}$ \\
\hline & & $\begin{array}{l}\text { Level to turn off upper air sparger } \\
\text { flow rate }\end{array}$ & 50 & $-10 /+0$ & in. & $<50$ & $\begin{array}{l}\text { Data Source: HMI } \\
\text { Upper air sparger is turned off when } \\
\text { UFP-VSL-T02A level is below } 52 \text { in. }\end{array}$ \\
\hline & & Level to stop PJMs & 15 & $-5 /+10$ & in. & $<15$ & Data Source: HMI \\
\hline & & UFP-VSL-T01B Operation & & & & & \\
\hline & & $\begin{array}{l}\text { Level to initiate steam ring air } \\
\text { purge and adjust flow rate }\end{array}$ & $<15$ & N.A. & in. & 0 & Data Source: HMI \\
\hline & & & & & & & $\begin{array}{l}\text { Steam ring air purge should be on before } \\
\text { the rising simulant level reaches the ring. }\end{array}$ \\
\hline & & Steam ring air purge flow rate & 0.20 & $-0.02 /+0.02$ & $\mathrm{~kg} / \mathrm{min}$ & 0.14 & Data Source: Local flow meter \\
\hline & & & & & & & $6.1 \mathrm{scfm}$ below $90^{\circ} \mathrm{C}$ in UFP-VSL-T01B \\
\hline & & Level to initiate PJM operation & 15 & $-2 /+10$ & in. & 16 & Data Source: HMI \\
\hline & & PJM jet velocity & 4.8 & $-0.3 /+0.3$ & $\mathrm{~m} / \mathrm{s}$ & 5.1 & Data Source: CCP-901 \\
\hline & & PJM stroke $(80 \%)$ & 29.0 & $-3 /+3$ & in. & 20.4 & Data Source: CCP-901 \\
\hline & & PJM cycle time & 35 & $-1 /+1$ & $\mathrm{~s}$ & 35.1 & Data Source: CCP-901 \\
\hline \multirow{6}{*}{$\begin{array}{l}\text { Transfer from } \\
\text { HLP-VSL-T22 } \\
\text { to UFP-VSL- } \\
\text { T01A ( } 2 \mathrm{nd} \\
\text { refill) (Section } \\
7.9 \text { ) }\end{array}$} & \multirow[t]{6}{*}{$\mathrm{N}$} & $\begin{array}{l}\text { Level in UFP-VSL-T01A to initiate } \\
\text { 2nd refill from HLP-VSL-T22 }\end{array}$ & 28 & $-5 /+5$ & in. & $\begin{array}{l}25.5- \\
31.4\end{array}$ & $\begin{array}{l}\text { Data Source: HMI } \\
\text { Refill is to be performed in preparation for } \\
\text { the } 2 \text { nd caustic-leach. }\end{array}$ \\
\hline & & $\begin{array}{l}\text { 2nd refill transfer volume } \\
\text { (endpoint) }\end{array}$ & 369 & $-10 /+10$ & gal & 369 & Data Source: HMI \\
\hline & & Refill transfer rate & 7.4 & $-1 /+1$ & gpm & 7.4 & Data Source: HMI \\
\hline & & & & & & & $\begin{array}{l}\text { This is to match plant Reynolds number of } \\
6,500\end{array}$ \\
\hline & & UFP-VSL-T01A Operation & & & & & \\
\hline & & $\begin{array}{l}\text { Volume of AFA to add to } \\
\text { UFP-VSL-T01A }\end{array}$ & 624 & $-10 /+10$ & $\mathrm{~mL}$ & 624 & $\begin{array}{l}\text { Data Source: Test Instruction } \\
\text { This volume is for undiluted AFA. }\end{array}$ \\
\hline
\end{tabular}


Table M.1. Integrated Test B Run Sheet

\begin{tabular}{|c|c|c|c|c|c|c|c|}
\hline & $\begin{array}{l}\text { Run } \\
\text { Sheet } \\
\text { Step }\end{array}$ & Operation & $\begin{array}{l}\text { Target or } \\
\text { Setpoint } \\
\text { Value }\end{array}$ & $\begin{array}{c}\text { Range } \\
(-/+)\end{array}$ & Units. & $\begin{array}{l}\text { Actual } \\
\text { Value }\end{array}$ & Comments \\
\hline \multirow{18}{*}{$\begin{array}{l}\text { Initial transfer } \\
\text { from UFP-VSL- } \\
\text { T01A to UFP- } \\
\text { VSL-T02A } \\
\text { (Section 7.10) }\end{array}$} & $\mathrm{O}$ & Transfer volume (endpoint) & 220 & $-3 /+3$ & gal & 219 & Data Source: WTP-RPT-186 \\
\hline & & & & & & & UFP-VSL-T02A level should be $\sim 52$ in. \\
\hline & & Transfer rate & 6.9 & $-1 /+1$ & gpm & 7.0 & Data Source: WTP-RPT-186 \\
\hline & & UFP-VSL-T02A Operation & & & & & \\
\hline & & Level to initiate PJM operation & 12 & $-2 /+10$ & in. & 16 & Data Source: HMI \\
\hline & & & & & & & $\begin{array}{l}12 \text { in. is the UFP-VSL-T02A low mixing } \\
\text { level. }\end{array}$ \\
\hline & & PJM jet velocity & 7.3 & $-0.4 /+0.4$ & $\mathrm{~m} / \mathrm{s}$ & 6.6 & Data Source: CCP-901 \\
\hline & & PJM stroke $(80 \%)$ & 31.5 & $-3 /+3$ & in. & 27.5 & Data Source: CCP-901 \\
\hline & & PJM cycle time & 33 & $-1 /+1$ & $\mathrm{~s}$ & 33.2 & Data Source: CCP-901 \\
\hline & & $\begin{array}{l}\text { Level to adjust steam ring air purge } \\
\text { rotameter }\end{array}$ & $<15$ & N.A. & in. & 0 & Data Source: HMI \\
\hline & & Steam ring air purge flow rate & 0.14 & $-0.02 /+0.02$ & $\mathrm{~kg} / \mathrm{min}$ & 0.14 & Data Source: Local flow meter \\
\hline & & & & & & & $\begin{array}{l}4.1 \mathrm{scfm} \text { in UFP-VSL-T02A whenever the } \\
\text { temperature is below } 90^{\circ} \mathrm{C} \text {. }\end{array}$ \\
\hline & & $\begin{array}{l}\text { Level to initiate and adjust lower } \\
\text { air spargers to full flow }\end{array}$ & 12 & $-2 /+10$ & in. & 0 & Data Source: HMI \\
\hline & & & & & & & $\begin{array}{l}\text { Full flow to lower spargers is required } \\
\text { whenever level is above lower mixing level } \\
\text { and filter-loop pumps are off. }\end{array}$ \\
\hline & & $\begin{array}{l}\text { Total lower air sparger flow rate } \\
\text { (for } 5 \text { sparge tubes) }\end{array}$ & 0.40 & $-0.05 /+0.05$ & $\mathrm{~kg} / \mathrm{min}$ & 0.4 & Data Source: Local flow meter \\
\hline & & & & & & & $\begin{array}{l}\text { Total of } 12 \text { scfm for the } 5 \text { sparge tubes, or } \\
2.4 \text { scfm for each sparge tube whenever } \\
\text { filter-loop pumps are not running. }\end{array}$ \\
\hline & & Upper air sparger flow rate & OFF & N.A. & $\mathrm{N} / \mathrm{A}$ & Off & Data Source: Test Instruction \\
\hline & & & & & & & $\begin{array}{l}\text { Upper air sparger is not turned on until } \\
\text { UFP-VSL-T02A level reaches } 52 \text { in. }\end{array}$ \\
\hline $\begin{array}{l}\text { Concentrate } \\
\text { solids to }\end{array}$ & $\mathrm{P}$ & $\begin{array}{l}\text { UFP-VSL-T02A level to initiate } \\
\text { make-up batch transfers }\end{array}$ & 28 & $-1 /+3$ & in. & 28 & Data Source: HMI \\
\hline
\end{tabular}


Table M.1. Integrated Test B Run Sheet

\begin{tabular}{|c|c|c|c|c|c|c|c|}
\hline & $\begin{array}{l}\text { Run } \\
\text { Sheet } \\
\text { Step }\end{array}$ & Operation & $\begin{array}{l}\text { Target or } \\
\text { Setpoint } \\
\text { Value }\end{array}$ & $\begin{array}{l}\text { Range } \\
(-/+)\end{array}$ & Units & $\begin{array}{l}\text { Actual } \\
\text { Value }\end{array}$ & Comments \\
\hline \multirow{12}{*}{$\begin{array}{l}20 \text {-wt } \% \text { in UFP- } \\
\text { VSL-T02A } \\
\text { (Section } 7.11 \\
\text { and } 7.12 \text { ) }\end{array}$} & & Make-up batch volume & 11 & $-1 /+1$ & gal & 11 & Data Source: Test Instruction \\
\hline & & $\begin{array}{l}\text { Total number of make-up batches } \\
\text { (endpoint) }\end{array}$ & 47 & $0 /+0$ & & 47 & Data Source: Test Instruction \\
\hline & & & & & & & $\begin{array}{l}\text { Concentrate a total of } 737 \text { gal of } 5-w t \% \\
\text { simulant to make } 173 \text { gal of } 20-w t \% \text { slurry. }\end{array}$ \\
\hline & & UFP-VSL-T02A Operation & & & & & \\
\hline & & $\begin{array}{l}\text { Total lower air sparger (idle) flow } \\
\text { rate (for } 5 \text { sparge tubes) }\end{array}$ & 0.010 & $\begin{array}{l}-0.005 / \\
+0.005\end{array}$ & $\mathrm{~kg} / \mathrm{min}$ & 0.012 & Data Source: Local flow meter \\
\hline & & & & & & & $\begin{array}{l}\sim 3.7 \text { scfh for each sparge tube whenever } \\
\text { filter-loop pumps are running and the } \\
\text { temperature is below } 90^{\circ} \mathrm{C} \text {. }\end{array}$ \\
\hline & & Upper air sparger flow rate & $\mathrm{OFF}$ & N/A & N/A & Off & $\begin{array}{l}\text { Data Source: Test Instruction } \\
\text { Upper air sparger is not turned on until } \\
\text { UFP-VSL-T02A level reaches } 52 \text { in. }\end{array}$ \\
\hline & & Ultrafiltration Loop Operation & & & & & \\
\hline & & Number of filter bundles & 1 & $-0 /+0$ & & 1 & Data Source: Test Instruction \\
\hline & & Filter loop flow rate & 109 & $-10 /+10$ & gpm & 109 & Data Source: HMI \\
\hline & & Transmembrane pressure & 40 & $-4 /+4$ & psi & 40 & $\begin{array}{l}\text { Data Source: HMI } \\
\text { Lower range not applicable if back } \\
\text { pressure valve is } 100 \% \text { open. }\end{array}$ \\
\hline & & $\begin{array}{l}\text { Temperature at inlet of UFP-FILT- } \\
\text { T01A }\end{array}$ & 25 & $-2 /+2$ & ${ }^{\circ} \mathrm{C}$ & 25 & Data Source: HMI \\
\hline \multirow{4}{*}{$\begin{array}{l}\text { Transfer from } \\
\text { HLP-VSL-T22 } \\
\text { to UFP-VSL- } \\
\text { T01A (3rd } \\
\text { refill) }\end{array}$} & Q & $\begin{array}{l}\text { Level in UFP-VSL-T01A to initiate } \\
\text { 3rd refill from HLP-VSL-T22 }\end{array}$ & 28 & $-10 /+1$ & in. & 28.1 & $\begin{array}{l}\text { Data Source: HMI } \\
\text { Level is reached after } \sim 33 \text { make-up batches } \\
\text { have been transferred (since } 2 \text { nd refill). }\end{array}$ \\
\hline & & $\begin{array}{l}\text { 3rd refill transfer volume } \\
\text { (endpoint) }\end{array}$ & 369 & $-10 /+10$ & gal & 369.4 & Data Source: HMI \\
\hline & & Refill transfer rate & 7.4 & $-1 /+1$ & gpm & 7.0 & $\begin{array}{l}\text { Data Source: HMI } \\
\text { This is to match plant Reynolds number of } \\
6,500\end{array}$ \\
\hline & & UFP-VSL-T01A Operation & & & & & \\
\hline
\end{tabular}


Table M.1. Integrated Test B Run Sheet

\begin{tabular}{|c|c|c|c|c|c|c|c|}
\hline & $\begin{array}{l}\text { Run } \\
\text { Sheet } \\
\text { Step }\end{array}$ & Operation & $\begin{array}{l}\text { Target or } \\
\text { Setpoint } \\
\text { Value }\end{array}$ & $\begin{array}{c}\text { Range } \\
(-/+)\end{array}$ & Units. & $\begin{array}{l}\text { Actual } \\
\text { Value }\end{array}$ & Comments \\
\hline & & $\begin{array}{l}\text { Volume of AFA to add to } \\
\text { UFP-VSL-T01A }\end{array}$ & 624 & $-10 /+10$ & $\mathrm{~mL}$ & 624 & $\begin{array}{l}\text { Data Source: Test Instruction } \\
\text { This volume is for undiluted AFA. }\end{array}$ \\
\hline \multirow{13}{*}{$\begin{array}{l}\text { Add caustic to } \\
\text { UFP-VSL- } \\
\text { T02A (Section } \\
7.13)\end{array}$} & \multirow[t]{13}{*}{$\mathrm{R}$} & $19 \mathrm{M}$ caustic to add (endpoint) & 720 & $-5 /+5$ & $\mathrm{~kg}$ & 721 & Data Source: WTP-RPT-186 \\
\hline & & Caustic addition rate & 10.2 & $-0.5 /+0.5$ & $\mathrm{~kg} / \mathrm{min}$ & 10.5 & $\begin{array}{l}\text { Data Source: WTP-RPT- } 186 \\
1.8 \text { gpm. Target applies to average transfer } \\
\text { rate. }\end{array}$ \\
\hline & & Tank level after caustic addition & 49.5 & $-2 /+2$ & in. & 52.9 & Data Source: WTP-RPT-186 \\
\hline & & $\begin{array}{l}\text { Volume of antifoam to add to } \\
\text { UFP-VSL-T02A }\end{array}$ & 330 & $-10 /+10$ & $\mathrm{~mL}$ & 330 & Data Source: Test Instruction \\
\hline & & & & & & & $\begin{array}{l}\text { Antifoam is added here to account for } \\
\text { caustic and steam additions. This value is } \\
\text { the undiluted AFA volume. }\end{array}$ \\
\hline & & Ultrafiltration Loop Operation & & & & & \\
\hline & & Filter-loop flow rate & 109 & $-10 /+10$ & gpm & 101 & Data Source: WTP-RPT-186 \\
\hline & & Permeate valves & CLOSED & & & Closed & Data Source: Test Instruction \\
\hline & & Maximum filter-loop temperature & 71 & $\mathrm{~N} / \mathrm{A}$ & ${ }^{\circ} \mathrm{C}$ & 57 & Data Source: HMI \\
\hline & & UFP-VSL-T02A Operation & & & & & \\
\hline & & Level to initiate upper air sparge & 50 & $-5 /+5$ & & 53 & Data Source: HMI \\
\hline & & Upper air sparger flow rate & 0.10 & $-0.03 /+0.03$ & $\mathrm{~kg} / \mathrm{min}$ & 0.1 & Data Source: Local flow meter \\
\hline & & & & & & & $\begin{array}{l}3.0 \mathrm{scfm} . \text { This applies whenever the level } \\
\text { is above } 52 \mathrm{in} \text {. and temperature is below } \\
90^{\circ} \mathrm{C} \text {. }\end{array}$ \\
\hline \multirow{4}{*}{$\begin{array}{l}\text { Initial heat-up } \\
\text { of UFP-VSL- } \\
\text { T02A (Section } \\
7.13 \text { ) }\end{array}$} & \multirow[t]{4}{*}{$\mathrm{S}$} & $\begin{array}{l}\text { Initial heat-up temperature } \\
\text { (endpoint) }\end{array}$ & 71 & $-1 /+1$ & ${ }^{\circ} \mathrm{C}$ & 71.8 & Data Source: WTP-RPT-186 \\
\hline & & Ultrafiltration Loop Operation & & & & & \\
\hline & & Filter-loop flow rate & 109 & $-10 /+10$ & gpm & 100 & Data Source: WTP-RPT-186 \\
\hline & & Permeate valves & CLOSED & & & Closed & Data Source: Test Instruction \\
\hline $\begin{array}{l}\text { Back flush } \\
\text { filter-loop to } \\
\text { UFP-VSL- }\end{array}$ & $\mathrm{T}$ & $\begin{array}{l}\text { Inhibited water addition for } \\
\text { filter-loop back-flush to UFP-VSL- } \\
\text { T02A (endpoint) }\end{array}$ & 74 & $-5 /+5$ & $\mathrm{~kg}$ & 77.6 & Data Source: WTP-RPT-186 \\
\hline
\end{tabular}


Table M.1. Integrated Test B Run Sheet

\begin{tabular}{|c|c|c|c|c|c|c|c|}
\hline & $\begin{array}{l}\text { Run } \\
\text { Sheet } \\
\text { Step }\end{array}$ & Operation & $\begin{array}{l}\text { Target or } \\
\text { Setpoint } \\
\text { Value }\end{array}$ & $\begin{array}{c}\text { Range } \\
(-/+)\end{array}$ & Units & $\begin{array}{l}\text { Actual } \\
\text { Value }\end{array}$ & Comments \\
\hline \multirow[t]{4}{*}{$\begin{array}{c}\text { T02A (Section } \\
7.13 \text { ) }\end{array}$} & & Flush water addition rate & $\begin{array}{l}\operatorname{maximum} \\
\text { achievable } \\
\text { flow }\end{array}$ & & $\mathrm{kg} / \mathrm{min}$ & 73 & $\begin{array}{l}\text { Data Source: WTP-RPT-186 } \\
\text { The pump capacity is } \sim 35 \mathrm{gpm} \\
(132 \mathrm{~kg} / \mathrm{min}) \text {; observed to be } \sim 22 \mathrm{gpm} \\
(83 \mathrm{~kg} / \mathrm{min}) \text { in November } 2008 .\end{array}$ \\
\hline & & Tank level after loop flush & 53.5 & $-3 /+3$ & in. & 56.3 & $\begin{array}{l}\text { Data Source: WTP-RPT-186 } \\
\sim 227 \text { gal in UFP-VSL-T02A. }\end{array}$ \\
\hline & & Ultrafiltration Loop Operation & & & & & \\
\hline & & $\begin{array}{l}\text { Filter-loop pumps UFP-PMP-T42A } \\
\text { and T43A }\end{array}$ & OFF & & & Off & Data Source: Test Instruction \\
\hline \multirow{13}{*}{$\begin{array}{l}\text { Final heat-up of } \\
\text { UFP-VSL- } \\
\text { T02A (Section } \\
7.13)\end{array}$} & \multirow[t]{13}{*}{$\mathrm{U}$} & Target final temperature (endpoint) & 98 & $-2 /+2$ & ${ }^{\circ} \mathrm{C}$ & 98.0 & Data Source: WTP-RPT-186 \\
\hline & & Heat-up time & 2.6 & $-0.5 /+0.5$ & $\mathrm{hr}$ & 2.4 & Data Source: WTP-RPT-186 \\
\hline & & UFP-VSL-T02A Operation & & & & & \\
\hline & & PJM jet velocity & 7.3 & $-0.4 /+0.4$ & $\mathrm{~m} / \mathrm{s}$ & 7.5 & Data Source: CCP-901 \\
\hline & & PJM stroke $(80 \%)$ & 31.5 & $-3 /+3$ & in. & 29.6 & Data Source: CCP-901 \\
\hline & & PJM cycle time & 33 & $-1 /+1$ & $\mathrm{~s}$ & 33.2 & Data Source: CCP-901 \\
\hline & & $\begin{array}{l}\text { Temperature limit to adjust air } \\
\text { sparger and steam ring air purge } \\
\text { rates }\end{array}$ & 90 & $-2 /+2$ & ${ }^{\circ} \mathrm{C}$ & 92.3 & Data Source: HMI \\
\hline & & $\begin{array}{l}\text { Steam ring air purge flow rate } \\
\text { (when above } 90^{\circ} \mathrm{C} \text { ) }\end{array}$ & 0.09 & $-0.02 /+0.02$ & $\mathrm{~kg} / \mathrm{min}$ & 0.09 & Data Source: CCP-783 \\
\hline & & & & & & & $2.6 \mathrm{scfm}$ above $90^{\circ} \mathrm{C}$ in UFP-VSL-T02A \\
\hline & & $\begin{array}{l}\text { Total lower air sparger flow rate } \\
\text { (total for } 5 \text { sparge tubes, when } \\
\text { above } 90^{\circ} \mathrm{C} \text { ) }\end{array}$ & 0.08 & $-0.02 /+0.02$ & $\mathrm{~kg} / \mathrm{min}$ & 0.08 & $\begin{array}{l}\text { Data Source: CCP-783 } \\
\text { Total of } 2.3 \mathrm{scfm} \text { for the } 5 \text { sparge tubes, or } \\
\sim 28 \text { scfh for each sparge tube. This applies } \\
\text { whenever level is above } 52 \text { in. and the } \\
\text { temperature is above } 90^{\circ} \mathrm{C} \text {. }\end{array}$ \\
\hline & & $\begin{array}{l}\text { Upper air sparger flow rate (when } \\
\text { above } 90^{\circ} \mathrm{C} \text { ) }\end{array}$ & 0.02 & $-0.03 /+0.03$ & $\mathrm{~kg} / \mathrm{min}$ & 0.02 & Data Source: CCP-783 \\
\hline & & & & & & & $\begin{array}{l}0.5 \mathrm{scfm} \text {. This applies whenever the level } \\
\text { is above } 52 \mathrm{in} \text {. and temperature is above } \\
90^{\circ} \mathrm{C} \text {. }\end{array}$ \\
\hline & & Level at end of heat-up & 60.4 & $-2 /+3$ & in & 61.8 & Data Source: HMI \\
\hline
\end{tabular}


Table M.1. Integrated Test B Run Sheet

\begin{tabular}{|c|c|c|c|c|c|c|c|}
\hline & $\begin{array}{l}\text { Run } \\
\text { Sheet } \\
\text { Step }\end{array}$ & Operation & $\begin{array}{l}\text { Target or } \\
\text { Setpoint } \\
\text { Value }\end{array}$ & $\begin{array}{l}\text { Range } \\
(-/+)\end{array}$ & Units & $\begin{array}{l}\text { Actual } \\
\text { Value }\end{array}$ & Comments \\
\hline \multirow{4}{*}{$\begin{array}{l}\text { Flush filter-loop } \\
\text { to UFP-VSL- } \\
\text { T62A/B } \\
\text { (Section 7.13) }\end{array}$} & \multirow[t]{4}{*}{$\mathrm{V}$} & $\begin{array}{l}\text { Inhibited water for filter-loop flush } \\
\text { to UFP-VSL-T62A/B (endpoint) }\end{array}$ & 690 & $-10 /+10$ & $\mathrm{~kg}$ & 682 & Data Source: HMI \\
\hline & & Flush water addition rate & $\begin{array}{l}\operatorname{maximum} \\
\text { achievable } \\
\text { flow }\end{array}$ & & & $\begin{array}{c}89 \\
\mathrm{~kg} / \mathrm{min}\end{array}$ & Data Source: HMI \\
\hline & & Ultrafiltration Loop Operation & & & & & - \\
\hline & & $\begin{array}{l}\text { Filter-loop pumps UFP-PMP-T42A } \\
\text { and T43A }\end{array}$ & OFF & & & Off & Data Source: Test Instruction \\
\hline \multirow{12}{*}{$\begin{array}{l}\text { Caustic-leach in } \\
\text { UFP-VSL- } \\
\text { T02A (Section } \\
7.13 \text { ) }\end{array}$} & \multirow[t]{12}{*}{$\mathrm{W}$} & Caustic-leach duration (endpoint) & 16 & $-0.1 /+1$ & $\mathrm{hr}$ & 16 & Data Source: CCP-783 \\
\hline & & UFP-VSL-T02A Operation & & & & & - \\
\hline & & Temperature & 98 & $-2 /+2$ & ${ }^{\circ} \mathrm{C}$ & 98 & Data Source: CCP-783 \\
\hline & & PJM jet velocity & 7.3 & $-0.4 /+0.4$ & $\mathrm{~m} / \mathrm{s}$ & 7.5 & Data Source: CCP-901 \\
\hline & & PJM stroke $(80 \%)$ & 31.5 & $-3 /+3$ & in. & 29.6 & Data Source: CCP-901 \\
\hline & & PJM cycle time & 33 & $-1 /+1$ & $\mathrm{~s}$ & 33 & Data Source: CCP-901 \\
\hline & & $\begin{array}{l}\text { Steam ring air purge flow rate } \\
\text { (automatically cycled on/off) }\end{array}$ & 0.09 & $-0.02 /+0.02$ & $\mathrm{~kg} / \mathrm{min}$ & 0.09 & Data Source: Local flow meter \\
\hline & & & & & & & $2.6 \mathrm{scfm}$ above $90^{\circ} \mathrm{C}$ in UFP-VSL-T02A \\
\hline & & $\begin{array}{l}\text { Total lower air sparger flow rate } \\
\text { (total for } 5 \text { sparge tubes, when } \\
\text { above } 90^{\circ} \mathrm{C} \text { ) }\end{array}$ & 0.08 & $-0.02 /+0.02$ & $\mathrm{~kg} / \mathrm{min}$ & 0.08 & $\begin{array}{l}\text { Data Source: Local flow meter } \\
\text { Total of } 2.3 \mathrm{scfm} \text { for the } 5 \text { sparge tubes, or } \\
\sim 28 \text { scfh for each sparge tube. This applies } \\
\text { whenever level is above } 52 \text { in. and the } \\
\text { temperature is above } 90^{\circ} \mathrm{C} \text {. }\end{array}$ \\
\hline & & $\begin{array}{l}\text { Upper air sparger flow rate (when } \\
\text { above } 90^{\circ} \mathrm{C} \text { ) }\end{array}$ & 0.02 & $-0.03 /+0.03$ & $\mathrm{~kg} / \mathrm{min}$ & 0.02 & Data Source: Local flow meter \\
\hline & & & & & & & $\begin{array}{l}0.5 \mathrm{scfm} . \text { This applies whenever the level } \\
\text { is above } 52 \mathrm{in} \text {. and temperature is above } \\
90^{\circ} \mathrm{C} \text {. }\end{array}$ \\
\hline & & Level at end of caustic-leach & 65.2 & $-5 /+5$ & in. & 69.3 & Data Source: HMI \\
\hline \multirow{3}{*}{$\begin{array}{l}\text { Cool-down of } \\
\text { UFP-VSL- } \\
\text { T02A (Section } \\
7.14)\end{array}$} & \multirow[t]{3}{*}{$\mathrm{X}$} & Final cool-down temperature & 25 & $-2 /+2$ & ${ }^{\circ} \mathrm{C}$ & 25 & Data Source: HMI \\
\hline & & $\begin{array}{l}\text { Time to reach final cool-down } \\
\text { temperature }\end{array}$ & 13.2 & $-1 /+1$ & $\mathrm{hr}$ & 13.2 & Data Source: Test Instruction \\
\hline & & UFP-VSL-T02A Operation & & & & & - \\
\hline
\end{tabular}


Table M.1. Integrated Test B Run Sheet

\begin{tabular}{|c|c|c|c|c|c|c|c|}
\hline & $\begin{array}{l}\text { Run } \\
\text { Sheet } \\
\text { Step }\end{array}$ & Operation & $\begin{array}{l}\text { Target or } \\
\text { Setpoint } \\
\text { Value } \\
\end{array}$ & $\begin{array}{c}\text { Range } \\
(-/+)\end{array}$ & Units & $\begin{array}{c}\text { Actual } \\
\text { Value }\end{array}$ & Comments \\
\hline & & $\begin{array}{l}\text { Temperature for air spargers and } \\
\text { steam ring air purge rate adjustment }\end{array}$ & 90 & $-2 /+2$ & ${ }^{\circ} \mathrm{C}$ & 78 & Data Source: HMI \\
\hline & & $\begin{array}{l}\text { Steam ring air purge flow rate } \\
\text { (when below } 90^{\circ} \mathrm{C} \text { ) }\end{array}$ & 0.14 & $-0.02 /+0.02$ & $\mathrm{~kg} / \mathrm{min}$ & 0.14 & Data Source: Local flow meter \\
\hline & & & & & & & $\begin{array}{l}4.1 \mathrm{scfm} \text { in UFP-VSL-T02A whenever the } \\
\text { temperature is below } 90^{\circ} \mathrm{C} \text {. }\end{array}$ \\
\hline & & $\begin{array}{l}\text { Total lower air sparger flow rate } \\
\text { (total for } 5 \text { sparge tubes, when }\end{array}$ & 0.40 & $-0.05 /+0.05$ & $\mathrm{~kg} / \mathrm{min}$ & 0.40 & Data Source: Local flow meter \\
\hline & & below $\left.90^{\circ} \mathrm{C}\right)$ & & & & & $\begin{array}{l}\text { Total of } 12 \mathrm{scfm} \text { for the } 5 \text { sparge tubes, or } \\
2.4 \mathrm{scfm} \text { for each sparge tube whenever } \\
\text { filter loop pumps are not running and the } \\
\text { temperature is below } 90^{\circ} \mathrm{C} \text {. }\end{array}$ \\
\hline & & $\begin{array}{l}\text { Upper air sparger flow rate (when } \\
\text { below } 90^{\circ} \mathrm{C} \text { ) }\end{array}$ & 0.10 & $-0.03 /+0.03$ & $\mathrm{~kg} / \mathrm{min}$ & 0.10 & Data Source: Local flow meter \\
\hline & & & & & & & $\begin{array}{l}3 \mathrm{scfm} \text {. This applies whenever the level is } \\
\text { above } 52 \mathrm{in} \text {. and temperature is below } \\
90^{\circ} \mathrm{C} \text {. }\end{array}$ \\
\hline \multirow{8}{*}{$\begin{array}{l}\text { Concentrate } \\
\text { leached solids } \\
\text { inUFP-VSL- } \\
\text { T02A and UFP- } \\
\text { VSL-T01B } \\
\text { (Section 7.15) }\end{array}$} & \multirow[t]{8}{*}{$\mathrm{Y}$} & $\begin{array}{l}\text { Total transfer volume via make-up } \\
\text { batches }\end{array}$ & 198 & $-5 /+5$ & gal & 198 & Data Source: Test Instruction \\
\hline & & Make-up batch volume & 11 & $-1 /+1$ & gal & 11 & Data Source: HMI \\
\hline & & $\begin{array}{l}\text { Number of make-up batches } \\
\text { (endpoint) }\end{array}$ & 18 & & & 18 & Data Source: Test Instruction \\
\hline & & Make-up batch flow rate & 6.9 & $-1 /+1$ & gpm & 6.9 & Data Source: HMI \\
\hline & & $\begin{array}{l}\text { UFP-VSL-T02A level to initiate } \\
\text { make-up batch additions from } \\
\text { UFP-VSL-T01B }\end{array}$ & 47 & $-1 /+1$ & in. & 47 & Data Source: HMI \\
\hline & & $\begin{array}{l}\text { Final UFP-VSL-T02A level } \\
\text { (endpoint) }\end{array}$ & 16.7 & $-1 /+1$ & in. & 16.7 & Data Source: HMI \\
\hline & & UFP-VSL-T02A Operation & & & & & \\
\hline & & $\begin{array}{l}\text { Total lower air sparger (idle) flow } \\
\text { rate (for } 5 \text { sparge tubes) }\end{array}$ & 0.010 & $\begin{array}{l}0.005 / \\
+0.005\end{array}$ & $\mathrm{~kg} / \mathrm{min}$ & 0.01 & $\begin{array}{l}\text { Data Source: Local flow meter } \\
\sim 3.7 \text { scfh for each sparge tube whenever } \\
\text { filter loop pumps are running and the } \\
\text { temperature is below } 90^{\circ} \mathrm{C} \text {. }\end{array}$ \\
\hline
\end{tabular}


Table M.1. Integrated Test B Run Sheet

\begin{tabular}{|c|c|c|c|c|c|c|c|}
\hline & $\begin{array}{l}\text { Run } \\
\text { Sheet } \\
\text { Step }\end{array}$ & Operation & $\begin{array}{l}\text { Target or } \\
\text { Setpoint } \\
\text { Value }\end{array}$ & $\begin{array}{c}\text { Range } \\
(-/+)\end{array}$ & Units & $\begin{array}{l}\text { Actual } \\
\text { Value }\end{array}$ & Comments \\
\hline & & Upper air sparger (idle) flow rate & 0.01 & $-0.01 /+0.01$ & $\mathrm{~kg} / \mathrm{min}$ & 0.01 & Data Source: Local flow meter \\
\hline & & & & & & & $\begin{array}{l}0.26 \mathrm{scfm} \text { or } \sim 16 \mathrm{scfh} \text {. This applies } \\
\text { whenever the level is less than } 52 \mathrm{in} .\end{array}$ \\
\hline & & Ultrafiltration Loop Operation & & & & & \\
\hline & & Number of filter bundles valved-in & 5 & $-0 /+0$ & & 5 & Data Source: Test Instruction \\
\hline & & $\begin{array}{l}\text { Number of filter bundles (use } \\
\text { UFP-FILT-T01A only) }\end{array}$ & 1 & $-0 /+0$ & & 1 & Data Source: Test Instruction \\
\hline & & Filter-loop flow rate & 109 & $-10 /+10$ & gpm & 109 & Data Source: HMI \\
\hline & & Transmembrane pressure & 40 & $-4 /+4$ & psi & 40 & Data Source: HMI \\
\hline & & $\begin{array}{l}\text { Temperature at inlet of } \\
\text { UFP-FILT-T01A }\end{array}$ & 25 & $-2 /+2$ & ${ }^{\circ} \mathrm{C}$ & 25 & Data Source: HMI \\
\hline \multirow{6}{*}{$\begin{array}{c}\text { Concentrate } \\
\text { leached solids } \\
\text { in UFP-VSL- } \\
\text { T01B (Section } \\
7.15)\end{array}$} & \multirow[t]{6}{*}{$\mathrm{Z}$} & & & & & & $\begin{array}{l}\text { Section } Z \text { was left blank in the original } \\
\text { document. }\end{array}$ \\
\hline & & & & & & & $\begin{array}{l}\text { Section } \mathrm{Z} \text { was left blank in the original } \\
\text { document. }\end{array}$ \\
\hline & & & & & & & $\begin{array}{l}\text { Section } \mathrm{Z} \text { was left blank in the original } \\
\text { document. }\end{array}$ \\
\hline & & & & & & & $\begin{array}{l}\text { Section } \mathrm{Z} \text { was left blank in the original } \\
\text { document. }\end{array}$ \\
\hline & & & & & & & $\begin{array}{l}\text { Section } \mathrm{Z} \text { was left blank in the original } \\
\text { document. }\end{array}$ \\
\hline & & & & & & & $\begin{array}{l}\text { Section } \mathrm{Z} \text { was left blank in the original } \\
\text { document. }\end{array}$ \\
\hline \multirow[t]{6}{*}{$\begin{array}{c}\text { Tracer test } \\
\text { (Section } 7.16)\end{array}$} & \multirow[t]{6}{*}{ A1 } & $\begin{array}{l}\text { Mass of } \mathrm{CsBr} \text { to inject into } \\
\text { filter-loop }\end{array}$ & 110 & $-5 /+5$ & g & 108.0 & $\begin{array}{l}\text { Data Source: Test Instruction } \\
\text { Solution to be added to the suction of } \\
\text { UFP-PMP-T42A. }\end{array}$ \\
\hline & & \multicolumn{6}{|l|}{ UFP-VSL-T02A Operation } \\
\hline & & PJM jet velocity & 12 & $-0.4 /+0.4$ & $\mathrm{~m} / \mathrm{s}$ & 11.7 & Data Source: CCP-901 \\
\hline & & PJM stroke $(80 \%)$ & 31.5 & $-3 /+3$ & in. & 16.7 & Data Source: CCP-901 \\
\hline & & PJM cycle time & 20 & $-1 /+1$ & S & 20.2 & Data Source: CCP-901 \\
\hline & & Steam ring air purge flow rate & 0.14 & $-0.02 /+0.02$ & $\mathrm{~kg} / \mathrm{min}$ & 0 & $\begin{array}{l}\text { Data Source: Test Instruction } \\
4.1 \mathrm{scfm} \text { in UFP-VSL-T02A whenever the } \\
\text { temperature is below } 90^{\circ} \mathrm{C} \text {. }\end{array}$ \\
\hline
\end{tabular}


Table M.1. Integrated Test B Run Sheet

\begin{tabular}{|c|c|c|c|c|c|c|c|}
\hline & $\begin{array}{l}\text { Run } \\
\text { Sheet } \\
\text { Step }\end{array}$ & Operation & $\begin{array}{l}\text { Target or } \\
\text { Setpoint } \\
\text { Value }\end{array}$ & $\begin{array}{c}\text { Range } \\
(-/+)\end{array}$ & Units & $\begin{array}{c}\text { Actual } \\
\text { Value }\end{array}$ & Comments \\
\hline & & $\begin{array}{l}\text { Total lower air sparger (idle) flow } \\
\text { rate (for } 5 \text { sparge tubes) }\end{array}$ & 0.010 & $\begin{array}{c}- \\
0.005 /+0.00 \\
5\end{array}$ & $\mathrm{~kg} / \mathrm{min}$ & 0 & $\begin{array}{l}\text { Data Source: Test Instruction } \\
\sim 3.7 \text { scfh for each sparge tube whenever } \\
\text { filter-loop pumps are running and the } \\
\text { temperature is below } 90^{\circ} \mathrm{C} \text {. }\end{array}$ \\
\hline & & Upper air sparger (idle) flow rate & 0.01 & $-0.01 /+0.01$ & $\mathrm{~kg} / \mathrm{min}$ & 0 & $\begin{array}{l}\text { Data Source: Test Instruction } \\
0.26 \mathrm{scfm} \text { or } \sim 16 \mathrm{scfh} \text {. This applies } \\
\text { whenever the level is less than } 52 \mathrm{in} \text {. }\end{array}$ \\
\hline & & Ultrafiltration Loop Operation & & & & & \\
\hline & & Number of filter bundles & 5 & $-0 /+0$ & & 5 & Data Source: Test Instruction \\
\hline & & Filter-loop flow rate & 109 & $-10 /+10$ & gpm & 67 & Data Source: HMI \\
\hline & & Permeate valves & CLOSED & $-4 /+4$ & psi & Closed & Data Source: Test Instruction \\
\hline & & $\begin{array}{l}\text { Temperature at inlet of } \\
\text { UFP-FILT-T01A }\end{array}$ & 25 & $-2 /+2$ & ${ }^{\circ} \mathrm{C}$ & 25 & Data Source: HMI \\
\hline \multirow[t]{6}{*}{$\begin{array}{l}\text { Post-caustic- } \\
\text { leach wash } \\
\text { (Section } 7.17)\end{array}$} & \multirow[t]{6}{*}{$\mathrm{B} 1$} & Total wash water & $\begin{array}{l}2,581 \\
2165 \text { (per } \\
\text { revised run } \\
\text { sheet) }\end{array}$ & $-20 /+20$ & $\mathrm{~kg}$ & 2204 & Data Source: WTP-RPT-187 \\
\hline & & Wash water batch flow rate & 28.0 & $-3 /+3$ & $\mathrm{~kg} / \mathrm{min}$ & 29.4 & $\begin{array}{l}\text { Data Source: WTP-RPT-187 } \\
\sim 7.4 \mathrm{gpm}\end{array}$ \\
\hline & & Wash water batch size & 41.6 & $-1 /+1$ & $\mathrm{~kg}$ & 42.4 & Data Source: WTP-RPT-187 \\
\hline & & $\begin{array}{l}\text { UFP-VSL-T02A level to initiate } \\
\text { wash water batch addition }\end{array}$ & 16.7 & $-1 /+1$ & in. & 16.7 & Data Source: HMI \\
\hline & & $\begin{array}{l}\text { Volume of antifoam to add initially } \\
\text { and after every 3rd wash water } \\
\text { batch to UFP-VSL-T02A }\end{array}$ & 44 & $-2 /+2$ & $\mathrm{~mL}$ & 44 & $\begin{array}{l}\text { Data Source: Test Instruction } \\
\text { Antifoam is added to account for wash } \\
\text { water dilution. This value is concentrate. } \\
\text { Dilute with } 3 \text { parts IW to } 1 \text { part AFA } \\
\text { concentrate. }\end{array}$ \\
\hline & & Estimated total permeate mass & $\begin{array}{l}2,800 \\
2400 \text { (per } \\
\text { revised run } \\
\text { sheet) }\end{array}$ & $-100 /+200$ & $\mathrm{~kg}$ & 2487 & $\begin{array}{l}\text { Data Source: WTP-RPT-187 } \\
\text { Estimate is rounded down to nearest } \\
\text { hundred. }\end{array}$ \\
\hline
\end{tabular}


Table M.1. Integrated Test B Run Sheet

\begin{tabular}{|c|c|c|c|c|c|c|c|}
\hline & $\begin{array}{l}\text { Run } \\
\text { Sheet } \\
\text { Step }\end{array}$ & Operation & $\begin{array}{c}\text { Target or } \\
\text { Setpoint } \\
\text { Value }\end{array}$ & $\begin{array}{c}\text { Range } \\
(-/+)\end{array}$ & Units & $\begin{array}{l}\text { Actual } \\
\text { Value }\end{array}$ & Comments \\
\hline & Estimated total permeate volume & $\begin{array}{l}682 \\
572 \text { (per } \\
\text { revised run } \\
\text { sheet) }\end{array}$ & $-35 /+35$ & gal & 597 & Data Source: WTP-RPT-187 \\
\hline & & UFP-VSL-T02A Operation & & & & & \\
\hline & & PJM jet velocity & 12 & $-0.4 /+0.4$ & $\mathrm{~m} / \mathrm{s}$ & 11.6 & Data Source: HMI \\
\hline & & PJM stroke ( $80 \%)$ & 31.5 & $-3 /+3$ & in. & 20 & Data Source: HMI \\
\hline & & PJM cycle time & 20 & $-1 /+1$ & $\mathrm{~s}$ & 20 & Data Source: HMI \\
\hline & & Steam ring air purge flow rate & 0.14 & $-0.02 /+0.02$ & $\mathrm{~kg} / \mathrm{min}$ & 0 & $\begin{array}{l}\text { Data Source: Test Instruction } \\
4.1 \mathrm{scfm} \text { in UFP-VSL-T02A whenever the } \\
\text { temperature is below } 90^{\circ} \mathrm{C} \text {. }\end{array}$ \\
\hline & & $\begin{array}{l}\text { Total lower air sparger (idle) flow } \\
\text { rate (for } 5 \text { sparge tubes) }\end{array}$ & 0.010 & $\begin{array}{c}-0.005 /+ \\
0.005\end{array}$ & $\mathrm{~kg} / \mathrm{min}$ & 0 & $\begin{array}{l}\text { Data Source: Test Instruction } \\
\sim 3.7 \text { scfh for each sparge tube whenever } \\
\text { filter-loop pumps are running and the } \\
\text { temperature is below } 90^{\circ} \mathrm{C} \text {. }\end{array}$ \\
\hline & & Upper air sparger (idle) flow rate & 0.01 & $-0.01 /+0.01$ & $\mathrm{~kg} / \mathrm{min}$ & 0 & $\begin{array}{l}\text { Data Source: Test Instruction } \\
0.26 \mathrm{scfm} \text { or } \sim 16 \mathrm{scfh} \text {. This applies } \\
\text { whenever the level is less than } 52 \mathrm{in} \text {. }\end{array}$ \\
\hline & & Ultrafiltration Loop Operation & & & & & \\
\hline & & Number of filter bundles & 5 & $-0 /+0$ & & 5 & Data Source: Test Instruction \\
\hline & & Filter-loop flow rate & 109 & $-10 /+10$ & gpm & $\begin{array}{l}81+/ \\
-7.4\end{array}$ & Data Source: WTP-RPT-187 \\
\hline & & Transmembrane pressure & 40 & $-4 /+4$ & psi & $\begin{array}{c}39.4+/ \\
-2.1\end{array}$ & $\begin{array}{l}\text { Data Source: WTP-RPT- } 187 \\
\text { Lower range not applicable if back } \\
\text { pressure valve is } 100 \% \text { open. }\end{array}$ \\
\hline & & $\begin{array}{l}\text { Temperature at inlet of } \\
\text { UFP-FILT-T01A }\end{array}$ & 25 & $-2 /+2$ & ${ }^{\circ} \mathrm{C}$ & $\begin{array}{c}23.0+1 \\
-1.5\end{array}$ & Data Source: WTP-RPT-187 \\
\hline
\end{tabular}


Table M.1. Integrated Test B Run Sheet

\begin{tabular}{|c|c|c|c|c|c|c|c|}
\hline & $\begin{array}{l}\text { Run } \\
\text { Sheet } \\
\text { Step }\end{array}$ & Operation & $\begin{array}{l}\text { Target or } \\
\text { Setpoint } \\
\text { Value }\end{array}$ & Range & Units & $\begin{array}{l}\text { Actual } \\
\text { Value }\end{array}$ & Comments \\
\hline \multirow[t]{8}{*}{$\begin{array}{l}\text { slurry (Section } \\
7.18)\end{array}$} & & add (endpoint) & $\begin{array}{l}366 \text { (per } \\
\text { revised run } \\
\text { sheet) }\end{array}$ & & & & $\begin{array}{l}\text { Chromium slurry vessel may be washed } \\
\text { out with as much as } 10 \text { gal of inhibited } \\
\text { water. }\end{array}$ \\
\hline & & Chromium slurry addition flow rate & $\begin{array}{l}\text { No } \\
\text { targeted } \\
\text { rate }\end{array}$ & & & & \\
\hline & & $\begin{array}{l}\text { Volume of AFA to add to } \\
\text { UFP-VSL-T02A }\end{array}$ & $\begin{array}{l}110 \\
130 \text { (per } \\
\text { revised run } \\
\text { sheet) }\end{array}$ & $-5 /+5$ & $\mathrm{~mL}$ & 130 & Data Source: Test Instruction \\
\hline & & Ultrafiltration Loop Operation & & & & & \\
\hline & & Number of filter bundles & 5 & $-0 /+0$ & & 5 & Data Source: Test Instruction \\
\hline & & Filter-loop flow rate & 109 & $-10 /+10$ & gpm & 88 & Data Source: HMI \\
\hline & & Permeate valves & CLOSED & $-4 /+4$ & psi & Closed & Data Source: Test Instruction \\
\hline & & $\begin{array}{l}\text { Temperature at inlet of } \\
\text { UFP-FILT-T01A }\end{array}$ & 25 & $-2 /+2$ & ${ }^{\circ} \mathrm{C}$ & 24 & Data Source: HMI \\
\hline \multirow{8}{*}{$\begin{array}{l}\text { Reconcentrate } \\
\text { and wash post- } \\
\text { chromium } \\
\text { slurry (Section } \\
\quad 7.18 \text { ) }\end{array}$} & \multirow[t]{8}{*}{ D1 } & $\begin{array}{l}\text { UFP-VSL-T02A level } \\
\text { (reconcentration endpoint) }\end{array}$ & 16.7 & $-1 /+1$ & in. & 19.7 & Data Source: HMI \\
\hline & & $\begin{array}{l}\text { UFP-VSL-T02A level to initiate } \\
\text { wash water batch additions }\end{array}$ & 16.7 & $-1 /+1$ & in. & 16.7 & Data Source: Test Instruction \\
\hline & & Number of wash water batches & $\begin{array}{l}19 \\
21 \text { (per } \\
\text { revised run } \\
\text { sheet) }\end{array}$ & $-10 /+10$ & $\mathrm{~kg}$ & 21 & $\begin{array}{l}\text { Data Source: Test Instruction } \\
\sim 209231 \text { gal }\end{array}$ \\
\hline & & Wash water batch size & 41.6 & $-1 /+1$ & $\mathrm{~kg}$ & 41.6 & Data Source: HMI \\
\hline & & Wash water batch flow rate & 28.0 & $-3 /+3$ & $\mathrm{~kg} / \mathrm{min}$ & 27 & $\begin{array}{l}\text { Data Source: HMI } \\
\sim 7.4 \mathrm{gpm}\end{array}$ \\
\hline & & $\begin{array}{l}\text { Volume of AFA to add initially and } \\
\text { after every 3rd wash water batch to } \\
\text { UFP-VSL-T02A }\end{array}$ & 44 & $-2 /+2$ & $\mathrm{~mL}$ & 44 & $\begin{array}{l}\text { Data Source: Test Instruction } \\
\text { AFA is added to account for wash water } \\
\text { dilution. This value is concentrate. Dilute } \\
\text { with } 3 \text { parts IW to } 1 \text { part AFA concentrate. }\end{array}$ \\
\hline & & Ultrafiltration Loop Operation & & & & & \\
\hline & & Number of filter bundles & 5 & $-0 /+0$ & & 5 & Data Source: Test Instruction \\
\hline
\end{tabular}


Table M.1. Integrated Test B Run Sheet

\begin{tabular}{|c|c|c|c|c|c|c|c|}
\hline & $\begin{array}{l}\text { Run } \\
\text { Sheet } \\
\text { Step }\end{array}$ & Operation & $\begin{array}{l}\text { Target or } \\
\text { Setpoint } \\
\text { Value }\end{array}$ & $\begin{array}{c}\text { Range } \\
(-/+)\end{array}$ & Units & $\begin{array}{l}\text { Actual } \\
\text { Value }\end{array}$ & Comments \\
\hline & & Filter-loop flow rate & 109 & $-10 /+10$ & gpm & 88 & Data Source: HMI \\
\hline & & Transmembrane pressure & 40 & $-4 /+4$ & psi & 41 & Data Source: HMI \\
\hline & & & & & & & $\begin{array}{l}\text { Lower range not applicable if back } \\
\text { pressure valve is } 100 \% \text { open. }\end{array}$ \\
\hline & & $\begin{array}{l}\text { Temperature at inlet of } \\
\text { UFP-FILT-T01A }\end{array}$ & 25 & $-2 /+2$ & ${ }^{\circ} \mathrm{C}$ & 23.1 & Data Source: HMI \\
\hline \multirow[t]{7}{*}{$\begin{array}{l}\text { Add sodium } \\
\text { permanganate } \\
\text { (Section } 7.18 \text { ) }\end{array}$} & \multirow[t]{7}{*}{ E1 } & $\begin{array}{l}\text { Volume of sodium permanganate to } \\
\text { add (endpoint) }\end{array}$ & $\begin{array}{l}17.8 \\
20.9 \text { (per } \\
\text { revised run } \\
\text { sheet) }\end{array}$ & $-0.3 /+0.3$ & gal & 20.9 & Data Source: WTP-RPT-188 \\
\hline & & Sodium permanganate addition rate & 5.0 & $-3 /+3$ & gpm & 5.2 & Data Source: WTP-RPT-188 \\
\hline & & Ultrafiltration Loop Operation & & & & & \\
\hline & & Number of filter bundles & 5 & $-0 /+0$ & & 5 & Data Source: Test Instruction \\
\hline & & Filter-loop flow rate & 109 & $-10 /+10$ & gpm & $86-89$ & Data Source: WTP-RPT-188 \\
\hline & & Permeate valves & CLOSED & & & Closed & Data Source: Test Instruction \\
\hline & & $\begin{array}{l}\text { Temperature at inlet of UFP FHT } \\
\text { T01A_UFP-VSL-T02A }\end{array}$ & 25 & $-2 /+2$ & ${ }^{\circ} \mathrm{C}$ & $\begin{array}{l}22.6- \\
26.0\end{array}$ & Data Source: WTP-RPT-188 \\
\hline \multirow[t]{4}{*}{$\begin{array}{l}\text { Conduct } \\
\text { oxidative-leach } \\
\text { (Section 7.18) }\end{array}$} & $\mathrm{F} 1$ & Oxidative leach duration (endpoint) & 6 & $-0.1 /+0.5$ & $\mathrm{hr}$ & 6 & $\begin{array}{l}\text { Data Source: Test Instruction } \\
\text { May be extended to accommodate } \\
\text { sampling. }\end{array}$ \\
\hline & & Ultrafiltration Loop Operation & & & & & \\
\hline & & Number of filter bundles & 5 & $-0 /+0$ & & 5 & Data Source: Test Instruction \\
\hline & & Filter-loop flow rate & $\begin{array}{c}109 \\
88 \text { (per } \\
\text { Steve } \\
\text { Barnes) }\end{array}$ & $-10 /+10$ & gpm & $86-89$ & Data Source: WTP-RPT-188 \\
\hline
\end{tabular}


Table M.1. Integrated Test B Run Sheet

\begin{tabular}{|c|c|c|c|c|c|c|}
\hline $\begin{array}{c}\text { Run } \\
\text { Sheet } \\
\text { Step }\end{array}$ & Operation & $\begin{array}{c}\text { Target or } \\
\text { Setpoint } \\
\text { Value }\end{array}$ & $\begin{array}{c}\text { Range } \\
(-/+)\end{array}$ & Units & $\begin{array}{c}\text { Actual } \\
\text { Value }\end{array}$ & Comments \\
\hline & Permeate valves & CLOSED & & & Closed & Data Source: Test Instruction \\
\hline & $\begin{array}{l}\text { Temperature at inlet of } \\
\text { UFP-FILT-T01A }\end{array}$ & 25 & $-2 /+2$ & ${ }^{\circ} \mathrm{C}$ & 24.7 & Data Source: HMI \\
\hline
\end{tabular}

\begin{tabular}{|c|c|c|c|c|c|c|c|}
\hline \multirow[t]{13}{*}{$\begin{array}{c}\text { Wash post- } \\
\text { oxidative-leach } \\
\text { slurry (Section } \\
7.19)\end{array}$} & G1 & $\begin{array}{l}\text { UFP-VSL-T02A level to initiate } \\
\text { wash water batch addition }\end{array}$ & $\begin{array}{c}20.7 \\
21.4 \text { (per } \\
\text { revised run } \\
\text { sheet) }\end{array}$ & $-1 /+1$ & in. & 21.4 & Data Source: HMI \\
\hline & & Number of wash water batches & 47 & $-20 /+20$ & $\begin{array}{c}\mathrm{Kg} \\
\text { Batch }\end{array}$ & 47 & Data Source: Test Instruction \\
\hline & & Wash water batch flow rate & 28.0 & $-3 /+3$ & $\mathrm{~kg} / \mathrm{min}$ & 31 & $\begin{array}{l}517 \text { gal } \\
\text { Data Source: WTP-RPT-187 }\end{array}$ \\
\hline & & & & & & & $\sim 7.4 \mathrm{gpm}$ \\
\hline & & Wash water batch size & 41.6 & $-1 /+1$ & $\mathrm{~kg}$ & 42.7 & Data Source: WTP-RPT-187 \\
\hline & & $\begin{array}{l}\text { Volume of antifoam to add initially } \\
\text { and after every 3rd 11-gal batch to } \\
\text { UFP-VSL-T02A }\end{array}$ & 44 & $-2 /+2$ & $\mathrm{~mL}$ & 44 & $\begin{array}{l}\text { Data Source: Test Instruction } \\
\text { Antifoam ( } \sim 14 \mathrm{~mL} \text { per } 11 \text { gallon wash } \\
\text { batch) is added to account for wash water } \\
\text { dilution. This value is concentrate. Dilute } \\
\text { with } 3 \text { parts IW to } 1 \text { part concentrate. }\end{array}$ \\
\hline & & Estimated total permeate mass & 2,000 & $-100 /+200$ & $\mathrm{~kg}$ & 2124 & WTP-RPT-187 \\
\hline & & Ultrafiltration Loop Operation & & & & & \\
\hline & & Number of filter bundles & 5 & $-0 /+0$ & & 5 & Data Source: Test Instruction \\
\hline & & Filter-loop flow rate & 109 & $-10 /+10$ & gpm & $\begin{array}{r}87+1 \\
-12.2\end{array}$ & Data Source: WTP-RPT-187 \\
\hline & & Transmembrane pressure & 40 & $-4 /+4$ & psi & $\begin{array}{c}37.3+1 \\
-4.2\end{array}$ & Data Source: WTP-RPT-187 \\
\hline & & & & & & & $\begin{array}{l}\text { Lower range not applicable if back } \\
\text { pressure valve is } 100 \% \text { open. }\end{array}$ \\
\hline & & $\begin{array}{l}\text { Temperature at inlet of } \\
\text { UFP-FILT-T01A }\end{array}$ & 25 & $-2 /+2$ & ${ }^{\circ} \mathrm{C}$ & $\begin{array}{c}24.6+1 \\
-0.2\end{array}$ & Data Source: WTP-RPT-187 \\
\hline
\end{tabular}


Table M.1. Integrated Test B Run Sheet

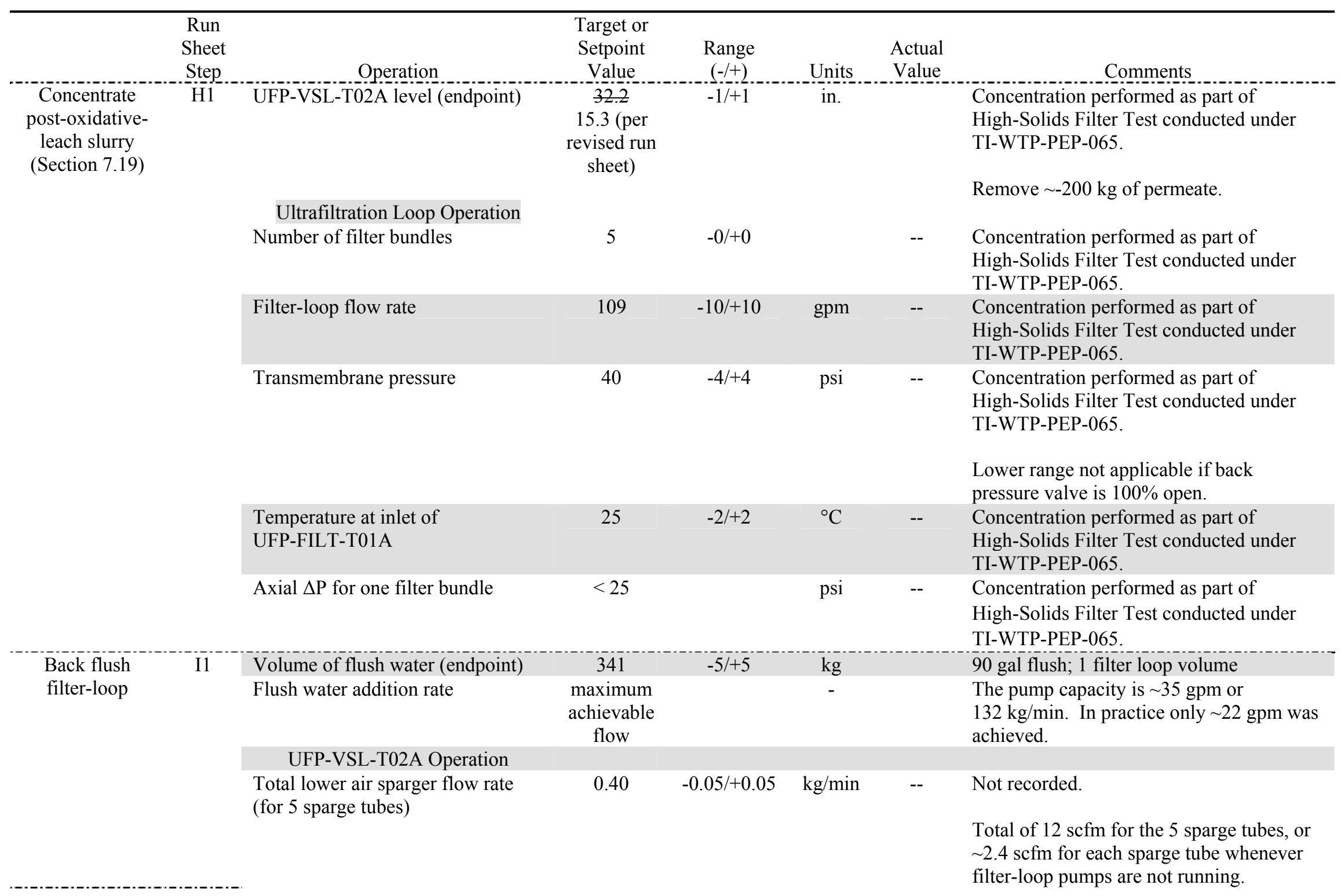


Table M.1. Integrated Test B Run Sheet

\begin{tabular}{|c|c|c|c|c|c|c|}
\hline $\begin{array}{r}\text { Run } \\
\text { Sheet } \\
\text { Step }\end{array}$ & Operation & $\begin{array}{l}\text { Target or } \\
\text { Setpoint } \\
\text { Value }\end{array}$ & $\begin{array}{c}\text { Range } \\
(-/+)\end{array}$ & Units & $\begin{array}{c}\text { Actual } \\
\text { Value }\end{array}$ & Comments \\
\hline & Upper air sparger flow rate & OFF & $\mathrm{N} / \mathrm{A}$ & $\mathrm{N} / \mathrm{A}$ & -- & $\begin{array}{l}\text { Not recorded. } \\
\text { Upper air sparger is not turned on until } \\
\text { UFP-VSL-T02A level reaches } 52 \text { in. }\end{array}$ \\
\hline & $\begin{array}{l}\text { Ultrafiltration Loop Operation } \\
\text { Number of filter bundles }\end{array}$ & 5 & $-0 /+0$ & - & -- & Not recorded. \\
\hline & Filter-loop flow rate & OFF & & & -- & Not recorded. \\
\hline & Permeate valves & CLOSED & & - & -- & Not recorded. \\
\hline
\end{tabular}

JTG Chair Signature

Date 

Appendix N

Reagent Analysis 



\section{Appendix N: Reagent Analysis}

The tables below provide the results of all the reagent analysis taken during Phase 1 testing. For Integrated Test $\mathrm{B}$, the following samples are most relevant:

19 M NaOH Analysis: Samples 19M NAOH 031709 1520, 19MNAOH 0317090700 (Sample taken 2 days after Integrated Test $\mathrm{B}$ batch 2 caustic addition). $2 \mathrm{M} \mathrm{HNO}_{3}$ Analysis: Sample: A 00C03 XXX XX 2609 ACT 2 (Sample taken as part of Integrated Test A. Acid cleaning conducted at conclusion of Test A).

$\mathrm{NaMnO}_{4}$ Analysis: Sample: A 00C04 XXX XX 2610 OST 2 (Sample taken as part of Integrated Test A). CsBr Tracer Analysis: Sample: B 0CSBR 020 XX 4155 ICP 2 (Sample taken as part of Integrated Test B). 
PEP Testing $19 \mathrm{M} \mathrm{NaOH}$ Analysis

\begin{tabular}{|c|c|c|c|c|c|c|c|c|c|c|c|c|c|c|c|c|c|c|c|c|c|}
\hline $\begin{array}{c}\text { Reagent } \\
\text { Description }\end{array}$ & $\begin{array}{c}\text { Analysis } \\
\text { Date }\end{array}$ & Sample name & Analysis & Reference file & $\begin{array}{c}\text { Free } \mathrm{OH} \\
\mathrm{M}\end{array}$ & $\begin{array}{c}\text { Density } \\
\mathrm{g} / \mathrm{cm}^{3}\end{array}$ & $\begin{array}{l}\mathrm{C}_{2} \mathrm{O}_{4} \\
\mu \mathrm{g} / \mathrm{g} \\
\end{array}$ & $\begin{array}{l}\mathrm{NO}_{3} \\
\mu \mathrm{g} / \mathrm{g}\end{array}$ & $\begin{array}{l}\mathrm{NO}_{2} \\
\mu \mathrm{g} / \mathrm{g}\end{array}$ & $\begin{array}{l}\mathrm{SO}_{4} \\
\mu \mathrm{g} / \mathrm{g}\end{array}$ & $\begin{array}{l}\mathrm{PO}_{4} \\
\mu \mathrm{g} / \mathrm{g}\end{array}$ & $\begin{array}{c}\mathrm{Al} \\
\mu \mathrm{g} / \mathrm{g}\end{array}$ & $\begin{array}{c}\mathrm{Ca} \\
\mu \mathrm{g} / \mathrm{g} \\
\end{array}$ & $\begin{array}{r}\mathrm{Cr} \\
\mu \mathrm{g} / \mathrm{g} \\
\end{array}$ & $\begin{array}{c}\mathrm{Fe} \\
\mu \mathrm{g} / \mathrm{g}\end{array}$ & $\begin{array}{c}\mathrm{Mg} \\
\mu \mathrm{g} / \mathrm{g}\end{array}$ & $\begin{array}{c}\mathrm{Mn} \\
\mu \mathrm{g} / \mathrm{g}\end{array}$ & $\begin{array}{c}\mathrm{Na} \\
\mu \mathrm{g} / \mathrm{g}\end{array}$ & $\begin{array}{c}\mathrm{Nd} \\
\mu \mathrm{g} / \mathrm{g}\end{array}$ & $\begin{array}{c}\mathrm{Sr} \\
\mu \mathrm{g} / \mathrm{g}\end{array}$ & $\begin{array}{c}\mathrm{Cl} \\
\mu \mathrm{g} / \mathrm{g}\end{array}$ \\
\hline \multirow{4}{*}{$\begin{array}{c}19 \mathrm{M} \\
\mathrm{NaOH}^{(\mathrm{a})}\end{array}$} & \multirow{4}{*}{$1 / 15 / 2009$} & $\begin{array}{c}\text { S 00C01 001 XX } \\
0173 \text { DEN } 2\end{array}$ & Density & 090115-14 DEN & $18.652 \mathrm{~N}$ & 1.522 & $<19.5$ & $<86.4$ & $<64.2$ & $<19.5$ & $<59.7$ & $<9.60$ & $<4.70$ & $<0.960$ & $<9.60$ & $<4.70$ & $<0.960$ & 285000 & $<0.0188$ & $<0.960$ & $<19.5$ \\
\hline & & $\begin{array}{c}\text { S 00C01 } 001 \text { XX } \\
0172 \text { ALK } 2\end{array}$ & $\begin{array}{c}\mathrm{OH} \\
\text { Titration }\end{array}$ & $\begin{array}{c}\text { Base Titration - } \\
\text { 090115-13 - Battelle } \\
\text { PNNL (011909) }\end{array}$ & & & & & & & & & & & & & & & & & \\
\hline & & $\begin{array}{l}\text { S } 00 C 01001 \mathrm{XX} \\
0171 \text { TFI } 2 \mathrm{D}\end{array}$ & IC & $\begin{array}{c}\text { 090115-151C_prelim, } \\
\text { 090115-15IC, } \\
\text { Battelle_090202- } \\
12131415\end{array}$ & & & & & & & & & & & & & & & & & \\
\hline & & $\begin{array}{l}\text { S 00C01 001 XX } \\
0171 \text { ICP 2 D }\end{array}$ & ICP & $\begin{array}{c}\text { 090115- } \\
\text { 16MetalsLIQUID }\end{array}$ & $18.6 \mathrm{~N}$ & 1.519 & $<16.9$ & $<74.7$ & $<55.5$ & $<16.9$ & $<51.6$ & $<9.94$ & $<9.94$ & $<0.994$ & $<19.9$ & $<9.94$ & $<0.994$ & 293000 & $<0.199$ & $<1.99$ & 23.7 \\
\hline \multirow{4}{*}{$\begin{array}{c}19 \mathrm{M} \\
\mathrm{NaOH}^{(\mathrm{a})}\end{array}$} & \multirow{4}{*}{$1 / 31 / 2009$} & $\begin{array}{l}\text { A 00C01 XXX XX } \\
2608 \text { ALK } 2\end{array}$ & IC & 090202-12IC & & & & & & & & & & & & & & & & & \\
\hline & & $\begin{array}{l}\text { A 00C01 XXX XX } \\
2608 \text { ALK } 2\end{array}$ & $\begin{array}{c}\mathrm{OH} \\
\text { Titration }\end{array}$ & $\begin{array}{l}\text { Base Titration - } \\
12090202-13\end{array}$ & & & & & & & & & & & & & & & & & \\
\hline & & $\begin{array}{l}\text { A 00C01 XXX XX } \\
2608 \text { ALK } 2\end{array}$ & $\mathrm{ICP}$ & 090202-12Metals & & & & & & & & & & & & & & & & & \\
\hline & & $\begin{array}{l}\text { A } 00 C 01 \text { XXX XX } \\
2608 \text { ALK } 2\end{array}$ & Density & 090202-12den & $18.6 \mathrm{~N}$ & 1.519 & $<16.9$ & $<74.7$ & $<55.5$ & $<16.9$ & $<51.6$ & $<9.94$ & $<9.94$ & $<0.994$ & $<19.9$ & $<9.94$ & $<0.994$ & 293000 & $<0.199$ & $<1.99$ & 23.7 \\
\hline $19 \mathrm{M} \mathrm{NaOH}$ & $2 / 6 / 2009$ & $\begin{array}{c}\text { A 00C } 01 \text { XXX XX } \\
2757 \text { ALK } 3\end{array}$ & $\mathrm{OH}$ & ASR $8351 \mathrm{OH}$ & 19.27 & -- & -- & -- & -- & -- & -- & -- & -- & -- & -- & -- & -- & -- & -- & -- & -- \\
\hline $19 \mathrm{M} \mathrm{NaOH}$ & $2 / 12 / 2009$ & $\begin{array}{c}\text { A 00CO1 XXX XX } \\
2763 \text { ALK } 3\end{array}$ & $\mathrm{OH}$ & ASR $8357 \mathrm{OH}$ & 19.22 & -- & -- & -- & -- & -- & -- & -- & -- & -- & -- & -- & -- & -- & -- & -- & -- \\
\hline $19 \mathrm{M} \mathrm{NaOH}$ & 2/18/2009 & $\begin{array}{c}\text { A 00CO1 XXX XX } \\
2807 \text { ALK } 3\end{array}$ & $\mathrm{OH}$ & ASR $8363 \mathrm{OH}$ & 18.86 & -- & -- & -- & -- & -- & -- & -- & -- & -- & -- & -- & -- & -- & -- & -- & -- \\
\hline $19 \mathrm{M} \mathrm{NaOH}$ & $2 / 18 / 2009$ & $\begin{array}{l}\text { A 00CO1 XXX XX } \\
2808 \text { ALK } 3\end{array}$ & $\mathrm{OH}$ & ASR $8363 \mathrm{OH}$ & 18.61 & -- & -- & -- & -- & -- & -- & -- & -- & -- & -- & -- & -- & -- & -- & -- & -- \\
\hline $19 \mathrm{M} \mathrm{NaOH}$ & $3 / 17 / 2009$ & $\begin{array}{l}\text { 19M NAOH } \\
0317091520\end{array}$ & $\mathrm{OH}$ & ASR $8388 \mathrm{OH}$ & 19.12 & -- & -- & -- & -- & -- & -- & -- & -- & -- & -- & -- & -- & -- & -- & -- & -- \\
\hline $19 \mathrm{M} \mathrm{NaOH}$ & $3 / 18 / 2009$ & $\begin{array}{c}\text { 19MNAOH } 0318 \\
090700\end{array}$ & $\mathrm{OH}$ & ASR $8388 \mathrm{OH}$ & 18.95 & -- & -- & -- & -- & -- & -- & -- & -- & -- & -- & -- & -- & -- & -- & -- & -- \\
\hline
\end{tabular}

PEP Testing $2 \mathrm{M} \mathrm{HNO}_{3}$ Analysis

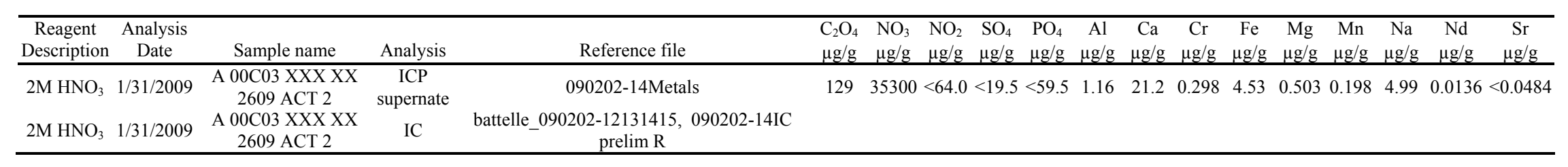


PEP Testing $\mathrm{NaMnO}_{4}$ Analysis

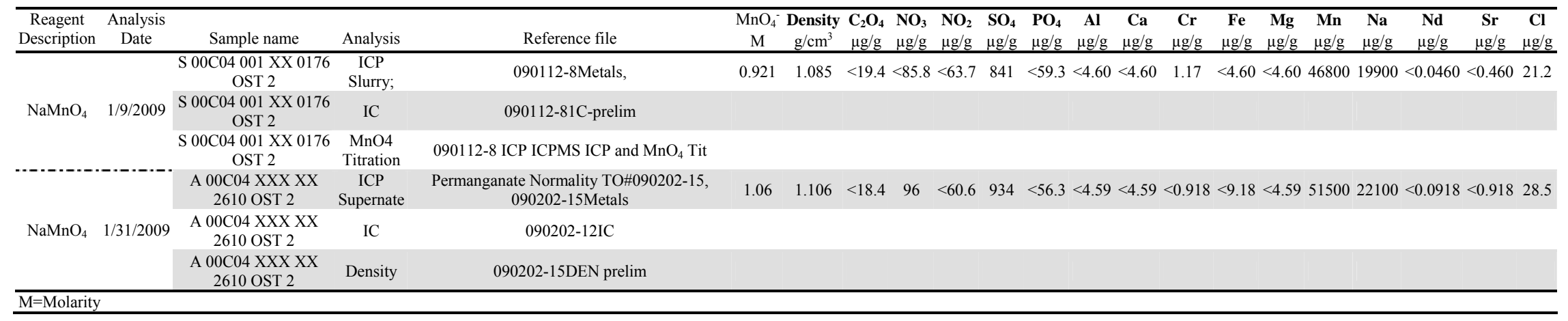

PEP Testing CsBr Tracer Analysis

\begin{tabular}{|c|c|c|c|c|c|}
\hline Reagent Description & Analysis Date & Sample name & Analysis & Reference file & $\begin{array}{l}\mathrm{CsBr} \\
\mu \mathrm{g} / \mathrm{g}\end{array}$ \\
\hline $\mathrm{CsBr}$ & $1 / 2 / 2009$ & S CsBr 0250990 ICP 2 & ICP solution & $090102-13 \mathrm{Cs}$ & 70300 \\
\hline $\mathrm{CsBr}$ & $2 / 4 / 2009$ & A CsBr XXX XX 2726 ICP 2 & ICP solution & 090204-4Cs & 181000 \\
\hline $\mathrm{CsBr}$ & $3 / 24 / 2009$ & B 0CSBR 020 XX 4155 ICP 2 & ICP solution & 090320-15MetalsLIQUID & 124000 \\
\hline $\mathrm{CsBr}$ & $3 / 24 / 2009$ & A 0CSBR 014 XX 6000 ICP 2 & ICP solution & 090324-35MetalsLIQUID & 198000 \\
\hline
\end{tabular}



PNNL-18723

WTP-RPT-192, Rev 0

\section{Distribution}

No. of

Copies

OFFSITE
No. of

Copies

ONSITE

1 Pacific Northwest National Laboratory (Authors will be notified electronically)

O.P. Bredt

K6-50

C.A. Burns

P7-25

J.G.H. Geeting

P7-28

C.E. Guzman-Leong

K5-22

B. D. Hanson

P7-27

J. L. Huckaby

H4-02

G.B. Josephson

K9-69

D. E. Kurath

K3-52

G.J. Sevigny

P7-27

Information Release (pdf)

Project File (1)

K3-52

2 Bechtel National Inc.

WTP R\&T Docs (1)

H4-02

S. Barnes

H4-02

Distr. 1 


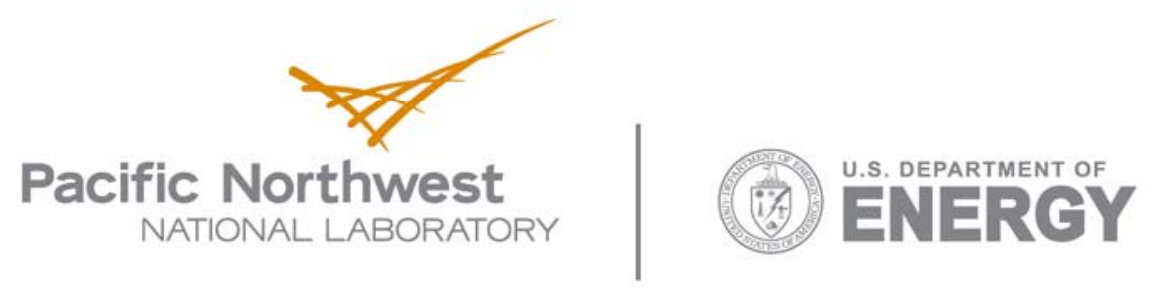

902 Battelle Boulevard

P.O. Box 999

Richland, WA 99352

1-888-375-PNNL (7665)

www.pnl.gov 